WSRC-TR-2003-00204, REV. 0

SRT-RPP-2003-00087, REV. 0

Key Words:

Precipitation

Filtration

Separation

Organic

Retention:

Permanent

Key WTP R\&T References:

Test Specification: 24590-WTP-TSP-RT-01-029, Rev 0

Test Plan: SRT-RPP-2002-00054, Rev 0

Test Scoping Statement(s): S-132

Test Exception: 24590-WTP-TEF-TR-03-027

\title{
FINAL REPORT: PILOT-SCALE CROSS-FLOW ULTRAFILTRATION TEST USING A HANFORD SITE TANK 241-AN-102 WASTE SIMULANT (U)
}

\author{
M. R. Duignan, 786-5A
}

MAY 6, 2003

Westinghouse Savannah River Company

Savannah River Site

Aiken, SC 29808

Prepared for the U.S. Department of Energy Under Contract Number DE-AC09-96SR18500

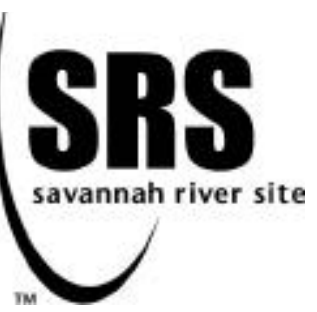


This page intentionally left blank 
This document was prepared in conjunction with work accomplished under Contract No. DE-AC09-96SR18500 with the U. S. Department of Energy.

\section{DISCLAIMER}

This report was prepared as an account of work sponsored by an agency of the United States Government. Neither the United States Government nor any agency thereof, nor any of their employees, makes any warranty, express or implied, or assumes any legal liability or responsibility for the accuracy, completeness, or usefulness of any information, apparatus, product or process disclosed, or represents that its use would not infringe privately owned rights. Reference herein to any specific commercial product, process or service by trade name, trademark, manufacturer, or otherwise does not necessarily constitute or imply its endorsement, recommendation, or favoring by the United States Government or any agency thereof. The views and opinions of authors expressed herein do not necessarily state or reflect those of the United States Government or any agency thereof.

This report has been reproduced directly from the best available copy.

Available for sale to the public, in paper, from: U.S. Department of Commerce, National Technical Information Service, 5285 Port Royal Road, Springfield, VA 22161, phone: (800) 553-6847, fax: (703) 605-6900

email: orders@ntis.fedworld.gov

online ordering: http://www.ntis.gov/help/index.asp

Available electronically at http://www.osti.gov/bridge

Available for a processing fee to U.S. Department of Energy and its contractors, in paper, from: U.S. Department of Energy, Office of Scientific and Technical Information, P.O. Box 62, Oak Ridge, TN 37831-0062,

phone: (865)576-8401,

fax: (865)576-5728

email: $\underline{\text { reports@ adonis.osti.gov }}$ 
This page intentionally left blank 


\section{ACKNOWLEDGMENTS}

The author would like to thank all that were involved in this task. Mike Armstrong's care and dedication in running this experiment allowed it to be successfully completed. Support from the other Engineering Development Laboratory \& other personnel: Vern Bush, Jimmy Mills, Jerry Corbett, Thaddeus Reown, and Mike Restivo, was instrumental in keeping the experiment on track and maintaining the test rig ready, from instrumental calibration to around-the-clock testing. A special thanks is in order to all those individuals just mentioned for the long hours they invested in the experiment, which at times disrupted their family life. The Lab supervisor, Susan Hatcher, is to be commended for keeping us safe, even when things seemed to be the most hectic. I would also like to thank Tim Steeper and Mike Williams for their assistance with the test and with providing the slurry simulant. The management of Dan Burns and Steve Wach made this task's successful completion a sure thing. Thanks is extended to Hector Guerrero, and Dan Burns who had the unenviable task of reviewing this report and to Linda-Lee DiVecchia who did a wonderful job of threading all the many pages into a usable form. Finally, I would like to thank my customer from the River Protection Project - Waste Treatment Plant Project, Paul Townson, for his support, which made this work possible. 


\section{TABLE OF CONTENTS}

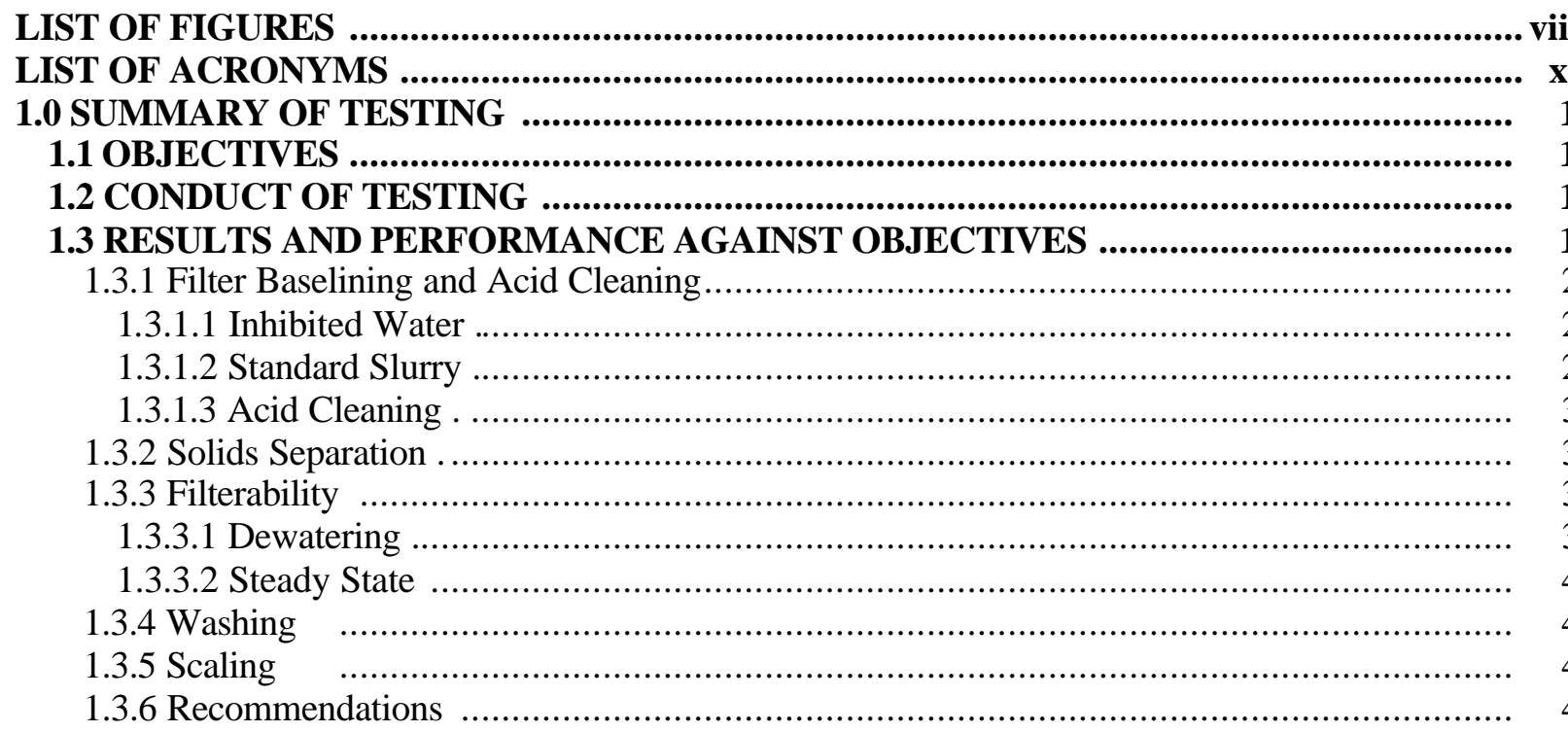

1.4 QUALITY REQUIREMENTS................................................................................... 4

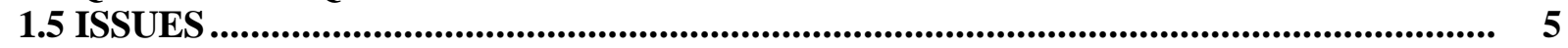

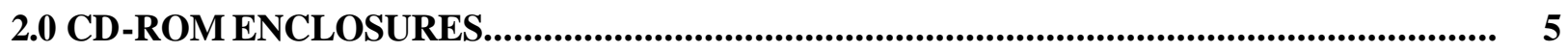

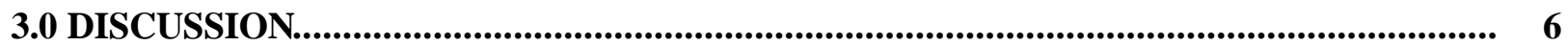

3.1 INTRODUCTION ................................................................................................................ 6

3.2 EXPERIMENTAL _........................................................................................................... 7

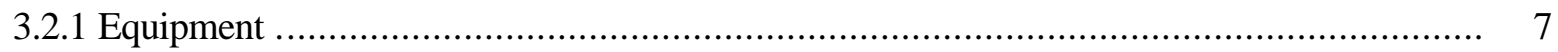

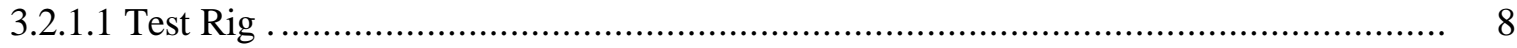

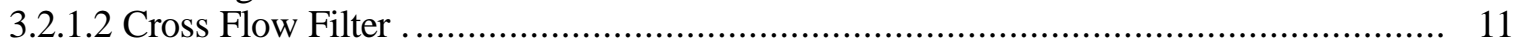

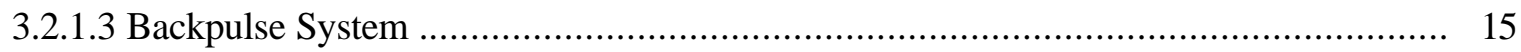

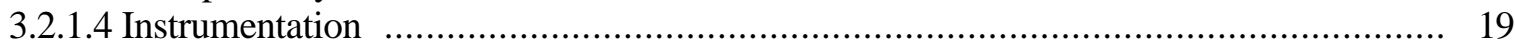

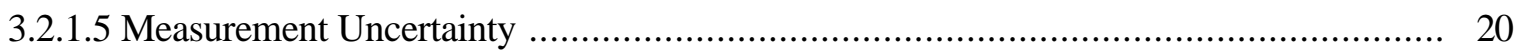

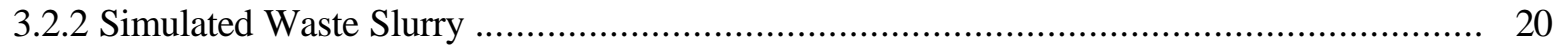

3.2.3 Test Procedure/Matrix ……………................................................................. 24

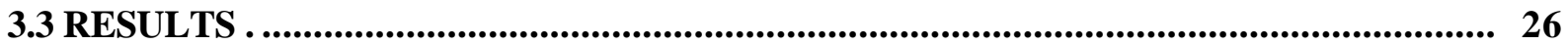

3.3.1 Insoluble Solids Separation ………............................................................. 26

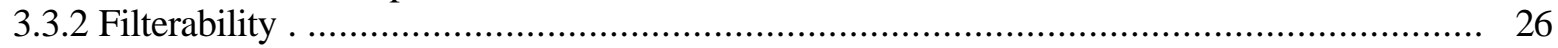

3.3.2.1 Baseline with Water ..................................................................................... 28

3.3.2.1.1 Water and Cross-flow Filtration ............................................................. 28

3.3.2.1.2 Overall Evaluation .......................................................................... 28

3.3.2.1.3 Individual Evaluations .................................................................... 30

3.3.2.1.3.1 New Filter and the Effect of Inhibited Water after Nitric Acid Cleaning ......... 31

3.3.2.1.3.2 Water Runs before and after Preconditioning with AN-107 Slurry Simulant 33

3.3.2.1.3.3 Water Runs before and after AN-102, Batch 3C Slurry Simulant ............... 34

3.3.2.1.3.4 Water Runs before and after AN-102, Batch 3B Slurry Simulant ............... 34

3.3.2.1.3.5 Water Runs before and after AN-102, Batch 3A and 4A Slurry Simulant .... 35

3.3.2.1.4 Relationship between Water and Slurry Filtrate Fluxes ...................................... 35

3.3.2.2 Baseline with Standard Slurry $\left(5 \mathrm{wt} \% \mathrm{SrCO}_{3}\right)$................................................. 36

3.3.2.3 Cleaning the 0.01-micron Mott Filter with 2 M Nitric Acid ..................................... 39

3.3.2.4 Dewatering of AN-102R2 Slurry Simulant ....................................................... 44

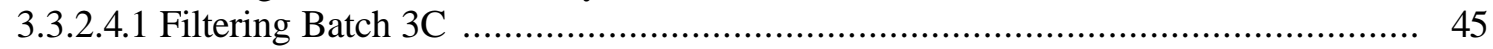




\section{TABLE OF CONTENTS (Continued)}

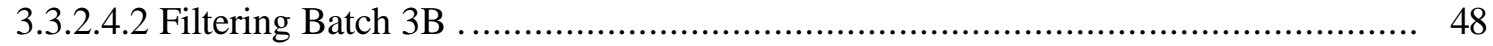

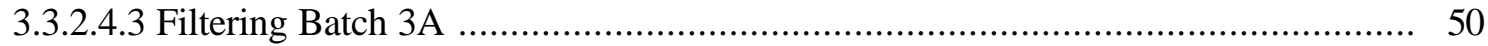

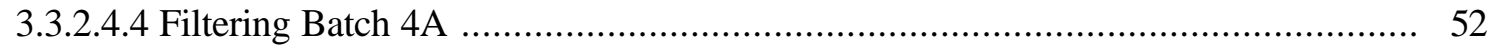

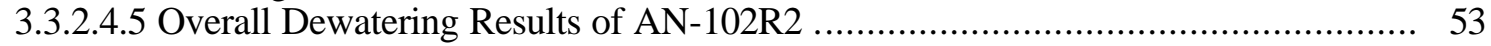

3.3.2.5 Steady State Filtering of AN-102R2 Slurry Simulant ...................................... 57

3.3.2.5.1 Typical Constant Insoluble Solids Concentration Steady-State Test Run ............. 57

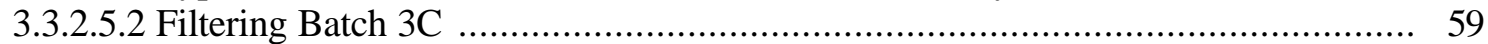

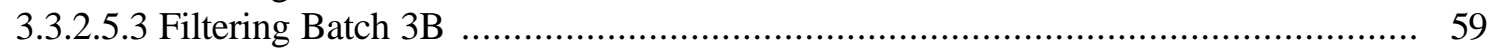

3.3.2.5.4 Filtering Batch 3A ............................................................................... 63

3.3.2.6 Washing and Concentration of Washed AN-102R2 Slurry Simulant ........................ 66

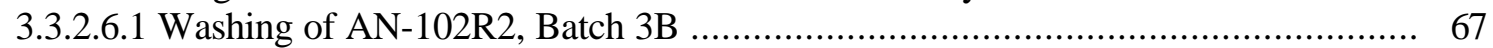

3.3.2.6.2 Concentration of Washed AN-102R2, Batch 3B ........................................... 69

3.3.3 Scaling: Differences between the PXU and Bench Top Cross-flow Test Facilities ............ 70

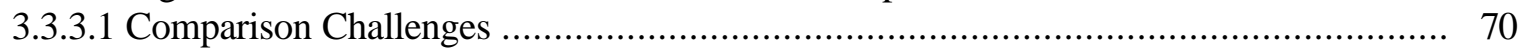

3.3.3.2 A First PXU-to-CUF Comparison with an Envelope C Simulant (AN-107) ................ 72

3.3.3.3 A Second PXU-to-CUF Comparison with an Envelope C Simulant (AN-102) ........... 72

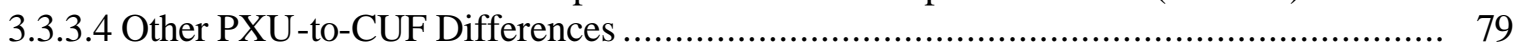

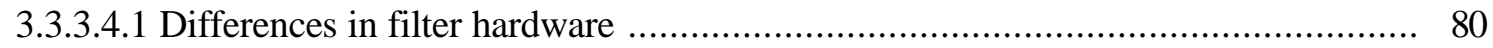

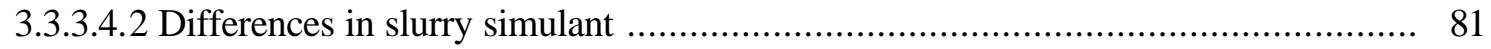

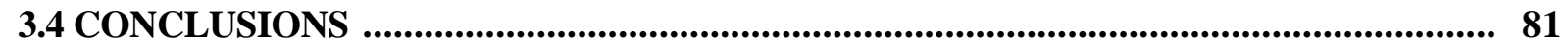

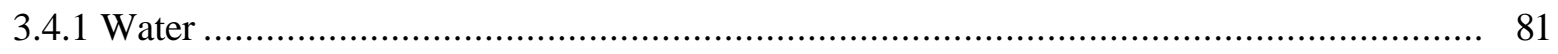

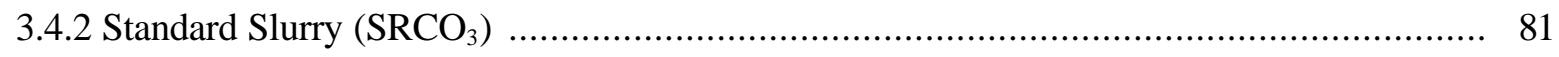

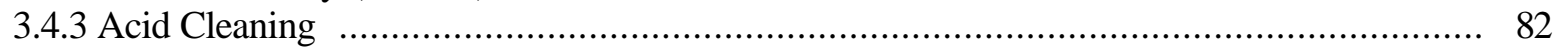

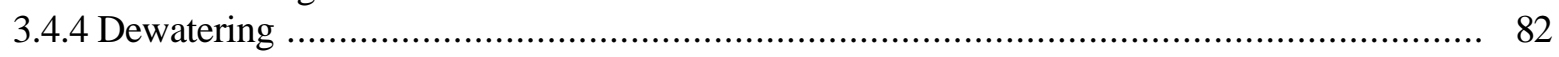

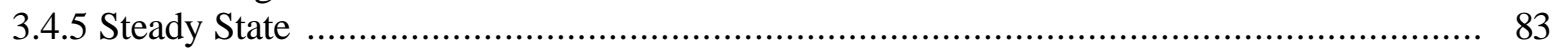

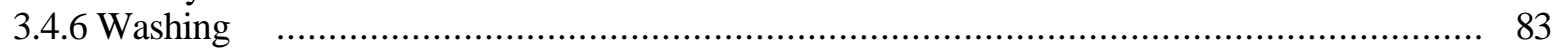

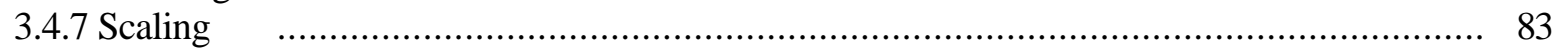

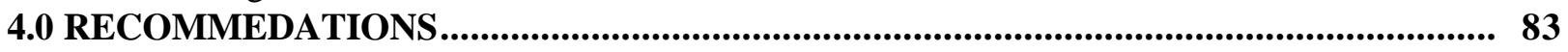

5.0 REFERENCES .......................................................................................................................... 84

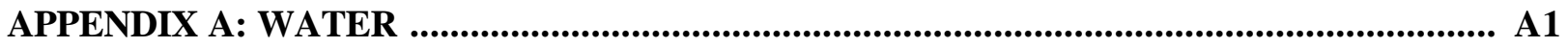

APPENDIX B: BASELINE SLURRY ................................................................................... B1

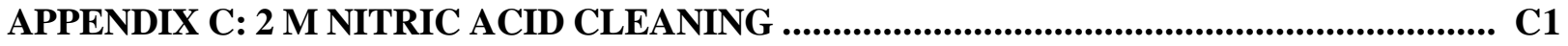

APPENDIX D: SLURRY DEWATERING, TEST RUN 1.16 - AN-102R2 ............................. D1

APPENDIX E: STEADY-STAE FILTERING, TEST RUNS 1.17 - 1.29 - AN-102R2 ............... E1

APPENDIX F: SLURRY WASH AND CONCENTRATION OF

WASHED SLURRY, AN-102R2, BATCH 3B ......................................... F1

APPENDIX G: ANALYTICAL, RHEOLOGICAL, AND PARTICAL-SIZE DATA ............... G1

APPENDIX H: INSTRUMENTATION AND MEASUREMENT UNCERTAINTY .................. H1 


\section{LIST OF FIGURES}

Figure 1. Partial drawing of the Pilot-scale Cross Flow Ultrafiltration Test Facility ............ 9

Figure 2. Schematic of the Pilot-scale Cross Flow Ultrafiltration Test Facility..................... $\quad 10$

Figure 3. 7-tube bundle of a nominal rated 0.1 micron filter, 1.2" i.d., 5/8" o.d., 90" long ... 12

Figure 4. Adjoined filter tube sections.............................................................................. 12

Figure 5. (a) Large-tube sheet, (b) tube-to-tube weldment, (c) tube supports,

(d) small-tube sheet

Figure 6. Magnified views (approximately 150x) of surfaces of two different

pore-size rated Mott filters .................................................................................. 13

Figure 7. Tube-sheet profiles (a) Downstream (b) Upstream .............................................. 13

Figure 8. Installed 90-inch tall cross-flow filter housing .................................................. 14

Figure 9. Planned RPP-WTP backpulse system ........................................................... 15

Figure 10. Procedure to operate backpulse system ......................................................... 15

Figure 11. Pilot test rig backpulse loop, see also Fig. 2 ................................................... 16

Figure 12. Pilot test rig backpulse operation with water ..................................................... 17

Figure 13. Pilot test rig backpulse duration (time to hold valve A open) ............................ 18

Figure 14. Pilot test rig backpulse operation with AN-102R2, Batch 3B............................. 18

Figure 15. Chemical make-up of the AN-102 simulant (AN-102R2) .................................. 21

Figure 16. Entrained solids added to the AN-102R2 simulant ........................................... 22

Figure 17. Nomenclature for the four AN-102R2 simulant batches .................................... 23

Figure 18. Test matrix used for AN-102R2 testing …………………………………...... 24

Figure 19. Water tests made before and after filtering several slurries ............................... $\quad 30$

Figure 20. Water tests made on the new filter and after filtering with the standard slurry $\quad \ldots \quad 31$

Figure 21. Water tests with and without caustic ................................................................. 32

Figure 22. Water tests with and without caustic. Tests done between Batches 3 and 4 ....... $\quad 32$

Figure 23. Water tests after cleaning the filter ................................................................. 33

Figure 24. Water tests before test slurry AN-102R2, Batch 3C ……………………........ 34

Figure 25. Water tests before test slurry AN-102R2, Batch 3B or after Batch 3C ……....... 35

Figure 26. New and Used Filter Standard Slurry Filter Tests ............................................ 36

Figure 27. Filtering with Time After a Backpulse for the New Mott Filter ......................... 37

Figure 28. Filtering with Time After a Backpulse for the Used Mott Filter ......................... 38

Figure 29. Used-Filter Standard Slurry Filter Tests ............................................................. 39

Figure 30. $2 \mathrm{M}$ nitric acid cleaning after AN-107 \& AN-102R2, Batch 3C ………............. $\quad 40$

Figure 31. $2 \mathrm{M}$ nitric acid cleaning after AN-102R2, Batch 3B ....................................... 41

Figure 32. $2 \mathrm{M}$ nitric acid cleaning after AN-102R2, Batches 3A ....................................... 41

Figure 33. $2 \mathrm{M}$ nitric acid cleaning after AN-102R2, Batches 3A \& 4 ................................ 42

Figure 34. $2 \mathrm{M}$ nitric acid cleaning after AN-102R2, before \& after 4A ……………......... 43

Figure 35. $2 \mathrm{M}$ nitric acid cleaning of the AN-107 and AN-102R2 slurry tests ................... 43

Figure 36. Comparison among the four AN-102R2 dewatering tests ................................. 45

Figure 37. Batch 3C: Dewatering filter flux of AN-102R2 precipitated with the

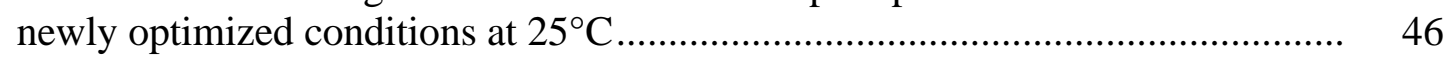

Figure 38. Pilot-scale X-flow UF Dewatering of AN-102R2, Batch 3C ............................. 47

Figure 39. Batch 3B: Dewatering filter flux of AN-102R2 precipitated with the baseline conditions at $50^{\circ} \mathrm{C}$ 


\section{LIST OF FIGURES \\ (Continued)}

Figure 40. Pilot-scale X-flow UF Dewatering of AN-102R2, Batch 3B

Figure 41. Batch 3A: Dewatering filter flux of AN-102R2 precipitated with the baseline conditions at $20^{\circ} \mathrm{C}$

Figure 42. Pilot-scale X-flow UF Dewatering of AN-102R2, Batch 3A

Figure 43. Batch 4A: Dewatering filter flux of AN-102R2 precipitated with the baseline conditions at $25^{\circ} \mathrm{C}$

Figure 44. Pilot-scale X-flow UF Dewatering of AN-102R2, Batch 4A

Figure 45. Pilot-scale X-flow UF Dewatering of AN-102R2, Batches $3 \& 4$.................... 54

Figure 46. Batch 3A filtrate flux with selected rheological properties ............................... 55

Figure 47. Batch 3\&4 Slurry Consistency ............................................................. 56

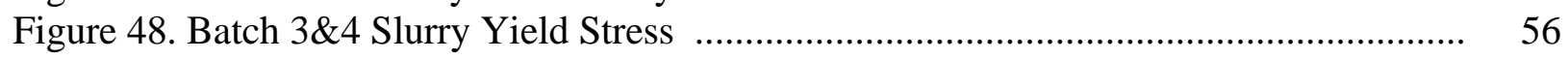

Figure 49. Batch 3\&4 Slurry Density ....................................................................... 56

Figure 50. Batch 3\&4 Mean Particle Size (by Number Distribution) .............................. 56

Figure 51. Batch 3\&4 Mean Particle Size (by Volume Distribution) ............................... 56

Figure 52. Batch 3B: Test Run 1.17 ....................................................................... 58

Figure 53. Batch 3B: Filtrate flux results averaged over the first hour of testing, including the five 2-hour test, i.e., 1.17, 1.18, 1.19, 1.24, and 1.29

Figure 54. Batch 3B: Filtrate flux results averaged over the first hour of testing for the five 2-hour test runs, i.e., 1.17, 1.18, 1.19, 1.24, and 1.29

Figure 55. Batch 3B: This figure repeats the data shown in Fig. 53 with depth fouling effect removed

Figure 56. Batch 3B: All the steady-state data that were adjusted for depth fouling effect removed (Fig. 55) as a function of transmembrane pressure, TMP

Figure 57. Batch 3B: All the steady-state data that were adjusted for depth fouling effect removed (Fig. 55) as a function of slurry axial velocity, V

Figure 58. Batch 3A: Filtrate flux results averaged over the one hour of each test

Figure 59. Batch 3A: Two filtrate flux data points shown are the average over the one hour test runs, i.e., 1.17 and 1.29

Figure 60. Batch 3A: This figure repeats of the data shown in Fig. 58 with depth fouling effect removed

Figure 61. Batch 3A: All the steady-state data that were adjusted for depth fouling effect removed (Fig. 60) as a function of transmembrane pressure, TMP

Figure 62. Batch 3A: All the steady-state data that were adjusted for depth fouling effect removed (Fig. 60) as a function of slurry axial velocity, V....

Figure 63. Washing of AN-102R2, Batch 3B after test run 1.29 and before concentration test run 1.30

Figure 64. Starting and ending slurry properties for the washing test run

Figure 65 . Reduction of $\mathrm{Na}^{+}$by washing with 21 mini-batches of inhibited water to AN-102R2, Batch 3B

Figure 66. Dewatering of washed slurry (AN-102R2, Batch 3B): Test Run 1.30

Figure 67. Comparison of Full-scale to scaled test facilities 


\section{LIST OF FIGURES \\ (Continued)}

Figure 68. PXU to CUF filtrate flux comparison using an AN-102R2 simulant ............... 73

Figure 69. PXU to CUF comparison AN-102R2, Batch 3B simulant ............................... 75

Figure 70. Operation of a multi-tube cross flow filter like the PXU ................................ 76

Figure 71. Parameters used in a wall shear CFD model .............................................. 77

Figure 72. Wall shear comparison for two different tube IDs at $12 \mathrm{ft} / \mathrm{s}$ and a viscosity of $4 \mathrm{cP}$

Figure 73. Wall shear comparison for two different tube IDs at $12 \mathrm{ft} / \mathrm{s}$ and a viscosity of $20 \mathrm{cP}$

Figure 74. Slurry flow through a cross-flow ultrafiltration tube

Figure 75. Dewatering results 3,82

This list only includes those figures in the body of this report, not those in the appendices. 


\section{LIST OF ACRONYMS}

ADS Analytical Development Section (part of WSRC/SRTC)

AN-102 Department of Energy Hanford Site Tank 241-AN-102

AN-102R2 Radioactive cold slurry simulant of AN-102 that was revised twice (R2) to be a better match to the actual radioactive waste. It was the principal simulant used for this test.

AN-107 Department of Energy Hanford Site Tank 241-AN-107. A simulant of this waste was used to precondition the cross-flow filter before testing began with AN-102R2

BC Baseline Conditions (see Fig. 17)

BNI Bechtel National, Inc.

CFD Computational Fluid Dynamics

$\mathrm{cP} \quad$ Centipoise

cSt Centistoke

CUF Cells Ultrafiltration facility (bench-top cross-flow filter used with both radioactively cold and hot wastes)

${ }^{\circ} \mathrm{C} \quad$ Degree Centigrade (or Celcius)

$\rho \quad$ density, $\mathrm{g} / \mathrm{ml}$

D Diameter

DF Decontamination Factor: (Slurry Molarity / Filtrate Molarity)

DIF Deionized and Filtered (0.1 micron) Water

DOE United States Department of Energy

dP Differential Pressure

$\mathrm{ft} \quad$ Foot

$\gamma \quad$ Shear Rate, $\mathrm{sec}^{-1}$ (Rheology Parameter)

i.d. or ID Inside Diameter

IS insoluble solids

in. inch

hr Time: Hour

HLW High Level (Activity) Waste

1 or L Liter

LAW Low (Level) Activity Waste

$\mu \quad$ Dynamic Viscosity, $\mathrm{cP}$, (Rheology Parameter)

$\mu_{0} \quad$ Consistency or Plastic Viscosity, cP, (Rheology Parameter)

NOC Newly Optimized Conditions (see Fig. 17)

m Meter

M Molar

$\min \quad$ Time: Minute

$\mathrm{ml}$ or $\mathrm{mL} \quad$ Milliliter

mm Millimeter

nominal The word "nominal" for a filter rating is a vague term because its meaning is manufacturer dependent. Further, a "nominal" rating does not give an exact size to a filter medium, but rather an approximation to the expected performance of a filter. In the case of Mott, a nominal rated $0.1-\mu \mathrm{m}$ filter means that approximately $95 \%$ of particles greater than $0.1 \mu \mathrm{m}$ will not pass the filter.

NTU Nephelometric Turbidity Unit 


\section{LIST OF ACRONYMS}

(Continued)

$\begin{array}{ll}\text { Pa } & \text { Pascal } \\ \text { PJM } & \text { Pulse Jet Mixer } \\ \text { PSD } & \text { Particle Size Distribution } \\ \text { psi } & \text { Pounds Per Square Inch } \\ \text { psig } & \text { Pounds Force Per Square Inch Gauge } \\ \text { psid } & \text { Pounds Force Per Square Inch Difference } \\ \text { PXU } & \text { Pilot-scale Cross- flow Ultrafiltration facility } \\ \text { QA } & \text { Quality Assurance } \\ \text { Re } & \text { Reynolds number, Re }=\rho \text { VD/ } \mu \text {, non-dimensional number } \\ \text { RPP } & \text { River Protection Project (at the DOE Hanford Site) } \\ \text { o.d. or OD } & \text { Outside Diameter } \\ \text { s or sec } & \text { Time: Second } \\ \text { SRTC } & \text { Savannah River Technology Center (part of WSRC) } \\ \text { Sr/TRU } & \text { Strontium/Transuranic } \\ \text { Std Dev } & \text { Standard Deviation } \\ \tau & \text { Shear Stress, dynes/cm }{ }^{2},(\text { Rheology Parameter) } \\ \tau & \text { Yield Stress (Shear Stress at Shear Rate }=0 \text { ), dynes/ cm }{ }^{2},(\text { Rheology Parameter) } \\ \text { TS } & \text { Total Solids } \\ \text { EDL } & \text { Engineering Development Laboratory (part of WSRC/SRTC) } \\ \text { TMP } & \text { Transmembrane Pressure (the average pressure drop across the thickness of the } \\ & \text { filter medium - perpendicular to the slurry flow.) } \\ \text { TRU } & \text { Trans uranic } \\ \text { TTR } & \text { Technical Task Request } \\ \text { UF } & \text { Ultrafiltration } \\ \text { ug } & \text { microgram } \\ \text { V } & \text { Velocity of the slurry flow along the length of the filter tubes } \\ \text { WGI } & \text { Washington Group International } \\ \text { WTP } & \text { Waste \& Immobilization Treatment Plant } \\ \text { WSRC } & \text { Westinghouse Savannah River Company } \\ & \end{array}$




\subsection{SUMMARY OF TESTING}

\subsection{OBJECTIVES}

This report discusses the results of cross-flow filter operation in a pilot-scale experimental facility that was designed, built, and run by the Engineering Development Laboratory of the Westinghouse Savannah River Company Savannah River Technology Center. This filter technology was evaluated for its inclusion in the pretreatment section of the nuclear waste stabilization plant being designed by Bechtel National, Inc. The waste treatment plant will be built at the U.S. Department of Energy's Hanford Site as part of the River Protection Project.

The test objectives, which are listed in the task plan (Duignan, 2002a), were to demonstrate using a simulated waste of AN-102R2:

- Separation of insoluble solids

- Filterability during simulant dewatering after precipitation

- Concentration of the simulant to above $15 \mathrm{wt} \%$

- Filterability of the concentrated simulant during a steady-state solids loading

- Washing the slurry

\subsection{CONDUCT OF TESTING}

The general test matrix was as follows:

- Baseline the filter with water

- Baseline the filter with a standard slurry

- Dewater simulant from 1 wt $\%$ to $>15 \mathrm{wt} \%$ insoluble solids

- Filter simulant at the highest concentration of solids at steady state solids loading

- Wash slurry

- Acid Clean Filter

Four batches of AN-102R2 simulant, precipitated under different conditions, were tested, but only one, Batch 3B, completed all the steps above due to its good filterability. This reports discusses in detail the problems and successes with each of those batches.

\subsection{RESULTS AND PERFORMANCE AGAINST OBJECTIVES}

The filter element under study was manufactured by the Mott Metallurgical Corporation in Connecticut. The filter unit was made of seven 316 stainless steel sintered metal tubes. Each tube was a nominal-rated 0.1 -micron filter with an inside diameter of $0.5 \mathrm{inch}$, an outside diameter of $0.625 \mathrm{inch}$, and a porous length of 90 inches. These dimensions give an active filter surface area of $6.7 \mathrm{ft}^{2}$. At the time of this task these aforementioned dimensions were given as prototypic; therefore, each filter tube was considered full size to the filter, which 
would be built for the plant. Only the number of tubes was expected to change. [Current plans call for 241 tubes ( $231 \mathrm{ft}^{2}$ of filter area) in each of the cross-flow filter bundles of which there will be 3 bundles in series for each of the two filtration flow loops.]

The filter was tested with a simulated nuclear waste of Tank 241-AN-102, which is referred to as an Envelope C waste. The recipe for the simulant was developed by the SRTC Waste Treatment Technology Department and the simulant was procured and prepared by EDL personnel specifically for this task. The supernatant portion of the simulant contained numerous inorganic salts, soluble organics, had a $\mathrm{pH} \approx 12$, and a sodium molarity of approximately $6.0 \mathrm{M}$. Insoluble solids were added to the supernatant to simulate entrained solids in the real waste. The particle size distribution was designed to be bimodal; one group ranged from 1 to 2 microns and the other ranged from 5 to 10 microns. Finally, to this slurry three precipitating agents were added: Sodium Hydroxide, Strontium Nitrate, and Sodium Permanganate, which raised the $\mathrm{pH}$ to approximately 14 . After all the additions, the insoluble solids loading started at 0.8 to $1.6 \mathrm{wt} \%$ and after dewatering it was eventually raised to about $25 \mathrm{wt} \%$. The total solids ranged from as low as $28 \mathrm{wt} \%$ for post-washed slurry to as high as $49 \mathrm{wt} \%$ for the pre-washed slurry. At $25^{\circ} \mathrm{C}$ and a solids loading of 1.0 $\mathrm{wt} \%$, this slurry had a density of approximately $1.29 \mathrm{~g} / \mathrm{mL}$, a viscosity of $4 \mathrm{cP}$, and Newtonian rheological characteristics. However, as the solids loading increased, a yield stress was present. At the highest loading, the slurry rheology showed time dependent characteristics of a thixotropic fluid, e.g., certain paints, inks, and foods like ketchup. The flow conditions for the test varied as follows: Axial slurry velocities ranged from $7 \mathrm{ft} / \mathrm{s}$ to 15 $\mathrm{ft} / \mathrm{s}(2.1 \mathrm{~m} / \mathrm{s}$ to $4.6 \mathrm{~m} / \mathrm{s})$ and transmembrane pressures ranged from $20 \mathrm{psid}$ to $60 \mathrm{psid}$ (138 $\mathrm{kPa}$ to $414 \mathrm{kPa}$ ) at a temperature of $25^{\circ} \mathrm{C}$.

\subsubsection{Filter Baselining and Acid Cleaning}

Before each test the filter was tested with inhibited $(0.01 \mathrm{M} \mathrm{NaOH})$ water and a standard slurry $\left(5 \mathrm{wt} \% \mathrm{SrCO}_{3}\right)$ to baseline its operation. After each test the filter was acid cleaned with $2 \mathrm{M} \mathrm{HNO}_{3}$ and retested with water and the standard slurry. The summary results are:

\subsubsection{Inhibited Water}

Inhibited water tests before and after each slurry test indicated a return to "clean" filter fluxes.

\subsubsection{Standard Slurry}

The use of a standard slurry to show a filter's return to cleanliness was confounded by the level of cleanliness of not just the filter but of the entire filter system. Unless the entire filtration loop was clean, then determining the cleanliness of just the in-line filter may not be possible. 


\subsubsection{Acid Cleaning}

The method used, two 90-minutes circulations of $2 \mathrm{M}$ nitric acid, cleaned most of the solids away from the filter but there were always some remaining solids. The complex wastes may need more targeted cleaning to remove all waste remnants

\subsubsection{Solids Separation}

With respect to insoluble solids separation the results were excellent. Turbidity for all filtrate samples measured considerable less than $1 \mathrm{NTU}$ and generally ranged from 0.1 to $0.3 \mathrm{NTU}$.

\subsubsection{Filterability}

With respect to filterability, the results ranged from unacceptable to good as compared to the target mean filtrate flux of $0.020 \mathrm{gpm} / \mathrm{ft}^{2}$ at $15 \mathrm{wt} \%$ insoluble solids and as explained below.

\subsubsection{Dewatering}

Under the flow conditions of a slurry velocity of $12.0 \mathrm{ft} / \mathrm{s}$ and a transmembrane pressure of $40 \mathrm{psid}(3.7 \mathrm{~m} / \mathrm{s}$ and $275 \mathrm{kPa})$, the observed filtrate flux during dewatering ranged from one unacceptable test (Batch 3C) to three acceptable tests (Batches 3A, 3B, and 4A, with 3B giving the best results) when compared to the target mean filtrate flux of $0.020 \mathrm{gpm} / \mathrm{ft}^{2}$. The results are summarized in Fig. 75, which is reproduced here for convenience. There can be many reasons why the filtration results varied among the batches, some of which are discussed herein, but the large st difference was between the poor filterability of Batch 3C, which had the lowest concentration of free hydroxide, and the good filterability of Batch 3B, which had a higher free hydroxide concentration and was precipitated at a higher temperature, i.e., $50^{\circ} \mathrm{C}$. The filterability of remaining two batches fell between the extremes. They both had the higher level of free hydroxide, but Batch 3A was precipitated at the lower temperature of $25^{\circ} \mathrm{C}$ and Batch $4 \mathrm{~A}$ used a pulse jet mixer during precipitation instead of an impeller agitator.

\begin{tabular}{|c|c|c|c|c|c|c|c|c|c|c|}
\hline $\begin{array}{l}\text { Batch } \\
\text { Number }\end{array}$ & $\begin{array}{l}\text { Precipitation } \\
\text { Conditions* }\end{array}$ & \begin{tabular}{|c|} 
Precipitation \\
Mixing
\end{tabular} & $\begin{array}{c}\text { Precipitation } \\
\text { Temp, }{ }^{\circ} \mathrm{C} \\
\end{array}$ & $\begin{array}{l}\text { Final Conc } \\
\text { of Free } \mathrm{OH}^{-}\end{array}$ & $\begin{array}{c}\text { Final Conc } \\
\text { of } \mathrm{SrNO}_{3}\end{array}$ & $\begin{array}{l}\text { Final Conc } \\
\text { of } \mathrm{NaMnO}_{4}\end{array}$ & at $2 \mathrm{wt} \%$ & $\begin{array}{l}\text { Filter Flux } \\
\text { at } 15 w t \%\end{array}$ & $\begin{array}{l}\left(\mathrm{gpm} / \mathrm{ft}^{2}\right) \\
\text { Avg to } 15 \text { wt\% }\end{array}$ & Location \\
\hline $3 C$ & $\mathrm{NOC}=$ & well & 25 & $0.3 \mathrm{M}$ & $0.03 \mathrm{M}$ & $0.03 \mathrm{M}$ & 0.019 & $0.005^{\star *}$ & $0.010^{\star \star \star}$ & (Fig. 38) \\
\hline $3 B$ & $\mathrm{BC}=$ & well & 50 & $1.0 \mathrm{M}$ & $0.075 \mathrm{M}$ & $0.050 \mathrm{M}$ & 0.066 & 0.024 & 0.049 & (Fig. 40) \\
\hline $3 A$ & $\mathrm{BC} @ 25^{\circ} \mathrm{C}=$ & well & 25 & $1.0 \mathrm{M}$ & $0.075 \mathrm{M}$ & $0.050 \mathrm{M}$ & 0.052 & 0.019 & 0.020 & (Fia. 42) \\
\hline $4 \mathrm{~A}$ & $\mathrm{BC} \mathrm{w} / \mathrm{PJM}=$ & PJM & $50>25$ & $1.0 \mathrm{M}$ & $0.075 \mathrm{M}$ & $0.050 \mathrm{M}$ & 0.021 & 0.014 & 0.020 & (Fig. 44) \\
\hline
\end{tabular}

Figure 75. Dewatering results 


\subsubsection{Steady State}

Steady state tests at high concentrations of insoluble solids (>20 wt $\%)$ indicated that slurry axial velocity is an important parameter in filtrate flux, while the transmembrane pressure is less so. Flow parameters of $12 \mathrm{ft} / \mathrm{s}$ and 40 psid are reasonable. With hourly backpulsing the filter depth fouling decreased the filtrate flux by approximately $60 \%$ after 24 hours of continuous operation.

\subsubsection{Washing}

A test to wash slurry with an equal volume of water showed good removal of sodium; approximately $65 \%$.

\subsubsection{Scaling}

Comparisons made with the small amount of existing data from similar small and pilot-scale tests show that relating small to plant-scale operation may not be possible.

\subsubsection{Recommendations}

It is recommended that:

1. Axial slurry velocity be greater than $11 \mathrm{ft} / \mathrm{s}$ and the transmembrane pressure more than 30 psid. Higher velocities will filter slurries faster, but higher transmembrane pressures will not increase filter fluxes significantly.

2. Baseline conditions (BC in Fig. 75) be used when precipitating Envelope C-type wastes like AN-102 to obtain the best filterability.

3. Backpulsing be minimized because increasing backpulsing increases depth fouling of the filter and thus reduces time between acid cleanings.

4. A more effective cleaning method be determined than the use of $2 \mathrm{M}$ nitric acid, alone.

\subsection{QUALITY REQUIREMENTS}

This work was conducted in accordance with the RPP-WTP Quality Assurance requirements specified for work conducted by SRTC as identified in DOE IWO MOSRLE60. Researchers followed the WSRC QA program, which has been approved by WTP, and the WSRC QA Management Plan (WSRC-RP-92-225). The program applied the appropriate QA requirements for this task, as indicated by the QA Plan Checklist in section IX of the Task Technical and Quality Assurance Plan (Duignan, 2002a).

Analytical sample labeling and tracking complied with established procedures (WSRC Manual L1, Procedure 7.15). The SRTC Analytical Development Section (ADS) conducted all analyses using the routine level QA program. Calibrated measuring and test equipment 
were utilized for all flow rate, pressure, and temperature measurements on the Pilot-scale Cross-flow Ultrafiltration Facility.

The Task Technical \& QA Plan provided the quality requirements for this work (Duignan, 2002a). NQA-1 1989, part 1, Basic and Supplementary Requirements and NQA-2a 1990, Part 2.7 were applied as appropriate.

\subsection{ISSUES}

Originally, this task was planned to filter only two slurry simulant batches, i.e., Batches ${ }^{\dagger} 3$ and 4. Those batches only differed by how well they would be mixed during the precipitation process. Batch 3 was to be well mixed with a mechanical agitator and Batch 4 was to be mixed with pneumatically driven pulse jet mixer (PJM). Previous work (Duignan, 2000b) with Envelope C-type simulants using well mixed baseline precipitation conditions has already been shown to attain an acceptable level of decontamination and filterability. Since RPP-WTP has been designed to use PJM to agitate the precipitating wastes; therefore, it was important to show its effects on decontamination and filterability. Unfortunately, the Batch 3 test demonstrated very poor filterability, which necessitated changes to the precipitation process and thus increased the number of tests to four batches ${ }^{\ddagger}$, i.e., $3 \mathrm{C}, 3 \mathrm{~B}, 3 \mathrm{~A}$, and 4A.

\subsection{CD-ROM ENCLOSURES}

The report is contained on a CD-ROM in the following file format:

Microsoft Word, Version 97

Adobe Acrobat, Version 5.0

\footnotetext{
${ }^{\dagger}$ Batches 1 and 2 were strictly related to the precipitation task (Steeper and Williams, 2003) and were not part of this task.

$\$$ The batch numbering nomenclature is arbitrary and the sequence shown above is the chronological order in which the batches were tested. Batch 4 is referred to $4 \mathrm{~A}$ because a $4 \mathrm{~B}$ was planned in the event Batch $4 \mathrm{~A}$ indicated that more testing was needed. Batch $4 \mathrm{~B}$ was not required.
} 


\subsection{DISCUSSION}

\subsection{INTRODUCTION}

Bechtel National, Inc. (BNI) has been contracted by the Department of Energy (DOE) to design a Waste Treatment and Immobilization Plant (WTP) to stabilize liquid radioactive waste that is stored at the Hanford Site as part of the River Protection Project (RPP). Because of its experience with radioactive waste stabilization, the Savannah River Technology Center (SRTC) of the Westinghouse Savannah River Company (WSRC) is working with BNI and Washington Group International (WGI), to help design and test certain parts of the waste treatment facility. One part of the process is the separation of radioactive solids from the liquid wastes by cross-flow ultrafiltration. This task tested a cross-flow filter, prototypic in porosity, length and diameter, with a simulated radioactive waste, made to prototypically represent the chemical and physical characteristics of a Hanford waste in tank 241-AN-102 (AN-102) and precipitated under prototypic conditions.

This technical baseline research and development work was initiated by a Technical Task Request (TTR) (Duignan, 2002a) that was issued in April of 2002. This TTR is a result of a WGI test specification (Townson, 2002a) to test a simulated radioactive waste in an existing pilot scale cross-flow filtration system in the Engineering Development Laboratory (EDL) of SRTC. With the initial documentation in place (Blunt, 2002; Duignan, 2002a,b,c,d; Edmunds, 2002) the task began by first preconditioning the filter. Since the first test would be using a brand new filter it was subjected to the flow of an archived simulant (AN-107) in order to attain a "used" filter state, which would be more prototypic of actual plant daily use. That is, a new filter could give falsely high filtrate flux rates, which would not be conservative. The preconditioned filter was then used to filter the AN-102R2 simulant. This waste simulant, along with other complexant containing wastes, like the preconditioning simulant, is referred to as Envelope C (see Eibling and Nash (2001) for a description of the entire range of radioactive wastes and their simulants). How well this simulant matched the actual waste is beyond the scope of this task and not addressed; it was used as given input. However, details can be found in Steeper and Williams (2003), which is a report that deals with the preparation and precipitation of $\mathrm{AN}-102 \mathrm{R} 2$. Finally, to this simulant several compounds were added to simulate the step of precipitating Strontium and Transuranic constituents. After precipitation, the simulant was ready for filtering, which began the test.

The simulant of AN-102R2 was the second in a series of two Envelope C-type wastes that have been tested. The first (AN-107) has been previously tested by Duignan (2000b) and these two tests followed an Envelope A (AN-105) simulant test (Duignan, 2000a). This report deals solely with the AN-102R2 simulant. 
The chosen filter was manufactured by the Mott Metallurgical Corporation to meet RPP-WTP specifications (Townson, 2002a), as follows:

7 filter tubes with each having an inside diameter of 0.5 -inch

90-inch porous length for each filter tube and made of stainless steel

Nominal rated 0.1 micron filter element (the List of Acronyms explains 'nominal')

and the pilot test rig was designed to have the following:

Maximum axial velocity through filter tubes of $4.6 \mathrm{~m} / \mathrm{s}(15 \mathrm{ft} / \mathrm{s})$

Maximum transmembrane pressure (TMP) of $60 \mathrm{psid}$

Maximum axial velocity to be achievable at the maximum TMP

Instrumentation to monitor the axial velocity, the filtrate flow rate, the

TMP, and the slurry temperature

All materials to be compatible with the high-caustic simulants and the $2 \mathrm{M}$ nitric acid cleaning solution

All specifications were met or exceeded.

A summary of EDL task activities is as follows:

Draft WGI Test Specification Received - May 8, 2001

Order placed (P.O. AC26481A) for filter from Mott Incorporated - May 17, 2001

Arrival of the filter from Mott Incorporated - August 15, 2001

Approved WGI Test Specification Received - January 16, 2002

WSRC Task Technical \& Quality Assurance Plan Approved - April 9, 2002

Principal shakedown activities began - October 1, 2002

Test Procedure Approved - September 5, 2002 (Revision 0)

Activities for preconditioning filter with AN-107 - September 9-29, 2002

Activities for AN-102R2, Batch 3C test - September 30 - October 17, 2002

Activities for AN-102R2, Batch 3B test - October 21 - November 5, 2002

Activities for AN-102R2, Batch 3A test - November 6 - November 26, 2002

Activities for AN-102R2, Batch 4A test - February 12-18 \& March 12-26, 2003

Final test activities (post calibrations) ended - April 15, 2003

Draft final report completed and sent for review to WGI - May 14, 2003

\subsection{EXPERIMENTAL}

\subsubsection{Equipment}

The equipment assembled for this task was done to conform to the Task Plan (Duignan, 2002a). To facilitate understanding of the experimental equipment an explanation of the salient features follows. 


\subsubsection{Test Rig}

Figure 1 is a partial drawing (Restivo, 2003) of the as-built test rig and Fig. 2 is a simplified schematic of the test rig. The facility stands approximately 25 -feet tall and is serviced by a two-level mezzanine. The test rig is taller than the 90-inch tall filter element because it originally was used to test a 10 -foot tall filter (Steimke, 1994). Several modifications were made in order to install the current cross-flow filter. The entire test rig was made of 300 series stainless steel with the majority being of 304 stainless steel.

The test rig is made up of three basic flow loops:

1. Slurry loop, which contains the filter and its housing and serves as the primary flow path for circulating slurries. This loop has an internal volume of approximately 26 liters, excluding the reservoir tank. It is made of primarily* 1.5 -inch sch 40 pipe, which has an inside diameter of 1.610 inches.

[*Some sch10 pipe was used, which has an inside diameter of 1.682 inches.]

2. Filtrate loop, which begins at the filter housing, allows the separated filtrate liquid to flow through the backpulse pulse pot before circulating back to the slurry reservoir to close the circuit. This loop has an internal volume of approximately 15 liters. It is made of primarily* 0.375 -inch tubing.

[*The pulse pot is made from 6 inch sch 80 pipe. The backpulse will be described in more detail after this section.]

3. Cleaning loop was not used for this test but is mentioned to point out valves that exist, but were not used, i.e., V8, V10, V12, and V19.

Two other flow circuits that are subsections of the other loops are the recirculation and the backpulse loops:

1. The recirculation loop is part of the slurry loop (by using valve V6) and is used to better control the slurry flow. The recirculation loop helps to increase mixing and to maintain a well mixed slurry.

2. The backpulse loop is part of the filtrate loop and stands ready to reverse the flow of filtrate. A pulse forces filtrate back through the seven filter elements in order to knock off built-up slurry cake on the inside diameter of the porous tubes. [The loop underwent significant changes to make it more prototypic to planned WTP operation; therefore, a more detailed explanation of the modified backpulse loop follows this section.] 
WSRC-TR-2003-00204, REV. 0

SRT-RPP-2003-00087, REV. 0

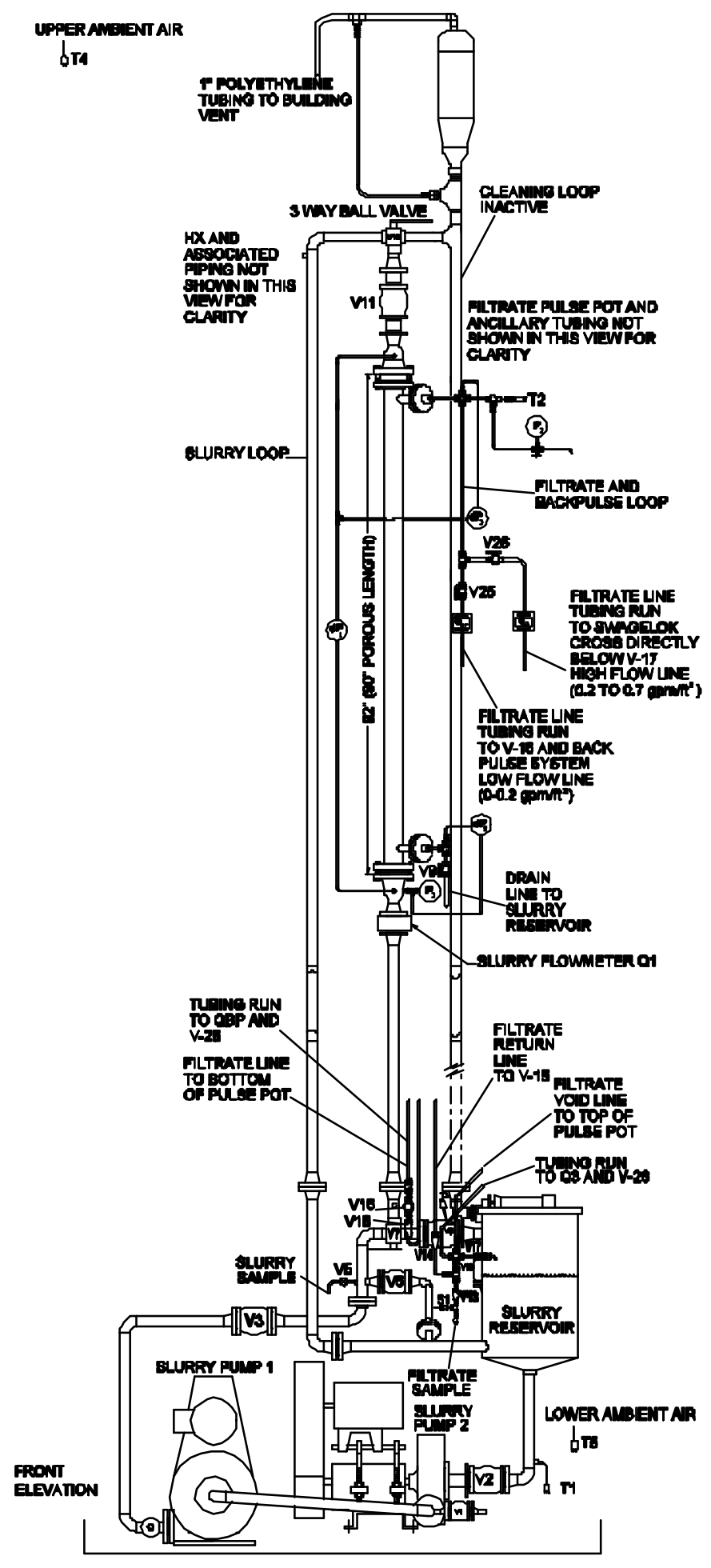

Figure 1. Partial drawing of the Pilot-scale Cross Flow Ultrafiltration Test Facility 
WSRC-TR-2003-00204, REV. 0

SRT-RPP-2003-00087, REV. 0

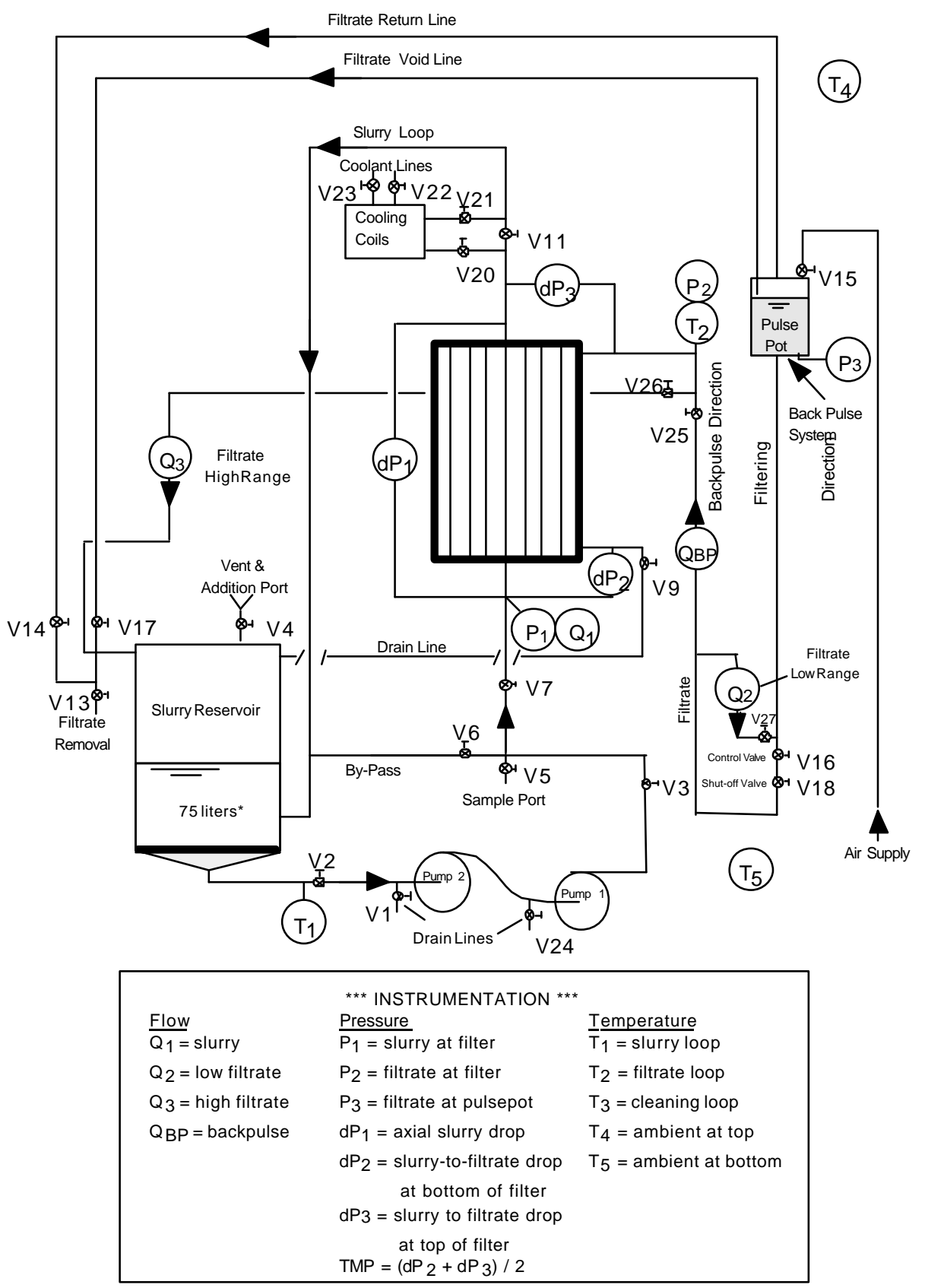

Figure 2. Schematic of the Pilot-scale Cross Flow Ultrafiltration Test Facility 
The test rig is controlled through a series of valves, which are described below:

V1: Drains slurry from the test rig at pump 2.

V2: Isolates the slurry reservoir from the pumps

V3: Isolates the slurry loop from the pumps

V4: Allows liquid to be introduced to the slurry reservoir

V5: Allows slurry to be sampled (not used for this test)

V6: Recirculates slurry to the reservoir

V7: Directs slurry to the filter; 3-way valve

V8: Not in use - see below

V9: Drains the filtrate loop

V10: Not in use - see below

V11: Controls the slurry flow downstream of the filter

V12: Not in use - see below

V13: Sample port for filtrate

V14: Shut off for filtrate return line

V15: Allows air to pressurize the pulsepot

V16: Preset pressure drop for backpulse filtrate line

V17: Allows liquid evacuation of pulsepot in preparation for backpulsing

V18: Actuates a backpulse

V19: Not in use - see below

V20: Upstream slurry flow to heat exchanger

V21: Downstream slurry flow from heat exchanger

V22: Upstream coolant flow to heat exchanger

V23: Downstream coolant flow from heat exchanger

V24: Drains slurry from the test rig at pump 1

V25: Shut low-flow filtrate line when high filtrate flows are needed (e.g. water)

V26: Directs high filtrate flow to slurry reservoir and flow meter

V27: Shuts filtrate flow to low-flow meter during a backpulse

Note: Valves V8, V10, V12, and V19 are for the cleaning loop, which was not used.

To circulate slurries in the test rig two $10 \mathrm{hp}$ Galigher centrifugal pumps were used. The impeller and impeller housing were made from EPDM to be compatible with both the $\mathrm{pH}=14$ slurry to be tested and the $2 \mathrm{M}$ nitric acid cleaning solutions. The two pumps were used in series on the slurry loop to attain a head of $70 \mathrm{psig}$ at $60 \mathrm{gpm}(\sim 4.6 \mathrm{~m} / \mathrm{s}$ in each filter tube).

\subsubsection{Cross Flow Filter}

The heart of this entire experimental task was the cross-flow filter element that was to be tested to define its operational characteristics under required flow conditions when using the Sr/TRU precipitation simulant. There were several candidates that could have been used for this test but due to availability and past experience in robust designs, a Mott filter was chosen. The specifications for the filter unit were: 
Material: $\quad 316$ stainless steel (sintered metal)

Porosity: nominal rated 0.1 micron

Length:

90 inches

Diameter:

Number:

1/2-inch I.D., 5/8-inch O.D.

7 tubes

The unit which was received from Mott met the requirements. Figure 3 and 4 show the 90-inch length was made from four 22.5-inch lengths that were welded together.

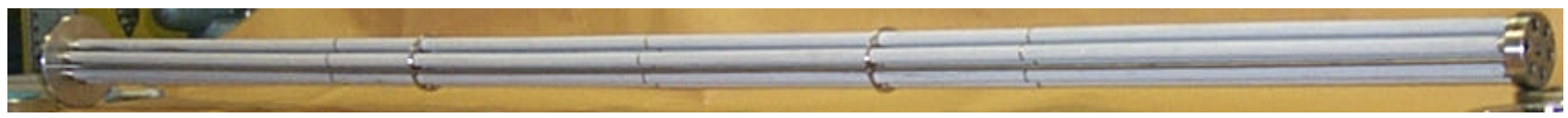

Figure 3. 7-tube bundle of a nominal rated 0.1 micron filter, 1.2” i.d., 5/8" o.d., 90" long

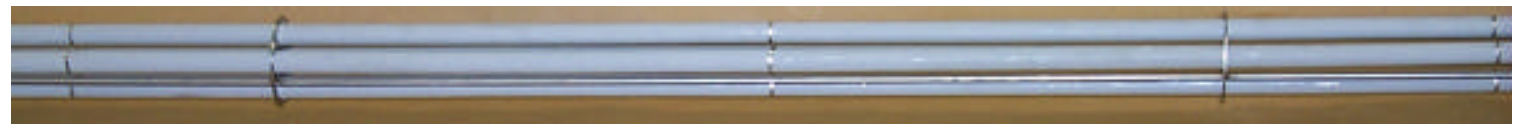

Figure 4. Adjoined filter tube sections

Besides the inter-tube weldments, Fig. 5b, the 7 tube bundle was welded together with the tube sheets, Figs 5a, d, and extra support was made with stabilizing plates and supporting solid metal 1/4-inch rods which ran the length of the tube bundle, Fig. 5c.

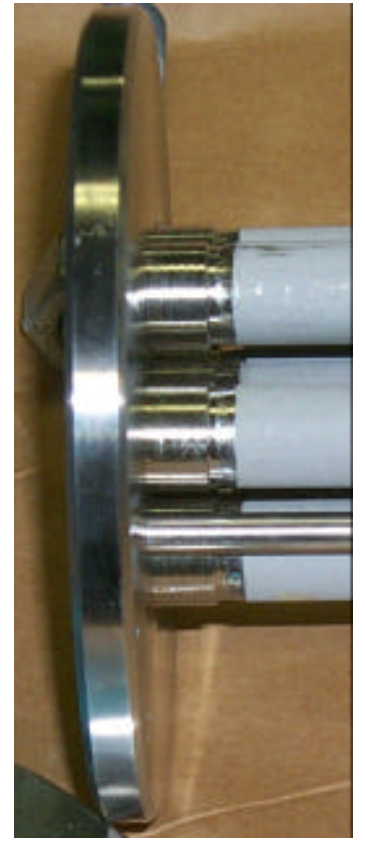

(a)

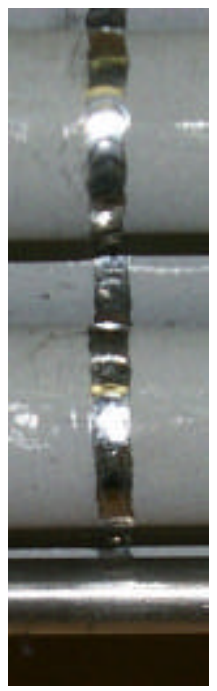

(b)

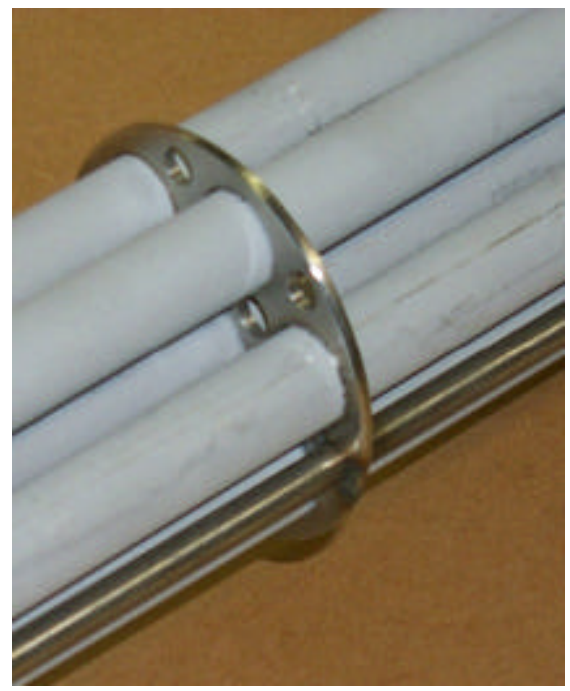

(c)

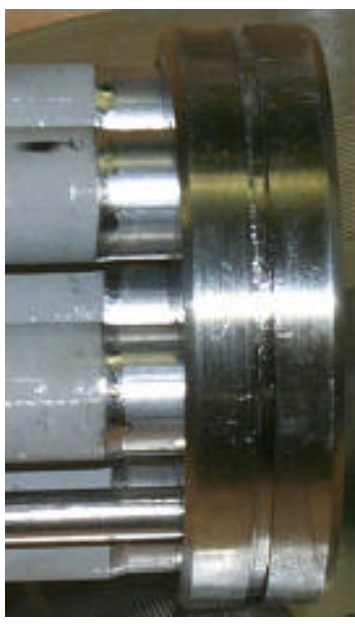

(d)

Figure 5: (a) Large-tube sheet, (b) tube-to-tube weldment, (c) tube supports, (d) small-tube sheet.

The stainless steel sintered surface has a fairly robust construction. Figure 6 shows an enlargement of two different pore-size filter elements, 0.5 and 100 microns. (Mott did not 
have a picture of a nominal rated 0.1 micron filter but stated that the appearance is identical to the larger sizes.)
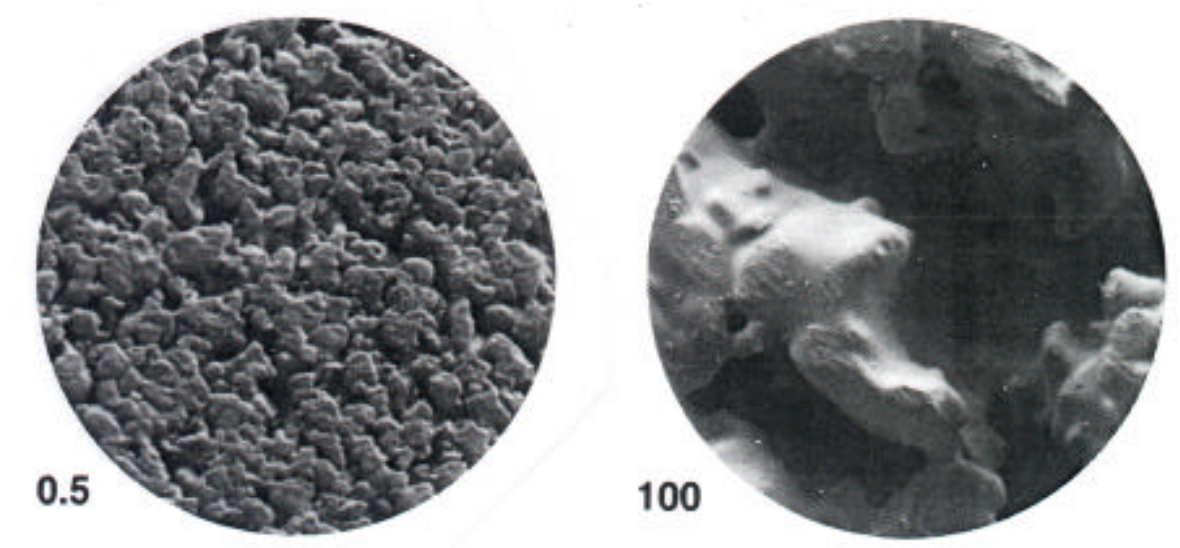

Figure 6. Magnified views (approximately 150x) of surfaces of two different pore-size rated Mott filters (the number to the left of each figure is the pore-size rating in microns)

When the test rig is in operation, slurry enters the porous tubes at the small tube sheet, Figs. $5 \mathrm{~d}$ and $7 \mathrm{~b}$, and exits the tubes at the large tube sheet, Figs. 5a and 7a. Each tube sheet is sealed to a 1.5 -inch to 3 -inch plenum.

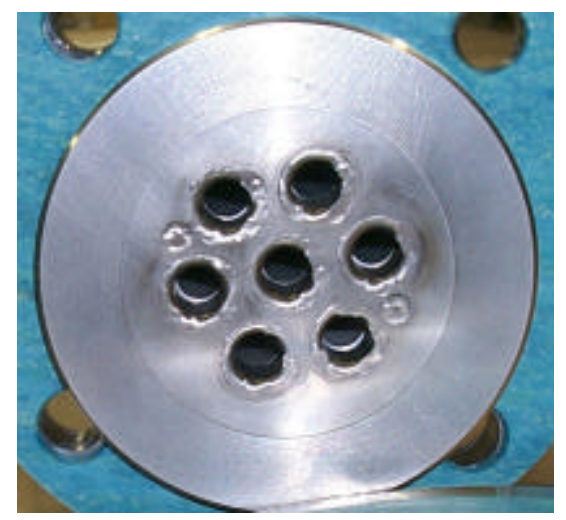

(a)

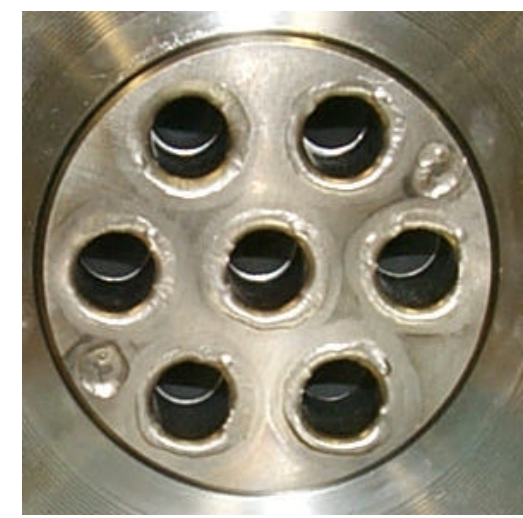

(b)

Figure 7: Tube-sheet profiles (a) Downstream (b) Upstream

The tube housing, Fig. 8, was made from a 3-inch schedule 10 pipe with two pipes connected at either end to remove filtrate. For this test the filter unit was oriented vertically in the test rig, see Figs. 1, 2, and 8. The tube bundle sat in the housing such that the large tube sheet, Figs. 5a and 7a, was secured to the top flange of the housing; this tube sheet also supported the weight of the assembly. The smaller, lower, tube sheet, Fig. $5 \mathrm{~d}$ and $7 \mathrm{~b}$, was able to pass through the housing and separated the slurry side of the flow channel from the filtrate side with an "O" ring between the outer perimeter of the lower tube sheet and the inside diameter of the filter housing. 
WSRC-TR-2003-00204, REV. 0

SRT-RPP-2003-00087, REV. 0

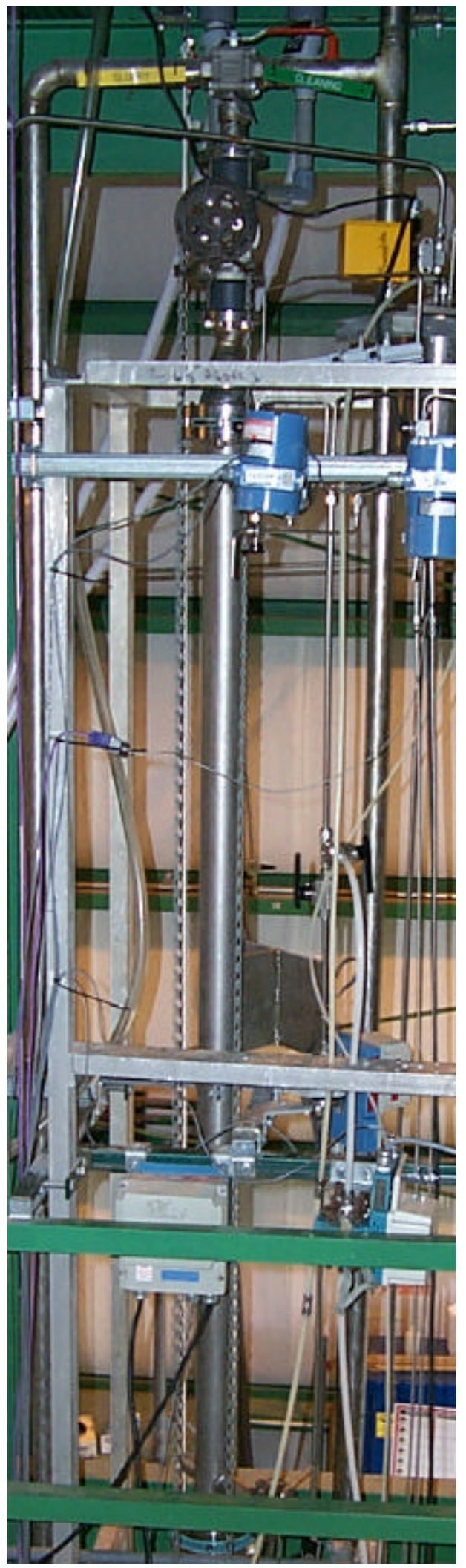

Figure 8. Installed 90-inch tall cross-flow filter housing 


\subsubsection{Backpulse System}

On March 20, 2002 the RPP-WTP customer requested that the backpulse system be changed from the piston-type, used for previous tests (Duignan, 2000a, b), to a pneumatic-type that would be more prototypic of the current RPP-WTP design. That is, a filtrate system, which is normally liquid solid, would introduce a gas into an small vessel, called a pulse pot to evacuate some of the liquid so that it can then be pressured. When ready, a valve is opened very fast to allow the pressurized liquid to flow back, "pulsed," into the crossflow filter. Figure 9 is a schematic of the RPP-WTP backpulse system and Fig 10 is a simplified operational procedure.

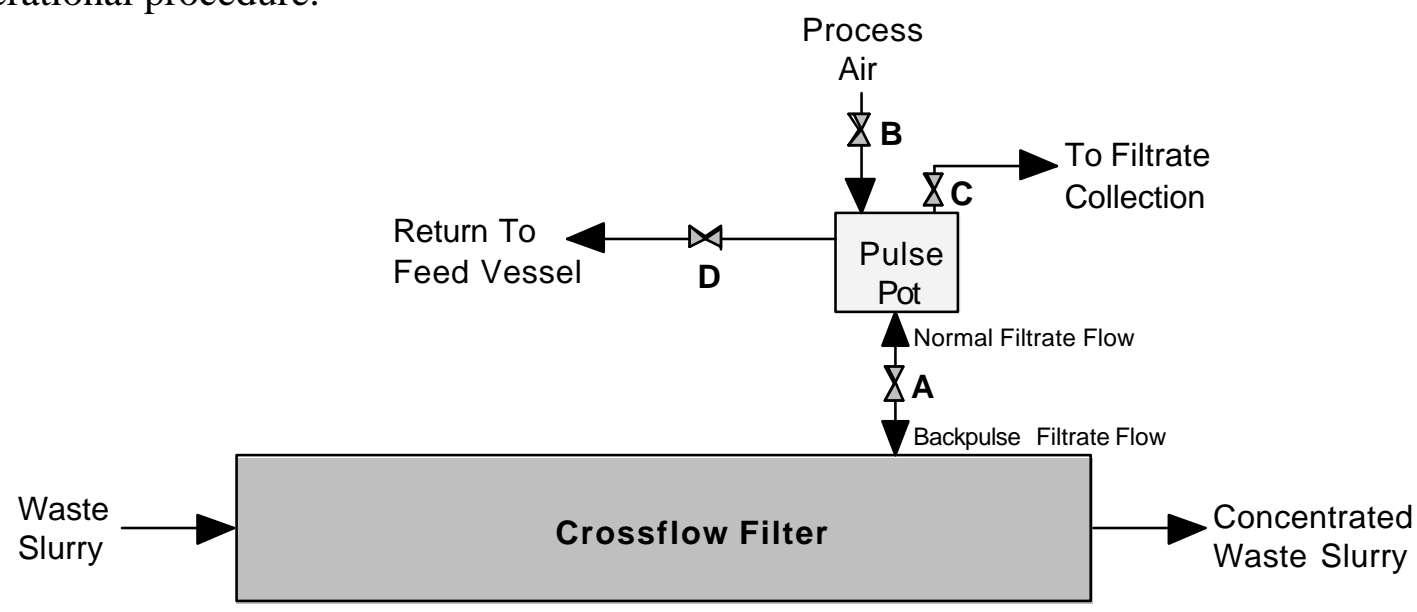

Figure 9. Planned RPP-WTP backpulse system

\begin{tabular}{|c|c|c|c|c|}
\hline V Mode V/Valve $>>$ & A & B & C & D \\
\hline Filtering & Open & Closed & Open & Closed \\
\hline Stop Filtration & Closed & Closed & Closed & Closed \\
\hline Void Pulsepot & Closed & Open & Closed & Open \\
\hline Pressure Pulsepot & Closed & Open & Closed & Closed \\
\hline Isolate Pulsepot & Closed & Closed & Closed & Closed \\
\hline Back Pulse & Open & Closed & Closed & Closed \\
\hline Resume Filtration & Open & Closed & Open & Closed \\
\hline
\end{tabular}

Figure 10. Procedure to operate backpulse system

The planned plant backpulse system is designed to have a:

1. Backpulse pressure 30 psi above the slurry pressure at the filter.

2. Pressure drop from the pulse pot to the filter of 10 psid during a backpulse.

3. Pulse liquid volume of at most $2 / 3^{\text {rds }}$ of the total pulse pot volume.

4. Volume of the pulse liquid enough for efficient back pulsing.

(This volume was stated to be a minimum of $70 \%$ of the volume of the porous tube walls.)

5. Back pulse cycle duration of 5 to 10 seconds.

The pilot test rig was changed to meet the above requirements. However, what could not be matched were the dynamic characteristics of the plant system. That is, because of differences 
in scale, and therefore mass, the momentum of the plant system will be considerably different. The operation of the pilot system followed the sequence shown in Fig. 10 and it was designed to meet all five criteria above. For the volume of pulse liquid, past experience (Duignan, 2000a, b) has shown an effective volume to be 0.036 gallons per square foot of inside area of the cross-flow filter. Therefore, the volume used was:

$\left(0.036 \mathrm{gal} / \mathrm{ft}^{2}\right) \times(7$ tubes $) \times \pi[(0.5 \mathrm{inch} / 12)] \times 90$ inches $/ 12=0.25$ gal.

By using the $4^{\text {th }}$ criterion above the volume would be:

$70 \% \times[(7$ tubes $) \times$ volume of porous tube wall $]=$

$70 \% \times\left[(7\right.$ tubes $) \times \pi / 4\left[(0.625 \mathrm{inch} / 12)^{2}-(0.5 \mathrm{inch} / 12)^{2}\right] \times 90$ inches $\left./ 12 \times 7.48 \mathrm{gal} / \mathrm{ft}^{3}\right]=0.21 \mathrm{gal}$.

Therefore, using 0.25 gallon would be conservative to effectively removing the filter cake.

Designing for the 10 psid pressure drop from the pulse pot to the filter housing, during the backpulse, was a little harder to obtain and, therefore, the pilot system was made to have an adjustable pressure drop using a valve. Several tests were done before the experiment began to estimate how to set that valve so that the backpulse pressure drop was near the target 10 psid. To illustrate the backpulse system, Figure 11 shows a subset of Fig. 2 and valve letters corresponding to those in Fig. 10 are included to compare operation. Note that for the pilot system, the voiding line, leading to valve $\mathrm{D}$, is through the top of the pulse pot instead of the side, Fig 9, in order to change the void space size if necessary.

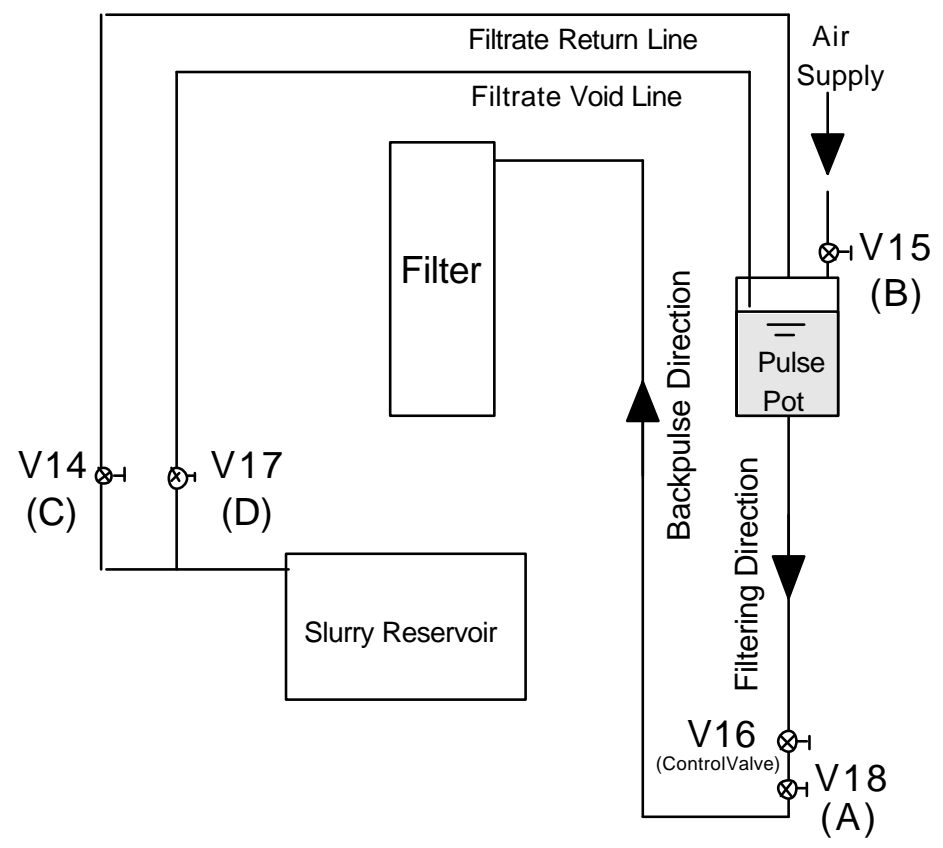

Figure 11. Pilot test rig backpulse loop, see also Fig. 2 


\section{WSRC-TR-2003-00204, REV. 0 SRT-RPP-2003-00087, REV. 0}

To see how the system of Fig. 11 operates and how the pressure drop control valve, V16 was set see Fig. 12, which shows the data from one of the many shakedown tests done with water. The instrumentation indicated in Fig. 12 can be found in Fig. 2. It shows the slurry (water) pressure in the filter to be approximately 50 psig; therefore, the air overpressure (P3) was set at $90 \mathrm{psig}$, or approximately $30 \mathrm{psig}$ at the filter housing when considering the pressure drop across the backpulse system at the highest backpulse flowrate. That is, as seen in Fig. 12, the backpulse flowrate peak after 2 seconds from opening valve A, at about $0.5 \mathrm{gpm} / \mathrm{ft}^{2}$ and the backpulse pressure drop, i.e., P3-P2, was at approximately 10 psid.

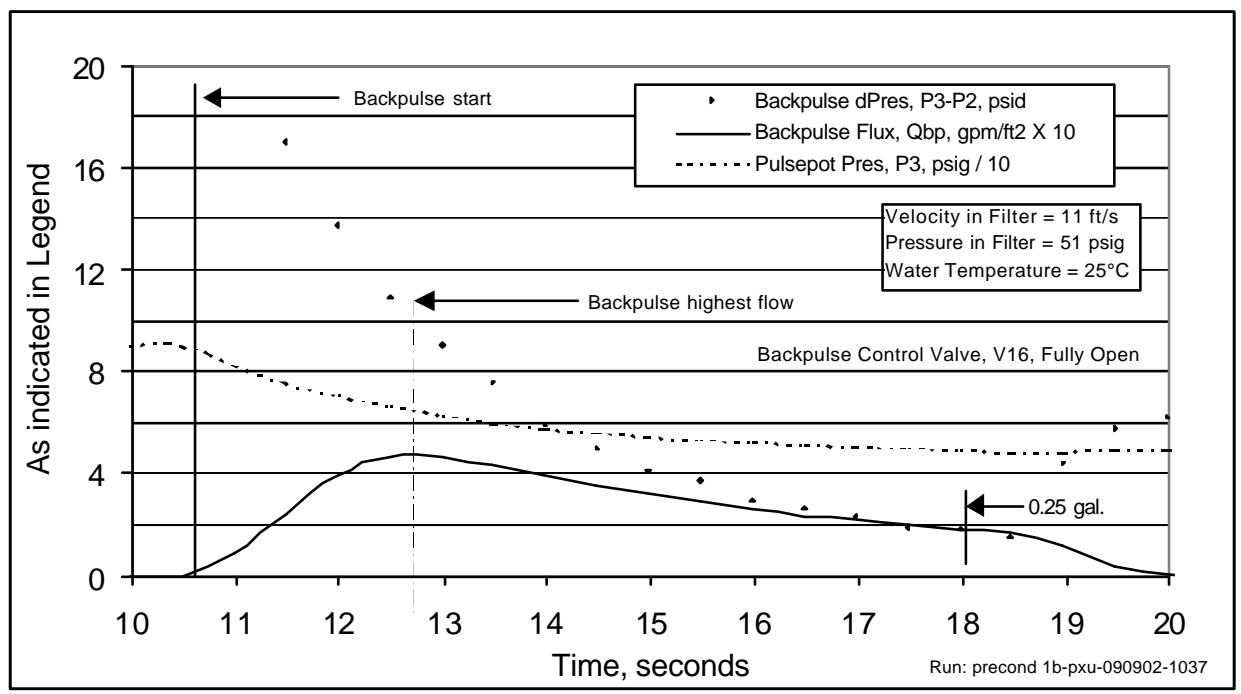

Figure 12. Pilot test rig backpulse operation with water

Figure 12 data show the sequence of events as indicated in Fig. 10, with the valves shown in Figs. 2 and 11:

1. Stop Filtration: Prior to data taking - all valves were closed.

2. Void Pulsepot: Prior to data taking - only valves B and D are open.

3. Pressurize Pulsepot: Prior to data taking - only valve B is left open.

4. Isolate Pulsepot: 0-10.5 seconds - all valves were closed.

5. Back Pulse: 10.5-18 seconds - valve A opened as fast as possible, then closed at some predetermined time, see Fig. 11.

6. Resume Filtration - valve C is opened, followed by opening valve A slowly.

Note that in step 6 valve $C$ is opened first to relieve any residual pneumatic pressure and prevent further, albeit weak, backpulsing. Valve A is opened very slowly, over 60 seconds to uniformly form the filter cake when reestablishing filtrate flow.

As Fig. 12 indicates, the backpulse control valve was fully opened; therefore, this would be the position set for future slurry tests. Many tests were done to determine the number of seconds to leave the backpulse flow open before the 0.25 gallon of filtrate passed through the filter wall. When slurry was introduced into the test rig, the backpulse conditions were checked again. By trial and error the times in Fig. 13 were used to obtain $0.25 \mathrm{gpm}$ of pulsed 
filtrate for each run, which must change due to pressure of the slurry in the filter and thus leading to different air overpressures, leading to different values of backpulse flowrates.

\begin{tabular}{|c|c|c|c|c|c|c|c|}
\hline Pressure at the filter (P1), psig & 10 & 20 & 30 & 40 & 50 & 60 & 70 \\
\hline Duration of Pulse, seconds & 9 & 11 & 12 & 14 & 15 & 17 & 18 \\
\hline
\end{tabular}

Figure 13. Pilot test rig backpulse duration (time to hold valve A open)

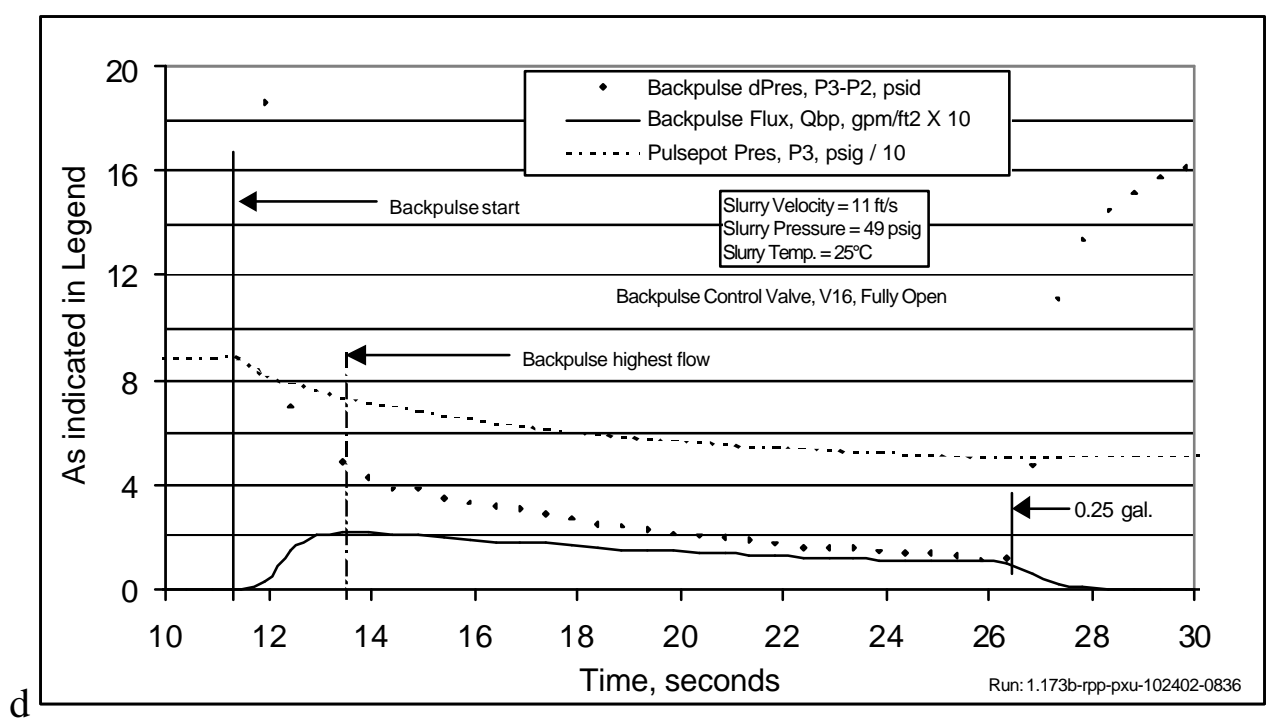

Figure 14. Pilot test rig backpulse operation with AN-102R2, Batch 3B

Figure 14 backpulse results were taken during one of the slurry tests (AN-102R2, Batch 3B) and indicates different results from those for water, basically because the slurry filtrate was 4 times more viscous than water. The peak backpulse flowrate occurred at almost the same time period as water, i.e., 2 seconds, but the rate only reached just under one half that of water. Moreover, at the peak flowrate, the backpulse system pressure drop was also about one half that of water, i.e., 5 psid. The times at which 0.25 gallon of filtrate passed back through the filter can be compared because both of the tests shown in Figs. 12 and 14 were done at a filter pressure of $50 \mathrm{psig}$. The water took 7.5 seconds and the slurry took 15 seconds. As can be verified in Fig. 13, the times shown are for slurry operation. Even though the backpulse system only presented a pressure drop of 5 psid at the peak filtrate flowrate, valve V16 was left open for all tests. The actual pressure that will exist in the fullsize RPP-WTP backpulse piping will be considerably less than the assumed 10 psid because the project began a design change, making the filtrate flow paths much bigger. It is unlikely that the plant pressure drop will be more than 1 or 2 psid. Besides, if V16 were closed enough to attain a 10 psid it is unlikely that the filtrate momentum would be enough to affect the filter cake. As it is, the filtrate backpulse flowrate was only $0.2 \mathrm{gpm} / \mathrm{ft}^{2}$ (or only 10 times greater that the target mean filtration rate of $0.02 \mathrm{gpm} / \mathrm{ft}^{2}$ ), which turned out not to be very effective in removing the Envelope C-type simulant filter cake. 


\subsubsection{Instrumentation}

The measurement equipment used for this experiment was:

5 Type $\mathrm{E}$ thermocouples with average accuracies from* 0.9 to $1.0^{\circ} \mathrm{C}$,

6 Variable capacitance pressure transducers with average accuracies from* 0.05 to $0.40 \mathrm{psi}$, and

4 Magnetic flow meters with accuracies from* 0.005 to $0.5 \mathrm{gpm}$.

*accuracies are a function of the instrument and calibration. The uncertainty introduced through the use of the 16-bit data acquisition system was insignificant $(<0.1 \%$ reading) and was not included in the values above.

Figure H1, Appendix H, shows several tables which list all those instruments and data acquisition system (DAS) channels for each of the non-thermocouple instruments. The thermocouples had their own dedicated computer card to interface and convert the temperatures properly. The calibration of the DAS was checked and that information can also be found in Fig. H1, which includes tables and graphs that show the results and the transfer functions used for each channel.

From Figs. 1, H1, and $\mathrm{H} 2$ the location and the usage of each instrument can be determined, however the following list will better describe the placement and usage of all the measurement instruments:

T1 - A thermocouple located in the exit pipe of the slurry reservoir to measure the slurry temperature on its way to the filter.

T2 - A thermocouple located in the filtrate line at the end of the upper filter housing filtrate exit pipe to measure the filtrate temperature as it leaves the filter housing.

T3 - A thermocouple located in the cleaning loop at the exit of the cleaning loop ${ }^{\dagger}$ pump.

T4 - A thermocouple located outside of the top of the test rig to measure ambient temperature.

T5 - A thermocouple located outside at the bottom of the test rig to measure ambient temperature.

For the 6 pressure transducers also refer to Fig. $\mathrm{H} 2$ which indicate pressure-line locations as well as their heights.

P1 - A gauge pressure transducer located at the beginning of the filter housing to measure the pressure of the slurry just before entering the filter tubes.

P2 - A gauge pressure transducer located in the filtrate line at the top filter housing filtrate exit pipe to measure the pressure of the filtrate as it leaves the filter housing. P3 - A gauge pressure transducer located at the bottom of the filtrate pulsepot to measure the pressure applied to produce a backpulse.

dP1 - A differential pressure transducer located across the slurry side of the filter to measure the drop in pressure along the filter tubes.

dP2 - A differential pressure transducer located across filter and housing at the filter to measure the transmembrane pressure at the beginning of the filter.

\footnotetext{
${ }^{\dagger}$ The cleaning loop in general was not used for this experiment, therefore its temperature measurement is generally irrelevant.
} 
dP3 - A differential pressure transducer located across filter and housing at the filter to measure the transmembrane pressure at the end of the filter.

[The transmembrane pressure is determined from the average of $\mathrm{dP} 2$ and $\mathrm{dP} 3$.]

Q1 - A magnetic flowmeter located at the entrance of the filter to measure the slurry flowrate. Q2 - A magnetic flowmeter located in the filtrate line between the exit of the filtrate housing and pulse pot to measure the low filtrate flowrates. This device was calibrated from 0 to $1.2 \mathrm{gpm}$ (or a filtrate flux of 0 to $0.17 \mathrm{gpm} / \mathrm{ft}^{2}$ ), which covers most of the filtrate flux range expected for slurries.

Q3 - A magnetic flowmeter located in the filtrate line between the exit of the filtrate housing and the slurry reservoir tank, downstream of valve V26 to measure the high filtrate flowrates. This device was calibrated from 0 to $5 \mathrm{gpm}$ (or a filtrate flux of $0.75 \mathrm{gpm} / \mathrm{ft}^{2}$ ), which covers all expected filtrate fluxes. This was used for water and nitric acid tests.

Qbp - A magnetic flowmeter located in the filtrate line between the filtrate housing and the pulsepot to measure the reversed filtrate flowrate during a backpulse. When a backpulse is made flowmeter, Q2, is isolated from the loop.

\subsubsection{Measurement Uncertainty}

Appendix $\mathrm{H}$ has all the pertinent information on the uncertainties. The measurement uncertainties (95\% confidence level) for the important calculated quantities are:

$\begin{array}{llll}\text { Slurry Velocity in a Filter Tube } & = & \mathrm{V} & \pm 6.2 \% \\ \text { Transmembrane Pressure } & = & \mathrm{TMP} & \pm 2.2 \% \\ \text { Temperature Corrected Filtrate Flux } & = & \mathrm{Fc} & \pm 5.4 \% \\ \text { Permeability } & = & \mathrm{P} & \pm 5.8 \%\end{array}$

These number are based on pre- and post-test calibrations of the instruments.

\subsubsection{Simulated Waste Slurry}

Beside the cross-flow filter, the most important aspect of this experiment was the slurry used to simulate a Hanford Site waste. The waste that was simulated is referred to as Envelope C. Envelope $\mathrm{C}$ are radioactive wastes that include tank 241-AN-102 from the Hanford Site, which is made up of organic and other complexants. The simulant used for this task was cold (non-radioactive), but chemically it was made as close as was known to actual waste. Once the base Envelope $\mathrm{C}$ simulant was ready for use, it was prepared for filtration by adding several precipitation reagents. Some information on the slurry tested will be given below but the goal of this task was to test filterability of a given waste simulant at a pilot scale and not to determine suitability of the simulant to the actual waste. Information of the actual waste can be found in Urie, et al. (2002), the development of the SRTC simulant can be found in Eibling (2003), and information on the preparation and precipitation of the simulant can be found in Steeper and Williams (2003). 


\section{WSRC-TR-2003-00204, REV. 0}

SRT-RPP-2003-00087, REV. 0

Filterability, notwithstanding, to understand how this experiment was run it is necessary to know the general aspects of the slurries that were tested. This task tested one base simulated waste, which was subjected to four different methods of precipitation. The pilot-scale cross-flow facility was then used to filter each prepared simulant to determine the effect the methods had on filterability. The base simulant was developed by SRTC and it was made of many compounds, Fig. 15. The resulting mixture was deluted to a sodium concentration of $6.0 \mathrm{M}$.

\begin{tabular}{|c|c|c|c|}
\hline Base Compound & Formula & Mol Wt & AN-102R2 \\
\hline Aluminum Nitrate & $\mathrm{Al}\left(\mathrm{NO}_{3}\right)_{3} .9 \mathrm{H}_{2} \mathrm{O}$ & 375.13 & 4.539E-01 M \\
\hline Cadmium Nitrate & $\mathrm{Cd}\left(\mathrm{NO}_{3}\right)_{2 .} 4 \mathrm{H}_{2} \mathrm{O}$ & 308.48 & 4.096E-04 M \\
\hline Calcium Nitrate & $\mathrm{Ca}\left(\mathrm{NO}_{3}\right)_{2 .} 4 \mathrm{H}_{2} \mathrm{O}$ & 236.15 & 9.257E-03 M \\
\hline Cerium Nitrate & $\mathrm{Ce}\left(\mathrm{NO}_{3}\right)_{3 .} 6 \mathrm{H}_{2} \mathrm{O}$ & 434.22 & $2.411 \mathrm{E}-04 \mathrm{M}$ \\
\hline Cesium Nitrate & $\mathrm{CsNO}_{3}$ & 194.91 & $9.054 \mathrm{E}-05 \quad \mathrm{M}$ \\
\hline Cobalt Nitrate & $\mathrm{Co}\left(\mathrm{NO}_{3}\right)_{3} \cdot 6 \mathrm{H}_{2} \mathrm{O}$ & 353.03 & $3.524 \mathrm{E}-05 \quad M$ \\
\hline Copper Nitrate & 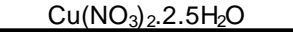 & 241.60 & 2.783E-04 M \\
\hline Ferric Nitrate & $\mathrm{Fe}\left(\mathrm{NO}_{3}\right)_{3} \cdot 9 \mathrm{H}_{2} \mathrm{O}$ & 403.99 & $5.662 \mathrm{E}-04 \quad \mathrm{M}$ \\
\hline Lanthanum Nitrate & $\mathrm{La}\left(\mathrm{NO}_{3}\right)_{3} \cdot 6 \mathrm{H}_{2} \mathrm{O}$ & 433.01 & 2.097E-04 M \\
\hline Lead nitrate & $\mathrm{Pb}\left(\mathrm{NO}_{3}\right)_{2}$ & 331.20 & $6.784 \mathrm{E}-04 \quad \mathrm{M}$ \\
\hline Manganous Chloride & $\mathrm{MnCl}_{2} .4 \mathrm{H}_{2} \mathrm{O}$ & 197.90 & $4.028 \mathrm{E}-04 \quad \mathrm{M}$ \\
\hline Neodymium Nitrate & $\mathrm{Nd}\left(\mathrm{NO}_{3}\right)_{2} \cdot 6 \mathrm{H}_{2} \mathrm{O}$ & 376.36 & 4.959E-04 M \\
\hline Nickel Nitrate & $\mathrm{Ni}\left(\mathrm{NO}_{3}\right)_{2} \cdot 6 \mathrm{H}_{2} \mathrm{O}$ & 290.81 & $5.381 \mathrm{E}-03 \quad \mathrm{M}$ \\
\hline Potassium Nitrate & $\mathrm{KNO}_{3}$ & 101.11 & $3.779 \mathrm{E}-02 \quad \mathrm{M}$ \\
\hline Rubidium Nitrate & $\mathrm{RbNO}_{3}$ & 147.48 & $7.376 \mathrm{E}-05 \quad \mathrm{M}$ \\
\hline Zinc Nit & $\mathrm{Zn}\left(\mathrm{NO}_{3}\right)_{2} .6 \mathrm{H}_{2} \mathrm{O}$ & 297.47 & $5.818 \mathrm{E}-05 \quad \mathrm{M}$ \\
\hline Zirconyl Nitrate & $\mathrm{ZrO}\left(\mathrm{NO}_{3}\right)_{2} \cdot \mathrm{H}_{2} \mathrm{O}$ & 249.23 & $1.111 \mathrm{E}-04 \quad \mathrm{M}$ \\
\hline EDTA & $\mathrm{Na}_{2} \mathrm{C}_{10} \mathrm{H}_{14} \mathrm{~N}_{2} \mathrm{O}_{8} .2 \mathrm{H}_{2} \mathrm{O}$ & 372.24 & $7.286 \mathrm{E}-03 \quad \mathrm{M}$ \\
\hline HEDTA & $\mathrm{Na}_{3} \mathrm{C}_{10} \mathrm{H}_{15} \mathrm{~N}_{2} \mathrm{O}_{7}$ & 278.26 & $1.524 \mathrm{E}-03 \quad \mathrm{M}$ \\
\hline Sodium Gluconate & $\mathrm{CH}_{2} \mathrm{OH}(\mathrm{CH}$ & 218.14 & $5.709 \mathrm{E}-03 \quad \mathrm{M}$ \\
\hline Citric Acid & $\mathrm{HOC}\left(\mathrm{CH}_{2} \mathrm{CO}_{2} \mathrm{H}\right)_{2} \mathrm{CO}_{2} \mathrm{H}$ & 192.13 & 2.038E-02 M \\
\hline Nitrilotriacetic Acid & $\mathrm{OOH})_{3}$ & 191.14 & $1.042 \mathrm{E}-03 \quad \mathrm{M}$ \\
\hline Iminodiacetic Acid & $\mathrm{SO}_{2} \mathrm{H}_{2}$ & 133.10 & $2.591 \mathrm{E}-02 \quad \mathrm{M}$ \\
\hline Succinic Acid & & 118.04 & 2.346E-04 M \\
\hline Glutaric Acid & & 132.12 & $3.786 \mathrm{E}-04 \quad \mathrm{M}$ \\
\hline Adipic Acid & $\mathrm{O}_{4}$ & 146.14 & $1.288 \mathrm{E}-03 \quad \mathrm{M}$ \\
\hline Azelaic Acid & $\mathrm{O}_{4}$ & 188.22 & $4.183 \mathrm{E}-03 \quad \mathrm{M}$ \\
\hline Suberic Acid & $\mathrm{C}_{8} \mathrm{H}_{14} \mathrm{O}_{4}$ & 174.20 & 7.949E-03 M \\
\hline Ammonium Acetate & $\mathrm{NH}_{4} \mathrm{CH}_{3} \mathrm{COO}$ & 77.08 & $6.162 \mathrm{E}-03 \mathrm{M}$ \\
\hline Boric acid & $\mathrm{O}_{3}$ & 61.83 & $2.575 \mathrm{E}-03 \quad \mathrm{M}$ \\
\hline Sodium Chloride & $\mathrm{NaCl}$ & 58.44 & $1.013 \mathrm{E}-01 \mathrm{M}$ \\
\hline Sodium Fluoride & $\mathrm{NaF}$ & 41.99 & $6.846 \mathrm{E}-02 \mathrm{M}$ \\
\hline Sodium Sulfate & $\mathrm{Na}_{2} \mathrm{SO}_{4}$ & 142.04 & 9.953E-02 M \\
\hline Potassium Molybdate & $\mathrm{K}_{2} \mathrm{MoO}_{4}$ & 238.14 & 3.561E-04 M \\
\hline Sodium Hydroxide & $\mathrm{OH}$ & 40.00 & $2.927 \mathrm{E}+00 \mathrm{M}$ \\
\hline Sodium Phosphate & $\mathrm{Na}_{3} \mathrm{PO}_{4.12 \mathrm{H}_{2} \mathrm{O}}$ & 380.12 & $3.476 \mathrm{E}-02 \quad \mathrm{M}$ \\
\hline Sodium Tungstate & $\mathrm{Na}_{2} \mathrm{WO}_{4} 2 \mathrm{H}_{2} \mathrm{O}$ & 329.86 & $6.903 \mathrm{E}-04 \mathrm{M}$ \\
\hline Sodium Metasilicate & $\mathrm{Na}_{2} \mathrm{SiO}_{3} .9 \mathrm{H}_{2} \mathrm{O}$ & 284.14 & $2.695 \mathrm{E}-04 \quad \mathrm{M}$ \\
\hline Sodium Formate & $\mathrm{NaHCOO}$ & 68.01 & $1.416 \mathrm{E}-01 \mathrm{M}$ \\
\hline Sodium Glycolate & $\mathrm{HOCH}_{2} \mathrm{COONa}$ & 98.01 & 1.056E-01 M \\
\hline Sodium Acetate & $\mathrm{NaCH}_{3} \mathrm{COO} .3 \mathrm{H}_{2} \mathrm{O}$ & 136.08 & 3.737E-03 M \\
\hline Sodium Oxalate & $\mathrm{Na}_{2} \mathrm{C}_{2} \mathrm{O}_{4}$ & 134.00 & 3.983E-03 M \\
\hline Sodium Chromate & $\mathrm{Na}_{2} \mathrm{CrO}_{4}$ & 161.97 & $3.663 \mathrm{E}-03 \quad \mathrm{M}$ \\
\hline Sodium Carbonate & $\mathrm{Na}_{2} \mathrm{CO}_{3}$ & 105.99 & $6.925 \mathrm{E}-01 \quad \mathrm{M}$ \\
\hline Sodium Nitrate & $\mathrm{NaNO}_{3}$ & 84.99 & $9.594 \mathrm{E}-01 \quad M$ \\
\hline Sodium Nitrite & $\mathrm{NaNO}_{2}$ & 69.00 & $1.086 \mathrm{E}+00 \mathrm{M}$ \\
\hline Strontium Nitrate & $\mathrm{Sr}\left(\mathrm{NO}_{3}\right)_{2}$ & 211.63 & $3.576 \mathrm{E}-05 \quad \mathrm{M}$ \\
\hline
\end{tabular}

Figure 15. Chemical make-up of the AN-102 simulant (AN-102R2) 
To the base simulant, a very small amount of other solids, to remain mostly insoluble, were added to represent the entrained solids expected in the real waste, Fig. 16.

\begin{tabular}{|c|c|c|c|}
\hline Entrained Solid $(E S)^{\star}$ & Formula & Mol Wt & Mass \% \\
\hline Aluminum Oxide & $\mathrm{Al}_{2} \mathrm{O}_{3}$ & 101.96 & $15.12 \%$ \\
\hline Barium Sulfate & $\mathrm{BaSO}_{4}$ & 233.4 & $0.02 \%$ \\
\hline Calcium Oxalate & $\mathrm{CaC}_{2} \mathrm{O}_{4} \cdot \mathrm{H}_{2} \mathrm{O}$ & 146.11 & $0.13 \%$ \\
\hline Calcium Tungstate & $\mathrm{CaWO}_{4}$ & 287.93 & $0.11 \%$ \\
\hline Cerium Oxalate & $\mathrm{Ce}\left(\mathrm{C}_{2} \mathrm{O}_{4}\right)_{3} \cdot 9 \mathrm{H}_{2} \mathrm{O}$ & 544.29 & $0.02 \%$ \\
\hline Chromic Oxide & $\mathrm{Cr}_{2} \mathrm{O}_{3}$ & 151.99 & $0.93 \%$ \\
\hline Ferric Hydroxide & $\mathrm{FeO}(\mathrm{OH})$ & 88.85 & $0.68 \%$ \\
\hline Lanthanum Oxalate & $\mathrm{La}_{2}\left(\mathrm{C}_{2} \mathrm{O}_{4}\right)_{3 .} 10 \mathrm{H}_{2} \mathrm{O}$ & 722.03 & $0.02 \%$ \\
\hline Lead Sulfate & $\mathrm{PbSO}_{4}$ & 303.25 & $0.08 \%$ \\
\hline Manganese Dioxide & $\mathrm{MnO}_{2}$ & 86.94 & $0.15 \%$ \\
\hline Neodymium Oxalate & $\mathrm{Nd}_{2}\left(\mathrm{C}_{2} \mathrm{O}_{4}\right)_{3} \cdot 10 \mathrm{H}_{2} \mathrm{O}$ & 732.69 & $0.04 \%$ \\
\hline Nickel Oxide & $\mathrm{NiO}$ & 74.71 & $0.01 \%$ \\
\hline Silicon Oxide & $\mathrm{SiO}_{2}$ & 60.09 & $0.05 \%$ \\
\hline Sodium Carbonate & $\mathrm{Na}_{2} \mathrm{CO}_{3} \cdot \mathrm{H}_{2} \mathrm{O}$ & 124.01 & $42.71 \%$ \\
\hline Sodium Fluoride & $\mathrm{NaF}$ & 41.99 & $3.15 \%$ \\
\hline Sodium Oxalate & $\mathrm{Na}_{2} \mathrm{C}_{2} \mathrm{O}_{4}$ & 134.00 & $16.10 \%$ \\
\hline Sodium Phosphate & $\mathrm{Na}_{3} \mathrm{PO}_{4.12 \mathrm{H}_{2} \mathrm{O}}$ & 380.12 & $12.28 \%$ \\
\hline Sodium Sulfate & $\mathrm{Na}_{2} \mathrm{SO}_{4} \cdot 10 \mathrm{H}_{2} \mathrm{O}$ & 322.04 & $8.35 \%$ \\
\hline Zinc Oxalate & $\mathrm{ZnC}_{2} \mathrm{O}_{4} \cdot 2 \mathrm{H}_{2} \mathrm{O}$ & 189.45 & $0.02 \%$ \\
\hline Zirconium Oxide & $\mathrm{ZrO} 2$ & 60.09 & $0.02 \%$ \\
\hline
\end{tabular}

Figure 16. Entrained solids added to the AN-102R2 simulant

The combination of the base simulant, Fig. 15, and the entrained solids, Fig. 16, represent the Hanford waste AN-102 that will be treated by the RPP-WTP. The initial treatment is precipitation. For organic-based wastes, like AN-102, three reagents are added to precipitate the strontium and transuranic components to decontaminate the wastes more effectively and to improve the wastes' filterability. Originally, this task was planned to filter only two slurry simulant batches, i.e., Batches ${ }^{\dagger} 3$ and 4 . Those batches differed only by how well they would be mixed during the precipitation process. Batch 3 was to be well mixed with a mechanical agitator and Batch 4 was to be mixed with a pneumatically driven pulse jet mixer (PJM). Previous work (Duignan, 2000b) ${ }^{\ddagger}$ with Envelope C-type simulants using well mixed baseline precipitation conditions, explained below, has already been shown to attain an acceptable level of decontamination and filterability. Since RPP-WTP has been designed to use PJM to agitate the precipitating wastes; therefore, it was important to show its effects on

\footnotetext{
Batches 1 and 2 were strictly related to the precipitation task (Steeper and Williams, 2003) and are not part of this task.

\# The previous dewatering test with an Envelope C-type waste was done with a simulant of AN-107 from 30 November to 1 December of 1999 and the average filtrate flux was $0.045 \mathrm{gpm} / \mathrm{ft}^{2}$ over the 28.5 hours of the test.
} 


\section{WSRC-TR-2003-00204, REV. 0} SRT-RPP-2003-00087, REV. 0

decontamination and filterability. Unfortunately, the Batch 3 test demonstrated very poor filterability', which necessitated changes to the precipitation process and thus increased the number of tests to four batches ${ }^{\ddagger}$, i.e., $3 \mathrm{C}, 3 \mathrm{~B}, 3 \mathrm{~A}$, and $4 \mathrm{~A}$.

The reason the Batch 3 test did not show the same level of acceptable filterability is that the precipitation recipe was changed to minimize the amount of reagents. Those changes are referred to as the newly optimized conditions (NOC). The quantities of precipitating reagents which were previously shown to give good filterability (Duignan, 2000b) for Envelope C-type simulants also produced decontamination factors many times greater than were necessary. Hallen, Brooks, and Jagoda (2000) showed exceptionally high decontamination factors and recommended lowering the concentration of precipitation reagents to minimize the amount of chemicals that would have to be added to the wastes, thus creating more wastes. Those new concentrations are referred to as NOC. Unfortunately, while the NOC did produce acceptable levels of decontamination they had a deleterious effect on filterability. Once the low filtrate flux was obtained with the NOC Batch 3 the test scope was expanded to revisit precipitation with the successful baseline conditions (BC). The next three tests used $\mathrm{BC}$ with slight variations on the precipitation preparation in the way of mixing temperatures and agitation. Figure 17 lists the differences among the four batches of AN-102R2 simulant, which were ultimately tested. This report documents the filtering results of each of these batches.

\begin{tabular}{|c|c|c|c|c|c|c|}
\hline $\begin{array}{l}\text { Batch } \\
\text { Number }\end{array}$ & $\begin{array}{l}\text { Precipitation } \\
\text { Conditions (1) }\end{array}$ & $\begin{array}{c}\text { Precipitation } \\
\text { Mixing (2) }\end{array}$ & \begin{tabular}{|l|} 
Precipitation \\
Temp, ${ }^{\circ} \mathrm{C}(3)$
\end{tabular} & $\begin{array}{l}\text { Final Conc } \\
\text { of Free } \mathrm{OH}^{-}\end{array}$ & $\begin{array}{c}\text { Final Conc } \\
\text { of } \mathrm{SrNO}_{3}\end{array}$ & $\begin{array}{l}\text { Final Conc } \\
\text { of } \mathrm{NaMnO}_{4}\end{array}$ \\
\hline $3 \mathrm{C}$ & $\mathrm{NOC}=$ & well & 25 & $0.3 \mathrm{M}$ & $0.03 \mathrm{M}$ & $3 \mathrm{M}$ \\
\hline $3 B$ & $\mathrm{BC}=$ & well & 50 & & $0.075 \mathrm{M}$ & \\
\hline $3 \mathrm{~A}$ & $\mathrm{BC} @ 25^{\circ} \mathrm{C}=$ & ell & 25 & & & \\
\hline $4 \mathrm{~A}$ & $\mathrm{BC} w / \mathrm{PJM}=$ & & $50>25$ & & & \\
\hline \multicolumn{7}{|c|}{$\begin{array}{l}\text { Notes } \\
\text { (1) - Condition nomenclature: } \\
\text { NOC = newly optimized conditions (to minimize volume of precipitating reagents) } \\
\text { BC = baseline condtions (known to obtain good decontamination and filterability) } \\
\text { BC @ } 25^{\circ} \mathrm{C}=\text { baseline conditions but precipitated at } 25^{\circ} \mathrm{C} \\
\mathrm{BC} \text { w/PJM = baseline conditions but mixed with a pulse jet mixer } \\
\text { (2) - Precipitation mixing nomenclature: } \\
\text { well = the precipitation solution was considered well mixed with a Lightin A-310 style } \\
\text { impeller in a } 42 \text {-inch diameter baffled tank rotating at } 508 \mathrm{rpm} \text { (an impeller tip } \\
\quad \text { speed of } 26.6 \mathrm{ft} / \mathrm{s} \text { ) imparting } 4.3 \mathrm{hp} / 1000 \text { gallons } \\
\text { PJM = pulse jet mixer which agitated the solution with pulses of air. The level of } \\
\quad \text { mixing is not know, but was less well mixed than with the impeller. } \\
\text { (3) Precipitation Temperature - after the last precipitating reagent was added } \\
\text { the mixture was maintained at the listed temperature for four hours before filtration } \\
\text { began. Additionally, batch } 4 \mathrm{~A} \text { was further mixed another } 18 \text { hours as its temperature } \\
\text { dropped from } 50^{\circ} \mathrm{C} \text { to } 25^{\circ} \mathrm{C} \text { before filtration began. }\end{array}$} \\
\hline
\end{tabular}

Figure 17. Nomenclature for the four AN-102R2 simulant batches

\footnotetext{
$\dagger$ The dewatering target was to have an average filtrate flux of $0.02 \mathrm{gpm} / \mathrm{ft}^{2}$ or higher, with a final concentration of better than $15 \mathrm{wt} \%$ insoluble solids. As will be seen, the first Batch 3 test resulted in an average flux of $0.011 \mathrm{gpm} / \mathrm{ft}^{2}$ over 45 hours of dewatering and the slurry was only concentrated to $8.5 \mathrm{wt} \%$ insoluble solids.

\# The batch numbering nomenclature is arbitrary and the sequence shown above is the chronological order in which the batches were tested. Batch 4 is referred to $4 \mathrm{~A}$ because a $4 \mathrm{~B}$ was planned in the event Batch $4 \mathrm{~A}$ indicated a larger quantity was needed to reach $15 \mathrm{wt} \%$ insoluble solids. Batch $4 \mathrm{~B}$ was not required.
} 
WSRC-TR-2003-00204, REV. 0

SRT-RPP-2003-00087, REV. 0

\subsubsection{Test Procedure/Matrix}

Details of the test matrix and procedure that were carried out can be found in the Test Procedure (Duignan, 2002e), but an illustrated matrix is shown as Fig. 18.

\begin{tabular}{|c|c|c|c|c|c|}
\hline Test No. & Test Activity & $\begin{array}{l}\text { Trans- } \\
\text { membrane } \\
\text { Pressure } \\
\text { (psid) }\end{array}$ & $\begin{array}{c}\text { Slurry } \\
\text { Velocity } \\
\text { (ft/s) }\end{array}$ & $\begin{array}{l}\text { Slurry } \\
\text { Temp } \\
\left({ }^{\circ} \mathrm{C}\right)\end{array}$ & $\begin{array}{l}\text { Run } \\
\text { Time } \\
\text { (min.) }\end{array}$ \\
\hline $1.00 \mathrm{~A}$ & Water (1) & 10 & 12 & 25 & 20 \\
\hline $1.00 \mathrm{~B}$ & Water & 20 & 12 & 25 & 20 \\
\hline $1.00 \mathrm{C}$ & Water & 30 & 12 & 25 & 20 \\
\hline $1.01 \mathrm{~A}$ & $5 \mathrm{wt} \% \mathrm{SrCO}_{3}+$ Water & 10 & 12 & 25 & 20 \\
\hline $1.01 \mathrm{~B}$ & $5 \mathrm{wt} \% \mathrm{SrCO}_{3}+$ Water & 20 & 12 & 25 & 20 \\
\hline $1.01 \mathrm{C}$ & $5 \mathrm{wt} \% \mathrm{SrCO}_{3}+$ Water & 30 & 12 & 25 & 20 \\
\hline 1.02 & Water Rinse & 20 & 12 & 25 & 60 \\
\hline $1.03-1.15$ & Low solids concentratio & $n$ tests are $n$ & o longer 1 & required & $(2)$ \\
\hline 1.16 & Dewater to $20 \mathrm{wt} \%$ (3) & 40 & 12 & 25 & $(4)$ \\
\hline 1.17 & $20 w t \%$ & 40 & 12 & 25 & 120 \\
\hline 1.18 & $20 \mathrm{wt} \%$ & 40 & 12 & 25 & 120 \\
\hline 1.19 & $20 \mathrm{wt} \%$ & 40 & 12 & 25 & 120 \\
\hline 1.20 & $20 \mathrm{wt} \%$ & 30 & 9 & 25 & 60 \\
\hline 1.21 & $20 \mathrm{wt} \%$ & 30 & 13 & 25 & 60 \\
\hline 1.22 & $20 \mathrm{wt} \%$ & 50 & 13 & 25 & 60 \\
\hline 1.23 & $20 w t \%$ & 50 & 9 & 25 & 60 \\
\hline 1.24 & 20 & 40 & 12 & 25 & 120 \\
\hline 1.25 & $20 w t \%$ & 40 & 7 & 25 & 60 \\
\hline 1.26 & $20 w t \%$ & 40 & 15 & 25 & 60 \\
\hline 1.27 & $20 \mathrm{wt} \%$ & 20 & 12 & 25 & 60 \\
\hline 1.28 & $20 \mathrm{wt} \%$ & 60 & 12 & 25 & 60 \\
\hline 1.29 & $20 w t \%$ & 40 & 12 & 25 & 120 \\
\hline Wash & & 40 & 12 & 30 & (6) \\
\hline 1.30 & $>20 \mathrm{wt} \%$ & 40 & 12 & 25 & (7) \\
\hline $1.31 \mathrm{~A}$ & Water Rinse & 40 & 12 & 25 & 60 \\
\hline $1.31 \mathrm{~B}$ & Acid clean (8) & 40 & 12 & 25 & 90 \\
\hline $1.31 \mathrm{C}$ & Acid clean & 40 & 12 & 25 & 90 \\
\hline 1.32 & Water Rinse & 20 & 12 & 25 & 60 \\
\hline $1.33 \mathrm{~A}$ & $5 \mathrm{wt} \% \mathrm{SrCO}_{3}+$ Water & 10 & 12 & 25 & 20 \\
\hline $1.33 \mathrm{~B}$ & $5 \mathrm{wt} \% \mathrm{SrCO}_{3}+$ Water & 20 & 12 & 25 & 20 \\
\hline $1.33 \mathrm{C}$ & $5 \mathrm{wt} \% \mathrm{SrCO}_{3}+$ Water & 30 & 12 & 25 & 20 \\
\hline $1.34 \mathrm{~A}$ & Water & 10 & 12 & 25 & 20 \\
\hline $1.34 \mathrm{~B}$ & Water & 20 & 12 & 25 & 20 \\
\hline $1.34 \mathrm{C}$ & Water & 30 & 12 & 25 & 20 \\
\hline \multicolumn{6}{|c|}{$\begin{array}{l}\text { Notes: } \\
\text { (1) - The water was deionized, filtered with a } 0.1 \text {-micron absolute filter, } \\
\text { then made caustic to } 0.01 \mathrm{M} \mathrm{NaOH} \text {. } \\
\text { (2) - Before testing began a test exception (Townson, 2002b) to the test } \\
\text { specification was issued to eliminate all low solids concentration tests. } \\
\text { (3) - wt } \% \text { = weight percent of insoluble solids } \\
\text { (4) - Run time is the time to dewater the simulant from the low wt\% to } \\
\text { the final concentration. The only test specification requirement } \\
\text { was to run for no less than } 12 \text { hours. } \\
\text { (5) - The concentrated slurry used for test } 1.29 \text { was washed with } \\
\text { a volume of water (see note } 1 \text { ) equal to } 21 / 18 \times \text { simulant volume } \\
\text { The water volume was broken down into } 21 \text { mini-washes. } \\
\text { (6) - The wash run time was the time necessary to introduce and } \\
\text { remove all } 21 \text { subvolumes. } \\
\text { (7) - Test } 1.30 \text { run time was not fixed. Filtering was to continue } \\
\text { until the simulant plugged the filter, or some test rig limit was reached. } \\
\text { (8) - } 2 \mathrm{M} \text { nitric acid was used. }\end{array}$} \\
\hline
\end{tabular}

Figure 18. Test matrix used for AN-102R2 testing 


\section{WSRC-TR-2003-00204, REV. 0}

SRT-RPP-2003-00087, REV. 0

Special attention should be given to test no. 1.16, dewatering. As will be seen in the results section and in the respective appendices, dewatering was the most important test. As previously mentioned, only 2 batches were originally planned, but as the number of batches grew to four, it was not necessary to carry out the entire test matrix listed in Fig. 18. The results section will discuss to what extent each batch was tested. Beside the test procedure, there were many Work Instructions issued to handle the daily changes that occurred. Those instructions can be found in the task notebook (Duignan, 2002b). To facilitate understanding the general operation of the test, a simplified version of the procedural steps is summarized below (see Figs. 1 and 2 for valve locations):

Daily pre-test activities -

1. Equipment is turned on to warm up if not already on.

2. The equipment was checked for functionality and after each of the four liquid-filled pressure sensing lines (see Fig. H2; Appendix H) were purged with $5 \mathrm{ml}$ of distilled and filtered water, the transducers were checked at their zeroes for drift. The zeroes are recorded for 2 to 3 minutes by the DAS. Those data are included in each day's data sheets.

Daily testing activities for constant solids runs (high concentrations) -

3. Begin circulating the slurry in the recirculation loop until the temperature reached $25^{\circ} \mathrm{C}$.

4. Turn on the reservoir cooling coil.

5. Allow the slurry to flow through the cross-flow filter.

6. Set the appropriate flow conditions as per the test procedure by iterating between the pumps' speeds and V11.

7. Set the DAS to read every minute.

8. Backpulse the filter. See the preceding backpulse section for its operation.

9. Allow the test rig to run for approximately 1 or 2 hours (as required).

10. Backpulse the filter once again.

11. Repeat from Step 6 for next set of flow conditions or shut down the test rig, if near the end of the work day.

12. End the test run.

Daily testing activities for wash test runs -

[Before beginning the test, prepare a volume of inhibited DIF water (i.e., deionized and filtered (0.1 micron filter) water to which caustic is added to obtain $0.01 \mathrm{M} \mathrm{NaOH}$ ). The volume is to be 21/18 times the volume of slurry to be washed, which is broken down into 21 small volumes to be added to the slurry one at a time. With the wash water ready, do Steps 3 to 8 from above, then continue below.]

9. Add a small volume of wash water to the slurry in the test rig.

10. Switch valve V13 to the open-loop position so that the filtrate is not returned to the slurry loop, but is collected outside the test rig.

11. Allow the test rig to run until a volume of filtrate is removed that is equal to the volume of water that was put in. (Slightly more mass is taken out than was put in because of the filtrate's higher density.)

12. Maintain the axial filter velocity and the TMP constant.

13. Repeat Steps 9 through 12 for all 21 small volumes. 
14. Switch valve V13 to the close-loop position.

15. Backpulse the filter once again.

16. End the test run.

Testing activities for pre-wash dewatering -

[Do Steps 3 to 8 from above.]

9. Switch valve V13 to the open-loop position so that the filtrate is not returned to the slurry loop, but is collected outside the test rig.

10. When the slurry level in the slurry reservoir drops to the 100-liter mark refill with more slurry from the precipitation test rig.

11. Repeat 10 until the entire contents of the prepared simulant is contained in the slurry reservoir of the filtration test rig.

12. If the filtrate flux drops below $0.015 \mathrm{gpm} / \mathrm{ft}^{2}$ do a backpulse.

13. Continue concentrating the slurry until there is less than 75 liters or as otherwise indicated by the task lead.

14. Close V13 to stop slurry concentration and do a backpulse.

15. End the test run.

\subsection{RESULTS}

\subsubsection{Insoluble Solids Separation}

The majority of the Results discussion concerns filterability because of the difficulties encountered, however, one absolute success of all the tests was the filter's ability to separate insoluble solids from the slurry simulants. The simulants' turbidity was measured at the beginning and end of each batch test and the result is:

Turbidity (for batches 3 and 4) $=0.17$ NTU \pm 0.06 NTU (one standard deviation)

One source (Martino, 2001) that deals with $5.8 \mathrm{M} \mathrm{Na}^{+}$simulated wastes, which was used in research connected to the SRS Defense Waste Processing Facility at DOE's Savannah River Site, states that 5 NTU represents less than $6 \mathrm{ppm}$ of insoluble solids. If this measure is used, than the 0.17 NTU would indicate that the filtrates obtained during the Batch 3 and 4 tests had less than $0.2 \mathrm{ppm}$ of insoluble solids.

\subsubsection{Filterability}

The actual events that took place to test all four simulated AN-102 batches did not follow the test matrix, Fig. $18^{\dagger}$. The most important aspect was the dewatering test, i.e., 1.16; therefore,

\footnotetext{
${ }^{\dagger}$ A slurry test not shown in Fig. 18 was one that was done with archived AN-107 simulated slurry. Before any testing was done the filter, which was new, was subjected to filter this old slurry to put the filter in a "used" condition. Prior to preconditioning the filter it was baselined with water and the standard slurry and then a steady state slurry run was done for several days. The test number terminology used for preconditioning was similar to that used in Fig. 18, except that each test number was preceded with the word "precond."
} 
that is the only test which all four simulants have data. In general, the matrix can be broken down into the following categories:

1. Baseline with water

2. Baseline with a standard slurry

3. Test slurry at a low concentration of solids (eliminated just before testing began)

4. Dewater

5. Test slurry at a high concentration of solids

6. Wash slurry

7. Dewater until pluggage

8. Acid Clean

9. Repeat step 2

10. Repeat step 1

Due to filterability difficulties and the change in the number of batches to test, the following is what actually occurred:

Batch 3C (test period: 1 to 3 October 2002)

1. Baseline with water

2. Baseline with a standard slurry

3. Dewatering from $0.8 \mathrm{wt} \%$ to $8.4 \mathrm{wt} \%$ insoluble solids (test stopped - poor filterability)

4. Acid Clean

5. Repeated step 2

6. Repeated step 1

Batch 3B (test period: 22 October to 5 November 2002)

The entire matrix was completed

Batch 3A (test period: 6 to 26 November 2002)

1. Baseline with water

2. Dewatering from $1.6 \mathrm{wt} \%$ to $25 \mathrm{wt} \%$ insoluble solids

3. Limited number of high concentration tests to verify operation

4. Acid Clean

5. Baseline with a standard slurry

6. Repeated step 1

Batch 4A (test period: 18 February \& 12 to 26 March 2003)

1. Baseline with water (18 February)

2. Dewatering from $1.5 \mathrm{wt} \%$ to $18 \mathrm{wt} \%$ insoluble solids

3. Limited number of high concentration tests to verify operation

4. Acid Clean

5. Baseline with a standard slurry

6. Repeated step 1 
The results for each of categories are discussed below. The discussion will first deal with baselining and cleaning, which will be then followed by the test data for the simulated waste.

\subsubsection{Baseline with Water}

\subsection{Water and Cross-flow Filtration}

Normally a crossflow filter would not be used with just a liquid because there is nothing to filter. However, flowing water through a filter is useful in the sense of determining the cleanliness of the filter element. That is, to show if the same water filtrate flux is obtained before use and after use and after cleaning. Since there are no solids to deposit on the filter surface, and thus challenge the filter, water will produce results that seem counter-intuitive. This section will not discuss the nature of filtering without solids, since this has been dealt with in some depth elsewhere (Duignan, 2000a or b). Discussed here will be the data obtained with water throughout the tests with the AN-102R2 slurry simulant in the Pilotscale Cross-flow Ultrafiltration (PXU) facility.

\subsection{Overall Evaluation}

At the start and end of each slurry test with the 0.1-micron Mott filter, it was required to test with water, as requested by the RPP-WTP customer (Townson, 2002a). Before use, the water was to be filtered with a 0.1-micron absolute filter and deionized. Further, the water was made mildly caustic to a concentration of $0.01 \mathrm{M} \mathrm{NaOH}$ (referred to as inhibited water). In most cases, the caustic addition was done, but there were some exceptions because the addition of even a small amount of caustic caused a significant reduction in filtrate flux when the inhibited water followed an acid cleaning. After using slurry, it was removed and then the slurry loop was cleaned with acid. The acid was followed with water rinses, however there could have been small pockets of slurry or acid solution left somewhere in the loop. The water with the mild caustic was thought to have caused some precipitation of solids that could not be totally removed from the slurry loop. Those solids would then affect the filtrate flux; confounding water-flux comparisons; therefore, some water runs did not include a caustic addition in order to see the effect.

To reiterate, the purpose of the water tests was to determine if the filter returned to the same level of cleanliness after being used with slurry. However, since water generally does not contain significant insoluble solids it does not act like a slurry; therefore it was followed by a standard slurry, $5 \mathrm{wt} \% \mathrm{SrCO}_{3}$, to better determine the level of filter cleanliness. The standard slurry tests had their own problems and they will be discussed in the next section.

Water tests were actually done at several places in the test matrix, as already mentioned, see Fig. 18: at the very beginning (test 1.00), a rinse after the initial standard slurry tests (test 1.02), a rinse immediately after doing an entire slurry matrix (test 1.31A), another rinse after the acid cleaning (test 1.32), and the final test that comes immediately after the final standard slurry tests (test 1.34). The purpose of this section is to describe the before and after water tests, i.e., 1.00 and 1.34, but some of the rinses are included to better explain the, 
sometimes conflicting, results as the filter went from a new state to some other level of cleanliness after its use.

For the testing of slurry simulant $\mathrm{AN}-102 \mathrm{R} 2$, there were a total of five batches of slurry runs: Preconditioning (with archived AN-107), AN-102R2 Batch 3C, AN-102R2 Batch 3B, AN-102R2 Batch 3A, AN-102R2 Batch 4A. [Note: For the preconditioning test, the test nomenclature is different. For example, while the initial water test number is 1.00 , the initial precond itioning water test was called PreCond 1.] As mentioned, originally, there were only two tests planned and with preconditioning it would have been three: Preconditioning, AN-102R2 Batch 3, and AN-102R2 Batch 4. However, due to problems with filtering Batch 3 it was done three different ways, which wound up being three completely different batches. With so many tests run close together, there was no point doing water runs before and after each slurry test. Between any two tests, the final water test (1.34) of one slurry test would be used for the initial water test (1.00) for the next slurry test. There were some exceptions, which will be seen throughout the following presentation.

Figure $19^{\dagger}$ illustrates some of the data sets taken before and after each slurry test. (Note, each datum point in the figure is the time average filtrate flux over the period of each test, which lasted approximately 20 minutes each.) Ideally, a clear indication was expected on how effective the filter cleaning was, or if depth fouling progressed with time. While there is a tendency which indicates a return to the same clean level, the data do not elicit straight forward information. The separation among the two data sets before-Batch-3C (open and closed circles) and after-Batch-3C data (triangles) is significant. The main reason was that the slurry loop was very well cleaned after the end of the filter preconditioning, before starting with Batch 3C. This cleaning was not planned but there was a pump-liner failure, which forced the more thorough cleaning. After preconditioning the filter with the AN-107 slurry simulant, the filter was cleaned with $2 \mathrm{M}$ nitric acid for 3 hours and then tested with the standard slurry, which was then followed by a water rinsing and the inhibited water test runs (open circles). The low filtrate flux was unexpected and was thought to be the result of chemicals that were left over from the cleaning that precipitated due to the caustic $(0.01 \mathrm{M} \mathrm{NaOH})$ added to the water. However, the acid cleaning caused the pump liners to fail during the water runs and needed replacing. Because pieces of the pump liner had to be removed from the entire filtration test rig, a very thorough cleaning was done. After the cleaning, the inhibited water runs were repeated before starting the slurry test with AN-102R2, Batch 3C. Those results show significantly higher filtrate fluxes (the closed circles). After filtering with Batch $3 \mathrm{C}$ slurry, the filter was cleaned once again with the standard method of 3 hours with $2 \mathrm{M}$ nitric acid, followed by the standard slurry test, and then by a water rinsing and the inhibited water tests (triangles). Those subsequent water tests showed, once again, a low water flux. This low flux seemed to confirm the assumption of the presence of precipitated solids (e.g. aluminum). One option to return to the higher water flux was to ultraclean the slurry loop each time a slurry run is done, but that would have been very time consuming and the actual plant will not do such extensive measures unless it is absolutely necessary.

\footnotetext{
$\dagger$ The data for all the figures in this section can be found in Appendix A.
} 


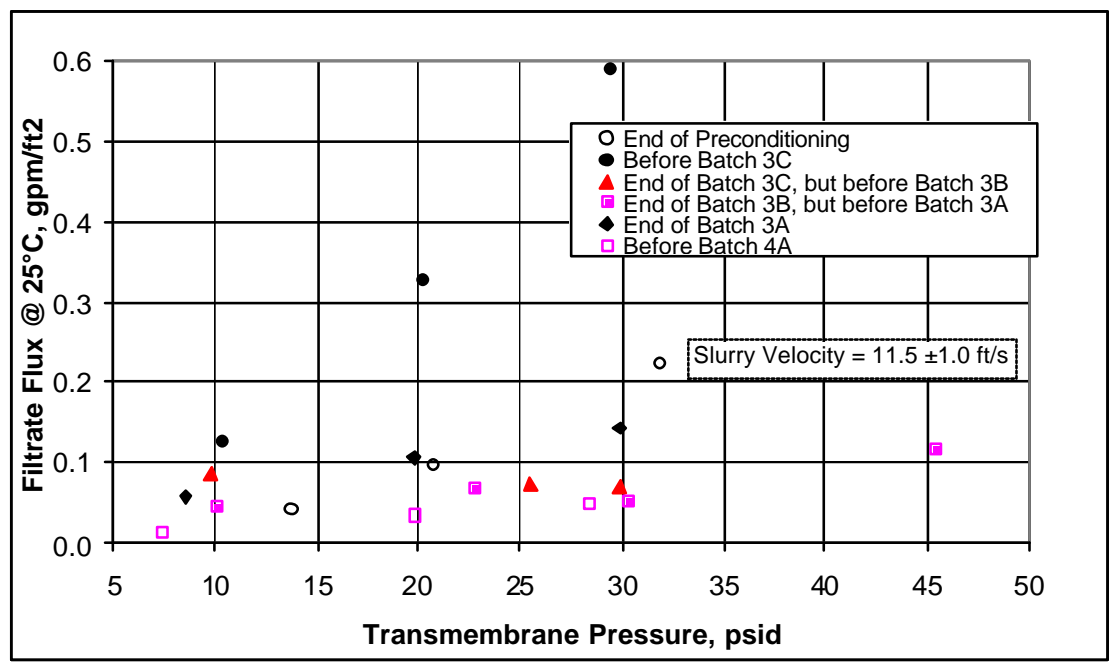

Figure 19. Water tests made before and after filtering several slurries. The filtered and deionized water was made mildly caustic $(0.01 \mathrm{M} \mathrm{NaOH})$.

The related data files are:

Open Circles=tests after Preconditioning with AN-107 and before Batch 3C, tests: 8a, 8b, 8c

Closed Circles=tests before slurry Batch 3C, tests: $1.00 \mathrm{a}-3 \mathrm{C}, 1.00 \mathrm{~b}-3 \mathrm{C}, 1.00 \mathrm{c}-3 \mathrm{C}$

Triangles=tests before slurry Batch $3 \mathrm{~B}$, tests: $1.00 \mathrm{a}-3 \mathrm{~B}, 1.00 \mathrm{~b}-3 \mathrm{~B}, 1.00 \mathrm{c}-3 \mathrm{~B}$

Square $=$ tests before slurry Batch $3 \mathrm{~A}$, test: $1.34 \mathrm{a}-3 \mathrm{~B}, 1.34 \mathrm{~b}-3 \mathrm{~B}, 1.34 \mathrm{c}-3 \mathrm{~B}$

Triangles=tests before slurry Batch $3 \mathrm{~B}$, tests: $1.00 \mathrm{a}-3 \mathrm{C}, 1.00 \mathrm{~b}-3 \mathrm{C}, 1.00 \mathrm{c}-3 \mathrm{C}$

Open Squares=tests before slurry Batch 4A, tests: 1.00a-4A, 1.00b-4A, 1.00c-4A

The bottom five sets of data (open circles, diamonds, triangles, open squares, and closed squares) are much closer to each other than to the initial data set (closed circles). Considering that the level of filter cleanliness can change from one cleaning to the next, and that the filtrate flux is very sensitive to any debris in the filter, then the scatter seems understandable. Unfortunately, it is not possible to determine if depth fouling was increasing with time because the data sets do not chronologically become lower and lower. For instance, the set indicated by diamonds (after Batch 3A) is higher than the triangles and squares even though it was obtained after the sequence of Batch 3C, 3B, and $3 \mathrm{~A}$ was complete. If depth fouling was increasing with time, its effect was confounded by other complications and not clearly observed.

\subsection{Individual Evaluations}

The different phases of testing where water runs were made are the following:

1. New Filter and the Effect of Inhibited Water after Nitric Acid Cleaning

2. Water Runs before and after Preconditioning with AN-107 slurry simulant

3. Water Runs before and after AN-102R2, Batch 3C slurry simulant

4. Water Runs before and after AN-102R2, Batch 3B slurry simulant

5. Water Runs before and after AN-102R2, Batch 3A slurry simulant

6. Water Runs before and after AN-102R2, Batch 4A slurry simulant 


\subsection{New Filter and the Effect of Inhibited Water after Nitric Acid Cleaning}

Figure 20 shows data (diamonds) taken with inhibited water (IW $=0.01 \mathrm{M} \mathrm{NaOH}$ ) just before testing it with the standard slurry of $5 \mathrm{wt} \% \mathrm{SrCO}_{3}$ and then data (squares) just after. However, the water after the standard slurry test did not have caustic and, in fact, was left mildly acidic $\left(0.03 \mathrm{HNO}_{3}\right)$ because the addition of caustic caused the filtrate flux to drop significantly. The TMP was very low (4.9 psid) because the filtrate loop had a significant pressure drop. After the initial test the loop was modified to reduce the pressure drop. However, by that time the filter already experienced flow with the standard slurry of $5 \mathrm{wt} \%$ $\mathrm{SrCO}_{3}$. After the $\mathrm{SrCO}_{3}$ tests were complete, and the slurry loop was flushed with water several times, the filtrate flux would not return to the higher values when using the IW; therefore a weak acid was used to prevent precipitation of solids to see if the filtrate flux could return to new-filter fluxes. Several water runs were done after adding the mild acid, as well as at the lower TMP to make a comparison to the new filter data. The two curves in Fig. 20 are significantly different but much closer to the each other than when the water was made caustic.

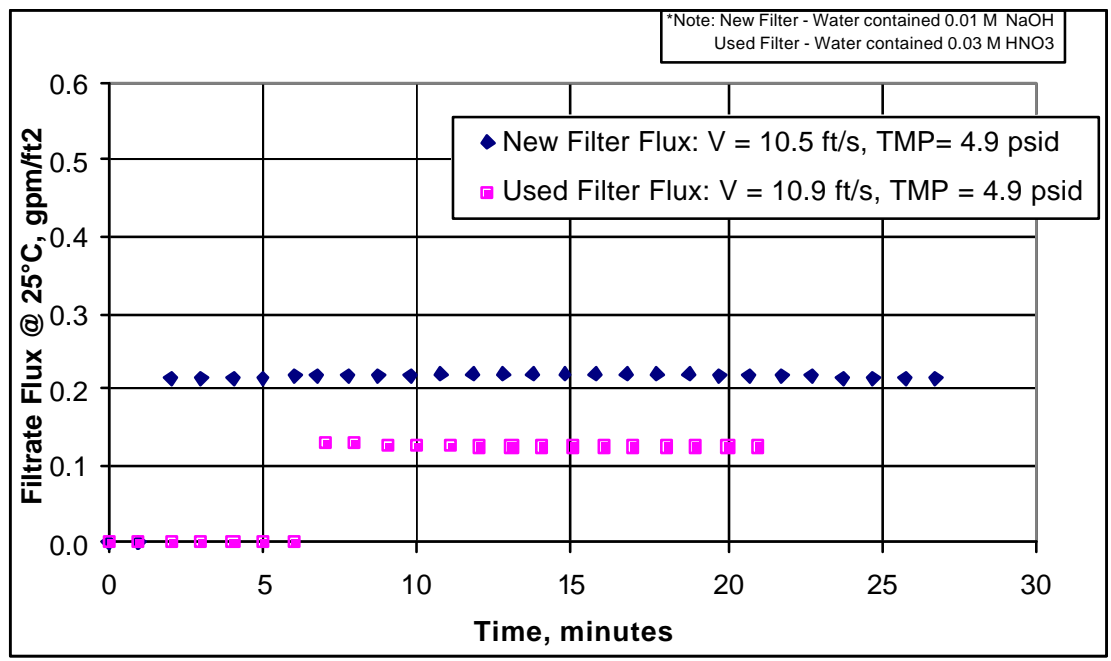

Figure 20. Water tests made on the new filter and after filtering with the standard slurry.

The water was filtered and deionized. The related data files are:

Diamonds=test on the new filter, and water had $0.01 \mathrm{M} \mathrm{NaOH}$ : PreCond 1a Squares=test after standard slurry, and water had $0.03 \mathrm{M} \mathrm{HNO}_{3}$ : PreCond 3

For example, see Fig. 21. After the standard slurry test and after rinsing the slurry with water, a fresh charge of IW was put into the test rig to do the water tests (PreCond 3a, 3b: squares in Fig 21, and 3c). Since the flux was very low, the test rig was cleaned with more acid and the runs were repeated (PreCond 3a;Rev.1, 3b;Rev1:diamonds in Fig. 21, and 3c) but the water was left slightly acidic $\left(0.03 \mathrm{~N} \mathrm{HNO}_{3}\right)$. Both test runs in Fig. 21 were done at approximately the same flow conditions, therefore it was only the addition of caustic that made the difference. The presence of solids is evident from the lower data set in Fig. 21 by the drop in filtrate flux with time. As time goes by, the solids in the water are slowly accumulating on the surface of the filter. 


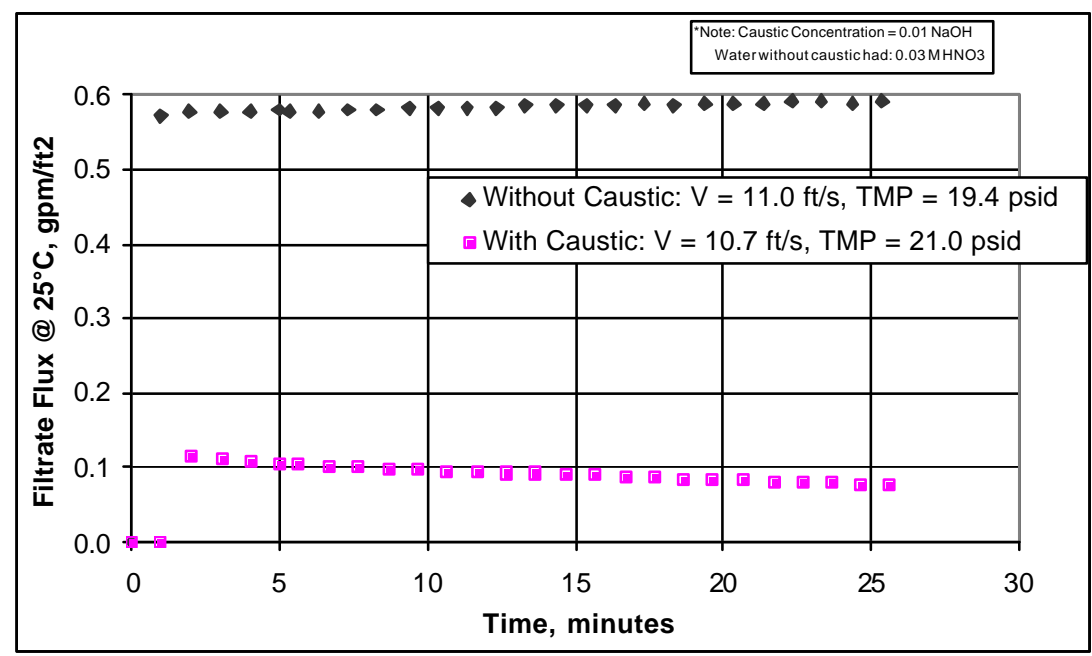

Figure 21. Water tests with and without caustic.

The water was filtered and deionized. The related data files are:

Diamonds=water had $0.03 \mathrm{M} \mathrm{HNO}_{3}$ : PreCond 3b, Rev1

Squares=water had 0.01 M NaOH: PreCond 3b

The effect of adding caustic to a freshly cleaned and rinsed filter is further illustrated by Fig. 22. After Batch 3 testing was complete in November 2002, the test rig sat idle for about 3 months, until February of 2003, while waiting for Batch 4 simulant and the new precipitation mixing equipment to be ready. Just before Batch 4 testing began, the test rig was once again "baselined" with inhibited water. Those data are shown by the open squares in Fig. 22. Not only was the flux low for water, it was lower than any of the previously inhibited water test runs, see Fig. 19.

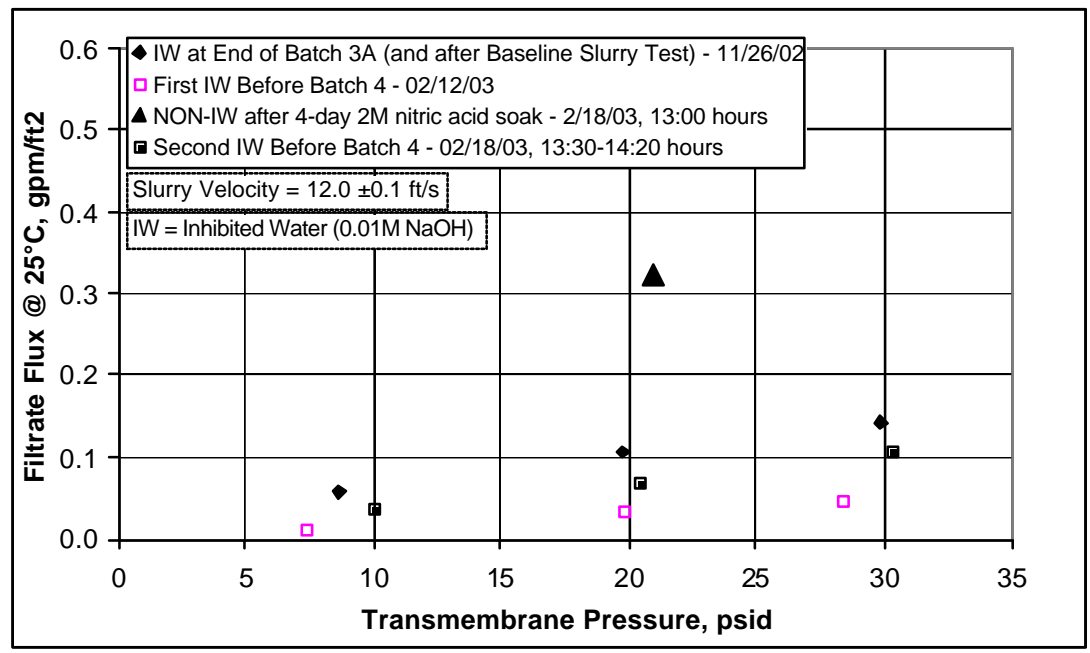

Figure 22. Water tests with and without caustic. Tests done between Batches 3 and 4. Large triangle was obtained with filtered ( 0.1 micron) and demineralized water only. For all other data the water had $0.01 \mathrm{M} \mathrm{NaOH}$

The filled diamonds in Fig. 22 are the water data at the end of Batch 3A testing done on $11 / 26 / 2002$. During time between the end of Batch 3A testing until next water data were 


\section{WSRC-TR-2003-00204, REV. 0}

SRT-RPP-2003-00087, REV. 0

taken on $2 / 12 / 2003$, the filter was sub merged in inhibited water. It is not known why the data were lower. In an attempt to bring the flux up, the filter was soaked in $2 \mathrm{M}$ nitric over a 4-day period ${ }^{\dagger}$ (2/14/03 to 2/17/03). Immediately before doing a new set of inhibited water runs an extra water test was done before adding the caustic. The single filled triangle at a TMP=20 psid shows the flux increased from about $0.05 \mathrm{gpm} / \mathrm{ft}^{2}$ to just above $0.3 \mathrm{gpm} / \mathrm{ft}^{2}$ ! Caustic was subsequently added to have a concentration of $0.01 \mathrm{M} \mathrm{NaOH}$ and the full set of water runs was done. The flux immediately dropped and at TMP $=20$ psid the flux was approximately $0.075 \mathrm{gpm} / \mathrm{ft}^{2}$, which fell between the post Batch $3 \mathrm{~A}$ water results and the water results of $2 / 12 / 02$. The 4 day cleaning improved the flux slightly, but it still did not return to the November 2002 flux of just above $0.1 \mathrm{gpm} / \mathrm{ft}^{2}$. It seems that unless every single surface is cleaned in this type of filtration system, the addition of caustic will always reduce the filtrate flux.

\subsection{Water Runs before and after Preconditioning with AN-107 Slurry Simulant}

To put the filter in a "steady state" condition, it was preconditioned with a used slurry simulant (AN-107) so that its performance would be closer to a "used" filter. As for all other slurry tests the filter was checked before and after the slurry run for its water flux. Figure 23 depicts the data. Like the data of top curve in Figure 21, no caustic was added to the water to prevent solids from precipitating out of solution. The top curve of Fig. 23, (diamonds) was obtained just before running slurry through the filter. The bottom curve (circles) represent the filtrate flux after the filter experienced the slurry for many hours and underwent acid cleaning. The lower post-slurry curve must be attributed to some of the slurry not being removed with the acid.

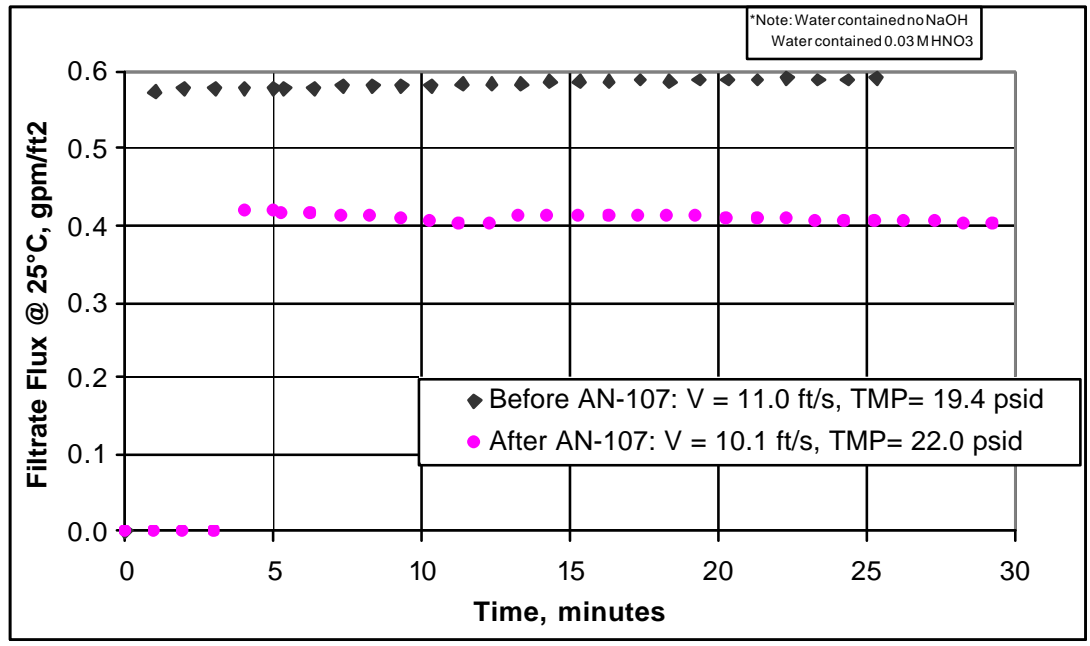

Figure 23. Water tests after cleaning the filter.

The water was filtered and deionized. The related data files are:Diamonds=water had $0.03 \mathrm{M} \mathrm{HNO}_{3}$ : PreCond 3b, Rev1 Circles=water had $0.03 \mathrm{M} \mathrm{HNO}_{3}$ : PreCond 6

\footnotetext{
${ }^{\dagger}$ This cleaning was atypical. Generally, the filter would be subjected to two 90 -minute cleanings with $2 \mathrm{M}$ nitric flowing at $12 \mathrm{ft} / \mathrm{s}$ and a TMP $=20$ psid. However, the upcoming Batch 4 slurry was expected to have poor filterability because of its preparation with poor mixing during the precipitation phase simulant using a Pulse Jet Mixer. SRTC and the RPP customer decided to clean the filter more thoroughly.
} 


\subsection{Water Runs before and after AN-102R2, Batch 3C Surry Simulant}

Just after the preconditioning slurry test and before the first Batch 3 test (3C) the filter was tested with water again. Figure 24 shows the data. These data sets are unique in that the test rig had a very thorough cleaning before the water test. During the first acid cleaning, after the preconditioning test, the coating on the impellers of the two slurry pumps began to break apart. It turned out that the 3-hour acid cleaning fatally degraded the pump liners, requiring replacement and upgrading of the liners.

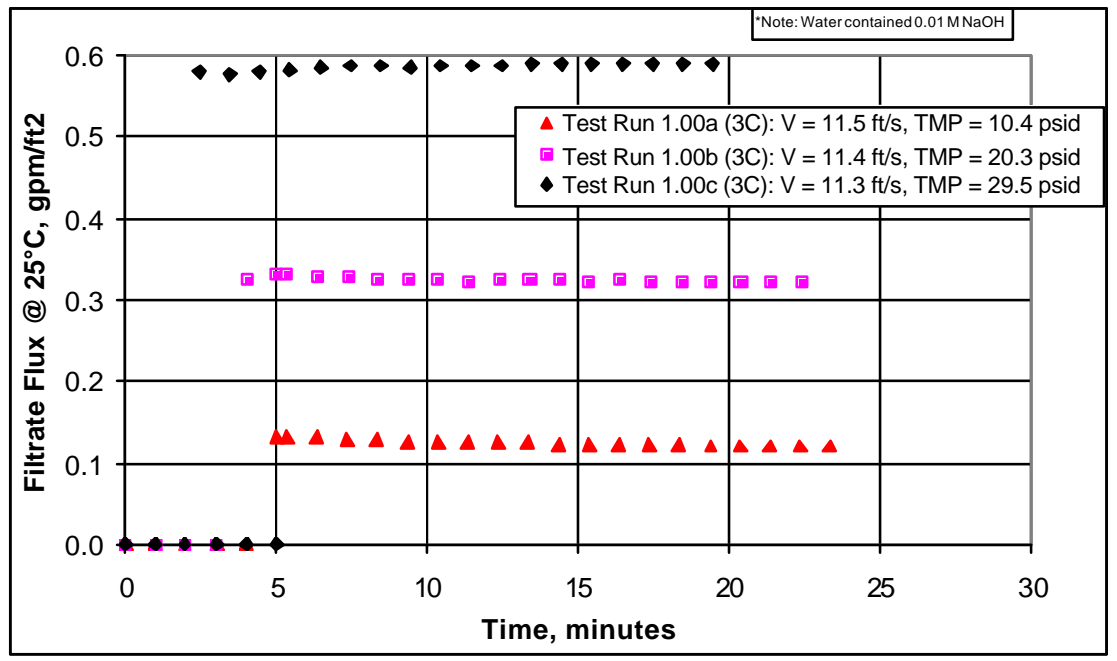

Figure 24. Water tests before test slurry AN-102R2, Batch 3C.

The water was filtered and deionized.

The related data files are:

Triangles=water had 0.01 M NaOH: Run 1a-3C

Squares=water had 0.01 M NaOH: Run $1 \mathrm{~b}-3 \mathrm{C}$

Diamonds=water had 0.01 M NaOH: Run $1 \mathrm{c}-3 \mathrm{C}$

During the replacement, the entire test rig underwent a thorough cleaning to remove all vestiges of the old pump liner. When it came time to test the filter with water again, IW water was used because it was thought that there would not be any solids in the slurry loop to precipitate when caustic was added. Indeed, this seems to be the case because the filtrate fluxes were relatively high, e.g., compare the middle data set of Fig. 24 (squares) to the data set with squares in Fig. 21, which had the same flow conditions, and had the same amount of caustic addition, i.e., 0.01 M NaOH. Also, evident in Fig. 24 is that the filtrate flux remained constant during the $20+$ minutes of the tests, indicating the lack of insoluble solids.

\subsection{Water Runs before and after AN102, Batch 3B Slurry Simulant}

In contrast to the data in Fig. 24, Fig. 25 indicates a significant reduction in filtrate flux. There are three significant features to note: 1. The lower filtrate flux as compared to Fig. 23, 2. the almost independence on TMP, and 3. that the flux decreases with time. 
WSRC-TR-2003-00204, REV. 0

SRT-RPP-2003-00087, REV. 0

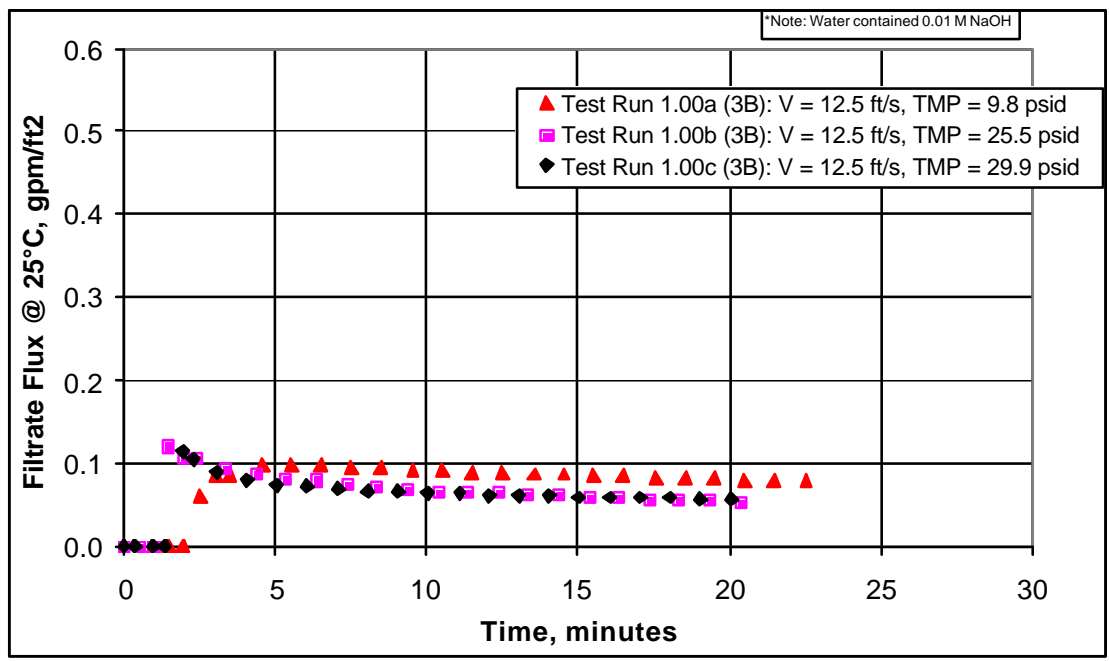

Figure 25. Water tests before test slurry AN-102R2, Batch 3B or after Batch 3C.

The water was filtered and deionized.

The related data files are:

Triangles=water had 0.01 M NaOH: Run 1a-3B

Squares=water had 0.01 M NaOH: Run $1 \mathrm{~b}-3 \mathrm{~B}$

Diamonds=water had 0.01 M NaOH: Run 1c $-3 \mathrm{~B}$

The inhibited water data in Fig. 25 were obtained between two AN-102R2 Batches, i.e., 3C and 3B. As per the test specification (Townson, 2002a) requirements, the water was inhibited with $0.01 \mathrm{M} \mathrm{NaOH}$, but as shown above, the caustic may have caused precipitation of solids that were left over from the 3 hours of nitric acid cleaning or the standard slurry test runs. Whether from precipitation or another cause, the filtrate flux was significantly reduced from clean test rig conditions (Fig. 24).

\subsection{Water Runs before and after AN-102R2, Batch 3A and 4A Slurry Simulant}

The water results after other slurry tests, e.g. Batches $3 \mathrm{~A}$ and $4 \mathrm{~A}$, were similar to those shown in Fig. 25, as can been seen from average data shown in Fig. 19. Those data are not shown here but the entire set of data can be found in Appendix A.

\subsection{Relationship between Water and Slurry Filtrate Fluxes}

One other important thing to note on the relationship of filtrate flux between water and slurries is that there appears to be none. This is described in the following two bullets:

- For the water test just before the testing of AN-102R2, Batch 3C, Fig. 19 and Fig. 24 show the highest filtrate flux was approximately $0.6 \mathrm{gpm} / \mathrm{ft}^{2}$ at an average slurry velocity of $11.3 \mathrm{ft} / \mathrm{s}$ and a TMP of $29.5 \mathrm{psid}$. Batch $3 \mathrm{C}$ slurry dewatering (Test 1.16 done on 1-3 Oct. 2002) with a velocity of $11.5 \mathrm{ft} / \mathrm{s}$ and a TMP of $41.8 \mathrm{psid}$, the filtrate flux started at $0.055 \mathrm{gpm} / \mathrm{ft}^{2}$. That is, the slurry filtrate flux started at an order of magnitude LESS than the water flux. 
- For the water test just before the testing of AN-102R2, Batch 3B, Fig. 19 and Fig. 25 show the highest filtrate flux for all three tests was under $0.1 \mathrm{gpm} / \mathrm{ft}^{2}$ at an average slurry velocity of $12.5 \mathrm{ft} / \mathrm{s}$ and a TMP from 9.8 to $29.9 \mathrm{psid}$. Batch 3B slurry dewatering (Test 1.16 done on $22-23$ Oct. 2002) with a velocity of $12.1 \mathrm{ft} / \mathrm{s}$ and a TMP of $40.5 \mathrm{psid}$, the filtrate flux started at $0.110 \mathrm{gpm} / \mathrm{ft}^{2}$. That is, the slurry filtrate flux started ABOVE the water flux, which further supports the theory that solids can stay in the test rig.

\subsubsection{Baseline with Standard Slurry (5 wt\% $\left.\mathrm{SrCO}_{3}\right)$}

Using a standard slurry to determine the level of cleanliness of the cross-flow filter was requested by the RPP-WTP customer. This standard slurry was a $5 \mathrm{wt} \%$ solution of Strontium Carbonate in water. Before making the slurry the water was to be deionized, filtered with a 0.1 - micron absolute filter, then made caustic to $0.01 \mathrm{M} \mathrm{NaOH}$.

The purpose of using a standard slurry was to determine if the filter, after being used and after it was cleaned with acid, would return to the same level of cleanliness. Using a standard slurry, instead of just water, was preferred because water generally does not contain any significant insoluble solids that will challenge the filter; therefore, it is not a true measure of filter performance. In concept, using a standard slurry is a good idea, however, in reality the filtrate flux obtained with any slurry is highly dependent not only on the level of filter cleanliness, but also on the slurry itself. The particles in the slurry are very important as to their size, shape, ability to deform, time stability, stickiness, etc.

The objective in conducting a standard slurry test before and after each test was to show that the filter returned to its original conditions before filtering. In this way permanent depth fouling could be measured. However, the variation from run to run was too large to make such a determination, except when the filter was new and after its first use. Figure $26^{\dagger}$ shows those data.

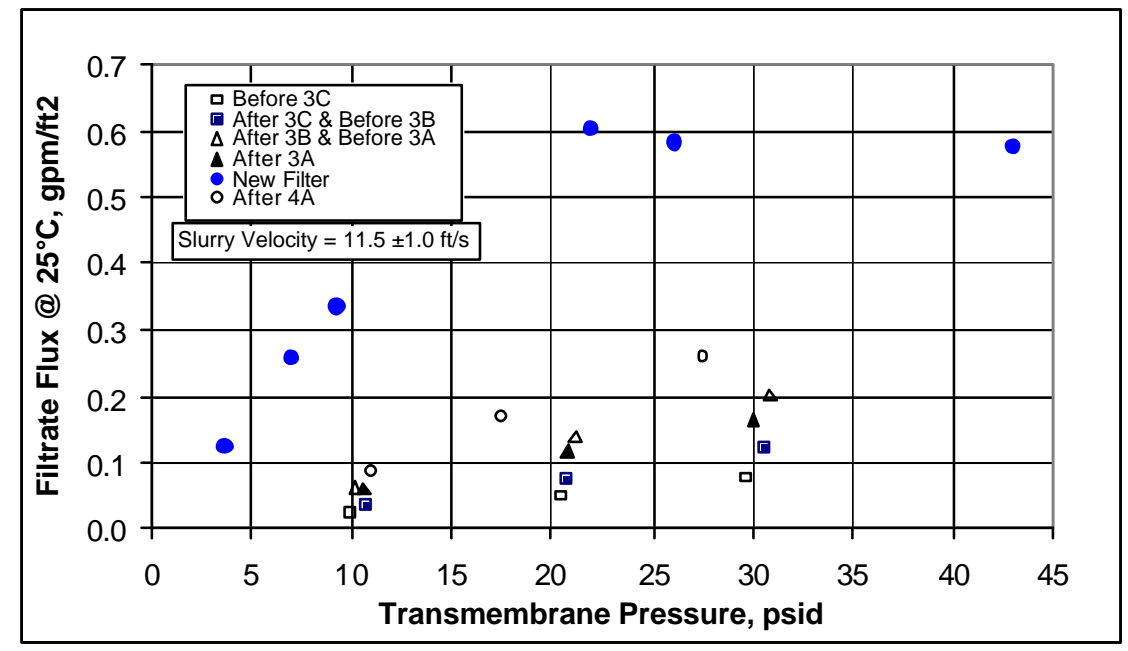

Figure 26. New and Used Filter Standard Slurry Filter Tests

\footnotetext{
${ }^{\dagger}$ The data for all the figures in this section can be found in Appendix B.
} 
A distinct feature in Fig. 26 is the large difference between the new-filter data and all the rest. With respect to the new-filter data, all used-filter data can be judged as the same. That is, the variation in the four sets of data is small compared to their filtrate flux magnitude, as compared to the new filter. This argument is made stronger by realizing that with a flux measurement uncertainty of approximately $5.4 \%$, the variation among the non-new filter data is still significant, but the significance is not large.

An interesting aspect of the new-filter data is the almost linear increase in flux with TMP up to $20 \mathrm{psid}$, at a constant slurry velocity of approximately $11 \mathrm{ft} / \mathrm{s}$. To understand this effect, see Fig. 27, which is the time data for new-filter data in Fig. 26. For the three test runs with the lowest TMP, below 10 psid, it appears as if there is no slurry at all. It is as if just a liquid is flowing because the filter is not challenged at all. As the TMP increases to above 20 psid the typical crossflow Filtrate Flux vs. Time decay appears. This indicates that the first three points for the new-filter data in Fig. 26 represent the constant filtrate flux value, while the last three points represent an average of the decaying filtrate flux. It is possible the TMP=20 psid cut-off point indicates that fast settling $\mathrm{SrCO}_{3}$ particles will not accumulate on the vertical filter surface until the TMP > 20 psid. (Note, from a well mixed condition the standard slurry solids settle out by gravity in only 1 minute and the particle density is $3.7 \mathrm{~g} / \mathrm{ml}$.). Another point of interest is for TMP> $20 \mathrm{psid}$, where it appears that the filtrate flux is independent of TMP. The last three data points for the new filter in Fig. 26 are statistically the same. This similarity tends to imply that at 11 to $12 \mathrm{ft} / \mathrm{s}$ of slurry velocity the slurry cake cannot build up further (or is compressed) with increasing pressure, only with increasing time. As time increases, the filter cake becomes thicker, but for different TMP, above 20 psid, the thickness is the same for a given time period.

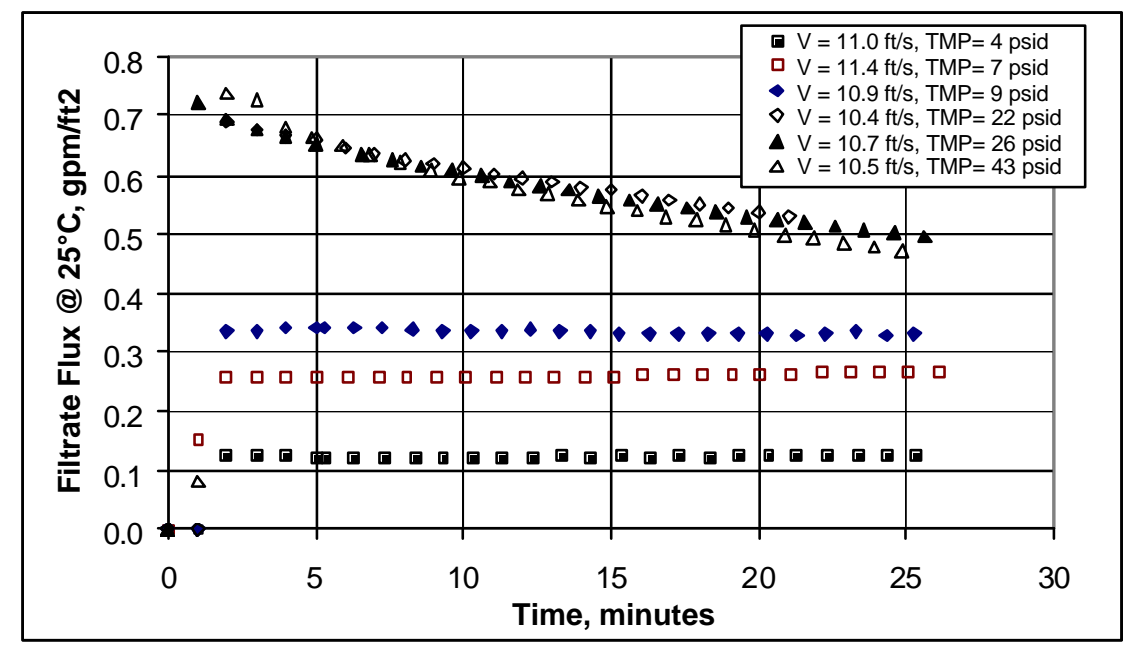

Figure 27. Filtering with Time After a Backpulse for the New Mott Filter

Because of the fast settling $\mathrm{SrCO}_{3}$ particles, and their difficulty in forming a filter cake (or ease in being compressed), this material may not be the best candidate for a standard. For the used filter, after it had experience several different slurries, and was cleaned, the temporal 
data are similar to the new filter, see Fig.28. The average values for these data are the black triangles in Fig. 26.

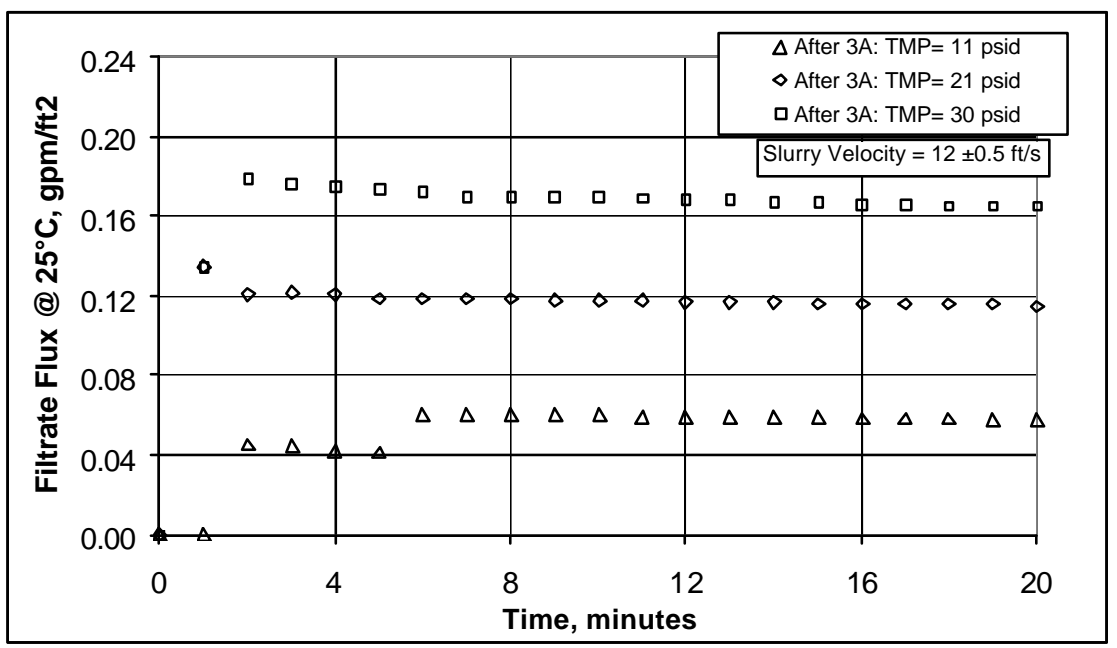

Figure 28. Filtering with Time After a Backpulse for the Used Mott Filter

The lowest data set shows that the filter is not being challenged, as well as the mid data set. However, the highest does show some reduction in flux with time. All other data sets for the used filter are similar, but the overall filtrate flux magnitude changes with the level of filter cleanliness.

A closer look at the used filter data is shown in Figure 29. Before and after each test with Batches 3 and 4 slurries, the standard slurry tests were done. To better understand the series of events, note the chronological order of the tests, i.e., 3C, 3B, 3A, and 4A. There were a total of 5 standard slurry tests.

As stated before, there is some significant difference among the five sets of data, but not by much, when compared to the new filter results shown in Fig. 26. In chronological order the earliest is the lowest set of data, before testing any of the slurry batches (i.e., before Batch 3C). The next lowest set was after completing sub-Batch $3 \mathrm{C}$, but just before doing the second sub-Batch, 3B. The second highest set was obtained just before doing the last sub-Batch, i.e., 3A. Then the next set, which was just after 3A, was lower than the pre-3A test. This implies that time is not an issue, but that it is the level of cleanliness of the filter, which is important. That is, just before each standard slurry, the filter was cleaned with $2 \mathrm{M}$ nitric acid for 3 hours. The acid does clean the filter but the filter does not return to its "new" state, as is evident in Fig. 26. How well the filter is cleaned is a function of several factors, like the level of fouling, and the type of material that is fouling the filter. The filter flux is very sensitive to any residual fouling or solids still in the slurry flow loop, therefore varying results of standard slurry testing are not surprising. The variation seen in the five data sets shown in Fig. 29 attest to the different level of filter cleanliness. This idea is further reinforced with the data obtained after the Batch 4A test. Just before the 4A test, the filter was soaked in $2 \mathrm{M}$ nitric acid for 4 days, which may have left it slightly cleaner and therefore the baseline data were 
slightly higher. With the limited cleaning protocol currently in practice, a standard slurry set of data cannot be expected to be more reproducible than that shown in Fig. 29.

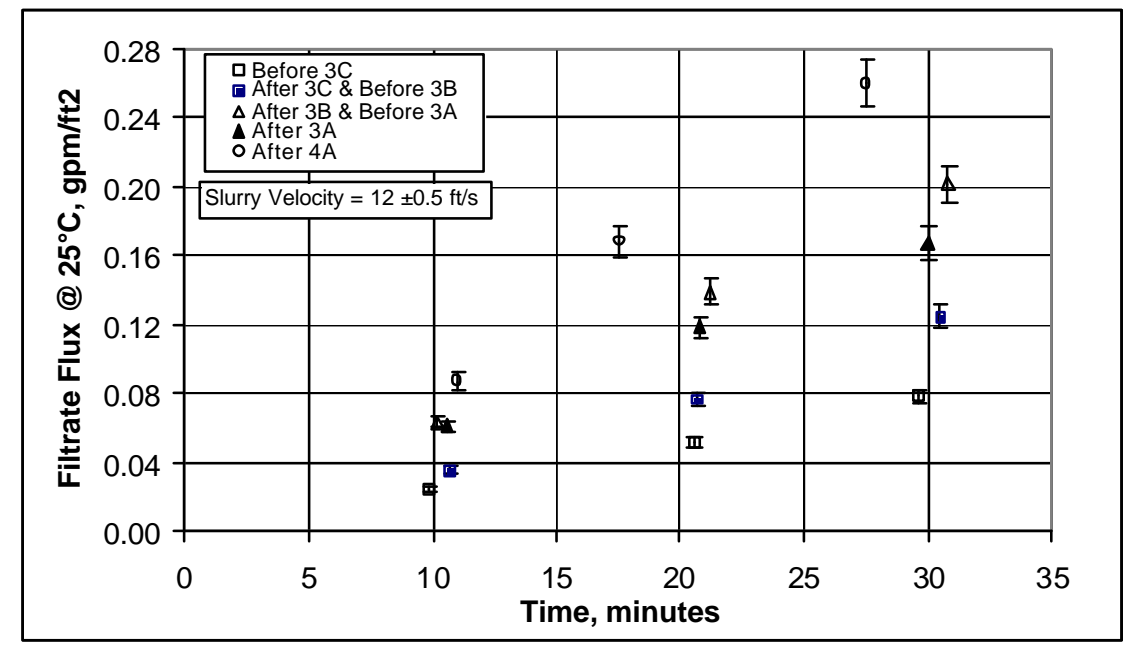

Figure 29. Used-Filter Standard Slurry Filter Tests

\subsubsection{Cleaning the 0.01-micron Mott Filter with 2 M Nitric Acid}

To clean the cross-flow filter a concentration of $2 \mathrm{M}$ nitric acid was requested by the RPPWTP customer; no other requirements were made except to document the results, which are herein recorded. No method to clean the filter was specified, so the one used was based on early bench-top filter testing by using two batches, each circulating for 90 minutes. Other researchers (i.e., Zamecnik, 2003) have used longer cleaning intervals, like soaking for 24 to 48 hours, using different chemicals, or different nitric acid concentrations, but other methods are beyond the scope of this task. Being limited to $2 \mathrm{M}$ nitric acid, then the 3-hour cleaning cycle was used because it was thought that the actual plant would not want to use longer cleaning periods due to expensive down time.

The purpose of cleaning the filter is to remove material, e.g., sludge, slurry, solids, etc., which would cause unacceptable filter fluxes, or operating pressures, that cannot be improved by either simply flushing with water or backpulsing. Backpulsing is the process of temporarily reversing the direction of filtrate flow by pulsing a fixed quantity through the filter wall to knock off built up filter cake from the inside surface of the filter tube. This material build-up occurs when fine slurry material is lodged in the filter wall, which is usually referred to as depth fouling. The wall thickness of the porous tubes used is 0.0625 inch or $1.5875 \mathrm{~mm}$, which is 15,875 times larger than the nominal pore size of 0.1 micron; that is, there is considerable space in which to deposit solids.

To prepare the filter for use with the test slurry (AN-102R2), it was preconditioned with a similar slurry, AN-107, in order to start with a "used" filter, which would be more typical of daily plant operation. That is, a new filter was first subjected to AN-107 for a predetermined time interval (several days), then the filter was cleaned with $2 \mathrm{M}$ nitric acid. For that first cleaning, the target acid flow parameters were an axial filter velocity (V) of $11 \mathrm{ft} / \mathrm{s}$ and a 
transmembrane pressure (TMP) of 20 psid. Figure $30^{\dagger}$ shows the actual parameters used (filled and open diamonds). During the first period (filled diamonds), the filter was challenged during the first 10 minutes with undissolved solids and then the filtrate flux improved from about $0.42 \mathrm{gpm} / \mathrm{ft}^{2}$ to above $0.45 \mathrm{gpm} / \mathrm{ft}^{2}$. After changing the nitric acid solution, the second period (open diamonds), there appears to be a reduced presents of solids, since the filter was not challenged as much. While there seemed to be a slight improvement in the filter cleanliness during the second period it appears that most of the cleaning occurred during the first period.

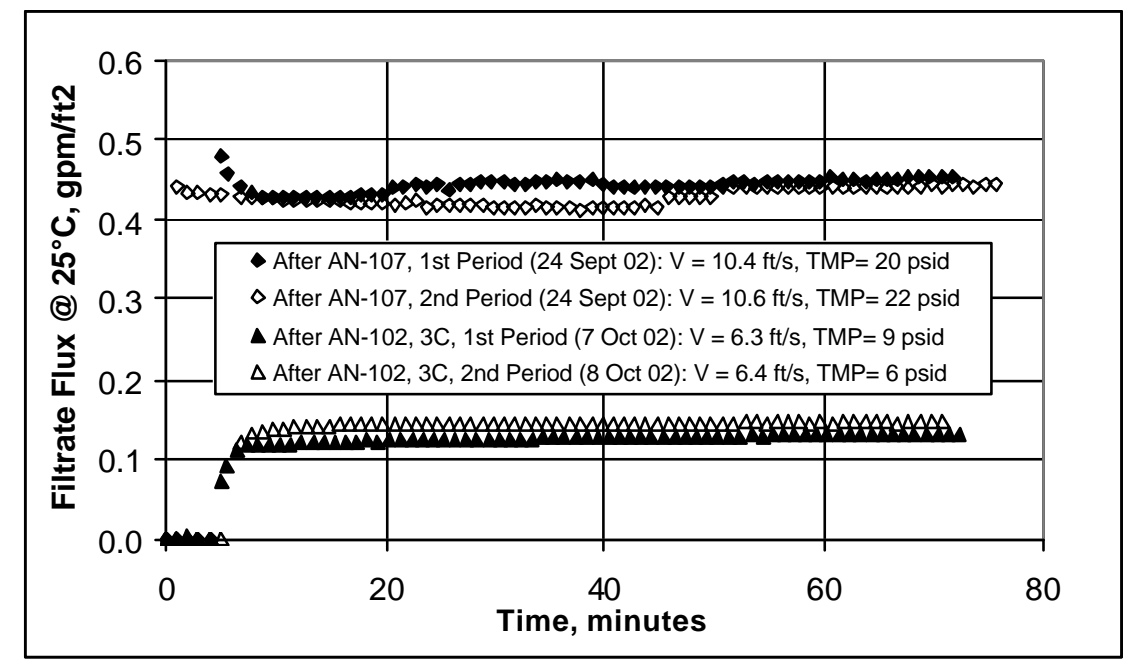

Figure 30. $2 \mathrm{M}$ nitric acid cleaning after AN-107 \& AN-102R2, Batch 3C

Unfortunately, the nitric acid cleaning after the preconditioning test damaged the pumps liners of the experimental test rig. While waiting for replacement liners that were of a material more impervious to nitric acid the filter had to be cleaned using a separate cleaning loop, which prevented acid from entering the pumps. That cleaning loop only had a small motor, which could not supply the energy necessary to attain the original flow parameters. Figure 30 shows that both $\mathrm{V}$ and TMP used for the Batch $3 \mathrm{C}$ acid cleaning were considerably less than those used for the preconditioning run. The post Batch $3 \mathrm{C}$ cleaning data are slowly increasing with time, but a plateau is not reached indicating that the filter may not have been brought to the same level of cleanliness as seen for the AN-107 cleaning.

Figure 31 shows a more dramatic effect from cleaning. After the second Batch 3 slurry test (3B), the first-period nitric acid cleaning shows how the filter was challenged during the first 60 minutes, after which the filtrate flux increased from 0.26 to $0.37 \mathrm{gpm} / \mathrm{ft}^{2}$. After replacing the nitric acid solution with a new charge, the second-period cleaning showed an improved filtrate flux and the filter was only mildly challenged for the first 15 minutes, then the flux increased to a steady value of approximately $0.56 \mathrm{gpm} / \mathrm{ft}^{2}$. This cleaning cycle implies that the two ninety-minute periods were necessary to clean the filter, but that further cleaning may not result in any significant filter performance. Note, the specified axial velocity was

\footnotetext{
${ }^{\dagger}$ The data for all the figures in this section can be found in Appendix C.
} 
increased by the customer from $\mathrm{V}=11$ to $\mathrm{V}=12 \mathrm{ft} / \mathrm{s}$ and the high TMP for the first period, Batch 3B, cleaning, i.e., 43 psid, was done in error.

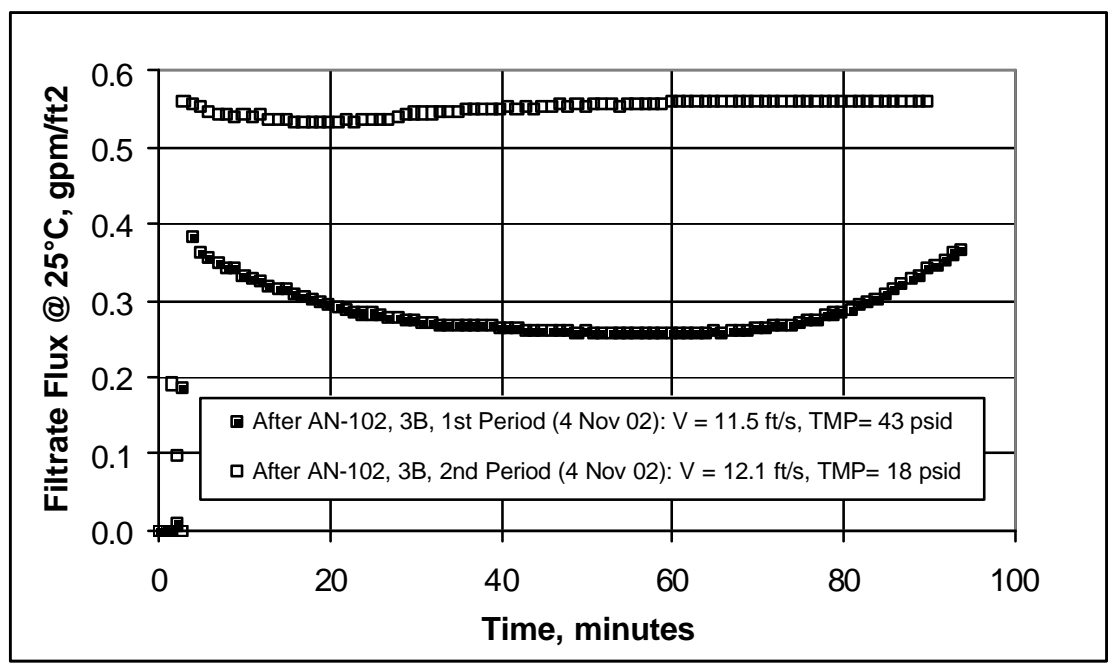

Figure 31. 2 M nitric acid cleaning after AN-102R2, Batch 3B

Figure 32 depicts another set of cleaning data, with the first period having the largest filtrate flux fluctuations and the second period indicating a basically clean filter. During the first cleaning period after Batch $3 \mathrm{~A}$ the flux appears rather steady for about 35 minutes, but then it dropped for about 15 minutes before increasing.

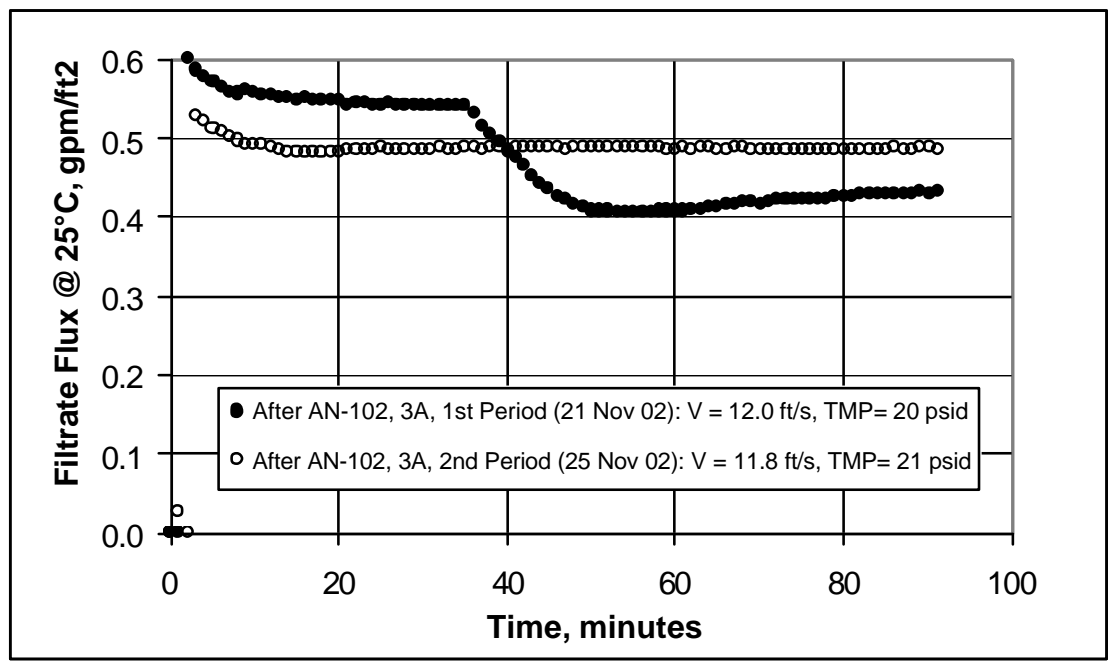

Figure 32. $2 \mathrm{M}$ nitric acid cleaning after AN-102R2, Batches 3A

The reason for the sudden drop is not known, however, the test rig had a lot of small locations where slurry could have been lodged. It is possible that some slurry broke loose within the test loop and began challenging the filter. Once the slurry was circulating the solids would be dissolving in the nitric acid causing the filtrate flux to rise. During the 
second cleaning period the slurry stops challenging the filter after 10 minutes and comes to steady state. This implies that there must have been some small amount of solids that were not dissolved by the nitric acid, but there was not enough to continue challenging the filter.

Just before doing the last AN-102R2 slurry test, Batch 4A, the filter was soaked for four days in $2 \mathrm{M}$ nitric acid in an attempt to obtain better water fluxes. The water runs displayed the lowest filtrate fluxes since the 0.1 micron filters were first used in September 2002 (see the section on water runs). Figure 33 shows the results.

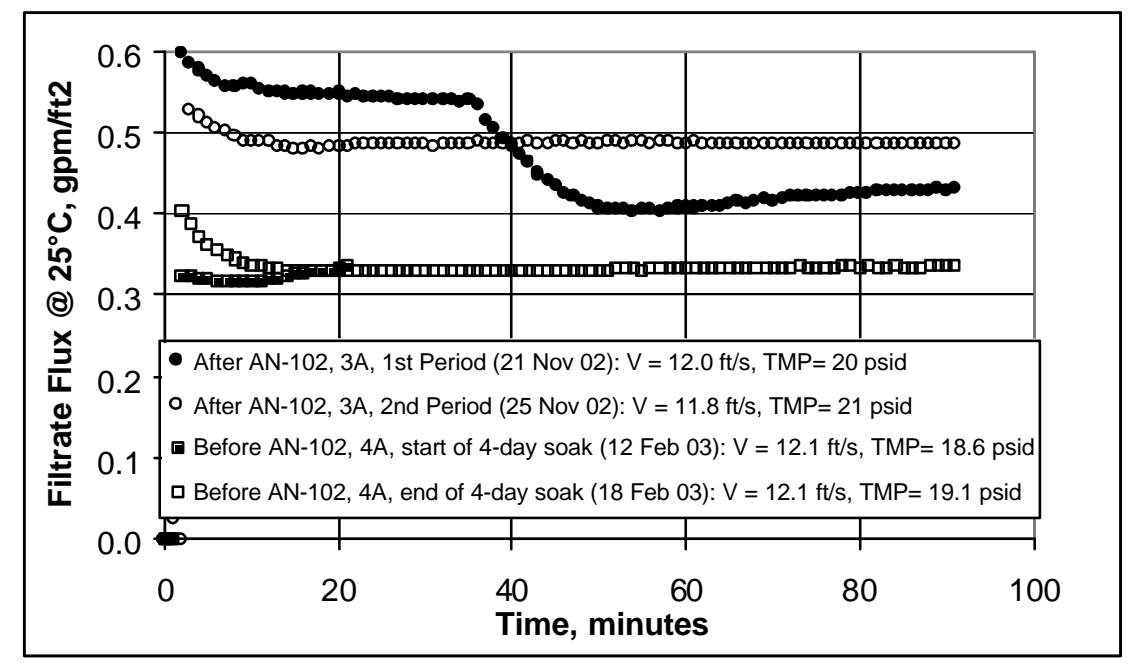

Figure 33. $2 \mathrm{M}$ nitric acid cleaning after AN-102R2, Batches 3A \& 4

Some interesting features of these pre-Batch $4 \mathrm{~A}$ cleaning data are the lack of significant effects of the 4day soaking and the lower filtrate flux values compared to the cleaning values done in November 2002. The test rig sat idle for approximately 3 months between the last Batch 3 slurry test (3A) and the acid cleaning done before the pre-Batch 4A test. During that time period, the filter was submerged in inhibited water. However, after the acid cleaning on 25 Nov 02 a standard slurry test $\left(5 \mathrm{wt} \% \mathrm{SrCO}_{3}\right.$ ) was performed, also on 25 of November. While the test rig was rinsed out several times and had final inhibited water test runs, it is possible that pockets of slurry remained in the test rig. This could have contributed to the lower filtrate flux obtained on $18 \mathrm{Feb} .03$, as shown in Fig. 33. The fact that the postsoak (18 Feb.) results show the filter was challenged during the first 15 minutes indicate that there were still some insoluble solids in the acid which coated the filter. Furthermore, since the pre-soak (12 Feb.) and post-soak (18 Feb.) results indicate approximately the same steady state flux value, then the soak itself did very little to dissolve the solids remaining in the acid or lodged in the filter wall.

Figure 34 shows some confusing results. After the last batch of AN-102R2, i.e., 4A, was complete the filter was cleaned again. The initial 90-minute cleaning gave similar flux results as the cleaning flux just before Batch 4A was tested. The filled circles show how the filter was initially challenged and as the solids dissolved the filtrate flux first leveled out, which then increased to asymptotically approach the before-Batch 4 A cleaning flux. Since this first period 
of final cleaning was at the end of a workweek the test rig was emptied of acid and filled with deionized and filtered water. On the following Monday nitric acid was added to the water to bring the concentration to $2 \mathrm{M}$ in order to begin the second 90-minute period of cleaning. Surprisingly, the filtrate flux was about double that from the previous period. Moreover, the very small flux drop during the first 30 minutes indicated the system had very little solids, after which the flux became stable. Somehow, the water soaking, over the weekend, and the second acid cleaning removed some material which caused to lower filtrate flux.

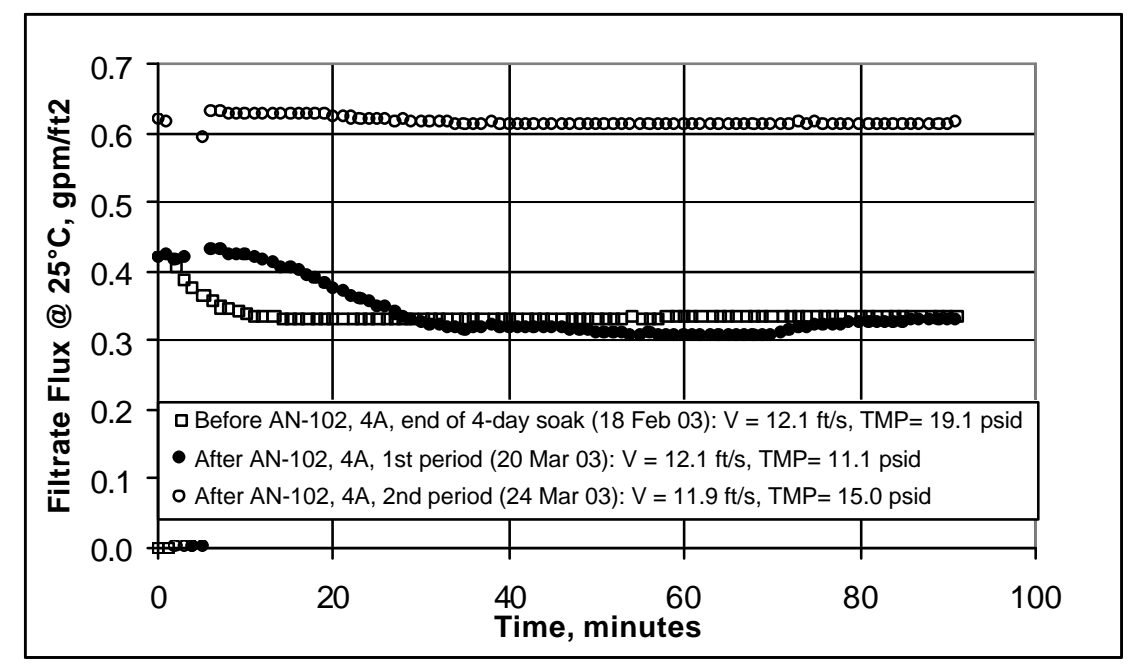

Figure 34. $2 \mathrm{M}$ nitric acid cleaning after AN-102R2, before \& after 4A

To conclude, Fig. 35 shows all the final period nitric acid cleanings that were done for the AN-102R2 simulant slurry tests, including the AN-107 filter preconditioning test.

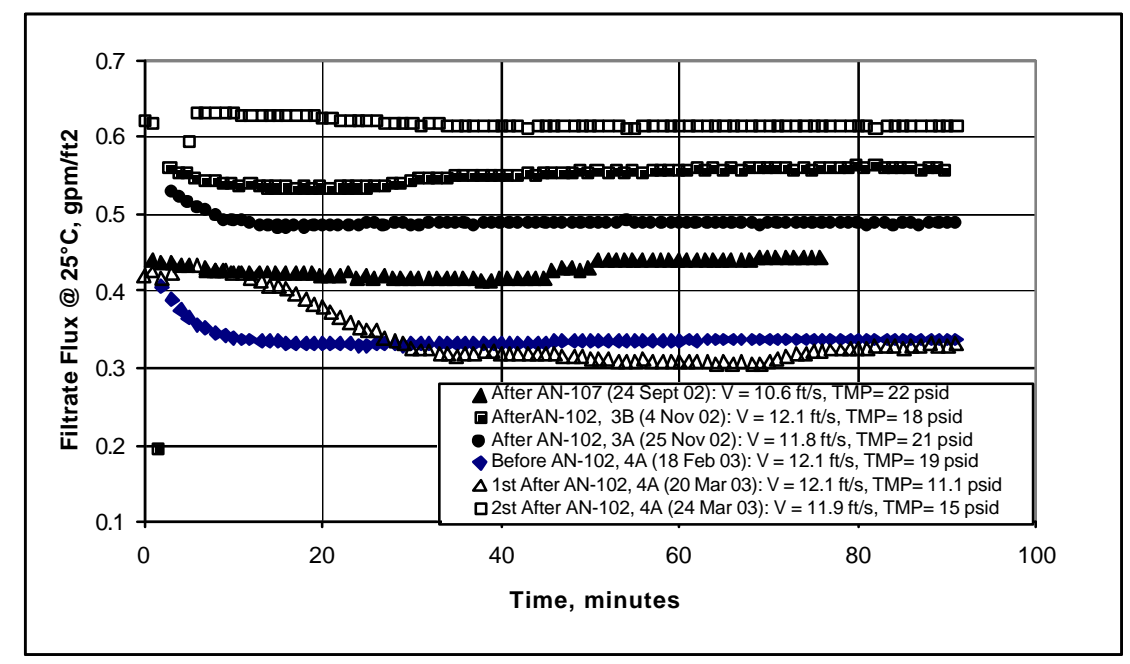

Figure 35. $2 \mathrm{M}$ nitric acid cleaning of the AN-107 and AN-102R2 slurry tests 
Neglecting the cleaning done on the filter after the preconditioning test the filtrate flux of acid chronologically becomes lower. The cleaning flux after the Batch $3 \mathrm{~B}$ test is about $0.56 \mathrm{gpm} / \mathrm{ft}^{2}$, then after the Batch $3 \mathrm{~A}$ test it goes to $0.49 \mathrm{gpm} / \mathrm{ft}^{2}$. Now from Batch $3 \mathrm{~A}$ to Batch $4 \mathrm{~A}$ the test rig sat idle so it was cleaned before 4A. The flux continued its downward trend to $0.33 \mathrm{gpm} / \mathrm{ft}^{2}$. Just after Batch $4 \mathrm{~A}$, the cleaning flux during the first cleaning period was slightly lower at about $0.32 \mathrm{gpm} / \mathrm{ft}^{2}$. This steady flux reduction with time may indicate that some of the solids are not soluble in $2 \mathrm{M}$ nitric acid and are building up in the porous wall of the filter tubes. However, when the second cleaning was done after Batch 4A the flux doubled to close to $0.6 \mathrm{gpm} / \mathrm{ft}^{2}$ ! The reason why is not known, but something had to have been dissolved, which was previously in the system.

\subsubsection{Dewatering of AN-102R2 Slurry Simulant}

To reiterate, the principal reason for the pilot-scale cross-flow ultrafiltration test was to determine the filterability of different preparations of an organic-based precipitated slurry. The slurry was a simulant of waste in the Hanford Tank 241-AN-102. The particular simulant used is referred to as $\mathrm{AN}-102 \mathrm{R} 2$ because its original recipe was revised twice. See the Simulated Waste Slurry section for more information on the recipe.

The test runs described in this section deal with four batches of AN-102R2:

1.16, Batch 3C This AN-102R2 simulant was precipitated under NOC*

1.16, Batch 3B This AN-102R2 simulant was precipitated under BC*

1.16, Batch 3A This AN-102R2 simulant was precipitated under $\mathrm{BC}$ at $25^{\circ} \mathrm{C}$

1.16, Batch 4A This AN-102R2 simulant was precipitated using PJM*

*See Fig. 17 for nomenclature and the number 1.16 refers to test 1.16 which is listed in the task plan (Duignan, 2002a) as the "dewatering" test.

After a slurry was made, all dewatering tests followed the same procedure:

1. Fill the pilot cross-flow test rig with slurry and take initial slurry and filtrate samples

2. Begin filtering while directing filtrate to a storage location so dewatering could commence.

3. Dewater until the insoluble solids concentration was > $15 \mathrm{wt} \%$ (but $>20 \mathrm{wt} \%$ was preferable).

4. Dewater for at least 12 hours and backpulse the filter when the filtrate flux $<0.015 \mathrm{gpm} / \mathrm{ft}^{2}$.

5. Stop dewatering and take final slurry samples.

Each of the dewatering tests result will be discussed in this section. Figure 36 shows the highlight results for each test as well as the simulant properties measured from samples taken before and after all four tests. They will be used throughout this section. 
WSRC-TR-2003-00204, REV. 0

SRT-RPP-2003-00087, REV. 0

\begin{tabular}{|c|c|c|c|c|}
\hline Batch of AN-102R2 Simulant>> & $3 C$ & $3 \mathrm{~B}$ & $3 \mathrm{~A}$ & $4 \mathrm{~A}$ \\
\hline $\begin{array}{l}\text { Date of Test } \\
\text { Duration of Test } \\
\text { Volume of Slurry Dewatered } \\
\text { Mixing Conditions } \\
\text { Slurry Precipitation Conditions } \\
\text { Highest Measured Filtrate Flux } \\
\text { Average Filtrate Flux over duration }\end{array}$ & $\begin{array}{l}1-3 \text { Oct. } 02 \\
46 \text { hours } \\
899 \text { liters } \\
\text { Well Mixed/Agitator } \\
\text { Newly Optimized } \\
0.06 \mathrm{gpm} / \mathrm{ft} 1 \\
0.011 \mathrm{gpm} / \mathrm{ft} 1\end{array}$ & $\begin{array}{l}22-23 \text { Oct. } 02 \\
12 \text { hours } \\
900 \text { liters } \\
\text { Well Mixed/Agitator } \\
\text { Baseline } \\
0.11 \mathrm{gpm} / \mathrm{ft} 2 \\
0.048 \mathrm{gpm} / \mathrm{ft} 2\end{array}$ & $\begin{array}{l}6-7,11 \text { Nov. } 02 \\
29 \text { hours } \\
920 \text { liters } \\
\text { Well Mixed/Agitator } \\
\text { Baseline }\left(\text { but } 25^{\circ} \mathrm{C}\right) \\
0.06 \mathrm{gpm} / \mathrm{ft} 2 \\
0.019 \mathrm{gpm} / \mathrm{ft} 2\end{array}$ & $\begin{array}{l}12-13 \text { Mar. } 02 \\
20 \text { hours } \\
650 \text { liters } \\
\text { Poorly Mixed/PJM } \\
\text { Baseline } \\
0.05 \mathrm{gpm} / \mathrm{ft} 2 \\
0.019 \mathrm{gpm} / \mathrm{ft} 2\end{array}$ \\
\hline \multicolumn{5}{|c|}{ Low Solids Concentrations at the start of dewatering } \\
\hline $\begin{array}{l}\text { Insoluble Solids } \\
\text { Total Solids } \\
\text { Slurry Consistency } \\
\text { Yield Stress } \\
\text { Filtrate Viscosity } \\
\text { Slurry Density } \\
\text { Filtrate Density } \\
\end{array}$ & $\begin{array}{l}0.8 \mathrm{wt} \% \\
33.6 \mathrm{wt} \% \\
3.6 \mathrm{cP} \\
0 \mathrm{~Pa} \\
2.7 \mathrm{cP} \\
1.29 \mathrm{~g} / \mathrm{ml} \\
1.25 \mathrm{~g} / \mathrm{ml} \\
\end{array}$ & $\begin{array}{l}1.2 \mathrm{wt} \% \\
32.7 \mathrm{wt} \% \\
4.0 \mathrm{cP} \\
0 \mathrm{~Pa} \\
3.8 \mathrm{cP} \\
1.29 \mathrm{~g} / \mathrm{ml} \\
1.279 \mathrm{~g} / \mathrm{ml} \\
\end{array}$ & $\begin{array}{l}1.6 \mathrm{wt} \% \\
33.0 \mathrm{wt} \% \\
4.1 \mathrm{cP} \\
0 \mathrm{~Pa} \\
3.7 \mathrm{cP} \\
1.29 \mathrm{~g} / \mathrm{ml} \\
1.276 \mathrm{~g} / \mathrm{ml} \\
\end{array}$ & $\begin{array}{l}1.5 \mathrm{wt} \% \\
33.3 \mathrm{wt} \% \\
4.2 \mathrm{cP} \\
0 \mathrm{~Pa} \\
3.7 \mathrm{cP} \\
1.29 \mathrm{~g} / \mathrm{ml} \\
1.277 \mathrm{~g} / \mathrm{ml} \\
\end{array}$ \\
\hline $\begin{array}{l}\text { PSD [2] (by volume dist.): Mean } \\
\text { PSD (by volume dist.): Mean } \\
\text { PSD (by volume dist.): Std Dev } \\
\text { PSD (by volume dist.): spread } \\
\text { PSD (by number dist.): Mean } \\
\text { PSD (by number dist.): Std Dev } \\
\text { PSD (by number dist.): spread }\end{array}$ & $\begin{array}{l}8.6 \text { micron }(68 \%)[1] \\
2.6 \text { micron }(32 \%)[1] \\
4.8 \text { micron } \\
1 \text { to } 74 \text { micron } \\
2.1 \text { micron } \\
0.7 \text { micron } \\
1 \text { to } 22 \text { micron } \\
\end{array}$ & $\begin{array}{l}8.6 \text { micron (48\%) [1] } \\
2.3 \text { micron }(52 \%)[1] \\
4.3 \text { micron } \\
1 \text { to } 52 \text { micron } \\
1.9 \text { micron } \\
0.5 \text { micron } \\
1 \text { to } 19 \text { micron } \\
\end{array}$ & $\begin{array}{l}9.8 \text { micron (42\%) [1] } \\
2.8 \text { micron (58\%) [1] } \\
4.4 \text { micron } \\
1 \text { to } 26 \text { micron } \\
2.0 \text { micron } \\
0.7 \text { micron } \\
1 \text { to } 19 \text { micron }\end{array}$ & $\begin{array}{l}2.7 \text { micron } \\
1.9 \text { micron } \\
0.8 \text { to } 37 \text { micron } \\
1.8 \text { micron } \\
0.6 \text { micron } \\
<0.7 \text { to } 16 \text { micron }\end{array}$ \\
\hline \multicolumn{5}{|c|}{ High Solids Concentration at the end of dewatering } \\
\hline $\begin{array}{l}\text { Insoluble Solids } \\
\text { Total Solids } \\
\text { Slurry Viscosity } \\
\text { Yield Stress } \\
\text { Filtrate Viscosity } \\
\text { Slurry Density } \\
\text { Filtrate Density } \\
\end{array}$ & $\begin{array}{l}8.4 \mathrm{wt} \%[3] \\
38.6 \mathrm{wt} \% \\
19 \mathrm{cP} \\
7.5 \mathrm{~Pa} \\
1.7 \mathrm{cP} \\
1.31 \mathrm{~g} / \mathrm{ml} \\
1.276 \mathrm{~g} / \mathrm{ml} \\
\end{array}$ & $\begin{array}{l}21.7 \mathrm{wt} \% \\
47.2 \mathrm{wt} \% \\
20 \mathrm{cP} \\
8.6 \mathrm{~Pa} \\
3.7 \mathrm{cP} \\
1.43 \mathrm{~g} / \mathrm{ml} \\
1.285 \mathrm{~g} / \mathrm{ml} \\
\end{array}$ & $\begin{array}{l}25.3 \mathrm{wt} \% \\
49.3 \mathrm{wt} \% \\
24 \mathrm{cP} \\
24 \mathrm{~Pa} \\
4.5 \mathrm{cP} \\
1.45 \mathrm{~g} / \mathrm{ml} \\
1.283 \mathrm{~g} / \mathrm{ml}\end{array}$ & $\begin{array}{l}18.3 \mathrm{wt} \% \\
45.8 \mathrm{wt} \% \\
14.8 \mathrm{cP} \\
7.7 \mathrm{~Pa} \\
3.8 \mathrm{cP} \\
1.38 \mathrm{~g} / \mathrm{ml} \\
1.286 \mathrm{~g} / \mathrm{ml} \\
\end{array}$ \\
\hline $\begin{array}{l}\text { PSD (by volume dist.): Mean } \\
\text { PSD (by volume dist.): Mean } \\
\text { PSD (by volume dist.): Std Dev } \\
\text { PSD (by volume dist.): spread } \\
\text { PSD (by number dist.): Mean } \\
\text { PSD (by number dist.): Std Dev } \\
\text { PSD (by number dist.): spread }\end{array}$ & $\begin{array}{l}6.7 \text { micron }(51 \%)[1] \\
2.5 \text { micron }(49 \%)[1] \\
3.0 \text { micron } \\
<0.7 \text { to } 52 \text { micron } \\
1.9 \text { micron } \\
0.7 \text { micron } \\
<0.7 \text { to } 16 \text { micron }\end{array}$ & $\begin{array}{l}8.2 \text { micron }(13 \%)[1] \\
0.9 \text { micron }(87 \%)[1] \\
0.25 \text { micron } \\
<0.7 \text { to } 44 \text { micron }[4] \\
0.8 \text { micron } \\
0.1 \text { micron } \\
<0.7 \text { to } 2 \text { micron }[4]\end{array}$ & $\begin{array}{l}2.5 \text { micron } \\
1.8 \text { micron } \\
<0.7 \text { to } 26 \text { micron } \\
1.8 \text { micron } \\
0.5 \text { micron } \\
<0.7 \text { to } 13 \text { micron }\end{array}$ & $\begin{array}{l}2.1 \text { micron } \\
0.8 \text { micron } \\
0.9 \text { to } 16 \text { micron } \\
1.8 \text { micron } \\
0.4 \text { micron } \\
0.9 \text { to } 11 \text { micron }\end{array}$ \\
\hline
\end{tabular}

Figure 36. Comparison among the four AN-102R2 dewatering tests

\subsection{Filtering Batch 3C}

Figure 37 is the time plot of the first Batch 3 test, which filtered very poorly and thus was stopped after about 46 hours of continuous filtering. The dewatering test began with the Envelope C-type slurry that was precipitated under what is referred to as "newly optimized conditions." In short, the optimization was done to minimize precipitation reagents of sodium hydroxide, strontium nitrate, and sodium permanganate while still obtaining the needed decontamination factors for strontium and transuranic components. Unfortunately, the reduced concentration of reagents had a negative impact on filterability. 
- Poor Filter Performance with Batch 3C

As stated above, at just under 46 hours of continuous filtering the cross-flow test rig was stopped and the test was terminated. The slurry that started out at about $1 \mathrm{wt} \%$ was only dewatered to an insoluble solids concentration of about $8.5 \mathrm{wt} \%$; far short of the $15 \mathrm{wt} \%$ target. The overall filtrate flux average was $0.011 \mathrm{gpm} / \mathrm{ft}^{2}$ for the 46 -hour test but for the last 25 hours of the test the flux was below that value and for the last 10 hours it remained at $0.005 \mathrm{gpm} / \mathrm{ft}^{2}$, well below the target mean value of $0.02 \mathrm{gpm} / \mathrm{ft}^{2}$. It was decided to repeat the test with another Batch 3 slurry but at the original precipitation conditions where this type (another organic type simulated slurry: AN-107, see Duignan $2000 \mathrm{~b}$ ) of slurry had already been shown to filter well. In fact, two more tests were added, so that now there would be four AN-102R2 tests, instead of two.

Due to the poor filter performance and the inability to concentrate the slurry to $20 \mathrm{wt} \%$ in a reasonable time period, no steady-state filtration runs were done with Batch $3 \mathrm{C}$, i.e., High Solids Concentration Test Runs Nos. 1.17 to 1.30 (Duignan, 2002a). These runs were to determine the filter operational parameters, V and TMP, that would give the highest filtrate flux. Steady state runs were put off until after the next slurry batch, or after the slurry batch that could be concentrated to at least $15 \mathrm{wt} \%$ of insoluble solids.

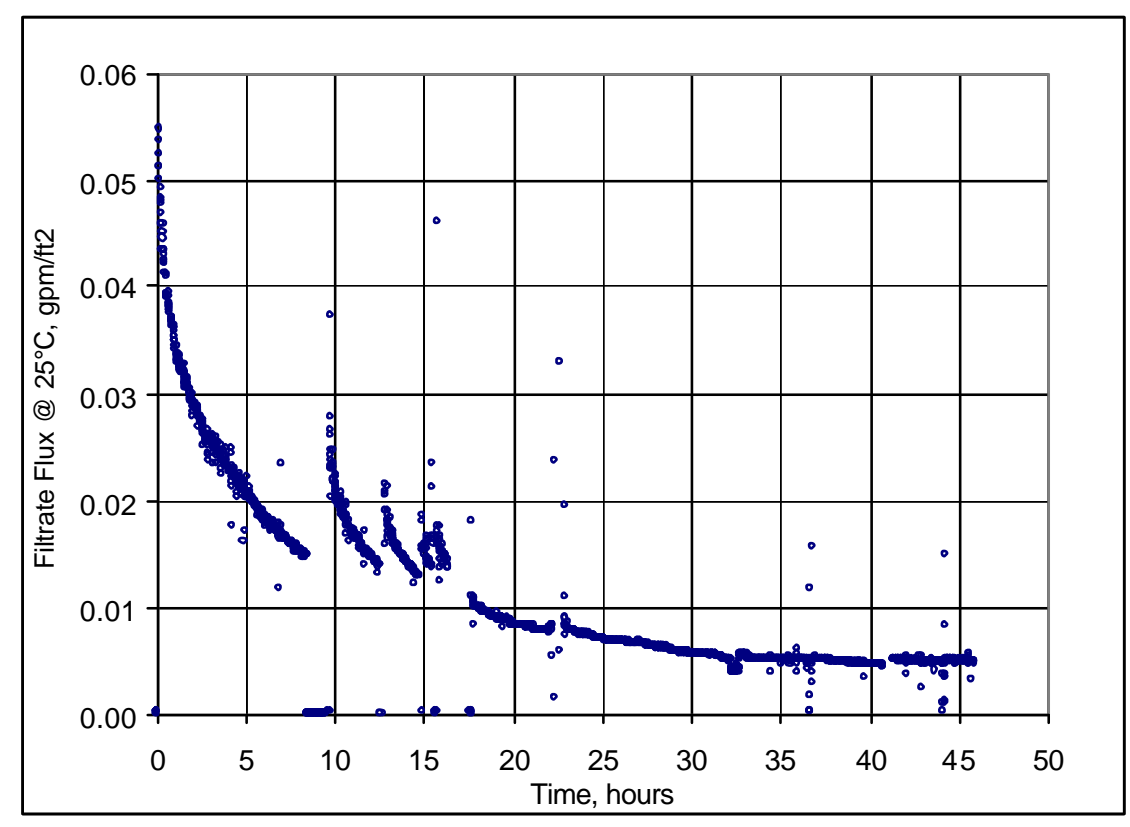

Figure 37. Batch 3C: Dewatering filter flux of AN-102R2 precipitated with the newly optimized conditions at $25^{\circ} \mathrm{C}$.

Over the filtering period the average slurry velocity was $\mathrm{V}=11.5 \mathrm{ft} / \mathrm{s}$, the average transmembrane pressure was TMP $=42 \mathrm{psid}$, and the average slurry temperature was $\mathrm{T}=26^{\circ} \mathrm{C}$ (the filtrate flux was adjusted to $\mathrm{T}=25^{\circ} \mathrm{C}$ ). The test was done from $10 / 1$ to $10 / 3 / 2002$ and concentrated the insoluble solids from approximately $1 \mathrm{wt} \%$ to $8.5 \mathrm{wt} \%$. 
- Backpulsing did not prove useful

Figure 37 shows that after 5 hours of dewatering, the flux dropped precipitously to the target mean flux of $0.02 \mathrm{gpm} / \mathrm{ft}^{2}$. The criterion to initiate a backpulse was when the filtrate flux dropped below $0.015 \mathrm{gpm} / \mathrm{ft}^{2}$ and this occurred after about 8.7 hours into the test and the solids concentration increased to just $2 \mathrm{wt} \%$, Fig. 38. The backpulse increased the flux rate but after only 4 hours it returned to the criterion level again. The approximate 1-hour period that appears to show no flow in Fig. 37, just before the tenth hour, occurred because the filtrate flowmeter could only measure flow after the entire backpulse system was purged of air. That is, during that period the system was refilling, there was filtrate flow but it could not be measured since no liquid was flowing through the filtrate system flowmeter. (Note, after this test the filtrate flowmeter was relocated to begin measuring the filtrate immediately after a backpulse.) During a backpulse the filtrate flow actually is stopped and reversed for only a few minutes, then the flow reestablishes almost immediately. After the first backpulse, the filter was backpulsed four more times, each being less effective than the preceding one. After four backpulses that action was stopped. The increase in the filtrate flux and the fast return to the original low flux indicated that backpulsing was not effective. Actually, backpulsing was intended to be stopped after the second one, but at about the $16^{\text {th }}$ hour (at $8 \mathrm{AM}$ on Wednesday, 10/2/2003) the RPP customer was present and made two requests: to increase the slurry velocity from $11 \mathrm{ft} / \mathrm{s}$ to $12 \mathrm{ft} / \mathrm{s}$ to conform to the bench-top tests and to perform a backpulse to observe its operation and how it was being performed. Unfortunately, the filtrate flux returned to a low flux in less than an hour's time and then one more backpulse was done at 8:45 AM, resulting in similar response. No further backpulsing was to be done, but at about the $22^{\text {nd }}$ hour a final backpulse was done with actuating air pressure increased from 30 psid to approximately 100 psid to see if the high pulse energy could dislodge the filter cake. As can be seen on Fig. 37 the increase of filtrate flow only lasted moments before it returned to the low filtrate flux. Backpulsing was totally ineffective and no longer used.

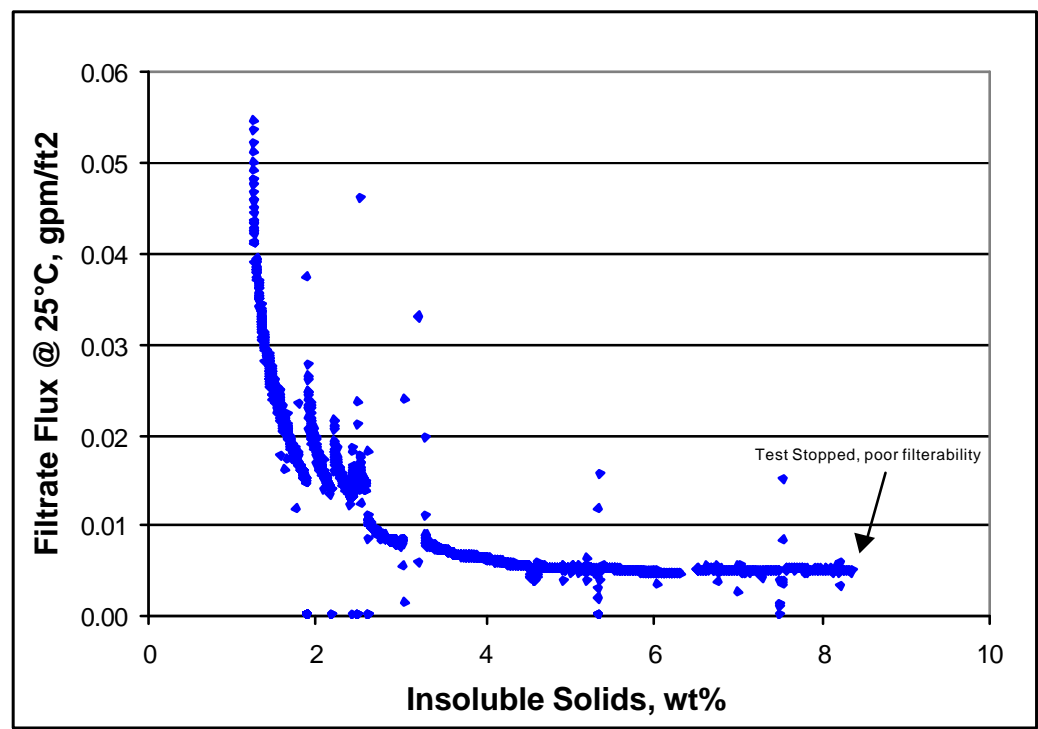

Figure 38. Pilot-scale X-flow UF Dewatering of AN-102R2, Batch 3C 


\subsection{Filtering Batch 3B}

The second Batch 3 test (called 3B) followed the first Batch 3 test (called 3C) to confirm that good filterability could be attained with the organic based slurry by using baseline conditions, $\mathrm{BC}$, during the precipitation step of the slurry preparation. The reluctance to initially use the $\mathrm{BC}$ slurry was because of the larger reagent quantities needed and the higher process temperature of $50^{\circ} \mathrm{C}$, which were not necessary to attain the decontamination of the Strontium and Transuranic constituents from the slurry. However, using the newly optimized conditions, NOC (see above), resulted in an unfilterable slurry, but with BC Batch 3B, using the same flow parameters of $\mathrm{V}=12 \mathrm{ft} / \mathrm{s}$ and $\mathrm{TMP}=40 \mathrm{psid}$, an acceptable filter flux resulted.

Figure 39 shows a filtrate flux above the target mean of $0.02 \mathrm{gpm} / \mathrm{ft}^{2}$ during the first 11.5 hours of the test. In fact, the entire test lasted just over 12 hours when the insoluble solids concentration went above $20 \mathrm{wt} \%$, Fig. 40 . The large drop off at the end was after the concentration was determined to be approximately $14 \mathrm{wt} \%$ and dewatering was continued until it went above $20 \mathrm{wt} \%$. Even at the highest concentration the filtrate flux was still higher than the overall average of $0.011 \mathrm{gpm} / \mathrm{ft}^{2}$ that was obtained during Batch $3 \mathrm{C}$; therefore, the test was consider a success. Over the entire 12.2 hours of dewatering, the filtrate flux averaged at $0.048 \mathrm{gpm} / \mathrm{ft}^{2}$, or better than a factor of 4 over Batch 3C. Subsequently, the decontamination factors were measured for Batch $3 \mathrm{~B}$ and were found to be acceptable (Steeper and Williams, 2003).

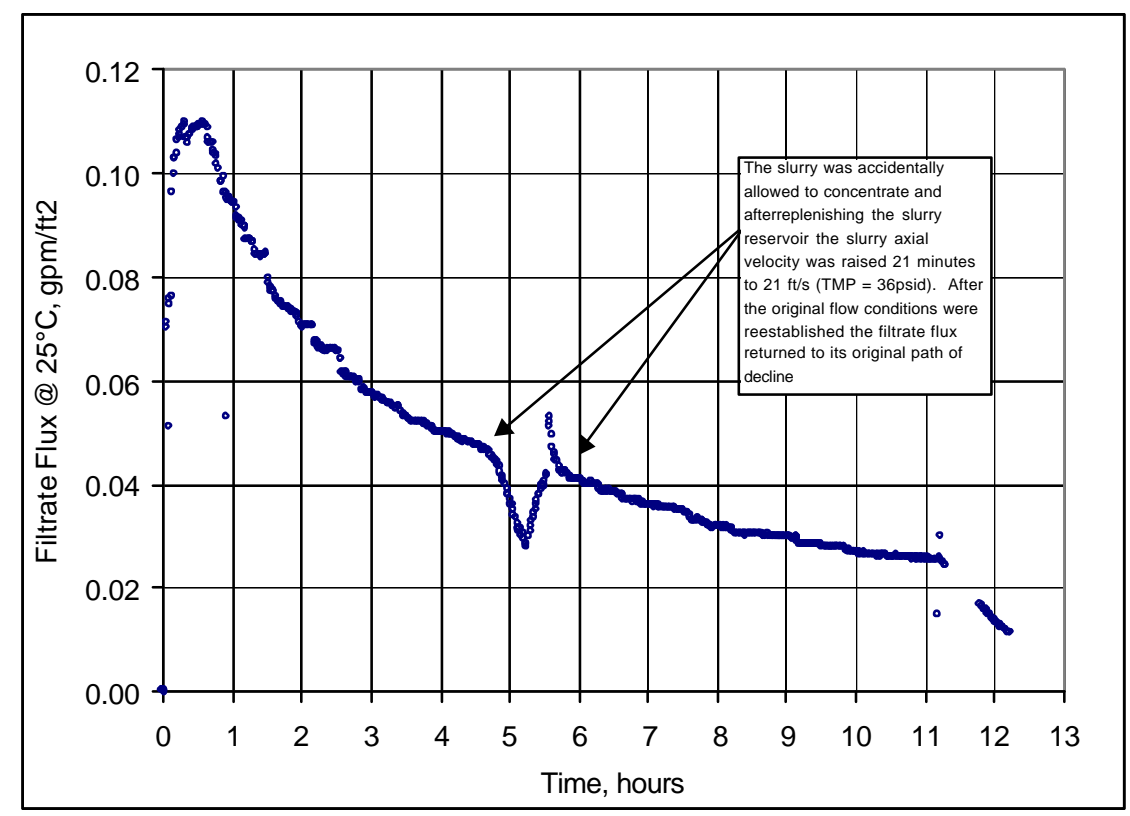

Figure 39. Batch 3B: Dewatering filter flux of AN-102R2 precipitated with the baseline conditions at $50^{\circ} \mathrm{C}$.

Over the filtering period the average slurry velocity was $V=12.1 \mathrm{ft} / \mathrm{s}$, the average transmembrane pressure was TMP $=41 \mathrm{psid}$, and the average slurry temperature was $\mathrm{T}=26^{\circ} \mathrm{C}$ (the filtrate flux was adjusted to $\mathrm{T}=25^{\circ} \mathrm{C}$ ). The test was done from $10 / 22$ to $10 / 23 / 2002$ and concentrated the insoluble solids from approximately $1 \mathrm{wt} \%$ to $22 \mathrm{wt} \%$. 
As a result of reaching the targeted solids concentration, this dewatering test run was followed by the steady-state runs, i.e., 1.17 to 1.29 . These runs will be discussed in the next section of this report. Some other aspects of the Batch 3B dewatering test were: that no backpulse was done, a temporary drop of filtrate flow occurred around the $5^{\text {th }}$ test hour, and a data gap at the 11.3 hour.

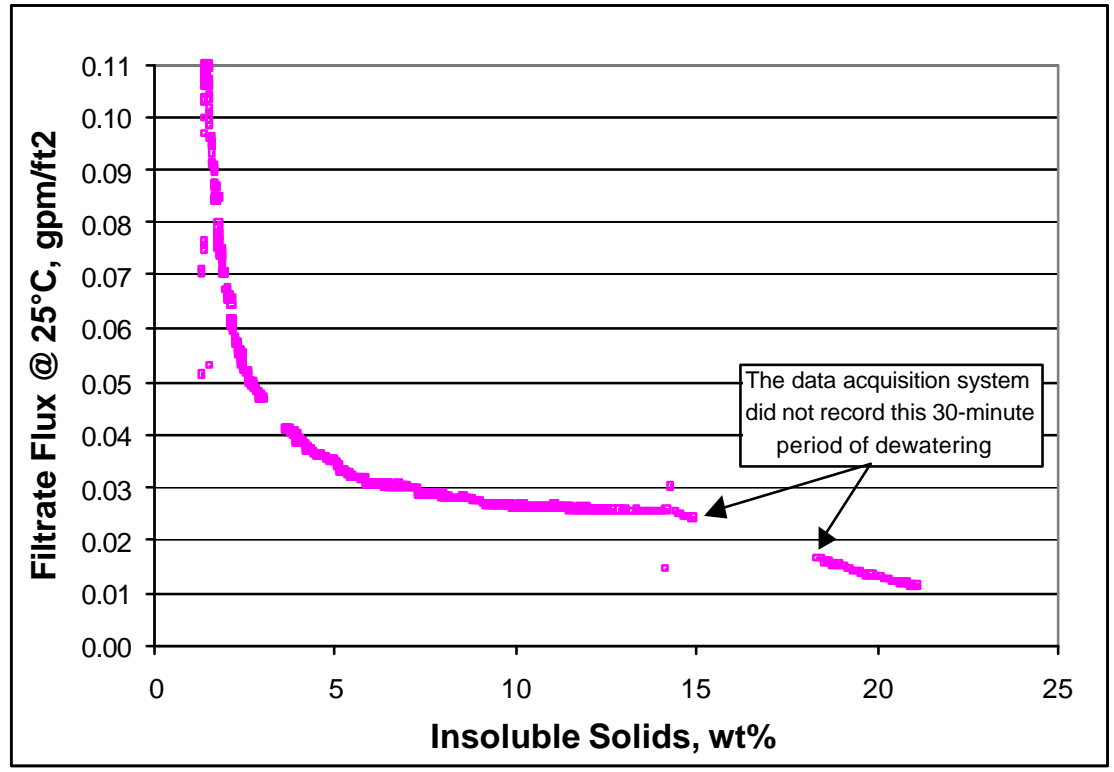

Figure 40. Pilot-scale X-flow UF Dewatering of AN-102R2, Batch 3B

- No Backpulse

No backpulse was done for Batch 3B because the criterion of a filtrate flux below $0.015 \mathrm{gpm} / \mathrm{ft}^{2}$ didn't occur during most of the test. At about the $12^{\text {th }}$ hour the backpulse criterion was reached but by that time the test was all but complete, so a backpulse was not done.

- Apparent data glitches were real events

At just past 4.5 hours into the test on Tuesday, 10/22/02, at around 8:15 PM the filtrate flux dropped precipitously, which continued until about 9:00 PM. During this time the slurry volume was not replenished and the slurry prematurely reached a high solids concentration. When the problem was discovered, more slurry was added to the test rig reservoir and the slurry axial velocity was increased from 12 to $21 \mathrm{ft} / \mathrm{s}$ for 21 minutes, in an attempt to recover the filtrate flux, without resorting to a backpulse which would have disturbed the filter cake. After 21 minutes, the filtrate flux appeared to be fully recovered, therefore the slurry velocity was returned to $12 \mathrm{ft} / \mathrm{s}$. However, the filtrate flux continued to increase and then decrease to the original flux. At about 9:30 PM the excursion had passed and the flux was recovered. It appeared that the filter cake had returned to its appropriate thickness, as if the upset never occurred. On Wednesday, 10/23/2002, at 3:00 AM, 11.3 hours after starting the test, dewatering was stopped to evaluate the solids concentration and wait for another batch of simulant to be made. However, the solids concentration, turned out to be slightly more than $14 \mathrm{wt} \%$. This was 


\section{WSRC-TR-2003-00204, REV. 0}

SRT-RPP-2003-00087, REV. 0

deemed sufficiently high such that the remaining slurry could be concentrated above 20 $\mathrm{wt} \%$. This was very important because it meant the remaining chemicals on hand could be saved to make enough slurry to carry out a third test and save the program considerable expense. At about 6:00 PM on the same day, the Batch 3B test restarted to further concentrate the slurry. That last concentration process took about one hour. Unfortunately, during the first 30 minutes of dewatering the data logger was not operating, but it did capture the last 30 minutes (from 6:30 PM to the end at 7:00 PM), when the concentration reached $22 \mathrm{wt} \%$. The actual final concentration was only known with a subsequent solids analysis. The actual filtrate flux, when the test rig was restarted at 6:00 PM, began above the final flux of $0.024 \mathrm{gpm} / \mathrm{ft}^{2}$, when the test was stopped at 3:00 AM earlier.

\subsection{Filtering Batch 3A}

After completing a successful Batch 3B test, it was important to know if filterability was sensitive to precipitation temperature. The reduced amount of simulant used for Batch $3 \mathrm{~B}$, approximately 1000 liters, enabled one more Batch 3 of approximately 900 liters to be made with the remaining chemicals. A third and final Batch 3 test (called 3A), was done, but the precipitation temperature of $50^{\circ} \mathrm{C}$ was lowered to $25^{\circ} \mathrm{C}$. If good filterability could also be obtained at a lower temperature, then less hardware and energy would be needed by the Waste Treatment Plant.

The use of $\mathrm{BC}$, while precipitating at $25^{\circ} \mathrm{C}$, did obtain a filtrate flux better than Batch $3 \mathrm{C}$, but unfortunately it was not as good as Batch $3 \mathrm{~B}$. Figure 41 shows the time plot of the entire data set as the insoluble solids concentration increased from 1.6 to $25 \mathrm{wt} \%$ as seen in Fig. 42, which plots filter flux as a function of insoluble solids wt $\%$. The average filtrate flux over that period was $0.019 \mathrm{gpm} / \mathrm{ft}^{2}$, which is a factor of 1.7 better than Batch $3 \mathrm{C}$, but only $40 \%$ that of Batch 3B. After only 8 hours of filtering, the flux dropped below the target mean of $0.02 \mathrm{gpm} / \mathrm{ft}^{2}$, and after 13 hours the flux dropped below the backpulsing criterion of 0.015 $\mathrm{gpm} / \mathrm{ft}^{2}$, which initiated backpulsing. Over the next 7 hours the filter was backpulsed six times with each successive backpulse less effective. At the $21^{\text {st }}$ hour, the RPP customer asked to stop backpulsing to see where the filtrate flux would end up as the concentration increased. At about the $26^{\text {th }}$ hour, the flux reduced to close to $0.01 \mathrm{gpm} / \mathrm{ft}^{2}$ and permission was obtained to stop the test on Thursday, 11/7/02. The test was then continued on the following Monday, 11/11/02. At that point the insoluble solids concentration was evaluated and found to be $15 \mathrm{wt} \%$. For about 3 hours on Monday the slurry was concentrated from 15 to $25 \mathrm{wt} \%$. Interestingly, the slurry filtrate flux increased from its value when the test was stopped on Thursday, 11/7/2003, of just above $0.01 \mathrm{gpm} / \mathrm{ft}^{2}$ to just below $0.02 \mathrm{gpm} / \mathrm{ft}^{2}$. Something occurred during the 88 hours it sat idle, from Thursday evening at 5 PM until Monday morning at 9 AM. However after about one hour of filtering the filtrate flux dropped very fast as the solids concentration approached $20 \mathrm{wt} \%$ and then onto $25 \mathrm{wt} \%$. It is possible that during the time period some of the filter cake fell off the filter surface since the filter was vertically oriented. At the end of Batch $3 \mathrm{~A}$ filtering the filtrate flux dropped to the same rate that Batch $3 \mathrm{C}$ ended, i.e., $0.005 \mathrm{gpm} / \mathrm{ft}^{2}$. It seems clear from the three Batch 3 filtering tests that if a flux of better than $0.02 \mathrm{gpm} / \mathrm{ft}^{2}$ is needed, it is very important to precipitate at the higher temperature, i.e., $50^{\circ} \mathrm{C}$. 


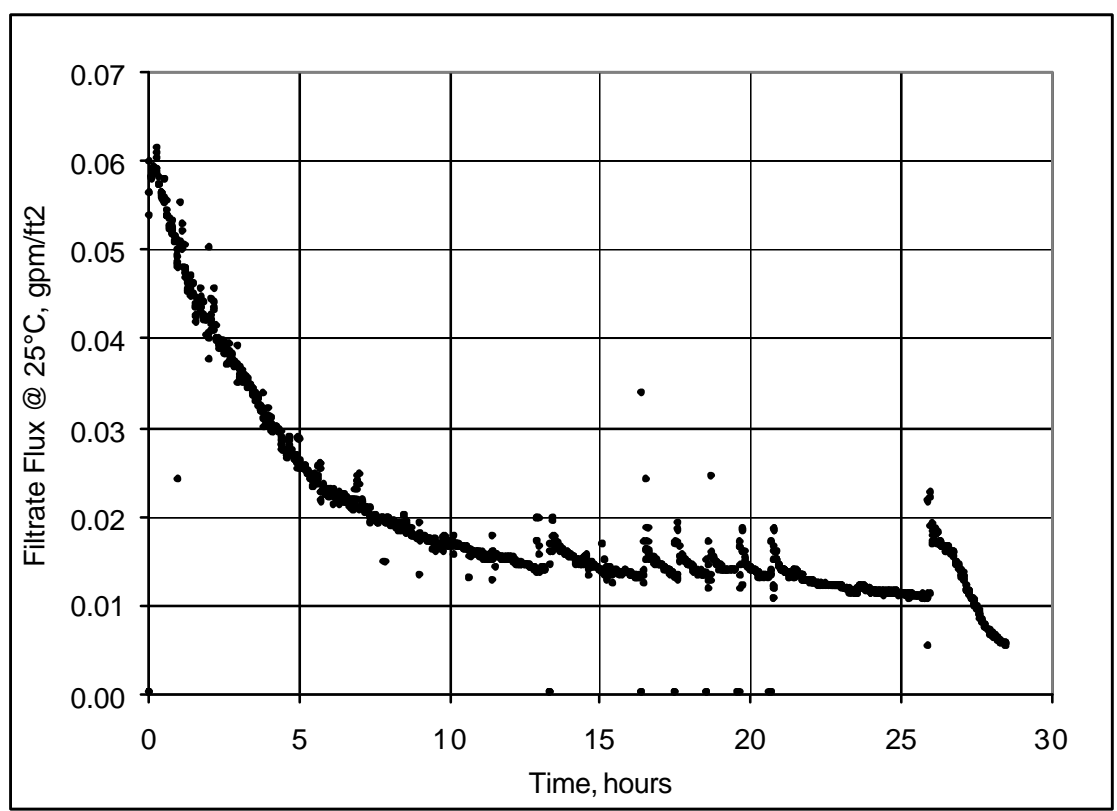

Figure 41. Batch 3A: Dewatering filter flux of AN-102R2 precipitated with the baseline conditions at $20^{\circ} \mathrm{C}$.

Over the filtering period the average slurry velocity was $\mathrm{V}=12.0 \mathrm{ft} / \mathrm{s}$, the average transmembrane pressure was $\mathrm{TMP}=40 \mathrm{psid}$, and the average slurry temperature was $\mathrm{T}=25^{\circ} \mathrm{C}$ (the filtrate flux was adjusted to $\mathrm{T}=25^{\circ} \mathrm{C}$ ). The test was done from $11 / 6$ to $11 / 11 / 2002$ and concentrated the insoluble solids from approximately $1.6 \mathrm{wt} \%$ to $25 \mathrm{wt} \%$.

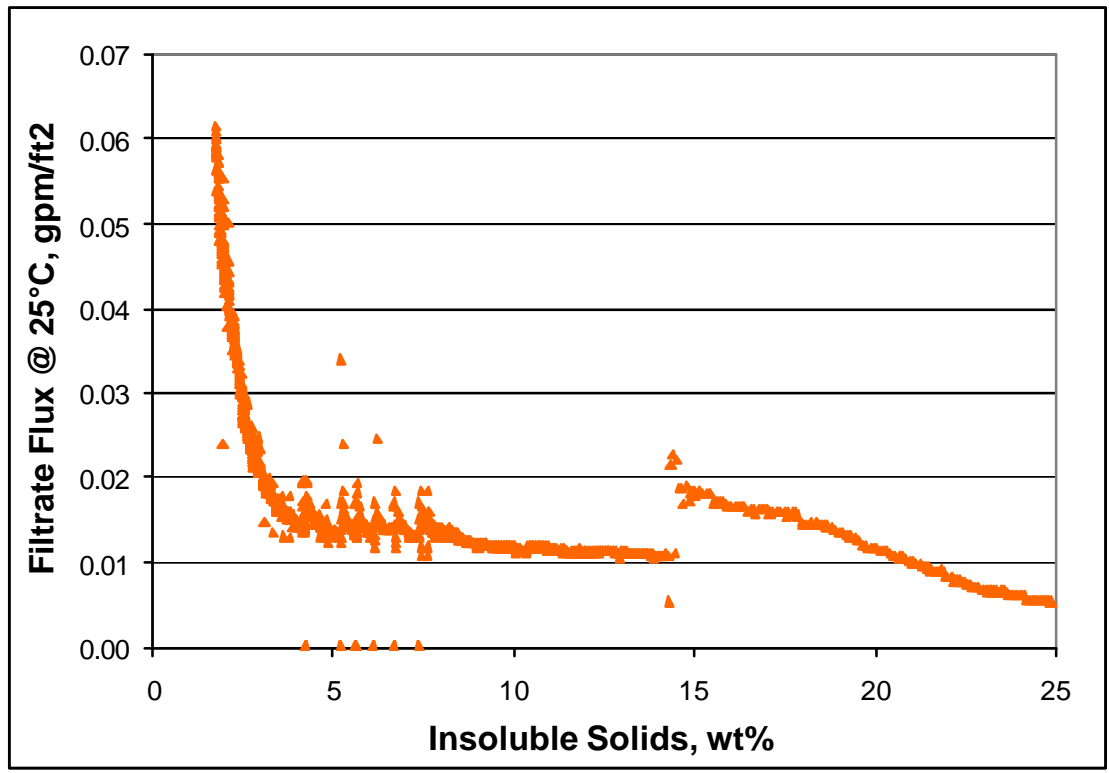

Figure 42. Pilot-scale X-flow UF Dewatering of AN-102R2, Batch 3A 


\subsection{Filtering Batch 4A}

After completing the Batch 3 tests, for which the simulants were all prepared under wellmixed precipitation conditions, a Batch 4 test was planned to precipitate under more WTPprototypic mixing conditions. That is, the WTP will use pulse jet mixers (PJM) that creates a completely different mixing environment as compared to standard impeller-type agitator. A new precipitation test rig was developed for this test (see Steeper and Williams, 2003). Two tests were planned, 1. Batch 4A, where the AN-102R2 simulant would be made at baseline conditions, and 2 . Batch $4 \mathrm{~B}$, which would be done depending on the results of $4 \mathrm{~A}$.

Figure 43 shows the results of Batch $4 \mathrm{~A}$ testing. The results were similar to Batch $3 \mathrm{~A}$, for which the filtrate flux was considered borderline acceptable. Figure 44 shows the entire data set as the insoluble solids concentration increased from 1.5 to $18 \mathrm{wt} \%$, over the 19.5 -hour period of dewatering. The average filtrate flux over that period was $0.019 \mathrm{gpm} / \mathrm{ft}^{2}$, which was the same as Batch 3A. That is, a factor of 1.7 better than Batch 3C, but only $40 \%$ that of Batch 3B. However, instead of only 8 hours of filtering, the flux dropped below the target mean of $0.02 \mathrm{gpm} / \mathrm{ft}^{2}$, in just under 5 hours! Conversely, even after 18 hours the flux still did not drop below the backpulsing criterion of $0.015 \mathrm{gpm} / \mathrm{ft}^{2}$. For this test the RPP-WTP customer requested a backpulse when the flux dropped below $0.02 \mathrm{gpm} / \mathrm{ft}^{2}$. This occurred at about 5.5 hours into the run. As before, the flux dropped very fast after the backpulse and after two more backpulses, around the $9^{\text {th }}$ hour of filtering, there seemed to be no further advantage to backpulsing, therefore it was stopped

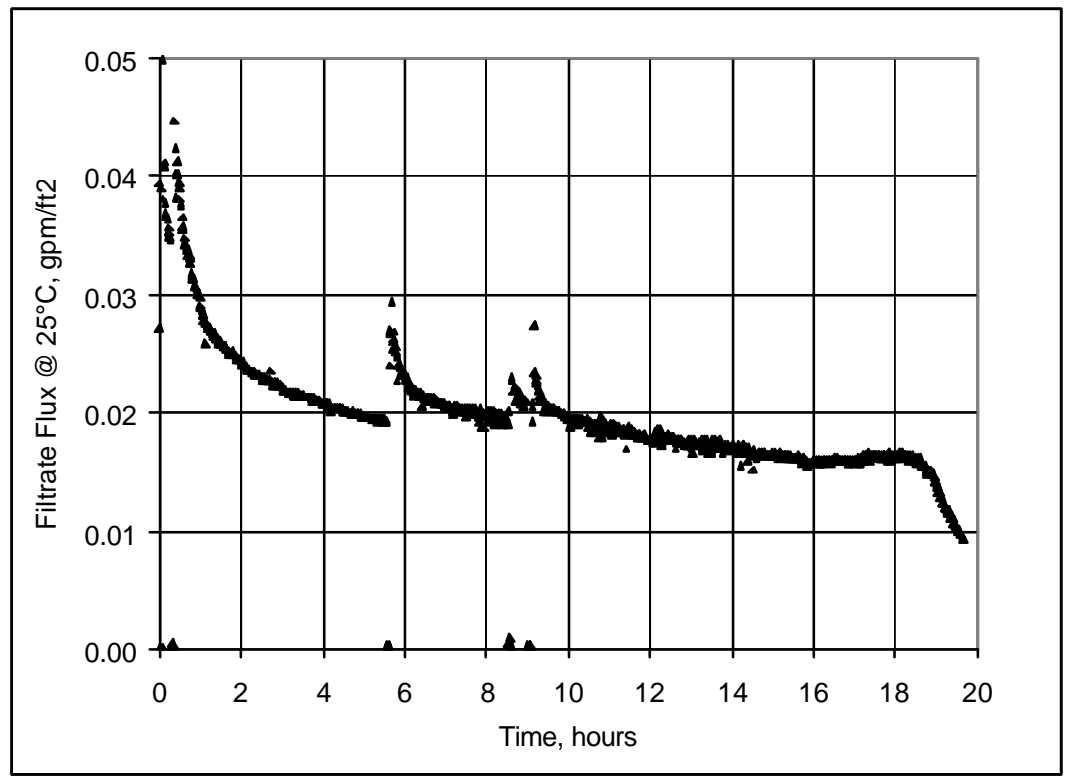

Figure 43. Batch 4A: Dewatering filter flux of AN-102R2 precipitated with the baseline conditions at $25^{\circ} \mathrm{C}$.

Over the filtering period the average slurry velocity was $\mathrm{V}=11.9 \mathrm{ft} / \mathrm{s}$, the average transmembrane pressure was TMP $=40 \mathrm{psid}$, and the average slurry temperature was $\mathrm{T}=25^{\circ} \mathrm{C}$.

The test was done from $03 / 12$ to $03 / 13 / 2003$ and concentrated the insoluble solids from approximately $1.5 \mathrm{wt} \%$ to $18 \mathrm{wt} \%$. 
Batch 4A was smaller than the Batch 3, i.e., 650 liters, see Fig. 36; therefore there was no stopping point to see if the target insoluble solids concentration was reached. Because the filterability was only borderline the RPP-WTP customer decided not to have another batch of simulant made and that Batch 4A should be concentrated as high as possible in the hope of reaching the target $15 \mathrm{wt} \%$. The slurry volume was reduced to just before the test rig reservoir began to aspirate air, which would have stopped slurry circulation and thus filtering. When the approximate 650 liters were reduced to 60 liters the test was stopped and slurry samples were taken. It turned out the target was exceeded, since the final concentration was $18.3 \mathrm{wt} \%$. For dewatering operation, the test was a success.

Once again, the conclusion for Batch $4 \mathrm{~A}$ is the same as for Batch $3 \mathrm{~A}$. That is, if a filtrate flux of better than $0.02 \mathrm{gpm} / \mathrm{ft}^{2}$ is required then the precipitation mixing environment needs to be better. While Batch $3 \mathrm{~A}$ needed to be mixed at the Batch $3 \mathrm{~B}$ temperature of $50^{\circ} \mathrm{C}$, Batch $4 \mathrm{~A}$ needed to be mixed with more vigorous agitation than was available with the single pulse jet mixer. While the pilot test used only one PJM in the precipitation tank, the WTP plant has multiple PJM units planned for its precipitation tank. Creative uses of different mixing cycles for the many PJMs in the WTP tank may overcome the poor mixing results for this single PJM test.

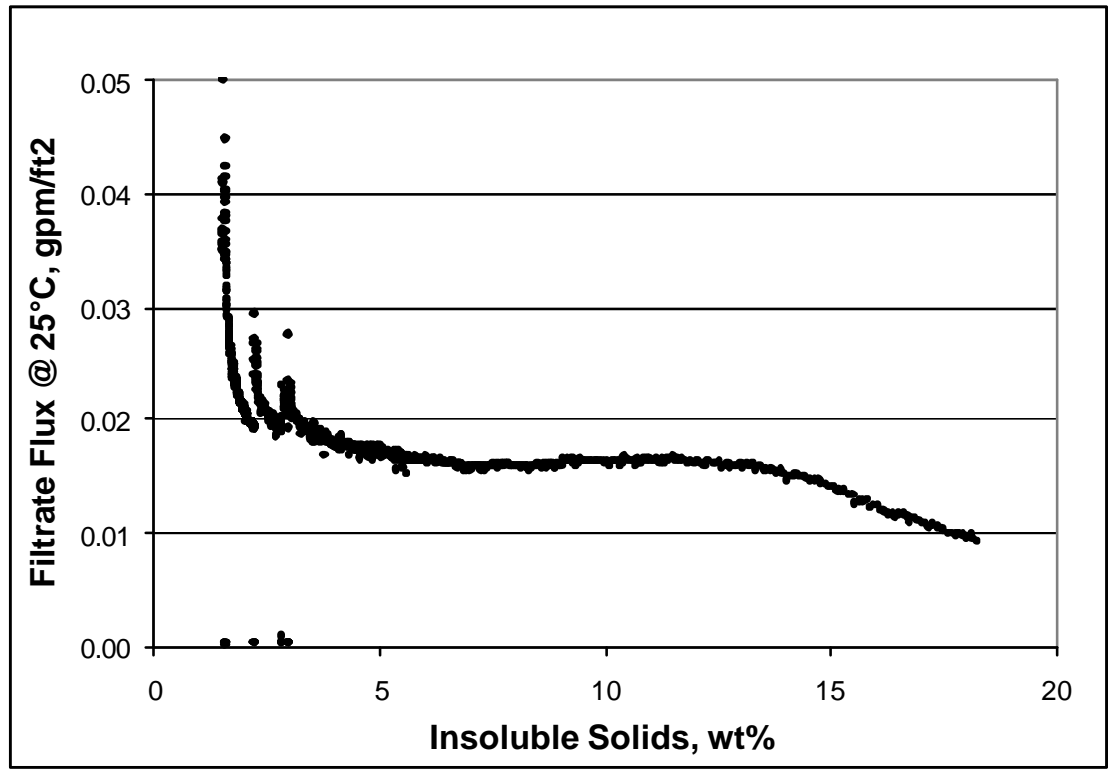

Figure 44. Pilot-scale X-flow UF Dewatering of AN-102R2, Batch 4A

\subsection{Overall Dewatering Results of AN-102R2}

For comparison Fig. 45 combines the filtrate flux curves for all four runs. The slurry simulant that filtered the best was Batch $3 \mathrm{~B}$, which was precipitated under the original baseline conditions, and the worst was Batch $3 \mathrm{C}$, which was done under a set of optimized conditions. Looking at the slurry data in Fig. 36 the reason for the disparity is not obvious. Out of all the simulants 3B had the smallest particles and was the only slurry where there was a significant reduction in the particle size after dewatering, i.e., from the low to high solids concentration. For all other properties the four simulants started at about the same 
conditions, i.e., total solids of $33 \mathrm{wt} \%$, viscosity of $4 \mathrm{cP}$ and no yield stress, slurry density of $1.29 \mathrm{~g} / \mathrm{ml}$, bi-modal particle volume distribution of about half with particles between 8 to 10 microns and half between 2 and 3 microns (the exception was 4A which only had one particle size mean of 2.7 microns). Also, a scan of the rheological data characteristics in Appendix G does not elicit significant differences. All the simulants act as time-dependent pseudoplastics at the highest solids concentrations.

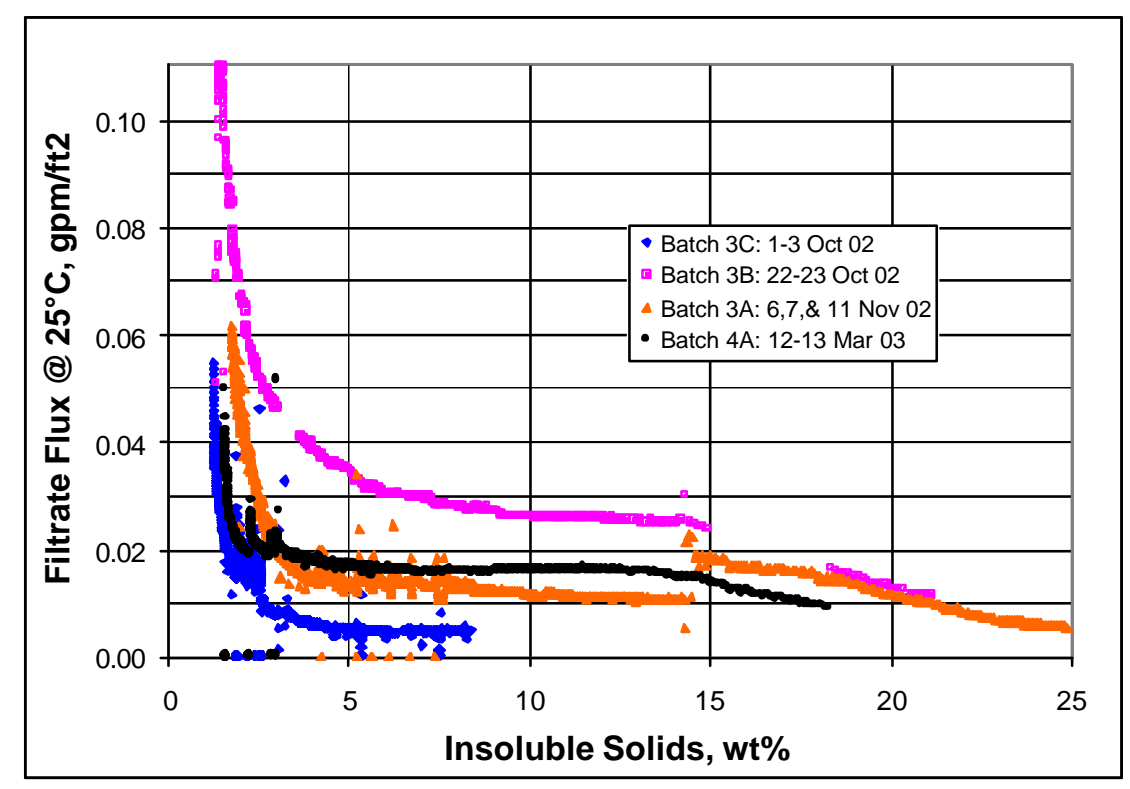

Figure 45. Pilot-scale X-flow UF Dewatering of AN-102R2, Batches $3 \& 4$

To definitively state why the filtrate fluxes differed among the simulants a lot more information would be needed about different chemical characteristics of the simulants. For instance, the particle-to-particle attractiveness. All the slurries appeared to be very sticky and thick as the solids concentration increased. How this affected the filter and the integrity of the filter cake is unknown. One telling feature was that backpulsing was not very affective. This indicates that much of the cake was not removed when a backpulse was made and as the cake became thicker backpulsing became completely ineffective. Small differences in a slurry's ability to adhere to the filter surface, and itself, very probably would lead to large differences in impeding filtrate from entering the porous substrate.

One notable feature in Fig. 45 is the sharp drop in filter flux when the insoluble solids concentration went beyond about $14 \mathrm{wt} \%$. Roughly speaking there appears to be three distinct filtering regions:

1. From 0 to $5 \mathrm{wt} \%$ the filter flux drops precipitously as the filter cake forms and grows fast, relative to its initially formed thickness.

2. From $5 \mathrm{wt} \%$ to $15 \mathrm{wt} \%$ the filter flux is relatively stable. The filter cake is formed and further growth is slow, relative to the established thickness. 
3. Greater than $15 \mathrm{wt} \%$, the filter flux begins to drop faster because the solids are close enough together to significantly increase the slurry viscosity, probably exponentially. See Fig. 46.

To understand these different regions it may help to look at some of the individual measured parameters shown in Fig. 36, or in Appendix G. Figure 46 shows one batch, Batch 3A, (using a modified Fig. 41) with some of the rheological data superimposed.

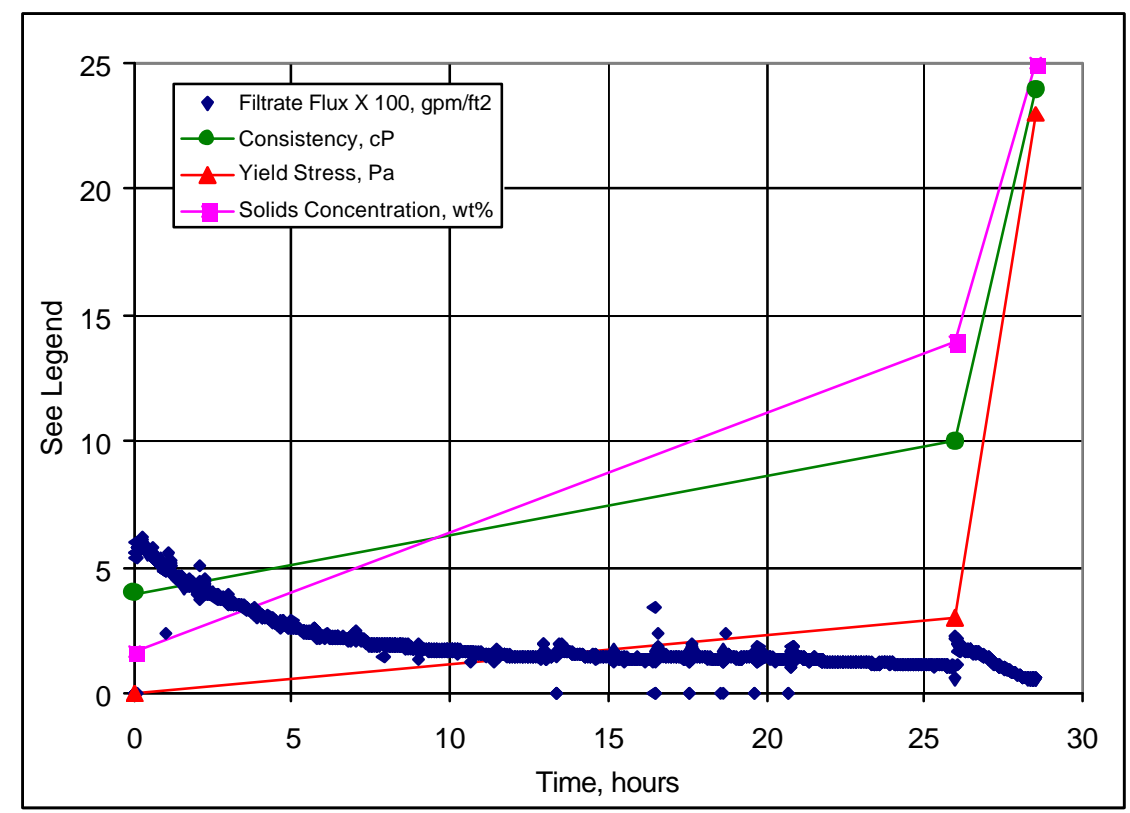

Figure 46. Batch 3A filtrate flux with selected rheological properties

It does appear that in region 3, after $14 \mathrm{wt} \%$ of insoluble solids, the rheological properties change significantly causing the filtrate flux to drop. Other differences among the different slurries are shown in Figs. 47 through 51 below.

Figures 47 and 48 show a distinct difference in the rheological properties of all the slurries made using baseline conditions (3B, 3A, and $4 \mathrm{~A}$ ) and the first test of $3 \mathrm{C}$ which was made with the newly optimized conditions. The much higher slurry consistency and yield stress was probably a major contributor in making $3 \mathrm{C}$ much harder to filter. This implies baseline conditions should be used during the precipitation of Envelope $\mathrm{C}$ slurries. However, the density for all the simulants seemed to be similar, Fig. 49.

From Figs. 50 and 51 it appears that all the slurries started with approximately the same size particles. That is, in the entire population of particles the mean particle size was very close to 2 microns and if particle volume is considered the particles were approximately equally distributed around two means, i.e., 2.5 microns and 9 microns. 
WSRC-TR-2003-00204, REV. 0

SRT-RPP-2003-00087, REV. 0

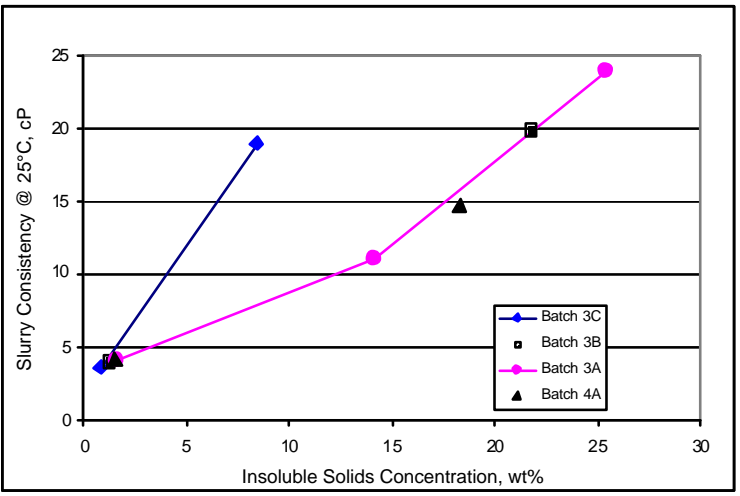

Figure 47. Batch $3 \& 4$ Slurry Consistency

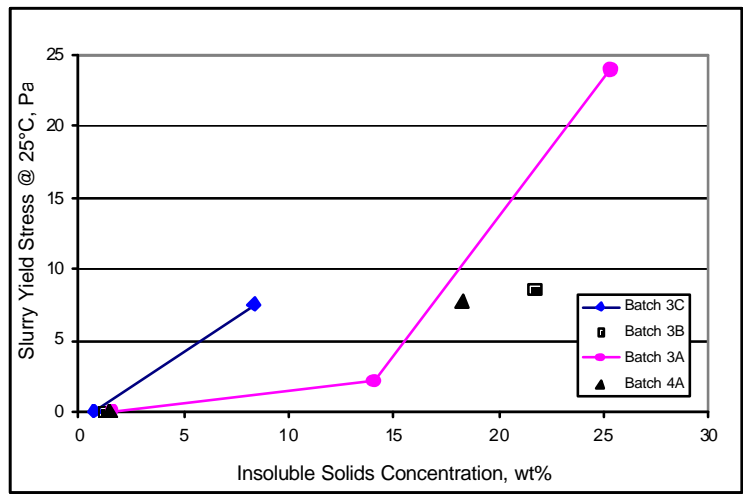

Figure 48. Batch $3 \& 4$ Slurry Yield Stress

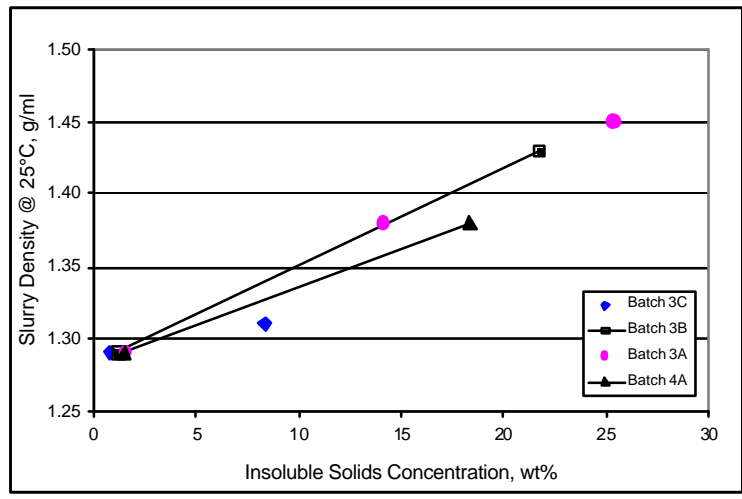

Figure 49. Batch $3 \& 4$ Slurry Density

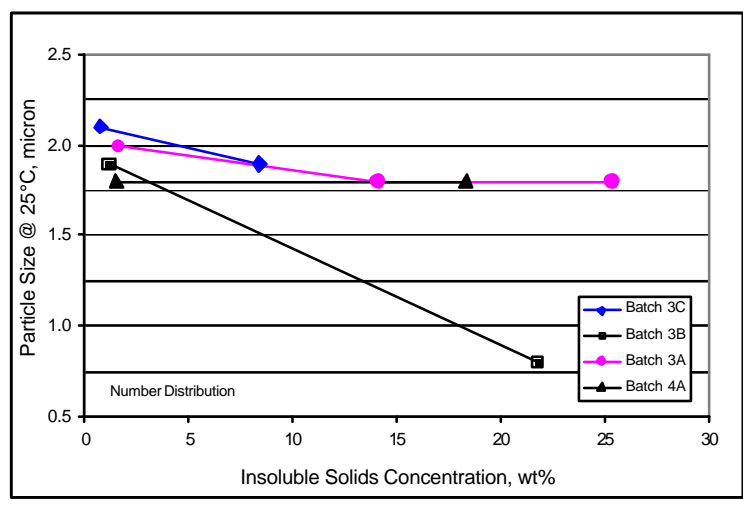

Figure 50. Batch 3\&4 Mean Particle Size (by Number Distribution)

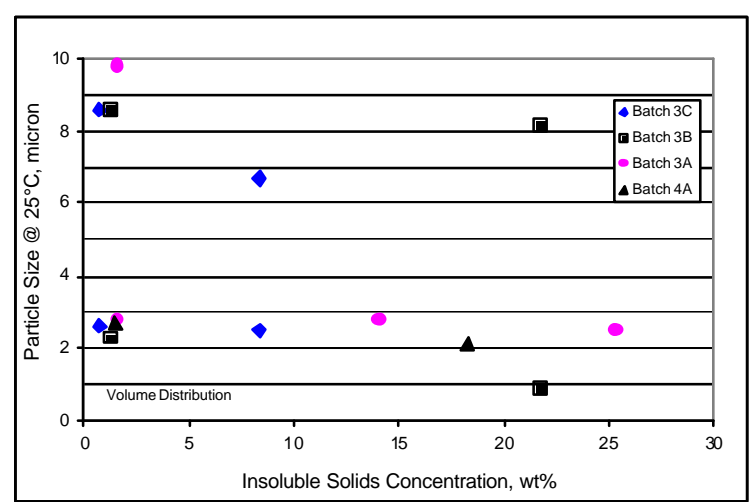

Figure 51. Batch $3 \& 4$ Mean Particle Size (by Volume Distribution) 
As the simulants were circulated in the filtration facility some particle changes are evident. Figure 50 shows that mean population particle size did not change significantly for most of the simulants. However, Batch $3 \mathrm{~B}$ stands out because its particles ended up to be less than one half as small. Remember, Batch $3 \mathrm{~B}$ had the best filterability. With respect to the volumetric distribution of particles, all but Batch 4A started with bi-modal distributions. After dewatering the particle distributions for all but Batch 3B became mono-modal. (Actually they all did because the bi-modal distribution of Batch 3B was weak, i.e., only $13 \%$ of the particles had a distribution around the mean of 8 microns.) A notable difference was Batch 4A, which started and ended with a single mean particle size, though the size became slightly smaller, i.e., 2.2 microns to 0.9 microns.

\subsubsection{Steady State Filtering of AN-102R2 Slurry Simulant}

The requirement of the Task Plan (Duignan, 2002a) was that after dewatering the Envelope C simulant to some high concentration of insoluble solids $\mathrm{s}^{\ddagger}$, it would be filtered while maintaining the solids concentration constant. In this way, a series of filter tests could be done to determine an optimum slurry velocity and transmembrane pressure.

\subsection{Typical Constant Insoluble Solids Concentration Steady-State Test Run}

Before discussing the overall steady-state results it is useful to look at one filter flux versus time to better understand the average data results. Figure 52 shows test run 1.17, for Batch $3 \mathrm{~B}$, which is a typical filter flux with time. While the other test runs have different magnitudes of filter fluxes the overall characteristics of filter flux with time are similar and therefore not shown here, but these can be found in Appendix E.

At the beginning of each steady-state run the typical operation is as follows: a backpulse is done in an attempt to start at the same point. However, due to depth fouling the filtrate flux will always decrease a small amount with each successive backpulse. This will be discussed later in this section. During the first few minutes there is no filtrate flux, while the filter is backpulsed. This then jumps to the highest flux, as a result of reestablishing filtrate flow after a backpulse. As soon as flow begins the flux drop precipitously as the filter cake begins to build. Within 30 to 40 minutes approximately $90 \%$ of the cake has been established (as indicated by the $90 \%$ drop in the filtrate flux from approximately $0.017 \mathrm{gpm} / \mathrm{ft}^{2}$, right after the backpulse, to $0.010 \mathrm{gpm} / \mathrm{ft}^{2}$ after about 40 minutes) and then there is a very slow reduction in flux with time. After about 2 hours an asymptote is reached (Fig. 29 in Duignan (2000a) shows that after 8 hours the 2 -hour flux is still basically the same). Note what appears to be a second backpulse just before the 10-minute mark. No second backpulse was given and this is a feature of how this particular filter test rig works. Just before the Batch 3B test the meter which measures filtrate flow was reoriented. Figure 2 shows the schematic of the test rig where the low flow filtrate flowmeter, Q2, is just downstream of valve V27. Originally the filtrate flowmeter was in the filtrate return line from the pulsepot, so that its large pressure drop would not affect the backpulse filtrate flow. Unfortunately, in that location it was necessary for the entire backpulse system to be liquid solid before the meter

\footnotetext{
${ }^{\ddagger}$ For dewatering the target insoluble solids concentration was $15 \mathrm{wt} \%$, but a high concentration was preferable.
} 
began registering a flow. For the unexpectedly very low flowrates experienced during the Batch $3 \mathrm{C}$ campaign, it took close to 30 minutes to replace the approximately 1.8 liters of filtrate evacuated during a backpulse. Having data during the first half an hour of operation is very important, as can be seen in Fig.52.

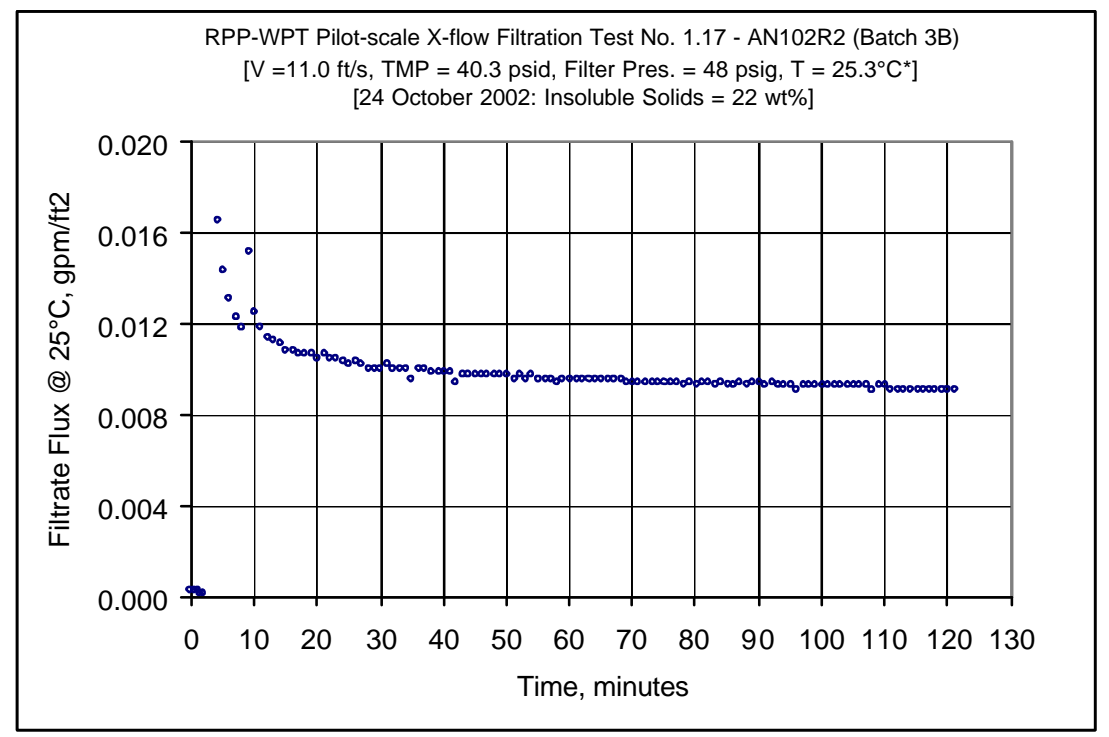

Figure 52. Batch 3B: Test Run 1.17

Over the filtering period the average slurry velocity was $\mathrm{V}=11.0 \mathrm{ft} / \mathrm{s}$, the average transmembrane pressure was TMP $=40.3 \mathrm{psid}$, and the average slurry temperature was $\mathrm{T}=25.3^{\circ} \mathrm{C}$

(the filtrate flux was adjusted to $\mathrm{T}=25^{\circ} \mathrm{C}$ ).

The low flow filtrate flowmeter was moved to the tubing between the filter housing and the pulsepot so immediate measurements could be taken; a special bypass line was used to valve out the flowmeter during a backpulse to avoid the high pressure drop that the meter would cause. Even though the new meter location allows the immediate measurement of filtrate flux, after filtrate flow is reestablished, the filtrate system still has a charge of air that needs to be expelled as the system is refilled with filtrate. In the case of test run 1.17 it took about 5 minutes to refill. Indeed, at 1.8 liters $(=0.48$ gallons $)$ the average flowrate over that time period of $0.014 \mathrm{gpm} / \mathrm{ft}^{2}$ gives a time of $0.48 / 0.014 \mathrm{gal} / \mathrm{min} / \mathrm{ft}^{2} / 6.707 \mathrm{ft}^{2}=5.1$ minutes $\left(6.707 \mathrm{ft}^{2}\right.$ is the flow area for the seven filter tubes). This happens because during refilling the air-filled system takes more pressure to flow, reducing the TMP. After becoming liquid solid the filtrate system pressure goes down and the TMP increased to its original setting before the backpulse was made.

In the figure caption of Fig. 52 some average measurements, i.e., V, TMP, and Slurry Temperature, $\mathrm{T}$, are given and are based on all the data points taken during the test run, excluding the first few minutes during the backpulse. For example, the data for Figure 52 can be found in Appendix E, Figs. E2-1 and E2-2. The averages were based on 119 data points from the $3^{\text {rd }}$ minute until the $121^{\text {st }}$ minute. The abscissa shows the filtrate flux at 
$25^{\circ} \mathrm{C}$, which indicates that while the actual slurry temperature may have been different from $25^{\circ} \mathrm{C}$ (it was actually $25.3 \pm 0.3^{\circ} \mathrm{C}$ ) it was adjusted for the effects of fluid viscosity and surface tension (Townson, 2002a) with the equation:

$$
\text { Flux@ } 25^{\circ} \mathrm{C}=\mathrm{P} / \mathrm{A} \times \mathrm{C}
$$

where

$$
\begin{array}{ll}
\text { Flux } & =\text { filtrate flux at } 25^{\circ} \mathrm{C}\left(\mathrm{gpm} / \mathrm{ft}^{2}\right) \\
\mathrm{P} & =\text { filtrate flow rate }(\mathrm{gpm}) \\
\mathrm{A} & =\text { filter surface area }\left(\mathrm{ft}^{2}\right) \\
\mathrm{C} & =\text { temperature correction factor }=\mathrm{e}^{\left(2500^{*}((1 /(273+\mathrm{T})-(1 / 298)))\right.} \\
\mathrm{T} & =\text { slurry/filtrate temperature in degrees Celsius }
\end{array}
$$

\subsection{Filtering Batch 3C}

No steady state tests were performed because the dewatering test was stopped after 46 hours because of low filterability and that the insoluble solids concentration only reached $8.5 \mathrm{wt} \%$.

\subsection{Filtering Batch 3B}

The second Batch 3 test (called 3B) experienced good filterability and at the end of the dewatering, test run 1.16 , it still was filtering at just above $0.01 \mathrm{gpm} / \mathrm{ft}^{2}$ when the insoluble solids concentration reached its highest concentration, i.e., $22 \mathrm{wt} \%$. The steady-state filter runs completed at $22 \mathrm{wt} \%$ insoluble solids were:

$$
\begin{aligned}
& 1.17,1.18,1.19,1.20 \\
& 1.21,1.22,1.23,1.24 \\
& 1.25,1.26,1.27,1.28,1.29
\end{aligned}
$$

done on 24 October 2002

done on 28 October 2002

done on 29 October 2002

Figure 53 depicts the average filtrate flux results for all the Batch 3B test runs. As per the Task Plant (Duignan, 2002a) five of the runs $(1.17,1.18,1.19,1.24$, and 1.29) were done for two hours and the remaining eight runs were done for one hour. However, the average filtrate flux values for all test runs are shown for one hour to make a better comparison. If the two-hour average were used it would only lower the average of those five test runs by 5 to $8 \%$, or about to the lower part of the error bars shown, which are $\pm 5.4 \%$; this can be seen in Fig. 53. Also note that the temperature for two runs (1.26 and 1.27) exceeded the target of $25^{\circ} \mathrm{C} \pm 5^{\circ} \mathrm{C}$. One had the highest slurry velocity, $\mathrm{V}=14.7 \mathrm{ft} / \mathrm{s}$ with a low TMP, i.e., $31 \mathrm{psid}$, and the other had the lowest TMP, i.e., 23 psid. At these pressures ${ }^{\dagger}$ the test rig cooling coil was unable to provide the necessary cooling.

\footnotetext{
$\dagger$ The temperature control problem is an aspect of the pilot-scale test rig. Figure 2 shows the slurry cooling coil to be on either side of the pressure control valve, V11, which is in parallel with the main slurry flow. In order to obtain low filter pressures V11 is run almost wide open, which starves slurry flow to the cooling coil and thus cuts down on cooling. The coil could not be put in series with the main slurry flow because the pressure drop would have been too large for most of the test runs.
} 


\section{WSRC-TR-2003-00204, REV. 0}

SRT-RPP-2003-00087, REV. 0

Figure 53 shows the Batch 3B test runs in chronological order and the total operational time the filter was active during these runs was about 18 hours. As time progressed the filtrate flux continually decreases. This is the result of depth fouling which is not removed by backpulsing. Fouling is actually increased because of backpulsing, which exposes the filter surface to the smallest particles in a slurry. For these tests the filter was backpulsed each time a new test began. Before a statement can be made about the dependence of filtrate flux on $\mathrm{V}$ and TMP, the effect of depth fouling needs addressing. Fortunately, the five 2-hour test runs were all done at the same V and TMP so a comparison can be made. By plotting only those five test runs the effect of fouling becomes more evident.

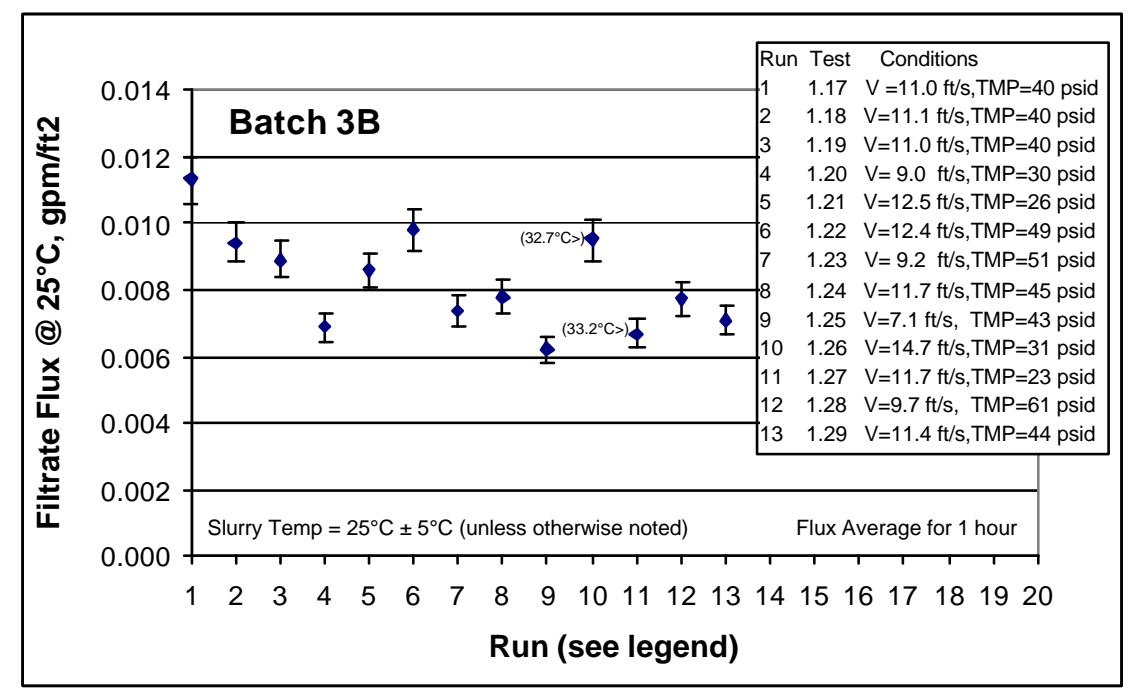

Figure 53. Batch 3B: Filtrate flux results averaged over the first hour of testing, including the five 2-hour test, i.e., 1.17, 1.18, 1.19, 1.24, and 1.29.

The tests were done from 10/24 to 10/29/2002 and at a constant insoluble solids concentration of $22 \boldsymbol{w t} \%$.

Figure 54 shows just the five test runs with the same V and TMP. There was a small variation in these parameters, which are shown on the figure. 


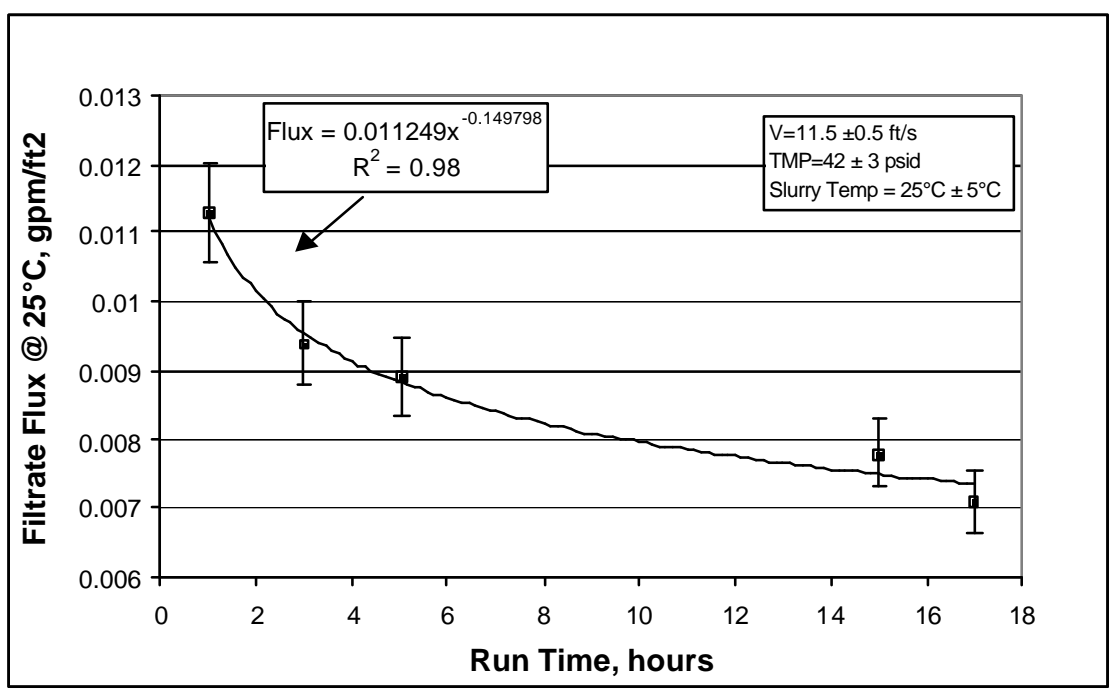

Figure 54. Batch 3B: Filtrate flux results averaged over the first hour of testing for the five 2-hour test runs, i.e., 1.17, 1.18, 1.19, 1.24, and 1.29.

These runs are unique in that they all have the same flow parameters $\mathrm{V} \sim 11 \mathrm{ft} / \mathrm{s}$ and TMP $\sim 40 \mathrm{psid}$.

The data indicate a decreasing average filtrate flux with time. Now this decrease could also indicate a slurry that is changing with time becoming more difficult to filter. It may be that the solid particles in the slurry were changing and making the filter cake less permeable. However, particle analyses do not support this theory ${ }^{\dagger}$. As shown on Fig. 54, the data were correlated to the power relation indicated and each point includes the measurement uncertainty. This relation can then be used to adjust the steady-state data to remove the effect of fouling.

Figure 55 repeats Fig. 53, but with the data adjusted with the relation shown in Fig. 54. A comparison between the data in Figs. 53 and 55 indicate a difference as to which are the most appropriate parameters to obtain the best filtrate flux. As expected, from past tests (e.g., Duignan, 2000a and b) the key factor was the slurry axial velocity. Figure 55 shows that for run 10 , test 1.26 , with $\mathrm{V}=14.7 \mathrm{ft} / \mathrm{s}$, the best filtrate flux occurred, despite the fact it had a very low TMP (31 psid). The next highest, but statistically the same filtrate flux, was run 6 , test 1.22 , where both the slurry velocity, $\mathrm{V}=12.4 \mathrm{ft} / \mathrm{s}$, and TMP (49 psid) were moderately high.

\footnotetext{
$\dagger$ From the particle size analysis results on the slurry done before the steady state test runs (Appendix G, sample 300188110) and after (Appendix G, sample 300189271): Before - the population distribution particle average diameter was $0.8 \pm 0.2$ and the volume distribution particle average diameter was $0.9 \pm 0.5$ micron. After - the population distribution particle average diameter was $1.2 \pm 0.4$ and the volume distribution particle average diameter was $1.3 \pm 0.5$ micron. The uncertainty given is twice the standard deviation representing the $95 \%$ confidence level. Statistically the particle did not change in size.
} 


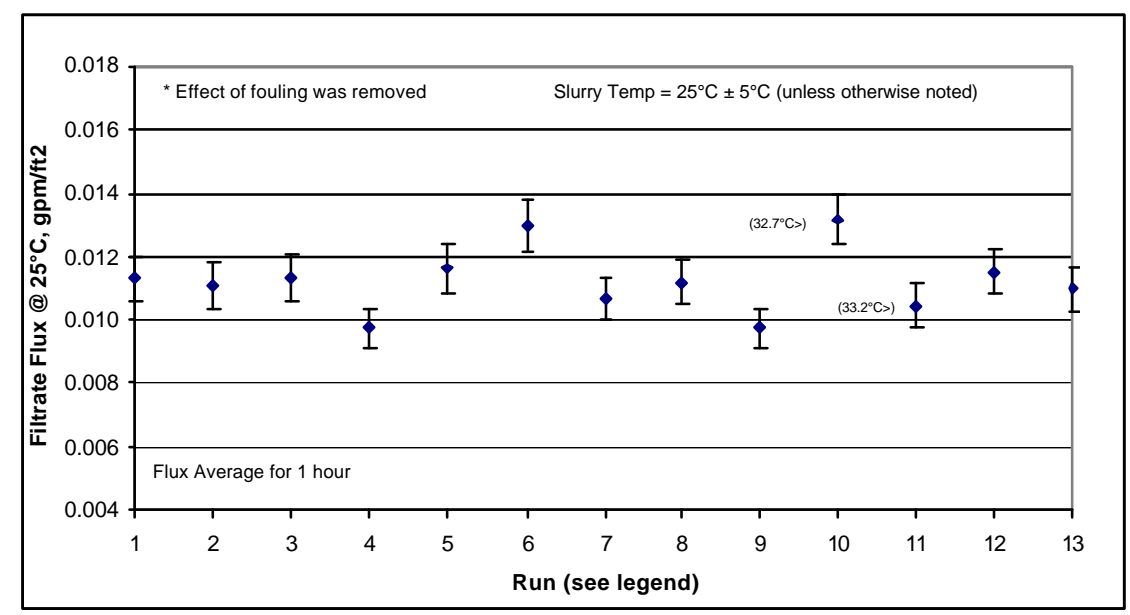

Figure 55. Batch 3B: This figure repeats the data shown in Fig. 53 with depth fouling effect removed. [The legend indicated in the abscissa title is that of Fig. 53.]

To more easily see the individual effects of V and TMP on the filtrate flux the data are shown for both. Figure 56 shows the effect of TMP and statistically there is none. The data were correlated with a linear least-squares fit and the correlation coefficient is near zero. Considering measurement uncertainty a coefficient of zero cannot be ruled out. Therefore, a decision of which operating TMP to use should be based on other consideration and not improvement of filtrate flux.

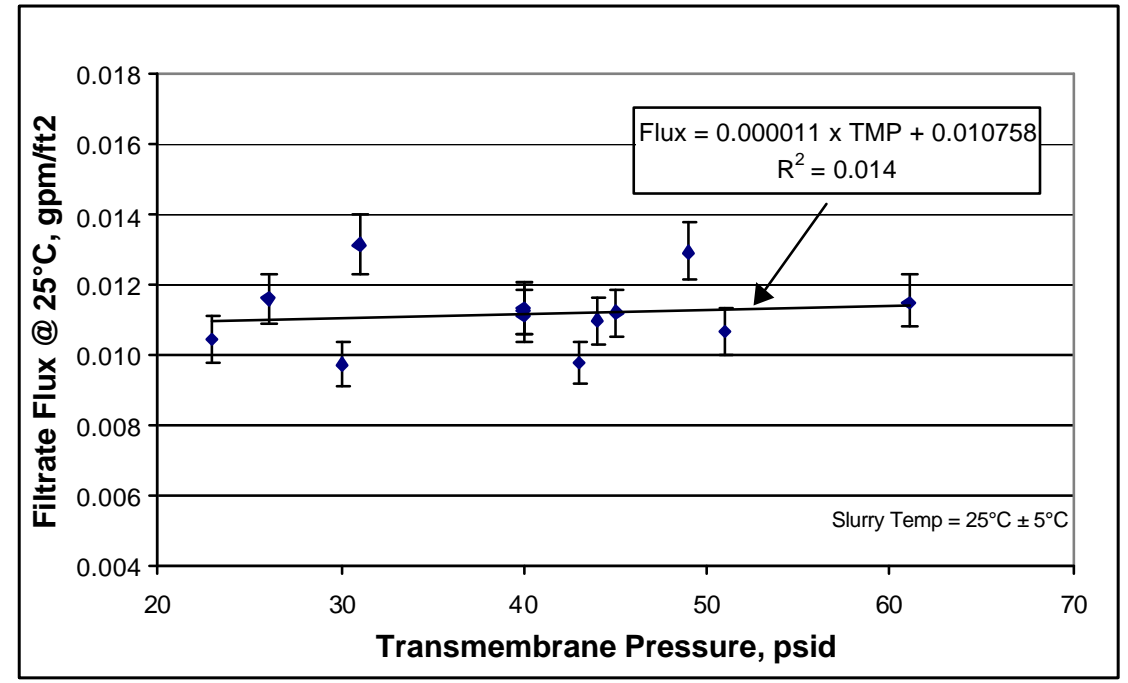

Figure 56. Batch 3B: All the steady-state data that were adjusted for depth fouling effect removed (Fig. 55) as a function of transmembrane pressure, TMP. 


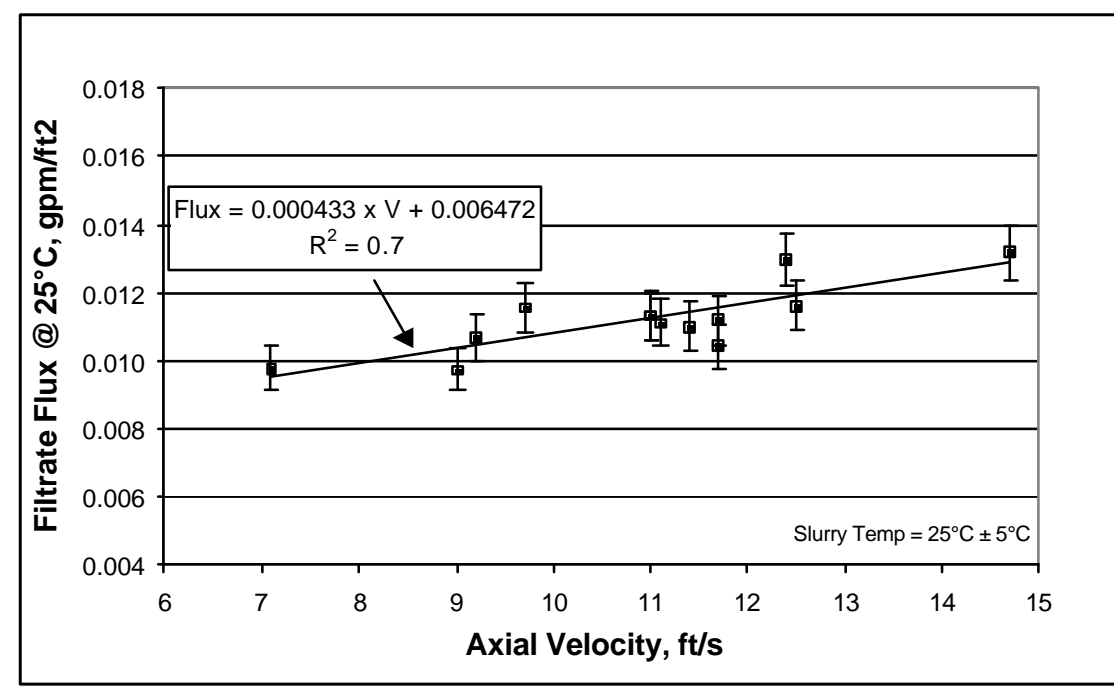

Figure 57. Batch 3B: All the steady-state data that were adjusted for depth fouling effect removed (Fig. 55) as a function of slurry axial velocity, V.

Figure 57 shows the Batch 3B data as a function of the slurry axial velocity, V. The figure includes a linear least-squares fit of the data with a correlation coefficient of 0.7 and therefore the filtrate flux is clearly dependent upon $\mathrm{V}$, even when considering the measurement uncertainty as shown on the figure. The conclusion that can be made from this analysis is that to increase filtrate flux, $\mathrm{V}$ must increase.

\subsection{Filtering Batch 3A}

The third Batch 3 test (called 3A) experienced better filterability then Batch 3C, but not as good as Batch 3B. Because of the length of the Batch 3A dewatering test, i.e., around 30 hours, and good Batch 3B results, the RPP customer did not require the full series of steady state test runs. However, due to the available time, slurry, and the desire to make some inter-Batch 3 comparisons, a limited number of steady-state runs were performed. Once the high insoluble solids concentration was attained (it ended up being $25 \mathrm{wt} \%$ ) the steady-state runs began. The runs completed were:

\section{$1.17,1.20$}

$1.23,1.25,1.28,1.29$ done on 11 November 2002

done on 12 November 2002

The choice of test runs to perform depended on the ability to keep the slurry within the temperature limit of $25^{\circ} \mathrm{C} \pm 5^{\circ} \mathrm{C}$. For instance, two attempts were made to do test run 1.27, which had the lowest TMP of 20 psid (see the first footnote in section 1.3.2.5.3). Unfortunately, within minutes of test initiation the temperature climbed above $30^{\circ} \mathrm{C}$ and reach $40^{\circ} \mathrm{C}$ before it could be controlled.

Figure 58 depicts the average filtrate flux results for all the Batch $3 \mathrm{~A}$ test runs. The six tests done were limited to one hour of filtering. The RPP customer did not required the extra hour because these tests were only to confirm filtrate fluxes and not to be used to optimize flow 
parameters. That is, all of the average filtrate flux values shown in Fig. 58 are over the entire run time for each test of one hour.

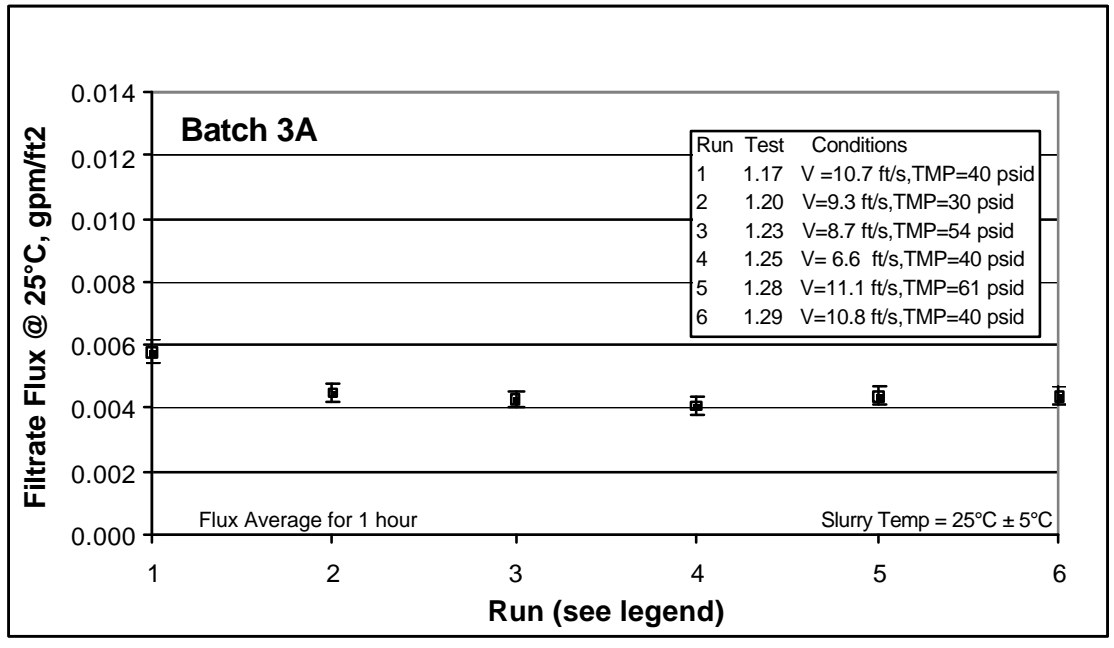

Figure 58. Batch 3A: Filtrate flux results averaged over the one hour of each test.

The tests were done from 11/11 to 11/12/2002 and at a constant insoluble solids concentration of $25 \mathrm{wt} \%$. The same filtrate flux range as shown on Fig. 53 was used for comparison.

Because the Batch 3A filtrate flux data are approximately a factor of 2 lower than the Batch 3B data (see Fig. 53) it's a bit harder to perceive trends, but both batches are similar. In fact, the depth fouling characteristics were very similar. Run 1, test 1.17 , and run 6 , test 1.29 for Batch 3A have the same flow conditions as those shown in Fig. 54. Since the Batch 3B and 3 A filtrate flux magnitudes are different, the data shown in Figure 59 were normalized by the first result (at the 1-hour mark) from each data.

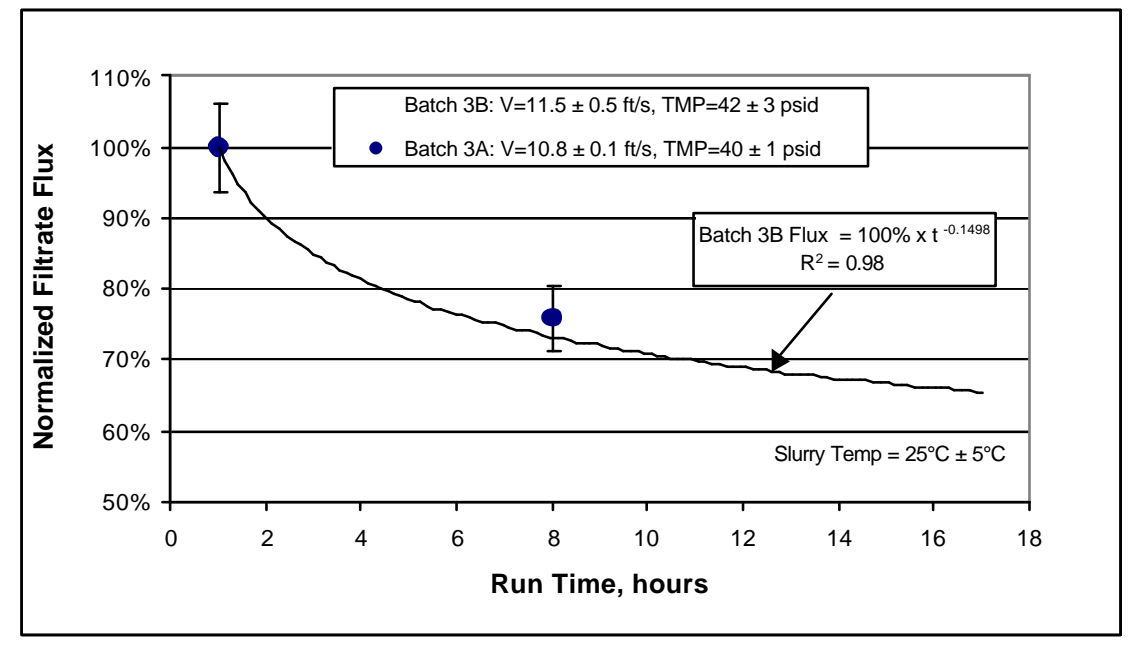

Figure 59. Batch 3A: Two filtrate flux data points shown are the average over the one hour test runs, i.e., 1.17 and 1.29. The Batch 3B curve fit is also shown for comparison. Both data sets were normalized by their respective first test run result (test run 1.17). 
For example, the 1-hour average filtrate flux for Batch 3B was $0.0113 \mathrm{gpm} / \mathrm{ft}^{2}$, so each of the five points shown in Fig. 54 were divided by 0.0113 ; along with the fitted curve equation. The same was done for the two data points of Batch 3A using the 1-hour average filtrate flux of $0.0058 \mathrm{gpm} / \mathrm{ft}^{2}$. While the slurry solids were precipitated in a slightly different way, making Batch 3A not as filterable as Batch 3B, the filter depth fouling mechanism seems to be the same. This similarity is seen in Fig. 59 by the closeness of the Batch 3A datum point at the $8^{\text {th }}$ hour to the Batch $3 \mathrm{~B}$ data curve fit. Of course, because of the normalization the first point of Batches $3 \mathrm{~B}$ and $3 \mathrm{~A}$ were forced to be $100 \%$ and thus that degree of freedom was lost.

Using the same process to adjust the Batch $3 \mathrm{~B}$ data to minimize the effects of depth fouling the Batch 3A data are shown in Figure 60. Note that the ordinate range has been reduced to better display the data because of the low magnitude and small differences.

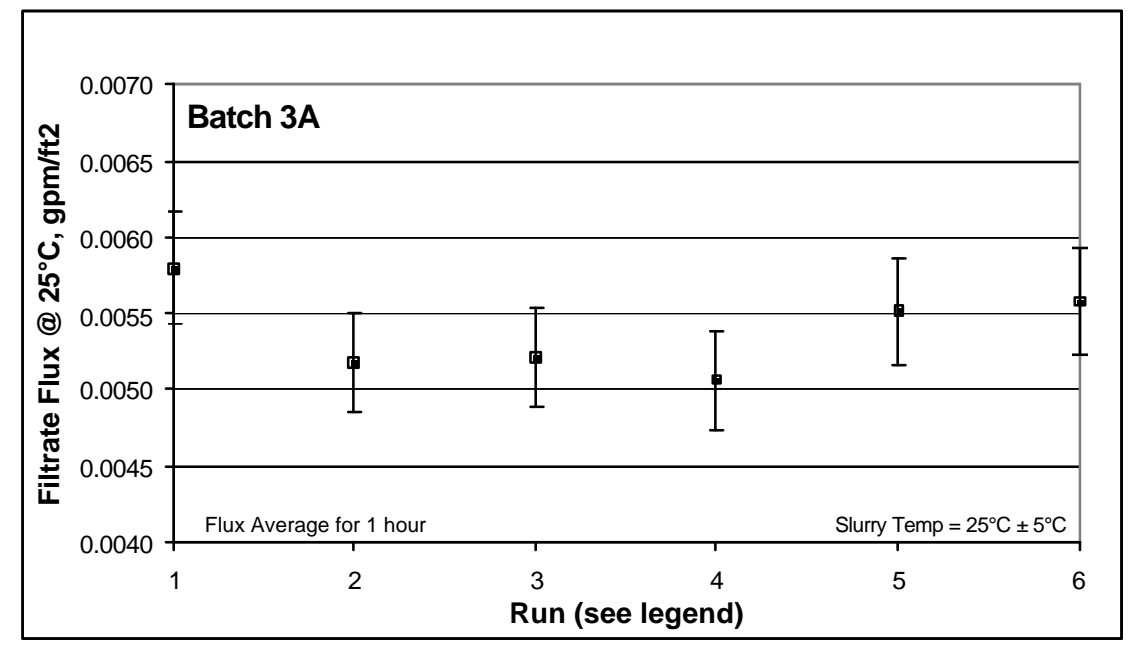

Figure 60. Batch 3A: This figure repeats of the data shown in Fig. 58 with depth fouling effect removed. [The legend indicated on the abscissa title is that of Fig. 58.]

As with Batch 3B data, if the Batch 3A results are shown as a function of TMP and V then filtrate flux dependence becomes evident. Figure 61 has TMP as the independent variable and Fig. 62 the axial velocity, V. 
WSRC-TR-2003-00204, REV. 0

SRT-RPP-2003-00087, REV. 0

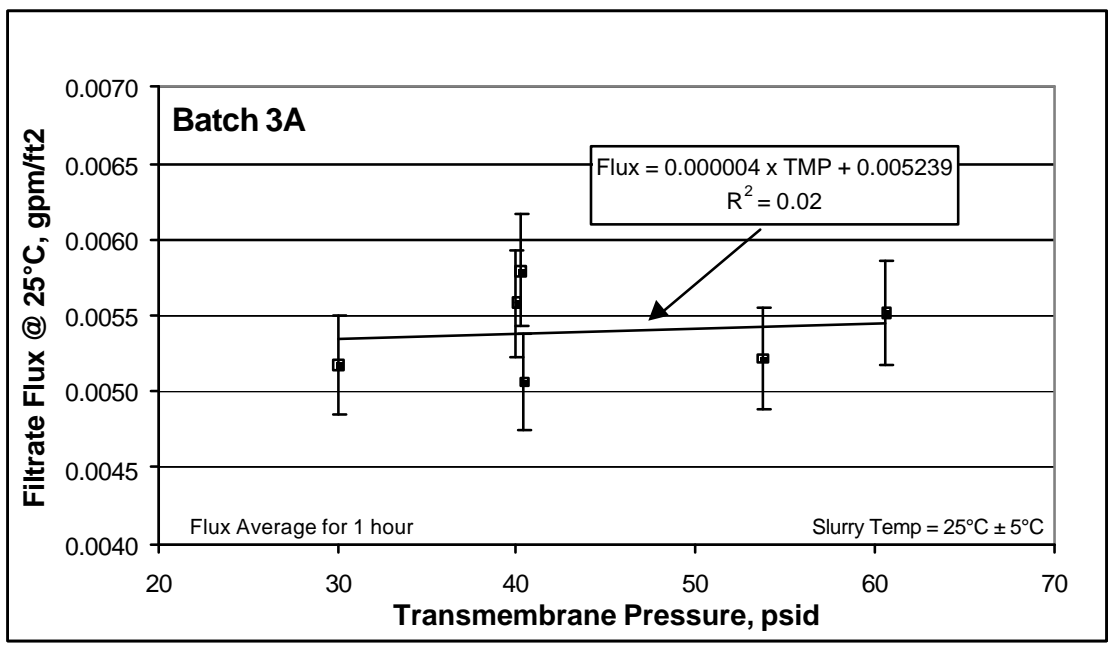

Figure 61. Batch 3A: All the steady-state data that were adjusted for depth fouling effect removed (Fig. 60) as a function of transmembrane pressure, TMP.

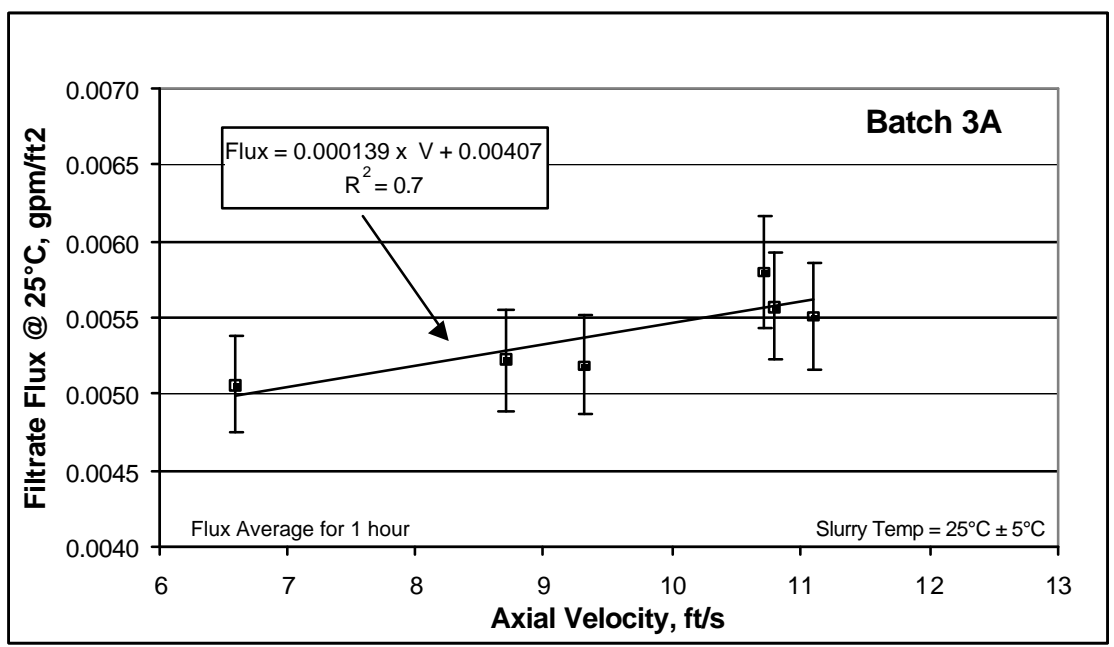

Figure 62. Batch 3A: All the steady-state data that were adjusted for depth fouling effect removed (Fig. 60) as a function of slurry axial velocity, V.

Once again, it appears that filtrate flux is not (or only very weakly) dependent upon the transmembrane pressure, while there is strong correlation with the slurry axial velocity in the filter tubes.

\subsubsection{Washing and Concentration of Washed ${ }^{\ddagger} A N-102 R 2$ Slurry Simulant}

To reduce the concentration of sodium of the waste slurry after being dewatered to some high concentration, e.g., between $15 \mathrm{wt} \%$ and $20 \mathrm{wt} \%$, WTP will wash the slurry. The current

\footnotetext{
${ }^{\dagger}$ All Steady State Filtering data can be found in Appendix F.
} 


\section{WSRC-TR-2003-00204, REV. 0}

SRT-RPP-2003-00087, REV. 0

WTP plan is to have a concentrated slurry volume of approximately 18,000 gallons. To this volume will be added 21,000 gallons of inhibited water to perform the washing. This wash water will be added in 21 mini-batches of 1,000 gallons. After the addition of each minibatch, the slurry will be dewatered to remove 1,000 gallons of diluted filtrate. This process will be repeated 21 times to complete the washing cycle.

The pilot-scale cross-flow test performed a similar washing cycle, but of course at a reduced scale. While this report deals with four different batches of AN-102R2 simulant, only after the first successful dewatering test could the steady state runs be done, and therefore, the washing test run. This occurred after the second batch, i.e., 3B. For subsequent batches most steady-state runs were not required, as well as the washing. After the last steady-state run with $\mathrm{AN}-102 \mathrm{R} 2$, Batch $3 \mathrm{~B}$, i.e., run 1.29 , the test rig was made ready to wash the slurry.

At the end of test run 1.29 there were approximately 67 liters of concentrated slurry. As required by the test specification (Townson, 2002a), the slurry insoluble solids concentration was reduced from what was attained during the dewater test run 1.16 to $20 \mathrm{wt} \%$, if it happened to be higher. The actual concentration for Batch 3B was approximately $22 \mathrm{wt} \%$; therefore, before washing some of the previously removed filtrate was added back to the slurry to reduce the solids concentration. The added filtrate was $7.5 \mathrm{~kg}$, or approximately 5.8 liters. The filtrate addition reduced the solids concentration of the simulant to $18.8 \mathrm{wt} \%$. The slurry volume was approximately 73 liters, so 85 liters (i.e., 73 x 21,000/18,000 = 73 x $1.16=85$ ) of wash water were prepared. The 85 liters were added in 4.04 -liter batches while 4.04 liters of washed filtrate were removed before adding the next batch. Of course this was done 21 times.

\subsection{Washing of AN-102R2, Batch 3B}

Figure 63 show the filtrate flux during the entire 3.7-hour process of washing the slurry. While the washing run was performed with a constant slurry velocity, $12 \mathrm{ft} / \mathrm{s}$, and TMP, 40 psid, the test is not really a steady-state run. This is because the properties of the slurry are constantly changing with the wash-water additions. Figure 64 shows the properties before and after the entire washing process.

Note that in Fig. 64 there is a significant drop in total slurry solids, which is basically due to the loss of soluble solids because there was very little change to the insoluble solids concentration. The loss of solids caused the density to drop, but more importantly the slurry consistency reduce to more that half, which means that the solution presents less shear for the same motive force. Interestingly, the yield stress almost doubled. The increase in yield stress would have little effect for the slurry in motion, but it does indicate that from rest it would take about double the energy to put the slurry in motion again. 


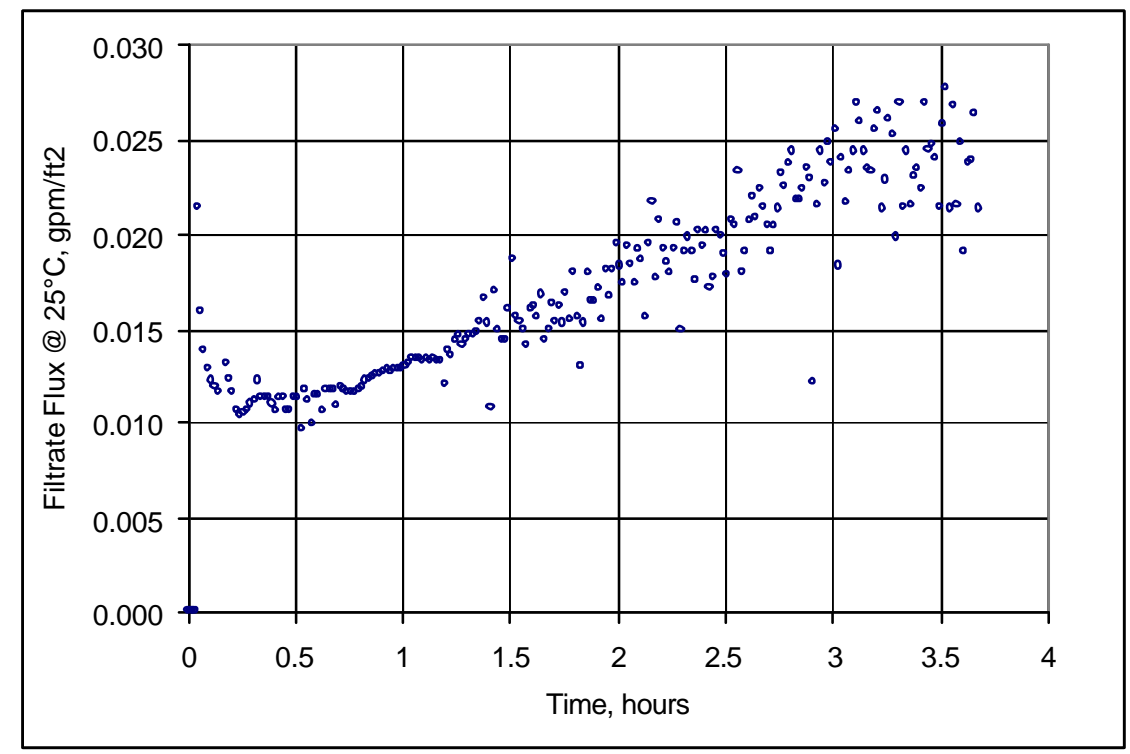

Figure 63. Washing of AN-102R2, Batch 3B after test run 1.29 and before concentration test run 1.30 .

Over the filtering period the average slurry velocity was $\mathrm{V}=12.1 \mathrm{ft} / \mathrm{s}$, the average transmembrane pressure was $\mathrm{TMP}=40.2 \mathrm{psid}$, and the average slurry temperature was $\mathrm{T}=29^{\circ} \mathrm{C}$ (the filtrate flux was adjusted to $\mathrm{T}=25^{\circ} \mathrm{C}$ ).

The insoluble solids started at $19.0 \mathrm{wt} \%$ and ended at $18.8 \mathrm{wt} \%]$

\begin{tabular}{|c|cccccc|}
\hline Vol. Wash & Total Solids & $\begin{array}{c}\text { Insoluble } \\
\text { Sol. Slurry }\end{array}$ & $\begin{array}{c}\text { Slurry } \\
\text { Selids }\end{array}$ & $\begin{array}{c}\text { Filtrate } \\
\text { Solids }\end{array}$ & $\begin{array}{c}\text { Slurry } \\
\text { Density }\end{array}$ & $\begin{array}{c}\text { Slurry } \\
\text { Consistency }\end{array}$ \\
Yield Stress \\
\hline
\end{tabular}

Figure 64. Starting and ending slurry properties for the washing test run. The total amount of inhibited water added was $21 / 18 \mathrm{X}$ the initial slurry volume or approximately $116 \%$.

These changes in slurry properties imply the scattering of filtrate flux data after about the first 60 minutes of washing. Figure 63 shows an increasing less stable flux as time (and added wash water) inc reased. The slurry velocity and TMP had to be continually trimmed to maintain that target values mentioned above. It is probable that as the slurry became successively more washed the filtrate cake was being affected and forcing the velocity and TMP to change. As the flux was trimmed to maintain it constant then the process repeated itself, leading to the shotgun scatter depicted in Fig. 63.

Of course, the reason for the washing was to remove some of the soluble salts, especially sodium. Figure 65 shows the result of the removal. 


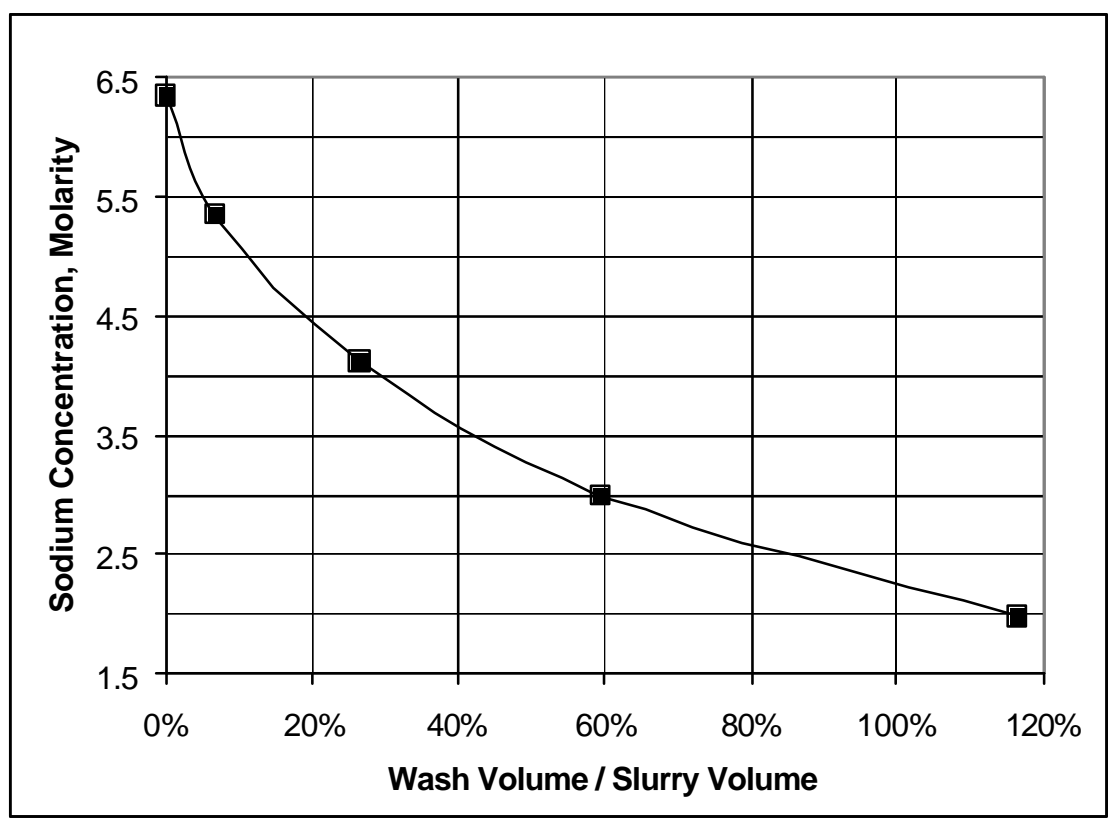

Figure 65. Reduction of $\mathrm{Na}^{+}$by washing with 21 mini-batches of inhibited water to AN-102R2, Batch 3B

\subsection{Concentration of Washed AN-102R2, Batch 3B}

Immediately following the washing test run the resulting slurry was concentrated under a constant slurry velocity of $12 \mathrm{ft} / \mathrm{s}$ and TMP $=40 \mathrm{psid}$. Figure 66 shows the results.

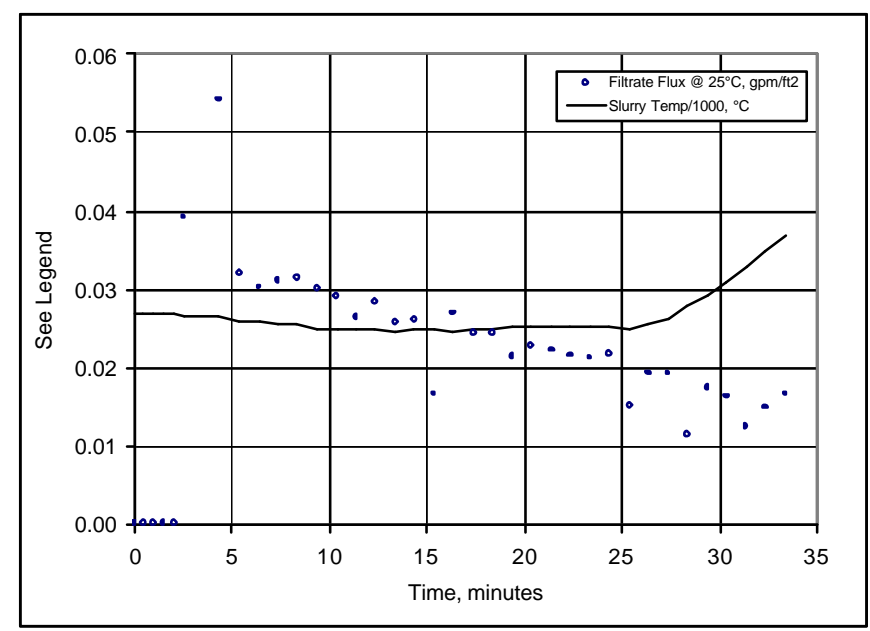

Figure 66. Dewatering of washed slurry (AN-102R2, Batch 3B): Test Run 1.30.

The average slurry velocity was $11.9 \mathrm{ft} / \mathrm{s}$ and the average transmembrane pressure was $\mathrm{TMP}=40.1 \mathrm{psid}$.

The insoluble solids increased from $19 \mathrm{wt} \%$ to $25 \mathrm{wt} \%$. The average slurry temperature was $\mathrm{T}=27^{\circ} \mathrm{C}$, but as is shown the temperature rose rapidly after 25 minutes. (The filtrate flux was adjusted to $\mathrm{T}=25^{\circ} \mathrm{C}$.) 
The slurry began at approximately $19 \mathrm{wt} \%$ insoluble solids but the test was stopped when it reached $25 \mathrm{wt} \%$. After about 25 minutes the slurry temperature began increasing rapidly and the test rig cooling system could no longer maintain the temperature stable. When the slurry temperature reached approximately $38^{\circ} \mathrm{C}$ the test was terminated. If fact, the thermal momentum caused the slurry temperature to continue rising until it reached $46^{\circ} \mathrm{C}$. While the slurry was definitely thick in appearance it flowed freely and no plugging was observed.

\subsubsection{Scaling: Differences between the PXU and Bench Top Cross-flow Test Facilities}

\subsubsection{Comparison Challenges}

The goal of the task at hand was to test several RPP WTP Envelope-C-type slurry simulants in a pilot-scale cross-flow ultrafiltration (PXU) ficility. In many ways the pilot test rig is prototypic of the planned plant cross-flow ultrafiltration system. Figure 67 shows a comparison among the planned WTP filter bundle with that of the PXU facility and to the bench top test facility referred to as Cells Unit Filtration (CUF) facility.

\begin{tabular}{|c|c|c|c|}
\hline Filter Tube Comparison & Planned WTP & Pilot Scale & Small Scale \\
\hline & & & \\
\hline Material & 316 stainless steel & same & same \\
\hline Pore Size & 0.1 micron & same & same \\
\hline Length & 90 inches & same & 24 inches \\
\hline Tube Inside Diameter & $1 / 2$ inch & same & $3 / 8$ inch \\
\hline Tube Outside Diameter & $5 / 8$ inch & same & $1 / 2$ inch \\
\hline Arrangement & multiple & same & sinqle \\
\hline Number & 241 per bundle & 7 & 1 \\
\hline Grid Pattern & $60^{\circ}$ Triangular & same & not applicable \\
\hline Center-to-Center Distance $^{\dagger}$ & $7 / 8$ inch & same & not applicable \\
\hline Orientation & Horizontal & Vertical & Horizontal \\
\hline & & & \\
\hline Other Features & & & \\
\hline Slurry Pump & centrifugal & same & low shear \\
\hline
\end{tabular}

Figure 67. Comparison of full-scale to scaled ${ }^{\dagger}$ test facilities

Details of the CUF work are not given here, but can be found in Zamecnik et al. (2003). Only salient features of the CUF results are discussed in an attempt to see the effect of scale on experimental results. The purpose of the CUF test was two-fold: 1. Test actual waste, which is radioactively hot, and 2. compare the real waste filterability to simulants of that waste. To test actual radioactive wastes at pilot or plant scales would have been impractical. The handling of radioactively hot waste is very time consuming and costly and as scale increases so do the costs and risks. The CUF facility can operate with only 3 liters of slurry

\footnotetext{
$\dagger$ Orientation is included in the scaled criteria for completeness, however, it is not considered a significant difference. During an Oct 17, 2001 meeting, Prof. Joseph L. Gaddis of the Dept. of Mechanical Engineering of Clemson University, an expert on cross-flow filtration, stated that at the high velocities, i.e., high turbulence, that will be used for the cross-flow filtration facilities, orientation will not affect operation. He further stated that it is only important during shut down when gravity can assist emptying vertical tubes.
} 


\section{WSRC-TR-2003-00204, REV. 0}

SRT-RPP-2003-00087, REV. 0

and has a small enough footprint that it can fit into most shielded cells to protect workers. Unfortunately, due to the complexities of the waste and the filter flow fields, extrapolating the small scale CUF data to the full-scale plant facility is not simple and at the very least would contain large uncertainties. The PXU facility was designed to be close to prototypic to minimize scaling issues so that confident full-scale data could be obtained. Of course, the drawback of the larger pilot scale is the amount of waste it needs and that it has to operate radioactively cold. This means a simulant must be used. For each waste a simulant, or simulants, is developed for large-scale testing. These simulants were made to match the real waste with respect to chemical and physical properties, while not being radioactive.

Because of the major differences between the PXU and CUF facilities filterability results were not expected to match. However, by showing statistically similar filter results for the actual waste and a simulant at the same scale, the CUF, then confidence was established for that simulated waste. A simulant verified with the CUF could then be used for testing at the pilot-scale to obtain results that would more closely match actual waste filterability at that large scale. The PXU results for the many conditions under which they were obtain, e.g., steady state, dewater, cleaning, rinsing, etc. have been previously discussed in this report. This section will be limited to discussing only comparable small-scale and pilot-scale results where possible. Even if results do not match between the scales, it is hoped that insight is obtained by making such a comparison.

Besides differences in scale of the CUF and PXU facilities, the other confounding feature is dissimilar simulants. This report deals with an organic based waste, classified as an Envelope C type waste. There are others, e.g., Envelopes A and B, which are non-organic and Envelope D, which is basically sludge based, containing most of the solids. All of these wastes are chemically complex and simulants are equally complex being comprised of dozens of compounds. Further, the Envelope $\mathrm{C}$ wastes are pre-treated to remove strontium and transuranics by precipitation before filtration occurs. This level of complexity makes one-to-one comparisons very difficult because no one simulant is the same. Even when two groups make the same simulant, following the same recipe, the resulting slurry may not be the same due to differences of how the recipe was applied and subsequent aging before filtration begins.

Because of differences in equipment of the CUF and PXU facilities and in formulations of waste simulants, there do not exist, to date, any data to make a quantitative side-by-side comparison of scale. Hwever, there are some data that can be utilized to make some qualitative estimations of the effect of scaling.

Going back to 1997 (Nash and Siler, 1997) RPP-WTP work began with Envelope C simulated slurries. [In 1997 the waste types were just being defined and Envelope-C-type wastes were referred to as Envelope A. The important feature to remember is that this type of waste contained organic complexants, which necessitated precipitation to remove strontium and transuranic components. An explanation of the different types of Hanford wastes and simulant slurries developed for the project can be found in Eibling and Nash (2001).] This early version of the benchtop filter unit contained a single porous tube that had a 0.5 -inch inside diameter, it was 4 feet long, and the 0.1 -micron porous tube was of a 
different design altogether. Besides the different filter structure, that 1997 test resulted in very poor filterability. After a little over a year of development SRTC recommended precipitation of Envelope $\mathrm{C}$ wastes with the additions of strontium nitrate and sodium permanganate. By the end of 1998 and into the early part of 1999 tests with the CUF showed that the new precipitation process with strontium and permanganate increased filterability (Nash, et al., 2000). Unfortunately, the CUF porous tube had been changed to a 0.2 porous tube and a 0.5 -inch inside diameter, which made comparisons with to 0.1 micron porous tube filtration difficult.

\subsubsection{A First PXU-to-CUF Comparison with an Envelope C Simulant (AN-107)}

After making some modifications to the SRTC CUF design, to conform to RPP-WTP needs, and refining the strontium and permanganate quantities, this new precipitation process was verified by PNNL by the end of 1999 with simulated Envelope C waste (AN-107) (Hallen, et al., 2000). The PNNL modified CUF had a two-foot long, 0.375-inch inside diameter filter tube with 0.1-micron porosity. That filter's initial filter flux was $0.03 \mathrm{gpm} / \mathrm{ft}^{2}$ (averaged over 1 hour) at filter slurry velocity of $12.2 \mathrm{ft} / \mathrm{s}$ and a TMP $=50 \mathrm{psid}$. PXU (Duignan, 2000b) testing for Envelope C simulated waste (AN-107) began in October 1999 and it too had a 0.375 -inch inside diameter filter tube with 0.1-micron porosity, but it was 3.3-feet long. That filter's initial filter flux was $0.08 \mathrm{gpm} / \mathrm{ft}^{2} \pm 0.01 \mathrm{gpm} / \mathrm{ft}^{2}$ (averaged over 1 hour ). Why was the PXU (AN-107) result higher than the CUF (AN-107)? The reason is not known. For the PXU 2000 test the Envelope C simulant was based on an SRTC recipe (Eibling and Nash, 2001). After precipitation steps and then mixing for 4 hours at $50^{\circ} \mathrm{C}$ the simulant had a density of $1.29 \mathrm{~g} / \mathrm{mL}$, a viscosity of $4 \mathrm{cP}$, insoluble solids of approximately $1.9 \mathrm{wt} \%$, sodium of $5.9 \mathrm{M}$, strontium of $0.075 \mathrm{M}$, and permanganate of $0.05 \mathrm{M}$. The CUF, used at PNNL (Hallen, et al., 2000) used the same recipe, that is, after precipitation it was mixed for 4 hours at $50^{\circ} \mathrm{C}$. The PNNL simulant had a density of $1.26 \mathrm{~g} / \mathrm{mL}$, a viscosity of 7 to $18 \mathrm{cP}$, insoluble solids of approximately $1.9 \mathrm{wt} \%$, sodium of $6 \mathrm{M}$, strontium of $0.075 \mathrm{M}$, and the permanganate of $0.05 \mathrm{M}$. It appears that both simulants were similar but for some reason the SRTC simulant did not start out as viscous. Now the differing viscosities could be attributed to measurement of rheological properties, but since the simulants were made from 3 dozen different compounds it would take significant analyses to understand the subtle differences and how those difference affect filtration.

\subsubsection{A Second PXU-to-CUF Comparison with an Envelope C Simulant (AN-102R2)}

The reason data exist for the first comparison was simply a coincidence. That is, those data are available due to simultaneous programs being done in the DOE Complex to meet RPP-WTP requirements. In both cases, the only driver was to have a simulant as close as possible to actual waste. No attempt was made to have exactly the same slurries so that such 
a comparison could be made. The only subsequent situation that occurred, which allowed ${ }^{\dagger} \mathrm{a}$ conscious effort to be made to make a better comparison was during the precipitation and dewatering tests at SRTC of another Envelope-C-type simulant (AN-102R2). The first dewatering test of an $\mathrm{AN}-102 \mathrm{R} 2$ simulant (called Batch $3 \mathrm{C}$ and previously discussed in this report) resulted in very low filter fluxes for both the CUF $\left(<0.02 \mathrm{gpm} / \mathrm{ft}^{2}\right.$ at $8 \mathrm{wt} \%$ insoluble solids: Zamecnik, et al., 2003) and the PXU facility $\left(=0.005 \mathrm{gpm} / \mathrm{ft}^{2}\right.$ at $8 \mathrm{wt} \%$ insoluble solids: this report). For this first AN-102R2 test both the CUF and the PXU personnel made their own slurry simulants. To increase the filterability another dewatering test was done with some changes to the simulant. However, this time, after making the PXU 1000-liter batch, then precipitating and mixing for 4 hours at $50^{\circ} \mathrm{C}, 75$ liters of simulant were removed and sent for use in a CUF facility. The hope was that differences in scale could be measured if the slurry were exactly the same in both facilities and operated under the same flow conditions. Unfortunately, while the CUF facility was the same as mentioned above, i.e., it had a two-foot long, 0.375 -inch inside diameter filter tube with 0.1 -micron porosity, the PXU facility had been changed to meet new RPP-WTP requirements of a 0.5 -inch inside diameter and a 90-inch long porous tube. Figure 68 shows the comparison of the PXU and CUF tests which dewatered the AN-102R2 slurry from $1.2 \mathrm{wt} \%$ to $22 \mathrm{wt} \%$ (for the PXU) and $1.6 \mathrm{wt} \%$ to $19 \mathrm{wt} \%$ (for the CUF)

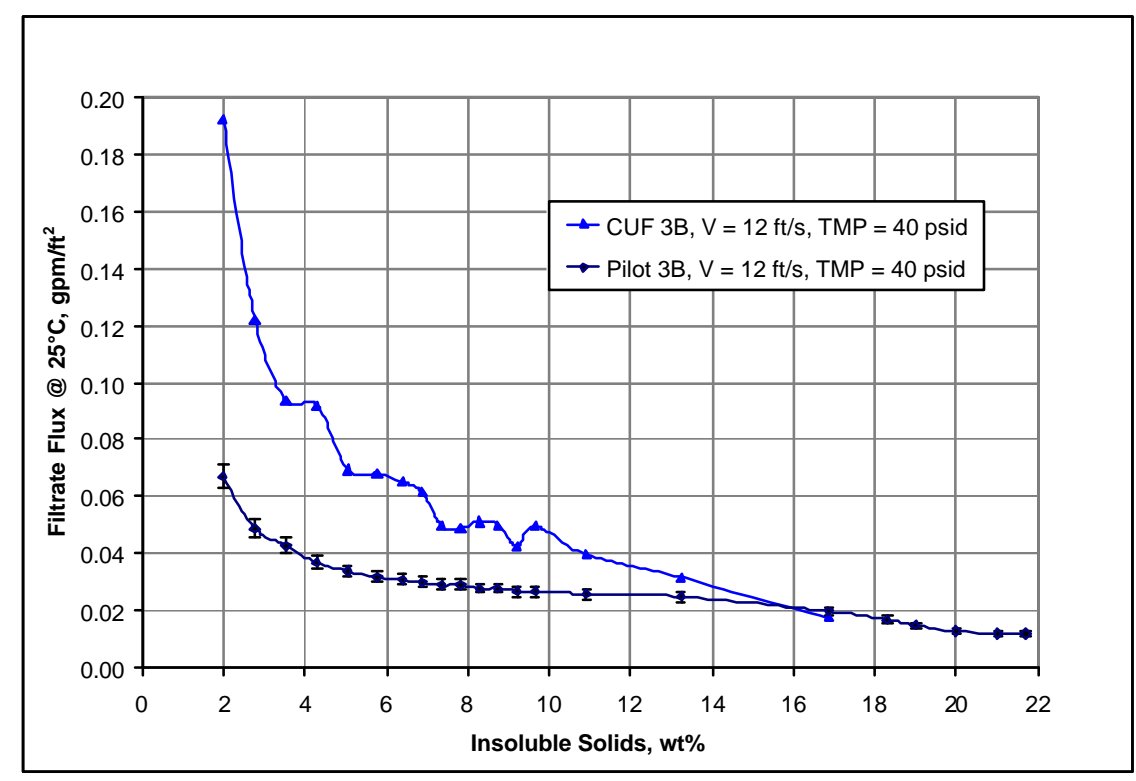

Figure 68. PXU to CUF filtrate flux comparison using an AN-102R2 simulant

It is important to note that the data in Figure 68 were obtained differently for each facility. For the CUF, each datum point was an average of a 3 liter batch of slurry. That is, filtrate flux is measured by the time it took for 3 liters to be filtered. The CUF test dewatered until

\footnotetext{
${ }^{\dagger}$ The RPP-WTP research and technology project did not plan for dedicated one-to-one comparison testing; therefore, any such work had to be done in conjunction with other planned and funded tasks and at the initiative of the principal investigators.

* The difference in the initial PXU and CUF insoluble solids concentrations may be due to analytical measurements or sampling techniques
} 


\section{WSRC-TR-2003-00204, REV. 0}

SRT-RPP-2003-00087, REV. 0

the slurry solids concentration reached approximately $19 \mathrm{wt} \%$ but the last average flux value was at approximately $17 \mathrm{wt} \%$. The PXU facility measured filtrate flux instantaneously and data were taken every minute. To make the Fig. 68 comparison the values of the PXU flux were taken at each of the averaged CUF insoluble solids points. This is the reason why all the abscissa points seem to line up. However, since the PXU dewatered up to $22 \mathrm{wt} \%$ insoluble solids, those points were also included. At about $16 \mathrm{wt} \%$ insoluble solids the two curve cross. At this point the CUF facility was close to its operational limit.

In general, it looks like the CUF filtrate flux is about a factor of 2 larger than the PXU facility up to approximately $10 \mathrm{wt} \%$ insoluble solids, after which the curves merge. At that point the thickness of the filter cake and increased slurry consistency minimize any scale differences. The data obtained from both tests do not give a clear indication as to why the curves are significantly different. Figure 69 includes some of the pertinent information of the facilities and the slurry used in both.

The PXU data have been taken from this report and those for the CUF have been estimated from data displayed in Zamecnic, et al. (2003). As already stated above, the starting slurry was exactly the same. It was made at one time and then 75 liters were separated for use in the CUF. The only starting difference is time. The PXU began filtration immediately after the newly precipitated slurry was mixed at $50^{\circ} \mathrm{C}$ for 4 hours. The CUF test began the following day, approximately 16 hours after the PXU test beginning; therefore, further precipitations reactions could have occurred. In fact, the particle-size distributions (PSD) for the initial CFU slurry appears to be slightly, but significantly, larger. However, all other characteristics, i.e., solids concentration, rheology, and density, appear to be the same ${ }^{\dagger}$. The PSD difference may have existed or it may have simply been how the PSD was performed. For instance, the PSD samples sit for a while before processing so they are given a mild amount of sonication to reduce the particles to their base size. The question is, "What is that base size?"

However, in both tests the particle-size distribution show three distinct changes:

1. The mean particle size was reduced to approximately one half.

2. The spread of particle sizes was reduce to approximately one half.

3. The bimodal volume distribution was reduced to predominately a single size.

In discussing the filtrate flux differences shown in Fig. 68 Zamecnik, et al. (2003); (on page 63 of that reference), hypothesized that the different pump types, i.e., PXU high shear centrifugal to CUF low shear progressive cavity, caused the particles in the PXU test to become smaller and therefore be harder to filter. Unfortunately, the data do not back this claim up. The mean particle size of the concentrated slurry for the PXU may be slightly smaller than the CUF, but the difference is minimally significant. Further, the same difference is apparent in the initial slurry, which was exactly the same before each facility began testing, therefore this size difference is questionable. However, even if the particle were significantly smaller the same report contradicts its hypothesis by stating, "Surprisingly, the most filterable slurries appear to have more smaller particles in the 1-4 micrometer range

\footnotetext{
${ }^{\dagger}$ See the preceding footnote.
} 


\section{WSRC-TR-2003-00204, REV. 0 SRT-RPP-2003-00087, REV. 0}

and, in general, have a flatter distribution of particle size." Indeed, Fig. 14 of that report shows that as the mean particle size became smaller the average filter flux increased. This counter-intuitive result makes sense when looking at the micrograph of that report, Fig. 17, which shows "sheet-like flat" particles in the 5 to 20 micron range for less filterable slurries.

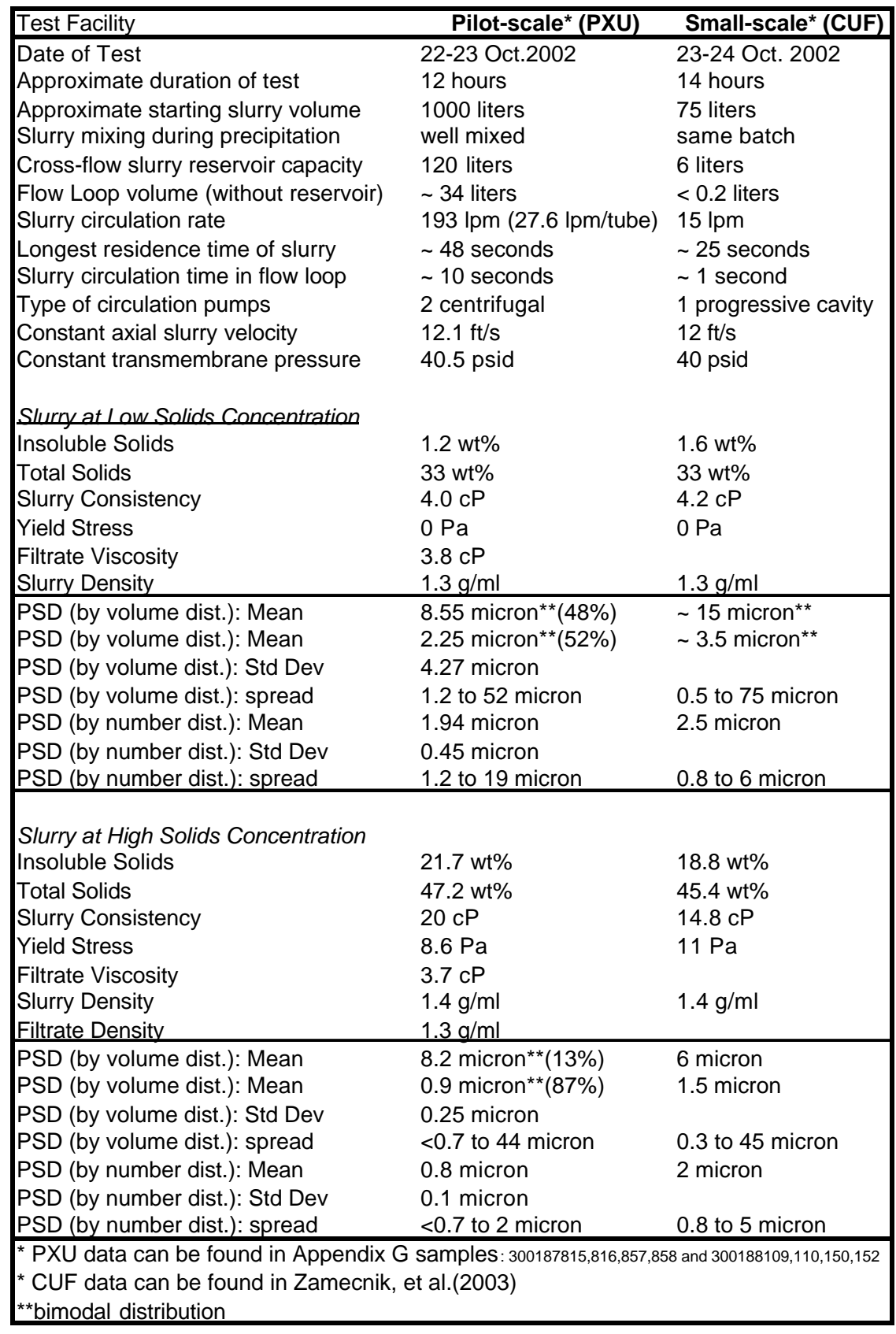

Figure 69. PXU to CUF comparison AN-102R2, Batch 3B simulant

As the slurry is processed in the filter facilities some of the large flat particle break up through shear. This breakup can happen in both the CUF and the PXU. Moreover, the fact that the CUF used a low shear pump does not mean the slurry does not experience significant 
shearing. By design the CUF was made to minimize the amount of slurry needed to filter because radioactive slurry samples are small; therefore, the circulation rate is high. Note that in Fig. 69 the slurry in the CUF takes under 1 second to traverse the loop. If the longest residence time, as shown in Fig. 69. is used, then for the first charge of 6 liters of slurry, of which 3 liters was dewatered over a 20.4-min period, the slurry was circulated 20.4*60/24= 51 times. By the end of the 14-hour test the initial batch of slurry had circulated $14 * 3600 / 24$ $=2,100$ times. For the PXU, the first slurry batch circulated only $12 * 3600 / 48=900$ times. Considering the high turn over rate of the slurry, the speed of the slurry, i.e., $12 \mathrm{ft} / \mathrm{s}$, and the pipe fittings and control valve in the CUF flow loop, the slurry does experience a considerable amount of shear. Many of the compounds that make up the slurry have strong oxide particles, which seem not to get any smaller than approximately $1 / 2$ micron. In fact, it appears (Nash and Siler, 1997) the particle breakup occurs mostly in the first few minutes of circulation and then remains fairly constant. The conclusion here is that when large flat particles are broken down to smaller, less flat, particles the slurry filters better. Since the smaller particles seem to be 3 to 20 times larger that the nominal pore rating of 0.1 micron, then the slurry becomes more filterable. That is, the flat particles are broken down so that they do not blind the filter element.

Another possible reason for differences in CUF and PXU filter fluxes is the difference in wall shear. Figure 70 shows a cartoon of how a cross-flow filter works. As the slurry and most of its liquor travels through the inside of the porous tubes a filter cake builds up on the inside. The thickness of the cake is exaggerated in Fig. 70 for illustration, but it could eventually build up to only about the thickness of a piece of paper.

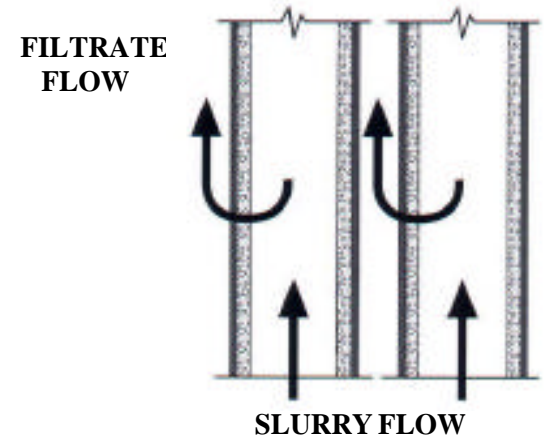

Figure 70. Operation of a multi- tube cross flow filter like the PXU

An advantage of the cross-flow filter over a dead-end filter is not needing periodic changing because the moving slurry constantly shears the cake, keeping it at reasonable thickness and a fairly constant filtering rate. Since the wall shear affects the cake formation on the porous wall, differing levels of wall shear may strongly influence that rate at which the slurry liquor can permeate through the porous wall, thus giving different filtration rates. As shown in Fig. 67 the CUF has a smaller diameter and shorter porous tube than the PXU, while Fig. 69 shows the axial slurry velocity was maintained the same at $12 \mathrm{ft} / \mathrm{s}$.

To see the wall-shear effect more quantitatively a simple computational fluid dynamic model was made for two slurries that represent the initial slurry and the concentrated slurry. 
Figure 71 shows the parameters assumed for the CUF and the PXU facilities. The slurry was assumed to be Newtonian and fully turbulent, i.e., Reynolds number greater than 2000 . The slurry at low solids concentration does indeed behave as a Newtonian solution, however, as the solids concentration increased it become slightly thixotropic, i.e., shear thinning with a time dependent shear stress. This non-Newtonian effect is not strong and as long the slurry is flowing it still behaves like a Newtonian solution. While the turbulence in the larger diameter tube is $1 / 3^{\text {rd }}$ more than the smaller tube for the same slurry velocity, i.e., $\operatorname{Re}($ large $) / \operatorname{Re}($ small $)=$ large ID / small ID $=0.5 / 0.375=1.333$, they are both much larger than the turbulence threshold of $\mathrm{Re}=2000$. By using some rough parameters, Fig. 71, for the two situations a comparison can be made between the two flow situations.

\begin{tabular}{|c|c|c|}
\hline Physical Parameters & $1.5 \mathrm{wt} \%$ Slurry & $25 \mathrm{wt} \%$ Slurry \\
\hline Porous Tube I.D. & $3 / 8$ and $1 / 2$ inch & $3 / 8$ and $1 / 2$ inch \\
\hline Axial Slurry Velocity & $12 \mathrm{ft} / \mathrm{s}$ & $12 \mathrm{ft} / \mathrm{s}$ \\
\hline Slurry Density & $1.3 \mathrm{~g} / \mathrm{ml}$ & $1.5 \mathrm{~g} / \mathrm{ml}$ \\
\hline Slurry Viscosity & $4 \mathrm{cP}$ & $20 \mathrm{cP}$ \\
\hline
\end{tabular}

Figure 71. Parameters used in a wall shear CFD model

Figure 72 shows the low solids concentration CFD results. Immediately obvious is the much higher wall shear for the small tube. At 24 inches $(0.6 \mathrm{~m})$ the steady state wall shear is $24 \%$ higher for the small tube. Further, it takes approximately $1 / 3^{\text {rd }}$ of the 24 -inch span for the wall shear to come to steady state because of entrance effects. There is approximately the same entrance effect on the larger tube, but since it is 90 inches long the effect is much less. When taking into account the entrance effects, the wall shear for the $3 / 8$-inch tube is approximately $30 \%$ higher than the $1 / 2$-inch tube.

As the slurry concentrates the wall shear difference decreases. Figure 73 shows the results. When taking into account the entrance effects, the wall shear for the $3 / 8$-inch tube is approximately $20 \%$ higher than the $1 / 2$-inch tube. However, since the filtrate cake built up when the slurry had a low concentration of insoluble solids, then the differences at the high concentrations may not be significant, as may be implied by the two filtrate flux curves coming together above approximately $16 \mathrm{wt} \%$ in Fig. 68. 


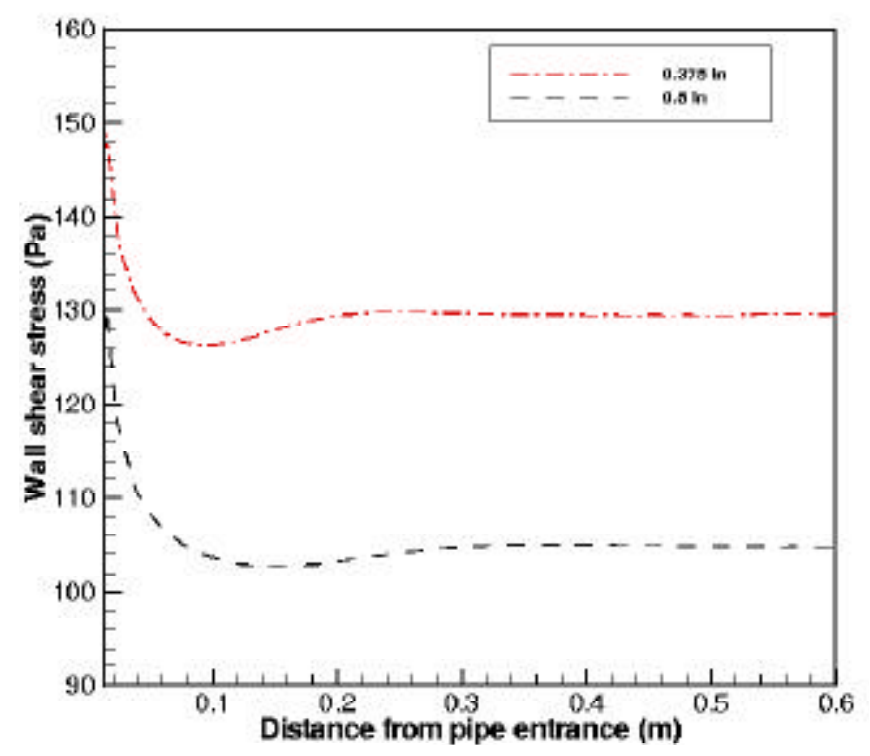

Figure 72. Wall shear comparison for two different tube IDs at $12 \mathrm{ft} / \mathrm{s}$ and a viscosity of $4 \mathrm{cP}$.

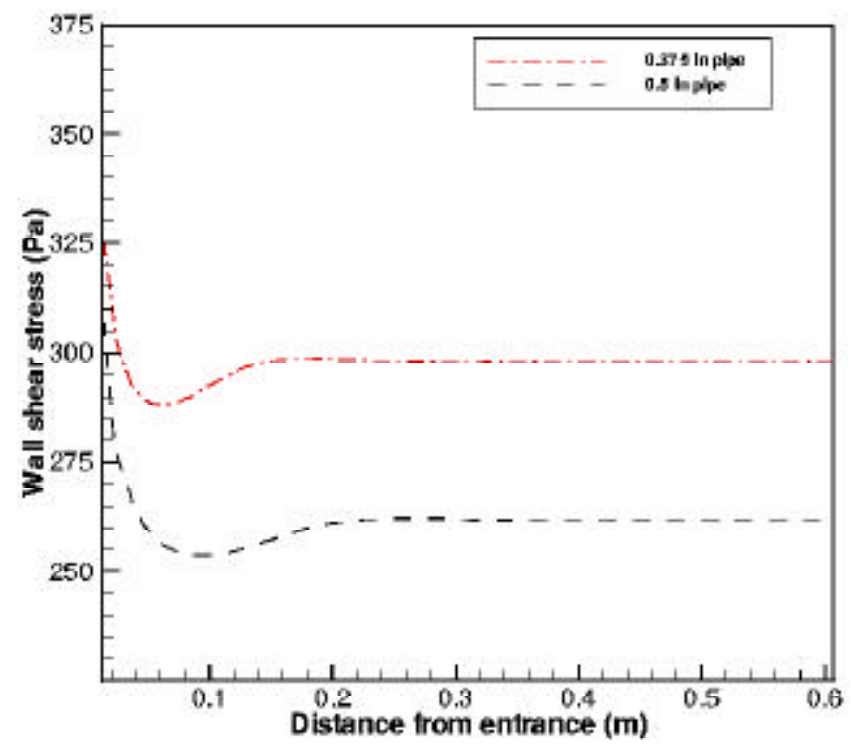

Figure 73. Wall shear comparison for two different tube IDs at $12 \mathrm{ft} / \mathrm{s}$ and a viscosity of $20 \mathrm{cP}$.

As stated by Murkes and Carlsson (1988) about crossflow filtration: "The method is basically a cake-free method (or intended to be such). Its purpose is to prevent the formation of the cake. Particles deposited on the filter medium are swept away by the feed flow. The cleankeeping efficiency of the flowing liquid increases with its velocity. Thus, the particle of the solute concentration polarization is controlled by the flow velocity." In the comparison between the PXU and the CUF facilities the velocities were kept the same, however, due to differences in porous tube channel size, the larger the channel the lower the wall shear, which is the mechanism that shears away the particles deposited on the filter medium. Shear stress is directly related to fluid velocity through Newton's law of viscosity, i.e., 


$$
\tau=\mu \mathrm{dv} / \mathrm{dy} \quad \text { and at the wall } \quad \tau_{\mathrm{wall}}=\mu[\mathrm{dv} / \mathrm{dy}]_{\mathrm{y}=0}
$$

where $\mathrm{v}$ is the local slurry velocity, $\mathrm{y}$ is the spatial coordinate perpendicular to the slurry flow, $\mu$ is the slurry dynamic viscosity, and $\tau$ is the local shear stress. Further Murkes and Carlsson show that the filtrate flux is related directly proportional to the filtrate cake permeability and inversely related to filtrate cake thickness. As the cake forms under the local wall shear both parameters will be affected. Figure 74 shows a schematic of slurry flow in a porous tube with an established filter cake. As the diameter, D, gets smaller so does the turbulence level, because it is directly proportional to the Reynolds number, which is directly proportional to this dimension. However, as D becomes smaller the boundary layer, where wall shear stress occurs, grows, and becomes a larger portion of the flow area, which is proportional to the square of the diameter. Therefore, wall shear stress is larger for smaller flow channels as long as the velocity remains constant.

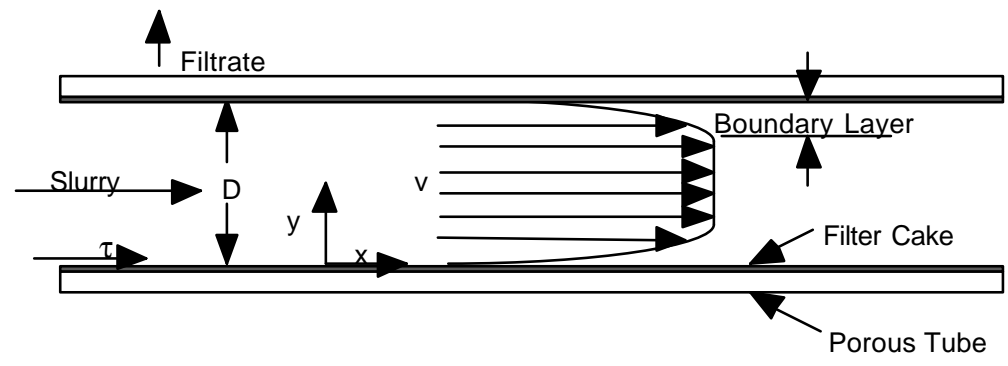

Figure 74. Slurry flow through a cross-flow ultrafiltration tube

Does the higher wall shear stress, and thus the higher "clean-keeping efficiency" of the CUF shown in Fig. 72 cause it to have a higher filtrate flux? The evidence seems to imply the affirmative. Is the initial 30\% higher wall shear stress in the CUF enough to make its filtrate flux approximately double that of the PXU? It is possible, but there is not enough quantitative data to show this fact. These organic-based waste slurries appear to be very sticky, that is, there seems to be sufficient inter-particle forces to make the solids cling to each other more than, for example, sand in water. These solids probably form some kind of a gel-like cake on the filter surface, which does not lend itself to available analyses. However, the changing wall shear stress with scale must have an effect and possibly an effect large enough to cause the differing results obtained with the CUF and the PXU facilities.

\subsubsection{Other PXU-to-CUF Differences}

Even if the wall shear differences could be accurately taken into account it may not be enough to show the differences between these two filter facilities. Other differences must exist and have not been quantified. Consider the differences in the hardware like the two porous tubes used and the pumps that circulate the slurries, or in the slurry simulants, etc. 


\subsection{Differences in filter hardware}

- Porosity - Both the CUF and the PXU facilities used 0.1 micron nominal porous tubes. However, each batch sintered-metal tubes is different. The 0.1 micron is an average value. At this ultrafiltration pore size small differences in porosity may have significant permeability effects. Besides, through the years a manufacturer like the Mott Corporation has changed, perfected, and enhanced their porous materials which sometimes has caused confusion of ordered products. For instance, the size 0.1 micron porosity sometimes means several difference things as described in Duignan (2001), and only with a closely monitored post-manufactured test can the porosity of any two tubes be equated.

- Tube Wall Thickness - While the inside diameter of tubes should be close to purchased size due to the process of forming sintered metal around a very accurately ground mandrel, the outside diameter depends on the force of a flexible surface to maintain the metal particle under pressure. This is evidenced by the measurements made on the seven-tube PXU tube assembly when it arrived from the manufacturer. Each of the seven tubes was made of four subsections (see Figs. 3 and 4) and a total of 100 measurements were made on the tubes to determine the dimensions. The manufacturer's stated inside diameter was 0.5 inch and the received tubes had an average diameter of 0.488 inch, with a maximum of 0.490 inch and a minimum of 0.485 inch. This 0.005 -inch variation is considered very good. . However, the manufacturer's stated outside diameter was 0.625 inch and the received tubes had an average diameter of 0.654 inch, with a maximum of 0.674 inch and a minimum of $0.640 \mathrm{inch}$. This 0.034 -inch variation is large but expected. While the 7-tube bundle of the PXU had 28 subsections, the CUF was comprised of a single 24-inch tube. The CUF would represent the PXU dimensionally only by coincidence, but more likely it was dimensionally different, which may have had some significant flux differences. The PXU will be more representative of the full-size plant filter unit because of the large variations in dimensions.

As the filter cake builds up on the inside of the porous tube the differences in porosity and wall thickness should become less evident because then the cake becomes the filtering medium and the largest source of pressure drop. However, it is probably very important how the filter cake is initially formed, which may persist for a significant amount of time until the cake is thick enough to make the two filters act the same. This seems to have happened as seen in Fig. 68.

- Feed Pumps - As mentioned in the section 3.3.3.3, the pumps for the two facilities were different. The CUF facility used a single air-driven progressive-cavity pump made by Oberdorfer. This pump had a stainless steel rotor, which was covered with a layer of hardened chrome. The rotor operated in a Buta-N stator, which has good resistance to caustic service but limited resistance to acid service, and moved the slurry in a piston-like fashion, through a helix-shaped cavity. Up to a threshold pressure this type of pump acts like a positive displacement pump, however, when the maximum pressure is reached (in this case it was approximately $90 \mathrm{psig}$ ) the elastomer liner gives. At a steady-state operation of a slurry velocity of $12 \mathrm{ft} / \mathrm{s}$, the CUF pump flow was $4 \mathrm{gpm}$. As mentioned in section 3.2.1.1, the PXU facility used two centrifugal pumps in series and were made by 
Galigher. The impellers and housings were made of EPDM, which has good resistance to both acid and caustic services. At a steady-state operation of a single filter tube slurry velocity of $12 \mathrm{ft} / \mathrm{s}$, the PXU pumps flow was $51 \mathrm{gpm}$. With respect to shear, the progressive-cavity pump imparted less than the centrifugal pumps, however, as seen in Fig. 69, the CUF circulated the slurry at a much higher rate due to the very small flow loop. Unfortunately, the high shear at the CUF control valve may have negated any benefit to the lower shear present by the pump.

\subsection{Differences in slurry simulant}

- $\quad$ Time - The simulant used in the AN-102R2 comparison for both the CUF and the PXU facilities was made at one time and when it was ready for filtration some of the large batch was removed and transported to the CUF location. However, the filtration of the separated sub-batch of simulant for the CUF only began about 15 or 16 hours after the PXU began. Figure 69 shows some characteristics of the simulant at the beginning filtration for the respective facility, like slightly larger particles for the CUF simulant, however, it does not show the complex number of reactions that occurred in making the simulant, nor the changes due to the reactions with time. The precipitation report (Steeper and Williams, 2003) shows that while most of the simulant was predominantly stable after 4 hours of mixing, just before feeding to the PXU facility, there are still some reactions occurring. For instance, about $10 \%$ of the aluminum available as a solid, when the PXU began filtering, is incorporated into the aqueous after about 20 hours. The presence of solid aluminum is known to inhibit filtration and being present for the PXU testing and not for the CUF testing may have had an effect. The point here is that chemically the simulants were slightly different due to aging, which could have contributed to the differing results.

\subsection{CONCLUSIONS}

\subsubsection{Water}

1. In general, inhibited water $(0.01 \mathrm{M} \mathrm{NaOH})$ tests before and after each slurry test indicate a return to "clean" filter fluxes (Fig. 19).

2. However, when water was used without the addition of caustic the filtrate flux was significantly higher (Fig. 21), which is assumed to be the result of having fewer precipitating solids left in the test rig.

\subsubsection{Standard Slurry $\left(\mathrm{SrCO}_{3}\right)$}

1. The use of a standard slurry to show a filter's return to cleanliness was confounded by the level of cleanliness of not just the filter but of the entire filter system (Fig. 29). That is, unless the entire filtration loop was clean then determining the cleanliness of just the inline filter may not be possible.

2. The heavy solids of the $5 \mathrm{wt} \% \mathrm{SrCO}_{3}$ standard slurry required transmembrane pressure above 20 psid for a filter to be challenged (Fig. 27). 


\section{WSRC-TR-2003-00204, REV. 0}

SRT-RPP-2003-00087, REV. 0

3. The standard slurry could only be effectively removed from the filter system with the used of acid because it dissolves carbonate. A concentration of $0.05 \mathrm{M} \mathrm{HNO}_{3}$ was used.

\subsubsection{Acid Cleaning}

1. The method used, two 90-minutes circulations of $2 \mathrm{M}$ nitric acid, cleaned most of the solids away from the filter, but there were always some remaining solids, which reduced the acid filtrate fluxes from that of a clean filter. (Fig. 35). That is, the complex wastes may need more targeted cleaning to remove all waste remnants.

2. Cleaning periods of more than three hours with the $2 \mathrm{M}$ nitric acid may not improve effectiveness. (Fig. 33).

\subsubsection{Dewatering}

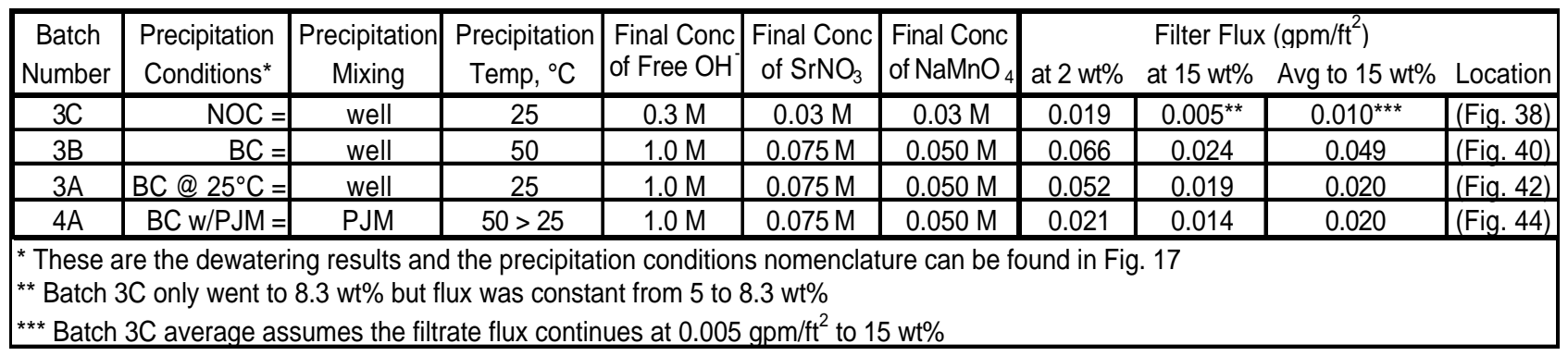

Figure 75. Overall dewatering results

1. Filtering to above $15 \mathrm{wt} \%$ insoluble was attainable. (Fig. 36), with results in the $18 \mathrm{wt} \%$ to $25 \mathrm{wt} \%$ range.

2. Precipitation with Newly Optimized Conditions left the wastes poorly filterable. (Figs. 37, 38, and the $1^{\text {st }}$ line in Fig. 75)

3. AN-102R2 had the best filterability when prepared at precipitation baseline conditions which included being well mixed and prepared at $50^{\circ} \mathrm{C}$. (Figs. 39, 40, and the $2^{\text {nd }}$ line in Fig. 75.)

4. AN-102R2 was more filterable when solid particles were broken down to near the micron size. (Compare Fig. 50 to the penultimate column in Fig. 75 and see section 3.3.3.3, which discusses how larger sheet-like particles may reduce filtration.)

5. Rheological properties of simulants underwent large changes as the slurry increases above 14 wt $\%$ insoluble solids. (Fig. 46)

6. Backpulsing the filter with this Envelope C-type slurry was not very effective and after reaching an insoluble solids concentration of approximately $5 \mathrm{wt} \%$ backpulsing became ineffective. (Figs. 38, 42, and 44)

7. Excellent solids separation was obtained with turbidities on the order of 0.2 NTU, possibly indicating that the separated filtrates had had less than $0.2 \mathrm{ppm}$ of insoluble solids (see section 3.3.1). 


\subsubsection{Steady State}

1. Increasing the slurry axial velocity by a factor of 2 increased the filtrate flux by approximately 20\%. (Figs. 57 and 62)

2. Filtrate flux was not significantly dependent upon transmembrane pressure. (Figs. 56 and 61)

3. Slurry axial velocity should not go below $11 \mathrm{ft} / \mathrm{s}$ or the transmembrane pressure below 30 psid. Doing so will cause a significant loss of filtrate flux. (Fig. 55, Batch 3B runs: 4, 7, 9, 11, and 12) (Fig. 60, Batch 3A runs: 2, 3, and 4)

4. With backpulsing at a frequency of one to two hours, a 0.1-micron filter will depth foul with the result of the filtrate flux loss of approximately $60 \%\left(24^{-0.15}\right)$ after 24 hours of continuous operation. (Figs. 54 and 59)

5. Excellent solids separation was obtained with turbidities on the order of 0.2 NTU, possibly indicating that the separated filtrates had had less than $0.2 \mathrm{ppm}$ of insoluble solids (see section 3.3.1).

\subsubsection{Washing}

1. While washing a volume of $A N-102 R 2$ with an equal volume of inhibited water cuts its consistency in approximately one half, its yield stress approximately doubles. (Fig. 64)

2. Washing a volume of $\mathrm{AN}-102 \mathrm{R} 2$ with an equal volume of inhibited water removes approximately $65 \%$ of soluble sodium. (Fig. 65)

\subsubsection{Scaling}

1. Not enough data are available to make quantitative comparison between the benchtop (CUF) data and the pilot-scale (PXF) data. Qualitatively the limited data sets are not comparable and caution is recommended when relating small-scale results to full-scale operation. A detailed discussion can be found in section 3.3.3.

\subsection{RECOMMENDATIONS}

SRTC recommends that:

1. Axial slurry velocity be greater than $11 \mathrm{ft} / \mathrm{s}$ and the transmembrane pressure more than 30 psid. Higher velocities will filter slurries faster, but higher transmembrane pressures will not increase filter fluxes significantly.

2. Baseline conditions (BC in Fig. 75) be used when precipitating Envelope C-type wastes like AN-102 to obtain the best filterability.

3. Backpulsing be minimized because increasing backpulsing increases depth fouling of the filter and thus reduces time between acid cleanings.

4. A more effective cleaning method be determined than the use of $2 \mathrm{M}$ nitric acid, alone. 


\subsection{REFERENCES}

Blunt, A. L. (2002),'Notice of NEPA Approval (NONA)," Westinghouse Savannah River Company Inter-office memorandum No. FSS-FSE-2002-301. (5 November).

Duignan, M. R. (2000a) "Final Report: Pilot-scale Cross-flow Ultrafiltration Test Using a Hanford Site Tank 241-AN-105 Waste Simulant - Envelope A + Entrained Solids," Westinghouse Savannah River Company Document No. BNF-003-98-0221, Rev. 0, (23 February).

Duignan, M. R. (2000b), "Final Report: Pilot-scale Cross-flow Ultrafiltration Test Using a Hanford Site Tank 241-AN-107 Waste Simulant - Envelope C + Entrained Solids + Strontium-Transuranic Precipitation," Westinghouse Savannah River Company Document No. BNF-003-0226, Rev. 0. (24 March)

Duignan, M. R. (2001), "Myths and Facts of Mott Tube Filters," Westinghouse Savannah River Company Inter-office Memorandum No. SRT-RPP-2001-00130. (28 August, cover letter 30 August)

Duignan, M. R. (2002a), "Pilot-scale cross-flow ultrafiltration test with a simulant of a LAW waste from Hanford Tank 241-AN-102 Sr/TRU Precipitants," Test Technical and Quality Assurance Plan, Westinghouse Savannah River Company Document No. WSRC-TR-200200112 (or SRT-RPP-2002-00054). (12 April)

Duignan, M. R. (2002b), “RPP-WTP Pilot Scale Cross Flow Filtration,” Westinghouse

Savannah River Company Notebook No. WSRC-NB-2002-00076. (began on 14 May)

Duignan, M. R. (2002c), “Analytical Study Plan: RPP-WTP Pilot-scale cross-flow filtration of AN-102," Document No. SRT-RPP-2002-00142. (11 June)

Duignan, M.R. (2002d), SRS Final Acceptance Inspection (SM-51), Approved on 6 September.

Duignan, M. R. (2002e), "RPP-WTP Pilot-scale Cross-flow Ultrafiltration Test Procedure," Westinghouse Savannah River Company Field Procedure No FP-932, (5 September: Rev.0, 12 September: Rev. 1, 21 October: Rev. 2)

Edmunds, J. (2002), "EPD Concurrence with Exemption Request for EEC-TC-A-2002-086, Rev. 0: Hanford Protection Project Cross-flow Filter Test," Westinghouse Savannah River Company Inter-office memorandum No. FSS-FSE-2002-300. (5 November).

Eibling, R. E. (2003), "Development of Supernate Simulant of Hanford Tank 241-AN-102 Waste (U)," Westinghouse Savannah River Company Document No. WSRC-TR-200300040, Rev. 0 (or SRT-RPP-00012). (February).

Eibling, R. E. and C. A. Nash, (2001), "Hanford Waste Simulants Created to Support the Research and Development on the River Protection Project - Waste Treatment Plant," Westinghouse Savannah River Company Document No. WSRC-TR-2001-00338, Rev. 0. (or SRT-RPP-2000-00017). (22 February)

Hallen, R. T, P. R. Bredt, K. P. Brooks, and L. K. Jagoda, (2000) "Combined Entrained Solids and Sr/TRU Removal from AN-107 Dilute Feed," Battelle Pacific Northwest

Division Document No. PNWD-3035 (or BNFL-RPT-027, Rev.0). (August)

Hallen, R. T, K. P. Brooks, and L. K. Jagoda (2000), "Demonstration of Entrained Solids and Sr/TRU Removal Processes with Archived AN-107 Waste,” Battelle Pacific Northwest Division Document No. PNWD-3033 (or BNFL-RPT-026, Rev.0). (July)

Mandel, J. (1964), The statistical Analysis of Experimental Data, Dover Publications, Inc. 


\section{WSRC-TR-2003-00204, REV. 0}

SRT-RPP-2003-00087, REV. 0

Martino, C. J., M. R. Poirier, F. F. Fondeur, and S. D. Fink (2001), "Flocculating, Settling, and Decanting for the Removal of Monosodium Titanate and Simulated High-Level waste sludge from salt supernate," Westinghouse Savannah River Company Document No.

WSRC-TR-2001-000413, Rev. 0. (16 October) [specifically: pp. 12-13, section 3.5.1 and p. 29, section 4.4.2]

Murkes, J. and C. G. Carlsson (1988), Crossflow Filtration: Theory and Practice, John Wiley \& Sons, Ltd.

Nash, C. A., S. W. Rosencrance, W. W. Walker, and W. R. Wilmarth (2000), "Investigation of Varied Stontium-Transuranic Precipitation Chemistries for Crossflow Filtration," Westinghouse Savannah River Company Document No. BNF-003-98-0171, Rev. 0. (18 April)

Nash, C. A., J. L. Siler (1997), "Filtration of Nonradioactive Hanford Waste Simulants," Westinghouse Savannah River Company Document No. SRTC-BNFL-016, Rev. 0. (30 September)

Restivo, M. L. (2003), "RPP part B2 pilot scale crossflow filter tests test rig assembly with 92" filter and Galigher pumps," As-built drawing: Equipment \& Engineered Systems Department (WSRC/SRTC) Document No. EES-22699-M6-005, Rev. B. (10 May)

Steeper, T. J. and M. R. Williams (2003), "Pilot Scale Precipitation Tests of Envelope C Simulants," Westinghouse Savannah River Company Document No. WSRC-TR-200300064, Rev. 0 (or SRT-RPP-2003-00019). (May)

Steimke, J. L., C. M. Hart, and S. E. Sponseller (1994), "Results from Tests of Du Pont Crossflow Filter (U)," Westinghouse Savannah River Company Document No. WSRC-TR94-0322. (October)

Townson, P. S. (2002a), "AN-102 Simulant Pilot Scale Ultrafiltration Test Specification," River Protection Project - Waste Treatment Plant Document No. 24590-WTP-TSP-RT-01029, Rev. 0. (16 January)

Townson, P. S. (2002b), Test Exception No. 24590-WTP-TEF-RT-03-027 (approved on 5/27/2003 by Reid Peterson) to Test Plan SRT-RPP-2002-00054, Rev. 0. (19 September)

Urie, M.W., J. A. Campbell, O. T. Farmer,. S. K. Fiskum, L. R. Greenwood, E. W. Hoppe, G. M. Mong, C. Z. Soderquist, R. G. Swoboda, M. P. Thomas, and J. J. Wagner (2002), "Chemical Analysis and Physical Properties Testing of 241-AN-102 Tank Waste," Battelle Pacific Northwest Division Document No. PNWD-3173 (or WTP-RPT-020). (July)

Zamecnik, J. R., M. A. Baich, E. K. Hansen, and M. R. Poirier (2003), "AN-102 Simulant SR/TRU Precipitation and Ultrafiltration," Westinghouse Savannah River Company Document No. WSRC-TR-2003-00056, Rev. 0 (or SRT-RPP-2002-00231, Rev. 0). (10 February) 
WSRC-TR-2003-00204, REV. 0

SRT-RPP-2003-00087, REV. 0

Page A- 1 of 83 


\section{Appendix A: Water}

As required by the RPP-WTP Test Specification each time the cross-flow filter experienced a slurry run the filter was to be tested with inhibited water. The water was distilled and filtered (to 0.1 micron absolute) and then made inhibited by adding caustic to a concentration of $0.01 \mathrm{M} \mathrm{NaOH}$. Those tests were noted in the test matrix as runs 1.00 before a slurry test and 1.34 after a slurry. Along with these tests there were other water rinses included that came after using the standard slurry (1.02), after the final slurry concentration test (1.31A) and after cleaning the filter with acid (1.32).

Run 1.02 was generally not done because initial tests with the slurry (during the preconditioning test with AN-107 and slurry AN-102R2 batch 3C) determined that only with considerable number of water rinses and acid cleaning could the baseline slurry be removed from the filter, costing time and money, therefore starting the AN-102R2 batch 3B test the RPP-WTP customer allowed baselining the filter only after each slurry test (run 1.33). Without the initial baseline slurry tests, then the rinse run, 1.02 was no longer necessary. This report deal with the testing of five separate batches of slurry (peconditioning with AN-107, AN-102R2:3C, AN-102R2:3B, AN-102R2:3A, and AN-102R2:4) therefore the water runs were:

Water test runs related to the Simulant Slurry AN-107 (Filter Preconditioning):
$1 \mathrm{~A}, 1 \mathrm{~B}, 1 \mathrm{C}$
Pre-Standard Slurry Test and Before Preconditioning the filter with AN-107;
pre-test rig modification
$3 \mathrm{~A}, 3 \mathrm{~B}, 3 \mathrm{C}, 3 \mathrm{D}$
Post-Standard Slurry Test Rinse and Before Preconditioning the filter with AN-
107; with INHIBITED water
3a, 3b, 3c, 3(TMP $=5$ psid) Post-Standard Slurry Test Rinse and Before Preconditioning the filter with AN- 107; with NON-INHIBITED water
5A After Preconditioning the filter with AN-107, but BEFORE acid cleaning
6 After Preconditioning the filter with AN-107. But AFTER acid cleaning
8A, 8B, 8C After Preconditioning the filter with AN-107, and after both acid cleaning and final standard slurry testing

Water test runs related to the Simulant Slurry AN-102R2 Batch 3C:

$1.00 \mathrm{~A}, 1.00 \mathrm{~B}, 1.00 \mathrm{C} \quad$ Before standard slurry test

$1.02 \quad$ After initial standard slurry test

1.31A After batch $3 \mathrm{C}$ test and before acid cleaning

1.32 After acid cleaning, not included do to pump liner change

$1.34 \mathrm{~A}, 1.34 \mathrm{~B}, 1.34 \mathrm{C} \quad$ Not done since $1.00 \mathrm{~A}, 1.00 \mathrm{~B}, 1.00 \mathrm{C}$ following tests give the same information

Water test runs related to the Simulant Slurry AN-102R2 Batch 3B:

$1.00 \mathrm{~A}, 1.00 \mathrm{~B}, 1.00 \mathrm{C} \quad$ Before standard slurry test

$1.02 \quad$ Not done since initial standard slurry test was no longer done

1.31A After batch 3B test and before acid cleaning

$1.32 \quad$ After acid cleaning, but before final standard slurry test

1.34A, 1.34B, 1.34C, 1.34D After final standard slurry test (extra test,TMP=40 psid was done in

error)

Water test runs related to the Simulant Slurry AN-102R2 Batch 3A:

$1.00 \mathrm{~A}, 1.00 \mathrm{~B}, 1.00 \mathrm{C} \quad$ Not done because preceding tests $1.34 \mathrm{a}, 1.34 \mathrm{~b}, 1.34 \mathrm{c}$ give the same information

1.02

$1.31 \mathrm{~A}$

1.32

$1.34 \mathrm{~A}, 1.34 \mathrm{~B}, 1.34 \mathrm{C}$

Not done since initial standard slurry test was no longer done

After batch 34 test and before acid cleaning

After acid cleaning, but before final standard slurry test

After final standard slurry test 


\section{WSRC-TR-2003-00204, REV. 0}

SRT-RPP-2003-00087, REV. 0

Water test runs related to the Simulant Slurry AN-102R2 Batch 4:

$1.00 \mathrm{~A}, 1.00 \mathrm{~B}, 1.00 \mathrm{C}$

Before batch 4 test

$1.00 \mathrm{r} 1$

Because the preceding tests $1.00 \mathrm{~A}, 1.00 \mathrm{~B}, 1.00 \mathrm{C}$ had a low filtrate flux the filter was soaked for 4 days in $2 \mathrm{M}$ nitric acid and this revision 1 (r1) water test at $\mathrm{TMP}=20$ psid was done with NO caustic, to compare to the following repeat test with caustic.

1.00Ar1, 1.00Br1, 1.00 $\mathrm{Cr} 1$ The preceding tests were repeated after an extra acid cleaning

1.02

$1.31 \mathrm{~A}$

1.32

$1.34 \mathrm{~A}, 1.34 \mathrm{~B}, 1.34 \mathrm{C}$
Not done since initial standard slurry test was no longer done After batch 4 test and before acid cleaning After acid cleaning, but before final standard slurry test After final standard slurry test

The letter A, B, C indicate different constant transmembrane pressure (TMP), generally, but not always: $\mathrm{A}=10$ psid, $\mathrm{B}=20$ psid, $\mathrm{C}=30$; psid.

For each water test this appendix contains a graph of the filtrate flux with time and tables of the data taken and calculated. To better understand the presented information important aspects of the graphs and tables are explained below:

Graphs - Each graph contains time averages of some of the imporant parameters taken during the run at the top of the figures, i.e. Water velocity in the filter tube, V; transmembrane pressure, TMP; water pressure at the entrance of the filter tube, Filter Pres; and the water temperature, T.

Tables - Each table is divided into raw data (the first 17 columns) and calculations (the last 10 columns) which are based on the raw data. The raw data section is divided into three parts:

Individual Entries - Raw Data (Note: Slurry = Water for these tests)

Temperature Filtrate - from the filtrate loop at the exit of the filter bundle

Cleaning - from the cleaning loop (this loop was not used)

Slurry - from the slurry loop in the slurry reservoir

Hi Amb. - outside ambient temperature at the top of the test rig

Lo Amb. - inside ambient temperature at the bottom of the test rig

Pressure BotTMP - differential pressure across the filter tube at the bottom of the vertical tube Filter - gauge pressure of the slurry at the entrance of the filter tube bundle

Filter $\mathrm{dP}$ - differential pressure of the slurry along the height of the vertical 90-inch tube TopTMP - differential pressure across the filter tube at the top the vertical 90-inch tube Filtrate - gauge pressure of the filtrate at the exit of the filter tube housing

Pulsepot - gauge pressure of the filtrate at the bottom of the pulsepot

Flow Slurry - rate of slurry just before entering the seven-filter-tube bundle Filtrate - rate of slurry filtrate under normal flow (max. is $1.2 \mathrm{gpm}$ )

Hi Filtrate - rate of any liquid which has a high flowrate (water, acid, etc.) (max. $5 \mathrm{gpm}$ ) Backpulse - rate of filtrate in opposite direction from normal flow, i.e., backpulse

Individual Entries - Calculated Data

Time - Minutes after start of test, including the backpulse (based on raw data TIME column)

Press. - Internal filter pressure of slurry at the filter bundle entrance (based on raw data P1 column)

Vel. - Slurry velocity in filter tube (based on raw data Q1 column and i.d. of the tubes of 0.488 inches)

TMP - Filter transmembrane pressure (based on average of raw data columns dP2 and dP3) in psi.

TMP - Filter transmembrane pressure (based on average of raw data columns dP2 and dP3) in bar. 


\section{WSRC-TR-2003-00204, REV. 0} SRT-RPP-2003-00087, REV. 0

Filtrate Flux - Filtrate flow rate (raw data columns Q2/Q3) divided by inside filter surface area $(6.707 \mathrm{ft} 2)^{\dagger}$ Filtrate Flux @ $25^{\circ} \mathrm{C}-$ Same as Filtrate flux, but correct for temperature with the following multiplier:

$\mathrm{T}($ corrected $)=\mathrm{T} \times \operatorname{Exp}[2500 /((1 / 273+\mathrm{T}))-(1 / 298)]$ as per Test Spec 24590-WTP-TSP-RT-01-029, Rev. 0

Permiability - Filterate flow rate per TMP (based on calculated columns Filtrate Flux @ $25^{\circ} \mathrm{C}$ and TMP)

Permiability x 1000 - Same as the above Permiability, but increase by 1000 to facilitate graphing

Permiability - Same as Permiability but in metric units, meter/day/bar

$\underline{\text { Statistics }}$

At the bottom of the last individual entry there are six rows with some data statistics to better understand the data and their fluctuations. The columns are self explained with titles of Averages, Maximum, Median, and Minimum. The next row $2 \times$ Std Dev, is two times the population stanard deviation based on the number of data points used, which is the last row. The Number of Points used generally do not included the points taken during the backpulse sequence of each test. This is explained below.

Raw Data Table Layout

The raw data is generally made up of three sections:

Zeros - These eight rows include approximately 15 to 20 seconds of data taken with the pressure transducers placed in the "zero" mode to determine if any zero drift occurred from one test to the next.

Data-Per Minute - On most of the tables the first six rows of these data are contained in a box which indicate data taken during the backpulse. This is were the filtrate flow is reversed temporarily to knock the filtrate cake of the filter surface. During these first few minutes the filtrate flow is stopped, then reversed, then stopped again, then it is finally returned to normal operation. The data during the backpulse operation were taken with a separate $\log$, therefore those data are marked with a box to note this fact. Most or all of those data points are not counted in the statistics at the bottom of the table. The tables of some of the later tests do not show the boxed data because separate logs of data were not taken, however since a backpulse for each run still occurred only those data were not included in the statistics at the bottom of the page.

\footnotetext{
${ }^{\dagger}$ For the water tests during preconditioning it will be noticed that $6.87 \mathrm{ft} 2$ was used. This was based on a tube inside diameter of 0.5 inch, which was based on the manufacturer's specification. After the tubes were measured the i.d. was changed to 0.488 inch and the area to $6.707 \mathrm{ft} 2$. This means that absolute axial velocity and filtrate flux are low by $2.5 \% \%$ for the preconditioning runs.
} 
WSRC-TR-2003-00204, REV. 0

SRT-RPP-2003-00087, REV. 0

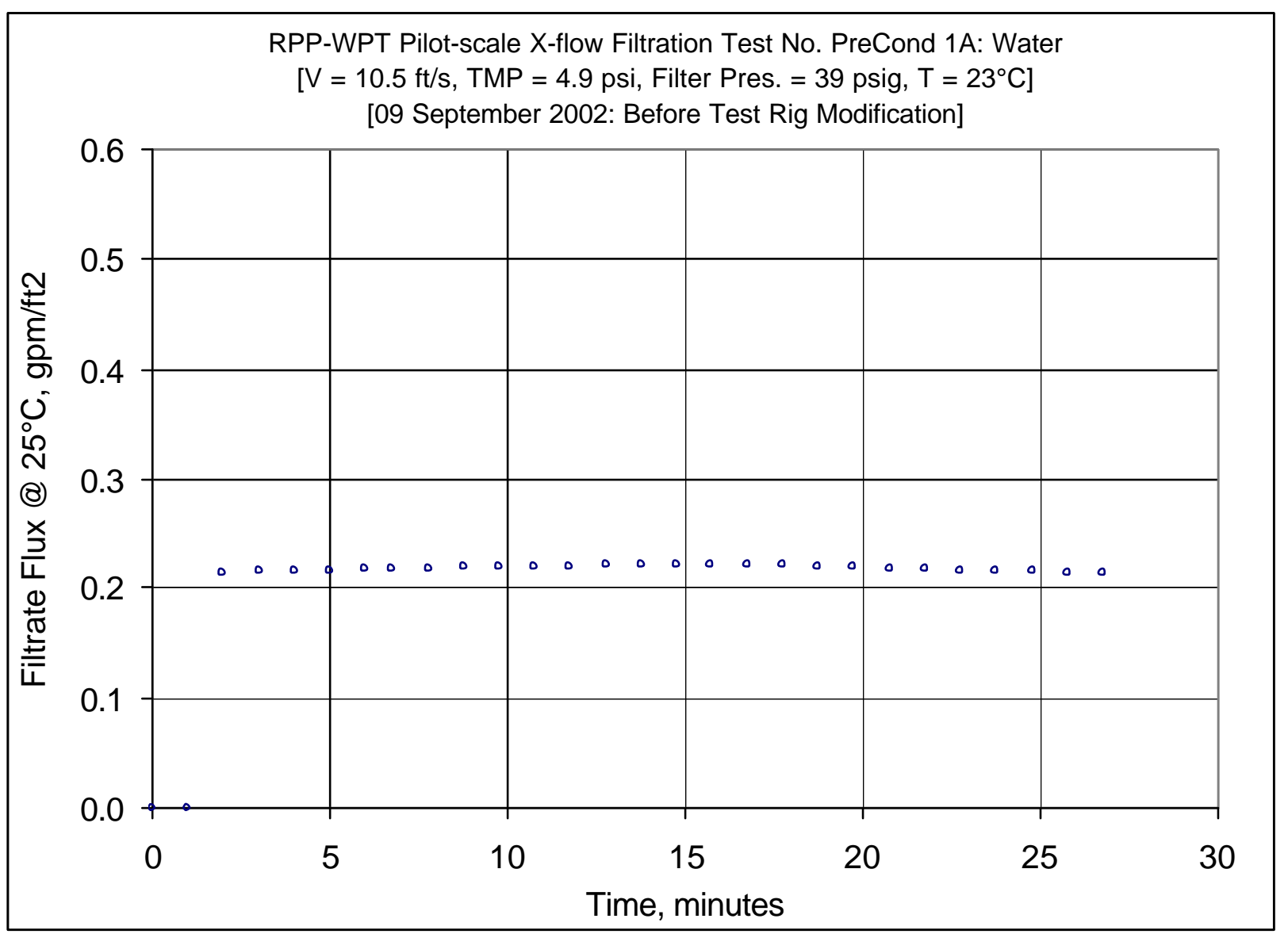

Figure A1: PreConditioning 1A - Inhibited Deionized \& Filtered Water - Before Test Rig Modification 
WSRC-TR-2003-00204, REV. 0

SRT-RPP-2003-00087, REV. 0

\begin{tabular}{|c|c|c|c|c|c|c|c|c|c|c|c|c|c|c|c|c|c|c|c|c|c|c|c|c|c|c|}
\hline \multirow{4}{*}{ DATE } & \multirow{4}{*}{ ME } & \multicolumn{5}{|c|}{$\ll \ll \ll \ll \ll \ll<$ Temperature Measurements 》1 } & \multicolumn{6}{|c|}{ 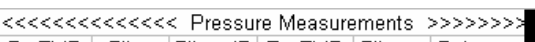 } & \multicolumn{4}{|c|}{$\mid \ll \ll \ll<\ll<<$ Flow Measurements $\gg$ 》 } & \multicolumn{10}{|c|}{ 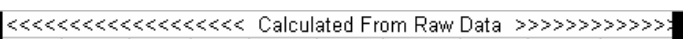 } \\
\hline & & Filtrate & Cleaning & Slurry & Hi Amb. & Lo Amb. & BotTMP & Filter & Filter $\mathrm{dP}$ & TopTMP & Filtrate & Pulsepot & Slurry & Filtrate & Hi Filtate & Backpulse & & & & & & & & & & \\
\hline & & $\operatorname{deg} \mathrm{C}$ & $\operatorname{deg} C$ & $\operatorname{deg} \mathrm{C}$ & $\operatorname{deg} C$ & $\operatorname{deg} C$ & psid & psig & psid & psid & psig & psig & $\mathrm{gpm}$ & $\mathrm{gpm}$ & $\mathrm{gpm}$ & $\mathrm{gpm}$ & & & & & & & & & & \\
\hline & & $\mathrm{T} 2$ & $\mathrm{~T} 3$ & $\mathrm{T1}$ & T4 & T5 & $\mathrm{dP} 2$ & $\mathrm{P} 1$ & $\mathrm{dP1}$ & dP3 & P2 & P3 & Q1 & Q2 & Q3 & Qbp & & & & & & & & & & \\
\hline \multicolumn{27}{|l|}{ Zeros } \\
\hline 9/9/2002 & 9:43:27 AM & 24.5 & 21.5 & 24.7 & 22.0 & 21.9 & -0.125 & -0.026 & -0.061 & 0.058 & -0.286 & 0.33 & 9.497 & 0.493 & 0.493 & & & \multirow{2}{*}{\multicolumn{2}{|c|}{$\begin{array}{l}\text { Filter Surface } \\
\text { Conversion }\end{array}$}} & $6.87 \mathrm{~F}$ & \multirow{2}{*}{\multicolumn{5}{|c|}{$\begin{array}{l}\mathrm{FT} 2 \\
\mathrm{~m} 3 / \mathrm{m} 2 / \text { day/barg } / \text { gpm/tt2/barg }\end{array}$}} & \\
\hline $9 / 9 / 2002$ & 9:45:35 AM & 24.5 & 21.5 & 24.7 & 22.0 & 21.9 & -0.125 & -0.026 & -0.061 & 0.058 & -0.286 & 0.33 & 9.497 & 0.493 & 0.493 & & & & & $851 n$ & & & & & & \\
\hline 9/9/2002 & 9:45:36 AM & 24.6 & 21.5 & 24.8 & 22.1 & 21.9 & -0.125 & -0.027 & -0.061 & 0.058 & -0.307 & 0.327 & 9.52 & 0.494 & 0.486 & & & & & & & & & & & \\
\hline 9/9/2002 & 9:45:37 AM & 24.6 & 21.5 & 24.8 & 22.0 & 21.9 & -0.127 & -0.027 & -0.06 & 0.058 & -0.307 & 0.33 & 9.516 & 0.493 & 0.49 & & & Note: $\mathrm{G}$ & Gauge & Pressur & re $\mathrm{P} 1$ is & $s$ approx & nat & 4 psig & & \\
\hline $9 / 9 / 2002$ & 9:45:38 AM & 24.6 & 21.5 & 24.8 & 22.1 & 21.9 & -0.127 & -0.027 & -0.061 & 0.058 & -0.307 & 0.33 & 9.541 & 0.493 & 0.489 & & & & & & & & & & & \\
\hline 9/9/2002 & 9:45:39 AM & 24.6 & 21.5 & 24.8 & 22.1 & 21.9 & -0.125 & -0.027 & -0.061 & 0.058 & -0.307 & 0.327 & 9.497 & 0.491 & 0.49 & & & Pressur & ure $\mathrm{P}$ lis & 5 correct & cted for & 40.88 in & inches of & f water tul & ubir & \\
\hline $9 / 9 / 2002$ & 9:45:40 AM & 24.6 & 21.5 & 24.8 & 22.0 & 21.9 & -0.125 & -0.026 & -0.061 & 0.056 & -0.307 & 0.327 & 9.486 & 0.494 & 0.488 & & & WN & & & & $<$ Filtra & rate Flux & $\ll$ PER & RMMABL & ILITY \\
\hline 9/9/2002 & 9:45:41 AM & 24.6 & 21.5 & 24.8 & 22.1 & 21.9 & -0.127 & -0.026 & -0.061 & 0.056 & -0.278 & 0.327 & 9.511 & 0.489 & 0.489 & & & & & & & & at $25 \mathrm{C}$ & & $\times 1000$ & \\
\hline & & & & & & & & & & & & & & & & & Time & Press. & Vel. & TMP T & TMP & gpm & $\mathrm{gpm}$ & gpm & $\mathrm{gpm}$ & met \\
\hline ta - Per M & Minute & & & & & & & & & & & & & & & & & & $\mathrm{f} / \mathrm{s}$ & psi b & bar & ift2 & $\mathrm{n} 2$ & $\mathrm{ft} 2 / \mathrm{psi} \mathrm{ft}$ & $\mathrm{ft} 2 / \mathrm{psi}$ & day/bar \\
\hline $9 / 9 / 2002$ & 10:03:54 AM & 25.591 & 21.841 & 23.961 & 23.749 & 22.145 & 2.402 & 41.016 & 3.876 & -1.473 & 33.552 & 80.564 & 44.799 & 0.002 & 0.001 & 0.003 & 0 & 39.5 & \begin{tabular}{|l|l|}
5 & 10.5 \\
\end{tabular} & 0.5 & 0.032 & 0.000 & 0.000 & 0.000 & 0.323 & 0.27 \\
\hline $9 / 9 / 2002$ & $10: 04: 54 \mathrm{AM}$ & 25.452 & 21.847 & 23.827 & 23.695 & 22.186 & 2.897 & 41.576 & 3.999 & -1.082 & 33.751 & 45.108 & 44.701 & 0.002 & 0.001 & 0.002 & 1 & 40.1 & $\begin{array}{ll}1 & 10.4 \\
\end{array}$ & 0.9 & & 0.000 & 0.000 & 0.000 & 0.166 & 0.14 \\
\hline 9/9/2002 & 10:05:54 AM & 24.623 & 21.884 & 23.653 & \begin{tabular}{l|l}
83.697 \\
\end{tabular} & 22.233 & 6.341 & 40.6 & & 2.883 & 29.112 & 667 & 45.59 & 1.192 & 1.419 & 0 & 2 & 39.1 & $1 \quad 10.6$ & 4.6 & 0.318 & 0.206 & 0.215 & 0.047 & 46.509 & 39.58 \\
\hline $9 / 9 / 2002$ & $10: 06: 54 \mathrm{AM}$ & 24.359 & 21.932 & 23.545 & 23.775 & 22.256 & 6.654 & 40.865 & 3. & 3.133 & 28.944 & 26.403 & 45.643 & 1.192 & 1.419 & 0 & 3 & 39.4 & \begin{tabular}{l|l}
4 & 10.7
\end{tabular} & 4.9 & 0.337 & 0.206 & 0.215 & 0.0444 & 43.968 & 37.42 \\
\hline 9/9/2002 & $10: 0$ & 24.176 & 21.939 & 23.401 & 23.686 & $\begin{array}{l} \\
\end{array} 22.293$ & 6.66 & 41.1 & & 2.922 & 29.048 & 589 & 45.55 & & & 0 & 4 & 39.6 & \begin{tabular}{|l|l|}
6 & 10.6 \\
\end{tabular} & 4.8 & & & & & & 38.38 \\
\hline $9 / 9 / 2002$ & 10:08:54 AM & 24.012 & 21.961 & 23.232 & 23.728 & 22.325 & 6.662 & 40.664 & 3.8 & 3.203 & 28.889 & 26.432 & 45.531 & 1.192 & 1.4 & 0 & 5 & 39.2 & $\begin{array}{ll}2 & 10.6 \\
\end{array}$ & 4.9 & 0.340 & 0.206 & 0.216 & 0.044 & 43.825 & 37.30 \\
\hline $9 / 9 / 2002$ & 10:09:54 AM & 23.889 & 21.988 & 23.169 & 23.761 & 22.347 & 6.6 & 40.858 & & & 28.849 & 26.357 & 45.548 & 1.192 & 1. & 0 & 6 & 39.4 & $\begin{array}{ll}4 & 10.6 \\
\end{array}$ & 4.7 & & & 0.217 & 0.046 & 45.952 & 39.11 \\
\hline $9 / 9 / 2002$ & 10:10:39 AM & 23.8 & 22.0 & 23.0 & 1) 23.9 & 22.4 & 6.478 & 40.515 & 3.7 & 3.121 & 28.976 & 26.432 & 45.641 & 1.192 & & & 6.75 & 39.0 & 10.7 & 4.8 & & & 0.218 & 0.0454 & 45.415 & 38.65 \\
\hline $9 / 9 / 2002$ & 10:11:39 AM & 23.7 & 22.0 & 23.0 & 23.8 & 22.4 & 6.528 & 40.614 & 3.7 & 3.133 & 28.91 & 26.441 & 45.515 & 1.1 & 1.411 & & 7.75 & 39.1 & $\begin{array}{ll}1 & 10.6 \\
\end{array}$ & 4.8 & 0.333 & 205 & \begin{tabular}{|l|}
5 \\
0.217
\end{tabular} & 0.0454 & 194 & 38.29 \\
\hline 9/9/2002 & 10:12:39 AM & 23.6 & & 5 & & 22.4 & 6.586 & 40.48 & & & 28.78 & 26.293 & 45.538 & 1.1 & 1.415 & & 8.75 & 39.0 & 10.6 & 4.9 & & 06 & $\begin{array}{l}0.219 \\
\end{array}$ & 0.0 & 45.069 & 38.35 \\
\hline $9 / 9 / 2002$ & & 23 & & & & 22.4 & 6.32 & 40. & & 2.826 & 28.991 & & & & & & 9.75 & 39.0 & 10 & 4.6 & & & & & & 40.74 \\
\hline 002 & $10: 1$ & 23.4 & & & & 22.4 & 6.55 & 40.5 & & 3.2 & 28.895 & 43 & 45.123 & 1.1 & & & 10.8 & 9.1 & $1 \quad 10.5$ & 4.9 & & & & & & 30 \\
\hline $9 / 9 / 2002$ & 10:15:39 AM & 23.3 & & & & 22.4 & & & & & 29. & & 45. & 1. & & & 11.8 & 39.4 & $\begin{array}{ll}4 & 10.6 \\
\end{array}$ & 5.1 & & & 0. & 0. & 35 & 36.88 \\
\hline & & 23 & & & & & & & & & & & & & & & 12.8 & & & 5.0 & & & & & & 37.24 \\
\hline 2002 & & 23 & & & & 22 & & & & & & & & & & & 13.8 & 16 & $\begin{array}{ll}6 & 10 \\
\end{array}$ & 5.0 & & & & & & 37.95 \\
\hline 9/9/2002 & 10:18:39 AM & 23 & & & & 22 & 72 & 40 & & & 28 & & 45. & & & & 14.8 & 9.4 & $4 \quad 10$ & 4.9 & & 16 & 0.221 & 0.0 & 44.747 & 38.08 \\
\hline 9) & & 23 & & & & & & & & & & & & & & & 15. & & & 5.1 & & & & & & 37.05 \\
\hline $9 /$ & & 22 & & & & & & & & & & & & & & & 16.8 & 39.4 & $\begin{array}{lll}4 & 10 \\
\end{array}$ & 5.1 & & & 0. & & & 37.03 \\
\hline 9/9/2002 & $10: 21$ & 22 & & & & & & 40. & & & 28. & & 6 & & & & 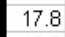 & & 10 & 4.8 & & & & & & 39.14 \\
\hline 2002 & 10:22: & 23 & & & & & & & & & & & & & & & & & & 4.7 & & & & & & 39.89 \\
\hline & 10 & 23.1 & & & & & & & & & 29. & & & & & & 19 & 39.1 & 110 & 4.8 & & & & & 99 & 39.06 \\
\hline 2002 & & 23.3 & & & & & & & & & & & & & & & 20.8 & 5 & $\begin{array}{ll}510 \\
\end{array}$ & 5.0 & & & & & & 36.89 \\
\hline & & 23 & & & & & & & & & & & & & & & & & & 4.6 & & & & & & 40.07 \\
\hline & $10: 26: 3$ & 23.5 & & & & 22.4 & & & & 2.996 & & 26 & & & & & & 39.3 & & 4.8 & & & & & & 38.63 \\
\hline 9/9/2002 & $10: 27: 39$ & 23.7 & 22.1 & & & 22.4 & & 40.652 & & 3.01 & 28.979 & & & & & & 23 & 39.2 & 210 & 4.8 & & & 0. & 0. & & 38.49 \\
\hline 9/9/2002 & 10:28:39 AM & 23. & & & & $22+2>+2>>0$ & & 40.7 & & & 29. & & & & & & & 39.3 & & 4.8 & & & & & & 38.46 \\
\hline & $10: 2$ & 23.9 & & & & 22 & & & & & 28. & 26.624 & & & & & 25. & 39.4 & \begin{tabular}{l|l|}
4 & 10.5 \\
\end{tabular} & 4.8 & & 0.206 & 0.214 & 0.0444 & 433 & 37.74 \\
\hline $9 / 9 / 2002$ & $10: 30: 39 \mathrm{AM}$ & 24.0 & 22.1 & 23.7 & 23.3 & 22.4 & 6.608 & 40.96 & 3.774 & 2.975 & 29.04 & 26.432 & 45.062 & 1.192 & 1.418 & & 26.8 & 39.5 & $\begin{array}{ll}5 & 10.5 \\
\end{array}$ & 4.8 & 0.330 & 0.206 & 0.214 & 0.0454 & 44.678 & 38.02 \\
\hline & & & & & & & & & & & & & & & & & & & & & & & & & & \\
\hline & & & & & & & & & & & & & & & & & & & & & & & & & & 38.3 \\
\hline & Maxi & 24.0 & 22.1 & 23.7 & 23 & 22.5 & 6.8 & & 3.9 & 3.4 & 29.2 & 26 & ${ }^{4}$ & 1.2 & 1. & & & 39.6 & \begin{tabular}{|l|l|}
6 & 10.7
\end{tabular} & 5.1 & 0.4 & 6 & 0.2 & 0.0 & 7.9 & 40.7 \\
\hline & & & & 22.8 & & 22 & & & & 0.7 & & & & 1.2 & 1.4 & & & 39.2 & $\begin{array}{ll}2 & 10.5\end{array}$ & 4.8 & 0.3 & & & & 0.1 & 38.4 \\
\hline & & 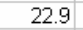 & & 22.3 & & 22 & 6 & & & 2. & & & & 1.2 & 1.4 & & & 38.9 & \begin{tabular}{|l|l|}
9 & 10.5 \\
\end{tabular} & 4.6 & 0.3 & & $\begin{array}{l}5.214 \\
\end{array}$ & 0.043 & 43.3 & 36.9 \\
\hline & & 0.632 & 0.084 & 0.795 & 0.580 & 0.066 & 0.261 & 0.359 & 0.087 & 0.369 & 0.190 & 0.251 & 0.442 & 0.000 & 0.004 & 0.000 & & 0.359 & 90.1030 & 0.295 & 0.020 & 0.001 & 0.005 & 0.002 & 2.407 & 2.048 \\
\hline mber of & f Points Used* & 21 & 21 & 21 & 21 & 21 & 21 & 21 & 21 & 21 & 21 & 21 & 21 & 21 & 21 & 21 & & 21 & 21 & 21 & 21 & 21 & 21 & 21 & 21 & 21 \\
\hline & & & & & & & & & & & & & & & & & & & & & & & & & & \\
\hline
\end{tabular}

Figure A2: PreConditioning 1A - Inhibited Deionized \& Filtered Water - Before Test Rig Modification - Raw \& Calculated Data 
WSRC-TR-2003-00204, REV. 0

SRT-RPP-2003-00087, REV. 0

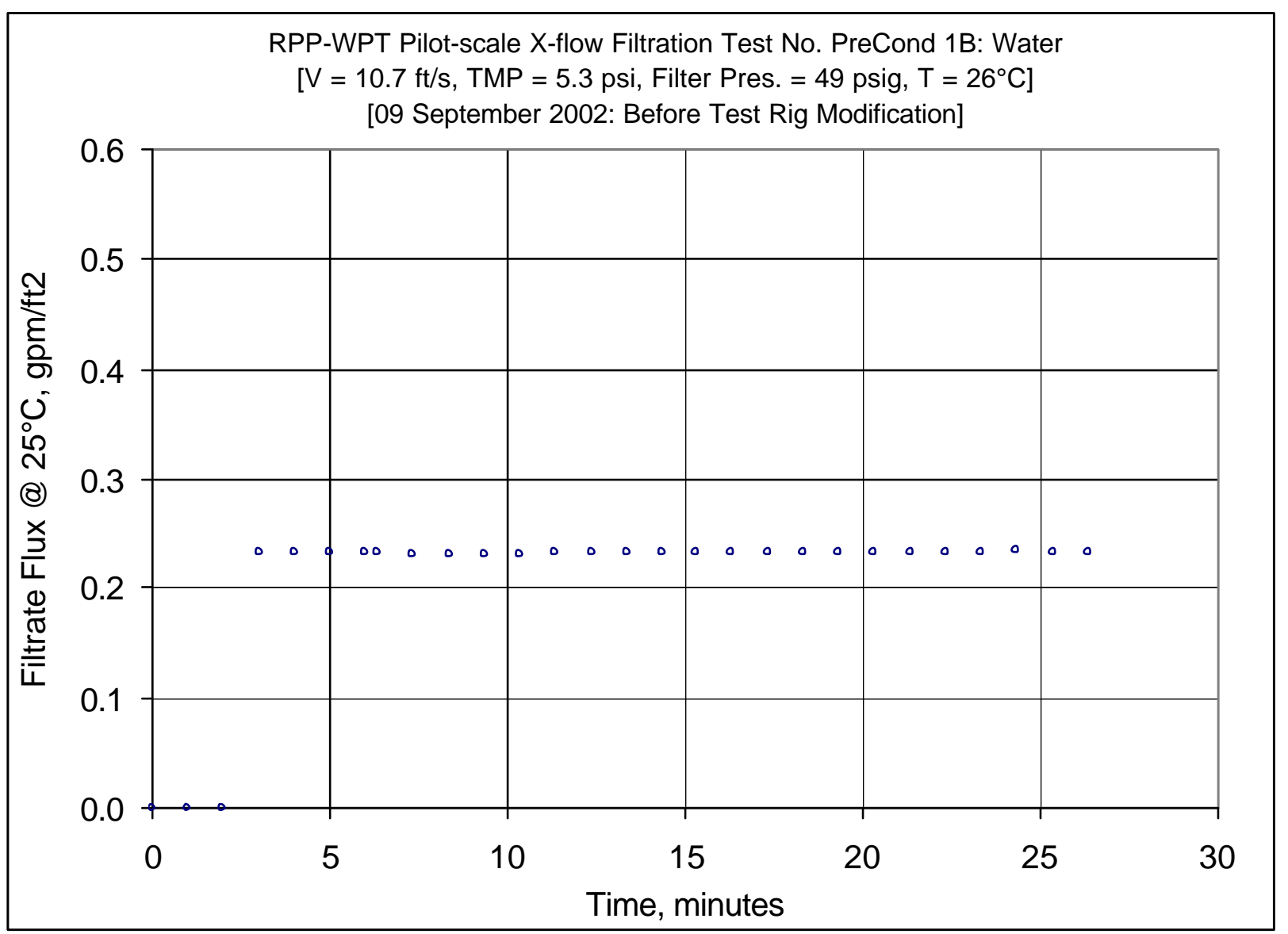

Figure A3: PreConditioning 1B - Inhibited Deionized \& Filtered Water - Before Test Rig Modification 
WSRC-TR-2003-00204, REV. 0

SRT-RPP-2003-00087, REV. 0

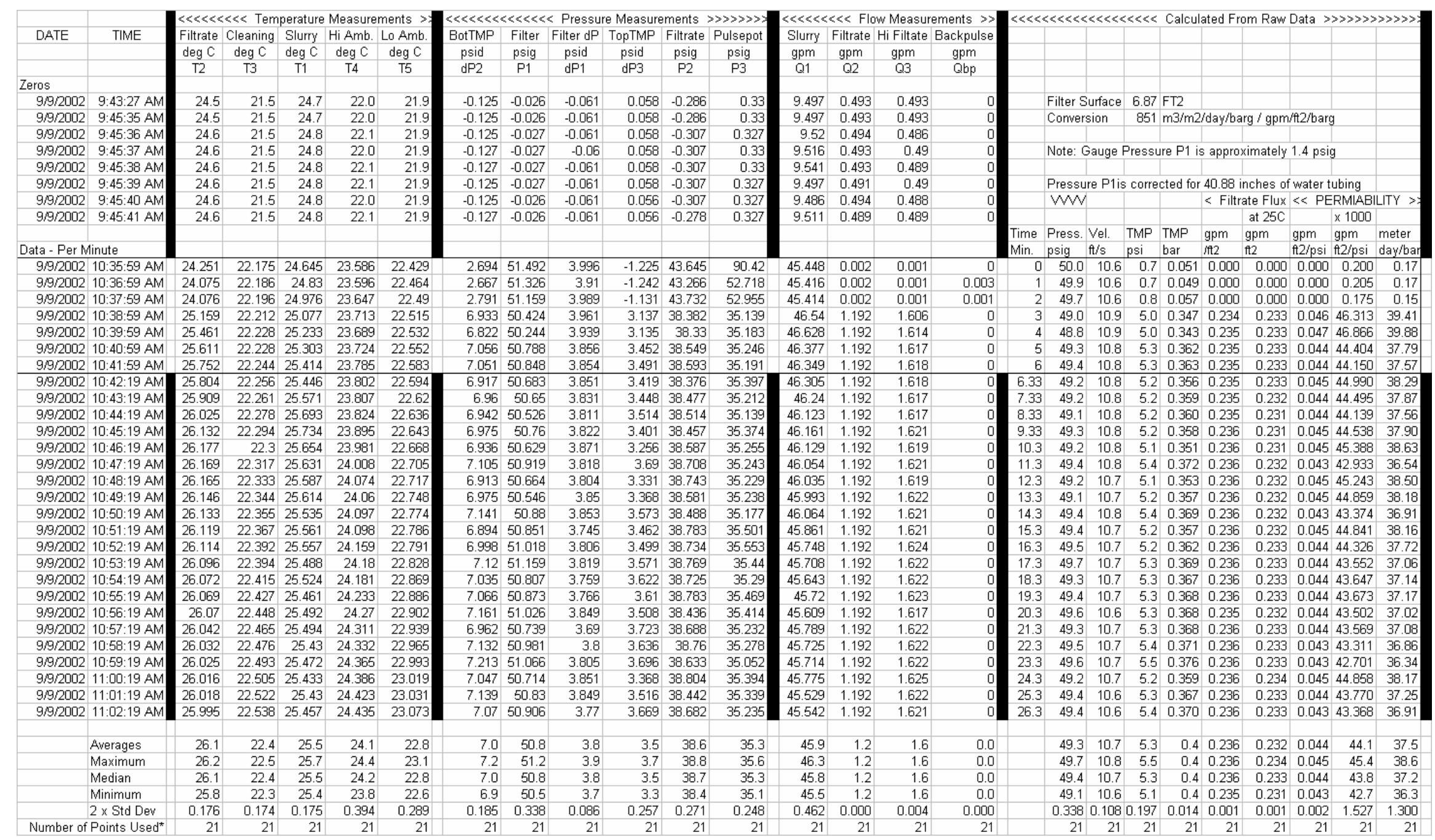

Figure A4: PreConditioning 1B - Inhibited Deionized \& Filtered Water - Before Test Rig Modification - Raw \& Calculated Data 
WSRC-TR-2003-00204, REV. 0

SRT-RPP-2003-00087, REV. 0

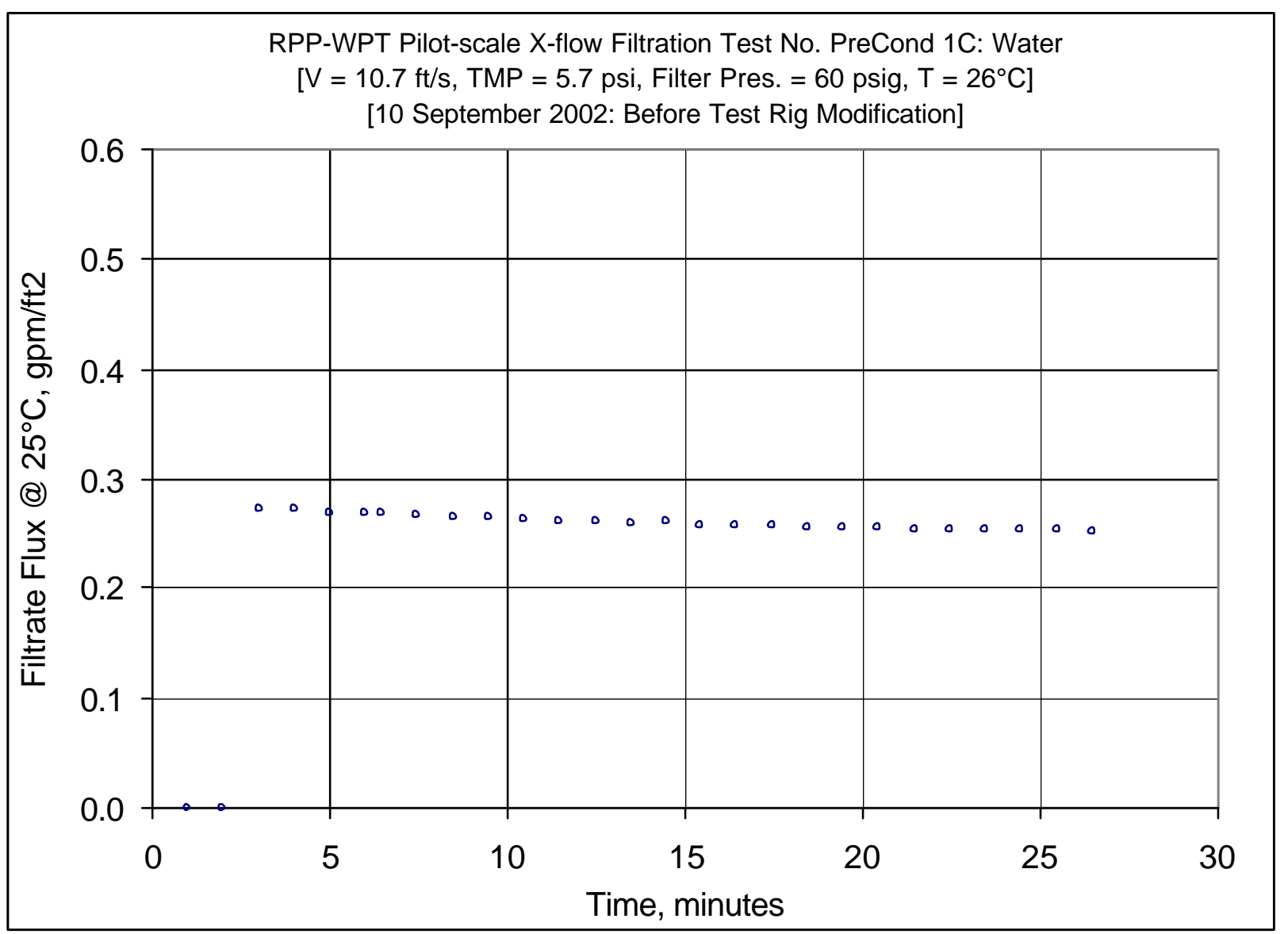

Figure A5: PreConditioning 1C - Inhibited Deionized \& Filtered Water - Before Test Rig Modification 
WSRC-TR-2003-00204, REV. 0

SRT-RPP-2003-00087, REV. 0

\begin{tabular}{|c|c|c|c|c|c|c|c|c|c|c|c|c|c|c|c|c|c|c|c|c|c|c|c|c|c|c|}
\hline \multirow{4}{*}{ DATE } & \multirow{4}{*}{ TIME } & \multicolumn{5}{|c|}{ 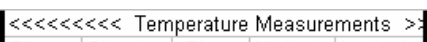 } & \multicolumn{6}{|c|}{ 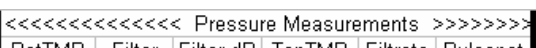 } & \multicolumn{4}{|c|}{$\ll \ll \ll \ll<\ll<<$ Flow Measurements 》> } & \multirow{2}{*}{\multicolumn{10}{|c|}{ 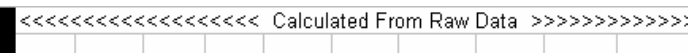 }} \\
\hline & & Filtrate & Cleaning & Slurry & Hi Amb. & Lo Amb. & BotTMP & & Filter $\mathrm{dP}$ & TopTMP & Filtrate & Pulsepot & & & & & & & & & & & & & & \\
\hline & & $\operatorname{deg} C$ & $\operatorname{deg} C$ & $\operatorname{deg} \mathrm{C}$ & $\operatorname{deg} C$ & $\operatorname{deg} C$ & psid & psig & psid & psid & psig & psig & $\mathrm{gpm}$ & $\mathrm{gpm}$ & gpm & $\mathrm{gpm}$ & & & & & & & & & & \\
\hline & & $\mathrm{T} 2$ & $\mathrm{~T} 3$ & $\mathrm{~T} 1$ & $\mathrm{~T} 4$ & 15 & $\mathrm{dP} 2$ & $\mathrm{P} 1$ & $\mathrm{dP1}$ & $\mathrm{dP3}$ & P2 & P3 & Q1 & Q2 & 03 & Qbp & & & & & & & & & & \\
\hline \multicolumn{27}{|l|}{ Zeros } \\
\hline $9 / 10 / 2002$ & 8:48:15 AM & 21.896 & 20.524 & 21.376 & 21.274 & 20.244 & -0.108 & -0.07 & -0.068 & 0.066 & -0.246 & 0.324 & 31.724 & $4 \quad 1.192$ & 1.231 & 0 & & \multirow{2}{*}{\multicolumn{2}{|c|}{ Filter Surface }} & $6.87 F$ & $\mathrm{FT} 2$ & & & & & \\
\hline $9 / 10 / 2002$ & 8:48:36 AM & 21.896 & 20.524 & 21.376 & 21.274 & $4 \quad 20.244$ & -0.108 & -0.07 & -0.068 & 0.066 & \begin{tabular}{|c|c|}
6 & -0.246
\end{tabular} & 0.324 & 31.724 & $4 \quad 1.192$ & 1.231 & 0 & & & & $851 \mathrm{r}$ & \multicolumn{5}{|c|}{$\mathrm{m} 3 / \mathrm{m} 2 /$ day $/$ barg $/ \mathrm{gpm} / \mathrm{ft} / \mathrm{barg}$} & \\
\hline $9 / 10 / 2002$ & 8:48:37 AM & 21.879 & 20.528 & 21.325 & \begin{tabular}{|l|l|} 
& 21.373 \\
\end{tabular} & 20.258 & -0.111 & -0.07 & -0.069 & 0.064 & $\begin{array}{ll}4 & -0.246\end{array}$ & 0.318 & 31.659 & \begin{tabular}{l|l}
9 & 1.192 \\
\end{tabular} & 1.231 & & & \multirow{2}{*}{\multicolumn{2}{|c|}{ Note: Gauge F }} & & & & & & & \\
\hline $9 / 10 / 2002$ & 8:48:38 AM & 21.885 & 5. 20.523 & 21.314 & 21.368 & 20.253 & -0.109 & -0.068 & -0.068 & 0.066 & $\begin{array}{ll}5 & -0.246\end{array}$ & 0.324 & 31.67 & 1.192 & 1.231 & 0 & & & & Pressur & ure $\mathrm{P} 1$ is & is approx & ximately & $1.4 \mathrm{psig}$ & & \\
\hline $9 / 10 / 2002$ & 8:48:39 AM & 21.879 & 9. 20.523 & 21.32 & 21.378 & 20.263 & -0.109 & -0.072 & -0.069 & 0.066 & 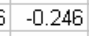 & 0.321 & 31.66 & f. 1.192 & 1.23 & & & & & & & & & & & \\
\hline $9 / 10 / 2002$ & 8:48:40 AM & 21.885 & $\begin{array}{l}5 \\
5\end{array}$ & 21.314 & 21.378 & 20.258 & -0.109 & -0.068 & -0.069 & 0.064 & $\begin{array}{ll}4 & -0.246\end{array}$ & 0.324 & 31.611 & 1.192 & 1.227 & 0 & & Pressul & ure $\mathrm{P} 1$ is & 5 correc & cted for & ir 40.88 in & inches of & f water $\mathrm{t}$ & tubing & \\
\hline $9 / 10 / 2002$ & $8: 48: 41 \mathrm{AM}$ & 21.89 & 20.523 & 21.309 & 21.388 & 20.263 & -0.109 & -0.07 & -0.069 & 0.064 & $4-0.246$ & 0.318 & 31.662 & 2. 1.192 & 1.233 & & & WW & & & & < Filtra & rate Flux & $\ll P E$ & ERMIABI & BILITY \\
\hline $9 / 10 / 2002$ & $8: 48: 42 \mathrm{AM}$ & 21.879 & 20.517 & 21.304 & $\begin{array}{l}41.383 \\
\end{array}$ & 20.257 & -0.109 & -0.07 & -0.069 & 0.066 & \begin{tabular}{|c|c|}
5 & -0.246 \\
\end{tabular} & 0.321 & 31.62 & 2. 1.192 & 1.232 & 0 & & & & & & & at $25 \mathrm{C}$ & & $\times 1000$ & \\
\hline & & & & & & & & & & & & & & & & & Time & Press. & Vel. & TMP & TMP & gpm & gpm & $\mathrm{gpm}$ & $\mathrm{gpm}$ & meter \\
\hline Data - Per M & Minute & & & & & & & & & & & & & & & & & psig & $\mathrm{f} / \mathrm{s}$ & & & ift2 & $\mathrm{n} 2$ & $\mathrm{f} 2 / \mathrm{psi}$ & $\mathrm{i} \mathrm{ft} / \mathrm{psi}$ & day/ba \\
\hline$\frac{1}{9 / 10 / 2002}$ & $8: 56: 11 \mathrm{AM}$ & 21.941 & 20.691 & 22.799 & 21.969 & 1.763 & 2.393 & 60.805 & 3.95 & -1.571 & 53.678 & 00.314 & 45.704 & $4 \quad 0.002$ & 0.001 & & 1 & 59.3 & 10.7 & 0.4 & 0.028 & 30.000 & 0.000 & 0.000 & 0.377 & $\begin{array}{ll}7 \quad 0.32 \\
\end{array}$ \\
\hline $9 / 10 / 2002$ & $8: 57: 11 \mathrm{AM}$ & 21.908 & 20.713 & 23.043 & 22.007 & 20.846 & 2.866 & 61.486 & 4.044 & -1.172 & 53.443 & 59.684 & 45.445 & 0.002 & 0.001 & 0.002 & 2 & 260.0 & 10.6 & 0.8 & 0.058 & 80.000 & 0.000 & 0.000 & 0.182 & 0.15 \\
\hline $9 / 10 / 2002$ & 8:58:11 AM & 23.335 & 20.737 & 23.277 & 22.141 & 20.93 & 7.636 & 61.014 & 4.04 & 3.843 & 48.082 & 44.051 & 46.857 & $\begin{array}{l}7.192 \\
\end{array}$ & 1.7 & & 3 & \begin{tabular}{ll|}
3 & 59.5 \\
\end{tabular} & 10.9 & 5.7 & 0.396 & 0.259 & 0.272 & 0.047 & 747.411 & $\begin{array}{lll}1 & 40.35\end{array}$ \\
\hline $9 / 10 / 2002$ & $8: 59: 11 \mathrm{AM}$ & 23.72 & 20.756 & 23.517 & 22.285 & 21.005 & 7.654 & 61.312 & 3.928 & 4.095 & 48.458 & 44.327 & 46.511 & 1.192 & 1.788 & 0 & 4 & $\begin{array}{ll}4 & 59.8\end{array}$ & 10.9 & 5.9 & 0.405 & 50.260 & 0.271 & 0.046 & 646.187 & $\begin{array}{ll}7 & 39.31 \\
\end{array}$ \\
\hline $9 / 10 / 2002$ & 9:00:11 AM & 24.005 & 20.795 & 23.727 & 22.455 & 21.089 & 7.462 & 60.919 & 3.857 & 4.025 & 48.495 & 44.321 & 46.394 & $4 \quad 1.192$ & 1.772 & & 5 & $\begin{array}{ll}5 & 59.4 \\
\end{array}$ & 10.8 & 5.7 & 0.396 & 0.258 & 0.267 & 0.047 & 746.539 & 939.61 \\
\hline $9 / 10 / 2002$ & 9:01:11 AM & 24.275 & 20.83 & 23.977 & $\begin{array}{r}22.455 \\
\end{array}$ & 21.175 & 7.582 & 61.019 & 3.897 & 3.985 & \begin{tabular}{|l|}
48.53 \\
\end{tabular} & 44.24 & 46.337 & 1.192 & 1.792 & U & 6 & 59.5 & 10.8 & 5.8 & 0.399 & 0.261 & 0.268 & 0.046 & 6.46 .409 & $\begin{array}{ll}9 & 39.49 \\
\end{array}$ \\
\hline $9 / 10 / 2002$ & 9:01:38 AM & 24.372 & 20.842 & 24.034 & 22.582 & 21.212 & 7.511 & 60.723 & 3.846 & 4.073 & 48.377 & 44.173 & 46.247 & 1.192 & 1.795 & & 6.45 & \begin{tabular}{|l|l|}
5 & 59.3 \\
\end{tabular} & 10.8 & 5.8 & 0.399 & 0.261 & 0.268 & 0.046 & 646.343 & $\begin{array}{ll}3 & 39.44 \\
\end{array}$ \\
\hline $9 / 10 / 2002$ & 9:02:38 AM & 24.583 & 20.867 & 24.28 & 22.632 & 21.282 & 7.199 & 60.568 & 3.846 & 3.813 & 48.692 & 44.59 & 46.129 & 3. 1.192 & 1.794 & 0 & 7.45 & $\begin{array}{ll}5 \quad 59.1 \\
\end{array}$ & 10.8 & 5.5 & 0.380 & 0.261 & 0.266 & 0.048 & 848.385 & $\begin{array}{ll}5 & 41.18 \\
\end{array}$ \\
\hline $9 / 10 / 2002$ & 9:03:38 AM & 24.788 & 20.907 & 24.449 & 22.722 & 21.327 & 7.636 & 61.213 & 3.8 & 4.052 & 48.414 & 44.28 & 46.15 & $\begin{array}{l}5 \\
5\end{array}$ & 1. & & 8.45 & \begin{tabular}{|l|l|}
5 & 59.7
\end{tabular} & 10.8 & 5.8 & 0.403 & 0.261 & 0.265 & 0.045 & 5.45 .395 & $\begin{array}{ll}538.63 \\
\end{array}$ \\
\hline $9 / 10 / 2002$ & 9:04:38 AM & 24.987 & 20.911 & 24.574 & 22.762 & 21.371 & 7.424 & 61.136 & 3.8 & 3.948 & 48.611 & 44.315 & 46.11 & $\begin{array}{l}1.192 \\
\end{array}$ & 1.7 & O & 9.45 & $\begin{array}{ll}5 & 59.7 \\
\end{array}$ & 10.8 & 5.7 & 0.392 & & 0.264 & 0.047 & & $\begin{array}{ll}8 \quad 39.59 \\
\end{array}$ \\
\hline $9 / 10 / 2002$ & 9:05:38 AM & 25.166 & 20.939 & 24.807 & 22.634 & 21.4 & 7.712 & 61.372 & 3.863 & 4.218 & 48.481 & 44.416 & 46.029 & 9. 1.192 & 1.792 & 0 & 10.5 & $\begin{array}{l}5 \\
5\end{array}$ & 10.7 & 6.0 & 0.411 & 0.261 & 0.262 & 0.044 & 443.953 & $\begin{array}{ll}3 & 37.40 \\
\end{array}$ \\
\hline $9 / 10 / 2002$ & 9:06:38 AM & 25.328 & 20.972 & 24.935 & $\begin{array}{l}52.717 \\
\end{array}$ & 21.422 & 7.545 & 61.058 & 3.872 & 3.884 & 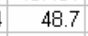 & 44.39 & 46.003 & \begin{tabular}{|l|}
1.192 \\
\end{tabular} & & & 11.5 & $\begin{array}{ll}5 & 59.6 \\
\end{array}$ & $\begin{array}{ll}6 & 10.7 \\
\end{array}$ & 5.7 & 0.394 & & 0.261 & 0.046 & 64 & $\begin{array}{ll}0 \quad 38.93 \\
\end{array}$ \\
\hline $9 / 10 / 2002$ & 9:07:38 AM & 25.506 & 20.995 & 25.073 & 22.654 & $\begin{array}{r}4 \\
4\end{array}$ & 7.478 & 61.027 & 3.8 & 3.768 & \begin{tabular}{|l|} 
\\
\end{tabular} & 44.353 & 45.909 & $\begin{array}{l}1.192 \\
\end{array}$ & & 0 & 12.5 & $\begin{array}{ll}5 & 59.6\end{array}$ & 10.7 & 5.6 & 0.388 & 0.261 & 0.260 & $\mid 0.046$ & 646.253 & $\begin{array}{lll}3 & 39.36 \\
\end{array}$ \\
\hline $9 / 10 / 2002$ & 9:08:38 AM & 25.638 & 21.006 & 25.239 & 22.585 & \begin{tabular}{|l|}
51.482 \\
\end{tabular} & 7.284 & 60.938 & 3.857 & 3.727 & 48.527 & 44.61 & 45.898 & 1.192 & 1.795 & 西 & 13.5 & \begin{tabular}{l|l|}
5 & 59.5 \\
\end{tabular} & \begin{tabular}{|l|}
5 \\
10.7
\end{tabular} & 5.5 & 0.380 & 0.261 & 0.259 & 0.047 & 7.47 .125 & $\begin{array}{ll}5 & 40.10\end{array}$ \\
\hline $9 / 10 / 2002$ & 9:09:38 AM & 25.795 & 21.039 & 25.322 & 22.593 & 21.534 & 7.37 & 61.05 & & 3.995 & 48.628 & 44.636 & 45.961 & 1.192 & 1. & ? & 14.5 & $\begin{array}{ll}5 & 59.6 \\
\end{array}$ & 10.7 & 5.7 & 0.392 & 0.262 & 0.260 & 0.046 & 645 & $\begin{array}{ll}3 \quad 38.89 \\
\end{array}$ \\
\hline 9/10/2002 & 9:10:38 AM & 25.937 & 21.071 & 25.499 & 22.575 & 21.577 & 7.317 & 60.923 & 3 & 3.944 & 48.588 & 44.434 & 45.941 & 1.1 & & 0 & 15.5 & $\begin{array}{ll}5 & 59.5 \\
\end{array}$ & 10.7 & 5.6 & 0.388 & 0.261 & 0.258 & 0.046 & & $\begin{array}{l}9 \\
9\end{array} 38.95$ \\
\hline $9 / 10 / 2002$ & 9:11:38 AM & 26.039 & 21.078 & 25.576 & 22.586 & 21.599 & 7.604 & 61.461 & 3.784 & 4.171 & 48.83 & 44.451 & 45.865 & 1.192 & 1.798 & & 16.5 & $\begin{array}{ll}5 & 60.0 \\
\end{array}$ & 10.7 & 5.9 & 0.406 & 0.262 & 0.257 & 0.044 & 4.43 .725 & $\begin{array}{ll}5 \quad 37.21 \\
\end{array}$ \\
\hline $9 / 10 / 2002$ & 9:12:38 AM & 26.2 & 21.119 & 25.677 & 22.633 & $\begin{array}{l}8 \quad 21.63 \\
\end{array}$ & & 61.049 & & & 48.553 & 44.408 & 45.844 & 1.192 & 1.8 & & 17.5 & $\begin{array}{ll}5 \quad 59.6 \\
\end{array}$ & \begin{tabular}{l|l} 
f 10.7 \\
\end{tabular} & 5.6 & & 0.262 & 0.257 & 0.046 & 64 & $\begin{array}{ll}5 & 38.89 \\
\end{array}$ \\
\hline $9 / 10 / 2002$ & 9:13:38 AM & 26.307 & 21.131 & 25.839 & $\begin{array}{r}22.64 \\
\end{array}$ & $\begin{array}{l}41.702 \\
4\end{array}$ & 7.513 & 61.019 & 3.8 & 3.786 & \begin{tabular}{|l|} 
\\
\end{tabular} & 44.173 & 45.727 & 1.192 & 1.798 & & 18.5 & $\begin{array}{ll}5 & 59.5 \\
\end{array}$ & \begin{tabular}{|l|l|}
5 & 10.7
\end{tabular} & 5.6 & 0.390 & 0.262 & 0.256 & 0.045 & 5.45 .233 & $\begin{array}{ll}3 & 38.49 \\
\end{array}$ \\
\hline $9 / 10 / 2002$ & 9:14:38 AM & 26.419 & 21.164 & 25.896 & 22.763 & 21.725 & 7.157 & 60.899 & 3.804 & 3.571 & 48.564 & 44.77 & 45.695 & 1.192 & 1.799 & 0 & 19.5 & $\begin{array}{ll}5 & 59.4 \\
\end{array}$ & $4 \quad 10.7$ & 5.4 & 0.370 & 0.262 & 0.255 & 0.048 & 847.591 & 140.50 \\
\hline $9 / 10 / 2002$ & 9:15:38 AM & 26.531 & 21.191 & 26.008 & 22.8 & 21.762 & & 60.497 & & & 48.654 & 44.442 & 45.854 & 1.1 & & & 20.5 & \begin{tabular}{l|l|}
5 & 59.0
\end{tabular} & 10.7 & 5.4 & & 0.262 & 0.255 & 0.047 & & $\begin{array}{ll}140.25 \\
\end{array}$ \\
\hline $9 / 10 / 2002$ & $9: 16: 38$ AM & 26.608 & 21.213 & 26.145 & 22.817 & 21.784 & 7.312 & 60.973 & 3.772 & 3.854 & 48.622 & 44.373 & 45.825 & 1.192 & 1.8 & & 21.5 & \begin{tabular}{l|l|}
5 & 59.5 \\
\end{tabular} & 10.7 & 5.6 & 0.385 & 0.262 & 0.254 & 0.045 & 5.45 .482 & 238.71 \\
\hline $9 / 10 / 2002$ & $9: 17: 38 \mathrm{AM}$ & 26.699 & 21.219 & 26.161 & 22.929 & 21.78 & 7.482 & 61.581 & 3.736 & 4.199 & 48.608 & 44.717 & 45.733 & 1.192 & 1.802 & 0 & 22.5 & \begin{tabular}{|l|l|}
5 & 60.1
\end{tabular} & \begin{tabular}{|l|} 
\\
$\mid$
\end{tabular} & 5.8 & 0.403 & 0.262 & 0.254 & 0.043 & 343.458 & $\begin{array}{ll}8 & 36.98\end{array}$ \\
\hline $9 / 10 / 2002$ & 9:18:38 AM & 26.805 & 21.23 & 26.297 & 22.869 & 21.786 & 7.505 & 61.256 & 3.772 & 4.034 & 48.721 & 44.411 & 45.743 & 1.192 & & 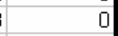 & 23.5 & $\begin{array}{ll}5 & 59.8 \\
\end{array}$ & 10.7 & 5.8 & 0.398 & 0.262 & 0.253 & 0.044 & 4.43 .850 & $\begin{array}{ll}0 & 37.32\end{array}$ \\
\hline $9 / 10 / 2002$ & 9:19:38 AM & 26.891 & 21.266 & 26.383 & 22.865 & 21.822 & 7.412 & 61.252 & 3.789 & 3.794 & 48.79 & 44.364 & 45.769 & 1.192 & 1.803 & & 24.5 & \begin{tabular}{|l|l|}
5 & 59.8 \\
\end{tabular} & 10.7 & 5.6 & 0.386 & 0.262 & 0.252 & 0.045 & 5.45 .045 & \begin{tabular}{l|l}
5 & 38.33 \\
\end{tabular} \\
\hline $9 / 10 / 2002$ & 9:20:38 AM & 26.99 & 21.276 & 26.422 & 22.98 & 21.812 & 7.364 & 61.01 & 3.743 & 3.95 & 48.654 & 44.486 & 45.689 & $\begin{array}{l}1.192 \\
\end{array}$ & 1.804 & 0 & 25.5 & $\begin{array}{ll}5 & 59.5 \\
\end{array}$ & 10.7 & 5.7 & 0.390 & 0.263 & 0.252 & 0.045 & 544.591 & 137.95 \\
\hline 9/10/2002 & 9:21:38 AM & 27.06 & 21.302 & 26.533 & 22.981 & 21797 & 7.598 & 61.629 & 3.784 & 4.073 & 48.761 & 44.622 & 45.727 & 1.192 & 1.804 & & 26.5 & $\begin{array}{ll}5 & 60.2 \\
\end{array}$ & 210.7 & 5.8 & 0.402 & 0.263 & 0.251 & 0.043 & 343.094 & $\begin{array}{ll}4 \quad 36.67 \\
\end{array}$ \\
\hline & & & & & & & & & & & & & & & & & & & & & & & & & & \\
\hline & Averages & 25.9 & 2 & 25.5 & 22 & & 7.4 & 61.1 & 3 & 3 & 48.6 & & 45.9 & 1.2 & 1.8 & 0 & & 59.6 & 10.7 & 5.7 & 0.4 & $\begin{array}{l}0.262 \\
0.763\end{array}$ & 0.258 & 0.046 & 45.5 & 38.8 \\
\hline & Maximum & 27 & 2 & 26.5 & & 2 & 7.7 & 61.6 & $a$ & & & 44 & & 1.2 & 1. & 0 & & 60.2 & $\begin{array}{l}2 \\
2\end{array}$ & 6.0 & 0.4 & 0.263 & 0.268 & 0.048 & 48.4 & 41.2 \\
\hline & Median & 26.0 & 21.1 & 25.6 & 22.7 & 21.6 & 7.4 & 61.0 & 3.8 & 3.9 & 48.6 & 44. & & 1.2 & 1.8 & 0. & & 59.6 & $\begin{array}{ll}\text { f } 10.7 \\
\end{array}$ & 5.7 & 0.4 & 0.262 & 0.257 & 0.046 & 45.7 & 38.9 \\
\hline & Minimum & 24.4 & 20.8 & 24.0 & 22.6 & 21.2 & 7.1 & 60.5 & 3.7 & 3.6 & 48.3 & 44.2 & 45.7 & 1.2 & 1.8 & 0.0 & & 59.0 & 10.7 & 5.4 & 0.4 & 0.261 & 0.251 & 0.043 & \begin{tabular}{|l|l}
3 & 43.1 \\
\end{tabular} & $\begin{array}{ll}1 & 36.7 \\
\end{array}$ \\
\hline & $2 \times$ Std Dev & 1.611 & 0.280 & 1.475 & 0.264 & 0.383 & 0.310 & 0.568 & 0.098 & 0.344 & 0.266 & 0.314 & 0.321 & 0.000 & 0.008 & .000 & & 0.569 & 0.0750 & 0.306 & 0.021 & 0.001 & 0.010 & 0.003 & $\begin{array}{ll}3 \quad 2.775 \\
\end{array}$ & $\begin{array}{ll}5 \quad 2.361 \\
\end{array}$ \\
\hline of & f Points Used ${ }^{*}$ & 21 & 21 & 21 & 21 & 21 & 21 & 21 & 21 & 21 & 21 & 21 & 21 & 21 & 21 & 21 & & 21 & 21 & 21 & 21 & 21 & 21 & 21 & 21 & 21 \\
\hline & *Backpulse p & 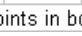 & 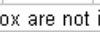 & luded & & & & & & & & & & & & & & & & & & & & & & \\
\hline
\end{tabular}

Figure A6: PreConditioning 1C - Inhibited Deionized \& Filtered Water - Before Test Rig Modification - Raw \& Calculated Data 
WSRC-TR-2003-00204, REV. 0

SRT-RPP-2003-00087, REV. 0

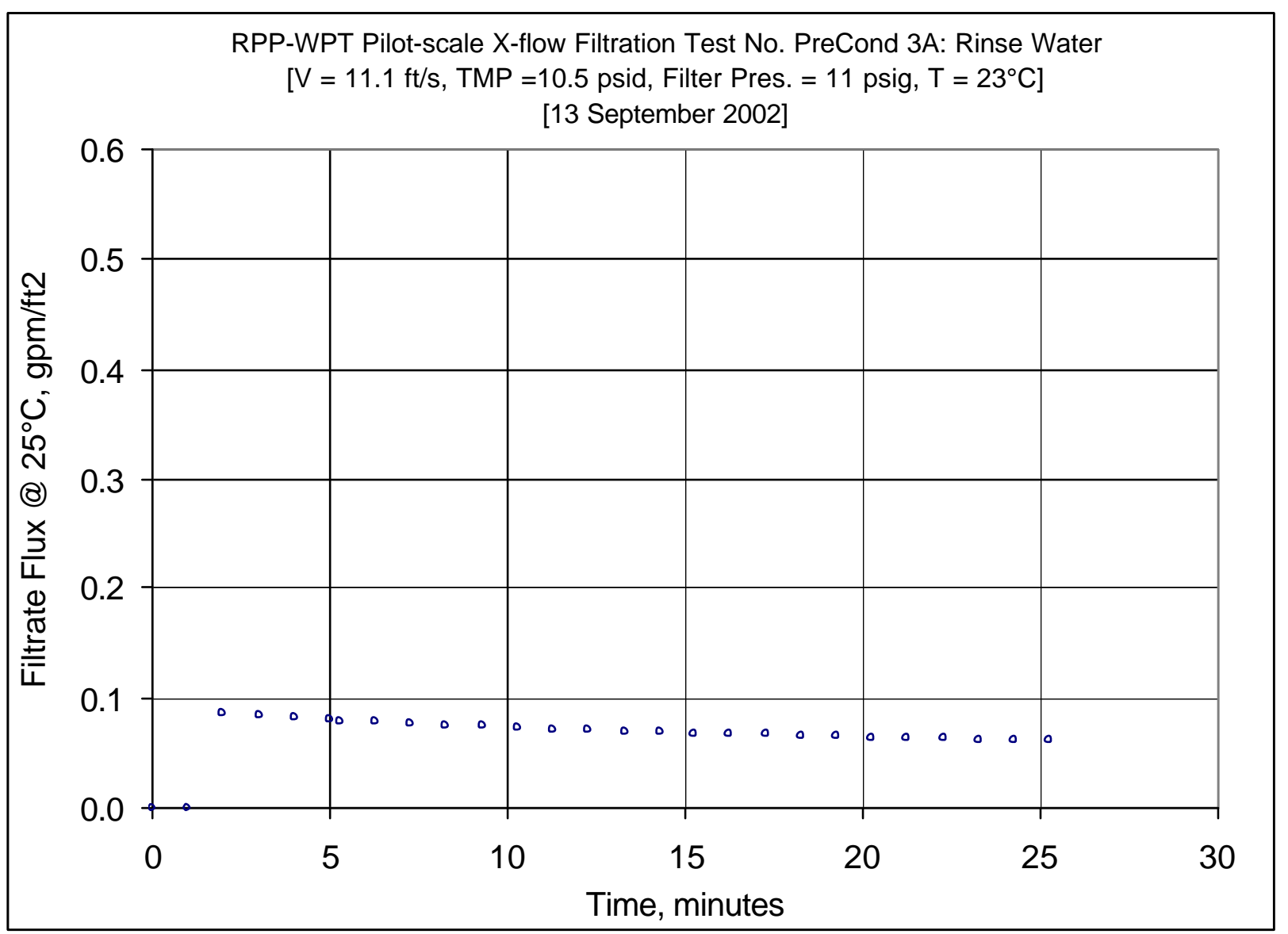

Figure A7: PreConditioning 3A - Inhibited Deionized \& Filtered Water - Post Standard Slurry Tests 
WSRC-TR-2003-00204, REV. 0

SRT-RPP-2003-00087, REV. 0

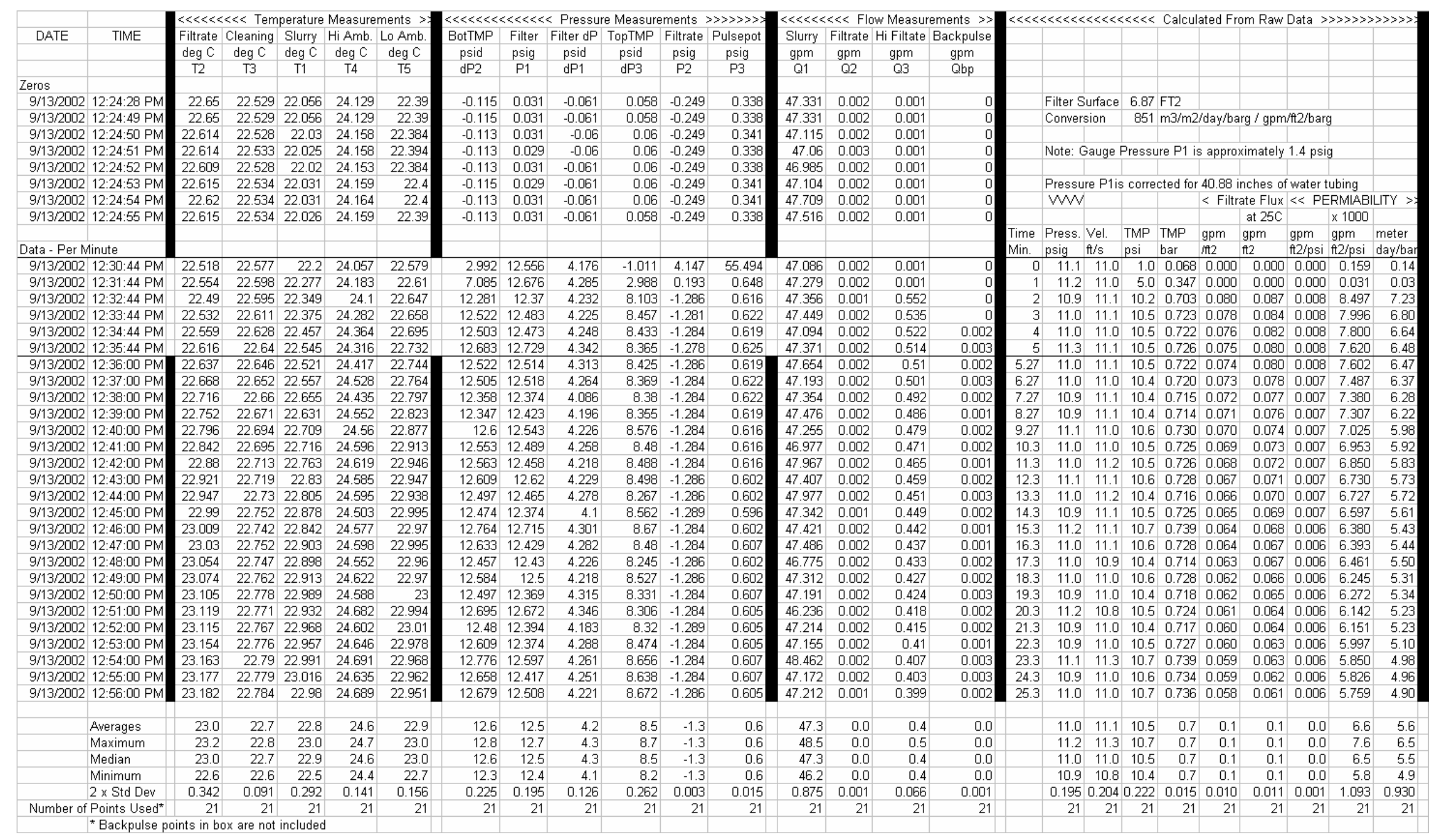

Figure A8: PreConditioning 3A - Inhibited Deionized \& Filtered Water - Post Standard Slurry Tests - Raw \& Calculated Data 
WSRC-TR-2003-00204, REV. 0

SRT-RPP-2003-00087, REV. 0

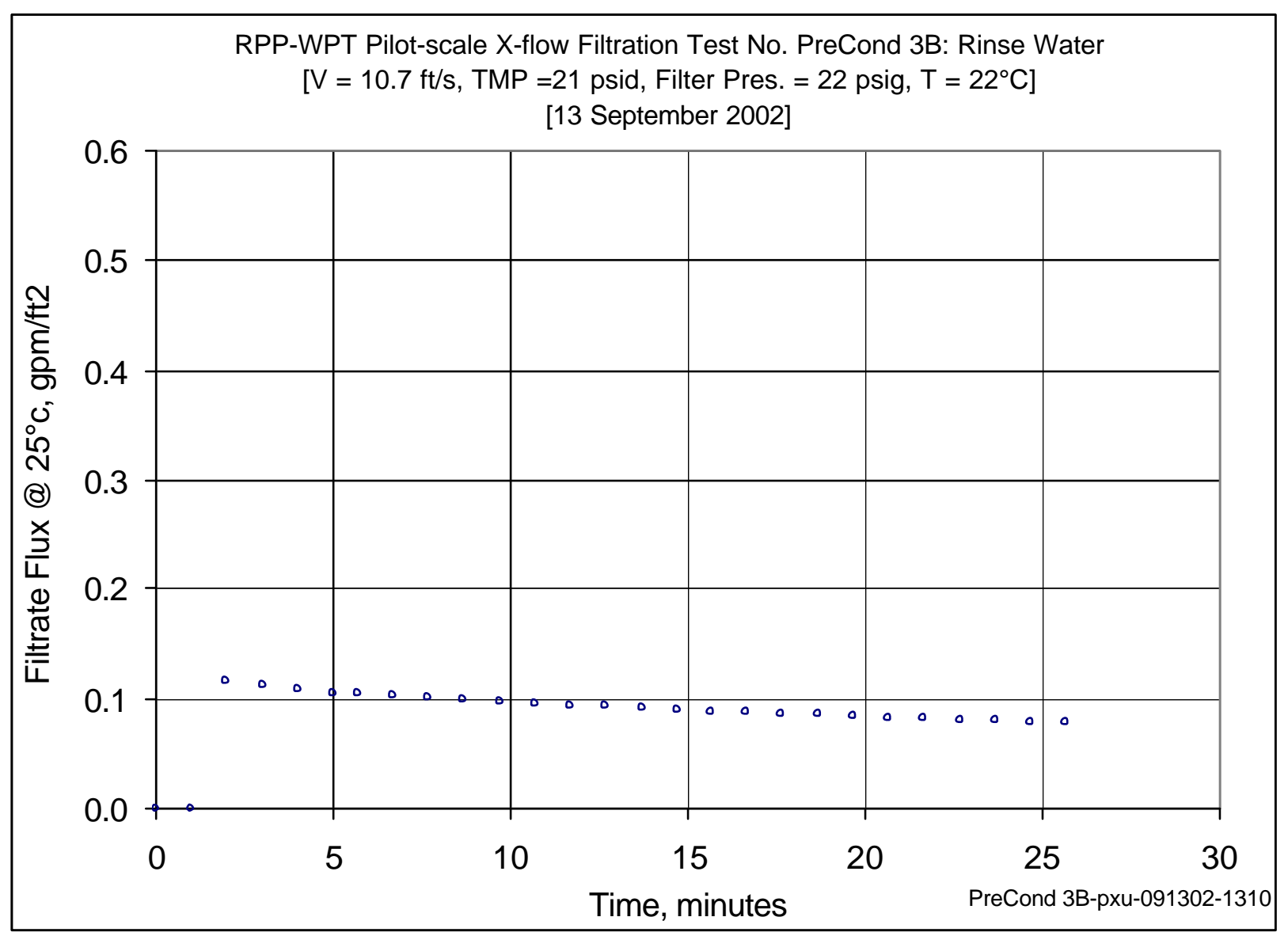

Figure A9: PreConditioning 3B - Inhibited Deionized \& Filtered Water - Post Standard Slurry Tests 
WSRC-TR-2003-00204, REV. 0

SRT-RPP-2003-00087, REV. 0

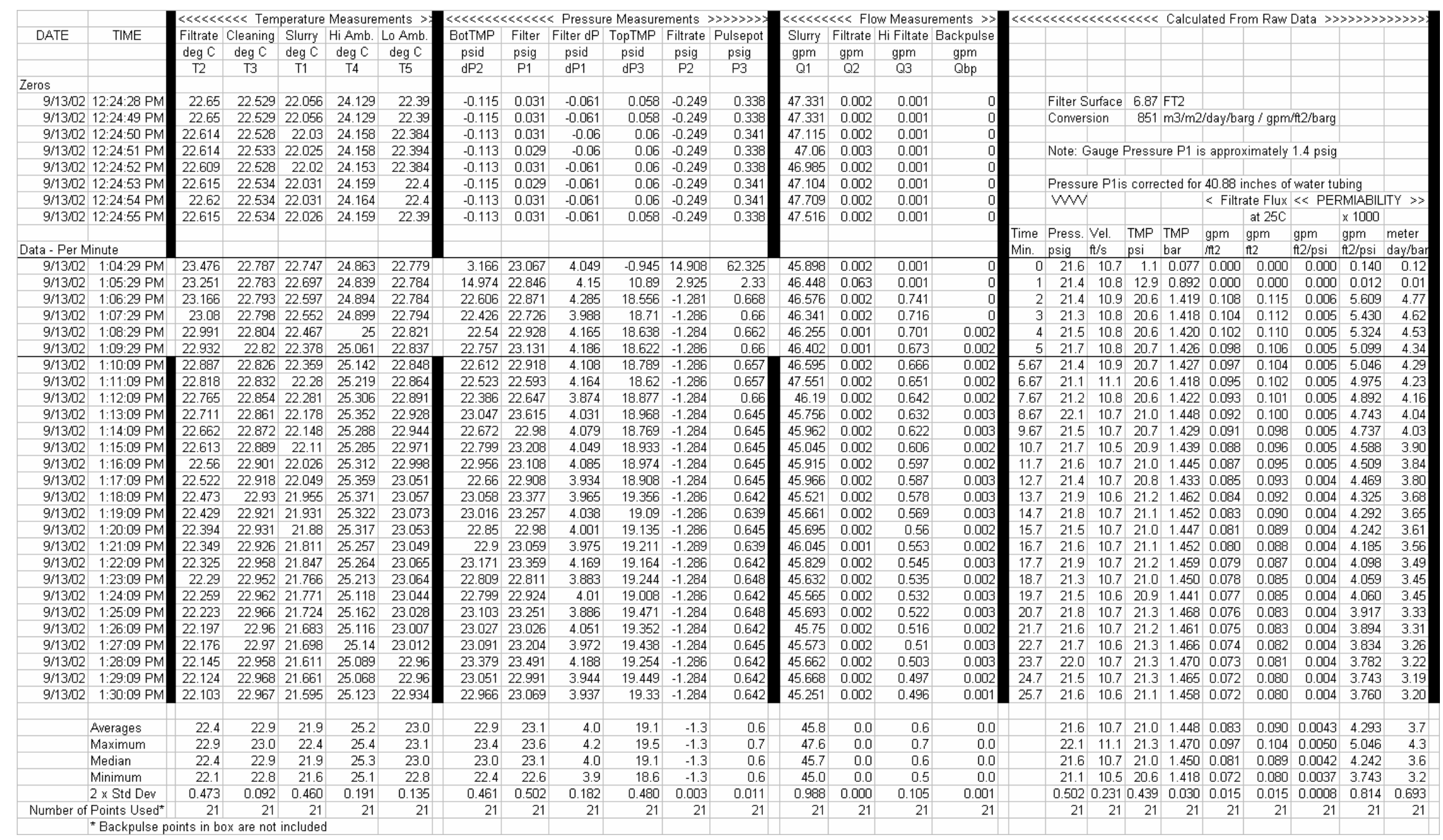

Figure A10: PreConditioning 3B - Inhibited Deionized \& Filtered Water - Post Standard Slurry Tests - Raw \& Calculated Data

Page A13 of A88 
WSRC-TR-2003-00204, REV. 0

SRT-RPP-2003-00087, REV. 0

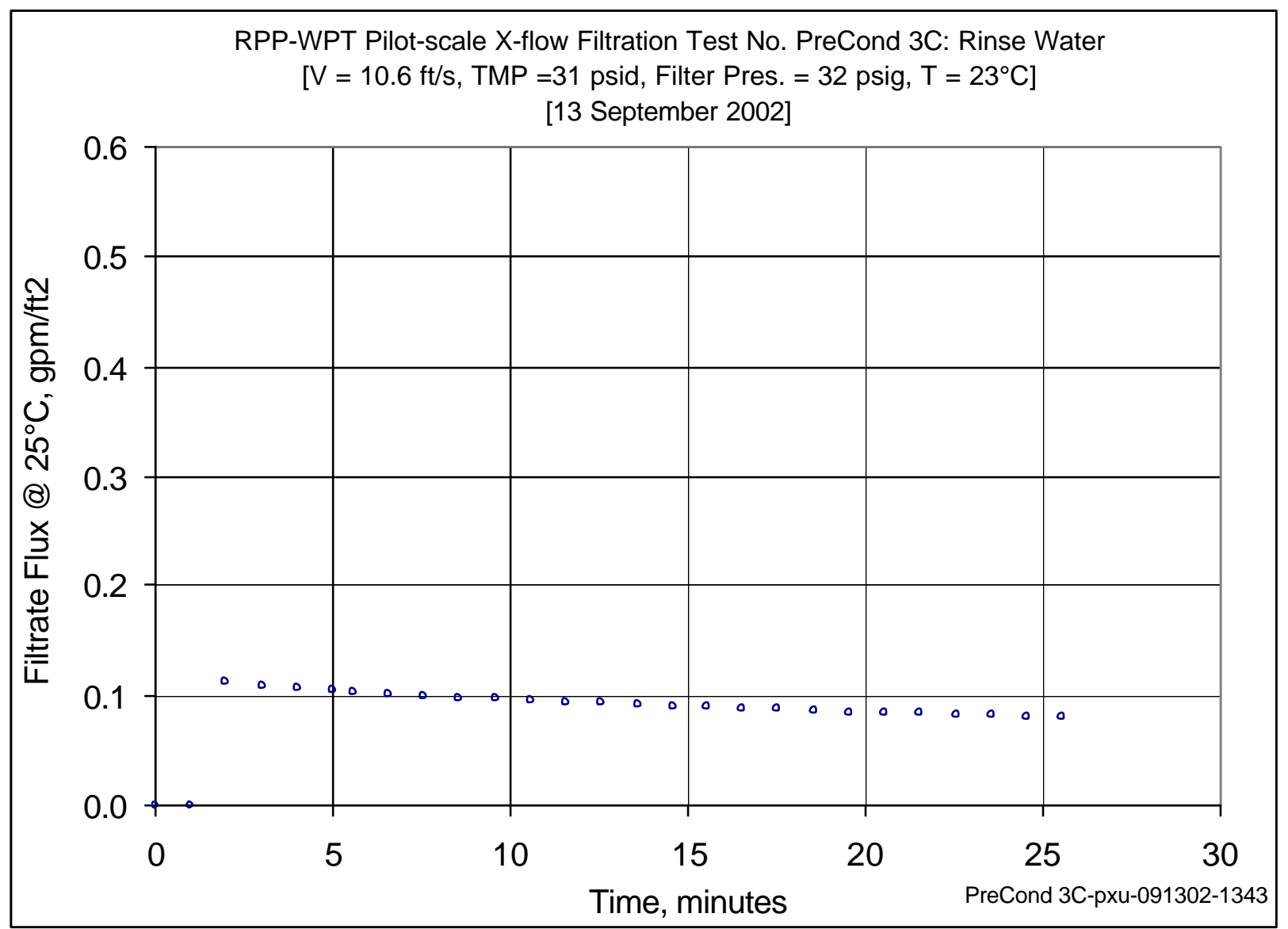

Figure A11: PreConditioning 3C - Inhibited Deionized \& Filtered Water - Post Standard Slurry Tests 
WSRC-TR-2003-00204, REV. 0

SRT-RPP-2003-00087, REV. 0

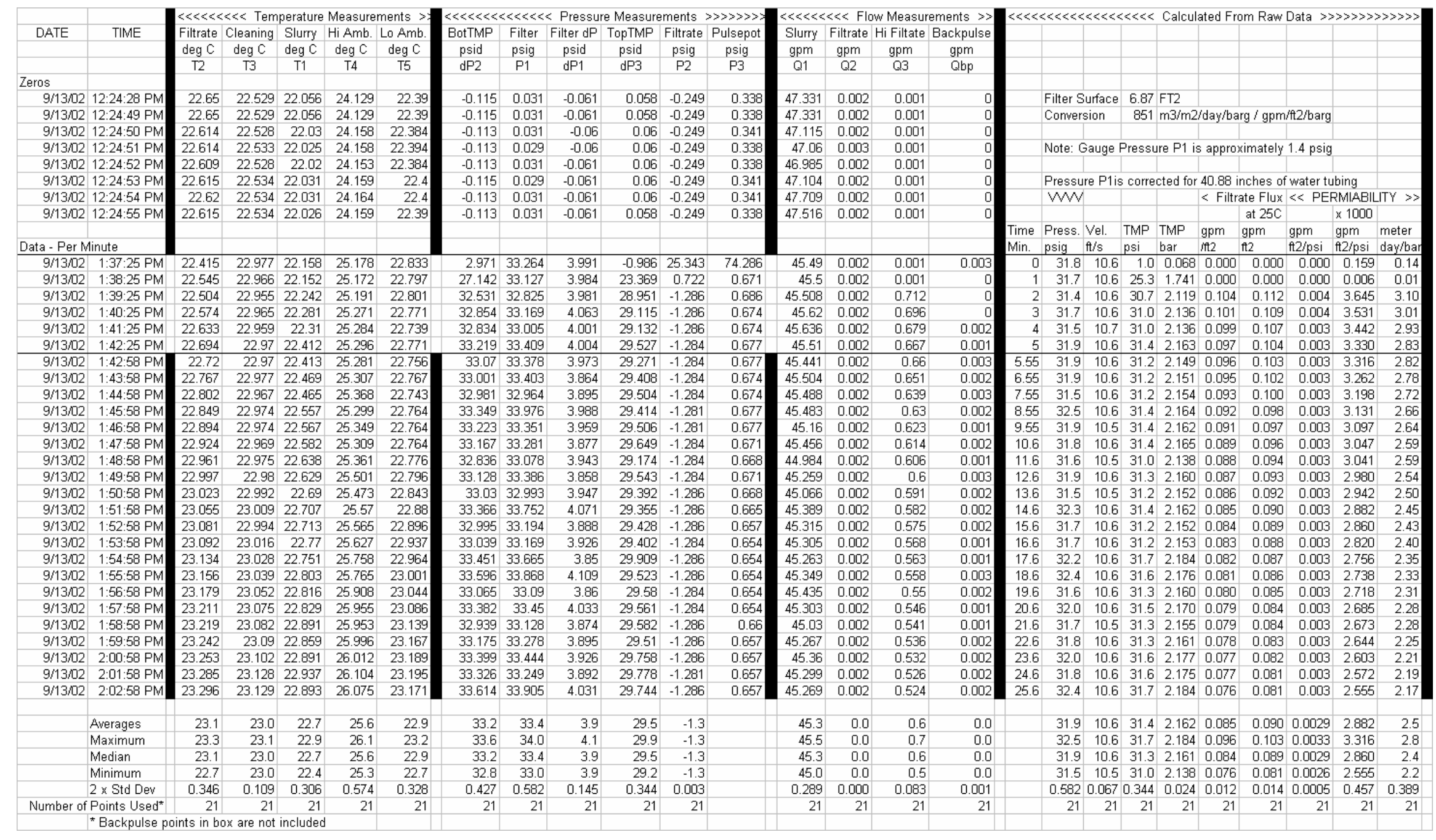

Figure A12: PreConditioning 3C - Inhibited Deionized \& Filtered Water - Post Standard Slurry Tests - Raw \& Calculated Data 
WSRC-TR-2003-00204, REV. 0

SRT-RPP-2003-00087, REV. 0

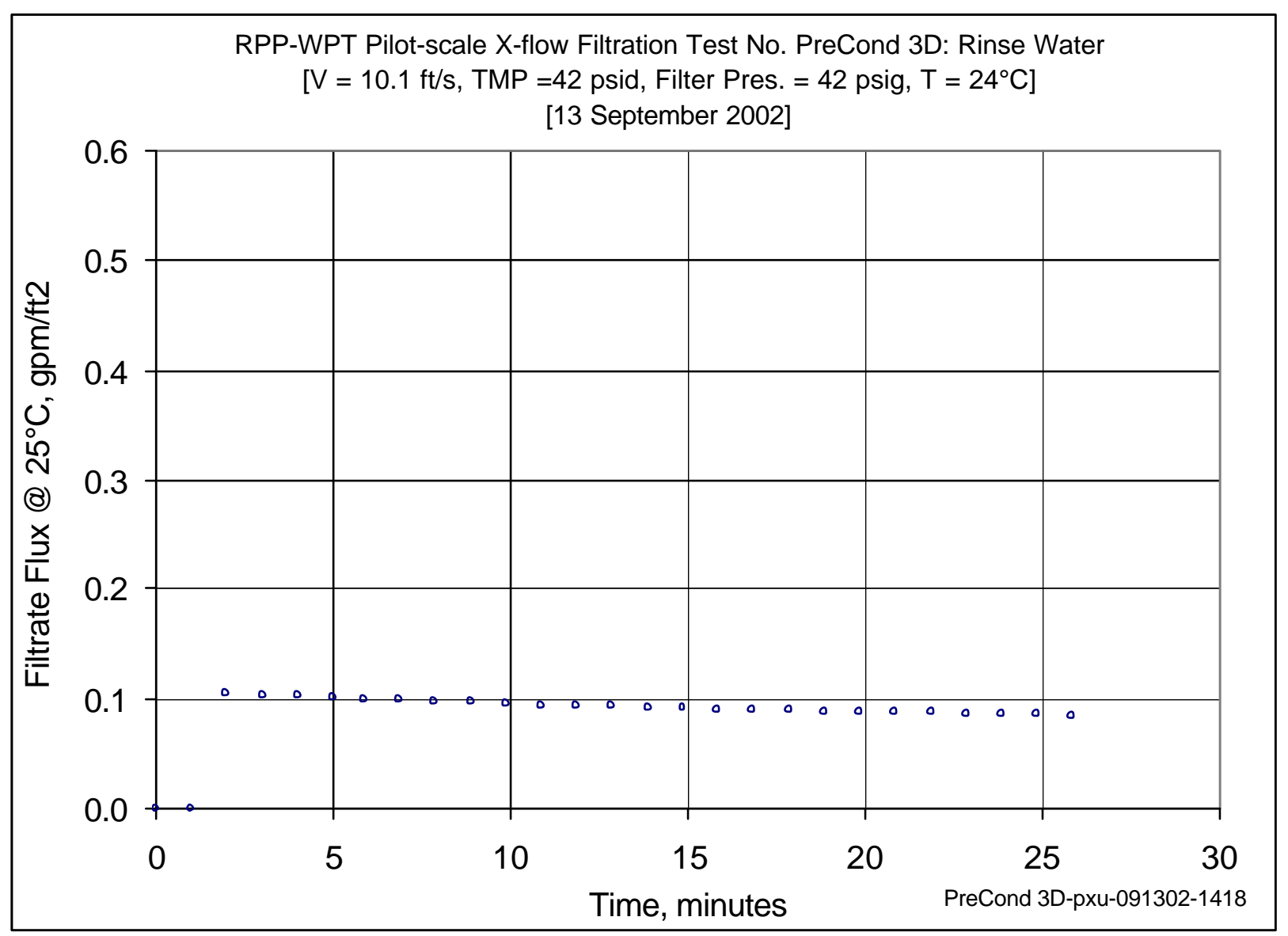

Figure A13: PreConditioning 3D - Inhibited Deionized \& Filtered Water - Post Standard Slurry Tests 
WSRC-TR-2003-00204, REV. 0

SRT-RPP-2003-00087, REV. 0

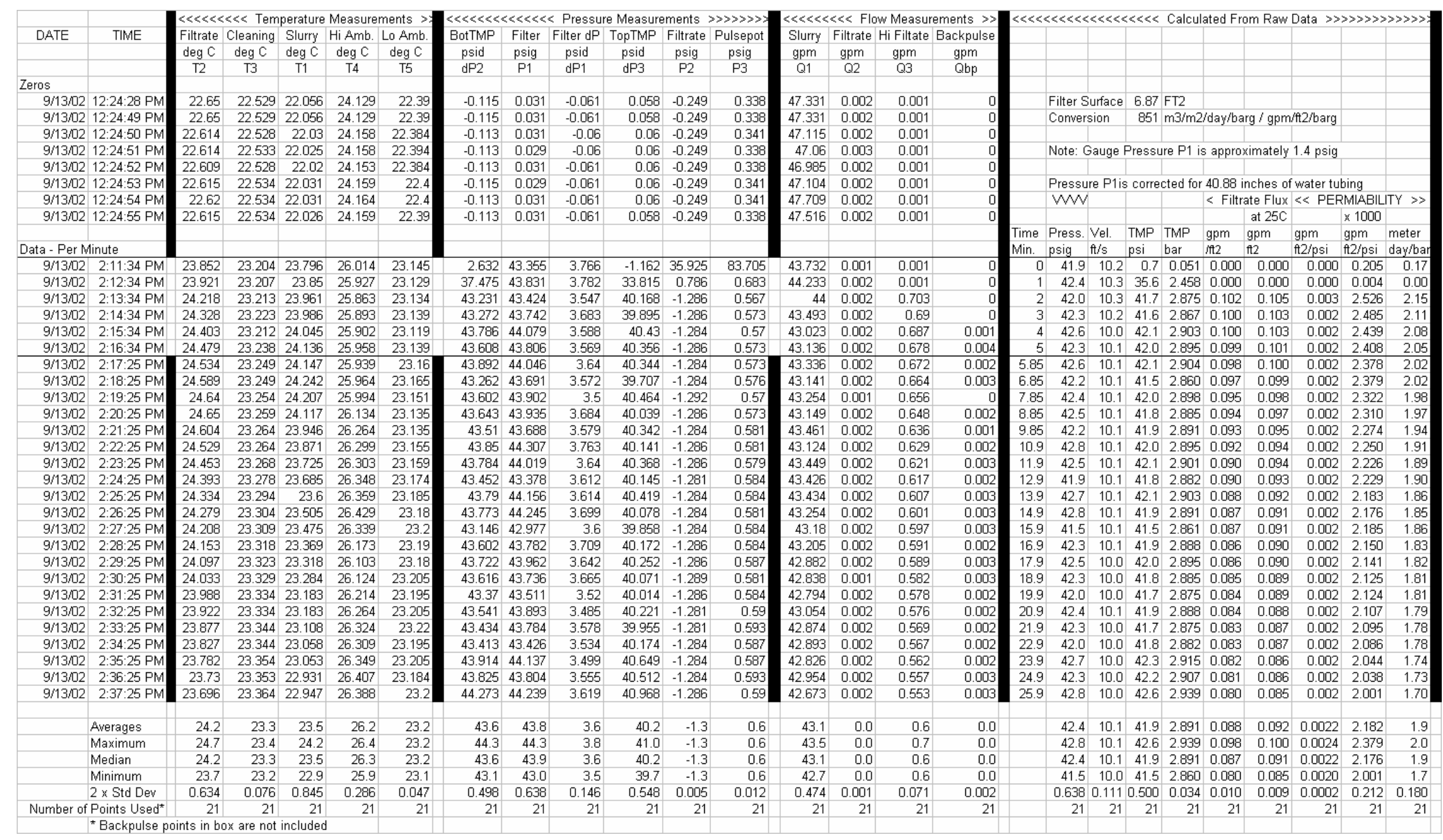

Figure A14: PreConditioning 3D - Inhibited Deionized \& Filtered Water - Post Standard Slurry Tests - Raw \& Calculated Data

Page A17 of A88 
WSRC-TR-2003-00204, REV. 0

SRT-RPP-2003-00087, REV. 0

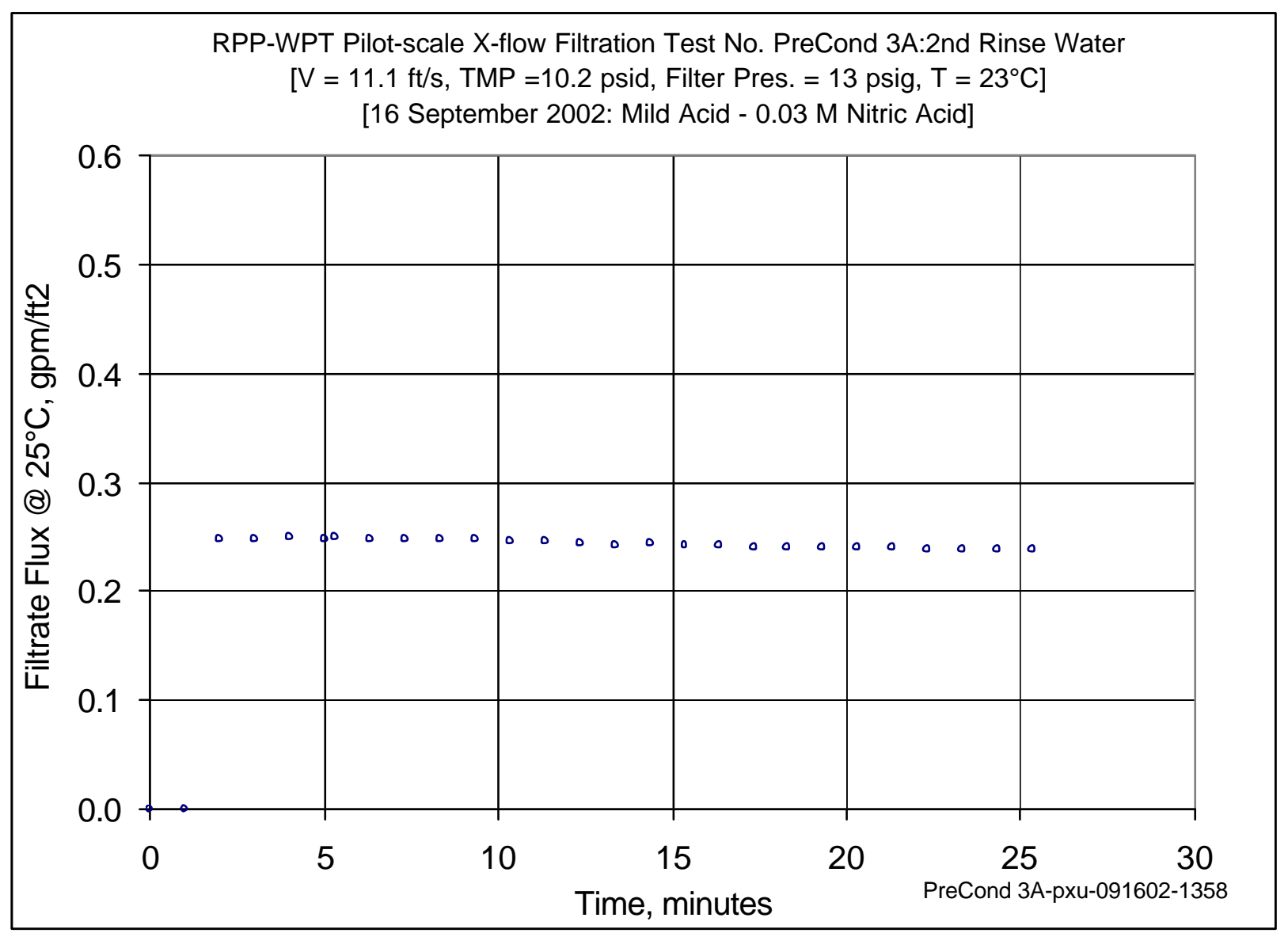

Figure A15: PreConditioning 3A - Deionized \& Filtered Water with 0.03 M Nitric Acid - Post Standard Slurry Tests 
WSRC-TR-2003-00204, REV. 0

SRT-RPP-2003-00087, REV. 0

\begin{tabular}{|c|c|c|c|c|c|c|c|c|c|c|c|c|c|c|c|c|c|c|c|c|c|c|c|c|c|c|}
\hline \multirow{3}{*}{ DATE } & \multirow{3}{*}{ TIME } & \multicolumn{5}{|c|}{$\ll<<<<<<$ Temperature Measurements $>$ - } & \multicolumn{6}{|c|}{ 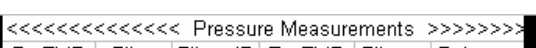 } & \multicolumn{4}{|c|}{ 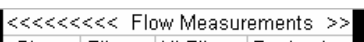 } & \multicolumn{4}{|c|}{ 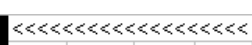 } & \multicolumn{6}{|c|}{ 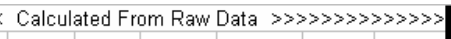 } \\
\hline & & Filtrate $\mathrm{C}$ - & Cleaning & Slurry 1 & Hi Amb. & Lo Amb. & BotTMP & Filter & Filter $\mathrm{dP}$ & TopTMP & Filtrate & Pulsepot & Slurry & Filtrate & Hi Filtate & Backpulse & & & & & & & & & & \\
\hline & & $\operatorname{deg} C$ & $\operatorname{deg} \mathrm{C}$ & $\operatorname{deg} C$ & $\operatorname{deg} \mathrm{C}$ & $\operatorname{deg} \mathrm{C}$ & psid & psig & psid & psid & psig & psig & $\mathrm{gpm}$ & $\mathrm{gpm}$ & $\mathrm{gpm}$ & $\mathrm{gpm}$ & & & & & & & & & & \\
\hline & & $\mathrm{T} 2$ & T3 & $\mathrm{T1}$ & T4 & T5 & $\mathrm{dP} 2$ & $\mathrm{P} 1$ & $\mathrm{dP1}$ & $\mathrm{dP} 3$ & $\mathrm{P} 2$ & $\mathrm{P} 3^{3}$ & Q1 & Q2 & Q3 & Qbp & & & & & & & & & & \\
\hline \multicolumn{27}{|l|}{ Zeros } \\
\hline $9 / 16 / 02$ & 2:19:17 PM & 23.65 & 22.68 & 23.513 & 24.56 & 22.656 & -0.115 & 0.038 & -0.059 & 0.062 & -0.205 & 0.33 & 47.558 & 0.002 & 1.564 & 0.001 & & \multicolumn{2}{|c|}{ Filter Surface } & 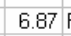 & $7 \mathrm{FT} 2$ & & & & & \\
\hline $9 / 16 / 02$ & 2:19:30 PM & 23.65 & 22.68 & 23.513 & 24.56 & 22.656 & -0.115 & 0.038 & -0.059 & 0.062 & -0.205 & 0.33 & 47.558 & 0.002 & 1.564 & 0.001 & & \multicolumn{2}{|c|}{ Conversion } & $851 \mathrm{r}$ & \multicolumn{4}{|c|}{$1 \mathrm{~m} 3 / \mathrm{m} 2 /$ day $/$ barg $/ \mathrm{gpm} / \mathrm{t} 2 /$ barg } & & \\
\hline 9/16/02 & 2:19:31 PM & 23.645 & 22.669 & 23.468 & 24.54 & 22.666 & -0.115 & 0.038 & -0.06 & 0.058 & -0.194 & 0.327 & 47.453 & 0.002 & 1.562 & 0.001 & & & & & & & & & & \\
\hline $9 / 16 / 02$ & 2:19:32 PM & 23.65 & 22.68 & 23.473 & 24.55 & 22.666 & -0.115 & 0.038 & -0.06 & 0.056 & -0.211 & 0.327 & 47.478 & 0.001 & 1.562 & 0.002 & & Note: $G$ & Gauge P & Pressur & ure $\mathrm{P} 1$ is & $s$ approxi & ximately & $1.4 \mathrm{psig}$ & & \\
\hline $9 / 16 / 02$ & 2:19:33 PM & 23.66 & 22.68 & 23.483 & 24.555 & 22.666 & -0.115 & 0.038 & -0.06 & 0.056 & -0.22 & 0.324 & 47.528 & 0.001 & 1.563 & 0.001 & & & & & & & & & & \\
\hline $9 / 16 / 02$ & 2:19:34 PM & 23.666 & 22.69 & 23.489 & 24.576 & 22.672 & -0.115 & 0.038 & -0.06 & 0.058 & -0.2 & 0.327 & 47.428 & 0.002 & 1.561 & & & Pressur & ure $\mathrm{P} 1$ is & $s$ correc & ected for 4 & 40.8 & inches of & f water tub & bing & \\
\hline 9/16/02 & 2:19:35 PM & 23.665 & 22.685 & 23.483 & 24.57 & 22.666 & -0.115 & 0.038 & -0.059 & 0.058 & -0.203 & 0.33 & 47.497 & 0.002 & 1.558 & 0.001 & & WW & & & & $<$ Filtrat & ate Flux & $\ll$ PERI & RMIABILIT & $T Y \gg$ \\
\hline $9 / 16 / 02$ & 2:19:36 PM & 23.665 & 22.685 & 23.478 & 24.575 & 22.666 & -0.115 & 0.038 & -0.059 & 0.058 & -0.179 & 0.33 & 47.578 & 0.002 & 1.562 & & & & & & & & at $25 \mathrm{C}$ & & $\times 1000$ & \\
\hline & & & & & & & & & & & & & & & & & Time & Press. & Vel. & TMP & TMP $g$ & gpm g & gpm & $\mathrm{gpm}$ & $\mathrm{gpm}$ & meter \\
\hline ta-Per M & Minute & & & & & & & & & & & & & & & & Min. & psig f & $\mathrm{f} / \mathrm{s}$ & psi & bar & ift2 & $\mathrm{n} 2$ & $\mathrm{ft} 2 / \mathrm{psi}$ & $\mathrm{ft} / \mathrm{psi}$ & day/bar \\
\hline $9 / 16 / 02$ & 1:52:31 PM & 23.1 & 22.661 & 22.501 & 24.557 & 22.819 & 2.87 & 14.769 & 4.19 & -1.422 & 6.57 & 55.738 & 46.666 & 0.002 & 0.001 & & 0 & 13.3 & 10.9 & 0.7 & 0.050 & 0.000 & 0.000 & 0.000 & 0.216 & 0.18 \\
\hline $9 / 16 / 02$ & 1:53:31 PM & 23.038 & 22.65 & 22.434 & 24.551 & 22.793 & 7.984 & 14.525 & 4.165 & 3.978 & 1.141 & 0.561 & 46.995 & 0.002 & 0.001 & & 1 & \begin{tabular}{|l|l|}
13.1 & 13.1 \\
\end{tabular} & 11.0 & 6.0 & 0.412 & 0.000 & 0.000 & 0.000 & 0.026 & 0.02 \\
\hline $9 / 16 / 02$ & 1:54:31 PM & 22.791 & 22.655 & 22.378 & 24.611 & 22.797 & 11.937 & 14.264 & 4.167 & 8.152 & -1.284 & 0.631 & 47.478 & 0.002 & 1.582 & & 2 & 212.8 & 11.1 & 10.0 & 0.693 & 0.230 & 0.248 & 0.025 & 524.690 & 21.01 \\
\hline $9 / 16 / 02$ & 1:55:31 PM & 22.725 & 22.674 & 22.428 & 24.62 & 22.801 & 12.113 & 14.484 & 4.263 & 8.136 & -1.284 & 0.634 & 47.386 & 0.002 & 1.584 & & 3 & \begin{tabular}{l|l}
3 & 13.0 \\
\end{tabular} & 11.1 & 10.1 & $\begin{array}{ll}0.698 \\
\end{array}$ & 0.230 & 0.248 & 0.024 & 424.491 & 20.84 \\
\hline $9 / 16 / 02$ & 1:56:31 PM & 22.674 & 22.673 & 22.312 & 24.639 & 22.781 & 11.962 & 14.209 & 4.208 & 8.128 & -1.286 & 0.631 & 47.513 & 0.002 & 1.589 & & 4 & \begin{tabular}{l|l}
4 & 12.7 \\
\end{tabular} & 11.1 & 10.0 & 0.693 & 0.231 & 0.250 & 0.025 & 524.845 & 21.14 \\
\hline $9 / 16 / 02$ & 1:57:31 PM & 22.629 & 22.668 & 22.341 & 24.629 & 22.78 & 12.078 & 14.364 & 4.105 & 8.279 & $\begin{array}{r}-1.284 \\
\end{array}$ & 0.619 & 47.453 & 0.002 & 1.58 & & 5 & \begin{tabular}{|l|l|}
5 & 12.9 \\
\end{tabular} & 11.1 & 10.2 & 20.702 & 0.230 & 0.248 & 0.024 & $\begin{array}{l}424.360 \\
\end{array}$ & 20.73 \\
\hline $9 / 16 / 02$ & 1:57:51 PM & 22.623 & 22.667 & 22.28 & 24.633 & 22.774 & 12.142 & 14.426 & 4.142 & 8.378 & -1.284 & 0.622 & 47.321 & 0.002 & 1.581 & & 5.33 & $\begin{array}{ll}3 & 13.0 \\
\end{array}$ & 11.0 & 10.3 & $\begin{array}{ll}30.707 \\
\end{array}$ & 0.230 & 0.249 & 0.024 & 424.224 & 20.62 \\
\hline $9 / 16 / 02$ & 1:58:51 PM & 22.583 & 22.677 & 22.305 & 24.613 & 22.779 & 11.892 & 14.194 & 4.075 & 8.095 & -1.284 & 0.622 & 47.463 & 0.002 & 1.575 & & 6.33 & \begin{tabular}{l|l}
3 & 12.7 \\
\end{tabular} & 11.1 & 10.0 & 0.689 & 0.229 & 0.247 & 0.025 & 524.758 & 21.07 \\
\hline $9 / 16 / 02$ & 1:59:51 PM & 22.557 & 22.677 & 22.214 & 24.628 & 22.784 & 12.064 & 14.325 & 4.157 & 55 & -1.286 & 0.622 & 47.327 & 0.002 & 1.574 & 0.002 & 7.33 & B 12.9 & 11.0 & 10.2 & 20.701 & 0.229 & 0.248 & 0.024 & 424.390 & 20.76 \\
\hline $9 / 16 / 02$ & 2:00:51 PM & 22.526 & 22.68 & 22.258 & 24.636 & 22.7 & 11.971 & 14.231 & & & -1.2 & & 47.36 & 0.002 & & $P$ & 8.33 & 12.8 & 11.1 & 10.1 & 10.693 & & 0.247 & 0.025 & & 20.89 \\
\hline $9 / 16 / 02$ & 2:01:51 PM & 22.495 & 22.675 & 22.172 & 24.636 & 22.787 & 12.074 & 14.296 & 4.1 & 8.326 & -1.286 & 0.622 & 47.465 & 0.002 & 1.563 & & 9.33 & \begin{tabular}{|l|l|}
3 & 12.8
\end{tabular} & 11.1 & 10.2 & 20.703 & 0.227 & 0.246 & 0.024 & 424.164 & 20.56 \\
\hline $9 / 16 / 02$ & 2:02:51 PM & 22.5 & 22.685 & 22.278 & 24.631 & 22.807 & 12.147 & 14.407 & 4.144 & 8.288 & -1.284 & 0.622 & 47.698 & 0.002 & 1.564 & & 10.3 & \begin{tabular}{l|l}
3 & 12.9 \\
\end{tabular} & 11.1 & 10.2 & 20.704 & 0.228 & 0.246 & 0.024 & 424.065 & 20.48 \\
\hline $9 / 16 / 22$ & 2:03:51 PM & 22.565 & 22.694 & 22.443 & 24.615 & 22.812 & 12.147 & 14.356 & 4.002 & 8.509 & -1.284 & 0. & 47.497 & 0.002 & & 0.001 & 11.3 & $\begin{array}{ll}3 & 12.9\end{array}$ & 11.1 & 10.3 & 30.712 & 0.228 & 0.245 & 0.024 & & 20.19 \\
\hline $9 / 16 / 02$ & 2:04:51 PM & 22.685 & 22.694 & 22.593 & 24.569 & 22.806 & 11.993 & 14.26 & 4.167 & 8.079 & -1.284 & 0.622 & 47.44 & 0.002 & 1.5 & 0.001 & 12.3 & $\begin{array}{ll}3 & 12.8 \\
\end{array}$ & 11.1 & 10.0 & 0 & 0.228 & 0.244 & 0.024 & 424 & 20.68 \\
\hline $9 / 16 / 02$ & 2:05:51 PM & 22.769 & 22.698 & 22.638 & 24.534 & 22.775 & 12.244 & 14.548 & 4.195 & 2 & -1.284 & 622 & 47.503 & 0.0 & 1.556 & 0.002 & 13.3 & \begin{tabular}{|l|l|} 
& 13.1 \\
\end{tabular} & 11.1 & 10.2 & 20.705 & 0.226 & 0.242 & 0.024 & 423.672 & 20.14 \\
\hline $9 / 16 / 02$ & 2:06:51 PM & 22.859 & 22.682 & 22.788 & 24.448 & 22.769 & & 14.345 & & & -1.2 & & 47.505 & & & & 14.3 & 3 12.9 & 11.1 & 10.1 & & & & & & 20.36 \\
\hline $9 / 16 / 02$ & 2:07:51 PM & 22.963 & 22.691 & 22.806 & 24.421 & 22.727 & 12.006 & 14.202 & & 8.296 & -1.2 & 0.622 & 47.4 & 0.002 & 1.565 & & 15.3 & \begin{tabular}{|l|l}
8 & 12.7
\end{tabular} & 11.1 & 10.2 & 20.700 & 0.228 & 0.242 & 0.024 & & 20.32 \\
\hline $9 / 16 / 02$ & 2:08:51 PM & 23.043 & 22.685 & 22.951 & 24.44 & 22.737 & 12.101 & 14.432 & 4.024 & 8.451 & -1.286 & 0.622 & 47.333 & 0.002 & 1.567 & & 16.3 & $\begin{array}{ll}3 & 13.0 \\
\end{array}$ & 11.0 & 10.3 & $\begin{array}{ll}30.708 \\
\end{array}$ & 0.228 & 0.242 & 4 & 423.516 & 20.01 \\
\hline 9/16/02 & 2:09:51 PM & 23.143 & 22.694 & 22.976 & & 22.726 & & 14.399 & & & -1.2. & 0. & 47.415 & 0.0 & 1. & 0.00 & 17.3 & $\begin{array}{l}32.9 \\
\end{array}$ & 11.1 & 10.2 & 20.7 & 0.226 & & & & 19.98 \\
\hline 9/16/02 & 2:10:51 PM & 23.21 & 22.694 & 23.101 & 24.364 & 22.736 & 12.084 & 14.351 & & 8.284 & -1.2 & & 47.446 & 0.002 & & 0.003 & 18.3 & 12.9 & 11.1 & 10.2 & 20.702 & 0.227 & 0.240 & & 423 & 20.06 \\
\hline $9 / 16 / 02$ & 2:11:51 PM & 23.281 & 22.677 & 23.094 & 24.333 & 22.694 & 12.004 & 14.204 & 4.1 & 8.21 & -1.2 & 0.622 & 47.725 & 0.002 & 1.558 & & 19.3 & \begin{tabular}{l|l}
3 & 12.7 \\
\end{tabular} & 11.1 & 10.1 & 10.697 & 0.227 & 239 & 0.024 & 423.676 & 20.15 \\
\hline $9 / 16 / 02$ & 2:12:51 PM & 23.32 & 22.676 & 23.199 & 24.407 & 22.688 & 11.958 & 14.293 & 4. & 99 & -1.284 & 10 & 47.532 & 0.002 & 1.567 & & 20.3 & 12.8 & 11.1 & 10.0 & 0.688 & 0.228 & 240 & 0.0 & 424.035 & 20.45 \\
\hline $9 / 16 / 02$ & 2:13:51 PM & 23.40 & 22.685 & 23.222 & 24.506 & 22.6 & 12.111 & 14.407 & & & -1.286 & & 47.434 & 0.002 & & 0. & 21.3 & B 12.9 & 11.1 & 10.2 & 20.704 & 0.228 & 0.239 & 0.023 & 323.454 & 19.96 \\
\hline $9 / 16 / 22$ & 2:14:51 PM & 23.438 & 22.684 & 23.296 & 24.494 & 22.665 & 12.116 & 14.308 & 4.075 & 8.404 & -1.286 & 0.8 & 47.493 & 0.001 & 1.5 & 0.001 & 22.3 & \begin{tabular}{|l|l|}
3 & 12.8
\end{tabular} & 11.1 & 10.3 & $\begin{array}{l}30.707 \\
\end{array}$ & 0.227 & 0.238 & 0.023 & 23.233 & 19.77 \\
\hline $9 / 16 / 02$ & 2:15:51 PM & 23.517 & 22.687 & 23.325 & 24.523 & 22.6 & 12.221 & 14.4 & 4.21 & 17 & -1.284 & & 47.503 & 0.0 & 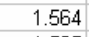 & & 23.3 & 13.0 & 11.1 & 10.3 & & 0.228 & 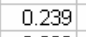 & & 323.204 & 19.75 \\
\hline $9 / 16 / 02$ & 2:16:51 PM & 23.537 & 22.687 & 23.385 & 24. & 22.6 & 12.002 & 14.2 & & & -1.286 & 0. & 47.516 & 0.002 & 1.565 & & 24.3 & \begin{tabular}{l|l}
3 & 12.7 \\
\end{tabular} & 11.1 & 10.1 & 10.695 & 0.228 & 0.238 & 0.024 & 423.634 & 20.11 \\
\hline $9 / 16 / 02$ & 2:17:51 PM & 23.595 & 22.675 & 23.388 & 24.511 & 22.647 & 12.026 & 14.234 & 4.138 & 8.224 & -1.286 & 0.619 & 47.348 & 0.002 & 1.561 & & 25.3 & $\begin{array}{ll}3 & 12.8 \\
\end{array}$ & 11.1 & 10.1 & 10.698 & 0.227 & 0.238 & 0.023 & 323.481 & 19.98 \\
\hline & & & & & & & & & & & & & & & & & & & & & & & & & & \\
\hline & Average & 23 & 22.7 & 22 & 24 & 22.7 & 12.1 & 14.3 & 4. & 8 & 3 & 0. & 47. & 0.0 & 1.6 & 0.0 & & 12 & 11. & 10.2 & 20.701 & 0.228 & 242 & & & 20.3 \\
\hline & Maximum & 23 & 22.7 & 23.4 & 24.6 & 22. & 12 & 14.5 & 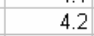 & 8.5 & -1.3 & 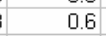 & & U. & 1. & 0.0 & & 13.1 & 11.1 & 10.3 & & 0.230 & 0.249 & & 24.758 & 21.1 \\
\hline & Median & 23.0 & 22.7 & 22.8 & 24.5 & 22.7 & 12.1 & 14.3 & 4.1 & 8.3 & -1.3 & 3 & 47.5 & 0.0 & 1.6 & 0. & & 12.9 & 11.1 & 10.2 & 20.702 & 0.228 & 0.242 & 0.0237 & 723.725 & 20.2 \\
\hline & Minimum & 22.5 & 22.7 & 22.2 & 24.3 & 22.6 & 11.9 & 14.2 & 4.0 & 8.0 & -1.3 & 0. & 47.3 & 0.0 & 1.6 & 0. & & 12.7 & 11.0 & 10.0 & 0.688 & 0.226 & 0.238 & 0.0232 & 223.204 & 19.7 \\
\hline & $2 \times 5$ & 0.770 & 0.016 & 0.850 & 0.187 & 0.109 & 0.174 & 0.193 & 0.111 & 0.240 & 0.003 & 0.003 & 0.207 & 0.001 & 0.012 & 0.002 & & 0.193 & 0.0480 & 0.188 & $\begin{array}{l}30.013 \\
\end{array}$ & 0.002 & 0.007 & 0.0008 & \begin{tabular}{|l|l|}
3 & 0.835 \\
\end{tabular} & 0.711 \\
\hline & Points Used ${ }^{*}$ & 21 & 21 & 21 & 21 & 21 & 21 & 21 & 21 & 21 & 21 & 21 & 21 & 21 & 21 & 21 & & 21 & 21 & 21 & 21 & 21 & 21 & 21 & 21 & 21 \\
\hline & 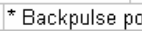 & & & & & & & & & & & & & & & & & & & & & & & & & \\
\hline
\end{tabular}

Figure A16: PreConditioning 3A - Deionized \& Filtered Water with 0.03 M Nitric Acid - Post Standard Slurry Tests - Raw \& Calculated Data 
WSRC-TR-2003-00204, REV. 0

SRT-RPP-2003-00087, REV. 0

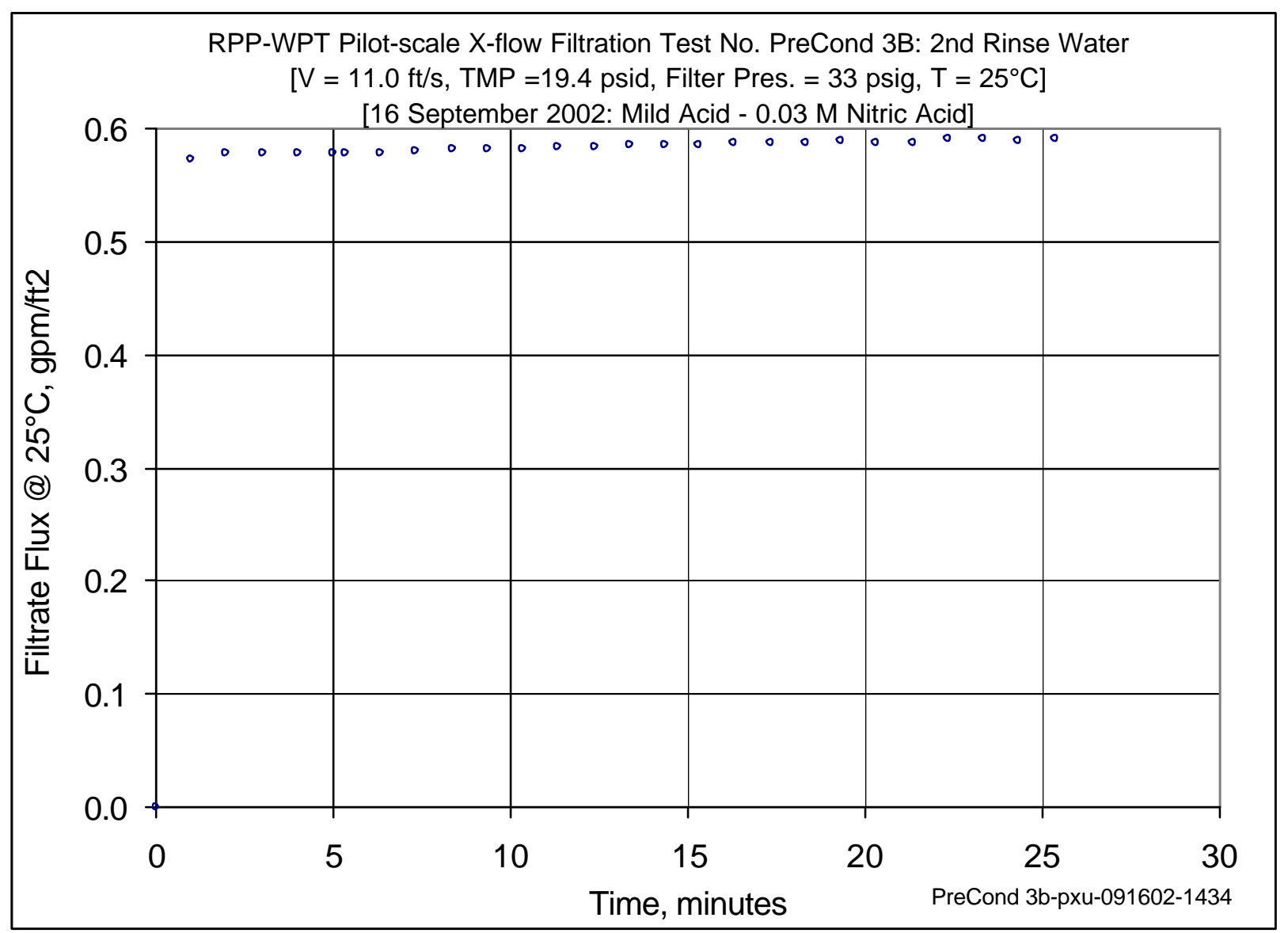

Figure A17: PreConditioning 3B - Deionized \& Filtered Water with 0.03 M Nitric Acid - Post Standard Slurry Tests 
WSRC-TR-2003-00204, REV. 0

SRT-RPP-2003-00087, REV. 0

\begin{tabular}{|c|c|c|c|c|c|c|c|c|c|c|c|c|c|c|c|c|c|c|c|c|c|c|c|c|c|c|}
\hline \multirow{2}{*}{ DATE } & \multirow[b]{2}{*}{ TIME } & \multicolumn{5}{|c|}{ 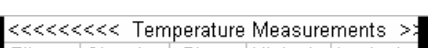 } & \multicolumn{6}{|c|}{ 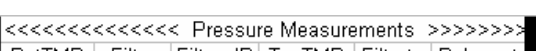 } & \multicolumn{4}{|c|}{ 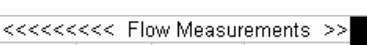 } & \multirow{2}{*}{\multicolumn{10}{|c|}{ 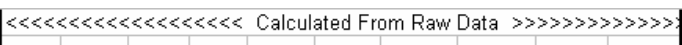 }} \\
\hline & & Filtrate & Cleaning & Slurry & Hi Amb. & Lo Amb. & BotTMP & Filter & Filter $\mathrm{dP}$ & TopTMP & Filtrate & Pulsepot & Slurry & Filtrate & Hi Filtate & Backpulse & & & & & & & & & & \\
\hline & & $\operatorname{deg} \mathrm{C}$ & $\operatorname{deg} C$ & $\operatorname{deg} C$ & $\operatorname{deg} C$ & $\operatorname{deg} \mathrm{C}$ & psid & psig & psid & psid & psig & psig & $\mathrm{gpm}$ & $\mathrm{gpm}$ & $\mathrm{gpm}$ & $\mathrm{gpm}$ & & & & & & & & & & \\
\hline & & 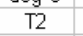 & T3 & $\mathrm{T} 1$ & T4 & T5 & $\mathrm{dP2}$ & P1 & $\mathrm{dP1}$ & $\mathrm{dP3}$ & $\mathrm{P} 2$ & P3 & Q1 & Q2 & 03 & Obp & & & & & & & & & & \\
\hline \multicolumn{27}{|l|}{ Zeros } \\
\hline $9 / 16 / 02$ & 19:17 PM & 23.65 & 22.68 & 23.513 & 24.56 & 22.656 & -0.115 & 0.038 & -0.059 & 0.062 & -0.205 & 0.33 & 47.558 & 0.002 & 1.564 & 0.001 & & \multicolumn{2}{|c|}{ Filter Surface } & $6.87 \mathrm{~F}$ & \multirow{2}{*}{\multicolumn{4}{|c|}{$\mathrm{FT2}$}} & & \\
\hline $9 / 16 / 02$ & 2:19:30 PM & 23.65 & 22.68 & 23.513 & 24.56 & 22.656 & -0.115 & 0.038 & -0.059 & 0.062 & -0.205 & 0.33 & 47558 & 0.002 & 1564 & 0.001 & & \multicolumn{2}{|c|}{ Conversion } & $851 n$ & & & & & & \\
\hline $9 / 16 / 02$ & 2:19:31 PM & 23.645 & 22.669 & 23.468 & 24.54 & 22.666 & -0.115 & 0.038 & -0.06 & 0.058 & -0.194 & 0.327 & 47.453 & 0.002 & 1.562 & 0.001 & & \multirow{2}{*}{\multicolumn{2}{|c|}{ A }} & & & & & & & \\
\hline $9 / 16 / 02$ & 2:19:32 PM & 23.65 & 22.68 & 23.473 & 24.55 & 22.666 & -0.115 & 0.038 & -0.06 & 0.056 & -0.211 & 0.327 & 47.478 & 0.001 & 1.562 & 0.002 & & & Gauge P & Pressure & ire $P 1$ is & is approx & oximately & y $1.4 \mathrm{psig}$ & & \\
\hline $9 / 16 / 02$ & 2:19:33 PM & 23.66 & 22.68 & 23.483 & 24.555 & 22.666 & -0.115 & 0.038 & -0.06 & 0.056 & -0.22 & 0.324 & 47.528 & 0.001 & 1.563 & 0.001 & & & & & & & & & & \\
\hline $9 / 16 / 02$ & 2:19:34 PM & 23.666 & 22.69 & 23.489 & 24.576 & 22.672 & -0.115 & 0.038 & -0.06 & 0.058 & -0.2 & 0.327 & 47.428 & 0.002 & 1.561 & & & Pressur & ure P1is & sc & 14 & or 40.88 ir & inches of & water tu & tubing & \\
\hline $9 / 16 / 02$ & $2: 19: 35 \mathrm{PM}$ & 23.665 & 22.685 & 23.483 & 24.57 & 22.666 & -0.115 & 0.038 & -0.059 & 0.058 & -0.203 & 0.33 & 47.497 & 0.002 & 1.558 & 0.001 & & WM & & & & $<$ Filtra & rate Flux & $x \ll$ PER & RMIABILI & LITY \\
\hline $9 / 16 / 02$ & 2:19:36 PM & 23.665 & 22.685 & 23.478 & 24.575 & 22.666 & -0.115 & 0.038 & -0.059 & 0.058 & -0.179 & 0.33 & 47.578 & 0.002 & 1.562 & & & & & & & & at $25 \mathrm{C}$ & & $\times 1000$ & \\
\hline & & & & & & & & & & & & & & & & & ime & Press. & Vel. & TMP T & TMP & $\mathrm{gpm}$ & & & $\mathrm{gpm}$ & meter \\
\hline ta - Per M & Minute & & & & & & & & & & & & & & & & (1) & psig & $\mathrm{f} / \mathrm{s}$ & psi b & bar & int2 & & $\mathrm{ft} 2 / \mathrm{psi}$ & $\mathrm{f} 2 / \mathrm{psi}$ & \\
\hline $9 / 16 / 02$ & $2: 28: 40 \mathrm{PM}$ & 24.506 & 22.773 & 24.564 & 25.159 & 22.845 & 2.58 & 36.084 & 3.732 & -1.006 & 28.548 & 76.342 & 44.099 & 0.002 & 0.001 & & 0 & 34.6 & 510.3 & 0.8 & 0.054 & 40.000 & 0.000 & 0.000 & 0.187 & 0.16 \\
\hline $9 / 16 / 02$ & 2:29:40 PM & 24.829 & 22.815 & 24.707 & 25.152 & 22.872 & 21.0693 & 34.785 & 3.796 & 18.808 & 7.471 & 1.137 & 47.163 & 0.002 & 3.901 & & 1 & 33.3 & $\begin{array}{ll}311.0 \\
\end{array}$ & 19.9 & 1.375 & 0.568 & 0.572 & 20.029 & 928.706 & \begin{tabular}{|l|l|}
5 & 24.43 \\
\end{tabular} \\
\hline $9 / 16 / 02$ & $2: 30: 40 \mathrm{PM}$ & 24.987 & 22.823 & 24.769 & 25.184 & 22.91 & 20.837 & 34.929 & 3.8 & 18.46 & 7.801 & 1.14 & 47.193 & 0.002 & & & 2 & 33.5 & $\begin{array}{lll}5 & 11.0\end{array}$ & 19.6 & 1.355 & 0.574 & 0.578 & & & 25.01 \\
\hline $9 / 16 / 02$ & $2: 31: 40 \mathrm{PM}$ & 25.115 & 22.851 & 24.807 & 25.333 & 22.933 & 20.817 & 34.975 & 3.7 & 18.495 & 7.847 & 1.143 & 47.113 & 0.002 & 3.954 & & 3 & 33.5 & $\begin{array}{lll}5 & 11.0\end{array}$ & 19.7 & 1.355 & 0.575 & 0.578 & 029 & & $\begin{array}{l}1 \quad 25.05 \\
1\end{array}$ \\
\hline $9 / 16 / 02$ & $2: 32: 40 \mathrm{PM}$ & 25.177 & 22.864 & 24.92 & 25.285 & 22.966 & 20.653 & 34.851 & 3.783 & 18.276 & 7.847 & 1.14 & 47.117 & 0.002 & 3.961 & & 4 & 33.4 & $\begin{array}{ll}4 & 11.0\end{array}$ & 19.5 & 1.342 & 0.576 & 0.578 & 30.030 & 29.679 & $\begin{array}{l}9 \\
9\end{array} 25.26$ \\
\hline $9 / 16 / 02$ & $2: 33: 40 \mathrm{PM}$ & 25.215 & 22.876 & $\begin{array}{r}24.92 \\
24.932\end{array}$ & 25.388 & 23.004 & 20.481 & 34.745 & 3.7 & 18.278 & 7.94 & 1.146 & 47.109 & 0.002 & 3.972 & & $\frac{4}{5}$ & 33.3 & $\begin{array}{ll}3 & 11.0\end{array}$ & 19.4 & 1.336 & 0.578 & 0.579 & $\begin{array}{ll}9 & 0.030\end{array}$ & 29.881 & $\begin{array}{ll}1 & 25.43 \\
1\end{array}$ \\
\hline 9/16/102 & $2: 34: 00 \mathrm{PM}$ & 25.241 & 22.888 & 25.009 & 25.389 & 23.01 & 20.576 & 34.758 & 3.694 & 18.439 & 7.934 & 1.146 & 47.04 & 0.003 & 3.974 & & 5.33 & 33.3 & $\begin{array}{ll}3 & 11.0 \\
\end{array}$ & 19.5 & 1.345 & 0.578 & 0.578 & \begin{tabular}{|l|l|}
3 & 0.030
\end{tabular} & 29.636 & \\
\hline $9 / 16 / 02$ & $2: 35: 00 \mathrm{PM}$ & 25.288 & 22.889 & 24.966 & 25.381 & 23.002 & 20.529 & 34.878 & 3.7 & 18.165 & 7.856 & 1.143 & 46.995 & 0.003 & 3.97 & 0003 & 6.33 & 33.4 & $\begin{array}{lll}4 & 11.0\end{array}$ & 19.3 & 1.334 & 0.578 & 0.578 & 0 & 888 & $\begin{array}{ll}3 & 25.43 \\
\end{array}$ \\
\hline $9 / 16 / 02$ & $2: 36: 00 \mathrm{PM}$ & 25.311 & 22.913 & 24.934 & 25.469 & 23.01 & & 34.65 & 3.7 & & 7.928 & 1.146 & & 0.002 & & & 7.33 & 33.2 & $\begin{array}{lll}2 & 11.0\end{array}$ & 19.3 & 1.333 & 0.579 & & & & 25.54 \\
\hline $9 / 16 / 102$ & $2: 37: 00 \mathrm{PM}$ & 25.207 & 22.913 & $\begin{array}{l}24.854 \\
24.844\end{array}$ & $\begin{array}{r}25.409 \\
25.47\end{array}$ & $\begin{array}{r}23.005 \\
\end{array}$ & & $\begin{array}{r}34.05 \\
34.739\end{array}$ & & 18. & $\begin{array}{l}0.9 \\
7.8 \\
5\end{array}$ & & & 0.002 & & & 8.33 & 33.3 & $\begin{array}{ll}311.0 \\
311.0\end{array}$ & 19.4 & & 0.579 & $\begin{array}{l}0.580 \\
0.582\end{array}$ & & & 25.51 \\
\hline $9 / 16 / 02$ & $2: 38: 00 \mathrm{PM}$ & 25.188 & 22.93 & 24.775 & 25.481 & 23.012 & 20.535 & 34.842 & 3.7 & 18.2 & 7.954 & 1.146 & 47.094 & 0.002 & 3.975 & 0.00 & 9.33 & 33.4 & $\begin{array}{lll}4 & 11.0\end{array}$ & 19.4 & 1.335 & 0.578 & $\begin{array}{l}0.582 \\
3\end{array}$ & 030 & 30.055 & $5 \quad 25.58$ \\
\hline $9 / 16 / 02$ & $2: 39: 00 \mathrm{PM}$ & 25.123 & 22.935 & 24.791 & 25.471 & 22.992 & 43 & 34.849 & & 18.353 & 7.96 & 1.152 & & 0.002 & & 0. & 10.3 & 33.4 & $\begin{array}{lll}4 & 11.0\end{array}$ & 19.5 & 1.342 & 0.579 & 0.582 & 5 & & 6. 25.46 \\
\hline $9 / 16 / 02$ & $2: 40: 00 \mathrm{PM}$ & & 22.952 & 24.672 & 25.413 & 22.983 & & & & & 7.8 & 1.149 & & 0.002 & & & \begin{tabular}{|l|l|}
11.3 \\
\end{tabular} & 33.6 & $\begin{array}{ll}5 & 10.9\end{array}$ & 19.6 & 1.352 & 0.579 & & & & $\begin{array}{ll}4 & 25.35\end{array}$ \\
\hline & $2: 41: 00 \mathrm{PM}$ & 25.055 & 22.947 & 24.652 & 25.352 & 22.978 & 20.553 & 34.7 & & & 7.97 & 1.146 & 46.889 & 0.002 & & & 12.3 & 33.3 & $\begin{array}{ll}3 & 10.9\end{array}$ & 19.4 & 1.336 & 0.579 & 0.5 & 4 & & 25.6 \\
\hline $9 / 16 / 02$ & $2: 42: 00 \mathrm{PM}$ & 24.995 & 22.957 & 24.662 & 25.362 & 22.5 & & 34.7 & 3. & 1 & 7.9 & 1. & 47.006 & 0.0 & 2 & & \begin{tabular}{|l|}
13.3 \\
13.3
\end{tabular} & 33.3 & $\begin{array}{lll}3 & 11.0\end{array}$ & 19.5 & 1.344 & 0.579 & 5 & $\frac{4}{5}$ & $\begin{array}{l}41 \\
99\end{array}$ & $\begin{array}{l}925.5 \\
9\end{array}$ \\
\hline $9 / 16 / 02$ & $2: 43: 00 \mathrm{PM}$ & 24 & 22.951 & 24. & 25.312 & 22.9 & & 34. & & & & & & & & & 14. & & $\begin{array}{ll}310.9 \\
\end{array}$ & 19.3 & & & & & & \\
\hline $9 / 16 / 02$ & $2: 44: 00 \mathrm{PM}$ & & 22.975 & $\begin{array}{l}24.6300 \\
24.601\end{array}$ & 25.326 & $\begin{array}{l}22.972 \\
\end{array}$ & & 34. & & 18. & 7.9 & 1.152 & & 0.002 & & & \begin{tabular}{|l|}
14.3 \\
15.3
\end{tabular} & 33.3 & $\begin{array}{ll}311.0 \\
3\end{array}$ & 19.4 & 1.336 & 0.579 & & & & $\begin{array}{ll}5 & 25.04 \\
5\end{array}$ \\
\hline $9 / 16 / 02$ & $2: 45: 00 \mathrm{PM}$ & 24.928 & 22.99 & 24.585 & 25.391 & 22.961 & 20.564 & 34.93 & 3. & 18.28 & 8. & 1.158 & 46. & 0.0 & 3.987 & 00 & \begin{tabular}{|l|l|}
16.3 \\
\end{tabular} & 33.5 & $\begin{array}{ll}5 & 11.0\end{array}$ & 19.4 & 1.339 & 0.580 & 0.587 & 0 & 23 & $\begin{array}{ll}3 & 25.72\end{array}$ \\
\hline $9 / 16 / 02$ & $2: 46: 00 \mathrm{PM}$ & 24 & 22.979 & 2 & 25.405 & 22 & & 34. & & & & 1. & & & & & 17. & 33.5 & \begin{tabular}{|l|l|}
5 & 10.9
\end{tabular} & 19.4 & & & & & & $\begin{array}{ll}65.81 \\
6\end{array}$ \\
\hline $9 / 16 / 02$ & $2: 47: 00 \mathrm{PM}$ & 24.891 & 22.984 & 24.549 & 25.374 & 22.5 & & 34 & 3. & & 8.0 & 1. & & 0.0 & & & 18.3 & 33.3 & $\begin{array}{ll}310.9 \\
3\end{array}$ & 19.4 & 1.339 & 0.580 & 0.587 & 7 & 30 & 125 \\
\hline $9 / 16 / 02$ & 2:48:00 PM & 24.85 & 22.972 & 24.442 & 25.413 & (2) & 43 & 34. & & & 8. & & & 0.0 & & & 19 & 33.4 & $\begin{array}{lll}4 & 11.0\end{array}$ & 19.5 & 1. & 50 & ח. & 9 & & 25.67 \\
\hline $9 / 16 / 02$ & $2: 49: 00 \mathrm{PM}$ & 24.854 & 22.977 & 24. & & 22 & & 34. & & & & & & & & & 20 & 33.5 & $\begin{array}{ll}510.9 \\
5\end{array}$ & 19.6 & 1. & & & & & 25.55 \\
\hline $9 / 16 / 02$ & $2: 50: 00 \mathrm{PM}$ & 24.8 & 22.992 & 24.502 & 25.453 & 22.9 & & 34 & 3.72 & & & & & 0.0 & & & 21. & 33.5 & $\begin{array}{ll}5 & 10.9\end{array}$ & 19.5 & 1.344 & 40 & & & 30 & \\
\hline $9 / 16 / 02$ & $2: 51: 00 \mathrm{PM}$ & 24.819 & 22.996 & $\begin{array}{l}24.302 \\
24.396\end{array}$ & 25.447 & 22.948 & 20.502 & 34.824 & 3 & 18.321 & 8.0 & 1.152 & 46.775 & 0.002 & 3.995 & & 22.3 & 33.4 & $\begin{array}{ll}4 & 10.9 \\
4\end{array}$ & 19.4 & 1.338 & 0 & 0.591 & 0 & 30.462 & 25.92 \\
\hline $9 / 16 / 02$ & 2:52:00 PM & 24.833 & 23.011 & 24.48 & 25.446 & $=0$ & 20.547 & 35.02 & & 10.51 & & & 46811 & 0.0 & & & & 33.5 & $\begin{array}{ll}5 & 10.9\end{array}$ & 19.3 & & & & & & \\
\hline $9 / 16 / 02$ & $2: 53: 00 \mathrm{PM}$ & 24. & 22.999 & 24.434 & 25. & 22.92 & & 35.0 & & & 8.0 & 1.15 & & 0.0 & & & 24 & 33.6 & $\begin{array}{ll}6 & 10.9\end{array}$ & 19.5 & 1.345 & 0.580 & 0.589 & & 30.198 & 25 \\
\hline $9 / 16 / 02$ & $2: 54: 00 \mathrm{PM}$ & 24.797 & 23.009 & 24.389 & 25.46 & 22.916 & 20.663 & 35.128 & 3.703 & 18.454 & $\begin{array}{l}\quad .024 \\
\end{array}$ & 1.158 & 46.844 & 0.002 & 3.992 & 0.002 & 25.3 & 33.7 & 10.9 & 19.6 & 1.348 & 0.581 & 0.591 & 0.030 & 30.216 & 25.71 \\
\hline & & & & & & & & & & & & & & & & & & & & & & & & & & \\
\hline & A: & & 23.0 & & & 23.0 & & & & & & 1. & & & & 0 & & 33.4 & 11.0 & 19.4 & & 79 & 86 & & & 25 \\
\hline & Maximum & 25.3 & 23.0 & 25 & 25 & 23.0 & 20 & 35.1 & 3. & 18 & 8 & 1. & 47 & 0 & 4.0 & 0. & & 33.7 & 711.0 & 19.6 & 1.352 & 0.582 & 0.591 & 0.0305 & 30.511 & 26. \\
\hline & & 25 & 23. & & 25 & 23.0 & & & 3 & & 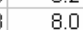 & 1.2 & & 0 & & & & 33.4 & $4 \quad 10.9$ & 19.4 & 1.339 & 0.579 & 0.586 & 0.0302 & 30.169 & 25 \\
\hline & & & & & & & & & & & & 1. & & & & & & 33.2 & 10.9 & 19.3 & & & & & & \\
\hline & & 0.336 & 0.073 & 0.374 & 0.096 & 0.059 & 0.152 & 0.246 & 0.083 & 0.222 & 0.141 & 0.010 & 0.234 & 0.001 & 0.014 & 0.00 & & 0.246 & 6.0550 & 0.167 & 0.012 & 0.002 & 0.008 & 0004 & $\begin{array}{r}0.421 \\
+0.03\end{array}$ & .358 \\
\hline f & f Points Used ${ }^{*}$ & 21 & 21 & 21 & 21 & 21 & 21 & 21 & 21 & 21 & 21 & 21 & 21 & 21 & 21 & 21 & & 21 & 21 & 21 & 21 & 21 & 21 & 21 & 21 & \\
\hline & & & & & & & & & & & & & & & & & & & & & & & & & & \\
\hline
\end{tabular}

Figure A18: PreConditioning 3B - Deionized \& Filtered Water with 0.03 M Nitric Acid - Post Standard Slurry Tests - Raw \& Calculated Data 
WSRC-TR-2003-00204, REV. 0

SRT-RPP-2003-00087, REV. 0

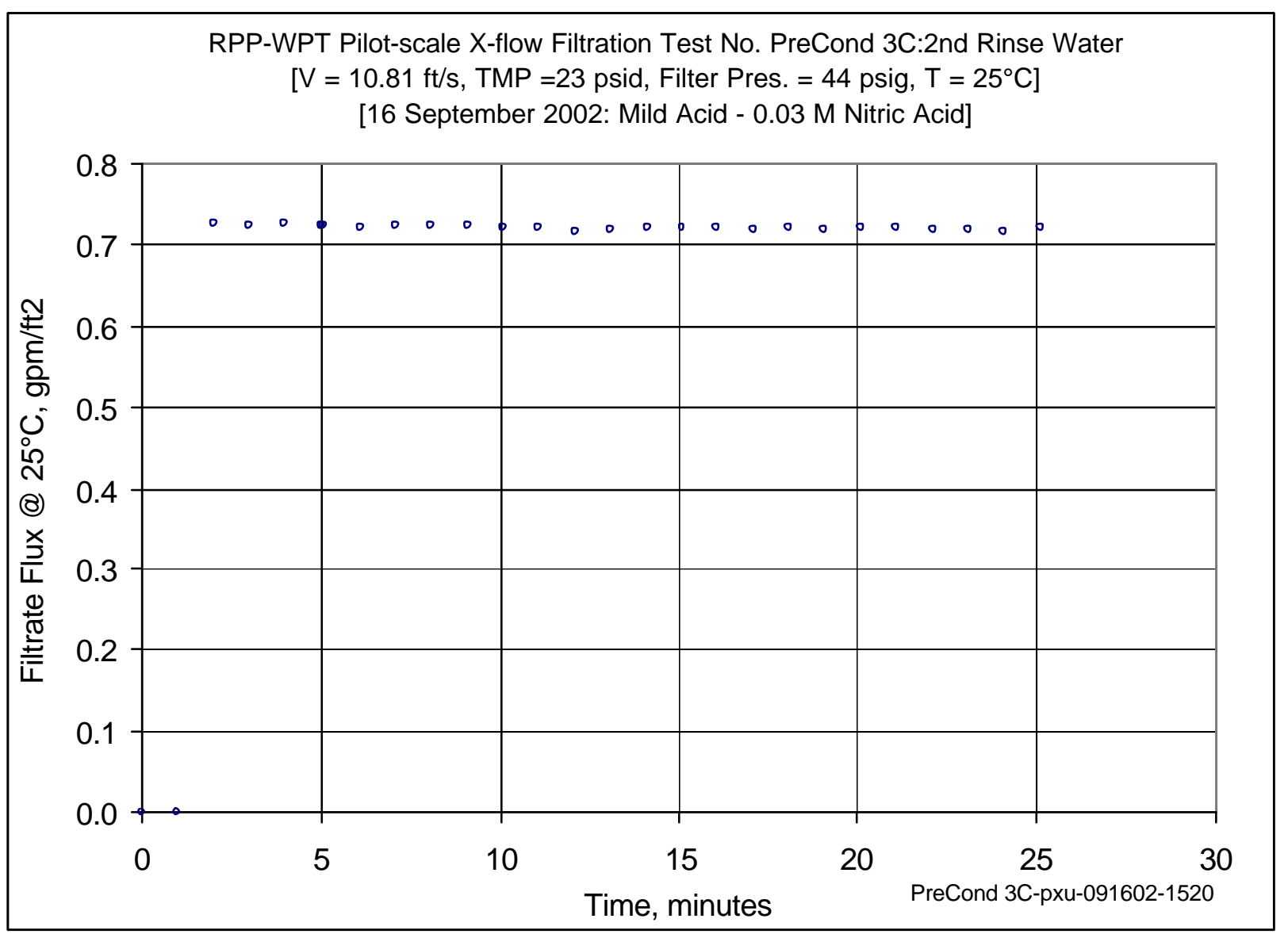

Figure A19: PreConditioning 3C - Deionized \& Filtered Water with 0.03 M Nitric Acid - Post Standard Slurry Tests 
WSRC-TR-2003-00204, REV. 0

SRT-RPP-2003-00087, REV. 0

\begin{tabular}{|c|c|c|c|c|c|c|c|c|c|c|c|c|c|c|c|c|c|c|c|c|c|c|c|c|c|c|}
\hline \multirow{3}{*}{ DATE } & \multirow[b]{2}{*}{ TIME } & \multicolumn{5}{|c|}{$\ll \ll \ll<\ll<<$ Temperature Measurements $>$} & \multicolumn{6}{|c|}{ 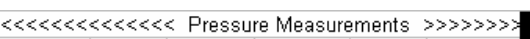 } & \multicolumn{4}{|c|}{$\ll \ll \ll<\ll<<$ Flow Measurements $\gg$} & \multicolumn{10}{|c|}{ 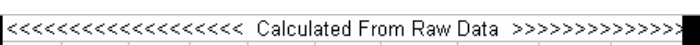 } \\
\hline & & Filtrate & Cleaning & Slurry & Hi Amb. & Lo Amb. & BotTMP & Filter & Filter $\mathrm{dP}$ & TopTMP & Filtrate & Pulsepot & Slurry & Filtrate & Hi Filtate & Backpulse & & & & & & & & & & \\
\hline & & $\operatorname{deg} \mathrm{C}$ & $\operatorname{deg} \mathrm{C}$ & $\operatorname{deg} C$ & $\operatorname{deg} \mathrm{C}$ & $\operatorname{deg} C$ & psid & psig & psid & psid & psig & psig & $\mathrm{gpm}$ & $\mathrm{gpm}$ & $\mathrm{gpm}$ & $\mathrm{gpm}$ & & & & & & & & & & \\
\hline & & $\mathrm{T} 2$ & $\mathrm{~T}_{3}$ & $\mathrm{~T} 1$ & T4 & T5 & $\mathrm{dP} 2$ & $\mathrm{P} 1$ & $\mathrm{dP1}$ & $\mathrm{dP} 3$ & P2 & $\mathrm{P} 3^{3}$ & Q1 & Q2 & Q3 & Qbp & & & & & & & & & & \\
\hline \multicolumn{27}{|l|}{ Zeros } \\
\hline $9 / 16 / 02$ & 2:19:17 PM & 23.65 & 22.68 & 23.513 & 24.56 & 22.656 & -0.115 & 0.038 & -0.059 & 0.062 & -0.205 & 0.33 & 47.558 & 0.002 & 1.564 & 0.00 & & \multicolumn{2}{|c|}{ Filter Surface } & 6.87 & \multirow{2}{*}{\multicolumn{4}{|c|}{$\begin{array}{ll}\mathrm{FT} 2 \\
\mathrm{~m} 3 / \mathrm{m} 2 / \mathrm{day} / \mathrm{barg} / \mathrm{gpm} / \mathrm{ft} 2 / \mathrm{harg}\end{array}$}} & & \\
\hline $9 / 16 / 02$ & 2:19:30 PM & 23.65 & 22.68 & 23.513 & 24.56 & 22.656 & -0.115 & 0.038 & -0.059 & 0.062 & -0.205 & 0.33 & 47.558 & 0.002 & 1.564 & 0.001 & & \multicolumn{2}{|c|}{ Conversion } & 851 & & & & & & \\
\hline $9 / 16 / 02$ & 2:19:31 PM & 23.645 & 22.669 & 23.468 & 24.54 & 22.666 & -0.115 & 0.038 & -0.06 & 0.058 & -0.194 & 0.327 & 47.453 & 0.002 & 1.562 & 0.001 & & \multirow{2}{*}{\multicolumn{2}{|c|}{ Note: Gauge P }} & & & & & & & \\
\hline $9 / 16 / 02$ & 2:19:32 PM & 23.65 & 22.68 & 23.473 & 24.55 & 22.666 & -0.115 & 0.038 & -0.06 & 0.056 & -0.211 & 0.327 & 47.478 & 0.001 & 1.562 & 0.002 & & & & Pressur & ure $\mathrm{P} 1$ is & is approx & ximately 1 & $1.4 \mathrm{psig}$ & & \\
\hline $9 / 16 / 02$ & 2:19:33 PM & 23.66 & 22.68 & 23.483 & 24.555 & 22.666 & -0.115 & 0.038 & -0.06 & 0.056 & -0.22 & 0.324 & 47.528 & 0.001 & 1.563 & & & & & & & & & & & \\
\hline $9 / 16 / 02$ & 2:19:34 PM & 23.666 & 22.69 & 23.489 & 24.576 & 22.672 & -0.115 & 0.038 & -0.06 & 0.058 & -0.2 & 0.327 & 47.428 & 0.002 & 1.561 & & & Pressur & re P1is & $s$ correc & cted for & r 40.88 it & inches of $\mathrm{v}$ & f water tub & ubing & \\
\hline $9 / 16 / 02$ & 2:19:35 PM & 23.665 & 22.685 & 23.483 & 24.57 & 22.666 & -0.115 & 0.038 & -0.059 & 0.058 & -0.203 & 0.33 & 47.497 & 0.002 & 1.558 & 0.00 & & WW & & & & $<$ Filtra & rate Flux & $\ll$ PER & RMIABILIT & ITY $\gg$ \\
\hline $9 / 16 / 02$ & 2:19:36 PM & 23.665 & 22.685 & 23.478 & 24.575 & 22.666 & -0.115 & 0.038 & -0.059 & 0.058 & -0.179 & 0.33 & 47.578 & 0.002 & 1.562 & & & & & & & & at $25 \mathrm{C}$ & & $\times 1000$ & \\
\hline & & & & & & & & & & & & & & & & & Time & Press. & Vel. & TMP & TMP & $\mathrm{gpm}$ & $\mathrm{gpm}$ & $\mathrm{gpm}$ & $\mathrm{gpm}$ & meter \\
\hline ta - Per Mi & linute & & & & & & & & & & & & & & & & Min. & psig & $\mathrm{f} / \mathrm{s}$ & psi & bar & ift2 & $\mathrm{f2} 2$ & $\mathrm{ft2/psi}$ & $\mathrm{ft} 2 / \mathrm{psi}$ & day/ban \\
\hline 9/16/02 & 3:14:48 PM & 24.916 & 23.295 & 24.639 & 26.365 & 23.472 & 2.501 & 46.916 & 3.569 & $\begin{array}{l}-1.084 \\
\end{array}$ & 39.202 & 86.337 & 42.637 & 0.002 & 0.001 & & 0 & 45.4 & 10.0 & 0.7 & 0.049 & 0.000 & 0.000 & 0.000 & $\begin{array}{ll}0.207 \\
0.07\end{array}$ & 0.18 \\
\hline $9 / 16 / 02$ & 3:15:48 PM & 24.95 & 23.319 & 24.692 & 26.393 & 23.481 & 19.398 & 44.855 & 3.585 & 17.174 & 19.392 & 1.068 & 45.701 & 0.005 & & & 1 & 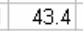 & 10.7 & 18.3 & 1.261 & 0.000 & 0.000 & 0.000 & 0.008 & 0.01 \\
\hline $9 / 16 / 02$ & 3:16:48 PM & 25.052 & 23.321 & 24.69 & 26.396 & 23.489 & 23.124 & 44.822 & 3.603 & 21.659 & 14.937 & -0.878 & 46.796 & 0.002 & 4.948 & & 2 & 43.3 & 10.9 & 22.4 & 1.544 & 0.720 & 0.726 & 0.032 & 232.437 & 27.60 \\
\hline $9 / 16 / 02$ & 3:17:48 PM & 25.111 & 23.344 & 24.728 & 26.429 & 23.512 & 23.134 & 44.768 & 3.529 & 21.856 & 14.908 & -0.889 & 46.45 & 0.002 & 4.943 & & 3 & 43.3 & 10.8 & 22.5 & 1.551 & 0.719 & 0.725 & 0.032 & 232.221 & 27.42 \\
\hline $9 / 16 / 02$ & 3:18:48 PM & 25.155 & 23.368 & 24.782 & 26.463 & 23.511 & 23.304 & 45.141 & 3.615 & 21.817 & 14.9 & -0.892 & 46.559 & 0.002 & 4. & & 4 & 43.7 & 10.9 & 22.6 & 1.555 & 0.721 & 0.725 & 0.032 & 232.143 & 27.35 \\
\hline 9/16/02 & 3:19:34 PM & 25.172 & 23.375 & 24.804 & 26.4 & 23.5 & 23.352 & 45.143 & 3.6 & 21.889 & 14.92 & -0.884 & 46.528 & 0.0 & & & 5 & 43.7 & 10.9 & 22.6 & 1.560 & 0.720 & 0.724 & & & \\
\hline $9 / 1602$ & 3:19:39 PM & \begin{tabular}{|l|}
25.177 \\
\end{tabular} & 23.381 & 24.81 & 26.485 & \begin{tabular}{|l|}
53.528 \\
\end{tabular} & 23.205 & 44.857 & 3.589 & 21.745 & 14.989 & -0.884 & 45.947 & 0.002 & 4.956 & & 5.08 & \begin{tabular}{|l|l|}
3 & 43.4 \\
\end{tabular} & 10.7 & 22.5 & 1.550 & 0.721 & 0.725 & 0.032 & 232.260 & 27.45 \\
\hline $9 / 16 / 02$ & 3:20:39 PM & 25.205 & 23.394 & 24.823 & 26.478 & 23.541 & 23.232 & 44.731 & 3.566 & 21.836 & 14.868 & -0.889 & 46.471 & 0.0 & & 0.002 & 6.08 & 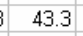 & 10.8 & 22.5 & 1.554 & 0.718 & 0.722 & 0.032 & 232.033 & 27.26 \\
\hline $9 / 16 / 02$ & 3:21:39 PM & 25.242 & 23.406 & 24.86 & 26.475 & 23.538 & 23.428 & 45.101 & 3.615 & 22.05 & 14.911 & -0.892 & 46.484 & 0.0 & & 0.002 & 7.08 & \begin{tabular}{|l|}
43.6 \\
\end{tabular} & 10.9 & 22.7 & & 0.721 & 0.724 & 0.032 & & 27.10 \\
\hline $9 / 16 / 02$ & 3:22:39 PM & 25.262 & 23.411 & 24.865 & 26.44 & 23.538 & 23.474 & 45.081 & 3.582 & 22.087 & 14.897 & -0.892 & 46.463 & 0.002 & 49 & 0.002 & 8.08 & \begin{tabular}{|l|}
3 \\
3
\end{tabular} & 10.8 & 22.8 & 1.571 & 0.720 & 0.723 & 0.032 & 231.733 & 27.01 \\
\hline $9 / 16 / 02$ & 3:23:39 PM & 25.271 & 23.415 & 24.879 & 26.384 & 23.5 & 23.362 & 45.172 & 3.546 & 22.118 & 15.151 & -0.892 & \begin{tabular}{|l|l|}
46.087 \\
\end{tabular} & 0.002 & 4. & 0.003 & 9.08 & \begin{tabular}{|l|l|}
3 & 43.7 \\
\end{tabular} & 10.8 & 22.7 & 1.568 & 0.722 & 0.725 & 0.032 & 231.873 & 27.12 \\
\hline $9 / 16 / 02$ & 3:24:39 PM & 25.317 & 23.436 & 24.91 & & 23.5 & & 45.027 & 3.477 & 22.075 & 14.877 & & & & & 0.00 & \begin{tabular}{|l|}
10.1 \\
\end{tabular} & 43.6 & 10.7 & 22.7 & & 0.720 & 0.722 & 0.032 & 231.778 & 27.04 \\
\hline $9 / 16 / 02$ & 3:25:39 PM & 25.321 & 23.435 & 24.919 & 26.319 & 23.5 & 23.486 & 45.155 & 3.5 & 22.046 & 14.851 & -0.8 & 46.048 & & & 0 & 11.1 & 43.7 & 10.7 & 22.8 & 1.570 & 0.720 & 0.722 & 0.032 & & 26.98 \\
\hline $9 / 16 / 02$ & 3:26:39 PM & 25.346 & 23.45 & 24.939 & 26.394 & 23.5 & 23.256 & 44.679 & 3.4 & 22.163 & 14.602 & -0.8 & 45.75 & 0.0 & 4.917 & 0.00 & 12.1 & 43.2 & 10.7 & 22.7 & 1.566 & 0.715 & 0.717 & 0.032 & 231.560 & 26. \\
\hline $9 / 16 / 02$ & 3:27:39 PM & 25.366 & 23.465 & 24.973 & & 23.5 & & 44.764 & & & 14.729 & & 45.766 & & & & 13 & 43.3 & 10.7 & 22.7 & & & & & 51 & 26. \\
\hline $9 / 16 / 02$ & 3:28:39 PM & 25.38 & 23.469 & 24.973 & 26.398 & 23.5 & 23. & 45.163 & & 22.308 & 14.897 & & 45.972 & & & & 14 & 43.7 & 10.7 & 23.0 & 1.582 & 0.720 & 0.720 & 0.031 & & 26.70 \\
\hline $9 / 16 / 02$ & 3:29:39 PM & 25.389 & 23.473 & 24.976 & 26.437 & 23.55 & 23.486 & 45.337 & 3.525 & 22.167 & 15.024 & -0.889 & 46.876 & 0.0 & 4.9 & 0.00 & 15.1 & 43.9 & 10.9 & 22.8 & 1.574 & 0.722 & 0.722 & 2 & 231.646 & 26.93 \\
\hline $9 / 16 / 02$ & 3:30:39 PM & 25.403 & 23.477 & 24.981 & 26.426 & 23.5 & 23.283 & 44.953 & & 1.944 & 14.946 & -0.887 & 46.026 & & & 0.00 & 16 & 43.5 & 10.7 & 22.6 & 1.559 & 0.720 & 0.7 & 2 & 776 & 27.13 \\
\hline $9 / 16 / 02$ & 3:31:39 PM & 25.421 & 23.505 & 25.019 & 26.359 & 23.5 & & 45.445 & & 22.368 & 14. & & 45.976 & 0. & & & 17 & 44.0 & 10.7 & 23.0 & & 19 & 0.7 & 1 & 63 & 26.61 \\
\hline $9 / 16 / 02$ & 3:32:39 PM & 25.43 & 23.504 & 25.018 & 26.338 & 23.5 & 23.472 & 45.153 & & 22.077 & 14.946 & & 46.012 & 0 & & 0 & 18 & 43.7 & 10.7 & 22.8 & 1.570 & 0.721 & 0.720 & 0.032 & 30 & 26.92 \\
\hline $9 / 16 / 02$ & 3:33:39 PM & 25.439 & 23.508 & 25.026 & 26.357 & 23. & 23.399 & 44.979 & & 22.235 & 14.9 & -0 & 46.047 & 0 & & & 19 & 43.5 & 10.7 & 22.8 & 1. & 0.720 & 0.720 & 0.032 & 231.539 & 26.84 \\
\hline $9 / 16 / 02$ & 3:34:39 PM & 25.443 & 23.522 & 25.02 & 26. & 23.5 & & 45.271 & & 22.442 & & & 45.94 & 0.0 & & & 20 & 43.8 & 10.7 & 23.0 & & 0.721 & 0.7 & 1 & 95 & 26.63 \\
\hline $9 / 16 / 02$ & 3:35:39 PM & 25.441 & 23.525 & 25.023 & 26.268 & 23.5 & 23.356 & 45.06 & 3. & 22.022 & 15.059 & -0.8 & 46.165 & 0.0 & 4. & 0. & 21 & 43.6 & 10.8 & 22.7 & 1.564 & 0.722 & 0.7 & 0.032 & 96 & 27.06 \\
\hline $9 / 16 / 02$ & 3:36:39 PM & 25.454 & 23.538 & 25.032 & 26.262 & 23.5 & 23.383 & 45.056 & & 21.962 & 14.943 & -0.875 & 45.92 & & & & & 43.6 & 10.7 & 22.7 & 1. & 0.720 & & & & 27.00 \\
\hline $9 / 16 / 02$ & 3:37:39 PM & 25.453 & 23.547 & 25.026 & & 23 & & 44.388 & & 9 & & & 45.838 & & & & & 42.9 & 10.7 & 22.4 & & & & & 129 & 27.2 \\
\hline $9 / 16 / 02$ & 3:38:39 PM & 25.461 & 23.565 & 25.059 & 26.244 & \begin{tabular}{|l|} 
\\
23.507 \\
\end{tabular} & 23.306 & 45.128 & 3.473 & 21.932 & 15.007 & -0.872 & 45.886 & 0.002 & 4. & 0.0 & 24 & 43.7 & 10.7 & 22.6 & 1.560 & 0.717 & 0.716 & 0.032 & 231.663 & 26.95 \\
\hline $9 / 16 / 02$ & 3:39:39 PM & 25.45 & 23.559 & 25.037 & 26.238 & 23.515 & 23.667 & 45.503 & 3.535 & 22.292 & 14.906 & -0.866 & 46.02 & 0.002 & 4.96 & & 25.1 & 44.0 & 10.7 & 23.0 & 1.584 & 0.722 & 0.721 & 0.031 & 131.376 & 26.70 \\
\hline & & & & & & & & & & & & & & & & & & & & & & & & & & \\
\hline & $A_{N}$ & & & & & & & & & & & -0.9 & & & & & & 43.6 & 10.8 & 22.7 & & & 0.7 & & & 27.0 \\
\hline & Maximum & 25 & 23.6 & & & & & 45 & 3 & 22.4 & 15 & -0 & 46.9 & 0. & 5 & 0. & & 44.0 & 10.9 & 23.0 & & 0.722 & 0.7 & 3 & 60 & 27.5 \\
\hline & & 25 & & & & & & & & & & 0 & & & & & & 43.6 & & 22.7 & 1. & & & & & 270 \\
\hline & Mir & 25.2 & 23.4 & 24 & & & 23.0 & 44.4 & 3.4 & & 14.0 & -0. & 45.8 & U.U & 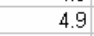 & & & 42.9 & 10.7 & 22.4 & 1.545 & 0.715 & $0.7 / 6$ & 0.0313 & 331.263 & 26.6 \\
\hline & $2 \times$ Std Dev & 0.174 & 0.110 & 0.149 & 0.157 & 0.027 & 0.311 & 0.510 & 0.100 & 0.354 & 0.229 & 0.015 & 0.536 & 0.000 & 0.024 & 0.002 & & 0.510 & $0.125 \square$ & 0.319 & 0.022 & 0.003 & 0.005 & 0.0005 & $\begin{array}{ll}5 & 0.488\end{array}$ & 0.416 \\
\hline ber of $\mathrm{F}$ & Points Used ${ }^{*}$ & 21 & 21 & 21 & 21 & 21 & 21 & 21 & 21 & 21 & 21 & 21 & 21 & 21 & 21 & 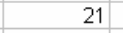 & & 21 & 21 & 21 & 21 & 21 & 21 & 21 & 21 & 20 \\
\hline & ${ }^{*}$ Backpulse po & in ho 30 & ant & 然 & & & & & & & & & & & & & & & & & & & & & & \\
\hline
\end{tabular}

Figure A20: PreConditioning 3C - Deionized \& Filtered Water with 0.03 M Nitric Acid - Post Standard Slurry Tests - Raw \& Calculated Data 
WSRC-TR-2003-00204, REV. 0

SRT-RPP-2003-00087, REV. 0

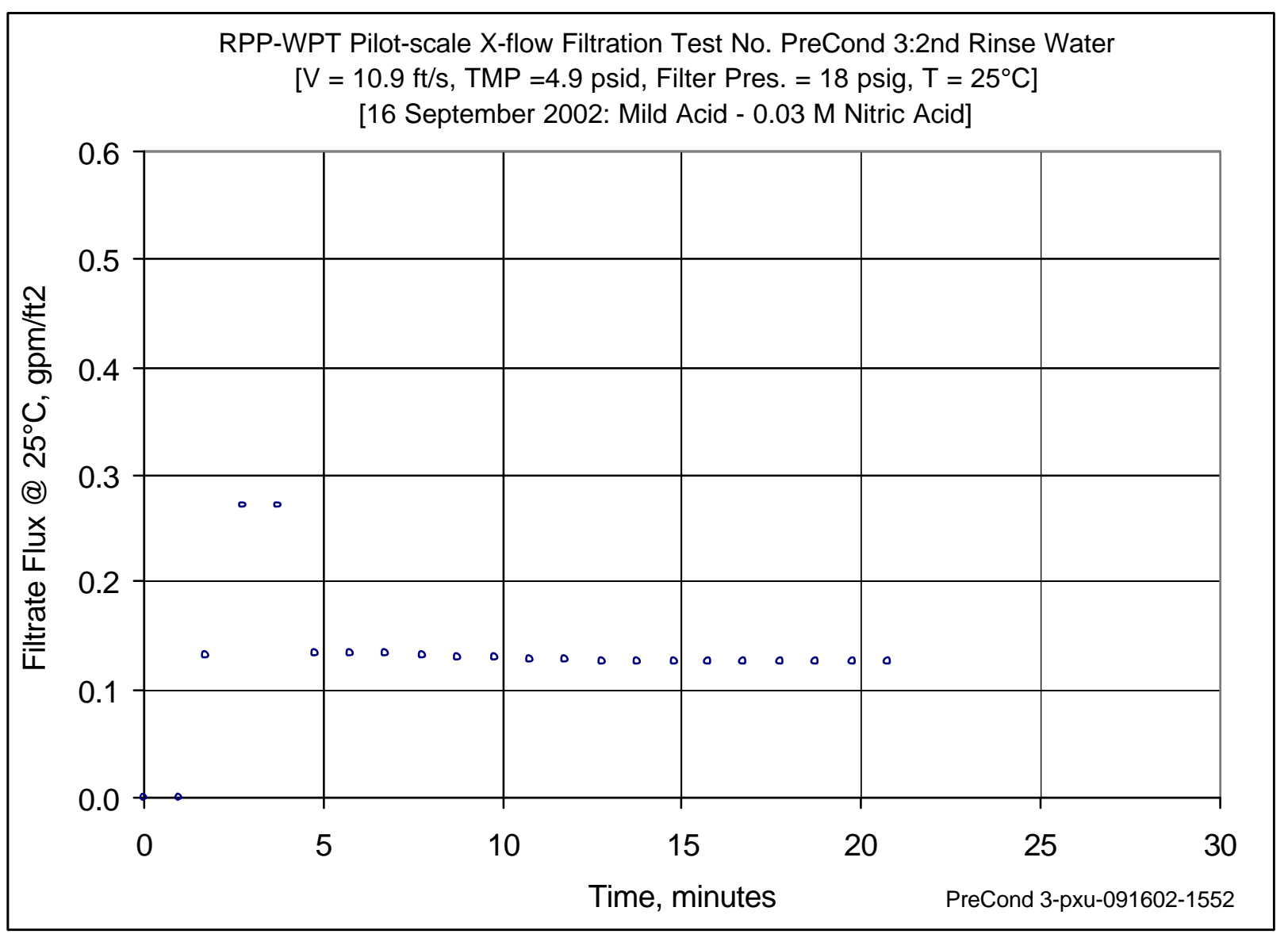

Figure A21: PreConditioning 3 - Deionized \& Filtered Water with 0.03 M Nitric Acid - Post Standard Slurry Tests 
WSRC-TR-2003-00204, REV. 0

SRT-RPP-2003-00087, REV. 0

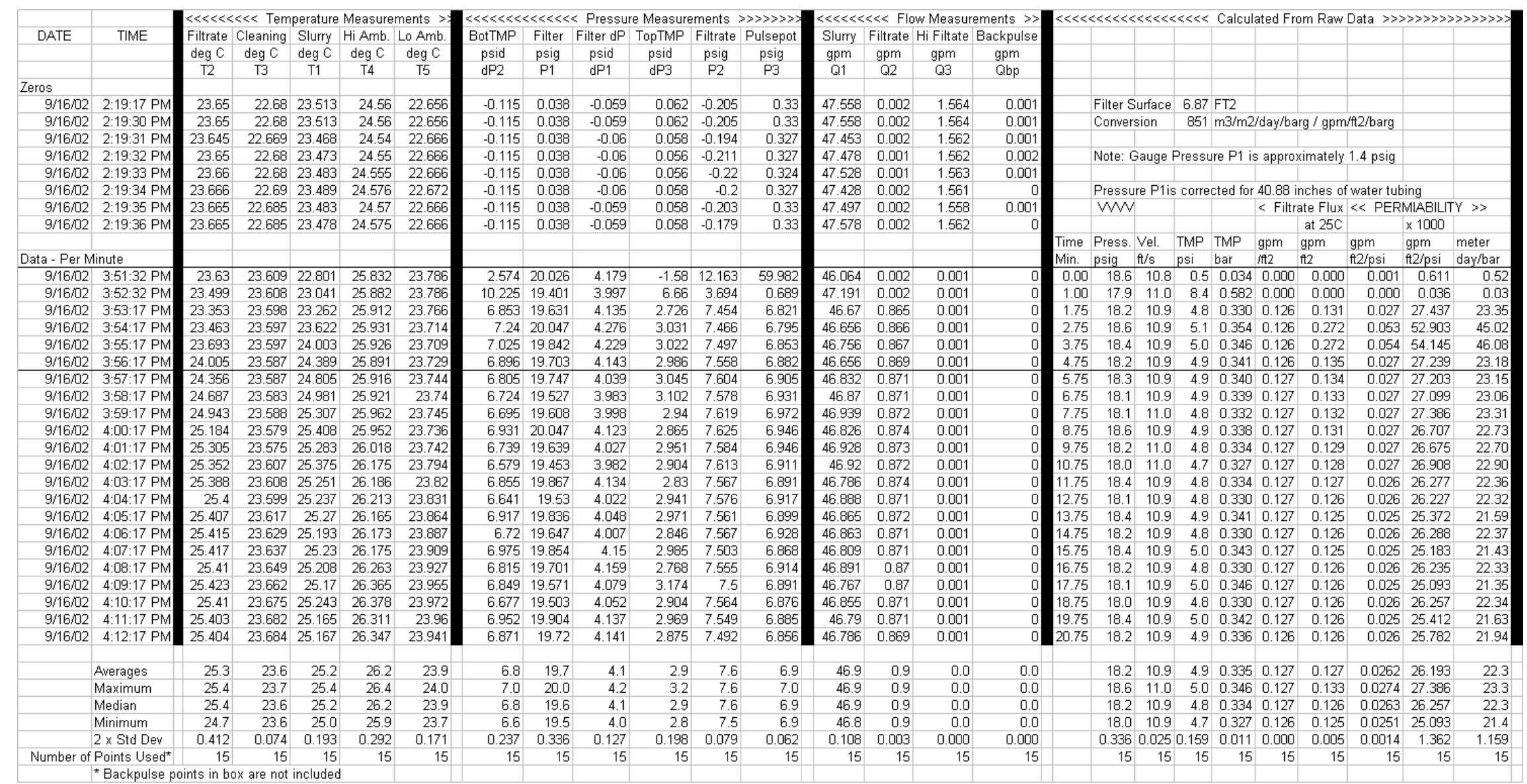

Figure A22: PreConditioning 3 - Deionized \& Filtered Water with 0.03 M Nitric Acid - Post Standard Slurry Tests - Raw \& Calculated Data 
WSRC-TR-2003-00204, REV. 0

SRT-RPP-2003-00087, REV. 0

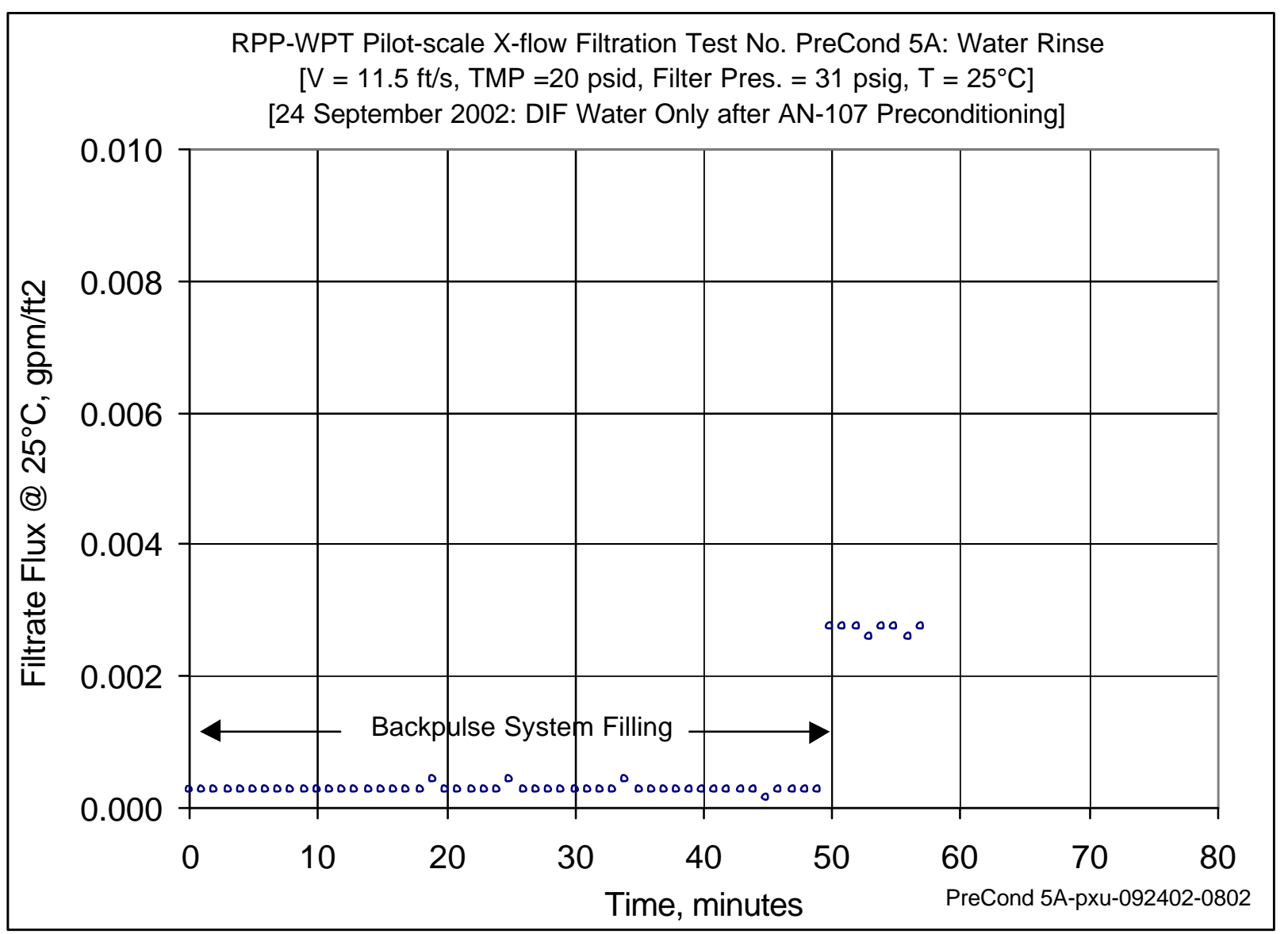

Figure A23: PreConditioning 5A - Deionized \& Filtered Water - Post AN-107 Preconditioning Slurry Test 
WSRC-TR-2003-00204, REV. 0

SRT-RPP-2003-00087, REV. 0

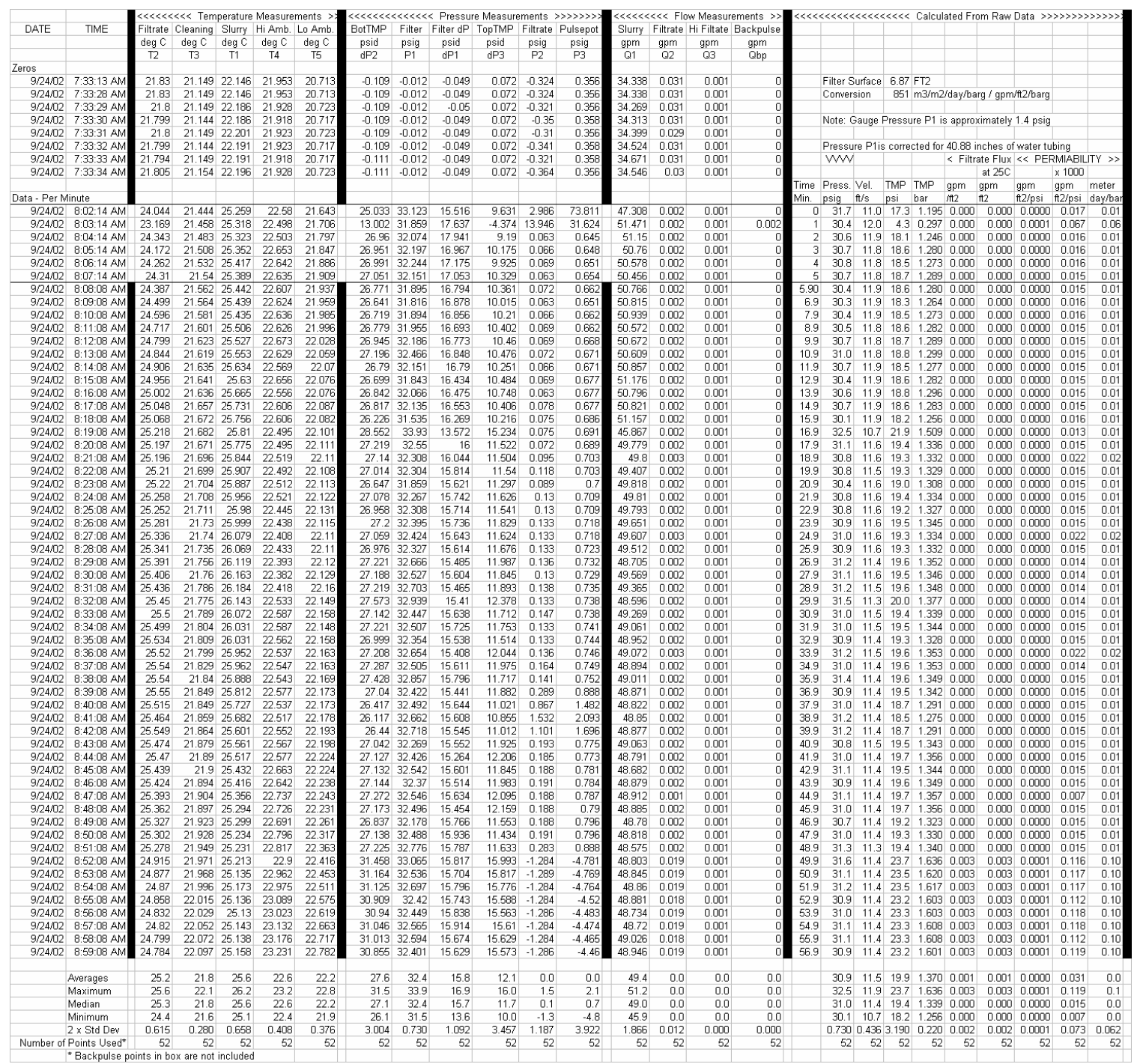

Figure B24: PreConditioning 5A - Deionized \& Filtered Water - Post AN-107 Preconditioning Slurry Test - Raw \& Calculated Data 
WSRC-TR-2003-00204, REV. 0

SRT-RPP-2003-00087, REV. 0

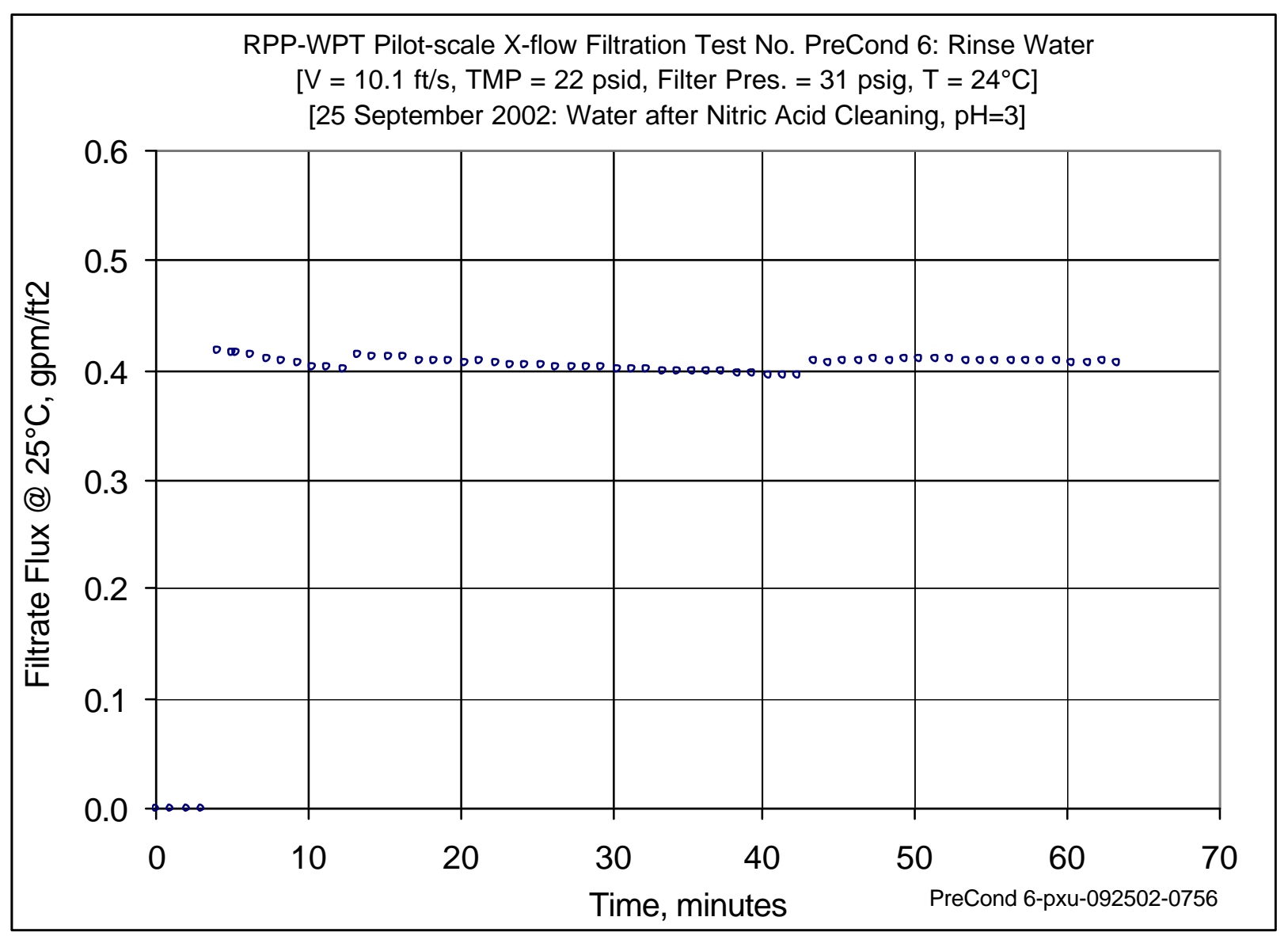

Figure A25: PreConditioning 6 - Deionized \& Filtered Water - Post Nitric Acid Cleaning 
WSRC-TR-2003-00204, REV. 0

SRT-RPP-2003-00087, REV. 0

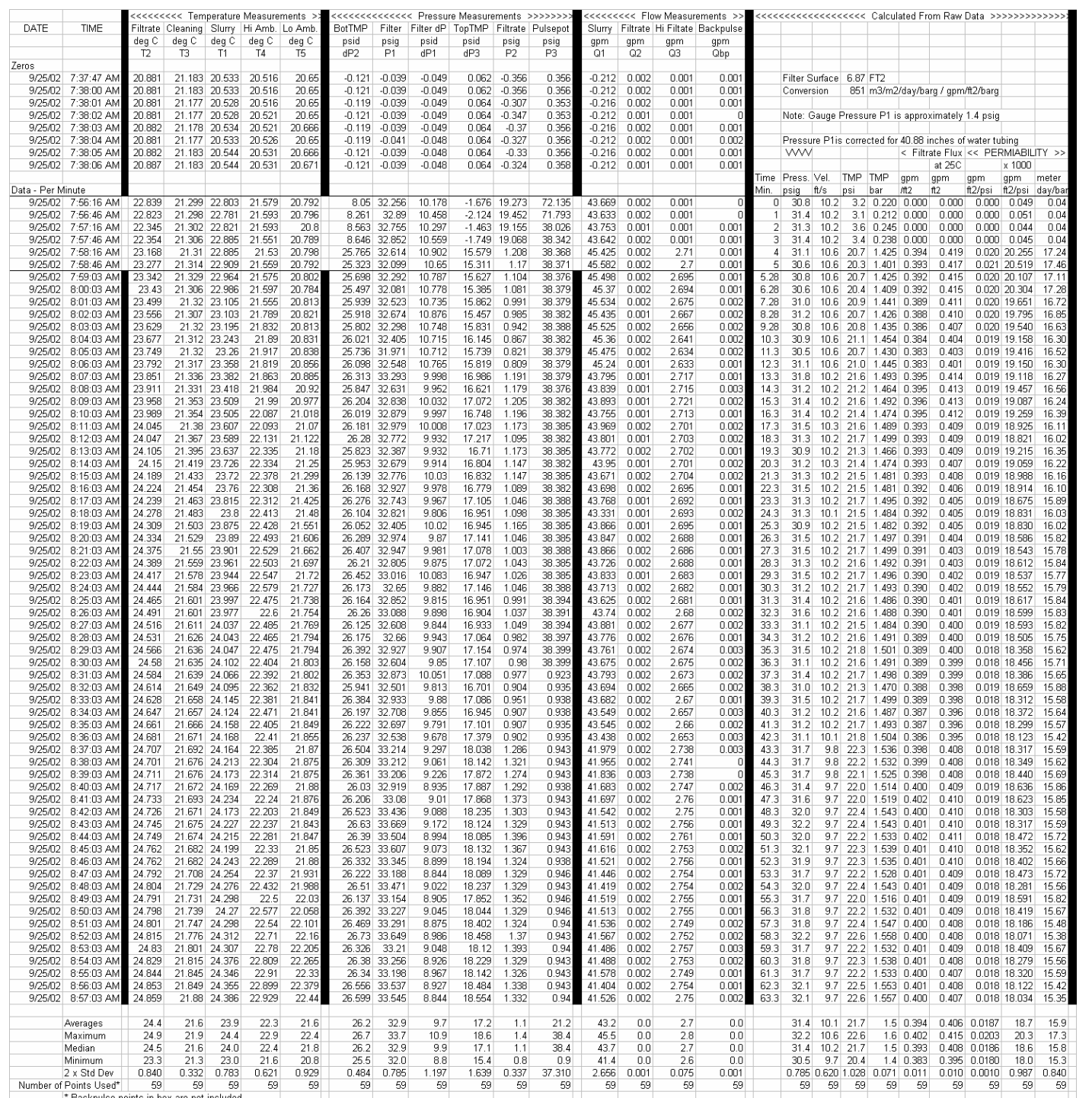

Figure A26: PreConditioning 6 - Deionized \& Filtered Water - Post Nitric Acid Cleaning - Raw \& Calculated Data 
WSRC-TR-2003-00204, REV. 0

SRT-RPP-2003-00087, REV. 0

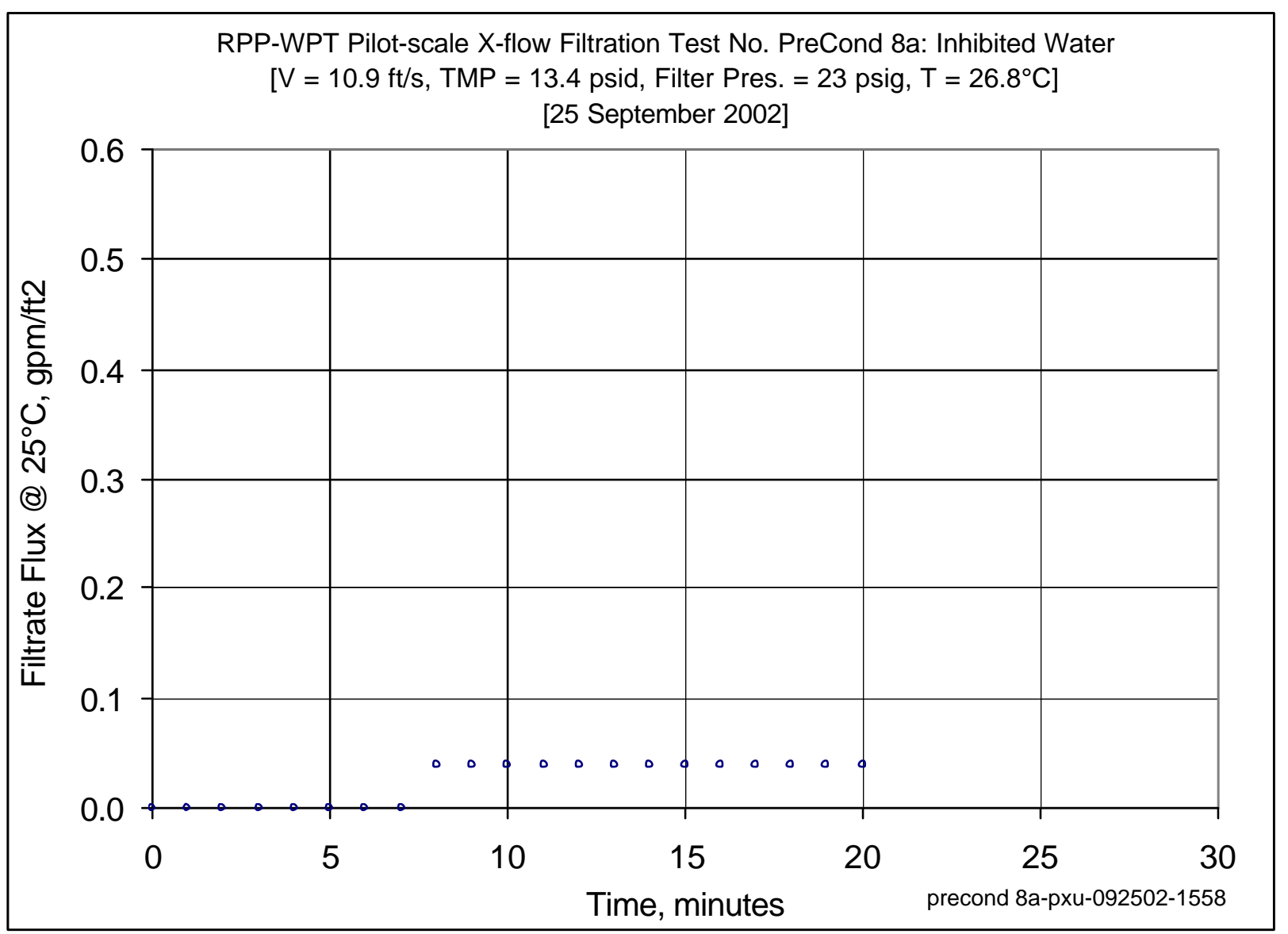

Figure A27: PreConditioning 8A - Deionized \& Filtered Water - Post Standard Slurry Tests 
WSRC-TR-2003-00204, REV. 0

SRT-RPP-2003-00087, REV. 0

\begin{tabular}{|c|c|c|c|c|c|c|c|c|c|c|c|c|c|c|c|c|c|c|c|c|c|c|c|c|c|c|}
\hline DATE & TIME & \multicolumn{5}{|c|}{$\ll \ll \ll \ll \ll<<$ Temperature Measurements $>$} & \multicolumn{6}{|c|}{$\ll<<<<<<<<<<<$ Pressure Measurements $\gg \gg \gg \gg \gg \gg>1$} & \multicolumn{4}{|c|}{$\ll \ll \ll \ll<\ll<$ Flow Measurements $\gg$} & \multicolumn{4}{|c|}{ 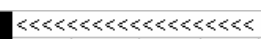 } & \multicolumn{6}{|c|}{ 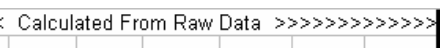 } \\
\hline & & $\operatorname{deg} C$ & $\operatorname{deg} C$ & $\operatorname{deg} C$ & $\operatorname{deg} C$ & $\operatorname{deg} C$ & psid & psig & psid & psid & psig & psig & $\mathrm{gmm}$ & $\mathrm{gpm}$ & $\mathrm{gpm}$ & gpm & & & & & & & & & & \\
\hline & & $\mathrm{T} 2$ & T3 & $\mathrm{T} 1$ & T4 & T5 & $\mathrm{dP2}$ & $\mathrm{P} 1$ & $\mathrm{dP1}$ & $\mathrm{dP3}$ & P2 & P3 & Q1 & Q2 & Q3 & Qbp & & & & & & & & & & \\
\hline \multicolumn{27}{|l|}{ Zeros } \\
\hline $9 / 25 / 02$ & 37:47 AM & 20.881 & 21.183 & 20.533 & 20.516 & 20.65 & -0.121 & -0.039 & -0.049 & 0.062 & -0.356 & 0.356 & -0.212 & 0.002 & 0.001 & 0.001 & & \multicolumn{2}{|c|}{ Filter Surface } & $6.87 \mid$ & \multirow{2}{*}{\multicolumn{5}{|c|}{$\begin{array}{l}7 \mathrm{FT} 2 \\
1 \mathrm{~m} 3 / \mathrm{m} 2 / \text { day/barg } / \mathrm{gpm} / \mathrm{f} 2 / \text { barg }\end{array}$}} & \\
\hline $9 / 25 / 02$ & 7:38:00 AM & 20.881 & 21.183 & 20.533 & 20.516 & 20.65 & -0.121 & -0.039 & -0.049 & 0.062 & -0.356 & 0.356 & -0.212 & 0.002 & 0.001 & 0.001 & & \multicolumn{2}{|c|}{ Conversion } & $851 !$ & & & & & & \\
\hline $9 / 25 / 02$ & 7:38:01 AM & 20.881 & 21.177 & 20.528 & 20.516 & 20.65 & -0.119 & -0.039 & -0.049 & 0.064 & -0.307 & 0.353 & -0.216 & 0.002 & 0.001 & 0.001 & & \multirow{2}{*}{\multicolumn{2}{|c|}{ Note: Gauge $\mathrm{F}$}} & & & & & & & \\
\hline $9 / 25 / 02$ & 7:38:02 AM & 20.881 & 21.177 & 20.528 & 20.521 & 20.65 & -0.121 & -0.039 & -0.049 & 0.064 & -0.347 & 0.353 & -0.212 & 0.001 & 0.001 & & & & & Pressur & ure $P 1$ is & is approx & oximately & $1.4 \mathrm{psig}$ & & \\
\hline $9 / 25 / 02$ & 7:38:03 AM & 20.882 & 21.178 & 20.534 & 20.521 & 20.666 & -0.119 & -0.039 & -0.049 & 0.064 & -0.37 & 0.356 & -0.216 & 0.002 & 0.001 & 0.001 & & & & & & & & & & \\
\hline $9 / 25 / 02$ & $7: 38: 04$ AM & 20.881 & 21.177 & 20.533 & 20.526 & 20.65 & -0.119 & -0.041 & -0.048 & 0.064 & -0.327 & 0.356 & -0.212 & 0.002 & 0.001 & 0.002 & & \multicolumn{8}{|c|}{ Pressure $\mathrm{P} 1$ is corrected for 40.88 inches of water tubing } & \\
\hline $9 / 25 / 02$ & $7: 38: 05 \mathrm{AM}$ & 20.882 & 21.183 & 20.544 & 20.531 & 20.666 & -0.121 & -0.039 & -0.048 & 0.064 & -0.33 & 0.356 & -0.216 & 0.002 & 0.001 & 0.001 & & \multicolumn{2}{|c|}{$\mathrm{WW}$} & & & $<$ Filtr & rate Flux & $\ll$ \&EF & EMMIABIL & ILITY \\
\hline $9 / 25 / 02$ & 7:38:06 AM & 20.887 & 21.183 & 20.544 & 20.531 & 20.671 & -0.121 & -0.039 & -0.048 & 0.064 & -0.324 & 0.358 & -0.212 & 0.001 & 0.001 & 0.001 & & & & & & & at $25 \mathrm{C}$ & & $\times 1000$ & \\
\hline & & & & & & & & & & & & & & & & & Time & Press. & 5. Vel. & TMP - & TMP & $\mathrm{gpm}$ & gpm & gpm & $\mathrm{gpm}$ & meter \\
\hline ta - Per M & Minute & & & & & & & & & & & & & & & & Min. & psig & $\mathrm{f} / \mathrm{s}$ & psi & bar & ift2 & $\mathrm{ft} 2$ & $\mathrm{f} 2 / \mathrm{psi}$ & $\mathrm{f} 2 / \mathrm{psi}$ & day/ban \\
\hline $9 / 25 / 02$ & 3:58:23 PM & 26.38 & 22.614 & 26.641 & 23.592 & 23.017 & 15.869 & 24.464 & 19.034 & -2.808 & 3.353 & 64.172 & 46.647 & 0.002 & 0.001 & & 0 & 23.0 & $0 \quad 10.9$ & 6.5 & 0.450 & 0.000 & 0.000 & 0.000 & 0.043 & $\begin{array}{ll}30.04 \\
\end{array}$ \\
\hline $9 / 25 / 02$ & 3:59:23 PM & 25.933 & 22.613 & 26.685 & 23.475 & 23.021 & 19.118 & 24.478 & 18.9 & 0.3 & 0.058 & 0.558 & 46.631 & 0.003 & & & 1 & 23.0 & $\begin{array}{ll}0 & 10.9\end{array}$ & $\begin{array}{l}9.7 \\
9\end{array}$ & 0.669 & 0.000 & 0.000 & 0.000 & 0.043 & 0.04 \\
\hline $9 / 25 / 02$ & 4:00:23 PM & 26.367 & 22.611 & 26.704 & 23.524 & 22.965 & 19.151 & 24.567 & 19.0 & 0.361 & 0.052 & 0.57 & 46.844 & 0.002 & 01 & & 2 & 23.1 & $\begin{array}{ll}1 & 10.9\end{array}$ & 9.8 & 0.673 & 0.000 & 0.000 & 0.000 & 28 & 0.02 \\
\hline $9 / 25 / 02$ & 4:01:23 PM & 26.681 & 22.606 & 26.758 & 23.413 & 22.979 & 18.979 & 24.356 & 18.729 & 0.41 & 0.066 & 0.587 & 46.704 & 0.001 & 01 & & 3 & 22.9 & $\begin{array}{ll}9 & 10.9\end{array}$ & $\begin{array}{l}9.7 \\
9\end{array}$ & 0.668 & 0.000 & 0.000 & 0.000 & 0.014 & 0.01 \\
\hline $9 / 25 / 02$ & 4:02:23 PM & 26.765 & 22.61 & 26.782 & 23.472 & 22.968 & 18.857 & 24.294 & 18 & 0.359 & 0.092 & 0. & 46.842 & 0.002 & 0. & & 4 & 22.8 & $\begin{array}{ll}8 & 10.9\end{array}$ & 9.6 & 0.662 & 0.000 & 0.000 & 0.000 & 0.029 & 0.02 \\
\hline $9 / 25 / 02$ & 4:03:23 PM & 26.851 & 22.621 & 26.848 & 23.524 & 23.04 & 18.901 & 24.435 & 18.6 & 0.466 & 0. & 0.657 & 46.874 & & & & 5 & 23.0 & $\begin{array}{l}0 \\
0\end{array}$ & 9.7 & & 0.000 & & 0.000 & & \\
\hline $9 / 25 / 02$ & 4:04:23 PM & \begin{tabular}{|l|}
26.867 \\
\end{tabular} & 22.637 & 26.894 & 23.49 & 23.061 & 18.638 & 24.13 & 18. & 0. & 0.1 & 0.697 & 46.888 & 0.0 & 0.0 & & 6.00 & 22.7 & \begin{tabular}{|l|l|}
7 & 10.9 \\
\end{tabular} & 9.5 & 0.652 & 0.000 & 0.000 & 0.000 & 0.029 & 0.02 \\
\hline $9 / 25 / 02$ & 4:05:23 PM & 26.914 & 22.643 & 26.93 & 23.651 & 23.102 & 5 & 24.555 & 19 & & 0.188 & 0.7 & & & & & 7 & 23.1 & $\begin{array}{ll}1 & 10.9\end{array}$ & 9.7 & & & & & & ( \\
\hline $9 / 25 / 02$ & 4:06:23 PM & 26.867 & 22.646 & 26.903 & 23.81 & 23.16 & 22.871 & 24.583 & & 3.763 & -1.284 & -3.3 & 46 & 0.2 & & & 8 & 23.1 & $\begin{array}{ll}1 & 10.9\end{array}$ & 13.3 & & & & 0.003 & & 2.55 \\
\hline $9 / 25 / 02$ & 4:07:23 PM & 27.04 & 22.67 & 26.862 & 23.999 & 23.204 & 22.565 & 24.573 & 18. & 3.87 & -1.2 & -2.98 & 46.748 & 0.2 & 0. & & 9 & 23.1 & $\begin{array}{ll}1 & 10.9\end{array}$ & 13.2 & 0.911 & 0.041 & \begin{tabular}{|l|l|}
1 & 0.039
\end{tabular} & 0.003 & 2.947 & 2.51 \\
\hline $9 / 25 / 02$ & 4:08:23 PM & 27.078 & 22.688 & 26.86 & 23.972 & 23.222 & & 24.246 & & 4.017 & -1.284 & -3. & 46.652 & 0. & & & 10 & 22.8 & $\begin{array}{ll}8 & 10.9\end{array}$ & 13.2 & 0. & 0.041 & & 0.003 & 2.964 & 2.52 \\
\hline $9 / 25 / 02$ & 4:09:23 PM & 27.082 & 22.702 & 26.829 & 24.011 & 23.272 & 22. & 24.362 & & & -1.2. & & & & & & 11 & 22.9 & $\begin{array}{ll}9 & 10.9\end{array}$ & 13.2 & & 0.041 & $\begin{array}{ll}1 & 0.039\end{array}$ & \begin{tabular}{|l|} 
\\
0.003 \\
\end{tabular} & 2.973 & 2.53 \\
\hline $9 / 25 / 02$ & 4:10:23 PM & 27.092 & 22.717 & 26.799 & 24.167 & 23.327 & 22.445 & 24.29 & 18. & 3.968 & -1.2 & -3.0 & 46.773 & 0.2 & 0. & & 12 & 22.8 & \begin{tabular}{ll|}
8 & 10.9
\end{tabular} & 13.2 & 0.911 & 0.041 & 139 & 0.003 & 2. & 2.51 \\
\hline $9 / 25 / 02$ & 4:11:23 PM & 27.106 & 22.747 & 26.808 & 24.1 & 23.376 & & 24.755 & & & $-1.2 .2 x+y$ & & 46. & & & & 13 & 23.3 & $\begin{array}{ll}3 & 10.9 \\
\end{array}$ & 13.4 & & & & & & 2.50 \\
\hline $9 / 25 / 02$ & 4:12:23 PM & 27.091 & 22.767 & 26.774 & 24.166 & 23.427 & 22. & 24.284 & 18. & & -1.2 & & & & & & 14 & 22.8 & \begin{tabular}{l|l|}
8 & 10.9
\end{tabular} & 13.4 & & & & & & 2.52 \\
\hline $9 / 25 / 12$ & 4:13:23 PM & 27.071 & 22.777 & 26.759 & 24.196 & 23.472 & 22.525 & 24.252 & 18.5 & & -1.2 & -3. & 46.601 & 0.2 & & & 15 & 22.8 & \begin{tabular}{|l|l|}
8 & 10.9
\end{tabular} & 13.4 & 0.921 & $\begin{array}{l}0.042 \\
0.042\end{array}$ & $\begin{array}{ll}2 & 0.040\end{array}$ & 0.003 & 2. & 2.54 \\
\hline $9 / 25 / 02$ & 4:14:23 PM & 27.071 & 22.791 & 26.748 & 24.226 & 23.481 & 22.931 & 24.654 & 19. & & -1. & -3 . & 46.53 & 0. & & & 16 & 23.2 & \begin{tabular}{|l|l|}
2 & 10.9
\end{tabular} & 13.5 & 0.929 & 0.041 & 39 & 0.003 & 2 & 2.49 \\
\hline $9 / 25 / 02$ & 4:15:23 PM & 27.069 & 22.799 & 26.70 & 24.184 & 23.479 & 22.819 & 24.455 & 18.646 & & -1.2 & & 46.324 & 0.2 & & & 17 & 23.0 & $\begin{array}{ll}0 & 10.8\end{array}$ & 13.7 & 0.945 & 0.042 & 20.040 & 0.003 & 2.904 & 2.47 \\
\hline $9 / 25 / 02$ & 4:16:23 PM & 27.051 & 22.807 & 26.708 & 24.156 & 23.451 & 22.896 & 24.561 & 19 & 4.177 & -1.286 & -3. & 46.398 & 0.28 & 0. & & 18 & 23.1 & \begin{tabular}{l|l}
1 & 10.8
\end{tabular} & 13.5 & 0.933 & 0.042 & 20.040 & 0.003 & 2.951 & 2.51 \\
\hline $9 / 25 / 02$ & 4:17:23 PM & 27.033 & 22.809 & 26.665 & 24.052 & 23.448 & 22.83 & 24.575 & 18.96 & 389 & -1.2 & -3.2 & 46.402 & 0.2 & & & 19 & 23.1 & $\begin{array}{ll}1 & 10.8 \\
\end{array}$ & 13.4 & 0.921 & 0.042 & 040 & 0.003 & 3.004 & 2.56 \\
\hline $9 / 25 / 02$ & 4:18:23 PM & 27.018 & 22.824 & 26.675 & 24.092 & 23.428 & 22.664 & 24.252 & 18.685 & 4.22 & -1.284 & -3.2 & 46.414 & 0.288 & 0.001 & & 20 & 22.8 & $\begin{array}{ll}8 & 10.8 \\
\end{array}$ & 13.4 & 0.927 & 0.042 & $\begin{array}{l}20.040 \\
\end{array}$ & 0.003 & 2.975 & 2.53 \\
\hline & & & & & & & & & & & & & & & & & & & & & & & & & & \\
\hline & Averages & 27. & 22 & 26 & & 23 & & & & & & & & & 0 & & & 23.0 & D 10.9 & 13.4 & 0.922 & 0.042 & 10 & 0.0030 & 2.960 & 2.519 \\
\hline & Maximum & 27 & 22 & & & 23 & & & & 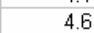 & -1.3 & -3 & 4 & & 0 & 0 & & 23.3 & $\begin{array}{ll}3 & 10.9\end{array}$ & 13.7 & 0.945 & & & 0.0030 & 3.004 & $\begin{array}{ll}4 & 2.557\end{array}$ \\
\hline & Median & 27.1 & 22.8 & 26 & 24.1 & 23.4 & 22.7 & 24.5 & 18 & 4.0 & -1.3 & -3 & 46. & 0.3 & 0.0 & 0 & & 23.0 & D 10.9 & 13.4 & 0.921 & 0.042 & 20.040 & 0.0030 & 2.957 & 2.516 \\
\hline & Minimum & 26.9 & 22.6 & 26.7 & 23 & 23.2 & 22.4 & 24.2 & 18.6 & 3.8 & -1.3 & -3 . & 46.3 & 0.3 & 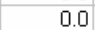 & 0.0 & & 22.8 & $\begin{array}{ll}8 & 10.8 \\
\end{array}$ & 13.2 & 0.911 & 0.041 & $\begin{array}{ll}1 & 0.039 \\
\end{array}$ & 0.0029 & 2.904 & $\begin{array}{l}4 \quad 2.471 \\
\text {. }\end{array}$ \\
\hline & $2 \times$ Std Dev & 0.117 & 0.113 & 0.145 & 0.226 & 0.221 & 0.337 & 0.340 & 0.309 & 0.420 & 0.003 & 0.19 & 0.324 & 0.005 & 0.000 & 0.000 & & 0.340 & 0.076 & 0.285 & 0.020 & 0.001 & 10.001 & 0.0001 & 0.052 & 20.045 \\
\hline of & Points Used ${ }^{*}$ & 13 & 13 & 13 & 13 & 13 & 13 & 13 & 13 & 13 & 13 & 13 & 13 & 13 & 13 & 13 & & 13 & 13 & 13 & 13 & 13 & 13 & 13 & 13 & 13 \\
\hline & 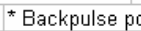 & $\therefore$ & & ed & & & & & & & & & & & & & & & & & & & & & & \\
\hline
\end{tabular}

Figure A28: PreConditioning 8A - Deionized \& Filtered Water - Post Standard Slurry Tests - Raw \& Calculated Data 
WSRC-TR-2003-00204, REV. 0

SRT-RPP-2003-00087, REV. 0

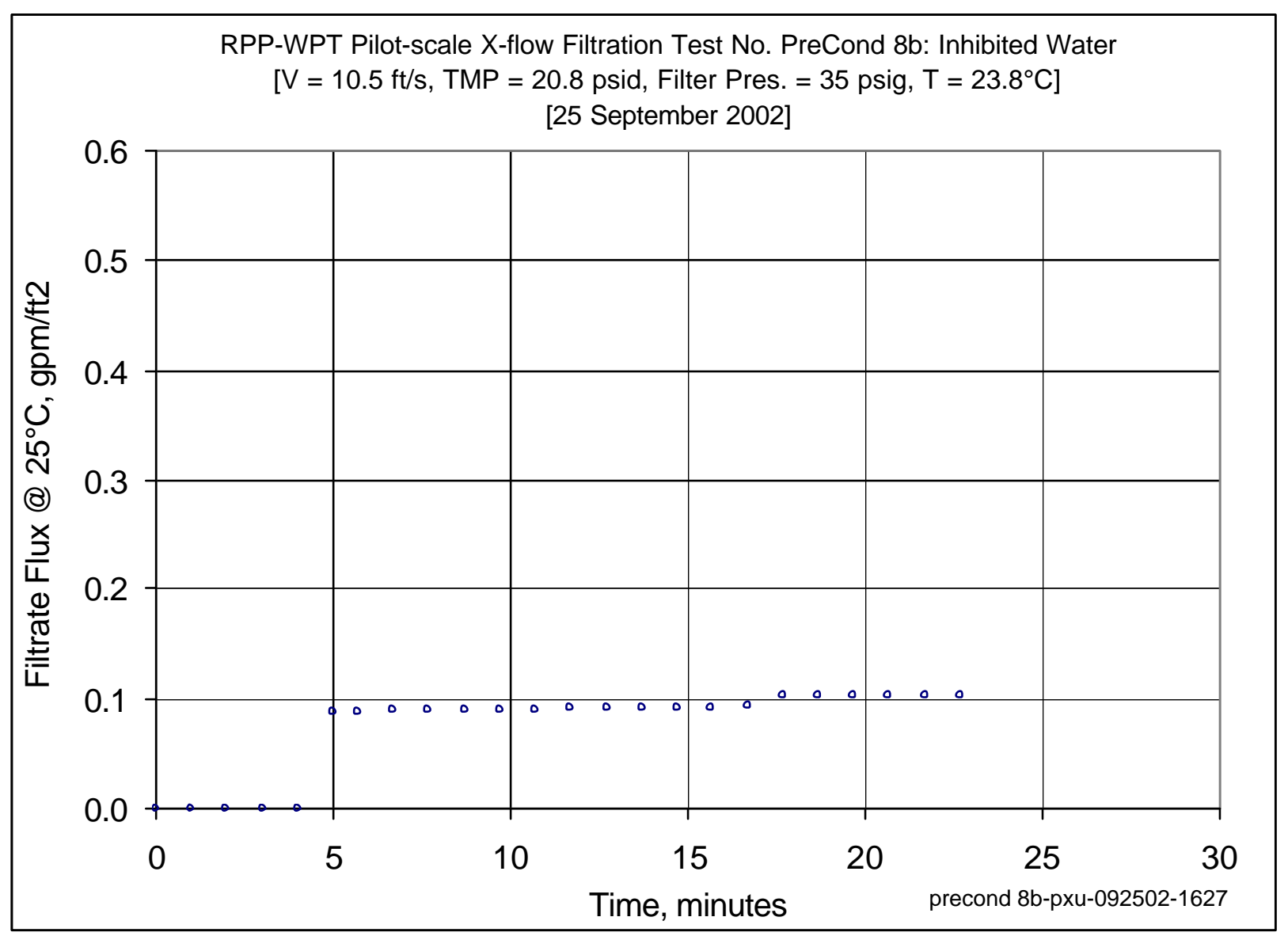

Figure A29: PreConditioning 8B - Deionized \& Filtered Water - Post Standard Slurry Tests 
WSRC-TR-2003-00204, REV. 0

SRT-RPP-2003-00087, REV. 0

\begin{tabular}{|c|c|c|c|c|c|c|c|c|c|c|c|c|c|c|c|c|c|c|c|c|c|c|c|c|c|c|}
\hline DATE & \multirow{2}{*}{ TIME } & \multicolumn{5}{|c|}{$\ll \ll \ll \ll \ll<<$ Temperature Measurements $>$} & \multicolumn{6}{|c|}{ 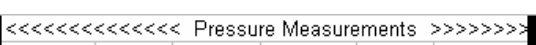 } & \multicolumn{4}{|c|}{ 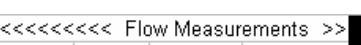 } & \multicolumn{4}{|c|}{ 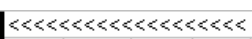 } & \multicolumn{6}{|c|}{ 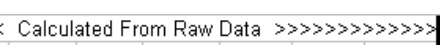 } \\
\hline & & $\operatorname{deg} C$ & $\operatorname{deg} C$ & $\operatorname{deg} C$ & $\operatorname{deg} C$ & $\operatorname{deg} C$ & psid & psig & psid & psid & psig & psig & $\mathrm{gpm}$ & $\mathrm{gpm}$ & $\mathrm{gpm}$ & gpm & & & & & & & & & & \\
\hline & & $\mathrm{T} 2$ & T3 & $\mathrm{T} 1$ & T4 & 15 & $\mathrm{dP} 2$ & P1 & $\mathrm{dP} 1$ & $\mathrm{dP3}$ & P2 & $\mathrm{P} 3$ & Q1 & $\mathrm{Q} 2$ & Q3 & Qbp & & & & & & & & & & \\
\hline \multicolumn{27}{|c|}{\begin{tabular}{l|l} 
Zeros & \\
905002 & $7.37 .47 . A M$ \\
\end{tabular}} \\
\hline $9 / 25 / 02$ & 7:37:47 AM & 20.881 & 21.183 & 20.533 & 20.516 & 20.65 & -0.121 & -0.039 & -0.049 & 0.062 & -0.356 & 0.356 & -0.212 & 0.002 & 0.001 & 0.001 & & \multicolumn{3}{|c|}{ Filter Surface } & FT2 & & & & & \\
\hline $9 / 25 / 02$ & $7: 38: 00 \mathrm{AM}$ & 20.881 & 21.183 & 20.533 & 20.516 & 20.65 & -0.121 & -0.039 & -0.049 & 0.062 & -0.356 & 0.356 & -0.212 & 0.002 & 0.001 & 0.001 & & \multicolumn{2}{|c|}{ Conversion } & $851 !$ & \multicolumn{5}{|c|}{$1 \mathrm{~m} 3 / \mathrm{m} 2 /$ day $/ \mathrm{barg} / \mathrm{gpm} / \mathrm{ta} 2 / \mathrm{barg}$} & \\
\hline $9 / 25 / 02$ & 7:38:01 AM & 20.881 & 21.177 & 20.528 & 20.516 & 20.65 & -0.119 & -0.039 & -0.049 & 0.064 & -0.307 & 0.353 & -0.216 & 0.002 & 0.001 & 0.001 & & & & & & & & & & \\
\hline $9 / 25 / 02$ & $7: 38: 02 \mathrm{AM}$ & 20.881 & 21.177 & 20.528 & 20.521 & 20.65 & -0.121 & -0.039 & -0.049 & 0.064 & -0.347 & 0.353 & -0.212 & 0.001 & 0.001 & & & \multicolumn{8}{|c|}{ approximately $1.4 \mathrm{psi}$} & \\
\hline $9 / 25 / 02$ & $7: 38: 03 \mathrm{AM}$ & 20.882 & 21.178 & 20.534 & 20.521 & 20.666 & -0.119 & -0.039 & -0.049 & 0.064 & -0.37 & 0.356 & -0.216 & 0.002 & 0.001 & 0.001 & & \multirow{2}{*}{\multicolumn{8}{|c|}{ Pressure $\mathrm{P} 1$ is corrected for 40.88 inches of wate }} & \\
\hline $9 / 25 / 02$ & 7:38:04 AM & 20.881 & 21.177 & 20.533 & 20.526 & 20.65 & -0.119 & -0.041 & -0.048 & 0.064 & -0.327 & 0.356 & -0.212 & 0.002 & 0.001 & 0.002 & & & & & & & & & & \\
\hline $9 / 25 / 02$ & $7: 38: 05 \mathrm{AM}$ & 20.882 & 21.183 & 20.544 & 20.531 & 20.666 & -0.121 & -0.039 & -0.048 & 0.064 & -0.33 & 0.356 & -0.216 & 0.002 & 0.001 & 0.001 & & WN & & & & $<$ Filtra & rate Flux & $\ll$ PEF & EMMABIL & ILITY \\
\hline $9 / 25 / 02$ & 7:38:06 AM & 20.887 & 21.183 & 20.544 & 20.531 & 20.671 & -0.121 & -0.039 & -0.048 & 0.064 & -0.324 & 0.358 & -0.212 & 0.001 & 0.001 & 0.001 & & & & & & & at $25 \mathrm{C}$ & & $\times 1000$ & \\
\hline & & & & & & & & & & & & & & & & & Time & Press. & Vel. & TMP & TMP & $\mathrm{gpm}$ & $\mathrm{gpm}$ & $\mathrm{gpm}$ & $\mathrm{gpm}$ & $\mathrm{me}$ \\
\hline ta - Per M & Minute & & & & & & & & & & & & & & & & Min. & & $\mathrm{f} / \mathrm{s}$ & psi & bar & int2 & $\mathrm{ft} 2$ & $\mathrm{fl} 2 / \mathrm{psi}$ & $\mathrm{ft} 2 / \mathrm{psi}$ & day/ban \\
\hline $9 / 25 / 02$ & $4: 27: 41 \mathrm{PM}$ & 27.094 & 22.88 & 25.492 & 23.973 & 23.339 & 16.25 & 35.8 & 18.783 & -2.022 & 14.645 & 76.747 & 46.221 & 0.002 & 0.001 & & 0 & 34.3 & 10.8 & 7.1 & 0.490 & 0.000 & 0.000 & 0.000 & 0.040 & 0.03 \\
\hline $9 / 25 / 02$ & 4:28:11 PM & 26.719 & 22.889 & 25.376 & 23.987 & 23.328 & 11.202 & 35.887 & 18.261 & -6.759 & 27.016 & 36.682 & 45.295 & 0.002 & 0.001 & 1.712 & 1 & $\begin{array}{ll}1 & 34.4 \\
\end{array}$ & 10.6 & 2.2 & 0.153 & 0.000 & 0.000 & 0.000 & 0.130 & 0.11 \\
\hline $9 / 25 / 02$ & 4:28:41 PM & 26.698 & 22.889 & 25.255 & 24.037 & 23.307 & 29.998 & 35.987 & 18.512 & 11.782 & 0.722 & 0.622 & 46.524 & 0.002 & & & 2 & $2 \quad 34.5$ & 10.9 & 20.9 & 1.440 & 0.000 & 0.000 & 0.000 & 14 & 0.01 \\
\hline $9 / 25 / 02$ & 4:29:11 PM & 26.743 & 22.889 & 25.135 & 24.007 & 23.332 & 29.874 & 35.763 & 18.582 & 11.686 & 0.719 & 0.668 & 46.591 & 0.002 & & & 3 & \begin{tabular}{|l|l|}
3 & 34.3
\end{tabular} & 10.9 & 20.8 & 1.433 & 0.000 & 0.000 & 0.000 & 14 & 0011 \\
\hline $9 / 25 / 02$ & 4:29:41 PM & 26.682 & 22.893 & 25.034 & 24.001 & 23.331 & 29.7 & 35.819 & 18.69 & 11.15 & 0.8 & & 46.475 & 0.0 & & & 4 & $\begin{array}{ll}4 & 34.3 \\
\end{array}$ & 10.8 & 20.4 & & & & 0.000 & & 0.01 \\
\hline $9 / 25 / 02$ & $4: 30: 11 \mathrm{PM}$ & 26.547 & 22.893 & 24.939 & 24.096 & 23.321 & 29.331 & 35.734 & 18.488 & 11.248 & 1.173 & 1.117 & 46.572 & 0.61 & 0.001 & & 5 & \begin{tabular}{|l|l|}
5 & 34.3 \\
\end{tabular} & 10.9 & 20.3 & 1.399 & 0.089 & $\begin{array}{l}9 \\
9\end{array}$ & 0.004 & $\begin{array}{ll}4 & 4.382 \\
\end{array}$ & 3.73 \\
\hline $9 / 25 / 02$ & $4: 30: 52 \mathrm{PM}$ & 26.362 & 22.903 & 24.773 & 24.076 & 23.321 & 29.71 & 35.964 & 18.871 & 11.149 & 1.162 & 1.082 & 46.589 & 0.606 & & & 5.68 & \begin{tabular}{|l|l|}
3 & 34.5 \\
\end{tabular} & 10.9 & 20.4 & 1.409 & 0.088 & \begin{tabular}{|l|l|}
3 & 0.089 \\
\end{tabular} & 0.004 & \begin{tabular}{|l|l|}
4 & 4.344 \\
\end{tabular} & 3.70 \\
\hline $9 / 25 / 02$ & $4: 31: 52 \mathrm{PM}$ & 26.096 & 22.897 & 24.577 & 24 & 23.305 & 29.677 & 36.067 & 18.8 & 11.28 & 1.141 & 1.068 & $\begin{array}{l}46.444 \\
46.0\end{array}$ & 0.609 & & & 6.68 & $\begin{array}{ll}3 & 34.6\end{array}$ & 10.8 & 20.5 & 1.412 & 0.089 & $\begin{array}{l}9 \\
9\end{array}$ & 0.004 & & 3.73 \\
\hline $9 / 25 / 02$ & $4: 32: 52 \mathrm{PM}$ & 25.911 & 22.901 & 24.441 & 24.05 & 23.3 & 29.244 & 35.587 & 18.623 & 10.98 & 1.098 & 1.059 & 46.456 & 0.60 & 0. & & 7.68 & \begin{tabular}{|l|l|} 
& 34.1
\end{tabular} & 10.8 & 20.1 & 1.387 & 0.088 & \begin{tabular}{|l|l|}
3 & 0.089 \\
\end{tabular} & 0.004 & \begin{tabular}{|l|l|}
4 & 4.447
\end{tabular} & 3.78 \\
\hline $9 / 25 / 02$ & $4: 33: 52 \mathrm{PM}$ & 25.705 & 22.901 & 24.255 & 24.059 & 23.264 & 29.822 & 36.239 & 18 & 11.457 & 1.0 & 1.033 & 46.381 & 0. & & & 8.68 & $\begin{array}{ll}3 & 34.8 \\
\end{array}$ & 10.8 & 20.6 & 1.423 & 0.088 & \begin{tabular}{l|l|}
3 & 0.090
\end{tabular} & 0.004 & $\begin{array}{l}+4.356 \\
\end{array}$ & 3.71 \\
\hline $9 / 25 / 02$ & $4: 34: 52$ PM & 25.529 & 22.895 & 24.139 & 24.018 & 23.248 & & 35.709 & 18. & & & 0.993 & & & & & 9.68 & $\begin{array}{ll}3 & 34.2 \\
\end{array}$ & 10.8 & 20.4 & 1.406 & 0.088 & \begin{tabular}{|l|l|} 
& 0.090
\end{tabular} & 0.004 & & 3.75 \\
\hline $9 / 25 / 02$ & $4: 35: 52 \mathrm{PM}$ & 25.328 & 22.905 & 23.988 & 23.962 & 23.248 & 29.669 & 35.978 & 18.908 & 11.137 & 1.0 & 1.01 & 46.391 & 0.6 & & & 10.7 & $\begin{array}{ll}7 & 34.5 \\
\end{array}$ & 10.8 & 20.4 & 1.407 & 0.088 & \begin{tabular}{|l|l|}
3 & 0.090
\end{tabular} & 0.004 & 4 & 3.76 \\
\hline $9 / 25 / 02$ & $4: 36: 52 \mathrm{PM}$ & 25.182 & 22.909 & 23.862 & 23.897 & 23.232 & 29.598 & 36.061 & 18. & 6 & 1.2 & 1.137 & 46.047 & 0. & & & 11.7 & $7 \quad 34.6$ & 10.7 & 20.5 & 1.414 & 0.088 & 91 & 0.004 & 54 & 3.79 \\
\hline $9 / 25 / 02$ & $4: 37: 52 \mathrm{PM}$ & 24.996 & 22.8 & 23.696 & 23.926 & 23.216 & 29.575 & 36.135 & & & & 1. & & & & & 12.7 & $\begin{array}{ll}7 & 34.7\end{array}$ & & 20.6 & & & & 0.004 & & 3.81 \\
\hline $9 / 25 / 02$ & 4:38:52 PM & 24.851 & 22.898 & 23.59 & 24.011 & 23.196 & 29.486 & 35.848 & & & 1. & & 45. & & & & 13.7 & $\begin{array}{ll}7 & 34.4\end{array}$ & 10.7 & 20.3 & 1. & & $\begin{array}{ll}3 & 0.092\end{array}$ & 0.005 & & 3.86 \\
\hline $9 / 25 / 02$ & 4:39:52 PM & 24.74 & 22.902 & 23.504 & 24.005 & 23.18 & 29.458 & 35.786 & 18 & 2 & & 1.0 & 45.806 & 0 & & & 14. & $\begin{array}{ll}7 & 34.3\end{array}$ & 10.7 & 20.5 & 1.410 & 0.088 & 992 & 0.004 & $\begin{array}{l}4.498 \\
+\end{array}$ & 3.83 \\
\hline $9 / 25 / 02$ & 4:40:52 PM & 24.594 & 22. & 23.373 & 24 & 23.174 & & 35.771 & & & & 1.0 & & & & & 15 & $\begin{array}{l}7 \quad 34.3 \\
\end{array}$ & 10.7 & 20.5 & & & & 0.005 & & 3.83 \\
\hline $9 / 25 / 02$ & $4: 41: 52 \mathrm{PM}$ & 24.479 & 22.902 & 23.373 & 23.864 & 23.184 & 30.274 & 37.13 & 16.1 & 14.298 & 1.52 & 1. & & & & & 16.7 & $\begin{array}{ll}7 & 35.7\end{array}$ & 10.2 & 22.3 & 1.537 & 0.089 & \begin{tabular}{|l|l|}
9 & 0.093
\end{tabular} & 0.004 & $\begin{array}{r}4.177 \\
+\quad 4.177 \\
\end{array}$ & 3.56 \\
\hline $9 / 25 / 02$ & 4:42:52 PM & 24.368 & 22.912 & 23.383 & 23.779 & 23.184 & 29.037 & 36.967 & 15. & 13.426 & 2.6 & 2.437 & 42.635 & 0 & & & 17 & $7 \quad 35.5$ & 10.0 & 21.2 & 1.464 & 0.098 & 03 & 0.005 & 5 & 4.12 \\
\hline $9 / 25 / 02$ & 4:43:52 PM & 24.272 & 22.896 & 23.358 & & 23.159 & & 36.899 & & & & & & & & & 18 & 35.4 & 9.9 & 21.2 & & & & 0.005 & & 4.16 \\
\hline $9 / 25 / 02$ & 4:44:52 PM & 24.217 & 22.901 & 23.313 & 23.723 & 23.159 & 28.851 & 36.752 & 16.0 & 13.238 & 2.746 & 2.5 & 42.522 & 0.6 & 0. & & 19 & $7 \quad 35.3$ & $\begin{array}{l}3.9 \\
3\end{array}$ & 21.0 & 1.451 & 0.099 & \begin{tabular}{|l|l|}
9 & 0.104
\end{tabular} & 0.005 & \begin{tabular}{|l|l|}
5 & 4.925 \\
\end{tabular} & 4.19 \\
\hline $9 / 25 / 02$ & 4:45:52 PM & 24.161 & 22.885 & 23.307 & 23.692 & 23.1 & 29.134 & 37.16 & & 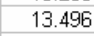 & & & 42. & & & & 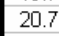 & 35.7 & 9.9 & 21.3 & 1. & 0.099 & 104 & 05 & & 4.14 \\
\hline $9 / 25 / 02$ & $4: 46: 52 \mathrm{PM}$ & 24.1 & & 23.30 & 23. & 23.12 & & 37.033 & 16. & & 2.7 & & 42.624 & & & & 21 & 35.6 & $\begin{array}{l}610.0 \\
\end{array}$ & 21.0 & 1.447 & 0.099 & $\begin{array}{ll}9 & 0.104\end{array}$ & 0.005 & 4.931 & 4.20 \\
\hline $9 / 25 / 02$ & 4:47:52 PM & 24.101 & 22.88 & 23.407 & 23.652 & 23.153 & 29.037 & 37.168 & 15.989 & 13.352 & 2.789 & 2.594 & 42.44 & 0.683 & 0.001 & & 22.7 & $7 \quad 35.7$ & 9.9 & 21.2 & 1.461 & 0.099 & \begin{tabular}{|l|l|}
9 & 0.104
\end{tabular} & 0.005 & \begin{tabular}{|l|} 
\\
\end{tabular} & 4.17 \\
\hline & & & & & & & & & & & & & & & & & & & & & & & & & & \\
\hline & Averag & 24 & 2 & 23.8 & 23 & 23.2 & 29.4 & 36.3 & & & 1. & 1. & & & 0 & 0 & & 34.9 & $\begin{array}{l}9 \\
9\end{array}$ & 20.8 & 1.433 & 0.092 & 5 & 0.0046 & 74 & $\begin{array}{l}4 \quad 3.893 \\
\end{array}$ \\
\hline & Maximum & 26.4 & 22.9 & 24.8 & 24.1 & 23.3 & 30.3 & 37.2 & 18 & 14 & 2.8 & 2. & 46.6 & 0 & 0.0 & 0 & & 35.7 & 10.9 & 22.3 & 1.537 & 0.099 & \begin{tabular}{|l|l|}
9 & 0.104 \\
\end{tabular} & 0.0049 & $\begin{array}{l}9.931 \\
\end{array}$ & $\begin{array}{ll}1 & 4.197\end{array}$ \\
\hline & Medi & 24. & 22 & 23.5 & 23 & 23.2 & 29.5 & 36 & 18 & 11 & 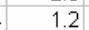 & 1 & 45.8 & 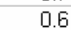 & 0 & 0 & & 34.6 & $\begin{array}{ll}6 & 10.7\end{array}$ & 20.6 & 1.418 & 0.089 & $\begin{array}{ll}9 & 0.092\end{array}$ & 0.0045 & \begin{tabular}{|l|l|}
5 & 4.486
\end{tabular} & $\begin{array}{ll}6 & 3.817\end{array}$ \\
\hline & Mir & 24 & 22 & 23.3 & 23 & 23.1 & 28 & 35.6 & & & 1. & & 42 & 0 & 0 & & & 34.1 & 9.9 & 20.1 & 1.387 & 0.088 & & 0.0042 & 4.177 & 3.5 \\
\hline & $2 \times$ Std Dev & 1.421 & 0.018 & 0.948 & 0.282 & 0.117 & 0.737 & 1.121 & 2.593 & 2.190 & 1.479 & 1.362 & 3.444 & 0.068 & 0.000 & 0.000 & & 1.120 & 0.804 & 1.024 & 0.071 & 0.010 & 0.012 & 0.0005 & \begin{tabular}{|l|l|}
5 & 0.475
\end{tabular} & $\begin{array}{ll}5 & 0.404\end{array}$ \\
\hline$f$ & f Points Used* & 18 & 18 & 18 & 18 & 18 & 18 & 18 & 18 & 18 & 18 & 18 & 18 & 18 & 18 & 18 & & 18 & 18 & 18 & 18 & 18 & 18 & 18 & 18 & 18 \\
\hline & & & & & & & & & & & & & & & & & & & & & & & & & & \\
\hline
\end{tabular}

Figure A30: PreConditioning 8B - Deionized \& Filtered Water - Post Standard Slurry Tests - Raw \& Calculated Data 
WSRC-TR-2003-00204, REV. 0

SRT-RPP-2003-00087, REV. 0

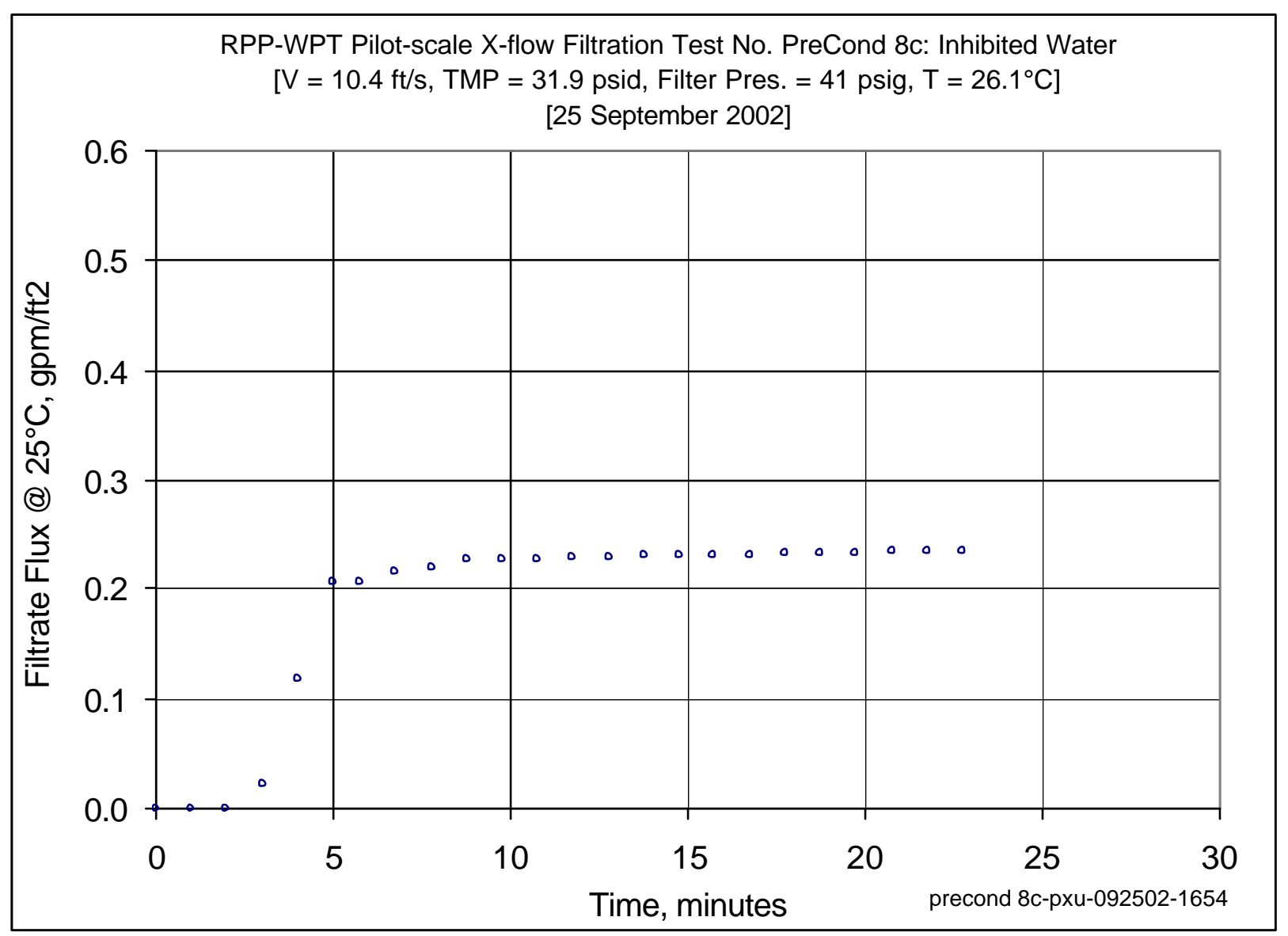

Figure A31: PreConditioning 8C - Deionized \& Filtered Water - Post Standard Slurry Tests 
WSRC-TR-2003-00204, REV. 0

SRT-RPP-2003-00087, REV. 0

\begin{tabular}{|c|c|c|c|c|c|c|c|c|c|c|c|c|c|c|c|c|c|c|c|c|c|c|c|c|c|c|}
\hline \multirow{3}{*}{ DATE } & \multirow{3}{*}{ TIME } & \multicolumn{5}{|c|}{$\ll \ll<\ll<\ll<$ Temperature Measurements $>$} & \multicolumn{6}{|c|}{ 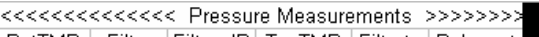 } & \multirow{2}{*}{\multicolumn{4}{|c|}{$\begin{array}{l}2 \ll \ll \ll \ll<\text { Flow Measurements } \gg \\
\text { Slury Filtrate Hi Filtate Backnulse }\end{array}$}} & \multicolumn{4}{|c|}{ 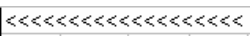 } & \multicolumn{6}{|c|}{ 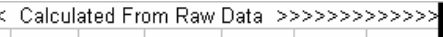 } \\
\hline & & Filtrate & Cleaning & Slurry & Hi Amb. & Lo Amb. & BotTMP & Filter & Filter $\mathrm{dP}$ & TopTMP & Filtrate & Pulsepot & Slurry & Filtrate & & & & & & & & & & & & \\
\hline & & $\operatorname{deg} \mathrm{C}$ & $\operatorname{deg} C^{\circ}$ & $\operatorname{deg} \mathrm{C}$ & $\operatorname{deg} C$ & $\operatorname{deg} C$ & psid & psig & psid & psid & psig & psig & $\mathrm{gpm}$ & gpm & gpm & $\mathrm{gpm}$ & & & & & & & & & & \\
\hline & & $\mathrm{T}$ & $\mathrm{T} 3$ & $T 1$ & T4 & $T 5$ & $\mathrm{dP2}$ & $\mathrm{P} 1$ & $\mathrm{dP1}$ & $\mathrm{dP3}$ & $\mathrm{P} 2$ & $\mathrm{P} 3^{3}$ & Q1 & Q2 & Q3 & Qbp & & & & & & & & & & \\
\hline \multicolumn{27}{|l|}{ Zeros } \\
\hline $9 / 25 / 02$ & 37:47 AM & 20.881 & 21.183 & 20.533 & 20.516 & 20.65 & -0.121 & -0.039 & -0.049 & 0.062 & -0.356 & 0.356 & -0.212 & 0.002 & 0.001 & 0.001 & & \multirow{2}{*}{\multicolumn{2}{|c|}{ Filter Surface }} & 6.87 & \multirow{2}{*}{\multicolumn{5}{|c|}{$\mathrm{m} 3 / \mathrm{m} 2 /$ day $/$ barg $/ \mathrm{gpm} / \mathrm{ft} 2 / \mathrm{barg}$}} & \\
\hline $9 / 25 / 02$ & 88:00 AM & 20.881 & 21.183 & 20.533 & 20.516 & 20.65 & -0.121 & -0.039 & -0.049 & 0.062 & -0.356 & 0.356 & -0.212 & 0.002 & 0.001 & 0.001 & & & & 851 & & & & & & \\
\hline $9 / 25 / 02$ & 7:38:01 AM & 20.881 & 21.177 & 20.528 & 20.516 & 20.65 & -0.119 & -0.039 & -0.049 & 0.064 & -0.307 & 0.353 & -0.216 & 0.002 & 0.001 & 0.001 & & & & & & & & & & \\
\hline $9 / 25 / 02$ & 7:38:02 AM & 20.881 & 21.177 & 20.528 & 20.521 & 20.65 & -0.121 & -0.039 & -0.049 & 0.064 & -0.347 & 0.353 & -0.212 & 0.001 & 0.001 & & & \multicolumn{8}{|c|}{ Note: Gauge Pressure P1 is approximately $1.4 \mathrm{psig}$} & \\
\hline $9 / 25 / 02$ & 7:38:03 AM & 20.882 & 21.178 & 20.534 & 20.521 & 20.666 & -0.119 & -0.039 & -0.049 & 0.064 & -0.37 & 0.356 & -0.216 & 0.002 & 0.001 & 0.001 & & & & & & & & & & \\
\hline $9 / 25 / 02$ & 7:38:04 AM & 20.881 & 21.177 & 20.533 & 20.526 & 20.65 & -0.119 & -0.041 & -0.048 & 0.064 & -0.327 & 0.356 & -0.212 & 0.002 & 0.001 & 0.002 & & Pressur & ure Plis & $s$ cor & ed for & r 40.88 in & inches of $w$ & f water th & tubing & \\
\hline $9 / 25 / 02$ & $7: 38: 05$ AM & 20.882 & 21.183 & 20.544 & 20.531 & 20.666 & -0.121 & -0.039 & -0.048 & 0.064 & -0.33 & 0.356 & -0.216 & 0.002 & 0.001 & 0.001 & & $\mathrm{WW}$ & & & & $<$ Filtra & rate Flux & $\ll P E$ & RMIABIL & \\
\hline $9 / 25 / 02$ & 7:38:06 AM & 20.887 & 21.183 & 20.544 & 20.531 & 20.671 & -0.121 & -0.039 & -0.048 & 0.064 & -0.324 & 0.358 & -0.212 & 0.001 & 0.001 & 0.001 & & & & & & & at $25 \mathrm{C}$ & & $\times 1000$ & \\
\hline & & & & & & & & & & & & & & & & & ne & Press. & Vel. T & TMP & TMP & $\mathrm{gpm}$ & & & gpm & ete \\
\hline a - Per M & Minute & & & & & & & & & & & & & & & & Min. & psig f & $\mathrm{ft} / \mathrm{s} \quad \mathrm{p}$ & psi & bar & ift2 & $\mathrm{f} 2$ & $\mathrm{ft} 2 / \mathrm{psi}$ & $\mathrm{ft} 2 / \mathrm{psi}$ & \\
\hline $9 / 25 / 02$ & 4:54:48 PM & 25.373 & 859 & 25.196 & 571 & .037 & 15.181 & 41.736 & 77 & -3.14 & 21.556 & 269 & 46.01 & 0.002 & 01 & & 0 & 40.3 & \begin{tabular}{|l|l|}
80.7 \\
\end{tabular} & 6.0 & 0.415 & 50.000 & 0.000 & 0.000 & \begin{tabular}{|l|l|}
0 & 0.048 \\
\end{tabular} & 0.04 \\
\hline $9 / 25 / 02$ & 4:55:18 PM & 24.396 & 22.869 & 25.251 & 23.666 & 23.037 & 16.237 & 42.04 & 18.833 & -2.3 & 21.027 & 38.759 & 45.949 & 0.002 & & 0.139 & 1 & 40.6 & $\begin{array}{ll}6 & 10.7\end{array}$ & 7.0 & 0.480 & 0.000 & 0.000 & 0.000 & 041 & 0.04 \\
\hline $9 / 25 / 02$ & 4:55:48 PM & 25.1 & 22.87 & 25 & 23. & 2 & 34.923 & 42.261 & & 6.106 & 1.907 & & 46.798 & & & & 2 & 40.8 & 10.9 & 25.5 & 1.759 & & & 000 & & \\
\hline $9 / 25 / 02$ & 4:56:18 PM & 25.584 & 22.875 & 25.396 & 23.727 & 23.082 & & 41.918 & 18.969 & 41 & 3.769 & 29 & 46.695 & & & & 3 & 40.4 & $4 \quad 10.9$ & 23.2 & 1.598 & 0.024 & 0.023 & 0.001 & 106 & 1.86 \\
\hline $9 / 25 / 02$ & 4:56:48 PM & 25.695 & 22.876 & 25.438 & 23.708 & 23.094 & 30.087 & 41.7 & 18. & 14 & 6.457 & 5.947 & 46.666 & 0.827 & 0.0 & & 4 & 40.2 & 210.9 & 20.9 & 1.441 & 0.120 & & 0.006 & \begin{tabular}{l|l}
6 & 5.689
\end{tabular} & \\
\hline $9 / 25 / 02$ & 4:57:18 PM & 25.826 & 22.882 & 25 & 23.829 & 23.109 & 40.066 & 41.738 & & 21.432 & -1.286 & -4.251 & 46.964 & & & & 5 & 40.3 & 311.0 & 30.7 & & & & & $\begin{array}{ll}7 & 6.724 \\
\end{array}$ & 4 \\
\hline $9 / 25 / 02$ & $4: 58: 03 \mathrm{PM}$ & 25.983 & 22.894 & 25.605 & 23.791 & 23.156 & 40.188 & 41.969 & 19.142 & 21.379 & -1.286 & -4.24 & 47.197 & 0.0 & 1.4 & & 5.75 & 40.5 & \begin{tabular}{|l|l|}
5 & 11.0 \\
\end{tabular} & 30.8 & 2.122 & 20.210 & 0.206 & 0.007 & 697 & 5.70 \\
\hline $9 / 25 / 02$ & 4:59:03 PM & 26.14 & 22.9 & 25.692 & 23.928 & 23.193 & 40.188 & 42.154 & 18. & 22.56 & -1.284 & -4.231 & 45. & & & & 6.75 & 40.7 & 10.7 & 31.4 & 2.163 & 21 & 6 & 0.007 & 00 & 5.87 \\
\hline $9 / 25 / 02$ & 5:00:03 PM & 26.288 & 22.908 & 25.82 & 24.057 & 23.242 & 39.965 & 41.794 & & & -1.286 & -4.231 & 45. & & & & 7.75 & 40.3 & 10.6 & 31.3 & & & & & & 5.97 \\
\hline $9 / 25 / 02$ & 5:01:03 PM & 26.401 & 22.922 & 25.943 & 24.11 & 23.285 & 40.431 & 42.462 & 17.3 & 23.434 & -1.284 & -4.225 & 44.462 & 0. & 1.5 & & 8.75 & 41.0 & 10.4 & 31.9 & 2.202 & & 0.227 & 0.007 & & 6.04 \\
\hline $9 / 25 / 02$ & 5:02:03 PM & 26.545 & 22.962 & 26.038 & 24.165 & 23.335 & 40.499 & 42.609 & & & -1.284 & -4.219 & 44.462 & & $1 . E$ & & 9.75 & 41.1 & $\begin{array}{l}10.4 \\
10\end{array}$ & 32.1 & 2. & & 0.2 & 0.007 & 58 & 0.0 \\
\hline $9 / 25 / 02$ & 5:03:03 PM & 26 . & & & & 23.4 & & 42. & & & -1.2 & & & & & & 10. & 41.2 & \begin{tabular}{l|l}
2 & 10.4 \\
\end{tabular} & 32.3 & & & & & & 6. \\
\hline $9 / 25 / 02$ & 5:04:03 PM & 26. & 22.983 & & & 13,47 & 40. & & & & -1.2 & & & & & & 11.8 & 41.1 & 10.3 & 32.0 & & & & & & 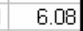 \\
\hline $9 / 25 / 02$ & 5:05:03 PM & 26 & 22.993 & 26 & 24.201 & 23 & 40. & 425 & 17 & & -1.2 & -4.2 & in & 0. & & & 12.8 & 41.1 & $\begin{array}{ll}10.4 \\
1\end{array}$ & 32.1 & 2.210 & 0 & 0.229 & 0.007 & 35 & 6.07 \\
\hline $9 / 25 / 02$ & 5:06:03 PM & & & & & 23 & & & & & & & & & & & 13.8 & 41.2 & $\begin{array}{ll}2 & 10.4\end{array}$ & 32.2 & & & & & & 6.07 \\
\hline $9 / 25 / 02$ & 5:07:03 PM & 26. & 23.0 & 26.227 & & & 40.5 & & & & $-1.2 .2 x+2$ & & & & & & 14.8 & 41.5 & $\begin{array}{ll}5 & 10.4\end{array}$ & 32.2 & & & & & & a \\
\hline $9 / 25 / 02$ & 5:08:03 PM & 26.8 & 23.019 & 26.22 & 2 & 23.5 & 40.354 & 42. & 16. & & -1.2 & -4. & 44.428 & 0.1 & & & 15.8 & 41.3 & \begin{tabular}{l|l}
3 & 10.4
\end{tabular} & 32.1 & & & & 0.007 & & i. \\
\hline $9 / 25 / 02$ & 5:09:03 PM & 26. & & & & & & & & & $-1.2 .2 x+1$ & & 44. & & & & 16 & 40.9 & 3 & 31.7 & & & & & & 6.22 \\
\hline $9 / 25 / 02$ & 5:10:03 PM & 26.8 & 23.043 & 26.164 & 24.26 & 23.5 & 40.325 & & 17. & 23. & -1.286 & & 44 & 0.0 & & & 17.8 & 41.3 & \begin{tabular}{l|l}
3 & 10.4
\end{tabular} & 31.9 & 2.2 & 20 & 0. & 0.007 & $\begin{array}{ll}7 & 7.262\end{array}$ & 6.18 \\
\hline $9 / 25 / 02$ & 5:11:03 PM & 26.892 & 23.054 & 26.19 & 20 & 23.5 & 40.578 & & & & -1.2 & & & & & & 18.8 & 41.4 & $\begin{array}{ll}4 & 10.4\end{array}$ & 32.2 & & & 33 & 07 & & 6.16 \\
\hline $9 / 25 / 02$ & 5:12:03 PM & 26.8 & 23.045 & 26.151 & & & & & & & -1.2 & & & & & & 15 & 41.5 & 4 & & & & & & & 6.22 \\
\hline $9 / 25 / 02$ & 5:13:03 PM & 26.8 & 23.06 & 26 . & 24.12 & 23.5 & 40.437 & 42.753 & 17. & & -1.286 & -4.1 & 44. & & & & 20. & 41.3 & \begin{tabular}{l|l}
3 & 10.4
\end{tabular} & & & 70 & 0.7 & 0.007 & 270 & 6.1 \\
\hline $9 / 25 / 02$ & 5:14:03 PM & 26.873 & 23.05 & 26.161 & 24.097 & 23.528 & 40.389 & 42.7 & 17.161 & 23.653 & -1.286 & -4.1 & 44.391 & 0.002 & 1.659 & & 21.8 & 41.2 & $\begin{array}{ll}2 & 10.4\end{array}$ & 32.0 & 2.208 & 80 & 0.2 & 0.007 & $\begin{array}{ll}7 & 7.297\end{array}$ & 6.21 \\
\hline $9 / 25 / 02$ & 5:15:03 PM & 26.867 & 23.049 & 26.115 & 24.046 & $7 ?$ & 40.097 & 42.383 & 16.969 & 23.659 & -1.284 & -4.17 & 44.258 & 0.002 & 1.658 & & 22. & 40.9 & $\begin{array}{ll}90.3 \\
\end{array}$ & 31.9 & 2.198 & 0.241 & 0.234 & 0.007 & $\begin{array}{ll}7 & 7.335 \\
\end{array}$ & 6.2 \\
\hline & & & & & & & & & & & & & & & & & & & & & & & & & & \\
\hline & & & & & & & & & & & & & & & & & & 1.1 & 10.4 & 31.9 & & 135 & & & 142 & .078 \\
\hline & & & & & & & & & & & & & & & & & & & & & & & & & & \\
\hline & & & & & & & & & & & & & & & 1. & & & 41.2 & $\begin{array}{ll}2 & 10.4 \\
\end{array}$ & 32.0 & & 0.238 & & & 17.143 & \\
\hline & Minimum & 26.0 & 22.9 & 25.6 & 23.8 & 23 & 40.0 & 41 & 16.9 & 2 & -1.3 & -4.2 & 44.2 & 0.0 & 1.4 & & & 40.3 & \begin{tabular}{|l|l|}
3 & 10.3 \\
\end{tabular} & 30.8 & 2.122 & 20.210 & 0.206 & 0.0067 & \begin{tabular}{l|l|}
7 & 6.697
\end{tabular} & 5.699 \\
\hline & $2 x$ & 0.574 & 0.111 & 0.385 & 0.292 & 0.250 & 0.379 & 0.623 & 1.057 & 1.238 & 0.002 & 0.041 & .451 & 0.000 & 113 & 0.00 & & 0.623 & 0.3390 & 0.742 & 0.051 & 0.016 & 0.014 & 0.0003 & 315 & 0.268 \\
\hline & f Points Used & 18 & 18 & & 18 & 18 & 18 & 18 & 18 & 18 & 18 & 18 & 18 & 18 & 18 & & & 18 & 18 & 18 & 18 & 18 & 18 & 18 & 18 & \\
\hline & & & & & & & & & & & & & & & & & & & & & & & & & & \\
\hline
\end{tabular}

Figure A32: PreConditioning 8C - Deionized \& Filtered Water - Post Standard Slurry Tests - Raw \& Calculated Data 
WSRC-TR-2003-00204, REV. 0

SRT-RPP-2003-00087, REV. 0

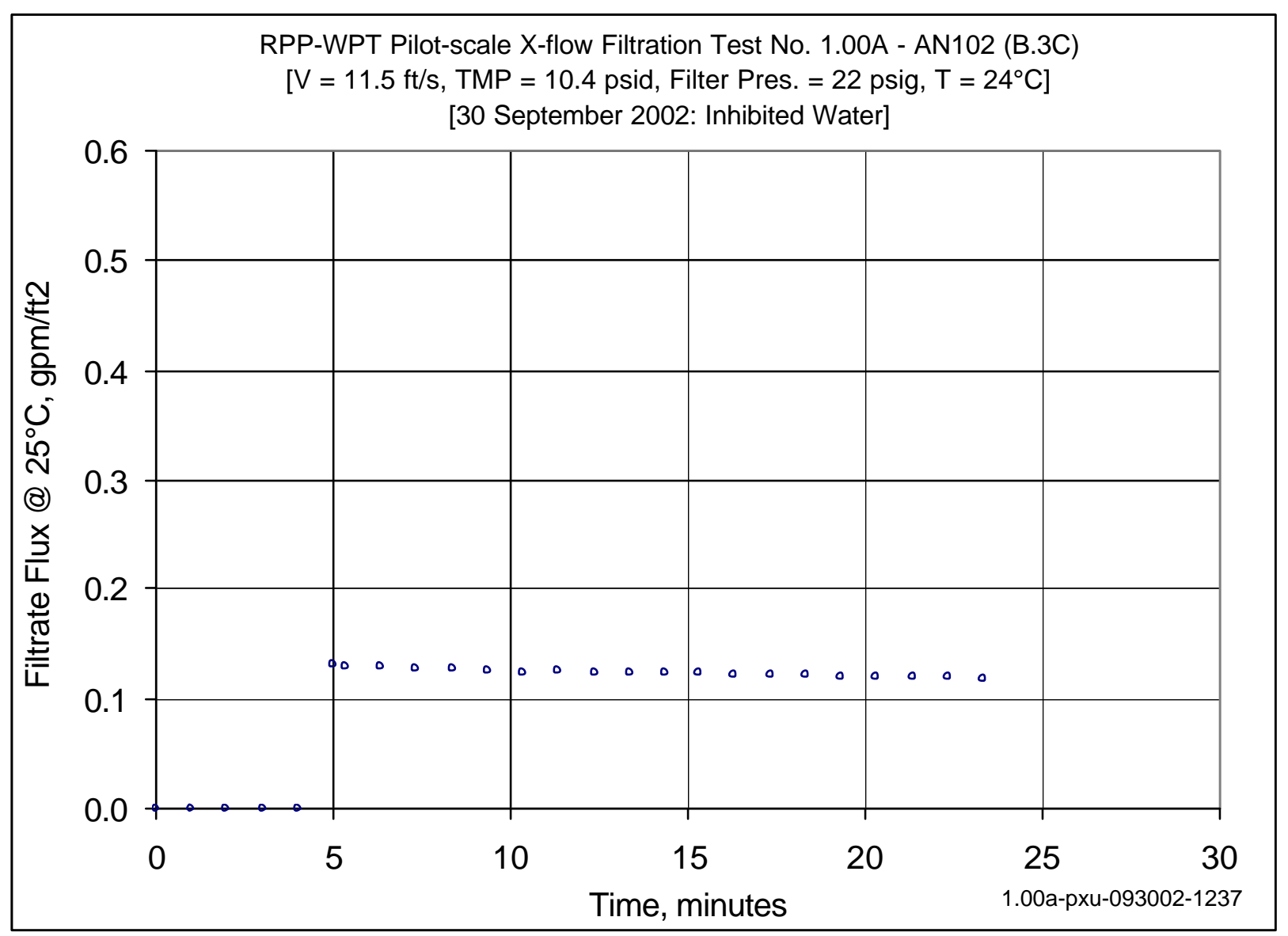

Figure A33: AN-102R2, Batch 3C Slurry Test 1.00A - Inhibited Deionized \& Filtered Water 
WSRC-TR-2003-00204, REV. 0

SRT-RPP-2003-00087, REV. 0

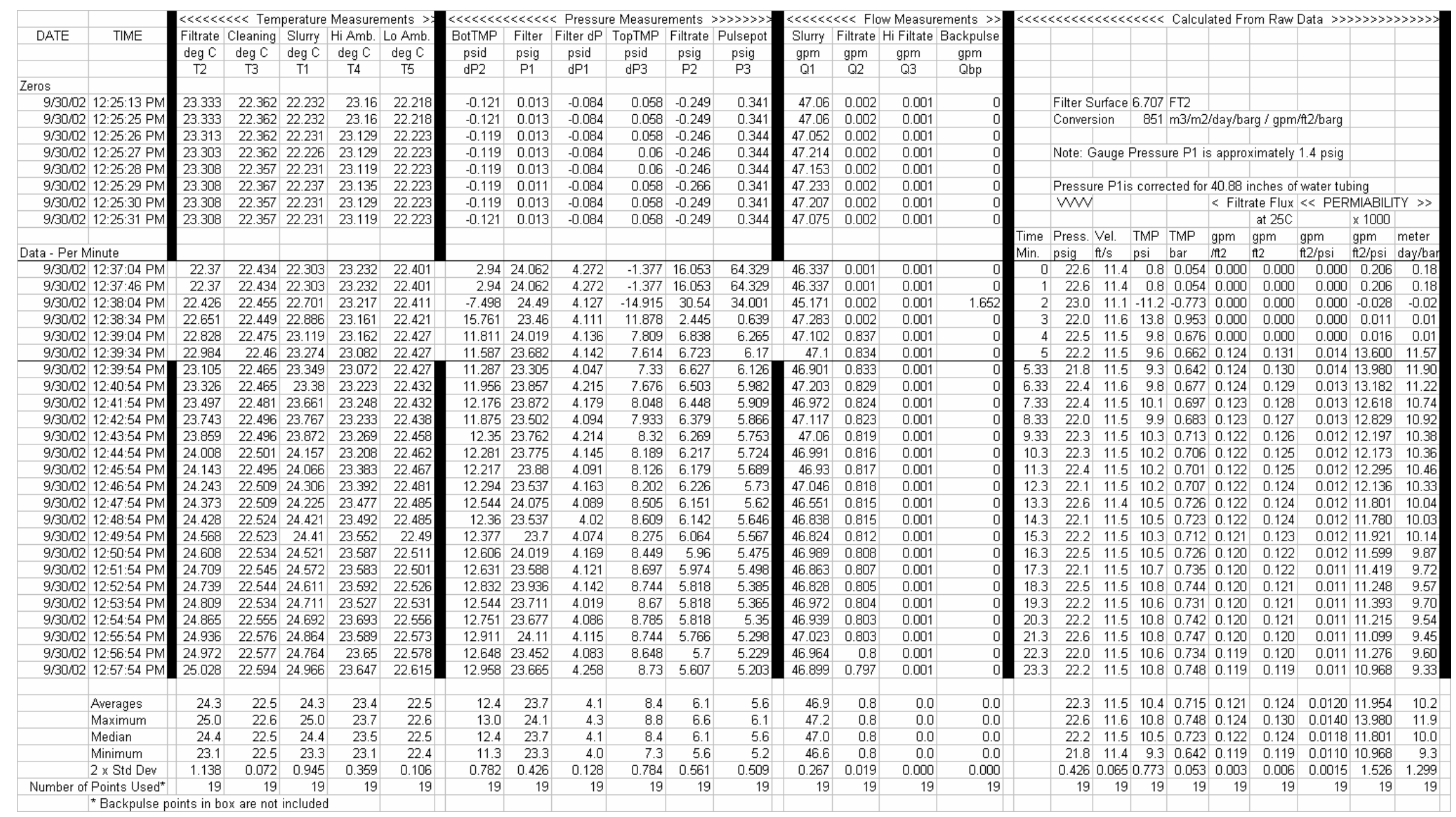

Figure A34: AN-102R2, Batch 3C Slurry Test 1.00A - Inhibited Deionized \& Filtered Water - Raw \& Calculated Data 
WSRC-TR-2003-00204, REV. 0

SRT-RPP-2003-00087, REV. 0

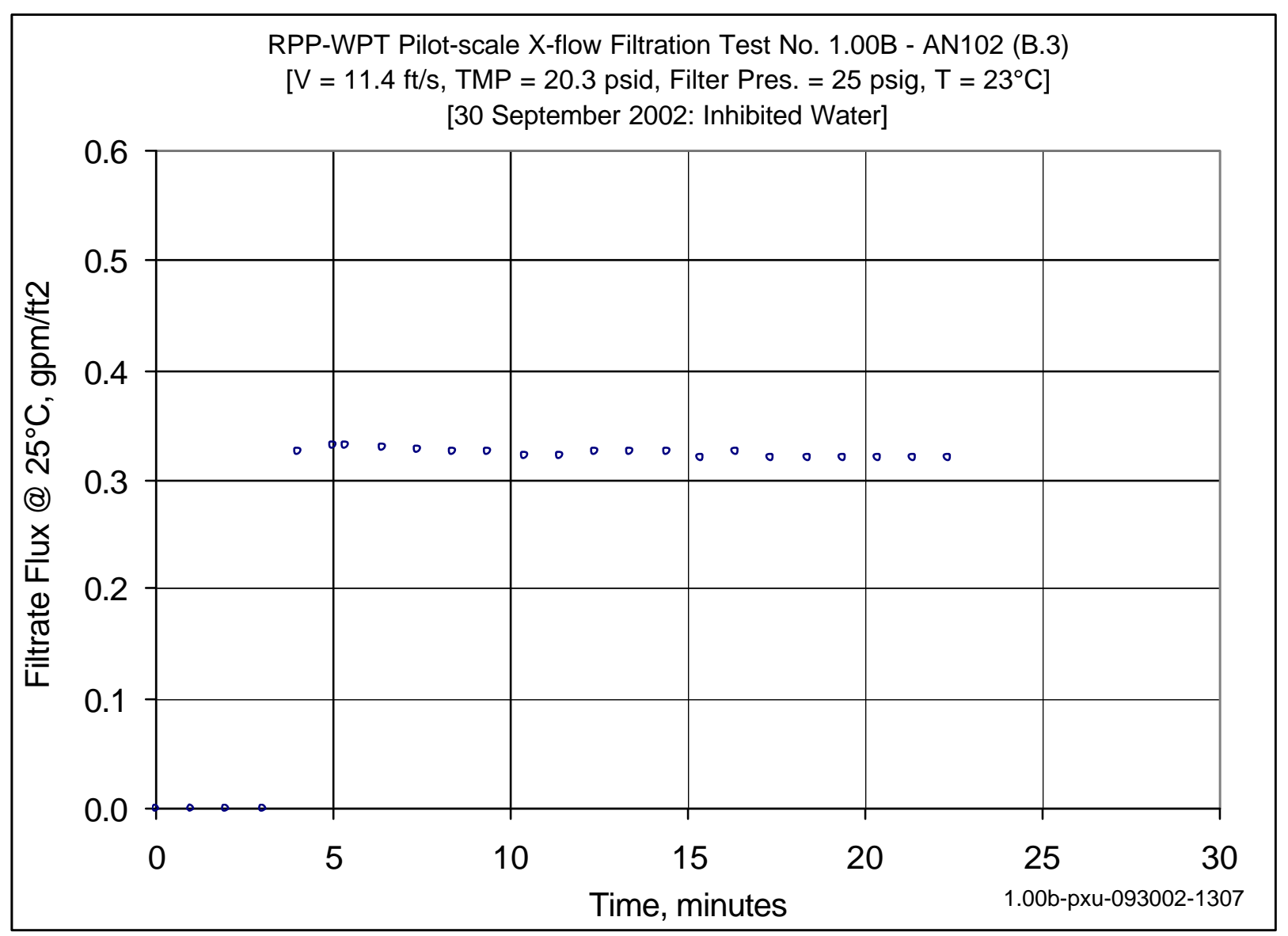

Figure A35: AN-102R2, Batch 3C Slurry Test 1.00B - Inhibited Deionized \& Filtered Water 
WSRC-TR-2003-00204, REV. 0

SRT-RPP-2003-00087, REV. 0

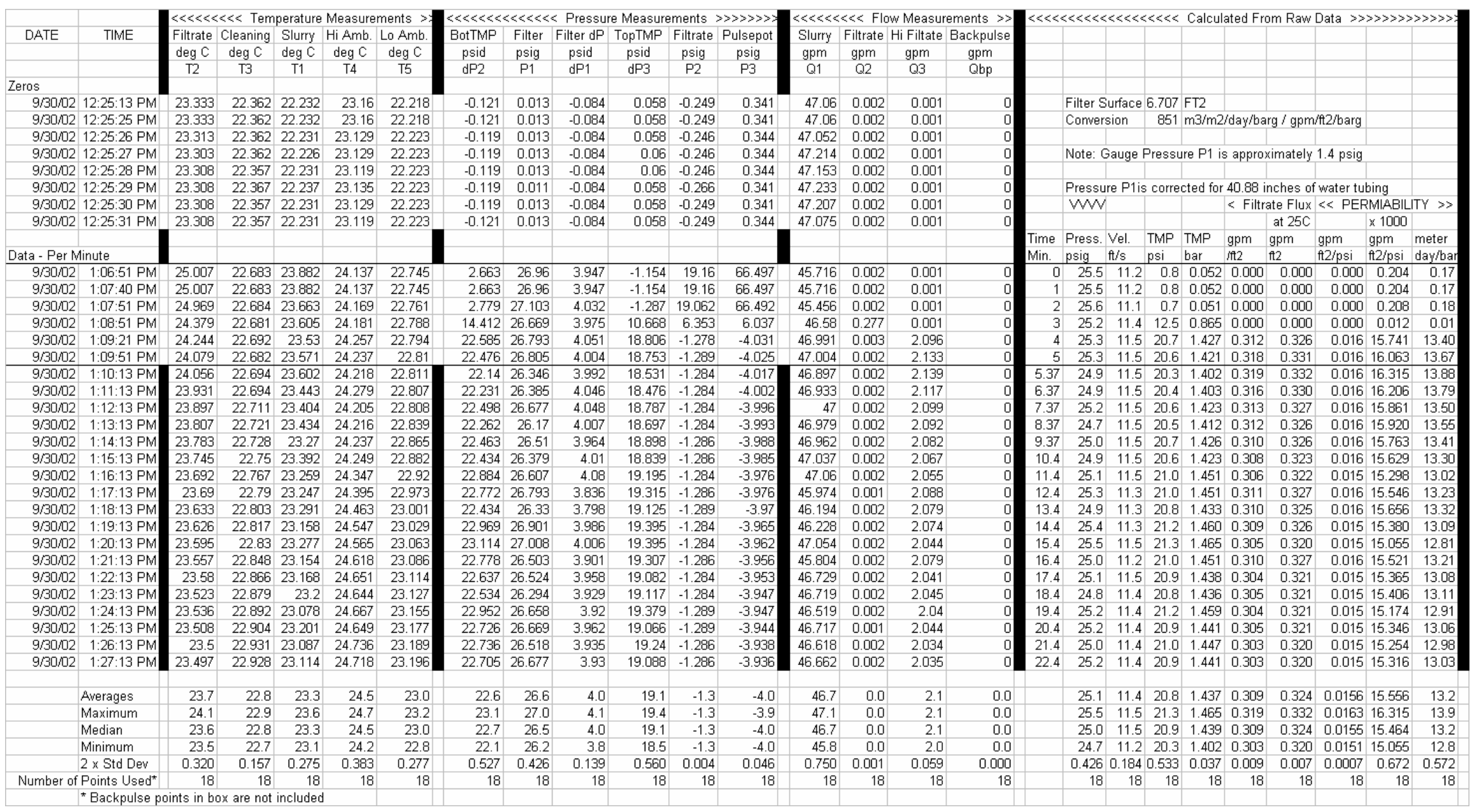

Figure A36: AN-102R2, Batch 3C Slurry Test 1.00B - Inhibited Deionized \& Filtered Water - Raw \& Calculated Data 
WSRC-TR-2003-00204, REV. 0

SRT-RPP-2003-00087, REV. 0

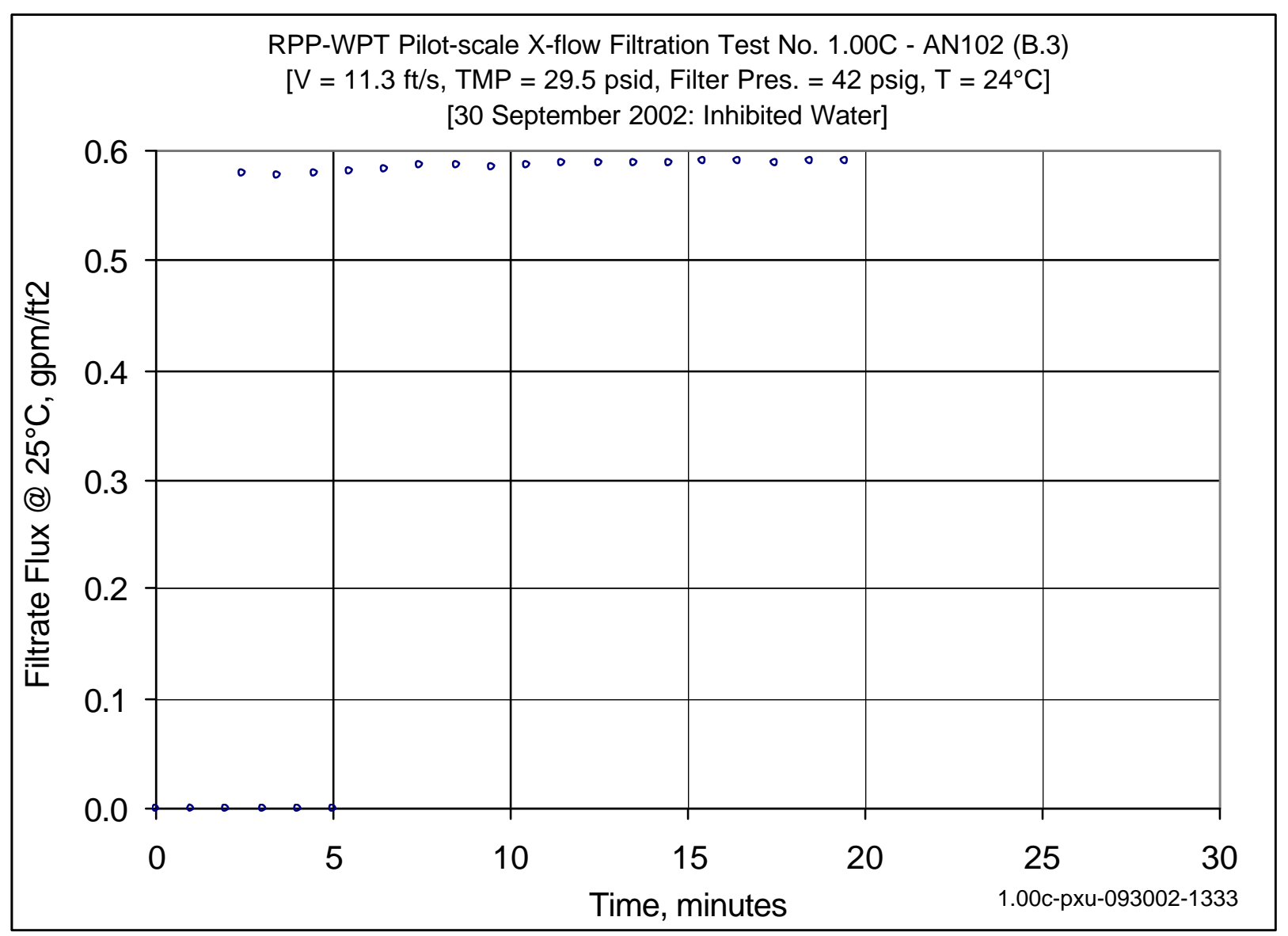

Figure A37: AN-102R2, Batch 3C Slurry Test 1.00C - Inhibited Deionized \& Filtered Water 
WSRC-TR-2003-00204, REV. 0

SRT-RPP-2003-00087, REV. 0

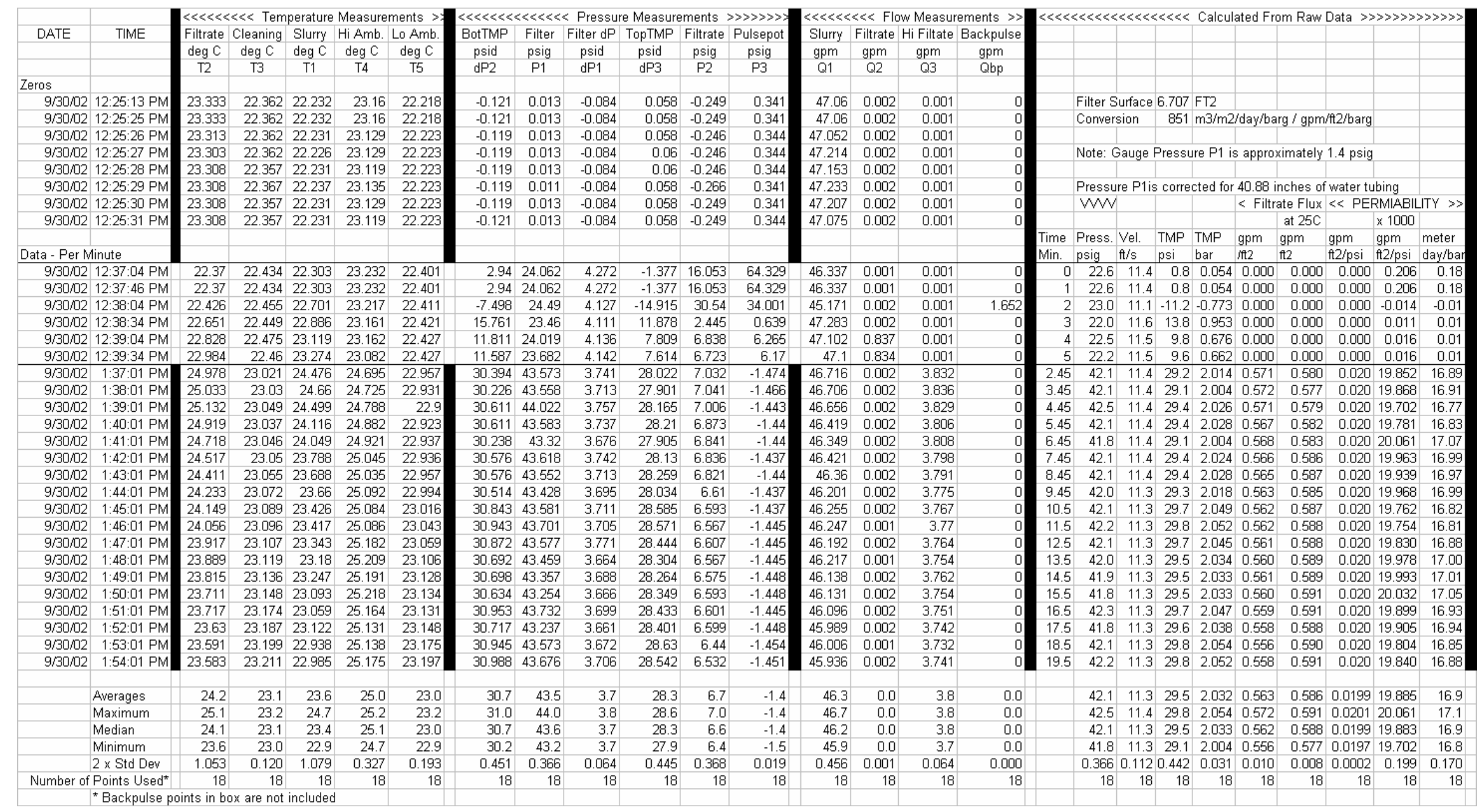

Figure A38: AN-102R2, Batch 3C Slurry Test 1.00C - Inhibited Deionized \& Filtered Water - Raw \& Calculated Data 
WSRC-TR-2003-00204, REV. 0

SRT-RPP-2003-00087, REV. 0

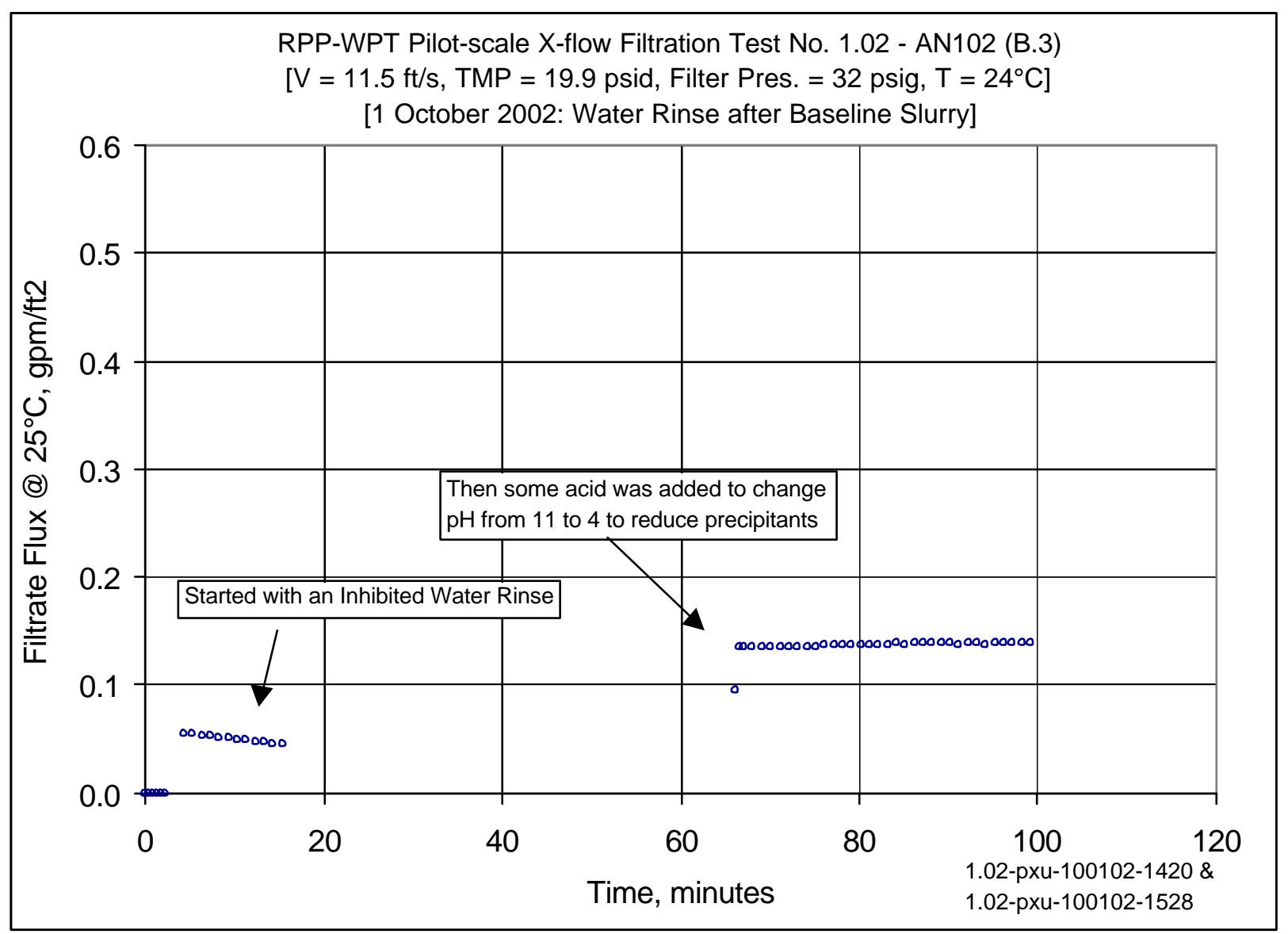

Figure A39: AN-102R2, Batch 3C Slurry Test 1.02 - Inhibited Deionized \& Filtered Water, Then a Mild Acid was Added 
WSRC-TR-2003-00204, REV. 0

SRT-RPP-2003-00087, REV. 0

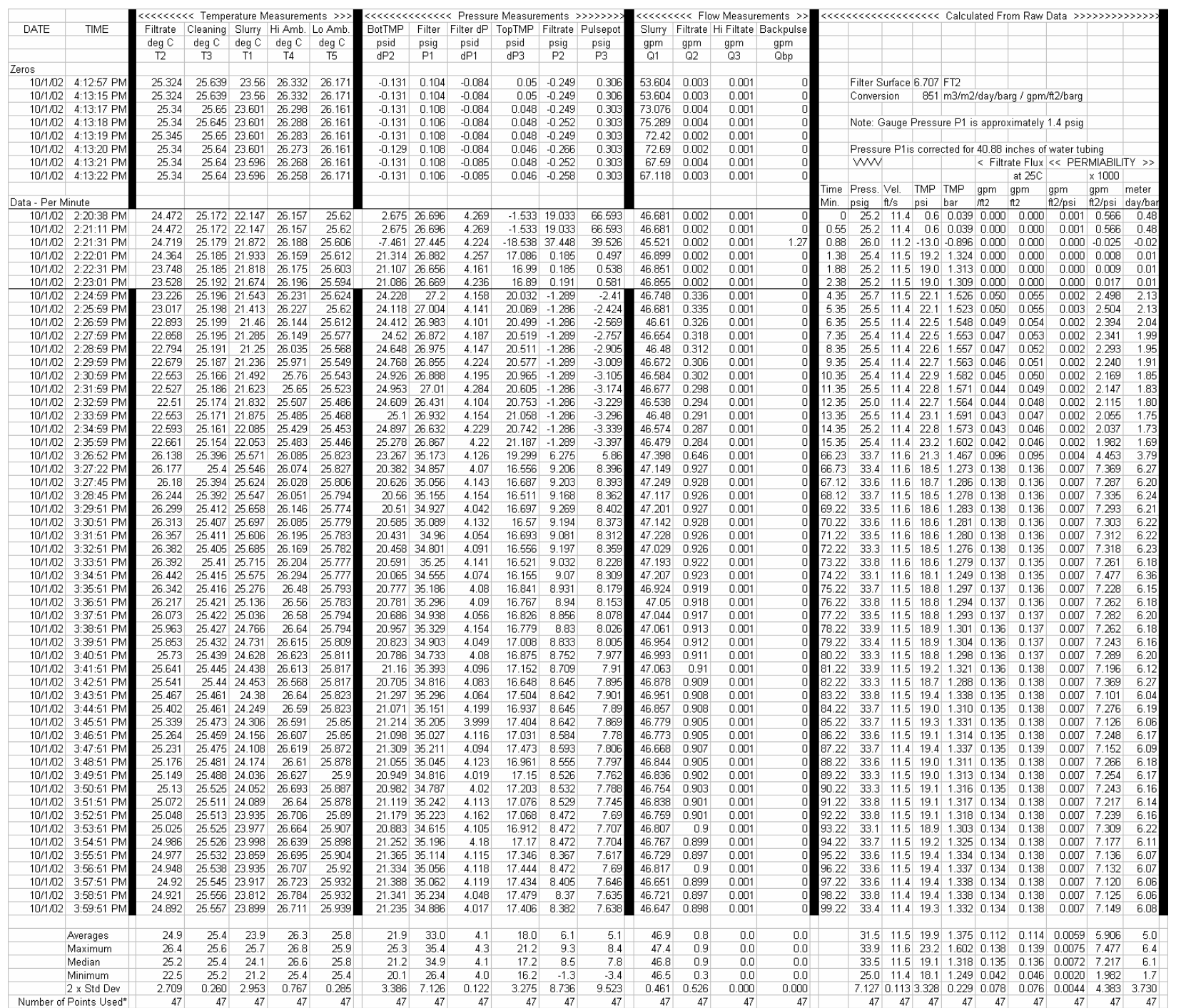

Figure A40: AN-102R2, Batch 3C Slurry Test 1.02 - Inhibited Deionized \& Filtered Water, Then a Mild Acid was Added - Raw \& Calculated Data 
WSRC-TR-2003-00204, REV. 0

SRT-RPP-2003-00087, REV. 0

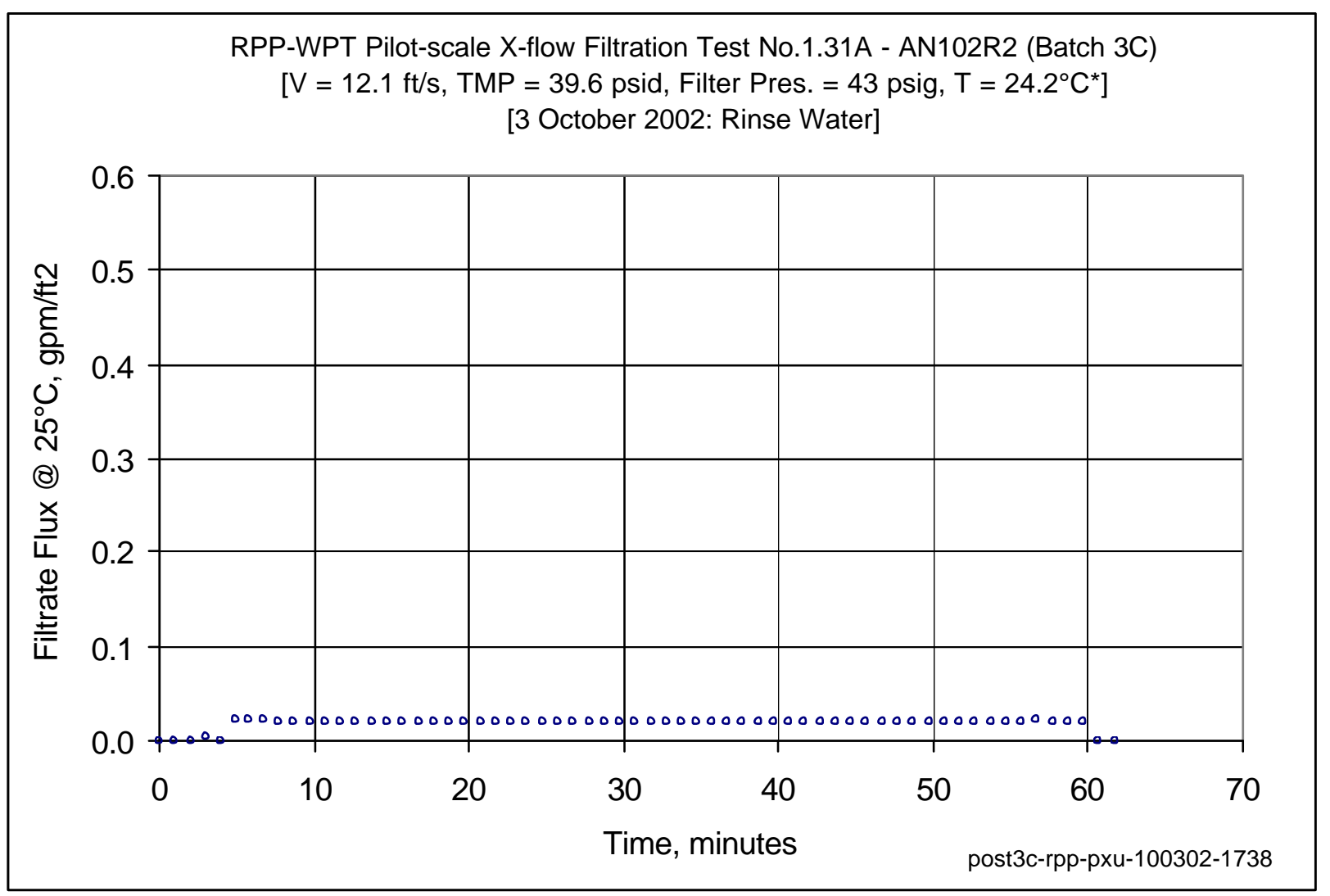

Figure A41: AN-102R2, Batch 3C Slurry Test 1.31A - Deionized \& Filtered Water Rinse after Dewatering Slurry 
WSRC-TR-2003-00204, REV. 0

SRT-RPP-2003-00087, REV. 0

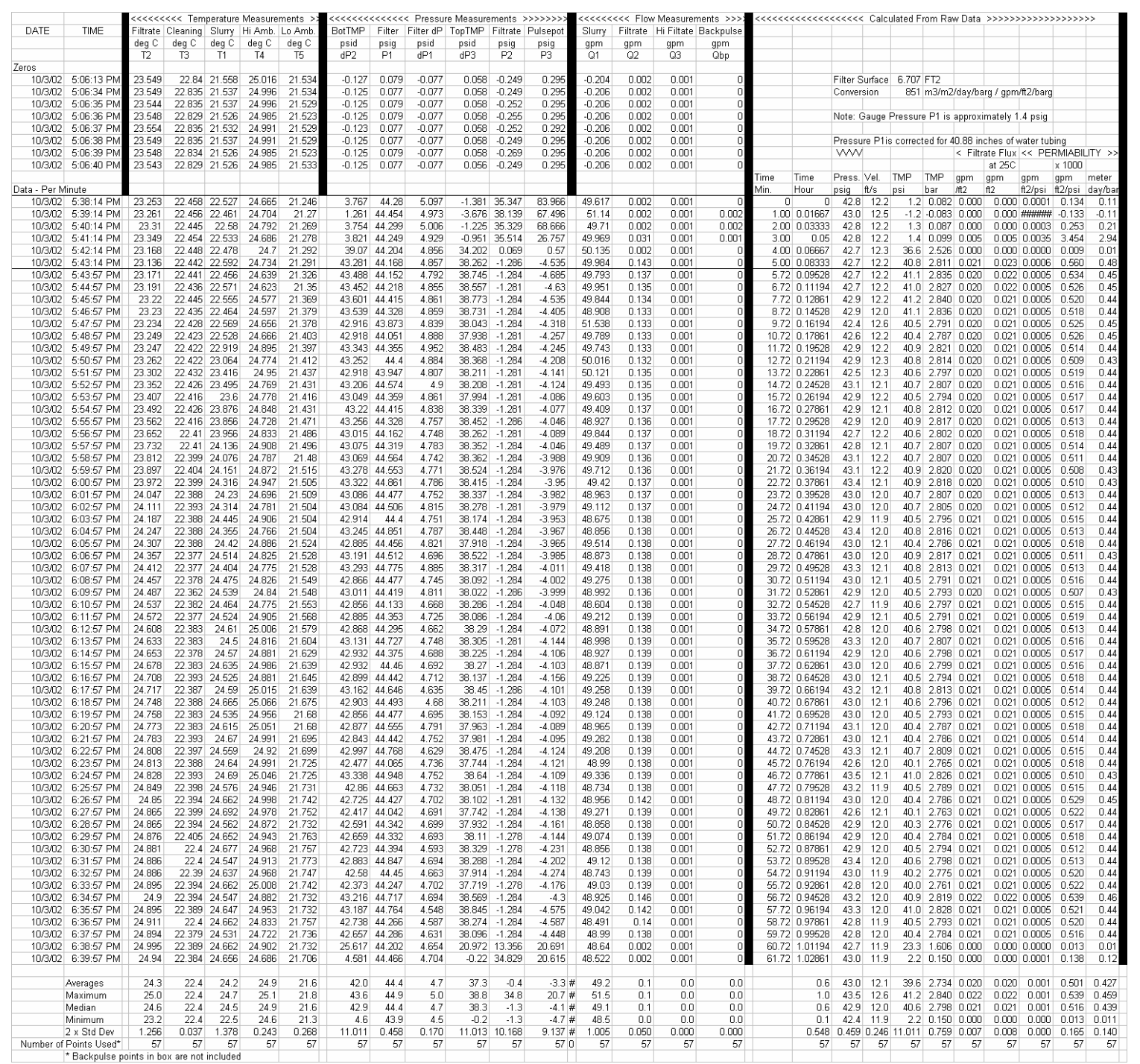

Figure A42: AN-102R2, Batch 3C Slurry Test 1.31A - Deionized \& Filtered Water Rinse after Dewatering Slurry - Raw \& Calculated Data 
WSRC-TR-2003-00204, REV. 0

SRT-RPP-2003-00087, REV. 0

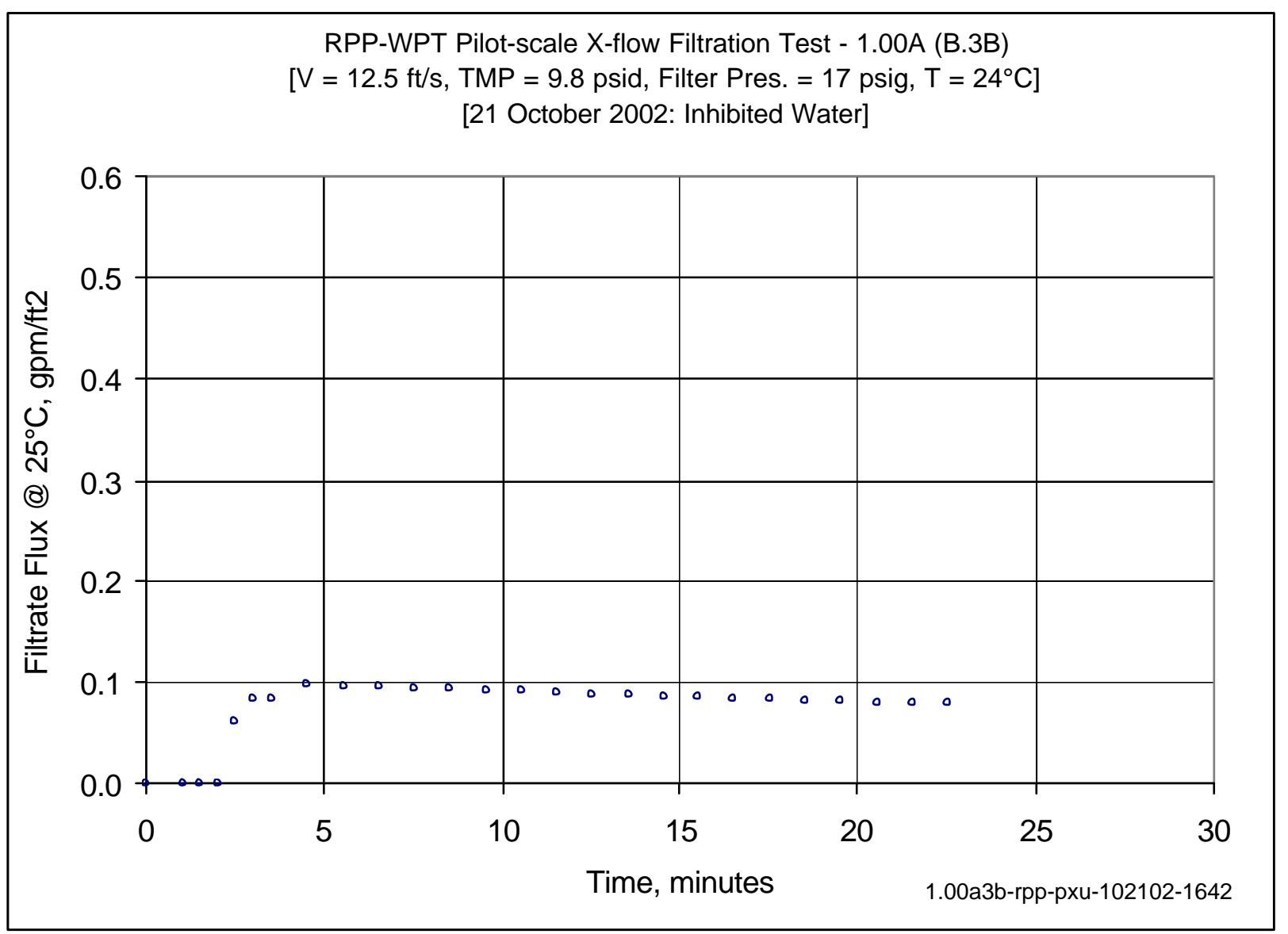

Figure A43: AN-102R2, Batch 3B Slurry Test 1.00A - Inhibited Deionized \& Filtered Water 
WSRC-TR-2003-00204, REV. 0

SRT-RPP-2003-00087, REV. 0

\begin{tabular}{|c|c|c|c|c|c|c|c|c|c|c|c|c|c|c|c|c|c|c|c|c|c|c|c|c|c|c|}
\hline \multirow{3}{*}{ DATE } & \multirow{3}{*}{ TIME } & \multicolumn{5}{|c|}{ 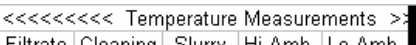 } & \multicolumn{6}{|c|}{ 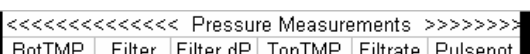 } & \multicolumn{4}{|c|}{ 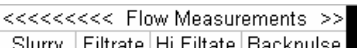 } & \multicolumn{4}{|c|}{$\cdots$} & \multicolumn{6}{|c|}{ Calculated From Raw Data } \\
\hline & & Filtrate & Cleaning & Slurry & Hi Amb. & Lo Amb. & BotTMP & Filter & Filter dP & TopTMP & Filtrate & Pulsepot & Slurry & Filtrate & Hi Filtate & Backpulse & & & & & & & & & & \\
\hline & & & $\operatorname{deg} C$ & $\operatorname{deg} C$ & $\operatorname{deg} C$ & $\operatorname{deg} C$ & psid & psig & psid & psid & psig & psig & gpm & gpm & gpm & gpm & & & & & & & & & & \\
\hline \multirow{2}{*}{\multicolumn{27}{|c|}{ Zeros }} \\
\hline 10/21/02 & 18.07 AM & & & & & & & & & & & & & & & & & & & & & & & & & \\
\hline $10 / 21 / 02$ & 23 & 307 & 22.682 & 964 & 23.022 & $39 \%$ & -0.125 & 0.079 & -0.0661 & 0.056 & -0.182 & 0.356 & 49.984 & 1.014 & 0.001 & & & \multicolumn{4}{|c|}{ Filter Surface 6.707 FT2 } & & & & & \\
\hline $10 / 21 / 02$ & $88: 24 \mathrm{AM}$ & 25.324 & 22.602 & $\begin{array}{l}24.964 \\
24.921\end{array}$ & 25.022 & 22419 & $\begin{array}{l}-0.125 \\
-0.127\end{array}$ & 0.077 & -0.061 & 0.0366 & -0.182 & 0.356 & 49.984 & 1.044 & 0.0001 & & & \multicolumn{2}{|c|}{ Conversion } & & & & & & & \\
\hline $10 / 21 / 02$ & $9 \cdot 08 \cdot 25 \mathrm{AM}$ & 25.319 & 22699 & 24921 & 23104 & 22419 & -0.125 & 0.077 & - 051 & . & 0 & 0.356 & 19766 & 1.012 & 年 & & & \multirow{2}{*}{\multicolumn{2}{|c|}{ Note: Gauge }} & & & & & & & \\
\hline $10 / 21 / 02$ & $68.26 \mathrm{AM}$ & 25324 & 22699 & 24921 & 23104 & & -0.127 & 0077 & (ח) & 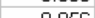 & & & & & & & & & & , ress & & & & 4 priy & & \\
\hline $10 / 21 / 02$ & 8:27 AM & 319 & 22704 & 921 & & & & & & & & 0.350 & 49.6044 & 1.071 & & & & Pressu & $P 1$ is & semerer & eted for & 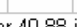 & incheas & fe watert & thing & \\
\hline $10 / 21 / 02$ & 9:08:28 AM & 25.314 & 22.705 & 24.926 & 23.125 & 22.43 & -0.125 & 0.079 & -0.061 & 0.058 & $\begin{array}{l}-0.179 \\
-0.179\end{array}$ & 0.356 & 49741 & 1.014 & 0.001 & & & Wh & & & & $<$ Filtra & ate Flux & $k<$ PEF & RMIABIL & ITY \\
\hline $10 / 21 / 02$ & 9:08:29 AM & 25.314 & 22.694 & 24.926 & 23.129 & 22.419 & -0.125 & 0.077 & -0.061 & 0.058 & -0.179 & 0.358 & 49.928 & 1.012 & 0.001 & & & & & & & & at $25 \mathrm{C}$ & & $\times 1000$ & \\
\hline & & & & & & & & & & & & & & & & & Time & Press. & Vel. & TMP T & TMP & gpm & gpm & $\mathrm{gpm}$ & $\mathrm{gpm}$ & meter \\
\hline ta - Per M & linute & & & & & & & & & & & & & & & & Min. & psig & $\mathrm{f} / \mathrm{s}$ & psi & bar & ift2 & $\mathrm{f} 2$ & $\mathrm{f} 2 / \mathrm{psi}$ & $\mathrm{ft2} / \mathrm{psi}$ & day/b \\
\hline $10 / 21 / 02$ & $4: 41: 58 \mathrm{PM}$ & 25.024 & 27.433 & 23.742 & 28.005 & 27.959 & 3.056 & 18.63 & 4.875 & -1.731 & 10.18 & 60.46 & 50.624 & 0.002 & 0.001 & & 0 & 17.2 & \begin{tabular}{|l|l|}
2 & 12.4 \\
\end{tabular} & 0.7 & 0.046 & 0.000 & 0.000 & 0.000 & 0.466 & 0.40 \\
\hline $10 / 21 / 02$ & 4:42:59 PM & 25.102 & 27.426 & 23.519 & 27.963 & 27.966 & 2.857 & 18.408 & 4.922 & -2.079 & 10.2 & 60.411 & 50.467 & 0.002 & & 0.001 & 1.02 & $\begin{array}{ll}26.9 \\
\end{array}$ & $\begin{array}{ll}9 & 12.4 \\
\end{array}$ & 0.4 & 0.027 & 0.000 & 0.000 & 0.001 & $\begin{array}{ll}1 & 0.799 \\
\end{array}$ & 80 \\
\hline $10 / 21 / 02$ & $\mathrm{PM}$ & 25.67 & 27.438 & 1 & & 00 & & & & & & 34.496 & 50.834 & 2 & & & 1.5 & 17.2 & $\begin{array}{lll}2 & 12 \\
\end{array}$ & 1.1 & & & & 000 & & \\
\hline $10 / 21 / 02$ & $4: 43: 58 \mathrm{PM}$ & 25.645 & 27.443 & 23.616 & 27.995 & 27.978 & 3.437 & 18.597 & 34 & -1.356 & 9.654 & 0.477 & 50.787 & 0.002 & & & 2.00 & 17.1 & $\begin{array}{ll}1 & 12.4 \\
\end{array}$ & 1.0 & 0.072 & 0.000 & 0.000 & 0.000 & 298 & 0.25 \\
\hline $10 / 21 / 02$ & 4:44:28 PM & 25.651 & 27.449 & 23.477 & 28.006 & 27.984 & 7.959 & 18.456 & (c) & 3.151 & 5.13 & 0.442 & 50.882 & 0.393 & & & 2.50 & 17.0 & 12.5 & 5.6 & 0.383 & 0.059 & 0.061 & 0.011 & 111.012 & 9. \\
\hline $10 / 21 / 02$ & $4: 44: 58 \mathrm{PM}$ & 24.721 & 27.46 & 23.484 & 33 & 27.996 & 10.096 & 18.274 & & 51 & 2.665 & 0.503 & 51.045 & 0.542 & & & 3.00 & 16.8 & $\begin{array}{ll}3 & 12.5\end{array}$ & 7.8 & 0.538 & 0.081 & 0.084 & 0.011 & 110 & \\
\hline $10 / 21 / 02$ & PM & 6 & 27.446 & 23.54 & & 28.022 & 10.256 & 18. & & & 2.662 & 0.579 & & 0.538 & & & 3.5 & 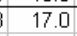 & 12.5 & 8.0 & & & & 1010 & & \\
\hline $10 / 21 / 02$ & 4:46:30 PM & 158 & 27.459 & 23.397 & 28.026 & 28.005 & 11.357 & 18.508 & 4.895 & 6.588 & 1.63 & -1.344 & 50.853 & 0.625 & & & 4.53 & 17.0 & 12.5 & 9.0 & 0.619 & 0.093 & 0.098 & 0.011 & 11 & 9.2 \\
\hline $10 / 21 / 02$ & 4:47:30 PM & 24 & 27.466 & 23.509 & 88 & 28.007 & 11 & 18.605 & 4 & & 1.598 & -1.382 & 04 & 0.62 & & & 5.53 & \begin{tabular}{|l|}
17.1 \\
\end{tabular} & 112.5 & 8.9 & 0.617 & 0.092 & 6 & 0.011 & & 9. \\
\hline $10 / 21 / 02$ & 4:48:30 PM & 24.018 & 27.469 & 23.392 & & 27.995 & 11. & 18.4 & & & 1.387 & -1.451 & & & & & 6.5 & 16.9 & $\begin{array}{ll}9 & 12.5\end{array}$ & 9.1 & & & & 0.011 & & \\
\hline & . & & 27.476 & 23.429 & & 27.997 & & 18.3 & & & 1.202 & & & & & & & 16.8 & & 9.2 & & & & 10 & & \\
\hline $10 / 21 / 02$ & 4:50:30 PM & 23.926 & 27.483 & 23.396 & 27 & 27.979 & 11.913 & 18.555 & & & 1.156 & -1.584 & 50. & 0.599 & & & 8.5 & 17.1 & 112.5 & 9.6 & 0.662 & 0.089 & 3 & .010 & 33 & 8.2 \\
\hline $10 / 21 / 02$ & 4:51:30 PM & & 27.48 & 23.353 & 22 & 27.976 & & 18 & & & & 657 & & 2 & & & 9.5 & 16.9 & $\begin{array}{ll}9 & 12.5\end{array}$ & 9.5 & & & & 10 & & \\
\hline $10 / 21 / 02$ & 4:52:30 PM & & 27.497 & 23.39 & & 27. & & & & & & & & & & & & 17.2 & $\begin{array}{ll}2 & 12.5\end{array}$ & 9.9 & & & & .009 & & \\
\hline & 4: & & 27.487 & & & 27.9 & & & & 7.2 & & & & & & & & 16.9 & & 9.7 & & & & 09 & & \\
\hline $10 / 21 / 02$ & $4: 54: 30 \mathrm{PM}$ & 23.934 & 27.491 & 23.93 & 27 & 28.01 & 12 & 18. & & 7.6 & & 25 & & 0.5 & & & 2.53 & 16.9 & $\begin{array}{ll}9 & 12.4\end{array}$ & 9.9 & 0. & 0 & 8 & 009 & & \\
\hline & 4:55:30 PM & 24 & 27.483 & 24.278 & & & & & & & & & & 0.5 & & & & 6.9 & $\begin{array}{ll}9 & 12.5\end{array}$ & 9.8 & & & & 19 & & \\
\hline 10 & $4: 56: 3$ & & 27.48 & & & 28. & & & & & & & & & & & & 17.0 & 12.5 & 10.0 & & & & .009 & & \\
\hline $10 / 21 / 02$ & 4:57:30 PM & 24.62 & 27.494 & & & 28.03 & & & & & 0.373 & -1 & & 0.5 & & & 15. & 17.0 & 12.5 & 10.1 & & & & 08 & & 7.1 \\
\hline $10 / 21 / 02$ & $4: 58: 30 \mathrm{PM}$ & 24.769 & 27.503 & 24.915 & 27 & 28.029 & 12 & 18 & & & & & & 0. & & & . & 8.1 & $\begin{array}{ll}1 & 12.5\end{array}$ & 10.2 & 0.705 & & & 18 & & \\
\hline & $4: 59: 3$ & 24.9 & 27.498 & & & 28. & & & & & & & & & & & & & \begin{tabular}{|l|l|}
3 & 12.6 \\
\end{tabular} & & & & & & & \\
\hline 16 & $5: 00: 30$ & 25 & 27.513 & 25.155 & 27.8 & 28.0 & 12. & 18.3 & & & & & & 0.5 & & & & 16.9 & \begin{tabular}{|l|l|}
9 & 12.6 \\
\end{tabular} & 10.3 & & & & 08 & & \\
\hline $10 / 21 / 02$ & 5:01:30 PM & 25.231 & 27.504 & 25.346 & 27.787 & 28.03 & 12.708 & 18.28 & & 7. & 0.0 & -2 & 51. & 0. & & & 19.5 & 16.8 & \begin{tabular}{|l|l|} 
& 12.5 \\
\end{tabular} & 10.3 & 0. & 30 & & 88 & 8 & 6. \\
\hline & $5: 02: 3$ & 25.371 & 27.514 & 25.411 & 27.787 & 28.0 & 12.766 & 18.3 & & & & 2. & & & & & & 169 & 125 & 10.4 & & & & 87 & & \\
\hline $10 \pi$ & 5:03:30 PM & 25.4 & 27.521 & & & 28.0 & & & & & & & & U. & & & 1.5 .5 & 17.0 & 12.5 & 10.6 & & & & .007 & 7 & \\
\hline $10 / 21 / 02$ & 5:04:30 PM & 25.583 & 27.521 & 25.698 & 27.814 & 28.022 & 12.828 & 18.265 & 4.848 & 8.155 & -0.162 & -2.25 & 51.155 & 0.536 & 0.001 & & 22.53 & 16.8 & $\begin{array}{ll}3 & 12.5\end{array}$ & 10.5 & 0.723 & 0.080 & 0.078 & \begin{tabular}{l|l}
3 & 0.007 \\
\end{tabular} & $\begin{array}{l}77.469 \\
\end{array}$ & 50 \\
\hline & & & & & & & & & & & & & & & & & & & & & & & & & & \\
\hline & Aw & 24 & 27.5 & & & & & & & & & & & & & & & 17.0 & 12.5 & 8 & & & & 990 & & \\
\hline & & 25 & 27.5 & & & & & & & & & & & . & & & & 17.2 & $\begin{array}{ll}2 & 12.6 \\
\end{array}$ & 10.6 & & & & 0.0109 & 910.867 & \\
\hline & & 24 & 27.5 & 24.1 & 27.8 & 26 & 12.2 & 18 & & 7.6 & 6 & -1 & & 0. & 0 & & & 16.9 & \begin{tabular}{ll|}
9 & 12.5
\end{tabular} & 9.9 & 0.683 & 0.085 & 0.087 & 0.0089 & $\begin{array}{ll}9 & 8.927\end{array}$ & 7.6 \\
\hline & & 23. & 27.4 & & & & & & & & & & & & & & & 16.8 & \begin{tabular}{|l|l|}
3 & 12.4
\end{tabular} & 8.0 & 0.553 & & & 0075 & & \\
\hline & & 1.17 & 0.04 & 17 & & & & & & & & & & & & & & . & 12 & 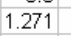 & & & & & & \\
\hline Number of & $\mathrm{Po}$ & 20 & 20 & 20 & 20 & 20 & 20 & 20 & 20 & 20 & 20 & 20 & 20 & 20 & 20 & 20 & & 20 & 20 & 20 & 20 & 20 & 20 & 20 & 20 & \\
\hline
\end{tabular}

Figure A44: AN-102R2, Batch 3B Slurry Test 1.00A - Inhibited Deionized \& Filtered Water - Raw \& Calculated Data 
WSRC-TR-2003-00204, REV. 0

SRT-RPP-2003-00087, REV. 0

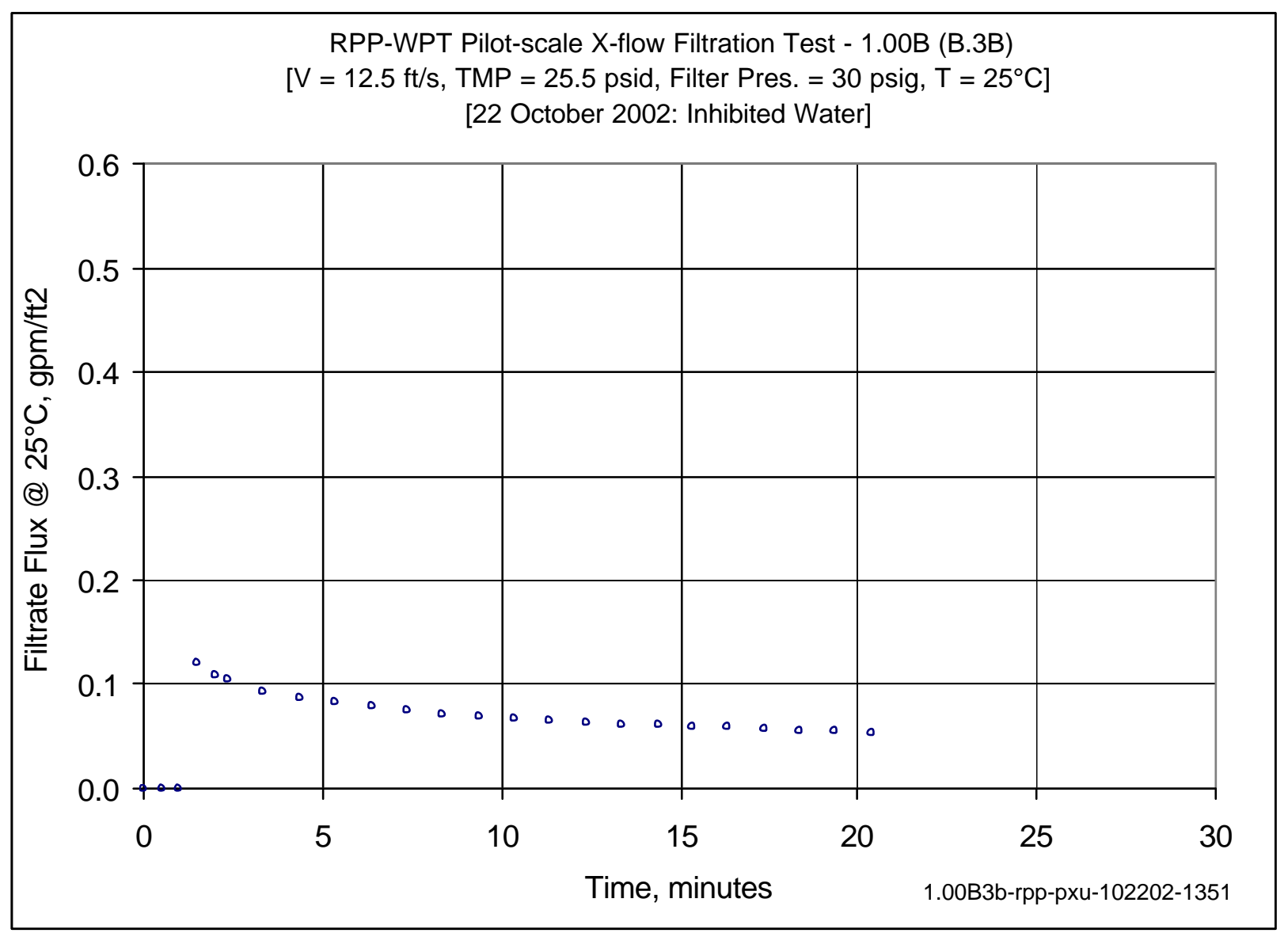

Figure A43: AN-102R2, Batch 3B Slurry Test 1.00B - Inhibited Deionized \& Filtered Water 
WSRC-TR-2003-00204, REV. 0

SRT-RPP-2003-00087, REV. 0

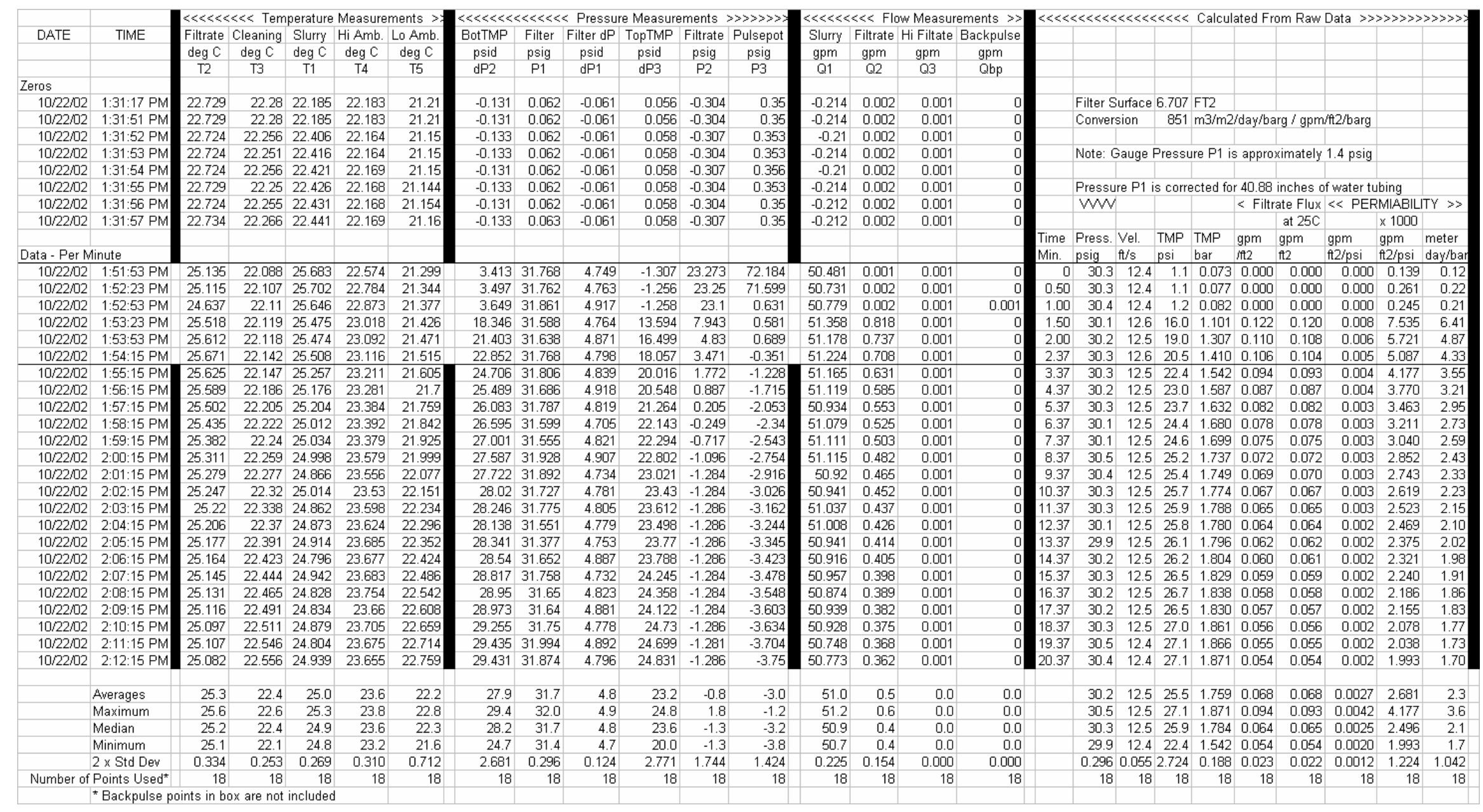

Figure A46: AN-102R2, Batch 3B Slurry Test 1.00B - Inhibited Deionized \& Filtered Water - Raw \& Calculated Data 
WSRC-TR-2003-00204, REV. 0

SRT-RPP-2003-00087, REV. 0

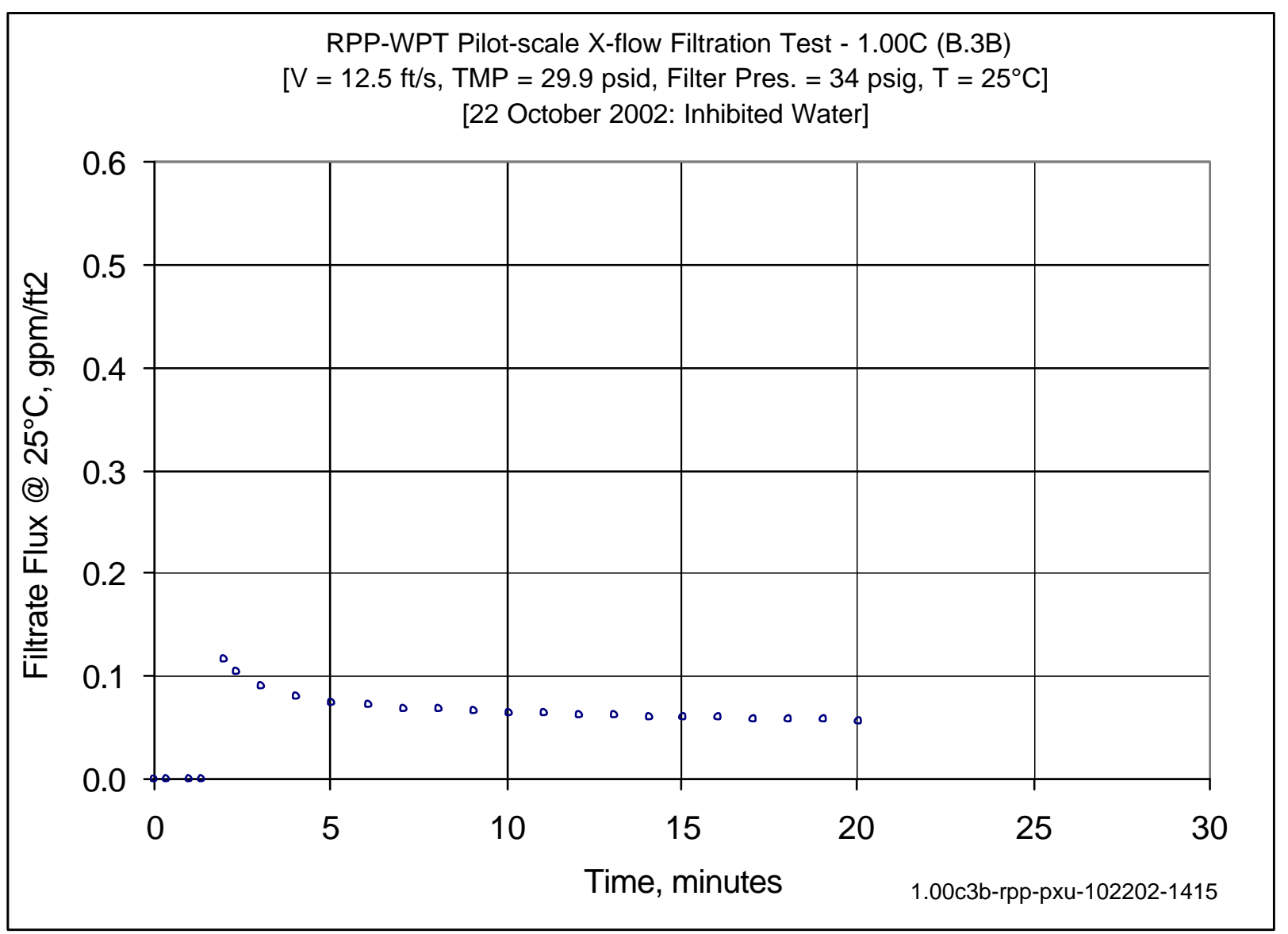

Figure A45: AN-102R2, Batch 3B Slurry Test 1.00C - Inhibited Deionized \& Filtered Water 
WSRC-TR-2003-00204, REV. 0

SRT-RPP-2003-00087, REV. 0

\begin{tabular}{|c|c|c|c|c|c|c|c|c|c|c|c|c|c|c|c|c|c|c|c|c|c|c|c|c|c|c|}
\hline \multirow[b]{2}{*}{ DATE } & \multirow[b]{2}{*}{ TIME } & \multicolumn{5}{|c|}{ 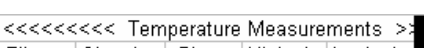 } & \multicolumn{6}{|c|}{ 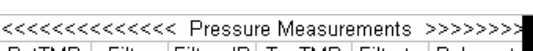 } & \multicolumn{4}{|c|}{ 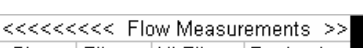 } & \multicolumn{4}{|c|}{ 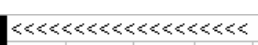 } & \multicolumn{6}{|c|}{ 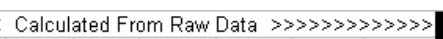 } \\
\hline & & Filtrate & Cleaning & Slury & Hi Amb. & Lo Amb. & BotTMP & Filter & Filter dP & TopTMP & Filtrate & Pulsepot & Slurry & Filtrate & Hi Filtate & Backpulse & & & & & & & & & & \\
\hline & & $\operatorname{deg} \mathrm{C}$ & $\operatorname{deg} C$ & $\operatorname{deg} C$ & $\operatorname{deg} \mathrm{C}$ & $\operatorname{deg} \mathrm{C}$ & psid & psig & psid & psid & psig & & $\mathrm{gpm}$ & $\mathrm{gpm}$ & $\mathrm{gpm}$ & $\mathrm{gpm}$ & & & & & & & & & & \\
\hline & & $\mathrm{T} 2$ & T3 & $\mathrm{T} 1$ & T4 & $\mathrm{T} 5$ & $\mathrm{dP2}$ & P1 & $\mathrm{dP1}$ & $\mathrm{dP3}$ & P2 & $\mathrm{P3}$ & Q1 & Q2 & Q3 & Qbp & & & & & & & & & & \\
\hline \multirow{2}{*}{\multicolumn{27}{|c|}{ Zeros }} \\
\hline & 31:17 PM & 22.729 & 22.28 & 22.185 & 22.183 & 21.21 & -0.131 & 0.062 & -0.061 & 0.056 & -0.304 & 0.35 & -0.214 & 0.002 & 0.001 & 0 & & \multicolumn{3}{|c|}{ Filter Surface 6.707} & \multirow{2}{*}{\multicolumn{4}{|c|}{$\begin{array}{l}\mathrm{FT} 2 \\
\mathrm{~m} 3 / \mathrm{m} 2 / \mathrm{da} / \mathrm{barg} / \mathrm{gmm} / \mathrm{f} 2 / \text { barg }\end{array}$}} & & \\
\hline $10 / 22 / 02$ & 1:31:51 PM & 22.729 & 22.28 & 22.185 & 22.183 & 21.21 & -0.131 & 0.062 & -0.061 & 0.056 & -0.304 & 0.35 & -0.214 & 0.002 & 0.001 & 0 & & \multicolumn{2}{|c|}{ Conversion } & 851 & & & & & & \\
\hline $10 / 22 / 02$ & 1:31:52 PM & 22.724 & 22.256 & 22.406 & 22.164 & 21.15 & -0.133 & 0.062 & -0.061 & 0.058 & -0.307 & 0.353 & -0.21 & 0.002 & 0.001 & 0 & & & & & & & & & \\
\hline $10 / 22 / 02$ & 1:31:53 PM & 22.724 & 22.251 & 22.416 & 22.164 & 21.15 & -0.133 & 0.062 & -0.061 & 0.058 & -0.304 & 0.353 & -0.214 & 0.002 & 0.001 & 0 & & Note: G & Gauge F & Pressu & ure $P 1$ is & $s$ approx & ximately & $1.4 \mathrm{psig}$ & & \\
\hline $10 / 22 / 02$ & 1:31:54 PM & 22.724 & 22.256 & 22.421 & 22.169 & 21.15 & -0.131 & 0.062 & -0.061 & 0.058 & -0.307 & 0.356 & -0.21 & 0.002 & 0.001 & 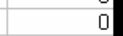 & & & & & & & & & & \\
\hline $10 / 22 / 02$ & 1:31:55 PM & 22.729 & 22.25 & 22.426 & 22.168 & 21.144 & -0.133 & 0.062 & -0.061 & 0.058 & -0.304 & 0.353 & -0.214 & 0.002 & 0.001 & & & Pressu & ure $\mathrm{P} 1$ is & is corre & rected for & $40.88 \mathrm{i}$ & inches of & of water $t$ & tubing & \\
\hline $10 / 22 / 02$ & 1:31:56 PM & 22.724 & 22.255 & 22.431 & 22.168 & 21.154 & -0.131 & 0.062 & -0.061 & 0.058 & -0.304 & 0.35 & -0.212 & 0.002 & 0.001 & 0 & & WW & & & & $<$ Filtra & ate Flux & $\ll$ PEF & ERMIABILI & LITY \\
\hline $10 / 22 / 02$ & 1:31:57 PM & 22.734 & 22.266 & 22.441 & 22.169 & 21.16 & -0.133 & 0.063 & -0.061 & 0.058 & -0.307 & 0.35 & -0.212 & 0.002 & 0.001 & 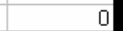 & & & & & & & at $25 \mathrm{C}$ & & $\times 1000$ & \\
\hline & & & & & & & & & & & & & & & & & ime & Press. & Vel. & TMP & TMP & $\mathrm{gpm}$ & & & & eteter \\
\hline ata - Per M & Ainute & & & & & & & & & & & & & & & & & psig & $\mathrm{ft} / \mathrm{s}$ & psi & bar & int2 & $\mathrm{ft} 2$ & $\mathrm{fl2} / \mathrm{psi}$ & $\mathrm{ft} 2 / \mathrm{psi}$ & /bar \\
\hline $10 / 22 / 02$ & 2:15:11 PM & 25.045 & 22.656 & 24.973 & 8.799 & 22.969 & 3.323 & 36.303 & 4.744 & -1.44 & 27.782 & 76.278 & 50.444 & 0.002 & 0.001 & & 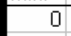 & 34.8 & 12.4 & 0.9 & 0.065 & 0.000 & 0.000 & 0.000 & ] 0.317 & 0.27 \\
\hline $10 / 22 / 02$ & 2:15:31 PM & 25.038 & 22.673 & 25.056 & 23.857 & 22.987 & 3.122 & 35.945 & 4.814 & -1.623 & 27.661 & 75.091 & 50.767 & 0.002 & 0.001 & f & 0.33 & 34.5 & 12.4 & $\begin{array}{ll}4 \\
4\end{array}$ & 0.052 & 0.000 & 0.000 & 0.000 & $\begin{array}{ll}0 & 0.397\end{array}$ & 0.34 \\
\hline $10 / 22 / 02$ & 2:16:11 PM & 24.805 & 22.666 & 25.099 & 23.78 & 23.025 & 3.468 & 35.846 & & -1.377 & 27.424 & 36.972 & 50.471 & 0.0 & & 002 & 1.00 & 34.4 & 12.4 & 1.0 & 0.072 & 0.000 & & 0.000 & & 0.12 \\
\hline $10 / 22 / 02$ & 2:16:31 PM & 24.802 & 22.673 & 25.016 & 23.832 & 23.037 & 3.448 & 35.815 & 4.856 & -1.483 & 27.323 & 0.454 & 50.683 & 0.002 & 0.001 & 0 & 1.33 & 34.3 & 12.4 & $\begin{array}{l}4.0 \\
1.0\end{array}$ & 0.068 & 0.000 & 0.000 & 0.000 & 0.303 & 0.26 \\
\hline $10 / 22 / 02$ & 2:17:11 PM & 25.167 & 22.693 & 25.105 & \begin{tabular}{|l|}
23.917 \\
\end{tabular} & 23.082 & 22.513 & 35.933 & 4.876 & 17.659 & 8.203 & 0.555 & 51.383 & 0.7 & 0. & & 2.00 & 34.5 & 12.6 & 20.1 & 1.385 & 0.116 & & 0.006 & $\begin{array}{ll}6 \quad 5.743 \\
\end{array}$ & 4.89 \\
\hline $10 / 22 / 02$ & 2:17:31 PM & 25.229 & 22.699 & 25.182 & 23.903 & 23.083 & 26.351 & 36.173 & 4.902 & 21.469 & 4.465 & 0.625 & 51.228 & 0.707 & 0. & & 2.33 & 34.7 & 12.6 & $\begin{array}{l}23.9 \\
6\end{array}$ & 1.649 & 0.105 & 0.105 & 0.004 & $\begin{array}{ll}4 & 4.386 \\
\end{array}$ & 3.73 \\
\hline $10 / 22 / 02$ & 2:18:15 PM & 25.338 & 22.714 & 25.066 & 23.953 & 23.133 & 29.178 & 35.635 & 4.804 & 24.613 & 1.272 & -1.506 & 51.201 & 0.607 & 0.0 & & 3.07 & 34.2 & 12.5 & 526.9 & 1.854 & 0.090 & 0.090 & 0.003 & \begin{tabular}{|l|l|}
3 & 3.359 \\
\end{tabular} & 2.86 \\
\hline $10 / 22 / 02$ & 2:19:15 PM & 25.349 & 22.739 & 25.246 & 23.983 & 23.153 & 30.814 & 35.958 & 4.938 & 26.004 & -0.096 & -2.227 & 51.241 & 0.5 & & 0 & 4.07 & 34.5 & 12.6 & 28.4 & 1.959 & 0.080 & 9 & 0.003 & $\begin{array}{ll}3 & 2.783\end{array}$ & 2.37 \\
\hline $10 / 22 / 02$ & 2:20:15 PM & 25.394 & 22.76 & 25.142 & 24.039 & 23.229 & 31.193 & 35.771 & & & -0.775 & -2.583 & & 0.4 & & & 5.07 & 34.3 & 12.5 & 28.8 & & & & 0.003 & $\begin{array}{ll}3 & 2.569\end{array}$ & 2.19 \\
\hline $10 / 22 / 02$ & 2:21:15 PM & 25.42 & 22.78 & 25. & 24.0 & 23.264 & 31. & 35.922 & & & & -2.751 & & & & 0 & 6.07 & 34.4 & 12.5 & 29.4 & & & & 0.002 & & 2.06 \\
\hline $10 / 22 / 02$ & 2:22:15 PM & 25.425 & 22.801 & 25.268 & $\begin{array}{r}\quad 24.11 \\
\end{array}$ & 23.295 & 31.883 & 35.85 & 4 & 27.234 & -1.284 & $\begin{array}{l}-2.893 \\
\end{array}$ & 51.077 & 0. & & 0 & 7.07 & $\begin{array}{l}34.4 \\
\end{array}$ & 12.5 & $\begin{array}{l}29.6 \\
5\end{array}$ & 2.038 & 0.069 & 69 & 0.002 & \begin{tabular}{|l|l|l|}
2 & 2.328
\end{tabular} & 1.98 \\
\hline $10 / 22 / 02$ & 2:23:15 PM & 25.451 & 22.822 & 25.169 & 24.081 & 23.3 & 31.98 & 35.724 & & & -1.2 & -3.003 & & & & & 8.07 & 34.3 & 12.5 & 29.6 & & 0.068 & & 0.002 & $2 \quad 2.276$ & 1.94 \\
\hline $10 / 22 / 02$ & 2:24:15 PM & 25.457 & 22.842 & 25.329 & 24.056 & 23.3 & 32.27 & 36.024 & & & -1.2 & & & & & 0 & 9.07 & 34.6 & 12.5 & 29.9 & & & & 0.002 & \begin{tabular}{|l|l|l|l|l|l|l|}
2 & 2.199
\end{tabular} & 1.87 \\
\hline $10 / 22 / 02$ & 2:25:15 PM & 25.477 & 22.863 & 25.255 & 24.116 & 23.437 & 32.434 & 35.906 & 4.814 & 27.725 & -1.2 & -3.168 & 51.085 & 0.4 & & 0 & 10.07 & 34.4 & 12.5 & 30.1 & 2 & & & 0.002 & 22.146 & 1.83 \\
\hline $10 / 22 / 02$ & 2:26:15 PM & 25.508 & 22.904 & 25.266 & 24.197 & 23.4 & 32.703 & 36.272 & & & -1.2 & -3.212 & & & & & 11.07 & 34.8 & 12.5 & 3 & & 0. & & 0.002 & $2 \quad 2.103$ & 1.79 \\
\hline $10 / 22 / 02$ & 2:27:15 PM & 25.498 & 22.909 & 25.371 & 24 & 23.5 & 32.535 & 35.8 & & & -1.2 & & & & & & 12. & 34.4 & 12.5 & & & & & & & 1.75 \\
\hline $10 / 22 / 02$ & 2:28:15 PM & 25.548 & 22.949 & 25.281 & 24.233 & 23.569 & 32.566 & 35.838 & 4.87 & & -1.2 & -3.336 & & 0.4 & & 0 & 13.07 & 34.4 & 12.5 & 30.2 & 2 & 0.062 & & 0.002 & \begin{tabular}{|l|l|}
2 & 2.032 \\
\end{tabular} & 1.73 \\
\hline $10 / 22 / 02$ & 2:29:15 PM & 25.538 & 22.944 & 25.366 & 24.173 & 23.589 & 32.865 & 35.968 & 4.799 & 28 & -1. & -3.377 & 51 & 0.4 & & 0 & 14.07 & 34.5 & 12.5 & 30.5 & 2. & 0.061 & 1 & 0.002 & \begin{tabular}{|l|l|}
2 & 1.981
\end{tabular} & 1.69 \\
\hline $10 / 22 / 02$ & 2:30:15 PM & 25.55 & 22.98 & 25.351 & 24. & 23. & & 35. & & & -1.2 & & & & & & & 34.5 & 12.5 & & & & & 02 & & 1.66 \\
\hline $10 / 22 / 02$ & 2:31:15 PM & 25.568 & 22.989 & 25.286 & 24.2 & 23.6 & 33.053 & 35.999 & & 28 & -1.2 & -3. & & 0.3 & & 0 & 57 & 34.5 & 12.5 & 30.6 & 2 & 0 & 9 & 0.002 & \begin{tabular}{|l|l|}
2 & 1.926 \\
\end{tabular} & 1.64 \\
\hline $10 / 22 / 02$ & 2:32: & 25.579 & 23.021 & 25.457 & 24. & 23.7 & 33.076 & 35 & & & -1. & . & & 0. & & & & 34.5 & 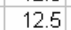 & & & & & 2 & 92 & 1.61 \\
\hline $10 / 22 / 02$ & 2:33:15 PM & 25.5 & 23.04 & 25. & & 23 & & 35. & & & -1. & -3 & & & & & & 34.4 & 12.5 & & & & & & & 1.59 \\
\hline $10 / 22 / 02$ & 2:34:15 PM & 25.608 & 23.059 & 25.381 & 24.393 & 23.779 & 33.111 & 35.778 & 4.764 & 28.556 & -1.286 & -3.559 & 50.916 & 0.387 & 0.0 & 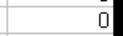 & 19.07 & 34.3 & 12.5 & 30.8 & 2.126 & 0.058 & .057 & 0.002 & \begin{tabular}{l|l}
2 & 1.851
\end{tabular} & 1.58 \\
\hline $10 / 22 / 02$ & 2:35:15 PM & 25.613 & 23.09 & 25.436 & \begin{tabular}{|l|}
24.474 \\
\end{tabular} & 23.84 & 33.281 & 35.852 & 4.746 & 28.655 & -1.286 & -3.594 & 50.718 & 0.384 & 0.001 & 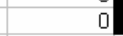 & 20.07 & 34.4 & 12.4 & 31.0 & 2.135 & 0.057 & 0.057 & 0.002 & \begin{tabular}{|l|l|}
2 & 1.826
\end{tabular} & 1.55 \\
\hline & & & & & & & & & & & & & & & & & & & & & & & & & & \\
\hline & $A_{N}$ & 25 & & & & 23 & & & & & -1 & & & & & 0. & & 34.4 & 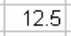 & & & & & & & 1.9 \\
\hline & Maximum & 25.6 & 23.1 & 25.5 & 24.5 & 23. & 33.3 & 36.3 & 4.9 & 28.7 & 1. & -1. & 51.2 & 0.6 & 0.0 & 0. & & 34.8 & 12.6 & 31.0 & 2. & 0.090 & 0 & 0.0034 & $\begin{array}{ll}4 & 3.359\end{array}$ & 2.9 \\
\hline & Med & 25.5 & 22.9 & 25.3 & 24 & 23 & 32.6 & 35 & & 7 & & . & & & & 0 & & 34.4 & 12.5 & 30.2 & & & & & & 1.8 \\
\hline & & 25 & 22.7 & 25 & & 23 & & & & & & & & & & 0 & & 34.2 & 12.4 & $\begin{array}{r}26.9 \\
+\end{array}$ & 1.854 & 0.057 & 0.057 & 0.0018 & \begin{tabular}{|l|l|}
8 & 1.826
\end{tabular} & 16 \\
\hline & $2 \times$ Std Dev & 0.167 & 0.226 & 0.198 & 0.286 & 0.429 & 2.015 & 0.269 & 0.099 & 1.991 & 1.262 & 1.051 & 0.230 & 0.114 & 0.000 & 0.000 & & 0.269 & 0.056 & 2.001 & 0.138 & 0.017 & 0.017 & 0.0008 & \begin{tabular}{l|l|}
8 & 0.760 \\
\end{tabular} & 0.647 \\
\hline imber of & Points Used ${ }^{*}$ & 18 & 18 & 18 & 18 & 18 & 18 & 18 & 18 & 18 & 18 & 18 & 18 & 18 & 18 & 10 & & 18 & 18 & 18 & 18 & 18 & 18 & 18 & 18 & 18 \\
\hline & & & & & & & & & & & & & & & & & & & & & & & & & & \\
\hline
\end{tabular}

Figure A48: AN-102R2, Batch 3B Slurry Test 1.00C - Inhibited Deionized \& Filtered Water - Raw \& Calculated Data 
WSRC-TR-2003-00204, REV. 0

SRT-RPP-2003-00087, REV. 0

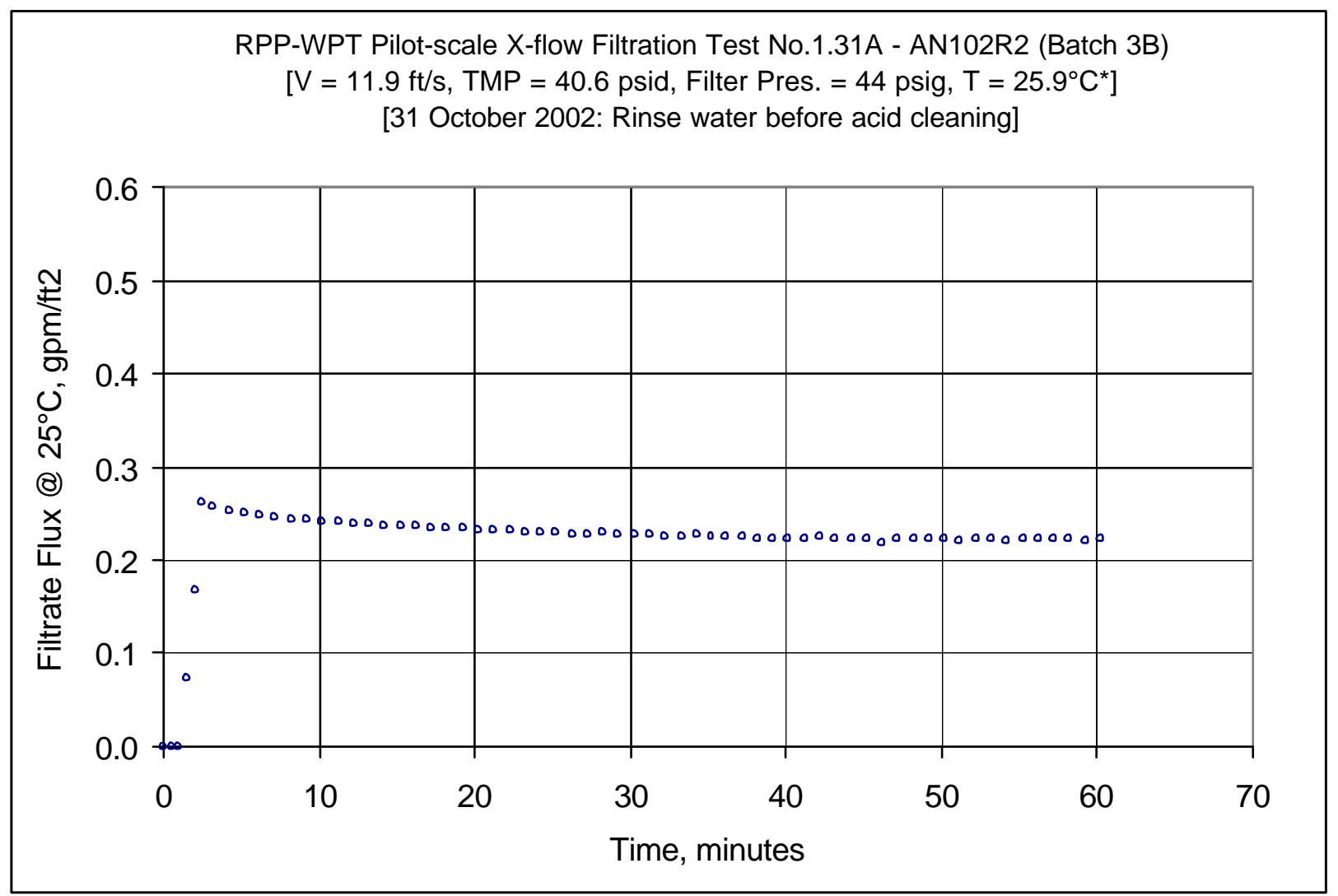

Figure A49: AN-102R2, Batch 3B Slurry Test 1.31A - Deionized \& Filtered Water Rinse after Dewatering Slurry 
WSRC-TR-2003-00204, REV. 0

SRT-RPP-2003-00087, REV. 0

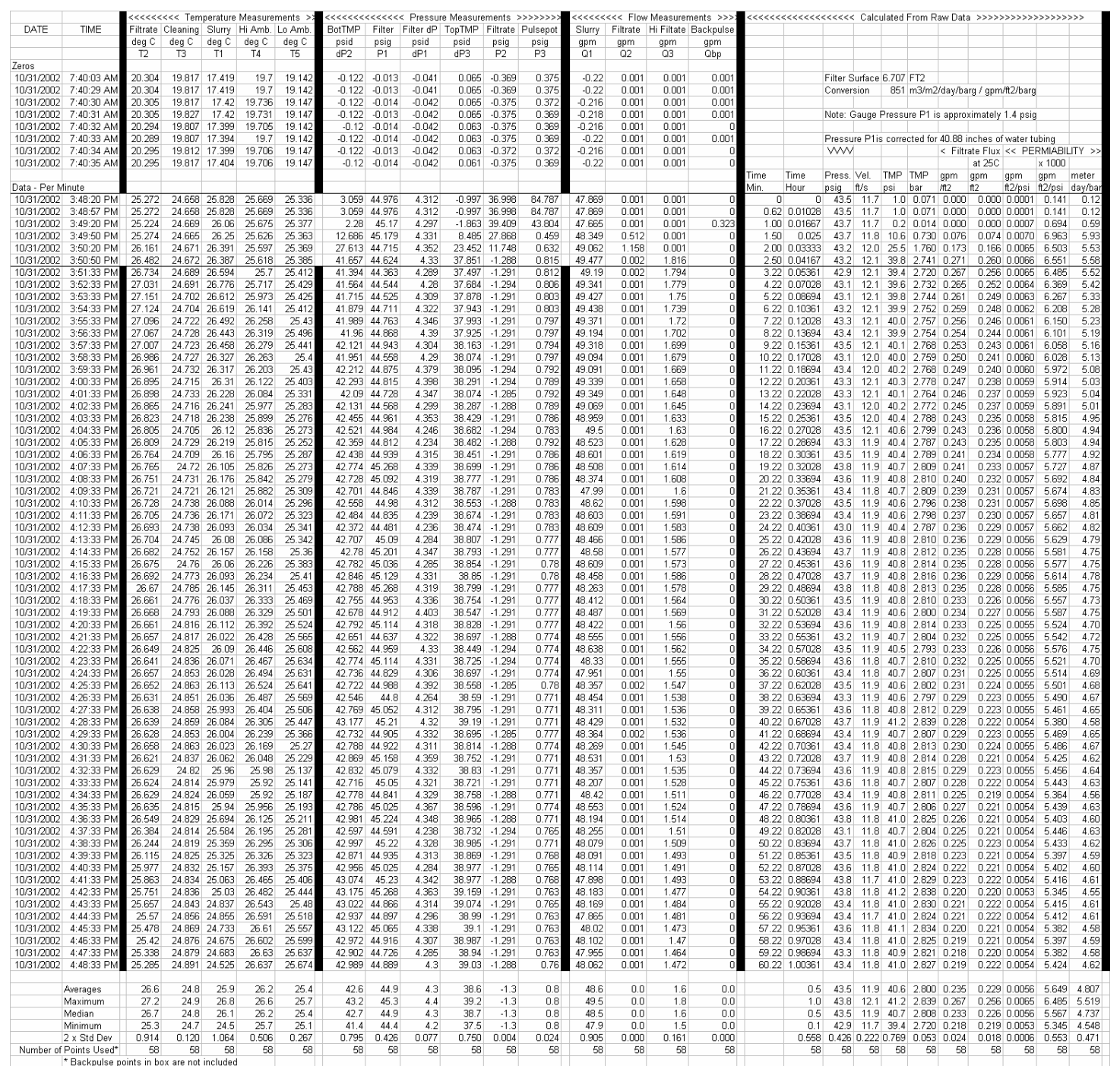

Figure A50: AN-102R2, Batch 3B Slurry Test 1.31A - Deionized \& Filtered Water Rinse after Dewatering Slurry - Raw \& Calculated Data 
WSRC-TR-2003-00204, REV. 0

SRT-RPP-2003-00087, REV. 0

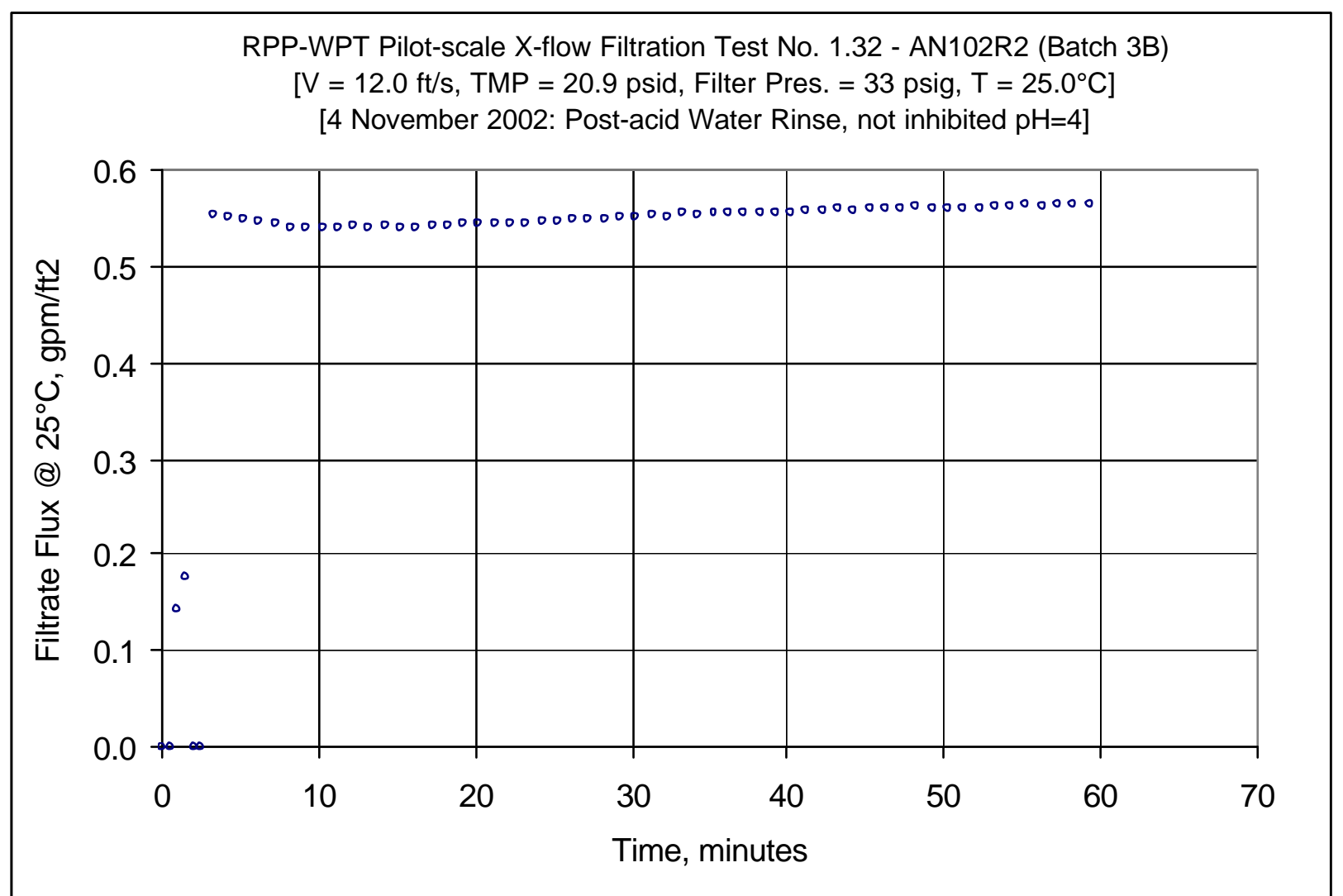

Figure A51: AN-102R2, Batch 3B Slurry Test 1.32 - Deionized \& Filtered Water (pH=4) Rinse after Acid Cleaning 
WSRC-TR-2003-00204, REV. 0

SRT-RPP-2003-00087, REV. 0

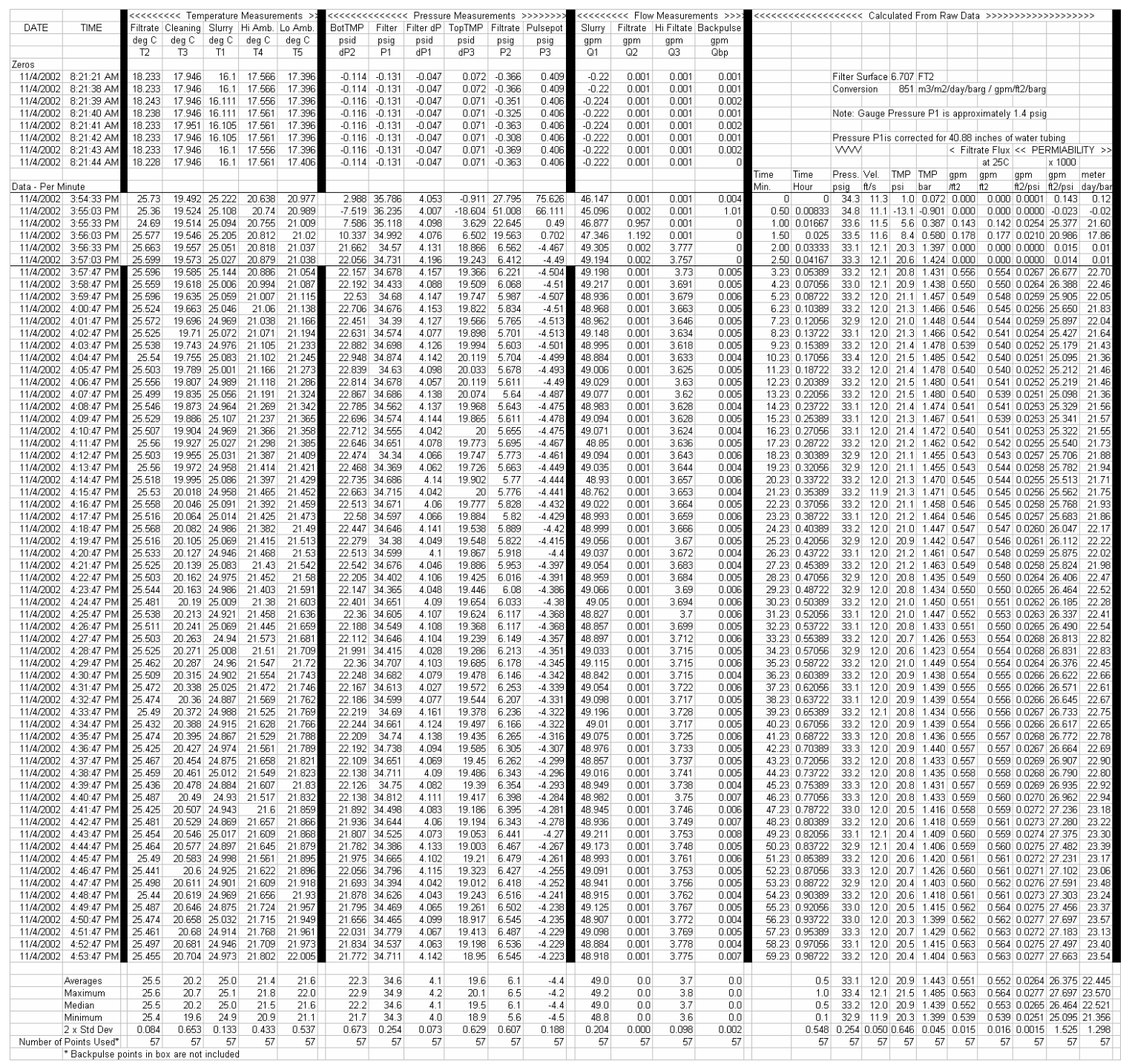

Figure A52: AN-102R2, Batch 3B Slurry Test 1.32 - Deionized \& Filtered Water (pH=4) Rinse after Acid Cleaning - Raw \& Calculated Data 
WSRC-TR-2003-00204, REV. 0

SRT-RPP-2003-00087, REV. 0

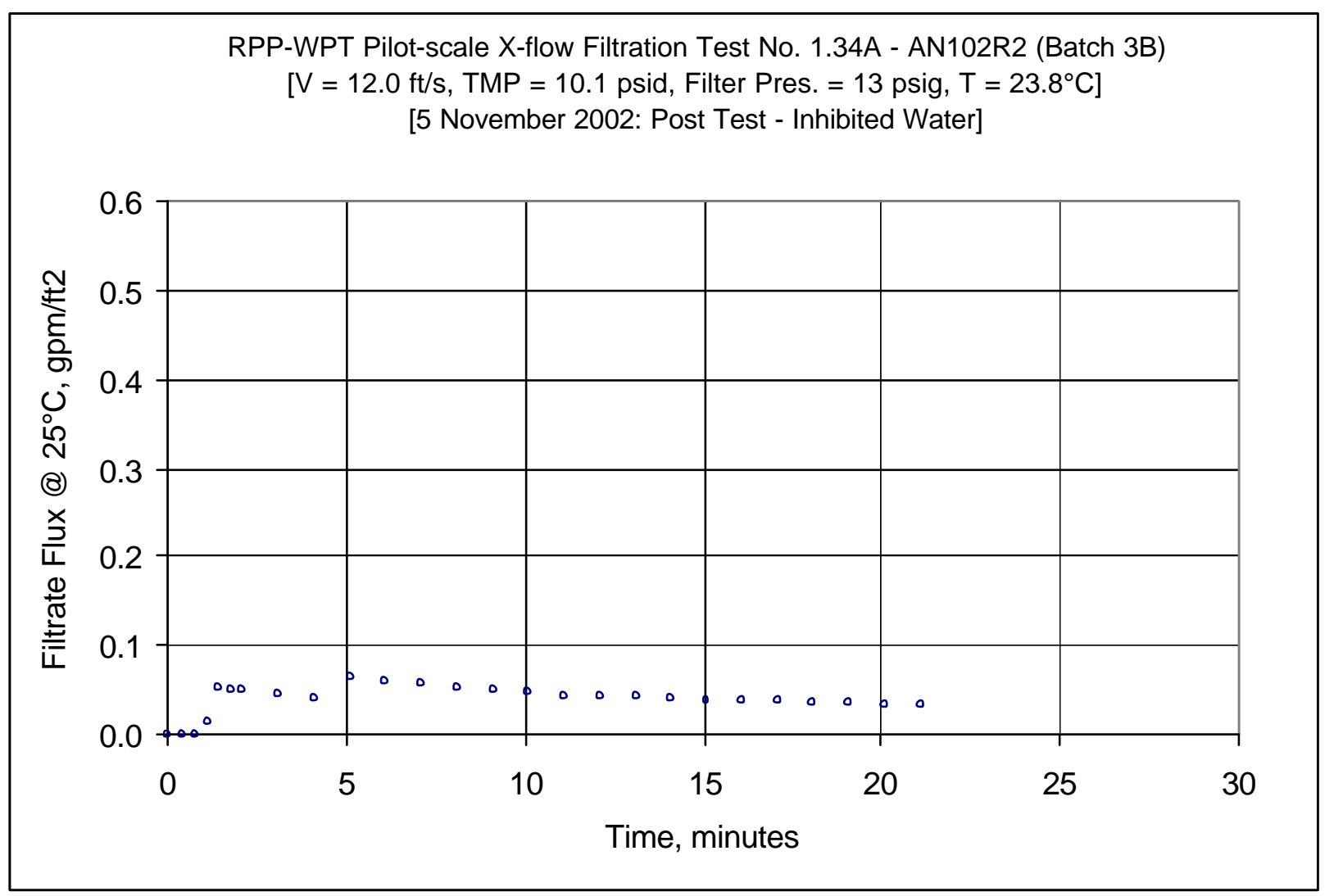

Figure A53: AN-102R2, Batch 3B Slurry Test 1.34A - Inhibited Deionized \& Filtered Water Test after Standard Slurry 
WSRC-TR-2003-00204, REV. 0

SRT-RPP-2003-00087, REV. 0

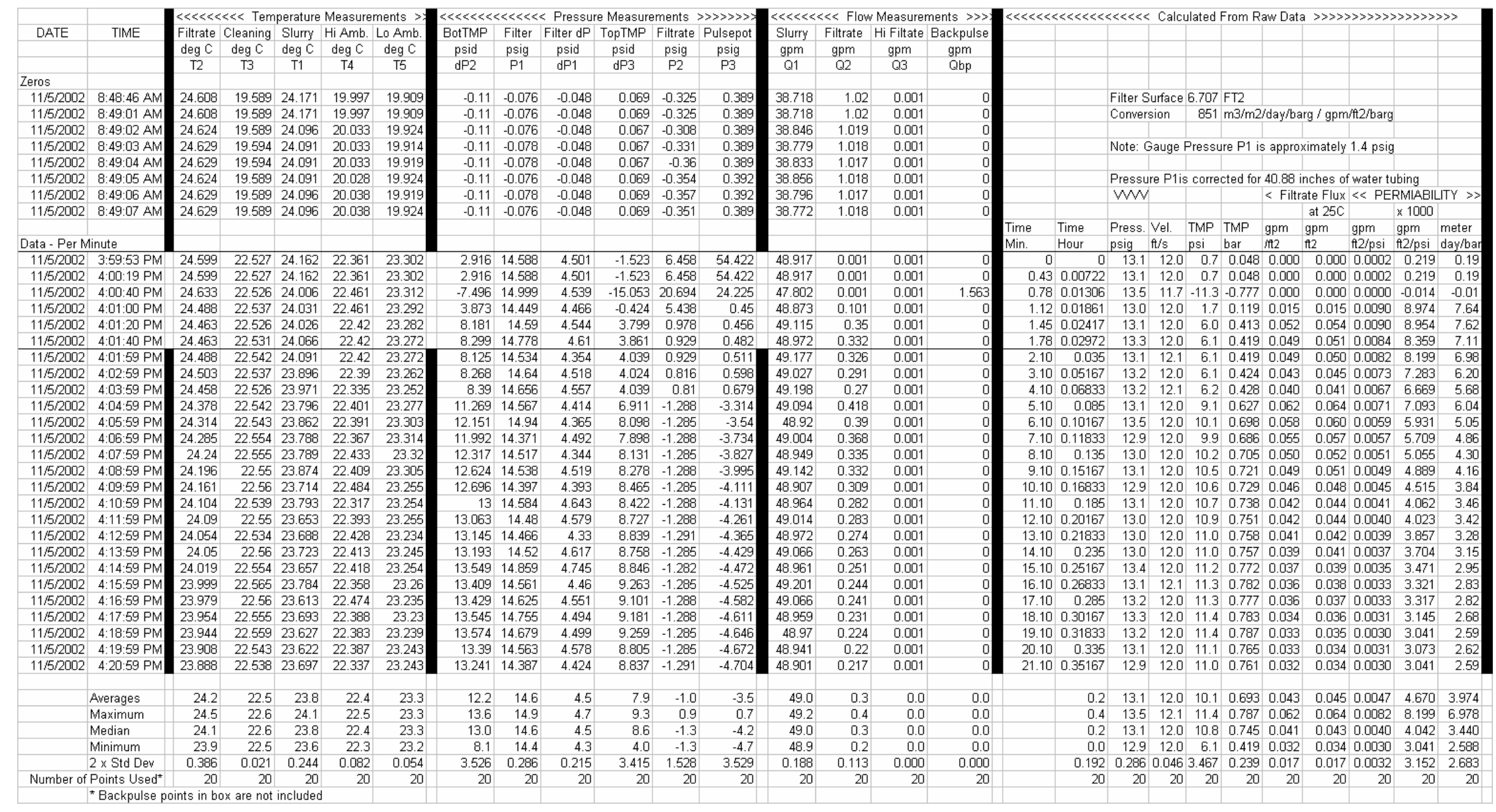

Figure A54: AN-102R2, Batch 3B Slurry Test 1.34A - Inhibited Deionized \& Filtered Water Test after Standard Slurry - Raw \& Calculated Data 
WSRC-TR-2003-00204, REV. 0

SRT-RPP-2003-00087, REV. 0

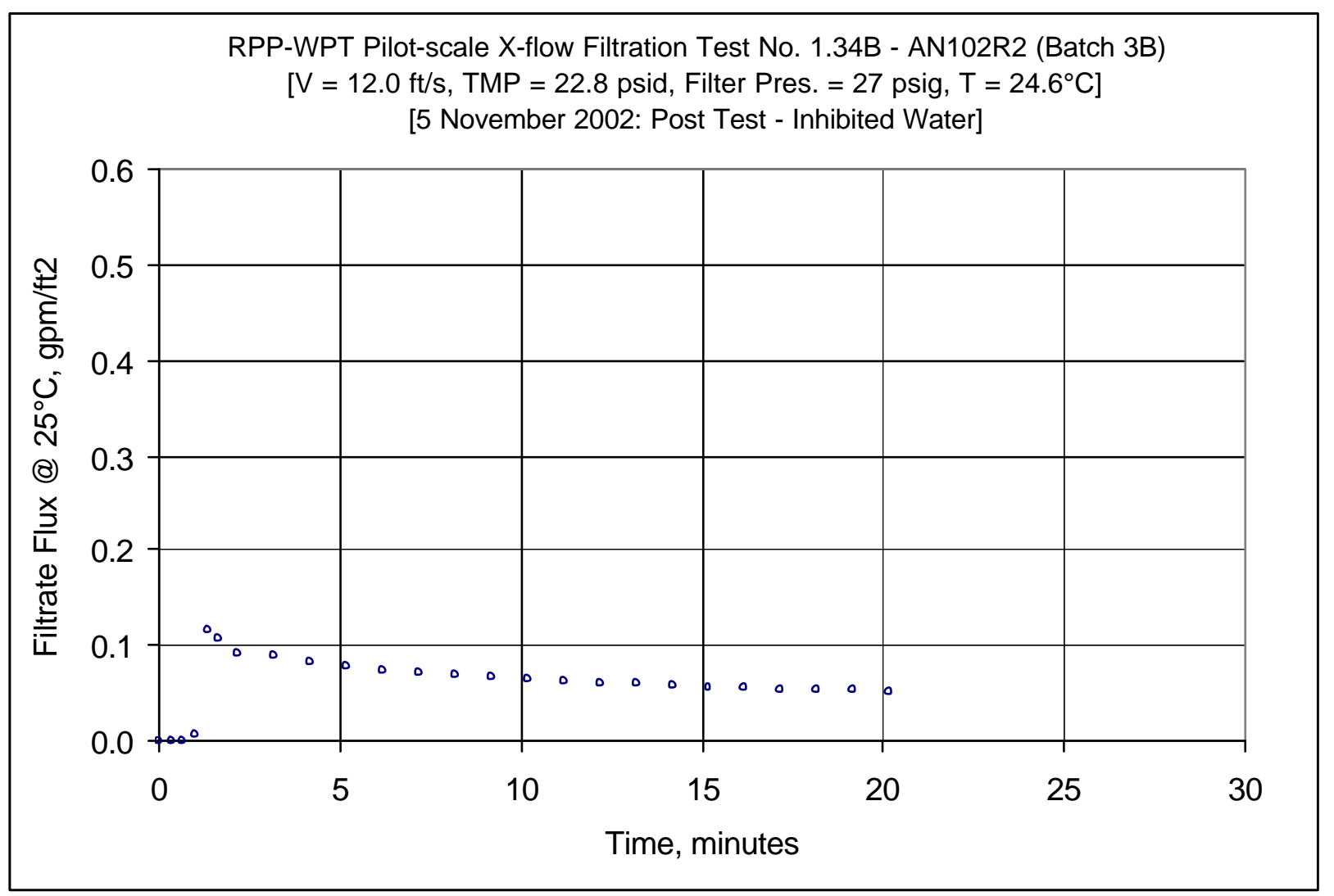

Figure A55: AN-102R2, Batch 3B Slurry Test 1.34B - Inhibited Deionized \& Filtered Water Test after Standard Slurry 
WSRC-TR-2003-00204, REV. 0

SRT-RPP-2003-00087, REV. 0

\begin{tabular}{|c|c|c|c|c|c|c|c|c|c|c|c|c|c|c|c|c|c|c|c|c|c|c|c|c|c|c|c|}
\hline \multirow{3}{*}{ DATE } & \multirow[b]{2}{*}{ TIME } & & \multirow{2}{*}{\multicolumn{6}{|c|}{ 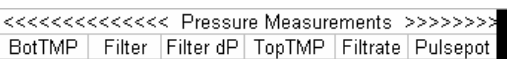 }} & & \multicolumn{11}{|c|}{ 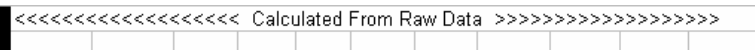 } \\
\hline & & \begin{tabular}{|l} 
Filtrate \\
$\operatorname{deg} C$
\end{tabular} & $\begin{array}{l}\text { Cleaning } \\
\operatorname{deg} C\end{array}$ & $\begin{array}{l}\text { Slurry } \\
\text { deg C }\end{array}$ & $\begin{array}{l}\text { HiAmb. } \\
\operatorname{deg} \mathrm{C}\end{array}$ & $\frac{\text { Lo Amb. }}{\operatorname{deg} C}$ & & & & & & & Slurry & Filtrate & Hi Filtate & Backpulse & & & & & & & & & & & \\
\hline & & & & & & & 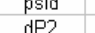 & psig & psid & psid & psig & psig & gpm & $\mathrm{gpm}$ & gpm & $\mathrm{gpm}$ & & & & & & & & & & & \\
\hline \multirow{2}{*}{\multicolumn{27}{|c|}{ Zeros }} & \\
\hline $11 / 5 / 2002$ & 48:46 AM & 24.608 & 19.589 & 24.171 & 19.997 & 19.909 & -0.11 & -0.076 & -0.048 & 0.069 & -0.325 & 0.389 & 38.718 & 1.02 & 0.001 & & & & \multicolumn{2}{|c|}{ Filter Surface 6.7} & 6.707 & 7FT2 & & & & & \\
\hline 11/5/2002 & 8:49:01 AM & 24.608 & 19.589 & 24.171 & 19.997 & 19.909 & -0.11 & -0.076 & -0.048 & 0.069 & -0.325 & 0.389 & 38.718 & 1.02 & 0.001 & & & & \multirow{2}{*}{\multicolumn{2}{|c|}{ Conversion }} & 851 & \multicolumn{5}{|c|}{$1 \mathrm{~m} 3 / \mathrm{m} 2 /$ day $/ \mathrm{barg} / \mathrm{gpm} / \mathrm{ta} 2 / \mathrm{barg}$} & \\
\hline 11/5/2002 & 8:49:02 AM & 24.624 & 19.589 & 24.096 & 20.033 & 19.924 & -0.11 & -0.076 & -0.048 & 0.067 & -0.308 & 0.389 & 38.846 & 1.019 & 0.001 & & & & & & & & & & & & \\
\hline $11 / 5 / 2002$ & 8:49:03 AM & 24.629 & 19.594 & 24.091 & 20.033 & 19.914 & -0.11 & -0.078 & -0.048 & 0.067 & -0.331 & 0.389 & 38.779 & 1.018 & 0.001 & & & & \multirow{2}{*}{\multicolumn{2}{|c|}{ Note: Gauge P }} & Pressul & 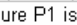 & is approxi & ximately & 4 psig & & \\
\hline 11/5/2002 & 8:49:04 AM & 24.629 & 19.594 & 24.091 & 20.033 & 19.919 & -0.11 & -0.078 & -0.048 & 0.067 & -0.36 & 0.389 & 38.833 & 1.017 & 0.0 & & & & & & & & & & & & \\
\hline $11 / 5 / 2002$ & 8:49:05 AM & 24.624 & 19.589 & 24.091 & 20.028 & 19.924 & -0.11 & -0.076 & -0.048 & 0.069 & -0.354 & 0.392 & 38.856 & 1.018 & 0. & & & & \multicolumn{8}{|c|}{ Pressure $P$ 1is corrected for 40.88 inches of water tubing } & \\
\hline $11 / 5 / 2002$ & 8:49:06 AM & 24.629 & 19.589 & 24.096 & 20.038 & 19.919 & -0.11 & -0.078 & -0.048 & 0.069 & -0.357 & 0.392 & 38.796 & 1.017 & 0.001 & & & & $\mathrm{~mW}$ & & & & Filtra & rate Flux & & ERMIABIL & LITY \\
\hline 11/5/2002 & 8:49:07 AM & 24.629 & 19.589 & 24.096 & 20.038 & 19.924 & -0.11 & -0.076 & -0.048 & 0.069 & -0.351 & 0.389 & 38.772 & 1.018 & 0.001 & & & & & & & & & at $25 \mathrm{C}$ & & $\times 1000$ & \\
\hline & & & & & & & & & & & & & & & & & & Time & Press. & Vel. & TMP & TMP & $\mathrm{gpm}$ & & gpm & $\mathrm{gnm}$ & meter \\
\hline a-Per M & & & & & & & & & & & & & & & & & & Hour & iin & $\mathrm{f} / \mathrm{s}$ & & 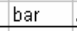 & & & $\mathrm{ft} 2 / \mathrm{ps}$ & $\mathrm{f} 2 / \mathrm{ps}$ & \\
\hline $11 / 5 / 2002$ & 25:03 PM & 23.915 & 22.555 & 24.135 & 22.389 & 23.205 & 3.204 & 28.664 & 4.468 & -1.319 & 20.442 & 3.714 & 18.651 & 0.00 & 0.001 & & & & 27 & 11.9 & & 00 & 0.00 & $\overline{0.0}$ & & 20.162 & 0.14 \\
\hline $11 / 5 / 2002$ & 4:25:24 PM & 23.915 & 22.555 & 24.135 & 22.389 & 23.205 & 3.204 & 28.664 & 4.468 & -1.319 & 20.442 & 3.714 & 48.651 & 0.0 & 1 & & 0.35 & 0.00583 & 27.2 & 11.9 & 0.9 & 0.065 & 0.000 & 0.000 & 0.0002 & 162 & 0.14 \\
\hline $11 / 5 / 2002$ & 25:43 PM & 23.876 & 22.552 & 24.141 & 22.395 & 23.216 & & 28.817 & & -14.238 & 33.923 & 36.687 & 47.479 & 0.001 & & 435 & 0.67 & 0.01111 & 27.3 & 11.6 & -10.9 & -0.749 & 0.000 & 0.000 & & 014 & -0.01 \\
\hline $11 / 5 / 2002$ & 26:03 PM & 1912 & 22.572 & 24.107 & 22.542 & 23.227 & 4.19 & 28.616 & 19 & -0.262 & 19.838 & 0.56 & 48.681 & 14 & & & 1.00 & 0.01667 & 27.1 & 11.9 & 2.0 & 0.135 & 0.006 & 06 & 0.0031 & 13.114 & 65 \\
\hline $11 / 5 / 2002$ & 26:23 PM & 24.122 & 22.572 & 24.132 & 22.481 & 23.217 & 16.565 & 28.577 & & 12.201 & 6.794 & 0.502 & 49 . & 0.754 & & & 1.33 & 0.02222 & 27.1 & 12.0 & 14.4 & 40.992 & 0.112 & 5 & 880 & 8.010 & \\
\hline $11 / 5 / 2002$ & 26:43 PM & 24.268 & 22.573 & 24.203 & 22.487 & 7370 & 19.094 & 28.629 & & 14.795 & 4.2 & 0.566 & & & & & 1.67 & & 27.2 & 12.1 & 16.9 & 1.168 & & 0.107 & & 6.308 & (0.JT \\
\hline $11 / 5 / 2002$ & M & \begin{tabular}{|l|}
24.359 \\
\end{tabular} & 22.573 & 24.333 & 22.487 & 23.208 & \begin{tabular}{|l|l|}
19.997 \\
\end{tabular} & 28.564 & & 15.699 & 3.2 & 0.653 & 49. & & & & 2.18 & & 27.1 & 12.1 & 17.8 & 1.231 & & 0.091 & & 82 & ( \\
\hline $11 / 5 / 2002$ & 4:28:14 PM & 24.481 & 22.57 & 24.25 & 22.635 & 23.22 & 22.482 & 28.6 & & 18.201 & 0.773 & -1.687 & 49 & 0.5 & & & 3.18 & & 27.1 & 12.0 & 20.3 & 3. 1.402 & 0. & & & & \\
\hline $11 / 5 / 2002$ & 4:29:14 PM & 24.517 & 22.566 & 24.436 & 22.57 & 23.196 & & 28.744 & & 18.619 & -0.045 & -2.101 & & 0.547 & & & 4.18 & 0.0 & 27.3 & 12.0 & 20.9 & 1.439 & 0.082 & 33 & 40 & 3.970 & 3.38 \\
\hline 11/5/2002 & 4:30:14 PM & & 22.583 & 24.339 & 22.719 & 23 & & 28. & & 19 & -0.609 & -2 & & & & & 5.18 & 339 & 27.1 & 12.0 & 21.5 & 51.485 & & & & 11 & 3.07 \\
\hline $11 / 5 / 2002$ & 31:14 PM & & 22.5 & 24.524 & 22.548 & 23. & & 28. & & & & & & & & & 8 & & 26.9 & 12.0 & 21.9 & & & & & & 187 \\
\hline $11 / 5 / 2002$ & 4:32:14 PM & 24.6 & 22. & & & & & & & & & & & & & & 7.18 & & 27.0 & 12.0 & 22.4 & 41. & & & & & 2.69 \\
\hline & & 24.7 & 22.5 & & & & & & & & & & & & & & & & 27.2 & 12.0 & 22.7 & & & & & & $2.55 \mathrm{~T}>\mathrm{C}$ \\
\hline $11 / 5 / 2002$ & 34:14 PM & 24.759 & 22.593 & 24 & 22.728 & 23. & & & & & -1. & & & & & & & & 27.1 & 12.0 & 23.0 & & & & & & \\
\hline 11/5/2002 & 4:35:14 PM & & 22.573 & & 22 & & & & & & -1 & & & & & & 10. & & 27.2 & 12.0 & 1 & 1. & & & & & 2.36 \\
\hline $11 / 5 / 2002$ & $6: 14$ & & & & & & & & & & & & & & & & & & 27.3 & & 23.5 & & & & & & 2.25 \\
\hline $11 / 5 / 2$ & 4:37:14 & & 22.5 & & & & & & & & & & & & & & & & 27.0 & & 23.4 & & & & & & 2.19 \\
\hline $11 / 5 / 2$ & 4:38:14 & 24.845 & 22.593 & 24.559 & 22.5 & & & 28. & & 21.681 & -1.2 & -3.5 & & & & & 13 & & 27.2 & 12.0 & 23.8 & & & & & 5. 2.494 & 2.12 \\
\hline $11 / 5 / 2002$ & $4:$ & 24,20 & 22.5 & 24 & 22.6 & & & 28. & & & 1 & & & & & & & & 27 & 12.0 & 23.7 & & & & & & 207 \\
\hline $11 / 5 / 2002$ & 4: & 24 & 22. & & 22 & & & 28 & & & & & & & & & & & 27 & & j & & & & & & \\
\hline $11 / 5$ & $4:$ & & & & & & & & & & & & & & & & & & 27 & & & & & & & & 8 \\
\hline 11/5/2002 & 4:42:14 & & 22. & & & & & & & & & & & & & & & & 27.1 & 12.0 & 24.2 & & & & & & 1.91 \\
\hline $11 / 5 / 2002$ & 4:43:14 PI & 24.899 & 22.627 & 24.693 & 22.622 & 23.2 & 26.235 & 28. & & 22.054 & -1.288 & & & 0.3 & & & 18.18 & 0.30306 & 27.0 & 11.9 & 24.1 & 1.665 & 0.053 & 0.054 & 40.0022 & 2.224 & 1.89 \\
\hline $11 / 5 / 2002$ & 4:44:14 PM & 24.92 & 22.644 & 24.689 & 22.769 & 23.2 & 26.242 & 28.37 & & 22.015 & -1.288 & -3.8 & & 0. & & & 19.18 & 0.31972 & 26.9 & 12.0 & 24.1 & 1.664 & 0.052 & 0.053 & 0.0022 & 2.182 & 1.86 \\
\hline 11/5/2002 & 4:45:14 PM & 24.916 & 22.664 & 24.685 & 22.805 & 23.2 & 26.42 & 28.448 & 4.464 & 22.217 & -1.288 & -3.9 & 48.609 & 0.345 & 0.001 & & 20.18 & 0.33639 & 27.0 & 11.9 & 24.3 & 3. 1.677 & 0.051 & 0.052 & 20.0021 & 2.134 & 4 \\
\hline & & & & & & & & & & & & & & & & & & & & & & & & & & & \\
\hline & & & & & & & & & & & & & & & & & & 0.3 & 27.3 & 12.1 & 24.3 & 31. & & & & 32 & 4.325 \\
\hline & $\mathrm{Me}$ & 24.8 & 22.6 & 24.6 & 22.7 & 23 & 25.6 & 28 & 4. & 21.3 & -1.3 & -3 & & 0. & 0. & & & 0.2 & 27.1 & 12.0 & 23.4 & 41.616 & 0.061 & 0. & 20.0026 & 2.645 & 2.251 \\
\hline & & 24.4 & 22.6 & 24.3 & 22.5 & & 20.0 & 28 & & 15.7 & -1.3 & -3. & & 0. & & & & 0.0 & 26.9 & 11.9 & 17.8 & $\begin{array}{ll}8 & 1.231\end{array}$ & 0.051 & 0.052 & 20.0021 & $\begin{array}{ll}1 & 2.134 \\
\end{array}$ & $\begin{array}{l}4.816 \\
\end{array}$ \\
\hline & & 0.319 & 0.054 & 0.281 & $011^{6}+20$ & & 20700 & 0.22 & 0 & ( & 208 & 19 & & 15 & & & & 183 & 0.223 & & 258 & 30.225 & & 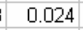 & 16 & 5 & 357 \\
\hline N & & 19 & 19 & & 19 & & 19 & 19 & 19 & 19 & 1 & & & & & & & & & & & & & & & & \\
\hline & ${ }^{*} \mathrm{Ba}$ & in $\mathrm{h}$ & $m_{0}$ & Ided & & & & & & & & & & & & & & & & & & & & & & & \\
\hline
\end{tabular}

Figure A56: AN-102R2, Batch 3B Slurry Test 1.34B - Inhibited Deionized \& Filtered Water Test after Standard Slurry - Raw \& Calculated Data 
WSRC-TR-2003-00204, REV. 0

SRT-RPP-2003-00087, REV. 0

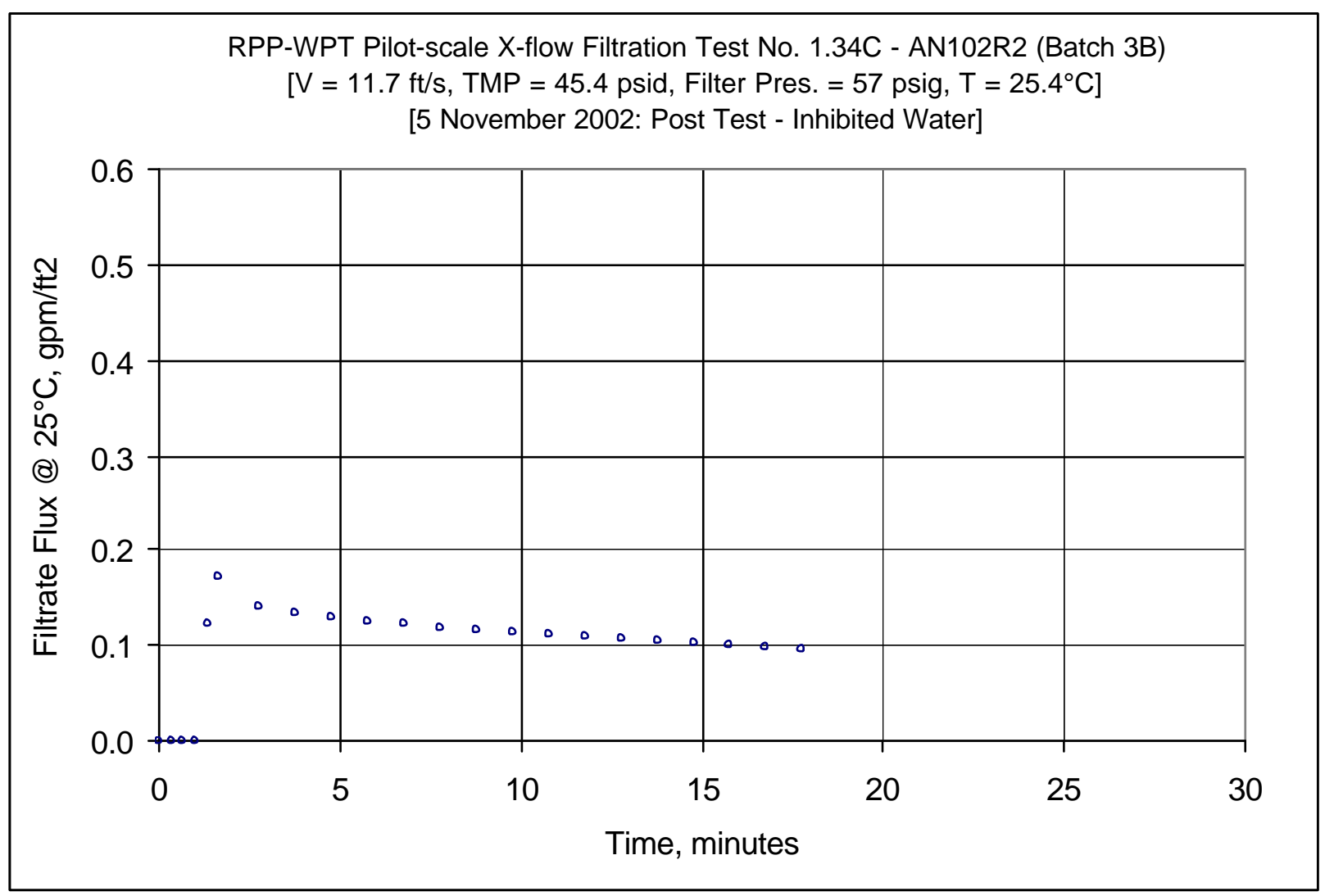

Figure A57: AN-102R2, Batch 3B Slurry Test 1.34C - Inhibited Deionized \& Filtered Water Test after Standard Slurry 
WSRC-TR-2003-00204, REV. 0

SRT-RPP-2003-00087, REV. 0

\begin{tabular}{|c|c|c|c|c|c|c|c|c|c|c|c|c|c|c|c|c|c|c|c|c|c|c|c|c|c|c|c|}
\hline \multirow{4}{*}{ DATE } & \multirow{3}{*}{ TIME } & \multicolumn{5}{|c|}{ « $\ll<\ll<<<$ Temperature Measurements $>$} & \multicolumn{6}{|c|}{ 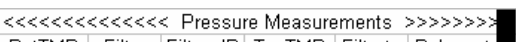 } & \multicolumn{4}{|c|}{$\ll \ll<\ll<<<<$ Flow Measurements $\gg \gg$} & \multirow{2}{*}{\multicolumn{11}{|c|}{ Calculated From Raw Data }} \\
\hline & & Filtrate & Cleaning & Slurry & Hi Amb. & Lo Amb. & BotTMP & Filter $\mathrm{F}$ & Filter dP & TopTMP & Filtrate & Pulsepot & Slurry & Filtrate & Hi Filtate & Backpulse & & & & & & & & & & & \\
\hline & & $\operatorname{deg} C$ & $\operatorname{deg} C$ & $\operatorname{deg} C$ & $\operatorname{deg} C$ & $\operatorname{deg} C$ & psid & psig & psid & psid & psig & psig & $\mathrm{gpm}$ & $\mathrm{gpm}$ & $\mathrm{gpm}$ & $\mathrm{gpm}$ & & & & & & & & & & & \\
\hline \multirow{2}{*}{\multicolumn{28}{|c|}{ Zeros }} \\
\hline & & & & & & & & & & & & & & & & & & & & & & & & & & & \\
\hline $11 / 5 / 2002$ & :48:46 AM & 1.608 & B 19.589 & 24.171 & 19.997 & 19.909 & -0.11 & -0.076 & -0.048 & 069 & -0.325 & 389 & .718 & 1.02 & 0.001 & & & & \multicolumn{4}{|c|}{ Filter Surfac } & & & & & \\
\hline $11 / 5 / 2002$ & 9:01 AM & 1.608 & 589 & 24.171 & 9.997 & 19.909 & -0.11 & -0.076 & -0.048 & 069 & -0.325 & 389 & 88.718 & 1.02 & 0.001 & & & & \multicolumn{2}{|c|}{ Conversion } & $851 \mathrm{r}$ & \multicolumn{5}{|c|}{$1 \mathrm{FT} 3 / \mathrm{m} 2 /$ day/barg / gpm/f2/barg } & \\
\hline $11 / 5 / 2002$ & 8:49:02 AM & 24.624 & $4 \quad 19.589$ & 24.096 & 20.033 & 19.924 & -0.11 & -0.076 & -0.048 & 0.067 & -0.308 & 0.389 & 38.846 & 1.019 & 0.001 & & & & & & & & & & & & \\
\hline $11 / 5 / 2002$ & 8:49:03 AM & 24.629 & 19.594 & 24.091 & 20.033 & 19.914 & -0.11 & -0.078 & -0.048 & 0.067 & -0.331 & 0.389 & 38.779 & 1.018 & 0.001 & & & & \multicolumn{8}{|c|}{ pproximately $1.4 \mathrm{psig}$} & \\
\hline $11 / 5 / 2002$ & 8:49:04 AM & 24.629 & 19.594 & 24.091 & 20.033 & 19.919 & -0.11 & -0.078 & -0.048 & 0.067 & -0.36 & 0.389 & 38.833 & 1.017 & 0.001 & & & & & & & & & & & & \\
\hline $11 / 150002$ & 8.49.05 AM & 24,624 & 19589 & 24091 & 20028 & 19924 & -011 & -0.076 & $-0,048$ & 0069 & -0.054 & 0392 & 38.856 & 1018 & & & & & \multicolumn{8}{|c|}{ nches of water tut } & \\
\hline $11 / 5 / 2002$ & 8:49:06 AM & 24.629 & 19.589 & 24.096 & 20.038 & 19.919 & -0.11 & -0.078 & -0.048 & 0.069 & -0.357 & 0.392 & 38.796 & 1.017 & 0.001 & & & & \multirow{2}{*}{\multicolumn{2}{|c|}{$\mathrm{m}$}} & & & $<$ Filtrat & te Flux & & IABILI & ILITY \\
\hline $11 / 5 / 2002$ & 8:49:07 AM & 24.629 & 19.589 & 24.096 & 20.038 & 19.924 & -0.11 & -0.076 & -0.048 & 0.069 & -0.351 & 0.389 & 38.772 & 1.018 & 0.001 & & & & & & & & & at $25 \mathrm{C}$ & & $\times 1000$ & \\
\hline & & & & & & & & & & & & & & & & & Time & Time & Press. $\mathrm{V}$ & Vel. & TMP T & TMP g & $\mathrm{gpm}$ & $\mathrm{gpm}$ & gpm & $\mathrm{gpm}$ & meter \\
\hline Data - Per M & Minute & & & & & & & & & & & & & & & & (n) & Hour & psig fl & $\mathrm{ft} / \mathrm{s}$ & psi b & bar & ift2 2 & $\pi 2$ & $\mathrm{f} 2 / \mathrm{psi}$ & $\mathrm{fl2} / \mathrm{psi}$ & \\
\hline$\frac{11 / 5 / 2002}{2}$ & 4:51:46 PM & 25.802 & 22.73 & 26.056 & 23.031 & 23.249 & 2.937 & 57.713 & 4.379 & -1.542 & 49.976 & 97.689 & 48.37 & 0.00 & 0.0 & & U & Hour & 56.2 & 11.9 & 0.7 & 0.0480 & 0.000 & 0.000 & 0.0002 & 20.208 & 0.18 \\
\hline $11 / 5 / 2002$ & $2: 06 \mathrm{PM}$ & 25.793 & 22.751 & 26.127 & 22.961 & 23.265 & 3.233 & 58.444 & & -1.03 & 50.338 & 96.769 & 47.529 & 0 & & & 33 & 0.00556 & 57.0 & 11.6 & 1.1 & & & & & & \\
\hline $11 / 5 / 2002$ & $52: 26 \mathrm{PM}$ & 25.217 & 22.736 & 26.057 & 22.951 & 23.255 & -2.848 & 58.367 & & -6.93 & 56.584 & 57.25 & 46.965 & 0.0 & & 0.63 & 0.67 & 0.01111 & 56.9 & 11.5 & -4.9 & $-0.337 \mathrm{C}$ & 00 & 0 & 000 & 30 & -0.03 \\
\hline $11 / 5 / 2002$ & 62:46 PM & 25.248 & 22.746 & 26.027 & 23.027 & 23.26 & & 57.999 & & -0.928 & 49.979 & 48.955 & 47.51 & & & & 1.00 & 0.01667 & 56.5 & 11.6 & 1.2 & 0.081 & 0.000 & 0.000 & 0.0002 & $\begin{array}{ll}2 & 0.248\end{array}$ & $\begin{array}{ll}3 & 0.21\end{array}$ \\
\hline $11 / 5 / 2002$ & 4:53:06 PM & 25.574 & 22.747 & 26.073 & 23.078 & 23.261 & 18.325 & 57.891 & 4. & 14.109 & 35.065 & 0.482 & 48.336 & 3 & & & 1.33 & 0.02222 & 56.4 & 11.8 & 16.2 & $1.118 \mathrm{c}$ & 0.127 & 0. & 176 & $\begin{array}{ll}6 & 7.610\end{array}$ & 10 \\
\hline $11 / 5 / 2002$ & $3: 26 \mathrm{PM}$ & 264 & & 26.168 & & 23.266 & 39.182 & 58.079 & & 34.979 & 13.725 & & & & & & 67 & & 56.6 & 11.9 & 37.1 & 2.557 & 0.178 & & & & 8 \\
\hline $11 / 5 / 2002$ & $4: 32 \mathrm{PM}$ & & & 26.085 & 23.281 & 23.283 & 42.161 & 57.885 & & 38.091 & & 3.319 & & & & & 2.77 & & 56.4 & 11.9 & 40.1 & 2.7670 & & & & & 2.97 \\
\hline $11 / 5 / 2$ & 5.370 & & 22 & & & & & & & & & & & & & & 3 & & 56.5 & 11.8 & 41.2 & & & & & & 2.77 \\
\hline $11 / 5 / 2002$ & 4:56:32 PM & 674 & 22.757 & 26.273 & 23.219 & 23.30 & 44.215 & 57.848 & 4. & 39.997 & 8.3 & 184 & 48. & 6 & & & 4.77 & 0.0 & 56.4 & 11.8 & 42.1 & 2.903 & 0.134 & 0.129 & 0.0031 & 061 & 2.61 \\
\hline $11 / 5 / 2002$ & $67: 32 \mathrm{PM}$ & & 22.769 & 26.185 & & & & 58.0 & & & 7. & 67 & & & & & 5.77 & & 56.6 & 11.8 & 43.0 & 2.966 & 0.130 & & & & 3 \\
\hline $11 / 5 / 2002$ & 4:58:32 PM & & 5 & 26.271 & 23 & & & & & & & 1.4 & & & & & & & 56.5 & 11.8 & 43.6 & & & & & & 2.38 \\
\hline $11 / 5 / 2002$ & $0.320 M$ & 26.7 & & & & & & & & & & & & & & & & & & & 44.5 & & & & & & 2.28 \\
\hline $11 / 5 /$ & $5: 00:$ & 26.7 & 22.7 & 25 & & & & & & & & & & & & & & & & 11.7 & 0 & & & & & $\begin{array}{ll}62 . \\
6\end{array}$ & 2.20 \\
\hline $11 / 5 / 2$ & 5:01: & 26501 & 22.748 & 25. & & & & & & & & & & & & & & & & 11.7 & 45.7 & & & & & & 2.20 \\
\hline $11 / 5 / 2002$ & 5:02:32 PM & & & & & & & 58 & & & & & & & & & & & 56 & 11.7 & 46.3 & & & & & & 04 \\
\hline & 5:03: & & & & & & & & & & & & & & & & & & & 11.7 & 46.8 & & & & & & 4 \\
\hline $11 / 5 / 2$ & $5: 04: 32$ & & 22.7 & 24.9 & & & & 57. & & & & & & & & & & & 56.4 & 11.7 & 47.2 & & & & & & .92 \\
\hline $11 / 5 / 2002$ & 5:05:32 P & 25.861 & 22.789 & 24.719 & 23 & 23. & 49.768 & 58.055 & 4.1 & & 3. & & 47.695 & $0.6-6-2$ & & & 13. & 0.2 & 56.6 & 11.7 & \begin{tabular}{|l|}
47.8 \\
\end{tabular} & 3.294 & 0. & 0.104 & 222 & 80 & 5 \\
\hline $11 / 5 / 2002$ & $5: 06: 32 \mathrm{PM}$ & 25.727 & 22.785 & & 23.869 & & 50.151 & 58.29 & & 48 & 2. & -0.7 & 47 & & & & 14 & & 56.8 & 11.7 & 48.1 & & & 2 & 21 & & 1.81 \\
\hline & & 25.593 & 22.791 & & & & & 58. & & & & & & & & & & & & & & & & & & & \\
\hline $11 / 5 /$ & $5: 0$ & 25 & & & & & & 57 & & & & & & & & & & & & 11.7 & 48.5 & & & & & & .72 \\
\hline $11 / 5 / 2002$ & 5:09:32 PM & 25.4 & 22.814 & 24.498 & 23.883 & 23.4 & 50.911 & 57.932 & 4.202 & 46.848 & 1.724 & -1.2 & 47.546 & 0.636 & 0.001 & & 17.77 & 0.29611 & 56.5 & 11.7 & 48.9 & 3.370 & 0.095 & 0.096 & 0.0020 & 1.968 & 67 \\
\hline & & & & & & & & & & & & & & & & & & & & & & & & & & & \\
\hline & & & & & & & & & & & & & & & & & & & 56 & 11.7 & 45.4 & 3. & 0.116 & 0.114 & 0.0025 & 2.5 & 2.1 \\
\hline & & & & & & & & & & & & & & & & & & & & 11.9 & & & & & & & \\
\hline & & & & & & & & & & & & & & & & & & & & & & & & & & & \\
\hline & & & & & & & & & & & & & & & & & & & 1.4 & 11. & & & & & & & \\
\hline & & 0.962 & 0.042 & 1.374 & 0.558 & 0.111 & 5.312 & 0.252 & 0.072 & 5.395 & 5.284 & 2.723 \# & 0.568 & 0.202 & 0.000 & 0.00 & & & 0.252 & 0.1395 & 5.353 & 0.369 & 0.030 & 0.026 & 0.0009 & $\begin{array}{ll}9 & 0.889\end{array}$ & 0.756 \\
\hline & Points Used ${ }^{*}$ & 16 & 16 & 16 & 16 & a & 16 & 16 & 16 & 16 & 16 & 100 & 16 & 16 & 16 & & & & 16 & 16 & 16 & 16 & 16 & 16 & 16 & 16 & \\
\hline & & & & & & & & & & & & & & & & & & & & & & & & & & & \\
\hline
\end{tabular}

Figure A58: AN-102R2, Batch 3B Slurry Test 1.34C - Inhibited Deionized \& Filtered Water Test after Standard Slurry - Raw \& Calculated Data 
WSRC-TR-2003-00204, REV. 0

SRT-RPP-2003-00087, REV. 0

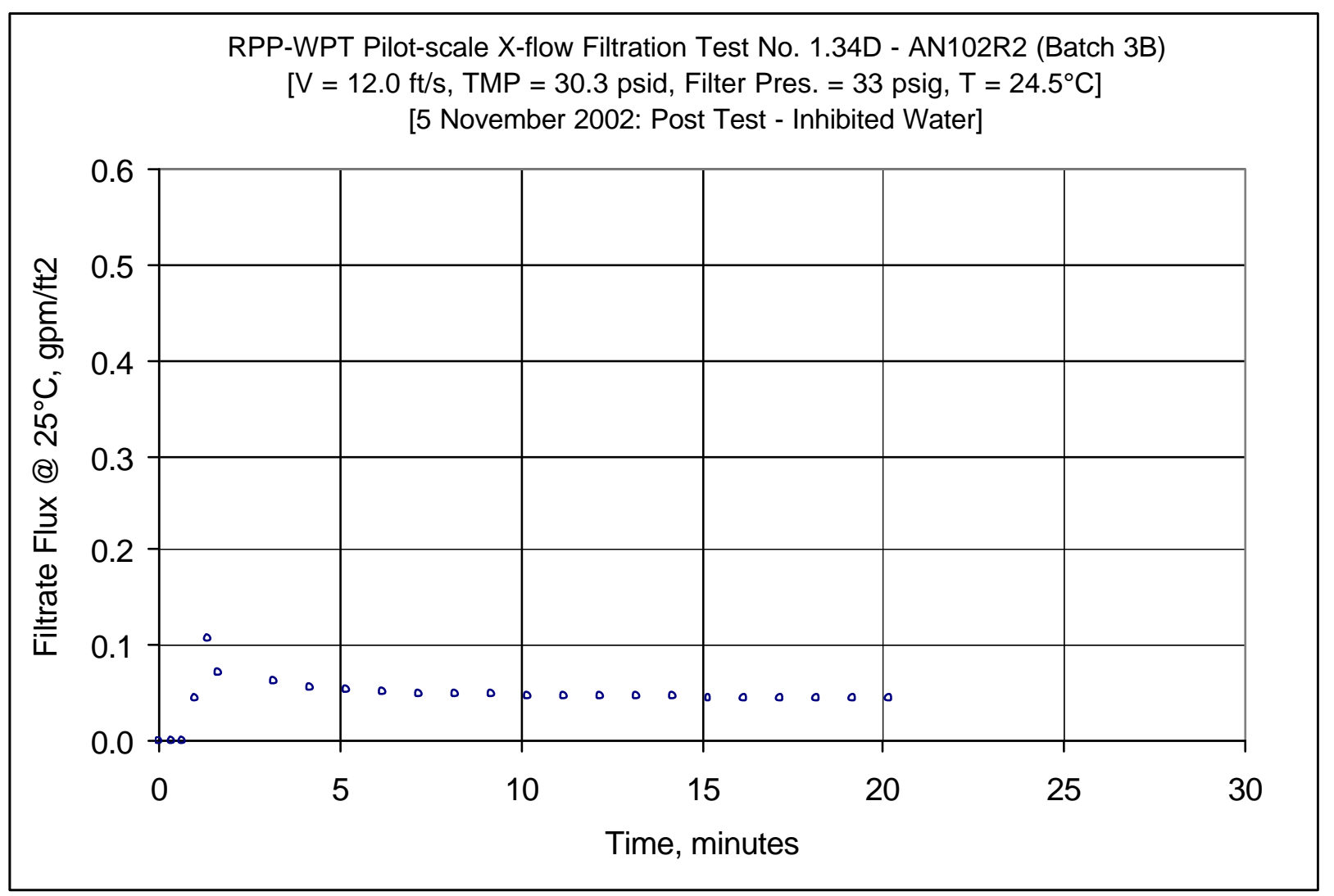

Figure A59: AN-102R2, Batch 3B Slurry Test 1.34D - Inhibited Deionized \& Filtered Water Test after Standard Slurry 
WSRC-TR-2003-00204, REV. 0

SRT-RPP-2003-00087, REV. 0

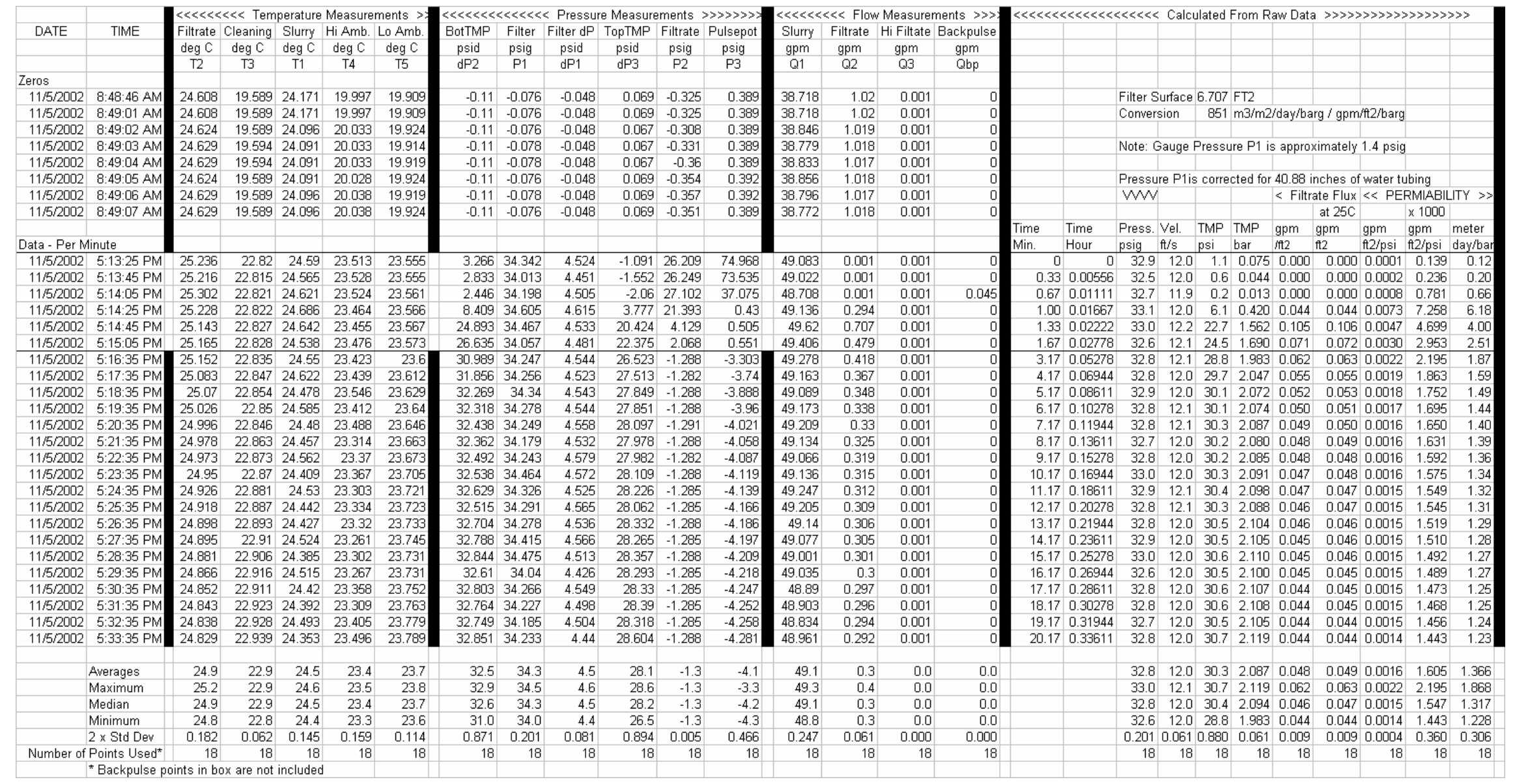

Figure A60: AN-102R2, Batch 3B Slurry Test 1.34C - Inhibited Deionized \& Filtered Water Test after Standard Slurry - Raw \& Calculated Data 
WSRC-TR-2003-00204, REV. 0

SRT-RPP-2003-00087, REV. 0

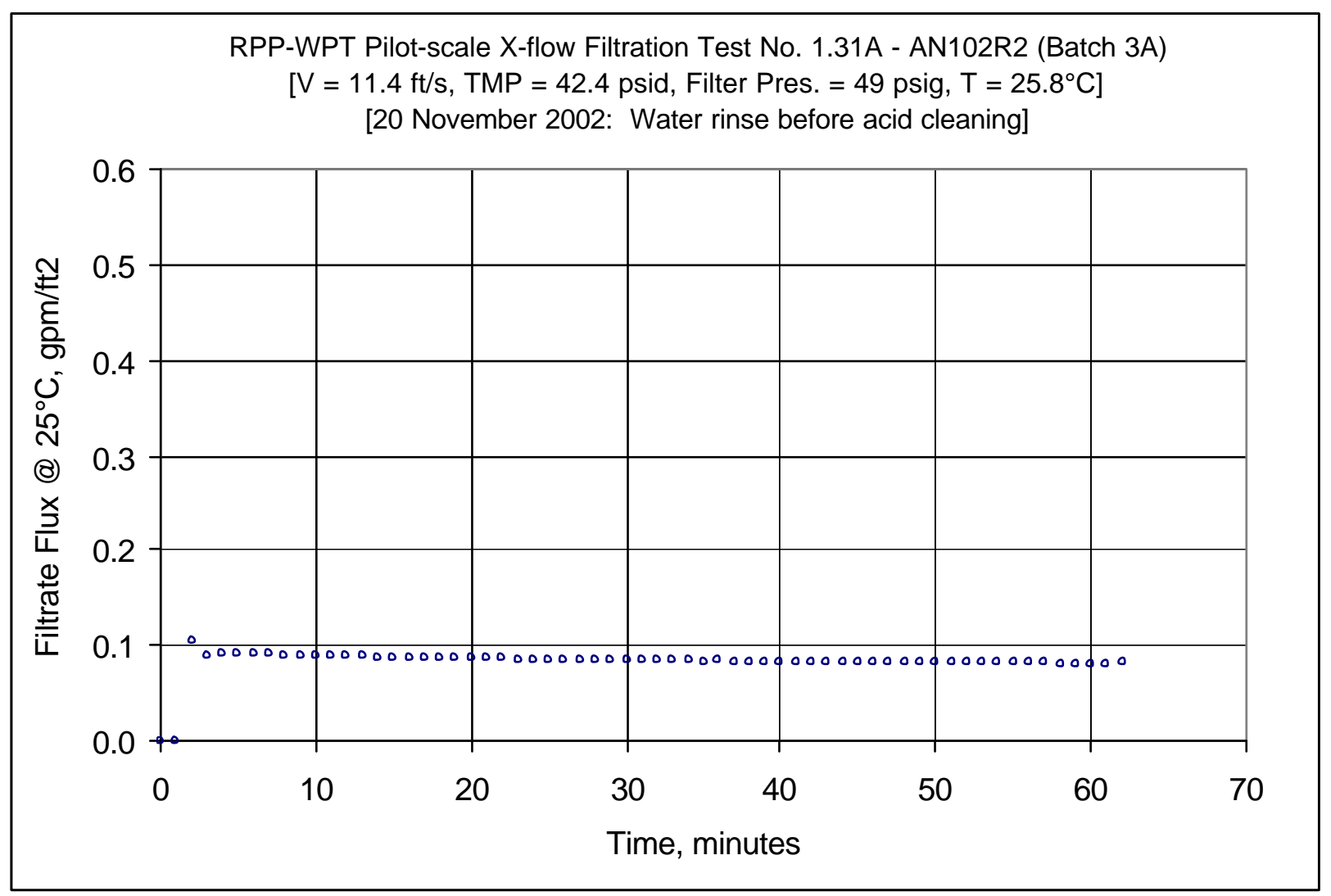

Figure A61: AN-102R2, Batch 3A Slurry Test 1.31A - Deionized \& Filtered Water Rinse after Dewatering Slurry 
WSRC-TR-2003-00204, REV. 0

SRT-RPP-2003-00087, REV. 0

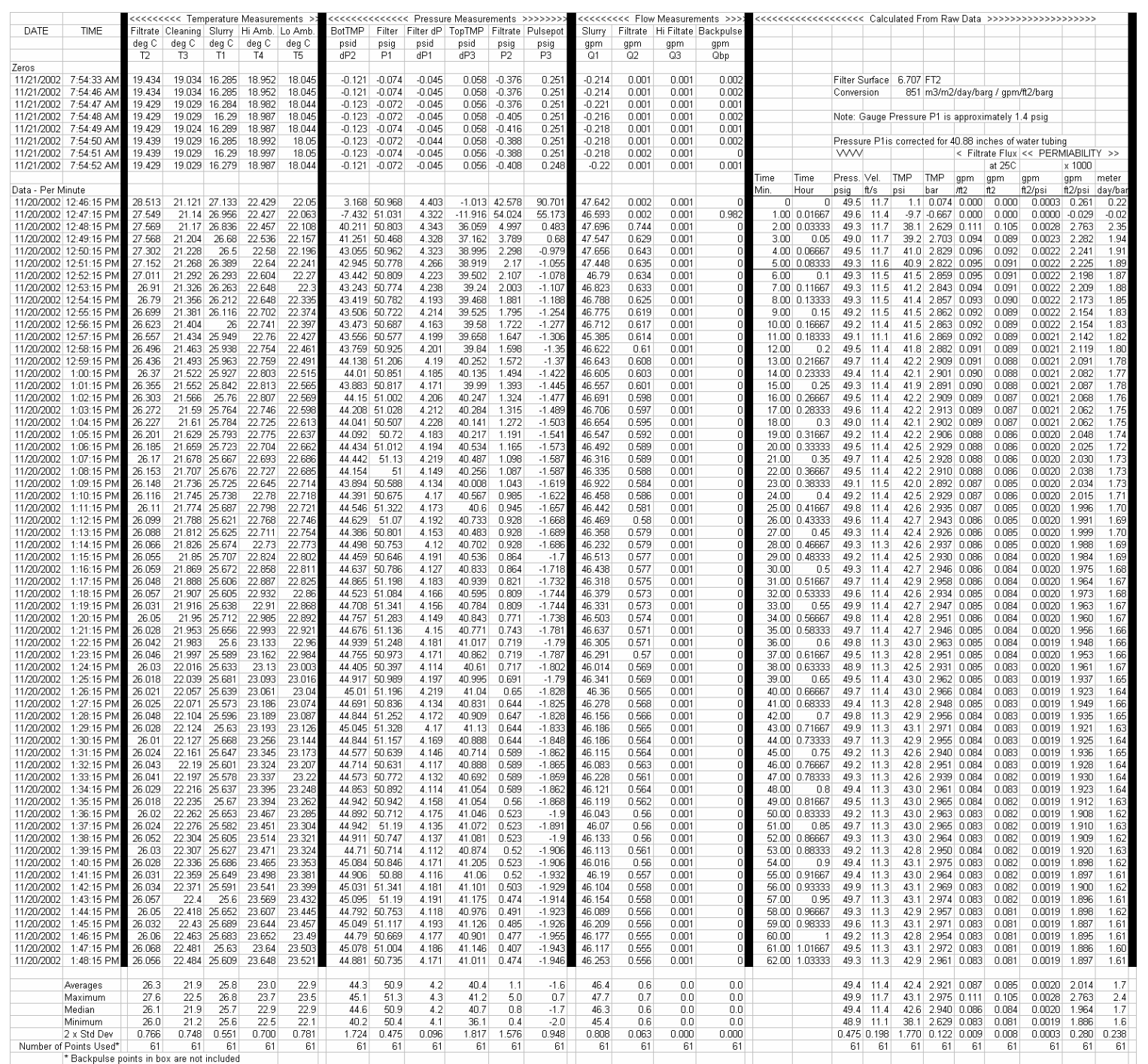

Figure A62: AN-102R2, Batch 3A Slurry Test 1.31A - Deionized \& Filtered Water Rinse after Dewatering Slurry - Raw \& Calculated Data 
WSRC-TR-2003-00204, REV. 0

SRT-RPP-2003-00087, REV. 0

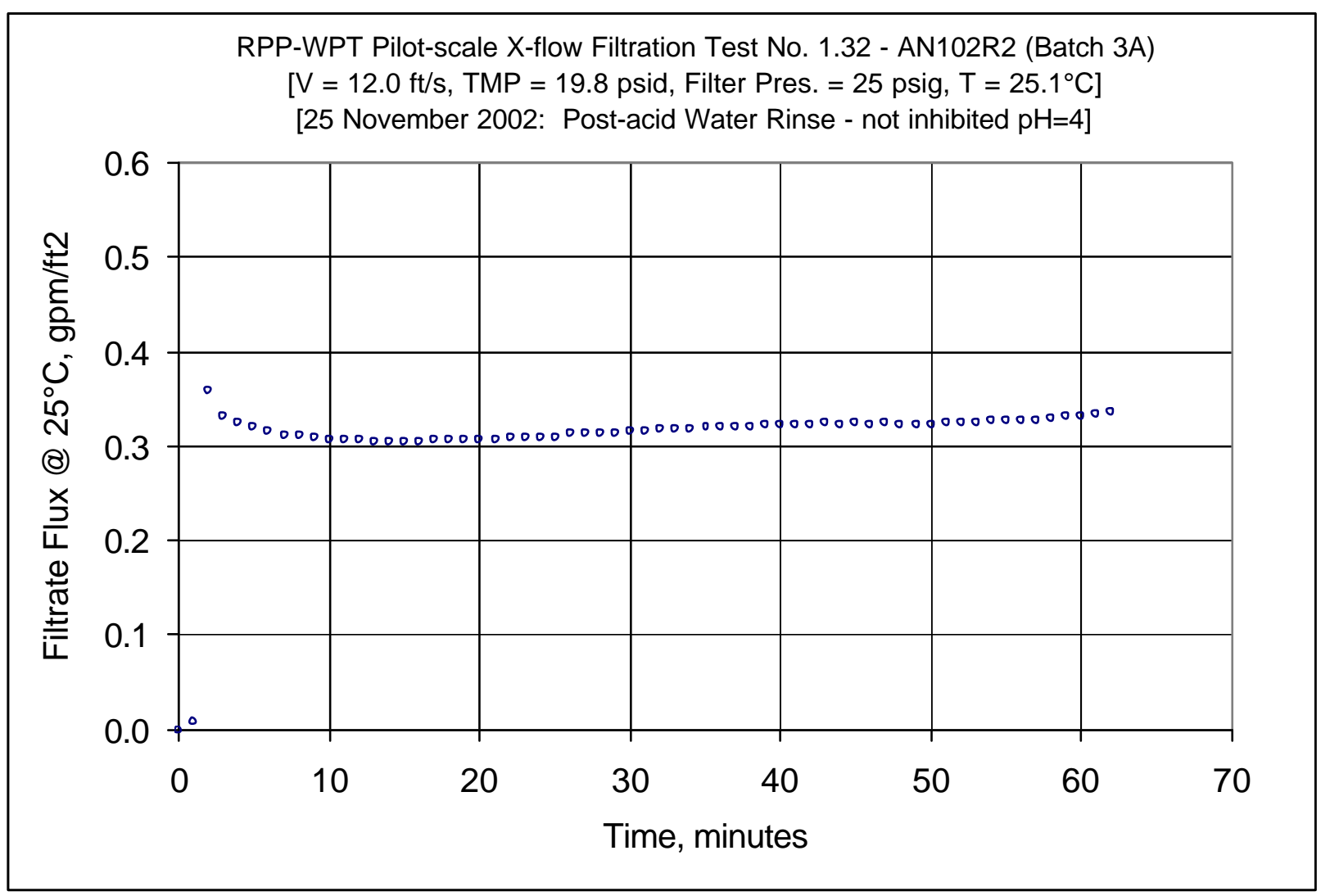

Figure A63: AN-102R2, Batch 3A Slurry Test 1.32 - Deionized \& Filtered Water $(\mathrm{pH}=4)$ Rinse after Acid Cleaning 
WSRC-TR-2003-00204, REV. 0

SRT-RPP-2003-00087, REV. 0

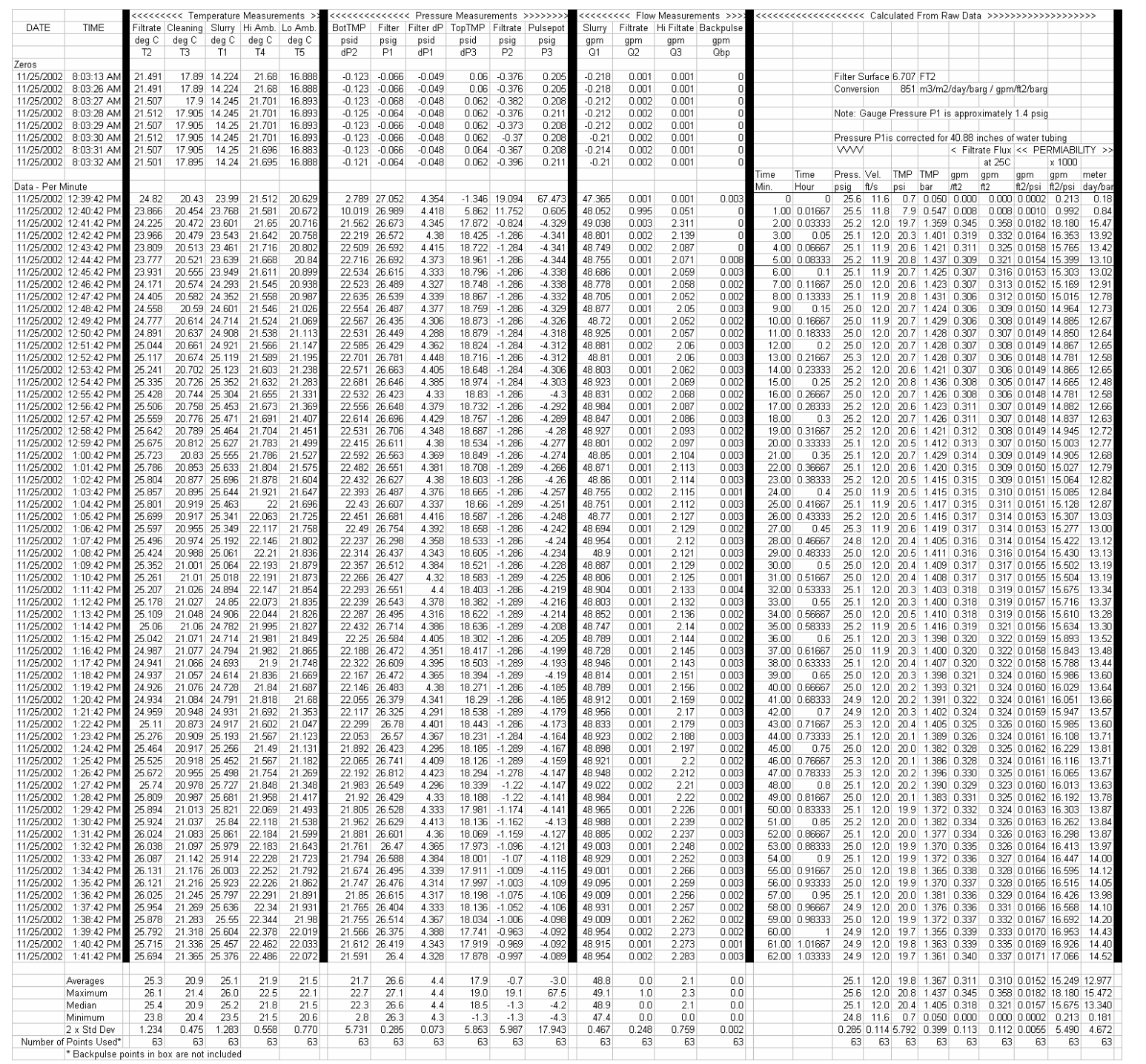

Figure A64: AN-102R2, Batch 3A Slurry Test 1.32 - Deionized \& Filtered Water (pH=4) Rinse after Acid Cleaning - Raw \& Calculated Data 
WSRC-TR-2003-00204, REV. 0

SRT-RPP-2003-00087, REV. 0

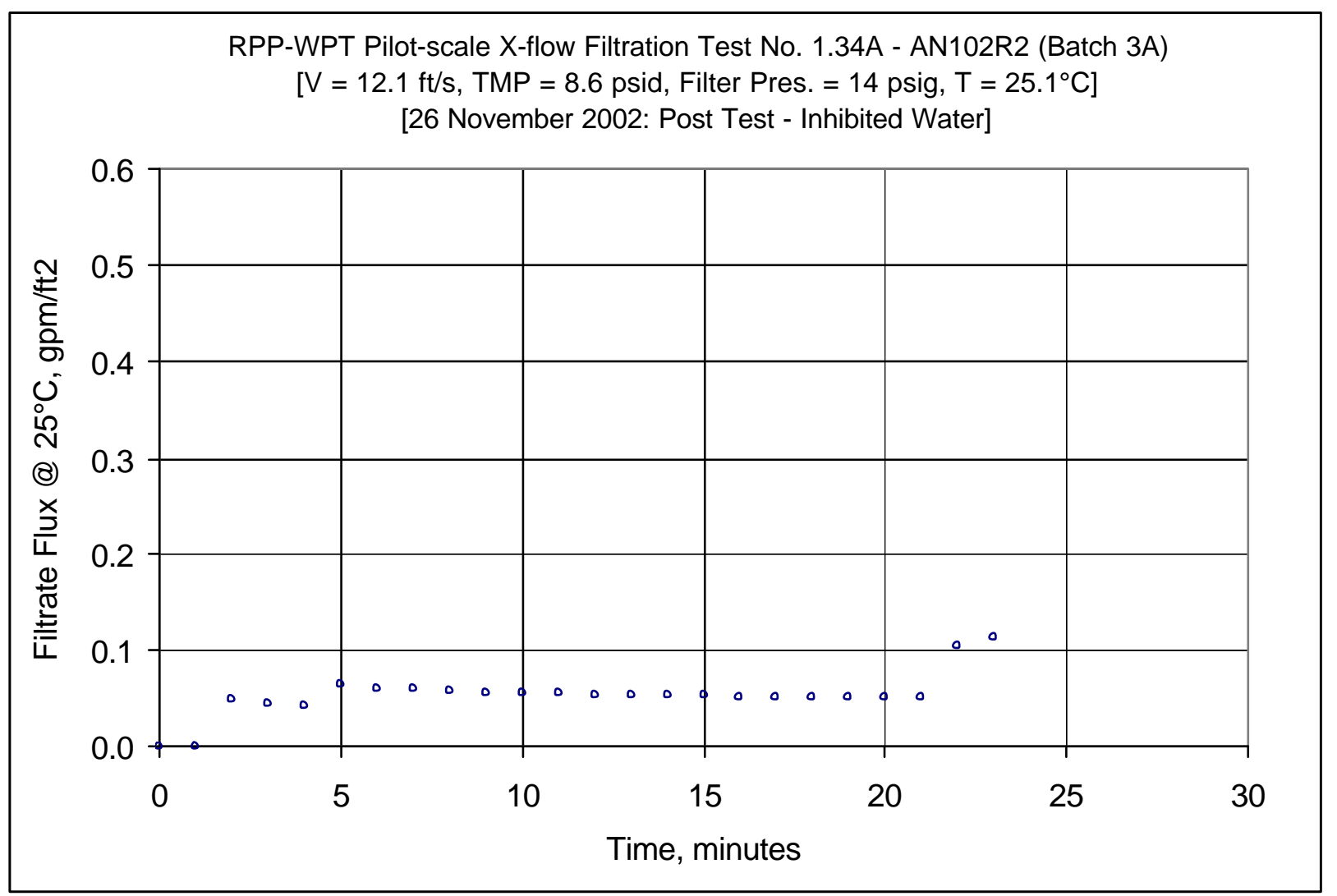

Figure A65: AN-102R2, Batch 3A Slurry Test 1.34A - Inhibited Deionized \& Filtered Water Test after Standard Slurry 
WSRC-TR-2003-00204, REV. 0

SRT-RPP-2003-00087, REV. 0

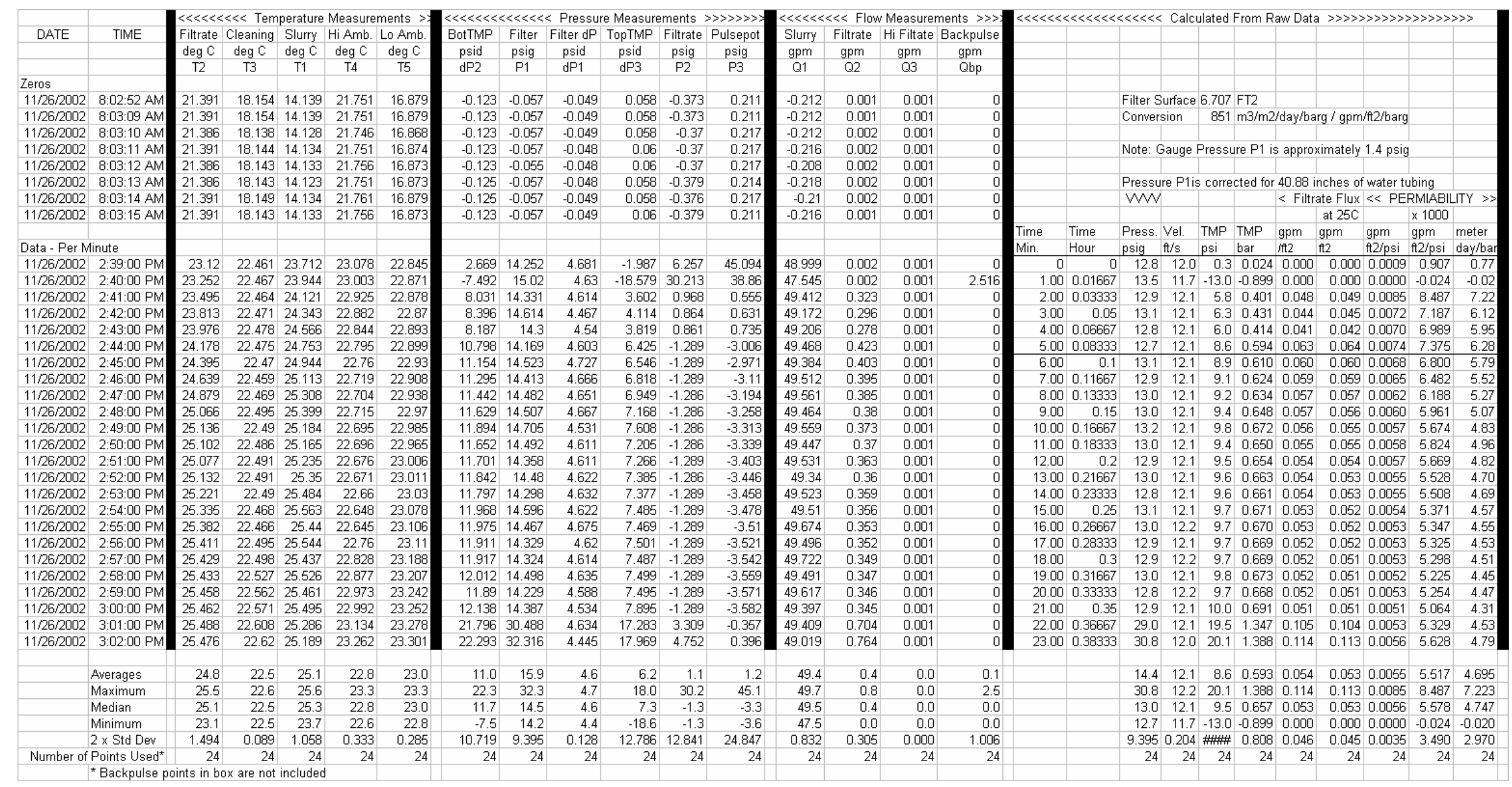

Figure A66: AN-102R2, Batch 3A Slurry Test 1.34A - Inhibited Deionized \& Filtered Water Test after Standard Slurry - Raw \& Calculated Data 
WSRC-TR-2003-00204, REV. 0

SRT-RPP-2003-00087, REV. 0

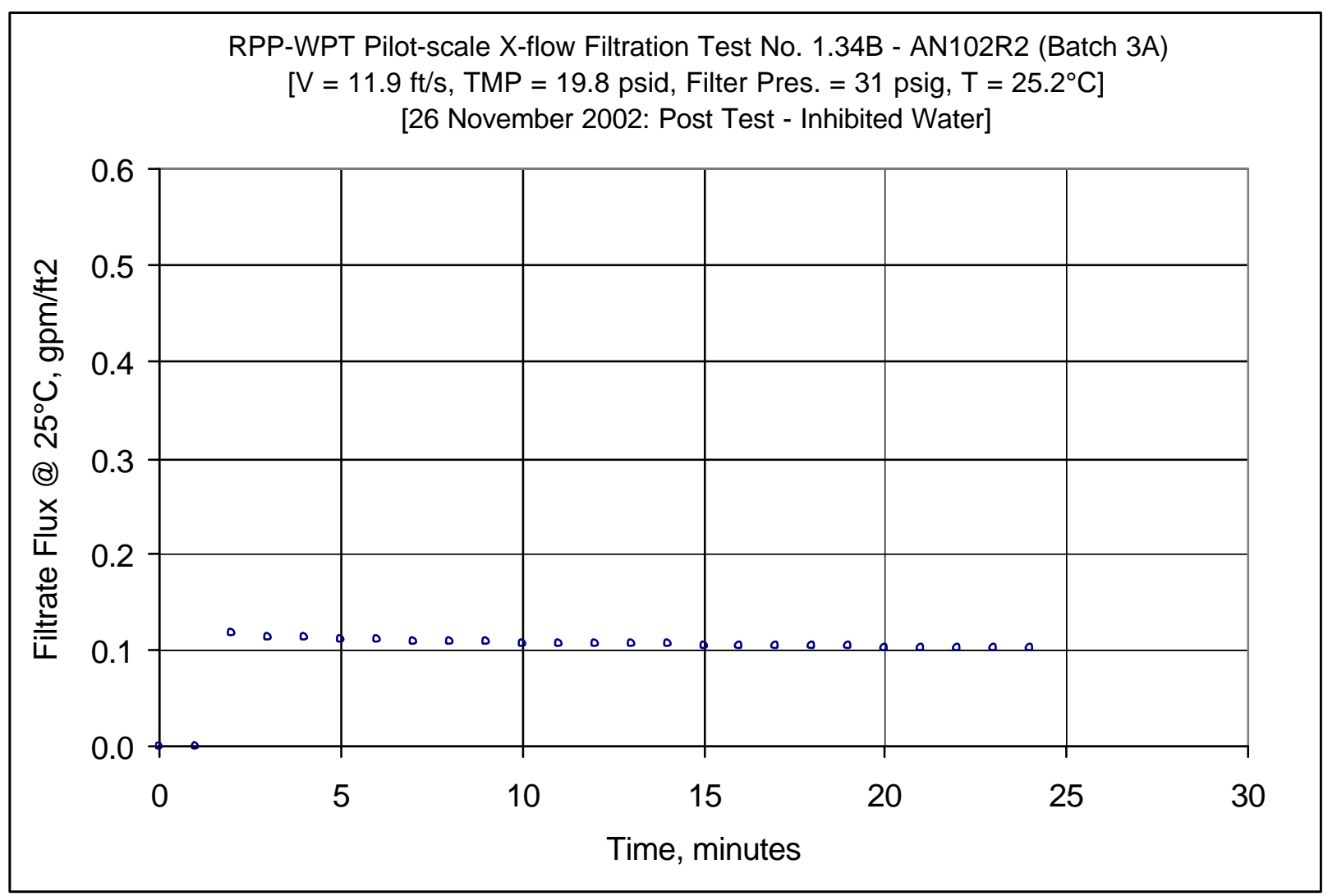

Figure A67: AN-102R2, Batch 3A Slurry Test 1.34B - Inhibited Deionized \& Filtered Water Test after Standard Slurry 
WSRC-TR-2003-00204, REV. 0

SRT-RPP-2003-00087, REV. 0

\begin{tabular}{|c|c|c|c|c|c|c|c|c|c|c|c|c|c|c|c|c|c|c|c|c|c|c|c|c|c|c|c|}
\hline \multirow{4}{*}{ DATE } & \multirow{3}{*}{ TIME } & \multicolumn{5}{|c|}{$\ll \ll<\ll<<$ Temperature Measurements $>1$} & \multicolumn{6}{|c|}{ 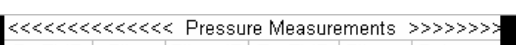 } & \multicolumn{4}{|c|}{ 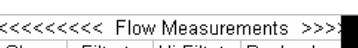 } & \multicolumn{11}{|c|}{ 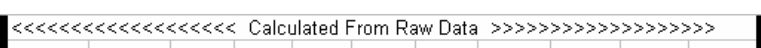 } \\
\hline & & Filtrate & Cleaning & Slurry & Hi Amb. & Lo Amb. & BotTMP & Filter & Filter $\mathrm{dP}$ & TopTMP & Filtrate & Pulsepot & Slurry & Filtrate & Hi Filtate & Backpulse & & & & & & & & & & & \\
\hline & & $\operatorname{deg} \mathrm{C}$ & $\operatorname{deg} C$ & $\operatorname{deg} \mathrm{C}$ & $\operatorname{deg} \mathrm{C}$ & $\operatorname{deg} \mathrm{C}$ & psid & psig & psid & psid & psig & psig & gpm & $\mathrm{gpm}$ & $\mathrm{gpm}$ & $\mathrm{gpm}$ & & & & & & & & & & & \\
\hline \multirow{2}{*}{\multicolumn{28}{|c|}{ Zeros }} \\
\hline & & & & & & & & & & & & & & & & & & & & & & & & & & & \\
\hline 11/26/2002 & 02:52 AM & 21.391 & 18.154 & 14.139 & 21.751 & 879 & -0.123 & -0.057 & -0.049 & 0.058 & -0.373 & 0.211 & -0.212 & 0.001 & 0.001 & & & & \multirow{2}{*}{\multicolumn{2}{|c|}{ Filter Surface }} & $6.707 \mathrm{~F}$ & \multirow{2}{*}{\multicolumn{5}{|c|}{$\begin{array}{l}\mathrm{FT} 2 \\
\mathrm{~m} 3 / \mathrm{m} 2 / \mathrm{day} / \mathrm{barg} / \mathrm{gpm} / \mathrm{t} 2 / \mathrm{barg}\end{array}$}} & \\
\hline $11 / 26 / 2002$ & 8:03:09 AM & 21.391 & 18.154 & 14.139 & 21.751 & 16.879 & -0.123 & -0.057 & $\begin{array}{l}-0.049 \\
-0.049\end{array}$ & 0.058 & -0.373 & 0.211 & -0.212 & 0.001 & 0.001 & 0 & & & & & $851 \mathrm{~m}$ & & & & & & \\
\hline $11 / 26 / 2002$ & 8:03:10 AM & 21.386 & 18.138 & 14.128 & 21.746 & 16.868 & -0.123 & -0.057 & -0.049 & 0.058 & -0.37 & 0.217 & -0.212 & 0.002 & 0.001 & & & & \multirow{2}{*}{\multicolumn{2}{|c|}{ Note: Gauge $\mathrm{F}$}} & & & & & & & \\
\hline $11 / 26 / 2002$ & 8:03:11 AM & 21.391 & 18.144 & 14.134 & 21.751 & 16.874 & -0.123 & -0.057 & -0.048 & 0.06 & -0.37 & 0.217 & -0.216 & 0.002 & 0.001 & & & & & & Pressure & re $\mathrm{P} 1$ is & $s$ approxin & imately 1. & $1.4 \mathrm{psig}$ & & \\
\hline $11 / 26 / 2002$ & 8:03:12 AM & 21.386 & 18.143 & 14.133 & 21.756 & 16.873 & -0.123 & -0.055 & -0.048 & 0.06 & -0.37 & 0.217 & -0.208 & 0.002 & 0.001 & & & & & & & & & & & & \\
\hline $11 / 26 / 2002$ & 8:03:13 AM & 21.386 & 18.143 & 14.123 & 21.751 & 16.873 & -0.125 & -0.057 & -0.048 & 0.058 & -0.379 & 0.214 & -0.218 & 0.002 & 0.001 & & & & Pressur & ure $\mathrm{P} 1$ is & is correcte & cted for 4 & $40.88 \mathrm{inc}$ & ches of $w$ & water tub & ubing & \\
\hline $11 / 26 / 2002$ & 8:03:14 AM & 21.391 & 18.149 & 14.134 & 21.761 & 16.879 & -0.125 & -0.057 & -0.049 & 0.058 & -0.376 & 0.217 & -0.21 & 0.002 & 0.0 & & & & $\mathrm{WM}$ & & & & < Filtrat & & & DOIL & 政 \\
\hline $11 / 26 / 2002$ & 8:03:15 AM & 21.391 & 18.143 & 14.133 & 21.756 & 16.873 & -0.123 & -0.057 & -0.049 & 0.06 & -0.379 & 0.211 & -0.216 & 0.001 & 0.001 & 0 & & & & & & & & at $25 \mathrm{C}$ & & $\times 1000$ & \\
\hline & & & & & & & & & & & & & & & & & me & Time & Press. & Vel. & TMP T & TMP & & gpm & gpm & $\mathrm{gpm}$ & \\
\hline Data - Per M & Minute & & & & & & & & & & & & & & & & & Hour & noia & $\mathrm{f} / \mathrm{s}$ & psi & bar & ift2 & $\mathrm{ft} 2$ & $\mathrm{fl} 2 / \mathrm{psi}$ & $\mathrm{ft2} / \mathrm{psi}$ & ay// \\
\hline $11 / 26 / 2002$ & 3:05:09 PM & .323 & 22.668 & 25.241 & 23.41 & 23.343 & 2.996 & 32.811 & 4.463 & -1.352 & 24.638 & 72.98 & 48.3 & 0.001 & 0.001 & & 0 & 0 & 31.3 & 3 11.8 & 0.8 & 0.057 & 0.000 & 0.000 & 0.0002 & 20.180 & S \\
\hline $11 / 26 / 2002$ & 3:06:09 PM & 25.265 & 22.68 & 25.168 & 23.417 & 23.36 & 3.077 & 32.945 & 4.535 & -1.453 & 24.661 & 71.964 & 48.283 & & & & 1.00 & 0.01667 & 31.5 & $\begin{array}{ll}5 & 11.8\end{array}$ & 0.8 & 0.056 & 0.000 & 0.000 & 0.0004 & 0.365 & 0. \\
\hline $11 / 26 / 2$ & 3:07:09 PM & 25.328 & 22.698 & 25.166 & 23 & 23.38 & 21.879 & 32.652 & 4.4 & 17.522 & 5.41 & 0.662 & 48.793 & 0.794 & & & 2.00 & 0.03333 & 31.2 & $\begin{array}{ll}2 & 12.0\end{array}$ & 19.7 & 1.358 & & 80 & & 5.981 & 5.09 \\
\hline $11 / 26 / 2$ & (19.6 & 25.4 & 22.716 & 25.243 & 23.458 & 2338 > & 22.256 & 32.532 & 4. & 7.811 & 4. & & 48.774 & 0.77 & & & 3.00 & 1.05 & 31.1 & $\begin{array}{ll}1 & 12.0 \\
\end{array}$ & 20.0 & 1.381 & & & 57 & 91 & 4.84 \\
\hline $11 / 26 / 2002$ & 3:09:09 PM & 25.403 & 22.723 & 25.206 & 23.5 & 23.408 & 22.718 & 32.583 & 4.454 & 18.409 & 4.636 & 0.338 & 48.728 & 0.759 & & & 4.00 & 0.06667 & 31.1 & 11.9 & 20.6 & 1.418 & 0.113 & 0.113 & 0.0055 & 5.5 .471 & 66 \\
\hline $11 / 26 / 2002$ & 3:10:09 PM & 25.416 & 22.742 & 25.134 & 23.499 & 23.416 & 22.813 & 32.482 & 4.461 & 18.45 & 4.35 & 0.228 & 48.728 & 5 & & & 5.00 & 0.08333 & 31.0 & 11.9 & 20.6 & 1.422 & 0.112 & 0.111 & 0.0054 & 45.399 & .59 \\
\hline $11 / 26 / 2002$ & 3:11:09 PM & 25.414 & 22.749 & 25.152 & 23.466 & 23.444 & 22.942 & 32.484 & 4.4 & 18.605 & 4.22 & 0.144 & 789 & & & & 6.00 & 0.1 & 31.0 & 12.0 & 20.8 & 1.432 & 0.111 & 0.110 & 0.0053 & 3 5.317 & \\
\hline $11 / 26 / 2$ & 3:12:09 PM & 25.411 & & 25.254 & 23.509 & 23.4 & & 32.72 & & & 4.10 & & 751 & & & & 7.00 & 0.11667 & 31.2 & $\begin{array}{ll}2 & 11.9\end{array}$ & 21.1 & 1.453 & & 0.109 & & & 41 \\
\hline $11 / 2$ & $3: 1$ & 20.4 & 22 & $\begin{array}{l}25.194 \\
25.192\end{array}$ & 23. & & & 32.453 & & & & & 65 & & & & 8.0 & & 31.0 & 11.9 & 21.1 & & & & & & $\begin{array}{r}4.41 \\
+\quad 4.38 \\
\end{array}$ \\
\hline & 1 & & 22.803 & 25.156 & 23.575 & & & 32.652 & & & & -0.0 & & & & & 0 & 15 & 31.2 & $\begin{array}{ll}2 & 11.9\end{array}$ & 21.2 & & & & & & 4.32 \\
\hline $11 / 26 / 2002$ & 3:15:09 PM & 432 & 317 & 25.205 & 23.609 & 23.532 & 6 & 32.449 & 4.455 & 17 & 3.778 & -0.09 & 48.678 & 0.7 & & & 10.00 & 0.16667 & 31.0 & 11.9 & 21.1 & 1.457 & 0.108 & 0.107 & 0.0051 & 5.079 & 4.32 \\
\hline $11 / 26 / 2002$ & 3:16:09 PM & & 25 & 25.272 & 23.592 & 23 & & 32.77 & & & 3.6 & -0.148 & 56 & & & & 11.00 & 0.18333 & 31.3 & $\begin{array}{ll}3 & 12.0 \\
\end{array}$ & 21.5 & 1.482 & 0.107 & 0.107 & 50 & 4.956 & $\begin{array}{l}6.22 \\
\end{array}$ \\
\hline $11 / 26 / 2002$ & 3:17:09 PM & & & 25.195 & 23.58 & 23.5 & & 32.888 & & & 3.5 & $5+2>$ & 2 & & & & 12.00 & 0.2 & 31.4 & $\begin{array}{ll}4 & 11.9\end{array}$ & 21.7 & 1.2 & & & & & .17 \\
\hline & 3:18:09 PM & 25. & & 25.169 & 23.583 & 23.5 & & 32.708 & & & 3.4 & & & & & & 13. & 67 & 31.2 & \begin{tabular}{|l|l|}
2 & 11.9
\end{tabular} & 21.7 & & & & & & 15 \\
\hline & & 23.4 & 22.869 & 25.236 & 23 & & & 32.6 & & & & & & & & & 14 & & 31.2 & $\begin{array}{ll}12.0 \\
2.0\end{array}$ & 21.8 & & & 5 & & & $\begin{array}{l}4.19 \\
4.12\end{array}$ \\
\hline & & & & 25.263 & & & & 32.7 & & & & & & & & & & 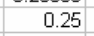 & 31.2 & $2 \quad 12.0$ & 21.8 & 2 & & & & & $\begin{array}{l}3.09 \\
\end{array}$ \\
\hline $11 / 26 / 2002$ & 3:21:09 P & 88 & 22 & 25.191 & 23 & & & 32.5 & & & 3. & & & & & & & 0.2 & 1 & 1 & 9 & & & & 48 & 4 & .06 \\
\hline & $3: 22:$ & & & 25.174 & & & & 32. & & & & & 48 & $0.7>->$ & & & & 0.28333 & 2 & \begin{tabular}{|l|l|}
2 & 11.9
\end{tabular} & 22.1 & & & & & & 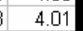 \\
\hline $11 / 2$ & 3:23:09 & $25.4>$ & & 25.276 & & & & & & & & & & & & & & 0.3 & 31.1 & 111.9 & 21.9 & & & & & 4.733 & .03 \\
\hline & $3: 24:$ & 25.44 & 22.926 & 25.258 & 23.693 & & & 32.6 & & & 3.1 & & 48.762 & & & 001 & 19 & 0.31667 & 31.2 & \begin{tabular}{|l|l|}
2 & 11.9
\end{tabular} & 21.9 & 1.510 & & 0.103 & 447 & 7.718 & $\begin{array}{r}3.01 \\
3\end{array}$ \\
\hline $11 / 2$ & $3: 25: 09$ & 25.447 & 22.944 & 25.18 & 23.725 & 23. & & 32.8 & 7. & & 3.0 & & & & & & 20.00 & 0.33333 & 31.4 & $\begin{array}{ll}4 & 11.9\end{array}$ & 22.1 & 1.525 & & 0.103 & 47 & 7.654 & $\begin{array}{l}7.96 \\
4\end{array}$ \\
\hline & & 25.474 & 22.961 & 25.202 & 23.753 & & & 32. & & & 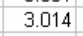 & & & & & & & & 312 & 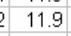 & ר? & & & & & & 96 \\
\hline & & 25.4 & & & & & & & & & & & & & & & & & & & & & & & & & \\
\hline & $3: 28: 0$ & 25. & 22. & 25. & & 23. & & 32. & & & 2.91 & & & & & & & 0.38 & 31.2 & $\begin{array}{ll}2 & 12.0\end{array}$ & 22.4 & & & & & 6.558 & \\
\hline $11 / 26 / 2002$ & 3:29:09 PM & 25.487 & 23.014 & 25.225 & 23.841 & 23.77 & 24.466 & 32.643 & 4.485 & 20.116 & 2.905 & -0.53 & 48.709 & 0.686 & 0.001 & & 24.00 & 0.4 & 31.2 & $\begin{array}{ll}2 & 11.9\end{array}$ & 22.3 & 1.537 & 0.102 & 0.102 & 0.0046 & 6.559 & . \\
\hline & & & & & & & & & & & & & & & & & & & & & & & & & & & \\
\hline & & & & & & & & & & & & & & & & & & & 31.2 & $\begin{array}{l}2 \\
2\end{array} 11.9$ & 19.8 & 5 & 99 & 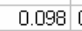 & & & \\
\hline & & & & & & & & & & & & & & & & & & & & & & & & & & & \\
\hline & & & & & & & & & & & & & & & & & & & & & & & & & & & \\
\hline & & & & & & & & & & & & & & & & & & & S1.U & & 0.8 & & & & & & 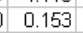 \\
\hline & $2 x$ & 0.097 & 0.201 & 0.091 & 0.237 & 0.260 & 11.260 & 0.263 & 0.069 & 11.298 & 11.453 & 39.406 & 0.261 & 0.394 & 0.000 & 0.00 & & & 0.263 & 30.064 & $\begin{array}{ll}0.0 \\
41.3\end{array}$ & 0.778 & 0.059 & 0.058 & 0.0027 & 655 & 2.259 \\
\hline & Points Used ${ }^{*}$ & 25 & 25 & 25 & 25 & 25 & 25 & 25 & 25 & 25 & 25 & 25 & 25 & 25 & 25 & 25 & & & 25 & 25 & 25 & 25 & 25 & 25 & 25 & 25 & 25 \\
\hline & & & & & & & & & & & & & & & & & & & & & & & & & & & \\
\hline
\end{tabular}

Figure A68: AN-102R2, Batch 3A Slurry Test 1.34B - Inhibited Deionized \& Filtered Water Test after Standard Slurry - Raw \& Calculated Data 
WSRC-TR-2003-00204, REV. 0

SRT-RPP-2003-00087, REV. 0

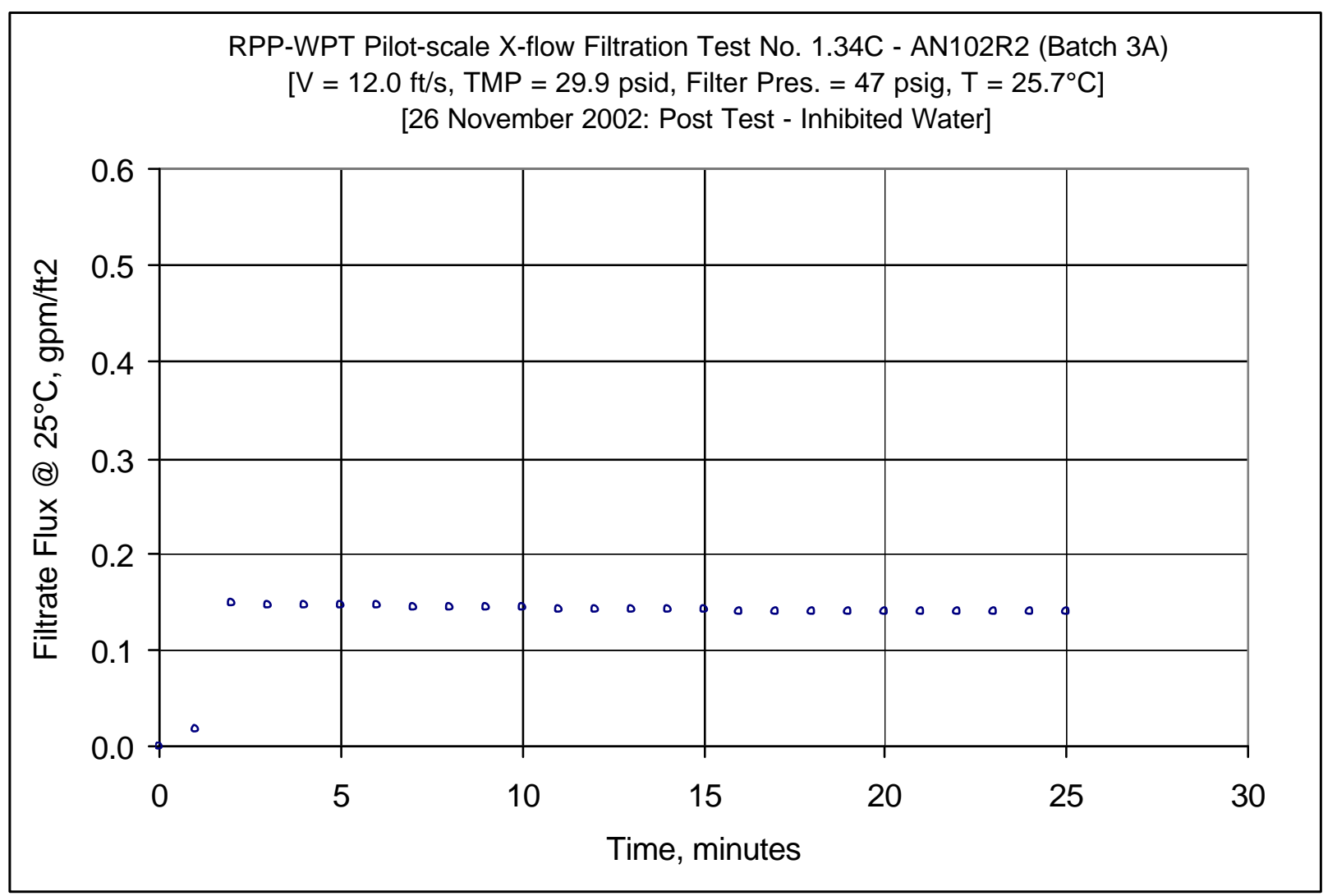

Figure A69: AN-102R2, Batch 3A Slurry Test 1.34C - Inhibited Deionized \& Filtered Water Test after Standard Slurry 
WSRC-TR-2003-00204, REV. 0

SRT-RPP-2003-00087, REV. 0

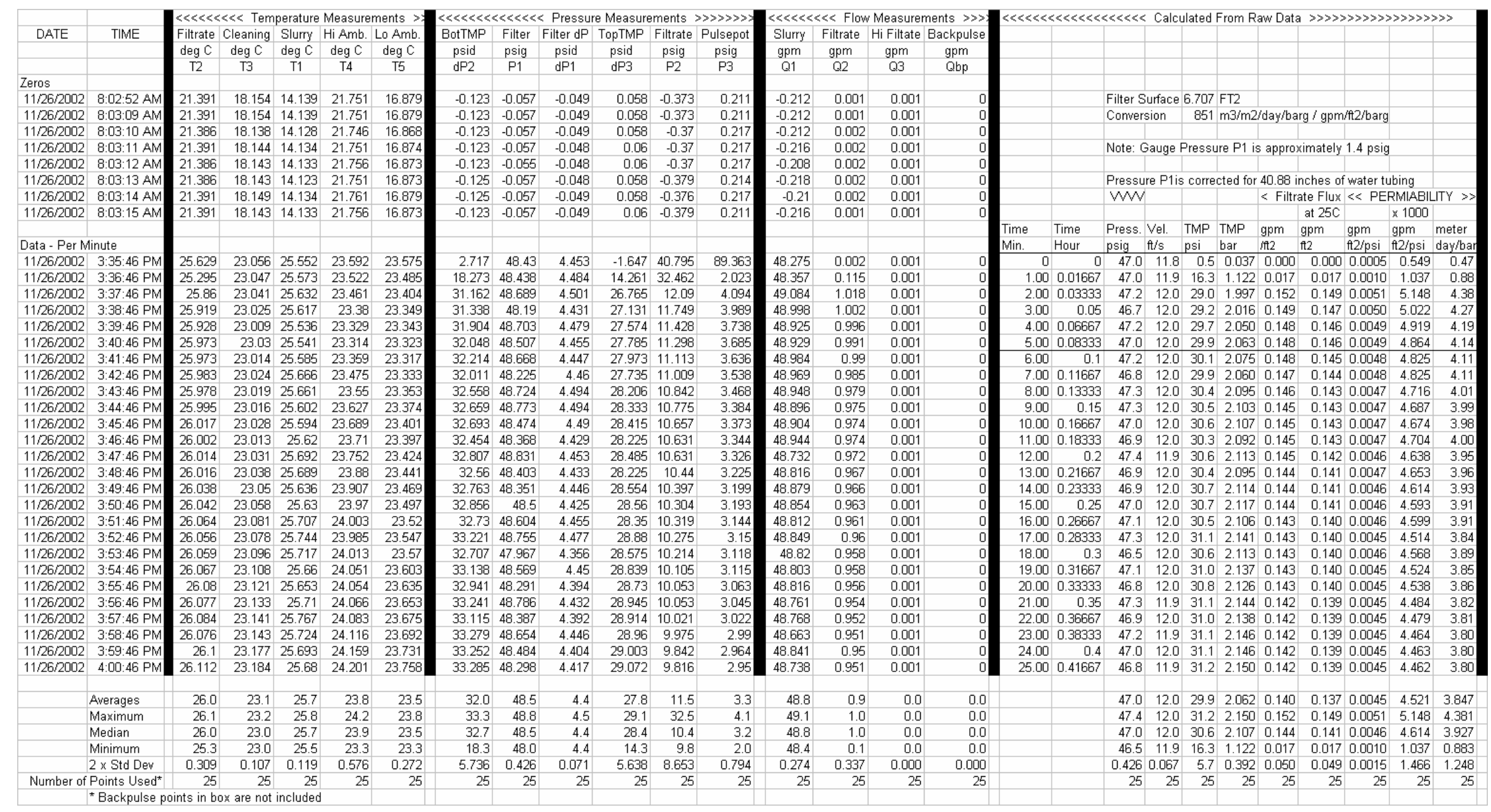

Figure A70: AN-102R2, Batch 3A Slurry Test 1.34C - Inhibited Deionized \& Filtered Water Test after Standard Slurry - Raw \& Calculated Data 
WSRC-TR-2003-00204, REV. 0

SRT-RPP-2003-00087, REV. 0

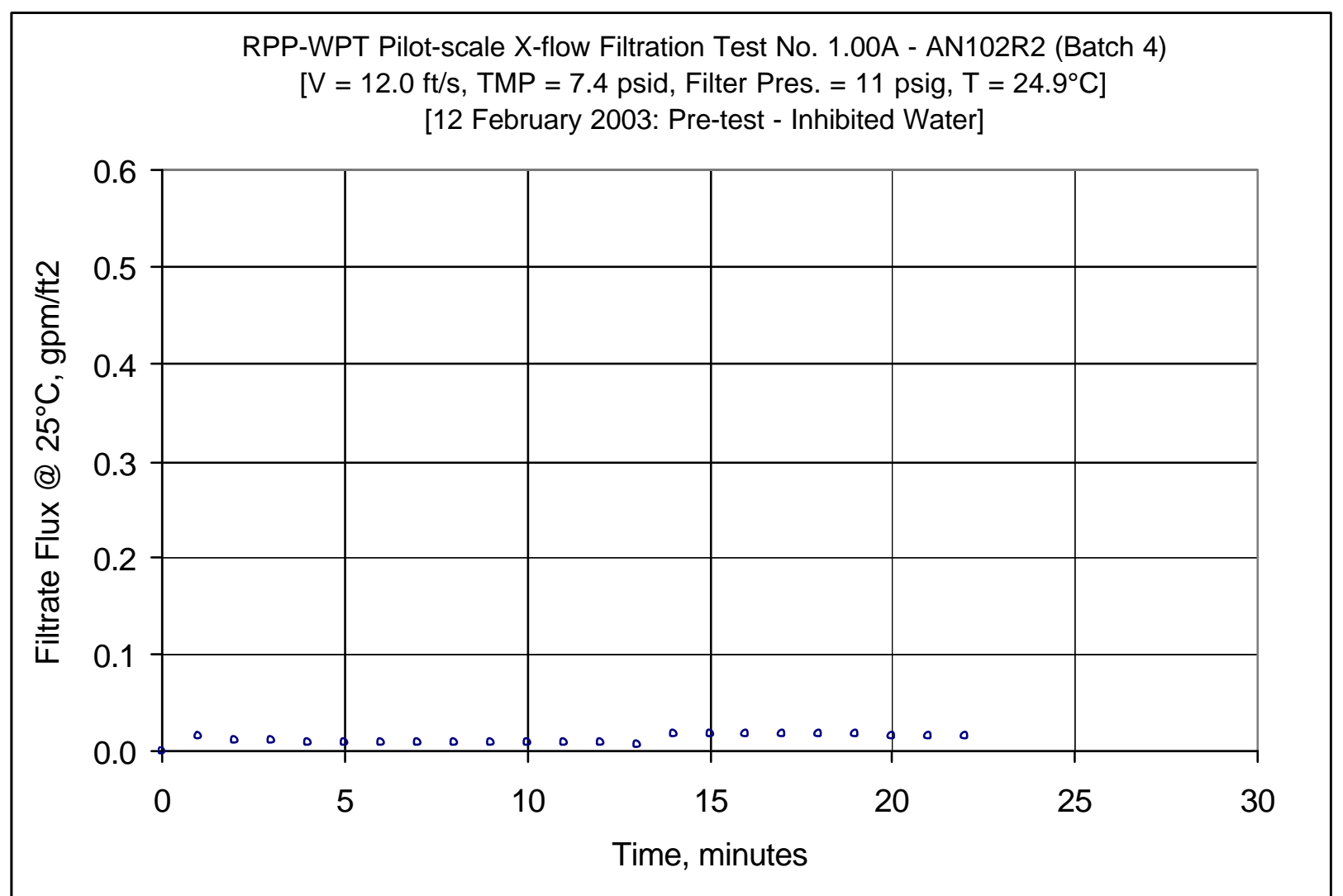

Figure A71: AN-102R2, Batch 4 Slurry Test 1.00A - Inhibited Deionized \& Filtered Water (Compare to 1.34A on 11/26/02) 
WSRC-TR-2003-00204, REV. 0

SRT-RPP-2003-00087, REV. 0

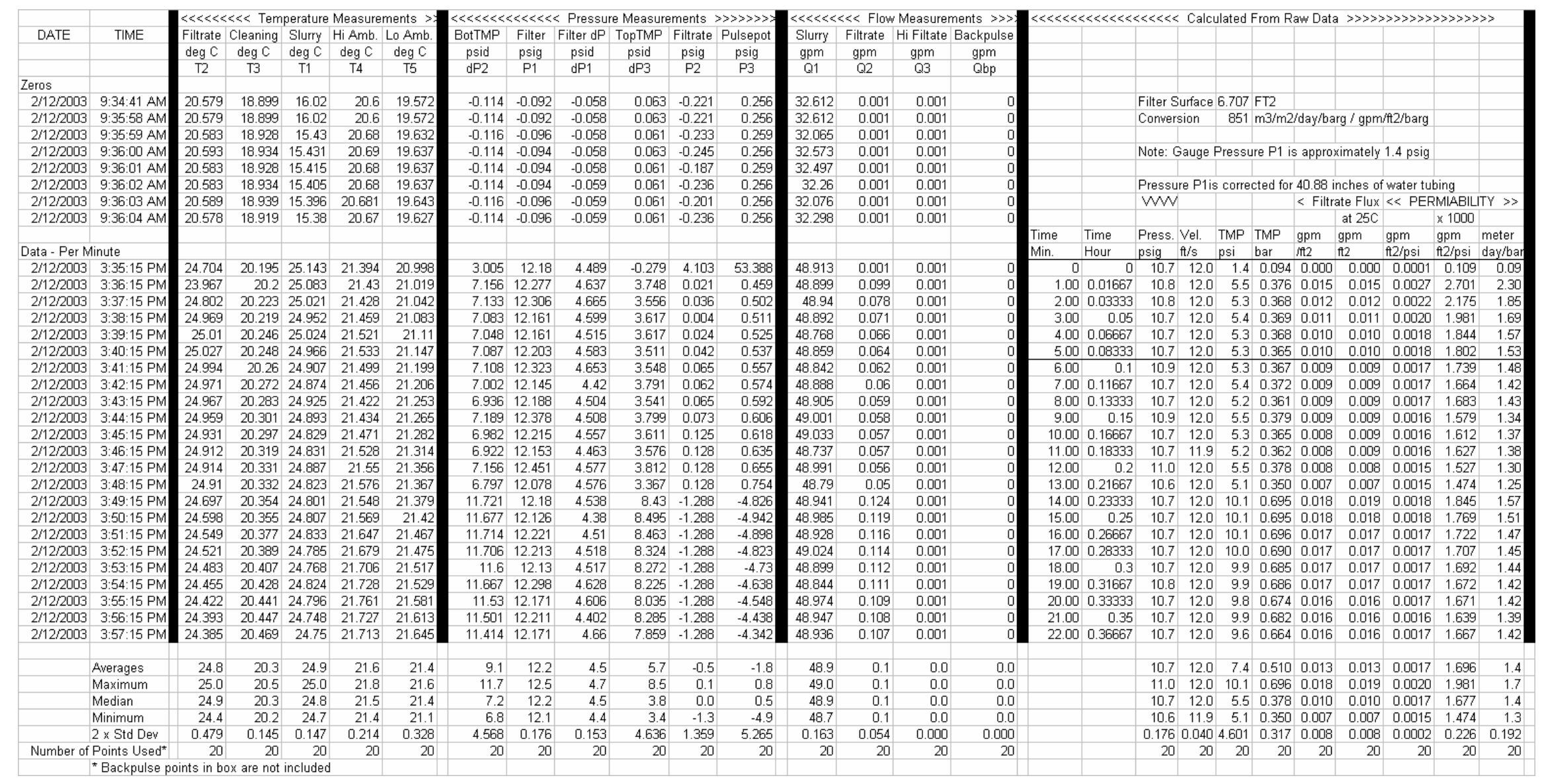

Figure A72: AN-102R2, Batch 4 Slurry Test 1.00A - Inhibited Deionized \& Filtered Water (Compare to 1.34A on 11/26/02) - Raw \& Calculated Data 
WSRC-TR-2003-00204, REV. 0

SRT-RPP-2003-00087, REV. 0

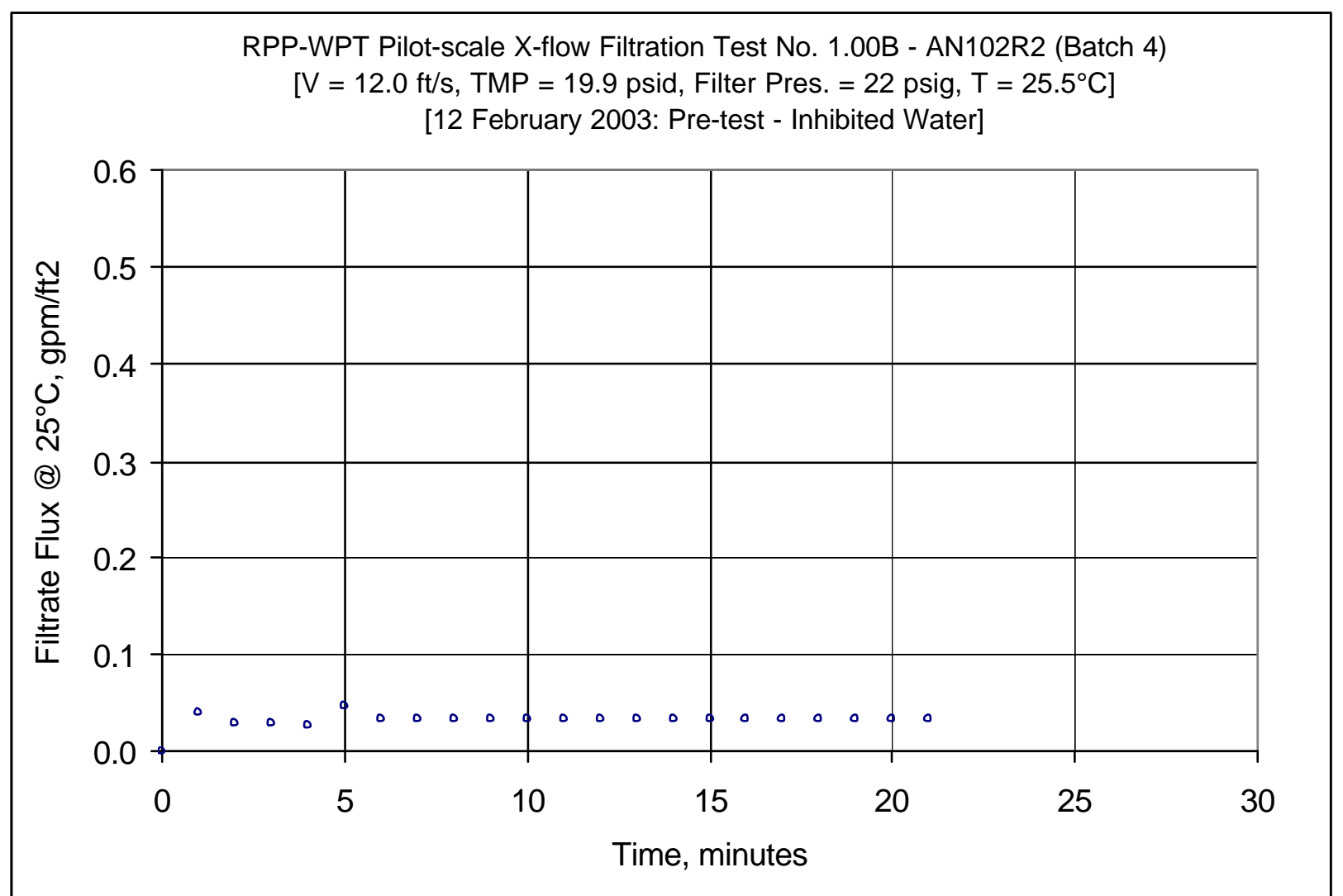

Figure A73: AN-102R2, Batch 4 Slurry Test 1.00B - Inhibited Deionized \& Filtered Water (Compare to 1.34B on 11/26/02) 
WSRC-TR-2003-00204, REV. 0

SRT-RPP-2003-00087, REV. 0

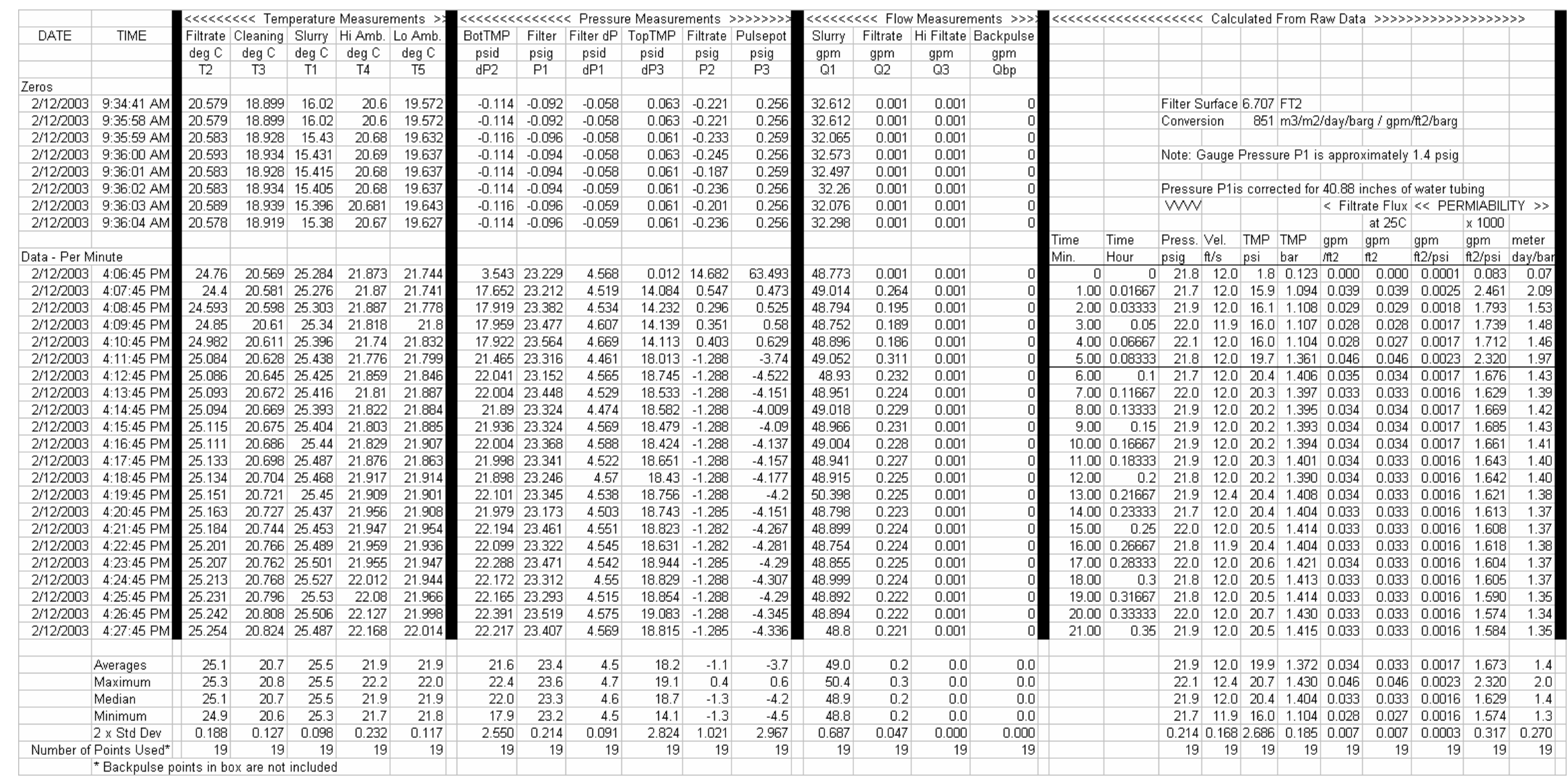

Figure A74: AN-102R2, Batch 4 Slurry Test 1.00B - Inhibited Deionized \& Filtered Water (Compare to 1.34B on 11/26/02) - Raw \& Calculated Data 
WSRC-TR-2003-00204, REV. 0

SRT-RPP-2003-00087, REV. 0

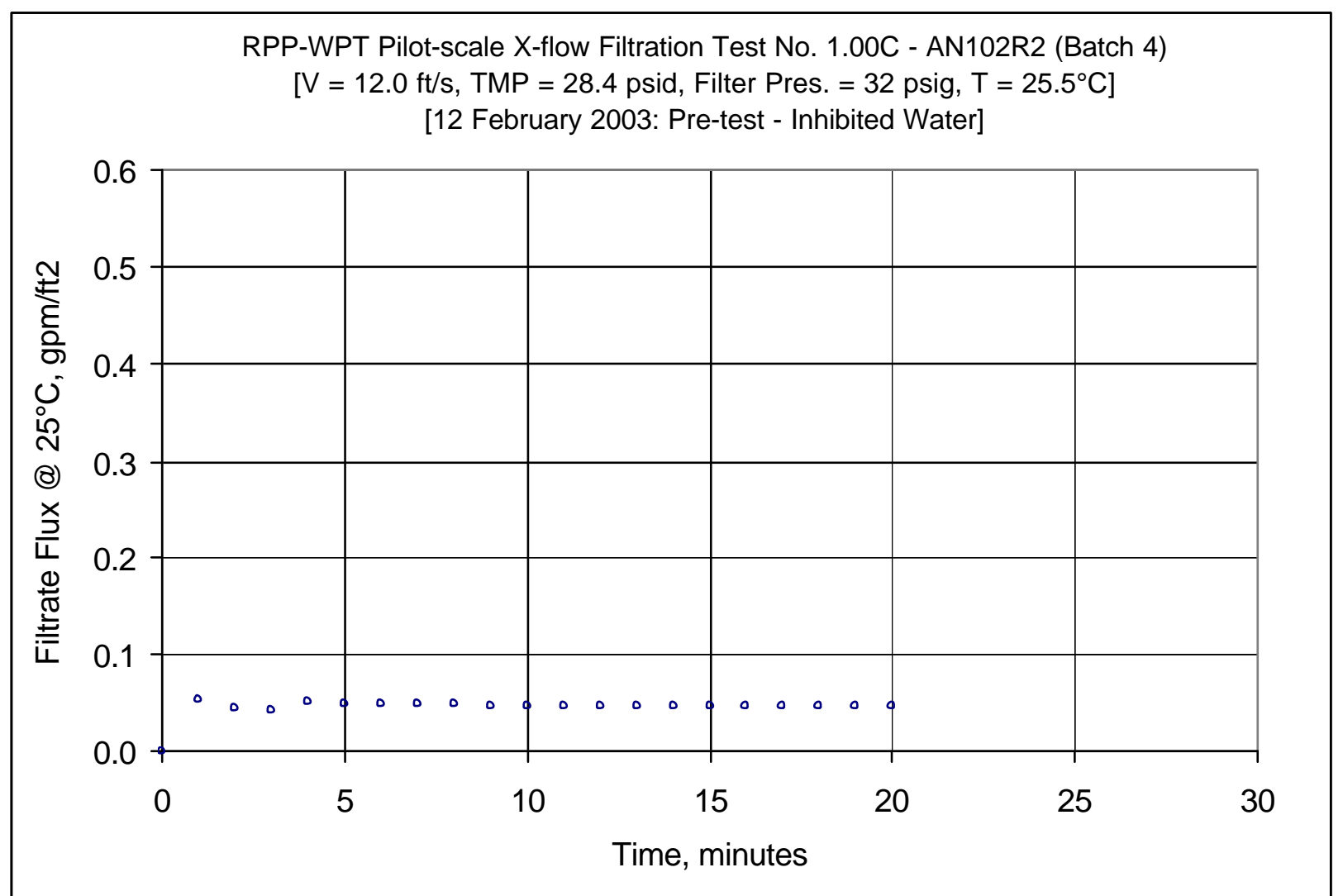

Figure A75: AN-102R2, Batch 4 Slurry Test 1.00C - Inhibited Deionized \& Filtered Water (Compare to 1.34C on 11/26/02) 
WSRC-TR-2003-00204, REV. 0

SRT-RPP-2003-00087, REV. 0

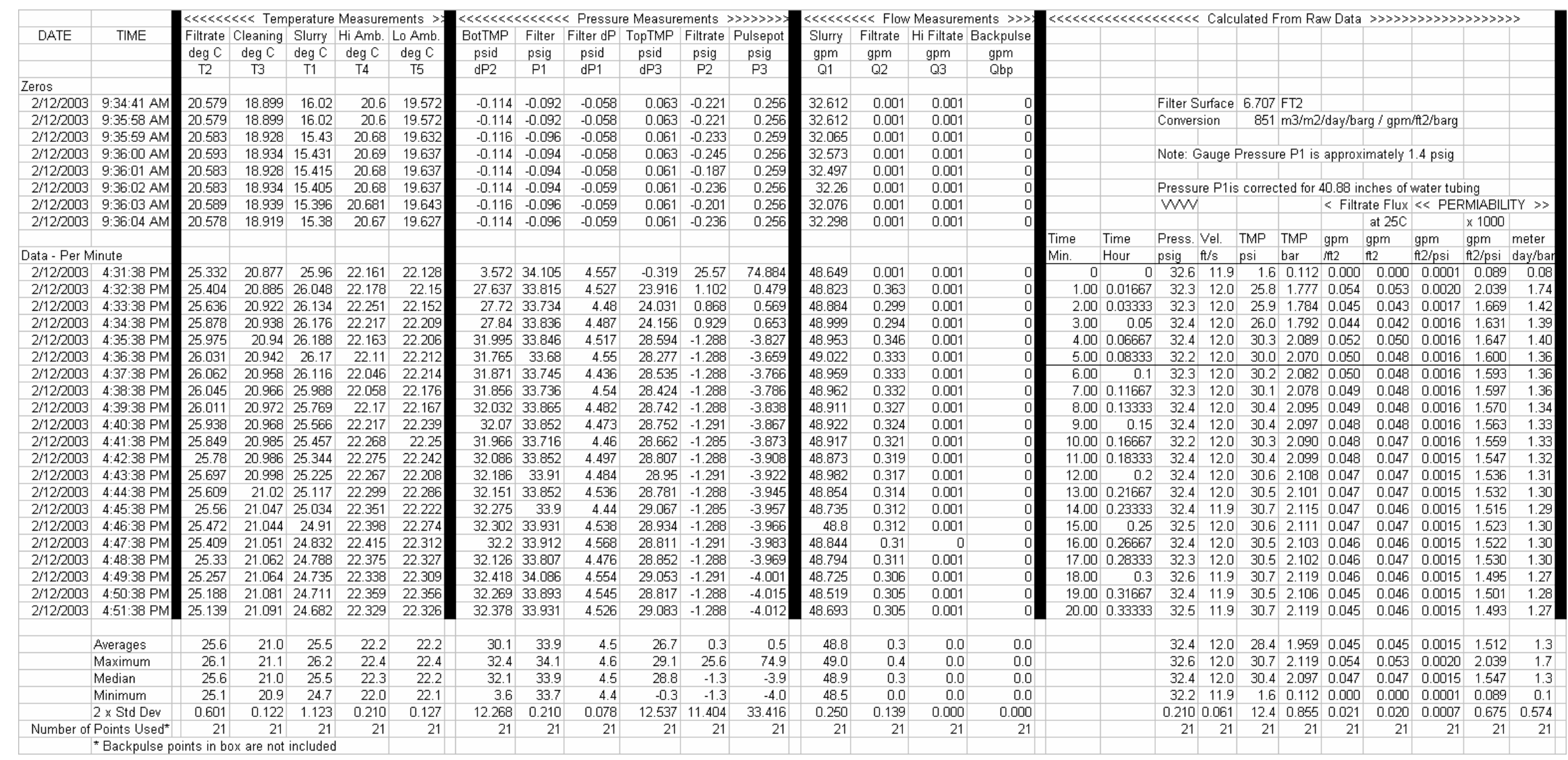

Figure A76: AN-102R2, Batch 4 Slurry Test 1.00C - Inhibited Deionized \& Filtered Water (Compare to 1.34C on 11/26/02) - Raw \& Calculated Data 
WSRC-TR-2003-00204, REV. 0

SRT-RPP-2003-00087, REV. 0

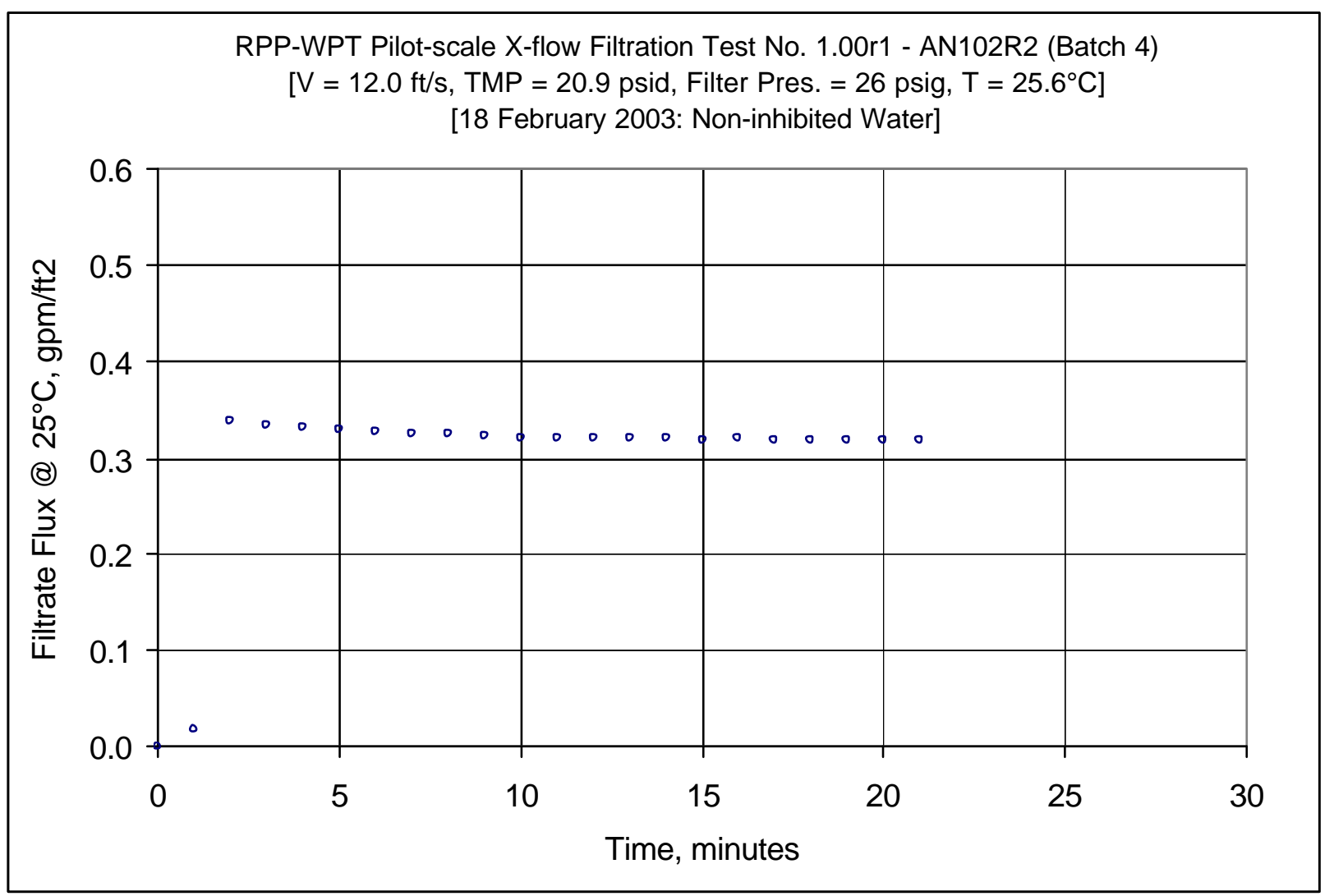

Figure A77: AN-102R2, Batch 4 Slurry Test 1.00r1 - Non-Inhibited Deionized \& Filtered Water 
WSRC-TR-2003-00204, REV. 0

SRT-RPP-2003-00087, REV. 0

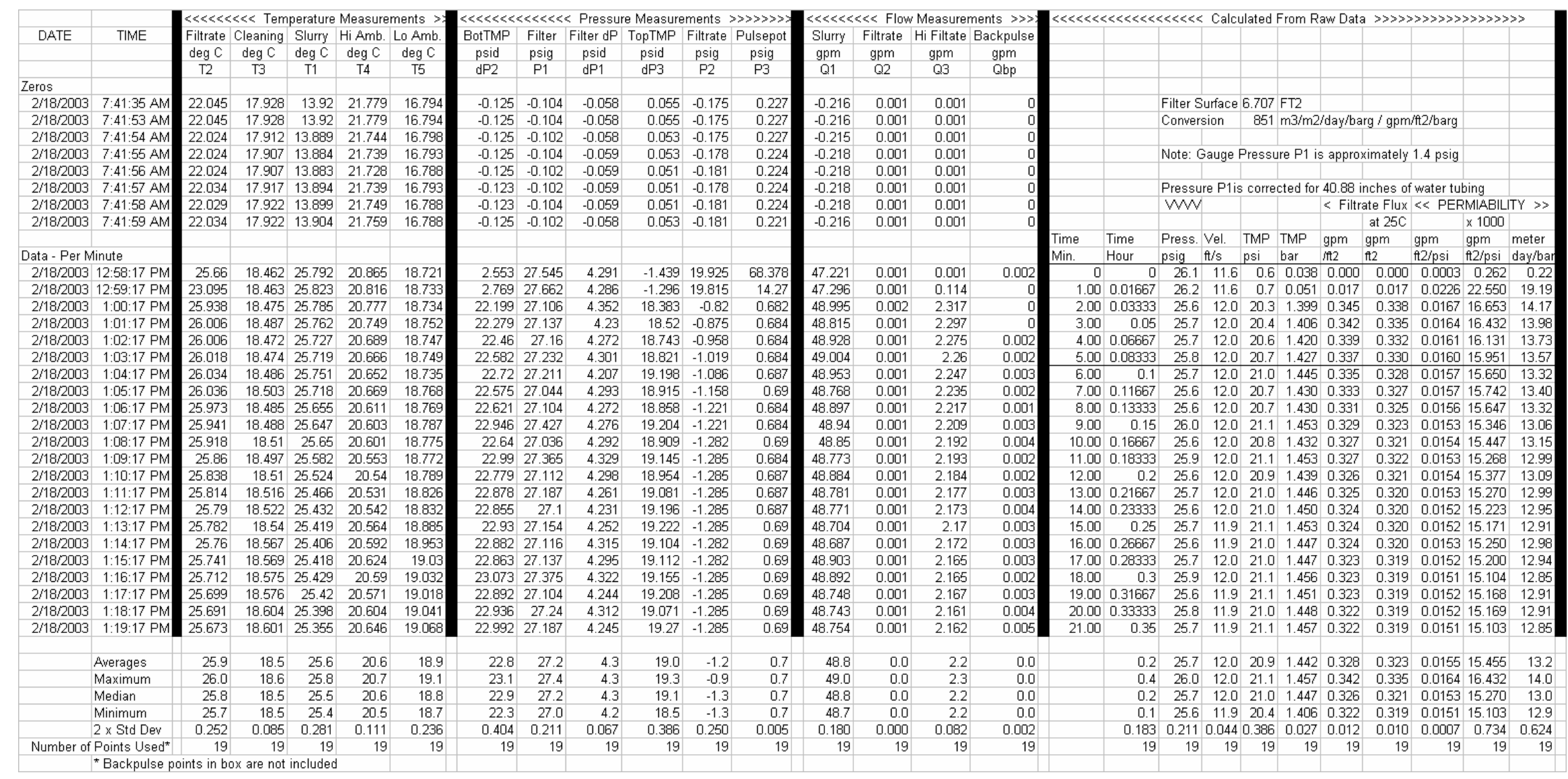

Figure A78: AN-102R2, Batch 4 Slurry Test 1.00r1 - Non-Inhibited Deionized \& Filtered Water - Raw \& Calculated Data 
WSRC-TR-2003-00204, REV. 0

SRT-RPP-2003-00087, REV. 0

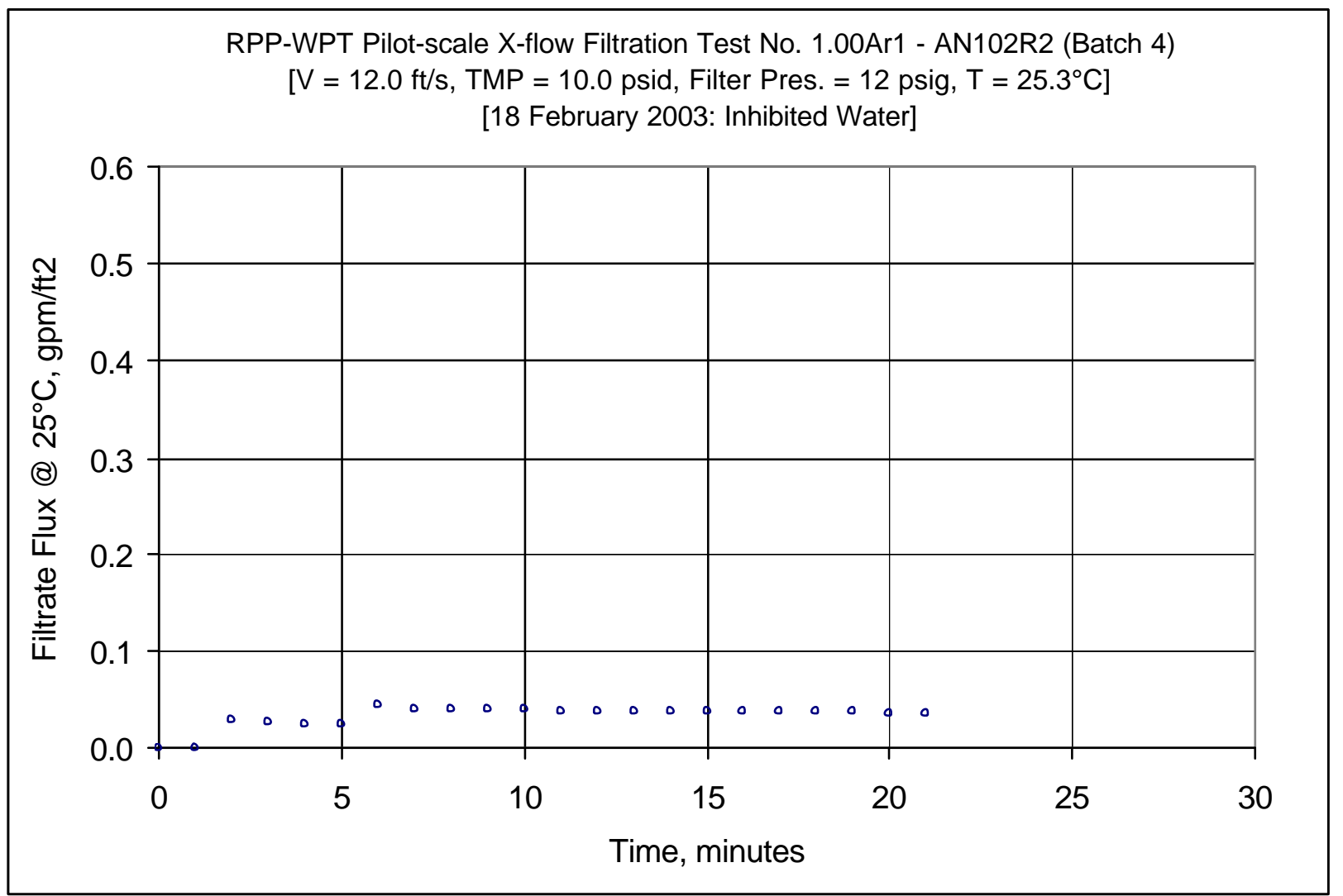

Figure A79: AN-102R2, Batch 4 Slurry Test 1.00Ar1 - Inhibited Deionized \& Filtered Water (compare to Test 1.00A done on 2/12/03) 
WSRC-TR-2003-00204, REV. 0

SRT-RPP-2003-00087, REV. 0

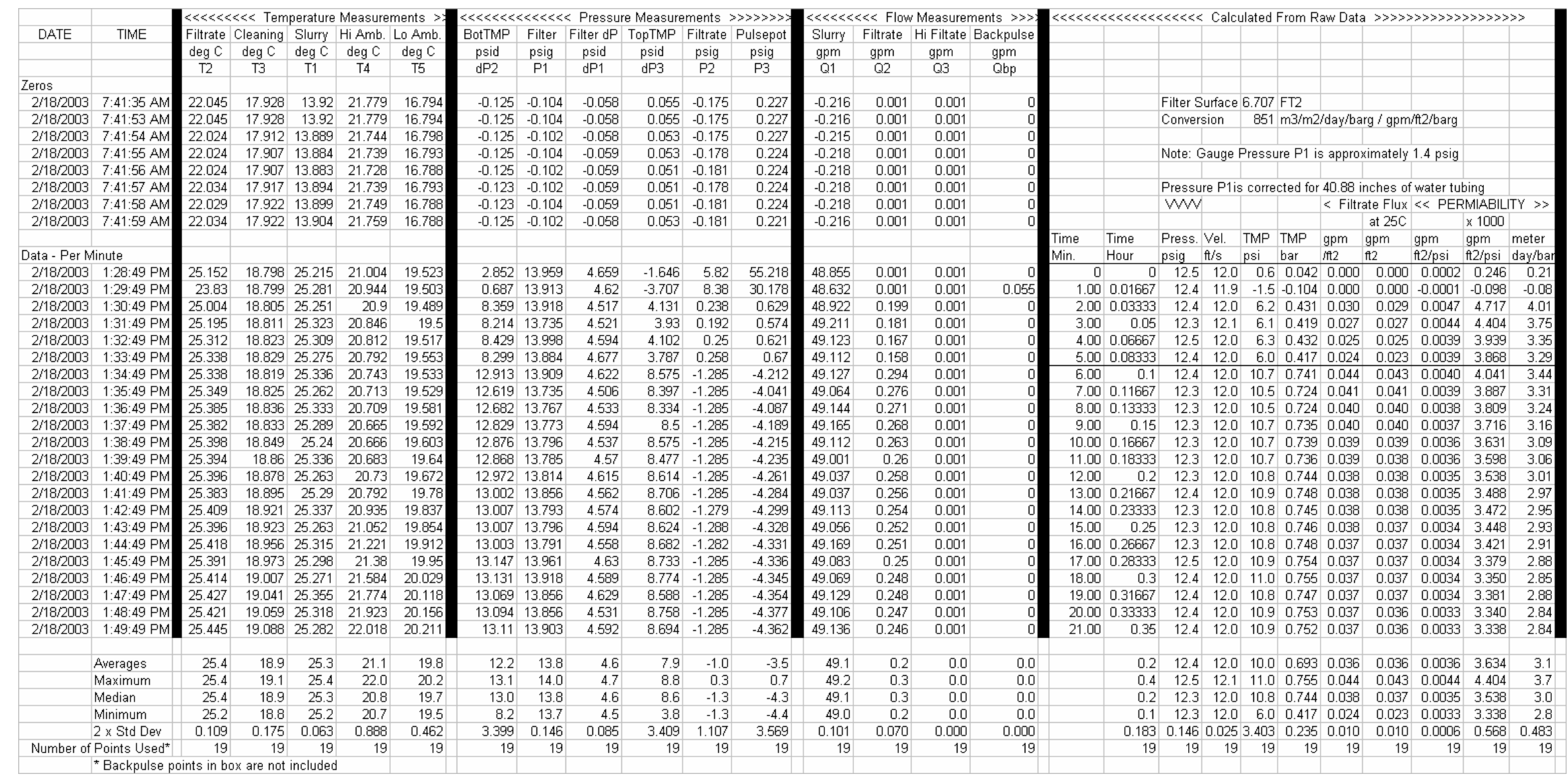

Figure A80: AN-102R2, Batch 4 Slurry Test 1.00Ar1 - Inhibited Deionized \& Filtered Water - Raw \& Calculated Data 
WSRC-TR-2003-00204, REV. 0

SRT-RPP-2003-00087, REV. 0

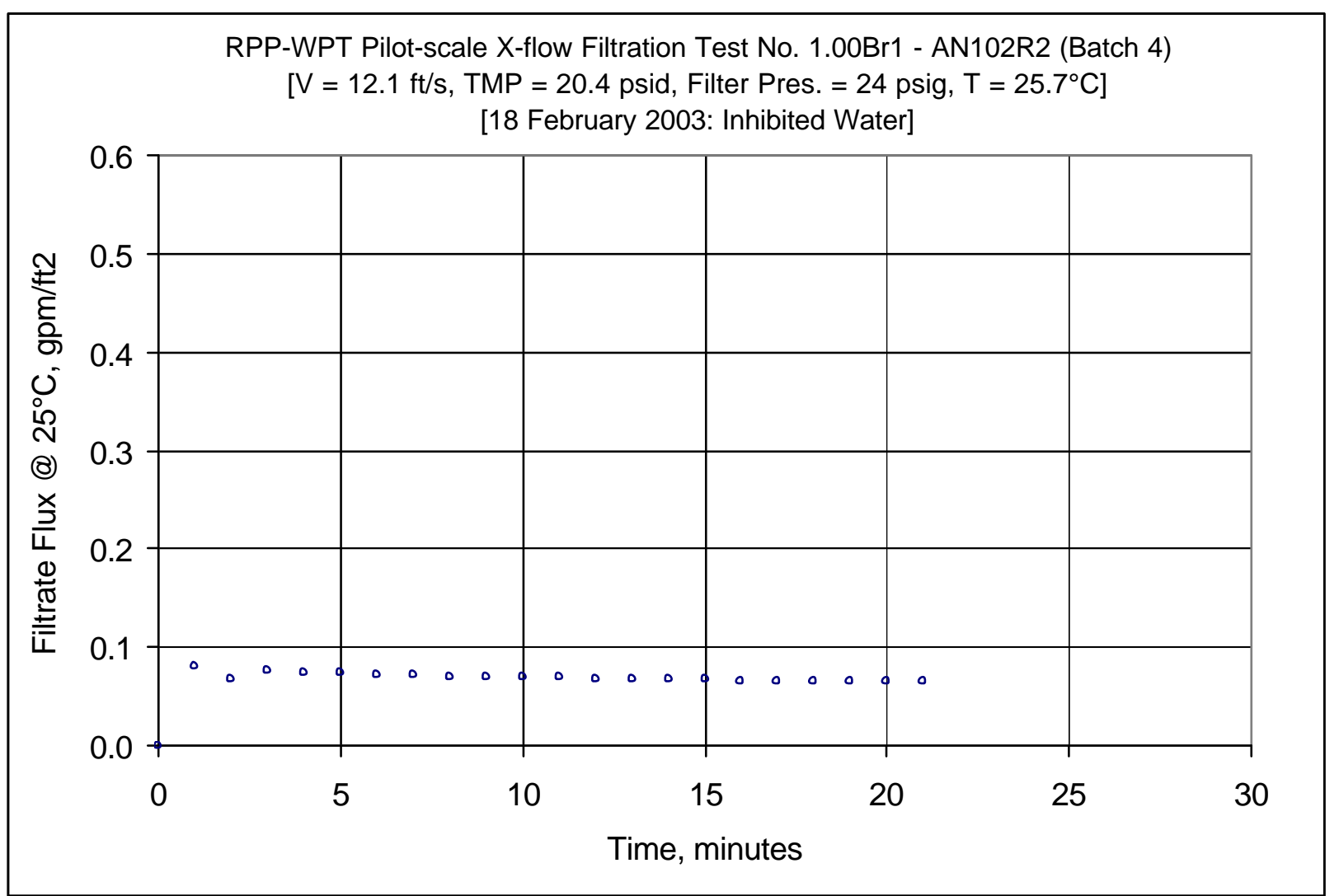

Figure A81: AN-102R2, Batch 4 Slurry Test 1.00Br1 - Inhibited Deionized \& Filtered Water (compare to Test 1.00B done on 2/12/03) 
WSRC-TR-2003-00204, REV. 0

SRT-RPP-2003-00087, REV. 0

\begin{tabular}{|c|c|c|c|c|c|c|c|c|c|c|c|c|c|c|c|c|c|c|c|c|c|c|c|c|c|c|c|}
\hline \multirow{3}{*}{ DATE } & \multirow[b]{2}{*}{ TIME } & \multicolumn{5}{|c|}{$\ll \ll \ll<\ll<\ll<$ Temperature Measurements $>$} & \multicolumn{6}{|c|}{ 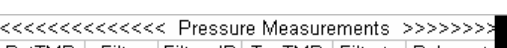 } & \multicolumn{4}{|c|}{$\mid \ll \ll<\ll<\ll<$ Flow Measurements 》》 } & \multicolumn{11}{|c|}{ 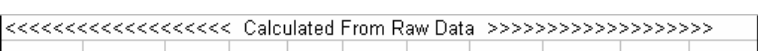 } \\
\hline & & Filtrate & Cleaning & Slurry & HiAmb. & Lo Amb. & BotTMP & Filter & Filter $\mathrm{dP}$ & TopTMP & Filtrate & Pulsepot & Slurry & Filtrate & Hi Filtate & Backpulse & & & & & & & & & & & \\
\hline & & $\operatorname{deg} C$ & $\operatorname{deg} C$ & $\operatorname{deg} C$ & $\operatorname{deg} C$ & $\operatorname{deg} C$ & psid & psig & psid & psid & psig & psig & gpm & $\mathrm{gpm}$ & $\mathrm{gpm}$ & $\mathrm{gpm}$ & & & & & & & & & & & \\
\hline & & $\mathrm{T} 2$ & T3 & T1 & T4 & T5 & $\mathrm{dP2} 2$ & P1 & $\mathrm{dP} 1$ & $\mathrm{dP3}$ & $\mathrm{P} 2$ & $\mathrm{P} 3^{3}$ & 01 & 02 & $\mathrm{Q3}$ & Obp & & & & & & & & & & & \\
\hline \multicolumn{28}{|l|}{ Zeros } \\
\hline $2 / 18 / 2003$ & $7: 41: 35 \mathrm{AM}$ & 22.045 & 17.928 & 13.92 & 21.779 & 16.794 & -0.125 & -0.104 & -0.058 & 0.055 & -0.175 & 0.227 & -0.216 & 0.001 & 0.001 & & & & \multicolumn{4}{|c|}{ Filter Surface 6.707 FT2 } & & & & & \\
\hline $2 / 18 / 2003$ & 7:41:53 AM & 22.045 & 17.928 & 13.92 & 21.779 & 16.794 & -0.125 & -0.104 & -0.058 & 0.055 & -0.175 & 0.227 & -0.216 & 0.001 & 0.001 & & & & \multicolumn{2}{|c|}{ Conversion } & $851 n$ & \multicolumn{4}{|c|}{$1 \mathrm{~m} 3 / \mathrm{m} 2 / \mathrm{day} / \mathrm{barg} / \mathrm{gpm} / \mathrm{t} 2 / \mathrm{barg}$} & & \\
\hline $2 / 18 / 2003$ & $7: 41: 54 \mathrm{AM}$ & 22.024 & 17.912 & 13.889 & 21.744 & 16.798 & -0.125 & -0.102 & -0.058 & 0.053 & -0.175 & 0.227 & -0.215 & 0.001 & 0.001 & & & & & & & & & & & & \\
\hline $2 / 18 / 2003$ & $7: 41: 55 \mathrm{AM}$ & 22.024 & 17.907 & 13.884 & 21.739 & 16.793 & -0.125 & -0.104 & -0.059 & 0.053 & -0.178 & 0.224 & -0.218 & 0.001 & 0.001 & & & & \multirow{2}{*}{\multicolumn{7}{|c|}{ Note: Gauge Pressure P1 is a }} & & \\
\hline $2 / 18 / 2003$ & $7: 41: 56 \mathrm{AM}$ & 22.024 & 17.907 & 13.883 & 21.728 & 16.788 & -0.125 & -0.102 & -0.059 & 0.051 & -0.181 & 0.224 & -0.218 & 0.001 & 0.001 & & & & & & & & & & $1.7 p$ & & \\
\hline $2 / 18 / 2003$ & $7: 41: 57 \mathrm{AM}$ & 22.034 & 17.917 & 13.894 & 21.739 & 16.793 & -0.123 & -0.102 & -0.059 & 0.051 & -0.178 & 0.224 & -0.218 & 0.001 & 0.001 & & & & \multicolumn{8}{|c|}{ Pressure $P 1$ is corrected for 40.88 inches of water tubi } & \\
\hline $2 / 18 / 2003$ & $7: 41: 58 \mathrm{AM}$ & 22.029 & 17.922 & 13.899 & 21.749 & 16.788 & -0.123 & -0.104 & -0.059 & 0.051 & -0.181 & 0.224 & -0.218 & 0.001 & 0.001 & & & & \multicolumn{2}{|l|}{ WN } & & & $<$ Filtrat & ate Flux. & $\ll$ PERN & MMIABILIT & ITY \\
\hline $2 / 18 / 2003$ & 7:41:59 AM & 22.034 & 17.922 & 13.904 & 21.759 & 16.788 & -0.125 & -0.102 & -0.058 & 0.053 & -0.181 & 0.221 & -0.216 & 0.001 & 0.001 & & & & & & & & & at $25 \mathrm{C}$ & & $\times 1000$ & \\
\hline & & & & & & & & & & & & & & & & & Time & Time & Press. & Vel. & TMP T & TMP & $\mathrm{gpm}$ & $\mathrm{gpm}$ & $\mathrm{gpm}$ & $\mathrm{gpm}$ & meter \\
\hline Data - Per M & Minute & & & & & & & & & & & & & & & & & Hour & & $\mathrm{f} / \mathrm{s}$ & & bar & & $\mathrm{f} 2 \mathrm{~m}$ & f $\mathrm{f} 2 / \mathrm{psi}$ & $\mathrm{ft} 2 / \mathrm{psi}$ & \\
\hline 2/18/2003 & 1:54:17 PM & 25.33 & 19.22 & 25.598 & 22.647 & 20.519 & 3.073 & 25.689 & 4.637 & -1.366 & 17.63 & 65.978 & 49.257 & 0.001 & 0.001 & & & 0 & $\frac{19 y}{24.2}$ & 212.1 & 0.9 & 0.059 & 0.000 & $\frac{12.000}{0.000}$ & 0.0002 & 0.172 & 0.15 \\
\hline $2 / 18 / 2003$ & 1:55:17 PM & 25.108 & 19.248 & 25.586 & 22.751 & 20.588 & 19.046 & 25.728 & 4.648 & 14.676 & 1.394 & 0.496 & 49.306 & 0.552 & 0.001 & & 1.00 & 0.01667 & 24.3 & \begin{tabular}{|l|} 
\\
\end{tabular} & 16.9 & 1.163 & 0.082 & 0.081 & 0.0048 & 4.801 & 4.09 \\
\hline $2 / 18 / 2003$ & 1:56:17 PM & 25.621 & 19.266 & 25.583 & 22.793 & 20.62 & 19.272 & 25.693 & 4.617 & 14.899 & 1.102 & 0.615 & 49.356 & 0.46 & 0.001 & & 2.00 & 0.03333 & 24.2 & 12.1 & 17.1 & 1.178 & 0.069 & 0.067 & 0.0039 & 3.949 & 3.36 \\
\hline $2 / 18 / 2003$ & 1:57:17 PM & 25.728 & 19.288 & 25.565 & 22.805 & 20.653 & 22.035 & 25.819 & 4.64 & 17.509 & -1.285 & -2.338 & 49.406 & 0.512 & 0.001 & & 3.00 & 0.05 & 24.3 & \begin{tabular}{|l|}
3 \\
\end{tabular} & 19.8 & 1.363 & 0.076 & 0.075 & 0.0038 & 3.800 & 3.23 \\
\hline $2 / 18 / 2003$ & 1:58:17 PM & 25.765 & 19.316 & 25.557 & 22.757 & 20.695 & 22.267 & 25.763 & 4.579 & 17.89 & -1.285 & -2.506 & 49.306 & 0.505 & 0.001 & & 4.00 & 0.06667 & 24.3 & \begin{tabular}{|l|}
12.1 \\
\end{tabular} & 20.1 & 1.384 & 0.075 & 0.074 & 0.0037 & 3.692 & 3.14 \\
\hline $2 / 18 / 2003$ & 1:59:17 PM & 25.761 & 19.342 & 25.599 & 22.708 & 20.727 & 22.271 & 25.672 & 4.632 & 17.765 & -1.285 & -2.588 & 49.316 & 0.497 & 0.0 & & 5.00 & 0.08333 & 24.2 & 12.1 & 20.0 & 1.380 & 0.074 & 0.073 & 0.0036 & 3.640 & 10 \\
\hline $2 / 18 / 2003$ & 2:00:17 PM & 25.823 & $\begin{array}{r}19.342 \\
19.38\end{array}$ & 25.686 & 22.735 & 20.779 & 22.259 & 25.488 & \begin{tabular}{|l|}
4.052 \\
4.563
\end{tabular} & 17.912 & $\begin{array}{l}-1.200 \\
-1.282 \\
\end{array}$ & $\begin{array}{l}-2.554 \\
-2.654\end{array}$ & $\begin{array}{l}49.10 \\
49.429\end{array}$ & 0.49 & 0.0 & & $\frac{5.00}{6.00}$ & 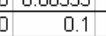 & $\begin{array}{l}24.2 \\
24.0\end{array}$ & $\begin{array}{l}12.1 \\
12.1\end{array}$ & \begin{tabular}{|l|}
20.0 \\
\end{tabular} & \begin{tabular}{|l|}
1.300 \\
1.385 \\
\end{tabular} & \begin{tabular}{|l|l|}
0.073 \\
\end{tabular} & 0.072 & \begin{tabular}{|l|l|}
0.0036 \\
\end{tabular} & 3.0468 & 3.04 \\
\hline $2 / 18 / 2003$ & $2: 01: 17 \mathrm{PM}$ & 25.825 & 19.402 & 25.683 & 22.712 & 20.816 & 22.344 & 25.639 & 4.627 & 17.662 & -1.282 & -2.706 & 49.377 & 0.486 & 0.0 & & 7.00 & 0.11667 & 24.2 & 12.1 & $\begin{array}{ll}120.0 \\
20\end{array}$ & 1.379 & 0.072 & 0.071 & 0.0036 & 3.554 & \\
\hline $2 / 18 / 2003$ & 2:02:17 PM & 25.832 & 19.419 & 25.649 & 22.643 & 20.843 & $\begin{array}{r}22.044 \\
22.54\end{array}$ & 25.736 & \begin{tabular}{|l|}
4.021 \\
\end{tabular} & 18.068 & -1.285 & -2.755 & 49.373 & 0.48 & 0.001 & & 8.00 & 0.13333 & $\begin{array}{l}24.2 \\
\end{array}$ & $\begin{array}{ll}12.1 \\
3\end{array}$ & 120.3 & 1.400 & 0.072 & 0.070 & 0.0035 & 3.461 & 2.95 \\
\hline $2 / 18 / 2003$ & 2:03:17 PM & 25.853 & 19.445 & 25.63 & 22.59 & 20.855 & 22.517 & 25.633 & 4.65 & 17.957 & -1.285 & -2.793 & 49.35 & 0.476 & 0.001 & & 9.00 & 0.15 & 24.2 & 12.1 & 120.2 & 1.395 & 0.071 & 0.070 & 0.0034 & 3.445 & 2.93 \\
\hline $2 / 18 / 2003$ & 2:04:17 PM & 25.865 & 19.462 & 25.612 & 22.516 & 20.912 & 22.712 & 25.832 & 4.645 & 18.164 & -1.285 & -2.828 & 49.318 & & 0.0 & & 10.00 & 0.16667 & 24.4 & $\begin{array}{l}12.1 \\
\end{array}$ & 120.4 & 1.409 & 0.070 & 0.069 & 0.0034 & 3.384 & 2.88 \\
\hline $2 / 18 / 2003$ & 2:05:17 PM & 25.881 & 19.479 & 25.613 & \begin{tabular}{|l|}
22.437 \\
\end{tabular} & 20.933 & 22.665 & 25.699 & \begin{tabular}{|l|}
4.045 \\
\end{tabular} & 18.096 & -1.285 & $\begin{array}{l}-2.051 \\
-2.851\end{array}$ & 49.326 & 0.469 & 0.0 & & 11.00 & 0.18333 & $\begin{array}{l}24.4 \\
\end{array}$ & 12.1 & $\begin{array}{l}120.4 \\
120.4\end{array}$ & 1.405 & 0.070 & 0.069 & 0.0034 & 3.372 & 2.87 \\
\hline $2 / 18 / 2003$ & 2:06:17 PM & 25.877 & 19.495 & 25.664 & 22.362 & 20.919 & 22.565 & 25.556 & 4.65 & 17.974 & -1.285 & -2.8 & 49.169 & 0.467 & 0.0 & & 12.00 & 0.2 & 24.1 & 12.0 & 20.3 & 1.398 & 0.070 & 0.068 & 0.0034 & 3.372 & 2.87 \\
\hline $2 / 18 / 2003$ & $2: 07: 17 \mathrm{PM}$ & 25.883 & 19.511 & 25.72 & 22.313 & 20.91 & 22.677 & 25.55 & 4.624 & 18.266 & -1.288 & -2.926 & 49.601 & 0.461 & 0.0 & & 13.00 & 0.21667 & 24.1 & 12.2 & 220.5 & 1.411 & 0.069 & 0.067 & 0.0033 & 3.290 & 2.80 \\
\hline $2 / 18 / 2003$ & 2:08:17 PM & 25.905 & 19.533 & 25.717 & 22.279 & 20.8 & 22.749 & 25.594 & $\begin{array}{l}4.024 \\
4.683\end{array}$ & 18.14 & -1.285 & $\begin{array}{l}-2.958 \\
-2.958\end{array}$ & $\begin{array}{l}49.0010 \\
49.729\end{array}$ & 0.46 & 0. & & 14.00 & 0.23333 & $\begin{array}{l}24.1 \\
\end{array}$ & 12.2 & 20.4 & 1.410 & $\begin{array}{l}0.0069 \\
0.069\end{array}$ & 0.067 & 0.0033 & 3.288 & 2.80 \\
\hline $2 / 18 / 2003$ & 2:09:17 PM & 25.901 & 19.554 & 25.693 & 22.25 & 20.897 & 22.737 & 25.625 & 4.62 & 18.375 & -1.282 & -2.973 & 49.582 & 0.455 & 0.0 & & 15.00 & 0.25 & 24.2 & 12.2 & 20.6 & 1.417 & 0.068 & 0.067 & 0.0032 & 3.236 & 2.75 \\
\hline $2 / 18 / 2003$ & 2:10:17 PM & 25.907 & 19.576 & 25.669 & 22.216 & 20.959 & 22.872 & 25.695 & 4.7 & 18.328 & -1.288 & -2.993 & 49.715 & 0.453 & 0.0 & & 16.00 & 0.26667 & 24.2 & 12.2 & 220.6 & 1.420 & 0.068 & 0.066 & 0.0032 & 3.218 & 2.74 \\
\hline $2 / 18 / 2003$ & 2:11:17 PM & 25.913 & 19.582 & $\begin{array}{r}25.0005 \\
25.65\end{array}$ & 22.172 & $\begin{array}{r}20.959 \\
\end{array}$ & 22.812 & 25.61 & 4.641 & $\begin{array}{l}10.320 \\
8.27\end{array}$ & $\begin{array}{l}-1.200 \\
-1.291\end{array}$ & $\begin{array}{l}-3.019 \\
-3.019\end{array}$ & 49.566 & 0.451 & 0.0 & & $\begin{array}{l}10.00 \\
17.00\end{array}$ & 0.28333 & \begin{tabular}{|l|}
24.2 \\
\end{tabular} & 12.1 & 120.5 & 1.416 & \begin{tabular}{|l|}
0.060 \\
0.067
\end{tabular} & 0.066 & 0.0032 & 3.214 & 2.74 \\
\hline $2 / 18 / 2003$ & $2: 12: 17 \mathrm{PM}$ & 25.924 & 19.603 & 25.661 & 22.163 & 20.941 & 22.865 & 25.544 & 4.659 & 18.356 & -1.285 & -3. & 49.643 & 0.448 & ח & & 18.00 & 0.3 & 24.1 & 12.2 & 220.6 & 1.421 & 67 & 0.066 & 0.0032 & 3.181 & 2.71 \\
\hline $2 / 18 / 2003$ & 2:13:17 PM & 25.936 & 19.614 & 25.688 & 22.124 & 20.9 & 22.851 & 25.573 & 4.629 & 18.414 & -1.2 & -3.0 & 49.222 & & & & 19.00 & 0.31667 & 24.1 & 12.1 & 120.6 & 1.423 & 77 & 0.066 & & 3.175 & 2.70 \\
\hline $2 / 18 / 2003$ & 2:14:17 PM & $\begin{array}{l}25.930 \\
25.942\end{array}$ & 19.636 & $\begin{array}{l}25.600 \\
25.759\end{array}$ & 22.106 & 20.914 & 22.838 & 25.546 & $\begin{array}{l}4.029 \\
4.653\end{array}$ & $\begin{array}{l}10.414 \\
18.414\end{array}$ & $\begin{array}{l}-1.200 \\
-1.282 \\
\end{array}$ & $\begin{array}{l}-3.0 \\
-3.0 \\
-10\end{array}$ & $\begin{array}{l}49.222 \\
49.272\end{array}$ & 0.446 & 0.0 & & 20.00 & 0.33333 & \begin{tabular}{|l|}
24.1 \\
\end{tabular} & 12.1 & $\begin{array}{l}120.6 \\
110.6\end{array}$ & 1.422 & \begin{tabular}{|l|l|}
0.066 \\
\end{tabular} & 0.065 & 0.0032 & 3.156 & 2.69 \\
\hline $2 / 18 / 2003$ & 2:15:17 PM & 25.929 & 19.638 & 25.766 & 22.067 & 20.91 & 22.95 & 25.621 & 4.625 & 18.612 & -1.285 & -3.071 & 49.524 & 0.444 & 0.001 & & 21.00 & 0.35 & 24.1 & 12.1 & 120.8 & 1.433 & 0.066 & 0.065 & 0.0031 & 3.118 & 2.65 \\
\hline & & & & & & & & & & & & & & & & & & & & & & & & & & & \\
\hline & Averages & 25.9 & 19 & 25.7 & 22 & & 22 & 25.6 & 4.6 & & -1.3 & & & 0. & 0.0 & & & 0.2 & 24.2 & 12.1 & 20.4 & 1.404 & 0.070 & 0.0 & 134 & 3.377 & 2.9 \\
\hline & Maximum & 25 & & 25.8 & & 21 & & & & & & -2 & & . & & & & 0.4 & 24.4 & 12 & 220 & 1.4 & 0.076 & & & 3.800 & 2 \\
\hline & Median & 25 & 19 & 25 & 22.4 & & 22 & & & & & -2 & & & U. & & & 0.2 & 24.2 & 12.1 & 120.4 & 1.4 & 0.070 & 0.068 & 0.0034 & 3.372 & 2.9 \\
\hline & Minimum & 25.7 & 19.3 & 25.6 & 22.1 & 20.7 & 22.0 & 25.5 & 4.6 & 17.5 & -1.3 & -3.1 & 49.2 & 0.4 & 0.0 & 0.0 & & 0.1 & 24.0 & 12.0 & 19.8 & 1.363 & 0.066 & 0.065 & 0.0031 & 3.118 & 2.7 \\
\hline & $2 \times$ Std Dev & 0.122 & 0.212 & 0.112 & 0.494 & 0.176 & 0.508 & 0.186 & 0.062 & 0.558 & 0.004 & 0.399 & 0.323 & 0.041 & 0.000 & 0.000 & & 0.183 & 0.186 & 0.079 & 90.525 & 0.036 & 0.006 & 0.006 & 0.0004 & 0.386 & 0.329 \\
\hline Number of & Points Used & 19 & 19 & 19 & 19 & . & 19 & 19 & 19 & 19 & 19 & 19 & 19 & 19 & 19 & 19 & & 19 & 19 & 19 & $9 \quad 19$ & 19 & 19 & 19 & 19 & 19 & 19 \\
\hline & & (1) & & & & & & & & & & & & & & & & & & & & & & & & & \\
\hline
\end{tabular}

Figure A82: AN-102R2, Batch 4 Slurry Test 1.00Br1 - Inhibited Deionized \& Filtered Water - Raw \& Calculated Data 
WSRC-TR-2003-00204, REV. 0

SRT-RPP-2003-00087, REV. 0

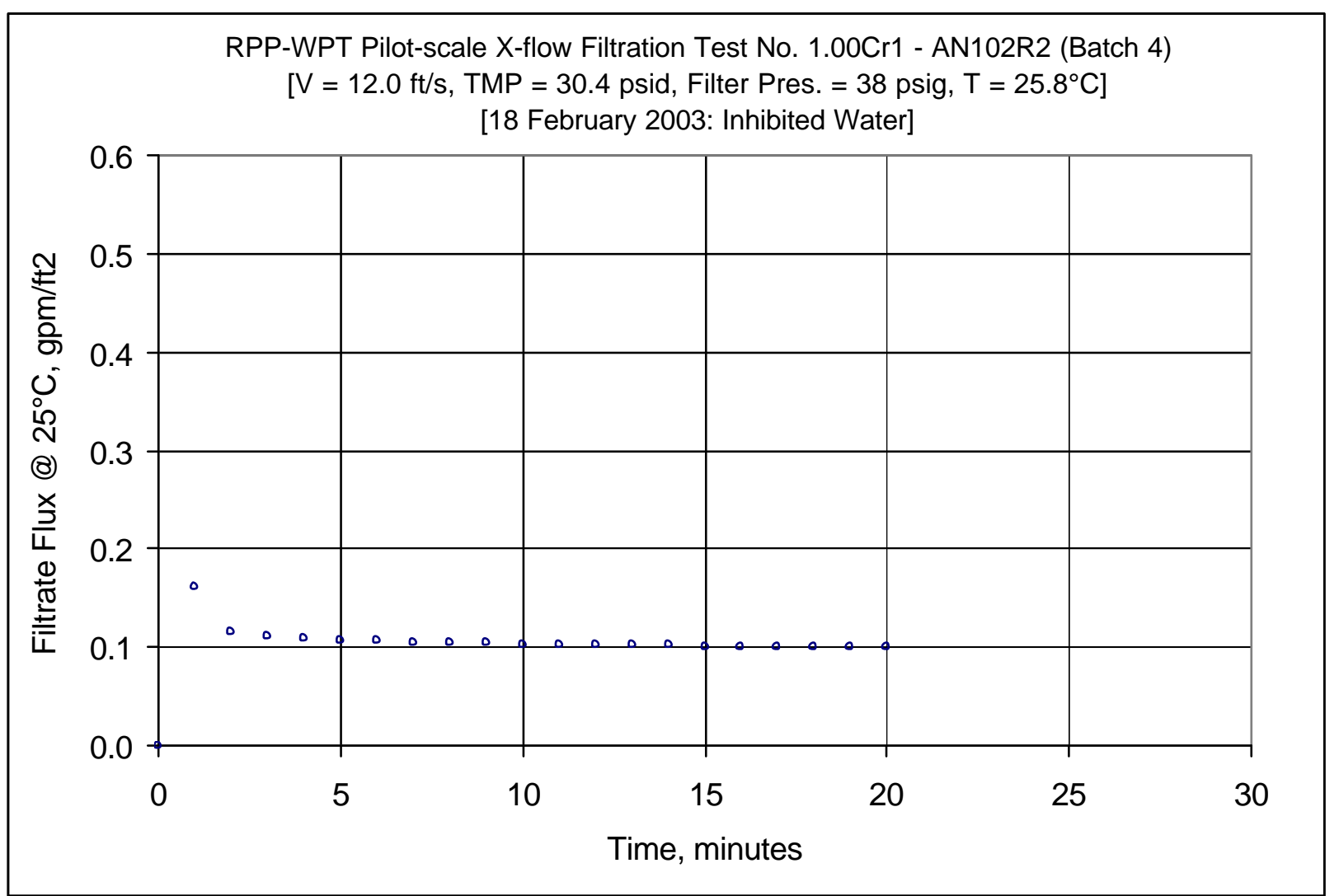

Figure A83: AN-102R2, Batch 4 Slurry Test 1.00Cr1 - Inhibited Deionized \& Filtered Water (compare to Test 1.00C done on 2/12/03) 
WSRC-TR-2003-00204, REV. 0

SRT-RPP-2003-00087, REV. 0

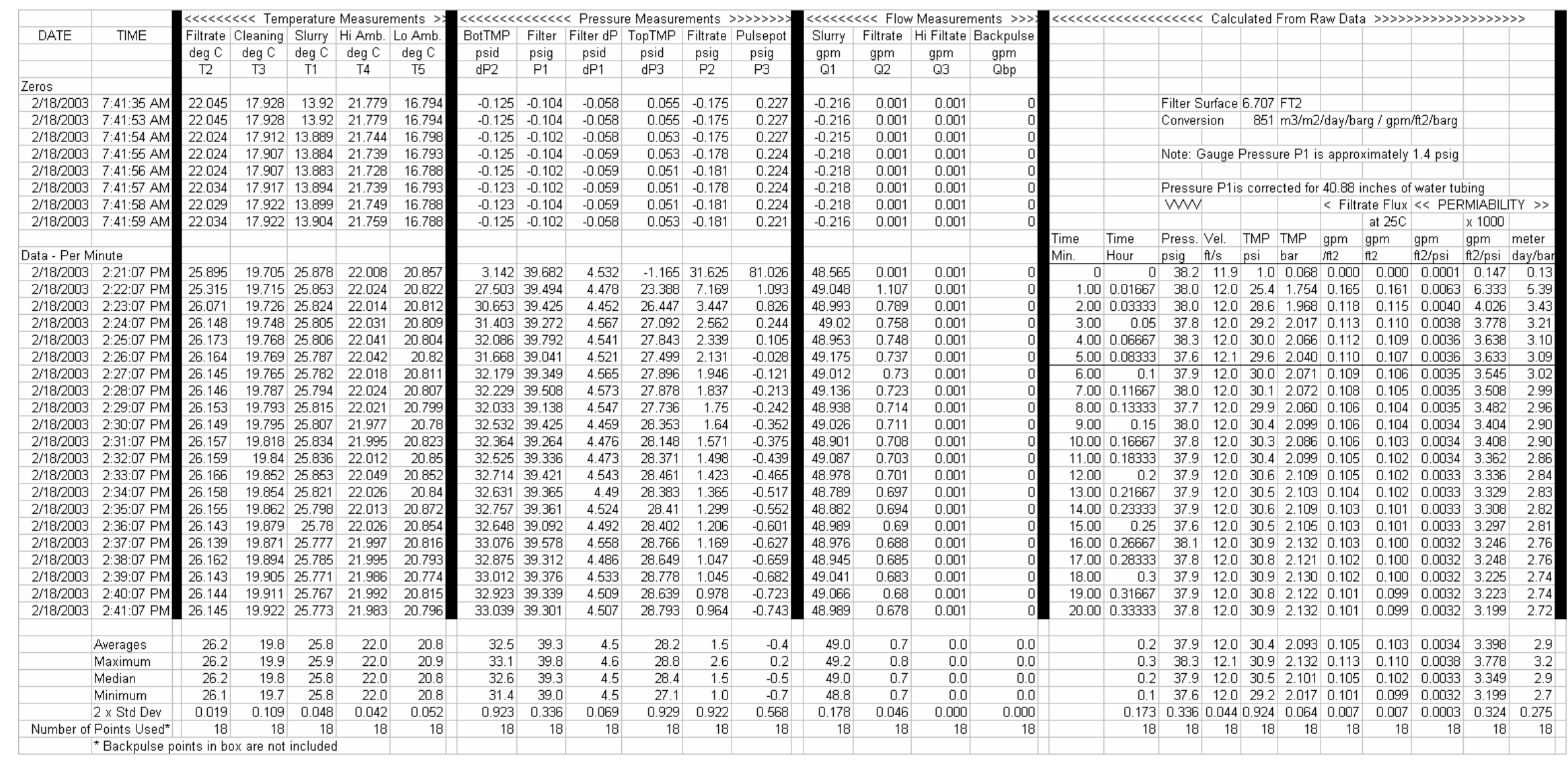

Figure A84: AN-102R2, Batch 4 Slurry Test 1.00Cr1 - Inhibited Deionized \& Filtered Water - Raw \& Calculated Data 
WSRC-TR-2003-00204, REV. 0

SRT-RPP-2003-00087, REV. 0

This page intentionally left blank 


\section{Appendix B: Baseline Slurry}

As required by the RPP-WTP Test Specification each time the cross-flow filter experienced a slurry run the filter was to be tested with a standard slurry, called here the Baseline Slurry, of $5 \mathrm{wt} \%$ of SrCo3 in distilled and filtered (to 0.1 micron absolute) water. Those tests were noted in the test matrix as runs 1.01 before a slurry test and 1.33 after a slurry. Initial tests with the slurry (during the preconditioning test with AN-107 and slurry AN-102R2 batch 3C) determined that only with considerable number of water rinses and acid cleaning could the baseline slurry be removed from the filter, costing time and money, therefore starting the AN-102R2 batch 3B test the RPP-WTP customer allowed baselining the filter only after each slurry test (run 1.33). As such, the baseline slurry run 1.33 after any slurry test could be used as the initial baseline run (1.01) for the next subsequential slurry test. This report deals with the testing of five separate batches of slurry (peconditioning with AN-107, AN-102R2:3C, AN-102R2:3B, AN-102R2:3A, and AN-102R2:4) therefore the baseline slurry runs were:

$2 \mathrm{~A}, 2 \mathrm{~B}$

$2 \mathrm{~A}, 2 \mathrm{~B}, 2 \mathrm{C}, 2 \mathrm{D}$

7A, 7B, 7C

$1.01 \mathrm{~A}, 1.01 \mathrm{~B}, 1.01 \mathrm{C}$

$1.33 \mathrm{~A}, 1.33 \mathrm{~B}, 1.33 \mathrm{C}$

$1.33 \mathrm{~A}, 1.33 \mathrm{~B}, 1.33 \mathrm{C}$

$1.33 \mathrm{~A}, 1.33 \mathrm{~B}, 1.33 \mathrm{C}$

$1.33 \mathrm{~A}, 1.33 \mathrm{~B}, 1.33 \mathrm{C}$
Before Preconditioning the filter with AN-107; pre-test rig modification After Preconditioning the filter with AN-107, post-test rig modification After Preconditioning the filter with AN-107

Before AN-102R2, batch 3C

After AN-102R2, batch 3C

After AN-102R2, batch 3B

After AN-102R2, batch 3A

After AN-102R2, batch 4A

The letter a, b, c indicate different constant transmembrane pressure (TMP), generally, but not always: $\mathrm{A}=10$ psid, $\mathrm{B}=20$ psid, $\mathrm{C}=30$; psid. Test runs that do not hold to this are 2A, 2B, (pre-test rig modification) because the high filtration pressure drop did not allow TMPs above 7 psid; $2 \mathrm{D}$ was done at above 40 psid to determine the limit the modified test rig could attain.

For each baseline slurry test this appendix contains a graph of the filtrate flux with time and tables of the data taken and calculated. To better understand the presented information important aspects of the graphs and tables are explained below:

Graphs - Each graph contains time averages of some of the imporant parameters taken during the run at the top of the figures, i.e. Slurry velocity in the filter tube, V; transmembrane pressure, TMP; slurry pressure at the entrance of the filter tube, Filter Pres; and the slurry temperature, T.

Tables - Each table is divided into raw data (the first 17 columns) and calculations (the last 10 columns) which are based on the raw data. The raw data section is divided into three parts:

\section{Individual Entries - Raw Data}

Temperature Filtrate - from the filtrate loop at the exit of the filter bundle

Cleaning - from the cleaning loop (this loop was not used)

Slurry - from the slurry loop in the slurry reservoir

Hi Amb. - outside ambient temperature at the top of the test rig

Lo Amb. - inside ambient temperature at the bottom of the test rig

Pressure $\quad$ BotTMP - differential pressure across the filter tube at the bottom of the vertical tube Filter - gauge pressure of the slurry at the entrance of the filter tube bundle

Filter $\mathrm{dP}$ - differential pressure of the slurry along the height of the vertical 90 -inch tube TopTMP - differential pressure across the filter tube at the top the vertical 90-inch tube Filtrate - gauge pressure of the filtrate at the exit of the filter tube housing

Pulsepot - gauge pressure of the filtrate at the bottom of the pulsepot

Flow Slurry - rate of slurry just before entering the seven-filter-tube bundle Filtrate - rate of slurry filtrate under normal flow (max. is $1.2 \mathrm{gpm}$ )

Hi Filtrate - rate of any liquid which has a high flowrate (water, acid, etc.) (max. $5 \mathrm{gpm}$ ) Backpulse - rate of filtrate in opposite direction from normal flow, i.e., backpulse 


\section{WSRC-TR-2003-00204, REV. 0} SRT-RPP-2003-00087, REV. 0

Individual Entries - Calculated Data

Time - Minutes after start of test, including the backpulse (based on raw data TIME column)

Press. - Internal filter pressure of slurry at the filter bundle entrance (based on raw data P1 column and corrected for a 40.9-inch water column that exists from the transducer to the point where P1 is measured.) Vel. - Slurry velocity in filter tube (based on raw data Q1 column and i.d. of the tubes of 0.488 inches) TMP - Filter transmembrane pressure (based on average of raw data columns dP2 and dP3) in psi. TMP - Filter transmembrane pressure (based on average of raw data columns dP2 and dP3) in bar. Filtrate Flux - Filtrate flow rate (raw data columns Q2/Q3) divided by inside filter surface area $(6.707 \mathrm{ft} 2)^{\dagger}$ Filtrate Flux @ $25^{\circ} \mathrm{C}$ - Same as Filtrate flux, but correct for temperature with the following multiplier:

$\mathrm{T}($ corrected $)=\mathrm{T} \times \operatorname{Exp}[2500 /((1 / 273+\mathrm{T}))-(1 / 298)]$ as per Test Spec 24590-WTP-TSP-RT-01-029, Rev. 0

Permiability - Filterate flow rate per TMP (based on calculated columns Filtrate Flux @ $25^{\circ} \mathrm{C}$ and TMP)

Permiability x 1000 - Same as the above Permiability, but increase by 1000 to facilitate graphing

Permiability - Same as Permiability but in metric units, meter/day/bar

\section{$\underline{\text { Statistics }}$}

At the bottom of the last individual entry there are six rows with some data statistics to better understand the data and their fluctuations. The columns are self explained with titles of Averages, Maximum, Median, and Minimum. The next row $2 \times$ Std Dev, is two times the population standard deviation based on the number of data points used, which is the last row. The Number of Points used generally do not included the points taken during the backpulse sequence of each test. This is explained below.

\section{Raw Data Table Layout}

The raw data is generally made up of three sections:

Zeros - These eight rows include approximately 15 to 20 seconds of data taken with the pressure transducers placed in the "zero" mode to determine if any zero drift occurred from one test to the next.

Data-Per Minute - On most of the tables the first six rows of these data are contained in a box which indicate data taken during the backpulse. This is were the filtrate flow is reversed temporarily to knock the filtrate cake of the filter surface. During these first few minutes the filtrate flow is stopped, then reversed, then stopped again, then it is finally returned to normal operation. The data during the backpulse operation were taken with a separate log, therefore those data are marked with a box to note this fact. Most or all of those data points are not counted in the statistics at the bottom of the table. The tables of some of the later tests (see Figs. B36, B38, and B40) do not show the boxed data because separate logs of data were not taken, however since a backpulse for each run still occurred only those data were not included in the statistics at the bottom of the page.

\footnotetext{
${ }^{\dagger}$ For the baseline slurry tests during preconditioning it will be noticed that $6.87 \mathrm{ft} 2$ was used. This was based on a tube inside diameter of 0.5 inch, which was based on the manufacturer's specification. After the tubes were measured the i.d. was changed to 0.488 inch and the area to $6.707 \mathrm{ft} 2$. This means that absolute axial velocity and filtrate flux are low by $2.5 \%$ for the preconditioning runs.
} 
WSRC-TR-2003-00204, REV. 0

SRT-RPP-2003-00087, REV. 0

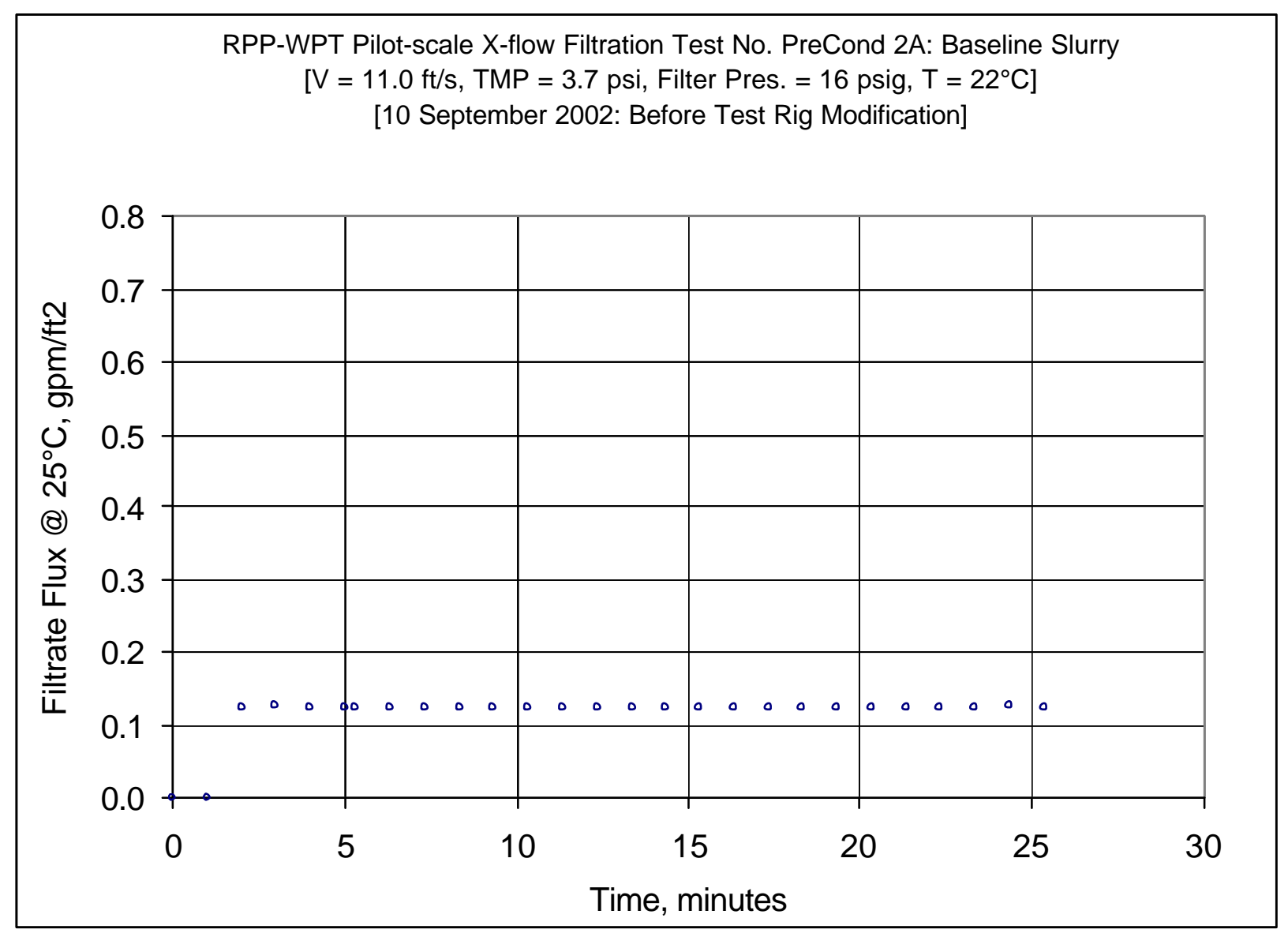

Figure B1: PreConditioning 2A - Baseline Slurry - Before Test Rig Modification 
WSRC-TR-2003-00204, REV. 0

SRT-RPP-2003-00087, REV. 0

\begin{tabular}{|c|c|c|c|c|c|c|c|c|c|c|c|c|c|c|c|c|c|c|c|c|c|c|c|c|c|c|}
\hline \multirow[b]{2}{*}{ DATE } & \multirow[b]{2}{*}{ TIME } & \multicolumn{5}{|c|}{$\ll \ll<\ll<\ll<$ Temperature Measurements > } & \multicolumn{6}{|c|}{ 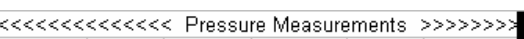 } & \multicolumn{4}{|c|}{ 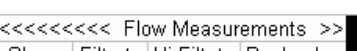 } & \multicolumn{4}{|c|}{ 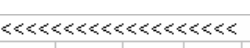 } & \multicolumn{6}{|c|}{ 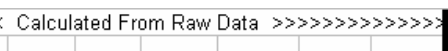 } \\
\hline & & Filtrate & Cleaning & Slurry & Hi Amb. & Lo Amb. & BotTMP & Filter & Filter $\mathrm{dP}$ & TopTMP & Filtrate & Pulsepot & Slurry & Filtrate & Hi Filtate & Backpulse & & & & & & & & & & \\
\hline & & & $\operatorname{deg} C$ & $\operatorname{deg} C$ & $\operatorname{deg} C$ & $\operatorname{deg} C$ & psid & psig & psid & psid & psig & psig & $\mathrm{gpm}$ & gpm & gpm & $\mathrm{gpm}$ & & & & & & & & & & \\
\hline \multirow{2}{*}{\multicolumn{27}{|c|}{ Zeros }} \\
\hline & & & & & & & & & & & & & & & & & & & & & & & & & & \\
\hline $9 / 10 / 2002$ & 8:48:15 AM & 21.896 & 20.524 & 21.376 & 27.274 & 20.244 & -0.108 & -0.07 & - 0.068 & 0.066 & -0.246 & 0.324 & 31.724 & 1.192 & 1.231 & & & \multicolumn{2}{|c|}{ Filter Surface } & $6.8 / \vdash$ & \multirow{2}{*}{\multicolumn{4}{|c|}{$\mathrm{m} 3 / \mathrm{m} 2 /$ day $/ \mathrm{barg} / \mathrm{gpm} / \mathrm{ft} 2 / \mathrm{barg}$}} & & \\
\hline $9 / 10 / 2002$ & $8.48 .37 \mathrm{AM}$ & 21.879 & 20.024 & 21.325 & 21.273 & 20258 & -0.111 & -0.07 & 0.069 & 0.0064 & 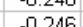 & 0.0318 & 31.724 & 1.192 & 1.201 & & & & & & & & & & & \\
\hline 9/10/2002 & 8:48:38 AM & 21.885 & 20.523 & 21.314 & 21.368 & 20.253 & -0.109 & -0.068 & -0.068 & 0.066 & -0.246 & 0.324 & 31.67 & 1.192 & 1.231 & & & \multicolumn{2}{|c|}{ Note: Gauge P } & Pressur & Ire $\mathrm{P} 1$ is & is approx & ximately 1 & $1.4 \mathrm{psig}$ & & \\
\hline $9 / 10 / 2002$ & 8:48:39 AM & 21.879 & 20.523 & 21.32 & 21.378 & 20.263 & -0.109 & -0.072 & -0.069 & 0.066 & -0.246 & 0.321 & 31.66 & 1.192 & 1.23 & & & & & & & & & & & \\
\hline $9 / 10 / 2002$ & 8:48:40 AM & 21.885 & 20.528 & 21.314 & 21.378 & 20.258 & -0.109 & -0.068 & -0.069 & 0.064 & -0.246 & 0.324 & 31.611 & 1.192 & 1.227 & & & Pressur & ure P1is & 5 correct & cted for & or 40.88 it & inches of $\mathrm{v}$ & f water tut & ubing & \\
\hline 9/10/2002 & 8:48:41 AM & 21.89 & 20.523 & 21.309 & 21.388 & 20.263 & -0.109 & -0.07 & -0.069 & 0.064 & -0.246 & 0.318 & 31.662 & 1.192 & 1.233 & & & WW & & & & $<$ Filtra & rate Flux & $\ll$ PER & RMIABILI & ITY $>>$ \\
\hline 9/10/2002 & $8: 48: 42 \mathrm{AM}$ & 21.879 & 20.517 & 21.304 & 21.383 & 20.257 & -0.109 & -0.07 & -0.069 & 0.066 & -0.246 & 0.321 & 31.62 & 1.192 & 1.232 & & & & & & & & at $25 \mathrm{C}$ & & $\times 1000$ & \\
\hline & & & & & & & & & & & & & & & & & Time & Press. & Vel. & TMP 1 & TMP & gpm & $\mathrm{gpm}$ & $\mathrm{gpm}$ & $\mathrm{gpm}$ & meter \\
\hline Data - Per M & Minute & & & & & & & & & & & & & & & & Min. & psig f & $\mathrm{ft} / \mathrm{s}$ & psi & bar & fit2 2 & $\mathrm{f} 2$ & $\mathrm{ft} 2 / \mathrm{psi}$ & $\mathrm{ft} 2 / \mathrm{psi}$ & day/bar \\
\hline 9/10/2002 & 2:26:27 PM & 25.663 & 25.076 & 22.321 & 28.75 & 25.313 & 3.23 & 17.506 & 4.845 & -1.694 & 8.966 & 58.048 & 47.069 & 0.002 & 0.001 & & 0 & 16.0 & 11.0 & 0.8 & 0.053 & 30.000 & 0.000 & 0.000 & \begin{tabular}{l|l|}
0 & 0.204 \\
\end{tabular} & 0.17 \\
\hline 9/10/2002 & 2:27:27 PM & 26.118 & 25.081 & 22.166 & 28.686 & 25.283 & 3.255 & 17.424 & 4.79 & -1.575 & 8.954 & 27.9 & 47.004 & 0.002 & 0.001 & 002 & 1 & 16.0 & 11.0 & 0.8 & 0.058 & 30.000 & 0.000 & 0.000 & $\begin{array}{l}0 \\
0.188\end{array}$ & 0.16 \\
\hline 9/10/2002 & 2:28:27 PM & 23.408 & 25.087 & 22.171 & 28.681 & 25.338 & 5.956 & 17.105 & 4.567 & 1.507 & 5.812 & 5.295 & 47.39 & 0.814 & 0.794 & & 2 & 15.6 & 11.1 & 3.7 & 0.257 & 70.116 & 0.125 & 0.034 & 433.555 & 28.56 \\
\hline 9/10/2002 & 2:29:27 PM & 23.049 & 25.119 & 22.124 & 28.594 & 25.461 & 5.788 & 16.995 & 4.676 & 1.112 & 5.885 & 5.373 & 47.413 & 0.818 & 0.798 & & 3 & 3 15.5 & 11.1 & 3.5 & 0.238 & 30.116 & 0.126 & 0.037 & 736.525 & 31.08 \\
\hline $9 / 10 / 2002$ & 2:30:27 PM & 22.876 & 25.153 & 22.333 & 28.588 & 25.47 & 6.03 & 17.318 & 4.658 & 1.305 & 5.885 & 5.336 & 47.415 & 0.816 & 0.796 & & 4 & 15.8 & 11.1 & 3.7 & 0.253 & 30.116 & 0.125 & 0.034 & 434.068 & 28.99 \\
\hline $9 / 10 / 2002$ & 2:31:27 PM & 22.799 & 25.167 & 22.382 & 28.332 & 25.448 & 6.066 & 17.231 & 4.752 & 1.35 & 5.835 & 5.35 & 47.22 & 0.814 & 0.794 & & 5 & $\begin{array}{ll}5 & 15.8 \\
\end{array}$ & 11.0 & 3.7 & 0.256 & 60.116 & 0.124 & 0.034 & 433.564 & \\
\hline $9 / 10 / 2002$ & 2:31:47 PM & 22.796 & 25.163 & 22.439 & 28.264 & 25.45 & 5.873 & 17.078 & 4.608 & 1.385 & 5.882 & 5.373 & 47.214 & 0.815 & 0.795 & & 5.33 & \begin{tabular}{|c|c|}
35.6 \\
\end{tabular} & 11.0 & 3.6 & 0.250 & 0.116 & 0.124 & 0.034 & 434.282 & 29.17 \\
\hline $9 / 10 / 2002$ & 2:32:47 PM & 22.774 & 25.161 & 22.391 & 27.987 & 25.422 & 6.059 & 17.221 & 4.819 & 1.286 & 5.885 & 5.339 & 47.316 & 0.815 & 0.795 & & 6.33 & \begin{tabular}{l|}
3 \\
3
\end{tabular} & 11.0 & 3.7 & 0.253 & 30.116 & 0.125 & 0.034 & 433.922 & 28.87 \\
\hline 9/10/2002 & 2:33:47 PM & 22.782 & 25.169 & 22.491 & 27.811 & $25.4 \mathrm{C}$ & 5.975 & 17.099 & & & 5.815 & 5.324 & & 0.813 & & & 7.33 & $\begin{array}{l}35.6 \\
\end{array}$ & 11.0 & 3.6 & & 0.116 & & 0.034 & & 29.14 \\
\hline 9/10/2002 & 2:34:47 PM & 22.79 & 25.162 & 22.458 & 27.61 & 25.364 & 5.898 & 17.045 & 4.671 & 1.344 & 5.928 & 5.414 & 47.333 & 0.816 & 0.796 & & 8.33 & 3 15.6 & 11.0 & 3.6 & 0.250 & 0.116 & 0.124 & 0.034 & 434.382 & 29.26 \\
\hline $9 / 10 / 2002$ & 2:35:47 PM & 22.802 & 25.159 & 22.475 & 27.582 & 25.341 & 6.03 & 17.363 & 61 & 411 & 6.02 & 5.489 & 47.018 & 0.82 & 0.798 & & 9.33 & $\begin{array}{l}35.9 \\
3\end{array}$ & 11.0 & 3.7 & 0.257 & 70.116 & 0.125 & 0.034 & 433.530 & 28.53 \\
\hline $9 / 10 / 2002$ & 2:36:47 PM & 22.805 & 25.157 & 22.488 & 27.645 & 25.334 & 6.082 & 17.384 & 4 & 1.45 & 6.044 & 5.481 & 47.082 & 0.819 & & & 10.3 & $3 \quad 15.9$ & 11.0 & 3.8 & & 0.116 & & 0.033 & 333.113 & 28.18 \\
\hline 9/10/2002 & 2:37:47 PM & 22.817 & 25.149 & 22.435 & 27.617 & 25.3 & 5.968 & 17.281 & & 1.297 & 6.02 & & 47.048 & & & & 11.3 & $\begin{array}{ll}35.8 \\
\end{array}$ & 11.0 & 3.6 & & 0.116 & & 0.034 & 434.339 & 9.22 \\
\hline $9 / 10 / 2002$ & 2:38:47 PM & 22.802 & 25.139 & 22.505 & 27.532 & 25.261 & 6.026 & 17.397 & 4.6 & 1.397 & 6.02 & 5.452 & 46.88 & 0.8 & 0.7 & & 12.3 & 15.9 & 10.9 & 3.7 & 0.256 & 60.116 & 0.125 & 0.034 & 433.583 & 28.58 \\
\hline 9/10/2002 & 2:39:47 PM & 22.817 & 25.129 & 22.415 & 27.337 & 25.181 & 5.89 & 17.177 & 4.552 & 1.44 & 6.006 & 5.449 & 47.081 & 0. & 0. & & 13.3 & 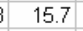 & 11.0 & 3.7 & 0. & 30.116 & 0.125 & 0.034 & 434.097 & 29.02 \\
\hline $9 / 10 / 2002$ & 2:40:47 PM & 22.827 & 25.129 & 22.521 & 27.287 & 25.1 & 6.066 & 17.392 & & 1.4 & & & 46. & & & & 14.3 & 3 15.9 & 11.0 & 3.8 & & 90.116 & & 3 & & 28.15 \\
\hline $9 / 10 / 2002$ & 2:41:47 PM & 22.819 & 25.105 & 22.401 & 27.194 & 25.067 & 6.003 & 17.359 & & 1.391 & & 5.452 & 47.23 & & & & 15.3 & 3 15.9 & 11.0 & 3.7 & 0. & 50.116 & & 4 & 857 & 28.81 \\
\hline 9/10/2002 & 2:42:47 PM & 22.817 & 25.089 & 22.495 & 27.272 & 25.03 & 5.896 & 17.107 & 4.59 & 1 & 6.026 & 5.472 & 47.018 & 0. & U & & 16.3 & $3 \quad 15.6$ & 11.0 & 3.6 & 0.251 & 10.116 & 0.125 & 0.034 & 434.266 & 29.16 \\
\hline $9 / 10 / 2002$ & 2:43:47 PM & 22.799 & 25.076 & 22.392 & 27.1 & 24.9 & 5.9 & 17.227 & & & & 5.428 & 47.094 & & & & 17.3 & 15.8 & 11.0 & 3.7 & & 20.116 & 0.125 & 0.034 & 434.199 & 29.10 \\
\hline $9 / 10 / 2002$ & 2:44:47 PM & 22.812 & 25.048 & 22.475 & 27.122 & 24.8 & 6.037 & 17.275 & & 6 & 5 & 5 & 46. & 0. & & & 18.3 & B 15.8 & 10.9 & 3.8 & 0.261 & 10.116 & 0.125 & 0.033 & 332.990 & 28.07 \\
\hline $9 / 10 / 2002$ & 2:45:47 PM & 22.793 & 25.035 & 22.391 & 27.019 & 24.8 & 5.801 & 17.163 & 4.51 & 6 & 6.049 & 5.489 & 46.886 & 0. & & & 19.3 & $\begin{array}{l}35.7 \\
\end{array}$ & 10.9 & 3.6 & 0. & 70.116 & 0.125 & 0.035 & 534.804 & 29.62 \\
\hline 9/10/2002 & 2:46:47 PM & 22.8 & 25.027 & 22.438 & 27.5 & 24.8 & & 17.272 & & & & & & & & & 20 & 8 & 11 & 7 & & 60.116 & & & & 28.57 \\
\hline $9 / 10 / 2002$ & 2:47:47 PM & 22.77 & 24.992 & 22.383 & 27.056 & 24.7 & 6.057 & 17.407 & & 1. & 5. & & 46 . & 0.8 & & & 21.3 & 15.9 & 10.9 & 3.7 & 0.254 & 16 & 0.125 & 0. & 433.888 & 28.84 \\
\hline $9 / 10 / 2002$ & 2:48:47 PM & 22.783 & 24.97 & 22.406 & 26.974 & 24.7 & 5.997 & 17.339 & 4.552 & 1.579 & 6.0. & 5 & 46.939 & 0. & 0.79 & & 22. & 15.9 & 11.0 & 3.8 & 0.261 & 16 & 0.125 & 3 & 332.998 & 28.08 \\
\hline $9 / 10 / 2002$ & 2:49:47 PM & 22.785 & 24.956 & 22.397 & 27.025 & 24.6 & 5.946 & 17.213 & & & & 5.51 & 46. & & 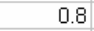 & & $23+2>3$ & B 15.7 & 11.0 & 3.7 & & 16 & & 0.1 & 433.622 & 28.61 \\
\hline $9 / 10 / 2002$ & 2:50:47 PM & 22.776 & 24.943 & 22.379 & 27.032 & 24.599 & 5.79 & 17.045 & 4.504 & 1.358 & 6.044 & 5.434 & 46.859 & 0.818 & 0.8 & & 24.3 & 15.6 & 10.9 & 3.6 & 0.246 & 0.116 & 0.125 & 0.035 & 535.089 & 29.86 \\
\hline $9 / 10 / 2002$ & 2:51:47 PM & 22.754 & 24.926 & 22.402 & 27.005 & 24.552 & 6.033 & 17.347 & 4.542 & 1.596 & 6.023 & 5.452 & 46.897 & 0.819 & 0.799 & & 25.3 & $\begin{array}{l}35.9 \\
\end{array}$ & 10.9 & 3.8 & 0.263 & 0.116 & 0.125 & 0.033 & 332.814 & 27.92 \\
\hline & & & & & & & & & & & & & & & & & & & & & & & & & & 700 \\
\hline & Maximum & 22.8 & 25.2 & 22.5 & 28.3 & 25.5 & 6.1 & 17.4 & 4.8 & 1.6 & 6.0 & 5.5 & 47.3 & 0.820 & 0 & 0. & & 159 & 110 & $\begin{array}{l}3.7 \\
3.8\end{array}$ & 34 & Ib & & 8 & 8.8 & 29.0 \\
\hline & Media & 22 & 25.1 & 22 & 27. & 25 & 6.0 & 17.3 & 4. & 1.4 & 6.1 & $5.4 \div-3$ & 47.0 & 0. & & 0. & & 15.8 & 11.0 & 3.7 & 0.254 & 0.116 & 0.125 & 0.0339 & 339 & $288,-3$ \\
\hline & & $70+2$ & 24.9 & 22 & & 24 & 5.8 & 17 & & 3 & 0.0 & & 8 & & & & & 15.6 & 10.9 & 3.6 & 0.246 & 0.116 & 0.124 & 0.0328 & 2.8 & 27. \\
\hline & $2 \times S t d$ & 0.037 & 0.162 & 0.089 & 0.710 & 0.577 & 0.166 & 0.239 & 0.157 & 0.174 & 0.131 & 0.094 & 0.307 & 0.003 & 0.003 & 0.000 & & 0.239 & $0.072 \square$ & 0.133 & 0.009 & 0.000 & 0.001 & 0.0012 & $\begin{array}{ll}2 & 1.207\end{array}$ & 1.028 \\
\hline & Point & 21 & 21 & 21 & 21 & 21 & 21 & 21 & 21 & 21 & 21 & 21 & 21 & 21 & 21 & 21 & & 21 & 21 & 21 & 21 & 21 & 21 & 21 & 21 & 21 \\
\hline & & & & & & & & & & & & & & & & & & & & & & & & & & \\
\hline
\end{tabular}

Figure B2: PreConditioning 2A - Baseline Slurry - Before Test Rig Modification - Raw \& Calculated Data 
WSRC-TR-2003-00204, REV. 0

SRT-RPP-2003-00087, REV. 0

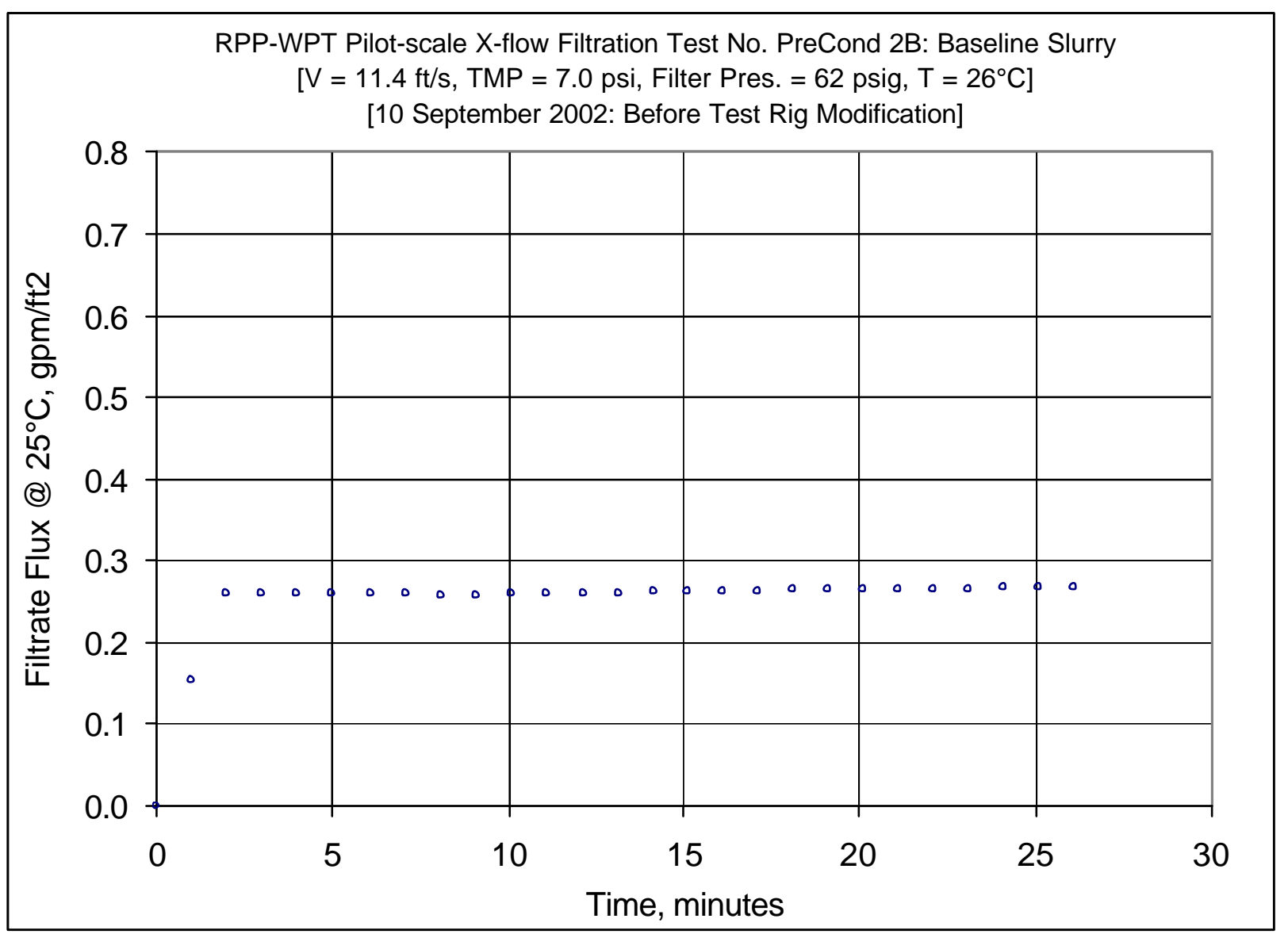

Figure B3: PreConditioning 2B - Baseline Slurry - Before Test Rig Modification 
WSRC-TR-2003-00204, REV. 0

SRT-RPP-2003-00087, REV. 0

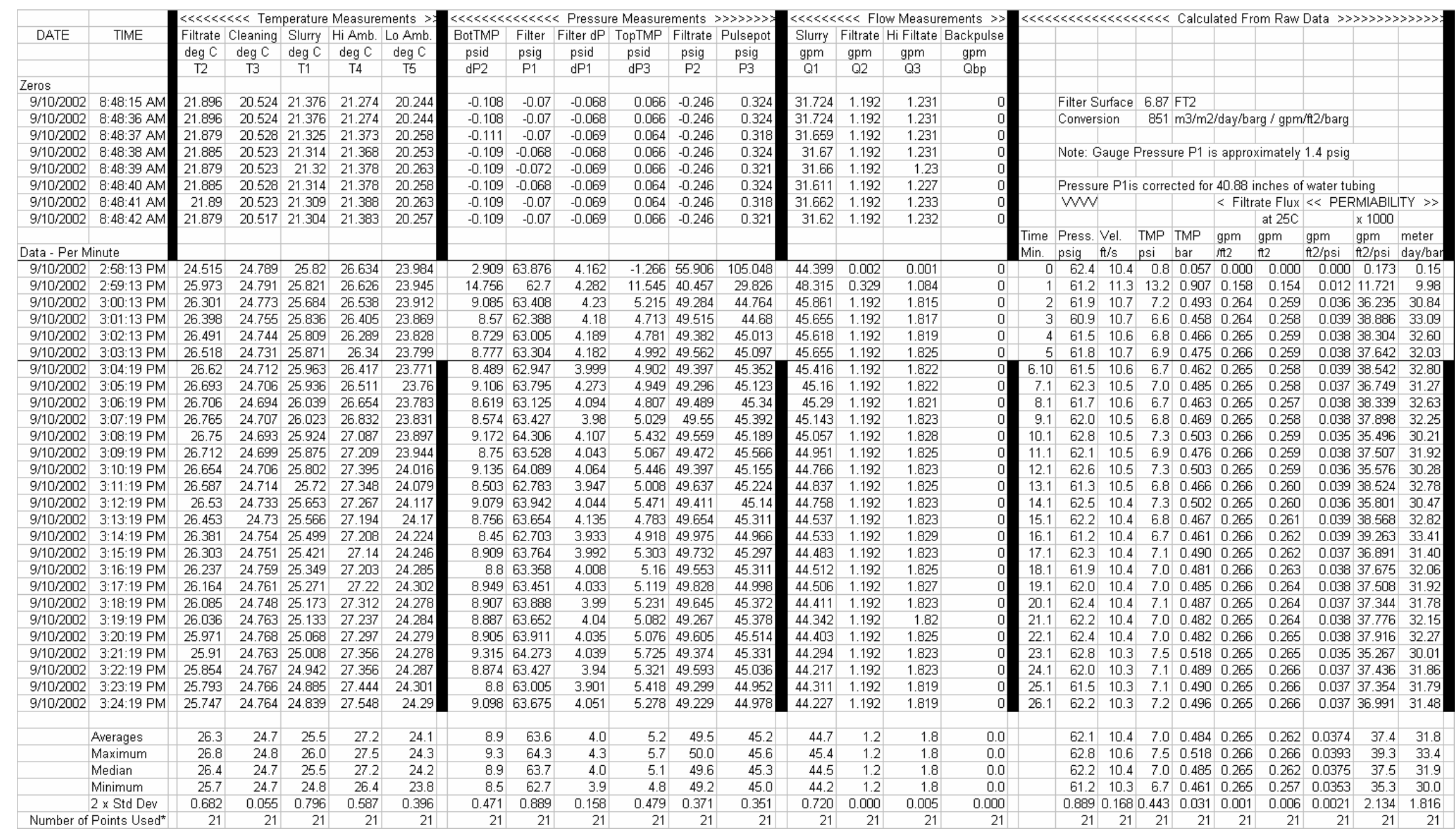

Figure B4: PreConditioning 2B - Baseline Slurry - Before Test Rig Modification - Raw \& Calculated Data 
WSRC-TR-2003-00204, REV. 0

SRT-RPP-2003-00087, REV. 0

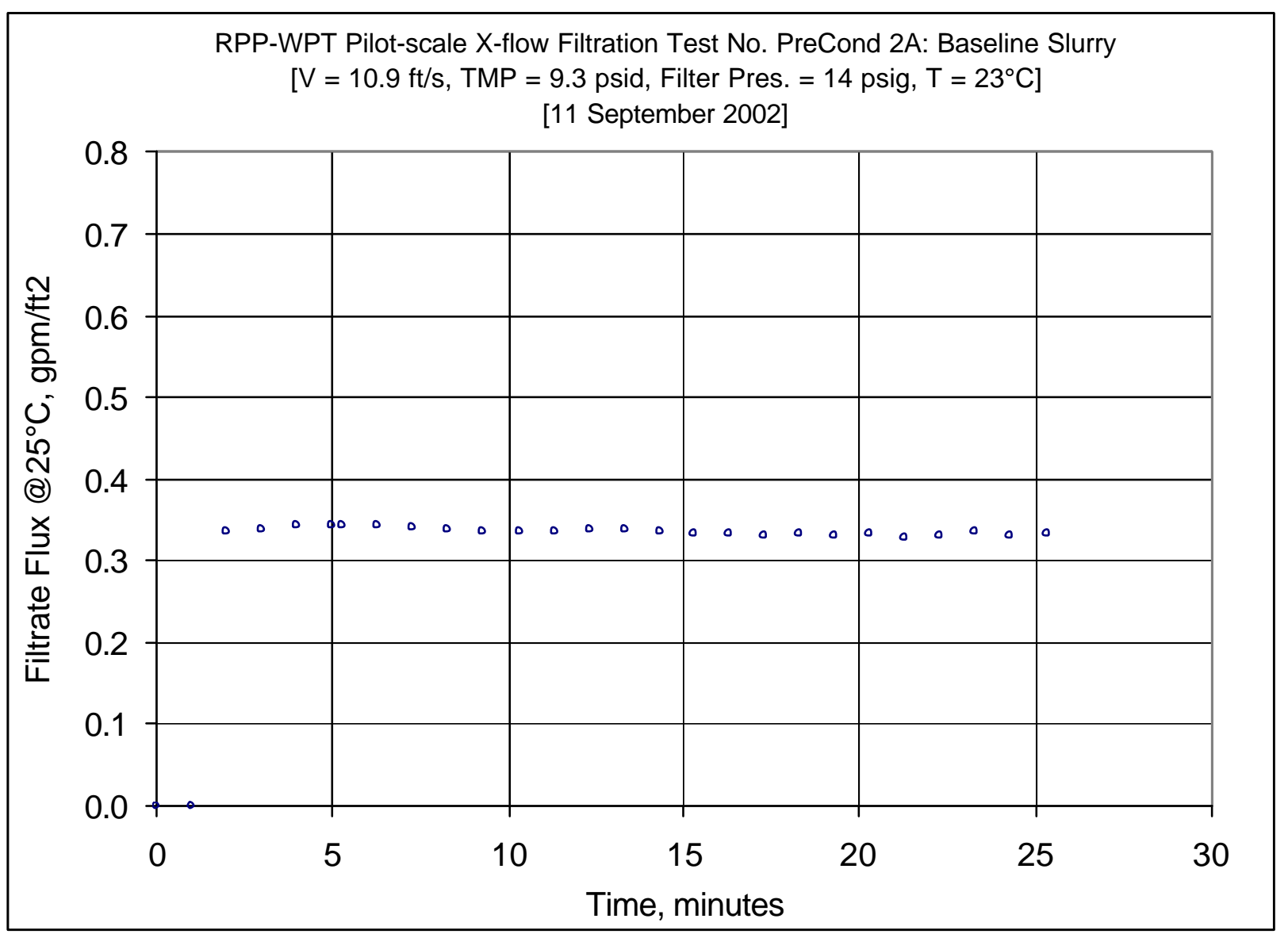

Figure B5: PreConditioning 2A - Baseline Slurry - After Test Rig Modification 
WSRC-TR-2003-00204, REV. 0

SRT-RPP-2003-00087, REV. 0

\begin{tabular}{|c|c|c|c|c|c|c|c|c|c|c|c|c|c|c|c|c|c|c|c|c|c|c|c|c|c|c|}
\hline \multirow{3}{*}{ DATE } & \multirow[b]{2}{*}{ TIME } & \multicolumn{5}{|c|}{$\ll \ll \ll \ll \ll<$ Temperature Measurements } & \multicolumn{6}{|c|}{ 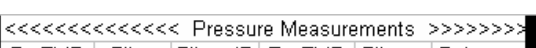 } & \multicolumn{4}{|c|}{$\ll \ll \ll \ll \ll \ll<$ Flow Measurements 》 } & \multicolumn{10}{|c|}{ 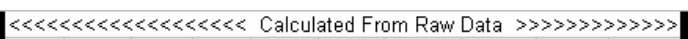 } \\
\hline & & Filtrate & Cleaning & Slurry & Hi Amb. & Lo Amb. & BotTMP & Filter & Filter $\mathrm{dP}$ & TopTMP F & Filtrate & Pulsepot & Slurry & Filtrate & Hi Filtate & Backpulse & & & & & & & & & & \\
\hline & & $\operatorname{deg} C$ & $\operatorname{deg} C$ & $\operatorname{deg} C$ & $\operatorname{deg} C$ & $\operatorname{deg} C$ & psid & psig & psid & psid & psig & & $\mathrm{gpm}$ & $\mathrm{gpm}$ & gpm & $\mathrm{gpm}$ & & & & & & & & & & \\
\hline & & $\mathrm{T} 2$ & $\mathrm{~T} 3$ & $\mathrm{~T} 1$ & T4 & $\mathrm{T} 5$ & $\mathrm{dP2}$ & P1 & $\mathrm{dP1}$ & $\mathrm{dP3}$ & P2 & $\mathrm{P3}$ & Q1 & Q2 & Q3 & Qbp & & & & & & & & & & \\
\hline \multicolumn{27}{|l|}{ Zeros } \\
\hline $9 / 11 / 2002$ & $0: 10 \mathrm{AM}$ & 20.716 & 20.836 & 20.261 & 20.264 & 20.354 & -0.121 & -0.035 & -0.059 & 0.06 & -0.226 & 0.361 & -0.216 & 0.002 & 0.001 & 0.002 & & \multicolumn{2}{|c|}{ Filter Surface } & \multicolumn{2}{|c|}{$6.87 \mathrm{FT}$} & \multirow{2}{*}{\multicolumn{3}{|c|}{ 2/day/barg / gpm/t2/barg }} & & \\
\hline $9 / 11 / 2002$ & $8: 10: 25 \mathrm{AM}$ & 20.716 & 20.836 & 20.261 & 20.264 & 20.354 & -0.121 & -0.035 & -0.059 & 0.06 & -0.226 & 0.361 & -0.216 & 0.002 & 0.001 & 0.002 & & \multicolumn{2}{|c|}{ Conversion } & $851 \mathrm{r}$ & $\mathrm{m} 3 / \mathrm{m} 2 /$ & & & & & \\
\hline 9/11/2002 & 10:26 AM & 20.716 & 20.841 & 20.256 & 20.269 & 20.359 & -0.117 & -0.037 & -0.059 & 0.06 & -0.246 & 0.358 & -0.214 & 0.002 & 0.001 & 0.002 & & & & & & & & & & \\
\hline $9 / 11 / 2002$ & 10:27 AM & 20.716 & 20.841 & 20.261 & 20.264 & 20.364 & -0.121 & -0.035 & -0.059 & 0.062 & -0.246 & 0.361 & -0.218 & 0.001 & 0.001 & 0.001 & & Note: $\mathrm{G}$ & Gaug & Pressur & ure $\mathrm{P} 1$ is & $x$ & ximat & $1.4 \mathrm{psig}$ & & \\
\hline 9/11/2002 & :10:28 AM & 20.711 & 20.841 & 20.256 & 20.264 & 20.359 & -0.119 & -0.037 & -0.059 & 0.06 & $\begin{array}{l}-0.249 \\
-0.249\end{array}$ & 0.358 & -0.216 & 0.001 & 0.001 & 0.00 & & & & The & & $\pi$ & & $4 \mathrm{~F}$ & & \\
\hline 9/11/2002 & 8:10:29 AM & 20.715 & 20.83 & 20.256 & 20.259 & 20.358 & -0.117 & -0.035 & -0.059 & 0.06 & -0.246 & 0.358 & -0.218 & 0.002 & 0.0 & 0.000 & & Pressur & Ire $\mathrm{P}$ 1is o & correct & ected for 4 & 40. & hes of & of water tu & bing & \\
\hline $9 / 11 / 2002$ & 10:30 AM & 20.721 & 20.841 & 20.261 & 20.264 & 20.359 & -0.121 & -0.035 & -0.059 & 0.06 & -0.246 & 0.358 & -0.218 & 0.002 & 0.001 & 0.001 & & WW & & & & $<$ Filtra & ate Flux & $\ll$ « PEF & EMMIABILI & LITY \\
\hline $9 / 11 / 2002$ & 8:10:31 AM & 20.71 & 20.835 & 20.256 & 20.254 & 20.358 & -0.119 & -0.035 & -0.059 & 0.06 & -0.246 & 0.356 & -0.218 & 0.002 & 0.001 & 0.002 & & & & & & & at $25 \mathrm{C}$ & & $\times 1000$ & \\
\hline & & & & & & & & & & & & & & & & & ime & Press. & Vel. & TMP T & TMP & $\mathrm{gpm}$ & gpm & gpm & $\mathrm{gpm}$ & meter \\
\hline Data - Per M & Min & & & & & & & & & & & & & & & & Min. & $p$ sig $f$ & $\mathrm{f} / \mathrm{s}$ & psi b & bar & ift2 & $\mathrm{ft} 2$ & $\mathrm{f} 2 / \mathrm{psi}$ & $\mathrm{ft} 2 / \mathrm{psi}$ & lay/ba \\
\hline $9 / 11 / 2002$ & $1: 12: 43 \mathrm{PM}$ & 24.537 & 22.427 & 22.297 & 24.63 & 21.775 & 3.415 & 15.754 & 4.873 & -1.34 & 7.269 & 55.688 & 46.698 & 0.002 & 0.0 & 0. & 0 & 14.3 & 10.9 & 1.0 & 0.072 & 0.000 & 0.000 & 0.000 & 0.151 & 0.13 \\
\hline $9 / 11 / 2002$ & 1:13:43 PM & 24.009 & 22.446 & 22.044 & 25.044 & 21.819 & 2.934 & 15.27 & & -1.426 & 7.26 & 8.249 & 46.845 & 0.2 & a & & 1 & $\begin{array}{ll}1 & 13.8 \\
\end{array}$ & 10.9 & 0.8 & 0.052 & 0.000 & 0.000 & 0.000 & 0.210 & 0.18 \\
\hline 9/11/2002 & 1:14:43 PM & 23.009 & 22.44 & 21.721 & 25.033 & 21.874 & 11.065 & 15.096 & 4.71 & 6.73 & -1.289 & & 48.088 & & & 003 & & 213.6 & 11.2 & 8.9 & 0.613 & 0.305 & 35 & 50.038 & & 32.04 \\
\hline $9 / 11 / 2002$ & 1:15:43 PM & 22.32 & 22.46 & 21.6 & 24.952 & 21.9 & 11.26 & 15.357 & 4.627 & 7.074 & -1.286 & & 47.53 & 0 & 2. & & 3 & \begin{tabular}{|l|l|} 
& 13.9 \\
\end{tabular} & 11.1 & 9.2 & 0.632 & 0.306 & 37 & 0.037 & 736.723 & 31.25 \\
\hline $9 / 11 / 2002$ & 1:16:43 PM & 21.972 & 22.479 & 21.363 & 24.9 & 21.9 & 11.409 & 1 & & & -1.2 & & & & 2.1 & & 4 & $\begin{array}{ll}4 & 14.1 \\
\end{array}$ & 11.0 & 9.3 & 0.642 & 0.308 & 2 & & 736.722 & 31.25 \\
\hline & & 21.755 & 22.488 & 21.135 & 24.8 & 21.977 & 4 & 1 & & & & & 17 & & & & 5 & & 11.0 & & & & & & & \\
\hline $9 / 11 / 2002$ & 8:00 PM & 21.679 & 22.493 & 21.07 & 24.8 & 21.987 & & 15.382 & & & -1.2 & 1.631 & 02 & 0.0 & 2. & & 5.28 & \begin{tabular}{|l|l|}
3 & 13.9 \\
\end{tabular} & 11.0 & 9.3 & 0.641 & 0.306 & $\frac{342}{342}$ & \begin{tabular}{|l|l|}
2 & 0.037
\end{tabular} & 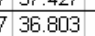 & 31.32 \\
\hline 9/11/2002 & 1:19:00 PM & 21.499 & 22.509 & 21.065 & 20 & 22. & 11.25 & 15.3 & & & & & & & & & 6.28 & \begin{tabular}{|l|l|} 
& 13.9
\end{tabular} & 11.0 & 9.2 & & 0.306 & & & & 31.71 \\
\hline $9 / 11 / 2002$ & $1: 20: 00 \mathrm{PM}$ & 21.52 & 22.525 & 21.464 & 24 & & 11.415 & 15 & & & & & & & & & 7.28 & \begin{tabular}{|l|l|}
3 & 14.1
\end{tabular} & 11 & 9.3 & & & & & & 31.23 \\
\hline 9/11/2002 & 1:21:00 PM & 21.763 & 22.536 & 21.8 & 24 & & 11.349 & 15.5 & & & & & & & & & 8.28 & \begin{tabular}{|l|l|} 
& 14.1
\end{tabular} & 11.0 & 9.3 & 0.641 & 0.309 & 38 & 36 & 38 & 30.92 \\
\hline $9 / 11 / 2002$ & 1:22:00 PM & 22 & 22.543 & 22.151 & 24 & & & 1 & & & -1. & & & & & & 9. & 14.1 & 11.0 & 9.5 & & 0.309 & & 5 & 535.278 & 30.02 \\
\hline $9 / 11 / 2002$ & 1:23:00 PM & 22.228 & 22.549 & 22.097 & & 22.1 & & 1 & & & & & & & & & 10.3 & 14.1 & 11.0 & 9.2 & & & & & & 30.5 \\
\hline 9/11/2002 & PM & 22.28 & 22.551 & 22.114 & 70 & 22 & 11.455 & 15.5 & & & & & & & 2. & & 11.3 & 14.0 & 11.0 & 9.5 & & 0 & & 5 & 34 & 29.90 \\
\hline $9 / 11 / 2002$ & PM & 22.33 & 22.561 & & 24.6 & & & 15 & & & & & & & & & 12.3 & 14.2 & 10.9 & 9.4 & & & & 5 & & 30.41 \\
\hline $9 / 11$ & $1:$ & 22 & 22. & & & & & 15. & & & & & & & & & 13.3 & & & 9.2 & & & & & & 31.22 \\
\hline $9 / 11 / 2002$ & 4. & 22.527 & 22.546 & 22. & & & & & & & & & & & & & 14.3 & 14.2 & 11.0 & 9.4 & & & & & & 30.28 \\
\hline 9/11/2002 & 1:28:00 PM & , & 22.551 & 22 & & & & 15. & & & & & & & & & 15.3 & 13.9 & 10.9 & 9.3 & 0 & & & 5 & 99 & 30.38 \\
\hline & & 22 & 22 & & & & & 15 & & & & & & & & & 16.3 & & & 9.1 & & & & & & 31.15 \\
\hline $9 / 1$ & & 22 & 22.561 & & 24.1 & & 11.36 & 155 & & & & & & & & & 17.3 & 14.0 & 11.0 & 9.4 & & & & & & 30. \\
\hline 9/11/2002 & $\mathrm{PM}$ & 199 & 22.56 & & $2-7$ & 22. & 11.268 & 15 & & & & & & & & & 18.3 & 14 & (1) & 9.1 & & & & & & 30. \\
\hline & & 23 & 22.559 & & & & & 15. & & & & & & & & & & & & 1 & & & & & & 30.83 \\
\hline $9 / 11 / 2002$ & $1: 33: \mathrm{C}$ & 23.57 & 22.563 & 23 & 24 & 22 . & 11.291 & 15 & & 7. & -1. & & & & & & 20.3 & 14 & 10 & 9.3 & 0 & 0. & 1 & & 34 & 30.32 \\
\hline $9 / 11 / 2002$ & & 23.7 & 22.558 & 23. & & & & 15. & & & & & & & & & & & 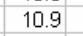 & 9.4 & & & & & & 29.58 \\
\hline & & & 22.545 & & & & & & & & & & & & & & & & & 5 & & & & & & 29.44 \\
\hline & 1:36:00 PM & 23.84 & 22.521 & 23.4 & 24.3 & 22.0 & 11.198 & 15.524 & & & -1. & & & & & & 23.3 & \begin{tabular}{|l|l|} 
& 14.1
\end{tabular} & 10.8 & 9.2 & & 0 & & & & 30.7 \\
\hline $9 / 11 / 2002$ & 1:37:00 PM & 23.714 & 22.532 & 23.356 & 24.423 & 22.076 & 11.283 & 15.568 & $\begin{array}{c}4.4 \\
4 .\end{array}$ & 7.213 & -1.28 & & 46.622 & 0.00 & & & 24.3 & 14.1 & 10.9 & 9.2 & 0.638 & 0 & 330 & 0.036 & 655 & 30.34 \\
\hline 9/11/2002 & 1:38:00 PM & 23.544 & 22.518 & 23.116 & 24.43 & 22.098 & 11.13 & 15.346 & 4.79 & 7.371 & -1.2 & & 46.574 & 00 & 2.15 & & 25.3 & 13.9 & 10.9 & 9.3 & 0.638 & 0.314 & 331 & 0.036 & 635.824 & 0.49 \\
\hline & & & & & & & & & & & & & & & & & & & & & & & & & & \\
\hline & & & & & & & & & & & & & & & & & & 14 & 10.9 & 9.3 & & & & 9 & 6.9 & 30.6 \\
\hline & & & & & & & & & & & & & & & & & & & & & & & & & & \\
\hline & & & & & & & & & & & & & & & & & & 14.1 & 10.9 & 9.3 & & & & & 5.8 & 30 \\
\hline & & 21.5 & 22.5 & 21.1 & 24.0 & 22 & 11.1 & 15.3 & & .0 & -1.3 & & 46.4 & & 2 & & & 13.9 & 10.8 & 9.1 & 0.626 & 0.306 & 328 & 0.0346 & $6 \quad 34.6$ & 29.4 \\
\hline & & 1.584 & 0.038 & 1.636 & 0.453 & 0.089 & 0.201 & 0.228 & 0.176 & 0.375 & 0.024 & 0.000 & 0.47 & 0.00 & 0.055 & .00 & & 0.228 & 0.1110 & 0.255 & 0.018 & 0.008 & 0.008 & 0.0014 & \begin{tabular}{|l|l|}
4 & 1.369 \\
\end{tabular} & \begin{tabular}{|l|l|} 
& 2.165 \\
\end{tabular} \\
\hline imber of & f Points Used & 21 & 21 & 21 & 21 & . & 21 & 21 & 21 & 21 & 21 & 21 & 21 & 21 & 1 & 21 & & 21 & 71 & 21 & 010 & 21 & 11 & 21 & 21 & \\
\hline
\end{tabular}

Figure B6: PreConditioning 2A - Baseline Slurry - After Test Rig Modification - Raw \& Calculated Data 
WSRC-TR-2003-00204, REV. 0

SRT-RPP-2003-00087, REV. 0

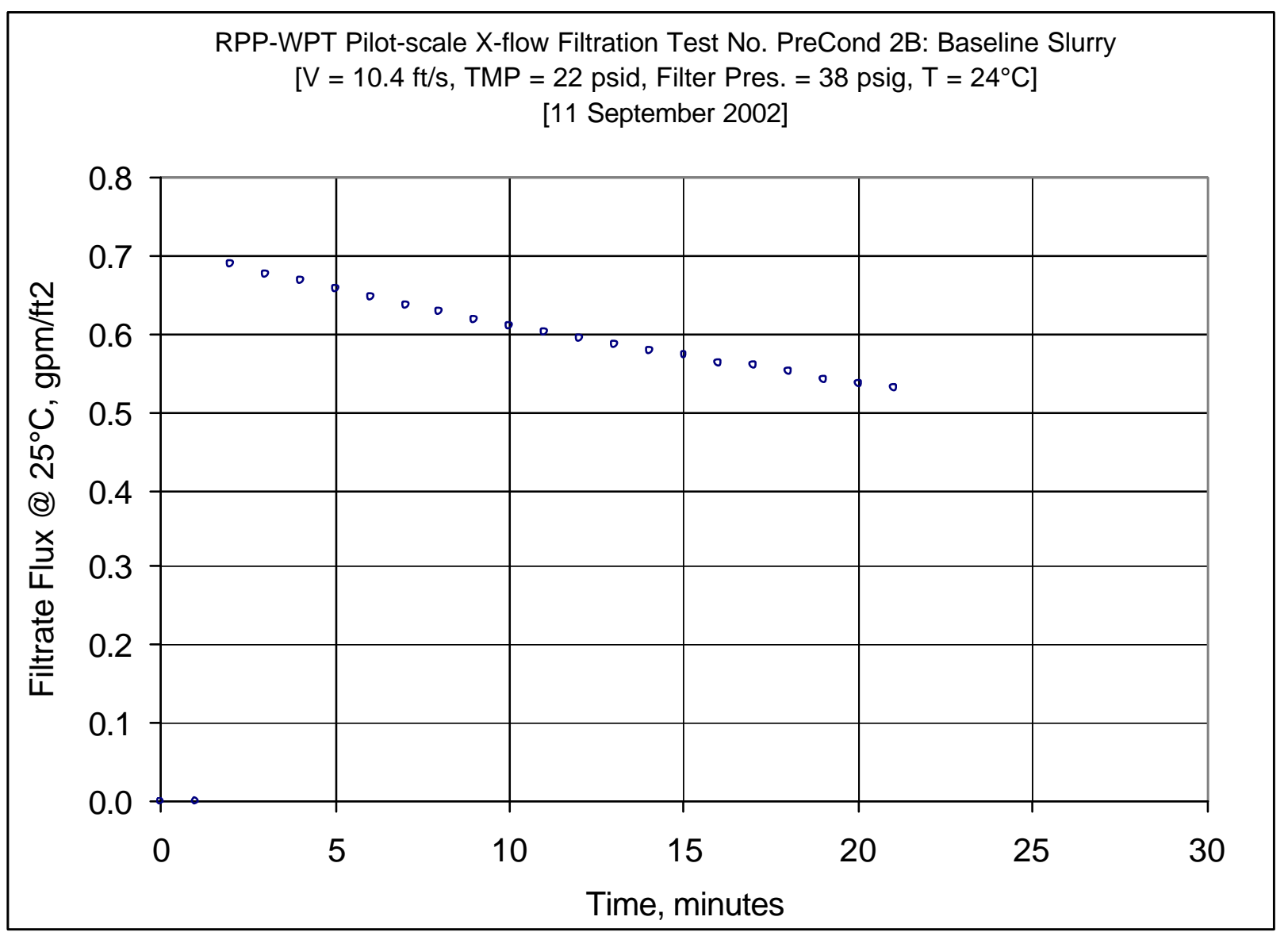

Figure B7: PreConditioning 2B - Baseline Slurry - After Test Rig Modification 
WSRC-TR-2003-00204, REV. 0

SRT-RPP-2003-00087, REV. 0

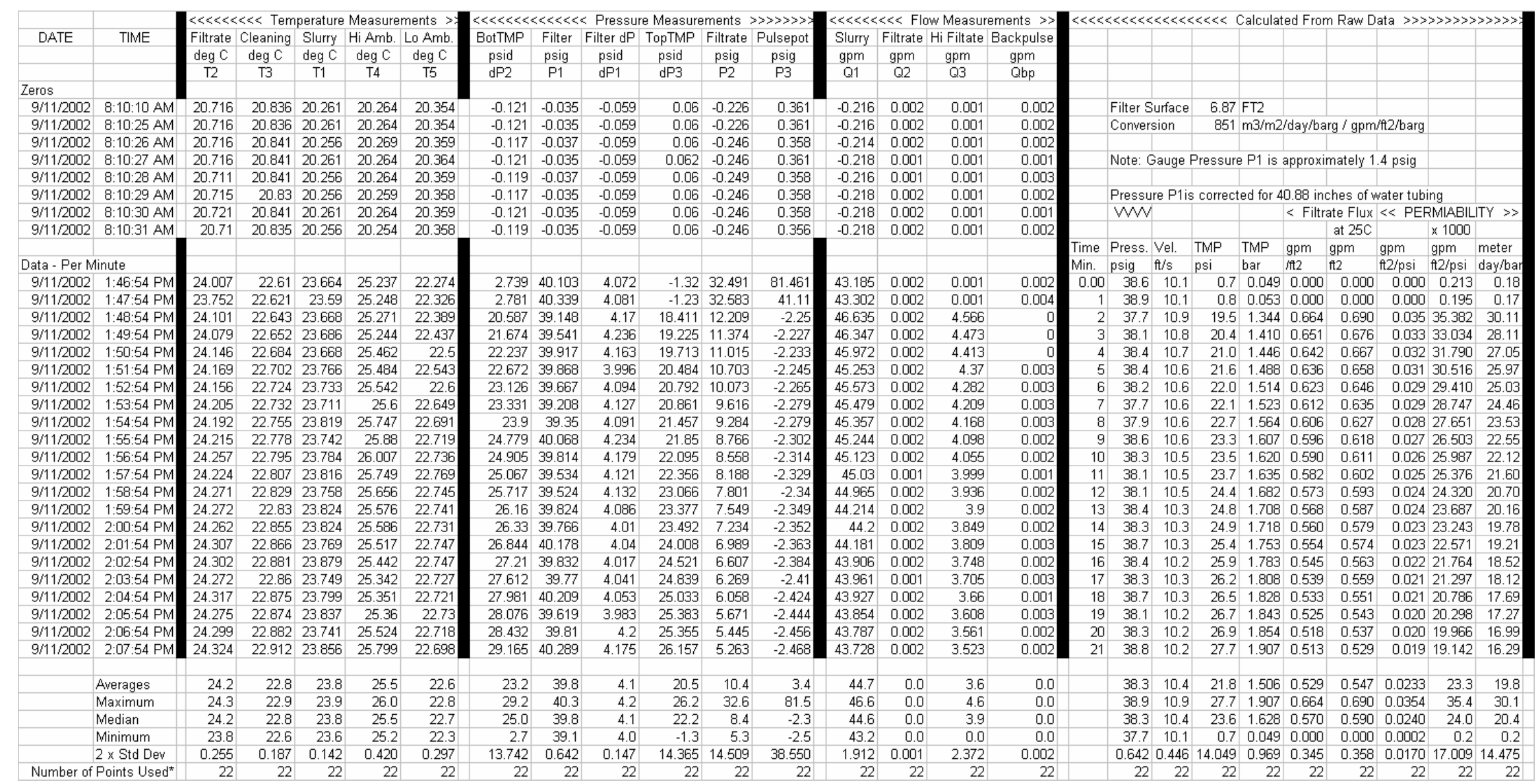

Figure B8: PreConditioning 2B - Baseline Slurry - After Test Rig Modification - Raw \& Calculated Data 
WSRC-TR-2003-00204, REV. 0

SRT-RPP-2003-00087, REV. 0

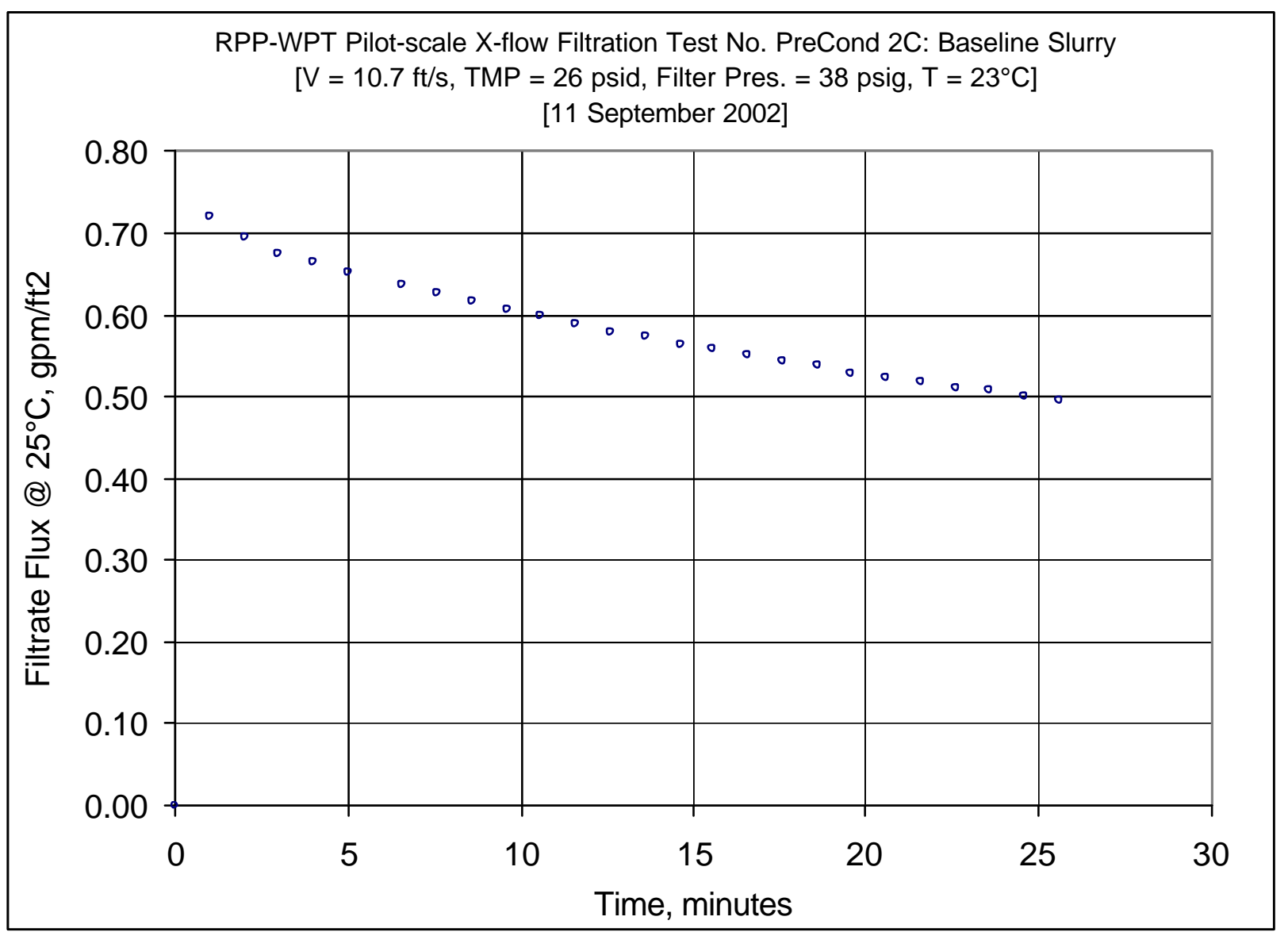

Figure B9: PreConditioning 2C - Baseline Slurry - After Test Rig Modification 
WSRC-TR-2003-00204, REV. 0

SRT-RPP-2003-00087, REV. 0

\begin{tabular}{|c|c|c|c|c|c|c|c|c|c|c|c|c|c|c|c|c|c|c|c|c|c|c|c|c|c|c|}
\hline \multirow{3}{*}{ DATE } & \multirow{3}{*}{ TIME } & \multicolumn{5}{|c|}{ 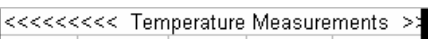 } & \multicolumn{6}{|c|}{ 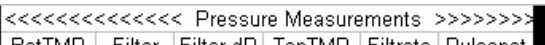 } & \multicolumn{4}{|c|}{$\mid \ll \ll<\ll<<<<$ Flow Measurements 》> } & \multicolumn{4}{|c|}{ 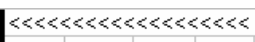 } & \multicolumn{6}{|c|}{ 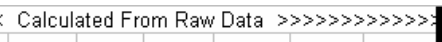 } \\
\hline & & Filtrate & Cleaning & Slurry & Hi Amb. & Lo Amb. & BotTMP & $\begin{array}{c}\text { Filter } \\
\text { psig }\end{array}$ & $\begin{array}{c}\text { Filter } \mathrm{dP} \\
\text { psid }\end{array}$ & TopTMP & $\begin{array}{c}\text { Filtrate } \\
\text { nsig }\end{array}$ & & & Filtrate & Hi Filtate & & & & & & & & & & & \\
\hline & & $\operatorname{deg} C$ & $\operatorname{deg} C$ & $\operatorname{deg} C$ & $\operatorname{deg} C$ & $\operatorname{deg} C$ & psid & psig & psid & psid & psig & psig & gpm & $\mathrm{gpm}$ & gpm & $\mathrm{gpm}$ & & & & & & & & & & \\
\hline & & T2 & $\mathrm{T} 3$ & T1 & T4 & 15 & $\mathrm{dP} 2$ & P1 & dP1 & $\mathrm{dP} 3$ & $\mathrm{P} 2$ & P3 & Q1 & Q2 & Q3 & Qbp & & & & & & & & & & \\
\hline \multicolumn{27}{|l|}{ Zeros } \\
\hline $9 / 11 / 2002$ & 10:10 AM & 20.716 & 20.836 & 20.261 & 20.264 & 20.354 & -0.121 & -0.035 & -0.059 & 0.06 & -0.226 & 0.361 & -0.216 & 0.002 & 0.001 & 0.002 & & \multirow{2}{*}{\multicolumn{2}{|c|}{ Filter Surface }} & 6.87 & FT2 & & & & & \\
\hline $9 / 11 / 2002$ & $8: 10: 25 \mathrm{AM}$ & 20.716 & 20.836 & 20.261 & 20.264 & 20.354 & -0.121 & -0.035 & -0.059 & 0.06 & -0.226 & 0.361 & -0.216 & 0.002 & 0.001 & 0.002 & & & & 851 & \multicolumn{5}{|c|}{$\mathrm{m} 3 / \mathrm{m} 2 /$ day $/$ barg $/ \mathrm{gpm} / \mathrm{t} 2 /$ barg } & \\
\hline $9 / 11 / 2002$ & $8: 10: 26 \mathrm{AM}$ & 20.716 & 20.841 & 20.256 & \begin{tabular}{l|l}
5 & 20.269 \\
\end{tabular} & 20.359 & -0.117 & -0.037 & -0.059 & 0.06 & -0.246 & 0.358 & -0.214 & 0.002 & 0.001 & 0.002 & & \multirow{2}{*}{\multicolumn{2}{|c|}{ Note: Gauge }} & & & & & & & \\
\hline 9/11/2002 & 8:10:27 AM & 20.716 & 20.841 & 20.261 & $1 \quad 20.264$ & 20.364 & -0.121 & -0.035 & -0.059 & 0.062 & -0.246 & 0.361 & -0.218 & 0.001 & 0.001 & 0.001 & & & & Pressur & ure $P 1$ is & is approx & ximately & y $1.4 \mathrm{psic}$ & & \\
\hline $9 / 11 / 2002$ & 8:10:28 AM & 20.711 & 20.841 & 20.256 & $\begin{array}{l}50.264 \\
\end{array}$ & 20.359 & -0.119 & -0.037 & -0.059 & 0.06 & -0.249 & 0.358 & -0.216 & 0.001 & 0.001 & 0.003 & & & & & & & & & & \\
\hline $9 / 11 / 2002$ & 8:10:29 AM & 20.715 & 20.83 & 20.256 & $\begin{array}{l}50.259 \\
\end{array}$ & 20.358 & -0.117 & -0.035 & -0.059 & 0.06 & -0.246 & 0.358 & -0.218 & 0.002 & 0.001 & 0.002 & & Pressur & ure P1is & con & cted for & 40.88 in & inches of & of water $t$ & tubing & \\
\hline $9 / 11 / 2002$ & $8: 10: 30 \mathrm{AM}$ & 20.721 & 20.841 & 20.261 & $1 \quad 20.264$ & 20.359 & -0.121 & -0.035 & -0.059 & 0.06 & -0.246 & 0.358 & -0.218 & 0.002 & 0.001 & 0.001 & & WW & & & & $<$ Filtra & rate Flux: & $x \ll<$ PEF & ERMIABIL & IILTYY \\
\hline $9 / 11 / 2002$ & 8:10:31 AM & 20.71 & 20.835 & 20.256 & 20.254 & 20.358 & -0.119 & -0.035 & -0.059 & 0.06 & -0.246 & 0.356 & -0.218 & 0.002 & 0.001 & 0.002 & & & & & & & at $25 \mathrm{C}$ & & $\times 1000$ & \\
\hline & & & & & & & & & & & & & & & & & Time & Press. & Vel. & TMP & TMP & $\mathrm{gpm}$ & $\mathrm{gpm}$ & gpm & gpm & meter \\
\hline Data - Per M & Minute & & & & & & & & & & & & & & & & Min & psig f & $\mathrm{f} / \mathrm{s}$ & psi & bar & int2 & $\mathrm{f} 2$ & $\mathrm{ft} 2 / \mathrm{psi}$ & $\mathrm{ft2} / \mathrm{psi}$ & day/bar \\
\hline $9 / 11 / 2002$ & 2:43:24 PM & 24.237 & 23.318 & 22.509 & 26.677 & 23.385 & 3.531 & 40.136 & 4.515 & -0.765 & 31.679 & 80.364 & 44.139 & 0.002 & 0.001 & .003 & 0 & 38.7 & 10.3 & \begin{tabular}{|l|l|}
8 & 1.4 \\
\end{tabular} & 40.095 & 0.000 & 0.000 & 0.000 & 0.226 & \begin{tabular}{|l|l|}
6 & 0.19 \\
\end{tabular} \\
\hline $9 / 11 / 2002$ & 2:44:24 PM & 23.257 & 23.346 & 22.567 & 26.6 & 23.433 & 19.887 & 38.845 & 4.373 & 17.395 & 12.55 & 1.077 & 47.755 & 0.002 & 4.62 & 0 & 1 & 37.4 & 11.1 & 18.6 & 51.285 & 0.672 & 0.720 & 0.039 & 938.643 & $\begin{array}{ll}3 & 32.89 \\
\end{array}$ \\
\hline $9 / 11 / 2002$ & 2:45:24 PM & 23.149 & 23.359 & 22.651 & 26.719 & 23.481 & 21.581 & 39.417 & 4.352 & 19.082 & 11.394 & 1.05 & 47.377 & 0.002 & 4.466 & 0 & 2 & 37.9 & 11.1 & 20.3 & $\begin{array}{l}31.402 \\
\end{array}$ & 0.650 & 0.695 & 0.034 & 434.166 & $\begin{array}{ll}6 & 29.08\end{array}$ \\
\hline $9 / 11 / 2002$ & 2:46:24 PM & 23.164 & 23.389 & 22.791 & 26.728 & 23.551 & 22.252 & 39.274 & 4.392 & 19.627 & 10.665 & 1.024 & 47.272 & 0.002 & 4.36 & 0 & 3 & 37.8 & 11.0 & 20.9 & 91.444 & 0.634 & 0.675 & 0.032 & 232.258 & $\begin{array}{ll}8 & 27.45 \\
\end{array}$ \\
\hline 9/11/2002 & 2:47:24 PM & 23.213 & 23.413 & 22.725 & 26.732 & 23.595 & 23.095 & 39.636 & 4.284 & 20.579 & 10.188 & 0.984 & 46.742 & 0.0 & & 0.002 & 4 & 38.2 & 10.9 & 921.8 & 31.506 & 0.624 & 0.665 & 0.030 & & 125.93 \\
\hline $9 / 11 / 2002$ & 2:48:24 PM & 23.256 & 23.421 & 22.833 & 26.67 & 23.623 & 23.532 & 39.568 & 4.46 & 20.538 & 9.619 & 0.969 & 46.689 & 0.002 & 4.214 & 0.002 & 5 & 38.1 & 10.9 & 22.0 & 1.519 & 0.613 & 0.652 & 0.030 & 29 & $\begin{array}{l}2 \quad 25.18 \\
\end{array}$ \\
\hline $9 / 11 / 2002$ & 2:49:59 PM & 23.296 & 23.44 & 22.868 & 26.624 & 23.643 & 24.286 & 39.652 & 4.351 & 21.406 & 9.029 & 0.943 & 46.7 & 0.001 & 4.123 & 0.003 & 6.58 & 38.2 & 10.9 & 22.8 & 31.575 & 5.600 & 0.637 & 0.028 & 327.897 & \begin{tabular}{|l|l|}
7 & 23.74 \\
\end{tabular} \\
\hline 9/11/2002 & $2: 50: 59 \mathrm{PM}$ & 23.309 & 23.464 & 22.856 & 26.508 & 23.631 & 24.706 & 39.537 & & & 8.509 & & 46.486 & 0.002 & & 0.003 & 7.58 & 38.1 & 10.9 & $\begin{array}{l}93.2 \\
9\end{array}$ & 21.600 & 0.589 & 0.626 & 0.027 & & $\begin{array}{ll}7 & 22.97\end{array}$ \\
\hline $9 / 11 / 2002$ & $2: 51: 59 \mathrm{PM}$ & 23.356 & 23.47 & 22.832 & 26 & 23.637 & 25.263 & 39.592 & 4. & 22.302 & 8.064 & 38 & 46.515 & 0.001 & 3 & 0.003 & 8.58 & 38.1 & 10.9 & 23.8 & $\begin{array}{ll}3 & 1.640\end{array}$ & 0.580 & 0.616 & 0.026 & 62 & $\begin{array}{ll}5 & 22.05\end{array}$ \\
\hline $9 / 11 / 2002$ & 2:52:59 PM & 23.352 & 23.481 & 22.949 & 26.285 & 23.654 & 25.292 & 39.193 & 4 & 22.257 & 7.801 & & 38 & 0. & & 0.004 & 9.58 & 37.7 & 10.9 & $\begin{array}{l}93.8 \\
2\end{array}$ & 31.639 & 0.573 & 0.607 & 0.026 & 52 & $\begin{array}{ll}6 & 21.72 \\
\end{array}$ \\
\hline $9 / 11 / 2002$ & 2:53:59 PM & 23.379 & 23.503 & 22.886 & 26 & 23.631 & 25. & 39.476 & & & & & & & & 003 & 10.6 & 38.0 & 10.8 & 24.5 & $\begin{array}{ll}5 & 1.691\end{array}$ & & & 0.024 & & $\begin{array}{ll}9 & 20.74\end{array}$ \\
\hline $9 / 11 / 2002$ & $2: 54: 59 \mathrm{PM}$ & 23.399 & 23.493 & 22.931 & 26.227 & 23.59 & 26.398 & 39.677 & 9 & 23.418 & 7.073 & & 46.328 & 0.0 & 3. & 0.003 & 11.6 & 38.2 & 10.8 & 24.9 & $\begin{array}{l}9 \\
1.717\end{array}$ & 0.556 & 0.589 & 0.024 & 423 & $\begin{array}{ll}5 & 20.14\end{array}$ \\
\hline $9 / 11 / 2002$ & 2:55:59 PM & 23.385 & 23.5 & 22.947 & 26 & 23.622 & & 39.356 & & 23 & 6.72 & & & & & 0001 & 12.6 & 37.9 & 10.8 & 24.9 & 91.716 & 0.547 & 0.580 & 0.023 & 32 & $\begin{array}{ll}4 & 19.82\end{array}$ \\
\hline $9 / 11 / 2002$ & $2: 56.59 \mathrm{PM}$ & 23. & & 22.8 & & & 27. & 39.648 & & & & & & & & & 13.6 & 38.2 & 10.8 & 25.4 & & & & 0.023 & & \begin{tabular}{|l|l|}
6 & 19.25
\end{tabular} \\
\hline $9 / 11 / 2002$ & $2: 57: 59 \mathrm{PM}$ & 23.426 & 23.51 & 22.988 & 26 & 7 & 27.219 & 39.373 & & 23.919 & 6.125 & & 46.003 & 0.0 & & 0.00 & 14.6 & 37.9 & 10.7 & 25.6 & 6 1.763 & 0.533 & 0.565 & 0.022 & 222.088 & $\begin{array}{ll}8 & 18.80\end{array}$ \\
\hline $9 / 11 / 2002$ & $2: 58: 59 \mathrm{PM}$ & 23.427 & 23.522 & 22.919 & 26 & & 27.8 & 39.712 & & & 5.726 & & & & & 5 & 15.6 & 38.2 & 10.7 & 26.1 & 1.801 & 0.526 & 0.558 & 0.021 & & $\begin{array}{ll}7 & 18.18 \\
\end{array}$ \\
\hline $9 / 11 / 2002$ & $2: 59: 59 \mathrm{PM}$ & 23. & 23.5 & & & & 28.416 & 40. & & & 5 & & & & & & 16.6 & 38.7 & 10.7 & 26.7 & 1.839 & 0.521 & 0.552 & 0.021 & 120 & $\begin{array}{ll}4 & 17.61\end{array}$ \\
\hline $9 / 11 / 2002$ & 3:00:59 PM & 23.453 & 23.542 & 23.025 & 26.071 & & 28.6 & 39.994 & 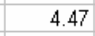 & 25 & 5.226 & & 45.899 & & & 0 & 17.6 & 38.5 & 10.7 & 26.9 & 9 1.855 & 0.513 & 0.542 & 0 & & $\begin{array}{ll}6 & 17.16\end{array}$ \\
\hline $9 / 11 / 2002$ & $3: 01: 59 \mathrm{PM}$ & & & 22.92 & & & & 40.1 & & & & & & & & & 18.6 & & 10.7 & 27.3 & & & & & & \begin{tabular}{|l|l|}
4 & 16.79
\end{tabular} \\
\hline $9 / 11 / 2002$ & $3: 02$ & & 23.537 & 23 & 26.126 & 23.56 & & 39.5 & & 25 & & & & 0.0 & & 0. & 19.6 & 38.1 & 10.7 & 27.1 & 1.869 & 0 & 0.529 & 20 & & $\begin{array}{ll}2 & 16.62\end{array}$ \\
\hline $9 / 11 / 2002$ & 3:03:59 PM & 23.457 & 23.537 & 22.979 & 26.0 & 23.55 & 29.321 & 40.0 & & 25.862 & 4.555 & & 87 & & & & $\pi$ & 36 & 10.7 & 27.6 & 61 & 20 & 0 & 9 & & $\begin{array}{ll}5 & 16.13\end{array}$ \\
\hline $9 / 11 / 2002$ & 3:04: & 23 & & & & & & 39. & & & & & & & & & & & & & & & & & & \\
\hline $9 / 11 / 2002$ & 3:05:59 PM & 23.486 & 23.54 & 23.048 & 26.459 & 23.5 & 29.75 & 39.919 & 4.482 & 26.126 & 4.101 & & 45.464 & 0.00 & 3.3 & 0.0 & 22.6 & 38.4 & 10.6 & 27.9 & 1.926 & 0.484 & 0.512 & 0.018 & 318 & $\begin{array}{ll}8 & 15.58\end{array}$ \\
\hline $9 / 11 / 2002$ & 3:06:59 PM & 23.5 & 23.559 & 22.977 & 26.328 & 23.551 & 30.174 & 40.263 & & 26.523 & & & 45.523 & 0.0 & & & ר? & 38 & 10.6 & 28.3 & 31.955 & 0.479 & 07 & 0.018 & 817.884 & $\begin{array}{ll}4 & 15.22\end{array}$ \\
\hline $9 / 11 / 2002$ & 3:07:59 PM & 23.514 & 23.549 & & & 23. & & & & & 3.7 & & & & & 0.0 & 24.6 & 38.3 & 10.6 & 28.2 & 21.942 & 0.474 & 0 & 0.018 & 317 & 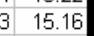 \\
\hline $9 / 11 / 2002$ & 3:08:59 PM & 23.494 & 23.569 & 23.036 & 26.207 & 23.52 & 30.371 & 39.979 & 4.494 & 26.89 & 3.581 & 0.703 & 45.399 & 0.002 & 3.223 & 0.003 & 25.6 & 38.5 & 10.6 & 28.6 & $\begin{array}{ll}6 & 1.974\end{array}$ & 0.469 & 0.496 & \begin{tabular}{|l|} 
\\
0.017 \\
\end{tabular} & 717.318 & $\begin{array}{ll}8 & 14.74\end{array}$ \\
\hline & & & & & & & & & & & & & & & & & & & & & & & & & & \\
\hline & & & & & & & & & & & & & & & & 0 & & 38.3 & 10.7 & 1 & & & & & 21.6 & 18.4 \\
\hline & Maximum & 23.5 & 23.6 & 23 & 26.6 & 23.7 & 30.4 & 40 & 4. & $2 E$ & 9.0 & 0 & 48 & 0.0 & 4. & 0. & & 38.8 & 10.9 & 28.6 & $\begin{array}{l}61.974 \\
\end{array}$ & 40.600 & 0.637 & 0.0279 & 27.9 & 23.7 \\
\hline & & 23. & 23 & 22 & & 23 & 28 & & & & & & & & & 0 & & 38.2 & 10.7 & 26.4 & 41.820 & 0.523 & 0.555 & 0.0210 & 21.0 & 17.9 \\
\hline & & 23 & 23 & 22 & & 23.5 & 24.3 & & & & b & o. & 45.4 & 0.0 & 3.2 & 0.0 & & 37.7 & 10.6 & 22.8 & $\begin{array}{ll}3 & 1.575\end{array}$ & 0.469 & 0.496 & 0.0173 & \begin{tabular}{|l|l|}
3 & 17.3
\end{tabular} & 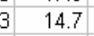 \\
\hline & $2 \times$ Std Dev & 0.126 & 0.066 & 0.120 & 0.302 & 0.080 & 3.769 & 0.579 & 0.148 & 3.312 & 3.277 & 0.138 & 0.760 & 0.001 & 0.541 & 0.001 & & 0.579 & 0.177 & 3.539 & 90.244 & 0.079 & 0.085 & 0.0063 & $\begin{array}{ll}3 & 6.344 \\
\end{array}$ & $\begin{array}{ll}4 & 5.399\end{array}$ \\
\hline Number of & f Points Used* & 20 & 20 & 20 & 20 & 20 & 20 & 20 & 20 & 20 & 20 & 20 & 20 & 20 & 20 & 20 & & 20 & 20 & 20 & 20 & 20 & 20 & 20 & 20 & 20 \\
\hline
\end{tabular}

Figure B10: PreConditioning 2C - Baseline Slurry - After Test Rig Modification - Raw \& Calculated Data 
WSRC-TR-2003-00204, REV. 0

SRT-RPP-2003-00087, REV. 0

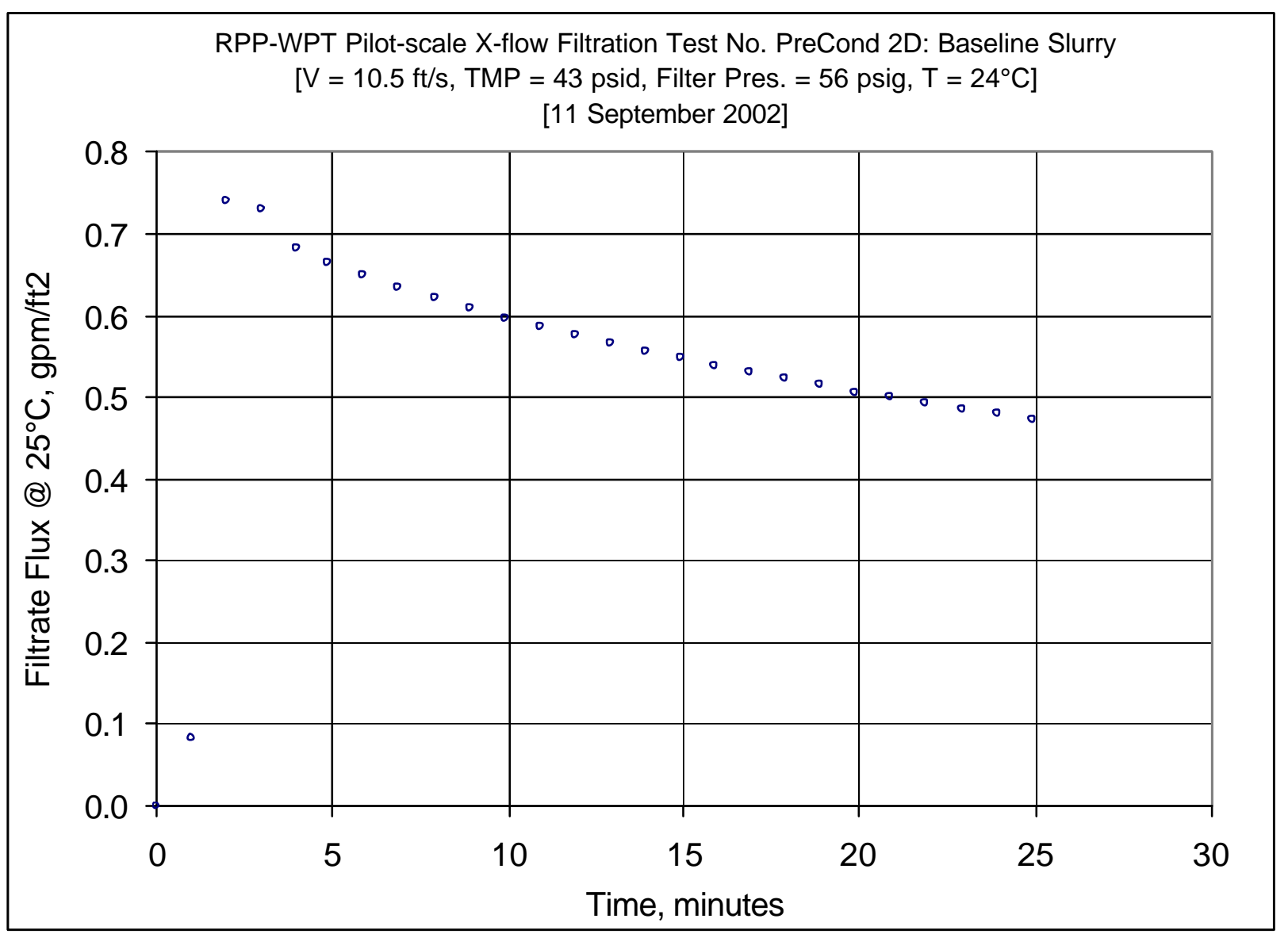

Figure B11: PreConditioning 2D - Baseline Slurry - After Test Rig Modification 
WSRC-TR-2003-00204, REV. 0

SRT-RPP-2003-00087, REV. 0

\begin{tabular}{|c|c|c|c|c|c|c|c|c|c|c|c|c|c|c|c|c|c|c|c|c|c|c|c|c|c|c|}
\hline \multirow[b]{2}{*}{ DATE } & \multirow[b]{2}{*}{ TIME } & \multicolumn{5}{|c|}{$\ll \ll \ll \ll \ll \ll<$ Temperature Measurements $>$} & \multicolumn{6}{|c|}{$\ll<\ll<\ll<<<<<<<<<$ Pressure Measurements $\gg \gg \gg \gg \gg$} & \multirow{2}{*}{\multicolumn{4}{|c|}{ 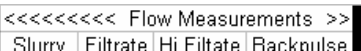 }} & \multicolumn{4}{|c|}{ 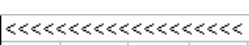 } & \multirow{2}{*}{\multicolumn{6}{|c|}{ 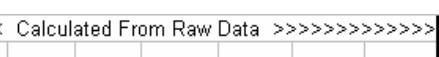 }} \\
\hline & & Filtrate & $\begin{array}{l}\text { Cleaning } \\
\text { deg } C\end{array}$ & $\begin{array}{l}\text { Slurry } \\
\operatorname{deg} C\end{array}$ & Hi Amb. & Lo Amb. & BotTMP & $\begin{array}{l}\text { Filter } \\
\text { psig }\end{array}$ & $\begin{array}{c}\text { Filter dP } \\
\text { nsid }\end{array}$ & $\begin{array}{c}\text { TopTMP } \\
\text { nsid }\end{array}$ & $\begin{array}{c}\text { Filtrate } \\
\text { nsig }\end{array}$ & Pulsepot & Slurry $F$ & Filtrate & Hi Filtate & & & & & & & & & & & \\
\hline & & $T^{2}$ & $T^{3}$ & $\mathrm{T1}$ & deg & 15 & dP2 & P1 & $\mathrm{dP} 1$ & $\mathrm{dP3}$ & $\mathrm{P} 2^{\mathrm{y}}$ & psig & 01 & 02 & $\begin{array}{ll}03 \\
3\end{array}$ & gpm & & & & & & & & & & \\
\hline \multicolumn{27}{|l|}{ Zeros } \\
\hline $9 / 11 / 2002$ & $0: 10 \mathrm{AM}$ & 1.716 & 20.836 & 20.261 & 20.264 & 20.354 & -0.121 & -0.035 & -0.059 & 0.06 & -0.226 & 0.361 & -0.216 & 0.002 & 0.001 & 0.0 & & \multicolumn{2}{|c|}{ Filter Surface } & $6.87 \mathrm{~F}$ & \multirow{2}{*}{\multicolumn{5}{|c|}{ gpm/ft2/barg }} & \\
\hline $9 / 11 / 2002$ & 10:25 AM & 1.716 & 20.836 & 20.261 & 20264 & 20.354 & -0.121 & -0.035 & $-0,059$ & 0.06 & -0.226 & 0.361 & -0.216 & 0.002 & 0.001 & 0.002 & & \multicolumn{2}{|c|}{ Conversion } & & & & & & & \\
\hline $9 / 11 / 2002$ & 10:26 AM & 0.716 & 20.841 & 20.256 & 20.269 & 20.359 & -0.117 & -0.037 & -0.059 & 0.06 & -0.246 & 0.358 & -0.214 & 0.002 & 0.001 & 0.002 & & & & & & & & & & \\
\hline $9 / 11 / 2002$ & 10:27 AM & 1.716 & 20.841 & 20.261 & 20.264 & 20.364 & -0.121 & -0.035 & -0.059 & 0.062 & -0.246 & 0.361 & -0.218 & 0.001 & 0.001 & 0.001 & & \multicolumn{2}{|l|}{ Note: G } & Pressure & ire $\mathrm{P} 1$ is & s approx & xima & y $1.4 \mathrm{ps}$ & & \\
\hline $9 / 11 / 2002$ & 10:28 AM & 0.711 & 20.841 & 20.256 & 20.264 & 20.359 & -0.119 & -0.037 & -0.059 & 0.06 & -0.249 & 0.358 & -0.216 & 0.001 & 0.001 & 0.003 & & & & & & & & & & \\
\hline $9 / 11 / 2002$ & 10:29 AM & 20.715 & 20.83 & 20.256 & 20.259 & 20.358 & -0.117 & -0.035 & -0.059 & 0.06 & -0.246 & 0.358 & -0.218 & 0.002 & 0.00 & 0.002 & & Pressur: & ure $\mathrm{P} 1$ is & correct & cted for. & 40.88 in & inches of & of water tut & ubing & \\
\hline $9 / 11 / 2002$ & $0: 30 \mathrm{AM}$ & 20.721 & 20.841 & 20.261 & 20.264 & 20.359 & -0.121 & -0.035 & & 0.06 & -0.246 & 0.358 & -0.218 & 0.002 & 0.001 & 0.001 & & WN & & & & $<$ Filtra & rate Flux & PER & RMIABIL & 니 \\
\hline $9 / 11 / 2002$ & 8:10:31 AM & 20.71 & 20.835 & 20.256 & 20.254 & 20.358 & -0.119 & -0.035 & -0.059 & 0.06 & -0.246 & 0.356 & -0.218 & 0.002 & 0.001 & 0.002 & & & & & & & at $25 \mathrm{C}$ & & $\times 1000$ & \\
\hline & & & & & & & & & & & & & & & & & Time & Press. & Vel. & TMP T & TMP & gpm & $\mathrm{gpm}$ & gpm & $\mathrm{gpm}$ & tele \\
\hline Data - Per M & Minute & & & & & & & & & & & & & & & & Min. & psig fi & $\mathrm{t} / \mathrm{s}$ & psi & bar & ift2 & $\mathrm{f} 2$ & $\mathrm{ft} 2 / \mathrm{psi}$ & $\mathrm{ft} 2 / \mathrm{psi}$ & $a y /$ \\
\hline $9 / 11 / 2002$ & 3:16:28 PM & 1.271 & 3.623 & 24.339 & 631 & 469 & 3.354 & 57.558 & 6 & 53 & 49.411 & .937 & 43.02 & 0.002 & 0.001 & 0. & 0 & 56.1 & 10.0 & 1.2 & 0.083 & 0.000 & 0.000 & 0.000 & 0.123 & \\
\hline $9 / 11 / 2002$ & 3:17:28 PM & 362 & 23.623 & 24.511 & 26.407 & 444 & 2.698 & 57.605 & & & 49.943 & 46.64 & 43.082 & 0.555 & & 0.003 & 1 & 56.1 & \begin{tabular}{|l|} 
\\
\end{tabular} & 0.7 & 0.049 & 0.081 & 0.082 & $\begin{array}{ll}20.116 \\
\end{array}$ & \#\# & 8.77 \\
\hline 9/11/2002 & 3:18:28 PM & 25 & 23.63 & & & & 31.29 & 5 & & & & & 47.256 & & & & 2 & 4.7 & 1.0 & 30.5 & 2.100 & & & & & . \\
\hline $9 / 11 / 2002$ & 3:19:28 PM & 5.225 & 23.63 & 708 & 39 & 23.4 & & 56.521 & & & 15.088 & 99 & 97 & 0.003 & 107 & & 3 & 5.0 & 10.9 & 33.5 & 2.308 & 0.723 & 29 & \begin{tabular}{l|l|}
9 & 0.0222 \\
\end{tabular} & 83 & 18. \\
\hline $9 / 11 / 2002$ & $3: 21: 28 \mathrm{PM}$ & 466 & 23.635 & 78 & 26.454 & 23.381 & 37.518 & 56.852 & & 18 & 12.677 & 0.428 & 15 & 0.002 & & 0.003 & 4 & 55.4 & 10.8 & 36.3 & 2.502 & 0.678 & 0.681 & \begin{tabular}{|l|l|}
1 & 0.019
\end{tabular} & 18.761 & 15.9 \\
\hline & PM & & 23.641 & & & 23.407 & 38.508 & 56.9 & & & & & & & & & 88 & 55.5 & & 37.2 & & & & & & 15.17 \\
\hline $9 / 11 / 2002$ & 3:23:21 PM & & 23.656 & & 265 & 23. & & 56.9 & & & 10.9 & 0.35 & & 0.0 & & 003 & 5.88 & 55.5 & $\begin{array}{ll}10.0 \\
10.7\end{array}$ & 38.0 & 2.622 & & 50 & 0.017 & & 14.5 \\
\hline 9/11/2002 & 3:24:21 PM & & 23.645 & 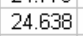 & 3 & & & 56 & & & 10.2 & 0.315 & 4 & & & & \begin{tabular}{|l|}
6.88 \\
\end{tabular} & & 0.7 & 38.9 & 2.679 & & & 50. & & 13.90 \\
\hline & $3: 25: 21 \mathrm{PM}$ & & 23.666 & & & & & 56. & & & & & & & & & & & & 39.5 & & & & & & 13.38 \\
\hline 9/11/2002 & 3:26:21 PM & 25.217 & 23.672 & & & & 42.073 & 57.1 & & & & & 45.4 & & & & 8.88 & 5.7 & 10.6 & 40.6 & 2.803 & & & 5 & & 12.75 \\
\hline $9 / 11 / 2002$ & 3:27:21 PM & & 677 & & & & & 5 & & & & & 2 & & & & 9.88 & 3 & 10.6 & 40.9 & 2. & 0.584 & 77 & 70. & & 12.4 \\
\hline & $3:$ & & 692 & & & & & & & & & & & & & & 10 & & 16 & 41.7 & & & & & & 11.97 \\
\hline 02 & 0.60 & & & & & & & & & & & & & & & & & & & 42.1 & & & & & & 11.6 \\
\hline $9 / 11 / 2002$ & $\mathrm{M}$ & & 22 & & & & & 5 & & & & & & & & & 12 & & 5 & 42.9 & & & & 3 & & 11. \\
\hline & & & & & & & & & & & & & & & & & & & & & & & & & & \\
\hline & & & 3 & & & & & & & & & & & & & & 14 & & 15 & 43.4 & & & & & & 10.72 \\
\hline 22 & 3 & & & & & & & & & & & & & & & & 15 & 8 & 0.4 & 43.9 & 3.0 & & 9 & 2 & & 10.45 \\
\hline & & & & & & & & & & & & & & & & & 16 & & & 44.1 & & & & & & 10.26 \\
\hline & & & 23.793 & & & 23. & & & & & & & & & & & 17 & 8 & $\begin{array}{l}10.4 \\
\end{array}$ & 45.1 & & & 3 & 2 & & 9.86 \\
\hline & & & & & & & & & & & & & & & & & & & & & & & & & & \\
\hline & & & & & & & & & & & & & & & & & & & & & & & & & & 9.52 \\
\hline $9 / 11 / 2002$ & $3: 38: 21$ & & 23.815 & & & 23. & & 56. & & & & & & & & & 20 & & 10.4 & & & & & & & 9.4 \\
\hline $9 / 11 / 2002$ & 3:39:21 PM & 24. & 23.821 & 23. & 8 & 23.6 & & 56. & & & & & & & & & 21.9 & 5.5 & 5 & 45.4 & & & & 11 & & \\
\hline & $3: 40: 2$ & & 23.8 & & & & & & & & & & & & & & & & & & & & & & & 80 \\
\hline 9/11/2002 & 3:41:21 PM & & 23.809 & 23.934 & 26.722 & 23.6 & 47.74 & 57. & & & 3.3 & & \begin{tabular}{|l|l|}
44.189 \\
\end{tabular} & 0.0 & & & 23.9 & 55.6 & 10.3 & 45.8 & 3.161 & 0.465 & 0.480 & 0.010 & 10.460 & 8.90 \\
\hline 9/11/2002 & 3:42:21 PM & 24.688 & 23.829 & 24.14 & 26.612 & 23. & 48.011 & 57.268 & .731 & 44.155 & 3.254 & 0.023 & 44.12 & 0.002 & 3.168 & . & 24.9 & 55.8 & 10.3 & 46.1 & 3.177 & 0.461 & 0.472 & \begin{tabular}{|l|l|}
2 & 0.0101
\end{tabular} & 10.249 & 8.72 \\
\hline & & & & & & & & & & & & & & & & & & & & & & & & & & \\
\hline & & & & & & & & & & & & & & & & & & & & & & & & & & 11. \\
\hline & & & 23 & & & & & & & & 11 & & & & & & & & 10.8 & 46.1 & 3. & 50 & 3 & & 8 & 15. \\
\hline & & & & & & & & & & & & & & & & & & & & & & & & & & \\
\hline & & & 23 & & & & & & & & & & & 0.0 & & & & 55.0 & 10.3 & $\begin{array}{l}4.4 \\
37.2\end{array}$ & & & & & 10.2 & $8.6 \quad 2$ \\
\hline & & -2 & 0.125 & 0.99 & 0.415 & 0.131 & 747 & 0.508 & 0.220 & 5.044 & 5.178 & 225 & 1.256 & 0.001 & 847 & 00 & & 0.508 & 0.293 & 5.394 & 0.372 & 0.123 & 113 & 80.0044 & 4.449 & 796 \\
\hline & & 21 & & & & & & & & & & & & & & & & & & & & & & & & \\
\hline
\end{tabular}

Figure B12: PreConditioning 2D - Baseline Slurry - After Test Rig Modification - Raw \& Calculated Data 
WSRC-TR-2003-00204, REV. 0

SRT-RPP-2003-00087, REV. 0

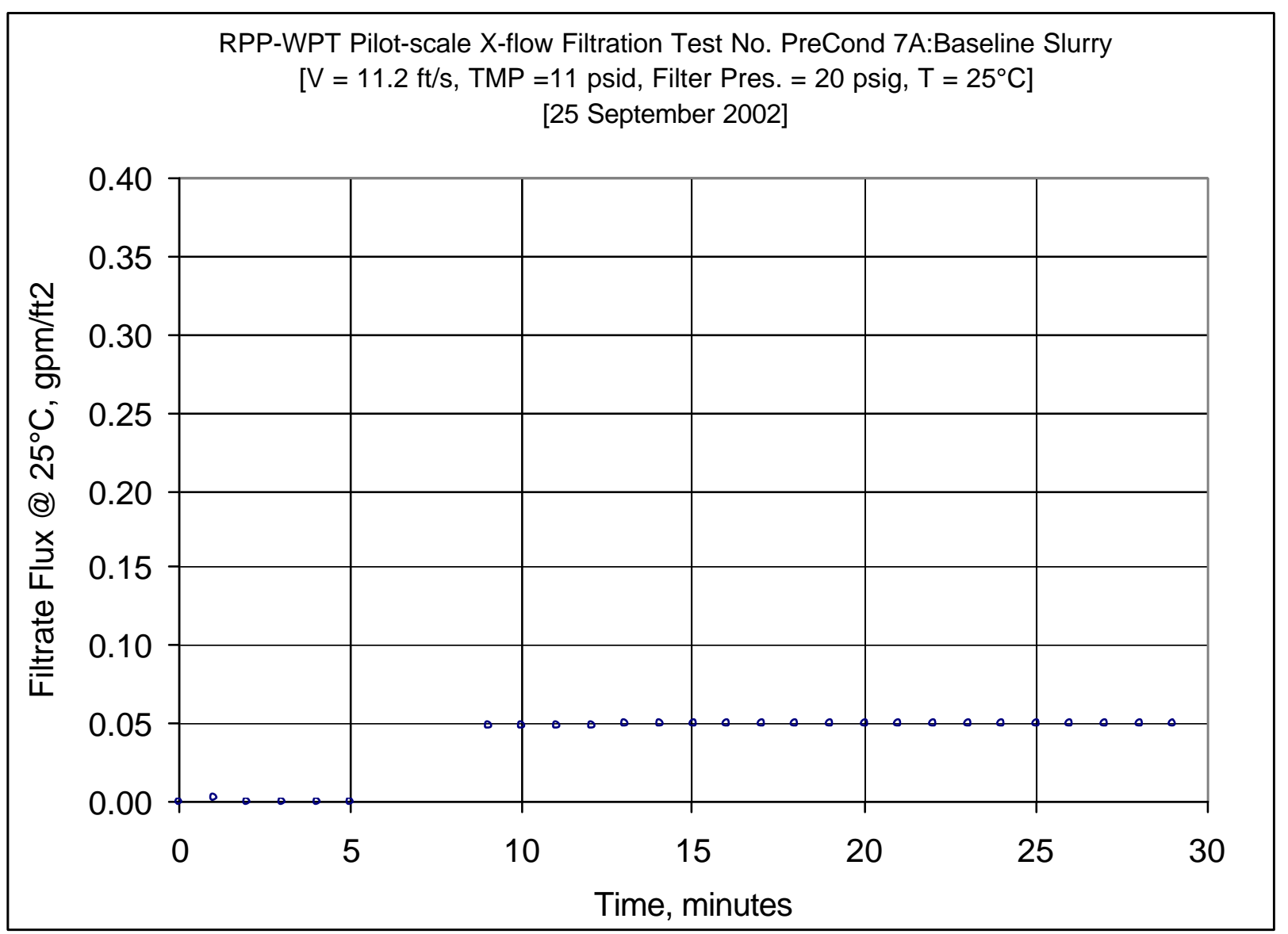

Figure B13: PreConditioning 7A - Baseline Slurry - After Preconditioning Filter with AN-107 
WSRC-TR-2003-00204, REV. 0

SRT-RPP-2003-00087, REV. 0

\begin{tabular}{|c|c|c|c|c|c|c|c|c|c|c|c|c|c|c|c|c|c|c|c|c|c|c|c|c|c|c|}
\hline \multirow{3}{*}{ DATE } & \multirow[b]{2}{*}{ TIME } & \multicolumn{5}{|c|}{ «《« } & \multicolumn{6}{|c|}{$\ll<<<<<<<<<<<<$ Pressure Measurements $\gg \gg \gg \gg \gg$} & \multicolumn{4}{|c|}{ 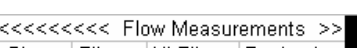 } & \multicolumn{10}{|c|}{ 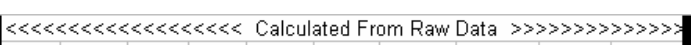 } \\
\hline & & Filtrate & Cleaning & Slurry & Hi Amb. & Lo Amb. & BotTMP & Filter $\mathrm{F}$ & Filter $\mathrm{dP}$ & TopTMP & Filtrate & Pulsepot & Slurry & Filtrate & Hi Filtate & Backpulse & & & & & & & & & & \\
\hline & & $\operatorname{deg} C$ & $\operatorname{deg} C^{\circ}$ & $\operatorname{deg} C$ & $\operatorname{deg} C$ & $\operatorname{deg} \mathrm{C}$ & psid & psig & psid & psid & noi & psig & $\mathrm{gpm}$ & $\mathrm{gpm}$ & gpm & $\mathrm{gpm}$ & & & & & & & & & & \\
\hline & & $\mathrm{T} 2$ & $\mathrm{~T} 3$ & $\mathrm{~T} 1$ & T4 & T5 & $\mathrm{dP2}$ & $\mathrm{P} 1$ & $\mathrm{dP1}$ & $\mathrm{dP3}$ & P2 & P3 & Q1 & Q2 & Q3 & Qbp & & & & & & & & & & \\
\hline \multicolumn{27}{|l|}{ Zeros } \\
\hline $9 / 25 / 2002$ & $7: 37: 47 \mathrm{AM}$ & 20.881 & 21.183 & 20.533 & 20.516 & 20.65 & -0.121 & -0.039 & -0.049 & 0.062 & -0.356 & 0.356 & -0.212 & 0.002 & 0.001 & 0.001 & & & 6.87 & \multirow{2}{*}{\multicolumn{4}{|c|}{$\begin{array}{l}7 \mathrm{FT} 2 \\
1 \mathrm{~m} 3 / \mathrm{m} 2 / \mathrm{day} / \mathrm{barg} / \mathrm{gpm} / \mathrm{f} 2 / \mathrm{barg}\end{array}$}} & & \\
\hline $9 / 25 / 2002$ & 7:38:00 AM & 20.881 & 21.183 & 20.533 & 20.516 & 20.65 & -0.121 & -0.039 & -0.049 & 0.062 & -0.356 & 0.356 & -0.212 & 0.002 & 0.001 & 0.001 & & \multicolumn{2}{|c|}{ Conversion } & 851 & & & & & & \\
\hline $9 / 25 / 2002$ & 7:38:01 AM & 20.881 & 21.177 & 20.528 & 20.516 & 20.65 & -0.119 & -0.039 & -0.049 & 0.064 & -0.307 & 0.353 & -0.216 & 0.002 & 0.001 & 0.001 & & \multirow{2}{*}{\multicolumn{2}{|c|}{ Note }} & & & & & & & \\
\hline $9 / 25 / 2002$ & 7:38:02 AM & 20.881 & 21.177 & 20.528 & 20.521 & 20.65 & -0.121 & -0.039 & -0.049 & 0.064 & -0.347 & 0.353 & -0.212 & 0.001 & 0.001 & & & & & Pressur & re $\mathrm{P} 1$ is & is approx & ximately & 1.4 psig & & \\
\hline $9 / 25 / 2002$ & 7:38:03 AM & 20.882 & 21.178 & 20.534 & 20.521 & 20.666 & -0.119 & -0.039 & -0.049 & 0.064 & -0.37 & 0.356 & -0.216 & 0.002 & 0.001 & 0. & & & & & & & & & & \\
\hline $9 / 25 / 2002$ & 7:38:04 AM & 20.881 & 21.177 & 20.533 & 20.526 & 20.65 & -0.119 & -0.041 & -0.048 & 0.064 & -0.327 & 0.356 & -0.212 & 0.002 & 0.001 & 0.002 & & Pressur & ure $\mathrm{P} 1$ is & cor & ted for & 40.88 & inches of & of water tut & bir & \\
\hline 9/25/2002 & 7:38:05 AM & 20.882 & 21.183 & 20.544 & 20.531 & 20.666 & -0.121 & -0.039 & -0.048 & 0.064 & -0.33 & 0.356 & -0.216 & 0.002 & 0.001 & 0.00 & & WW & & & & $<$ Filtra & rate Flux & $x \ll$ PER & RMIABILIT & ITY $>$ \\
\hline $9 / 25 / 2002$ & 7:38:06 AM & 20.887 & 21.183 & 20.544 & 20.531 & 20.671 & -0.121 & -0.039 & -0.048 & 0.064 & -0.324 & 0.358 & -0.212 & 0.001 & 0.001 & 0.001 & & & & & & & at $25 \mathrm{C}$ & & $\times 1000$ & \\
\hline & & & & & & & & & & & & & & & & & Time & Press. & el. & TMP & TMP & $\mathrm{gpm}$ & & & gpm & meter \\
\hline $\operatorname{ta}-$ Per M & Minute & & & & & & & & & & & & & & & & & & $\mathrm{f} / \mathrm{s}$ & & bar & st2 & & & & 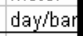 \\
\hline $9 / 25 / 2002$ & 10:06:18 AM & 24.833 & 2.383 & 25.707 & 22.773 & 455 & 12.103 & 20.314 & 5.493 & -3.311 & 3.02 & 1.104 & 47.04 & 0.002 & 0.001 & & 0 & 18.8 & 311.0 & 4.4 & 0.303 & 30.000 & 10.000 & 0.000 & 0.065 & 0.06 \\
\hline $9 / 25 / 2002$ & 10:07:18 AM & 24.301 & 22.391 & 25.841 & 22.737 & 22.509 & 15.431 & 20.392 & 15.292 & 0.308 & -0.229 & 0.573 & 47.01 & 0.015 & 0.196 & & 1 & 18.9 & $\begin{array}{ll}9 & 11.0\end{array}$ & 7.9 & 0.543 & 0.002 & 0.002 & 0.000 & 0.271 & 0.23 \\
\hline 9/25/2002 & 10:08:18 AM & 25.316 & 22.394 & 25.914 & 22.755 & 22.537 & 14.754 & 19.939 & 15.2 & -0.274 & -0.041 & 0.5 & 46.511 & 0.1. & & & 2 & 8.5 & \begin{tabular}{|l|l|}
5 & 10.9
\end{tabular} & 7.2 & 0.499 & 0.000 & 10 & 0.000 & 0.039 & 0.03 \\
\hline $9 / 25 / 2002$ & 10:09:18 AM & 25.631 & 22.399 & 26.024 & 22.72 & 22.592 & 15.676 & 20.986 & 16.142 & -0.237 & -0.041 & 0.538 & 48.103 & 0. & & & 3 & 19.5 & \begin{tabular}{|l|l|}
5 & 11.2
\end{tabular} & 7.7 & & 20.000 & & 0.000 & 0.018 & 0.02 \\
\hline $9 / 25 / 2002$ & 10:10:18 AM & 25.882 & 22.426 & 26.115 & 22.741 & 22.659 & 77 & 20.781 & 15.944 & 0.058 & 0.046 & 0.584 & 48.105 & & & & it & 19.3 & \begin{tabular}{|l|l|} 
& 11.2 \\
\end{tabular} & 7.8 & 0.539 & 0.000 & 00 & 0.000 & 0.018 & 0.02 \\
\hline $9 / 25 / 2002$ & 10:11:18 AM & 25.987 & 22.436 & 26.185 & 22.927 & 22.714 & & 20.974 & 15. & & 0.089 & & 47.971 & & & & 5 & 19.5 & \begin{tabular}{|l|l|}
5 & 11.2 \\
\end{tabular} & 7.8 & 0.537 & 0.000 & & 0.000 & 0.036 & \\
\hline 9/25/2002 & 10:15:18 AM & 26.073 & 22.492 & 26.425 & 23.118 & 22.886 & 18.969 & 21.245 & 16.2 & 2.975 & -1.284 & -2.624 & 48.017 & 0.3 & & & 9.00 & 19.8 & \begin{tabular}{|l|l|}
3 & 11.2 \\
\end{tabular} & 11.0 & 0.756 & 50.051 & & 0.004 & 4.486 & 3.82 \\
\hline 9/25/2002 & 10:16:18 AM & 26.116 & 22.5 & 26.479 & 23.096 & 22.879 & 18.884 & 21.124 & 16.31 & 3.014 & -1.284 & -2.641 & 48.155 & 0.3 & 0. & & 10 & 19.7 & $\begin{array}{ll}7 & 11.2\end{array}$ & 10.9 & 0.755 & 50.052 & 0.049 & 0.005 & 4.514 & 3.84 \\
\hline $9 / 25 / 2002$ & 10:17:18 AM & 193 & 22.501 & 26.495 & 23.063 & 22.885 & 18.909 & 21.093 & 16. & & -1.2 & -2.662 & 48.246 & 0. & & & 11 & 19.6 & \begin{tabular}{|l|l|}
6 & 11.3 \\
\end{tabular} & 11.0 & 0.756 & 0.052 & 19 & 0.005 & 4.507 & 3.84 \\
\hline $9 / 25 / 2002$ & 10:18:18 AM & 242 & 22.511 & 9 & & 22.8 & & & 16. & & & & & & & & 12 & 20.2 & \begin{tabular}{|l|l|}
2 & 11.3
\end{tabular} & 11.3 & & 20.052 & & 04 & 4.366 & \\
\hline $9 / 25 / 2002$ & $10: 19: 18 \mathrm{AM}$ & 36 & 22 & $2 E$ & & 22. & & & & & & & & & & & 13 & 19.8 & \begin{tabular}{|l|l|}
3 & 11.2 \\
\end{tabular} & 11.1 & & & & & & 3.81 \\
\hline $9 / 25 / 2002$ & $10: 20: 18 \mathrm{AM}$ & 5.299 & 22.512 & 26.021 & 23.385 & 22.8 & 19.071 & 21.307 & 16.1 & 3.315 & -1.2 & -2 & 47.815 & 0.3 & & & 14 & 19.8 & \begin{tabular}{|l|l|} 
& 11.2
\end{tabular} & 11.2 & 0.772 & 20.051 & 0.050 & 0.004 & $\begin{array}{l}4.447 \\
\end{array}$ & 3.78 \\
\hline $9 / 25 / 2002$ & 10:21:18 AM & 246 & 22.51 & 25.814 & 23.287 & 22. & & 21.453 & 16. & 2 & -1.2 & -2 & & & & & 15 & 20.0 & 11.2 & 11.1 & & & & 0.004 & 4.488 & 3.82 \\
\hline 9/25/2002 & 10:22:18 AM & 4 & 22.517 & 25.611 & & & & 20 & & & -1.2 & & & & & & 16 & 19.5 & \begin{tabular}{|l|l|}
5 & 11.2 \\
\end{tabular} & 10.8 & & & & 0.005 & 4.604 & $\begin{array}{l}3.92 \\
\end{array}$ \\
\hline $9 / 25 / 2002$ & 10:23:18 AM & 151 & 22.52 & 25. & & & & 21. & 16. & & & & & & & & 17 & 20.0 & 11.2 & 11.2 & & & & 0.004 & 36 & 3.77 \\
\hline $9 / 25 / 2002$ & 10:24:18 AM & 109 & 22.527 & 25 & 23 & & & 21.232 & 16. & & -1. & -2 & & & & & 18 & 8 & \begin{tabular}{|l|l|} 
& 11.2 \\
\end{tabular} & 11.0 & 0 & 5.050 & 0 & 0.005 & 4.569 & 3.89 \\
\hline $9 / 25 / 2002$ & 10:25:18 AM & 26.0 & 22. & 25. & & 22. & & & & & & & & & & & 19 & 19.6 & \begin{tabular}{|l|l|}
6 & 11.2
\end{tabular} & 11.0 & & & & & 4.512 & 3.84 \\
\hline 9/25/2002 & 10:26:18 AM & 200 & 22.529 & 25. & & 22.8 & & 21.371 & & & -1. & & & & & & 20 & 19.9 & \begin{tabular}{|l|l|}
9 & 11.2
\end{tabular} & 11.0 & & & & 0.005 & 4.550 & $\begin{array}{l}3.87 \\
\end{array}$ \\
\hline $9 / 25 / 2002$ & 10:27:18 AM & 25.907 & 22.531 & 24.974 & 23.197 & 22.8 & 19.025 & 21.135 & 16.2 & & -1. & & 75 & & & & 21 & 19.7 & \begin{tabular}{|l|l|}
7 & 11.2
\end{tabular} & 11.2 & 0. & 50 & f & 4 & 74 & 3.81 \\
\hline $9 / 25 / 2002$ & 10:28:18 AM & 5.808 & 22.507 & & & & & & & & & & & & & & 22 & 7 & \begin{tabular}{l|l}
7 & 11.2
\end{tabular} & 11.1 & & & & & 4 & 3.85 \\
\hline $9 / 25 / 22$ & $10: 29: 18 \mathrm{AM}$ & 25.731 & 22 & 24.758 & & 22. & & 21.776 & & & & & & & & & 23 & 20.3 & \begin{tabular}{|l|l|}
3 & 11.2
\end{tabular} & 11.3 & & 0.050 & & 0.004 & 4.424 & 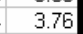 \\
\hline $9 / 25 / 2002$ & 10:30:18 AM & 25.644 & 22.517 & 24.715 & 23.1 & 22.7 & 19.097 & 21.176 & 16.2 & 3.264 & -1. & -2. & & 0. & & & 24 & 19.7 & $\begin{array}{ll}7 & 11.2\end{array}$ & 11.2 & 0.771 & 49 & 0 & 14 & $\begin{array}{l}4.44 \\
4.448 \\
\end{array}$ & 3.78 \\
\hline 9/25/2002 & 10:31:18 AM & 25.567 & 22.521 & 24 & 23.088 & 22.7 & & 21.352 & & & & & & & & & 25 & 19.9 & \begin{tabular}{|l|l|}
9 & 11.2 \\
\end{tabular} & 11.1 & & & & & 90 & 3.82 \\
\hline $9 / 25 / 2002$ & $10: 32: 18 \mathrm{AM}$ & 25.47 & & 24 & & & & & & & & & & & & & 2 & 19.9 & \begin{tabular}{|l|l}
9 & 11.1 \\
\end{tabular} & 11.3 & & & & & $\begin{array}{l}4.399 \\
\end{array}$ & 3.74 \\
\hline $9 / 25 / 2002$ & 10:33:18 AM & 25.408 & 22.522 & 24.535 & 23.044 & 22.7 & 19.01 & 21.093 & 16. & 2. & -1.2 & -2.9 & \begin{tabular}{|l|}
47.87 \\
\end{tabular} & 0. & & & 2 & 19.6 & \begin{tabular}{ll|}
6 & 11.2
\end{tabular} & 11.0 & 0.756 & 49 & 0 & 0.005 & 519 & 3.85 \\
\hline $9 / 25 / 2002$ & 10:34:18 AM & 25.315 & 22.509 & 24.427 & 23.5 & 22.7 & 19.224 & 21.145 & 16. & & & -2 & 47.9 & & & & 28 & 19.7 & $\begin{array}{ll}7 & 11.2 \\
\end{array}$ & 11.0 & 0.760 & 50 & 0.050 & 0.005 & 4.507 & 3.84 \\
\hline 9/25/2002 & 10:35:18 AM & 25.248 & 22.507 & 24.415 & 22.988 & & 19.485 & 21.627 & 16.6 & 3.203 & -1.286 & & 47.803 & 0.33 & 0.001 & & 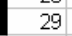 & 20.2 & \begin{tabular}{|l|l|}
2 & 11.2
\end{tabular} & 11.3 & 0.782 & 20.049 & 0.050 & 0.004 & 4.369 & 3.72 \\
\hline & & & & & & & & & & & & & & & & & & & & & & & & & & \\
\hline & & & & & & & & & & & & & & & & & & 19.8 & \begin{tabular}{|l|l|}
3 & 11.2 \\
\end{tabular} & 11.1 & 0.8 & 50 & 50 & & 4.5 & 3.0 \\
\hline & & & & & & & & & & & & & & & & & & & \begin{tabular}{|l|l|} 
& 11.3 \\
\end{tabular} & & & & & & & \\
\hline & & $2 E$ & & & & & & & & & -1.3 & -2 & & & & & & 19.8 & \begin{tabular}{ll|}
8 & 11.2
\end{tabular} & 11.1 & 8 & 0.050 & & & 4.5 & 3.8 \\
\hline & Minimum & 25.2 & 22.5 & 24.4 & 23.0 & 22.8 & 18.8 & 21.0 & 16.1 & 2.8 & -1.3 & -3 & 47.7 & 0.3 & 0.0 & & & 19.5 & \begin{tabular}{|l|l|}
5 & 11.1 \\
\end{tabular} & \begin{tabular}{|l|}
10.8 \\
\end{tabular} & 0.7 & 0.049 & 0.049 & \begin{tabular}{l|l}
9 & 0.0044
\end{tabular} & 4.4 & 3.7 \\
\hline & $2 \times \operatorname{Std} \mathrm{Dev}$ & 0.669 & 0.020 & 1.461 & 0.176 & 0.084 & 0.370 & 0.421 & 0.353 & 0.333 & 0.003 & 0.206 & 0.301 & 0.013 & 0.000 & 0.000 & & 0.421 & 10.070 & 0.284 & 0.020 & 0.002 & 0.000 & 0.0001 & 0.119 & 0.101 \\
\hline $\mathrm{N}$ & Points Used f $^{*}$ & 21 & 21 & 21 & 21 & 21 & 21 & 1 & 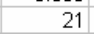 & 21 & 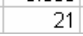 & 21 & 0 & & 2 & & & 21 & 21 & 21 & 21 & 2 & 21 & 21 & 21 & 7 \\
\hline
\end{tabular}

Figure B12: PreConditioning 7A - Baseline Slurry - After Preconditioning Filter with AN-107- Raw \& Calculated Data 
WSRC-TR-2003-00204, REV. 0

SRT-RPP-2003-00087, REV. 0

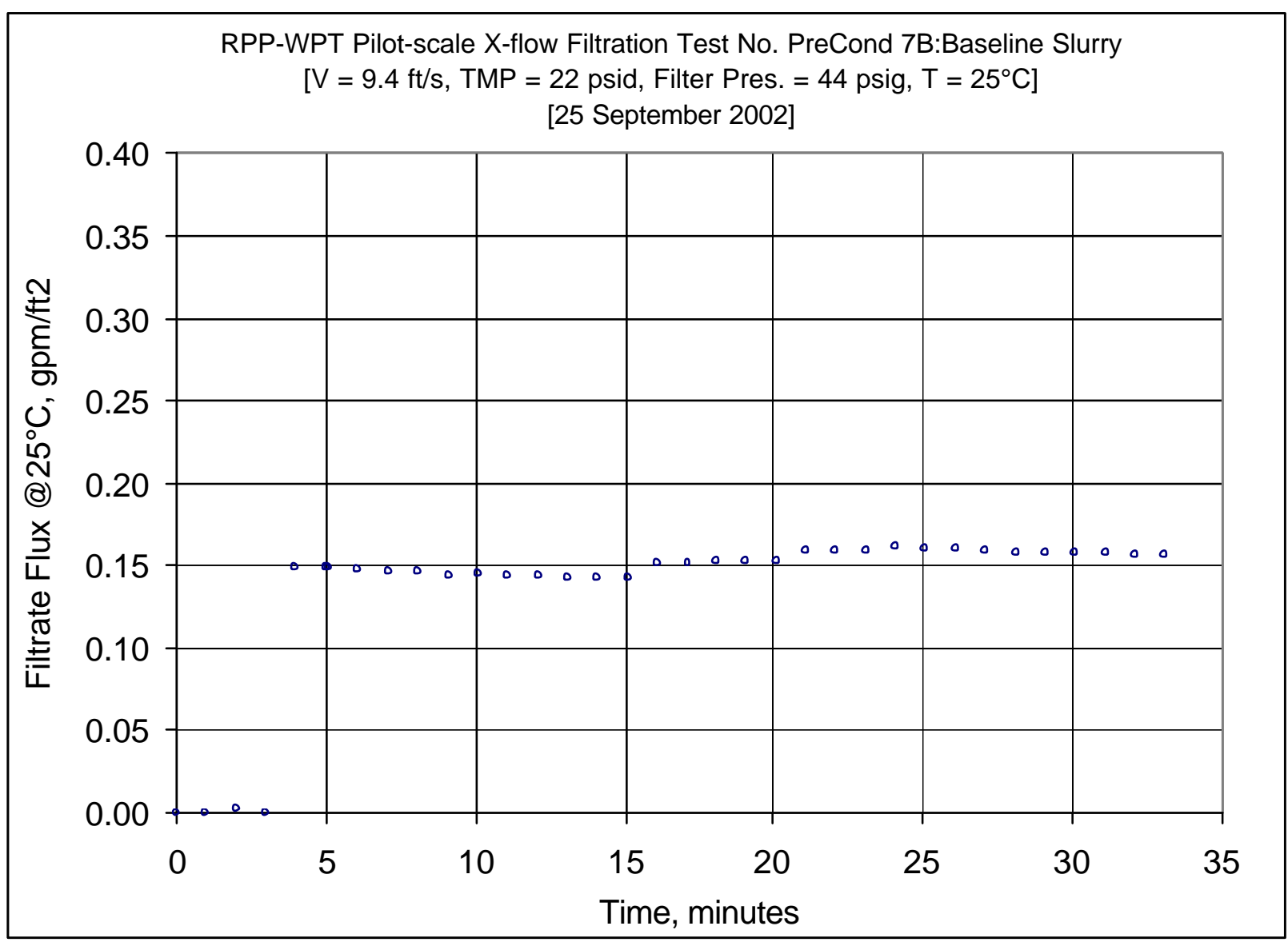

Figure B15: PreConditioning 7B - Baseline Slurry - After Preconditioning Filter with AN-107 
WSRC-TR-2003-00204, REV. 0

SRT-RPP-2003-00087, REV. 0

\begin{tabular}{|c|c|c|c|c|c|c|c|c|c|c|c|c|c|c|c|c|c|c|c|c|c|c|c|c|c|c|}
\hline \multirow[t]{3}{*}{ DATE } & \multirow{2}{*}{ TIME } & & & \multicolumn{6}{|l|}{ T } & \multicolumn{4}{|c|}{$\begin{array}{l}\ll \ll \text { Flow Measurements } \\
\text { Filtrate Hi Filtate Backpuls }\end{array}$} & & & & & \\
\hline & & $\mathrm{gC}$ & $\operatorname{deg} C$ & $\operatorname{deg} C$ & $\operatorname{deg} C$ & $\operatorname{deg} C$ & psid & psig & psid & psid & psig & psig & $\mathrm{gpm}$ & & $\mathrm{gpm}$ & $\mathrm{gpm}$ & & & & & & & & & & \\
\hline & & T2 & $\mathrm{T} 3$ & $\mathrm{~T} 1$ & T4 & & $\mathrm{dP2}$ & $\mathrm{P} 1$ & $\mathrm{dP} 1$ & $\mathrm{dP} 3$ & $\mathrm{P} 2$ & P3 & $\mathrm{Q1}$ & $\mathrm{Q} 2$ & Q3 & Qbp & & & & & & & & & & \\
\hline \multicolumn{27}{|l|}{ Zeros } \\
\hline 9/ & $7: 47 \mathrm{~A}$ & 881 & 21.183 & 533 & .516 & 20.65 & -0.121 & -0.039 & -0.049 & 0057 & -0.356 & & .212 & 0.002 & 0.001 & & & \multicolumn{2}{|c|}{ Filter Surface } & & $7 \mathrm{FT} 2$ & & & & & \\
\hline 002 & $: 38: 00 \mathrm{~A}$ & 881 & 21.183 & 20.533 & $\begin{array}{ll}3 & 20.516\end{array}$ & 20.6 & -0.121 & -0.039 & .049 & 0.062 & -0.356 & 35 & -0.212 & 002 & 0.001 & 0.6 & & Convers & & & & & & & & \\
\hline $9 / 25 / 2002$ & $7: 38: 01 \mathrm{Al}$ & 20.881 & 21.177 & 20.528 & 20.516 & 20.65 & $\begin{array}{l}-0.1219 \\
-0.119\end{array}$ & $\begin{array}{l}-0.039 \\
-0.039\end{array}$ & $\begin{array}{l}-0.049 \\
-0.049\end{array}$ & 0.064 & $\begin{array}{l}-0.307 \\
-0.307\end{array}$ & 353 & $\begin{array}{l}-0.216 \\
-0.216\end{array}$ & 0.002 & 0.001 & 0.001 & & & & & & & & & & \\
\hline $9 / 25 / 2002$ & $7: 38: 02$ & 0.881 & 21.177 & 628 & 21 & 20.65 & -0.121 & -0.039 & -0 & 0.064 & -0.347 & & -0.212 & 0.001 & 0.001 & & & Note: $G$ & Gauge P & Pressu & sure $P 1$ is & is approx & oximately & y $1.4 \mathrm{psig}$ & & \\
\hline $9 / 25 / 2002$ & 7:38:03 AM & 20.882 & 21.178 & 20.534 & $\begin{array}{l}4 \quad 20.521 \\
\end{array}$ & 20.666 & -0.119 & -0.039 & -0 & 0.064 & -0.37 & 356 & -0.216 & 0.002 & 0.001 & 0.001 & & & & & & & & & & \\
\hline $9 / 25 / 2002$ & 7:38:04 AM & 20.881 & 21.177 & 20.533 & \begin{tabular}{|l|l}
3 & 20.526
\end{tabular} & 20.65 & -0.119 & -0.041 & -0.048 & 0.064 & -0.327 & 0.356 & -0.212 & 0.002 & 0.001 & 0.002 & & & ure $\mathrm{P} 1$ is & is corre & ected for & & & of water tut & & \\
\hline $9 / 25 / 2002$ & 7:38:05 AM & 20.882 & 21.183 & 20.544 & \begin{tabular}{|l|l|}
4 & 20.531
\end{tabular} & $1 \quad 20.666$ & -0.121 & -0.039 & -0.048 & 0.064 & -0.33 & 0.35 & -0.216 & 0.002 & 0.001 & & & WN & & & & $<$ Filtra & rate Flux & $x \ll$ PER & RMAABILI & IITY $>$ \\
\hline $9 / 25 / 2002$ & 7:38:06 AM & 20.887 & 21.183 & 20.544 & $\begin{array}{ll}4 & 20.531\end{array}$ & 20.671 & -0.121 & -0.039 & -0.048 & 0.064 & $\begin{array}{l}-0.324 \\
\end{array}$ & 0.358 & -0.212 & 0.001 & 0.001 & 0.001 & & & & & & & at $25 \mathrm{C}$ & & $\times 1000$ & \\
\hline & & & & & & & & & & & & & & & & & & Press. & 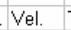 & TMP & TMP & $\mathrm{am}$ & & & & eter \\
\hline Per $\mathrm{N}$ & Minute & & & & & & & & & & & & & & & & & & & & & & & & & \\
\hline $9 / 25$ & A & 831 & 1.501 & .074 & 1.152 & 477 & 11.242 & 4.621 & 13.52 & -2.222 & 28.233 & 3.61 & 384 & & & & & 43.1 & 10 & & & 000 & 000 & & 664 & \\
\hline $9 / 25 / 2002$ & $10: 43: 39 \mathrm{AM}$ & 4.795 & 22.495 & 25.063 & 52 & 222.482 & -7.339 & 45.896 & 12 & -18.509 & 43.154 & 76.608 & 43.612 & 02 & & 0.416 & 1 & 44.4 & $\begin{array}{l}410.2 \\
4\end{array}$ & $2-12.9$ & $9-0.891$ & 0.000 & .000 & 0.000 & 122 & -0.02 \\
\hline $9 / 25 / 2002$ & $10: 44: 09 \mathrm{AM}$ & 24.8 & 22.495 & 25.148 & 57 & 22.492 & 19 & 44.574 & 13.833 & 5.911 & 19.782 & 19.362 & 44.323 & 17 & & & 2 & 43.1 & 10.3 & $\begin{array}{ll}3 & 12.7 \\
\end{array}$ & & 002 & 002 & 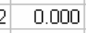 & 94 & 016 \\
\hline $9 / 25 / 2002$ & $10: 44: 39 \mathrm{AM}$ & 5.342 & 22.521 & 25.255 & \begin{tabular}{|l|l|}
5 & 23.198
\end{tabular} & 22.528 & 34.379 & 44.144 & 14.161 & & 4.376 & 0.79 & 44.982 & & & & 3 & 42.7 & 10.5 & 527.5 & $\begin{array}{ll}5 & 1.895\end{array}$ & 0.000 & 000 & 0.000 & 011 & \\
\hline $9 / 25 / 2002$ & $10: 45: 09 \mathrm{AM}$ & 25.437 & 22.526 & 25.265 & $\begin{array}{l}5 \quad 23.274 \\
\end{array}$ & $4 \quad 22.533$ & 26.102 & 44.022 & 14.114 & 12.202 & 12.758 & 11.677 & 45.148 & 1.025 & & & 4 & 42.5 & $\begin{array}{ll}6 & 10.5 \\
\end{array}$ & $\begin{array}{ll}5 & 19.2 \\
\end{array}$ & $\begin{array}{lll}2 & 1.320\end{array}$ & 0.149 & 0.148 & $\begin{array}{l}8 \\
8.008\end{array}$ & 87.730 & 6.58 \\
\hline $9 / 25 / 2002$ & $10: 45: 39 \mathrm{AM}$ & 25.563 & 22.517 & 25.24 & \begin{tabular}{|l|l|}
4 & 23.289 \\
\end{tabular} & 9. 22.549 & 25.742 & 43.542 & 13.92 & 12.218 & 12.689 & 11.605 & 45.02 & 1.025 & 1 & & 5 & 42.1 & 10.5 & $\begin{array}{ll}5 & 19.0 \\
\end{array}$ & $\begin{array}{ll}0 & 1.309 \\
0\end{array}$ & 0.149 & 0.148 & 88 & 805 & \\
\hline $9 / 25 / 2002$ & $10: 45: 44 \mathrm{AM}$ & 25.569 & 22.528 & 25.241 & 123.295 & \begin{tabular}{|l|l|}
5 & 22.545 \\
\end{tabular} & 25.966 & $\begin{array}{l}43.502 \\
43.602\end{array}$ & 14.219 & 11.964 & $\begin{array}{l}12.695 \\
\end{array}$ & \begin{tabular}{|l|l|}
11.619 \\
\end{tabular} & \begin{tabular}{|l|l}
45.16 \\
5.16
\end{tabular} & 1.027 & & & 5.08 & 42.1.1. & 10.5 & $5 \quad 19.0$ & $\begin{array}{lll}0 & 1.308 \\
\end{array}$ & 0.149 & 0.148 & 88 & & 6.06 \\
\hline $9 / 25 / 2002$ & $10: 46: 44 \mathrm{AM}$ & 25.68 & 22.524 & & & $5 \quad 22.596$ & & 44.404 & 14.348 & & 12.527 & 11.492 & & & & & 6.08 & 42.9 & 10.6 & 519.5 & & & & & & 6.43 \\
\hline & $10: 47$ & 25.773 & 22. & 25.4 & & 22. & & & 14. & & & 11.353 & & & & & 7.08 & 42.6 & 10.5 & $\begin{array}{ll}5 & 19.6\end{array}$ & & & & & & 6.34 \\
\hline & & 25.832 & 22. & 25.455 & 523. & 22. & & 44.034 & & & 12 & . & & & & & 8.08 & 426 & 10.5 & & & & & & & 6.27 \\
\hline & & 25.902 & 22.5 & 25.514 & 423.5 & 22.7 & & 44.148 & 14. & & 11. & & & & & & 9.08 & 42.7 & 10.5 & 520.0 & 0 & & & & & \\
\hline $9 / 25 / 2002$ & $10: 50: 44 \mathrm{AM}$ & 25.94 & 22.564 & 25.457 & 23.597 & 22.782 & 26.645 & 43.693 & 13 & & 11.908 & 10.892 & 54 & & & & 10.1 & 42.2 & $\begin{array}{l}210.0 \\
20.4\end{array}$ & $\begin{array}{ll}4 & 19.9 \\
\end{array}$ & $\begin{array}{lll}9 & 1.374\end{array}$ & & & & & 6.15 \\
\hline $9 / 25$ & $10: 51: 44 \mathrm{AM}$ & 25.979 & & 25.612 & 22 & 22. & & & 13. & & 11 & 10. & 44.665 & 1 & & & 11.1 & 42.9 & $\begin{array}{l}30.4 \\
3\end{array}$ & 420.3 & $\begin{array}{ll}3 & 1.399 \\
\end{array}$ & 0.146 & t & 7 & 056 & \\
\hline $9 / 25 / 2002$ & $10: 52: 44 \mathrm{AM}$ & 26.029 & 22 & 25.552 & $2 \quad 23.737$ & 22.8 & 26 . & & 13. & & 11 & & 3 & 0 & & & 12.1 & 42.2 & .4 & $\begin{array}{ll}420.2 \\
\end{array}$ & $\begin{array}{ll}2 & 1.389\end{array}$ & & & & 88 & 6.03 \\
\hline $9 / 25 / 2002$ & $10: 53: 44$ & 26 & 22 & 25.507 & 23.883 & $\begin{array}{ll}3 & 22.942\end{array}$ & 27. & & 13. & & 11 & 10. & & & & & 13.1 & 42.9 & 4 & $\begin{array}{ll}420.6 \\
\end{array}$ & 51. & & & & & \\
\hline $9 / 25$ & $10: 54: 4$ & & 22 & 25.391 & 23.947 & 22. & & & & & & 10. & & & & & 14.1 & 42.5 & 1.4 & & & & & & & \\
\hline & & & & & & & & & & & & & & & & & & 42.9 & 10.4 & & & & & & & \\
\hline & & & & & & & & & & & & & & & & & 16 & 43.9 & $\begin{array}{r}9.4 \\
9.5\end{array}$ & & & & & & & \\
\hline & & & 2 & 25 & & 23. & & & & & & & & & & & & & 9.5 & & & & & & & 5. \\
\hline & & & & & & & & & & & & & & & & & 18 & 4 & 9.5 & 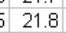 & & & & & & 5. \\
\hline & $10: 59: 4$ & 27 & 22 & & & & & 45 & & & & & & & & & & 4.2 & 9.4 & 42 & & & & & & \\
\hline & $11: 00: 4$ & & & 2 & & 23. & & & 10. & & & & & & & & & 4.3 & 9.4 & & & & & & & \\
\hline & & & & & & & & & & & & & & & & & & & 8.7 & & & & & & & \\
\hline & & & & & & & & & & & & & & & & & & & 7 & & & & & & & \\
\hline & & & & & & 23. & & & & & & & & & & & & 3 & 7 & & & & & & & \\
\hline & $11:$ & & & & & & & & & & & & & & & & & 5.2 & 8.5 & & & & & & & \\
\hline & $11: 0$ & 25 & & & 23. & & & & & & & & & & & & & 5.8 & 8.4 & & & & & & & \\
\hline & & 24.8 & 22.7 & 2 & 23 & $\begin{array}{ll}4 & 23 \\
4 & 23 .\end{array}$ & 27. & $\frac{47}{46}$ & & & & & & & & & & 45.3 & $\begin{array}{l}0.4 \\
8.4\end{array}$ & $\begin{array}{ll}42 \\
4\end{array}$ & & & & & & \\
\hline & & & 22.7 & & & 2. & & 47. & & & & & & & & & & & & & & & & & & \\
\hline & & & & & & & & & & & & & & & & & & & & & & & & & & \\
\hline & & & & & & & & & & & & & & & & & & & & & & & & & & \\
\hline & & & & & & & & & & & & & & & & & & & & & & & & & & \\
\hline & & & & & & 23. & & & & & & & & 1.0 & & & & & 8.4 & & & & & & & \\
\hline & $11: 12: 44 \mathrm{~A}$ & 25.322 & 22.773 & 24.824 & 23.806 & 623.0 & 27.734 & 47. & & 19.363 & 14.174 & 12. & 35.968 & 1.07 & & & 32.1 & 5.6 & 8.4 & 23.5 & $5 \quad 1.624$ & 0.156 & 0.156 & 0.007 & $7 \quad 6.645$ & 5.65 \\
\hline $9 / 25$ & 11:13:44 An & 25.376 & 22.781 & 24.798 & 23.865 & 23.0 & 27.699 & 46.881 & 8.824 & 19.082 & 14.122 & 12.876 & \begin{tabular}{|l|}
36.081 \\
\end{tabular} & 1.069 & 0.00 & & 33.1 & 45.4 & 8.4 & 23.4 & $\begin{array}{ll}4 & 1.613 \\
\end{array}$ & 0.156 & 0.156 & 0.007 & $\begin{array}{lll}7 & 6.6888\end{array}$ & \\
\hline & & & & & & & & & & & & & & & & & & & & & & & & & & \\
\hline & & & & & & & & & & & & & & & & & & 4.2 & 9.4 & 21.7 & & & & & & \\
\hline & & & & & & & & & & & & & & & & & & & & & & & & & & \\
\hline & & & & & & & & & & & & & & & & & & & & & & & & & & \\
\hline & & & & & & & & & & & & & & & & & & 1 & 8.4 & & & & & & & \\
\hline & & 0.709 & 0. & 0.915 & 0.546 & 0.3 & & 2.7 & & 5.1 & 2.1 & & & 0.0 & & & & 2.781 & 1.786 & 2.939 & 90.203 & 19 & 13 & 05 & 47 & 6 \\
\hline & $\mathrm{fP}$ & 29 & & 29 & & & 20 & & & 29 & 29 & & 29 & 29 & 29 & & & 29 & 29 & 29 & 29 & 29 & 29 & 29 & 29 & \\
\hline
\end{tabular}

Figure B14: PreConditioning 7B - Baseline Slurry - After Preconditioning Filter with AN-107- Raw \& Calculated Data 
WSRC-TR-2003-00204, REV. 0

SRT-RPP-2003-00087, REV. 0

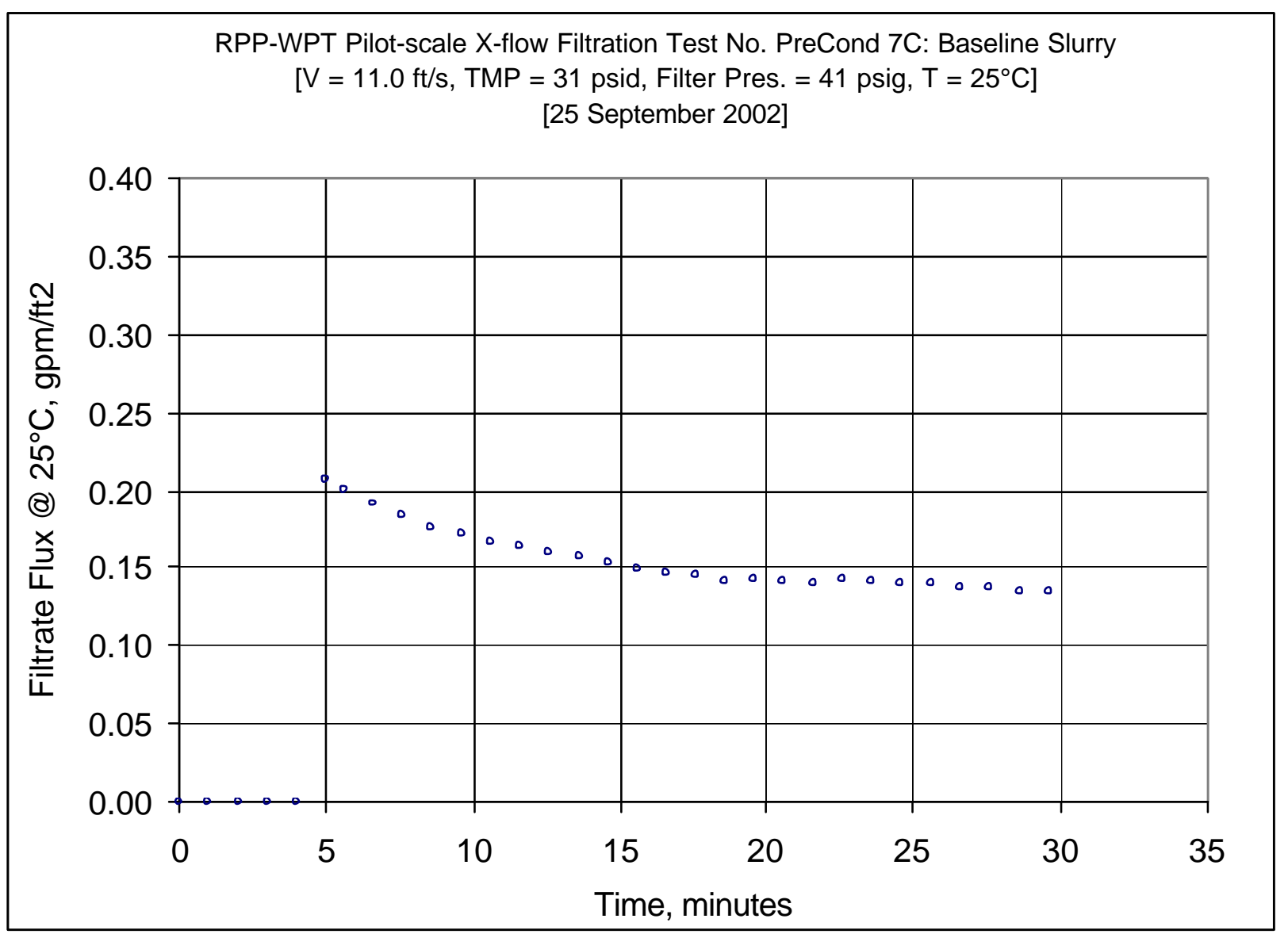

Figure B15: PreConditioning 7C - Baseline Slurry - After Preconditioning Filter with AN-107 
WSRC-TR-2003-00204, REV. 0

SRT-RPP-2003-00087, REV. 0

\begin{tabular}{|c|c|c|c|c|c|c|c|c|c|c|c|c|c|c|c|c|c|c|c|c|c|c|c|c|c|c|}
\hline \multirow{4}{*}{ DATE } & \multirow{4}{*}{ TIME } & \multicolumn{5}{|c|}{ «« } & \multicolumn{6}{|c|}{ 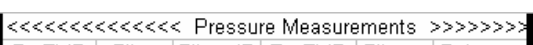 } & \multicolumn{4}{|c|}{ 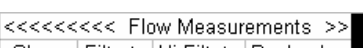 } & \multicolumn{10}{|c|}{ 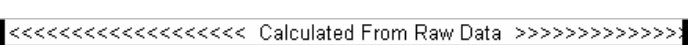 } \\
\hline & & Filtrate & Cleaning & Slurry & Hi Amb. & Lo Amb. & BotTMP & & Filter $\mathrm{dP}$ & TopTMP F & Filtrate & Pulsepot & Slurry $\mathrm{F}$ & Filtrate & Hi Filtate & Backpulse & & & & & & & & & & \\
\hline & & $\operatorname{deg} C$ & $\operatorname{deg} C$ & $\operatorname{deg} C$ & $\operatorname{deg} C$ & $\operatorname{deg} C$ & psid & psig & psid & psid & psig & psig & $\mathrm{gpm}$ & $\mathrm{gpm}$ & $\mathrm{gpm}$ & $\mathrm{gpm}$ & & & & & & & & & & \\
\hline \multirow{2}{*}{\multicolumn{27}{|c|}{ Zeros }} \\
\hline & & & & & & & & & & & & & & & & & & & & & & & & & & \\
\hline $9 / 25 / 2002$ & 37:47 AM & 1.881 & 21.183 & 20.533 & 1.516 & 20.65 & -0.121 & -0.039 & -0.049 & 0.062 & -0.356 & 0.356 & -0.212 & 0.002 & 0.001 & 0.00 & & & 6.8 & FT2 & & & & & \\
\hline $9 / 25 / 2002$ & 7:38:00 AM & 0.881 & 21.183 & 20.533 & 1.516 & 20.65 & -0.121 & -0.039 & -0.049 & 0.062 & -0.356 & 0.356 & -0.212 & 0.002 & 0.001 & 0.001 & & \multicolumn{2}{|c|}{ Conversion } & 851 & $\mathrm{~m} 3 / \mathrm{m} 2 / \mathrm{c}$ & /day/ba & arg / gpm/ & $\mathrm{m} / \mathrm{ft} 2 / \mathrm{barg}$ & & \\
\hline $9 / 25 / 2002$ & 7:38:01 AM & 20.881 & 21.177 & 20.528 & 0.516 & 20.65 & -0.119 & -0.039 & -0.049 & 0.064 & -0.307 & 0.353 & -0.216 & 0.002 & 0.001 & 0.001 & & & & & & & & & & \\
\hline $9 / 25 / 2002$ & 7:38:02 AM & 20.881 & 21.177 & 20.528 & 20.521 & 20.65 & -0.121 & -0.039 & -0.049 & 0.064 & -0.347 & 0.353 & -0.212 & 0.001 & 0.001 & & & Note: $G$ & Sauge & Pressur & ure $P 1$ is & S approx & oximate & $1.4 \mathrm{psig}$ & & \\
\hline $9 / 25 / 2002$ & 7:38:03 AM & 20.882 & 21.178 & 20.534 & 521 & 20.666 & -0.119 & -0.039 & -0.6 & 064 & -0.37 & 356 & -0.216 & 0.002 & 0.001 & 0.001 & & & & & & & & & & \\
\hline $9 / 25 / 2002$ & 7:38:04 AM & 20.881 & 21.177 & 20.533 & 20.526 & 20.65 & -0.119 & -0.041 & -0.048 & 64 & -0.327 & 356 & -0.212 & 0.002 & 0.001 & 0.002 & & Pressur: & ure & $s \mathrm{co}$ & cted & 40.88 ir & inches of & f water tu & at & \\
\hline $9 / 25 / 2002$ & 7:38:05 AM & 20.882 & 21.183 & 20.544 & 20.531 & 20.666 & -0.121 & -0.039 & -0.048 & 0.064 & -0.33 & 0.356 & -0.216 & 0.002 & 0.001 & 0.001 & & m & & & & $<$ Filtra & trate Flux & $\ll$ PEF & RMMABILIT & LITY $\gg$ \\
\hline $9 / 25 / 2002$ & 7:38:06 AM & 20.887 & 21.183 & 20.544 & 20.531 & 20.671 & -0.121 & -0.039 & -0.048 & 0.064 & -0.324 & 0.358 & -0.212 & 0.001 & 0.001 & 0.001 & & & & & & & at $25 \mathrm{C}$ & & $\times 1000$ & \\
\hline & & & & & & & & & & & & & & & & & Time & Press. & Vel. & TMP & TMP & $\mathrm{gpm}$ & $\mathrm{gpm}$ & gpm & $\mathrm{gpm}$ & meter \\
\hline Data - Per Mi & Minute & & & & & & & & & & & & & & & & (1) & & $\mathrm{ft} / \mathrm{s}$ & & bar & & & $\mathrm{f} 2 / \mathrm{psi}$ & $\mathrm{ft} 2 / \mathrm{psi}$ & ay/bal \\
\hline 905007 & $12: 12$ & 24.835 & 2.922 & 608 & .513 & 517 & 19.29 & 1747 & 2.876 & 469 & 8.51 & 2.857 & 47.23 & 02 & 0.001 & & 0 & 41 . & 11.0 & 7.9 & 0.545 & 0.000 & 0.0 & 0.000 & 0.019 & 0.02 \\
\hline $9 / 25 / 2002$ & 12:13:56 PM & 24.539 & 22.942 & 24.707 & 593 & 2.566 & 20.019 & 42.867 & 22. & & 17.55 & 39.72 & 47.304 & & & & 1 & 41.4 & 11.0 & 8.9 & 11 & 0.000 & 00 & 0.000 & & 0.01 \\
\hline $9 / 25 / 2002$ & 12:14:56 PM & 24.537 & 22.93 & 24.701 & 23.647 & 22.6 & 53 & 42.1 & & -2.462 & 17.212 & 40.154 & 46.847 & & & 0.00 & 2 & 40.6 & 10.9 & 8.7 & 0.603 & & 00 & 00 & D 0.017 & 0.01 \\
\hline $9 / 25 / 2002$ & 12:15:56 PM & 24.528 & 22.951 & 24.792 & 23.8 & 22.656 & 20.141 & 42.396 & 22. & -2.654 & 17.25 & & 47.2 & & & 0.002 & 3 & 40 & 11.0 & 8.7 & & & & & & 0.01 \\
\hline $9 / 25 / 2002$ & 12:16:56 PM & 25.115 & 22.952 & 24.777 & 23.753 & 22.712 & 35.309 & 42.526 & 22.7 & 13.129 & 1.948 & 0.752 & 47.662 & & & & 4 & 41.1 & 11.1 & 24.2 & & & & & & .01 \\
\hline $9 / 25 / 2$ & 12:17:56 PM & 25.255 & 22.957 & 24.832 & 23.844 & $\begin{array}{l}42.767 \\
\end{array}$ & 84 & 42.3 & 22.791 & & -1.284 & -4.196 & 48.002 & 0. & & & 5 & 40.8 & 11.2 & 29.3 & 2.022 & 0.207 & 08 & \begin{tabular}{|l|l|}
3 & 0.007 \\
\end{tabular} & $\begin{array}{l}7 \quad 7.089 \\
\end{array}$ & 6.03 \\
\hline $9 / 25$ & 12:18:31 PM & 25.308 & 22.965 & 24.795 & 7 & 22.776 & 43 & 42.278 & 22.482 & 55 & -1.281 & & 47.788 & & & & 5.58 & 40.8 & 11.2 & 29.7 & 2. & & & 0.007 & \begin{tabular}{|l|l|}
7 & 6.770 \\
\end{tabular} & 5.76 \\
\hline & & 6 & 22.9 & & & 22.8 & & 41.965 & & & & & & & & & 6.58 & 40 & 11.1 & & & & & & & 52 \\
\hline $9 / 25 / 2002$ & 12:20:31 PM & 25.396 & 22.968 & 24.829 & 24.0 & 22.79 & 41.385 & 2.708 & 23. & & -1. & -4.205 & & & & & 7.58 & 41.2 & 11.1 & 30.0 & 2. & & & 06 & & 5.20 \\
\hline $9 / 25 /$ & 12:21:31 PM & 25.413 & 22.965 & 24.936 & & & & 5 & & & -1. & & & & & & 8.5 & 41. & 11.1 & & & & & & & 4.96 \\
\hline $9 / 25$ & $12: 22$ & 25.449 & 22.96 & 2 & & & & 2 & & & & & & & & & 9.5 & 40 & 11.1 & & & & & & & 4.92 \\
\hline $9 / 25 / 2$ & 12:23:31 PM & 25.46 & 22.966 & 24.947 & 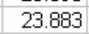 & 22 & 41.271 & 42.319 & 22. & & & -4 & 47 & & & & 10.6 & 40.8 & 11.0 & 30.3 & & 0 & & 06 & 02 & 4.68 \\
\hline $9 / 25 / 2$ & 12:24:31 PM & 25.495 & 22.961 & 24 & 3 & & & 42.24 & & & -1. & & & & & & 11.6 & 40.8 & 11.1 & 30.2 & & & & 15 & $5 \quad 5.403$ & 4.60 \\
\hline & 12:25: & 25.477 & & & & & & & & & & & & & & 0.001 & 12 & 40 & 11.1 & & & & & & & 4.47 \\
\hline & 12:26: & 25 & & & & & & & & & & & & & & & 13 & & 11.1 & & & & & & & we \\
\hline $9 / 25 /$ & 12:27:31 PM & 5.5 & 22. & 2 & 20 & & & 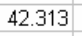 & 22. & & -1. & & & & & 002 & 14 & 40 & 11.1 & 30 & 2. & & & & 55. & 4.29 \\
\hline & PM & 25.3 & & & & & & & & & & & & & & & & & & & & & & & & \\
\hline & & 25 & 22.9 & 24 & 23.8 & & & 42. & & & & & & & & 101 & 16 & & 11.1 & & & & & & & .06 \\
\hline $9 / 25 / 2$ & 12:30:31 PM & 25.561 & 22.932 & 24.893 & 23 & 22 & & 69 & 22. & & & & & & & & 17 & 40 & 11.1 & 3 & & & & 5 & 33 & 4.07 \\
\hline & 12:31:31 PM & $5.56 \quad-$ & & & & & & & & & & & & & & & 18 & & & & & & & & & 401 \\
\hline & & 2 & 22. & & & & & & & & & & & & & & 19 & 40 & 11.0 & & & & & & & 95 \\
\hline & $12: 3$ & 6.5 & 22. & & & & & & & & & & & & & & 0 & & 10.9 & & & & & & & \\
\hline & 12:34:31 PM & 2 & & & & & & & & & & & & & & & & & & & & & & & & \\
\hline & $12: 35$ & 25.60 & 22 & & & & & & & & & & & & & & & & & & & & & & & 3.88 \\
\hline $9 / 25$ & PM & 25.60 & 22.945 & 24 & 24.0 & 22.8 & & & 20. & & & & & & & & 23 & 40.8 & 10.9 & 3 & & & & 4 & & 1 \\
\hline & 12:37:31 PM & 25.606 & 22.942 & 24 & & 22.8 & & & & & & & & & & & & & 10.8 & & & & & & & 380 \\
\hline & $12: 38$ & $=0$ & & & & & & & & & & & & & & & & 40.5 & & & & & & & & 3.79 \\
\hline & 12:39:31 PM & 5.599 & 22.951 & 24.9 & 23.8 & 22. & 41 & & 21. & 6 & -1.2 & & & & & & 26 & 41.0 & 10.8 & 31.4 & 2 & 8 & 38 & 0.004 & 380 & 3.73 \\
\hline & $12: 4$ & 25.5 & 22.9 & & & & & & & & & & & & & & & & & & & & & & & \\
\hline & & 25.5 & & & & & & & & & & & & & & & & & & & & & & & 325 & \\
\hline $9 / 25 / 2002$ & 12:42:31 PM & 25.566 & 22.942 & 24.902 & 23.904 & 22.97 & 41.733 & 42.147 & 20.893 & 21.244 & -1.289 & -4.135 & 46.029 & 0.001 & 0.925 & & 29.6 & 40.7 & 10.7 & 31.5 & 2.171 & 0.135 & 0.135 & 0.004 & \begin{tabular}{|l|l|}
4 & 4.286 \\
\end{tabular} & . \\
\hline & & & & & & & & & & & & & & & & & & & & & & & & & & \\
\hline & & & & & & & & & & & & & & & & & & & & & & & & & & \\
\hline & & & & & & & & & & & -1 & -4 & & & & & & 41.2 & 11.2 & 31.5 & 2. & & 0.201 & & 6.8 & 5.8 \\
\hline & & & & & & & & & & & -1.3 & -4 & & & & & & 40.8 & 11.1 & 30.4 & 2.098 & 0.145 & 0.145 & 0.0048 & 4.8 & 4.1 \\
\hline & & & & & & & & & & & & & & & & & & 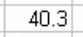 & & 07 & & & & & 3 & 0.0 \\
\hline & & 0.1 & 020 & 0.103 & & 0.1 & 15 & 0.4. & $1.4 E$ & 2 & 0.00 & & 1. & & & & & 40 & 0.285 & 1.272 & 8 & 36 & 37 & & 381 & 175 \\
\hline of $\mathrm{F}$ & f Points Used* & $2 J$ & 25 & & & & 25 & & 20 & 20 & & & 20 & & & & & 25 & 25 & 25 & 25 & 25 & 25 & & 25 & \\
\hline
\end{tabular}

Figure B14: PreConditioning 7C - Baseline Slurry - After Preconditioning Filter with AN-107- Raw \& Calculated Data

Page B20 of B50 
WSRC-TR-2003-00204, REV. 0

SRT-RPP-2003-00087, REV. 0

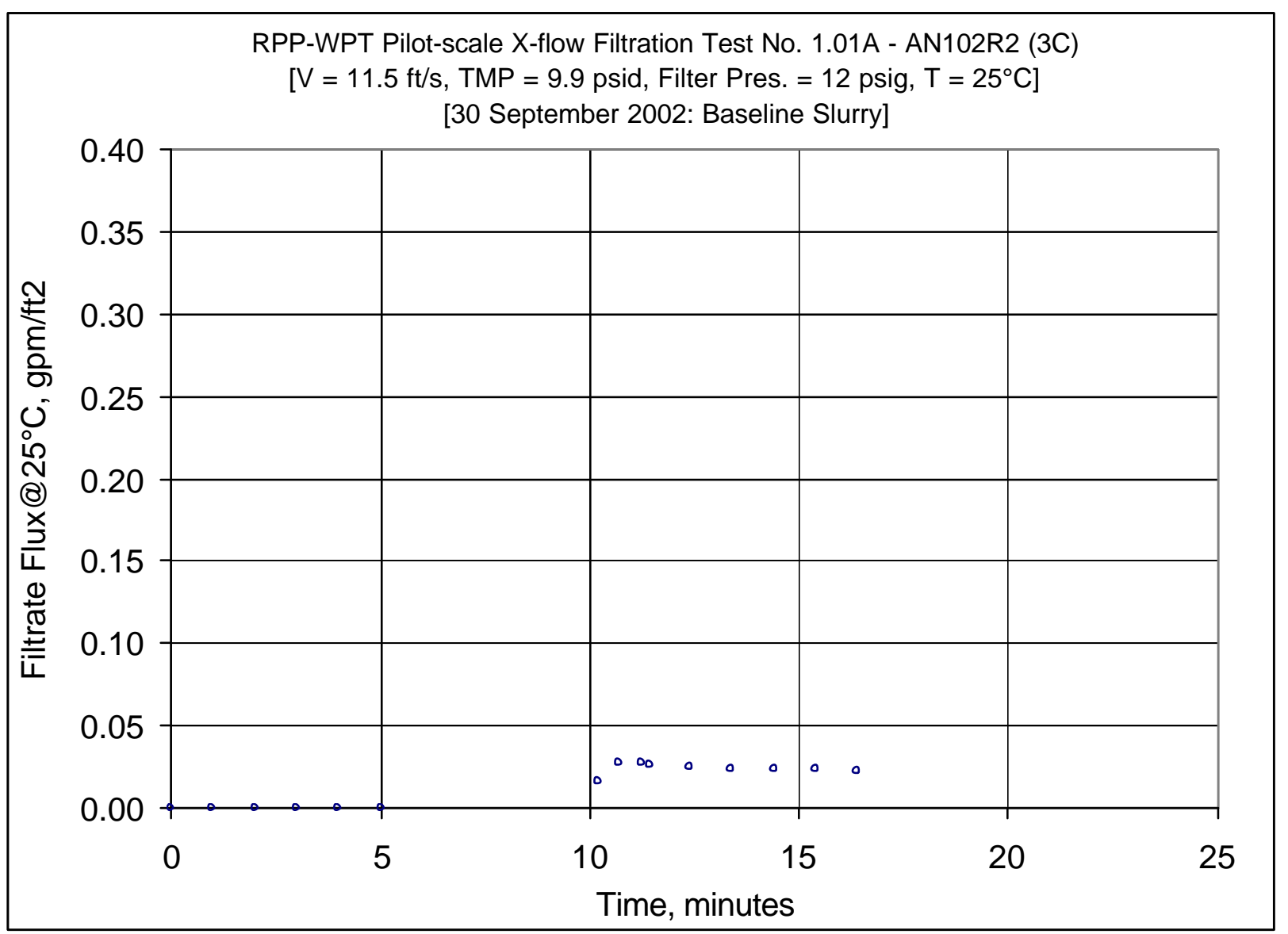

Figure B17: Run 1.01A - Baseline Slurry - Before Using Slurry AN-102R2 Batch 3C 
WSRC-TR-2003-00204, REV. 0

SRT-RPP-2003-00087, REV. 0

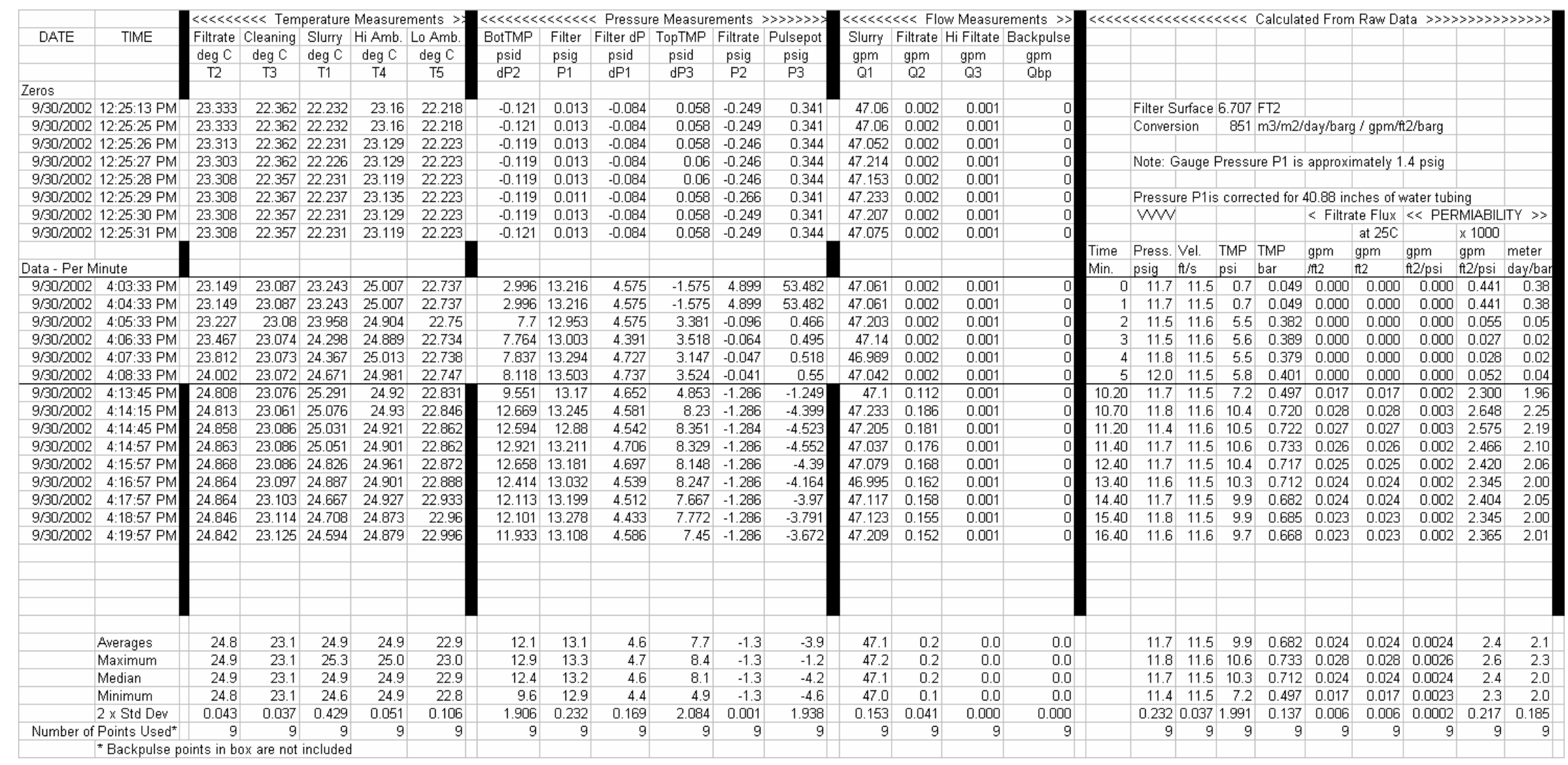

Figure B18: Run 1.01A - Baseline Slurry - Before Using Slurry AN-102R2 Batch 3C - Raw \& Calculated Data 
WSRC-TR-2003-00204, REV. 0

SRT-RPP-2003-00087, REV. 0

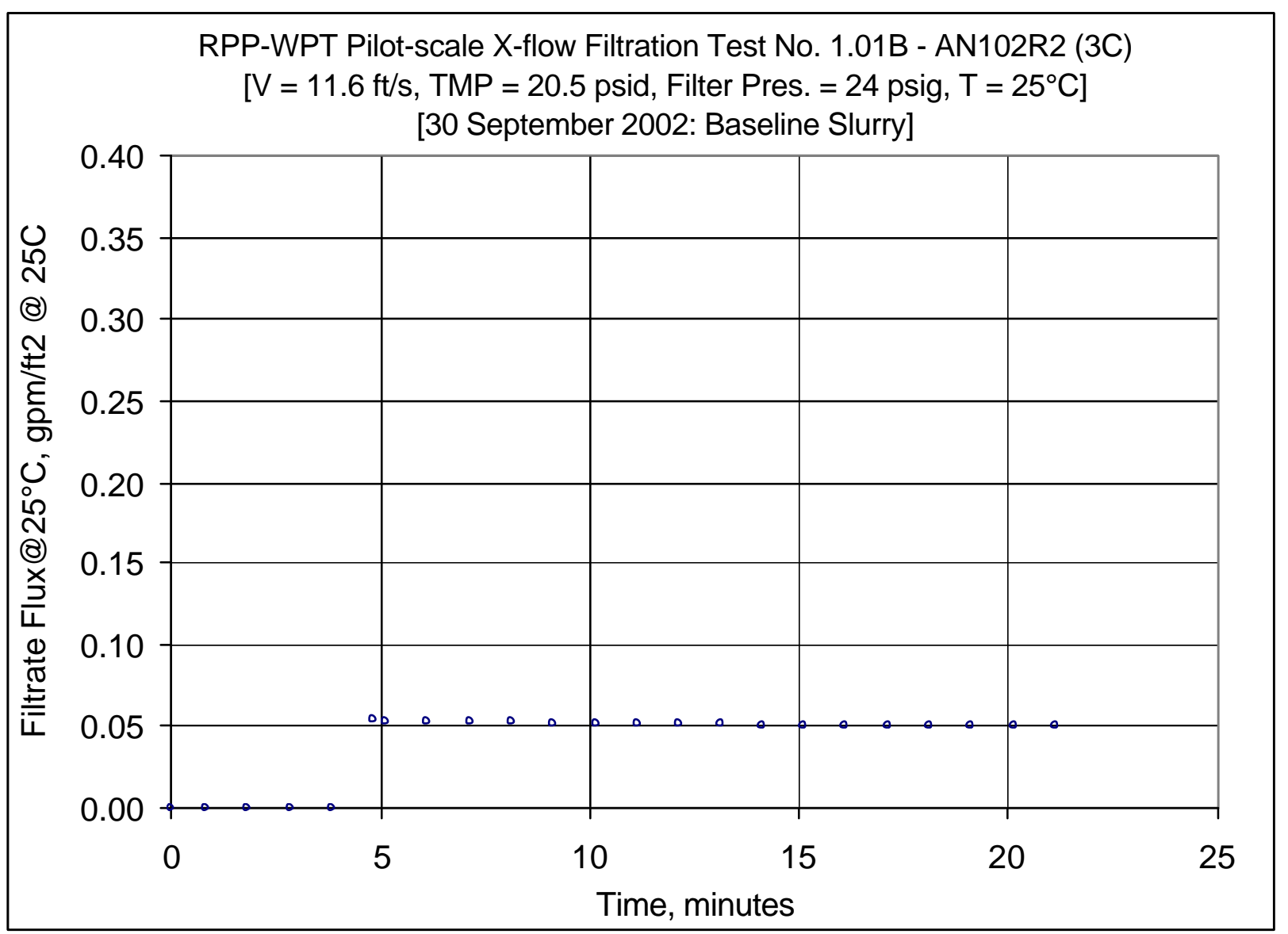

Figure B19: Run 1.01B - Baseline Slurry - Before Using Slurry AN-102R2 Batch 3C 
WSRC-TR-2003-00204, REV. 0

SRT-RPP-2003-00087, REV. 0

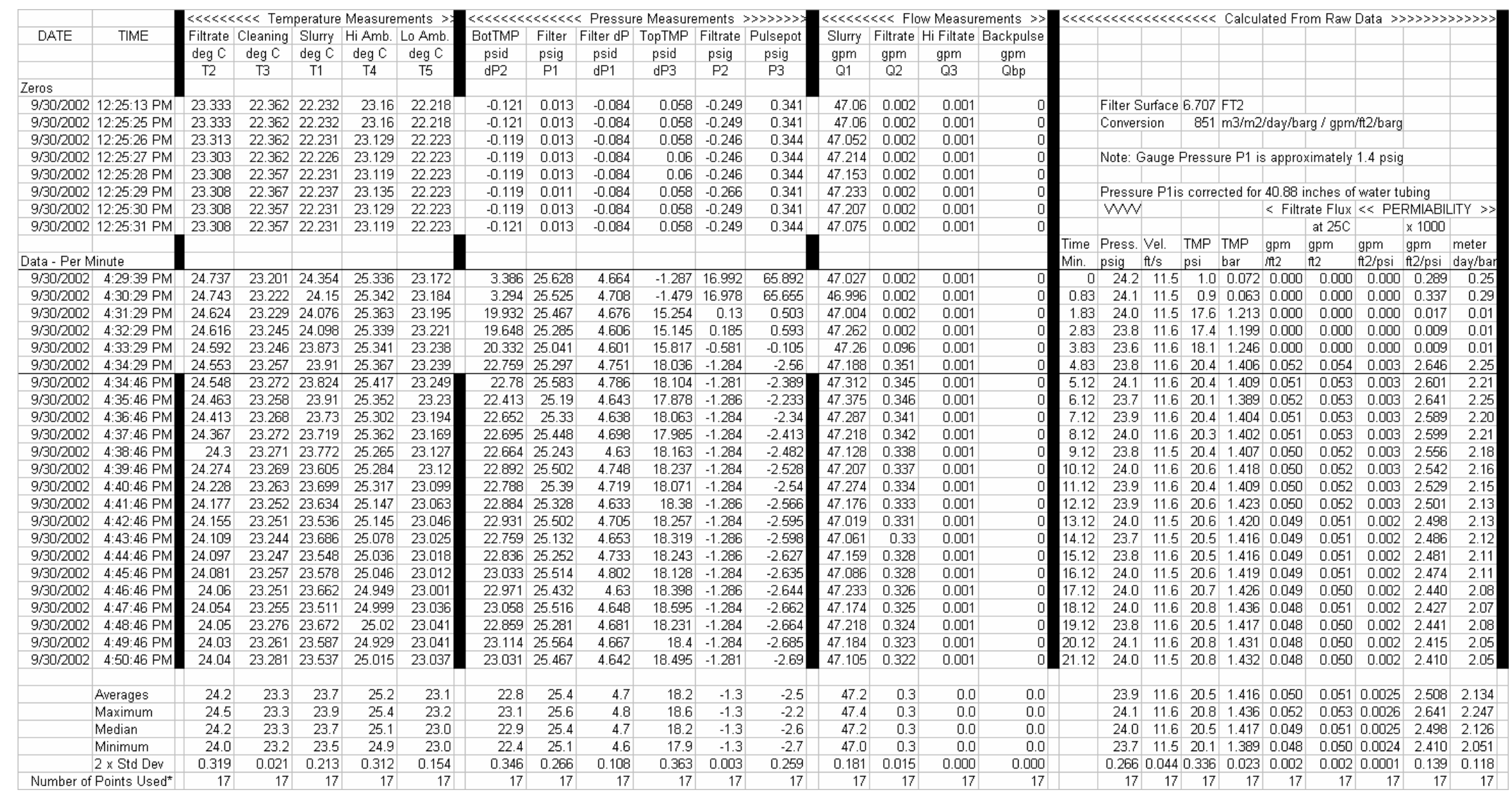

Figure B20: Run 1.01B - Baseline Slurry - Before Using Slurry AN-102R2 Batch 3C - Raw \& Calculated Data 
WSRC-TR-2003-00204, REV. 0

SRT-RPP-2003-00087, REV. 0

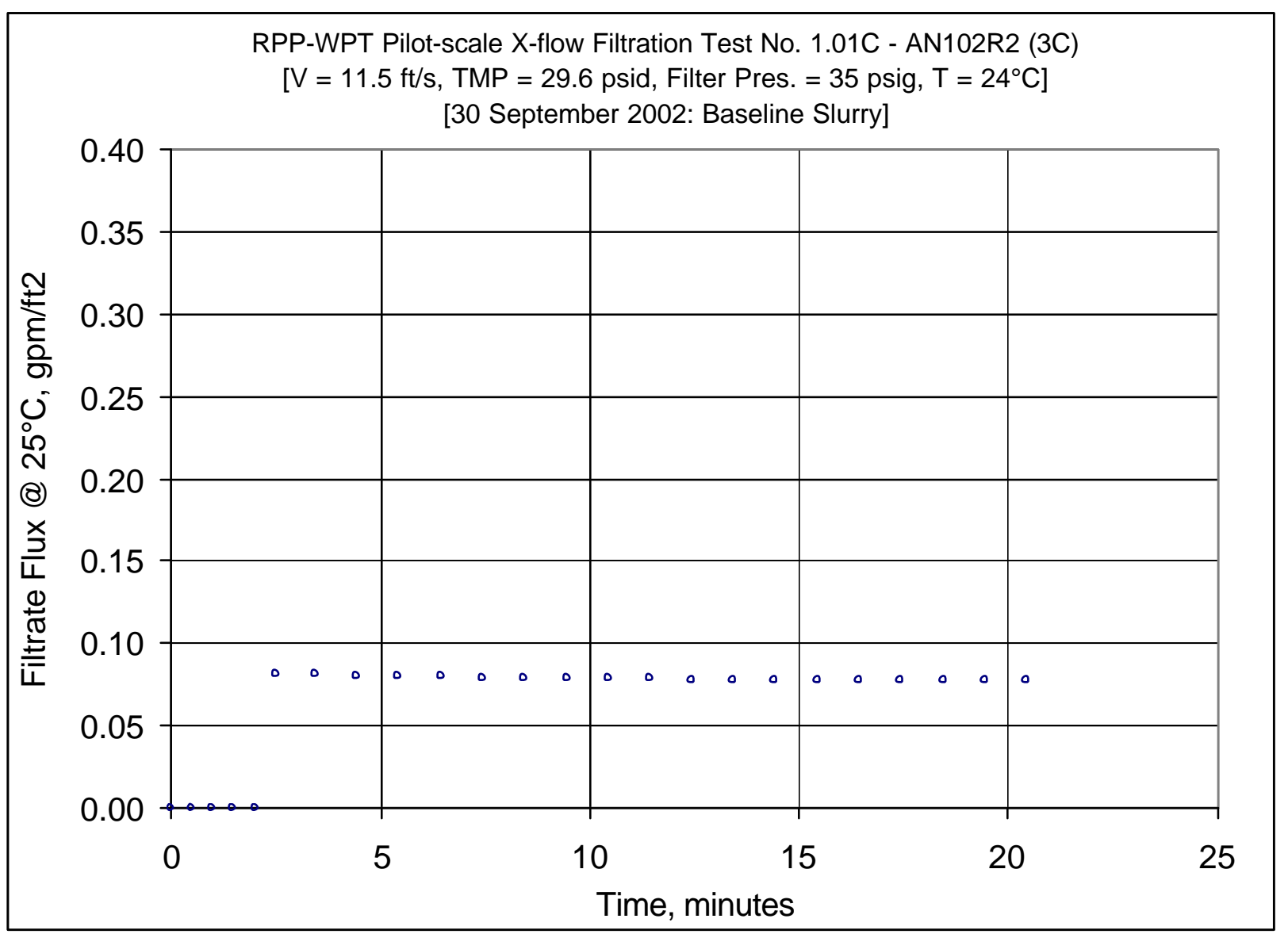

Figure B21: Run 1.01C - Baseline Slurry - Before Using Slurry AN-102R2 Batch 3C 
WSRC-TR-2003-00204, REV. 0

SRT-RPP-2003-00087, REV. 0

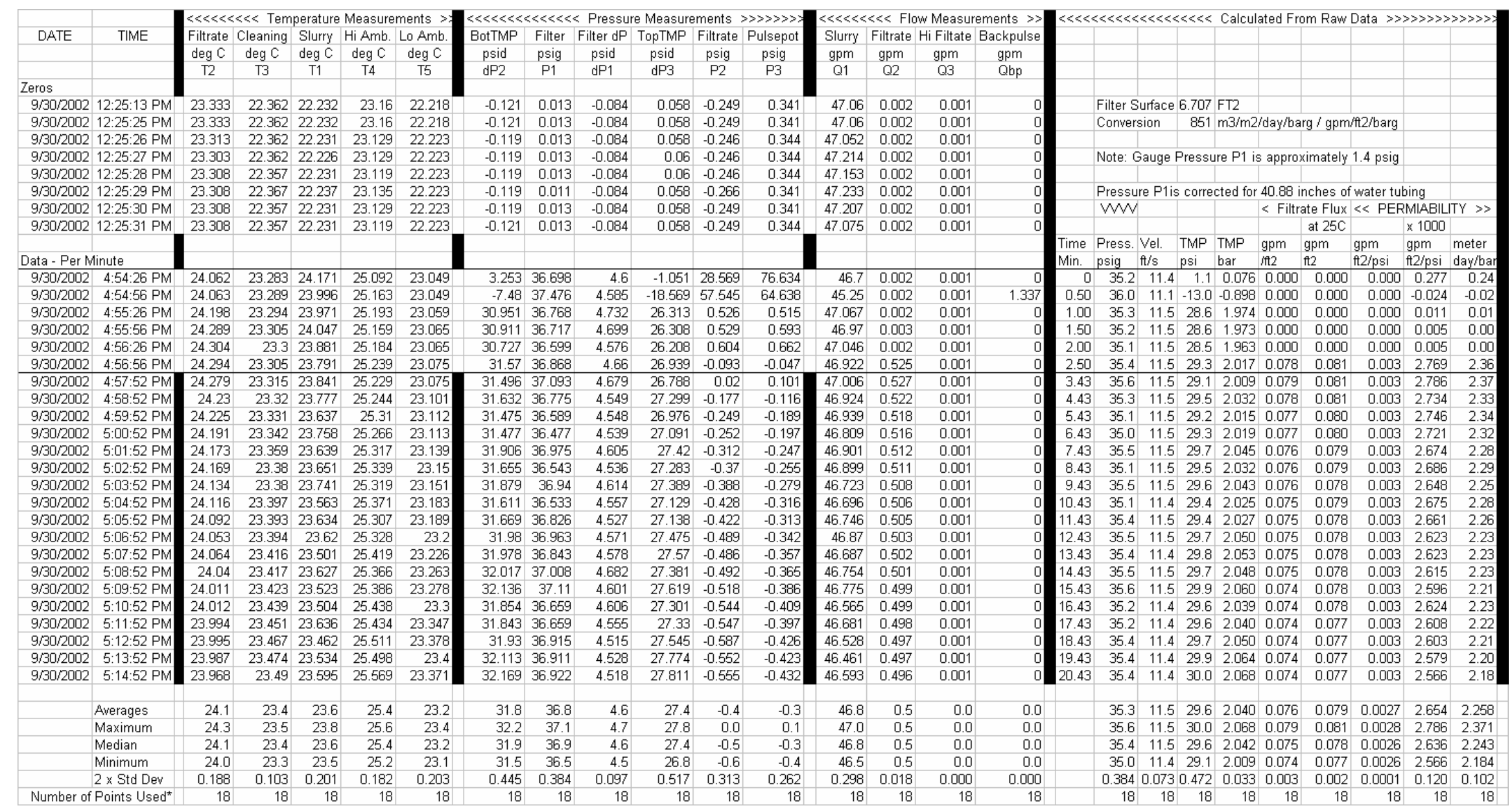

Figure B22: Run 1.01C - Baseline Slurry - Before Using Slurry AN-102R2 Batch 3C - Raw \& Calculated Data 
WSRC-TR-2003-00204, REV. 0

SRT-RPP-2003-00087, REV. 0

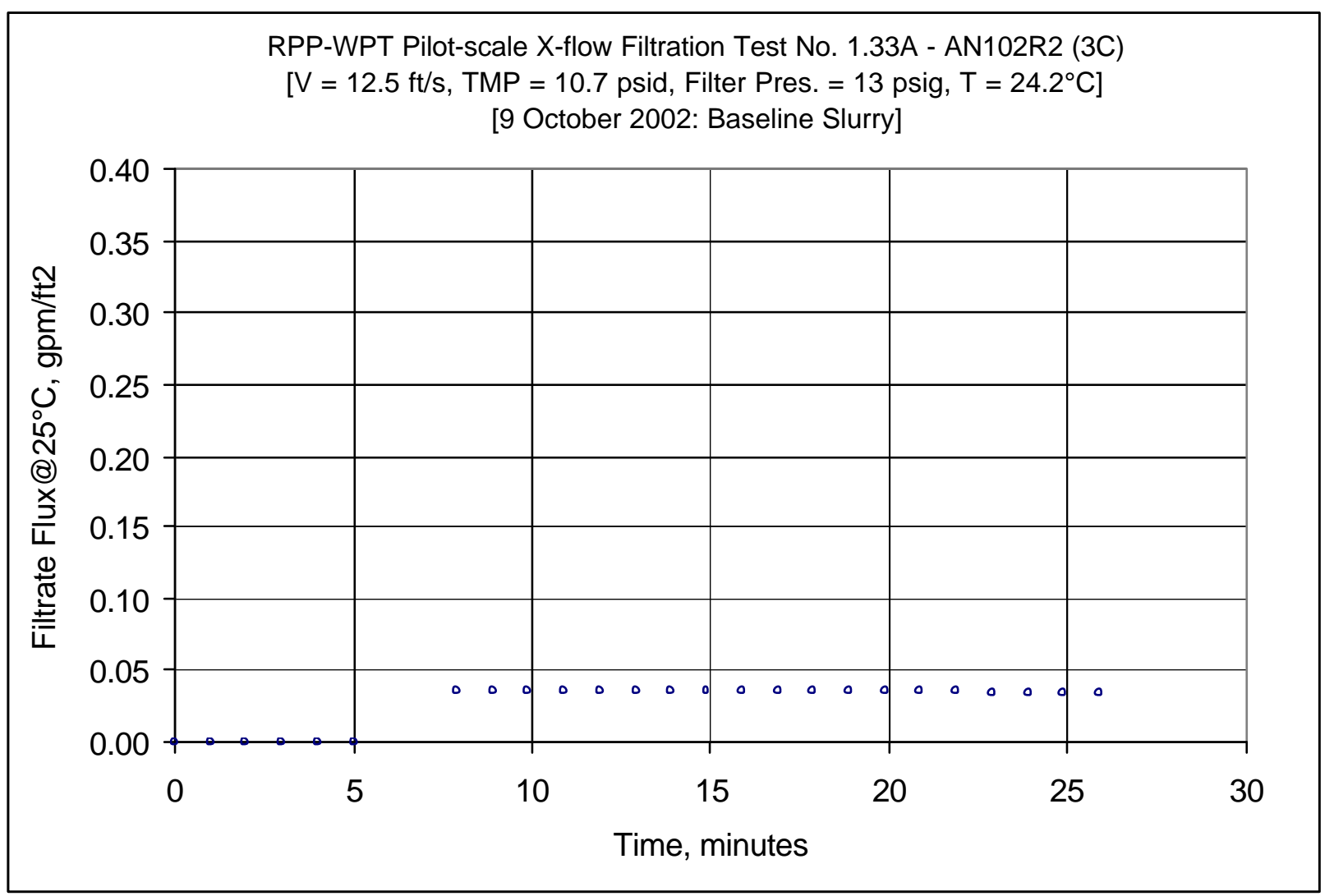

Figure B23: Run 1.33A - Baseline Slurry - After AN-102R2 Batch 3C and Before Batch 3B 
WSRC-TR-2003-00204, REV. 0

SRT-RPP-2003-00087, REV. 0

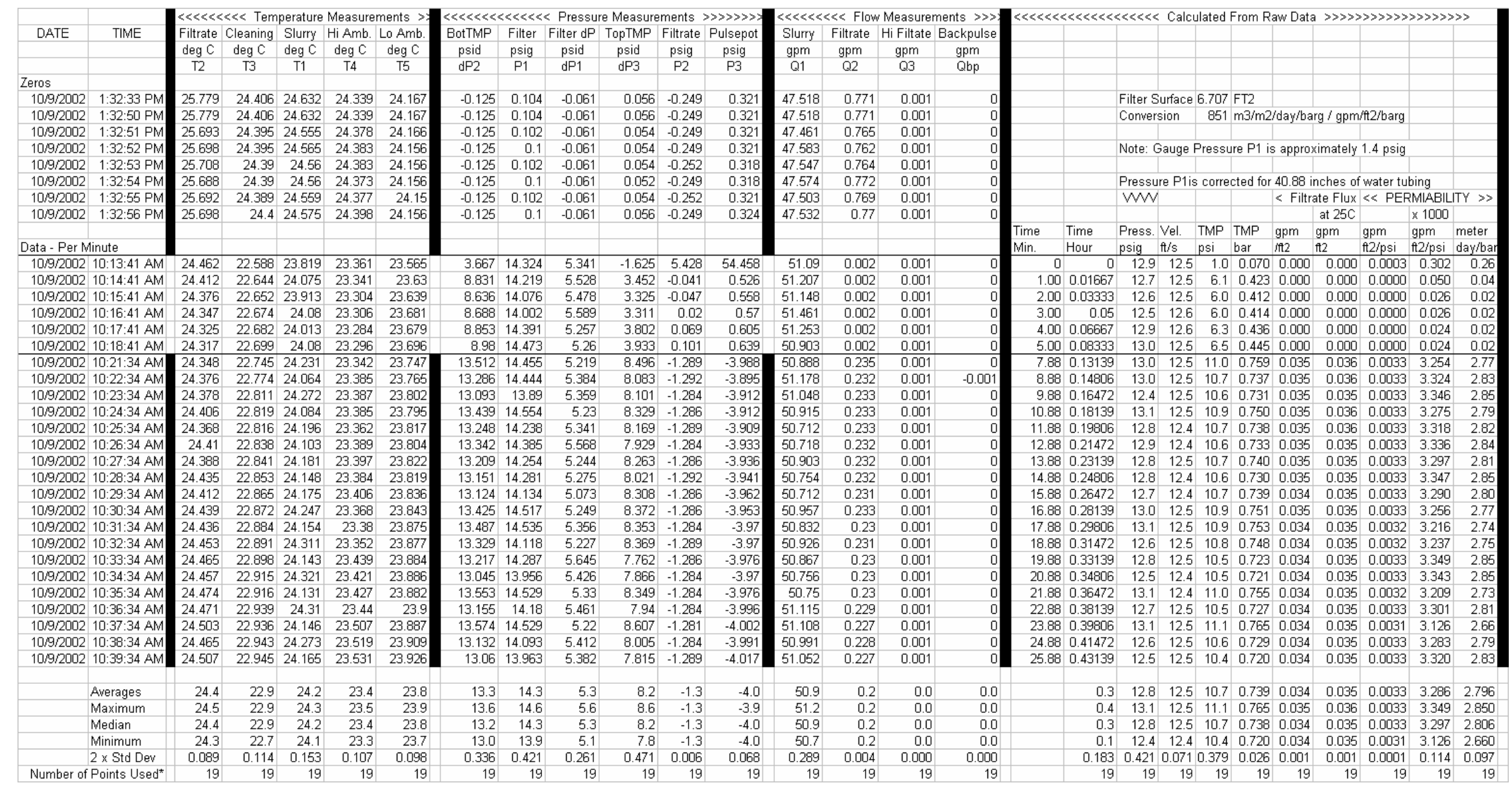

Figure B24: Run 1.33A - Baseline Slurry - After AN-102R2 Batch 3C and Before Batch 3B - Raw \& Calculated Data 
WSRC-TR-2003-00204, REV. 0

SRT-RPP-2003-00087, REV. 0

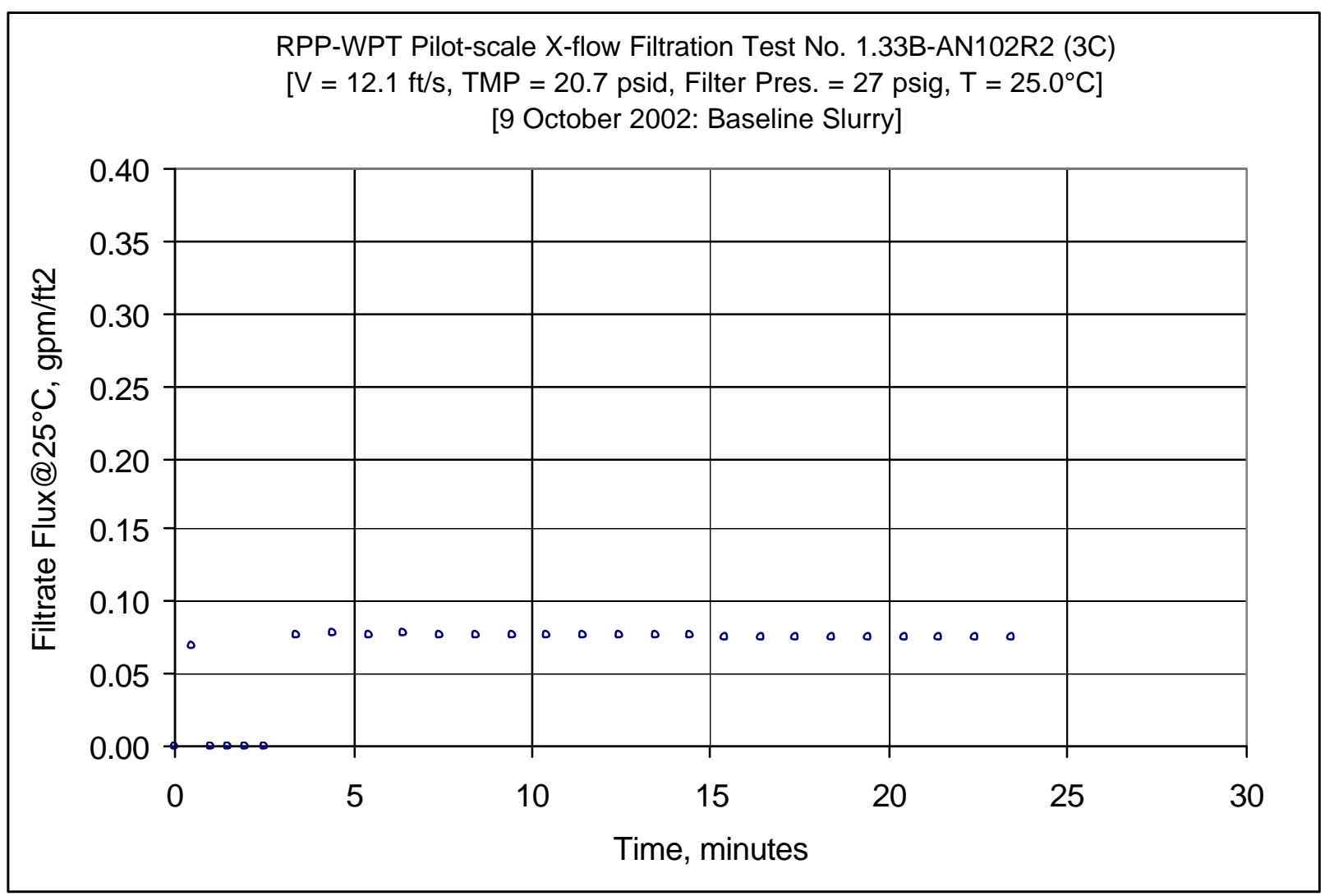

Figure B25: Run 1.33B - Baseline Slurry - After AN-102R2 Batch 3C and Before Batch 3B 
WSRC-TR-2003-00204, REV. 0

SRT-RPP-2003-00087, REV. 0

\begin{tabular}{|c|c|c|c|c|c|c|c|c|c|c|c|c|c|c|c|c|c|c|c|c|c|c|c|c|c|c|c|}
\hline \multirow{2}{*}{ DATE } & \multirow{2}{*}{ TIME } & & \multicolumn{6}{|c|}{ BotTMP $\quad$ Filter Filter dP TopTMP Filtrate } & \multicolumn{4}{|c|}{ 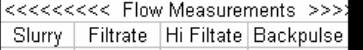 } & \multicolumn{11}{|c|}{$\gg \gg \gg$} \\
\hline & & $\operatorname{deg} C$ & $\operatorname{deg} C$ & $\operatorname{deg} \mathrm{C}$ & $\operatorname{deg} C$ & $\operatorname{deg} C$ & psid & psig & psid & psid & psig & psig & $\mathrm{gpm}$ & gpm & & $\mathrm{gpm}$ & & & & & & & & & & & \\
\hline & & & $T^{2}$ & & $\mathrm{~T} 4$ & & $\mathrm{dP} 2$ & $\mathrm{P}_{1}$ & $\mathrm{dP} 1$ & $\mathrm{dP3}$ & $0^{\circ}$ & 83 & Q1 & Q2 & 3 & & & & & & & & & & & & \\
\hline \multicolumn{28}{|l|}{ Zeros } \\
\hline 10/9/2002 & 1:32:33 PM & 25.779 & 24.406 & 24.632 & 24.339 & 24.167 & -0.125 & 0.104 & -0.061 & 0.056 & -0.249 & 0.321 & 47.518 & 0.771 & 0.001 & 0 & & & \multicolumn{4}{|c|}{ Filter Surface 6.707 FT2 } & \multirow{2}{*}{\multicolumn{3}{|c|}{ 2/day/barg / gpm/tit2/barg }} & & \\
\hline 10/9/2002 & 1:32:50 PM & 25.779 & 24.406 & 24.632 & 24.339 & 24.167 & -0.125 & 0.104 & -0.061 & 0.056 & -0.249 & 0.321 & 47.518 & 0.771 & 0.001 & & & & \multirow{2}{*}{\multicolumn{3}{|c|}{\begin{tabular}{l|l} 
Conversion 85 \\
\end{tabular}}} & $\mathrm{~m} 3 / \mathrm{m} 2 / \mathrm{d}$ & & & & & \\
\hline 10/9/2002 & 1:32:51 PM & 25.693 & 24.395 & 24.555 & 24.378 & 24.166 & -0.125 & 0.102 & -0.061 & 0.054 & -0.249 & 0.321 & 47.461 & 0.765 & 0.001 & & & & & & & & & & & & \\
\hline 10/9/2002 & 1:32:52 PM & 25.698 & 24.395 & 24.565 & 24.383 & 24.156 & -0.125 & 0.1 & -0.061 & 0.054 & -0.249 & 0.321 & 47.583 & 0.762 & 0.001 & 0 & & & \multicolumn{8}{|c|}{ Note: Gauge Pressure P1 } & \\
\hline 10/9/2002 & 1:32:53 PM & 25.708 & 24.39 & 24.56 & 24.383 & 24.156 & -0.125 & 0.102 & -0.061 & 0.054 & -0.252 & 0.318 & 47.547 & 0.764 & 0.001 & & & & & & & & & & & & \\
\hline $10 / 9 / 2002$ & 1:32:54 PM & 25.688 & 24.39 & 24.56 & 24.373 & 24.156 & -0.125 & 0.1 & -0.061 & 0.052 & -0.249 & 0.318 & 47.574 & 0.772 & 0.001 & 0 & & & \multicolumn{8}{|c|}{ Pressure $P$ lis corrected for 40.88 inches of water tubing } & \\
\hline 10/9/2002 & 1:32:55 PM & 25.692 & 24.389 & 24.559 & 24.377 & 24.15 & -0.125 & 0.102 & -0.061 & 0.054 & -0.252 & 0.321 & 47.503 & 0.769 & 0.001 & 0 & & & WW & & & & $<$ Filtrat & date $\Gamma$ IOR 8 & & & ITY \\
\hline 10/9/2002 & 1:32:56 PM & 25.698 & 24.4 & 24.575 & 24.398 & 24.156 & -0.125 & 0.1 & -0.061 & 0.056 & -0.249 & 0.324 & 47.532 & 0.77 & 0.001 & 0 & & & & & & & & at $25 \mathrm{C}$ & & $\times 1000$ & \\
\hline & & & & & & & & & & & & & & & & & ne & Time & Press. & Vel. & TMP & TMP & $\mathrm{gpm}$ & gpm & $\mathrm{gpm}$ & $\mathrm{gnm}$ & mete \\
\hline Data - Per M & Minute & & & & & & & & & & & & & & & & n. & Hour & & $\mathrm{f} / \mathrm{s}$ & $\mathrm{psi}$ & bar & & & $\mathrm{fl} 2 / \mathrm{psi}$ & $\mathrm{ft2} / \mathrm{psi}$ & \\
\hline 10/9/2002 & 11:11:41 AM & 25.154 & 23.402 & 24.957 & 24.104 & 24.237 & 3.41 & 28.03 & 5.264 & -1.903 & 19.38 & 8.255 & 50.443 & 0.002 & 0.00 & & & & 6 & 12.4 & 0.8 & 0.052 & 0.000 & 0.000 & & & \\
\hline $10 / 9 / 2002$ & 11:12:11 AM & 25.059 & 23.423 & 25.068 & 24.079 & 24.238 & 3.794 & 28.02 & 5.257 & -1.354 & 19.068 & 41.613 & 50.263 & 0.47 & 0. & & 0.50 & 0.00833 & 26.5 & \begin{tabular}{|l|l|} 
& 12.3 \\
\end{tabular} & 1.2 & 0.084 & 0.070 & 0.070 & 0.0573 & 357.327 & 48.79 \\
\hline $10 / 9 / 2002$ & 11:12:41 AM & 25.201 & 23.42 & 24.889 & 24.071 & 24.255 & 16.026 & 28.007 & 5.247 & 10.859 & 6.682 & 0.61 & 50.704 & 0.002 & 0.001 & 0 & 1.00 & 0.01667 & 26.5 & 512.4 & 13.4 & 0.927 & 0.000 & 0.000 & & 011 & 1001 \\
\hline $10 / 9 / 2002$ & 11:13:11 AM & 25.252 & 23.431 & 24.986 & 24.102 & 24.266 & 22.045 & 28.096 & 5.212 & 17.066 & 0.592 & 0.671 & 50.89 & 102 & & & 1.50 & 0.025 & 26.6 & 12.5 & 19.6 & 1.348 & 0.000 & 0.000 & 0.0000 & 008 & \\
\hline $10 / 9 / 2002$ & 11:13:41 AM & 25.278 & 23.432 & 25.107 & 24.073 & 24.262 & 22.138 & 28.105 & 5.278 & 17.053 & 0.523 & 0.654 & 50.628 & 12 & 0.001 & & 2.00 & 0.03333 & 26.6 & 12.4 & 19.6 & 1.351 & 0.000 & 0.000 & 0 & 0.008 & \\
\hline $10 / 9 / 2002$ & 11:14:11 AM & 25.284 & 23.433 & 24.902 & 24.073 & 24.267 & 22.19 & 27.833 & 5.2 & 17.256 & 0.271 & 0.356 & 50.899 & 0.533 & 0.0 & & 2.50 & 0.04167 & 26.4 & 12.5 & 19.7 & 1.360 & 0.000 & 0.000 & 0 & 0.008 & \\
\hline $10 / 9 / 2002$ & & 25.3 & 23.448 & 25.053 & 24.104 & 24.283 & 22.596 & 28.096 & & 17.733 & & 0.107 & \begin{tabular}{|l|l|}
49.657 \\
\end{tabular} & 0.52 & & & 3.42 & & 26.6 & 12.2 & 20.2 & 1.390 & \begin{tabular}{|l|}
0.078 \\
\end{tabular} & 0.077 & & & 3.27 \\
\hline $10 / 9$ & 11:1 & 25.316 & 23.455 & 24.864 & 24.091 & 24.295 & 22.768 & 28.227 & 5.0 & 17. & -0. & 0.112 & 49.695 & 0.522 & & & 4.42 & & 26.8 & 12.2 & 20.4 & 1.404 & & & & & \\
\hline $10 / 9 / 2002$ & 11:17:06 AM & 25.338 & 23.482 & 25.087 & 24.138 & 24.317 & 22.89 & 28.297 & 5.099 & 18.024 & -0.049 & 0.098 & 49.716 & 0.521 & 0. & 0 & 5.42 & 20.09028 & 26.8 & 32.2 & 20.5 & 1.410 & 0.078 & 0.077 & 0.0038 & 3.788 & 3.22 \\
\hline 10/9/2002 & 11:18:06 AM & 25.345 & 23.499 & 24.953 & 24.2 & 24.339 & 23.047 & 28.504 & & 18.194 & -0.047 & 0.069 & 49.653 & 0.52 & & & 6.42 & 20.10694 & 27.0 & 12.2 & 20.6 & 1.422 & 0.078 & 8 & 0 & 3.765 & \begin{tabular}{|l|}
5.20 \\
\end{tabular} \\
\hline $10 / 9 / 2002$ & 11:19:06 AM & 25.372 & 23.531 & 24.955 & 24.167 & 24.34 & & 28.071 & & & & & 49.615 & & & & 7.42 & 20.12361 & 26.6 & 12.2 & 20.4 & 1.405 & 0.077 & & & & \\
\hline $10 / 9 / 2002$ & 11:20:06 AM & 25.343 & 23.547 & 25.061 & 24.223 & 24.3 & 23. & 28.415 & & & & & & & & & 8.4 & & 26.9 & 12.2 & 20.6 & 1.424 & 0.077 & & & & \\
\hline $10 / 9 /$ & $11: 2$ & 25.375 & 23.564 & 24.913 & 24.2 & & & 28.562 & & & & & & & & & 9.42 & & 27.1 & & & & & & & & \\
\hline & 11:22:06 AM & 25.367 & 23.586 & 25.1 & 24.2 & 24. & & 28.3 & & & & & 7. & & & & 10.42 & & 9 & 12.1 & 20.5 & & & & & & \\
\hline $10 / 9 / 2002$ & 11:23:06 AM & 25.353 & 23.582 & 24.941 & 24.253 & 24.397 & 23.124 & 28.4 & 5. & 18 & -0.1 & -0 & \begin{tabular}{|l|l|}
49.517 \\
\end{tabular} & & & & 11.42 & 20.19028 & 26.9 & 12.1 & 20.7 & 1.428 & 0.076 & 6 & 0.0037 & 3.685 & 3.14 \\
\hline 10/9. & 11:24:06 AM & 25.374 & 23.598 & 24.957 & 24.244 & 24.3 & & 28.208 & & & -0 & & 49 & & & & 12 & 20.2 & 26.7 & 12.1 & 20.5 & 1.415 & & & & & \\
\hline $10 / 9 / 2002$ & 11:25:06 AM & 25.371 & & 25.069 & 24. & & & 28. & & & & & & & & & & & 27.0 & 12 & & & & & & & \\
\hline & 11:26:06 AM & 25.402 & 23.651 & 24.935 & 24. & & & 28. & & & & & & & & & & & 27.2 & & & & & & & & \\
\hline $10 / 9$ & $11: 27: 0$ & 25.373 & 23.638 & 25.091 & 24.2 & 24. & & 28 & & & & & 49 & & & & 15.42 & & 27.1 & 12.1 & 21.0 & 1.447 & & 0. & & $\begin{array}{l}6.622 \\
\end{array}$ & 3.08 \\
\hline $10 / 9$ & 11:28:06 AM & 25.384 & 23.663 & 24.967 & 24. & 24. & & 28.2 & & & & & 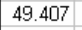 & & & & 16 & & 8 & 12.1 & 20.8 & 1.436 & & & & & \\
\hline $10 / 9 /$ & 11:29:06 AM & 25.406 & 23.686 & 24.989 & 24.286 & 24 & & 28.3 & & & -0 & & 49.3 & & & & 17 & 20.2 & 9 & 312 & 20.8 & 1.432 & & & 37 & 7 & \\
\hline & $11: 3$ & 25.397 & & 25 & 24. & & & & & & & & & & & & & & & & & & & & & & \\
\hline & $11: 31: 06$ & 25.409 & & 24. & & & & & & & & & \begin{tabular}{|l|l|}
49.107 \\
\end{tabular} & & & & & & 27.0 & 12.0 & & 1.445 & & & & & 05 \\
\hline & 11:32:06 A & 25.396 & 23.731 & 25.099 & 24.316 & & 23.194 & 28.328 & & & -0.2 & & \begin{tabular}{|l|l|}
48.77 \\
\end{tabular} & 0.5 & & & 20.4 & 20.34028 & 26.9 & 12.0 & 20.9 & 1.443 & & 0.076 & 0.0036 & 6. 3.608 & \\
\hline $10 / 9$ & $11: 33: 06 \mathrm{Al}$ & 25.393 & 23.748 & 24.966 & 24.343 & 24.5 & 23.113 & 28.256 & 4.8 & 18.466 & -0.2 & -0. & 48.703 & 0. & & 001 & 21.42 & 20.35694 & 26.8 & $\begin{array}{ll}31.9 \\
\end{array}$ & 20.8 & 1.433 & 75 & 6 & 36 & $\begin{array}{l}6.632 \\
\end{array}$ & 99 \\
\hline & AM & 25.41 & 23.754 & 24.973 & 24.32 & 24.5 & 23.132 & 28.2 & 4.82 & 18 & -0.2 & -0 & 48.7 & & & & 22. & & 6.8 & 92 & 20.9 & 1.438 & & & & & \\
\hline 10/9/2002 & 11:35:06 AM & 25.387 & 23.772 & 25.06 & 24.422 & 24.5 & 23.352 & 28.562 & 4.000 & 18.644 & -0.266 & -0.1 & 48.524 & 0.50 & 0.001 & & 23.42 & 3028 & 27.1 & 11.9 & 21.0 & 1.448 & 0.075 & 0.075 & 0.0036 & $\begin{array}{l}6.587 \\
\end{array}$ & \\
\hline & & & & & & & & & & & & & & & & & & & & & & & & & & & \\
\hline & Maximum & 25.4 & & 25.1 & 24.4 & 2 & & & 5. & 18.7 & 0 & 0 & 45 & 0.5 & 0. & U. & & 0.4 & 27.2 & 12.2 & 21.0 & 1.448 & 0.078 & 0.078 & 0.0038 & $\begin{array}{l}3.039 \\
3.839\end{array}$ & 3.267 \\
\hline & & 25. & & & & & & 28 & 5. & 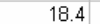 & -0 & & & 0 & 0. & o. & & 0.2 & 26.9 & 12.12 & 20.8 & 1.432 & 0.076 & 76 & 0.0037 & 600 & 3.115 \\
\hline & & 25 . & & & & & & & & & & & & & & & & 0.1 & 26.6 & 110 & 20.2 & 139000 & & & & & $=00$ \\
\hline & & 0.059 & 0.198 & 0.146 & 0.170 & 15 & 0.393 & 0.313 & $0.1:$ & 0.523 & & 0.19 & 0.70 & & 0.00 & 0 & & 0.202 & 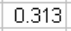 & 0.1740 & 0.451 & .031 & 0.002 & 002 & 0.0002 & 153 & 3 \\
\hline of & Points Uses & 21 & 21 & 21 & 21 & 21 & 21 & 21 & 21 & 21 & 21 & 21 & 21 & 211 & 21 & 21 & & 21 & 21 & 21 & 21 & 21 & 21 & 21 & 21 & 21 & 21 \\
\hline
\end{tabular}

Figure B26: Run 1.33B - Baseline Slurry - After AN-102R2 Batch 3C and Before Batch 3B - Raw \& Calculated Data 
WSRC-TR-2003-00204, REV. 0

SRT-RPP-2003-00087, REV. 0

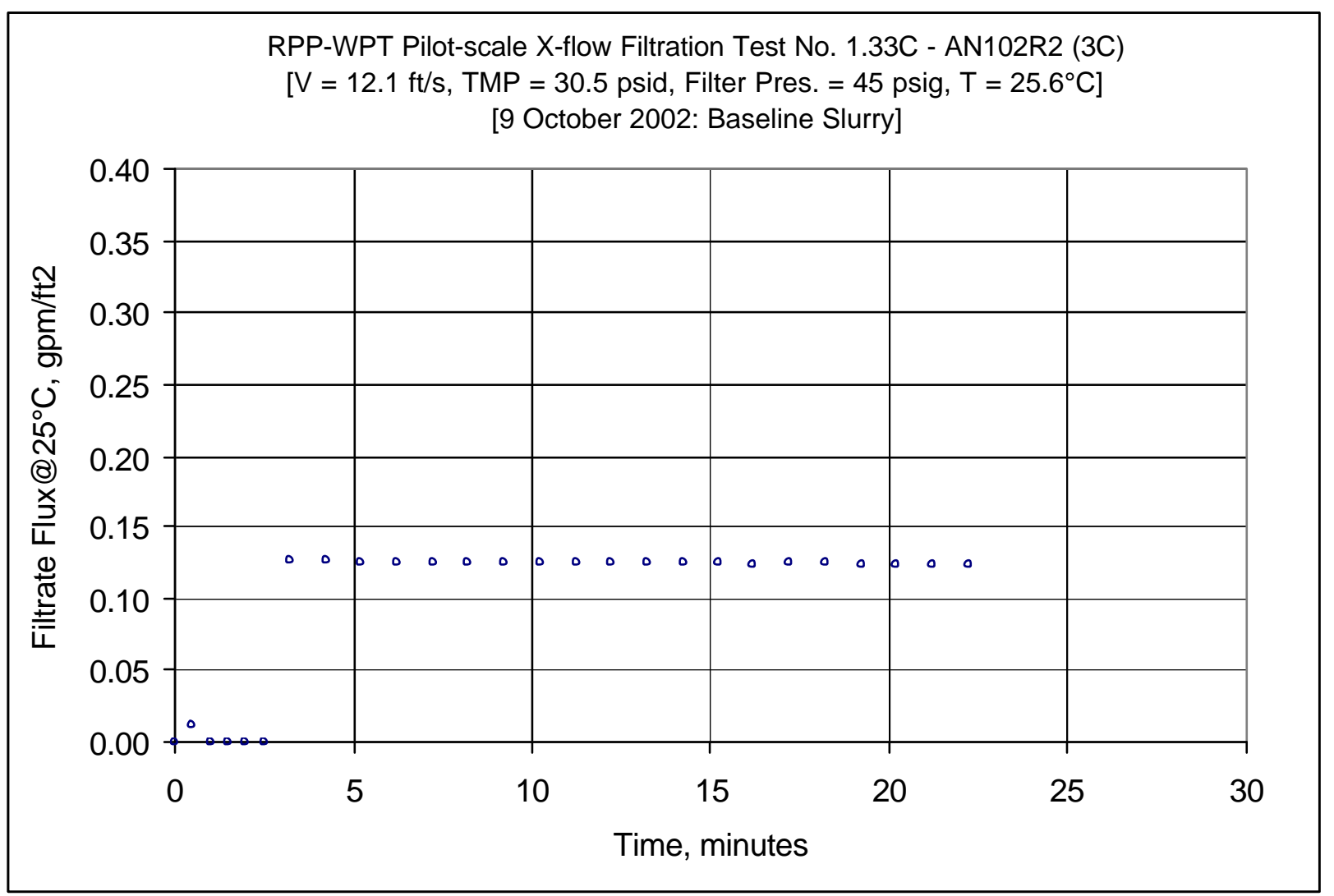

Figure B27: Run 1.33C - Baseline Slurry - After AN-102R2 Batch 3C and Before Batch 3B 
WSRC-TR-2003-00204, REV. 0

SRT-RPP-2003-00087, REV. 0

\begin{tabular}{|c|c|c|c|c|c|c|c|c|c|c|c|c|c|c|c|c|c|c|c|c|c|c|c|c|c|c|c|}
\hline \multirow{2}{*}{ DATE } & \multirow{2}{*}{ TIME } & & \multicolumn{6}{|c|}{ 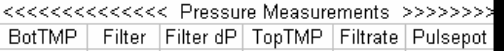 } & \multicolumn{4}{|c|}{ 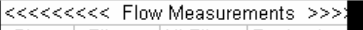 } & \multicolumn{11}{|c|}{ 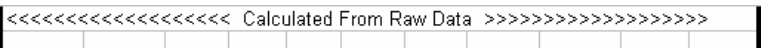 } \\
\hline & & $\operatorname{deg} C$ & $\operatorname{deg} C$ & $\operatorname{deg} \mathrm{C}$ & $\operatorname{deg} C$ & $\operatorname{deg} C$ & psid & psig & psid & psid & psig & psig & $\mathrm{gpm}$ & $\mathrm{gpm}$ & $\mathrm{gpm}$ & $\mathrm{gpm}$ & & & & & & & & & & & \\
\hline & & & $T 3$ & $\mathrm{~T} 1$ & T4 & & $\mathrm{dP} 2$ & $\mathrm{P} 1$ & $\mathrm{dP1}$ & $\mathrm{dP} 3$ & P2 & P3 & Q1 & Q2 & Q3 & & & & & & & & & & & & \\
\hline \multicolumn{28}{|l|}{ Zeros } \\
\hline 10/9/2002 & 1:32:33 PM & 25.779 & 24.406 & 24.632 & 24.339 & 24.167 & -0.125 & 0.104 & -0.061 & 0.056 & -0.249 & 0.321 & 47.518 & 0.771 & 0.001 & & & & \multicolumn{4}{|c|}{ Filter Surface 6.707 FT2 } & & & & & \\
\hline 10/9/2002 & 1:32:50 PM & 25.779 & 24.406 & 24.632 & 24.339 & 24.167 & -0.125 & 0.104 & -0.061 & 0.056 & -0.249 & 0.321 & 47.518 & 0.771 & 0.001 & & & & \multicolumn{2}{|c|}{ Conversion } & 851 & \multirow{2}{*}{\multicolumn{4}{|c|}{$1 \mathrm{~m} 3 / \mathrm{m} 2 / \mathrm{day} / \mathrm{barg} / \mathrm{gpm} / \mathrm{ft} 2 / \mathrm{barg}$}} & & \\
\hline 10/9/2002 & 1:32:51 PM & 25.693 & 24.395 & 24.555 & 24.378 & 24.166 & -0.125 & 0.102 & -0.061 & 0.054 & -0.249 & 0.321 & 47.461 & 0.765 & 0.001 & & & & & & & & & & & & \\
\hline 10/9/2002 & 1:32:52 PM & 25.698 & 24.395 & 24.565 & 24.383 & 24.156 & -0.125 & 0.1 & -0.061 & 0.054 & -0.249 & 0.321 & 47.583 & 0.762 & 0.001 & & & & \multirow{2}{*}{\multicolumn{2}{|c|}{ Note: Gaug }} & Pressu & re $\mathrm{P} 1$ is & is approx & oximately & 1.4 psig & & \\
\hline $10 / 9 / 2002$ & 1:32:53 PM & 25.708 & 24.39 & 24.56 & 24.383 & 24.156 & -0.125 & 0.102 & -0.061 & 0.054 & -0.252 & 0.318 & 47.547 & 0.764 & 0.001 & & & & & & & & & & & & \\
\hline 10/9/2002 & 1:32:54 PM & 25.688 & 24.39 & 24.56 & 24.373 & 24.156 & -0.125 & 0.1 & -0.061 & 0.052 & -0.249 & 0.318 & 47.574 & 0.772 & 0.001 & & & & \multicolumn{8}{|c|}{ Pressure $\mathrm{P}$ 1is con } & \\
\hline 10/9/2002 & 1:32:55 PM & 25.692 & 24.389 & 24.559 & 24.377 & 24.15 & -0.125 & 0.102 & -0.061 & 0.054 & -0.252 & 0.321 & 47.503 & 0.769 & 0.001 & & & & WW & & & & & trate Flux & $x \ll P E$ & RMIABILIT & ITY $\gg$ \\
\hline 10/9/2002 & 1:32:56 PM & 25.698 & 24.4 & 24.575 & 24.398 & 24.156 & -0.125 & 0.1 & -0.061 & 0.056 & -0.249 & 0.324 & 47.532 & 0.77 & 0.001 & 0 & & & & & & & & at $25 \mathrm{C}$ & & $\times 1000$ & \\
\hline & & & & & & & & & & & & & & & & & Time & Time & Press. & Vel. & TMP & TMP & $\mathrm{gpm}$ & $\mathrm{gpm}$ & $\mathrm{gpm}$ & $\mathrm{gpm}$ & $\mathrm{me}$ \\
\hline Data - Per M & Ainute & & & & & & & & & & & & & & & & & Hour & psig & $\mathrm{f} / \mathrm{s}$ & noi & bar & ift2 & itt2 & $\mathrm{ft} 2 / \mathrm{psi}$ & $\mathrm{ft} 2 / \mathrm{psi}$ & ay/l \\
\hline $10 / 9 / 2002$ & 11:43:02 AM & 26.022 & 23.871 & 25.725 & 24.803 & 24.636 & 3.166 & 46.446 & 4.975 & -1.758 & 38.275 & 87.061 & 49.439 & 01 & 0.001 & & 0 & sor & 45.0 & 12.1 & 0.7 & 70.049 & 0.000 & 0.000 & 1) 0.0002 & 20.208 & \\
\hline 10/9/2002 & 11:43:32 AM & 25.862 & 23.892 & 25.891 & 24.843 & 24.631 & 3.742 & 46.752 & 4.899 & -1.101 & 37.968 & 54.996 & 49.409 & & & & 0.50 & 0.00833 & \begin{tabular}{|l|l|}
3 & 45.3 \\
\end{tabular} & 12.1 & 1.3 & 30.091 & 0.011 & $\begin{array}{ll}1 \quad 0.011 \\
\end{array}$ & $\begin{array}{ll}1 & 0.0085 \\
\end{array}$ & $\begin{array}{ll}5 & 8.479 \\
\end{array}$ & 8 \\
\hline $10 / 9 /$ & & 25.858 & 23.893 & 25.731 & 24. & 24.6 & 9.823 & 46.8 & 497 & 4.92 & 32.251 & 19.385 & 49.2 & & & & 1.00 & 0.01667 & $\begin{array}{ll}7 & 45.4 \\
\end{array}$ & 12.1 & 7.4 & 608 & & & & & 0.02 \\
\hline 10/9/2002 & 11:44:32 AM & 26.258 & 23.918 & 25.716 & 24.884 & 24.657 & 30.622 & 46.628 & 4.968 & 25.707 & 10.931 & 4.383 & 49 . & 02 & & & 1.50 & 0.025 & \begin{tabular}{|l|l|}
5 & 45.2 \\
\end{tabular} & 12.2 & 28.2 & 21.942 & 000 & 00 & 0 . 0 . & 005 & 0.00 \\
\hline 10/9/2002 & 11:45:02 AM & 26.295 & 23.919 & 25.838 & 24.866 & 24.669 & 37.852 & 46.014 & 4.978 & 33.117 & 2.91 & 1.656 & 49.969 & & & & 2.00 & 0.03333 & $\begin{array}{ll}3 & 44.5 \\
\end{array}$ & 12.2 & 35.5 & 52.447 & 0.000 & 00 & & 0.004 & \\
\hline $10 / 9 / 2002$ & 11:45:32 AM & 26.316 & 23.926 & 25.689 & 24.912 & 24.69 & 32.21 & 46.233 & 4.994 & 27.285 & 8.833 & 8.173 & 49. & 0.863 & 11 & & 2.50 & 0.04167 & $7 \quad 44.8$ & 12.2 & 29.7 & 72.051 & 0.000 & 0.000 & 0.0000 & 0.005 & \\
\hline 10/9/2002 & 11:46:15 AM & 26.287 & \begin{tabular}{|l|l|}
23.947 \\
\end{tabular} & 25.686 & 24.974 & 24.707 & 32.343 & 46.368 & & 27.369 & & & & 0.861 & & & 3.22 & 0.05361 & 144.9 & 12.2 & 29.9 & 92.058 & 0.128 & 26 & \begin{tabular}{|l|l|}
5 & 0.0042 \\
\end{tabular} & 24.218 & \\
\hline $10 / 9 /$ & 11:47:15 AM & 26.289 & 23.964 & 25.587 & 24.98 & 24.72 & 32.541 & 46.641 & & & & & & & & & 4.22 & & \begin{tabular}{|l|l|}
3 & 45.2 \\
\end{tabular} & 12.1 & 30.1 & 12.074 & & & & & \\
\hline & & 26.23 & 23.96 & 25.698 & 24.9 & & & 46.289 & & & & & & & & & 5.22 & & $\begin{array}{ll}4 & 44.8 \\
\end{array}$ & 12.1 & 30.0 & & & & & & 3.56 \\
\hline & & 26.221 & 23.981 & 25.539 & 25.0 & & & 46.4 & & & & & & & & & & & $\begin{array}{ll}1 & 45.0 \\
\end{array}$ & 12.1 & 30.1 & & & & & & \\
\hline $10 / 9 / 2002$ & 11:50:15 AM & 26.228 & 24.009 & 25.572 & 25.075 & 24.76 & 32.896 & 46.761 & 4.911 & 28.083 & 8 & 7.916 & 49. & & & & 7.22 & 0.12028 & \begin{tabular}{|l|l|}
3 & 45.3 \\
\end{tabular} & 12.1 & 30.5 & 52.102 & 0.127 & \begin{tabular}{l|l|}
7 & 0.125 \\
\end{tabular} & $\begin{array}{l}5 \quad 0.0041 \\
\end{array}$ & $\begin{array}{ll}1 & 4.114 \\
\end{array}$ & 50 \\
\hline $10 / 9 / 2$ & 11:51:15 AM & 26.19 & 24.02 & 25.683 & 25.116 & 24.78 & & 46.632 & & 28 & 8 & & & & & & 8.22 & 994 & $\begin{array}{l}4 \quad 45.2 \\
\end{array}$ & 12.1 & 30.5 & 52.103 & 0 & 5 & & 14.091 & \\
\hline $10 / 9 / 2002$ & 11:52:15 AM & 26.197 & 24.047 & 25.5 & & 24. & & 46.266 & & & & & & & & & 9. & & $\begin{array}{ll}1 & 44.8 \\
\end{array}$ & 12.1 & 30.3 & & & & & & \\
\hline $10 / 9 /$ & 11:53:15 AM & 26.193 & & 25.582 & & & & 47.5 & & & & & & & & & 10.22 & & & 12.0 & 30.8 & & & & & & \\
\hline & & 26.191 & 24. & 25.689 & 25 & & & 46.7 & & & & & & & & & 11.22 & & $\begin{array}{l}4 \quad 45.3 \\
\end{array}$ & 12.1 & 30.6 & & & & & & 3.46 \\
\hline & & 26.182 & 24.098 & 25.47 & 25 & & & 46 . & & & & & & & & & 12.22 & & $\begin{array}{ll}1 & 45.0 \\
\end{array}$ & 12.1 & 30.4 & & & & & & \\
\hline & 11:56:15 AM & 26.174 & 24.099 & 25.602 & 25 & & & 46 & & & & & & & & & 13 & & $\begin{array}{l}3 \\
3\end{array}$ & 12.1 & 7 & & & & & & \\
\hline 1019 & 11:57:15 AM & 66 & 24.131 & 25.609 & 25 & & & 46 & & & & & & & & & 14 & & 1 & 12.0 & 30.6 & & & & & & \\
\hline & $11: 5$ & & & 25. & & & & & & & & & & & & & 15. & & $\begin{array}{ll}1 & 45.0 \\
\end{array}$ & $\mid 12.1$ & & & & & & & \\
\hline & 11:59:15 & 26.169 & 24. & 25.647 & & & 32. & & & & & & & & & & & & \begin{tabular}{|l|l|}
3 & 45.1 \\
\end{tabular} & 12.1 & & & & & & & \\
\hline & $12: 0$ & 26.155 & 24.171 & 25.554 & 25.187 & & 33.097 & 46.566 & 4.815 & & & & & & & & 17.22 & & $\begin{array}{ll}4 & 45.1 \\
\end{array}$ & 12.0 & & & & $\begin{array}{l}6 \\
5\end{array}$ & 40.1 & & \\
\hline $10 / 9$ & & 26.183 & 24.199 & 25.497 & 25.22 & 24.9 & 33 & 46.659 & & & & & & & & & 18.22 & & $\begin{array}{lll}1 & 45.2 \\
1\end{array}$ & 12.1 & 30.8 & & & $\begin{array}{ll}6 & 0 . \\
0\end{array}$ & & 3 & 3 \\
\hline $10 / 9 /$ & $12: 0$ & 26.165 & 24.221 & 25.684 & 25.262 & & 32 & 46. & & & & & & & & & & & \begin{tabular}{l|l}
3 & 45.0 \\
\end{tabular} & 12.1 & 30.6 & & & & & & \\
\hline & $12: 0$ & 26.161 & 24. & & & & & & & & & & & & & & & & & & & & & & & & \\
\hline & & 26.1 & 24. & 25. & & 25 . & & & & & & 7. & & & & & 27. & & $\begin{array}{ll}1 & 45.1 \\
\end{array}$ & 12.1 & 30.6 & & & & & & a \\
\hline $10 / 9 / 2002$ & 12:05:15 PM & 26.16 & 24.271 & 25.683 & 25.247 & 25.039 & 33.113 & 46.49 & 4.851 & 28.456 & 8.162 & 7.568 & 49.202 & 0.843 & 0.001 & & 22.22 & 0.37028 & $\begin{array}{ll}3 & 45.0 \\
\end{array}$ & 12.1 & 30.8 & B 2.122 & 0.126 & \begin{tabular}{|l|l|}
6 & 0.123 \\
\end{tabular} & $\begin{array}{ll}3 & 0.0040 \\
\end{array}$ & 4.005 & 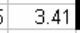 \\
\hline & & & & & & & & & & & & & & & & & & & & & & & & & & & \\
\hline & & & & & & & & & & & & & & & & & & & & 12.1 & 0.5 & 52 & & & & & \\
\hline & & & & & & & & & & & & & & & & & & & & & & & & & & & \\
\hline & & & & & & & & & & & & & & & & & & & & & & & & & & & \\
\hline & & & & & & & & & & & & & & & & & & 0.1 & .0 & & & & & & & 5 & \\
\hline & & 0.075 & 0.199 & 0.154 & 0.175 & 0.204 & 0.493 & 0.345 & 0.115 & 0.607 & 0.349 & 0.314 & 0.351 & 0.011 & 0.000 & 0.00 & & 0.192 & 20.345 & 0.0860 & 0.544 & 40.038 & 0.002 & $\begin{array}{l}20.001 \\
\end{array}$ & $\begin{array}{l}10.0001 \\
\end{array}$ & .117 & 0.099 \\
\hline Number of & Points Used & 20 & 20 & 20 & 20 & 20 & 20 & 20 & 20 & 20 & 20 & 20 & 20 & 20 & 20 & 20 & & 20 & 20 & 20 & 20 & 20 & 20 & 20 & 20 & 20 & 20 \\
\hline
\end{tabular}

Figure B28: Run 1.33C - Baseline Slurry - After AN-102R2 Batch 3C and Before Batch 3B - Raw \& Calculated Data 
WSRC-TR-2003-00204, REV. 0

SRT-RPP-2003-00087, REV. 0

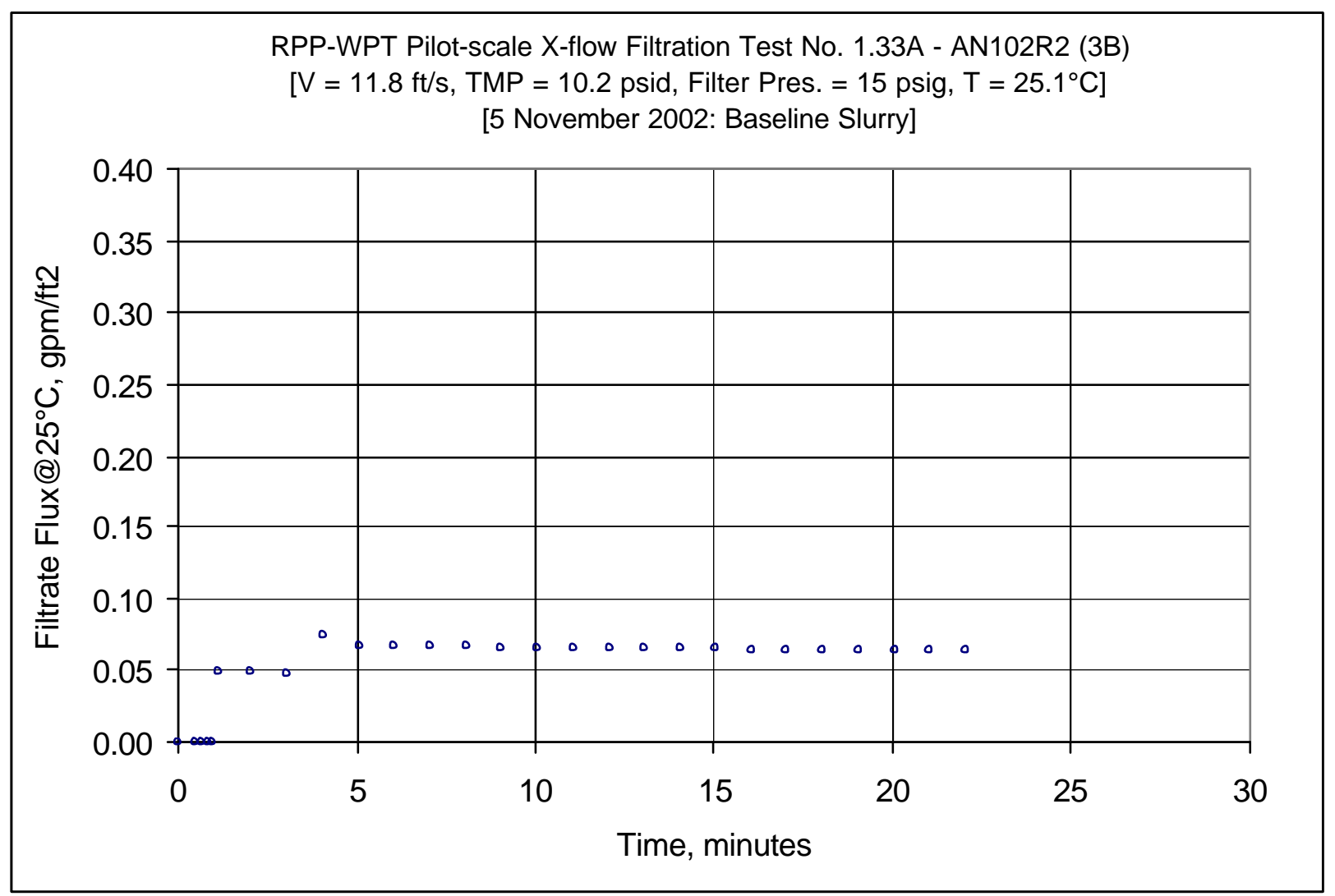

Figure B29: Run 1.33A - Baseline Slurry - After AN-102R2 Batch 3B and Before Batch 3A 
WSRC-TR-2003-00204, REV. 0

SRT-RPP-2003-00087, REV. 0

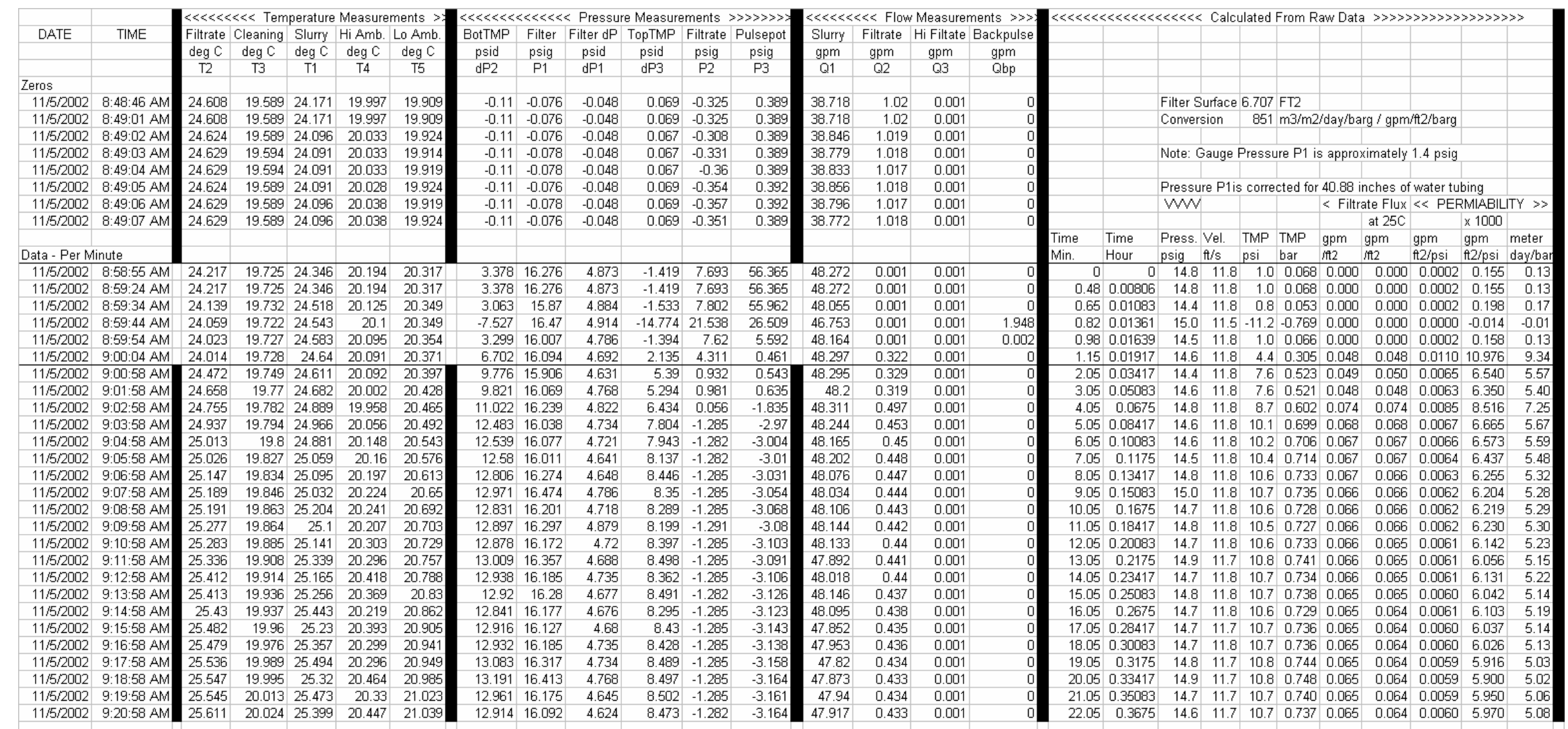

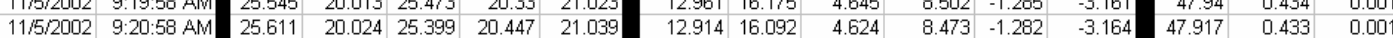

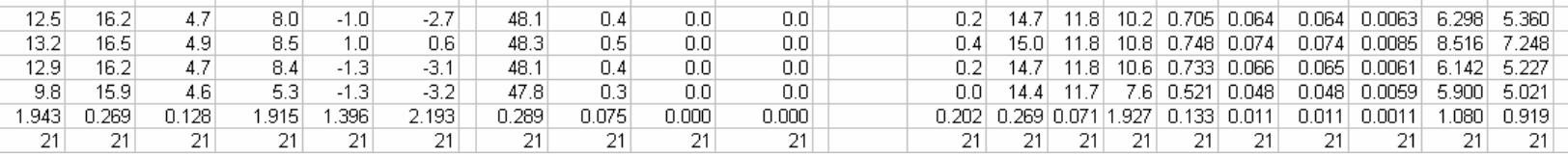

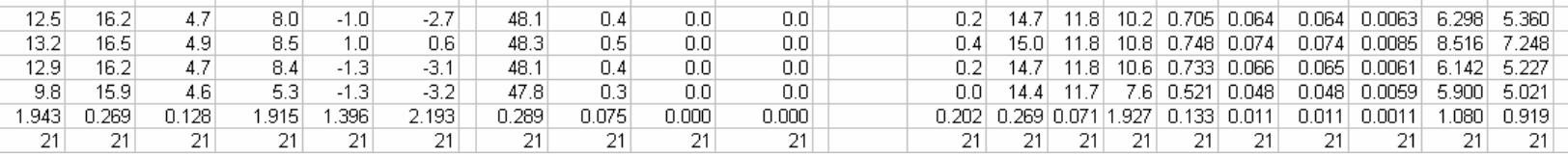

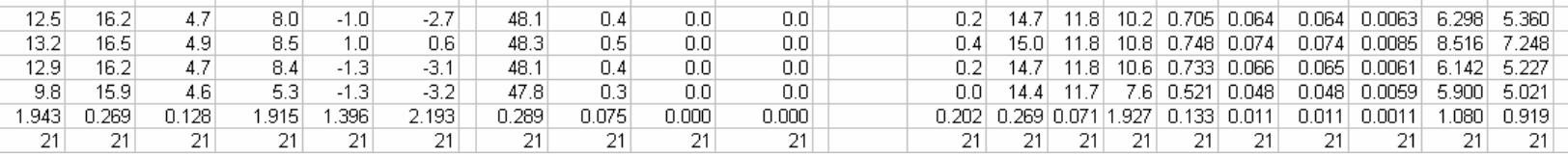

Minimum \begin{tabular}{|l|r|r|r|r|}
\hline 25.2 & 19.9 & 25.1 & 20.2 & 20.7 \\
\hline 25.6 & 20.0 & 25.5 & 20.5 & 21.0 \\
\hline 24.3 & 19.9 & 25.2 & 20.2 & 20.7 \\
\hline 24.5 & 19.7 & 24.6 & 20.0 & 20.4 \\
\hline
\end{tabular} \begin{tabular}{r|r|r|r|r|r|}
\hline $2 \times$ Std Dev & 0.617 & 0.166 & 0.480 & 0.275 & 0.393 \\
\hline Number of Points Used & 21 & 21 & 21 & 21 & 21 \\
\hline
\end{tabular}

Figure B30: Run 1.33A - Baseline Slurry - After AN-102R2 Batch 3B and Before Batch 3A - Raw \& Calculated Data 
WSRC-TR-2003-00204, REV. 0

SRT-RPP-2003-00087, REV. 0

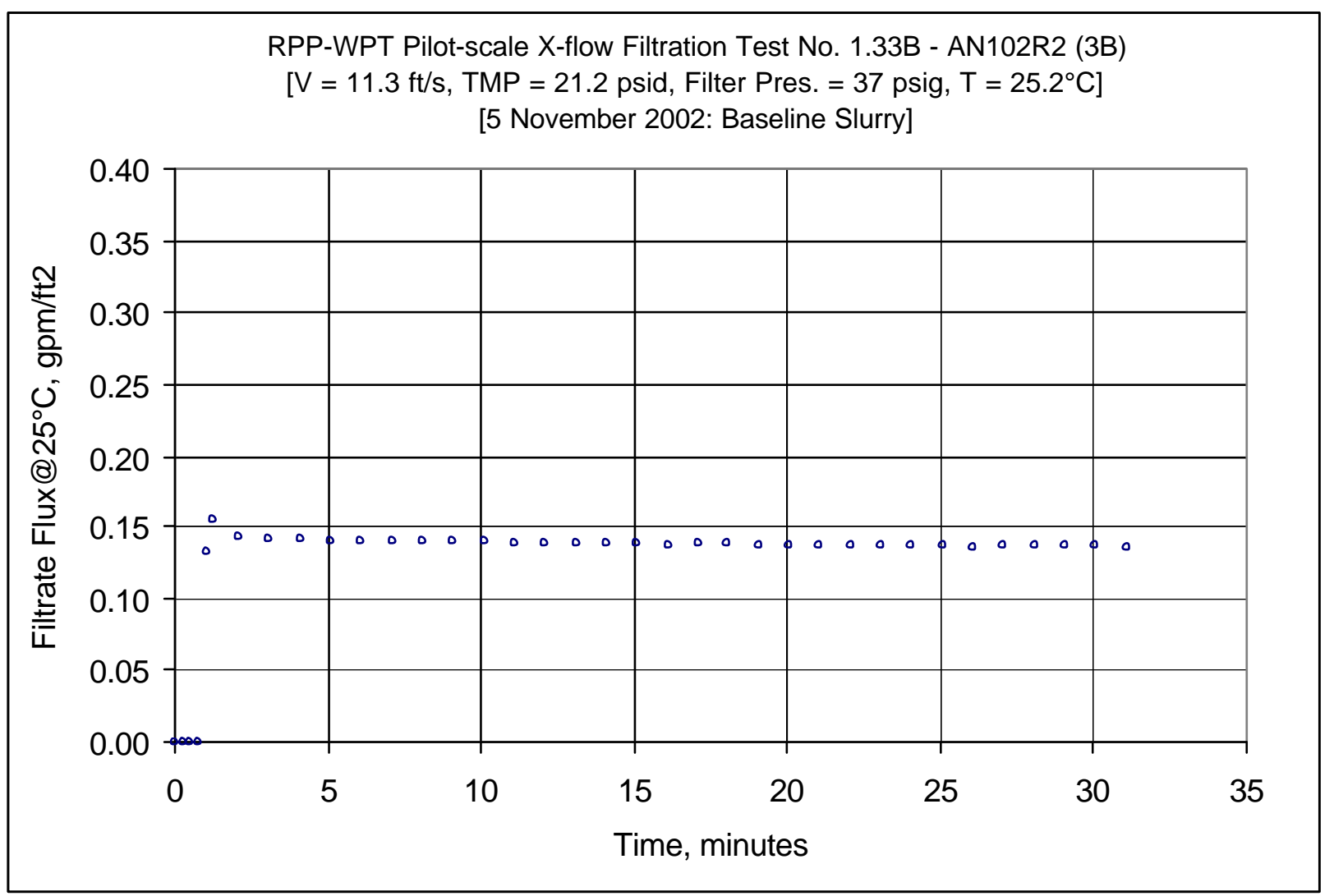

Figure B31: Run 1.33B - Baseline Slurry - After AN-102R2 Batch 3B and Before Batch 3A 
WSRC-TR-2003-00204, REV. 0

SRT-RPP-2003-00087, REV. 0

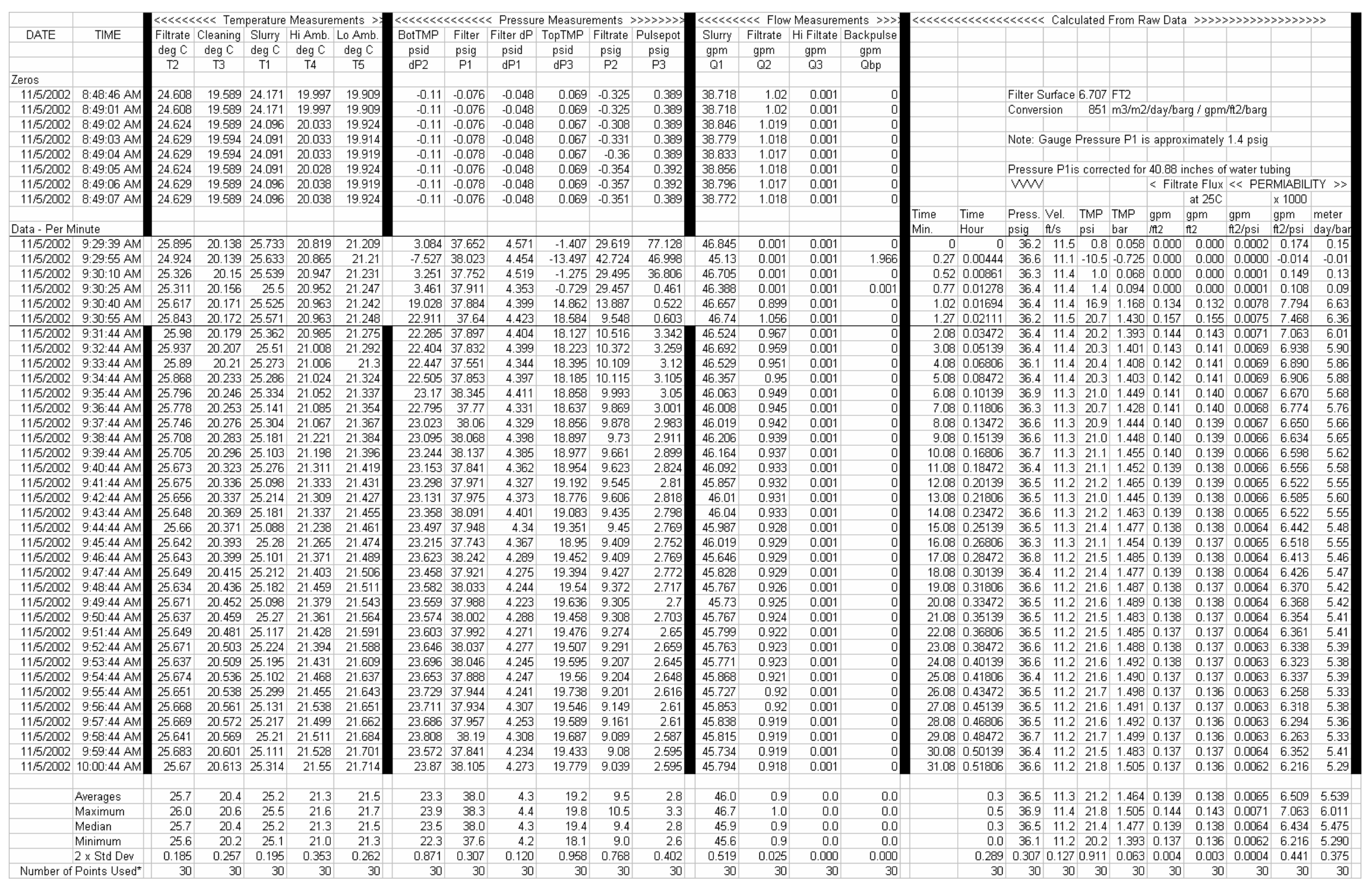

Figure B32: Run 1.33B - Baseline Slurry - After AN-102R2 Batch 3B and Before Batch 3A - Raw \& Calculated Data 
WSRC-TR-2003-00204, REV. 0

SRT-RPP-2003-00087, REV. 0

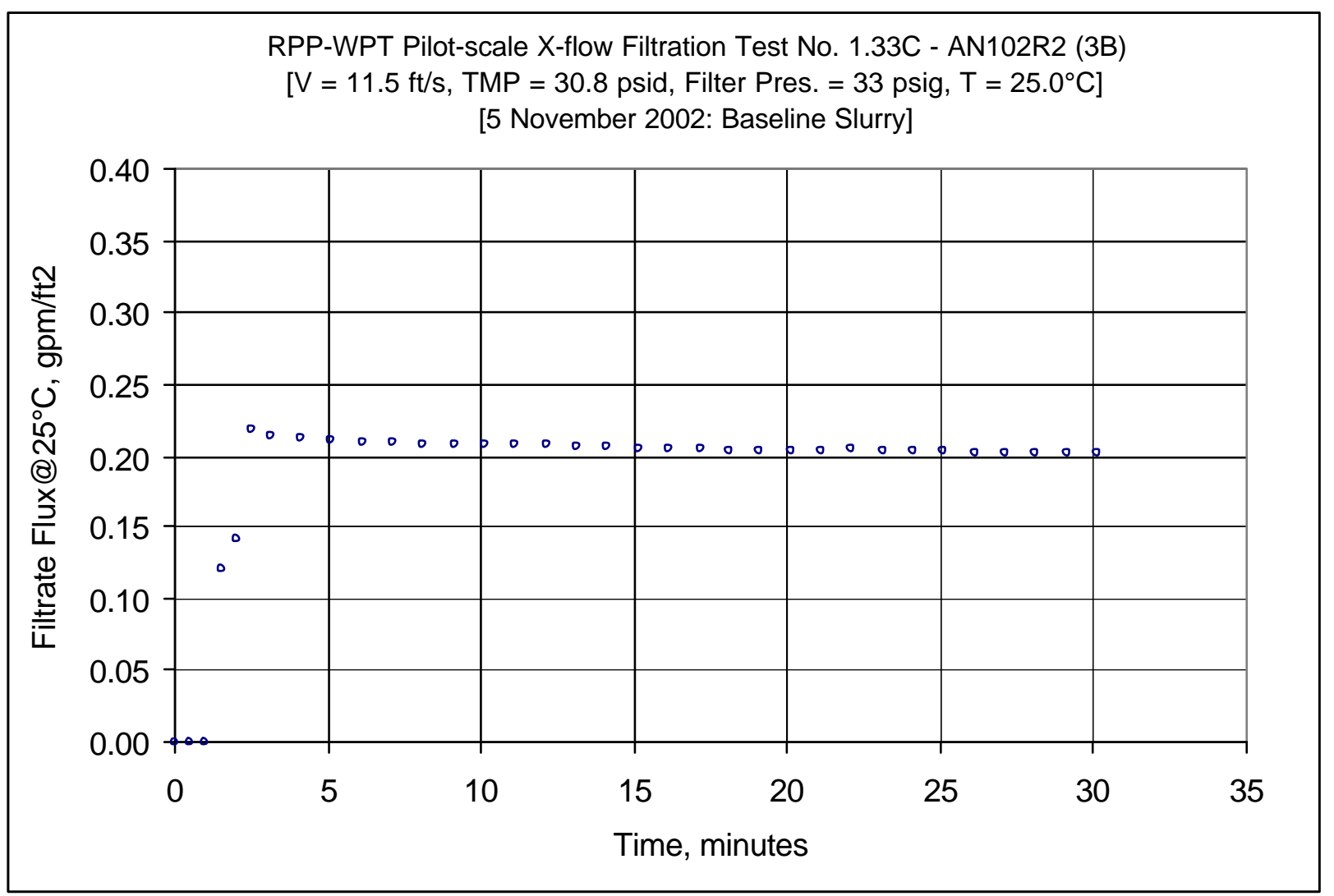

Figure B33: Run 1.33C - Baseline Slurry - After AN-102R2 Batch 3B and Before Batch 3A 
WSRC-TR-2003-00204, REV. 0

SRT-RPP-2003-00087, REV. 0

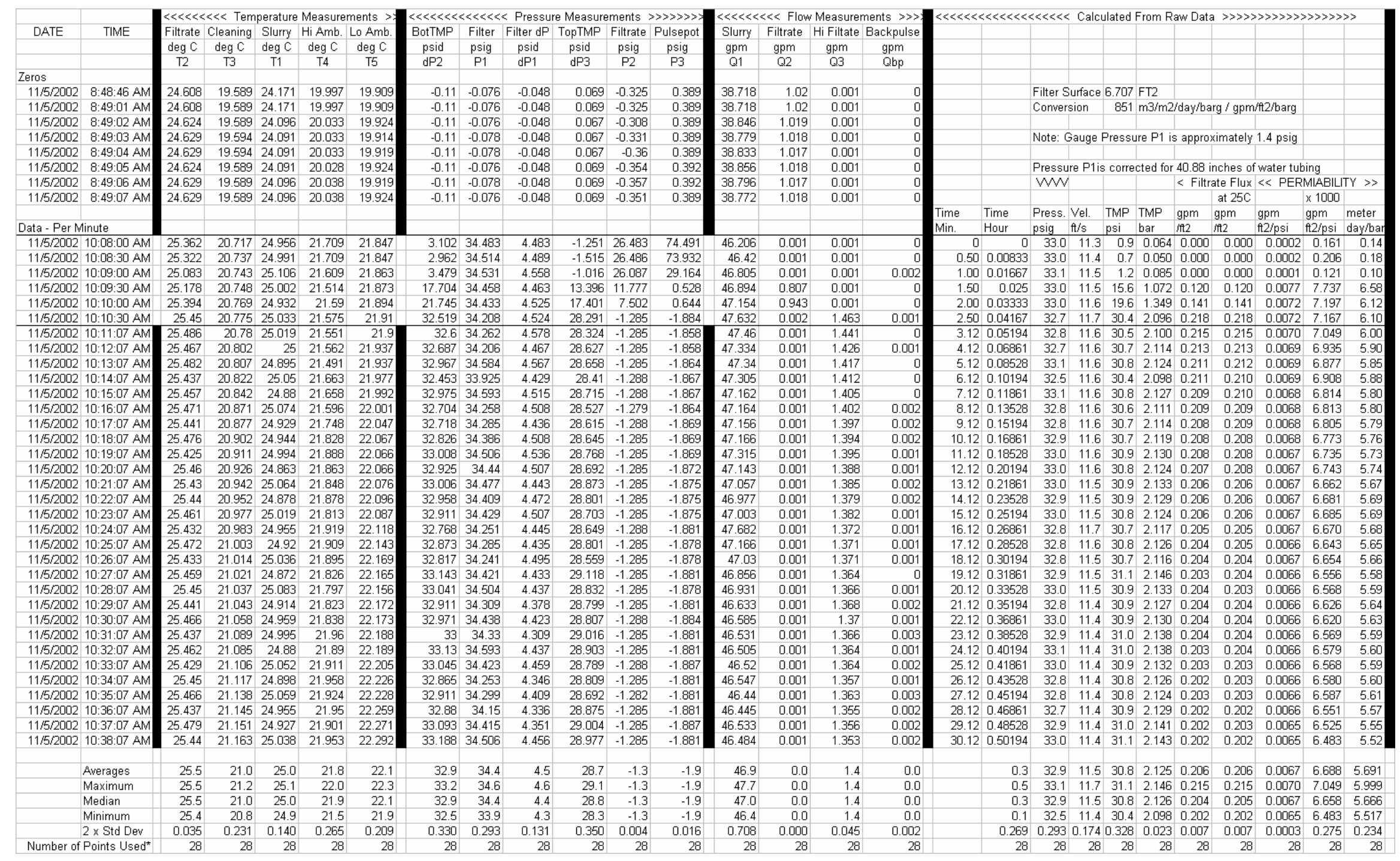

Figure B34: Run 1.33C - Baseline Slurry - After AN-102R2 Batch 3B and Before Batch 3A - Raw \& Calculated Data 
WSRC-TR-2003-00204, REV. 0

SRT-RPP-2003-00087, REV. 0

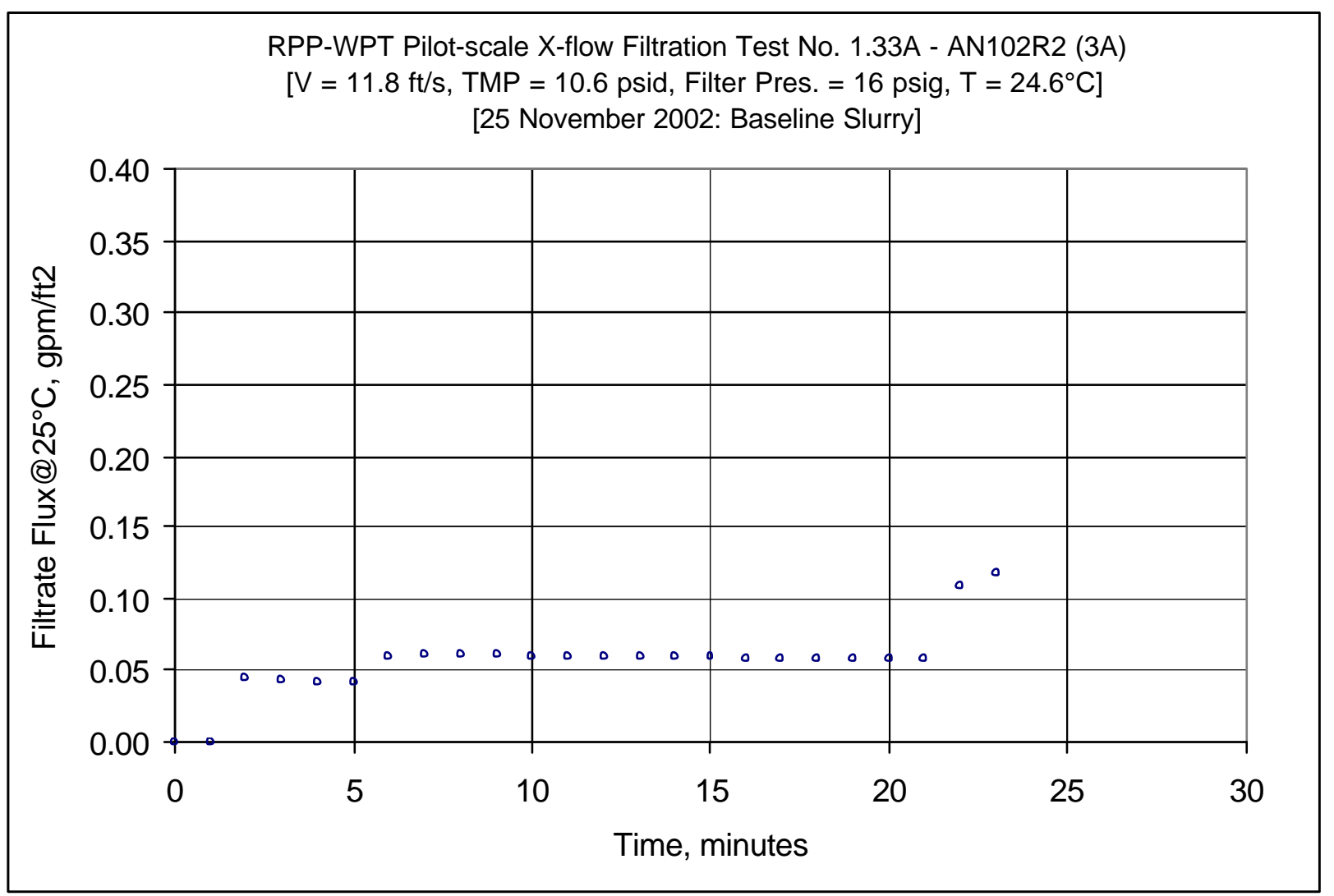

Figure B35: Run 1.33A - Baseline Slurry - After AN-102R2 Batch 3A and Before Batch 4A 
WSRC-TR-2003-00204, REV. 0

SRT-RPP-2003-00087, REV. 0

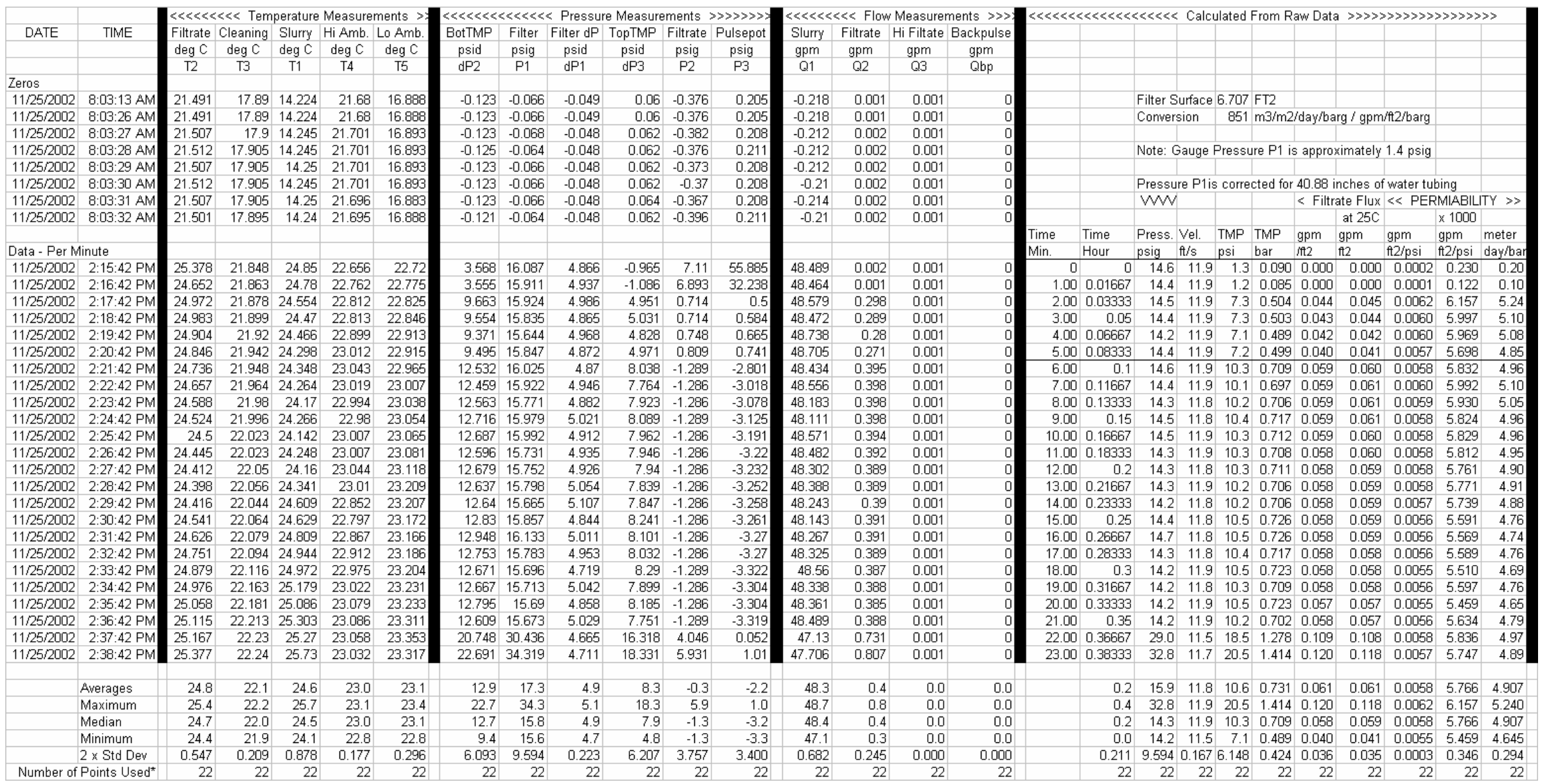

Figure B36: Run 1.33A - Baseline Slurry - After AN-102R2 Batch 3A and Before Batch 4A - Raw \& Calculated Data 
WSRC-TR-2003-00204, REV. 0

SRT-RPP-2003-00087, REV. 0

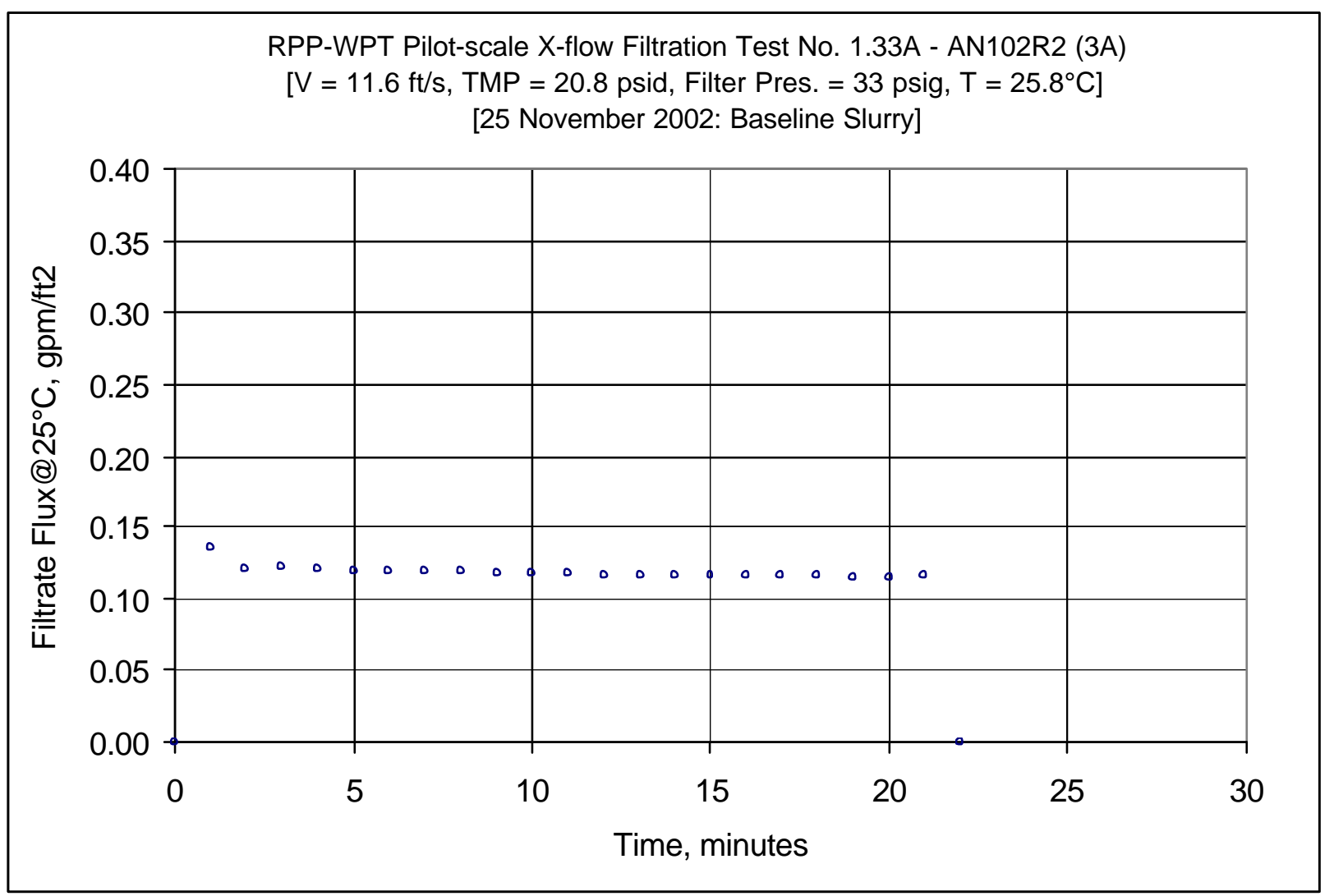

Figure B37: Run 1.33B - Baseline Slurry - After AN-102R2 Batch 3A and Before Batch 4A 
WSRC-TR-2003-00204, REV. 0

SRT-RPP-2003-00087, REV. 0

\begin{tabular}{|c|c|c|c|c|c|c|c|c|c|c|c|c|c|c|c|c|c|c|c|c|c|c|c|c|c|c|c|}
\hline \multirow{2}{*}{ DATE } & \multirow{2}{*}{ TIME } & \multicolumn{5}{|c|}{ 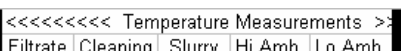 } & \multicolumn{6}{|c|}{ 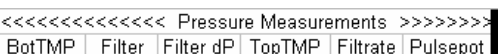 } & \multicolumn{4}{|c|}{ 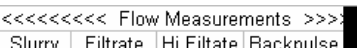 } & \multicolumn{11}{|c|}{ Calculated From Raw Data } \\
\hline & & $\begin{array}{l}\text { Filftrate } \\
\operatorname{deg} C\end{array}$ & $\begin{array}{c}\text { Cleaning } \\
\operatorname{deg} \mathrm{C}\end{array}$ & $\begin{array}{l}\text { Slurry } \\
\operatorname{deg} \mathrm{C}\end{array}$ & $\begin{array}{l}\text { Hi Amb. } \\
\operatorname{deg} \mathrm{C}\end{array}$ & $\begin{array}{l}\text { Lo Amb. } \\
\operatorname{deg} C\end{array}$ & BotTMP & $\begin{array}{l}\text { Fititer } \\
\text { psig }\end{array}$ & $\begin{array}{c}\text { Filter dP } \\
\text { psid }\end{array}$ & $\begin{array}{l}\text { TopTMP } \\
\text { psid }\end{array}$ & $\begin{array}{c}\text { Filtrate } \\
\text { psig }\end{array}$ & $\begin{array}{l}\text { Pulsepot } \\
\text { psig }\end{array}$ & $\begin{array}{l}\text { Slurry } \\
\text { gpm }\end{array}$ & $\begin{array}{c}\text { Filtrate } \\
\text { gpm }\end{array}$ & $\begin{array}{l}\text { HiFiftate } \\
\text { gpm }\end{array}$ & $\begin{array}{l}\text { Backpulse } \\
\text { gpm }\end{array}$ & & & & & & & & & & & \\
\hline & & $\mathrm{T} 2$ & T3 & T1 & T4 & & $\mathrm{dP2}$ & P1 & $\mathrm{dP1}$ & $\mathrm{dP3}$ & P2 & $\mathrm{P} 3$ & Q1 & 02 & Q3 & Qbp & & & & & & & & & & & \\
\hline \multicolumn{28}{|l|}{ Zeros } \\
\hline /25/20002 & 13:13 AM & 1.491 & 17.89 & 14.224 & 21.68 & 886 & -0.123 & -0.066 & -0.049 & 0.06 & -0.376 & 0.205 & -0.218 & 0.001 & 0.001 & & & & \multicolumn{4}{|c|}{$707 \mathrm{FT2} 2 \mathrm{2}$} & & & & & \\
\hline $11 / 25 / 2002$ & :26 Al & 21.491 & 17.89 & 14.224 & 21.68 & 888 & -0.123 & -0.066 & -0.049 & 0.06 & -0.376 & 0.20 & -0.218 & 0.001 & 0.001 & & & & \multirow{2}{*}{\multicolumn{2}{|c|}{ Conversion }} & 51 & & & & & & \\
\hline $11 / 25 / 2002$ & 8:03:27 A & 21.507 & 17.9 & 14.245 & 21.701 & 16.893 & -0.123 & -0.068 & -0.048 & 0.062 & -0.382 & 0.208 & -0.212 & 0.002 & 0.001 & & & & & & & & & & & & \\
\hline $11 / 25 / 2002$ & 8:03:28 AM & 21.512 & 17.905 & 14.245 & 21.701 & 16.893 & -0.125 & -0.064 & -0.048 & 0.062 & -0.376 & 0.211 & -0.212 & 0.002 & 0.001 & & & & \multirow{2}{*}{\multicolumn{7}{|c|}{ Note: Gauge Pressure $\mathrm{P} 1$ is approximately 1.4 psig }} & & \\
\hline $11 / 25 / 2002$ & 8:03:29 AM & 21.507 & 17.905 & 14.25 & 21.701 & 16.893 & -0.123 & -0.066 & -0.048 & 0.062 & -0.373 & 0.208 & -0.212 & 0.002 & 0.001 & & & & & & & & & & & & \\
\hline $11 / 25 / 2002$ & $8: 03: 30 \mathrm{AM}$ & 21512 & 17.905 & 14.245 & 21701 & 6.893 & -0.123 & -0.066 & -0.048 & 0.062 & -0.37 & 0.208 & -0.21 & 0.002 & 0.001 & & & & \multicolumn{8}{|c|}{ ted for 40.88 inches of water tut } & \\
\hline $11 / 25 / 2002$ & 8:31 & 21.507 & 17.905 & 4.25 & 21.696 & 6.88 & -0.123 & 0.066 & -0.0 & 0.064 & -0.367 & 0.208 & -0.214 & 0.002 & 0.1 & & & & \multicolumn{2}{|l|}{ m } & & & \multicolumn{2}{|c|}{$\begin{aligned} r \text { 4 } 40.88 \text { inches of } \\
<\text { Filtrate Flux }\end{aligned}$} & & 3L & ITY \\
\hline $11 / 25 / 2002$ & $3: 32 \mathrm{~A}$ & 1.501 & 17.895 & 14.24 & 21.695 & & -0.121 & -0.064 & -0.048 & 0.062 & -0.396 & 071 & -0.21 & 0.002 & 0.001 & & & & & & & & & at $25 \mathrm{C}$ & & 1000 & \\
\hline & & & & & & & & & & & & & & & & & Time & Time & Press. & Vel. T & TMP & TMP & m & $\mathrm{gpm}$ & $\mathrm{gpm}$ & gpm & me \\
\hline Data - Per M & Ain & & & & & & & & & & & & & & & & & Hour & psig $\mathrm{ft}$ & $f / s \quad p$ & psi & bar & int2 & $\mathrm{ft} 2$ & $\mathrm{ft} 2 / \mathrm{psi}$ & $\mathrm{ft2} / \mathrm{psi}$ & avel \\
\hline $11 / 25 / 2002$ & 2:41:57 PM & 25.458 & 22.351 & 25.811 & 23.209 & 3.378 & 3.197 & 34.04 & 03 & -1.365 & 25.748 & 74.715 & 47.641 & 0.002 & 01 & & 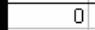 & T & 32.6 & 11.7 & 0.9 & 0.063 & 30.000 & 0.000 & 0.0003 & $\begin{array}{l}30.318 \\
\end{array}$ & \\
\hline $11 / 25 / 2002$ & 2:42:57 PM & 25.331 & 22.39 & 25.939 & 23.218 & 3.38 & 21.923 & 34.478 & & 17.604 & 6.966 & 0.564 & 47. & & & & 1.00 & 0.01667 & 33.0 & 11.7 & 19.8 & 1.363 & 30.138 & 0.135 & $5 \quad 0.0068$ & $3 \quad 6.811$ & \\
\hline & 2 & 25.89 & 22.418 & 12 & 23.246 & & 21.796 & 34.331 & & 17.446 & & & 47. & & & & 2.00 & 0.03333 & 32.9 & 11.7 & 19.6 & & & & & & \\
\hline & 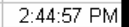 & 25.972 & 22. & 2 & 23.2 & & 22.167 & & & & & & & & & & 3.00 & & & 11.7 & & & & & & & \\
\hline $11 / 2$ & 2:45:57 PM & 25.989 & 22.463 & 25.752 & 23.27 & 23.3 & 52 & 34.245 & 25 & 1 & 2 & 1.207 & 47. & & & & 4.00 & 0.06667 & 32.8 & 11.6 & 20.3 & 1.397 & 23 & 20 & & 38 & J \\
\hline $11 / 25$ & 2:46:57 PM & 26.017 & 22.511 & 25.905 & 23.369 & 23. & & 34.284 & & 51 & & 1.14 & & & & & 5.00 & 0.08333 & 32.8 & 11.7 & 20.4 & 1.404 & 40.122 & 0.119 & $\begin{array}{l}9 \quad 0.0058 \\
\end{array}$ & 35.839 & 3 \\
\hline $11 / 25 / 2002$ & 2:47:57 PM & 14 & 22.5 & 25.802 & 23.396 & & 6 & 34.412 & & & & 1.05 & & & & & 6.00 & 0.1 & 32.9 & 11.7 & 20.6 & 1.421 & 10.121 & 0.118 & $\begin{array}{ll}80 . \\
80 .\end{array}$ & 45 & \\
\hline $11 / 25$ & 2:48:57 PM & & 22.5 & 25.755 & 23. & & & 34.482 & & & & 1.00 & & & & & 7.00 & 0.11667 & 33.0 & 11.7 & 20.7 & 1.425 & & & & & \\
\hline & PM & & & & & & & 34. & & & & & & & & & 8.00 & & 1 & 11.5 & & & & & & & 87 \\
\hline & $2.5+3$ & & & & & & & & & & & & & & & & & & & 11.6 & & & & & & & \\
\hline & & & & & & & & & & & & & & & & & & & 32. & 11.6 & & & & & & & \\
\hline $11 / 2$ & 2:52:57 PM & & & 25 & & & & 34 & & & 5.763 & & & & & & 5 & 0.18333 & 33.2 & 11.5 & & 1.451 & 1 & & & & 4 \\
\hline & 2:53:57 P & & & 25. & 23. & & & 34 & & & H & & & & & & & 0.2 & & 11.5 & & & & & & & \\
\hline & $2: 54: 5$ & & & 25. & & & & 34. & & & & & & & & & & 0 & & 11.5 & & & & & & & \\
\hline & $2:$ & & & & & & & & & & & & & & & & & & 9 & 11.5 & & & & & & & \\
\hline & $2: 5$ & & 22.8 & 25 & 23.522 & 23. & 23. & & & & & & & & & & & 25 & 33.0 & 11.5 & & & & & & & \\
\hline & $2:$ & & & 25 & & & 2 & & & & & & & & & & & & & Sat & & & & & & & \\
\hline & & & & & & & & & & & & & & & & & & & & & & & & & & & \\
\hline & 2.5 & & & & & & & & & & & & & & & & & the & & & & & & & & & \\
\hline & 3:00: & 26. & 22. & & & & & & & & 5.4 & & & & & & & & 33.0 & 11.5 & & & & & & & 58 \\
\hline & 3:01:57 P & 26.131 & 22.921 & 25.863 & 23.708 & 23. & 23.463 & 34.452 & 4.529 & 19.295 & 5.434 & & & & & & 20.00 & 0.33333 & 33.0 & 11.5 & 21.4 & 1.474 & 40 & 15 & 1054 & 77 & 4.58 \\
\hline & 3:02:57 PM & 26.083 & 22.934 & 25.596 & 23.806 & 23. & 23.613 & 34.422 & & 19.446 & 5.33 & 0. & & 0. & & & 21.00 & 0.35 & 32.9 & 11.5 & 21.5 & 1.484 & 40.117 & 0.115 & 154 & 359 & $\begin{array}{l}3.56 \\
3\end{array}$ \\
\hline $25 / 2002$ & 3:03:57 P & $0700+5$ & 22.946 & 25.408 & 23.869 & 20. & 32.744 & 33 & 5.143 & 27.916 & -1.289 & -4.732 & 50 & 0.0 & 1.104 & & 22.00 & 0.36667 & 32.5 & 12.3 & 30.3 & 2.091 & 10.000 & 0.000 & 0.0000 & 0.010 & 0.0 \\
\hline & & & & & & & & & & & & & & & & & & & & & & & & & & & \\
\hline & & & & & & & & & & & & & & & & & & 0.4 & 33.2 & 11.7 & & & 4. & 35 & & & \\
\hline & $\mathrm{Me}$ & & 22.7 & 25.9 & 23.4 & & & 34.4 & & 18.8 & 5. & 0. & & 0 & 0 & & & 0.2 & 33.0 & 11.5 & 20.9 & 1.443 & $\begin{array}{ll}30.120 \\
\end{array}$ & 0.117 & 156 & 5.569 & 4.739 \\
\hline & & 25.3 & 22.4 & 25.6 & 23.2 & 23 & 18 & & & & 3 & & & & & & & 0.0 & 32.7 & 11.4 & 19.6 & 1.353 & $\begin{array}{ll}3 & 0.117 \\
\end{array}$ & 0.115 & 054 & 45.359 & 4.561 \\
\hline & & 0.338 & 502 & 156 & 0.311 & 0.16 & 998 & 00 & & 1.099 & & & & & & & & 02 & 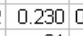 & 11 & 1.046 & 72 & 00 & & 107 & 1665 & 66 \\
\hline & & $21,2>3$ & 21 & & & 21 & 21 & & & & & & & & & & & & & & & & & & & & \\
\hline
\end{tabular}

Figure B38: Run 1.33B - Baseline Slurry - After AN-102R2 Batch 3A and Before Batch 4A - Raw \& Calculated Data 
WSRC-TR-2003-00204, REV. 0

SRT-RPP-2003-00087, REV. 0

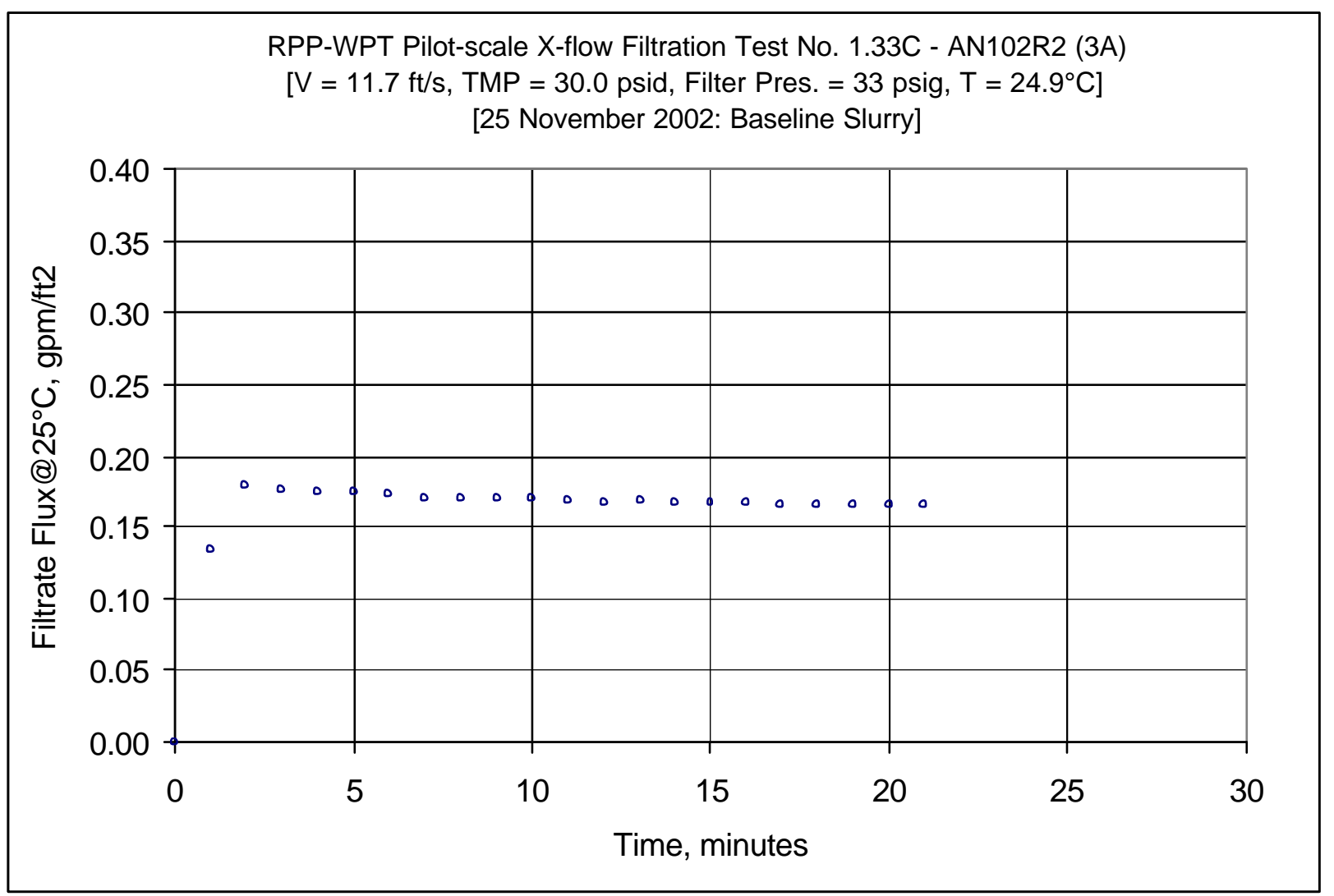

Figure B39: Run 1.33C - Baseline Slurry - After AN-102R2 Batch 3A and Before Batch 4A 
WSRC-TR-2003-00204, REV. 0

SRT-RPP-2003-00087, REV. 0

\begin{tabular}{|c|c|c|c|c|c|c|c|c|c|c|c|c|c|c|c|c|c|c|c|c|c|c|c|c|c|c|c|}
\hline \multirow{3}{*}{ DATE } & \multirow{3}{*}{ TIME } & \multicolumn{5}{|c|}{$\ll \ll \ll \ll<<$ Temperature Measurements $\gg$} & & & \multicolumn{11}{|c|}{ 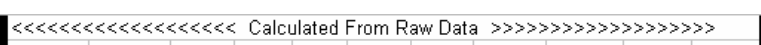 } \\
\hline & & Filtrate & Cleaning & Slurry & Hi Amb. & Lo Amb. & iotTMP & Filter & Filter dP & TорTMP & Filtrate & Pulsepot & Slurry & Filtrate $\mathrm{l}$ & Hi Filtate & Backpulse & & & & & & & & & & & \\
\hline & & of & $c$ & $\operatorname{deg} \mathrm{C}$ & $\operatorname{deg} C$ & $\operatorname{deg} C$ & psid & psig & psid & psid & psig & psig & gpm & gpm & $\mathrm{gpm}$ & $\mathrm{gpm}$ & & & & & & & & & & & \\
\hline \multirow{2}{*}{\multicolumn{28}{|c|}{ Zeros }} \\
\hline & & & & & & & & & & & & & & & & & & & & & & & & & & & \\
\hline $11 / 25 / 2002$ & 03:13 AM & 21.491 & 17.89 & 14.224 & $4 \quad 21.68$ & 16.888 & -0.123 & -0.066 & -0.049 & 0.06 & -0.376 & 0.205 & -0.218 & 0.001 & 0.001 & 5 & & & \multicolumn{4}{|c|}{ Filter Surface 6.707 FT2 } & & & & & \\
\hline 11/25/2002 & 8:03:26 AM & 21.491 & 17.89 & 14.224 & $4 \quad 21.68$ & 16.888 & -0.123 & -0.066 & -0.049 & 0.06 & -0.376 & 0.205 & -0.218 & 0.001 & 0.001 & 0 & & & \multicolumn{2}{|c|}{ Conversion } & $851 !$ & \multicolumn{4}{|c|}{$51 \mathrm{~m} 3 / \mathrm{m} 2 /$ day/barg $/ \mathrm{gpm} / \mathrm{ft} / \mathrm{h}$ barg } & & \\
\hline $11 / 25 / 2002$ & 8:03:27 AM & 21.507 & 17.9 & 14.245 & $5 \quad 21.701$ & 16.893 & -0.123 & -0.068 & -0.048 & 0.062 & -0.382 & 0.208 & -0.212 & 0.002 & 0.001 & & & & & & & & & & & & \\
\hline $11 / 25 / 2002$ & 8:03:28 AM & 21.512 & 17.905 & 14.245 & 621.701 & 16.893 & -0.125 & -0.064 & -0.048 & 0.062 & -0.376 & 0.211 & -0.212 & 0.002 & 0.001 & 0 & & & \multirow{2}{*}{\multicolumn{7}{|c|}{ e Pressure $\mathrm{P} 1$ is approximately $1.4 \mathrm{psig}$}} & & \\
\hline $11 / 25 / 2002$ & 8:03:29 AM & 21.507 & 17.905 & 14.25 & $\begin{array}{l}51.701 \\
\end{array}$ & 16.893 & -0.123 & -0.066 & -0.048 & 0.062 & -0.373 & 0.208 & -0.212 & 0.002 & 0.001 & & & & & & & & & & & & \\
\hline $11 / 25 / 2002$ & 8:03:30 AM & 21.512 & 17.905 & 14.245 & $\begin{array}{l}51.701 \\
\end{array}$ & 6.8 & -0.123 & -0.066 & -0.048 & 0.062 & -0.37 & 0709 & -0.21 & 0.002 & 0.0 & & & & \multicolumn{8}{|c|}{ Pressure P1is corrected for 40.8} & \\
\hline $11 / 25 / 2002$ & 8:03:31 AM & 21.507 & 17.905 & 14.25 & 21.696 & 16.883 & -0.123 & -0.066 & -0.048 & 0.064 & -0.367 & 0.208 & -0.214 & 0.002 & 0.001 & 0 & & & \multicolumn{2}{|c|}{ WW } & & & \multicolumn{2}{|c|}{$<$ Filtrate Flux } & $\ll$ PER & RMIABILI & LTTY : \\
\hline 11/25/2002 & 8:03:32 AM & 21.501 & 17.895 & 14.24 & 421.695 & 16.888 & -0.121 & -0.064 & -0.048 & 0.062 & -0.396 & 0.211 & -0.21 & 0.002 & 0.001 & & & & & & & & & at $25 \mathrm{C}$ & & $\times 1000$ & \\
\hline & & & & & & & & & & & & & & & & & Time & Time & Press. V & Vel. & TMP & TMP & $\mathrm{gpm}$ & gpm & $\mathrm{gpm}$ & $\mathrm{gpm}$ & $\mathrm{me}$ \\
\hline Data - Per M & Ainute & & & & & & & & & & & & & & & & & Hour & & $f / s$ & noi & bar & if2 & $\mathrm{A} 2$ & $\mathrm{ft} 2 / \mathrm{psi}$ & $\mathrm{f} 2 / \mathrm{psi}$ & th \\
\hline $11 / 25 / 2002$ & 3:07:54 PM & 25.716 & 23.027 & 25.088 & $\begin{array}{l}83.789 \\
\end{array}$ & 23.702 & 3.377 & 34.042 & 4.832 & -1.223 & 25.406 & 74.364 & 48 & 0.002 & 0.001 & & 0 & 0 & 32.6 & 11.8 & B 1.1 & 0.074 & 40.000 & 0.000 & 0.0003 & 0.276 & \\
\hline $11 / 25 / 2002$ & 3:08:54 PM & 25.534 & 23.051 & 25.037 & 23.797 & 23.7 & 21.508 & 33.698 & 4.839 & 17.057 & 6.795 & & 48.558 & 0.894 & & & 1.00 & 0.01667 & 32.2 & 11.9 & 19.3 & 1.329 & 90.133 & 0.133 & 0.0069 & & \\
\hline & 3:09:54 P & 25.501 & 23.068 & 25.114 & 23.814 & 23.7 & 32.312 & 33.825 & 4.8 & 27.836 & -1.2 & & 48.571 & & & & 2.00 & 0.03333 & 32.4 & 1.9 & 30.1 & & & & & & 6.0b \\
\hline $11 / 2$ & 3:10:54 PM & 25.397 & 23.069 & 25.045 & 23.8 & 23.72 & 32.529 & 33.864 & 4.71 & 69 & $-1.28<x=$ & 0.596 & 3.082 & & 1.1 & & 3.00 & 05 & 32.4 & 11.8 & 30.3 & 2.092 & & & & & 492 \\
\hline $11 / 25 / 2002$ & 3:11:54 PM & 25.36 & 23.087 & 24.917 & 23.853 & 23.751 & 32.602 & 33.959 & 4.796 & 28.173 & -1.289 & 0.593 & 48.04 & 0.001 & 1.168 & & 4.00 & 0.06667 & 32.5 & 11.8 & 30.4 & 2.095 & 50.174 & 0.175 & 50.0057 & 5.744 & \\
\hline $11 / 25 / 2002$ & 3:12:54 PM & 25.327 & 23.094 & 24.884 & \begin{tabular}{|l|l|} 
& 23.87 \\
\end{tabular} & 23.788 & 32.655 & 33.965 & 4.702 & 41 & -1.289 & 0.593 & 47.996 & 12 & 1.16 & & 5.00 & 0.08333 & 32.5 & 11.8 & 30.4 & 2.099 & 90.173 & 0.174 & $4 \quad 0.0057$ & 5.699 & 4.85 \\
\hline $11 / 25 / 2002$ & 3:13:54 PM & 25.278 & 23.095 & 24.956 & \begin{tabular}{|l|l|}
6.837 \\
\end{tabular} & 23.78 & 32.635 & 33.963 & 4.7 & & -1.289 & 0.5 & 47.941 & & 1.1 & 0.00 & 6.00 & 0.1 & 32.5 & \begin{tabular}{ll|}
11.7 \\
\end{tabular} & 30.4 & 2.097 & 0.172 & 0.172 & 20.0057 & 5.660 & 4.82 \\
\hline $11 / 25 / 2002$ & $3: 14: 54 \mathrm{PM}$ & 25.219 & 23.091 & 24.912 & 23.808 & 23.7 & & 34.228 & 4.7 & & -1.2 & & 47.866 & & & & 7.00 & 0.11667 & 32.8 & 11.7 & 6 & 2.112 & & & 0.0055 & & 72 \\
\hline & $3: 15:$ & 25.217 & 23.119 & & & 25.1 & & 33.872 & 4.7 & & & & & & & & 8.00 & & 32.4 & 11.8 & 4 & & & & & & 4.76 \\
\hline & 3:16:54 F & 25.235 & 23.158 & 24.823 & 323 & 238 & & 33.9 & & & -1.2 & & 178 & & & 00 & 9.00 & 15 & 32.5 & 11.8 & 6 & 2.113 & & & & & 4.70 \\
\hline $11 / 25 / 2002$ & 3:17:54 PM & 25.188 & 23.145 & 24.905 & \begin{tabular}{|l|}
53.882 \\
\end{tabular} & 23. & 32 & 33.959 & 4. & 64 & -1.2 & 0.587 & 47.99 & & 1.132 & & 10.00 & 0.16667 & 32.5 & 11.8 & 30.6 & 2.107 & 70 & 9 & 0.0055 & 36 & 4.7 \\
\hline $11 / 25 / 2002$ & 3:18:54 PM & 25.155 & 23.153 & 24.873 & & & & 33.5 & & & -1. & & 47.86 & & & 0.00 & 11 & 0.18333 & 32.5 & 11.7 & 30.6 & 2.108 & 0.168 & 8 & 0 & & 4.6 \\
\hline $11 / 25 / 2002$ & 3:19:54 PM & 25.153 & & 24.781 & & & & 33. & & & & & 47. & & & & & 0.2 & 32.2 & 11.7 & & & & & & & J \\
\hline $11 / 25$ & $3: 20: 5$ & 25.156 & 9 & & & & & 33.9 & & & -1.2 & & 47 & & & & 13.00 & 0. & 32.4 & 11.7 & & 2.109 & & & 0.0055 & & 4 \\
\hline & $3: 21: 5$ & 25.134 & 23.212 & & 23.9 & 23. & & 33.8 & & & -1.2 & & & & & & 14 & 0.23 & 32.4 & 11.7 & 30.6 & 2.110 & & & 0 & 5.448 & 4.64 \\
\hline & 3:22:54 $\mathrm{F}$ & 25.12 & 23.245 & 24. & 24 & 72 & & 34. & 4.7 & & -1. & & & & & & 15 & te & 32.7 & 7 & 7 & 2 & & 7 & & & 4.63 \\
\hline & 3:23:54 $\mathrm{F}$ & 25.12 & 23.257 & 6 & 24 & & & 33. & $4.6 c$ & & -1. & & & & & & & 0.2 & 32.3 & 11.7 & 5 & 2 & & $=$ & & & 107 \\
\hline & $3: 24: 54 \mathrm{~F}$ & 25. & 23 & 24 & & & & 34. & & & -1. & & & & & & & 0.2 & 32.6 & & & & & & & & 459 \\
\hline $11 / 25 / 2002$ & 3:25:54 $\mathrm{F}$ & 25.103 & 23.296 & 24. & & & & & & & -1 & & & & & & & 0.3 & 32.6 & 11.7 & & & & 0.164 & 40.0054 & 5.364 & 4.5 \\
\hline $11 / 2$ & 3:26:54 PM & 25.1 & 23.314 & 24.773 & 3.24 & 23.9 & 32.8 & 34.139 & 4 & 28.352 & -1.28 & 0.5 & 47.642 & 0. & 1.0 & 0. & 19.00 & 0.31667 & 32.7 & \begin{tabular}{|l|l|}
11.7 \\
\end{tabular} & 30.6 & 2.108 & 30.164 & 0.165 & 0.0054 & 5.383 & 4.58 \\
\hline $11 / 25 / 2002$ & 3:27:54 PM & 25.091 & 23.324 & 24.688 & 24.051 & 23.9 & 32.8 & 33.957 & 4.5 & 7 & -1.2 & 0. & 47.644 & 0. & 1. & 0.00 & 20.00 & 0.33333 & 32.5 & 11.7 & 30.8 & 2.121 & 10.163 & 0.164 & 153 & 5.343 & 4.55 \\
\hline $11 / 25 / 2002$ & 3:28:54 PM & 25.086 & 23.33 & 24.729 & 24.046 & 23.929 & 32.769 & 33.856 & 4.691 & 28.444 & -1.289 & 0.579 & 47.746 & 0.001 & 1.094 & 0.002 & 21.00 & 0.35 & 32.4 & 11.7 & 30.6 & 2.110 & 0.163 & 0.164 & 0.0054 & 5.370 & 4.57 \\
\hline & & & & & & & & & & & & & & & & & & 0.2 & & & 00 & 2068 & & 167 & 156 & 5.599 & $y$ \\
\hline & Maximum & & & & & & & & & & & & & 0. & 1. & & & 0.4 & 32.8 & 11.9 & 8 & & & 79 & 69 & 905 & $\begin{array}{l}5 \quad 5.876 \\
\end{array}$ \\
\hline & Median & 25.2 & 23.2 & 24.8 & 23 & & 32 & 34 & 4. & 28 & -1.5 & 0 & & 0.0 & 1.1 & 0. & & 0.2 & 32.5 & \begin{tabular}{|l|}
11.7 \\
\end{tabular} & 30.6 & 2.108 & 30.166 & 0.167 & 0.0055 & 5.506 & \begin{tabular}{|l|l|}
6 & 4.6866
\end{tabular} \\
\hline & Minimum & 25.1 & 23.1 & 24.7 & 23.8 & 23 & 21.5 & 33.7 & 4.6 & 17.1 & -1.3 & 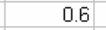 & 47.5 & 0.0 & 0.0 & 0.0 & & 0.0 & 32.2 & \begin{tabular}{|l|}
11.7 \\
\end{tabular} & 19.3 & 1.329 & 90.133 & 0.133 & 0.0053 & 5.343 & \begin{tabular}{|l|l|}
3 & 4.547
\end{tabular} \\
\hline & & 0.259 & 0.183 & 0.220 & 0.195 & 0.128 & 4.782 & 0.263 & 0.115 & 823 & 3.443 & 04 & 0.548 & .380 & 0.484 & 002 & & 0.202 & 0.2630 & 0.134 & 4.802 & 0.331 & 0.017 & 0.017 & 0.0007 & 0.659 & 560 \\
\hline Number & hints lserd? & 21 & 21 & 21 & 21 & 2 & 21 & 21 & 21 & 21 & 21 & 21 & 21 & 21 & 21 & 21 & & 21 & 04 & & 21 & & 21 & 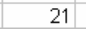 & & 4 & \\
\hline
\end{tabular}

Figure B40: Run 1.33C - Baseline Slurry - After AN-102R2 Batch 3A and Before Batch 4A - Raw \& Calculated Data 
WSRC-TR-2003-00204, REV. 0

SRT-RPP-2003-00087, REV. 0

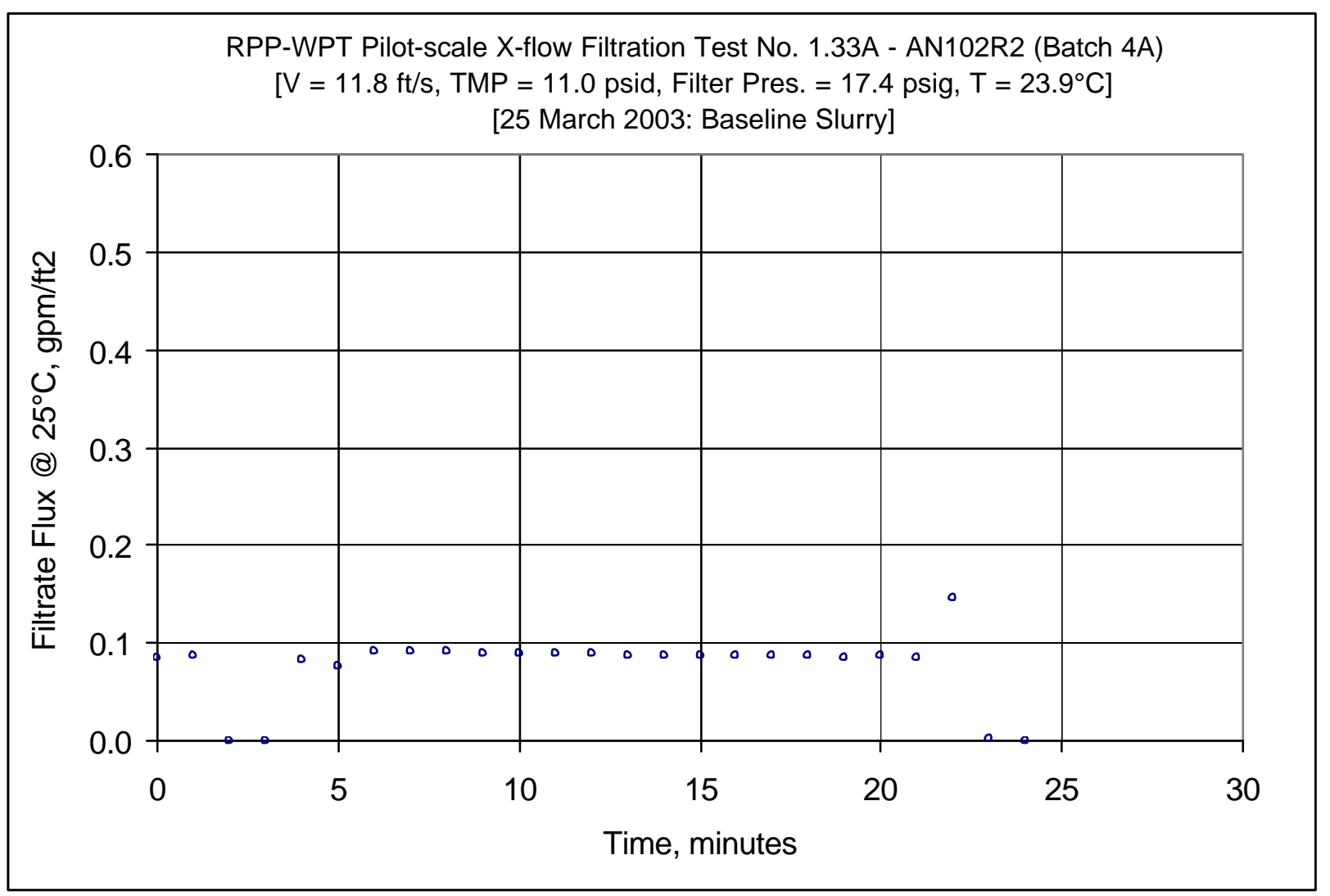

Figure B41: Run 1.33A - Baseline Slurry - After AN-102R2 Batch 4A 
WSRC-TR-2003-00204, REV. 0

SRT-RPP-2003-00087, REV. 0

\begin{tabular}{|c|c|c|c|c|c|c|c|c|c|c|c|c|c|c|c|c|c|c|c|c|c|c|c|c|c|c|c|}
\hline \multirow{4}{*}{ DATE } & \multirow{3}{*}{ TIME } & \multicolumn{5}{|c|}{$\ll \ll \ll \ll \ll \ll<$ Temperature Measurements $>$. } & \multicolumn{6}{|c|}{ 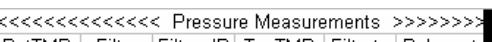 } & \multicolumn{4}{|c|}{ 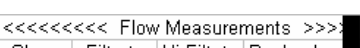 } & \multicolumn{3}{|c|}{ 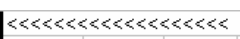 } & \\
\hline & & Filtrate & Cleaning & Slurry & Hi Amb. & Lo Amb. & BotTMP & Filter & Filter dP & TopTMP & Filtrate & Pulsepot & Slurry & Filtrate & Hi Filtate & Backpulse & & & & \multicolumn{8}{|c|}{ 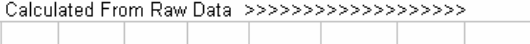 } \\
\hline & & $\operatorname{deg} C$ & $\operatorname{deg} \mathrm{C}$ & $\operatorname{deg} \mathrm{C}$ & $\operatorname{deg} \mathrm{C}$ & $\operatorname{deg} \mathrm{C}$ & psid & psig & psid & psid & psig & psig & $\mathrm{gpm}$ & $\mathrm{gpm}$ & $\mathrm{gpm}$ & $\mathrm{gpm}$ & & & & & & & & & & & \\
\hline \multirow{2}{*}{\multicolumn{28}{|c|}{ Zeros }} \\
\hline & & & & & & & & & & & & & & & & & & & & & & & & & & & \\
\hline 305 & 9:00:50 AM & 22.653 & 19.599 & 23.314 & 21.549 & 18.6 & -0.123 & -0.068 & -0.062 & 0.06 & -0.156 & 0.234 & 37.838 & 1.192 & 0.001 & 0.0 & & & & $07 \mathrm{~F}$ & $7 \mathrm{FT2}$ & & & & & \\
\hline $3 / 25 / 2003$ & 9:01:12 AM & 22.653 & 19.599 & 23.314 & 21.549 & 18.6 & -0.123 & -0.068 & -0.062 & 0.06 & -0.156 & 0.234 & 37.838 & 1.192 & 0.001 & & & & \multicolumn{2}{|c|}{$\begin{array}{l}\text { Filter Surface } A \\
\text { Conversion }\end{array}$} & & $1 \mathrm{~m} 3 / \mathrm{m} 2 / \mathrm{s}$ & $2 /$ day/ $/ 2$ la & $\mathrm{ag} / \mathrm{gpm} / \mathrm{t}$ & /ft2/barg & & \\
\hline $3 / 25 / 2$ & 9:01:13 AM & 22.91 & 19.594 & 23.544 & 21.529 & 18.595 & -0.121 & -0.07 & -0.062 & 0.058 & -0.179 & 0.228 & 7.979 & 1.192 & 0.001 & & & & & & & & & & & & \\
\hline $3 / 25 / 2003$ & 9:01:14 AM & 22.904 & 19.583 & 23.564 & 1.513 & 18.589 & -0.123 & -0.068 & -0.062 & 0.058 & -0.177 & 0.231 & 37.958 & 1.192 & 0.001 & 0.001 & & & \multicolumn{7}{|c|}{ Note: Gauge Pressure P1 is approximately $1.4 \mathrm{psig}$} & & \\
\hline $3 / 25 / 2003$ & 9:01:15 AM & 22.924 & 19.573 & 23.538 & 21.508 & 18.584 & -0.123 & -0.068 & -0.062 & 0.06 & -0.179 & 0.231 & 37.912 & 1.192 & 0.001 & 0.001 & & & & & & & & & & & \\
\hline $3 / 25 / 2003$ & 9:01:16 AM & 22.939 & 19.578 & 23.574 & 21.523 & 18.574 & -0.123 & -0.068 & -0.062 & 0.06 & -0.177 & 0.231 & 37.956 & 1.192 & 0.001 & 0.001 & & & Pressure & $\mathrm{eP}$ 1is $\mathrm{c}$ & corrected & ted for 40 . & 0.88 inch & hes of wat & ater tubi & & \\
\hline $3 / 25 / 2003$ & 9:01:17 AM & 22.974 & 19.583 & 23.609 & 21.538 & 18.574 & -0.123 & -0.068 & -0.061 & 0.062 & -0.177 & 0.234 & 38 & 1.192 & 0.001 & 0.001 & & & WN & & & & $<$ Filtrat & ate Flux & $\ll$ PERI & RMIABILIT & ITY \\
\hline $3 / 25 / 2003$ & 9:01:18 AM & 22.984 & 19.578 & 23.614 & 21.533 & 18.574 & -0.123 & -0.068 & -0.061 & 0.058 & -0.174 & 0.231 & 37.977 & 1.192 & 0.001 & 0.002 & & & & & & & & at $25 \mathrm{C}$ & & $\times 1000$ & \\
\hline & & & & & & & & & & & & & & & & & ime & Time & Press. & Vel. & TMP & TMP & $\mathrm{gpm}$ & & & $\mathrm{gpm}$ & eter \\
\hline M & Minute & & & & & & & & & & & & & & & & & & & & & & & & & & \\
\hline m & $12: 3$ & .058 & 22.835 & 2 & 24.108 & 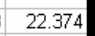 & 3 & 16.927 & 5.161 & 7366 & -0.951 & -1.998 & 9108 & 0.567 & 0001 & & & & & & 100 & 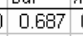 & 0.085 & 0.086 & & 991 & \\
\hline $3 / 25 / 2003$ & $12: 39: 29 \mathrm{PM}$ & 941 & 22.827 & 24.338 & 24.111 & 22.382 & 12.733 & 17.026 & 4.999 & 7.772 & -0.94 & $\begin{array}{l}-1.99 \\
\end{array}$ & 48.772 & 0.568 & & & 1.00 & 667 & 15.6 & 12.0 & 10.3 & 0.707 & 0.085 & 0.086 & 0.0084 & 116 & 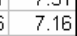 \\
\hline $3 / 25 / 2003$ & 12:40:29 PM & & 22.819 & 24.17 & 24.169 & 22.379 & 3.454 & 17.275 & 4.98 & -1.545 & 8.648 & 18.177 & & & & & 2.00 & 0.03333 & 15.8 & 11.8 & 1.0 & 0.066 & 0.000 & 0.000 & 0.0003 & 320 & 0.27 \\
\hline $3 / 25 / 2003$ & $12: 41: 29 \mathrm{PM}$ & & 22.823 & 24.027 & 24.167 & 22.362 & 3.276 & 17.072 & & & 8.648 & 145 & & 02 & & & 3.00 & 0.05 & 15.6 & \begin{tabular}{|l|l|}
11.7 \\
\end{tabular} & 0.8 & 0.055 & 0.000 & 0.000 & 104 & 0.381 & $\begin{array}{ll}1 & 0.32 \\
\end{array}$ \\
\hline $3 / 25 / 2003$ & $12: 42: 29 \mathrm{PM}$ & & 22.8 & 23.889 & 99 & 22.38 & 10.579 & 17.043 & & & 1.2 & & & & & & 4.00 & 0.06667 & 15.6 & 11.8 & 8.1 & 0.559 & 0.079 & 0.082 & & 10.106 & \\
\hline $3 / 25 / 2003$ & $12: 43: 29 \mathrm{PM}$ & & 22.804 & & 24. & 22.408 & 10.6 & 17. & & & & & & & & & 5.00 & & 6 & 11.8 & 8.1 & & & & 0 & 25 & \\
\hline & & & 22.8 & & & 22.4 & & & & & & & & & & & $\frac{3.00}{6.00}$ & 0.1 & & \begin{tabular}{|l|}
11.0 \\
11.8
\end{tabular} & $\frac{0.1}{9.8}$ & & & & & $\frac{25}{10}$ & \\
\hline & & & & 23.607 & & 27 & & & & & & & & & & & 7.00 & & & 1.8 & 10.1 & & & & & & 1 \\
\hline & $12: 46: 29 \mathrm{PM}$ & 24 & 22.82 & 23.548 & 24.515 & 22.5 & 12.47 & 17.1 & & & & & & & & & 8.00 & & & 11.7 & 10.0 & & & & & & 7.70 \\
\hline $3 / 25$ & 12:47:29 PM & 923 & 22.833 & 23.511 & 24.678 & 22.618 & 12.608 & 17.1 & & 7.657 & -0. & -1 & 47 & 0.5 & & & $\begin{array}{l}0.000 \\
9.00\end{array}$ & 15 & .7 & 11.7 & 10.1 & 0.699 & 0.086 & 0 & 0 & 36 & 7. \\
\hline $3 / 25 / 2003$ & 12:48:29 PM & & 22. & & & & & & & & & -1.8 & & & & & 10.00 & 0.16667 & 6 & 11.7 & 10.3 & & 0.086 & & & & 7.42 \\
\hline $3 / 25 /$ & $12: 49: 29 \mathrm{PM}$ & & 22.8 & 11 & 24. & 22.724 & & 17.2 & & & & & & & & & 11.00 & 0. & & 11.7 & 10.3 & & & & & & \\
\hline $3 / 25$ & $12: 50: 29 \mathrm{PN}$ & & & & & 22.75 & & & & & & & & & & & & 0.2 & & & 3 & & & & & & \\
\hline & & & & 23.591 & & & & & & & & & & & & & & 0.2 & & & 10.4 & & & & & & 7. \\
\hline & & & 22.8 & 23.618 & & 22 & & & & & & & & & & & & & & 8 & 4 & & & & & & $\begin{array}{ll}3.2 \\
3\end{array}$ \\
\hline & $12: 5$ & & 22. & 20 & 24 & & 12 & 17 & & & -0 & & & & & & 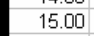 & 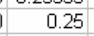 & 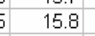 & 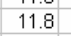 & 4 & 40 & & & & & \\
\hline & & & 22. & 23 & & & & & & & & & & & & & & & & & & & & & & & \\
\hline & 125 & & & 23 & 24.7 & 22 & & & & & & & & & & & & & & 8 & 4 & & & & & & $\begin{array}{l}4.7 . \\
\end{array}$ \\
\hline & $12: 56: 29$ & & & & & & & & & & -0.9 & & & & & & & 0.3 & & 11.8 & & & & & & & $\begin{array}{ll}3 & 6.90 \\
\end{array}$ \\
\hline & $12: 57: 29$ & & 22.9 & 23.9 & 24.7 & & & & & & & & & & & & & 0.31667 & & 11.8 & 10.4 & & & & & 8.209 & \\
\hline & $12: 58: 29$ & & 22.928 & 23.992 & 24.778 & 22.8 & 12. & 17.1 & & & & & 47 & & & & 20.00 & 0.3 & 15.7 & 11.7 & & & & & & & 6 \\
\hline $3 / 25$ & $12: 59: 29 \mathrm{P}$ & $\left.\right|^{25.0900}$ & $\begin{array}{l}22.926 \\
22.939\end{array}$ & 23.992 & 24 & 22.9 & 13. & 17.3 & & & & & & & & & 21.00 & ) & 15.9 & 11.6 & $\begin{array}{l}10.5 \\
10.6\end{array}$ & 0 & & & & 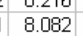 & 88 \\
\hline & $1: 0$ & & 22.929 & & 24. & & & 31. & & & & & & & & & & 0.3 & & 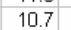 & & & & & & & 7.34 \\
\hline & & & & & & & & & & & & & & & & & & & & & & & & & & & 0.06 \\
\hline $3 / 25 / 2003$ & 1:02:29 F & 24.692 & 22.935 & $\begin{array}{l}24.001 \\
24.976\end{array}$ & 24.84 & 22.90 & 22.652 & 23.433 & 5.384 & 17.404 & $\begin{array}{l}-1.28 \\
-1.28 \\
\end{array}$ & & 51.4 & 0.002 & 1.12 & & 24.00 & 0.4 & 22.0 & 12.6 & 20.0 & 1.381 & 0.000 & 000 & 0.0000 & 0.015 & 5 \\
\hline & & & & & & & & & & & & & & & & & & & & & & & & & & & \\
\hline & & & & & & & & & & & & & & & & & & & 17.4 & & & & & & & & 6.2 \\
\hline & & 25.1 & 22 & 25 & 25.0 & 22 & 35 & 39 & 5 & 31.4 & 8. & & & & 1. & & 24 & 0.4 & 38.1 & 12.6 & 33.5 & 2 & 0. & 9 & 0 & 10 & 8.6 \\
\hline & & 23.9 & & & 24.8 & 22 & 12.8 & 17 & 4. & & -0. & & & & 0. & & 12.0 & 0.2 & 15.7 & 11.8 & 10.3 & 0 & 4 & 6 & 0 & & 7.2 \\
\hline & & 23 & 22.8 & 23. & & 22 & 3.3 & 9 & & & & & & & & & 0.0 & 0.0 & 15.5 & \begin{tabular}{|l|}
10.7 \\
\end{tabular} & 0.8 & & & & & & 0.0 \\
\hline & & & & & & & 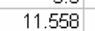 & & & & & & & & & & & & & & & & & & & & \\
\hline & & 25 & 25 & 25 & 25 & & 25 & 25 & 25 & 25 & 25 & & 25 & 25 & 2 & 25 & 25 & & & & & & & & & 25 & \\
\hline
\end{tabular}

Figure B42: Run 1.33A - Baseline Slurry - After AN-102R2 Batch 4A - Raw \& Calculated Data 
WSRC-TR-2003-00204, REV. 0

SRT-RPP-2003-00087, REV. 0

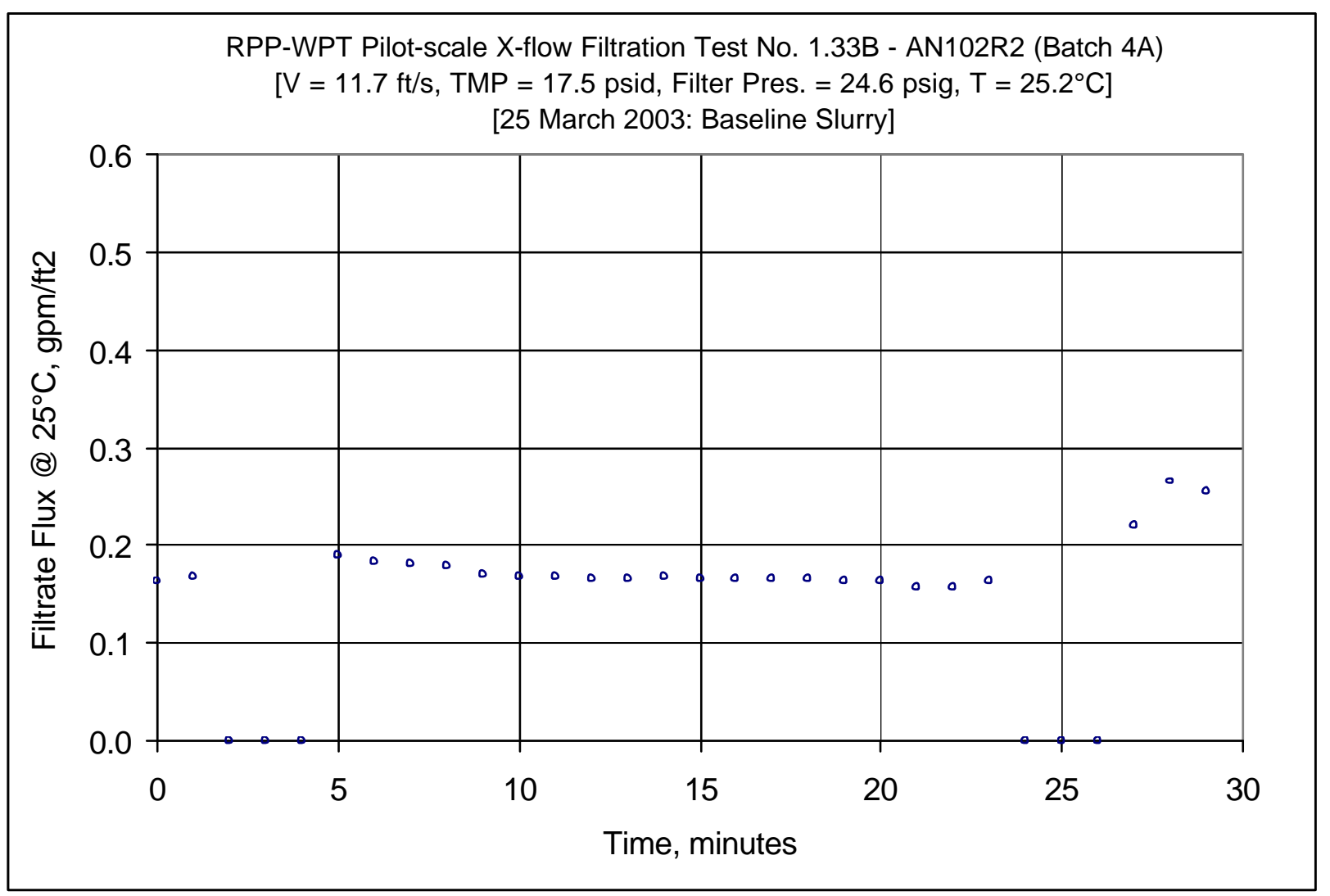

Figure B43: Run 1.33B - Baseline Slurry - After AN-102R2 Batch 4A 
WSRC-TR-2003-00204, REV. 0

SRT-RPP-2003-00087, REV. 0

\begin{tabular}{|c|c|c|c|c|c|c|c|c|c|c|c|c|c|c|c|c|c|c|c|c|c|c|c|c|c|c|c|}
\hline \multirow{3}{*}{ DATE } & \multirow{3}{*}{ TIME } & \multicolumn{5}{|c|}{ 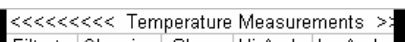 } & \multicolumn{6}{|c|}{ 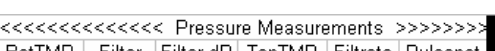 } & \multirow{2}{*}{\multicolumn{4}{|c|}{ 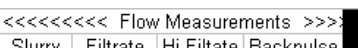 }} & \multicolumn{11}{|c|}{ 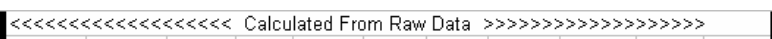 } \\
\hline & & Filtrate & Cleaning & Slurry & Hi Amb. & Lo Amb. & BotTMP & Filter & Filter $d P$ & TopTMP & Filtrate & Pulsepot & Slurry & Filtrate & & & & & & & & & & & & & \\
\hline & & $\operatorname{deg} C$ & $\operatorname{deg} C^{\circ}$ & $\operatorname{deg} \mathrm{C}$ & $\operatorname{deg} \mathrm{C}$ & $\operatorname{deg} C$ & psid & psig & psid & psid & psig & psig & gpm & gpm & $\mathrm{gpm}$ & $\mathrm{gpm}$ & & & & & & & & & & & \\
\hline \multirow{2}{*}{\multicolumn{28}{|c|}{ Zeros }} \\
\hline & & & & & & & & & & & & & & & & & & & & & & & & & & & \\
\hline $3 / 25 / 2003$ & 9:01:12 AM & 22.653 & $\begin{array}{l}19.599 \\
19.599\end{array}$ & $\frac{23.314}{23.314}$ & 21.549 & $\begin{array}{l}18.6 \\
18.6\end{array}$ & $\begin{array}{l}-0.123 \\
-0.123\end{array}$ & $\begin{array}{ll}-0.068 \\
-0.068\end{array}$ & -0.062 & 0.06 & $\begin{array}{l}-0.156 \\
-0.156\end{array}$ & $\begin{array}{l}0.234 \\
0.234\end{array}$ & $\begin{array}{l}3.856 \\
37.838\end{array}$ & $\begin{array}{l}1.192 \\
1.92\end{array}$ & $\begin{array}{l}0.001 \\
0.001\end{array}$ & 0.001 & & & \multicolumn{2}{|c|}{ Conversion } & $\begin{array}{r}6.707 \\
851.01\end{array}$ & $\mathrm{FT} 2$ & 2/daw/ba & 10 & $2 / \mathrm{harg}$ & & \\
\hline $3 / 25 / 2003$ & 9:01:13 AM & 22.91 & 19.594 & 23.544 & 21.529 & 18.595 & -0.121 & -0.07 & -0.062 & 0.058 & -0.179 & 0.228 & 37.979 & 1.192 & 0.001 & & & & & & & & & & & & \\
\hline $3 / 25 / 2003$ & 9:01:14 AM & 22.904 & 19.583 & 23.564 & 21.513 & 18.589 & -0.123 & -0.068 & -0.062 & 0.058 & -0.177 & 0.231 & 37.958 & 1.192 & 0.001 & 0.001 & & & \multirow{2}{*}{\multicolumn{7}{|c|}{ Note: Gauge Pressure P1 is approximately 1.4 psig }} & & \\
\hline $3 / 25 / 2003$ & 9:01:15 AM & 22.924 & 19.573 & 23.538 & 21.508 & 18.584 & -0.123 & -0.068 & -0.062 & 0.06 & -0.179 & 0.2 & 37.912 & 1.192 & 0.001 & 0.001 & & & & & & & & & & & \\
\hline $3 / 25 / 2003$ & 9:01:16 AM & 22.939 & 19.578 & 23.574 & 21.523 & 18.574 & -0.123 & -0.068 & -0.062 & 0.06 & -0.177 & 0.231 & 37.956 & 1.192 & 0.001 & 0.001 & & & Pressur: & re $\mathrm{P} 1$ is $\mathrm{C}$ & $s$ correcte & ed for 40 & $0.88 \mathrm{incl}$ & ches of wat & ater tubing & & \\
\hline $3 / 25 / 2003$ & 9:01:17 AM & 22.974 & 19.583 & 23.609 & 21.538 & 18.574 & -0.123 & -0.068 & -0.061 & 0.062 & -0.177 & 0.234 & 38 & 1.192 & 0.001 & 0.001 & & & $\mathrm{~m}$ & & & & $<$ Filtr & trate Flux & $\ll$ PERI & RMIABILIT & LITY \\
\hline $3 / 25 / 2003$ & 9:01:18 AM & 22.984 & 19.578 & 23.614 & 21.533 & 18.574 & -0.123 & -0.068 & -0.061 & 0.058 & -0.174 & 0.231 & 37.977 & 1.192 & 0.001 & 0.002 & & & & & & & & at $25 \mathrm{C}$ & & $\times 1000$ & \\
\hline & & & & & & & & & & & & & & & & & & Time & Press. & Vel. & TMP & TMP & & -2 & & & eter \\
\hline Data - Per M & Ainute & & & & & & & & & & & & & & & & & & & & & & & & & & \\
\hline 03 & 1:03:12 PM & 798 & 22.92 & 25.096 & 765 & 912 & 22.917 & 7 & .99 & 971 & -1.286 & -5.192 & .126 & 0.002 & 997 & & & & 3 & 12.0 & 20.4 & 1.4 & 0. & 4 & & & \\
\hline $3 / 25 / 2003$ & 1:04:12 PM & 24.933 & 22.93 & 25.146 & .735 & $\begin{array}{l}22.922 \\
\end{array}$ & 23.337 & 24.106 & $\begin{array}{l}4.952 \\
4.957\end{array}$ & 18.48 & $\begin{array}{l}-1.280 \\
-1.289 \\
\end{array}$ & -5.184 & 48.285 & 22 & 28 & 0.002 & 1.00 & 11667 & 22.6 & 11.8 & 20.9 & $\begin{array}{l}1.440 \\
1.442\end{array}$ & 20.168 & 167 & 0.0080 & 010 & 6.82 \\
\hline $3 / 25 / 2003$ & 75:12 PM & 24.957 & 22.929 & 25.196 & 24779 & 22.906 & 3.348 & 24.457 & 483 & & 15.992 & 21432 & 47.197 & & & & 2.00 & 0.03333 & $\begin{array}{l}3 \\
3\end{array} 23.0$ & 11.6 & 1.0 & 0.066 & 0.000 & 0.000 & & 155 & $5 \quad 0.13$ \\
\hline $3 / 25 / 2003$ & 1:06:12 PM & 24.892 & 22.924 & 25.23 & 24.729 & 22.9 & 3.197 & 24.312 & 4.862 & -1.733 & 16.013 & 21.192 & 47.321 & & 11 & & 3.00 & 0.05 & 22.8 & 11.6 & 0.7 & 0.050 & 0.000 & 0.000 & 0.0002 & 0.202 & $\begin{array}{ll}2 & 0.17\end{array}$ \\
\hline $3 / 25 / 2003$ & 1:07:12 PM & & 22.938 & 25.33 & 24.753 & 22.905 & 3.39 & 24.534 & 4.808 & -1.375 & 16.091 & 002 & 46 & & 0.0 & 04 & 4.00 & 0.06667 & 23.1 & 11.5 & 1.0 & 0.069 & 0.000 & 0.000 & 0.0001 & \begin{tabular}{|l|l|} 
& 0.147 \\
\end{tabular} & 0.12 \\
\hline $3 / 25 / 2003$ & 1:08:12 PM & & 22.932 & 25.398 & 24.727 & 22.9 & 22.981 & 24.238 & & & -1.289 & & & & & & 5.00 & 0.08333 & 22.8 & 11.7 & 20.7 & 1.426 & & 89 & & & \\
\hline $3 / 25 /$ & $99: 1$ & 25.274 & 22.93 & 25.452 & & & 23.01 & & 4. & & & & & & & & $\frac{3.00}{6.00}$ & 0.1 & 122.6 & \begin{tabular}{|l|l|}
11.7 \\
\end{tabular} & $\frac{20.8}{20.8}$ & & & & & & \\
\hline $3 / 25$ & $10: 12 \mathrm{P}$ & 25.343 & 22.925 & 25.461 & 24 & 22. & 23.113 & 24. & 47. & & & & & & & & 7.00 & 0. & 22.7 & 11.7 & 20.8 & & & & & & \\
\hline $3 / 25 / 2003$ & 1:11:12 PM & 397 & 22.923 & 25.475 & 24.794 & 22.9 & 23.372 & 24.416 & & & -1.286 & 0.468 & 47.423 & & 1.208 & & 8.00 & 33 & $\begin{array}{ll}3 & 22.9 \\
\end{array}$ & 11.6 & 21.1 & $\begin{array}{l}1.452 \\
\end{array}$ & 20.180 & 78 & & & \\
\hline $3 / 25 / 2003$ & 1:12:12 PM & 25.446 & 22.927 & 25.509 & 24.722 & & 22.792 & 23.7 & & & -1.289 & & & & 1.153 & & 9.00 & 0.15 & $\begin{array}{ll}5 & 22.2 \\
\end{array}$ & 12.1 & 0.3 & 1.401 & 0.172 & $\begin{array}{ll}2 & 0.169\end{array}$ & & 341 & 10 \\
\hline $3 / 25 / 2003$ & $13: 12 \mathrm{PM}$ & & 22.932 & 25.553 & 24.767 & 22.9 & 22.828 & 23.682 & & 17.989 & -1.2 & & 23 & & 3 & & 10.00 & 0.16667 & $7 \quad 22.2$ & 12.0 & 20.4 & 1.407 & 0.170 & 0.168 & 0.8 & 8.221 & $\begin{array}{ll}1 & 7.00\end{array}$ \\
\hline $3 / 25 / 2003$ & 1:14:12 PM & & 22.93 & 25.607 & 24.78 & 22 & 22.987 & 23.828 & & & & & & & & 0.00 & 11.00 & 0.18333 & 22.4 & 12.0 & 20.5 & & & & & & 6.91 \\
\hline $3 / 25 / 2003$ & 1:15:12 P & & & & 24.7 & & 23.103 & 23.8 & & & & & & & & & & 0.2 & $2 \quad 22.4$ & 11.9 & & & & & & & \\
\hline $3 / 25$ & 1:16:12 P & & 22. & & & & & & & & & & & & & & & 0. & & 11.9 & & & & & & & \\
\hline & 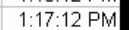 & 2 & 22.9 & & 24.7 & & & 24. & & & & & & & & & & & & 11. & & & & & & & 16 \\
\hline & 1:18:1 & & 22.923 & 25.689 & & & 23.55 & & & & & & & & & & & & & 11.6 & $\frac{2}{3}$ & & & & & & \\
\hline $3 / 25 / 2003$ & 1:19:12 P & 5 & 22.921 & 25 & 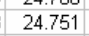 & & 23.391 & 24.2. & & & -1. & & & & & & b & 0.26667 & 22.7 & 11.6 & 1 & 1.453 & & & & & \\
\hline & 1:20:12 & & 22. & & & & & & & & & & & & & & & 0 & 9 & 11.6 & & & & & & & \\
\hline $3 / 25$ & $1: 21: 12 \mathrm{~F}$ & & 22.9 & & & & & 24.2 & & & & & & & & & & 0.3 & & 11.6 & & & & & & & \\
\hline & 1:22:12 & & 22.8 & & & & & & & & & & & & & & & 0.3 & & 11.5 & & & & & & & 9 \\
\hline & 1:23:12 & & 22.9 & & & & & 24. & & & & & & & & & & & & 11.6 & & & & & & & 51 \\
\hline & $1.24: 1$ & & 22.9 & 25 & 25.098 & 23 & 22. & 24. & & & \begin{tabular}{|l}
-1.2 \\
-1.2
\end{tabular} & & & & & & & & $\begin{array}{l}5 \\
5 \\
5\end{array}$ & 11.6 & & & & & & & ja \\
\hline & 1:25:1 & & 22.916 & 24 & 25.1 & 23. & 23.1 & 24.8 & & & & & & & & & & & 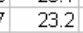 & 11 & & & & & & & \\
\hline & 1:26: & 25 & 22.92 & & 25 & & 23. & 24. & & & & & & & & & & & 5 & 11 & & & & & & & \\
\hline & 1:27:1 & & & & & & & & & & & & & & & & & & & & 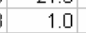 & & & & & & \\
\hline & 1:28:1 & & 22. & & & & & & & & & & & & & & & 0.4 & & 11 & 1.1 & & & & & & 0. \\
\hline & 1:29:12 & & 22.937 & 24.357 & 25.323 & 23.0 & -5.2 & 24.8 & & & 25.1 & & & & & & & 0.43333 & $\begin{array}{l}3 \\
3\end{array} 23.4$ & 11.1 & -7.6 & & & & & -0.020 & $\begin{array}{l}\mid \\
-0.02\end{array}$ \\
\hline & 1:30:12 & & 22.927 & 24.322 & 25 & 23.1 & 28.469 & 37.855 & & 24. & 4.0 & & 46. & & & & 27.00 & 0.45 & $\begin{array}{ll}5 & 23.4 \\
\end{array}$ & 11.3 & 26.4 & 1 & 0 & 0 & 0 & 8.340 & 3 \\
\hline $3 / 25$ & $1: 31: 12$ & 24.588 & 22.931 & 24.501 & 25. & 23.1 & 34. & 45.4 & 5.7 & 28. & 5.8 & & 53 & & & & 28.00 & 0.46667 & .0 & 13.0 & 31.5 & 2.174 & 40.2 & 5 & 84 & 92 & 7.14 \\
\hline $3 / 25 / 2003$ & 1:32:12 PM & 24.681 & 22.959 & 24.634 & 25.465 & 23.22 & 33.459 & 44.311 & 4.877 & 28.82 & 5.48 & 33.63 & 48.266 & 0.002 & 1.688 & & 29.00 & 0.48333 & 42.8 & 11.8 & 31.1 & 2.147 & 0.252 & $\begin{array}{ll}2 & 0.254\end{array}$ & 0.0082 & 8.166 & 6.95 \\
\hline & & & & & & & & & & & & & & & & & & & & & & & & & & & \\
\hline & & & & & & & & & & & & & & & & & & 0 & & & & & & & & & 8 \\
\hline & & & & & & & & & & & & & & & 1. & & 14.5 & 0.2 & 22.9 & 11.6 & 20.8 & & & 5 & & 8.839 & 6.7 \\
\hline & Minimum & 24.0 & 22.9 & 24.3 & 24.7 & 22 & -5.3 & 23.7 & 4.4 & -9.9 & -1.3 & & 45.4 & 0 & 0 & & 0.0 & 0.0 & 22.2 & 11.1 & -7.6 & -0.523 & 0.000 & 0.000 & 0.0000 & -0.020 & 0.0 \\
\hline & & 0.956 & 0.021 & 0.897 & 0.468 & 0.156 & 18.996 & 11.181 & 0.443 & .966 & 15.291 & .6 & 2.7 & 0.001 & 1.01 & 0.427 & 17.311 & 0.289 & 911.181 & 0.670 & 18.980 & 1.309 & 90 & $\begin{array}{ll}1 & 0.150\end{array}$ & 0.0064 & 6.394 & 4 \\
\hline Tvumt & & 30 & 30 & 30 & 30 & 30 & 30 & 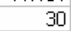 & 30 & 30 & 30 & & 30 & 30 & 30 & 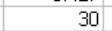 & 30 & & & & 30 & & & 30 & . & 30 & \\
\hline
\end{tabular}

Figure B44: Run 1.33B - Baseline Slurry - After AN-102R2 Batch 4A - Raw \& Calculated Data 
WSRC-TR-2003-00204, REV. 0

SRT-RPP-2003-00087, REV. 0

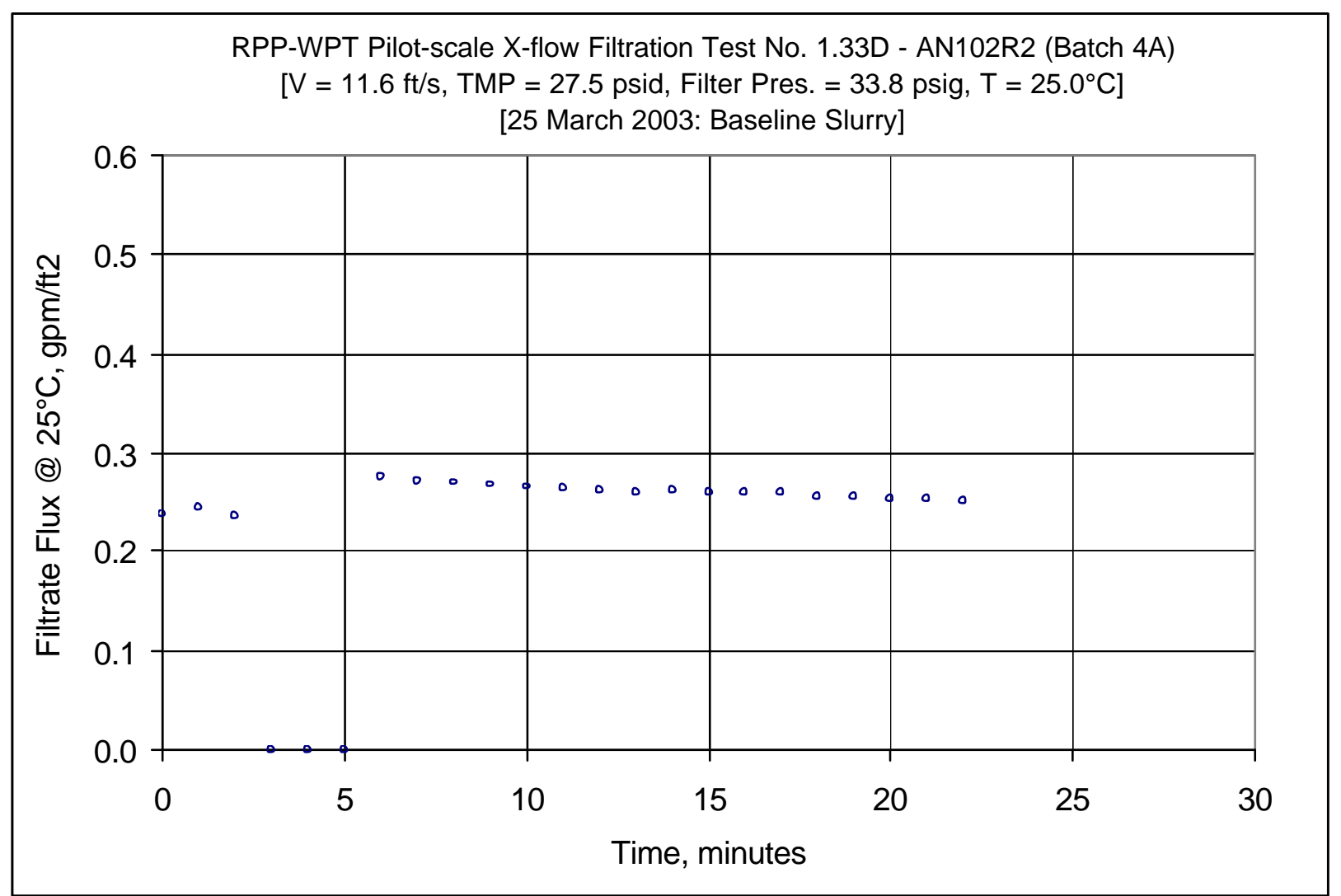

Figure B45: Run 1.33D - Baseline Slurry - After AN-102R2 Batch 4A (replaced 1.33C which had control problems) 
WSRC-TR-2003-00204, REV. 0

SRT-RPP-2003-00087, REV. 0

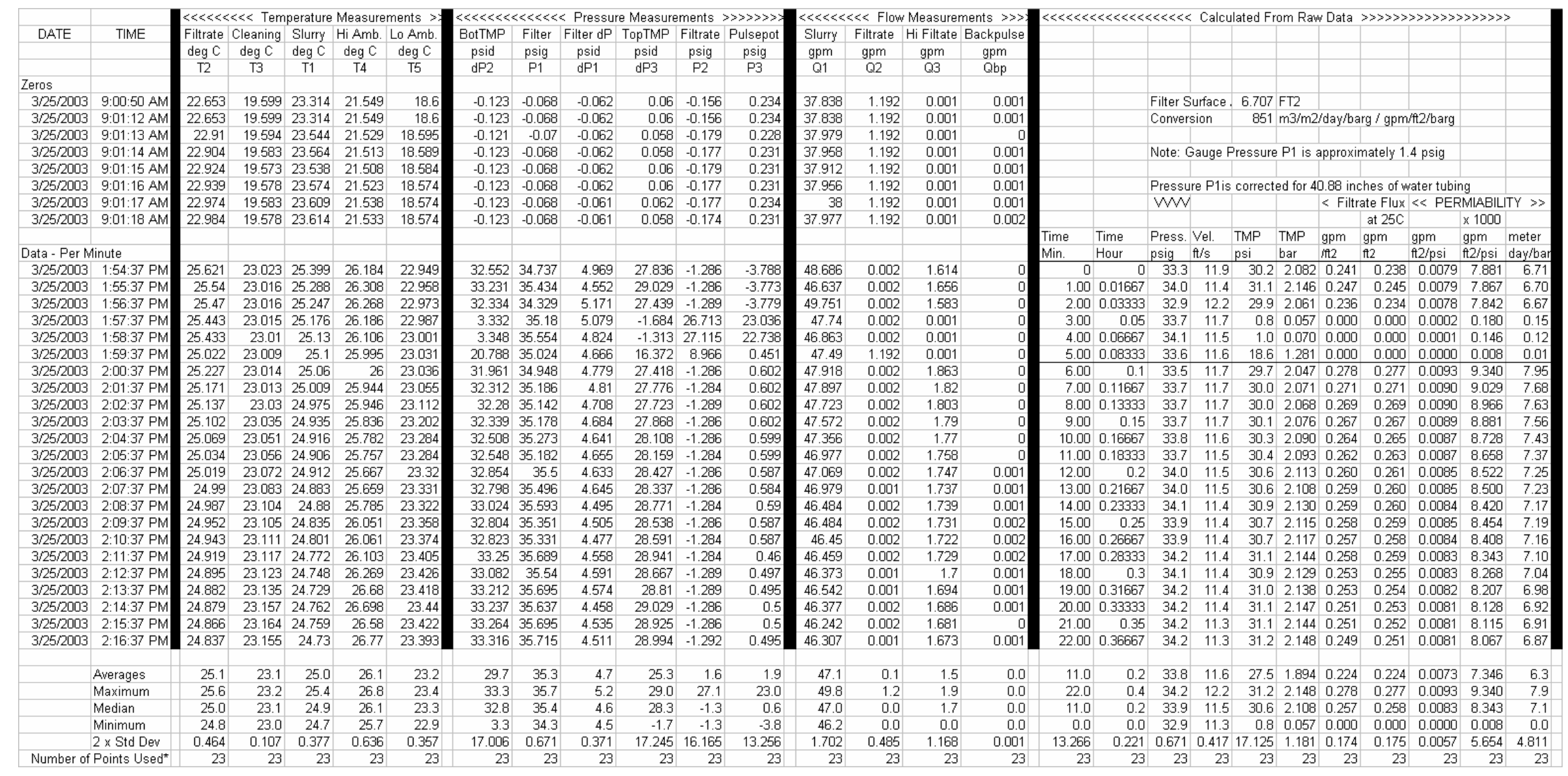

Figure B46: Run 1.33D - Baseline Slurry - After AN-102R2 Batch 4A (replaced 1.33C which had control problems) - Raw \& Calculated Data 
WSRC-TR-2003-00204, REV. 0 SRT-RPP-2003-00087, REV. 0

\section{Appendix C: 2 M Nitric Acid Cleaning}

As required by the RPP-WTP Test Specification each time the cross-flow filter experienced a slurry run the filter was to be cleaned with $2 \mathrm{M}$ Nitric Acid. Those tests were noted in the test matrix as runs $1.31 \mathrm{~B}$ and $1.31 \mathrm{C}, 1.31 \mathrm{~A}$ was a water rinse that began the process of cleaning. (Note that prior to slurry testing the filter was preconditioned with an AN-107 simulant slurry during which the acid cleaning steps were referred to as 5B and 5C.) Since the slurries tested for this task were (in chronological order): an AN-107simulant slurry (to precondition the filter) and $\mathrm{AN}-102 \mathrm{R} 2$ simulant slurries, Batches $3 \mathrm{C}, 3 \mathrm{~B}, 3 \mathrm{C}$, and 4, the filter was cleaned with acid at least 5 times. This Appendix includes the following cleaning test runs

$\begin{array}{ll}\text { 5B, 5C } & \text { After Preconditioning the filter with AN-107 } \\ \text { Clean 1, Clean } 2^{\dagger} & \text { After AN-102R2, batch 3C (using the cleaning loop) } \\ 1.31 \mathrm{~B}, 1.31 \mathrm{C} & \text { After AN-102R2, batch 3B } \\ 1.31 \mathrm{~B}, 1.31 \mathrm{C} & \text { After AN-102R2, batch 3A } \\ \text { ACIDA \& ACIDB } \text { A }^{\ddagger} & \text { Before AN-102R2, batch 4A (4-day acid soak) } \\ 1.31 \mathrm{~B}, 1.31 \mathrm{C} & \text { After AN-102R2, batch 4A }\end{array}$

The letter B and C (or numbers 1 and 2) simply indicate different cleaning cycle. When this task was done there was no set protocol for cleaning. The only requirement was to use a nitric acid with a concentration of 2 molar. While there were some exceptions, as shown in the data in this Appendix, in general, the filter was cleaned by the following method:

1. Rinse the filter once with water (test $1.31 \mathrm{~A})$.

2. Circulate 100 liters of 2 M HNO3 for 90 minutes at a circulation axial velocity of 11 to $12 \mathrm{ft} / \mathrm{s}$ and a transmembrane pressure of 20 psid, then discard the 100 liters.

3. Repeat step 2 once.

4. Rinse with water (test 1.32)

These cleaning steps were basically arbitrarily chosen. At the time of this task some bench top tests used two 90-minute steps, however, there seemed to be no set rule. For instance, some bench-top tests allowed the filter to soak for several days in acid to bring it to a very clean condition. For this test it was thought more important to clean the filter similar to how the actual plant may operate. To spend several days cleaning the filter did not seem practical. The exception is between slurry test AN-102R2, batch 3A and 4 during which the filter sat idle for almost 3 months. Just before batch 4 the filter was given a much more thorough cleaning by letting it soak over a 4-day period before use.

Each acid-cleaning test in this Appendix contains a graph of the filtrate flux with time and tables of the data taken and calculated. To better understand the presented information important aspects of the graphs and tables are explained below:

Graphs - Each graph contains time averages of some of the imporant parameters taken during the run at the top of the figures, i.e. Slurry velocity in the filter tube, V; transmembrane pressure, TMP; slurry pressure at the entrance of the filter tube, Filter Pres; and the slurry temperature, T.

Tables - Each table is divided into raw data (the first 17 columns) and calculations (the last 10 columns) which are based on the raw data. The raw data section is divided into three parts:

$\dagger$ Clean 1 and Clean 2 would have been called $1.31 \mathrm{~B}$ and $1.31 \mathrm{C}$, like the other test run cleaning, but a different nomenclature was given to those cleaning because the there were pump lining problems, which forced the use of the Test Rig cleaning loop. The cleaning loop had a much smaller pump then the Test Rig, therefore the nitric acid solution circulation velocity and transmembrane pressure could not meet the test specification requirements of $12 \mathrm{ft} / \mathrm{s}$ and $20 \mathrm{psid}$, respectively. The actual values used are listed in this Appendix.

* Because the filter sat idle, submerged in inhibited water, for 3 months between test $3 \mathrm{~A}$ and $4 \mathrm{~A}$ it was more extensively cleaned. 


\title{
WSRC-TR-2003-00204, REV. 0
} SRT-RPP-2003-00087, REV. 0

\author{
Individual Entries - Raw Data \\ Temperature Filtrate - from the filtrate loop at the exit of the filter bundle \\ Cleaning - from the cleaning loop (this loop was not used, exc ept for tests Clean $1 \& 2$ ) \\ Slurry - from the slurry loop in the slurry reservoir \\ Hi Amb. - outside ambient temperature at the top of the test rig \\ Lo Amb. - inside ambient temperature at the bottom of the test rig \\ Pressure $\quad$ BotTMP - differential pressure across the filter tube at the bottom of the vertical tube \\ Filter - gauge pressure of the slurry at the entrance of the filter tube bundle \\ Filter $\mathrm{dP}$ - differential pressure of the slurry along the height of the vertical 90-inch tube \\ TopTMP - differential pressure across the filter tube at the top the vertical 90-inch tube \\ Filtrate - gauge pressure of the filtrate at the exit of the filter tube housing \\ Pulsepot - gauge pressure of the filtrate at the bottom of the pulsepot \\ Flow Slurry - rate of slurry just before entering the seven-filter-tube bundle \\ Filtrate - rate of slurry filtrate under normal flow (max. is $1.2 \mathrm{gpm}$ ) \\ Hi Filtrate - rate of any liquid which has a high flowrate (water, acid, etc.) (max. $5 \mathrm{gpm}$ ) \\ Backpulse - rate of filtrate in opposite direction from normal flow, i.e., backpulse
}

Individual Entries - Calculated Data

Time - Minutes after start of test, including the backpulse (based on raw data TIME column)

Press. - Internal filter pressure of slurry at the filter bundle entrance (based on raw data P1 column and corrected for a 40.9-inch water column that exists from the transducer to the point where P1 is measured.)

Vel. - Slurry velocity in filter tube (based on raw data Q1 column and i.d. of the tubes of 0.488 inches)

TMP - Filter transmembrane pressure (based on average of raw data columns dP2 and dP3) in psi.

TMP - Filter transmembrane pressure (based on average of raw data columns dP2 and dP3) in bar.

Filtrate Flux - Filtrate flow rate (raw data columns Q2/Q3) divided by inside filter surface area $(6.707 \mathrm{ft} 2)^{\dagger}$

Filtrate Flux @ $25^{\circ} \mathrm{C}$ - Same as Filtrate flux, but correct for temperature with the following multiplier:

$\mathrm{T}($ corrected $)=\mathrm{T} \times \operatorname{Exp}[2500 /((1 / 273+\mathrm{T}))-(1 / 298)]$ as per Test Spec 24590-WTP-TSP-RT-01-029, Rev. 0

Permiability - Filterate flow rate per TMP (based on calculated columns Filtrate Flux @ $25^{\circ} \mathrm{C}$ and TMP)

Permiability x 1000 - Same as the above Permiability, but increase by 1000 to facilitate graphing

Permiability - Same as Permiability but in metric units, meter/day/bar

$\underline{\text { Statistics }}$

At the bottom of the last individual entry there are six rows with some data statistics to better understand the data and their fluctuations. The columns are self explained with titles of Averages, Maximum, Median, and Minimum. The next row $2 \times$ Std Dev, is two times the population standard deviation based on the number of data points used, which is the last row. The Number of Points used generally do not included the points taken during the backpulse sequence of each test. This is explained below.

\section{Raw Data Table Layout}

The raw data is generally made up of three sections:

Zeros - These eight rows include approximately 15 to 20 seconds of data taken with the pressure transducers placed in the "zero" mode to determine if any zero drift occurred from one test to the next.

Data-Per Minute - On most of the tables the first six rows of these data are contained in a box which indicate data taken during the backpulse. This is were the filtrate flow is reversed temporarily to knock the filtrate cake of the filter surface. During these first few minutes the filtrate flow is stopped, then reversed, then stopped again, then it is finally returned to normal operation. The data during the backpulse operation

${ }^{\dagger}$ For the acid-cleaning tests during preconditioning it will be noticed that $6.87 \mathrm{ft} 2$ was used. This was based on a tube inside diameter of 0.5 inch, which was based on the manufacturer's specification. After the tubes were measured the i.d. was changed to 0.488 inch and the area to $6.707 \mathrm{ft} 2$. This means that absolute axial velocity and filtrate flux are low by $2.5 \% \%$ for the preconditioning runs. 
WSRC-TR-2003-00204, REV. 0

SRT-RPP-2003-00087, REV. 0

were taken with a separate log, therefore those data are marked with a box to note this fact. Most or all of those data points are not counted in the statistics at the bottom of the table. The tables of some of the later tests do not show the boxed data because separate logs of data were not taken, however since a backpulse for each run still occurred only those data were not included in the statistics at the bottom of the page. 
WSRC-TR-2003-00204, REV. 0

SRT-RPP-2003-00087, REV. 0

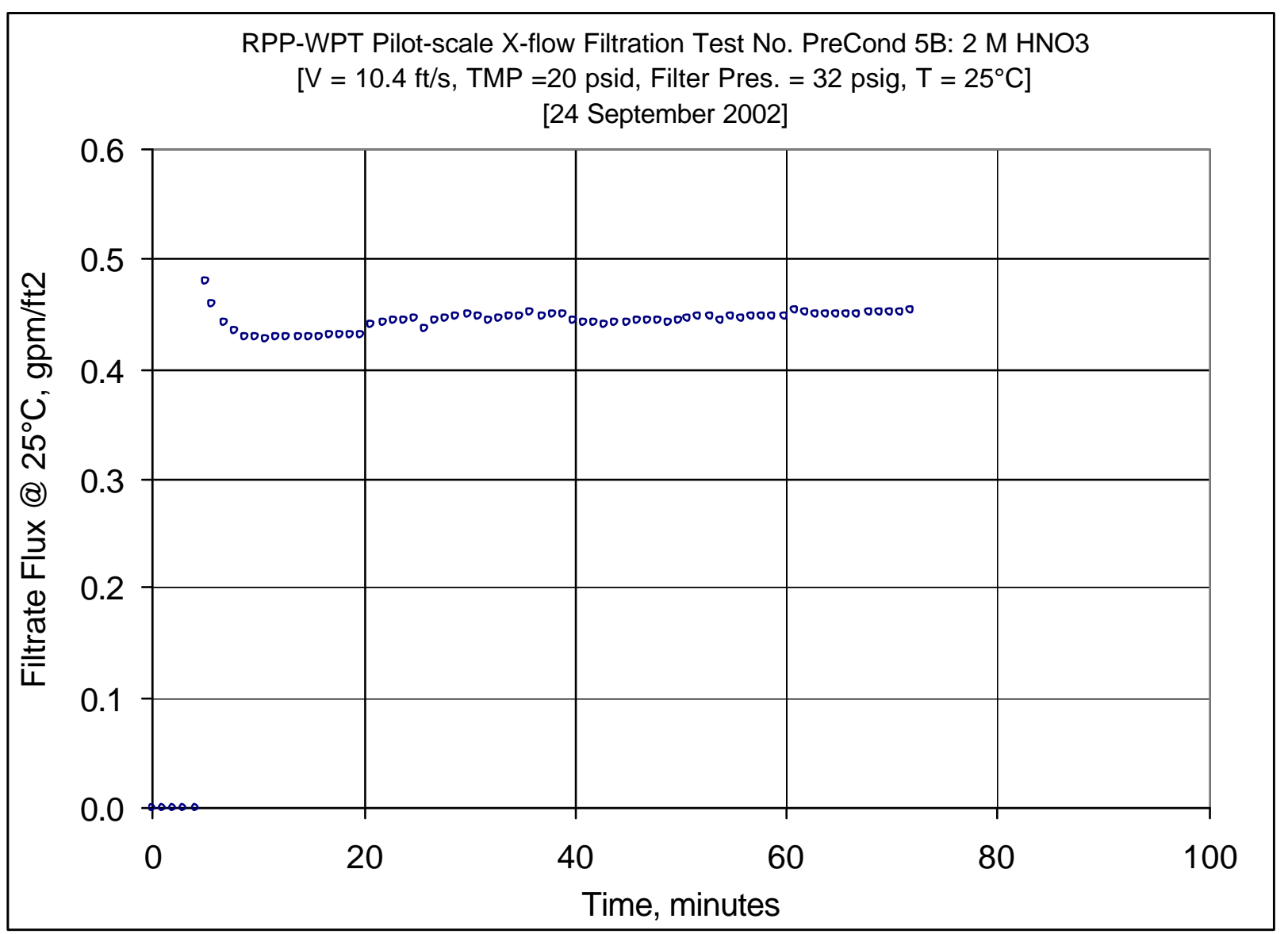

Figure C1: PreConditioning 5B - 2 M Nitric Acid Cleaning - FirstCleaning 
WSRC-TR-2003-00204, REV. 0

SRT-RPP-2003-00087, REV. 0

\begin{tabular}{|c|c|c|c|c|c|c|c|c|c|c|c|c|c|c|c|c|c|c|c|c|c|c|c|c|c|c|}
\hline \multirow{2}{*}{ DATE } & \multirow{2}{*}{ ME } & & $\begin{array}{l}\ll \ll \text { Tem } \\
\text { Cleaning }\end{array}$ & Slurry & Hi Amb. & Lo Amb. & BotTMP & Fiter & Filter dP & TopTMP & Filtrate & Pulsepot & \multicolumn{4}{|r|}{ kpulse } & & Calcula & llated Fro & rom Raw D & Data 》> & 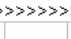 & 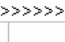 \\
\hline & & & $\operatorname{deg} C$ & $\operatorname{deg} C$ & $\operatorname{deg} C$ & $\operatorname{deg} C$ & & psig & psid & & psig & & & $\mathrm{gpm}$ & $\mathrm{gpm}$ & & & & & & & & & & & \\
\hline & & & & & & & $\mathrm{dP} 2$ & $\mathrm{P1}$ & $\mathrm{dP1}$ & $\mathrm{dP} 3$ & P? & 3 & Q1 & 02 & 03 & & & & & & & & & & & \\
\hline 002 & & & & & & & & & & & & & & & & & & & & & & & & & & \\
\hline $\begin{array}{l}9 / 2 / 242002 \\
9 / 24 / 2002\end{array}$ & $\begin{array}{l}7: 3: 313 \mathrm{AM} \\
7: 33: 28 \mathrm{AM}\end{array}$ & & $\begin{array}{l}321.149 \\
21.149\end{array}$ & $\begin{array}{l}22.146 \\
22146\end{array}$ & $\begin{array}{l}21.953 \\
21.953\end{array}$ & $\begin{array}{l}20.713 \\
20713 \\
\end{array}$ & $\begin{array}{l}-0.109 \\
-0.109\end{array}$ & \begin{tabular}{|l|l|}
-0.012 \\
-0.012
\end{tabular} & $\begin{array}{l}-0.0499 \\
-0.049\end{array}$ & 0.0722 & $\begin{array}{r}-0.324 \\
-0.324\end{array}$ & $\begin{array}{l}0.356 \\
0.356\end{array}$ & $\begin{array}{l}34.338 \\
34338\end{array}$ & 0.031 & $\begin{array}{l}0.001 \\
0.001\end{array}$ & & & Filter Su & Surface & 6.87 & FT2 & & & & & \\
\hline 9/24/2002 & 7:33:29 AM & 21.8 & $\begin{array}{ll}37.149 \\
\end{array}$ & 22.186 & 21.928 & 20.723 & -0.109 & \begin{tabular}{|l|}
-0.012 \\
-
\end{tabular} & $\begin{array}{l}-0.049 \\
-0.05\end{array}$ & $\begin{array}{l}0.072 \\
0.072\end{array}$ & \begin{tabular}{|}
-0.024 \\
-0.321 \\
\end{tabular} & 0.356 & $\begin{array}{l}34.538 \\
34.269\end{array}$ & 0.031 & 0.001 & & & & & & & & $g / g p$ & zorarg & & \\
\hline $9 / 24 / 2002$ & 7:33:30 AM & $21.79 \mathrm{~S}$ & 21.144 & 22.186 & 21.918 & 20.717 & -0.109 & -0.012 & -0.049 & 0.072 & -0.35 & & \begin{tabular}{|l|}
34.313 \\
\end{tabular} & 0.031 & 0.001 & & & Note: $G$ & Gauge & Press & ure P1 & ap & natel & $4 \mathrm{psig}$ & & \\
\hline $24 / 2002$ & 7:33:31 AM & 21. & 21.149 & 22.201 & 21.923 & 20.723 & -0.109 & \begin{tabular}{|l|l|} 
& -0.012 \\
\end{tabular} & -0.049 & 0.072 & -0.31 & & 34.399 & 0.029 & 0.001 & & & & & & & & & & & \\
\hline $9 / 24 / 2002$ & 7:33:32 AM & 21.79. & 21.144 & 22.191 & 21.923 & 20.717 & -0.109 & \begin{tabular}{|l|l|} 
& -0.012 \\
\end{tabular} & -0.049 & 0.072 & -0.341 & & 34.524 & 0.031 & 0.001 & & & Pressur & ure P1 & s correc & ctes & & & tu & tut & \\
\hline & 7:33:33 AM & $21.79 \mathrm{r}$ & $\begin{array}{l}41.149 \\
\end{array}$ & 22.191 & 21.918 & 20.717 & -0.111 & -0.012 & -0.049 & 0.072 & -0.321 & & 34.671 & 0.031 & 0.001 & & & W & & & & < Filtral & rate $F$ & PEF & ERM/ABIL & \\
\hline $9 / 24 / 2002$ & 7:33:34 AM & 21.805 & $5 \quad 21.154$ & 22.196 & 21.928 & 20.723 & -0.111 & -0.012 & -0.049 & 0.072 & -0.364 & 0.356 & 34.546 & 0.03 & 0.001 & & & & & & & & at $25 \mathrm{C}$ & & $\times 1000$ & \\
\hline & & & & & & & & & & & & & & & & & & Press. & Vel. & TMP & TMP & $\mathrm{gpm}$ & & gpm & & meter \\
\hline - Per M & & & & & & & & & & & & & & & & & & & & & & & & & & \\
\hline $9 / 24 / 2002$ & 10:21:26 AM & 24.62 & 22.631 & 23.79 & 23.559 & 22.909 & 9.28 & 33.452 & 11.798 & -2.042 & \begin{tabular}{|l|l|}
18.998 \\
\end{tabular} & & 44.441 & 0.002 & 0.001 & & & 32.0 & 10.4 & 3.6 & 0.250 & 0.000 & 0.000 & 0.000 & 0.042 & 20.04 \\
\hline $9 / 24 / 2002$ & 10:22:00 AM & 24.6 & 22.631 & 23.79 & 23.559 & 22.909 & 9.28 & 33.452 & 11.798 & -2.042 & 18.998 & 73.16 & 44.441 & 0.00 & & 0.00 & 1 & 32.0 & 10.4 & 3.6 & 0.250 & 0.000 & 0.000 & 0.000 & 0.042 & $2 \quad 0.04$ \\
\hline $9 / 24 / 2002$ & 10:22:26 AM & 24.7 & 22.636 & 23.846 & 23.543 & 22.889 & 8.837 & 33.698 & 11.3 & -2.042 & 19.666 & $16.4 \mathrm{Cl}$ & & & & 0.007 & & 32.2 & 10.2 & 3.4 & 0.234 & $\begin{array}{ll}4 & 0.000\end{array}$ & 0.000 & 0.000 & 0.044 & 54 \\
\hline $9 / 24 / 2002$ & 10:22:56 AM & 24.472 & 22.646 & 23.956 & 23.549 & 22.874 & 13.226 & 34.015 & 11 & 2.234 & 15.177 & 13.7 & & & & & 3 & 32.5 & 10.4 & 7.7 & 0.533 & 30.000 & 0.000 & 0.000 & 019 & $\begin{array}{ll}9 & 0.02\end{array}$ \\
\hline $9 / 24 / 2$ & 10:23:26 AM & 24.505 & 22.651 & 23.951 & 23.533 & 22.889 & 13.182 & $\begin{array}{l}33.407 \\
\end{array}$ & 11.36 & 2.349 & 14.868 & 13 & 44.237 & & & & & 31.9 & 10.3 & 7.8 & 535 & 50.000 & 0.000 & 000 & & 9 \\
\hline $9 / 4$ & 3:56 AM & 24.52 & 22.651 & 23.976 & 23.508 & 22.884 & 23.345 & 33.223 & 11.525 & 13.367 & 3.647 & -3. & 45.964 & 0.0 & 3.2 & & & 31.8 & 10.7 & 18.4 & 1.266 & 50.466 & 0.479 & & & \\
\hline 9/24/2 & 10:24:41 AM & 24.6 & 22.656 & 24.106 & 23.468 & 22.9 & 24.052 & $\begin{array}{l}33.072 \\
\end{array}$ & 11.588 & 13.862 & 3.014 & & 45.366 & 0.6 & 3.082 & & 5.75 & 31.6 & 10.6 & 9.0 & 307 & 70.448 & $\begin{array}{l}30.460 \\
3\end{array}$ & & & 220. \\
\hline 9/24/2 & 10:25:41 AM & 24.685 & 22.661 & 24.116 & 23.4 & 22.909 & 24.735 & $\begin{array}{l}33.175 \\
\end{array}$ & 11.757 & 14.333 & 2.303 & & & 0.6 & 2.97 & & 75 & 31.7 & 10.7 & 19.5 & 347 & 0.432 & $\begin{array}{l}2 \\
2.443\end{array}$ & & & \\
\hline $9 / 24 / 2$ & 10:26:41 AM & 24.77 & 22.661 & 24.176 & 23.463 & $22.8 \mathrm{Z}$ & 24.973 & 33.128 & $11.6 \mathrm{C}$ & 14.855 & 2.168 & & & 0.0 & 2.922 & 0.002 & 7.75 & 31.7 & 10.6 & 19.9 & 373 & $\begin{array}{l}30.425 \\
\end{array}$ & $\begin{array}{l}5 \\
5\end{array}$ & & & \\
\hline $9 / 24$ & 10:27:41 AM & 24.805 & 22.666 & 24.302 & 23.4 & 22.9 & 25.342 & 33.278 & 11.615 & 15.211 & 1.5 & -3. & 45 & 0.0 & 2.899 & & 8.75 & 31.8 & 10.7 & 20.3 & 1.398 & $\begin{array}{l}30.422 \\
\end{array}$ & 20.430 & & 21.218 & \\
\hline $9 / 24 \lambda$ & 10:28:41 AM & 24.876 & 22.66 & 24.271 & 23.432 & 22. & 25.261 & 33.442 & 11.562 & 14.947 & 1.9 & -3 & & & & 0.022 & 9.75 & 32.0 & 10.6 & 20.1 & 1.386 & 6. 0.421 & $\begin{array}{ll}1 & 0.430\end{array}$ & & 21.375 & \\
\hline $9 / 24$ & 10:29:41 AM & 24.935 & 22.66 & 24.386 & 23.3 & 22.8 & 25. & : 33.392 & $11.6 \mathrm{C}$ & 14.974 & 1.9 & -3. & & & & & 10.8 & 31.9 & 10.6 & 20.2 & 1.392 & 20.421 & $\begin{array}{ll}1 & 0.428\end{array}$ & & 21.207 & \\
\hline $9 / 24 / \lambda$ & 10:30:41 AM & 24.999 & 22.675 & 24.476 & 23.422 & $22.8 \mathrm{P}$ & 25.379 & 33.562 & 11.609 & 15.025 & 1.997 & -3. & & & & 0.001 & 11.8 & 32.1 & 10.5 & 20.2 & 1.393 & $\begin{array}{l}30.422 \\
\end{array}$ & 0.429 & & 21.214 & 4 \\
\hline 9/24/ & 10:31:41 AM & $25.07 \mathrm{~s}$ & 22.674 & \begin{tabular}{|l|}
24.49 \\
\end{tabular} & 23.426 & $22.8 \mathrm{r}$ & 25.238 & 33.425 & 11.4 & 15.033 & 1.968 & & & & 2.9 & & 12.8 & 32.0 & 10.5 & 20.1 & 1.388 & 80.423 & $\begin{array}{l}30.429 \\
\end{array}$ & & 21.305 & \\
\hline $9 / 24 / 2$ & 10:32:41 AM & 25.113 & 22.664 & 24.615 & 23.406 & 22.8 & 25. & 33.065 & $11.4 \mathrm{r}$ & 14.998 & 2.1 & & & & & & 13.8 & 31.6 & 10.9 & 20.0 & 1.379 & 90.425 & $\begin{array}{c}5 \\
5\end{array}$ & & 21.471 & $\begin{array}{ll}1 & 18.27\end{array}$ \\
\hline $9 / 24 /$. & 10:33:41 AM & 25.184 & 22.669 & 24.606 & 23.436 & $22.8 \mathrm{r}$ & 25. & $\begin{array}{l}33.076 \\
\end{array}$ & 11.5 & & 2.0 & & & & 2.912 & & 14.8 & 31.6 & 10.7 & 20.1 & 1.384 & & $\begin{array}{ll}4 & 0.428\end{array}$ & & 21.345 & $.17 \mathrm{~T}$ \\
\hline 9/24i & 10:34:41A & 25.248 & 22.679 & 24.625 & 23.466 & 22.8 & & 33.554 & 11.4 & 15.455 & 2.1 & & & & 2.9 & & 15.8 & 32.1 & 10.7 & 20.4 & 409 & & & & & \\
\hline 9/24. & 10:35:41. & 25.197 & 22.672 & 24.418 & 23.696 & 22.855 & 25 . & 33.268 & $11.4 \mathrm{r}$ & 14.849 & 2.0 & & & & & & 16.8 & 31.8 & 10.5 & 20.0 & 1.376 & & 130 & & 21.564 & \\
\hline $9 / 24 / /$ & 10:36:41: & 25.082 & 22.672 & 24.368 & 23.605 & 22 & 25.178 & 33.343 & $11.3 \mathrm{~s}$ & 15.086 & 2.014 & & & & & & 17.8 & 31.9 & 10.1 & 20.1 & 1.368 & & & & & \\
\hline & 10:37: & 24.987 & 22.677 & 24.243 & 23.675 & $22.8 \mathrm{r}$ & & 33.084 & 11.379 & & & & & & 2.905 & & 18.8 & 31.6 & 10.5 & & 1.384 & & & & & \\
\hline & 10:38:4 & 24.9 & 22.671 & 24.162 & 23.684 & 22. & & & & & 1.5 & & & & & & 19.8 & 31.9 & 10.5 & .2 & & & & & & \\
\hline & 10:39:4 & 24.8. & 22.677 & 24.147 & 23.635 & $22.8 \mathrm{~s}$ & & 33.2 & 10.8 & & & & & & & & 20.8 & 31.7 & & & & & & & & $\begin{array}{ll}2 & 18 .\end{array}$ \\
\hline & 10:4L & & 22.693 & 24.027 & 23.675 & 22. & & & & & & & & & & 0.001 & & 32.2 & & & & & & & & 9 \\
\hline & 10:4 & & 22.693 & 24.007 & 23 & 22. & & & & & & & & & & & & 32.1 & 10.5 & & & & & & & \\
\hline & $10: 4$ & & 22.688 & 23.952 & 23.565 & 22. & & & & 15. & & & & & & & & 31.9 & & & & & & & & \\
\hline & & & 22.693 & & & & & & & & & & & & & & & 32.3 & & & & & & & & \\
\hline & & & 22. & & & & & & & & & & & & & & & & & & & & & & & \\
\hline & & & 22. & 23.826 & 23.5 & & & & & & & & & & & & & 32.2 & & & & & & & & 1 \\
\hline & & & 22.691 & & & & & & & & & & & & & & & 32.2 & & & & & & & & \\
\hline & & & & & & & & & & & & & & & & & & & & & & & & & & \\
\hline & & & & & & & & & & & & & & & & & & & & & & & & & & \\
\hline & & & 22. & & & & & & & & & & & & & & & .5 & & & & & & & & \\
\hline & & & & & & & & & & & & & & & & & & .8 & & & & & & & & \\
\hline & & & & & & & & & & & & & & & & & & & & & & & & & & \\
\hline & & & & & & & & & & & & & & & & & & .5 & & & & & & & & \\
\hline & & & & & & & & & & & & & & & & & & & & & & & & & & \\
\hline & & & & & & & & & & & & & & & & & & 14 & & & & & & & & \\
\hline & & & & & & & & & & & & & & & & & & & & & & & & & & \\
\hline & & & & & & & & & & & & & & & & & & & & & & & & & & \\
\hline & & & & & & & & & & & & & & & & & & & & & & & & & & \\
\hline & & & & & & & & & & & 2.6 & & & & & & & & & & & & & & & \\
\hline & & & & & & & & & & & & & & & & & & 2 & & & & & & & & \\
\hline & & & & & & & & & & & & & & & & & & 2 & & & & & & & & \\
\hline & & & & & $23.456 \mathrm{~b}$ & & & & & & & & & & & & & 1 & & & & & & & & \\
\hline & & $\begin{array}{ll}25.24 \\
5.200\end{array}$ & 2.770 & & 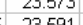 & 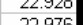 & & & & & & & & & . & & & 32.4 & & & & & & & & \\
\hline $24 / 2002$ & 11:03:41 AM & $25.298 \mathrm{C}$ & 22.734 & 24.685 & 23.591 & 22.976 & 24.965 & 33.698 & 11.255 & 15.021 & 2.564 & -3.036 & 44.334 & 0.002 & 3.015 & & 44.8 & 32.2 & 10.3 & 20.0 & 1.378 & 0.439 & 0.443 & 0.022 & 22.139 & $\begin{array}{ll}9 & 18.84\end{array}$ \\
\hline
\end{tabular}

Figure C2a: PreConditioning 5B - 2 M Nitric Acid Cleaning - FirstCleaning (first 45 minutes) - Raw \& Calculated Data 
WSRC-TR-2003-00204, REV. 0

SRT-RPP-2003-00087, REV. 0

\begin{tabular}{|c|c|c|c|c|c|c|c|c|c|c|c|c|c|c|c|c|c|c|c|c|c|c|c|c|c|c|}
\hline $9 / 24 / 2002$ & 11:04:41 AM & 25.3 & 22.741 & 24.742 & 23.649 & 23.019 & 24.878 & 33.616 & 11.02 & 15.227 & 2.636 & -3.032 & 44.372 & 0.002 & 3.026 & 0.002 & 45.8 & 32.1 & 10.4 & 20.1 & 1.383 & 0.440 & 0.444 & 0.022 & 22.119 & 18.82 \\
\hline $9 / 24 / 2002$ & 11:05:41 AM & 25.334 & 22.755 & 24.701 & 23.727 & 23.063 & 25.052 & 33.698 & 11.041 & 15.401 & 2.575 & -3.032 & 44.216 & 0.002 & 3.024 & 0.002 & 46.8 & 32.2 & 10.3 & 20.2 & 1.395 & 0.440 & 0.444 & 0.022 & 21.939 & 18.67 \\
\hline $9 / 24 / 2002$ & $211: 06: 41 \mathrm{AM}$ & 25.348 & 22.769 & 24.77 & 23.752 & 23.112 & 25.168 & 33.878 & 11.177 & 15.612 & 2.65 & -3.029 & 44.401 & 0.001 & 3.028 & 0 & 47.8 & 32.4 & 10.4 & 20.4 & 1.406 & 0.441 & 0.443 & 0.022 & 21.750 & 18.51 \\
\hline $9 / 24 / 2002$ & 11:07:41 AM & 25.363 & 22.794 & 24.8 & 23.852 & 23.167 & 25.118 & 33.829 & 11.117 & 15.539 & 2.627 & -3.018 & 44.195 & 0.002 & 3.025 & 0.004 & 48.8 & 32.4 & 10.3 & 20.3 & 1.402 & 0.440 & 0.443 & 0.022 & 21.776 & 18.53 \\
\hline $9 / 24 / 2002$ & 11:08:41 AM & 25.388 & 22.789 & 24.76 & 23.907 & 23.212 & 25.046 & 33.794 & 11.008 & 15.485 & 2.65 & -3.018 & 44.183 & 0.002 & 3.03 & 0.002 & 49.8 & 32.3 & 10.3 & 20.3 & 1.397 & 0.441 & 0.444 & 0.022 & 21.904 & 18.64 \\
\hline $9 / 24 / 2002$ & 11:09:41 AM & 25.409 & 22.82 & 24.861 & 23.948 & 23.279 & 25.052 & 34.023 & 11.12 & 15.428 & 2.775 & -3.018 & 44.489 & 0.002 & 3.052 & 0.002 & 50.8 & 32.6 & 10.4 & 20.2 & 1.395 & 0.444 & 0.446 & 0.022 & 22.028 & 18.75 \\
\hline $9 / 24 / 2002$ & 11:10:41 AM & 25.429 & 22.835 & 24.826 & 24.059 & 23.319 & 25.042 & 34.12 & 11.146 & 15.189 & 2.798 & -3.015 & 44.63 & 0.002 & 3.06 & 0.002 & 51.8 & 32.6 & 10.4 & 20.1 & 1.387 & 0.445 & 0.447 & 0.022 & 22.244 & 18.93 \\
\hline $9 / 24 / 2002$ & 11:11:41 AM & 25.444 & 22.82 & 24.811 & 24.023 & 23.328 & 25.006 & 33.876 & 11.058 & 15.428 & 2.815 & -3.015 & 44.47 & 0.002 & 3.06 & & 52.8 & 32.4 & 10.4 & 20.2 & 1.394 & 0.445 & 0.448 & 0.022 & 22.142 & 18.84 \\
\hline $9 / 24 / 2002$ & 21::12:41 AM & 25.467 & 22.853 & 24.914 & 24.081 & 23.337 & 24.708 & 33.49 & 11.04 & 15.103 & 2.737 & -3.012 & 44.583 & 0.002 & 3.05 & 0.002 & 53.8 & 32.0 & 10.4 & 19.9 & 1.372 & 0.444 & 0.445 & 0.022 & & 19.02 \\
\hline $9 / 24 / 2002$ & 211:13:41 AM & 25.485 & 22.856 & 24.837 & 24.039 & 23.345 & 25.104 & 34.048 & 11.098 & 15.496 & 2.827 & -3.012 & 44.38 & 0.002 & 3.065 & 0.001 & 54.8 & 32.6 & 10.4 & 20.3 & 1.400 & 0.446 & 0.448 & 0.022 & 22.071 & 18.78 \\
\hline $9 / 24 / 2002$ & 211:14:41 AM & 25.512 & 22.868 & 24.904 & 24.056 & 23.336 & 24.654 & 33.5 & 10.989 & 15.113 & 2.902 & -3.012 & 44.351 & 0.0 & 30 & 0.003 & 55.8 & 32.0 & 10.4 & 19.9 & 1.371 & 0.446 & 0.447 & 0.022 & & 19.13 \\
\hline $9 / 24 / 2002$ & 11:15:41 AM & 25.508 & 22.864 & 24.91 & 24.032 & 23.343 & 24.853 & 33.814 & 11.087 & 15.207 & 2.91 & -3.006 & 44.497 & 0.002 & 3. & 0.001 & 56.8 & 32.3 & 10.4 & 20.0 & 1.381 & 0.447 & 0.448 & 0.022 & & 19.03 \\
\hline $9 / 24 / 2002$ & $11: 16: 41 \mathrm{AM}$ & 25.544 & 22.885 & 24.881 & 24.043 & 23.339 & 25.075 & 34.172 & 11.015 & 15.608 & 2.916 & & 44.468 & 0.002 & 3.071 & 0.003 & 57.8 & 32.7 & 10.4 & 20.3 & 1.402 & 0.447 & 0.448 & 0.022 & 22.042 & 18.76 \\
\hline $9 / 24 / 2002$ & $21: 17: 41$ AM & 25.545 & 22.871 & 24.928 & 23.995 & 23.35 & 25.357 & 34.211 & 11.095 & 15.897 & 2.957 & -3.006 & 44.466 & 0.0 & 3. & 0.001 & 58. & 32.7 & 10.4 & 20.6 & 1.422 & 0.448 & 0.449 & 0.022 & 21.751 & 18.51 \\
\hline $9 / 24 / 2002$ & 11:18:41 AM & 25.551 & 22.891 & 24.928 & 24.005 & 23.335 & 25 & 34.013 & 11.091 & 15.465 & 2.91 & -3.003 & 44.388 & 0.002 & 3.0 & & 59.8 & 32.5 & 10.4 & 20.2 & 1.395 & 0.448 & 0.449 & & 22.168 & 18.86 \\
\hline $9 / 24 / 2002$ & 21:19:41 AM & 25.581 & 22.902 & 24.938 & 24.02 & 23.345 & 25.06 & 34.241 & 11.024 & 15.446 & 3.026 & -3.003 & 44.153 & 0.002 & 3. & & 60.8 & 32.8 & 10.3 & 20.3 & 1.396 & 0.452 & 0.453 & 0.022 & 22.362 & 19.03 \\
\hline $9 / 24 / 2002$ & 11:20:41 AM & 25.611 & 22.907 & 25.033 & 23.99 & 23.341 & 24.982 & 34.094 & 11.199 & 15.244 & 3.017 & -3.006 & 44.286 & 0.0 & 3. & 0.001 & 61.8 & 32.6 & 10.3 & 20.1 & 1.387 & 0.451 & 0.451 & 0.022 & 21 & 19.08 \\
\hline $9 / 24 / 2002$ & 11:21:41 AM & 25.641 & 22.922 & 24.988 & 24.02 & 23.321 & 25.031 & 34.214 & 11.173 & 15.344 & 2.983 & -2.997 & 44.372 & 0.002 & 3.087 & 0.001 & 62.8 & 32.7 & 10.4 & 20.2 & 1.392 & 0.449 & 0.449 & 0.022 & 22.259 & 18.94 \\
\hline $9 / 24 / 2002$ & 11:22:41 AM & 25.656 & 22.922 & 25.003 & 24.02 & 23.336 & 24.934 & 33.978 & 10.958 & 15.563 & 2.983 & -2.997 & 44.651 & 0.0 & 3.0 & 0.001 & 63.8 & 32.5 & 10.4 & 20.2 & 1.396 & 0.449 & 0.449 & 0.022 & 22.190 & 18.8 \\
\hline $9 / 24 i$ & 2 $11: 23: 41$ AM & 25.646 & 22.927 & 25.048 & 24.03 & 23.3 & 24.888 & 34 & 11 & 15.287 & 3.069 & & 44.464 & 0.0 & & 0.00 & 64 & 32.5 & 10.4 & 20.1 & 1.385 & & & & & 19.06 \\
\hline $9 / 24 / 2002$ & 211:24:41 AM & 25.651 & 22.922 & 24.968 & 24.015 & 23.3 & 24.969 & 34.166 & 11.235 & 15.082 & 3.02 & -2.997 & 44.573 & 0.0 & 3. & 0.001 & 65 & 32.7 & 10.4 & 20.0 & 1.381 & 0.4 & 0.451 & 0.023 & & 19.16 \\
\hline $9 / 24 / 2002$ & 11:25:41 AM & 25.665 & 22.926 & 25.027 & 23.989 & 23.309 & 24.613 & 33.715 & 11.074 & 14.971 & 3.023 & -2.997 & 44.518 & 0.0 & 3.1 & & 66. & 32.2 & 10.4 & 19.8 & 1.365 & 0.451 & 0.451 & 0.023 & 22.782 & 19.39 \\
\hline $9 / 24 / 2002$ & $11: 26: 41 \mathrm{AM}$ & 25.654 & 22.93 & 25.021 & 24.0 & 23.3 & 24.65 & 33.8 & 11.0 & 1 & 3.1 & -2 & 44.535 & 0.0 & & & 67 & 32.4 & 10.4 & 19.9 & 1.373 & 0.2 & 0.452 & 0.023 & & 19.33 \\
\hline $9 / 24 /$ & $211: 27: 41 \mathrm{AM}$ & 25.669 & 22.935 & 24.976 & 23.998 & 23.299 & 24.758 & 33.821 & 11.028 & 15.299 & 3.066 & -2.995 & 44.422 & 0.0 & 3.10 & 0.001 & 68.8 & 32.3 & 10.4 & 20.0 & 1.381 & 0.452 & 0.453 & 0.023 & 22.603 & 19.24 \\
\hline $9 / 24 / 2002$ & 11:28:41 AM & 25.668 & 22.934 & 25.05 & 23.972 & 23.272 & 24.698 & 33.94 & 11. & 14.949 & 3.081 & -2.995 & 44.42 & 0.0 & 3.1 & 0.001 & 69 & 32.5 & 10.4 & 19.8 & 1.367 & 0.453 & 0.452 & 0.023 & 22.819 & 19.42 \\
\hline $9 / 24 / 2002$ & 11:29:41 A & 25.663 & 22.934 & 25.015 & 23.991 & 23.27 & 24.882 & 34.18 & 11.0 & 15.3 & 3.092 & -2.99 & 44.445 & 0.002 & 3.1 & 0.002 & 70.8 & 32.7 & 10.4 & 20.1 & 1.387 & 0.453 & 0.453 & 0.023 & 22.504 & 19.15 \\
\hline \multirow{7}{*}{$9 / 24 / 2002$} & 11:30:41 AM & 25.673 & 22.924 & 24.985 & 23.961 & 23.267 & 24.864 & 34.04 & 11.286 & 15.111 & 3.081 & -2.995 & 44.386 & 0.001 & 3.118 & & 71.8 & 32.6 & 10.4 & 20.0 & 1.378 & 0.454 & 0.454 & 0.023 & 22.709 & 19.33 \\
\hline & & & & & & & & & & & & & & & & & & & & & & & & & & \\
\hline & A: & 25 & 2 & 24.5 & & & 25.0 & 3 & & 1 & 2.6 & -3 & 44.5 & 0 & 3. & 0.0 & & 32 & 10 & 20.2 & 1.4 & 0. & 0 & 0 & 20 & 18.7 \\
\hline & Maximum & 25.7 & 22.9 & 25.1 & 24.1 & 23 & 25.4 & 34.2 & 11.8 & 15.9 & 3.1 & -3 & 46.5 & 0.0 & 3. & 0. & & 32.8 & 10.9 & 20.6 & 1.4 & 0.454 & 0.460 & 0.024 & 1.3 & 20.6 \\
\hline & & 25.2 & 22.7 & 24.6 & 23. & 22 & 25.1 & 33. & 11 & 15.3 & 2 & -3 & 44.4 & 0.0 & 2 & 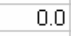 & & 32.2 & 10.4 & 20 & 1.4 & 0.4 & 0.445 & 0.022 & & 18 \\
\hline & & 24.4 & 22.7 & 23.7 & & 22 & 24 & 3 & 10 & 13.9 & 1.9 & -3 & 43.3 & 0.0 & 2. & 0.0 & & 31.6 & 10.1 & 19.0 & 1.3 & 0.421 & 0.428 & 0.021 & 21.0 & 17.9 \\
\hline & $2 \times$ Std Dev & 0.771 & 0.198 & 0.782 & 0.468 & 0.422 & 0.462 & 0.654 & 0.458 & 0.643 & 0.734 & 0.074 & 1.181 & 0.001 & 0.138 & 0.005 & & 0.655 & 0.276 & 0.489 & 0.034 & 0.020 & 0.015 & 0.001 & 1.001 & 0.852 \\
\hline Number of & f Points Used ${ }^{*}$ & 67 & 67 & 67 & 67 & $\begin{array}{r}6.47 \\
67\end{array}$ & 67 & 67 & 67 & 67 & 67 & 67 & 67 & 67 & 67 & 67 & & 67 & 67 & 67 & 67 & 67 & 67 & 67 & 67 & 67 \\
\hline
\end{tabular}

Figure C2b: PreConditioning 5B - 2 M Nitric Acid Cleaning - FirstCleaning (remaining 22 minutes) - Raw \& Calculated Data 
WSRC-TR-2003-00204, REV. 0

SRT-RPP-2003-00087, REV. 0

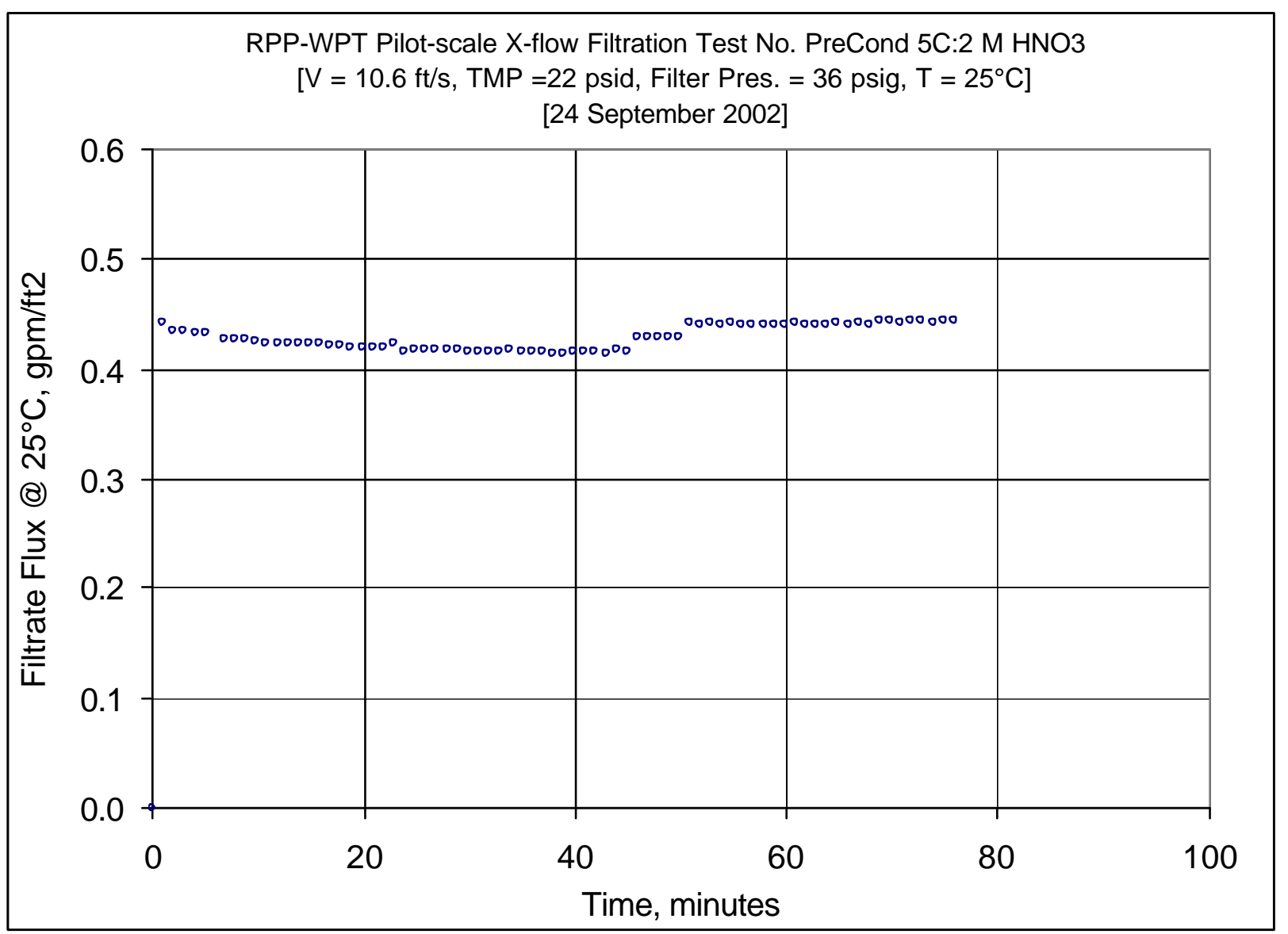

Figure C3: PreConditioning 5C - 2 M Nitric Acid Cleaning - Second Cleaning 
WSRC-TR-2003-00204, REV. 0

SRT-RPP-2003-00087, REV. 0

\begin{tabular}{|c|c|c|c|c|c|c|c|c|c|c|c|c|c|c|c|c|c|c|c|c|c|c|c|c|c|c|}
\hline \multirow[t]{2}{*}{ DATE } & \multirow[t]{2}{*}{ TIME } & \multirow{3}{*}{$\begin{array}{l}\text { Filtrate } \\
\text { deg C } \\
\frac{T}{\mathrm{~T} 2}\end{array}$} & \multirow{2}{*}{$\begin{array}{c}\text { Cleaning } \\
\operatorname{deg} C\end{array}$} & \multirow{3}{*}{$\begin{array}{l}\text { Slurry } \\
\text { deg } \mathrm{C}\end{array}$} & \multirow{3}{*}{$\begin{array}{c}\text { Hi Amb. } \\
\text { deg C } \\
\text { T4 }\end{array}$} & & BotTMP & \begin{tabular}{|l|} 
Filter \\
\end{tabular} & Filter dP & TopTMP & Ements s & Pulsepot & Slurry & Filtrate & Hi Filtate & $\begin{array}{l}\text { Ements }>> \\
\text { Backpulse }\end{array}$ & & & & & Calculate & ated From & m Raw L & Data & & \\
\hline & & & & & & deg & psid & psig & psid & psid & psig & psig & $\mathrm{gpm}$ & $\mathrm{gpm}$ & $\mathrm{gpm}$ & & & & & & & & & & & \\
\hline & & & & & & & $\mathrm{dP} 2$ & P1 & $d P 1$ & $\mathrm{dP3}$ & P2 & P3 & Q1 & Q2 & Q3 & & & & & & & & & & & \\
\hline $\begin{array}{l}\text { Zeros } \\
\text { 9/24/2002 }\end{array}$ & $7: 33: 13 \mathrm{AM}$ & & & & & & & & & & & & & & & & & & & & & & & & & \\
\hline $\begin{array}{l}9 / 24 / 2002 \\
9 / 21202\end{array}$ & 7:33:28 AM & 21.83 & 21.149 & $\frac{22.146}{22.146}$ & 21.953 & 20.713 & $\begin{array}{l}-0.109 \\
-0.09\end{array}$ & -0.012 & $\begin{array}{l}-0.049 \\
-0.049\end{array}$ & 0.072 & $\begin{array}{l}-0.324 \\
-0.324 \\
-12\end{array}$ & 0.356 & \begin{tabular}{|l|}
34.330 \\
34.338
\end{tabular} & 0.031 & $\begin{array}{l}0.001 \\
0.001\end{array}$ & 竞 & & Conversi & $\begin{array}{l}\text { sion } \\
\text { sion }\end{array}$ & $851 \mathrm{~m}$ & & dday/barg & & & & \\
\hline 9/24/2002 & 7:33:29 & 21.8 & 21.149 & 22.186 & 21.928 & 20.723 & -0.109 & 0.012 & -0.05 & 0.072 & -0.321 & 0.356 & 34.269 & 0.031 & 0.001 & & & & & & & . & gr gromint & titz/barg & & \\
\hline $9 / 24 / 2002$ & 3:30 & 21.799 & 21.144 & 22.186 & 21.918 & & -0.109 & 0.012 & -0.049 & 0.072 & -0.35 & 0.35 & 34.313 & 0.031 & 0.001 & & & Note: $\mathrm{Ga}$ & Sauge & Pressure & ire $\mathrm{P}$ & sapk & $y 1$ & 1.4 psig & & \\
\hline $9 / 24 / 2002$ & $33: 31 \mathrm{AM}$ & 21.8 & 21.149 & 22.201 & 21.923 & 0.72 & $\begin{array}{l}-0.109 \\
-0.9\end{array}$ & -0.012 & -0.049 & 0.072 & -0.31 & & 34.399 & 0.029 & 0.0 & & & & & & & & & mis & & \\
\hline $9 / 24 / 2002$ & $3: 32 \mathrm{AM}$ & 21799 & 21.144 & 22.191 & 21.923 & & $\begin{array}{r}-0.109 \\
-\end{array}$ & 0.012 & -0.049 & 0.072 & 341 & & 34.524 & 0.03 & 0. & & & Press & P P1is & $s$ correcte & cted & & & Water tw & tubing & \\
\hline $9 / 24 / 2002$ & 7:33:33 AM & 1.794 & 21.149 & 22.191 & 21.918 & & -0.111 & 0.012 & -0.049 & 0.072 & 321 & & 34.671 & 0.031 & 0.001 & & & $m$ & & & & $<$ Filtra & & & & \\
\hline 9/24/2002 & 7:33:34 AM & 21.805 & 21.154 & 22.196 & 21.928 & 20.72 & -0.111 & -0.012 & -0.049 & 0.072 & -0.364 & 0.35 & 34.546 & 0.03 & 0.001 & & & & & & & & at $25 \mathrm{C}$ & & UOU & \\
\hline & & & & & & & & & & & & & & & & & Time & Press. $V$ & Vel. & TMP TI & TMP gl & & & & gpm & \\
\hline Data - Per M & & & & & & & & & & & & & & & & & & & & & & & & & & \\
\hline 9/24/2002 & 12:18:12 PM & 28.88 & 92 & 25.922 & 1.55 & 23.154 & 14.682 & 38.273 & 415 & 265 & 397 & 78.783 & 45.137 & 0.002 & 0.001 & 0.002 & 0 & 8 & & & & 0.000 & & & 23 & \\
\hline & & 27.075 & & & 24.575 & 154 & 28.608 & & 922 & 12.056 & 2.789 & 35.915 & 46.964 & 0.002 & 3.077 & 0. & & 36.0 & 11.0 & 0.31 & & 0.448 & & & & \\
\hline & 12:20:12 PM & 26.455 & 23.102 & 25.277 & 24.586 & 23.204 & 28.861 & 37.439 & 17.99 & 12.181 & 2.506 & & 46.716 & 0.002 & 3.019 & 0.005 & 2 & 36.0 & 10.9 & & & 0.439 & 136 & & & \\
\hline & 12:21:12 PM & 26.121 & & $\begin{array}{l}24.902 \\
2.777\end{array}$ & 24.576 & 219 & & & 28 & & & & 46.931 & 0.002 & 984 & 0.014 & 3 & 35.9 & 11.0 & & & 0.434 & 135 & & & \\
\hline $9 / 24$ & 12:22: & 25.771 & 23.107 & 24.737 & 24.556 & 23.219 & 29.393 & 37.683 & $\begin{array}{l}18.09 \\
17.07\end{array}$ & & 2.26 & 36.158 & 47.006 & & 2.95 & 0.003 & & 36.2 & 11.0 & .01 & & & 32 & & & \\
\hline & & 25.495 & & & & & & $\frac{37.418}{37.27}$ & & & & 36.158 & $\begin{array}{l}46.754 \\
\end{array}$ & 0.002 & 2.923 & & & 35.9 & & & & & & & & \\
\hline $9 / 24$ & 12:25:00 PM & $\begin{array}{l}25.191 \\
25197\end{array}$ & 23.129 & 24.483 & 24.392 & 23.221 & & & $\begin{array}{l}17.784 \\
1773\end{array}$ & & & & \begin{tabular}{|l|l|}
46.727 \\
\end{tabular} & 0.002 & 901 & & 6.80 & 35.7 & & & & & & & & \\
\hline & & $\begin{array}{l}25.187 \\
0.07\end{array}$ & & 24.429 & & 23.232 & & 37.482 & 17.73 & 13.088 & & 36.15 & & & & 0.001 & 7.8 & 36.0 & 10.9 & 1.31 & & & & & & .06 \\
\hline & & 25.208 & & 24.465 & & & & & & & & & & & 2.892 & & 8.8 & 36.4 & 10.9 & 21.51 & & & & & & 16.92 \\
\hline & & \begin{tabular}{|l}
25.219 \\
7.27
\end{tabular} & & & & 23.244 & & & & & & & & & & & 9.8 & 36.3 & 10.9 & 21.41 & & & 0.426 & & & .90 \\
\hline & & 25.225 & & & & 23.234 & & 37.728 & & & & 36.155 & & & & 0.002 & 10.8 & 36.3 & 10.9 & 21.61 & $1.486 \mathrm{O}$ & & 0.424 & & & 16.76 \\
\hline & & \begin{tabular}{|l}
25.235 \\
0.72
\end{tabular} & & & & & & $\begin{array}{l}37.689 \\
27.55\end{array}$ & & & & 36.15 & & & & & 11.8 & 36.2 & 11.1 & 21.61 & $1.487 \mathrm{O}$ & & 23 & & 06 & 16.68 \\
\hline & 12:31:00 PM & 25.23 & & 24.582 & & $\begin{array}{r}23.22 \\
\end{array}$ & & & & & & 36.153 & & & & 0.003 & 12.8 & 36.0 & 10.8 & 21.01 & 1.4510 & & 124 & & 68 & \\
\hline & 12:32: & 25.255 & & 24.511 & 24.235 & 23.234 & & $\begin{array}{l}37.346 \\
37.47\end{array}$ & 17.798 & & & 36.153 & & 0.002 & & 0.001 & 13.8 & 35.9 & 10.8 & 21.41 & 1770 & & 24 & & 78 & .83 \\
\hline & $2: 33$ & 25.28 & & 24.577 & 45 & 23.224 & 29 & 37.447 & & & & 36.147 & & & & 001 & 14.8 & 6.0 & 10.9 & 21.1 & 580 & & 23 & & 007 & 7.03 \\
\hline $9 / 24 / 2$ & 12:34:00 PM & 25.29 & 23.122 & 24.612 & 24.265 & 23.244 & 29.497 & $\begin{array}{r}37.273 \\
27.09\end{array}$ & 17.774 & 12.865 & 1.855 & 36.147 & 46.454 & 0.002 & 2.877 & 001 & 15.8 & 35.8 & 10.8 & 21.21 & 1.4600 & & 23 & 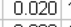 & 982 & 7.01 \\
\hline & 12:35: & 25.319 & & 24.565 & 24.349 & 23.238 & 29.762 & 37.395 & 17.773 & & & 36.147 & & & & 002 & & 35.9 & 10.9 & & & & & & & \\
\hline & & \begin{tabular}{|l|l|}
25.334 \\
\end{tabular} & 23.121 & 24.661 & 24.344 & 23.223 & 29.944 & & 17.827 & & 1.8 & & & & & & 17. & 36.2 & 10.8 & & & & & & & \\
\hline & & 25.333 & & 24.635 & 24.333 & 23.218 & 29.959 & 37.726 & & & 1.7 & & & 0.4 & & & 18.8 & 36.3 & 10.9 & & & & & & & \\
\hline & & 25.373 & & & 24.298 & & 29.7 & \begin{tabular}{|l|}
37.617 \\
\end{tabular} & & & & & & & & & & 36.1 & 10.9 & & & & & & & \\
\hline & & 25.378 & 23. & $\begin{array}{l} \\
\end{array} 4.709$ & 24.273 & & 30.271 & 38.055 & $18 . \mathrm{C}$ & & & & & 0.8 & 2.8 & & & 36.6 & 0.8 & & & & & & & 5 \\
\hline & & 25.398 & & 24.669 & 24.273 & & 30.087 & 38.039 & 17. & & & & & & & & & 36.6 & 10.9 & & & & & & & 16.36 \\
\hline & & 25.413 & 23.1 & 24.689 & 24.303 & & 29. & \begin{tabular}{|l|}
37.929 \\
\end{tabular} & 17. & & & & & & & & & 36.5 & 10. & & & & & & & 16.51 \\
\hline & & 25.428 & 23 & 24.779 & 24.248 & & 30.137 & 37.801 & 17. & 13. & 1.7 & & & 0.0 & & & & 36.3 & 10. & 1.7 & & & & & & 16.3 \\
\hline & & 25.433 & 23. & 24.684 & $\mid 24.223$ & & 29.836 & 37.602 & 17.6 & 13. & 1.7 & & & 0.0 & & & & 36.1 & IU. & 6 & & & & & & 16.5 \\
\hline & & 25.468 & 23.14 & 24.749 & 24.228 & & 29.807 & \begin{tabular}{|l|}
37.67 \\
\end{tabular} & $17:$ & & 1. & & & 0.0 & & & & 36.2 & & & & & & & & 16.5 \\
\hline & & 25.467. & 23.129 & 24.804 & 24.257 & & 29.737 & 37.342 & 17.7 & 13.203 & 1.7 & & & 0.0 & & & & 35.9 & & & & & & & & 16.5 \\
\hline & & 25.487. & 23.134 & 24.744 & 24.2 & & 29.917 & 37.633 & 17.9 & 13. & 1.6 & & & 0.0 & & & & 36.2 & 10. & & & & & & & 16.5 \\
\hline & & 5.503 & 23.14 & 24.795 & 24.243 & 23. $\mathrm{.}$ & 29.8 & \begin{tabular}{|l|}
37.53 \\
\end{tabular} & 17.5 & 13. & 1.7 & & & 0.0 & & & & & & & & & & & & 16 \\
\hline & & 5.49 & 23.14 & 24.8 & 24.273 & & 29.789 & 37.488 & $17.7 \mathrm{C}$ & 13. & 1.8 & & & 0.0 & 2.8 & & & 0 & & & & 0. & & & & 5 \\
\hline & & 5.506 & 23.13 & 24.749 & 24.318 & 23. & 29.8 & 37.4 & 17 & 13. & 1.8 & & 46. & 0.0 & 2.8 & & & 36.0 & & 1.7 & & 0. & & & & 216 \\
\hline & & $5.52 \mathrm{Z}$ & 23.13 & 24.8 & 24.258 & & 29.7 & 37.5 & 17.6 & 13. & 1. & & & 0.0 & 2.8 & & & & & 1.4 & & 0. & & & & 16.5 \\
\hline & & 5.523 & 23.1 & 24.8. & 24.278 & & 30. & 37.7 & 17. & 13 & 1. & & & 0.0 & 2. & & & & & & & 0 & & & & \\
\hline & & 5.543 & 23.1 & 24 & 24.378 & & 29 & 37.3 & 17. & 13. & 1. & & & 0. & & & & 35.9 & & & & 0 & & & & \\
\hline & & 5.538 & 23.146 & 24.855 & 24.2 & & 30. & 37.8 & 17. & 13 & 1.7 & & 46 & 0.0 & & & & +1 & & 1.8 & & 0 & & & 20 & \\
\hline & & 5.533 & 23.141 & 24.775 & 24.2 & & 30. & 37.7 & 17. & 13. & 1.6 & & & 0. & & & & 3 & & & & 0 & & & & \\
\hline $9 / 24 / 2$ & 2:55: & 5.554 & 23.141 & 24.81 & 24.2 & & 30. & 37.7 & 17 & 13 & 1. & & & 0.0 & & & & $3+2$ & & & & c & & & & \\
\hline $9 /$ & $: 56$ & .535 & 23.142 & 24.86 & 24.2 & & 29. & 37.39 & 17.7 & 12. & 1.6 & & 46.391 & 0.0 & & & & 9 & & & & 0.2 & & & & \\
\hline $9 / 2 \quad-\quad$ & $2: 5$ & 5.551 & 23.143 & 24.813 & 24.281 & & 30. & 87.722 & 17.5 & 13.839 & 1.6 & & & 0.0 & & & & 2 & & 2.0 & & 0. & & & 60 & \\
\hline $9 /$ & 2:58: & 561 & 23.144 & 24.85 & 24.3 & & $29.8 \mathrm{r}$ & 7.4 & 17. & 13 & 1.682 & & & 0.00 & 2 . & & & .0 & & & & 0.415 & & & & \\
\hline $9 /$ & 2:59 & i.548 & 23.14 & 24.885 & 24.328 & & $29.8 \mathrm{r}$ & 7.702 & 17.7 & 13.6 & 1.679 & & 9 & 0.00 & 2.8 & & & 2.2 & & & .482 0 & 0.4 & & & & \\
\hline $9 /$ & : $00: 4$ & 553 & 23.146 & 24.81 & 24.3 & & $29.7 \mathrm{r}$ & 7.298 & 17.6 & 13. & 1.662 & & 3 & 0.00 & 2.8 & & & 35.8 & & & & 0.413 & & & & \\
\hline $9 f$ & $: 01: 00 \mathrm{~F}$ & 565 & 23.1. & 24.876 & $24.4 \mathrm{C}$ & & 30.015 & 7.493 & $17.5 \mathrm{~S}$ & 13.7 & 1.653 & & 2 & 0.00 & 2.8 & & & 36.0 & & & 508 & 0.414 & & & & \\
\hline $9 /$ & & & 23.1 & 4.86 & 24. & & 30.0 & 7.679 & 17.7 & 13 & 1.7 & & 5.186 & 0.00 & 2.8 & & & 362 & & & & 1416 & & & & \\
\hline & & & 158 & 4.858 & & & 298 & & & 13768 & 167 & & & 000 & 28 & & & & & & & & & & & \\
\hline 9/24/2002 & 1:04:00 PM & 586 & 23.149 & 24.933 & .472 & & 29.764 & 37.863 & 16.762 & 14.23 & 2.173 & & 5.229 & 0.002 & 2.941 & & & 36.4 & & & (3) & 0.428 & .42 & & .492 & \\
\hline
\end{tabular}

Figure C4a: PreConditioning 5C - 2 M Nitric Acid Cleaning - Second Cleaning (first 45 minutes) - Raw \& Calculated Data 
WSRC-TR-2003-00204, REV. 0

SRT-RPP-2003-00087, REV. 0

\begin{tabular}{|c|c|c|c|c|c|c|c|c|c|c|c|c|c|c|c|c|c|c|c|c|c|c|c|c|c|c|}
\hline $9 / 24 / 2002$ & 1:05:00 PM & 25.607 & 23.149 & 24.919 & 24.462 & 23.306 & 29.617 & 37.884 & 16.816 & 14.108 & 2.22 & 36.118 & 45.143 & 0.002 & 2.943 & 0.002 & 46.8 & 36.4 & 10.5 & 21.9 & 1.507 & 0.428 & 0.429 & 0.020 & 19.633 & 16. \\
\hline $9 / 24 / 2002$ & 1:06:00 PM & 25.652 & 23.149 & 24.934 & 24.502 & 23.296 & 29.582 & 37.97 & 16.722 & 13.942 & 2.217 & 36.121 & 45.125 & 0.002 & 2.943 & 0.002 & 47.8 & 36.5 & 10.5 & 21.8 & 1.500 & 0.428 & 0.429 & 0.020 & 19.715 & 16.78 \\
\hline $9 / 24 / 2002$ & 1:07:00 PM & 25.673 & 23.155 & 25.04 & 24.539 & 23.328 & 30.021 & 38.279 & 17.068 & 14.169 & 2.225 & 36.118 & 45.192 & 0.002 & 2.95 & 0.002 & 48.8 & 36.8 & 10.5 & 22.1 & 1.523 & 0.429 & 0.429 & 0.019 & 19.406 & 16.51 \\
\hline $9 / 24 / 2002$ & 1:08:00 PM & 25.708 & 23.155 & 25.005 & 24.549 & 23.318 & 29.513 & 37.836 & 16.667 & 14.034 & 2.222 & 36.118 & 45.167 & 0.002 & 2.951 & 0.003 & 49.8 & 36.4 & 10.5 & 21.8 & 1.501 & 0.429 & 0.429 & 0.020 & 19.719 & 16.78 \\
\hline 9/24/2002 & 1:09:00 PM & 25.749 & 23.151 & 25.041 & 24.504 & 23.333 & 30.039 & 38.922 & 16.016 & 15.322 & 2.67 & 36.112 & 44.109 & 0.001 & 3.04 & 0.002 & 50.8 & 37.4 & 10.3 & 22.7 & 1.564 & 0.442 & 0.442 & 0.019 & 19.481 & 16.58 \\
\hline $9 / 24 / 2002$ & 1:10:00 PM & 25.775 & 23.152 & 25.112 & 24.46 & 23.339 & 29.911 & 38.601 & 16.183 & 15.029 & 2.595 & 36.115 & 43.982 & 0.002 & 3.035 & 0.00 & 51.8 & 37.1 & 10.3 & 22.5 & 1.549 & 0.442 & 0.440 & 0.020 & 19.592 & 16.67 \\
\hline $9 / 24 / 2002$ & 1:11:00 PM & 25.81 & 23.162 & 25.057 & 24.485 & 23.334 & 29.712 & 38.461 & 16.081 & 15.039 & 2.688 & 36.115 & 44.122 & 0.002 & 3.041 & 0.00 & 52.8 & 37.0 & 10.3 & 22.4 & 1.543 & 0.443 & 0.442 & 0.020 & 19.745 & 16.80 \\
\hline $9 / 24 / 2002$ & 1:12:00 PM & 25.815 & 23.152 & 25.117 & 24.455 & 23.32 & 29.665 & 38.452 & 15.941 & 15.088 & 2.673 & 36.112 & 44.147 & 0.002 & 3.038 & 0.002 & 53.8 & 37.0 & 10.3 & 22.4 & 1.543 & 0.442 & 0.441 & 0.020 & 19.691 & 16.76 \\
\hline $9 / 24 / 2002$ & 1:13:00 PM & 25.825 & 23.162 & 25.127 & 24.445 & 23.335 & 29.505 & 38.378 & 16.173 & 14.703 & 2.757 & 36.109 & 44.044 & 0.001 & 3.048 & 0.00 & 54.8 & 36.9 & 10.3 & 22.1 & 1.524 & 0.444 & 0.442 & 0.020 & 19.994 & 17.01 \\
\hline $9 / 24 / 2002$ & 1:14:00 PM & 25.856 & 23.163 & 25.108 & 24.531 & 23.325 & 29.604 & 38.477 & 16.081 & 14.64 & 2.679 & 36.115 & 44.067 & 0.002 & 3.04 & & 55.8 & 37.0 & 10.3 & 22.1 & 1.525 & 0.442 & 0.441 & 020 & 19.936 & \\
\hline $9 / 24 / 2002$ & 1:15:00 PM & 25.866 & 23.169 & 25.179 & 24.577 & 23.346 & 29.342 & 38.235 & 16.105 & 14.533 & 2.734 & 36.112 & 44.047 & 0.002 & 3.047 & 0.00 & 56.8 & 36.8 & 10.3 & 21.9 & 1.513 & 0.443 & 0.441 & 0.020 & & \\
\hline $9 / 24 / 2002$ & 1:16:00 PM & 25.856 & 23.174 & 25.133 & 24.592 & 23.336 & 29.482 & 38.45 & 16.297 & 14.243 & 2.754 & 36.118 & 44.074 & 0.002 & 3.043 & 0.00 & 57.8 & 37.0 & 10.3 & 21.9 & 1.507 & 0.443 & 0.441 & 0.020 & 20.178 & \\
\hline $9 / 24 / 2002$ & 1:17:00 P & 25.883 & 23.185 & 25.13 & 24.598 & 23.347 & 29.87 & 38.75 & 16.2 & 14.961 & 2.679 & 36.115 & 44.191 & 0.002 & 3.0 & & 58.8 & 37.3 & 10.3 & 22.4 & 1.545 & 0.443 & 0.441 & 0.020 & 19.682 & 16.75 \\
\hline $9 / 24 / 2002$ & 1:18:00 P & 25.868 & 23.175 & 25.19 & 24.598 & 23.332 & 29.304 & 37.89 & 16.013 & 14.66 & 2.728 & 36.109 & 44.131 & 0.001 & 3.04 & 0.0 & 59.8 & 36.4 & 10.3 & 22.0 & 1.516 & 0.444 & 0.441 & 0.020 & & \\
\hline $9 / 24 / 2002$ & 1:19:00 PM & 25.883 & 23.191 & 25.15 & 24.629 & 23.343 & 29.342 & 38.061 & 15.954 & 14.818 & 2.746 & 36.118 & 44.208 & 0.002 & 3.051 & 0.00 & 60.8 & 36.6 & 10.3 & 22.1 & 1.522 & 0.444 & 0.442 & 0.020 & 20.022 & \\
\hline $9 / 24 / 2002$ & 1:20:00 PM & 25.894 & 23.186 & 25.171 & 24.614 & 23.358 & 29.731 & 38.726 & 16.155 & 14.853 & 2.728 & 36.112 & 44.122 & 0.002 & 3.046 & 0.00 & 61.8 & 37.3 & 10.3 & 22.3 & 1.537 & 0.443 & 0.441 & 0.020 & 19.788 & |b.84 \\
\hline $9 / 24 / 2002$ & 1:21:00 F & 25.884 & 23.186 & 25.206 & 24.589 & 23.3 & 29.337 & 38.183 & 15.905 & 14.775 & 2.772 & 36.112 & 44.023 & 0.002 & 3.0 & & 62.8 & 36.7 & 10.3 & 22.1 & 1.521 & 0.444 & 0.441 & 0.020 & 20.006 & \\
\hline $9 / 24 / 2002$ & 1:22:00 PM & 25.895 & 23.187 & 25.137 & 24.65 & 23.354 & 29.354 & 38.221 & 15.946 & 14.599 & 2.682 & 36.109 & 44.21 & 0.002 & 3.0 & 0.0 & 63.8 & 36.7 & 10.3 & 22.0 & 1.515 & 0.443 & 0.441 & 0.020 & 20.084 & \\
\hline $9 / 24 / 2002$ & 1:23:00 PM & 25.905 & 23.192 & 25.202 & 24.6 & 23.349 & 29.358 & 38.252 & 16.079 & 14.572 & 2.746 & 36.109 & 44.0 & 0.0 & 3. & & 64.8 & 36.8 & 10.3 & 22.0 & 1.514 & 0.444 & 0.442 & & & \\
\hline $9 / 24 / 2002$ & 1:24:00 PM & 25.895 & 23.192 & 25.192 & 24.585 & 23.359 & 29.449 & 38.368 & 15.9 & 14. & 2.734 & 36.106 & 44.0 & 0.0 & 3. & & 65.8 & 36.9 & 10.3 & 22.1 & 1.521 & & 0.441 & 0.020 & & 17.00 \\
\hline $9 / 24 / 2002$ & 1:25:00 PM & 25.92 & 23.197 & 25.157 & 24.54 & 23.364 & 29.192 & 38.124 & 16.075 & 14.509 & 2.786 & 36.112 & 43.971 & 0.002 & 3.051 & 0.00 & 66.8 & 36.7 & 10.3 & 21.9 & 1.507 & 0.444 & 0.442 & 0.020 & 20.229 & 17.21 \\
\hline $9 / 24 / 2002$ & $1: 26: 00 \mathrm{PM}$ & 25.925 & 23.202 & 25.237 & 24.53 & 23.384 & 29.17 & 37.888 & 16 & 14.556 & 2.743 & 36.109 & 43.969 & 0.0 & 3.0 & 0.00 & 67.8 & 36.4 & 10.3 & 21.9 & 1.507 & 0.443 & 0.440 & 0.020 & 20.119 & \\
\hline $9 / 24 / 2002$ & 1:27: & 25.93 & 23.207 & 25.197 & 24.5 & 23 & 29.435 & 38.531 & 15.7 & & 2.893 & & & 0.0 & 3.0 & & 68.8 & 37.1 & 10.2 & 22.2 & 1.531 & 0.446 & 0.444 & 0.020 & 19.988 & \\
\hline $9 / 24 / 2002$ & 1:28:00 PM & 25.95 & 23.212 & 25.217 & 24.586 & 23.37 & 29.518 & 38.496 & 16.0 & 14. & 2.861 & 36. & 43.824 & 0.0 & 3.0 & 0.0 & 69.8 & 37.0 & 10. & 22.2 & 1.528 & 0.4 & 0.444 & 0.020 & 20.043 & \\
\hline 9/24/2002 & 1:29:00 PM & 25.966 & 23.218 & 25.303 & 24.556 & 23.395 & 29.188 & 38.074 & 15. & 14 & 2.91 & 36. & 43.893 & 0.0 & 3. & & 70 & 36. & 10.2 & 21.8 & 1.502 & \begin{tabular}{|l|}
0.441 \\
0.447
\end{tabular} & 0.443 & 0.0 & 20.347 & 17. \\
\hline $9 / 24 / 2002$ & $1: 30$ & 25.981 & 23.214 & 25.254 & 24.6 & 23.4 & 29.143 & 38.02 & 15.8 & 14.5 & 2.922 & 36 . & & 0.0 & 3.0 & & 71.8 & 36.5 & 10.2 & 21.8 & 1.506 & 0.448 & 0.445 & & 2 & \\
\hline $9 / 24 / 2002$ & $1: 31: 0$ & 26.031 & 23.224 & 25.304 & 24.657 & 23.416 & 29.257 & 38.246 & 15.921 & 14.7 & 2.936 & 36.109 & 43.717 & 0.00 & 3.0 & 0.00 & 72.8 & 36.8 & 10.2 & 22.0 & 1.515 & 0.448 & 0.444 & 0.020 & 20.218 & \\
\hline 9/24/2002 & 1:32:00 PM & 26.052 & 23.239 & 25.399 & 24.572 & 23.407 & 29.327 & 38.301 & 15.744 & 14.922 & 2.977 & 36.106 & 43.789 & 0.0 & 3. & 0.002 & 73.8 & 36.8 & 10.2 & 22.1 & 1.525 & 0.448 & 0.443 & 0.020 & 20.031 & \\
\hline $9 / 24 / 2002$ & $1: 33: 0$ & 26.077 & 23.234 & 25.334 & 24.5 & 23.41 & 29.238 & 38.268 & 15.4 & 15.23 & 2.983 & 36.103 & 43.7 & 0.00 & 3.083 & 0.00 & 74.8 & 36.8 & 10.2 & 22.2 & 1.533 & 0.449 & 0.444 & 0.020 & 19.987 & \\
\hline $9 / 24 / 2002$ & 1:34:00 PI & 26.113 & 23.245 & 25.395 & 24.558 & 23.412 & 29.186 & 38.113 & 15.775 & 14.924 & 2.968 & 36.103 & 43.537 & 0.001 & 3.088 & 0.002 & 75.8 & 36.6 & 10.2 & 22.1 & 1.521 & 0.449 & 0.444 & 0.020 & 20.149 & 17. \\
\hline & & & & & & & & & & & & & & & & & & & & & & & & & & \\
\hline & Ave & 25 & 2 & 2 & 24.4 & & 29.7 & 37 & $1 t$ & 13 & 2.2 & $3 t$ & 45.5 & 0. & 2. & & & 36.4 & 10.6 & 21.8 & 1.5 & 0.4 & 0.429 & 0.020 & 19.7 & 16 \\
\hline & Maximun & 26.1 & 23.2 & 25.4 & 24 & 23 & 30.3 & 38. & 18 & 15.3 & 3.0 & 36. & 47.4 & 0.0 & 3. & & & 37.4 & 11.1 & 22.7 & 1.6 & 0.449 & 0.445 & 0.020 & 20.4 & 17 \\
\hline & Media & 25.6 & 23.1 & 24.9 & 24 & 23.3 & 29.7 & 37.8 & 17. & 13.7 & 1.9 & 36.1 & 46.3 & 0.0 & 2 & & & 36.4 & 10.8 & 21.8 & 1.5 & 0.420 & 0.425 & 0.020 & 197 & \\
\hline & Minim & 25.2 & 23.1 & 24.4 & 24 & 703 & 29.1 & 37.2 & 15 & 10 & 1.6 & 1 & 43.5 & 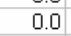 & 2. & & & 35.7 & 10.2 & 21.0 & 1.5 & 0.413 & 0.414 & 0.019 & 18.9 & \\
\hline & & 0.513 & 0.065 & 0.516 & 0.275 & 0.125 & 0.570 & 0.813 & 1.687 & 1.520 & 0.992 & 0.031 & 2.376 & 0.001 & 0.187 & 0.00 & & 0.813 & 0.555 & 0.681 & 0.047 & 0.027 & 0.022 & 0.001 & 0.769 & 0.654 \\
\hline Number of & Points Used ${ }^{*}$ & 70 & 70 & 70 & 70 & 70 & 70 & 70 & 70 & 70 & 70 & 70 & 70 & 70 & 70 & 7 & & 70 & 70 & 70 & 70 & 70 & 70 & 70 & 70 & \\
\hline
\end{tabular}

Figure C4b: PreConditioning 5C - 2 M Nitric Acid Cleaning - Second Cleaning (remaining 36 minutes) - Raw \& Calculated Data 
WSRC-TR-2003-00204, REV. 0

SRT-RPP-2003-00087, REV. 0

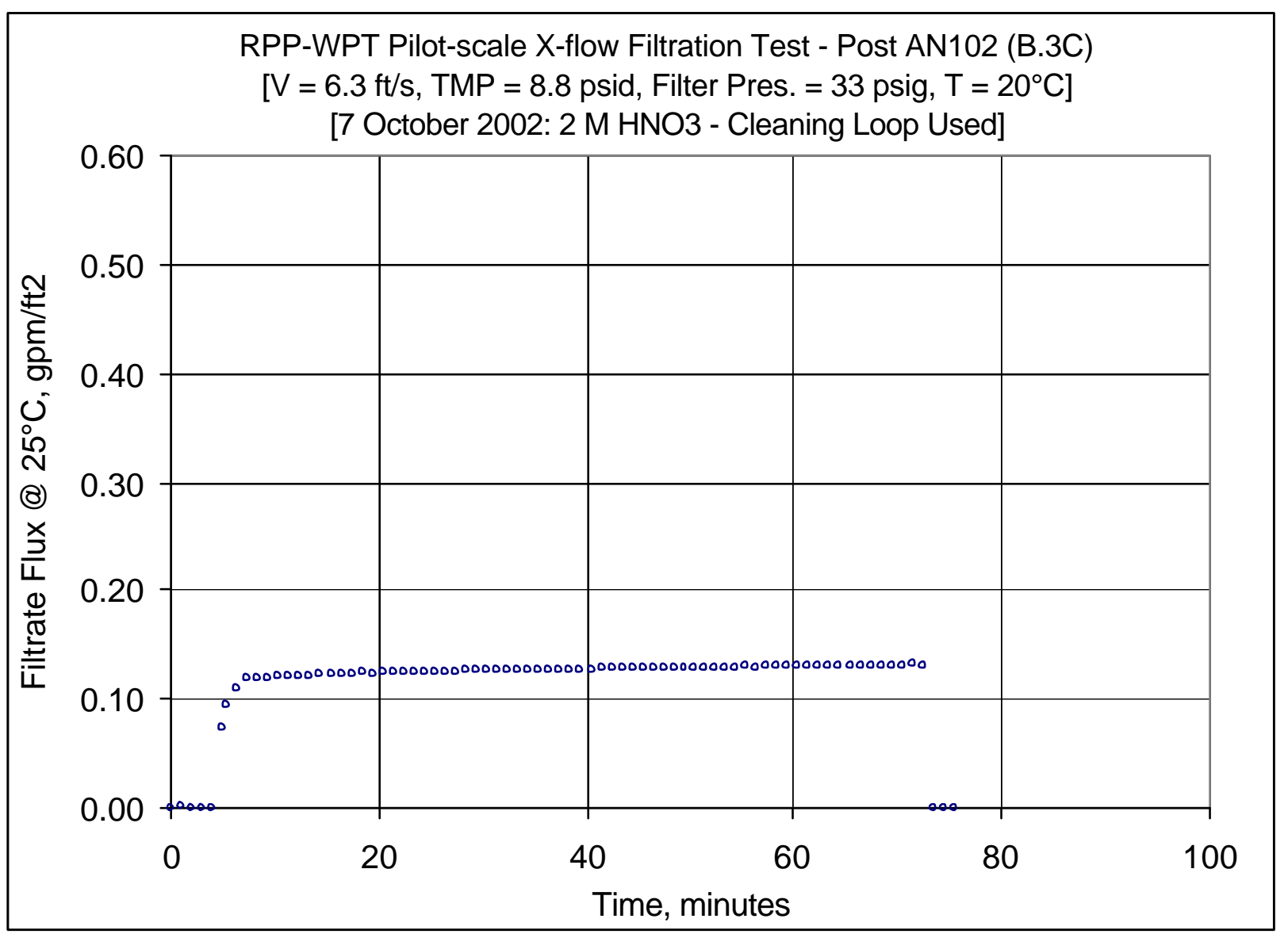

Figure C5: Clean 1: After AN-102R2, Batch 3C-2 M Nitric Acid Cleaning - First Cleaning 
WSRC-TR-2003-00204, REV. 0

SRT-RPP-2003-00087, REV. 0

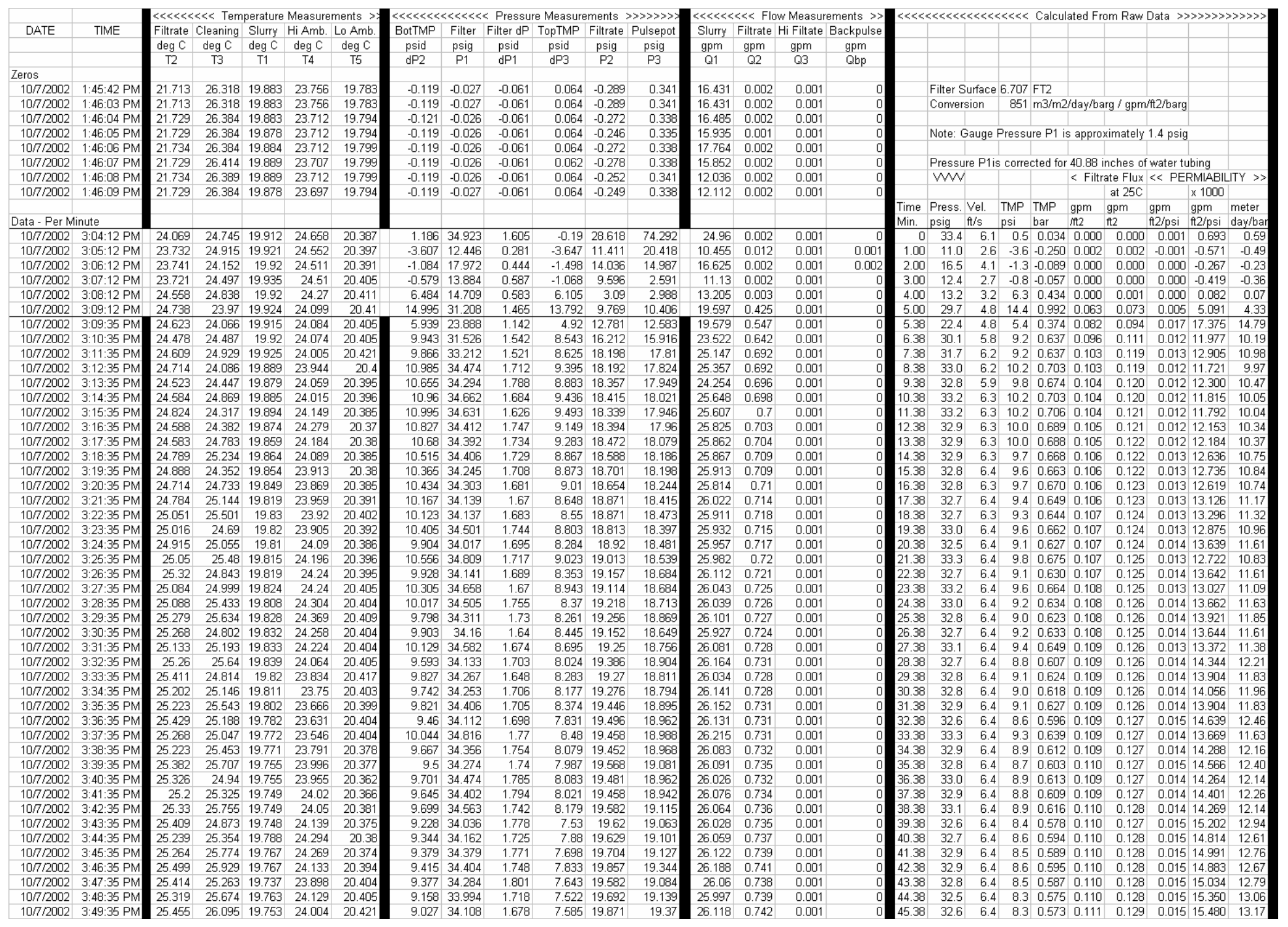

Figure C6a: Clean 1: After AN-102R2, Batch 3C - 2 M Nitric Acid Cleaning - First Cleaning (first 45 minutes) - Raw \& Calculated Data 
WSRC-TR-2003-00204, REV. 0

SRT-RPP-2003-00087, REV. 0

\begin{tabular}{|c|c|c|c|c|c|c|c|c|c|c|c|c|c|c|c|c|c|c|c|c|c|c|c|c|c|c|}
\hline $10 / / 2002$ & 3:50:35 PM & 25.736 & 25.4 & 19.779 & 23.904 & 20.411 & 9.425 & 34.383 & 1.613 & 8.066 & \begin{tabular}{|l|l|}
19.724 \\
\end{tabular} & 19.24 & 26.049 & 0.741 & 0.001 & c & 46.38 & 32.9 & 6.4 & 8.7 & 0.603 & 0.110 & 0.128 & 0.015 & 14.671 & 12.49 \\
\hline $10 / / 2002$ & 3:51:35 PM & 25.465 & 25.549 & 19.743 & 23.959 & 20.395 & 9.017 & 34.079 & 1.756 & 7.379 & 19.808 & 19.336 & 26.164 & 0.743 & 0.001 & 0 & 47.38 & 32.6 & 6.4 & 8.2 & 0.565 & 0.111 & 0.129 & 0.016 & 15.709 & \\
\hline $10 / / 2002$ & 3:52:35 PM & 25.471 & 25.941 & 19.749 & 23.91 & 20.412 & 9.172 & 34.288 & 1.708 & 7.624 & 19.92 & 19.472 & 26.16 & 0.745 & 0.001 & 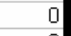 & 48.38 & 32.8 & 6.4 & 8.4 & 0.579 & 0.111 & 0.129 & 0.015 & 15.374 & 13.08 \\
\hline $10 \pi / 2002$ & 3:53:35 PM & 25.662 & 26.257 & 19.74 & 23.886 & 20.433 & 8.945 & 34.135 & 1.674 & 7.389 & 20.004 & 19.503 & 26.2 & 0.746 & 0.001 & 0 & 49.38 & 32.7 & 6.4 & 8.2 & 0.563 & 0.111 & 0.129 & 0.016 & 15.834 & 13.48 \\
\hline $10 / / 2002$ & 3:54:35 PM & 25.632 & 25.406 & 19.735 & 24.001 & 20.422 & 8.884 & 34.127 & 1.786 & 7.137 & 19.932 & 19.466 & 26.139 & 0.744 & 0.001 & & 60.38 & 32.7 & 6.4 & 8.0 & 0.552 & 0.111 & 0.129 & 0.016 & 16.103 & 13.70 \\
\hline $10 / 7 / 2002$ & 3:55:35 PM & 25.491 & 25.776 & 19.724 & 24.156 & 20.422 & 9.032 & 34.185 & 1.69 & 7.555 & 19.923 & 19.472 & 26.101 & 0.745 & 0.00 & 0 & 1.38 & 32.7 & 6.4 & 8.3 & 0.572 & 0.111 & 0.129 & 0.016 & 15.579 & 13.26 \\
\hline $10 / / 2002$ & 3:56:35 PM & 25.622 & 26.207 & 19.73 & 24.207 & 20.448 & 9.125 & 34.414 & 1.839 & 7.385 & 19.958 & 19.448 & 26.141 & 0.745 & 0.0 & 0 & 52.38 & 32.9 & 6.4 & 8.3 & 0.569 & 0.111 & 0.129 & 0.016 & 15.649 & 13.32 \\
\hline $10 / / 2002$ & 3:57:35 PM & 25.812 & 25.416 & 19.75 & 24.252 & 20.448 & 9.172 & 34.452 & 1.724 & 7.653 & 20.042 & 19.538 & 26.213 & 0.748 & 0.001 & 0 & 53.38 & 33.0 & 6.4 & 8.4 & 0.580 & 0.112 & 0.130 & 0.015 & 15.409 & 13.11 \\
\hline $10 / / 2002$ & 3:58:35 PM & 25.592 & 25.681 & 19.75 & 24.462 & 20.442 & 8.891 & 34.141 & 1.67 & 7.454 & 20.036 & 19.59 & 26.171 & 0.746 & 0.0 & & 4.38 & 32.7 & 6.4 & 8.2 & 0.563 & 0.111 & 0.129 & 0.016 & 15.819 & 13.46 \\
\hline $10 / / 2002$ & 3:59:35 PM & 25.585 & 26.085 & 19.764 & 24.456 & 20.451 & 8.963 & 34.441 & 1.71 & 7.481 & 20.169 & 19.706 & 26.252 & 0.75 & 0.0 & 0 & .38 & 33.0 & 6.4 & 8.2 & 0.567 & 0.112 & 0.130 & 0.016 & & 13.45 \\
\hline $10 / / 2002$ & 4:00:35 PM & 25.8 & 25.839 & 19.763 & 24.46 & 20.45 & 9.139 & 34.551 & 1.785 & 7.555 & 20.103 & 19.64 & 26.099 & 0.748 & 0.0 & 0 & 56.38 & 33.1 & 6.4 & 8.3 & 0.575 & 0.112 & 0.130 & 0.016 & 15.524 & 13.21 \\
\hline $10 / / 2002$ & 4:01:35 PM & 25.639 & 25.568 & 19.757 & 24.354 & 20.455 & 8.646 & 33.974 & 1.652 & 7.188 & 20.16 & 19.645 & 26.217 & 0.751 & 0.0 & 0 & 7.38 & 32.5 & 6.4 & 7.9 & 0.546 & 0.112 & 0.130 & 0.016 & 16.435 & 13.99 \\
\hline $10 / / 2002$ & 4:02:35 PM & 25.558 & 25.948 & 19.746 & 24.429 & 20.459 & 8.837 & 34.228 & 1.7 & 7.473 & 20.204 & 19.7 & 70 & 0. & & & & 32.8 & 6. & 8.2 & & & & & & 13.56 \\
\hline $10 / / 2002$ & 4:03:35 PM & 25.728 & 26.323 & 19.736 & 24.499 & 20.459 & 8.561 & 34.011 & 1.6 & 7.131 & 20.204 & 19.75 & 26.166 & 0.752 & 0.0 & 0 & 88 & 32.5 & 6.4 & 7.8 & 0.541 & 0.112 & 0.130 & 0.017 & 516 & 14.14 \\
\hline $10 / / 2002$ & $4: 04: 35 \mathrm{PM}$ & 25.703 & 25.447 & 19.736 & 24.433 & 20.464 & 9.204 & 34.582 & 1.7 & 7.774 & 20.131 & 19.628 & 26.06 & 0.75 & 0.0 & 0 & 60.38 & 33.1 & 6.4 & 8.5 & 0.585 & 0.112 & 0.130 & 0.015 & 15.317 & 13.03 \\
\hline $10 / / 2002$ & $4: 05: 35 \mathrm{PM}$ & 25.567 & 25.832 & 19.75 & 24.598 & 20.463 & 8.715 & 34.044 & 1.5 & 7.418 & 20.207 & 19.695 & 26.236 & 0.75 & 0.0 & 0 & 1.38 & 32.6 & 6.4 & 8.1 & 0.556 & 0.112 & 0.130 & 0.016 & 16.113 & 13.71 \\
\hline $10 / / 2002$ & 4:06:35 PM & 25.682 & 26.252 & 19.75 & 24.623 & 20.473 & 8.965 & 34.596 & 1.72 & 7.516 & 20.348 & 19.79 & 26.206 & 0.754 & 0.1 & 0 & 238 & 33.1 & 6.4 & 8.2 & 0.568 & & 0.131 & 0.016 & 15.857 & 13.49 \\
\hline $10 / / 2002$ & 4:07:35 PM & 25.826 & 25.411 & 19.744 & 24.567 & 20.467 & 8.646 & 34.129 & 1.78 & 6.953 & 20.204 & 19.732 & 26.12 & 0.752 & 0.0 & 0 & 63.38 & 32.7 & 6.4 & 7.8 & 0.538 & 0.112 & 0.130 & 0.017 & 16.712 & 14.22 \\
\hline $10 / / 2002$ & 4:08:35 PM & 25.617 & 25.717 & 19.75 & 24.527 & 20.473 & 8.541 & 34.12 & 1.66 & 7.182 & 20.4 & 19.865 & 26. & 0.7 & & 0 & 64.38 & & 6.4 & 7.9 & 0.542 & & & 0.017 & & 14.16 \\
\hline $10 / / 2002$ & 4:09:35 PM & 25.6 & 26.122 & 19.745 & 24.36 & 20.458 & 8.549 & 34.13 & 1 & 7.084 & 20. & 19.8 & & 0.7 & & & 65. & 32 & 6.4 & 7.8 & & & & 0.017 & & 14.25 \\
\hline $10 / / 2002$ & 4:10:35 PM & 25.823 & 25.853 & 19.746 & 24.373 & 20.479 & 8.52 & 34.08 & 1.66 & 7.135 & 20.37 & 19.828 & 26.124 & 0.754 & 0.1 & 0 & 66.38 & 32.6 & 6.4 & 7.8 & 0.540 & 0.112 & 0.131 & 0.017 & 16.695 & 14.21 \\
\hline $10 / / 2002$ & 4:11:35 PM & 25.668 & 25.597 & 19.731 & 24.428 & 20.484 & 8.831 & 34.3 & 1.6 & 7.547 & 20.3 & 19.79 & 26 . & 0.7 & & 0 & 67.3 & 32.9 & 6.4 & 8.2 & 0.565 & & 0. & 0. & 886 & 13.60 \\
\hline $10 / / 2002$ & 4:12:35 PM & 25.608 & 25.998 & 19.751 & 24.6 & 20.484 & 8.468 & 34. & 1.8 & & & 19.9 & & & & 0 & 68. & 32 & 6. & 7.8 & & & & & & 14.27 \\
\hline $10 / / 2002$ & 4:13:35 PM & 25.748 & 26.383 & 19.721 & 24.7 & 20.4 & 8.719 & 34 & 1.7 & 7.284 & 20. & 19. & & & & & & 33 & 6. & 8.0 & & & & & & 13.95 \\
\hline $10 \pi / 2002$ & 4:14:35 PM & 25.748 & 25.497 & 19.731 & 24.619 & 20.484 & 8.63 & 34.257 & 1.7 & 7.194 & 20. & 19 & 54 & 0.756 & 0.0 & ( & 70.3 & 32.8 & 6.4 & 7.9 & 0.546 & 0.113 & 0.131 & 0.017 & 16.568 & 14.10 \\
\hline $10 / / 2002$ & $4: 15: 3$ & 25.6 & 25.882 & 19.745 & 24.7 & 20.473 & 8.418 & 34. & $1 . E$ & & 20.4 & 19 & & & & 0 & & 32.5 & 6.4 & 7.8 & 0.535 & & & 0.017 & & 14.43 \\
\hline $10 \pi / 2002$ & $4: 16: 3$ & 25.702 & 26.297 & 19.735 & 24.7 & 20.478 & 8.483 & 34.1 & 1.7 & 6.998 & 20.449 & 19.9 & 26.101 & 0.7 & 0. & 0 & 72. & 32.7 & 6.4 & 7.7 & 0.534 & 0.113 & 0.131 & 0.017 & 16.955 & 14.43 \\
\hline $10 / / 2002$ & 4:17:35 PM & 25.883 & 25.487 & 19.74 & 24.6 & 20. & 11.022 & 34.1 & $1 . E$ & 9. & 17. & 17. & 26.1 & 0. & & & 70 & 32 & 6.4 & 10.3 & 0.713 & & 0.1 & 0.000 & 034 & -1 \\
\hline $10 / / 2002$ & 4:18:35 PM & 25.627 & 25.767 & 19.735 & 24. & 20.483 & 10.9 & 34.1 & $1 . E$ & 9 & 17 & 17. & 26 . & & & & 74 & 32.7 & 6.4 & 10.2 & 0.706 & & 0. & 0.000 & 334 & 0.03 \\
\hline \multirow[t]{7}{*}{$10 / / 2002$} & 4:19:35 PM & 25.667 & 26.162 & 19.735 & 24.588 & 20.489 & 10.579 & 33.76 & 1.618 & 9.295 & 17.961 & 17.071 & 26.041 & 0.002 & 1.035 & 1 & 75.38 & 32.3 & 6.4 & 9.9 & 0.685 & 0.000 & 0.000 & 0.000 & 0.035 & 0.03 \\
\hline & & & & & & & & & & & & & & & & & & & & & & & & & & \\
\hline & Avera & 25 & 25.4 & 19 & 24.2 & 2 & 0 & 34 & 1. & 8.0 & 19 & 10 & 2 & 0. & 0.0 & 0.0 & & 32.6 & 6.3 & 8.8 & 0.605 & 0.104 & 0. & & 14.0 & 11.9 \\
\hline & Ma & 25 & 26.4 & & & 20.5 & 11.0 & 34 & 1. & 9.7 & 20 & & 26 & 0 & 1. & & & 33.3 & 6.4 & 10.3 & & & 0. & 0.0174 & 7.4 & 14.8 \\
\hline & Median & 25.4 & 25.5 & 19.8 & 24 & 20.4 & 9.5 & 34.3 & 1.7 & 8.0 & 19.6 & $1:$ & 26.1 & 0.7 & 0.0 & 0. & & 32.8 & 6.4 & 8.7 & 0.603 & 0.110 & 0.127 & 0.0146 & 14.6 & 12.5 \\
\hline & Mir & 24.5 & 24.1 & 19.7 & 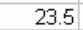 & 20.4 & 5.9 & 23 & & 4.9 & 17 & 12 & 19 & 0.0 & 0 & & & 22.4 & 4.8 & 5.4 & 0.374 & & 0.000 & 0.0000 & 0.0 & 0 \\
\hline & $2 \times$ & 0.760 & 1.127 & 0.109 & 0.576 & 0.074 & 1.710 & 2.57 & 0.17 & 1.647 & 2.25 & 2.19 & 1.718 & 0.299 & 0.4 & J.00 & & 2.570 & 0.421 & 1.7 & 0.115 & 0.045 & 0.052 & 066 & 6.602 & 5.619 \\
\hline & Points Used ${ }^{*}$ & 71 & 71 & 71 & 71 & 71 & 71 & 71 & 71 & 71 & 71 & 71 & 71 & 71 & 71 & 71 & & 71 & 71 & 71 & 71 & 71 & 71 & 71 & 71 & 71 \\
\hline & Dackpuse & & & & & & & & & & & & & & & & & & & & & & & & & \\
\hline & & & & & & & & & & & & & & & & & & & & & & & & & & \\
\hline
\end{tabular}

Figure C6b: Clean 1: After AN-102R2, Batch 3C - 2 M Nitric Acid Cleaning - First Cleaning (remaining 30 minutes) - Raw \& Calculated Data 
WSRC-TR-2003-00204, REV. 0

SRT-RPP-2003-00087, REV. 0

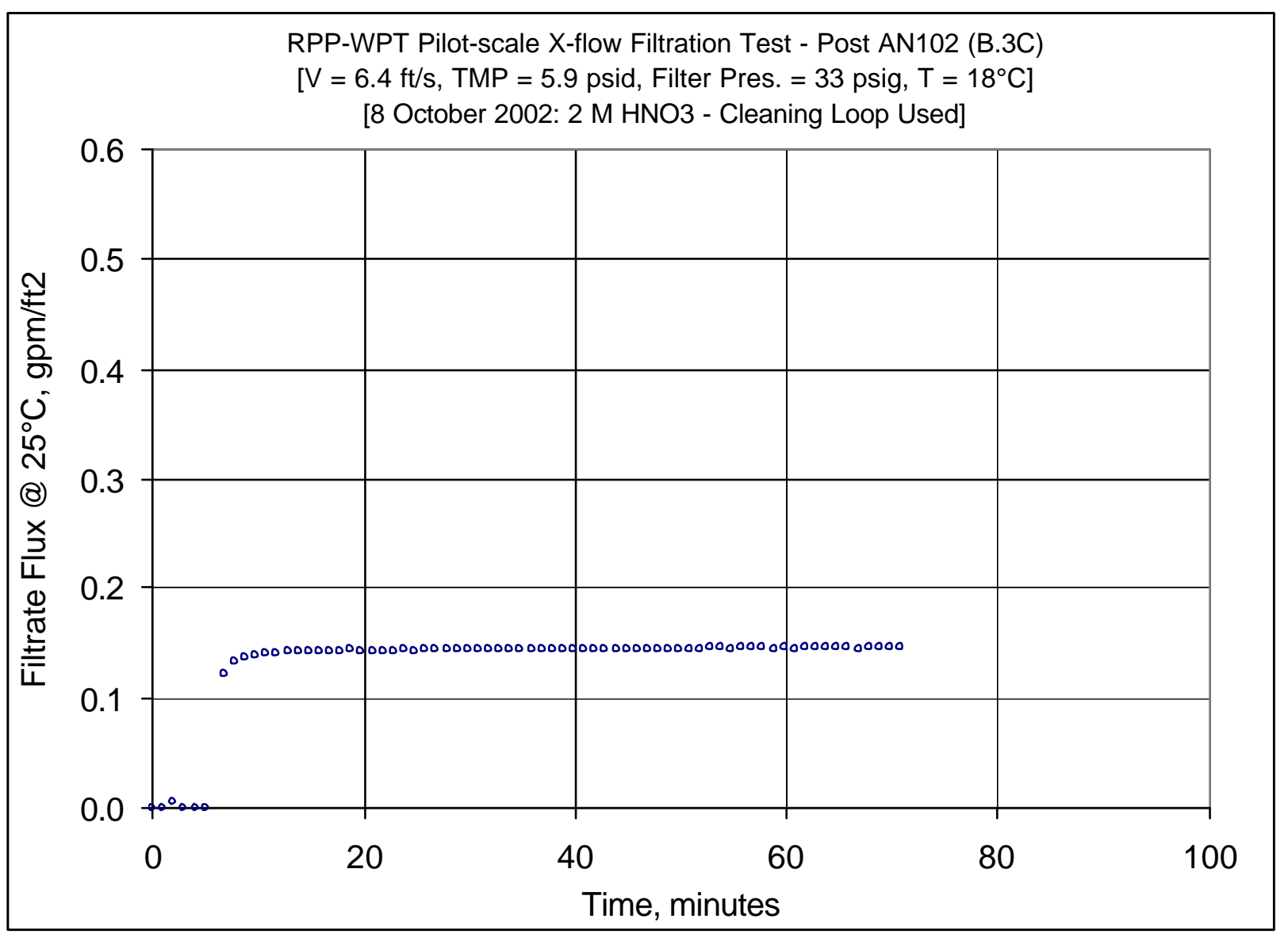

Figure C7: Clean 2: After AN-102R2, Batch 3C-2 M Nitric Acid Cleaning - Second Cleaning 
WSRC-TR-2003-00204, REV. 0

SRT-RPP-2003-00087, REV. 0

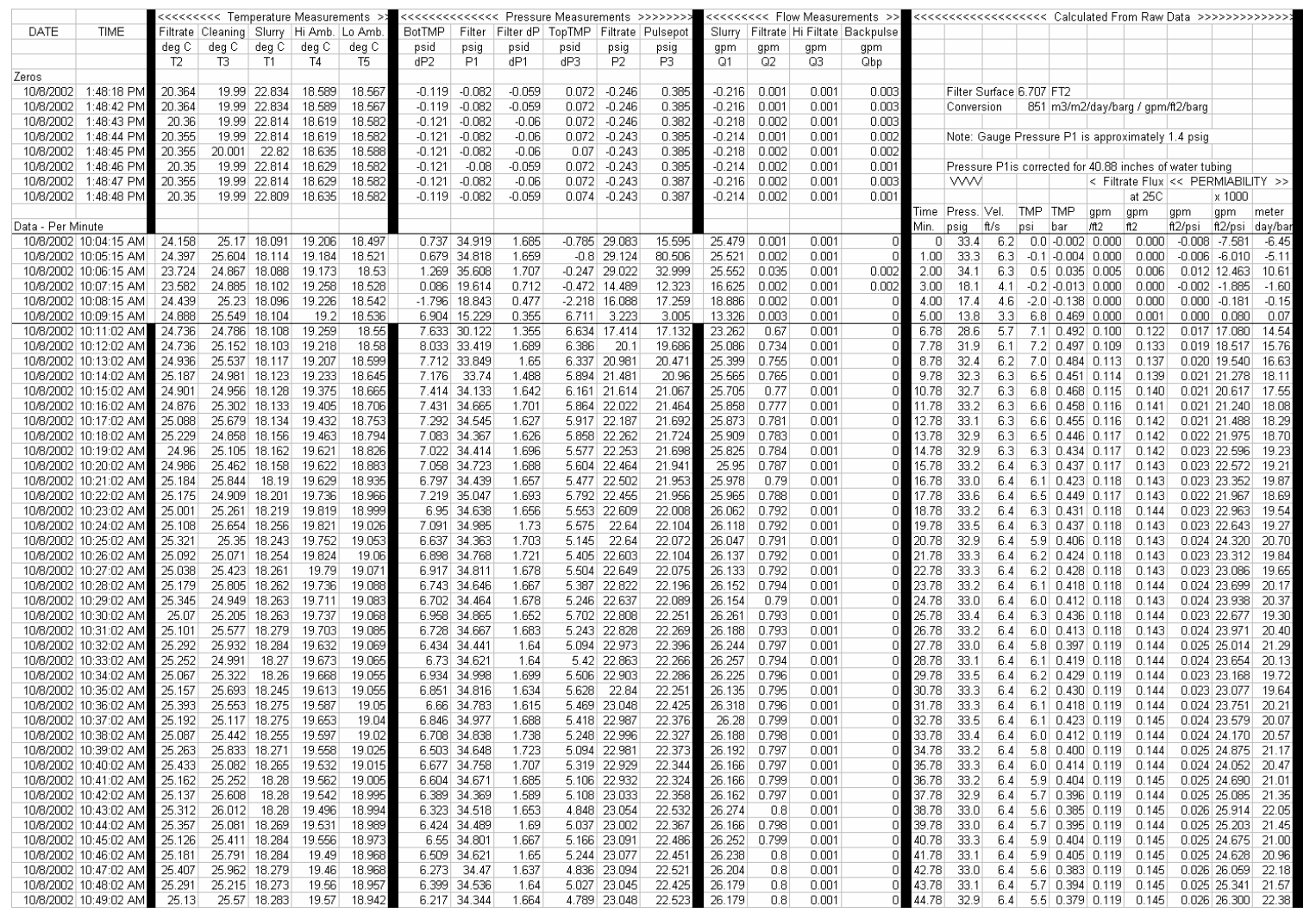

Figure C8a: Clean 2: After AN-102R2, Batch 3C - 2 M Nitric Acid Cleaning - Second Cleaning (first 45 minutes) - Raw \& Calculated Data 
WSRC-TR-2003-00204, REV. 0

SRT-RPP-2003-00087, REV. 0

\begin{tabular}{|c|c|c|c|c|c|c|c|c|c|c|c|c|c|c|c|c|c|c|c|c|c|c|c|c|c|c|}
\hline 10. & 10:50:02 AM & 25.256 & 25.966 & 18.288 & 19.515 & 18.957 & 6.411 & 34.627 & 1.683 & 5.024 & 23.21 & 22.538 & 26.16 & 0.801 & 0.001 & 0 & 45.78 & 33.2 & 6.4 & 5.7 & 0.394 & 0.119 & 0.145 & 0.025 & 25.341 & 21.57 \\
\hline $10 / 8 / 2002$ & $10: 51: 02 \mathrm{AM}$ & 25.496 & 25.511 & 18.293 & 19.484 & 18.952 & 6.496 & 34.7 & 1.695 & 5.031 & 23.065 & 22.434 & 26.171 & 0.8 & 0.001 & 0 & 46.78 & 33.2 & 6.4 & 5.8 & 0.397 & 0.119 & 0.145 & 0.025 & 25.104 & 21,36 \\
\hline $10 / 8 / 2002$ & 10:52:02 AM & 25.235 & 25.394 & 18.272 & 19.569 & 18.936 & 6.171 & 34.404 & 1.674 & 4.732 & 23.135 & 22.495 & 26.177 & 0.801 & 0.001 & 0 & 47.78 & 32.9 & 6.4 & 5.5 & 0.376 & 0.119 & 0.145 & 0.027 & 26.591 & 22.63 \\
\hline $10 / 8 / 2002$ & 10:53:02 AM & 25.159 & 25.76 & 18.282 & 19.574 & 18.921 & 6.246 & 34.435 & 1.671 & 4.787 & 23.094 & 22.495 & 26.215 & 0.801 & 0.001 & 0 & 48.78 & 33.0 & 6.4 & 5.5 & 0.380 & 0.119 & 0.145 & 0.026 & 26.269 & 22.36 \\
\hline $10 / 8 / 2002$ & $10: 54: 02 \mathrm{AM}$ & 25.31 & 26.125 & 18.257 & 19.504 & 18.926 & 6.235 & 34.555 & 1.693 & 4.865 & 23.204 & 22.683 & 26.219 & 0.802 & 0.001 & 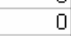 & 49.78 & 33.1 & 6.4 & 5.6 & 0.383 & 0.120 & 0.145 & 0.026 & 26.163 & 22.26 \\
\hline $10 / 8 / 2002$ & $10: 55: 02 \mathrm{AM}$ & 25.505 & 25.334 & 18.277 & 19.549 & 18.936 & 6.368 & 34.708 & 1.754 & 4.875 & 23.172 & 22.538 & 26.242 & 0.803 & 0.0 & 0 & 0.78 & 33.2 & 6.4 & 5.6 & 0.388 & 0.120 & 0.145 & 0.026 & 25.847 & 22.00 \\
\hline $10 / 8 / 2002$ & $10: 56: 02 \mathrm{AM}$ & 25.199 & 25.619 & 18.272 & 19.609 & 10.020 & 6.457 & 34.745 & 1.689 & 5.18 & 23.1 & 22.5 & 26.152 & 0.8 & 0.0 & 0 & 1.78 & 33.3 & 6.4 & 5.8 & 0.401 & 0.119 & 0.145 & 0.025 & 24.882 & 21.18 \\
\hline $10 / 8 / 2002$ & $10: 57: 02 \mathrm{AM}$ & 25.205 & 26.005 & 18.262 & 19.559 & 18.936 & 6.184 & 34.35 & 1.64 & 4.799 & 23.103 & 22.521 & 26.217 & 0.803 & 0.001 & 0 & 52.78 & 32.9 & 6.4 & 5.5 & 0.379 & 0.120 & 0.145 & 0.026 & 26.471 & 22.53 \\
\hline $10 / 8 / 2002$ & $10: 58: 02 \mathrm{AM}$ & 25.395 & 26.38 & 18.282 & 19.529 & $18.93 \mathrm{R}$ & 6.509 & 34.975 & 1.736 & 5.026 & 23.256 & 22.654 & 26.263 & 0.804 & 0.1 & 0 & 3.78 & 33.5 & 6.4 & 5.8 & 0.398 & 0.120 & 0.145 & 0.025 & 25.220 & 21.46 \\
\hline $10 / 8 / 2002$ & 10:59:02 AM & 25.449 & 25.419 & 18.256 & 19.564 & 18.92 & 6.484 & 34.849 & 1.71 & 5.082 & 23.1 & 22. & 26.206 & 0.801 & 0. & c & 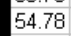 & 33.4 & 6.4 & 5.8 & 0.399 & 0.119 & 0.145 & & 25.078 & 21.34 \\
\hline $10 / 8$ & $11: 00: 02 \mathrm{AM}$ & 25.199 & 25.724 & 18.241 & 19.553 & 18.91 & 6.412 & 34.772 & 1.761 & 4.902 & 23.27 & 22.55 & 26.25 & 0.804 & & 0 & 78 & 33.3 & 6.4 & 5.7 & 0.390 & 0.120 & 0.146 & 0.026 & 25.744 & 21.91 \\
\hline $10 / 8 /$ & $11: 01: 02 \mathrm{AM}$ & 25.254 & 26.099 & 18.266 & 19.558 & 18.9 & 6.302 & 34.648 & 1.638 & 5.022 & 23.346 & 22.668 & 26.32 & 0.806 & 0. & & 6.78 & 33.2 & 6.5 & 5.7 & 0.390 & 0.120 & 0.146 & 0.026 & 25.766 & 21.93 \\
\hline $10 / 8 / 2002$ & $11: 02: 02 \mathrm{AM}$ & 25.443 & 26.309 & 18.266 & 19.492 & & 6.42 & 34.878 & 1.7 & 4.861 & 23.233 & 22. & 26.1 & 0.8 & & & & 33 & 6.4 & 5.6 & & & 0.1 & & & 21.98 \\
\hline $10 / 8 / 2002$ & $11: 03: 02 \mathrm{AM}$ & 25.393 & 25.488 & 18.285 & 19.562 & 18.8 & 6.32 & 34.547 & 1.6 & 5.041 & 23.221 & 22.5 & 26.179 & 0.802 & & 0 & 3.78 & 33. & 6.4 & 5.7 & 0.392 & & 0.145 & 0.026 & 25.541 & 21.74 \\
\hline $10 / 8 / 2002$ & 11:04:02 AM & 25.217 & 25.822 & 18.26 & 19.526 & 18.88 & 6.258 & 34.532 & 1.654 & 4.803 & 23.181 & 22.59 & 26.133 & 0.803 & 0 & 0 & 9.78 & 33.1 & 6.4 & 5.5 & 0.381 & 0.120 & 0.145 & 0.026 & 26.285 & 22.37 \\
\hline $10 / 8 / 2002$ & 11:05:02 AM & 25.327 & 26.183 & 18.254 & 19.466 & & 6.283 & 34.536 & 1.6 & 4.986 & 23.181 & 22.5 & 26.2 & 0.80 & & & & 33 & 6.4 & 5.6 & 0.388 & & 0.145 & 0.026 & & 21.93 \\
\hline & $11: 06: 02 \mathrm{AM}$ & 25.532 & 25.918 & 18.265 & 19.451 & 18.8 & 5.991 & 34.383 & 1.6 & 4.691 & 23.36 & 22.70 & 26.24 & 0.80 & 0 & ( & 0. & 32 & 6.4 & 5.3 & 0.368 & 0 & 0.146 & 0.027 & 27.282 & 23.22 \\
\hline $10 / 8 / 2002$ & $11: 07: 02$ AM & 25.331 & 25.556 & 18.253 & 19.53 & 18.8 & 6.097 & 34.408 & 1.6 & 4.715 & 23.221 & 22.60 & 26.179 & 0.8 & & ( & 62.78 & 32 & 6.4 & 5.4 & 0.373 & 0 & 0.146 & 0.027 & 26.930 & 22.92 \\
\hline $10 \%$ & $11: 08: 02$ AM & 25.252 & 25.902 & 18.279 & 19.556 & 18 & 6.167 & 34.561 & 1.7 & & 23.2 & 22. & & 0.8 & & & & & 6 & 5.4 & & & & & & \\
\hline $10 / 8 /$ & & 25.367 & 26.267 & 18.249 & 19.485 & & 6.31 & 34.636 & 1.6 & 5.076 & 23.2 & 22 & 26 & 0.8 & & & & 3 & 6 & 5.7 & & & & 0.026 & & 21.79 \\
\hline $10 / 8 / 2002$ & $11: 10: 02$ AM & 25.583 & 25.577 & 18.265 & 19.491 & 18 & 6.242 & 34.652 & 1.69 & 4.871 & 23.32 & 22.68 & 26.286 & 0.80 & 0 & 0 & 65.78 & 33.2 & 6.4 & 5.6 & 0.383 & 0 & 0.146 & 0.026 & 26.256 & 22.34 \\
\hline $10 / 8 / 2002$ & 11:11:02 AM & 25.338 & 25.653 & 18.275 & 19.6 & 110 & 6.209 & 34.503 & 1.5 & 4.949 & 23. & 22 & 26. & 0.8 & & ( & 66.78 & 33 & 6.4 & 5.6 & 0. & & & 0.026 & & 22.17 \\
\hline $10 / 8$ & $11: 1$ & 25.288 & 26.014 & 18.271 & 19.674 & & 6.221 & 34.623 & $1 . E$ & 4.857 & 23.298 & 22. & 26.21 & 0.80 & & & 7.78 & 33.1 & 6.4 & 5.5 & 0.382 & 0. & 0.146 & 0.026 & 26.335 & 22.41 \\
\hline $10 / 0$ & $11: 13: 02 \mathrm{AM}$ & 25.449 & 26.364 & 18.251 & 19.624 & 19.0 & 6.03 & 34.507 & 1. & 4.683 & 23.3 & 22 & 26.2 & 0.8 & & & 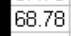 & 3 & 6. & 5.4 & & 0 & 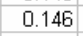 & 0.027 & 214 & 7 \\
\hline & 11:14:02 AM & 25.529 & 25.429 & 18.272 & 19.725 & 19. & 6.246 & 34.687 & $1 . E$ & 4.779 & 23. & 22 & 26. & 0.8 & & & 69. & 33 & 6.4 & 5.5 & 0 & & 0.146 & 0.026 & & 22.49 \\
\hline \multirow{7}{*}{$10 / 8 / 2002$} & $11: 15: 02 \mathrm{AM}$ & 25.301 & 25.771 & 18.283 & 19.818 & 19.1 & 6.405 & 35.039 & 1.658 & 5.09 & 23.464 & 22.842 & 26.303 & 0.807 & 0.001 & c & 70.78 & 33.6 & 6.4 & 5.7 & 0.396 & 0.120 & 0.146 & 0.025 & 25.402 & 21.62 \\
\hline & & & & & & & & & & & & & & & & & & & & & & & & & & \\
\hline & $A_{v}$ & 2 & 25 & 18 & 1 & & 6. & 34.5 & 1 & 2 & 2 & & 26.1 & 0 & 0 & 0. & & 33 & 6.4 & 5.9 & 0.409 & 0.118 & 0.144 & 0.0243 & 2 & 20.716 \\
\hline & Max & 25 & 26.4 & 18.3 & 19 & & 8.0 & 35.0 & 1.8 & 6 & 23 & 2 & & 0. & 0 & 0 & & 33.6 & 6.5 & 7.2 & 0.497 & 0.120 & 0.146 & 0.0273 & 2 & 23.217 \\
\hline & & 25.2 & 25.6 & 18.3 & 19 & & 6.5 & 34.6 & 1.7 & ats & 23.0 & 22.4 & 26.2 & 0.8 & 0 & 0. & & 33.1 & 6.4 & 5.8 & 0.400 & 0.119 & 0.145 & 0.0249 & 24.882 & 21.175 \\
\hline & & 24 & 24.8 & 18 & 19 & & 6 & 30 & & & 17 & & & 0.7 & & & & 28.6 & 5.7 & 5.3 & 0.368 & 0. & 0177 & 00171 & & 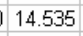 \\
\hline & & 0.359 & 0.791 & 0.103 & 0.271 & 0.28 & 0.860 & 1.234 & 0.117 & 0.882 & 1.764 & 1.66 & 0.822 & 0.040 & 0.000 & .000 & & 1.234 & 0.202 & 0.865 & 0.060 & 0.006 & 0.007 & 0.0042 & .151 & 1.533 \\
\hline Number of & f Point & 65 & 65 & 65 & 65 & & 65 & 65 & 65 & 65 & 65 & 65 & 65 & 65 & 65 & 6 & & 65 & 65 & 65 & 65 & 65 & 65 & 65 & 65 & \\
\hline
\end{tabular}

Figure C8b: Clean 2: After AN-102R2, Batch 3C - 2 M Nitric Acid Cleaning - Second Cleaning (remaining 25 minutes) - Raw \& Calculated Data 
WSRC-TR-2003-00204, REV. 0

SRT-RPP-2003-00087, REV. 0

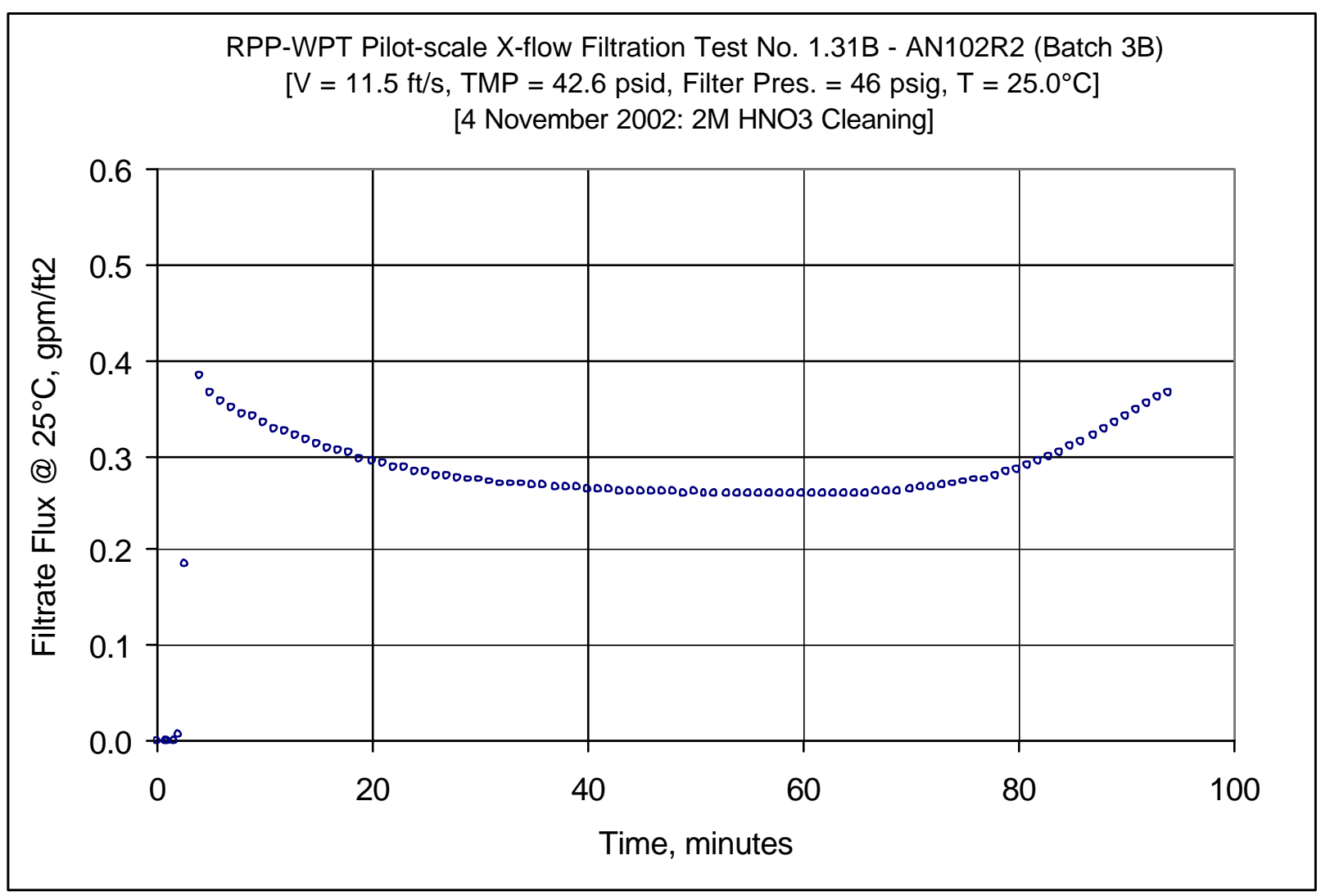

Figure C9: 1.31B: After AN-102R2, Batch 3B - 2 M Nitric Acid Cleaning - First Cleaning 
WSRC-TR-2003-00204, REV. 0

SRT-RPP-2003-00087, REV. 0

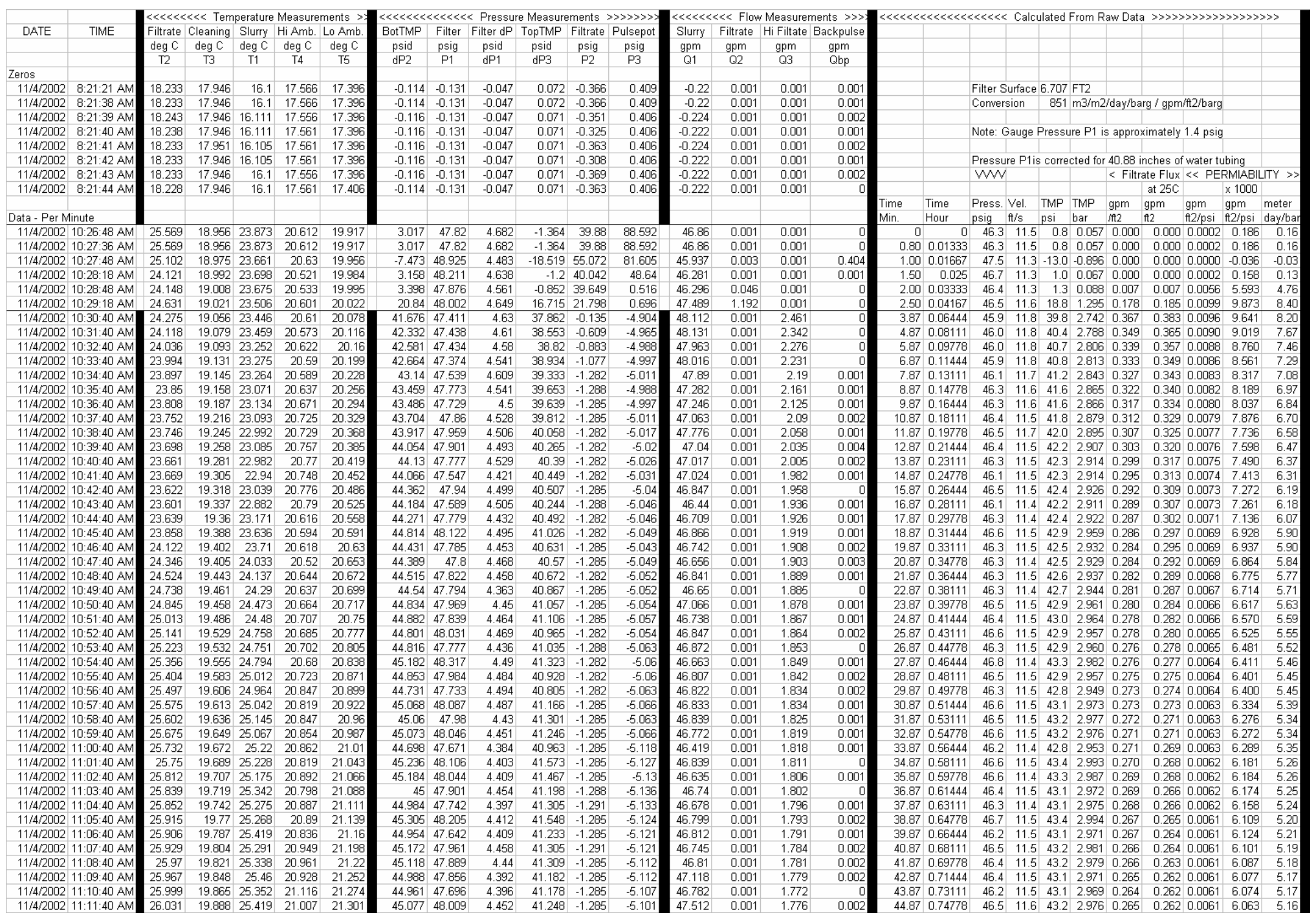

Figure C10a: 1.31B: After AN-102R2, Batch 3B - 2 M Nitric Acid Cleaning - First Cleaning (first 45 minutes) - Raw \& Calculated Data 
WSRC-TR-2003-00204, REV. 0

SRT-RPP-2003-00087, REV. 0
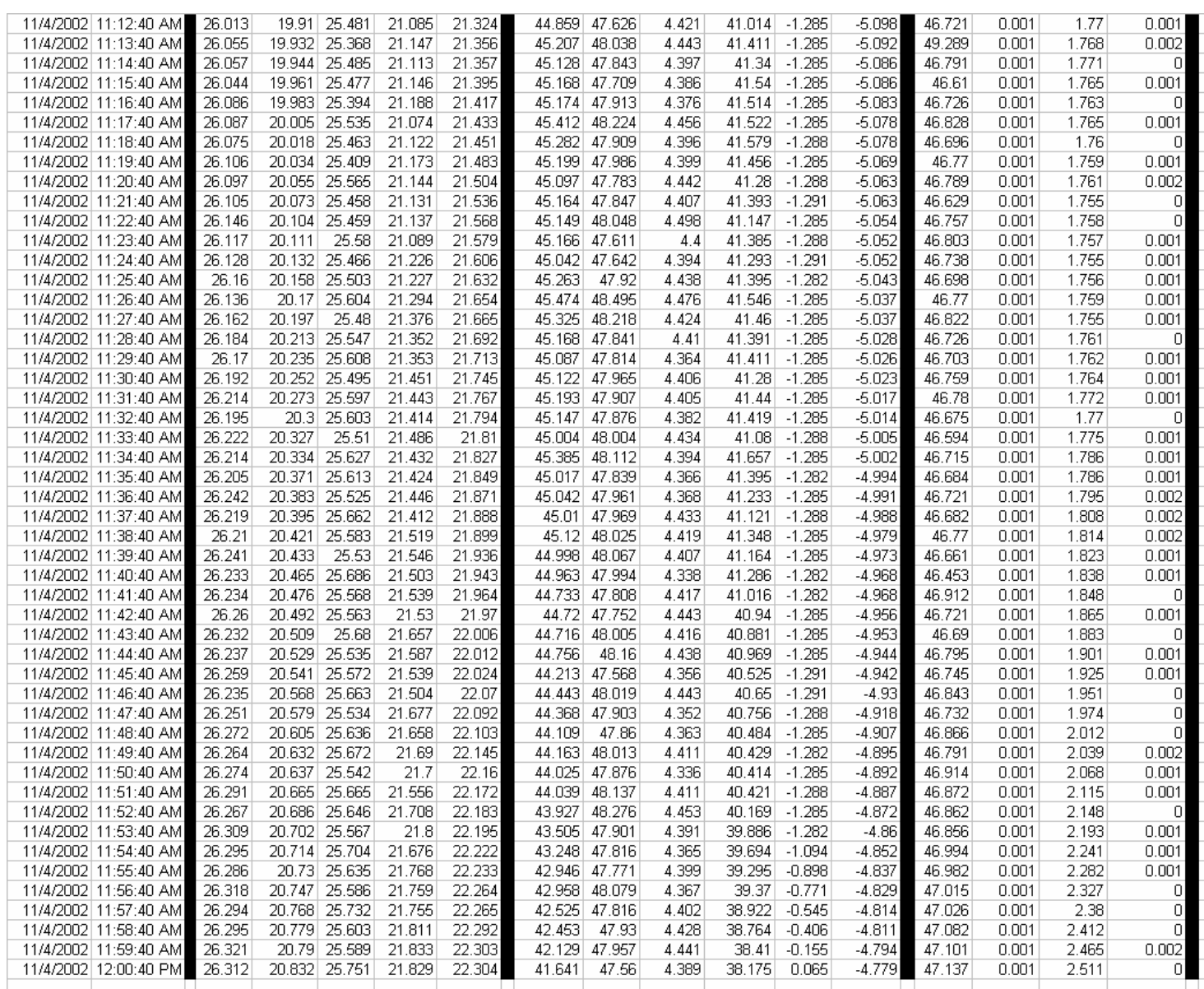

\begin{tabular}{lll|l|l|l|l|l|l|l|l}
45.87 & 0.76444 & 46.2 & 11.4 & 42.9 & 2.960 & 0.264 & 0.260 & 0.0061 & 6.064
\end{tabular} \begin{tabular}{|l|l|l|l|l|l|l|l|l|l|l|}
466.87 & 0.78111 & 46.6 & 12.1 & 43.3 & 2.986 & 0.264 & 0.261 & 0.0060 & 6.024 & 51 \\
\hline 47.87 & 0.79778 & 46.4 & 115 & 432 & 2981 & 0.264 & 0260 & 0.0060 & 6025 & 51
\end{tabular}

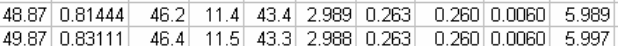
$\begin{array}{llllllllll}49.87 & 0.83111 & 46.4 & 11.5 & 43.3 & 2.988 & 0.263 & 0.260 & 0.0060 & 5.997 \\ 50.87 & 0.84778 & 46.8 & 11.5 & 43.5 & 2.997 & 0.263 & 0.259 & 0.0060 & 5.964\end{array}$

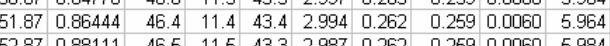

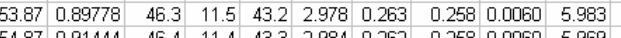
$\begin{array}{llllllllllll}54.87 & 0.91444 & 46.4 & 11.4 & 43.3 & 2.984 & 0.262 & 0.258 & 0.0060 & 5.969 \\ 5587 & 0.93111 & 46 . & 11.5 & 431 & 2.975 & 0262 & 0259 & 0.0060 & 5997\end{array}$ \begin{tabular}{lllllllllll}
55.87 & 0.93111 & 46.6 & 11.5 & 43.1 & 2.975 & 0.262 & 0.259 & 0.0060 & 5.997 \\
\hline 56.87 & 0.94778 & 46.1 & 11.5 & 43.3 & 2.984 & 0.262 & 0.258 & 0.0060 & 5.955
\end{tabular} $\begin{array}{lllllllllll}56.87 & 0.94778 & 46.1 & 11.5 & 43.3 & 2.984 & 0.262 & 0.258 & 0.0000 & 5.955 & 50 \\ 57.87 & 0.96444 & 46.2 & 11.5 & 43.2 & 2.976 & 0.262 & 0.258 & 0.0060 & 5.983 & 50\end{array}$

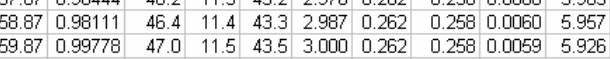
\begin{tabular}{lllllllllllll}
60.87 & 1.01444 & 46.7 & 11.5 & 43.4 & 2.992 & 0.262 & 0.258 & 0.0059 & 5.949 & 50 \\
\hline
\end{tabular} $\begin{array}{lllllllllll}61.87 & 1.03111 & 46.4 & 11.5 & 43.3 & 2.984 & 0.263 & 0.259 & 0.0060 & 5.974 \\ 62.87 & 1.04778 & 46.3 & 11.4 & 43.2 & 2.982 & 0.263 & 0.258 & 0.0060 & 5.971\end{array}$

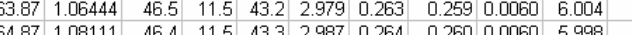

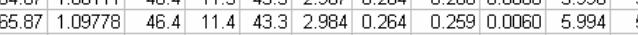
$\begin{array}{lllllllllllll}66.87 & 1.11444 & 46.5 & 11.4 & 43.0 & 2.968 & 0.265 & 0.261 & 0.0061 & 6.061 & 5\end{array}$ $\begin{array}{llllllllllll}67.87 & 1.13111 & 46.6 & 11.4 & 43.5 & 3.001 & 0.266 & 0.262 & 0.0060 & 6.012 \\ 68.87 & 1.14778 & 46.4 & 11.4 & 43.2 & 2.979 & 0.266 & 0.262 & 0.0061 & 6.058\end{array}$ $\begin{array}{llllllllllll}69.87 & 1.16444 & 46.5 & 11.4 & 43.1 & 2.974 & 0.268 & 0.264 & 0.0061 & 6.113 & 5 \\ 70.87 & 1.18111 & 46.5 & 11.4 & 43.1 & 2969 & 0270 & 0.265 & 0.0061 & 6.144 & 523\end{array}$

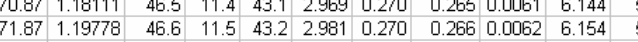
\begin{tabular}{llllllllllll}
72.87 & 1.21444 & 46.6 & 11.4 & 43.1 & 2.970 & 0.272 & 0.268 & 0.0062 & 6.216 \\
\hline 73.87 & 123111 & 46.5 & 11.4 & 431 & 2973 & 0.274 & 0.269 & 0.0062 & 6233 &
\end{tabular} \begin{tabular}{llllllllllll}
74.87 & 1.24778 & 46.3 & 11.5 & 42.9 & 2.956 & 0.276 & 0.271 & 0.0063 & 6.324 & 53 \\
\hline 7587 & 126444 & 463 & 11.4 & 428 & 2953 & 0.278 & 0274 & 0.0064 & 6.390 & 54
\end{tabular} $\begin{array}{lllllllllll}75.87 & 1.26444 & 46.3 & 11.4 & 42.8 & 2.953 & 0.278 & 0.274 & 0.0064 & 6.390 \\ 76.87 & 1.28111 & 46.5 & 11.4 & 42.8 & 2.951 & 0.281 & 0.275 & 0.0064 & 6.435\end{array}$ \begin{tabular}{lllllllllll|l}
77.87 & 1.29748 & 46.7 & 11.5 & 42.9 & 2.955 & 0.283 & 0.279 & 0.0005 & 6.514 \\
\hline 78.87 & 1.31444 & 46.1 & 11.5 & 42.4 & 2.921 & 0.287 & 0.282 & 0.0067 & 6.666
\end{tabular} $\begin{array}{lllllllllllll}79.87 & 1.33111 & 46.5 & 11.5 & 42.5 & 2.933 & 0.291 & 0.286 & 0.0067 & 6.711\end{array}$ $\begin{array}{llllllllllll}80.87 & 1.34778 & 46.4 & 11.5 & 42.6 & 2.935 & 0.294 & 0.290 & 0.0068 & 6.812 & 58\end{array}$ \begin{tabular}{llllllllllll}
81.87 & 1.36444 & 46.4 & 11.5 & 42.3 & 2.916 & 0.300 & 0.295 & 0.0070 & 6.967 & 593 \\
\hline 2.87 & 133111 & 465 & 11.5 & 423 & 2915 & 0304 & 0298 & 0.0071 & 7.053 &
\end{tabular} \begin{tabular}{lllllllllllll}
82.87 & 1.38111 & 46.5 & 11.5 & 42.3 & 2.916 & 0.304 & 0.298 & 0.0071 & 7.053 & 6.0 \\
\hline 83.87 & 1.39778 & 46.4 & 11.5 & 422 & 2911 & 0.308 & 0304 & 0.0072 & 7.192 & 6.12
\end{tabular} \begin{tabular}{l|llllllllllll}
83.87 & 1.3977 & 46.4 & 11.5 & 42.2 & 2.911 & 0.308 & 0.304 & 0.0072 & 7.192 & 6.12 \\
\hline 84.87 & 1.41444 & 46.7 & 11.5 & 42.2 & 2.912 & 0.315 & 0.309 & 0.0073 & 7.329 & 6.24 \\
\hline
\end{tabular} \begin{tabular}{llllllllllll}
85.87 & 1.43111 & 46.8 & 11.5 & 42.0 & 2.899 & 0.320 & 0.314 & 0.0075 & 7.479 & 6 \\
86.87 & 1.44778 & 46.4 & 11.5 & 41.7 & 2.875 & 0.327 & 0.322 & 0.0077 & 7.718 & 657 \\
\hline
\end{tabular} \begin{tabular}{llll|l|l|l|l|l|l|l}
87.87 & 1.46444 & 46.3 & 11.5 & 41.5 & 2.859 & 0.334 & 0.328 & 0.0079 & 7.899 & 672
\end{tabular} \begin{tabular}{lllllllllll}
88.87 & 1.48111 & 46.3 & 11.5 & 41.1 & 2.835 & 0.340 & 0.334 & 0.0081 & 8.128 \\
\hline 89.87 & 1.49778 & 456 & 11.5 & 412 & 2838 & 0347 & 0341 & 0.0083 & 8290
\end{tabular} \begin{tabular}{llllllllllllll}
89.87 & 1.49778 & 46.6 & 11.5 & 41.2 & 2.838 & 0.347 & 0.341 & 0.0083 & 8.290 & 7.00 \\
\hline 90.87 & 151444 & 46.3 & 11.5 & 407 & 2808 & 0355 & 0.348 & 0.0085 & 8.536 & \\
\hline
\end{tabular} $\begin{array}{llllllllll}90.87 & 1.51444 & 46.3 & 11.5 & 40.7 & 2.808 & 0.355 & 0.348 & 0.0085 & 8.536 \\ 91.87 & 1.53111 & 46.5 & 11.5 & 40.6 & 2.800 & 0.360 & 0.354 & 0.0087 & 8.707\end{array}$

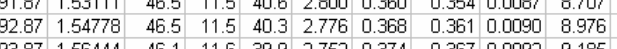
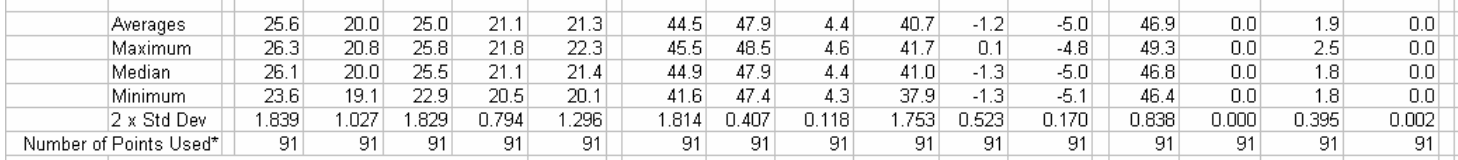

$\begin{array}{llllllllllll}0.8 & 46.4 & 11.5 & 42.6 & 2.937 & 0.287 & 0.288 & 0.0068 & 6.774 & 5.764\end{array}$ $\begin{array}{lllllllllll}47.0 & 12.1 & 43.5 & 3.001 & 0.374 & 0.383 & 0.0096 & 9.641 & 8.205\end{array}$ \begin{tabular}{llllllllllll}
\hline & 46.4 & 11.5 & 42.9 & 2.960 & 0.275 & 0.274 & 0.0064 & 6.390 & 5.438
\end{tabular}

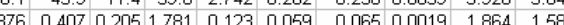
* Backpulse points in box are not included

Figure C10b: 1.31B: After AN-102R2, Batch 3B - 2 M Nitric Acid Cleaning - First Cleaning (Remaining 45 minutes) - Raw \& Calculated Data 
WSRC-TR-2003-00204, REV. 0

SRT-RPP-2003-00087, REV. 0

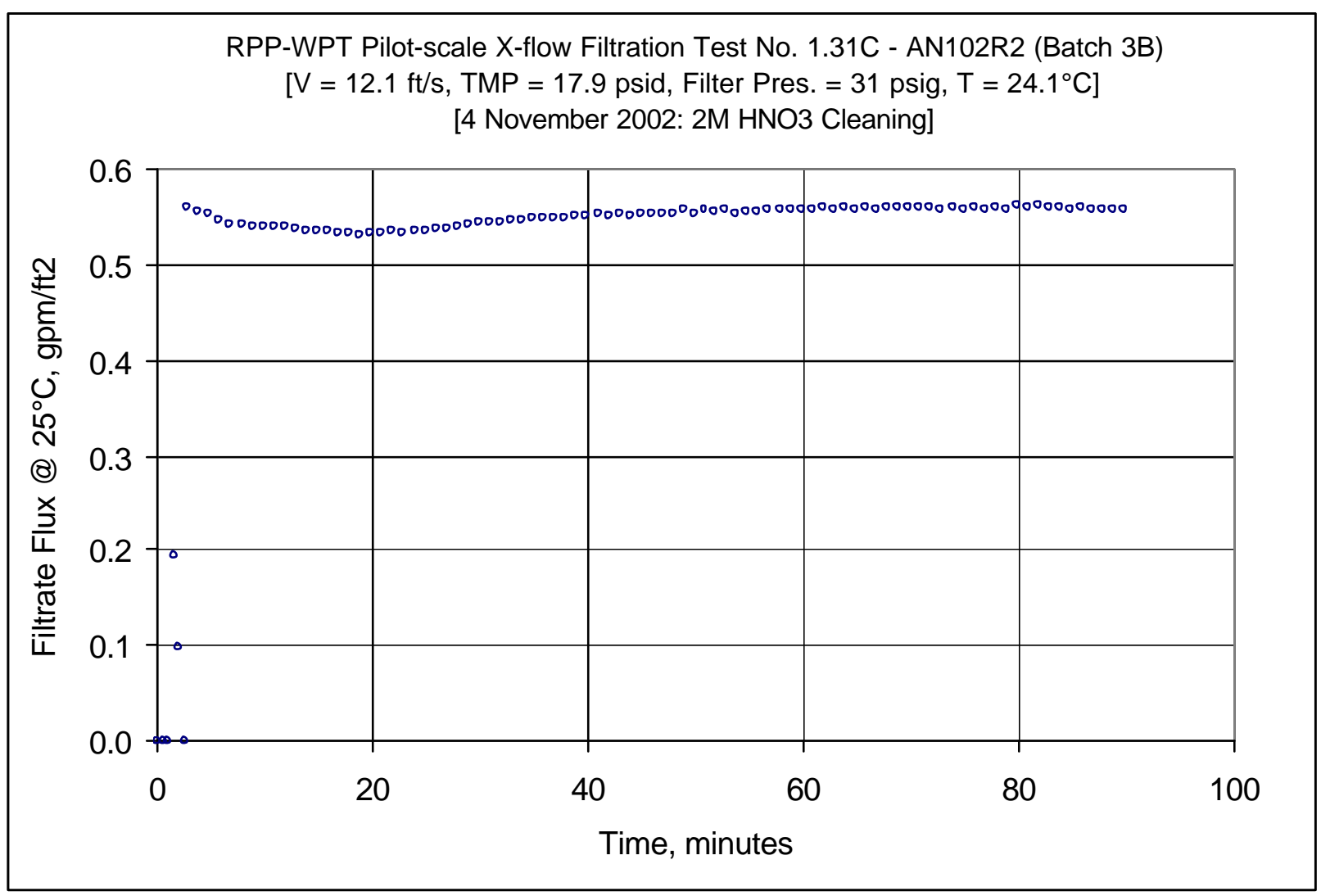

Figure C11: 1.31C: After AN-102R2, Batch 3B - 2 M Nitric Acid Cleaning-Second Cleaning 
WSRC-TR-2003-00204, REV. 0

SRT-RPP-2003-00087, REV. 0

\begin{tabular}{|c|c|c|c|c|c|c|c|c|c|c|c|c|c|c|c|c|c|c|c|c|c|c|c|c|c|c|c|}
\hline \multirow{3}{*}{ DATE } & \multirow{3}{*}{ TIME } & \multicolumn{5}{|c|}{ ti Amb. Lo Amb. } & \multirow{3}{*}{$\begin{array}{c}\text { BotTMP } \\
\text { psid } \\
\mathrm{dP2}\end{array}$} & \multirow{3}{*}{$\begin{array}{l}\text { Filter } \\
\text { psigi } \\
\text { P1 }\end{array}$} & \multirow{3}{*}{$\begin{array}{l}\text { CPressure } \\
\text { Filter dP } \\
\text { psid } \\
\text { dP1 }\end{array}$} & $\begin{array}{l}\text { Treasuret } \\
\text { TopTMP }\end{array}$ & & & & Filtrete & Hi Filtot & the & & & & Calcul & Ulated $\mathrm{Fr}_{\mathrm{I}}$ & From Ra & Raw Data & ta $>$ & . & & \\
\hline & & Filtrate & deg & $\operatorname{deg} \mathrm{C}$ & $\operatorname{deg} C$. & Deg $\mathrm{C}$ & & & & $\begin{array}{c}\text { TopTMP } \\
\text { psid }\end{array}$ & $\begin{array}{l}\text { Filtrate } \\
\text { spig }\end{array}$ & $\begin{array}{l}\text { Pulsepot } \\
\text { psig }\end{array}$ & \begin{tabular}{|l} 
Slurry \\
gom
\end{tabular} & Filtrate & Hi Filtate & $\begin{array}{l}\text { Backpulse } \\
\text { gmm }\end{array}$ & & & & & & & & & & & \\
\hline & & & & $\mathrm{T} 1$ & & & & & & & P2 & & & & $\begin{array}{l}\text { gpm } \\
\text { Q3 }\end{array}$ & & & & & & & & & & & & \\
\hline & & 233 & & & & & & & & & & & & & & & & & & & & & & & & & \\
\hline & & & & & & & & & & & & & & & & & & & & & & & & & & & \\
\hline $\begin{array}{l}11 / 4 / 2002 \\
11 / 42002\end{array}$ & $\begin{array}{l}8: 21: 38 \mathrm{AM} \\
8.21 .9 \mathrm{AM}\end{array}$ & $\begin{array}{l}18.233 \\
18243 \\
\end{array}$ & $\begin{array}{l}17.946 \\
17.946\end{array}$ & $\begin{array}{r}16.1 \\
16111\end{array}$ & $\begin{array}{l}17.566 \\
17.556\end{array}$ & 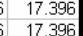 & $\begin{array}{l}-0.114 \\
-0.116\end{array}$ & $\mid \begin{array}{l}-0.131 \\
-0.131\end{array}$ & $\begin{array}{l}-0.047 \\
-0.047\end{array}$ & 0.072 & $\begin{array}{l}-0.366 \\
-0.351 \\
-0.03\end{array}$ & 0.44 & $\begin{array}{r}-0.22 \\
-0.224\end{array}$ & 0.001 & 0.0011 & 0.00 & & & Convers & & $851 \mathrm{n}$ & & 2/day/bar! & $\mathrm{g} / \mathrm{gpm} /$ & n/fit/harg & & \\
\hline $11 / 4 / 4 / 2002$ & 8.21.40 AM & 18.238 & 17.946 & 16.111 & 17.561 & $\begin{array}{lll}117396 \\
17\end{array}$ & -0.116 & -0.131 & $\begin{array}{l}-0.047 \\
-0.047 \\
-0.04\end{array}$ & 0.071 & $\mid-0.325$ & & -0.2222 & 0.001 & 001 & & & & Note: $G$ & Gauge Pr & Pressu & & is appr & & 4 & & \\
\hline $11 / 4 / 2002$ & 8:21:41 AM & & 17.951 & 16.105 & 17.561 & 17.396 & -0.116 & -0.131 & -0.047 & 0.071 & -0.363 & & -0.224 & 0.001 & 0.001 & & & & & & & & & & & & \\
\hline 11/4/2002 & $21: 42 \mathrm{AM}$ & 233 & 17.946 & $\begin{array}{l}16.105 \\
\end{array}$ & 17.561 & 17.39 & -0.116 & \begin{tabular}{|l|}
-0.131 \\
\end{tabular} & -0.047 & 0.071 & -0.308 & & -0.222 & 0.001 & 0.001 & & & & Pressur & sure $\mathrm{P} 1$ is & correct & cted for & & inches of & water tu & th & \\
\hline 1/4/2002 & $21: 43 \mathrm{AM}$ & 233 & 17.946 & \begin{tabular}{|l|} 
\\
\end{tabular} & 17.556 & 17.3 & -0.116 & $\mid-0.131$ & -0.047 & 0.071 & -0.369 & & -0.222 & 0.001 & 0.001 & 0. & & & WW & & & & $<$ Filtrat & rate Flux & $<\mathrm{PEF}$ & & \\
\hline 11/4/2002 & $21: 44 \mathrm{AM}$ & .228 & 17.946 & 16.1 & 17.561 & 17.40 & -0.114 & -0.131 & -0.047 & 0.071 & -0.363 & 0.4 & -0.222 & 0.001 & 0.001 & & & & & & & & & at $25 \mathrm{C}$ & & $\times 1000$ & \\
\hline & & & & & & & & & & & & & & & & & & Time & Press. & Vel. T & TMP & TMF & & & & & \\
\hline$\frac{\text { Data - Per M }}{1140007}$ & & & & & & & & & & & & & & & & & & & & & & & & & & & \\
\hline $\begin{array}{l}111 / 4 / 2002 \\
11 / 4 / 2002\end{array}$ & $\begin{array}{l}\text { 1:1:22:29 PM } \\
\text { 1.11:29 PM }\end{array}$ & 24.0255 & $\begin{array}{l}21.413 \\
21.413\end{array}$ & $\begin{array}{l}22.423 \\
22.48\end{array}$ & $\begin{array}{l}22.3399 \\
22.309\end{array}$ & & $\begin{array}{l}2.972 \\
3.038\end{array}$ & $\begin{array}{l}32.776 \\
3337\end{array}$ & $\begin{array}{l}4.6344 \\
4.665\end{array}$ & $\begin{array}{l}-1.433 \\
-1.361\end{array}$ & $\begin{array}{l}24.627 \\
24.662\end{array}$ & & & 0.001 & 0.0011 & & & & 1.3 & .5 & & & & & & & \\
\hline $11 / 4 / 2002$ & 1:13:29 PM & 23.0294 & 21.424 & 22.253 & 22.289 & 22.316 & $\begin{array}{r}.3 .906 \\
2.926\end{array}$ & 32.778 & $\begin{array}{l}4.0650 \\
4.668\end{array}$ & $\begin{array}{l}-1.301 \\
-1.464\end{array}$ & $\begin{array}{l}24.062 \\
4\end{array}$ & 0.557 & $\begin{array}{l}46.2455 \\
46.256\end{array}$ & 0.001 & 0.001 & 0.002 & 1.00 & 100.01667 & $\begin{array}{ll}7 & 31.6 \\
\end{array}$ & $\begin{array}{lll}3 & 11.3 \\
\end{array}$ & 0.7 & 050 & 0.000 & .000 & 0.0002 & 221 & \\
\hline & 1:13:59 PM & 23.459 & 21.429 & 22.092 & 22.37 & 22.316 & 10.111 & 32.503 & 4.717 & 5.799 & 16.994 & $0=$ & 47.388 & 1.192 & 001 & & 1.50 & $50 \quad 0.025$ & $\begin{array}{ll}5 & 31.0\end{array}$ & & & & & & & 324.266 & \\
\hline $11 / 4$ & :14:29 P & 23.057 & 21.444 & 22.127 & 22.37 & 22. & 21.565 & 32.069 & 4.7 & 18.469 & 9. 4.103 & -0.454 & 49.129 & 0.609 & 3.086 & & & 0.03333 & $3 \quad 30.6$ & 612.0 & 20.0 & .380 & 0.091 & 0.099 & 90.0049 & 94.922 & \\
\hline & 1:14:59 PM & 22.878 & & 21.993 & 22.296 & 22.326 & 20.482 & 31.934 & 4.728 & 17.1 & 5.279 & -4.658 & 48.876 & 0.002 & 3.453 & & & 0.04167 & $\begin{array}{l}7 \quad 30.5 \\
\end{array}$ & & 188 & & & & & & \\
\hline 11/4/2002 & 1:15:18 PM & 2.792 & 21.445 & 21.892 & 22.346 & 22.33 & 20.627 & 32.11 & 4.748 & 17.202 & 5.172 & -4.664 & 49.083 & 0.002 & 3.438 & & & 20.04694 & $\begin{array}{ll}4 & 30.6 \\
\end{array}$ & 2.0 & 18.9 & 304 & 40.513 & & 8 & 629.605 & \\
\hline 11/4/2002 & 1:16:18 PM & 22.557 & 21.452 & 21.858 & 22.362 & 22.319 & 20.906 & 32.133 & 4.615 & & 4.912 & -4.665 & & 0.002 & 3.405 & & 3.82 & & $\begin{array}{lll}1 & 30.7 \\
\end{array}$ & 12.0 & 19.3 & 332 & 20.508 & & & 28.744 & \\
\hline 11/4/2002 & 1:17:18 PM & 22.392 & 21.457 & 21.698 & 22.312 & 22.339 & 20.887 & 31.959 & 4.701 & 17.462 & $\begin{array}{l}4.796 \\
6.701\end{array}$ & -4.664 & & 0.002 & & & 4.82 & & $\begin{array}{ll}8 & 30.5 \\
\end{array}$ & & 19.2 & 322 & & & & & \\
\hline $\begin{array}{l}11 / 4 / 2002 \\
11 / 40\end{array}$ & $\begin{array}{l}1: 18: 18 \text { PM } \\
1.19 .18\end{array}$ & $\begin{array}{l}22.393 \\
225993\end{array}$ & $\begin{array}{l}21.474 \\
21.47\end{array}$ & $\begin{array}{l}21.9116 \\
22199\end{array}$ & $\begin{array}{r}22.042 \\
21.208\end{array}$ & $\begin{array}{l}22.371 \\
22393\end{array}$ & $\begin{array}{r}20.935 \\
2087\end{array}$ & $\begin{array}{l}31.719 \\
31.948\end{array}$ & 4.685 & $\begin{array}{l}17.556 \\
17.394\end{array}$ & $\begin{array}{l}5.724 \\
4 \\
4\end{array}$ & -4.661 & \begin{tabular}{|l}
48.951 \\
48947
\end{tabular} & 0.001 & & & 5.82 & & $\begin{array}{ll}4 & 30.2 \\
1\end{array}$ & 2.0 & 19.2 & 327 & & & 84 & $\begin{array}{l}428.433 \\
4\end{array}$ & $\begin{array}{ll}3 & 24.20 \\
6 & 2\end{array}$ \\
\hline $\begin{array}{r}11 / 4 / 2002 \\
1142002\end{array}$ & $\begin{array}{l}1: 19: 18 \mathrm{PM} \\
1.2018 \mathrm{PM}\end{array}$ & $\begin{array}{l}22.586 \\
22.582\end{array}$ & $\begin{array}{r}21.47 \\
21.476\end{array}$ & $\begin{array}{r}22.199 \\
2218\end{array}$ & $\begin{array}{r}21.928 \\
211969\end{array}$ & $\begin{array}{l}22.393 \\
22389\end{array}$ & $\begin{array}{r}20.887 \\
21144\end{array}$ & $\begin{array}{r}31.848 \\
31998\end{array}$ & $\begin{array}{l}4.685 \\
4.682\end{array}$ & $\begin{array}{c}17.394 \\
1771\end{array}$ & $\begin{array}{l}4 \quad 4.689 \\
4 \\
4\end{array}$ & $\begin{array}{l}-4.652 \\
-4649\end{array}$ & $\begin{array}{l}48.947 \\
4859\end{array}$ & 0.001 & $\begin{array}{r}3.363 \\
3.36 \\
\end{array}$ & & $\begin{array}{l}6.82 \\
7.82\end{array}$ & & $\begin{array}{ll}1 & 30.4 \\
8 & 30.5\end{array}$ & & 19.1 & .320 329 & 0.501 & & & $\begin{array}{l}428.366 \\
49.77 .938\end{array}$ & $\begin{array}{ll}6 & 24.14 \\
8 & 2378\end{array}$ \\
\hline & $\begin{array}{l}1: 21: 18 \mathrm{PM} \\
1: 21: 18 \mathrm{PM}\end{array}$ & $\begin{array}{l}22.682 \\
22.829\end{array}$ & $\begin{array}{l}21.468 \\
21.487\end{array}$ & \begin{tabular}{|l|}
22.18 \\
22.452
\end{tabular} & $\begin{array}{r}21.1699 \\
21.92\end{array}$ & $\begin{array}{l}22.369 \\
22.349\end{array}$ & $\begin{array}{l}21.144 \\
20.985\end{array}$ & \begin{tabular}{|l|}
31.998 \\
31.982
\end{tabular} & $\begin{array}{l}4.6622 \\
4.6899\end{array}$ & $\begin{array}{l}17.11 \\
17.617\end{array}$ & $\begin{array}{l}4.715 \\
4.692\end{array}$ & $\begin{array}{l}-4.649 \\
-4.638\end{array}$ & \begin{tabular}{|l|}
44.959 \\
49.075
\end{tabular} & $\begin{array}{l}0.001 \\
0.002\end{array}$ & $\begin{array}{r}3.36 \\
3.364\end{array}$ & & $\begin{array}{l}7.82 \\
8.82\end{array}$ & $\begin{array}{ll}2 & 0.13018 \\
2 & 0.14694\end{array}$ & $\begin{array}{ll}8 & 30.5 \\
4 & 30.5\end{array}$ & $\begin{array}{l}512.0 \\
5 \\
5\end{array}$ & $\begin{array}{l}19.4 \\
193\end{array}$ & $\begin{array}{l}1.399 \\
1331\end{array}$ & $\begin{array}{l}9.501 \\
1 \\
10.502\end{array}$ & $\begin{array}{l}0.543 \\
0.539\end{array}$ & 90.0279 & 927.938 & $\begin{array}{lll}8 & 23.78 \\
5 & 237\end{array}$ \\
\hline $11 / 4 / 4 / 2002$ & 1:22:18 PM & 22.91 & 21.488 & 22.387 & 21.89 & 22.375 & 21.192 & 32.154 & 4.697 & 17.798 & $\begin{array}{r}34.052 \\
4.707\end{array}$ & $\begin{array}{l}-4.050 \\
-4.638 \\
\end{array}$ & \begin{tabular}{|l|}
49.045 \\
49.047
\end{tabular} & 0.002 & $\begin{array}{l}3.364 \\
3.365\end{array}$ & & $\begin{array}{l}0.82 \\
9.82\end{array}$ & $\begin{array}{ll}2 \\
2 & 0.14694 \\
\end{array}$ & $\begin{array}{ll}4 & 30.5 \\
1 & 30.7\end{array}$ & $\begin{array}{l}72.0 \\
12.0\end{array}$ & 19.5 & .344 & $\begin{array}{l}0.502 \\
4 \\
4\end{array}$ & & & $\begin{array}{l}927.955 \\
7\end{array}$ & $\begin{array}{lll}7 & 23.11 \\
7 & 23.59\end{array}$ \\
\hline 11/4/2002 & 1:23:18 PM & 3.031 & 21.499 & 22.614 & 21.831 & 22.386 & 20.989 & 31.827 & 4.618 & 17.783 & $\begin{array}{l}3.791 \\
\end{array}$ & & & 0.001 & 8 & 0.001 & 10. & 0.18028 & $\begin{array}{ll}8 & 30.4\end{array}$ & $\begin{array}{ll}412.0 \\
-12\end{array}$ & 19.4 & 337 & 70.504 & & & 827.799 & \\
\hline $11 / 4 / 2002$ & 1:24:18 PM & & 21.495 & 22.565 & 21.842 & 22.3 & 20.946 & 32.027 & 4.668 & 17.593 & 3 & & & 0.001 & 4 & & & & & 2.0 & & 329 & & & & & \\
\hline & & 23.22 & & 22.893 & 21.813 & & 20.761 & 31.816 & & & & & & & & & & & 30.3 & $\begin{array}{ll}312.0 \\
\end{array}$ & & & & & & & \\
\hline & & 3.482 & 21.507 & 23.241 & 21.683 & & 20.805 & 32.176 & 4.697 & 17.3 & 5.002 & & & 0.0 & & & & & 30.7 & & & & & & & & \\
\hline & 1:27: & 784 & 21.518 & 23.316 & 21.638 & & 20.827 & 32.269 & & 17.3 & 5.059 & & & & & & & & $\begin{array}{ll}4 & 30.8 \\
\end{array}$ & & & & & & & & \\
\hline & 1:28:18 & 3.889 & 21.523 & 23.557 & 21.694 & & 20.455 & 31.713 & 4.637 & & 5.146 & & & 0.001 & & & & & 30.2 & $\begin{array}{ll}212.0 \\
\end{array}$ & 18.8 & & & & & & \\
\hline & 1:29:18 F & 4.17. & 21.543 & 23.783 & 21.669 & 22. & 20.595 & 32.04 & 4.675 & $17.2 \mathrm{C}$ & 5.241 & & & 0.001 & 3.4 & 0.00 & & & $\begin{array}{lll}8 & 30.6\end{array}$ & & & 306 & & & & & \\
\hline & 1:30: & 24.24 & 21.533 & 23.828 & 21.734 & 22. & 20. & 32.104 & & 17. & 3. & & & 0.001 & & & & & $\begin{array}{ll}4 & 30.6\end{array}$ & $\begin{array}{lll}5 & 12.1\end{array}$ & & & & & & & \\
\hline $11 / 4$ & 1:31: & 4.432 & 21.539 & 24.095 & 21.645 & 22. & 20.161 & 31.86 & 4.679 & 16.8 & 5.357 & & & 0.001 & & & & & $\begin{array}{ll}1 & 30.4\end{array}$ & 2.0 & 5 & 174 & & & & & \\
\hline & & 4.506 & 21.54 & 24.049 & 21.6 & & 20. & 31.895 & & & 5. & & & & & 0.00 & & & 8 & & & & & & & & \\
\hline & & 4.632 & 21.544 & & 21.6 & & & & & & & & & & & & & & & & & & & & & & \\
\hline $11 / 4$ & & 4.703 & 21.555 & 24.201 & $\begin{array}{l}21.701 \\
21716\end{array}$ & & 20.142 & \begin{tabular}{|l|l|}
32.005 \\
11.079
\end{tabular} & & & $\begin{array}{l}4 \quad 5.542 \\
-5507 \\
\end{array}$ & & & & & & & &. & & & & & & & 55 & 5 \\
\hline $\begin{array}{r}11 / 4 / 2002 \\
11 / 42002\end{array}$ & $\begin{array}{l}1: 35 \\
1.36\end{array}$ & $\frac{4}{8}$ & 21.571 & $\begin{array}{l}24.452 \\
2431\end{array}$ & $\begin{array}{l}21.716 \\
21756\end{array}$ & & 20.132 & $\begin{array}{l}31.978 \\
32009\end{array}$ & $\begin{array}{l}4.676 \\
4.653\end{array}$ & & & & & & & & & & 5 & & & & & & & $\begin{array}{l}28.821 \\
29.197\end{array}$ & $\begin{array}{l}1 \\
7\end{array}$ \\
\hline & $\begin{array}{l}1.36 .1 \\
1.37 .1\end{array}$ & 4 & $\begin{array}{r}21.156 \\
21.576\end{array}$ & 24.357 & $\begin{array}{r}21.1766 \\
21736\end{array}$ & & & $\begin{array}{l}32.009 \\
31905\end{array}$ & 4.6 & & & & & & & & & & $\begin{array}{l}4 \quad 50.5 \\
1 \\
1\end{array}$ & 4 & & & & & & & $\begin{array}{ll}7 & 24.85 \\
8 & 2482\end{array}$ \\
\hline & 1:38:1 & 24.944 & 21.57 & & 21. & & & & & & & $\begin{array}{l}-4.4961 \\
-4.496\end{array}$ & & & & & & & $\begin{array}{ll}8 & 30.4 \\
8\end{array}$ & $\begin{array}{ll} & 2.1 \\
& 12.1\end{array}$ & & 63 & & & & & 3 \\
\hline $11 / 4$ & 1:39 & 25.039 & 21.586 & 24.593 & 21. & 22 & 19. & 32.193 & 4.729 & & & -4.49 & & & & & & & $\begin{array}{ll}4 & 30.7\end{array}$ & 12.1 & 3.3 & 1.262 & & & & & \\
\hline & 1:40: & 25.008 & 21.569 & 24. & 21. & 22 & & & 4.644 & & & -4 & & & & & & & $\begin{array}{ll}1 & 30.6\end{array}$ & 5 & 2 & 254 & & & & & \\
\hline & & 25.073 & $\begin{array}{l}21.464 \\
\end{array}$ & & 21.5 & 21.7 & & 32.102 & & & & & & & & & & & . & 2112 & & 558 & & & & & \\
\hline 11/4/2002 & 1:42:1 & 25.018 & 21.373 & 24.406 & $\begin{array}{r}21.378 \\
\end{array}$ & 21.2. & $\begin{array}{r}19.562 \\
19.61\end{array}$ & $\begin{array}{l}31.74 \\
\end{array}$ & 4.637 & $\begin{array}{r}16.43 \\
15.44\end{array}$ & 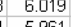 & -4.478 & 49.215 & & 3. & & & & $\begin{array}{ll}4 \quad 30.3 \\
\end{array}$ & 2.1 & $I_{1}$ & 241 & 34 & & & 86 & 6 \\
\hline $11 / 4 / 2002$ & $\begin{array}{l}1: 43: 18 \mathrm{~F} \\
1.44 \cdot 18 \mathrm{P}\end{array}$ & 5.106 & $\begin{array}{r}21.25 \\
21.77\end{array}$ & 24.388 & $\begin{array}{r}21.1899 \\
22109\end{array}$ & 20.8 & $\begin{array}{r}19.61 \\
19899\end{array}$ & $\begin{array}{r}32.027 \\
32191\end{array}$ & $\begin{array}{l}4.628 \\
4.672\end{array}$ & $\begin{array}{l}16.444 \\
165399\end{array}$ & 5.961 & -4.47 & & & & & & & 30.6 & 2.1 & .0 & 243 & & & & $\begin{array}{l}230.242 \\
2.3027\end{array}$ & \\
\hline & 1.44 & & $\begin{array}{l}21.1 / 16 \\
21.093\end{array}$ & $\begin{array}{r}24.4 \\
24388\end{array}$ & $\begin{array}{r}21.095 \\
21032\end{array}$ & & 19.839 & $\mid \begin{array}{l}31.91 \\
31.814\end{array}$ & $\begin{array}{l}4.6 .62 \\
4.641\end{array}$ & & $\begin{array}{ll}6 \\
3 \\
3\end{array}$ & & & & & & & & & & & 8 & & & & & $\frac{2}{2}$ \\
\hline & & 25.0 & & $\begin{array}{l}24.000 \\
24.419\end{array}$ & 20. & & & & & & & & & & & & & & 5 & 12.1 & & 241 & & & & & \\
\hline & & 25.133 & 20. & & & & & & & & & & & & & & & & $\begin{array}{ll}8 & 30.3\end{array}$ & 12.1 & & & & & & & \\
\hline & & & & & 20. & & & & & & & -4.4 .5 & & & & & & & $\begin{array}{ll}4 & 30.3 \\
\end{array}$ & 2.1 & & & & & & & \\
\hline & 1:49: & 25.156 & 20.604 & & 20.79 & & & & & & & & & & & & & & 30.4 & & & 226 & & & & & \\
\hline & & 25.104 & 20.502 & 24.446 & 20.723 & 1. & & 31.901 & 4.612 & 16.244 & $\begin{array}{l}4 \quad 6.155 \\
4.550\end{array}$ & 50 & & & & & & & $\begin{array}{ll}8 & 30.4\end{array}$ & 12.1 & & 228 & & & & & \\
\hline & & & 20.40 & 24.427 & 20.7 & & & & & & $\begin{array}{l}6.259 \\
\end{array}$ & & & & & & & & & & & & & & & & \\
\hline & & & 20 & 24.501 & 20. & & & & $4.6 \mathrm{C}$ & & 6.2 & & & & & & & & & & & & & & & & \\
\hline & & & 20.1 & 24. & 20. & & & 31 & & & 6. & & & & 3 & & & & & & & & & & & & \\
\hline & & & 19. & 24 & 20 & & & & & & 6.38 & & & & & & & & & & & & & & & & \\
\hline & & & 19. & 24 & 20 & & & & & & 6. & & & & & & & & & & & & & & & & 6.7 \\
\hline & & & & 24 & & & & & & & & & & & & & & & & & & & & & & & \\
\hline & & & & 24 & 20.548 & & & 31.6 & 4.5 & & & & & & $65 /$ & & & & & & & & & & & 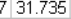 & \\
\hline
\end{tabular}

Figure C12a: 1.31C: After AN-102R2, Batch 3B - 2 M Nitric Acid Cleaning - First Cleaning (first 45 minutes) - Raw \& Calculated Data 
WSRC-TR-2003-00204, REV. 0

SRT-RPP-2003-00087, REV. 0

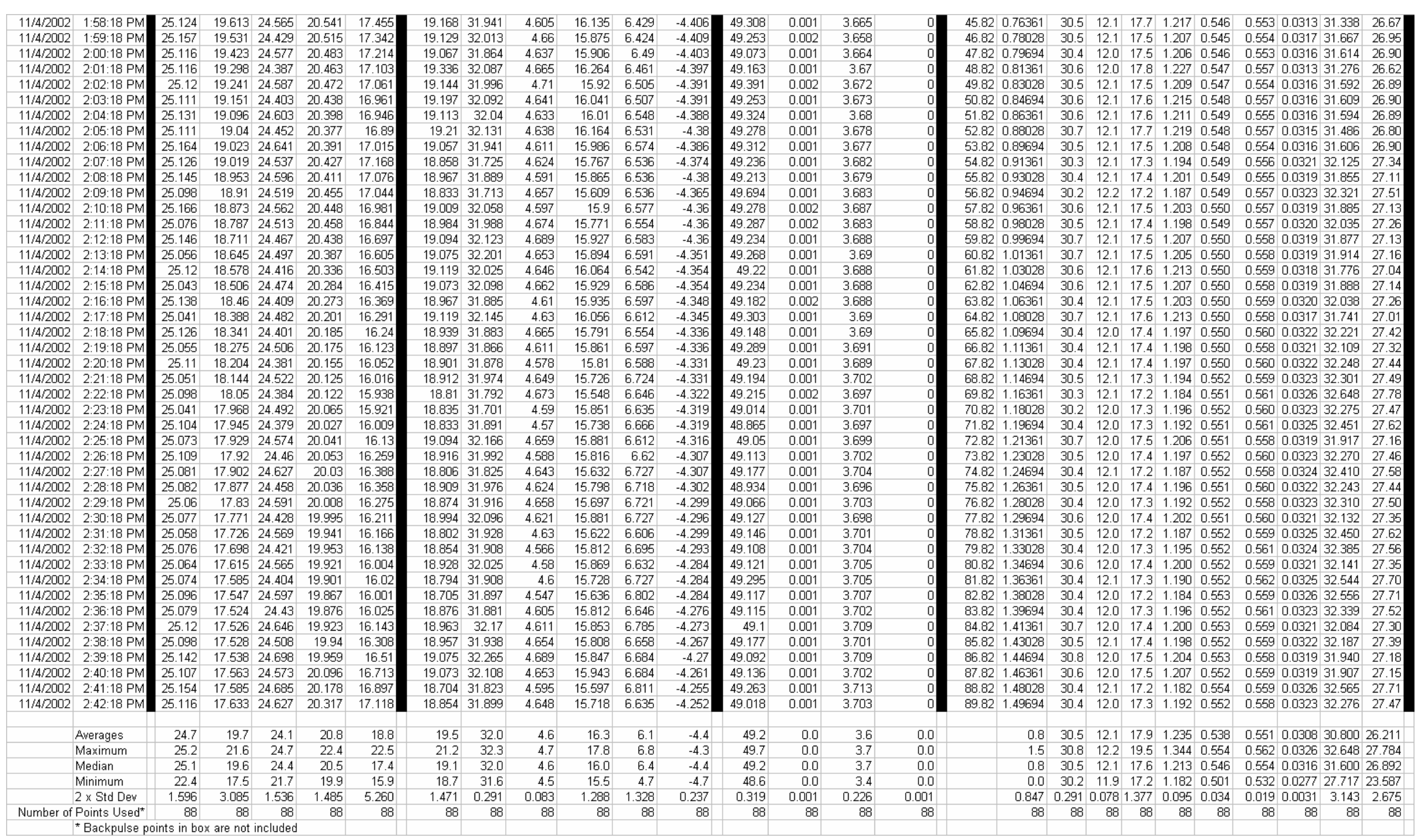

Figure C12b: 1.31C: After AN-102R2, Batch 3B - 2 M Nitric Acid Cleaning - Second Cleaning (remaining 45 minutes) - Raw \& Calculated Data 
WSRC-TR-2003-00204, REV. 0

SRT-RPP-2003-00087, REV. 0

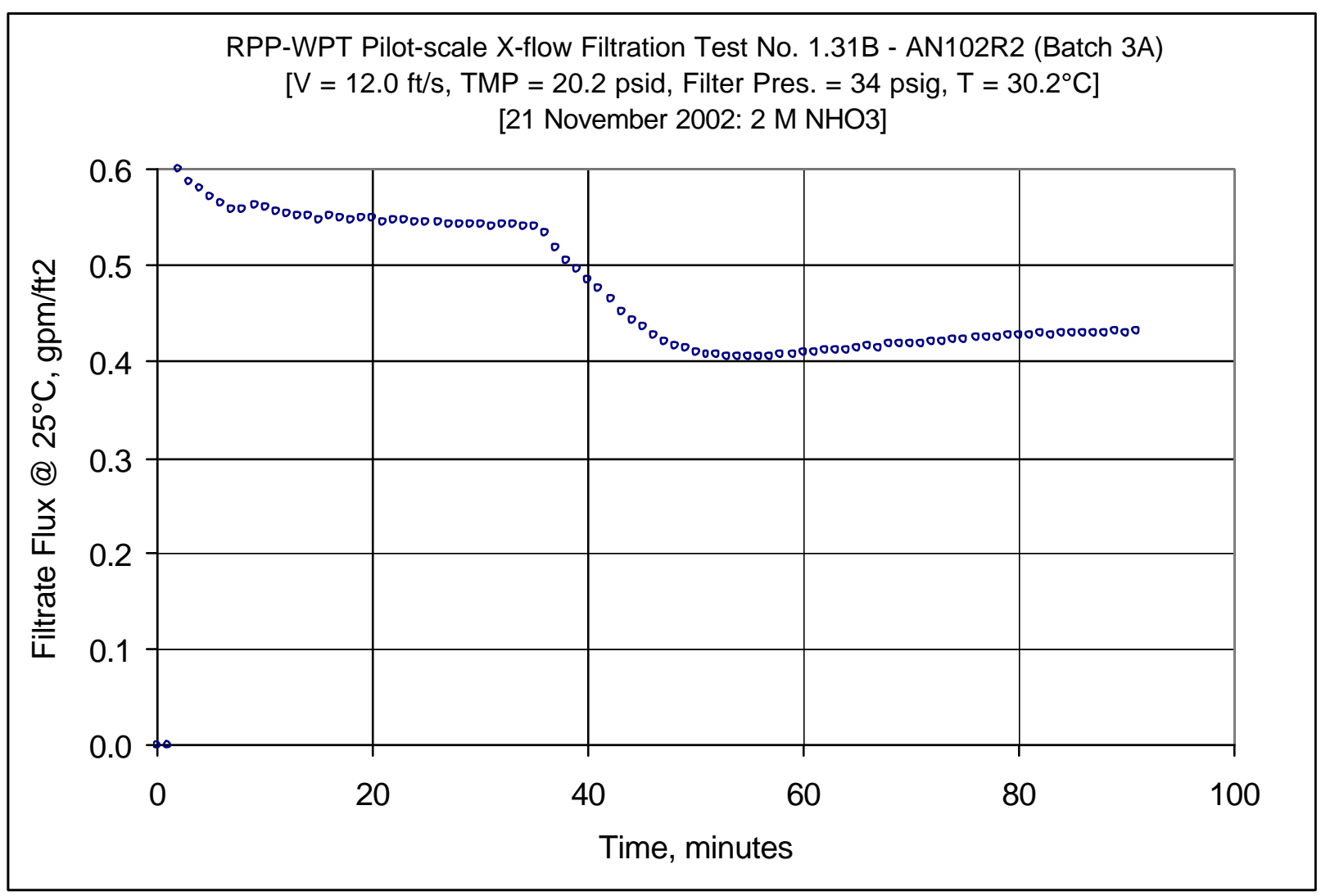

Figure C13: 1.31B: After AN-102R2, Batch 3A - 2 M Nitric Acid Cleaning - First Cleaning 
WSRC-TR-2003-00204, REV. 0

SRT-RPP-2003-00087, REV. 0

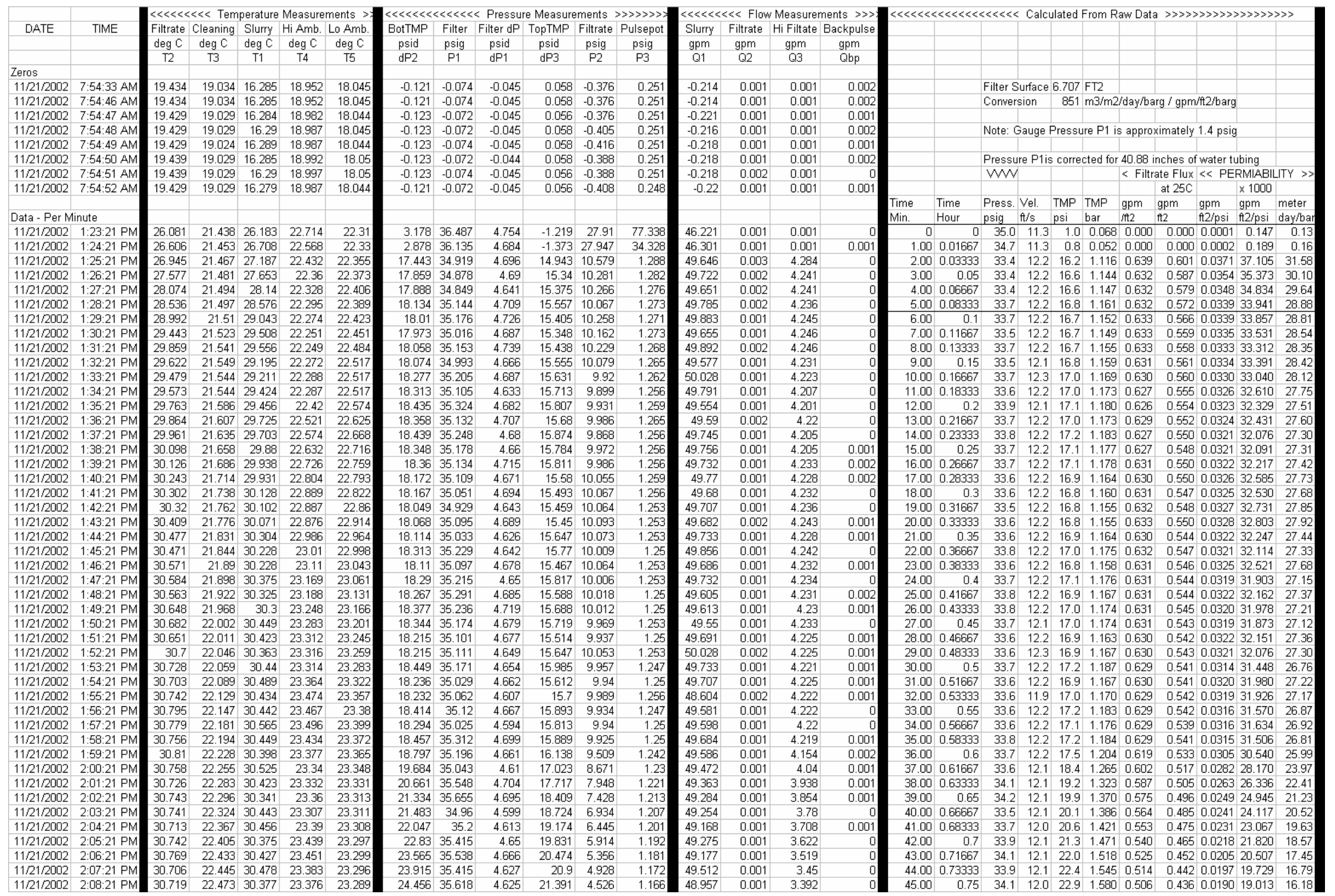

Figure C14a: 1.31B: After AN-102R2, Batch 3A - 2 M Nitric Acid Cleaning - First Cleaning (first 45 minutes) - Raw \& Calculated Data 
WSRC-TR-2003-00204, REV. 0

SRT-RPP-2003-00087, REV. 0

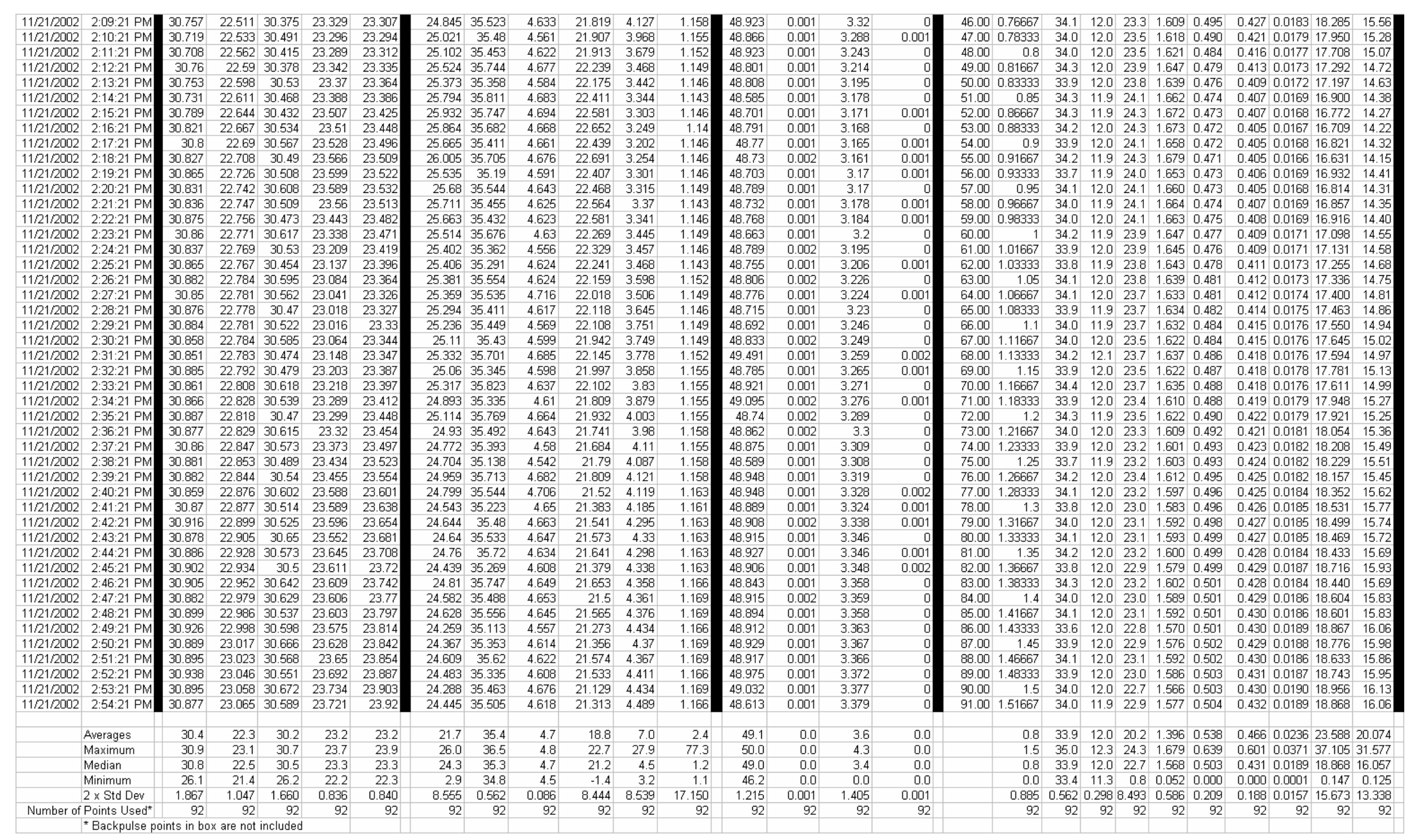

Figure C14b: 1.31B: After AN-102R2, Batch 3A - 2 M Nitric Acid Cleaning - First Cleaning (remaining 45 minutes) - Raw \& Calculated Data 
WSRC-TR-2003-00204, REV. 0

SRT-RPP-2003-00087, REV. 0

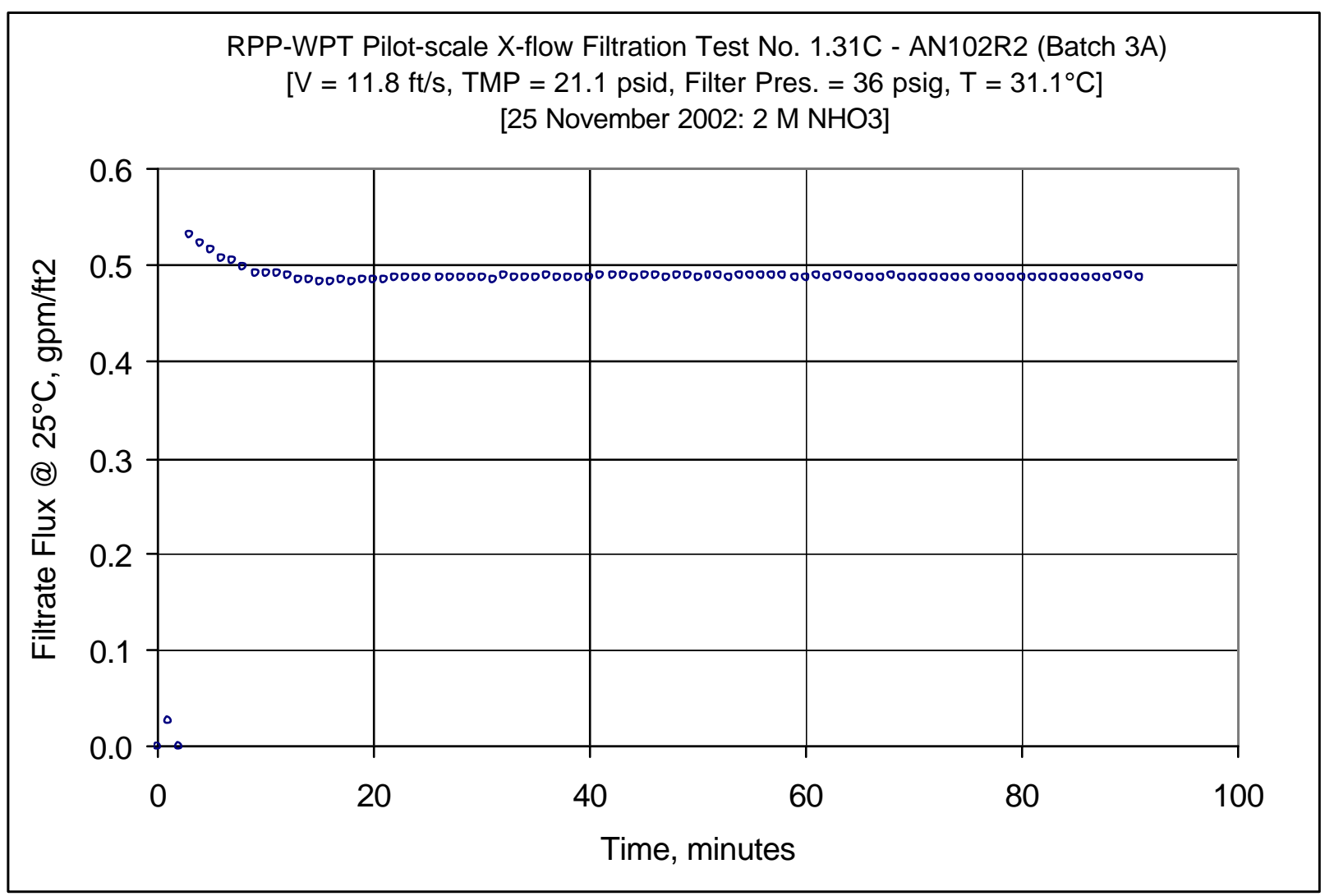

Figure C15: 1.31C: After AN-102R2, Batch 3A-2 M Nitric Acid Cleaning-Second Cleaning 
WSRC-TR-2003-00204, REV. 0

SRT-RPP-2003-00087, REV. 0

\begin{tabular}{|c|c|c|c|c|c|c|c|c|c|c|c|c|c|c|c|c|c|c|c|c|c|c|c|c|c|c|c|}
\hline \multirow{3}{*}{ DATE } & \multirow{2}{*}{ TIME } & \multicolumn{5}{|c|}{ 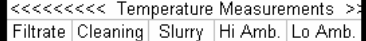 } & \multirow{3}{*}{ 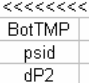 } & \multirow{3}{*}{$\begin{array}{l}\text { Filter } \\
\text { psig }\end{array}$} & \multirow{3}{*}{$\begin{array}{l}\text { Filtes dPre } \\
\text { psid } \\
\text { dP1 }\end{array}$} & $\begin{array}{l}\text { re Measure } \\
\text { TopTMP }\end{array}$ & Filtrate & Pulsepot & & $\begin{array}{c}\text { \&iltrate } \\
\text { Fitate }\end{array}$ & $\begin{array}{l}\text { Measuruer } \\
\text { Hi Filtate }\end{array}$ & $\begin{array}{l}\text { ments } \gg> \\
\text { Backpulse }\end{array}$ & & & & S Lalcul & llated $F$ & From R & taw Lata & $a \gg$ & $>$ & & \\
\hline & & . & $\operatorname{deg} C$ & $\operatorname{deg} \mathrm{C}$ & $\operatorname{deg} C$ & $\operatorname{deg} C$ & & & & psid & psig & psig & $\begin{array}{l}\text { ofury } \\
\mathrm{gpm}\end{array}$ & gpm & gpm & $\mathrm{gpm}$ & & & & & & & & & & & \\
\hline & & & & & & & & & & & & & & ? & Q3 & & & & & & & & & & & & \\
\hline & & & & & & & & & & & & & & & & & & & & & & & & & & & \\
\hline & 3:13 AM & 21.491 & 17.89 & 14.224 & 68 & 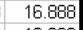 & -0.123 & -0.066 & -0.049 & 0.06 & 76 & 5 & -0.218 & 001 & 0.001 & & & & Filter $S$ & Surface 6 & 6.707 म & FT2 & & & & & \\
\hline 11/25/2002 & 8:03:26 AM & 21.491 & 17.89 & 14.224 & \begin{tabular}{|l}
21.68 \\
\end{tabular} & 16.888 & -0.123 & -0.066 & 49 & 0.06 & 776 & 0.205 & \begin{tabular}{|c|}
-0.218 \\
\end{tabular} & 101 & 001 & & & & Convers & rsion & $851 \mathrm{r}$ & & /day/bard & $\mathrm{arg} / \mathrm{gpm} /$ & miftzibarg & & \\
\hline & 8:03:27 AM & 21.507 & 17.9 & $\begin{array}{l}14.245 \\
\end{array}$ & 21.701 & 893 & & -0.068 & & 0.062 & 862 & 0.208 & & & & & & & & & & & & & & & \\
\hline $5 / 2002$ & 8:03:28 AM & 21.512 & 17.905 & 14.245 & 21,701 & 993 & -0.125 & -0.064 & -0.048 & 0.062 & 76 & 0.211 & \begin{tabular}{|c|}
-0.212 \\
\end{tabular} & 0.002 & 0.001 & & & & Note: $G$ & & & ure P1 i & roxi & ximately & y 1.4 psig & & \\
\hline $11 / 25 / 2002$ & 8:03:29 AM & $\begin{array}{l}21.507 \\
21.517\end{array}$ & 17.905 & $\begin{array}{r}14.25 \\
14.25\end{array}$ & 21.701 & 16.893 & -0.123 & -0.066 & -0 & 0.062 & $\begin{array}{ll}2 & -0.373 \\
\end{array}$ & 0.208 & \begin{tabular}{|c|}
-0.212 \\
\end{tabular} & 0.002 & 0.001 & & & & & & & & & & & & \\
\hline $\begin{array}{l}11 / 25 / 2002 \\
11 / 25 / 2002\end{array}$ & $8: 00: 30 \mathrm{AM}$ & 12.512 & $\begin{array}{l}17.955 \\
17905\end{array}$ & $\begin{array}{r}14.245 \\
14.25\end{array}$ & $\begin{array}{l}21.701 \\
21.696\end{array}$ & $\begin{array}{l}16.893 \\
16883\end{array}$ & $\begin{array}{l}-0.123 \\
-0.023 \\
-12\end{array}$ & $\begin{array}{l}-0.066 \\
-0.066\end{array}$ & $\begin{array}{l}-0.048 \\
-0.048\end{array}$ & 0.0662 & 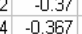 & $\begin{array}{l}0.208 \\
0.208\end{array}$ & $\begin{array}{r}-0.21 \\
-0.214\end{array}$ & 0.0022 & 0.001 & & & & $\begin{array}{l}\text { Pressur } \\
\text { my }\end{array}$ & ure Plis & 5 correc & ected for & $\begin{array}{l}40.88 \text { in } \\
<\text { <iltrat }\end{array}$ & $\begin{array}{l}\text { inches of } \\
\text { rate Flux }\end{array}$ & $\begin{array}{l}\text { of water tu } \\
x \leqslant<P E F\end{array}$ & tu & \\
\hline $11 / 25 / 2002$ & 8:03:32 AM & 21501 & & 14.24 & $\begin{array}{l}1.6965 \\
1.695\end{array}$ & .888 & $\begin{array}{l}-0.121 \\
-0.121\end{array}$ & \begin{tabular}{|l|l}
-0.060 \\
-0.064
\end{tabular} & -0.048 & 0.062 & & & & & & & & & & & & & & at 250 & & & \\
\hline & $0.00 .02 \mathrm{~min}$ & 1.50 & & & 1.005 & & & 1.0 .004 & & 0.002 & $2=0.596$ & 0.2 & -0.21 & 0.002 & 0.001 & & & & Press. & Vel. & TMP 1 & TMP & $\mathrm{gpm}$ & $\mathrm{gpm}$ & om & gpm & \\
\hline - Per Mi & & & & & & & & & & & & & & & & & & pur & & & & & & & & & \\
\hline & & 2.324 & & \begin{tabular}{|l|}
31.84 \\
\end{tabular} & 22.308 & 18.842 & 2.764 & 38.105 & 4.478 & -1.328 & 830.051 & 78.823 & 45.768 & 0.002 & 0.001 & & & & & & & & & & & 172 & \\
\hline & : 33 AM & 937 & 18.416 & $31.762 \mid$ & 22.253 & 188. & 4.11 & 37.869 & 4.43 & 0.163 & $\begin{array}{ll}3 & 29.384\end{array}$ & & 45.89 & 0.12 & & & 1.00 & 0116 & 36.4 & & & & & & & & \\
\hline $1 / 25 / 2002$ & 11:33 AM & 1.929 & 18.442 & $\begin{array}{l}31.634 \\
\end{array}$ & \begin{tabular}{|l|l|} 
& 22.189 \\
\end{tabular} & 18.5 & 7.735 & 38.041 & 4.479 & 3.71 & 124.721 & $10.32 \mathrm{r}$ & \begin{tabular}{|l|}
46.824 \\
\end{tabular} & 1.192 & 0.001 & & 2.00 & 0.03333 & $\begin{array}{ll}3 & 36.6\end{array}$ & $\begin{array}{l}5 \\
5\end{array}$ & 5.7 & 0.395 & 0.000 & 0.000 & 00.0000 & 122 & 02 \\
\hline 002 & 12:33 AM & .919 & 18.463 & 31.798 & 22.149 & 9.016 & 19.824 & 36.928 & 4.397 & 17.553 & $\begin{array}{ll}3 & 10.35\end{array}$ & 5 & \begin{tabular}{|l|}
49.003 \\
\end{tabular} & 0.002 & & & 3.00 & 0.05 & $\begin{array}{l}5 \\
5\end{array}$ & $\begin{array}{l}5 \\
5\end{array}$ & 18.7 & 1.289 & 0.639 & 0.530 & 00.0284 & 428.377 & 7.24 .15 \\
\hline /25/2/2002 & 13:33 AM & 866 & 18.489 & 31.704 & 22.12 & 77 & 20.292 & 36.746 & 4. & 17.786 & 85 & 5 & $\mid$\begin{tabular}{|l|}
$\mid 48.904$ \\
$\mid$
\end{tabular} & 02 & & & 4.00 & 0.06667 & $7 \mid 35.3$ & $\begin{array}{l}8 \\
3\end{array}$ & 19.0 & 1.313 & 0.627 & 0.522 & 20.0274 & 427.392 & 23.31 \\
\hline 02 & 4:33 AM & 931 & 18.515 & 31.661 & 22.055 & 19 & 20.99 & 37.095 & 4. & 78 & $\begin{array}{lll}8 & 9.313\end{array}$ & 812 & & 12 & & & 5.00 & 0.08333 & 35.6 & 5 & 19.7 & 1.357 & & 515 & & & 26 \\
\hline & 5:33 & 912 & 18.536 & 31.83 & 22.046 & 19.19 & 21.486 & ; 37.207 & 4. & & $\begin{array}{l}28.946 \\
2\end{array}$ & 5 & & & & & 6.00 & 0.1 & 35.7 & & 0.2 & & 12 & & & & 35 \\
\hline 2002 & 6:33 & 898 & 18.562 & 31.737 & 22.017 & 19.251 & 21.691 & 37.151 & & 107 & $\begin{array}{ll}7 & 8.81 \\
\end{array}$ & -2.858 & & & & & 7.00 & 0.11667 & 35.7 & 11.9 & 20.4 & .406 & 0.606 & 0.504 & $\begin{array}{l}40.024 \\
-\end{array}$ & 693 & 321.01 \\
\hline $1 / 25 / 2002$ & 7:33 & .953 & 18.603 & 31.688 & 22.032 & 19. & 21.916 & 37.029 & & 238 & $\begin{array}{ll}8.451 \\
\end{array}$ & & & & & & 8.00 & 0.13333 & 35.6 & 511.9 & 20.6 & 1.419 & 98 & 0.498 & 80.0242 & 186 & 6. 20.58 \\
\hline $11 / 25 / 2002$ & s & .939 & 18.624 & 838 & 21.978 & & 22.476 & 37.157 & 4. & & $\begin{array}{lll}8 & 8.099\end{array}$ & & & & & & 9.00 & 0.15 & 35.7 & $7 \quad 11.9$ & 21.2 & .460 & 92 & 0.491 & 10 & 173 & 3 \\
\hline $11 / 25 / 2002$ & 8 & 911 & 18.651 & 55 & 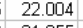 & & 22.865 & 37.712 & & & & & & & & & 10.00 & 0.16667 & 36.2 & 11.9 & 1.5 & 483 & & 0. & 10 & 809 & 41 \\
\hline & s & 341 & 18.677 & 31.676 & 5 & & & 37.164 & & & & & & & & & 11.00 & 0.18333 & 35.7 & 1.9 & 1.2 & .464 & & & & 141 & \\
\hline & 8 & $4{ }_{42}$ & 18.708 & 31.83 & 21.905 & & & 37.12 & & & & & & & & & 12.00 & 0.2 & 35.7 & .9 & .3 & & & & & & \\
\hline & & & & & 21.8 & & & 37.199 & & & & & & & & & & & 35.7 & 9 & & & & & & & \\
\hline & & 962 & 18.754 & & 21.679 & & & $\begin{array}{l}37.044 \\
3.077\end{array}$ & & & & & & & & & & & $\begin{array}{ll}3 & 35.6 \\
\end{array}$ & .9 & & & & & & & \\
\hline & & 6 & 18. & & 21. & & & 36.977 & & & & & & & & & & & 5 & & & & & & & 08 & \\
\hline & & & 18. & & & & & & & & & & & & & & & & 35.6 & & & & & & & & \\
\hline & & & 18.816 & 31.699 & 21. & & 22. & $\begin{array}{l}36.893 \\
\end{array}$ & & & & & & & & & & 333 & $\begin{array}{l}5 \\
6\end{array}$ & & & & & & & & 18 \\
\hline & & & & & 21. & & & & & & & & & & & & & . & 35.6 & & & & & & & & 16 \\
\hline 2 & 8 & & 8 & 3 & 2 & & & & & & & & & & & & 20 & 833 & 8 & & & & & & & & $\begin{array}{l}95 \\
94 \\
94\end{array}$ \\
\hline & & & & & 21.247 & & & & & & & & & & & & 21.00 & 0.35 & $\begin{array}{ll}5 & 35.9\end{array}$ & $\begin{array}{lll}9 & 11.9\end{array}$ & & & & 0. & & & \\
\hline & & & & & 21 & & & & & & & & & & & & & & 36.0 & & & & & & & & \\
\hline & & & & & 21 . & & & & & & & & & & & & & 00.38333 & .0 & & & & & & & & \\
\hline & & & 18. & & 21. & & & & & & & & & & & & & 0.4 & & & & & & & & & \\
\hline & & & & & 21. & & & & & & & & & & & & & 0.41667 & .7 & & & & & 0. & & & \\
\hline & & & 18. & & 20 & & & 37. & & & & & & & & & & 0.43333 & $\begin{array}{ll}3 & 35.7\end{array}$ & & & 1. & & & & & \\
\hline & & & & & & & & & & & & & & & & & 00 & 45 & $\begin{array}{l}5 \\
5\end{array}$ & & & & & & & & \\
\hline & & & & & & & & & & & & & & & & & & & 35.7 & & & & & & & & \\
\hline & & & & & & & & & & & & & & & & & & 833 & .5 & & & & & & & & \\
\hline & & & & & & & & & & & & & & & & & & & & & & & & & & & \\
\hline & & & & & & & & & & & & & & & & & & 0.5 & .4 & & & & & & & & \\
\hline & & & & & & & & & & & & & & & & & & & & & & & & & & & \\
\hline & & & & & & & & & & & & & & & & & & & & & & & & & & & \\
\hline & & & & & & & & & & & & & & & & & & & & & & & & & & & \\
\hline & & & & & & & & & & & & & & & & & & & & & & & & & & & \\
\hline & & & & & & & & & & & & & & & & & & & & & & & & & & & \\
\hline & & & & & & & & & & & & & & & & & & & & & & & & & & & \\
\hline & & & & & & & & & & & & & & & & & & & & & & & & & & & \\
\hline & & & & & & & & & & & & & & & & & & & & & & & & & & & \\
\hline & & & 19. & & & & & & & & & & & & & & & & & & & & & & & & \\
\hline & & & 19. & & 21.3 & & & & & & & & & & & & & & & & & & & & & & \\
\hline & & & 19 & 30. & 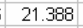 & & 23. & 37. & 4 & & & & & & & & 42.00 & & & & & & & & & & \\
\hline & & & 1 & & 21 & & & 3 & & & & & & & & & & & 9 & & & & & & & & \\
\hline & & & 193 & & & & & & & & & & & & & & & 73333 & 35.9 & & & & & & & & \\
\hline 12002 & 9:24:33 AM & 1.068 & 19.359 & \begin{tabular}{|l|}
30.803 \\
\end{tabular} & 21.526 & 20 & 202 & 37.197 & 409 & 486 & $\begin{array}{lll}6 & 7.341\end{array}$ & 963 & 327 & 0.002 & 3.853 & & 45.00 & 0.75 & 35.7. & 11.8 & 21.8 & 1.5[ & 574 & $.4 c^{6}$ & $.02 \mathrm{CZ}$ & 2.404 & 19.0 \\
\hline
\end{tabular}

Figure C16a: 1.31C: After AN-102R2, Batch 3A - 2 M Nitric Acid Cleaning - Second Cleaning (first 45 minutes) - Raw \& Calculated Data 
WSRC-TR-2003-00204, REV. 0

SRT-RPP-2003-00087, REV. 0

\begin{tabular}{|c|c|c|c|c|c|c|c|c|c|c|c|c|c|c|c|c|c|c|c|c|c|c|c|c|c|c|c|}
\hline $11 / 25 / 2002$ & 9:25:33 AM & 31.081 & 19.362 & 30.841 & 21.469 & 20.278 & 23.435 & 37.373 & 4.477 & 20.667 & 7.37 & -2.966 & 48.048 & 0.001 & 3.853 & & 46.00 & 0.76667 & 35.9 & 11.8 & 22.1 & 1.520 & 0.574 & 0.489 & 0.0222 & 22.171 & 18.87 \\
\hline $11 / 25 / 2002$ & 9:26:33 AM & 31.05 & 19.401 & 30.92 & 21.463 & 20.307 & 23.497 & 37.422 & 4.473 & 20.642 & 7.292 & -2.96 & 48.239 & 0.002 & 3.844 & & 47.00 & 0.78333 & 36.0 & 11.8 & 22.1 & 1.522 & 0.573 & 0.487 & 0.0221 & 22.053 & 18.77 \\
\hline $11 / 25 / 2002$ & $9: 27: 33 \mathrm{AM}$ & 31.053 & 19.41 & 30.794 & 21.431 & 20.34 & 23.227 & 36.992 & 4.333 & 20.593 & 7.336 & -2.9 & 48.214 & 0.002 & 3.85 & & 48.00 & 0.8 & 35.5 & 11.8 & 21.9 & 1.511 & 0.574 & 0.489 & 0.0223 & 22.325 & \\
\hline $11 / 25 / 2002$ & 9:28:33 AM & 31.116 & 19.449 & 30.857 & 21.374 & 20.359 & 23.364 & 37.246 & 4.489 & 20.575 & 7.379 & -2.957 & 48.277 & 0.002 & 3.852 & & 49.00 & 0.81667 & 35.8 & 11.8 & 22.0 & 1.515 & 0.574 & 0.489 & 0.0222 & 22.238 & 18.92 \\
\hline $11 / 25 / 2002$ & 9:29:33 AM & 31.06 & 19.457 & 30.934 & 21.322 & 20.383 & 23.2 & 37.102 & 4.411 & 20.488 & 7.445 & -2.96 & 48.218 & 0.002 & 3.856 & & 50.00 & 0.83333 & 35.6 & 11.8 & 21.8 & 1.506 & 0.575 & 0.488 & 007 & 22.342 & 19.01 \\
\hline $11 / 25 / 2002$ & 9:30:33 AM & 31.052 & 19.465 & 30.803 & 21.294 & 20.411 & 23.316 & 37.265 & 4.423 & 20.603 & 7.339 & -2.957 & 48.122 & 0.002 & 3.853 & 0 & 51.00 & 0.85 & 35.8 & \begin{tabular}{|l|}
11.8 \\
\end{tabular} & 22.0 & 1.514 & 0.574 & 0.489 & 0.0223 & 22.286 & 18.97 \\
\hline $11 / 25 / 2002$ & 9:31:33 AM & 31.115 & 19.492 & 30.811 & 21.211 & 20.413 & 23.329 & 37.25 & 4.448 & 20.566 & 7.373 & -2.957 & 48.224 & 0.001 & 3.851 & & 52.00 & 0.86667 & 35.8 & 11.8 & 21.9 & 1.513 & 0.574 & 0.489 & 0.0223 & 22.282 & 18.96 \\
\hline $11 / 25 / 2002$ & 9:32:33 AM & 31.053 & 19.495 & 30.927 & 21.229 & 20.431 & 23.151 & 37.151 & $\begin{array}{l}4.384 \\
4.387\end{array}$ & 20.484 & 7.48 & -2.957 & $\begin{array}{l}48.139 \\
4\end{array}$ & 0.001 & 3.856 & & 53.00 & 0.88333 & 35.7 & 11.8 & 21.8 & 1.504 & 0.575 & 0.488 & 0.0224 & 22.373 & 19.04 \\
\hline $11 / 25 / 2002$ & 9:33:33 AM & 31.051 & 19.508 & 30.807 & 21.182 & 20.449 & 23.091 & 37.04 & 4.405 & 20.364 & 7.365 & -2.95 & 48.189 & 0.001 & 3.8 & & 54.00 & 0.9 & 35.6 & \begin{tabular}{|l|}
11.8 \\
\end{tabular} & 21.7 & 1.498 & 0.575 & 0.490 & 0.0225 & & \\
\hline $11 / 25 / 2002$ & 9:34:33 AM & 31.113 & 19.536 & 30.799 & 21.149 & 20.487 & 23.196 & 37.19 & 4.453 & 20.4 & 7.336 & -2.954 & 48.214 & 0.0 & 3. & & 55.00 & 0.91667 & 35.7 & 11.8 & 21.8 & 1.503 & 0.574 & 0.4 & 0224 & 6 & \\
\hline $11 / 25 / 2002$ & 9:35:33 AM & 31.09 & 19.559 & 30.96 & 21.131 & 0.499 & 23.178 & 37.1 & 4.335 & 20.531 & 7.431 & -2.951 & 48.151 & 0. & 3.86 & & 56.00 & 0.93333 & 35.6 & 11.8 & 21.9 & 1.507 & 0.575 & 0.488 & & 839 & 19.01 \\
\hline $11 / 25 / 2002$ & 9:36:33 AM & 31.063 & 19.556 & 30.843 & 21.108 & 20.512 & 23.335 & 37.265 & 4.444 & 20.64 & 7.307 & -2.951 & 48.195 & 0.002 & 3.853 & & 57.00 & 0.95 & 35.8 & \begin{tabular}{|l|l|}
11.8 \\
\end{tabular} & 22.0 & 1.516 & 0.574 & 0.489 & 0.0222 & 22.234 & 18.92 \\
\hline $11 / 25 / 2002$ & 9:37:33 AM & 31.11 & 19.564 & 30.761 & 21.03 & 20.509 & 23.337 & 37.251 & 4.487 & 20.546 & 7.307 & -2.951 & 48.386 & 0.001 & 3.843 & & 58.00 & 0.96667 & 35.8 & 11.9 & 21.9 & 1.513 & 0.573 & 0.489 & 0.0223 & 22.272 & 18.95 \\
\hline $11 / 25 / 2002$ & 9:38:33 AM & 31.097 & 19.586 & 30.952 & 21.058 & 20.542 & 23.124 & 36.998 & 4.433 & 20.417 & 7.341 & -2.95 & 48.376 & 0.002 & 3.85 & & 59.00 & 0.98333 & 35.5 & 11.9 & 21.8 & 1.501 & 0.574 & 0.487 & 0.0224 & 22.372 & .04 \\
\hline $11 / 25 / 2002$ & $9: 39: 33 \mathrm{~A}$ & 31.07 & 19.598 & 30.865 & 21.009 & 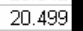 & 23.348 & 37.323 & 4.4 & & 7.321 & & & 00 & 3.8 & & 60.00 & & 35 & 11.8 & 22.0 & & & & & & \\
\hline $11 /$ & $9: 40: 3$ & 31.127 & 19.621 & 30.803 & 20.992 & 582 & 23.43 & 37.296 & 4.486 & 20. & 7.312 & -2.951 & 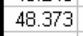 & 0.0 & 3.8 & & 0 & 1.01667 & 35 & 11.9 & 22.0 & 0 & & & & & \\
\hline $11 / 25 / 2002$ & 9:41:33 AM & 31.104 & 19.623 & 30.934 & 20.988 & .578 & 23.341 & 37.18 & 4.445 & 20.638 & 7.281 & -2.945 & 48 & 0.001 & 3.8 & & 62.00 & 1.03333 & 35.7 & 11.9 & 22.0 & 1.516 & 0. & 0.487 & & 54 & 18.85 \\
\hline $11 / 25 / 2002$ & 9:42:33 AM & 31.076 & 19.63 & 30.876 & 21.016 & 20.555 & 23.29 & 37.294 & 4.462 & 20.46 & 7.339 & -2.948 & 48.222 & 0.002 & 3.852 & & 63.00 & 1.05 & 35.8 & 11.8 & 21.9 & 1.508 & 0.574 & 0.488 & 0.0223 & 322 & 19.00 \\
\hline $11 / 25 / 2002$ & 9:43:33 AM & 31.102 & 19.642 & 30.784 & 20.982 & 20.547 & 23.294 & 37.168 & 4.47 & 20.501 & 7.336 & -2.945 & 48 & 0.001 & 3.845 & & 64.00 & 1.06667 & 35 & \begin{tabular}{|l|}
11.8 \\
\end{tabular} & 21.9 & 1.510 & 0.573 & 0.489 & 0.0223 & 22.314 & \\
\hline $11 / 25 / 2002$ & 9:44:33 AM & 31.129 & 19.664 & 30.915 & 21.005 & 20.569 & 23.095 & 36.762 & 4.413 & 20.433 & 7.26 & -2.942 & 48 & 0.0 & 3.8 & & 65.00 & 1.08333 & 35.3 & 11.9 & 21.8 & 1.501 & 0 & 0.487 & 0.0224 & 83 & 19.05 \\
\hline & & 11.087 & 19.676 & 30.902 & .... & -1 & 23.093 & 37.0 & 4.4 & & 7.34 & & & & & & & 1.1 & 35 & 11.9 & 21.7 & & & & & & \\
\hline & & 31.113 & 19.688 & 4 & 20.973 & 20 & 23 & 37 & 4 & 20 & 7.3 & & & & & & & 1667 & 35.7 & 11 & 22 & & & & & & \\
\hline $11 / 25 / 2002$ & 9:47:33 AM & 31.13 & 19.695 & 30.886 & 20.985 & 20.58 & 23.209 & 37.18 & 4.482 & 20. & 7.333 & -2.94 & 48 & 0.002 & 3.8 & & 68.00 & 1.13333 & 35.7 & 11.8 & 21.8 & 1.501 & 0 & 0.488 & 0.0224 & 142 & 15 \\
\hline $11 / 25 / 2002$ & 9:48:33 AM & 31.092 & 19.717 & 30.932 & 21.012 & 20.567 & 23.076 & 36.911 & 4.432 & 20.294 & 7.289 & -2.9 & 48 & 0. & 3.8 & & 69.00 & 1.15 & 35 & 11.9 & 21.7 & 1.495 & 0 & 0.486 & 0.0224 & 22.420 & 19.08 \\
\hline $11 / 25 / 2002$ & 9:49:33 A & 31.109 & 19.734 & 30.83 & 21.049 & 20.584 & 23.397 & 37.182 & 4.48 & 20. & 7.27 & -2.9 & & 0. & 3.8 & & 70.00 & 1.16667 & 35 & 11.9 & 22.0 & 1.518 & & 0.2 & 0.02 & & 18.85 \\
\hline $11 / 25 / 2002$ & $9: 50: 3$ & 31.145 & 19.751 & 30.876 & 21.0 & 20.57 & 23.221 & 37.097 & 4.4 & & 7.30 & -2.5 & & & 3.8 & & 71.00 & 1.18333 & 35 & 11.8 & 21.8 & 1.504 & & 0.487 & & & \\
\hline & & & 19.757 & 30.937 & & $30=$ & 23 & & 4.4 .5 & & 7.2 & & & & & & & 1.2 & 3 & 11.8 & 21.9 & & & & & & 88 \\
\hline & & 31.123 & 19.799 & 30.859 & & 2 & 23. & 36. & & & 7.2 & & & & & & & 1667 & & 11.9 & 21.8 & & & & & & \\
\hline $11 / 25 / 2002$ & $9: 53: 33 \mathrm{~A}$ & 31.145 & 19.806 & 30.846 & 21.005 & 5.631 & 23.401 & 37.147 & 4. & 20.705 & 7.27 & -2.9 & 48 & 0.0 & 3.8 & & 74.00 & 1.23333 & 35 & 11.8 & 22.1 & 1.520 & 0 & $\begin{array}{l}0.487 \\
0.487\end{array}$ & 0.0221 & 03 & 18.8 \\
\hline $11 / 25 / 2002$ & $9: 54: 33$ & 31.102 & 19.823 & 30.962 & 21 & 20.6 & 23.153 & 36.984 & 4 & 20 & 7.3 & -2.9 & & & . & & 75.00 & 1.25 & 35 & 11.9 & 21.8 & 1. & & 0. & 0. & 49 & 19.02 \\
\hline & $9: 55: 3$ & 31.104 & 19.85 & 30.845 & 21. & 20. & 23. & 37.265 & 4.4 & 20.7 & 7.278 & -2.94 & & & 3.8 & & & 1.26667 & & \begin{tabular}{|l|}
11.8 \\
\end{tabular} & 22.1 & & & & & & 18.82 \\
\hline & $9: 56$ & 3111 & 19.888 & 3 & 21. & 20. & 23. & 37.0 & 4.4 & 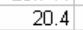 & 7.3 & -2.9 & & & & & & 1.28333 & & 11.9 & 21.8 & & & & & & \\
\hline & & & 19.889 & 30.968 & 21. & 20. & 23 & 37. & & 20. & & & & & 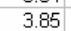 & & & 1.3 & 35.6 & 11.8 & 21.8 & & & & & & 899 \\
\hline & & 31.09 & 19.917 & 30.871 & & 2 & 23.318 & 36. & & & 7.2 & & & & 3.8 & & 10 & 1667 & 35 & 11.8 & 22.0 & & & & & & \\
\hline $11 / 2$ & 9:59:3 & 31.146 & 19.934 & 33 & 21 & 20 & 23 & 37 & 4.461 & 20 & 7.2 & -2.9 & & & & & 0 & 333 & & 11.9 & 22.0 & & & & & & 18.83 \\
\hline & & 31.1 & 19.5 & 3 & & 20 & & 37. & & & & 2 & & & & & & & & 11.8 & 2 & & & & & & 18. \\
\hline & & 31 & 19.973 & 34 & 21. & 30 & 23.2 & 37. & 4. & 20. & 7.3 & -2 & & & 3. & & & 1.36667 & & \begin{tabular}{|l|}
11.8 \\
\end{tabular} & 21.8 & & & & & & \\
\hline & & 31.1 & 19. & 30.8 & & 20.7 & 23. & 36. & & & 7.2 & & & & & & & 1.38333 & & 11.8 & & & & & & & \\
\hline & & 31.129 & 20.012 & 30.964 & 21 & , & 23.412 & 37.4 & 4. & 20. & 7.3 & & & & & & & 1.4 & 35.9 & 11.8 & 22.0 & & & & & & 18.80 \\
\hline & 10:0 & 31.09 & 20.023 & 30.8 & 21. & 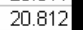 & 23 & 37 & 4 & & 7.2 & & & & & & & 667 & 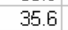 & $1 .$. & 211 & & & & & & \\
\hline & & 31. & 20 & & & & & & & & & & & & & & & & & & & & & & & & \\
\hline & & 31. & & 30. & 21. & & 23. & 37 & & & 7.2 & -2.9 & & & & & & & & 9 & & & & & & & \\
\hline & $10: 0$ & 31.09 & 20.072 & 30.914 & 2 & 20.8 & 23.296 & 37. & 4.433 & 20.6 & 2 & -2.9 & 48. & & 3. & & 88 & 1.46667 & 35.6 & 11.9 & 22.0 & & & & & 79 & \\
\hline & 10:08 & 31.125 & 20.093 & 30.826 & 21.086 & 20.87 & 23.273 & 37.222 & 4.4 & 20.5 & 7.278 & & 46 & 0.0 & & & 89.00 & 1.48333 & 35.8 & 11.8 & 21.9 & & 0.5 & 0.488 & 0 & 304 & \\
\hline & 10:09 & 31.156 & 20.115 & 30.902 & 21.112 & 20.909 & 23.196 & 37.248 & 4.415 & 20.468 & 7.466 & -2.928 & 48.38 & 0.001 & 3.8 & & 90.00 & 1.5 & 35.8 & 11.9 & 21.8 & 1.505 & 0.575 & 0.489 & 0.0224 & 22.380 & 19.05 \\
\hline 11 & 10:10:33 An & 31.113 & 20.132 & 30.939 & 21.144 & . & 23.196 & 37.021 & 4.434 & 20.552 & 7.443 & & 48.3 & 0.001 & 3. & & 91.00 & 1.51667 & 35.6 & 11.9 & 21.9 & 1.508 & 0.574 & $\begin{array}{l}0.4867 \\
\end{array}$ & 0.0223 & 22.274 & \\
\hline & & & & & & & & & & & & & & 0.0 & & & & 0.8 & 35.7 & & & & & & & & 8.697 \\
\hline & & 32 & 20 & 31 & 22 & & 23 & & 4. & 2 & 30.1 & 78 & & 1 & 4 & & & 1.5 & 36.6 & 12.0 & 22.1 & 1. & 0 & 0. & & & 724 \\
\hline & Med & 31 & 19.4 & 30 & 21.1 & 20 & 23.2 & 37.2 & 4.4 & 20. & 7.3 & -2 & 4 & 0 & 3. & & & 0.8 & 35.7 & 11.8 & 21.8 & & 0.574 & 0.488 & 0.0223 & 22.296 & 74 \\
\hline & & 28.9 & $\begin{array}{l}18.4 \\
\end{array}$ & 30.8 & 20.9 & 18 & 2.8 & 36.7 & & -1.3 & 7.2 & $\begin{array}{l}-3.0 \\
-3.0\end{array}$ & & & $\sqrt{3}$ & & & 0.0 & 35.3 & 11.2 & 0.7 & 0.050 & 0.000 & $\begin{array}{l}0.4000 \\
0.000\end{array}$ & 000 & 0.022 & 018 \\
\hline & & 0.857 & 0.984 & & & & & & 08 & 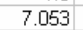 & & & & & & & & 895 & & & & & & & & & \\
\hline & & 92 & 92 & & 92 & & 92 & 92 & 92 & 92 & 92 & & 92 & 92 & & & & 92 & & & & & & 92 & & & \\
\hline
\end{tabular}

Figure C16b: 1.31C: After AN-102R2, Batch 3A - 2 M Nitric Acid Cleaning - Second Cleaning (remaining 46 minutes) - Raw \& Calculated Data 
WSRC-TR-2003-00204, REV. 0

SRT-RPP-2003-00087, REV. 0

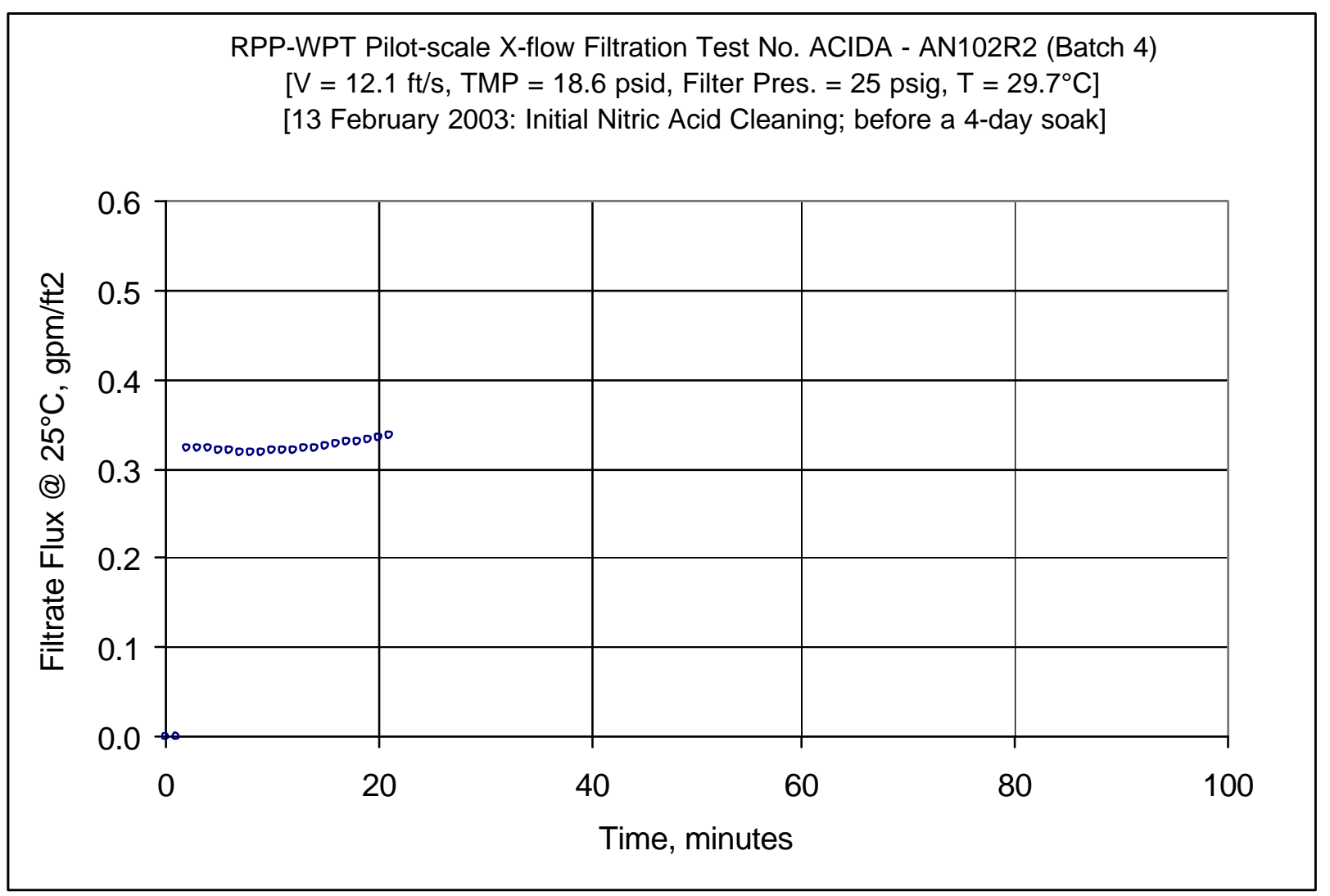

Figure C17: ACIDA: Before AN-102R2, Batch 4-2 M Nitric Acid Cleaning - First 20 minutes of a 4-day soak 
WSRC-TR-2003-00204, REV. 0

SRT-RPP-2003-00087, REV. 0

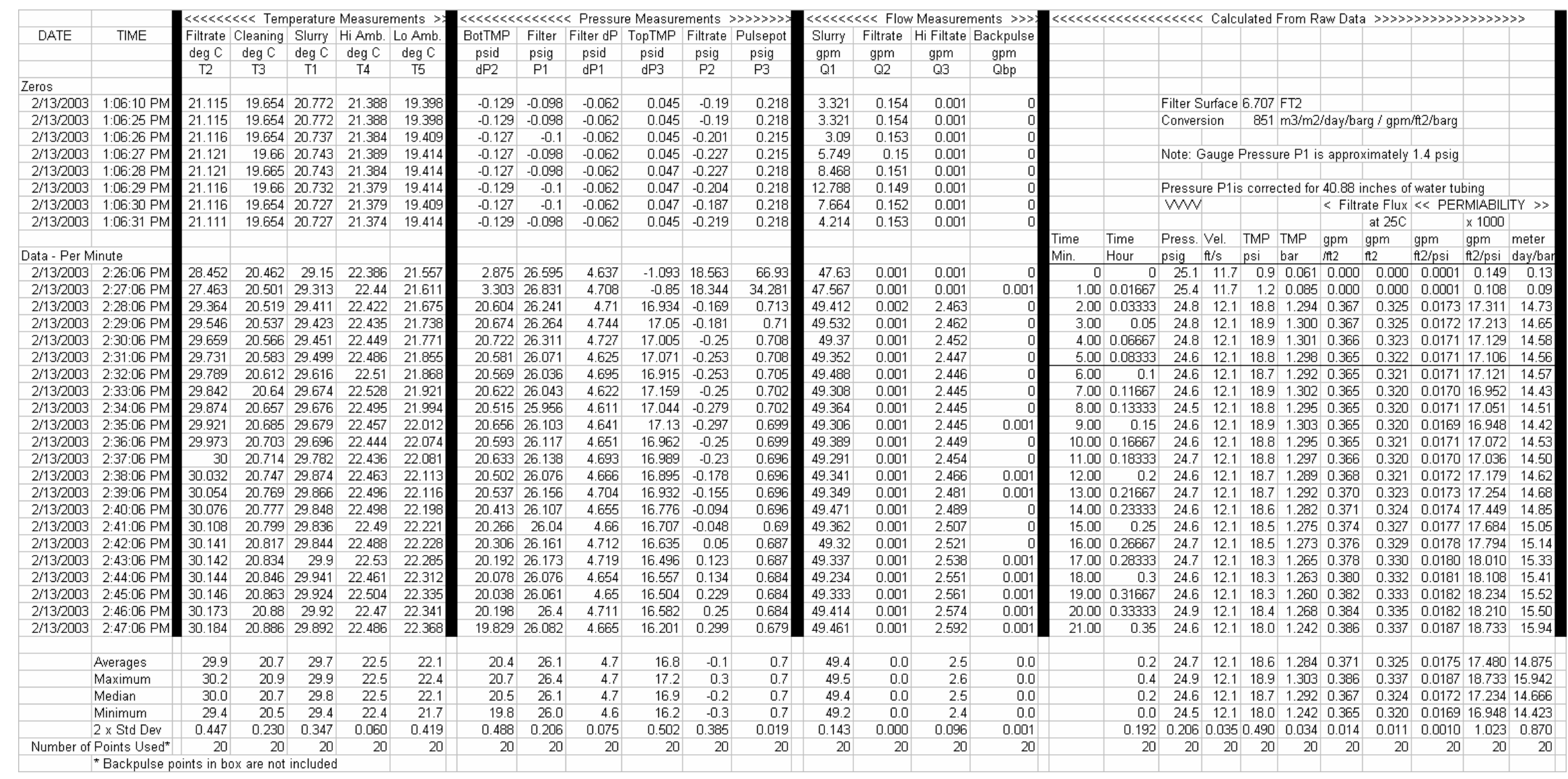

Figure C18: ACIDA: Before AN-102R2, Batch 4-2 M Nitric Acid Cleaning - First 20 minutes of a 4-day soak - Raw \& Calculated Data 
WSRC-TR-2003-00204, REV. 0

SRT-RPP-2003-00087, REV. 0

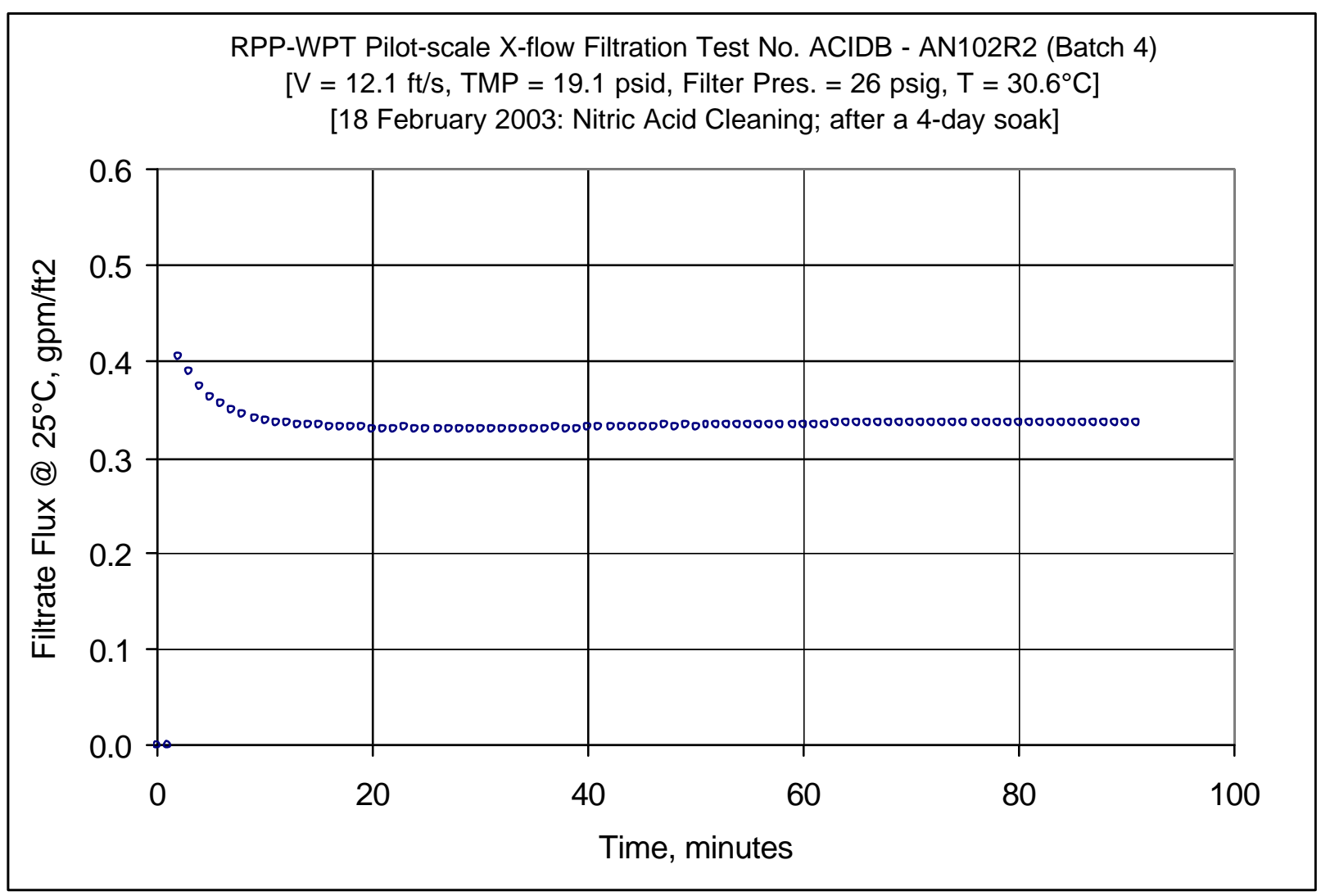

Figure C19: ACIDB: Before AN-102R2, Batch 4 - 2 M Nitric Acid Cleaning - Last 90 minutes of a 4-day soak 
WSRC-TR-2003-00204, REV. 0

SRT-RPP-2003-00087, REV. 0

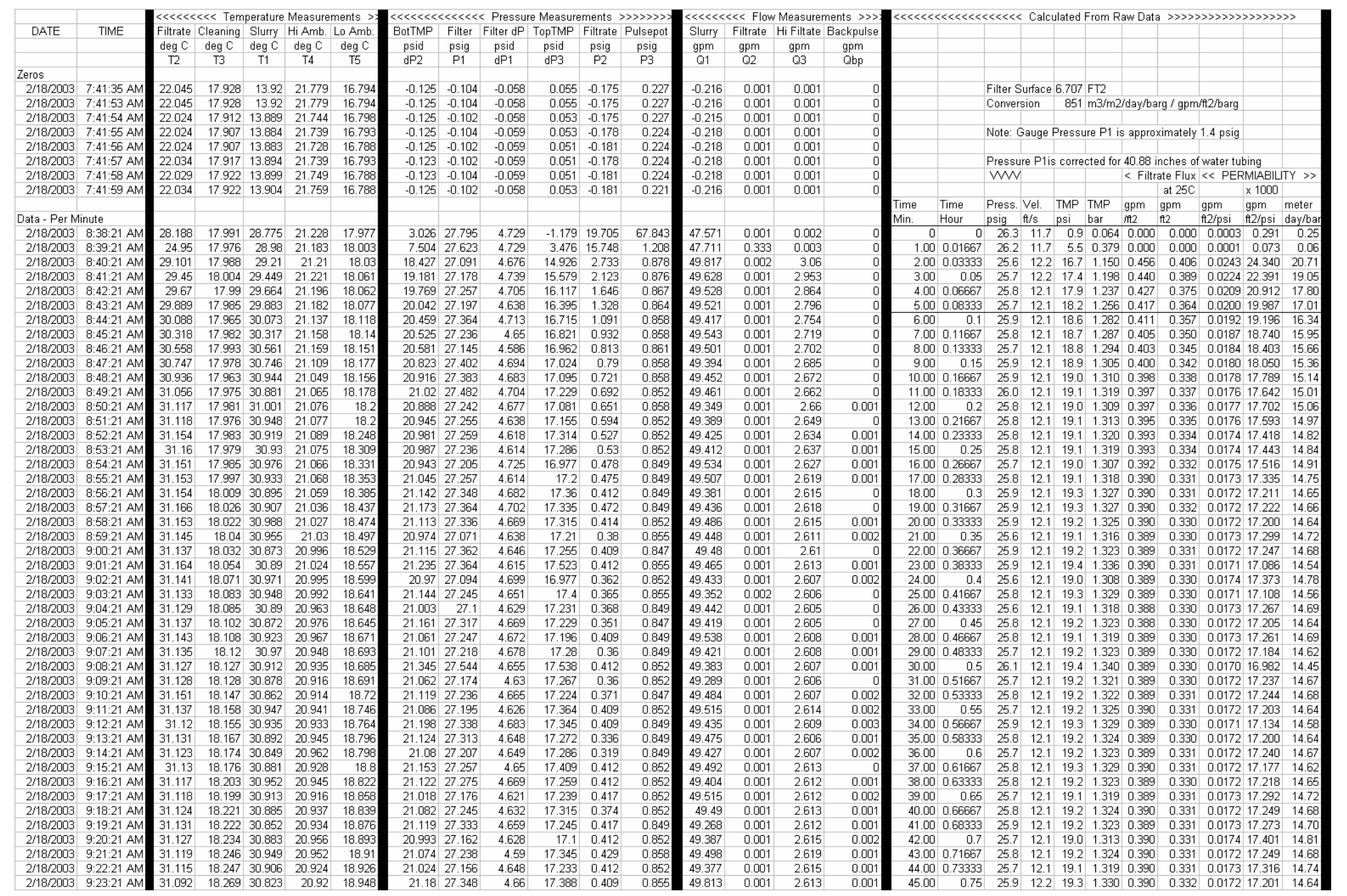

Figure C20a: ACIDB: Before AN-102R2, Batch 4 - 2 M Nitric Acid Cleaning (first 45 minutes) - Last 90 minutes of a 4-day soak 
WSRC-TR-2003-00204, REV. 0

SRT-RPP-2003-00087, REV. 0

\begin{tabular}{|c|c|c|c|c|c|c|c|c|c|c|c|c|c|c|c|c|c|c|c|c|c|c|c|c|c|c|c|}
\hline $2 / 18 / 2003$ & 9:24:21 AM & 81.034 & 18.266 & 30.72 & 20.886 & 18.95 & 21.167 & 27.354 & 4.669 & 17.466 & 0.472 & 0.849 & 49.226 & 0.001 & 2.616 & & 46.00 & 0.76667 & 25.9 & 12.1 & 19.3 & 1.332 & 0.390 & 0.333 & 0.0172 & 17.240 & 14.67 \\
\hline $2 / 18 / 2003$ & 9:25:21 AM & 31.011 & 18.288 & 30.673 & 20.924 & 18.962 & 21.01 & 27.207 & 4.637 & 17.186 & 0.412 & 0.852 & 49.263 & 0.001 & 2.616 & 0.001 & 47.00 & 0.78333 & 25.7 & 12.1 & 19.1 & 1.317 & 0.390 & 0.333 & 0.0175 & 17.460 & 14.86 \\
\hline $2 / 18 / 20003$ & $9.26 .21 \mathrm{AM}$ & 30.969 & 18305 & 30.65 & 20916 & 18974 & 21111 & 27286 & 4669 & 1729 & 0.426 & 0849 & 49398 & 0.001 & 2612 & & 4800 & 08 & 258 & 121 & 192 & 1324 & 0389 & 0333 & 00174 & 17351 & 1477 \\
\hline $2 / 18 / 2003$ & 9:27:21 AM & 30.917 & 18.313 & 30.672 & 20.949 & 18.997 & 21.049 & 27.209 & 4.663 & 17.274 & 0.478 & 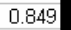 & 49.087 & 0.001 & 2.615 & & 49.00 & 1667 & 25.7 & 12.0 & 19.2 & 1.321 & 0.390 & 0.333 & 0.0174 & & \\
\hline $2 / 18 / 2003$ & 9:28:21 AM & 30.869 & 18.32 & 30.664 & 20.95 & 19.019 & 21.08 & 27.267 & 4.676 & 17.243 & 0.414 & 85 & 49.308 & 0.001 & 2.61 & & 50.00 & 0.83333 & 25.8 & 12.1 & 19.2 & 1.321 & 0.389 & 0.333 & 0.0174 & 7.366 & 14.78 \\
\hline $2 / 18 / 2003$ & 9:29:21 AM & 30.84 & 18.336 & 30.606 & 20.982 & .015 & 21.068 & 27.203 & 4.66 & 17.241 & 0.412 & 355 & 49.406 & 0.001 & 2.61 & 0.001 & 51.00 & 0.85 & 25.7 & 12.1 & 19.2 & 1.321 & 0.389 & 0.333 & 0.0174 & 17.400 & 14.81 \\
\hline $2 / 18 / 2003$ & 9:30:21 AM & 30.817 & 18.338 & 30.533 & 20.949 & 19.027 & 21.093 & 27.275 & 4.612 & 17.317 & 0.412 & 0.852 & 49.404 & 0.001 & 2.612 & & 52.00 & 0.86667 & 25.8 & 12.1 & 19.2 & 1.324 & 0.389 & 0.334 & 0.0174 & 17.402 & 14.81 \\
\hline $2 / 18 / 2003$ & 9:31:21 AM & 30.805 & 18.345 & 30.506 & 20.956 & 19.04 & 21.105 & 27.185 & 4.604 & 17.4 & 0.409 & 0.852 & 49.406 & 0.001 & 2.611 & 0.001 & 53.00 & 0.88333 & 25.7 & 12.1 & 19.3 & 1.327 & 0.389 & 0.334 & 0.0174 & 17.365 & 14.78 \\
\hline $2 / 18 / 2003$ & 9:32:21 AM & 30.791 & 18.352 & 30.502 & 20.937 & 19.041 & 21.136 & 27.327 & 4.582 & 17.36 & 0.409 & 0.057 & 49.282 & 0.001 & 2.613 & 0.00 & 54.00 & 0.9 & 25.9 & 12.1 & 19.2 & 1.327 & 0.390 & 0.335 & 0.0174 & 17.384 & 14.79 \\
\hline $2 / 18 / 2003$ & 33:21 AM & 30.768 & 18.364 & 30.564 & 20.964 & 19.07 & 20.966 & 27.098 & 4.584 & 17.278 & 0.481 & 4 & 19.383 & 0.0 & 28 & & 55.00 & 0.91667 & 25.6 & 12.1 & 19.1 & 1.318 & & 0.334 & 0.0175 & 17.469 & \\
\hline $2 / 18 / 2003$ & $34: 21 \mathrm{~A}$ & 30.745 & 18.371 & 30.531 & 20.936 & 19.085 & 21.153 & 27.313 & 4.671 & 17.278 & 0.42 & 1849 & 9. & 0.001 & 2.0 & .002 & 56.00 & 0.93333 & 25.8 & 12.1 & 19.2 & 1.3 & 0.3 & 4 & 74 & & \\
\hline $2 / 18 / 2003$ & 9:35:21 AM & 30.733 & 18.389 & 30.484 & 20.933 & 108 & 21.111 & 27.253 & 4.595 & 17.392 & 0.4 & 40 & & 11 & 2.61 & 0.001 & 57.00 & 0.95 & 25.8 & 12.1 & 19.3 & 1.327 & 0.389 & 0.334 & 0.0174 & 70 & 14.78 \\
\hline $2 / 18 / 2003$ & 9:36:21 AM & 30.714 & 18.38 & 30.416 & 20.884 & 19.094 & 21.215 & 27.402 & 4.677 & 17.39 & 0.469 & 849 & 49.377 & 0.001 & 2.612 & & 58.00 & 0.96667 & 25.9 & 12.1 & 19.3 & 1.331 & 0.389 & 0.335 & 0.0174 & 69 & 14.78 \\
\hline $2 / 18 / 2003$ & 9:37:21 AM & 30.726 & 18.396 & 30.417 & 20.835 & 19.091 & 21.155 & 27.346 & 4.704 & 17.319 & 0.458 & 0.849 & 49.312 & 0.001 & 2.61 & 0.0 & 59.00 & 0.98333 & 25.9 & 12.1 & 19.2 & 1.326 & 0.389 & 0.335 & 0.0174 & 17.414 & 14.82 \\
\hline $2 / 18 / 2003$ & 9:38:21 AM & 30.701 & 18.386 & 30.467 & 20.755 & 19.07 & 21.01 & 27.135 & 4.646 & 17.192 & 0.409 & 849 & 49. & 0.001 & 2.6 & 0.0 & 60.00 & 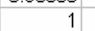 & 25.7 & 12.1 & 19.1 & 1.317 & 0.389 & 0.335 & 0.0175 & 17.528 & 14.92 \\
\hline $2 / 18 / 2003$ & 20.7 & 30,682 & 18.397 & 30.463 & 20 & 19.0 & 21.163 & 27.35 & 4.6 & & 0.4 & & & & 2. & & 61.00 & 1.01667 & 25.9 & 12.1 & 107 & & & & 0.0174 & & \\
\hline $2 / 18 /$ & $10: 2$ & .672 & 18.402 & 30.4 & 20. & 19 & 21. & 27.1 & 4.6 & 17 & 0.41 & & & & & & jo & 333 & 25.7 & 12.1 & 19.1 & & & & & & \\
\hline $2 / 18 / 2003$ & 9:41:21 AM & 30.677 & 18.403 & 30.374 & 20.484 & .057 & 21.219 & 27.367 & 4.6 & $\begin{array}{l}17.229 \\
17.409\end{array}$ & 0.4 & 849 & & 0.001 & 2. & 0.001 & 63.00 & 1.05 & 25.9 & 12.1 & 19.3 & 1.332 & 0 & 86 & 174 & & 14.81 \\
\hline $2 / 18 / 2003$ & 9:42:21 AM & 30.677 & 18.402 & 30.358 & 20.408 & 19.051 & 21.047 & 27.158 & 4.615 & 17.317 & 0.409 & $202-2$ & & & & 0.001 & 64.00 & 1.06667 & 25.7 & 12.1 & 19.2 & 1.323 & 0.390 & 0.336 & 0.0175 & 26 & 14.91 \\
\hline $2 / 18 / 2003$ & 9:43:21 AM & 30.657 & 18.408 & 30.423 & 20.337 & 19.016 & 21.101 & 27.28 & 4.641 & 17.327 & 0.412 & 849 & & 0.00 & 2. & & 65.00 & 1.08333 & 25.8 & 12.1 & 19.2 & 1.325 & & 0.336 & 0.0175 & 17. & 14.86 \\
\hline $2 / 18 / 2003$ & 9:44:21 & 30.647 & 18.413 & 30.433 & 20.2 & 18.9 & 21.093 & 27.28 & 4.6 & 17. & 0.4 & & & & & 0.00 & 66.00 & 1.1 & 25.8 & 12.1 & 19.2 & 1.325 & & & 0.0175 & & 14.86 \\
\hline $2 / 18 / 2003$ & & 30.8 & 18.413 & 30.3 & 20. & 19.0 & 21.217 & 27.37 & 4.6 & & 0.4 & & & & & & 67.00 & 1.11667 & 25.9 & 12.1 & 19.3 & & & & 0.0174 & & 14.80 \\
\hline $2 / 18 / 2003$ & 9:46 & 30.647 & 18. & 30.3 & 20 & & 21. & 27.30 & 4. & & 0. & & & & & & 68.00 & & 258 & 12.1 & 19.2 & & & & & & \\
\hline $2 / 18 / 2003$ & 9:47:21 AM & 30.661 & $\begin{array}{l}18.4007 \\
\end{array}$ & 30.343 & 20.155 & 18.93 & 20.943 & 27.081 & 4.598 & 17.173 & 0.44 & & & & 2.6 & & 69.00 & 1.15 & 25.6 & 12.1 & 19.1 & 1.314 & 0. & 0.336 & 0.0177 & 54 & 15.02 \\
\hline $2 / 18 / 2003$ & 9:48:21 AM & 30.647 & 18.428 & 30.453 & 20.221 & 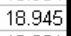 & 21.074 & 27.276 & 4.6 & 17.306 & 0.51 & 8 & 49 & 0.0 & 2.6 & 0.003 & 70.00 & 1.16667 & 25.8 & 12.1 & 19.2 & 1.323 & 0 & 0.336 & 0.0175 & 17.493 & 14.89 \\
\hline $2 / 18 / 2003$ & $9: 49: 21$ & 30.619 & 18.414 & 30.409 & 20.243 & 10.9 & 21.188 & 27.302 & 4.64 & 17. & 0.4 & & & & & & 71.00 & 1.18333 & 25.8 & 12.1 & 19.3 & 1.3 & & & 0.0174 & & 14.84 \\
\hline $2 / 18 / 2003$ & 9:50:2 & 30. & 18.414 & 30.36 & & 18.9 & 21.179 & 27.36 & & & & & & & & & 72.00 & 1.2 & 25.9 & 12.1 & 19.2 & & & & 0.0174 & & \\
\hline $2 / 18 /$ & & 30. & & 30 & & & & 27.2 & & & & & & & & 01 & & 1.21667 & 25.8 & 12.1 & 19.3 & & & & & & 14.87 \\
\hline & & & 18. & 30.3 & 20. & & & 27.32 & & & & & & & & & & & 25.9 & 12.1 & 19.2 & & & & & & \\
\hline $2 / 18 / 2003$ & 9:53:21 AM & 30.626 & 18.437 & 30.436 & 20.4 & 19.0 & 20.941 & 27.149 & 4.6 & 17.052 & 0.4 & & 49 & & 2.6 & 0.002 & 75.00 & 1.25 & 25.7 & 12.1 & 19.0 & 1.310 & & 0.336 & 0.0177 & 17.687 & 15.05 \\
\hline $2 / 18 / 2$ & $9: 54: 21$ & 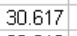 & 18. & 30.4 & 20 & 19 & 21. & 27.35 & 4. & 17. & 0.4 & & & & & 0.0 & 76 & 1.26667 & 25.9 & 12.1 & 19.2 & 1. & & 6 & 0.0175 & & 14. \\
\hline $2 / 18 /$ & 9:55: & & 18. & 30.3 & & 19. & 20. & 27.11 & 4.75 & & & & & & & & & 1.28333 & 25.6 & 12.1 & 19 & & & & & & \\
\hline $2 / 18 /$ & & & 18. & & & & & 27.18 & 4. & & & & & & & & & & & 12.1 & & & & & & & \\
\hline & & 30. & 18. & 30.3 & & & & 27 & 4.6 & & & & & & & & & & 25.8 & 12.1 & 19.2 & & & & & & \\
\hline & & & 18.46 & 30.425 & & & & 27.23 & 4. & & & & & & & & jo & 33 & 25.8 & 12.1 & 10.2 & & & & fo & & \\
\hline $2 / 18 /$ & 9:59:2 & 30. & 18.446 & 30.3 & 20 & & 21 . & 27.32 & 4.5 & & & & & & & & & 35 & 25.9 & 12.1 & 19.4 & & & & & & 14.82 \\
\hline & & 30. & 18. & 30. & & & & 27.3 & & & & & & & & 0.0 & & 1.36667 & 25.9 & 12.1 & 1 & & & & & & 14. \\
\hline & & 30. & 18. & 30. & 20. & & & 27. & & & & & & & & & & 1.38333 & 25.9 & 12.1 & 19.3 & & & & & & \\
\hline & & & 18. & 30.3 & & & & 27. & & & & & & & & & & 1.4 & 26.0 & 12.1 & 19.2 & & & & & & 14.90 \\
\hline & & 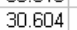 & 18.4 & 30.425 & 20. & & 21. & 27.24 & 4.6 & & & & & & 2.8 & & & & 25.8 & 12.1 & 19.2 & & & & 0. & & 14.91 \\
\hline $2 / 18$ & 10:0 & 30.595 & 18 & 30.3 & 20 & & 21. & 27.19 & 1 & t & & & 49. & & & & & & 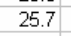 & 121 & 2 & & & & & & 1 \\
\hline & & & 18 & & & & & 27. & & & & & & & & & & & & & & & & & & & \\
\hline & & & 18. & & & & & 27. & & & & & & & & & & 1.4 & & 12.1 & & & & & & & \\
\hline & & 30.8 & 18.5 & 30.379 & & & & 27.2 & 4. & 17.2 & & & & & 2. & & & 1.48333 & 25 & 12.1 & 19 & & & 0.337 & 0.0176 & & 15 \\
\hline & 10:08:21 Al & 30.595 & 18.506 & 30.4 & 20.758 & 19.1 & 20.999 & 27.23 & 4.648 & 17.2 & 0.524 & 0.84 & 49.324 & 0.00 & 2.622 & 0.002 & 90.00 & & 25.8 & 12.1 & 19.1 & 1.317 & 0.391 & 0.337 & 0.0176 & 17.629 & 15.00 \\
\hline $2 / 18 / 2003$ & 10:09:21 AM & 30.601 & 18.527 & 30.377 & 20.804 & 19 & 21.055 & 27.269 & 4.584 & 17.366 & 0.501 & 0.8 & 49.404 & 0.001 & 2.622 & 0.002 & 91.00 & 1.51667 & 25.8 & 12.1 & 19.2 & 1.324 & 0.391 & 0.337 & 0.0175 & 17.538 & 14.92 \\
\hline & & & & & & & & & & & & & & & & & & & & & & & & & & & \\
\hline & & & & 31. & & & & & & & 2 & & & & & & & 1.5 & 1 & 12.2 & & & & & & & 20.7 \\
\hline & & & 18.3 & 30. & 20 & & 21 & 27 & 4 & 17 & 0 & & & 0. & & & & 0.8 & 25.8 & 12.1 & 19.2 & 1.3 & 0.3 & 0.335 & 0.0174 & 17.418 & 4.8 \\
\hline & & 29.1 & 18.0 & 29.2 & 20.2 & 18 & 18.4 & 27.1 & 4 & 14.9 & 0.3 & 0 & 49 & 0.0 & 2.6 & 0. & & 0.0 & 25.6 & 12.0 & 16.7 & 1.150 & 0.388 & 0.330 & 0.0170 & 16.982 & 14.5 \\
\hline & & 0.749 & 0.367 & 0.677 & 0.560 & 0.654 & 0.794 & 0.187 & 0.073 & 0.720 & 0.694 & 0.01 & J.2 & 00 & 0.1. & 00 & & 0.866 & 187 & 0.0500 & 0.753 & 0.052 & 0.021 & 0.023 & 0.0021 & 2.053 & 1.747 \\
\hline & & & & & & & & & & & & & & & & & & & & & & & & & & & \\
\hline
\end{tabular}

Figure C20b: ACIDB: Before AN-102R2, Batch 4- 2 M Nitric Acid Cleaning (remaining 46 minutes) - Last 90 minutes of a 4-day soak 
WSRC-TR-2003-00204, REV. 0

SRT-RPP-2003-00087, REV. 0

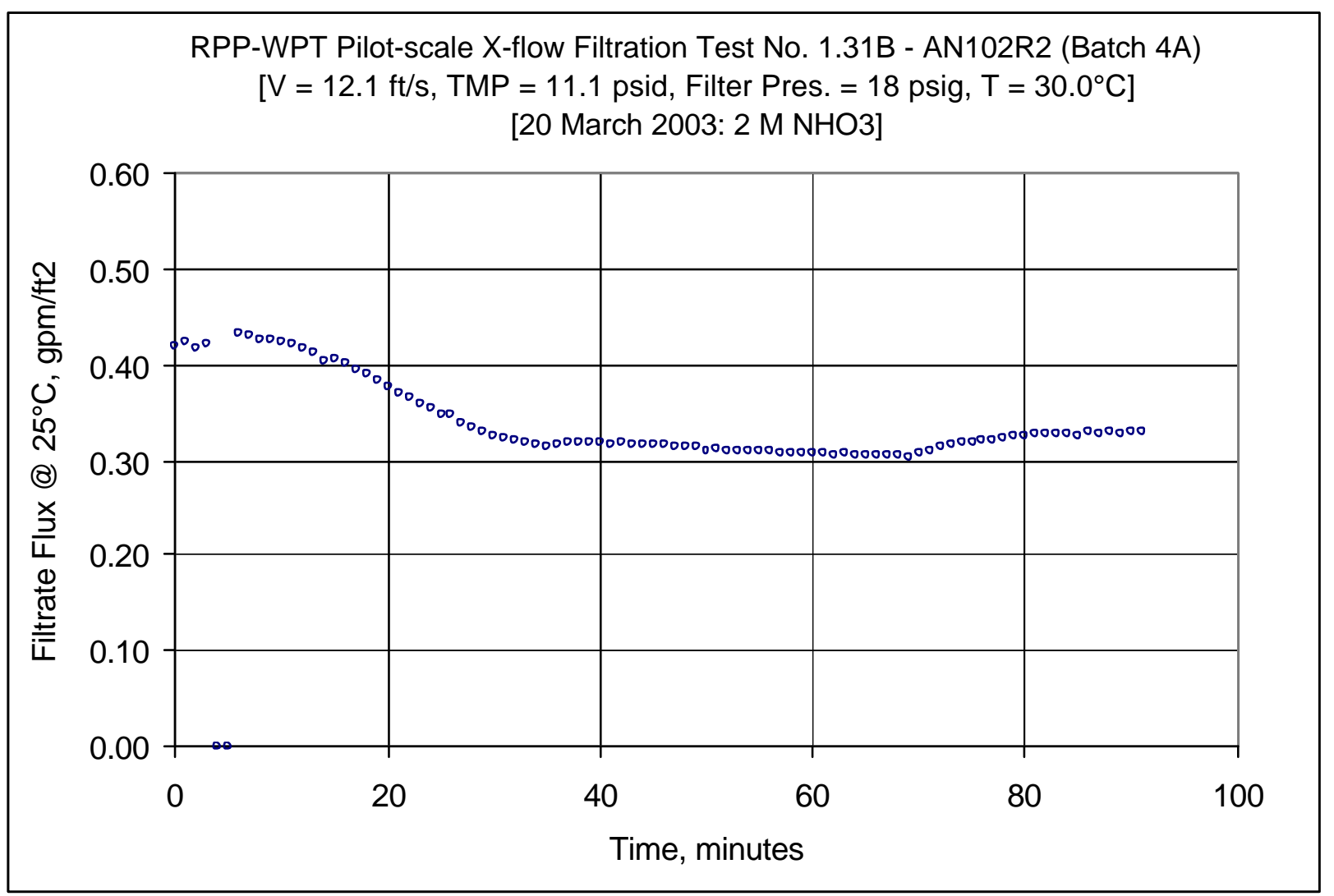

Figure C21: 1.31B: After AN-102R2, Batch 4A - 2 M Nitric Acid Cleaning - First Cleaning 
WSRC-TR-2003-00204, REV. 0

SRT-RPP-2003-00087, REV. 0

\begin{tabular}{|c|c|c|c|c|c|c|c|c|c|c|c|c|c|c|c|c|c|c|c|c|c|c|c|c|c|c|c|}
\hline \multirow{3}{*}{ DATE } & \multirow{3}{*}{ TIME } & «« & & & & & & Ne & & & & & \multirow{2}{*}{\multicolumn{4}{|c|}{$\begin{array}{l}\mid<\ll<<<<<<\text { Flow Measurements } \gg> \\
\text { Slurry Filtrate Hi Filtate Backpulse }\end{array}$}} & \multicolumn{11}{|c|}{ 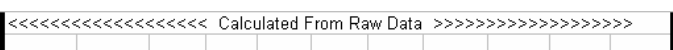 } \\
\hline & & \begin{tabular}{|l|} 
Filtrate \\
degc
\end{tabular} & Cleaning & Slurry & Hi Amb. & Lo Amb. & BotTMP & \begin{tabular}{|l|} 
Filter \\
isig
\end{tabular} & Filter $d P$ & TopTMP & Filtrate & $P$ & & & & & & & & & & & & & & & \\
\hline & & 2 & & $\frac{\operatorname{deg} \mathrm{C}}{\mathrm{T} 1}$ & & $\frac{\operatorname{deg} C}{\mathrm{~T}}$ & $\frac{\mathrm{ps}}{\mathrm{dP}}$ & $\begin{array}{l}\text { psig } \\
\text { P11 }\end{array}$ & $\begin{array}{l}\text { psid } \\
\mathrm{dP} 1\end{array}$ & $\begin{array}{l}\text { psid } \\
\text { dP3 }\end{array}$ & $\begin{array}{l}\text { Psig } \\
\text { P2 }\end{array}$ & $\begin{array}{l}\text { psig } \\
\text { P3 }\end{array}$ & $\mathrm{gpm}$ & $\begin{array}{c}\mathrm{gpm} \\
02\end{array}$ & gpm & & & & & & & & & & & & \\
\hline & & & & & & & & & & & & & & & & & & & & & & & & & & & \\
\hline & 8:33:49 AM & 20.344 & 19.646 & 17.583 & 19.665 & $18.8 \mathrm{z}$ & -0.127 & -0.057 & -0.054 & 0.052 & -0.174 & & -0.216 & 0.002 & 0.001 & & & & & ene & $.707 \mathrm{~F}$ & & & & & & \\
\hline $3: 2$ & $20 \mathrm{AM}$ & 20.344 & 19.646 & 17.583 & 19.665 & & -0.127 & -0.057 & -0.054 & 0.052 & -0.174 & & -0.216 & 0.002 & 0.001 & & & & & & $851 \mathrm{n}$ & $\mathrm{m} 3 / \mathrm{m}$ & day/barg & $\mathrm{rg} / \mathrm{gpm} / \mathrm{t}$ & /ft2/barg & & \\
\hline & 8:34:2 & 20.341 & 19.643 & 17.576 & 19.657 & & -0.129 & -0.057 & -0.054 & & -0.162 & & -0.216 & 0.002 & 0.001 & & & & & & & & & & & & \\
\hline $3 / 20 /$ & 8:34:22 & 20.341 & 19.648 & 17.581 & 19.657 & & -0.127 & -0.057 & -0.054 & 0.052 & -0.174 & & -0.212 & 0.002 & 0.001 & & & & Note: $G a$ & Gaug & Pres: & re $\mathrm{P}$ & & & 4 psig & & \\
\hline 3/20/2003 & 8:34:23 AM & 20.341 & 19.648 & 17.581 & 19.657 & 18.842 & $\begin{array}{l}-0.127 \\
-.077\end{array}$ & \begin{tabular}{|c|}
-0.055 \\
\end{tabular} & -0.054 & 0.05 & -0.174 & $.22 \mathrm{P}$ & \begin{tabular}{|c|}
-0.216 \\
\end{tabular} & 0.002 & 0.001 & & & & & & & & & & & & \\
\hline \begin{tabular}{|l|}
$3 / 20 / 2003$ \\
$3 / 202003$
\end{tabular} & $\begin{array}{l}8: 34424 \mathrm{AM} \\
8: 34: 25 \mathrm{AM}\end{array}$ & $\begin{array}{l}20.341 \\
20.346\end{array}$ & $\begin{array}{l}19.643 \\
19.643\end{array}$ & $\begin{array}{r}17.57 \\
17.581\end{array}$ & $\begin{array}{l}\begin{array}{l}19.657 \\
19.662\end{array} \\
\text {. }\end{array}$ & $\begin{array}{l}18.841 \\
18.847\end{array}$ & $\begin{array}{l}-0.127 \\
-0.127\end{array}$ & $\mid \begin{array}{l}-0.057 \\
-0.057\end{array}$ & $\begin{array}{l}-0.054 \\
-0.054\end{array}$ & $\begin{array}{l}0.052 \\
0.052\end{array}$ & $\begin{array}{r}-0.174 \\
-0.174\end{array}$ & 22. & $\begin{array}{l}-0.212 \\
-0.216\end{array}$ & $\begin{array}{l}0.002 \\
0.001\end{array}$ & $\begin{array}{l}0.001 \\
0.001\end{array}$ & & & & $\begin{array}{l}\text { Pressure } \\
\text { my }\end{array}$ & & & 4 & $\begin{array}{l}40.88 \text { inch } \\
<\text { < Fitrate }\end{array}$ & $\begin{array}{l}\text { chese of of } \\
\text { ate Flux }\end{array}$ & $\begin{array}{l}\text { er tub } \\
\text { PER }\end{array}$ & & \\
\hline $3 / 20 / 2003$ & 8:34:26 AM & 20.336 & 19.648 & 17.58 & 19.662 & 18.846 & -0.127 & \begin{tabular}{|c|}
-0.057 \\
\end{tabular} & -0.054 & 0.052 & -0.171 & & -0.21 & 0.002 & 0.001 & & & & & & & & & at $25 \mathrm{C}$ & & $\times 1000$ & \\
\hline & & & & & & & & & & & & & & & & & & Time & Press. & & TMP & TMP & & & & & ter \\
\hline Data-Per M & & & & & & & & & & & & & & & & & & Hour & & & & & & & & & \\
\hline & 2:29:50 PM & 24.067 & 23.765 & 23.595 & 25.638 & 24.62 & 12.261 & 19.414 & 4.827 & 8.517 & 0.977 & & 49.449 & 0.002 & 2.704 & & & & & & & & & & & & \\
\hline & $2.30 .50 \mathrm{P}$ & & 23.775 & 23.485 & 25.623 & & 12.376 & 19.685 & 4.759 & & 1.133 & & 48.671 & & 2.727 & 0. & & 667 & 18.2 & 11.9 & & & & & & & \\
\hline $3 / 20$ & 2:31:50 PM & 23.875 & 23.778 & 23.694 & 25.551 & 24.713 & 12.296 & 19.672 & 4.788 & 8.597 & 1.251 & & 48.812 & 02 & 2.691 & & 2.00 & 00.03333 & 18.2 & 12.0 & 10.4 & 0.720 & 401 & 0.416 & & 939.850 & \\
\hline & $2: 32: 50 \mathrm{PM}$ & 24.009 & 23.797 & 23.788 & $\begin{array}{l}25.499 \\
\end{array}$ & 24.752 & 12.273 & 19.519 & 4.741 & 8.603 & 1.167 & & \begin{tabular}{|l|l|} 
& 48.841 \\
\end{tabular} & & 2.728 & & 3.00 & 0.05 & 18.0 & 12.0 & d) 10.4 & & 0.407 & 0.421 & & 340.323 & \\
\hline & & & 23.836 & 23.861 & & & & 20.347 & 4.64 & & & & 47.79 & & & & 4.00 & & 18.9 & 117 & & & & & & & \\
\hline & & 26.394 & 23.839 & 23.925 & & 24.818 & 6.219 & 20.043 & 4.674 & & 8.521 & & 47.306 & & & & 5.00 & 8333 & 18.6 & 11.6 & 4.2 & & 00 & 000 & & & \\
\hline & 2:35:50 PM & 24.218 & 23.846 & 24.007 & 25.448 & 24.836 & $\begin{array}{r}11.9 \\
\end{array}$ & 19.59 & 4.804 & & 1.569 & 0. & 49.129 & 0.002 & 2.816 & & 6.00 & 0.1 & 18.1 & 12.0 & 10.1 & 94 & 20 & 1432 & & 391 & 50 \\
\hline $3 / 20 / 2003$ & $\begin{array}{l}2: 36: 50 \mathrm{PM} \\
\end{array}$ & \begin{tabular}{|l|}
24.246 \\
2.393
\end{tabular} & $\begin{array}{r}23.874 \\
23\end{array}$ & 24.05 & 25.496 & 24.864 & 11.718 & 19.378 & 4.786 & 8.105 & 1.552 & 0.5 & 49.177 & 0.002 & 2.81 & & 7.00 & 11667 & 17.9 & 12.1 & 9.9 & 0.683 & 119 & 0.430 & & & 95 \\
\hline $\begin{array}{l}3 / 20 / \\
3000\end{array}$ & 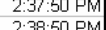 & \begin{tabular}{|l}
24.393 \\
2464
\end{tabular} & $\begin{array}{r}23.89 \\
23892\end{array}$ & $\begin{array}{r}24.342 \\
2459\end{array}$ & 25.443 & $\begin{array}{r}24.87 \\
1\end{array}$ & $\begin{array}{r}11.78 \\
11747\end{array}$ & 19.529 & 4.816 & 8.081 & $\begin{array}{l}1.581 \\
1565\end{array}$ & 0.5 & $\begin{array}{r}49.275 \\
48.98\end{array}$ & & $\begin{array}{r}2.81 \\
23835\end{array}$ & & 8.00 & $\begin{array}{lll}0 & 0.13333 \\
0.15\end{array}$ & 18.1 & 12.1 & 9.9 & 0.685 & 50.419 & 0.427 & & 42.978 & .58 \\
\hline $\begin{array}{l}3 / 20201003 \\
3 / 202003 \\
\end{array}$ & $\begin{array}{l}2: 38: 50 \text { PM } \\
2.39 \cdot 50\end{array}$ & $\begin{array}{r}24.64 \\
24.943\end{array}$ & $\begin{array}{l}23.892 \\
23.924\end{array}$ & $\begin{array}{l}24.6399 \\
2492\end{array}$ & $\begin{array}{l}25.294 \\
25.196\end{array}$ & $\begin{array}{l}24.877 \\
2484\end{array}$ & $\begin{array}{l}11.747 \\
11759\end{array}$ & $\begin{array}{r}19.67 \\
19.633\end{array}$ & $\begin{array}{l}4.736 \\
4.725\end{array}$ & $\begin{array}{r}8.04 \\
8.071\end{array}$ & $\begin{array}{l}1.656 \\
1553\end{array}$ & 0.56 & $\begin{array}{l}48.982 \\
48025\end{array}$ & 0.001 & $\begin{array}{r}2.835 \\
2834\end{array}$ & & $\begin{array}{r}9.00 \\
1000\end{array}$ & $\begin{array}{l}0.15 \\
0 \\
0\end{array}$ & $\begin{array}{l}18.2 \\
182\end{array}$ & $\begin{array}{l}12.0 \\
118\end{array}$ & $\begin{array}{r}9.9 \\
9\end{array}$ & 0.682 & $\begin{array}{l}20.423 \\
40.433\end{array}$ & & 0.0432 & $\begin{array}{l}243.159 \\
7260\end{array}$ & 73 \\
\hline $3 / 20$ & $2: 40: 50 \mathrm{PM}$ & $\begin{array}{l}\text { 24.943 } \\
25.228\end{array}$ & $\begin{array}{l}23.924 \\
23.918\end{array}$ & $\begin{array}{l}25.452 \\
25.237\end{array}$ & 25.025 & $\begin{array}{l}24.0 \\
24.8\end{array}$ & $\begin{array}{l}11.599 \\
11.695\end{array}$ & $\begin{array}{l}19.6535 \\
19.625\end{array}$ & $\begin{array}{l}4.725 \\
4.714\end{array}$ & 8.028 & 1.673 & & $\begin{array}{l}48.025 \\
49.158\end{array}$ & 0.0 & 2.8 & 0.0 & $\begin{array}{l}10.00 \\
11.00\end{array}$ & $\begin{array}{ll}0.1 .1666 \\
0\end{array}$ & $\begin{array}{l}18.2 \\
18.2\end{array}$ & $\begin{array}{l}11.8 \\
12.0\end{array}$ & $\begin{array}{l}9.9 \\
\end{array}$ & $\begin{array}{l}0.6644 \\
0.680\end{array}$ & $\begin{array}{l}4 \\
4 \\
0\end{array} 0.4243$ & $\begin{array}{l}0.423 \\
0.421\end{array}$ & 0.0 & $\begin{array}{l}42.660 \\
42.681\end{array}$ & $\begin{array}{l}30 \\
32\end{array}$ \\
\hline & $2: 41$ & 25. & 23.934 & 25.533 & 24.93 & 24. & 11.739 & 19.612 & 4.767 & & & & . & & & & 12.00 & 0.2 & 18.1 & 12.1 & 90 & & 20.424 & & & & \\
\hline & 2:42 & & 23.928 & 25.786 & 24.819 & 24. & 11.724 & 19.619 & 4.787 & & 1.659 & & 49.059 & & & 0. & 13.00 & 0.21667 & 18.1 & 12.0 & 9.9 & 80 & 0.422 & 0.413 & & 941.881 & 1 \\
\hline $3 / 2$ & $2: 43: 50 \mathrm{~F}$ & 26.067 & 23.942 & 26.081 & 24.733 & & 12.076 & 19.765 & 4.791 & 33 & 1.448 & & 49.37 & & 2.797 & & 14.00 & 0.23333 & 18.3 & \begin{tabular}{|l|}
12.1 \\
\end{tabular} & 10.3 & \begin{tabular}{|l|l|} 
\\
\end{tabular} & 70.417 & 0.405 & & 539.451 & \\
\hline & & 26.346 & 23.951 & 26.369 & 24.666 & & & 19.776 & 4.72 & & & & & & & & 15.00 & 0.25 & 18.3 & \begin{tabular}{|l|}
12.1 \\
\end{tabular} & 10.1 & & 9.421 & 0.405 & & & \\
\hline & & & 23.944 & 26.622 & 2 & & & 19.339 & 4.77 & & & & & & & & 16. & & 7.9 & \begin{tabular}{|l|}
12.1 \\
\end{tabular} & 9.8 & & & & & & \\
\hline & & 26.86 & 23.943 & 26.88 & 24.543 & & & 19.6 & & & & & & & & & 17.00 & 33 & 18.1 & 12.0 & 10.2 & & & & & & \\
\hline & 2:47:50 PM & \begin{tabular}{|l|}
27.137 \\
7.700
\end{tabular} & 23.963 & 27.15 & 24.508 & 24.80 & 12.167 & 19.718 & 4.728 & & 1.3 & & & & & 0.0 & 18.00 & 0.3 & 3 & 12.0 & 10.3 & & & & & & \\
\hline $3 / 2 \mathrm{C}$ & 2:48:50 PM & \begin{tabular}{|l|}
27.382 \\
77631
\end{tabular} & $\begin{array}{r}23.968 \\
23.968\end{array}$ & $\begin{array}{l}27.414 \\
27659\end{array}$ & 24.483 & 24.81 & $\begin{array}{r}12.265 \\
12.347\end{array}$ & 19.5 & $\begin{array}{l}4.723 \\
4.652\end{array}$ & & 1. & & & & & 0.0 & 19.00 & & & 1 & $\begin{array}{l}10.5 \\
106\end{array}$ & & & & & & \\
\hline & $\begin{array}{l}2: 49: 50 \mathrm{PM} \\
2.55 .50 \mathrm{P}\end{array}$ & \begin{tabular}{|}
27.631 \\
27.876
\end{tabular} & 23.968 & $\begin{array}{r}27.659 \\
27.913\end{array}$ & 24.473 & 24.83 & $\begin{array}{l}12.347 \\
12.484\end{array}$ & $\begin{array}{l}19.538 \\
11955\end{array}$ & 4.652 & $\begin{array}{r}8.76 \\
8771\end{array}$ & 1.042 & & 49.112 & & 2.72 & 0.0 & 20.00 & $\begin{array}{lll}0 & 0.33333 \\
0\end{array}$ & 3.1 & 12.0 & 10.6 & 28 & & & & & 8 \\
\hline & 2.50 & $2 \pi$ & $\begin{array}{l}23.973 \\
23984\end{array}$ & $\begin{array}{l}27.913 \\
28169\end{array}$ & $\begin{array}{l}24.496 \\
24544\end{array}$ & & 12.484 & $\begin{array}{l}19.525 \\
19639\end{array}$ & 4.728 & & 0. & & & & & & 21.00 & $\begin{array}{l}0.35 \\
0 \\
0\end{array}$ & & & 10.6 & & 8 & & & & $\begin{array}{l}329.72 \\
0.8855\end{array}$ \\
\hline & 2.52 & & 23.99 & 28.408 & & & 12. & 19.728 & & & 0 & & & & & 0. & 23.00 & 0.368333 & & 12.1 & 11.1 & & 60.395 & & & & $\begin{array}{l}28.65 \\
27.55\end{array}$ \\
\hline & & & 24.001 & 28.663 & & & & 19.697 & & & & & & & & & 24.00 & & & \begin{tabular}{|l|} 
\\
\end{tabular} & | 11. & & & & & & \\
\hline & & & 24.002 & 28.884 & & & & & & & & & & & & & & 0.41667 & & 8 & 11.3 & & & & & & \\
\hline & & & 24.019 & 29. & & & & 19.6 & & & & & & & & & 26.00 & 0.4 & 2 & 12.0 & 11. & & & & & & \\
\hline & & & 24.04 & & & & & & & & & & & & & & & & & \begin{tabular}{|l|}
12.0 \\
\end{tabular} & 11.3 & & & & & & \\
\hline & & & 24.057 & 29.624 & & & & 19. & & & & & & & & & & & & 1 & 11.6 & & & & & & \\
\hline & $2: 58$ & 29.792 & 24.058 & 29.864 & 24 & & & 19.7 & & & 0. & & & & & & & 33 & & 12.1 & 11.8 & & & & & & \\
\hline & $2: 59$ & 30. & 24.079 & 30.109 & 24 & 200 & 13. & 19.6 & & & 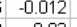 & & & & & & 30.00 & & 2 & 5 & 111.7 & & & & & & \\
\hline $3 / 2$ & 3:00: & 30.2 & 24.091 & $\begin{array}{l}30.324 \\
30.53\end{array}$ & 24.836 & 25 & 13. & 19 & 4. & 10.058 & -0 & & & & & 0. & 31 & & 4 & 1 & 1.9 & & & & & & \\
\hline & $\begin{array}{l}3: 01: 50 \\
3.50 .50\end{array}$ & $\begin{array}{l}30.48 \\
30.71\end{array}$ & 24.106 & $\begin{array}{r}30.553 \\
30791\end{array}$ & 24.792 & 25 & $\begin{array}{r}13.64 \\
13799\end{array}$ & 19. & $\begin{array}{l}4.656 \\
4708\end{array}$ & $\begin{array}{r}9.979 \\
9.981\end{array}$ & & & & & & & & 0.53333 & 2 & 12.1 & $\begin{array}{l}11.8 \\
119\end{array}$ & & 75 & & & 45 & 5 \\
\hline & & & $\begin{array}{l}24.126 \\
24.136\end{array}$ & $\begin{array}{l}30.791 \\
31009\end{array}$ & 24.777 & & & $\begin{array}{r}19.85 \\
19623\end{array}$ & 4.7 & 9.981 & & & & & 25 & & & & & & & & & & & & 22.7 \\
\hline & & & 24.1 & 31.221 & & & & 19.993 & & & & & & & 2.5 & & 35.00 & 0.0 .58333 & 18.5 & 1 & 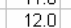 & & 50.374 & & & 27 & \\
\hline & & & 24.1 & & & & & & & & & & & & 2. & & 36 & & & & & & & & & & \\
\hline & & & & & & & & & & & & & & & & & & & & & & & & & & & \\
\hline & & & & & & & & & & & & & & & & & & & & 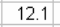 & 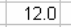 & & & & & & \\
\hline & & & 24. & & & & & & & & & & & & & & & & & 1 & 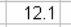 & & & & & & \\
\hline & $3: 0$ & & 24. & 30.082 & & & & 19.7 & & & -0.2 & & & & & & & & & & & & & & & & \\
\hline $3 / 20 / 2003$ & 3:10:50 F & 30.344 & 24.198 & 30.206 & 24.482 & 25. & 13.905 & 19.759 & 4.656 & 10.155 & -0.2 & & 49 & & & 0.001 & 41.00 & 0.68333 & 3.3 & 12.1 & 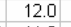 & & 90.367 & & & & \\
\hline & 3:11 & \begin{tabular}{|c|}
30.458 \\
\end{tabular} & 24.212 & 30.379 & 24.527 & 25.11 & 13.694 & 19.55 & 4.68 & 9.878 & -0.182 & & 49. & & 2. & & 42.00 & & 18.1 & 0 & & & 69 & & & & \\
\hline $\begin{array}{r}3 / 20 / 2003 \\
300003\end{array}$ & $\begin{array}{l}3: 12: 50 \mathrm{~F} \\
3.13: 50 \mathrm{P}\end{array}$ & 30.587 & 24.217 & $\begin{array}{l}30.538 \\
3067\end{array}$ & 24.552 & 25.13 & 13.899 & 19.821 & 4.678 & 10.091 & -0.179 & & $\begin{array}{l}49.078 \\
5039\end{array}$ & $0 !$ & 2.47 & 0.0 & 43. & 0.71667 & 3.4 & 0 & & & 69 & & & 29 & \\
\hline & & & 24233 & & & & & 1995 & & & & & & & & & & & & & & & & & & & \\
\hline & & 1.915 & & & .618 & & 3.897 & 19.95 & 4.659 & .06 & 775 & & & 002 & 2.497 & & & & & 11.8 & & & 372 & & & . & \\
\hline
\end{tabular}

Figure C22a: 1.31B: After AN-102R2, Batch 4A - 2 M Nitric Acid Cleaning - First Cleaning (first 45 minutes) - Raw \& Calculated Data 
WSRC-TR-2003-00204, REV. 0

SRT-RPP-2003-00087, REV. 0

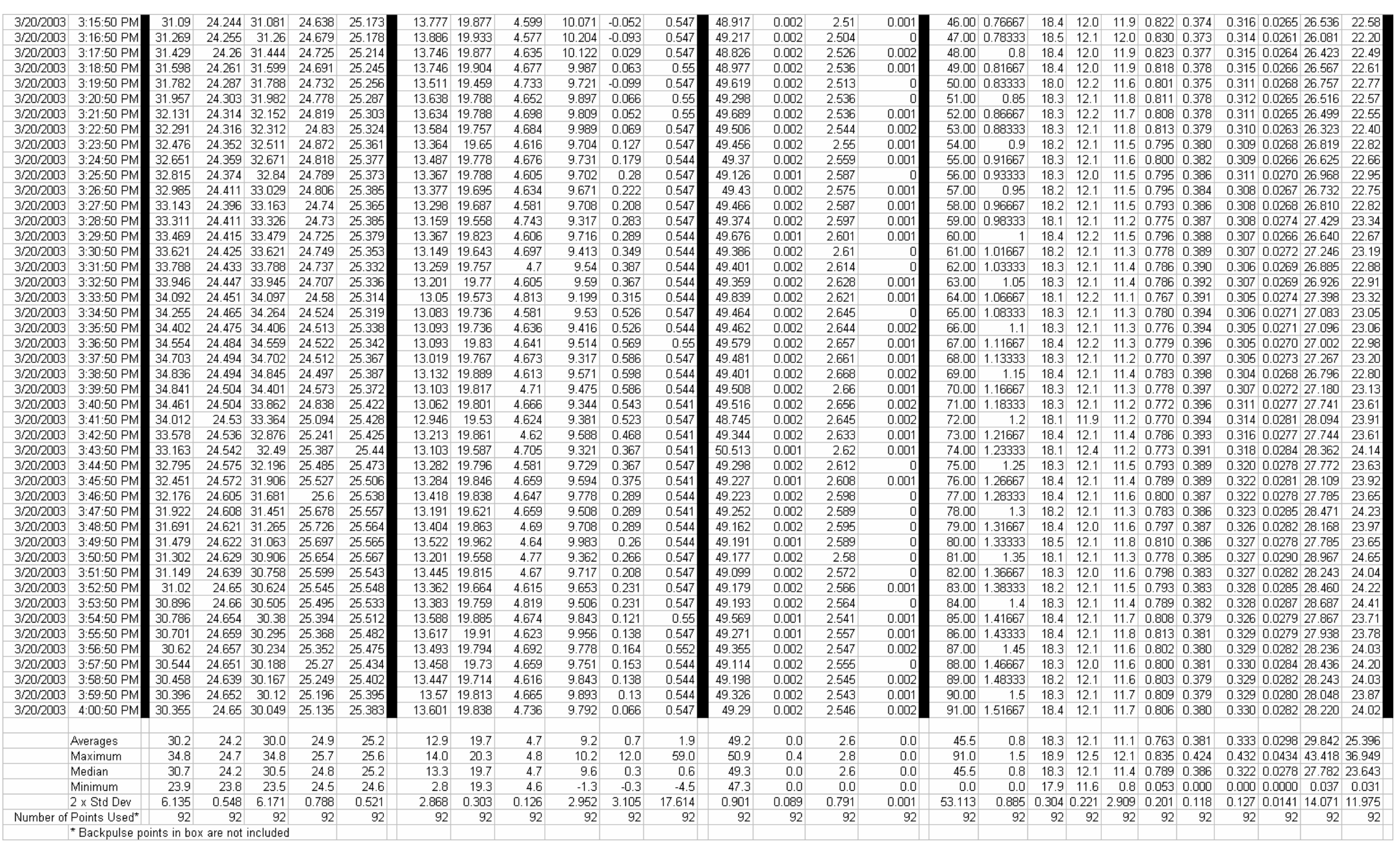

Figure C22b: 1.31B: After AN-102R2, Batch 4A - 2 M Nitric Acid Cleaning - First Cleaning (remaining 45 minutes) - Raw \& Calculated Data 
WSRC-TR-2003-00204, REV. 0

SRT-RPP-2003-00087, REV. 0

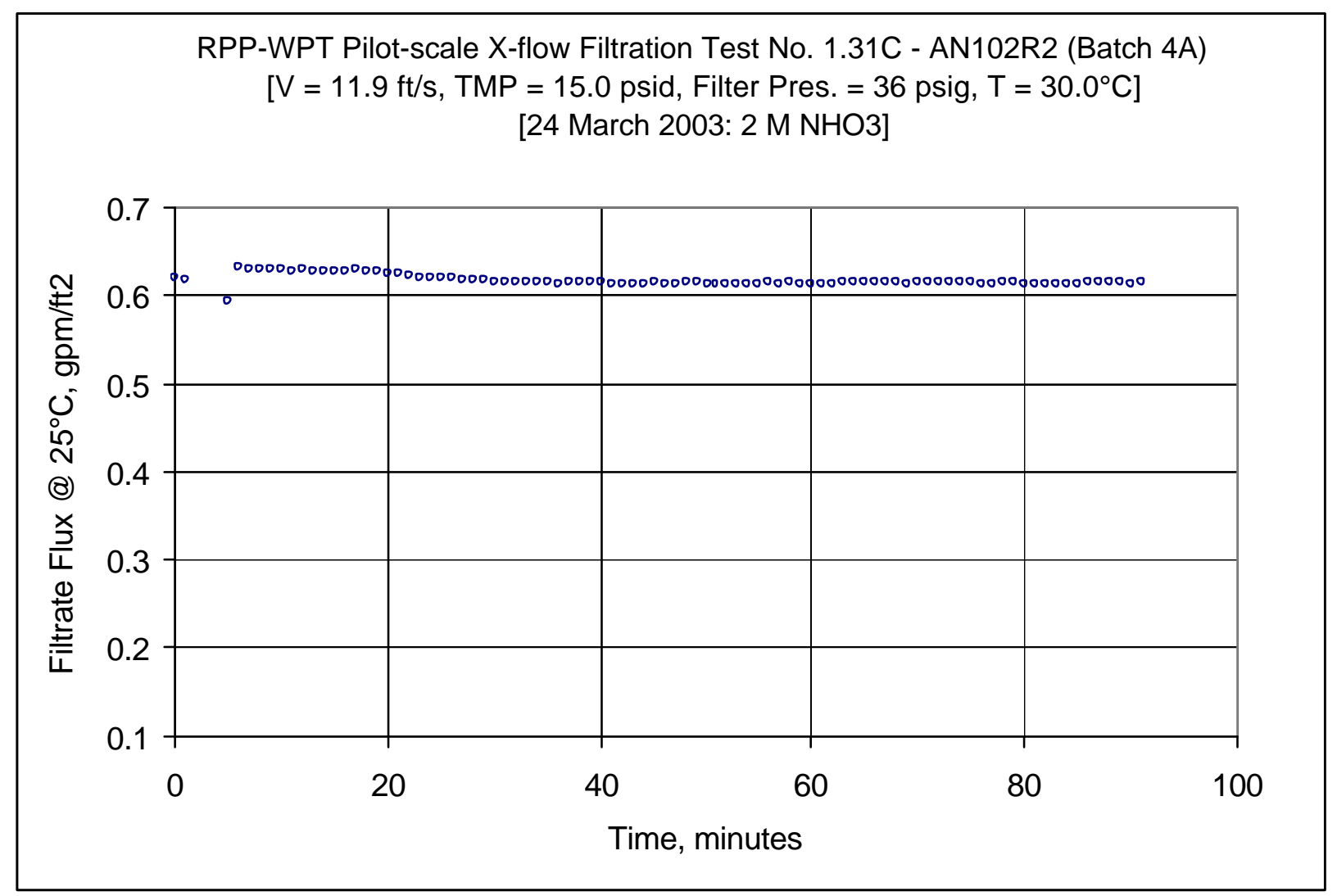

Figure C23: 1.31C: After AN-102R2, Batch 4A - 2 M Nitric Acid Cleaning - Second Cleaning 
WSRC-TR-2003-00204, REV. 0

SRT-RPP-2003-00087, REV. 0

\begin{tabular}{|c|c|c|c|c|c|c|c|c|c|c|c|c|c|c|c|c|c|c|c|c|c|c|c|c|c|c|c|}
\hline \multirow{3}{*}{ DATE } & \multirow{3}{*}{ TIME } & \multicolumn{5}{|c|}{ 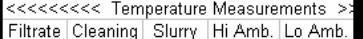 } & \multicolumn{6}{|c|}{ 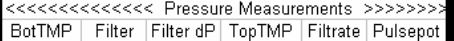 } & \multicolumn{4}{|c|}{ 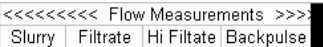 } & \multicolumn{11}{|c|}{ 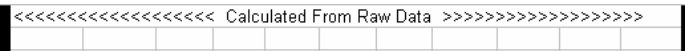 } \\
\hline & & $\operatorname{deg} \mathrm{C}$ & $\operatorname{deg} C$ & $\operatorname{deg} \mathrm{C}$ & & $\operatorname{deg} \mathrm{C}$ & psid & psig & psid & psid & psig & psig & $\begin{array}{l}\text { Slurry } \\
\text { gpm }\end{array}$ & gpm & gpm & & & & & & & & & & & & \\
\hline & & & & & & & $\mathrm{dP2}$ & & $\mathrm{dP1}$ & $\mathrm{dP3}$ & & & & 2 & & & & & & & & & & & & & \\
\hline & & & & & & & & & & & & & & & & & & & & & & & & & & & \\
\hline & :57:40 & 463 & 19.765 & 18.083 & 818 & & 0.129 & & -0.056 & 0.05 & 174 & & 0.216 & & & & & & & & & & & & & & \\
\hline & & & 19.765 & 18.083 & & & -0.129 & -0.0 & -0.056 & 0.05 & 174 & & 0.216 & 0.001 & & & & & & & & & & & & & \\
\hline $3 / 24 / 2003$ & 8:57:57 & 20.462 & 19.76 & 18.093 & $\begin{array}{l}320.828 \\
3\end{array}$ & 819.55 & -0.133 & -0.053 & -0.056 & 0.052 & $2-0.174$ & & 0.212 & 0.002 & & & & & & & & & & & & & \\
\hline 3/24/2003 & $8: 57: 58$ AM & 20.462 & 19.76 & 18.093 & $\begin{array}{ll}320.823 \\
3\end{array}$ & | & -0.131 & -0.053 & -0.056 & 0.054 & $\begin{array}{cc}4 & -0.171\end{array}$ & & -0.216 & 0.002 & 0.001 & & & & Note: & Gauge F & essure & re $F$ & is approxi & ximately & $1.4 \mathrm{psig}$ & & \\
\hline $3 / 24 / 2003$ & 8:57:59 AM & 20.467 & 19.765 & 18.093 & $\begin{array}{ll}320.823 \\
3\end{array}$ & 819.55 & -0.131 & -0.055 & -0.056 & 0.05 & $\begin{array}{lll}5 & -0.174\end{array}$ & & -0.212 & 0.002 & 0.001 & & & & & & & & & & & & \\
\hline $3 / 24 / 2003$ & 8:58:00 AM & 20.462 & 19.76 & 18.098 & $\begin{array}{l}320.828 \\
\end{array}$ & $\begin{array}{l}8 \\
0\end{array}$ & -0.131 & -0.053 & -0.056 & 0.054 & $\begin{array}{lll}4 & -0.174\end{array}$ & & -0.218 & 0.002 & 0.001 & & & & res & ref & s correcte & cted for 4 & & & vater tub & & \\
\hline $3 / 24 / 2003$ & 8:58:01 AM & 20.462 & 19.765 & 18.093 & $\begin{array}{ll}3 & 20.833 \\
\end{array}$ & $\begin{array}{l}8 \\
8\end{array}$ & -0.131 & -0.053 & $\begin{array}{l}8 \\
8\end{array}$ & 0.054 & $\begin{array}{lll}44 & -0.174\end{array}$ & 0.222 & -0.212 & 0.002 & 0.001 & & & & WW & & & & $<$ Filtra & rate Flux & $x \ll P E F$ & & ILITY $>$ \\
\hline 3/24/2003 & 8:58:02 AM & 20.467 & 19.765 & 18.093 & $\begin{array}{l}320.828 \\
\end{array}$ & $\begin{array}{l}8 \\
8\end{array}$ & -0.131 & -0.055 & -0.056 & 0.052 & $\begin{array}{lll}2 & -0.174\end{array}$ & 0.215 & -0.218 & 0.002 & 0.001 & & & & & & & & & & & $\times 1000$ & \\
\hline & & & & & & & & & & & & & & & & & & Time & Press. & & TMP T & TMP & & & & & \\
\hline - Per M & & & & & & & & & & & & & & & & & & & & & & & & & & & \\
\hline 3/24/2003 & 9:29:37 AM & 29.896 & 20.187 & 29.855 & 21.365 & 20.497 & 16.444 & 37.352 & 4.424 & 14.458 & $8 \quad 14.03$ & -4.35 & 49.03 & 0.001 & 4.758 & & & 0 & 35.9 & 912.0 & & & $5 \quad 0.709$ & & & 140.134 & \\
\hline 3/24/2003 & 9:30:37 A & & 20.203 & 30.09 & 21.397 & 20.569 & 16.508 & 37.4 & 4.42 & 14.535 & $5 \quad 14.03$ & -4.35 & 48.994 & 0.002 & 4.764 & & 1.00 & 667 & 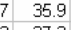 & 912.0 & 15.5 & & & & & 39.747 & 33 \\
\hline $3 / 24 / 2$ & & & & 30.186 & 21.449 & & & 38.746 & 4.323 & & & 15.018 & & 0.003 & & & 2.00 & & $\begin{array}{ll}3 \quad 37.3 \\
-17\end{array}$ & 1.1 & 0.7 & & & & & & \\
\hline $3 / 24$ & $9: 32$ & 29.957 & 20.255 & 30.334 & \begin{tabular}{|l|l|} 
\\
\end{tabular} 1.5999 & & 2.806 & $\begin{array}{l}38.829 \\
\end{array}$ & $\begin{array}{l}3.346 \\
\end{array}$ & & $\begin{array}{lll}4 & 30.881\end{array}$ & & \begin{tabular}{|l|l|}
44.774 \\
15007
\end{tabular} & 0.002 & & & 3.00 & & & 1.0 & 0.8 & & & & & & \\
\hline $3 / 24$ & 9:33:3 & 29.757 & $\begin{array}{l}20.284 \\
20.717\end{array}$ & 30.358 & 21.729 & 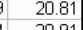 & $\begin{array}{r}2.704 \\
\end{array}$ & $\begin{array}{l}38.781 \\
77\end{array}$ & 4.3 & & 830 & $\begin{array}{r}78.563 \\
\end{array}$ & \begin{tabular}{|l|}
45.003 \\
\end{tabular} & 0.002 & & & 4.00 & 06667 & & 1.0 & 0.7 & & & & & & 16 \\
\hline $\begin{array}{l}3 / 24 / 2 \\
3 / 24 / 2\end{array}$ & $\begin{array}{l}9: 34: \\
9355\end{array}$ & $\begin{array}{l}30.015 \\
30.179 \\
\end{array}$ & $\begin{array}{l}20.313 \\
20.354\end{array}$ & $\begin{array}{l}30.119 \\
29.945\end{array}$ & $\begin{array}{r}21.924 \\
22.06\end{array}$ & 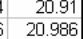 & $\begin{array}{l}15.489 \\
15.583\end{array}$ & $\begin{array}{l}37.399 \\
37.371\end{array}$ & $\begin{array}{l}\mid 4.384 \\
1\end{array}$ & $\begin{array}{l}13.563 \\
13.684\end{array}$ & $\begin{array}{ll}3 & 14.926 \\
4 & 14.871\end{array}$ & 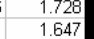 & & $\begin{array}{l}0.887 \\
0.002\end{array}$ & $\begin{array}{l}4.593 \\
4.862\end{array}$ & & $\frac{5.00}{6.00}$ & $\frac{8333}{0.1}$ & 5.9 & 2.0 & \begin{tabular}{|l|}
14.5 \\
14.6
\end{tabular} & & & & & $\begin{array}{ll}14 \\
96\end{array}$ & 4.82 \\
\hline $3 / 24 / 2003$ & 9:36:37 AM & 30.07 & 20.385 & 29.826 & 22.162 & & 15.728 & 37.325 & \begin{tabular}{|l|l}
5 & 4.332 \\
\end{tabular} & 14.024 & $\begin{array}{lll}4 & 14.631\end{array}$ & & & 0.002 & 4.836 & & 7.00 & 0.11667 & $\begin{array}{ll}7 & 35.9\end{array}$ & $\begin{array}{lll}9 & 12.0\end{array}$ & 14.9 & 1.026 & $\begin{array}{ll}6 & 0.721\end{array}$ & & & & \\
\hline $3 / 24$ & $9: 37: 37 \mathrm{~A}$ & 29.946 & 20.421 & 29.732 & 22.308 & \begin{tabular}{ll|}
8 & 21.164 \\
\end{tabular} & 15.788 & 37.265 & \begin{tabular}{|l|l|}
5 & 4.351
\end{tabular} & 13.884 & $\begin{array}{ll}4 & 14.539\end{array}$ & & 48.877 & 0.001 & 4.82 & & 8.00 & 00.13333 & $\begin{array}{ll}3 & 35.8\end{array}$ & $\begin{array}{ll}8 & 12.0\end{array}$ & 14.8 & .023 & $\begin{array}{ll}3 & 0.719\end{array}$ & & & & \\
\hline & 9:38:37 & & 20.457 & 29.669 & 22.389 & 3 & 5.955 & |37.288 & 4.30 & 14.175 & $\begin{array}{llll}5 & 14 .\end{array}$ & & & & 4.8 & & 9.00 & & $\begin{array}{ll}5 & 35\end{array}$ & 11.9 & & & & & & & \\
\hline $124 /$ & 9:39:37 & 29.76 & 20.488 & 29.605 & 22.486 & $\begin{array}{ll}5 & 21.3 \\
\end{array}$ & 16.214 & 37.49 & 4.3 .3 & 14.443 & $\begin{array}{ll}3 & 14.359\end{array}$ & & & & 4.8 & & 10.00 & 0.166 & & $\begin{array}{ll}0 & 12.0\end{array}$ & 15.3 & & $\begin{array}{lll}7 & 0.716\end{array}$ & & & & \\
\hline $3 / 24 / 2$ & 9:40:37 An & 29.6. & 20.52 & 29.547 & 22.568 & $\begin{array}{ll}8 & 21.3\end{array}$ & 16.2 & | 37.437 & 4.408 & 14.316 & 6 14.296 & 1.62 & & 0.0 & 4.78 & 0.0 & 11.00 & 0.0 .18333 & $\begin{array}{ll}3 & 36.0\end{array}$ & $\begin{array}{lll}0 & 11.9\end{array}$ & 15.3 & 52 & $\begin{array}{ll}20.713 \\
\end{array}$ & & & 2 41.209 & \\
\hline 24. & $\mathrm{Al}$ & & 20.541 & 29.494 & 22.594 & 421. & 16.17 & 37.203 & 4.368 & 14.257 & $\begin{array}{ll}7 & 14.14\end{array}$ & & & & 4. & & 12.00 & 0 & 35.7 & 11.9 & $5 ?$ & & & & & 341.342 & \\
\hline $3 / 24$ & & 74 & 20.583 & 29.47 & 22.621 & & 16.202 & 37.203 & & 14.218 & $\begin{array}{llll}8 & 14.171\end{array}$ & & & 0.6 & 4. & & 13 & 21667 & 35.7 & 11.9 & 15.2 & .049 & 90.711 & 0.62 & & & \\
\hline 3/24/2003 & 9:43:37 AN & 29.537 & 20.631 & 29.427 & 22.633 & 3 & 16.264 & 37.284 & $\begin{array}{l}4 \\
4\end{array}$ & 14.212 & $\begin{array}{ll}2 & 14.166\end{array}$ & 5 & 76 & 0. & 4.7 & & 14.00 & 100.23333 & 35.8 & $\begin{array}{lll}8 & 11.9\end{array}$ & 15.2 & 1.051 & 10.711 & & & 41.243 & \\
\hline $3 / 24 / 2$ & 9:44:37 & 29.503 & 20.666 & 29.388 & 22.649 & 3 & 16.361 & \begin{tabular}{|l|}
37.273 \\
\end{tabular} & $\begin{array}{l}3 \\
3\end{array}$ & 14.519 & 913.958 & & 48 & 0. & 4.7 & & 15.00 & 0.25 & $\begin{array}{ll}5 & 35.8 \\
5\end{array}$ & $\begin{array}{lll}8 & 11.9\end{array}$ & 15.4 & 1.065 & $\begin{array}{ll}5 & 0.709 \\
\end{array}$ & & & & \\
\hline $3 / 24 / 2$ & 9:45:37 & & & 29.35 & 22.661 & & & 37.228 & & & & & & & 7 & & 16.00 & & $\begin{array}{ll}7 & 35.8 \\
\end{array}$ & $\begin{array}{l}3 \\
\end{array}$ & 15.4 & 60 & 0.709 & & & & \\
\hline & & & & & & & & 37.213 & & & & & & & & & & & 7 & 1.9 & 4 & & & & & & \\
\hline & & & & 29.263 & 22.733 & & & & & & & & & & & & & 0.3 & 7 & 711.9 & 4 & 61 & & & & & \\
\hline & & 29.379 & 20.808 & 29.299 & $\begin{array}{r}22.74 \\
\end{array}$ & 948 & & 37.3 & $\begin{array}{l}4.315 \\
\end{array}$ & & & & & & & 0.001 & & & 8.8 & B 11.9 & 15.5 & & & & & & \\
\hline & $\begin{array}{l}9: 49: 37 \\
0.57: 37\end{array}$ & 29.524 & $\begin{array}{l}1 \\
-20.85 \\
\end{array}$ & $\begin{array}{r}29.4 \\
\end{array}$ & $\begin{array}{l}22.801 \\
20.862\end{array}$ & & & $\begin{array}{l}37.337 \\
37.30\end{array}$ & $\begin{array}{r}4.37 \\
3.361\end{array}$ & & & & & & & & & & 99 & $\begin{array}{l}9 \\
9 \\
9\end{array}$ & 15.5 & 68 & & & & & .42 \\
\hline $\begin{array}{r}3 / 24 / 2000 \\
324003\end{array}$ & $\begin{array}{l}9: 50: 37 \\
9.51 \cdot 17\end{array}$ & 29.61 & $\begin{array}{l}20.886 \\
20.027\end{array}$ & 29.496 & $\begin{array}{l}22.862 \\
20.819\end{array}$ & 2 & & $\begin{array}{r}37.342 \\
37.3\end{array}$ & & & & & & & & & & 35 & & $\begin{array}{l}911.9 \\
6119\end{array}$ & & 74 & & & & & \\
\hline $\begin{array}{r}3 / 24 / 2000 \\
3 / 24 / 2003\end{array}$ & $\begin{array}{l}9: 51: 37 \\
9.52 \cdot 37\end{array}$ & $\begin{array}{r}29.68 \\
29736 \\
\end{array}$ & $\begin{array}{l}20.922 \\
20.959\end{array}$ & $\begin{array}{l}29.596 \\
20992\end{array}$ & $\begin{array}{r}22.848 \\
2288\end{array}$ & 8 & & $\begin{array}{r}37.3 \\
37.306\end{array}$ & $\begin{array}{l}8.342 \\
537\end{array}$ & & & & & & & & 22.00 & 6337 & 8.8 & 1.9 & 4 & 169 & & & & & $\begin{array}{lll}2 & 34.55 \\
2 & 3\end{array}$ \\
\hline $\begin{array}{l}3 / 24 / 20005 \\
3 / 24 / 2003\end{array}$ & $\begin{array}{l}\text { 9.5.2.77 AMM } \\
9.55377\end{array}$ & $\begin{array}{l}29.136 \\
29792\end{array}$ & 20.959 & $\begin{array}{l}29.692 \\
29762\end{array}$ & $\begin{array}{l}22.86 \\
22896\end{array}$ & $\begin{array}{l}22.184 \\
222245 \\
0\end{array}$ & 16 & $\begin{array}{l}37.7006 \\
37.236\end{array}$ & $\begin{array}{ll}4.57 \\
6\end{array}$ & $\begin{array}{l}14.596 \\
14699\end{array}$ & 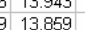 & 009 & & 0. & 4. & & 23.00 & $\begin{array}{l}0.38333 \\
0\end{array}$ & $\begin{array}{ll}1 & 35.8 \\
4 & 358\end{array}$ & $\begin{array}{l}811.9 \\
3 \\
3 \\
120\end{array}$ & \begin{tabular}{|l}
15.4 \\
156
\end{tabular} & 1703 & $\begin{array}{ll}08 \\
07\end{array}$ & 8 & 03 & $\begin{array}{l}840.283 \\
839776\end{array}$ & $\begin{array}{l}3.34 .28 \\
6 \\
6\end{array}$ \\
\hline & $9: 5$ & & 21.025 & 29.787 & & & & & $\begin{array}{rr}4 & 4.04 \\
4 & 4.302\end{array}$ & & & & & & & & 25.00 & 0.41667 & $\begin{array}{ll}\begin{array}{l}7 \\
7\end{array} & 35.7 \\
\end{array}$ & 7.11 .9 & 15.4 & & & & & & $\begin{array}{ll}6 & 34.16 \\
\end{array}$ \\
\hline $3 / 24$ & $9: 5$ & & 21.057 & & & & & |37.625 & & & & & & & & & & 0.43333 & 2.2 & $2 \quad 11.9$ & 15.8 & & & & & 339.305 & 5 \\
\hline & & & 21. & & 23.185 & & & & & & & & & & & & 27.00 & 45 & .9 & 9 9 11.9 & & & & & & & \\
\hline & & & & & & & & & & & & & & & & & & 0.466667 & & 9 & & & & & & & \\
\hline & & & & & & & & & & & & & & & & & & 00 & 9 & $\begin{array}{l}9 \\
9\end{array}$ & 15.5 & 68 & & & & & \\
\hline & & & & & & & & & & & & & & & & & & 0.5 & 7 & $7 \quad 11.9$ & 15.4 & & & & & & \\
\hline & & & & & 23.209 & & & & & & & & & & & & & & & 1.9 & & & & & & & \\
\hline & & & 21. & 29.959 & 23.2 & & & & & & & & & & & & & & & 911.9 & & & & & & & \\
\hline & 10: & & 21.301 & 30 & $\begin{array}{l}23.236 \\
\end{array}$ & & & & & & & & & & & & & & & 11.9 & & & & & & & \\
\hline & & & $\begin{array}{l}21.348 \\
.21 .371\end{array}$ & $\begin{array}{l}30.031 \\
30.037\end{array}$ & 23.293 & $\begin{array}{l}322.77 \\
1\end{array}$ & 16 & $\begin{array}{r}37.284 \\
37.486\end{array}$ & $\begin{array}{l}4.369 \\
4\end{array}$ & 14. & & & & & & & & & 7 & 1.9 & 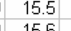 & 69 & & & & & \\
\hline & 10:04 & 30. & 21.374 & 30.037 & $\begin{array}{r}23.334 \\
23.52\end{array}$ & $\begin{array}{ll}422 \\
5\end{array}$ & 16. & \begin{tabular}{|l|}
37.468 \\
37.277
\end{tabular} & 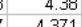 & $\begin{array}{l}14.634 \\
1.611\end{array}$ & & & & & & & & & & 1.9 & & & & & & & \\
\hline $3 / 24$ & & & 21.41 & 30.043 & $\begin{array}{r}23.35 \\
23.356\end{array}$ & 5 & & 37. & 4. & 14.611 & & & & & & & & & & 1.9 & & & & & & & \\
\hline & $\begin{array}{l}10: 0.0673 \\
10.73: 37\end{array}$ & 30. & $\begin{array}{l}21.436 \\
21.472\end{array}$ & $\begin{array}{l}30.049 \\
30.054\end{array}$ & $\begin{array}{l}23.506 \\
230101\end{array}$ & $\begin{array}{l}522.5 \\
11 \\
1\end{array}$ & & \begin{tabular}{|}
33.386 \\
37777
\end{tabular} & 7 & $\begin{array}{l}14.5 / 6 \\
14556\end{array}$ & $\begin{array}{l}6 \\
6 \\
6\end{array}$ & & & & & & 37.0 & U. 0.6766 & 1 & 11.9 & $\begin{array}{l}6 \\
5\end{array}$ & 罗 & & & & $=0$ & 33 \\
\hline & & & & & 23. & & & & & & & & & & & & & & & 9 & & & & & & & \\
\hline & & & 21.54 & & 23.519 & & & & 4. & 14. & & & & & & & 44 & & 7 & 711.9 & $\begin{array}{l}15.4 \\
\end{array}$ & & & & & & \\
\hline & & & 21.5 & & 23. & & & 37.296 & & & & & & & & & & 00.6 & 3 & 1.9 & 1 & & & & & & \\
\hline & & & & & 23 . & & & & & & & & & & & & 42.00 & & 7 & .9 & & & & & & & \\
\hline & & & & 30.114 & & & & & & & & & & & & & & & & 1.9 & & & & & & & \\
\hline $3 / 24 / 2$ & 10:13 & & 21.684 & 30.1 & 23.6 & 823 & & & & & & 1.6 & & & & & & 00. & 5 & $\begin{array}{l}9 \\
9\end{array}$ & 6 & 76 & & & & & \\
\hline $3 / 24 / 2003$ & 10:14:37, & 30.17 & 21.71 & 30.096 & 23.729 & & 16.571 & 37.381 & 4.364 & 14.626 & $\begin{array}{lll}6 & 13.926\end{array}$ & & |48.665 & 0.001 & 4.746 & & 45.00 & 0.75 & 35.9 & 11.9 & 15.6 & 1.075 & $\begin{array}{l}50.708 \\
\end{array}$ & $\begin{array}{ll}8 & 0.6\end{array}$ & 50.0394 & 439.395 & \\
\hline
\end{tabular}

Figure C24a: 1.31B: After AN-102R2, Batch 4A - 2 M Nitric Acid Cleaning - First Cleaning (first 45 minutes) - Raw \& Calculated Data 
WSRC-TR-2003-00204, REV. 0

SRT-RPP-2003-00087, REV. 0
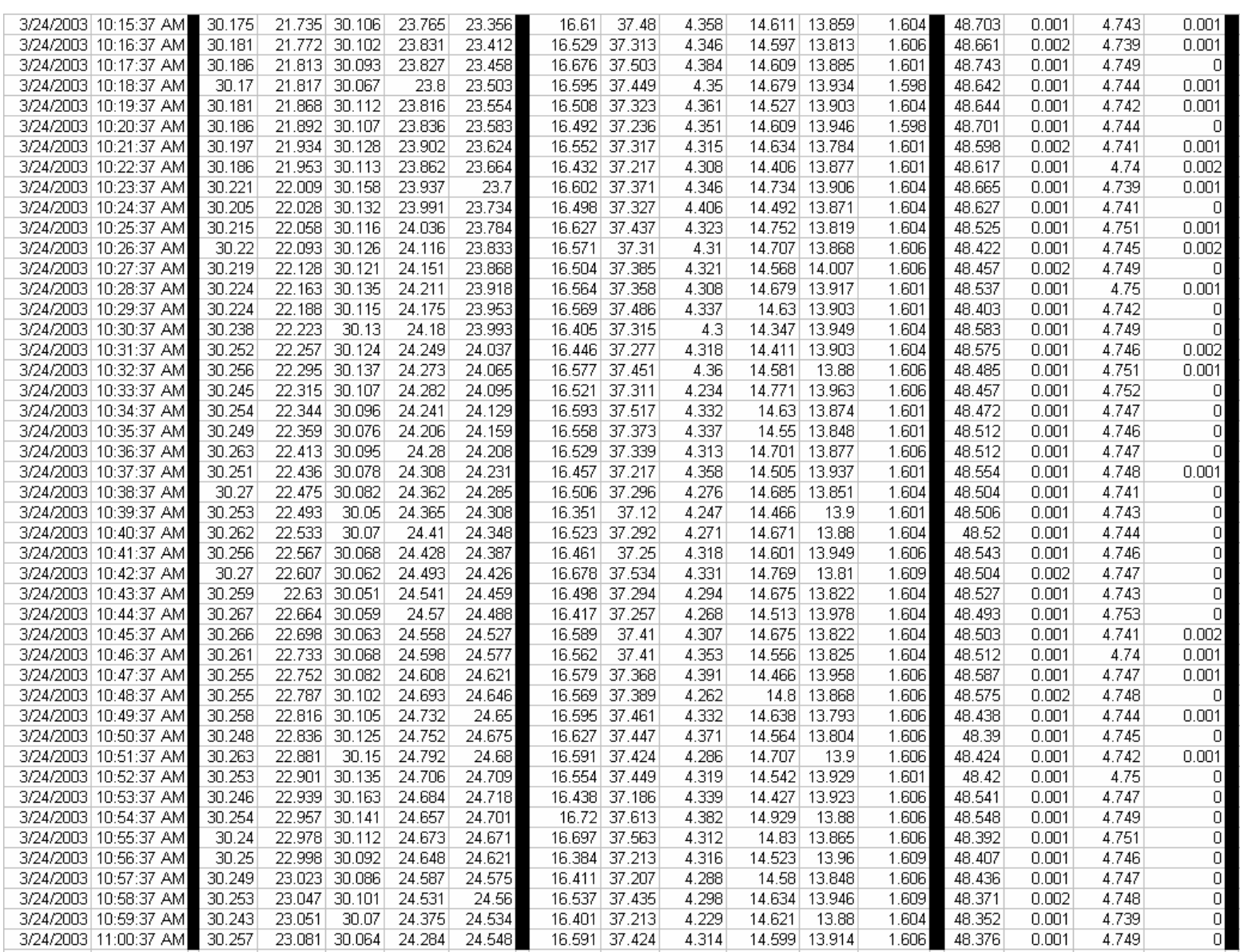

\begin{tabular}{|l|l|l|l|l|l|l|l|l|l|l|}
\hline 46.00 & 0.76667 & 36.0 & 11.9 & 15.6 & 1.076 & 0.707 & 0.614 & 0.0393 & 39.329 & 33.47 \\
\hline 47.00 & 078333 & 35.8 & 11.9 & 15.6 & 1.073 & 0.707 & 0.613 & 0.0394 & 39.420 & 33.55 \\
\hline
\end{tabular}

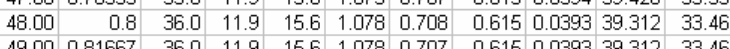
\begin{tabular}{l|l|l|l|l|l|l|l|l|l|l|}
49.00 & 0.81667 & 36.0 & 11.9 & 15.6 & 1.078 & 0.707 & 0.615 & 0.0393 & 39.312 & 33.46 \\
\hline 50.00 & 0.83333 & 35.9 & 11.9 & 15.5 & 1.070 & 0.707 & 0.614 & 0.0395 & 39.550 & 33.66 \\
\hline
\end{tabular}

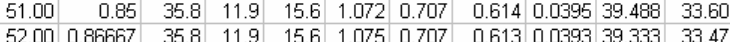
\begin{tabular}{llllllllllll}
52.00 & 0.86667 & 35.8 & 11.9 & 15.6 & 1.075 & 0.707 & 0.613 & 0.0393 & 39.333 & 33.47 \\
\hline 53.00 & 0.88333 & 35.7 & 11.9 & 15.4 & 1.063 & 0.707 & 0.613 & 0.0398 & 39.785 & 33.86 \\
\hline
\end{tabular}

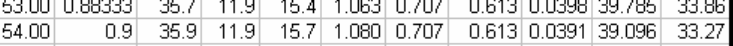

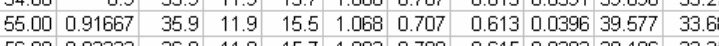

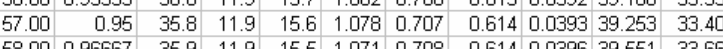
\begin{tabular}{lllllllllll}
0.96667 & 35.9 & 11.9 & 15.5 & 1.071 & 0.708 & 0.614 & 0.0396 & 39.551 & 33.66 \\
\hline
\end{tabular}

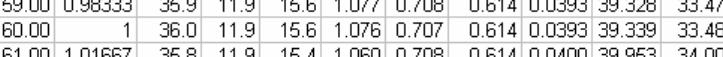

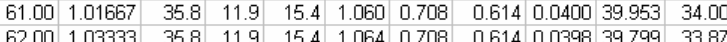
\begin{tabular}{rrrrrrrrrrr}
62.00 & 1.03333 & 35.8 & 11.9 & 15.4 & 1.064 & 0.708 & 0.614 & 0.0398 & 39.799 & 33.87 \\
\hline 6300 & 105 & 36.0 & 119 & 156 & 1074 & 0.708 & 0.614 & 0.0394 & 39.442 & 33.57 \\
\hline
\end{tabular} \begin{tabular}{rrrrrrrrrrrr}
63.00 & 1.05 & 36.0 & 11.9 & 15.6 & 1.074 & 0.708 & 0.614 & 0.0394 & 39.442 & 33.57 \\
\hline 64.00 & 1.06667 & 35.8 & 11.9 & 15.6 & 1.079 & 0.708 & 0.515 & 0.0393 & 39.313 & 33.46
\end{tabular} \begin{tabular}{llllllllllll}
64.00 & 1.06667 & 35.8 & 11.9 & 15.6 & 1.079 & 0.708 & 0.615 & 0.0393 & 39.313 & 33.46 \\
\hline 65.00 & 1.08333 & 36.0 & 11.9 & 15.6 & 1.076 & 0.708 & 0.615 & 0.0394 & 39.370 & 33.50
\end{tabular}

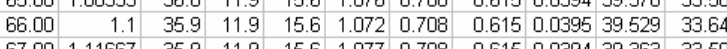

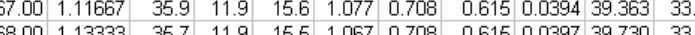

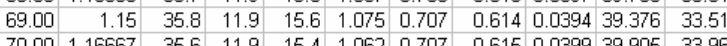

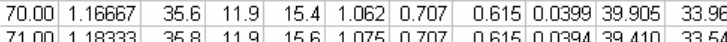
\begin{tabular}{llllllllllllll}
71.00 & 1.18333 & 35.8 & 11.9 & 15.6 & 1.075 & 0.707 & 0.615 & 0.0394 & 39.410 & 33.54 \\
\hline 7200 & 12 & 358 & 11.9 & 155 & 1.071 & 0.708 & 0.615 & 0.0396 & 39.596 & 3370
\end{tabular}

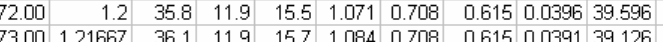

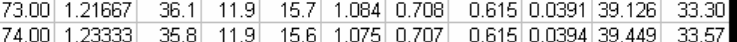

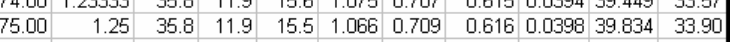

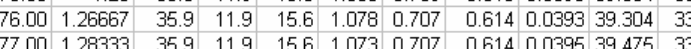

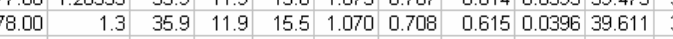
$\begin{array}{llllllllllll}79.00 & 1.31667 & 35.9 & 11.9 & 15.7 & 1.081 & 0.708 & 0.615 & 0.0392 & 39.189 & 33 & 33.4\end{array}$

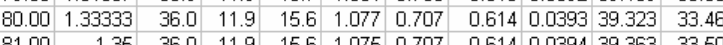

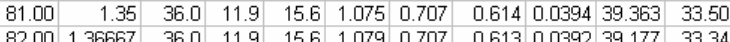

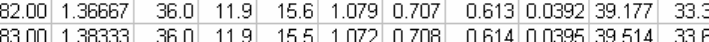
\begin{tabular}{r|rrrrrrrrrr}
84.00 & 1.4 & 35.7 & 11.9 & 15.4 & 1.064 & 0.708 & 0.614 & 0.0398 & 39.755 & 33
\end{tabular} \begin{tabular}{l|l|l|l|l|l|l|l|l|l|l}
85.00 & 1.41667 & 36.1 & 11.9 & 15.8 & 1.091 & 0.708 & 0.614 & 0.0388 & 38.809 & 33.03 \\
\hline
\end{tabular}

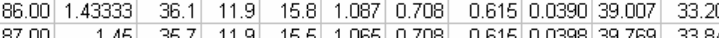

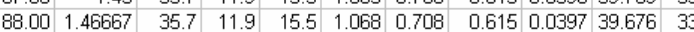
$\begin{array}{lllllllllll}89.00 & 1.48333 & 36.0 & 11.9 & 15.6 & 1.075 & 0.708 & 0.615 & 0.0394 & 39.439 & 33\end{array}$

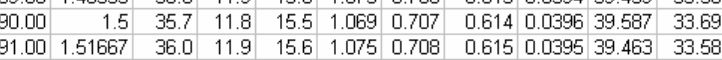
3/24/2003 11:00:37 AM

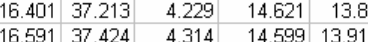
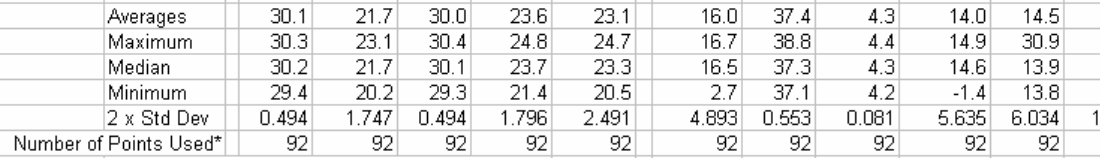

Figure C24b: 1.31C: After AN-102R2, Batch 4A - 2 M Nitric Acid Cleaning - Second Cleaning (remaining 45 minutes) - Raw \& Calculated Data 


\section{Appendix D: Slurry Dewatering, Test Run 1.16 - AN-102R2}

While this task filtered slurry under steady state conditions, i.e., both the flow parameters and slurry solids concentration were held constant, the most important test runs dealt with "dewatering" the slurry, which means allowing the solids concentration to increase with time. Initially there were to be just two dewatering tests for this organic based slurry: Batch 3 and Batch 4, which would differ only in mixing regimes while the slurry was prepared before filtration. However, problems with filtering Batch 3 led to reformulating the slurry; therefore it was followed with a Batch 3B and a Batch 3A. The first batch was named Batch 3C. Instead of having two batch it turned out to be four, for which the data are included herein.

The general test matrix was to first test the cross filter with water and then a standard slurry. The slurry dewatering test run, No. 1.16, began by preparing the test slurry and introducing into the test at a insoluble solids concentration near $1.0 \mathrm{wt} \%$. During the dewatering test the slurry axial velocity, V, was maintained at approximately $12 \mathrm{ft} / \mathrm{s}$ and the Transmembrane Pressure, TMP, at approximately $40 \mathrm{psid}$. This process continued until the solids concentration reached the target of $20 \mathrm{wt}$. This Appendix includes the following dewatering test runs:

1.16 , Batch 3C

1.16, Batch 3B

1.16 , Batch $3 \mathrm{~A}$

1.16 , Batch $4 \mathrm{~A}$
This AN-102 simulant was precipitated under NOC, see below This AN-102 simulant was precipitated under BC, see below This AN-102 simulant was precipitated under $\mathrm{BC}$ at $25^{\circ} \mathrm{C}$, see below This AN-102 simulant was precipitated using PJM, see below

NOC - Newly Optimized Conditions - Initially there was to be only a single Batch 3 simulant prepared under the newly optimized conditions, however poor filterability forced a formulation of the slurry simulant and two more Batch 3 test. The conditions that were being "optimized" are called Baseline Conditions, explained below, and basically means using less reagents during the precipitation phase of the slurry preparation, i.e., are $0.03 \mathrm{M} \mathrm{SrCO}$, $0.03 \mathrm{M} \mathrm{NaMn} 4$, bring the free hydroxide concentration to $0.3 \mathrm{M}$, and precipitate at $20^{\circ} \mathrm{C}$. Unfortunately only the decontamination factors were optimized while the filterability of the slurry became worse. This slurry was made while being well mixed.

$\mathrm{BC}$ - Baseline Conditions - These were the precipitation conditions used to test another organic based slurry in the past, i.e., AN-107 slurry (Duignan, 2000), which were $0.075 \mathrm{M} \mathrm{SrCO3,0.05} \mathrm{M,} \mathrm{bring} \mathrm{the} \mathrm{free}$ hydroxide concentration to $1 \mathrm{M}$, and precipitate at $50^{\circ} \mathrm{C}$. This slurry was made while being well mixed.

$\mathrm{BC}$ at $20^{\circ} \mathrm{C}-$ Baseline Conditions but at the lower temperature of $20^{\circ} \mathrm{C}$. This slurry was made while being well mixed.

PJM - Pulse Jetpump Mixer - This AN-102 simulated slurry was precipitated at the baseline conditions, however, the mixing environment during precipitation was made to mock that which was planned for actual plant operation. A scaled version of the air driven jetpumps were used to determine its effect on the overall process.

Each dewatering test in this Appendix contains a graph of the filtrate flux with time and tables of the data taken and calculated. To better understand the presented information important aspects of the graphs and tables are explained below:

Graphs - Each graph contains time averages of some of the imporant parameters taken during the run at the top of the figures, i.e. Slurry velocity in the filter tube, V; transmembrane pressure, TMP; slurry pressure at the entrance of the filter tube, Filter Pres; and the slurry temperature, T.

Tables - Each table is divided into raw data (the first 17 columns) and calculations (the last 10 columns) which are based on the raw data. The raw data section is divided into three parts: 


\title{
WSRC-TR-2003-00204, REV. 0
} SRT-RPP-2003-00087, REV. 0

\author{
Individual Entries - Raw Data \\ Temperature Filtrate - from the filtrate loop at the exit of the filter bundle \\ Cleaning - from the cleaning loop (this loop was not used, except for tests Clean $1 \& 2$ ) \\ Slurry - from the slurry loop in the slurry reservoir \\ Hi Amb. - outside ambient temperature at the top of the test rig \\ Lo Amb. - inside ambient temperature at the bottom of the test rig \\ Pressure BotTMP - differential pressure across the filter tube at the bottom of the vertical tube \\ Filter - gauge pressureof the slurry at the entrance of the filter tube bundle \\ Filter $\mathrm{dP}$ - differential pressure of the slurry along the height of the vertical 90-inch tube \\ TopTMP - differential pressure across the filter tube at the top the vertical 90-inch tube \\ Filtrate - gauge pressure of the filtrate at the exit of the filter tube housing \\ Pulsepot - gauge pressure of the filtrate at the bottom of the pulsepot \\ Flow Slurry - rate of slurry just before entering the seven-filter-tube bundle \\ Filtrate - rate of slurry filtrate under normal flow (max. is $1.2 \mathrm{gpm}$ ) \\ Hi Filtrate - rate of any liquid with has a high flowrate (water, acid, etc.) (max. 5 gpm) \\ Backpulse - rate of filtrate in opposite direction from normal flow, i.e., backpulse
}

Individual Entries - Calculated Data

Time - Minutes after start of test, including the backpulse (based on raw data TIME column)

Press. - Internal filter pressure of slurry at the filter bundle entrance (based on raw data P1 column and corrected for a 40.9-inch water column that exists from the transducer to the point where P1 is measured.)

Vel. - Slurry velocity in filter tube (based on raw data Q1 column and i.d. of the tubes of 0.488 inches)

TMP - Filter transmembrane pressure (based on average of raw data columns dP2 and dP3) in psi.

TMP - Filter transmembrane pressure (based on average of raw data columns dP2 and dP3) in bar.

Filtrate Flux - Filtrate flow rate (raw data columns Q2/Q3) divided by inside filter surface area $(6.87 \mathrm{ft} 2)$

Filtrate Flux @ $25^{\circ} \mathrm{C}$ - Same as Filtrate flux, but correct for temperature with the following multiplier:

$\mathrm{T}($ corrected $)=\mathrm{T} \times \operatorname{Exp}[2500 /((1 / 273+\mathrm{T}))-(1 / 298)]$ as per Test Spec 24590-WTP-TSP-RT-01-029, Rev. 0

Permiability - Filterate flow rate per TMP (based on calculated columns Filtrate Flux @ $25^{\circ} \mathrm{C}$ and TMP)

Permiability x 1000 - Same as the above Permiability, but increase by 1000 to facilitate graphing

Permiability - Same as Permiability but in metric units, meter/day/bar

$\underline{\text { Statistics }}$

At the bottom of the last individual entry there are six rows with some data statistics to better understand the data and their fluctuations. The columns are self explained with titles of Averages, Maximum, Median, and Minimum. The next row $2 \times$ Std Dev, is two times the population standard deviation based on the number of data points used, which is the last row. The Number of Points used generally do not included the points taken during the backpulse sequence of each test. This is explained below.

\section{Raw Data Table Layout}

The raw data is generally made up of three sections:

Zeros - These eight rows include approximately 15 to 20 seconds of data taken with the pressure transducers placed in the "zero" mode to determine if any zero drift occurred from one test to the next.

Data-Per Minute - On most of the tables the first six rows of these data are contained in a box which indicate data taken during the backpulse. This is were the filtrate flow is reversed temporarily to knock the filtrate cake of the filter surface. During these first few minutes the filtrate flow is stopped, then reversed, then stopped again, then it is finally returned to normal operation. The data during the backpulse operation were taken with a separate log, therefore those data are marked with a box to note this fact. Most or all of those data points are not counted in the statistics at the bottom of the table. The tables of some of the later tests do not show the boxed data because separate logs of data were not taken, however since a backpulse for each run still occurred only those data were not included in the statistics at the bottom of the page. 


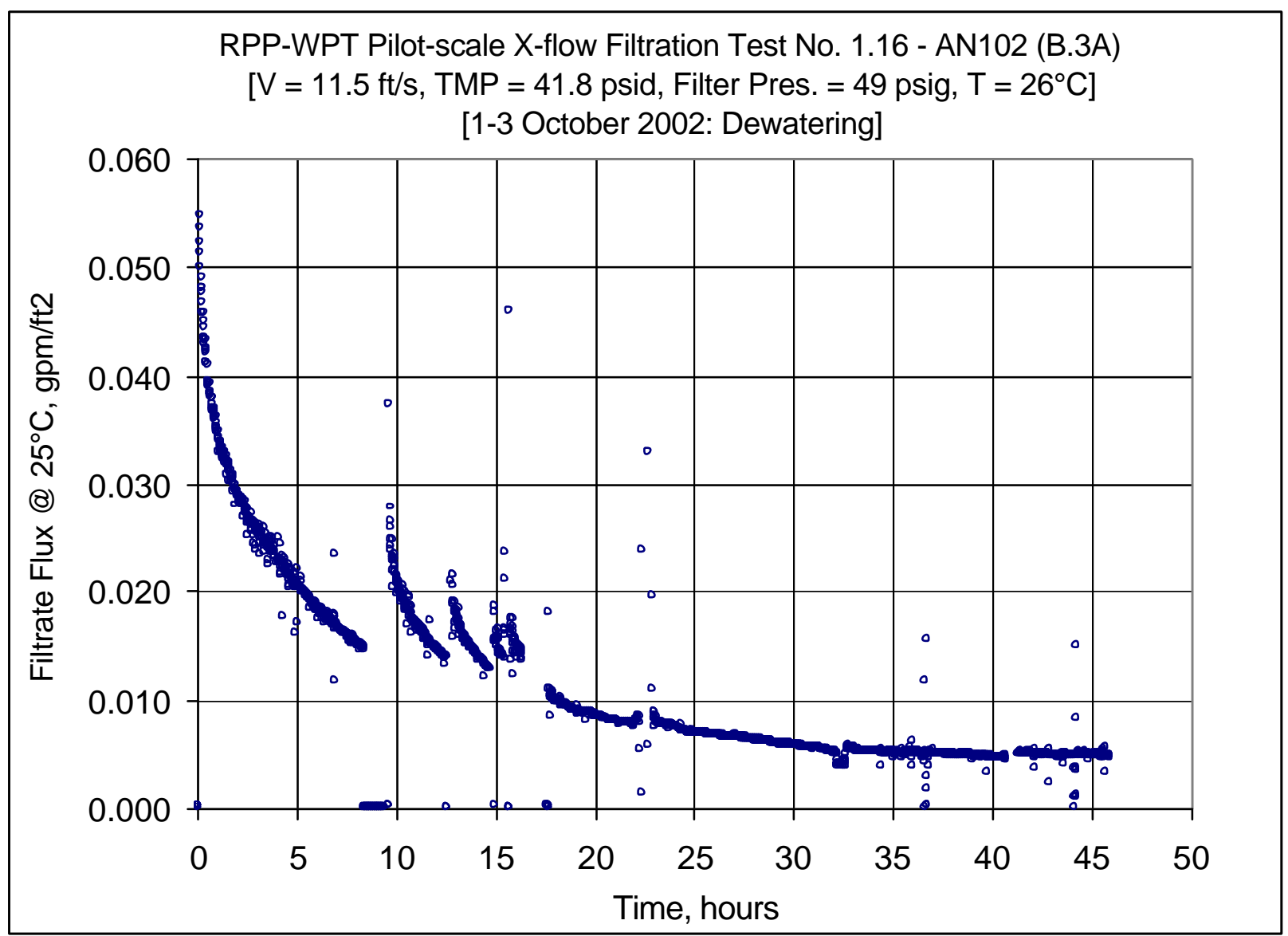

Figure D1: Dewatering of the AN-102R2, batch 3C, insoluble solids concentrations from 1 to $8.5 \mathrm{wt} \%$

(Data from 26 Files)

(1.16-rpp-pxu - 100102-1710, -1910, -2110, -2310)

(1.16-rpp-pxu-100202-0132, -0240, -0335, -0534, -0541, -0549, -0756, -0840, -1040)

(1.16-rpp-pxu-100202-1251, -1501, -1524, -1600, -1800, -2000, -2359)

(1.16-rpp-pxu-100302-0200, -0400, -0834, -1031, -1248) 
WSRC-TR-2003-00204, REV. 0

SRT-RPP-2003-00087, REV. 0

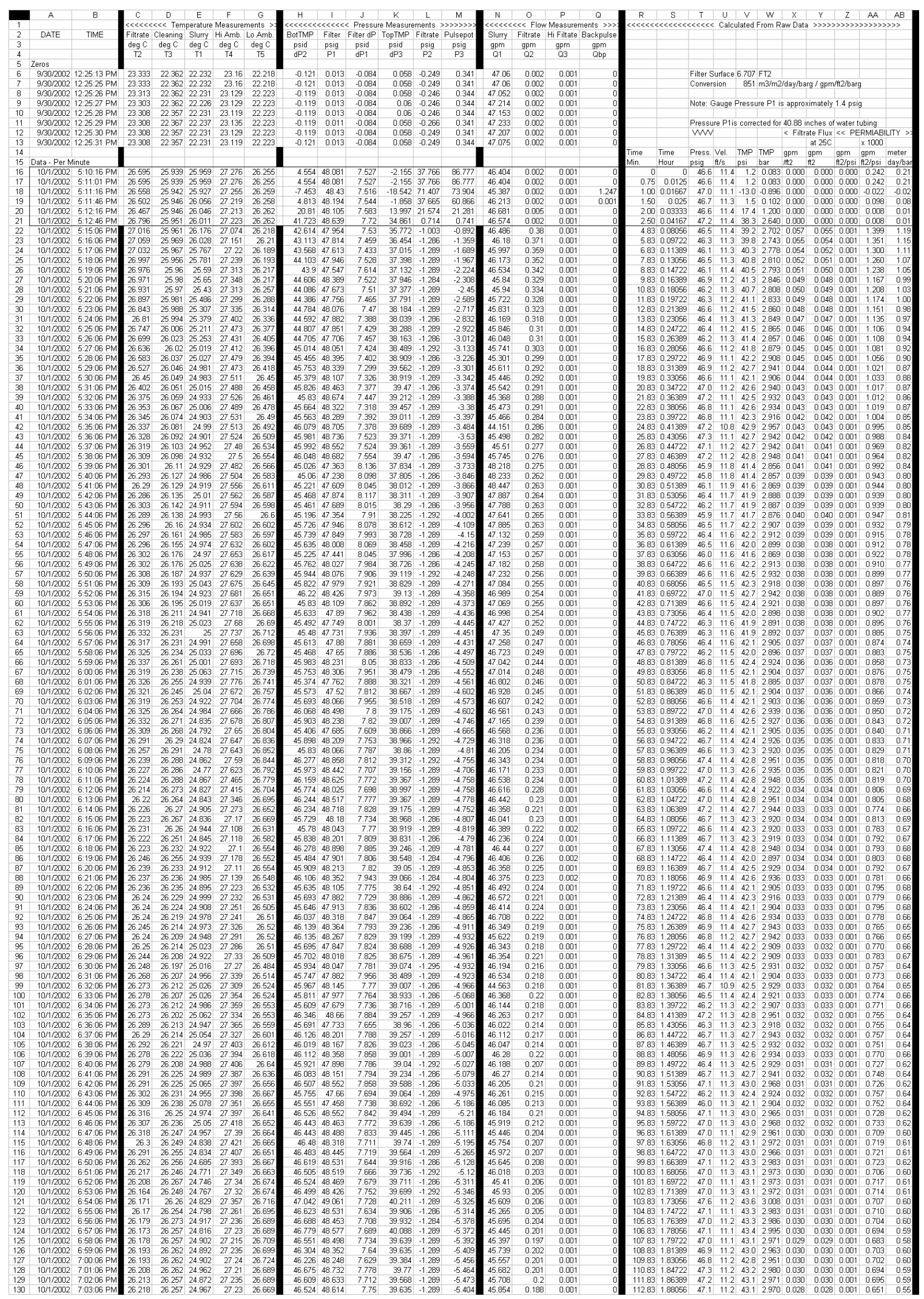

Figure D2-1: Dewatering of the AN-102R2, batch 3C, insoluble solids concentrations from 1 to 8.5 wt $\%$ Raw \& Calculated Data 
WSRC-TR-2003-00204, REV. 0 SRT-RPP-2003-00087, REV. 0
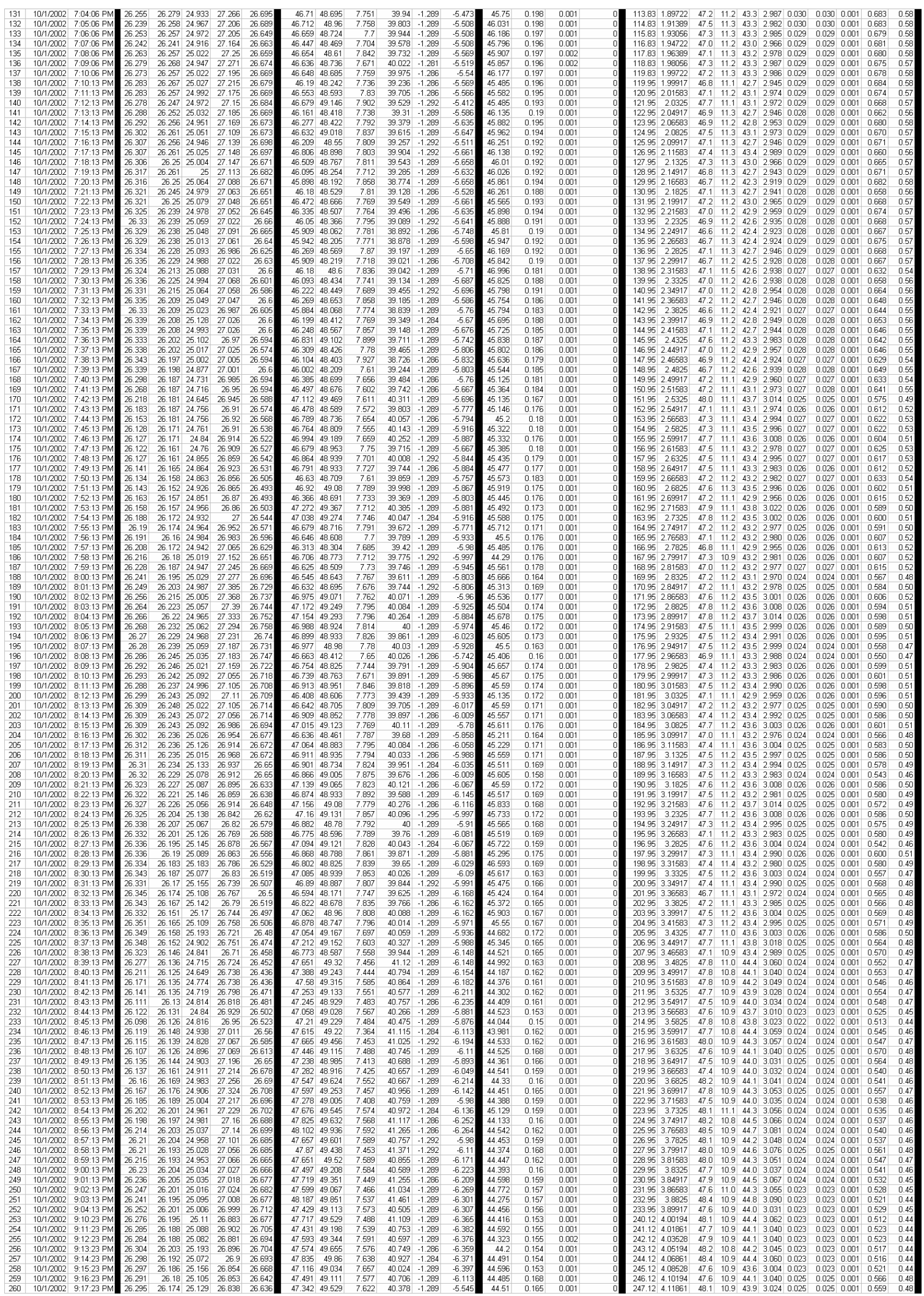

Figure D2-2: Dewatering of the AN-102R2, batch 3C, insoluble solids concentrations from 1 to 8.5 wt\% Raw \& Calculated Data 
WSRC-TR-2003-00204, REV. 0 SRT-RPP-2003-00087, REV. 0
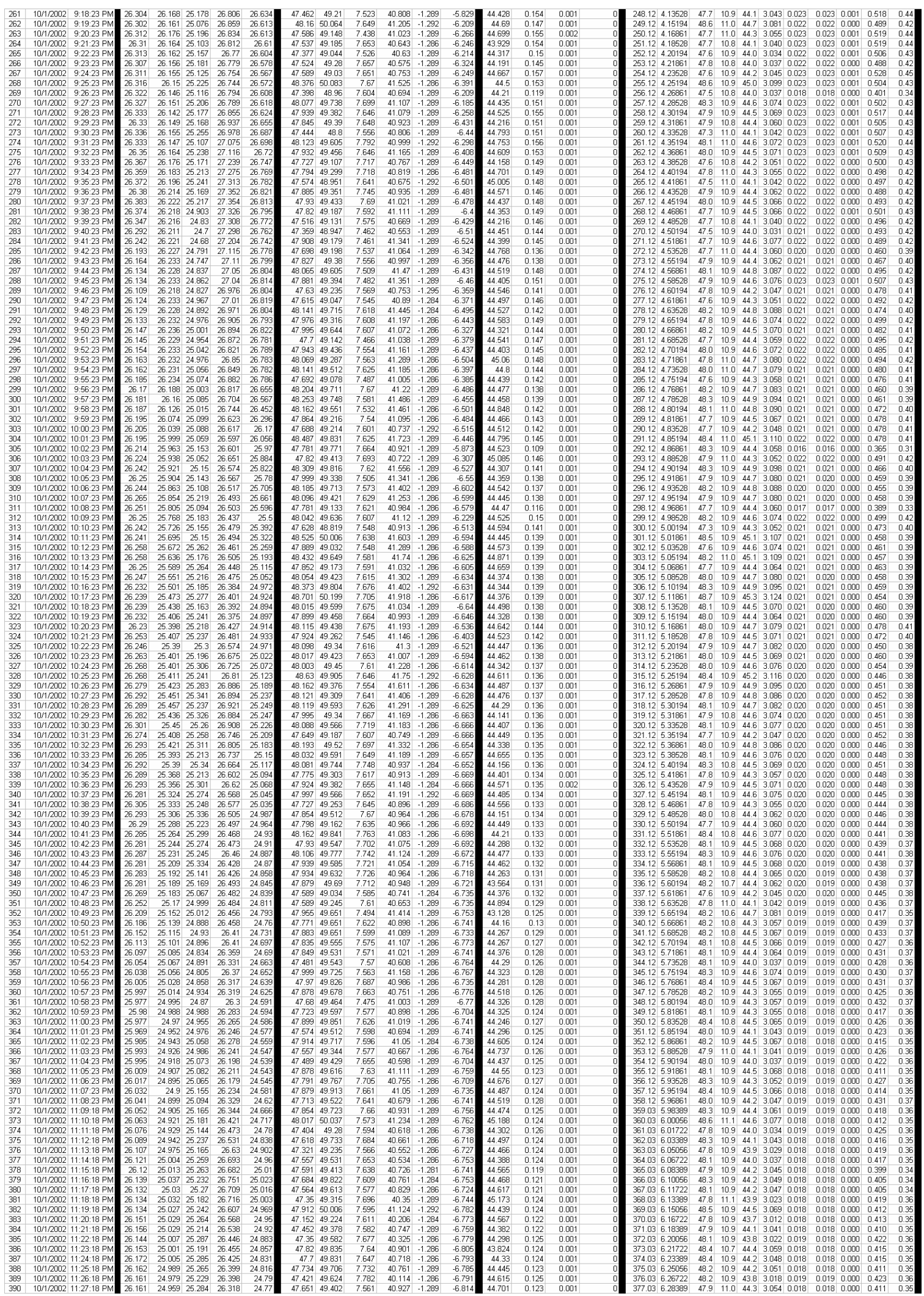

Figure D2-3: Dewatering of the AN-102R2, batch 3C, insoluble solids concentrations from 1 to 8.5 wt $\%$ Raw \& Calculated Data 
WSRC-TR-2003-00204, REV. 0 SRT-RPP-2003-00087, REV. 0
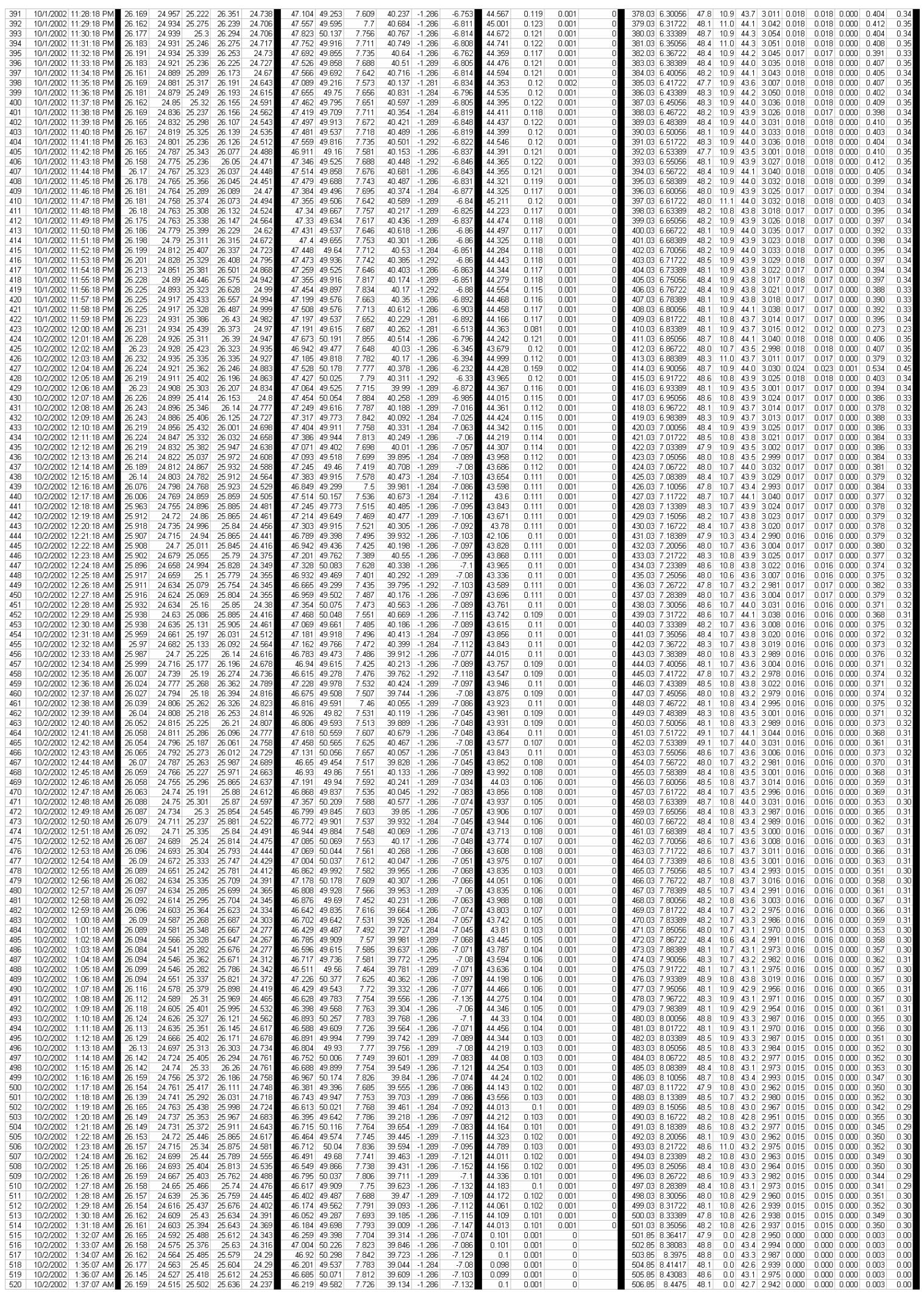

Figure D2-4: Dewatering of the AN-102R2, batch 3C, insoluble solids concentrations from 1 to 8.5 wt\% Raw \& Calculated Data 
WSRC-TR-2003-00204, REV. 0 SRT-RPP-2003-00087, REV. 0

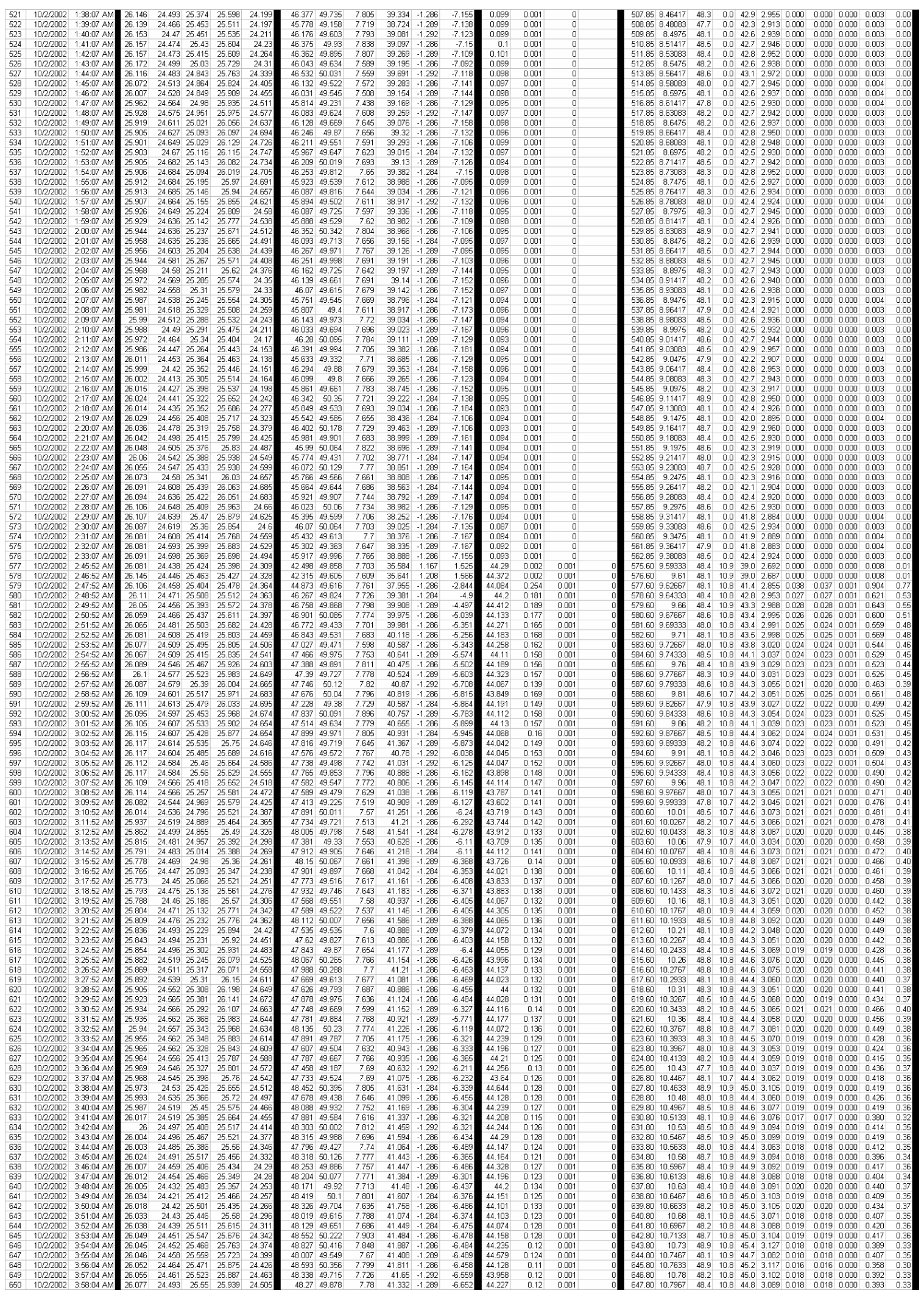

Figure D2-5: Dewatering of the AN-102R2, batch 3C, insoluble solids concentrations from 1 to 8.5 wt\% Raw \& Calculated Data 
WSRC-TR-2003-00204, REV. 0 SRT-RPP-2003-00087, REV. 0
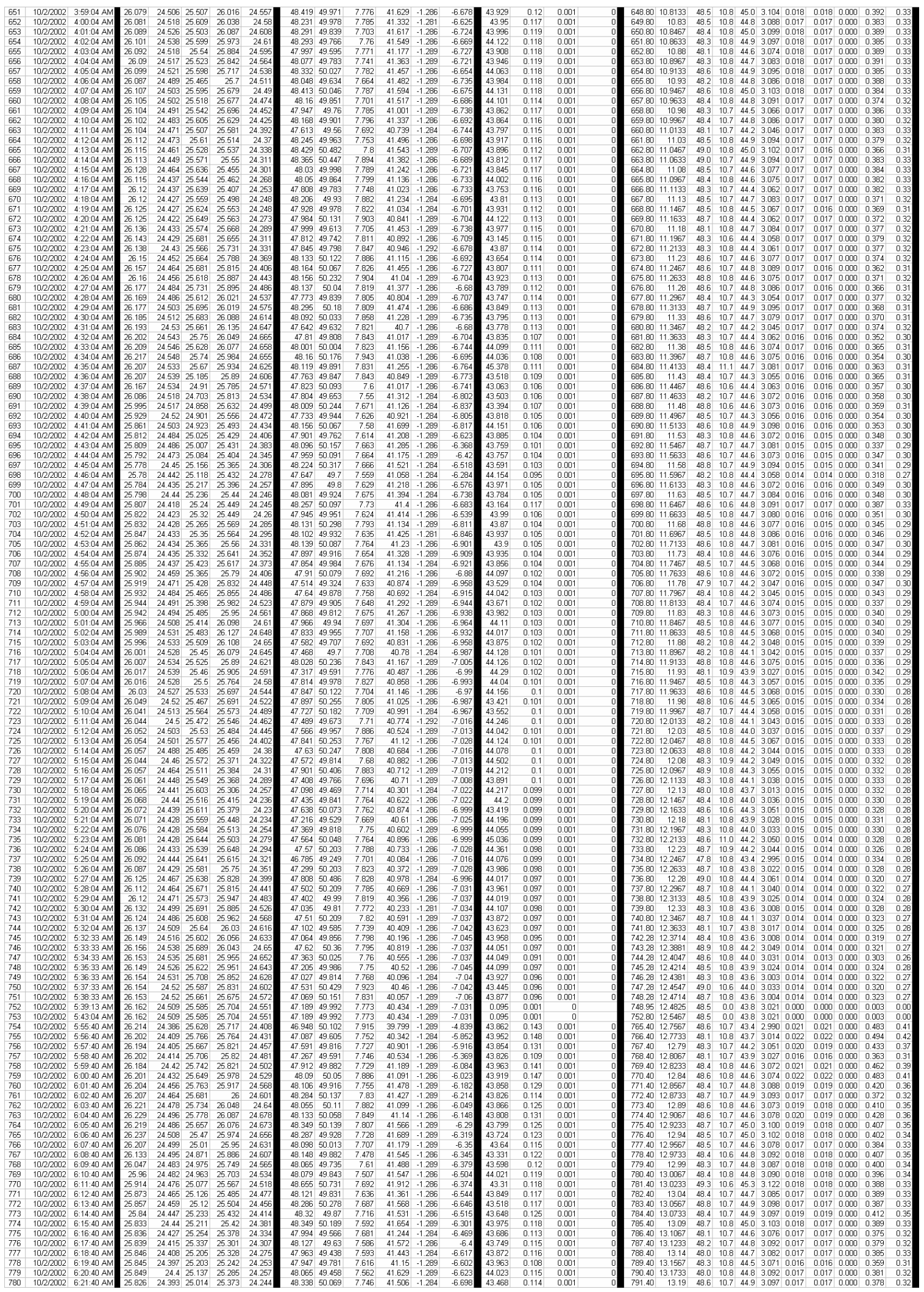

Figure D2-6: Dewatering of the AN-102R2, batch 3C, insoluble solids concentrations from 1 to 8.5 wt\% Raw \& Calculated Data 
WSRC-TR-2003-00204, REV. 0 SRT-RPP-2003-00087, REV. 0
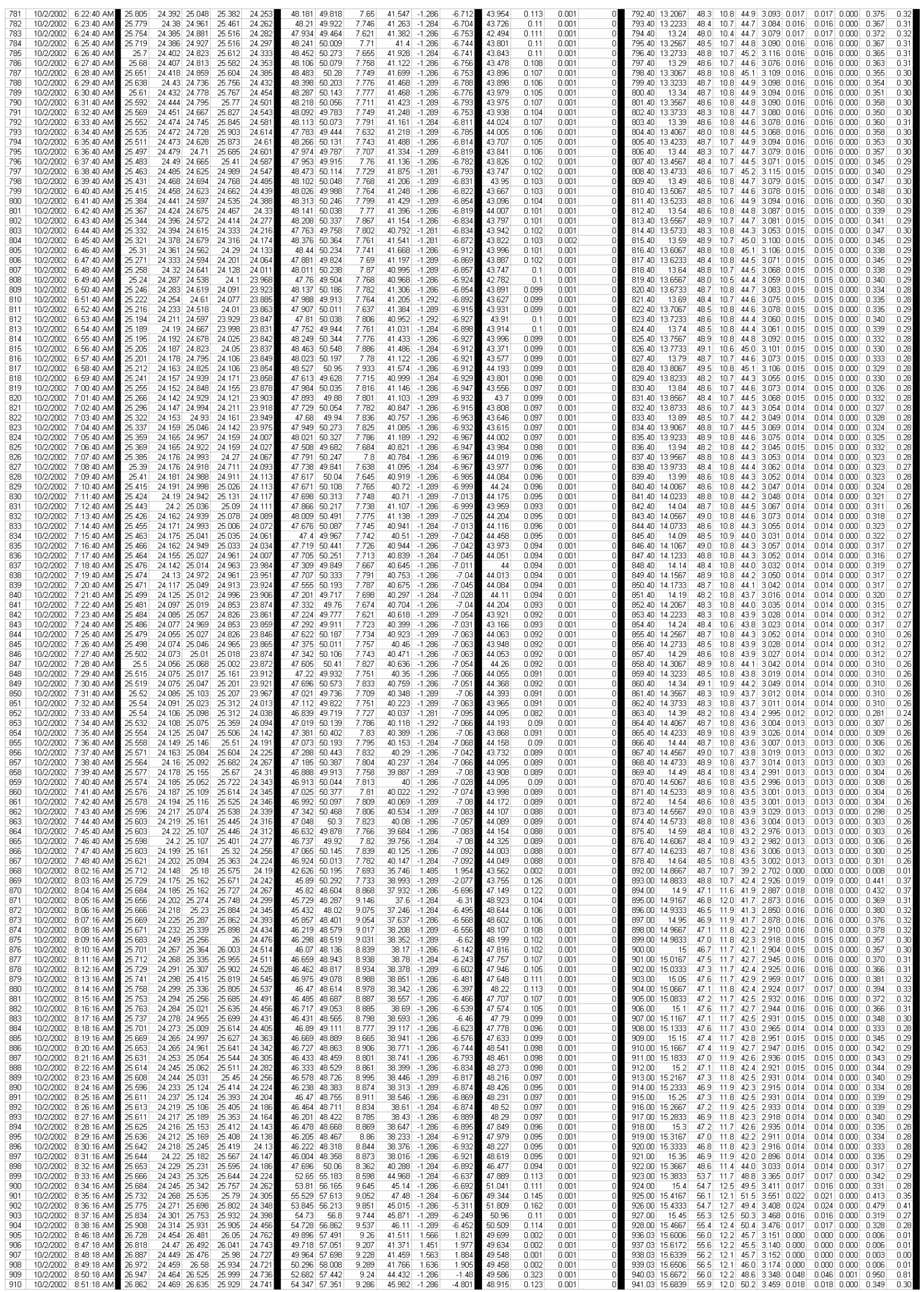

Figure D2-7: Dewatering of the AN-102R2, batch 3C, insoluble solids concentrations from 1 to 8.5 wt\% Raw \& Calculated Data 
WSRC-TR-2003-00204, REV. 0 SRT-RPP-2003-00087, REV. 0
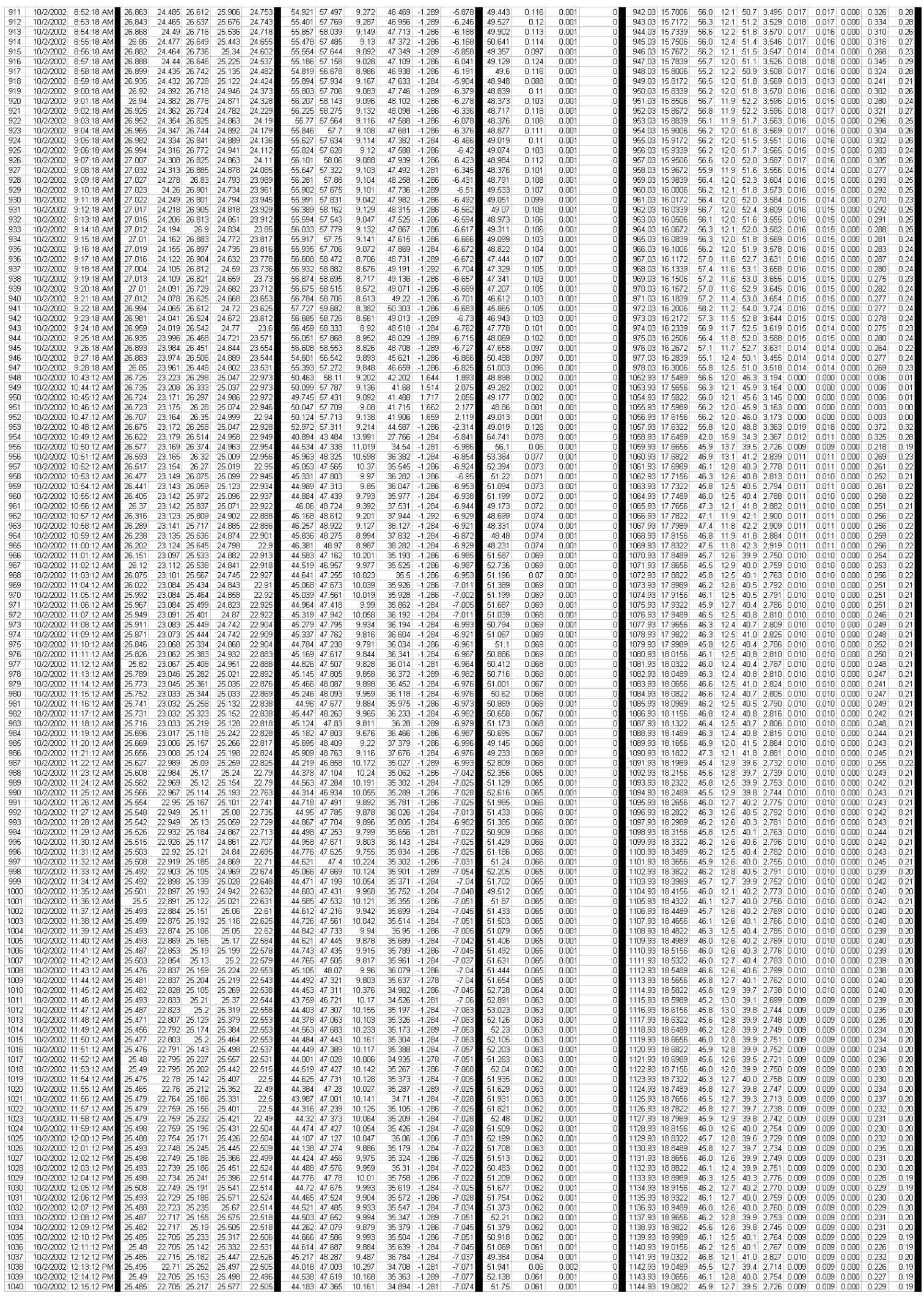

Figure D2-8: Dewatering of the AN-102R2, batch 3C, insoluble solids concentrations from 1 to 8.5 wt\% Raw \& Calculated Data 
WSRC-TR-2003-00204, REV. 0 SRT-RPP-2003-00087, REV. 0

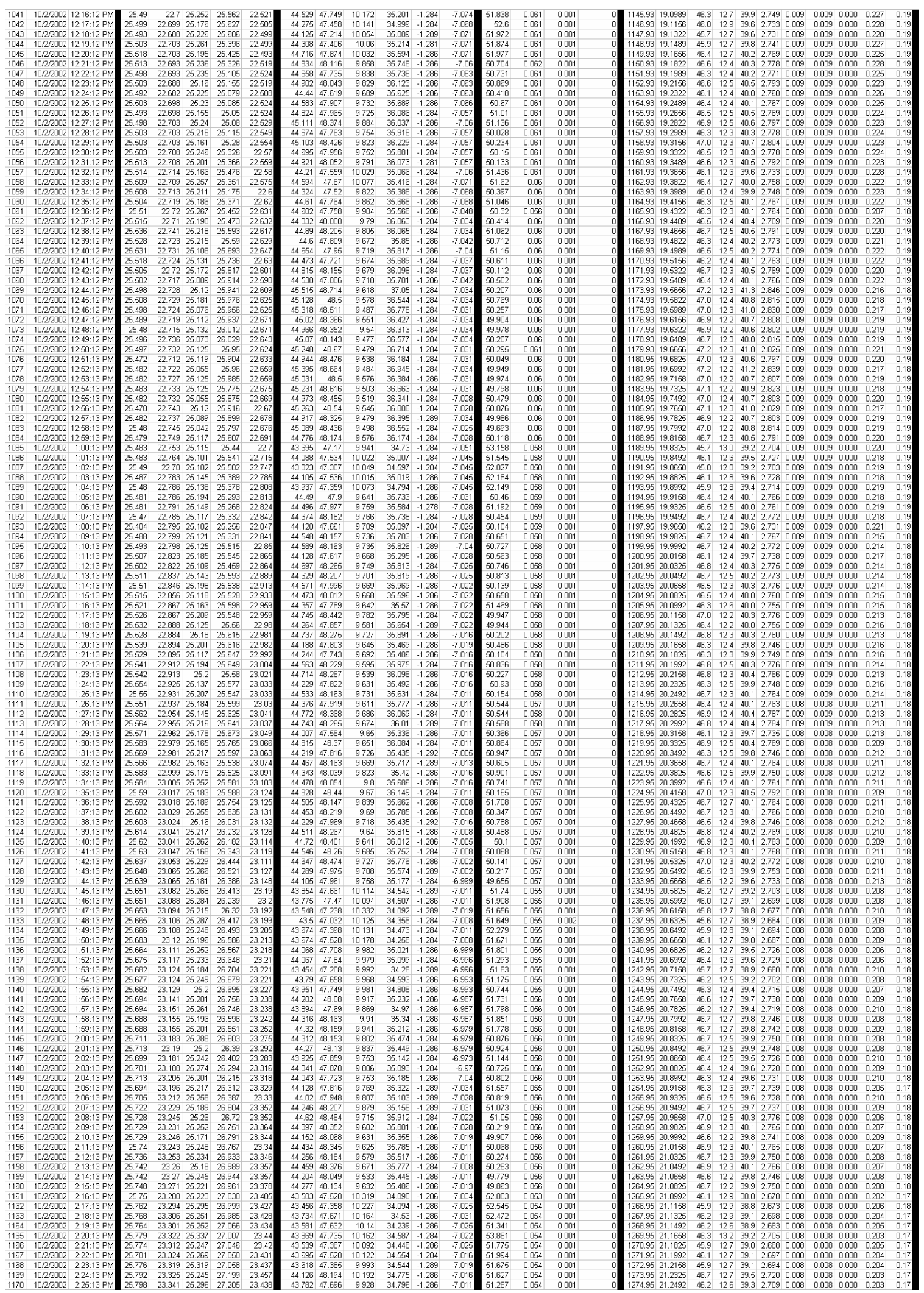

Figure D2-9: Dewatering of the AN-102R2, batch 3C, insoluble solids concentrations from 1 to 8.5 wt\% Raw \& Calculated Data 
WSRC-TR-2003-00204, REV. 0 SRT-RPP-2003-00087, REV. 0
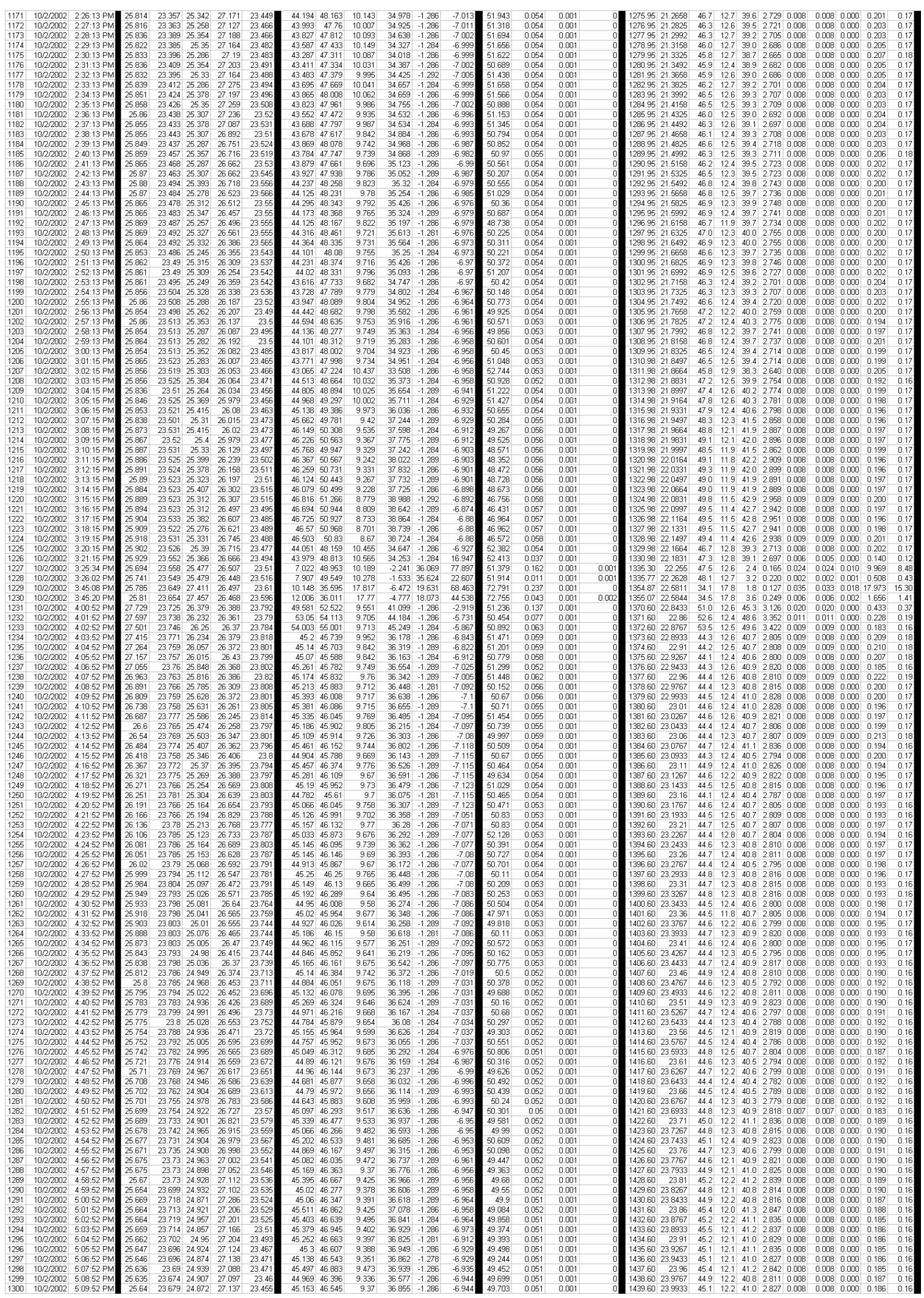

Figure D2-10: Dewatering of the AN-102R2, batch 3C, insoluble solids concentrations from 1 to 8.5 wt $\%$ Raw \& Calculated Data 
WSRC-TR-2003-00204, REV. 0 SRT-RPP-2003-00087, REV. 0
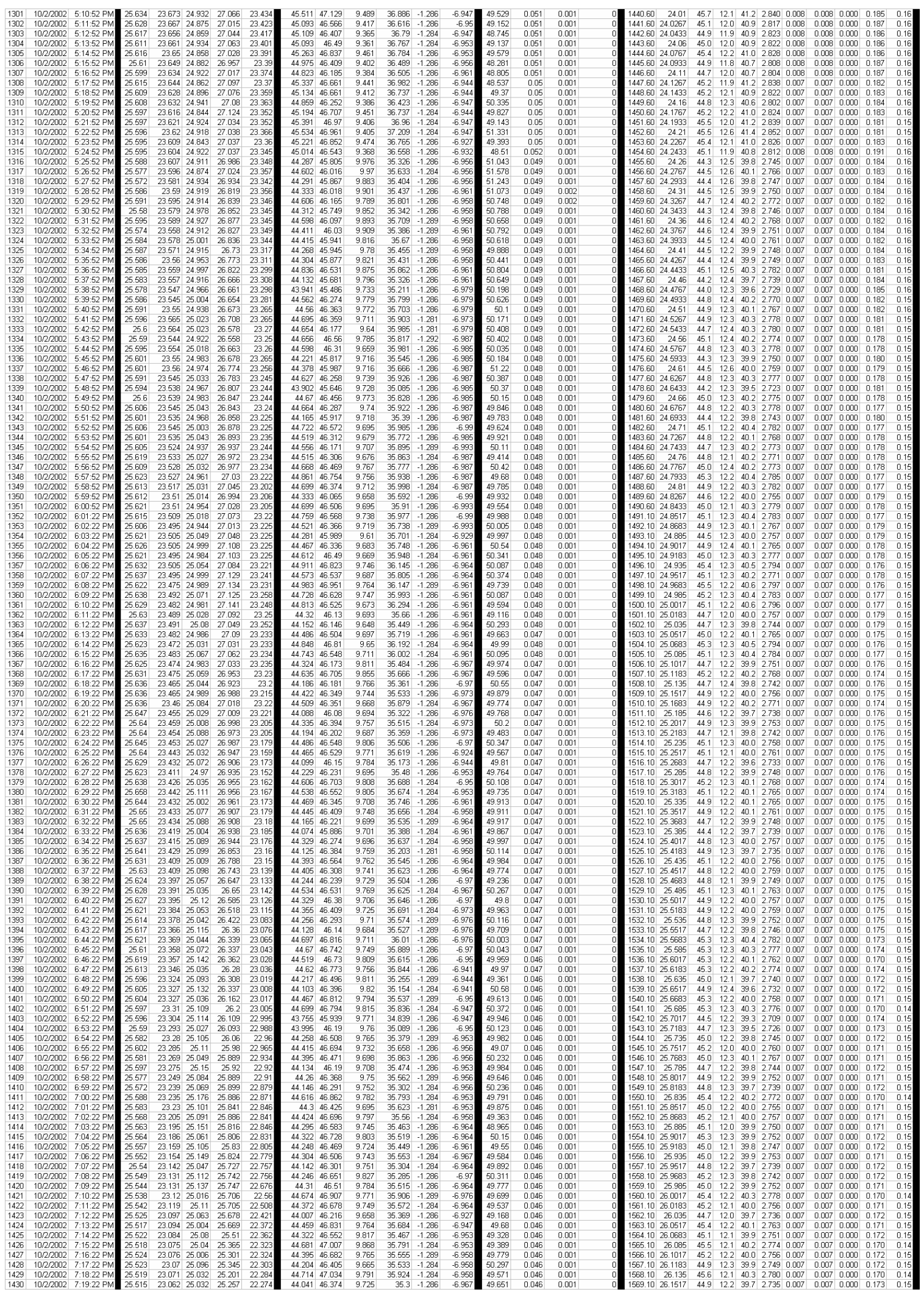

Figure D2-11: Dewatering of the AN-102R2, batch 3C, insoluble solids concentrations from 1 to 8.5 wt $\%$ Raw \& Calculated Data 
WSRC-TR-2003-00204, REV. 0 SRT-RPP-2003-00087, REV. 0

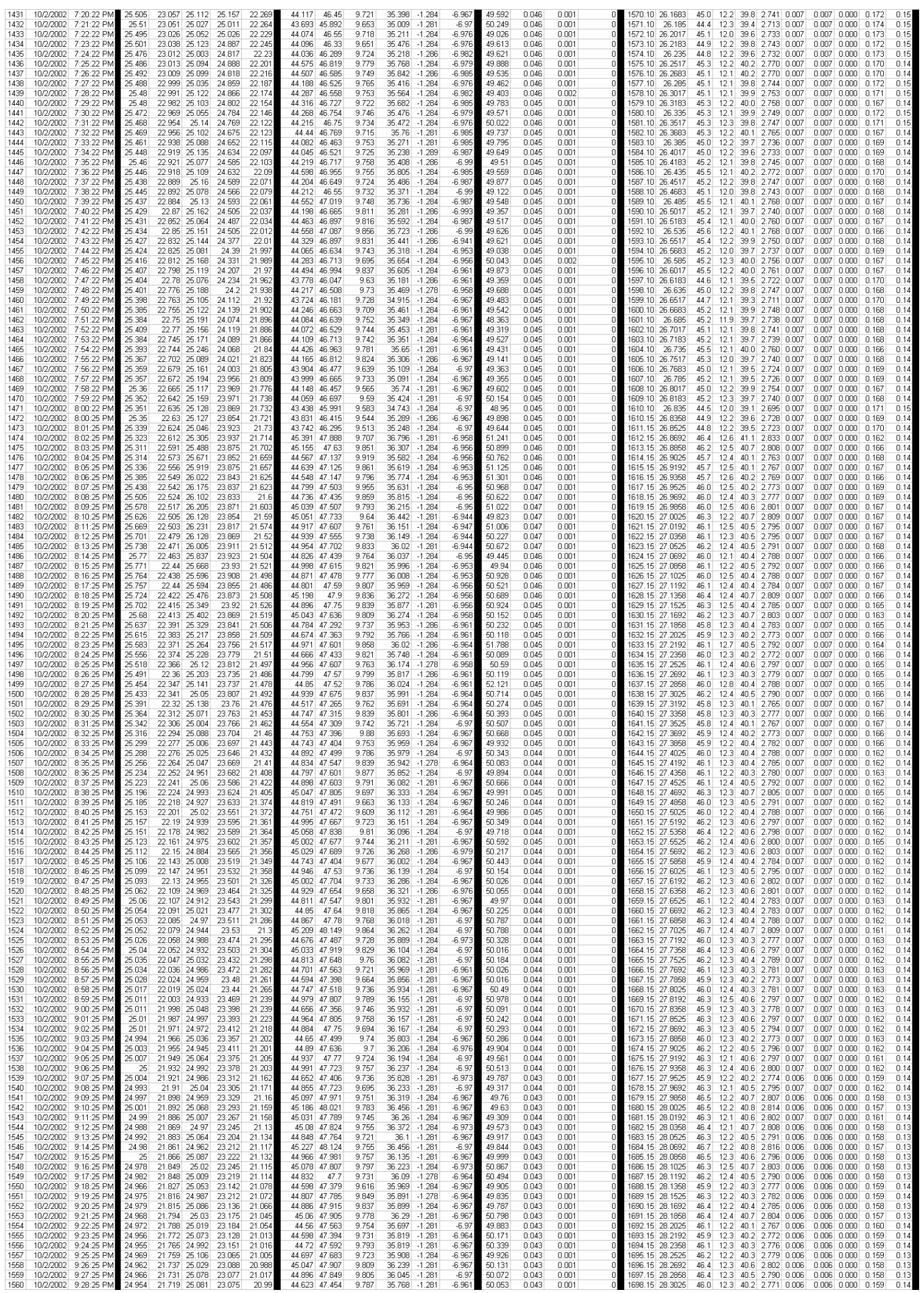

Figure D2-12: Dewatering of the AN-102R2, batch 3C, insoluble solids concentrations from 1 to 8.5 wt $\%$ Raw \& Calculated Data 
WSRC-TR-2003-00204, REV. 0 SRT-RPP-2003-00087, REV. 0
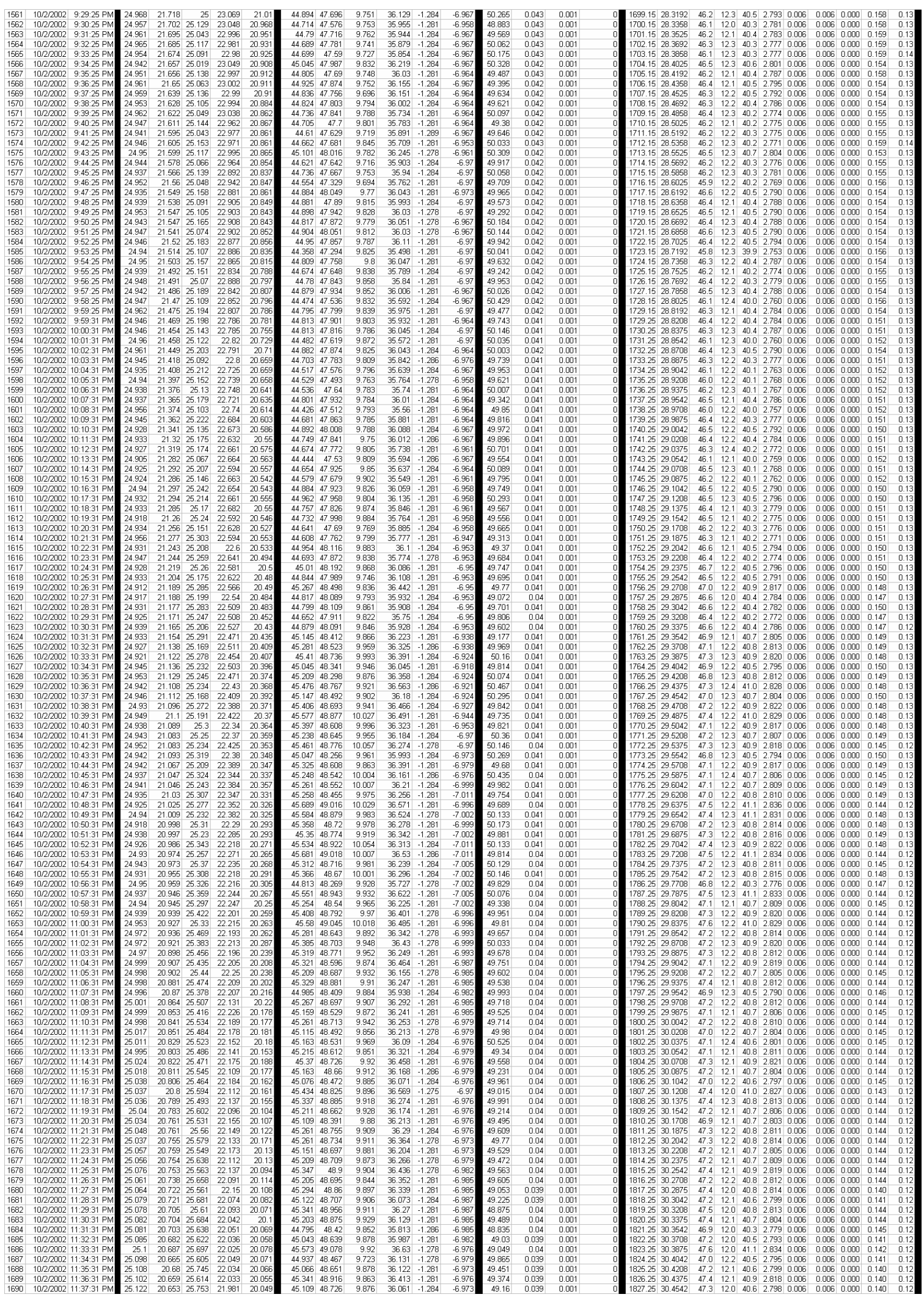

Figure D2-13: Dewatering of the AN-102R2, batch 3C, insoluble solids concentrations from 1 to 8.5 wt $\%$ -

Raw \& Calculated Data 
WSRC-TR-2003-00204, REV. 0 SRT-RPP-2003-00087, REV. 0
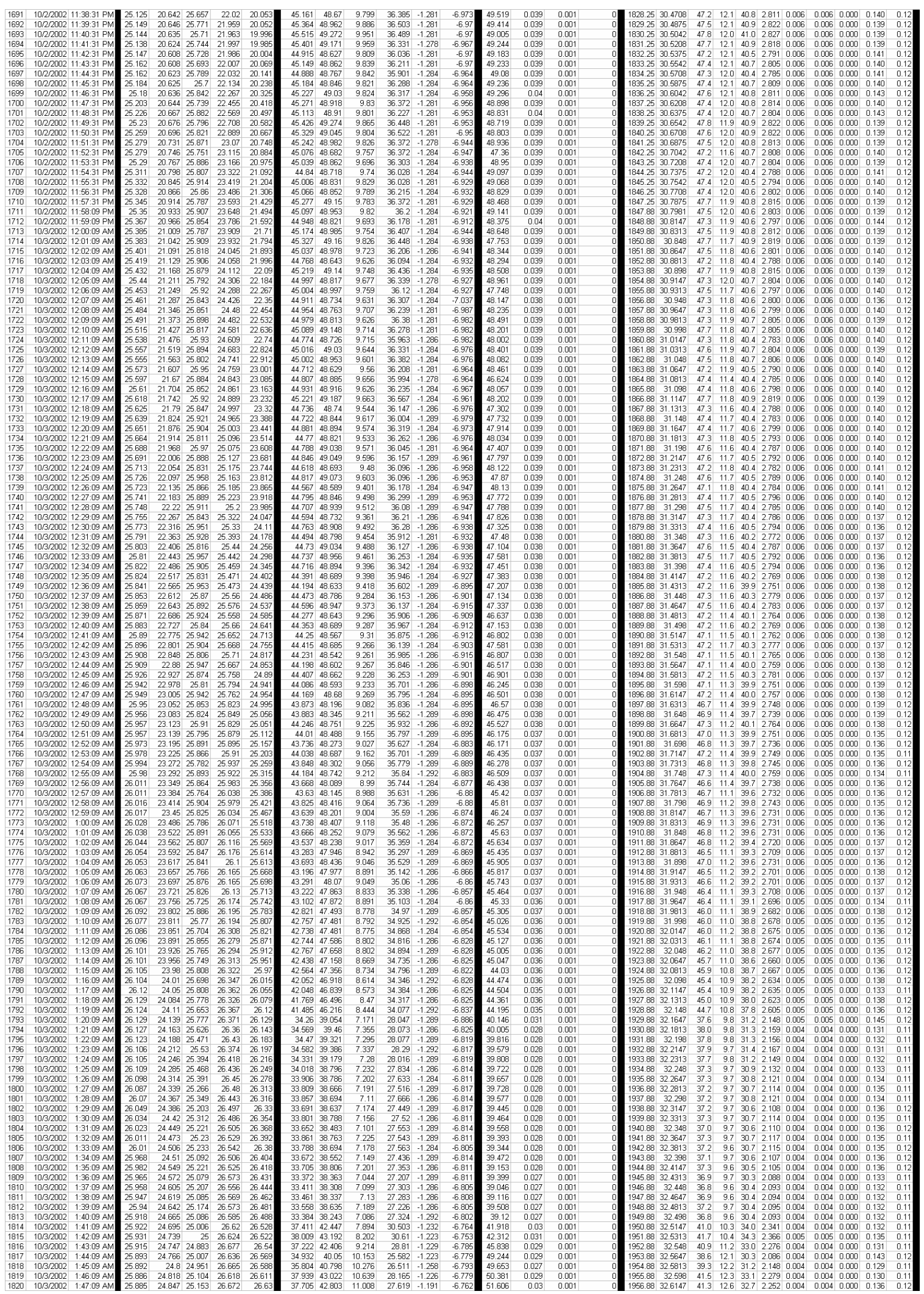

Figure D2-14: Dewatering of the AN-102R2, batch 3C, insoluble solids concentrations from 1 to 8.5 wt $\%$ Raw \& Calculated Data 
WSRC-TR-2003-00204, REV. 0 SRT-RPP-2003-00087, REV. 0

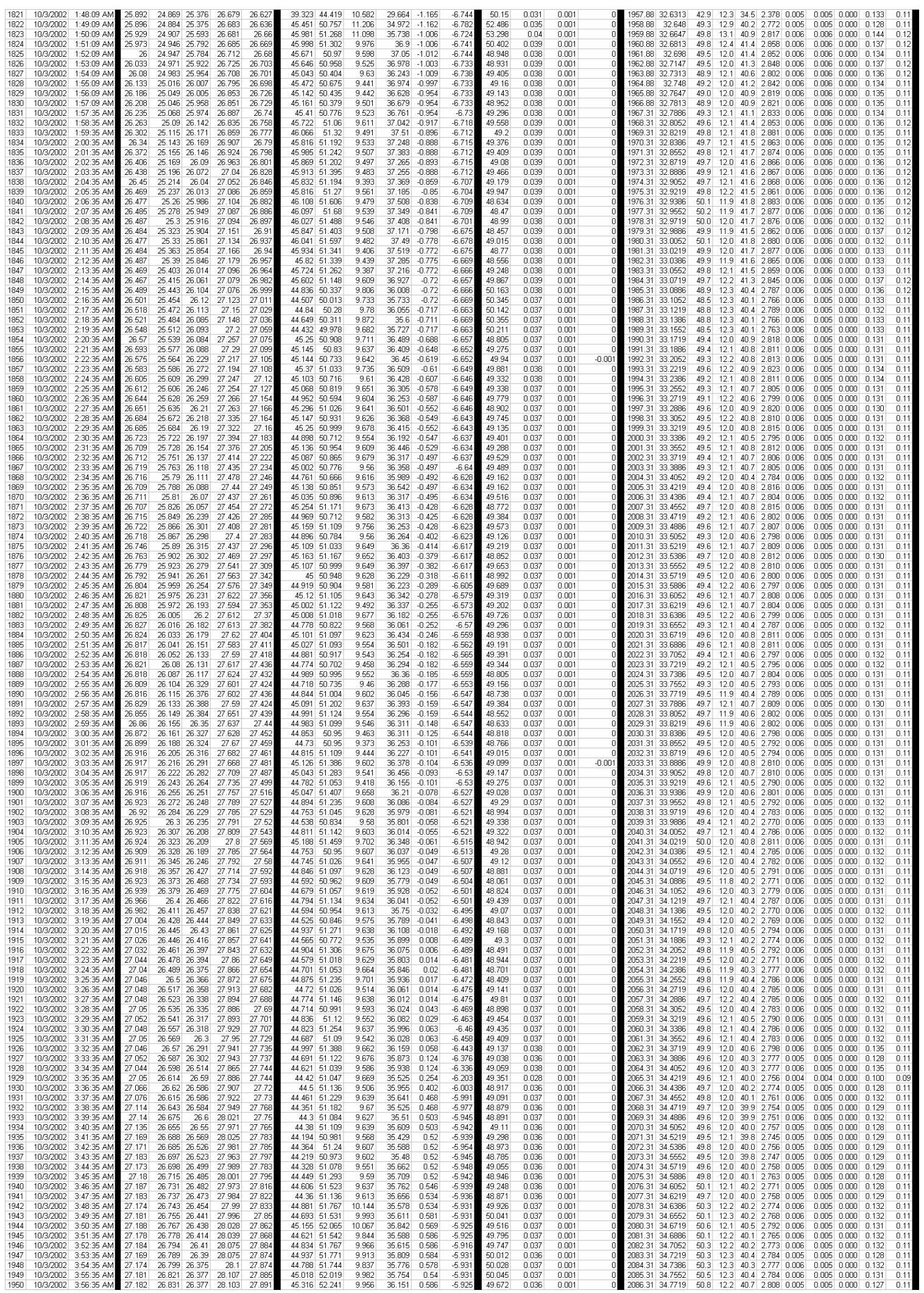

Figure D2-15: Dewatering of the AN-102R2, batch 3C, insoluble solids concentrations from 1 to 8.5 wt $\%$ Raw \& Calculated Data 
WSRC-TR-2003-00204, REV. 0 SRT-RPP-2003-00087, REV. 0
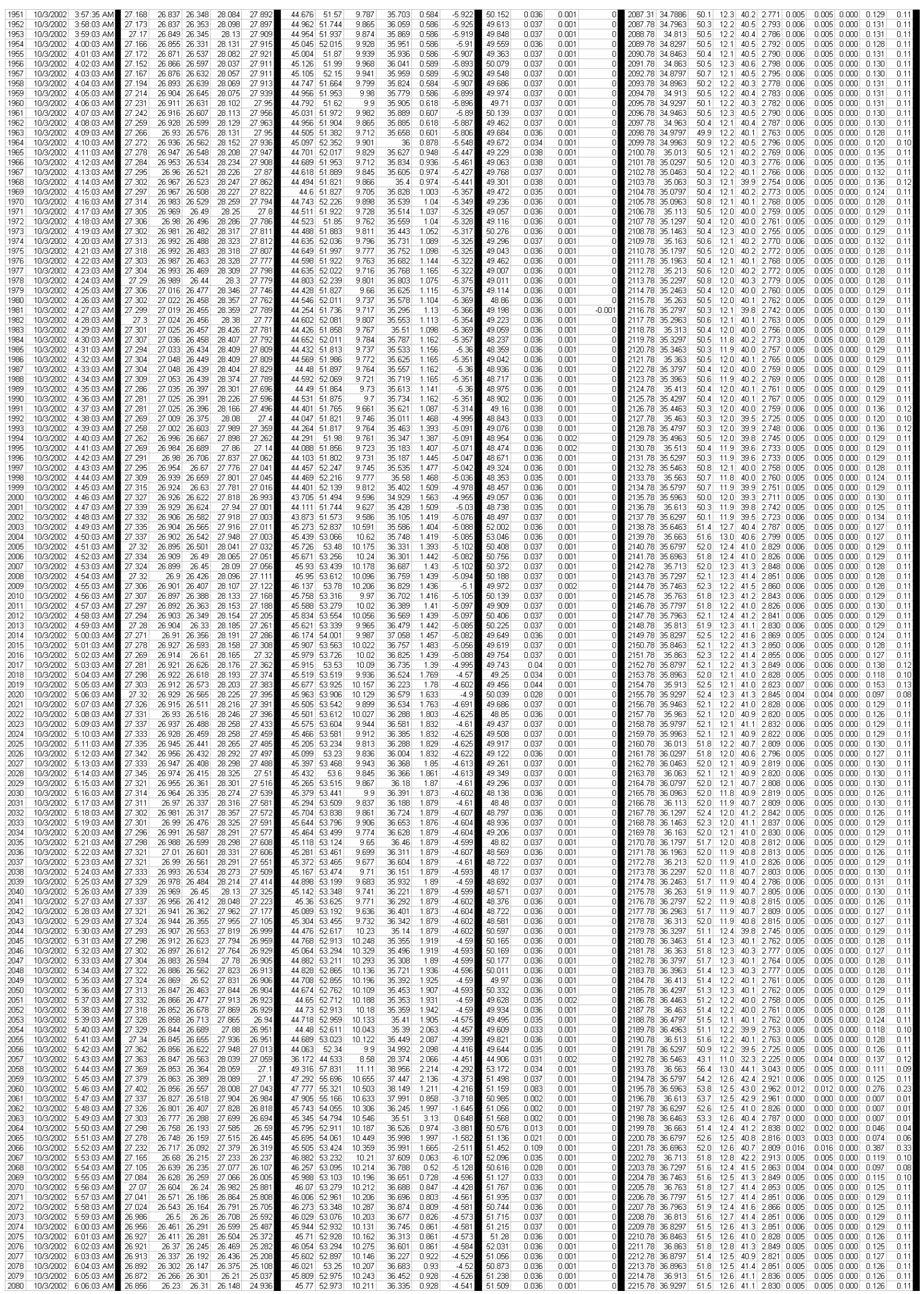

Figure D2-16: Dewatering of the AN-102R2, batch 3C, insoluble solids concentrations from 1 to 8.5 wt $\%$ Raw \& Calculated Data 
WSRC-TR-2003-00204, REV. 0 SRT-RPP-2003-00087, REV. 0

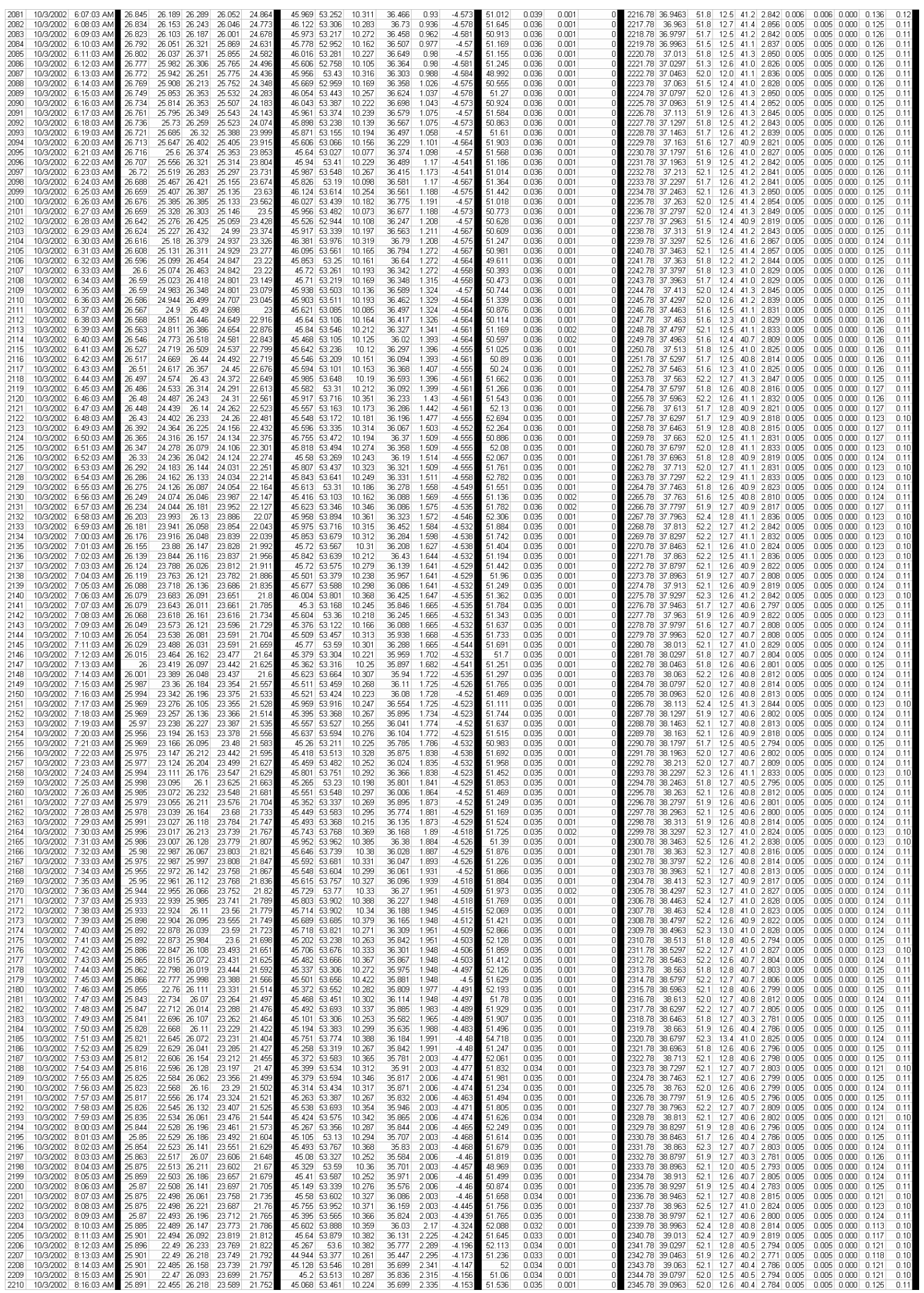

Figure D2-17: Dewatering of the AN-102R2, batch 3C, insoluble solids concentrations from 1 to $8.5 \mathrm{wt} \%$ Raw \& Calculated Data 
WSRC-TR-2003-00204, REV. 0 SRT-RPP-2003-00087, REV. 0
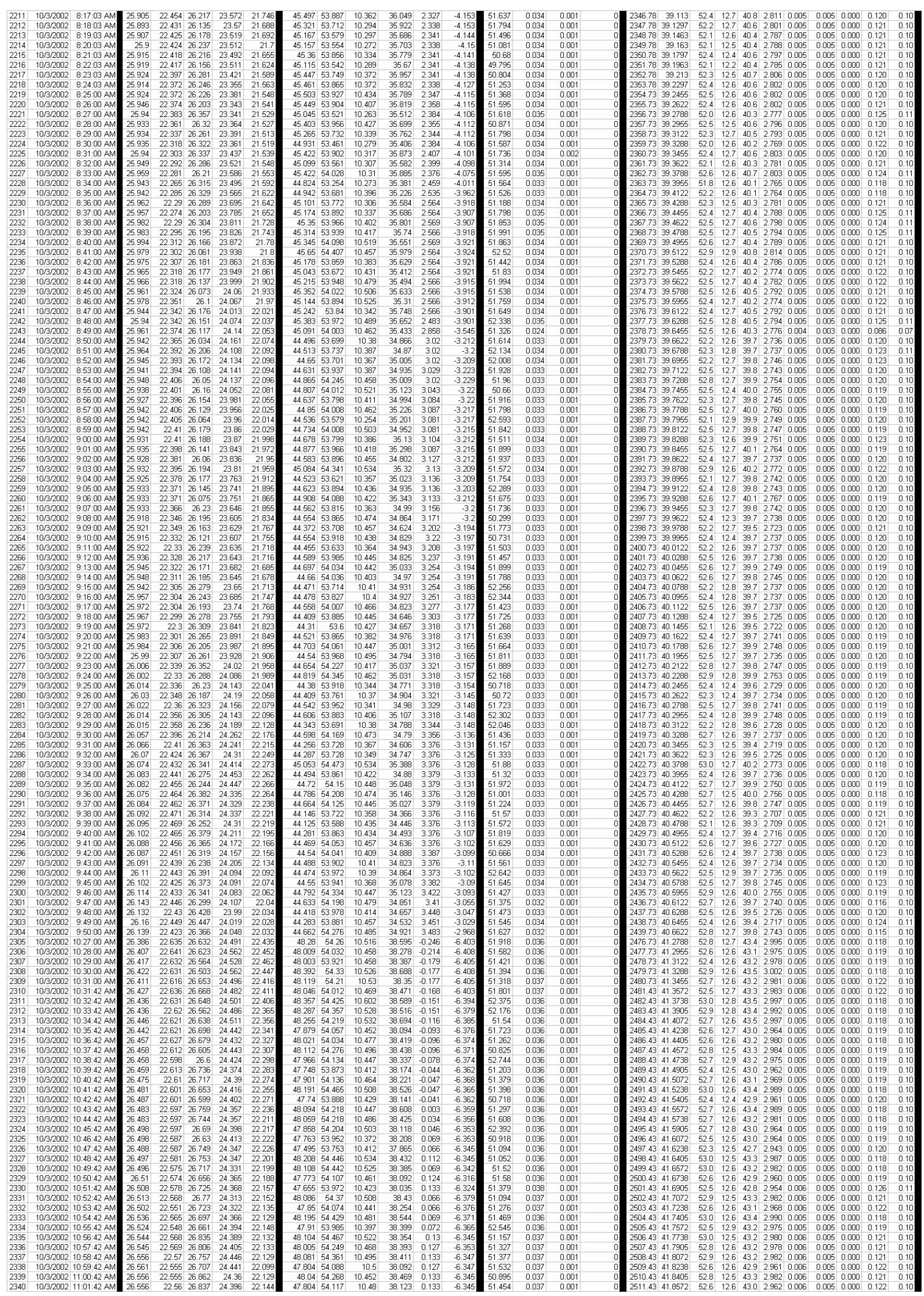

Figure D2-18: Dewatering of the AN-102R2, batch 3C, insoluble solids concentrations from 1 to 8.5 wt $\%$ Raw \& Calculated Data 
WSRC-TR-2003-00204, REV. 0 SRT-RPP-2003-00087, REV. 0

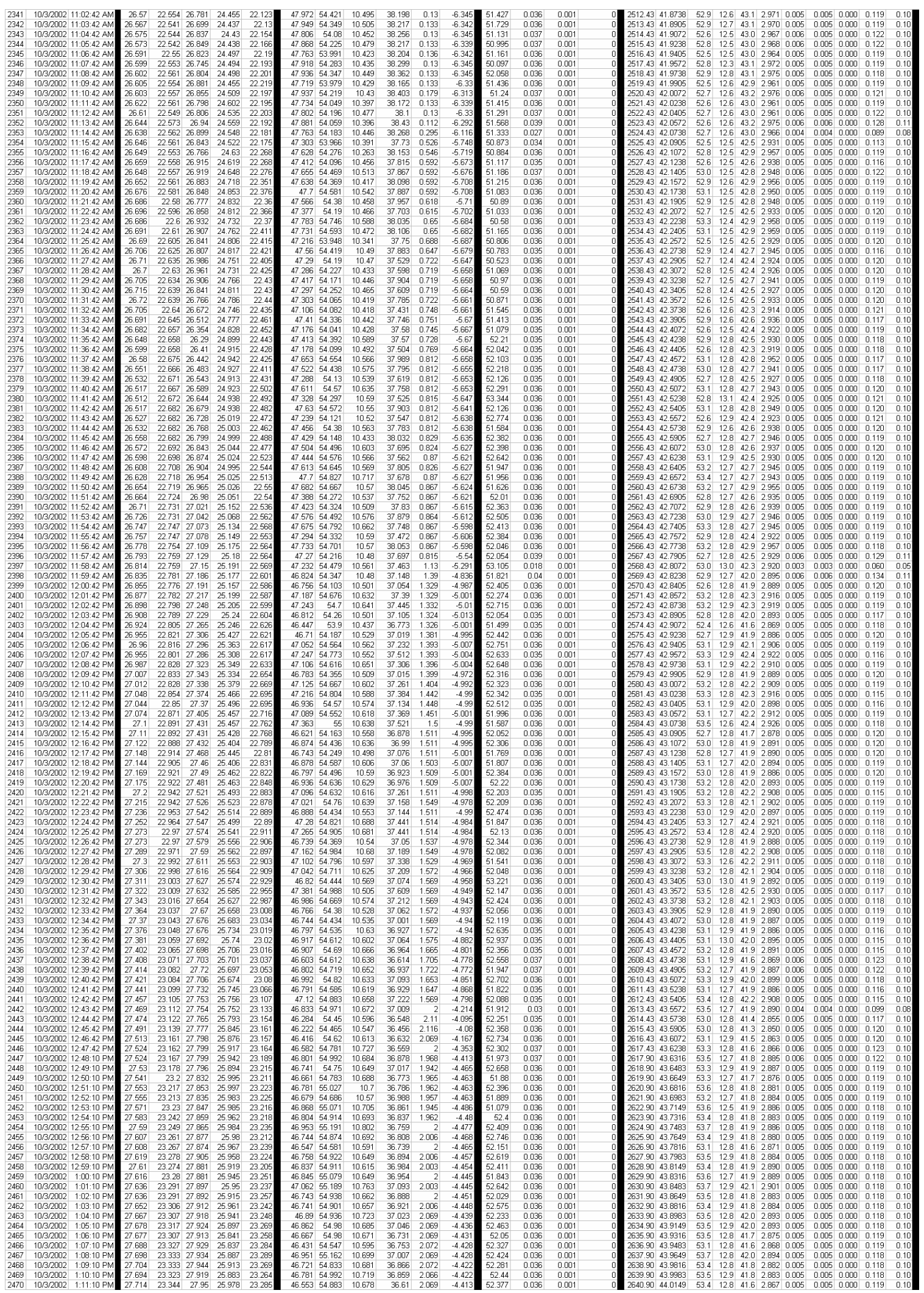

Figure D2-19: Dewatering of the AN-102R2, batch 3C, insoluble solids concentrations from 1 to 8.5 wt $\%$ Raw \& Calculated Data 
WSRC-TR-2003-00204, REV. 0 SRT-RPP-2003-00087, REV. 0

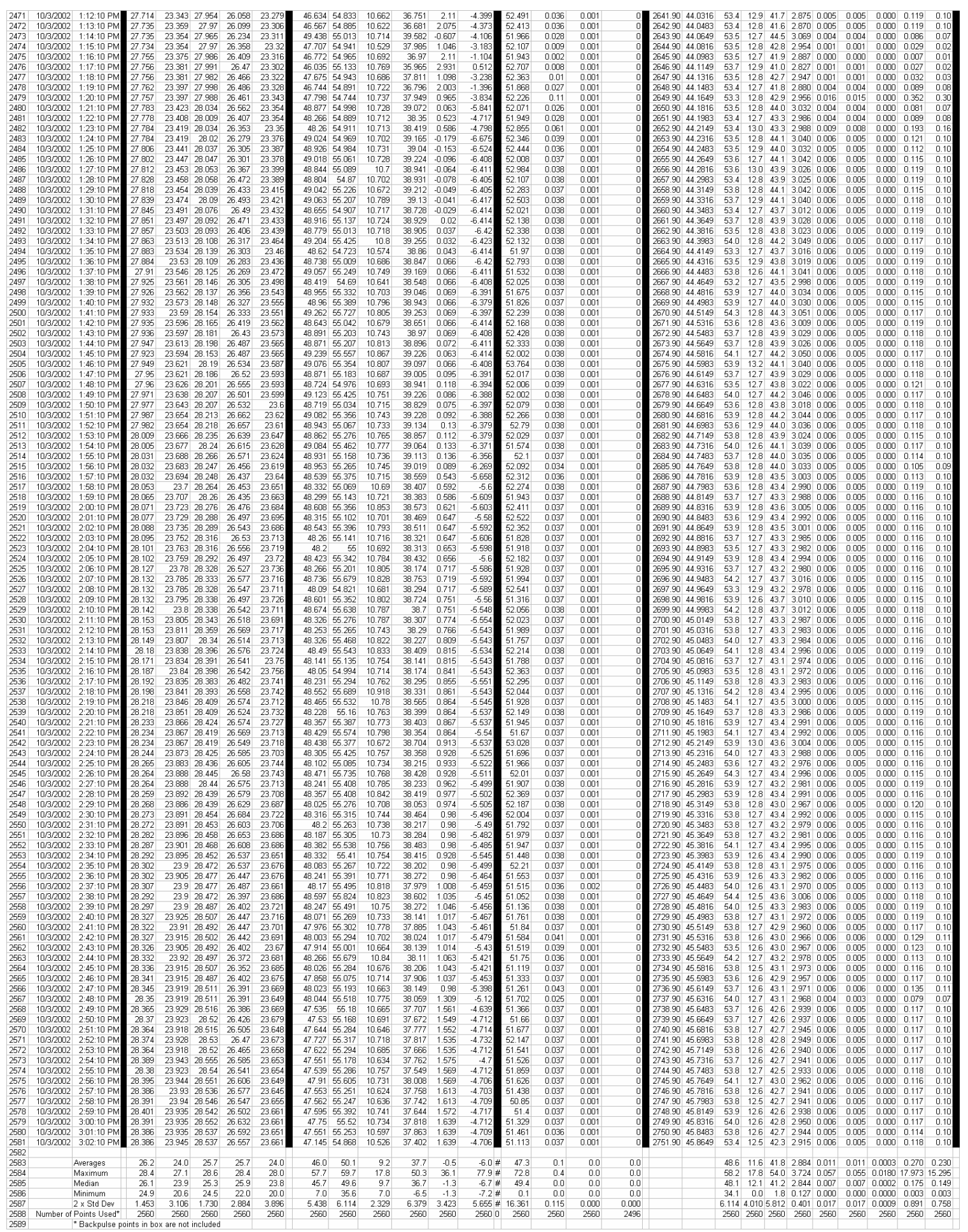

Figure D2-20: Dewatering of the AN-102R2, batch 3C, insoluble solids concentrations from 1 to $8.5 \mathrm{wt} \%$ Raw \& Calculated Data 


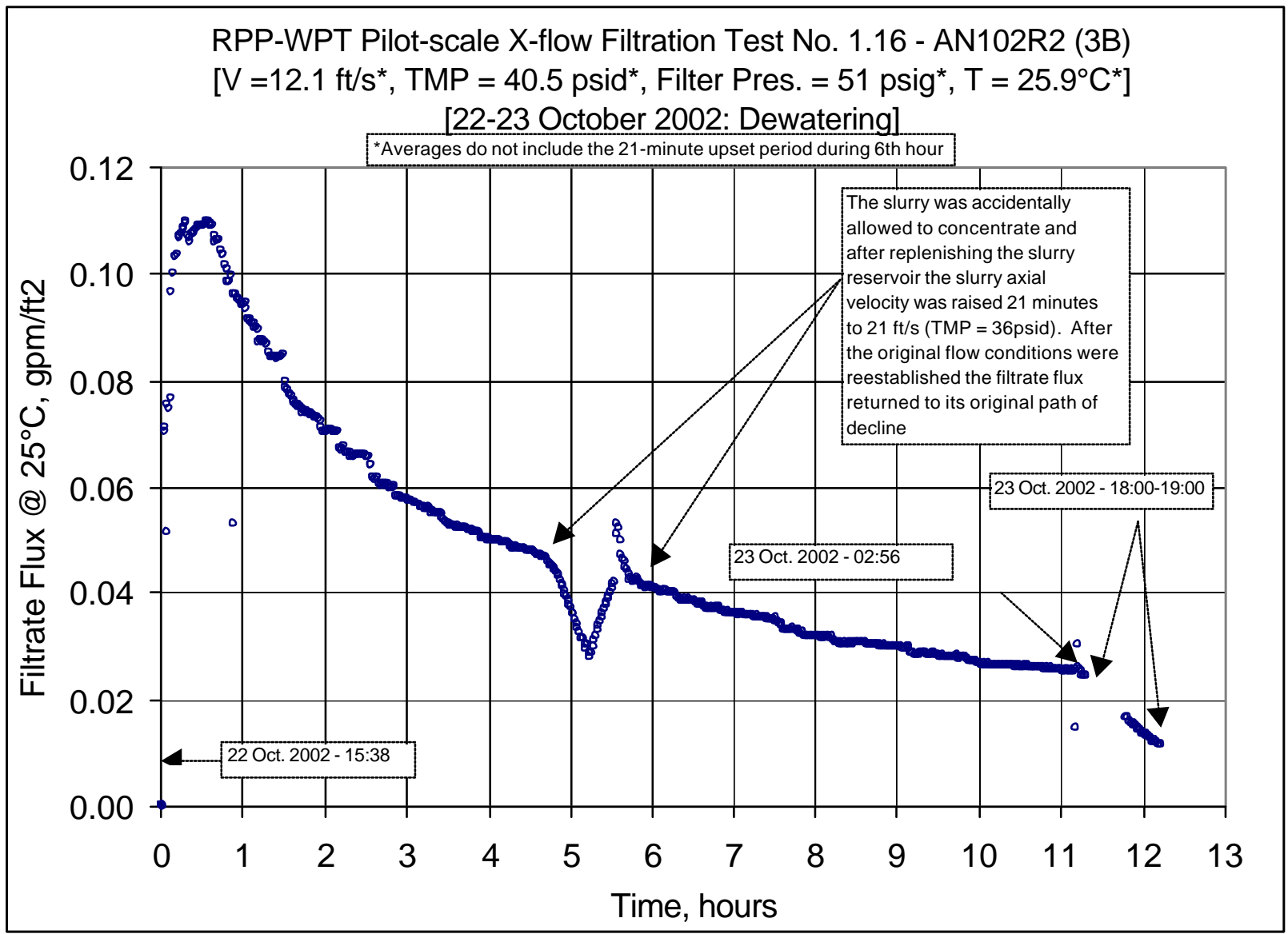

Figure D3: Dewatering of the AN-102R2, batch 3B, insoluble solids concentrations from 1 to $22 \mathrm{wt} \%$

(Data from 6 Files)

(1.163b -rpp-pxu-102202-1539, -1739, -1939, -2139)

(1.163b-rpp-pxu-102302-0139, -1835) 
WSRC-TR-2003-00204, REV. 0

SRT-RPP-2003-00087, REV. 0

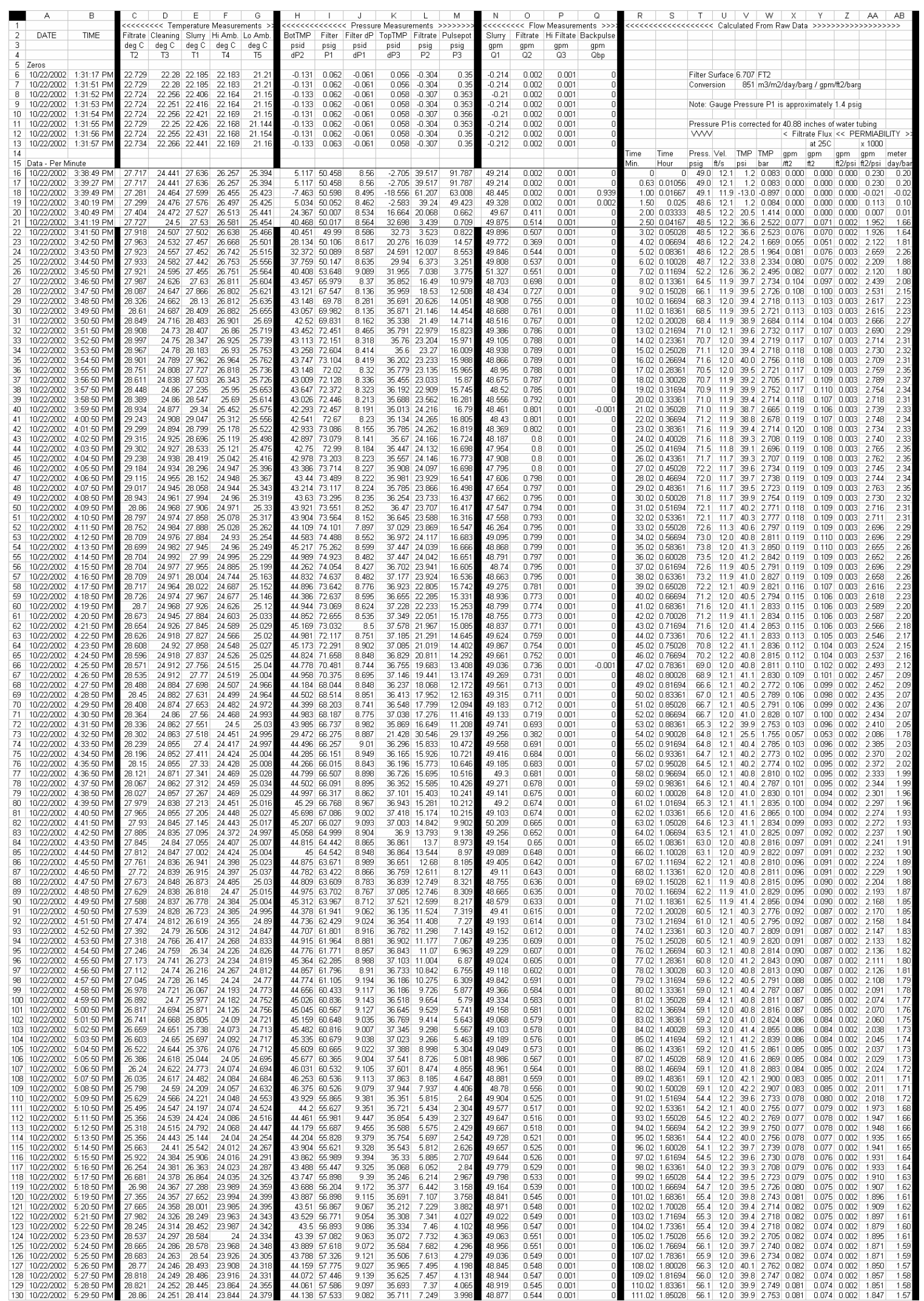

Figure D4-1: Dewatering of the AN-102R2, batch 3B, insoluble solids concentrations from 1 to 22 wt $\%$ Raw \& Calculated Data 
WSRC-TR-2003-00204, REV. 0

SRT-RPP-2003-00087, REV. 0

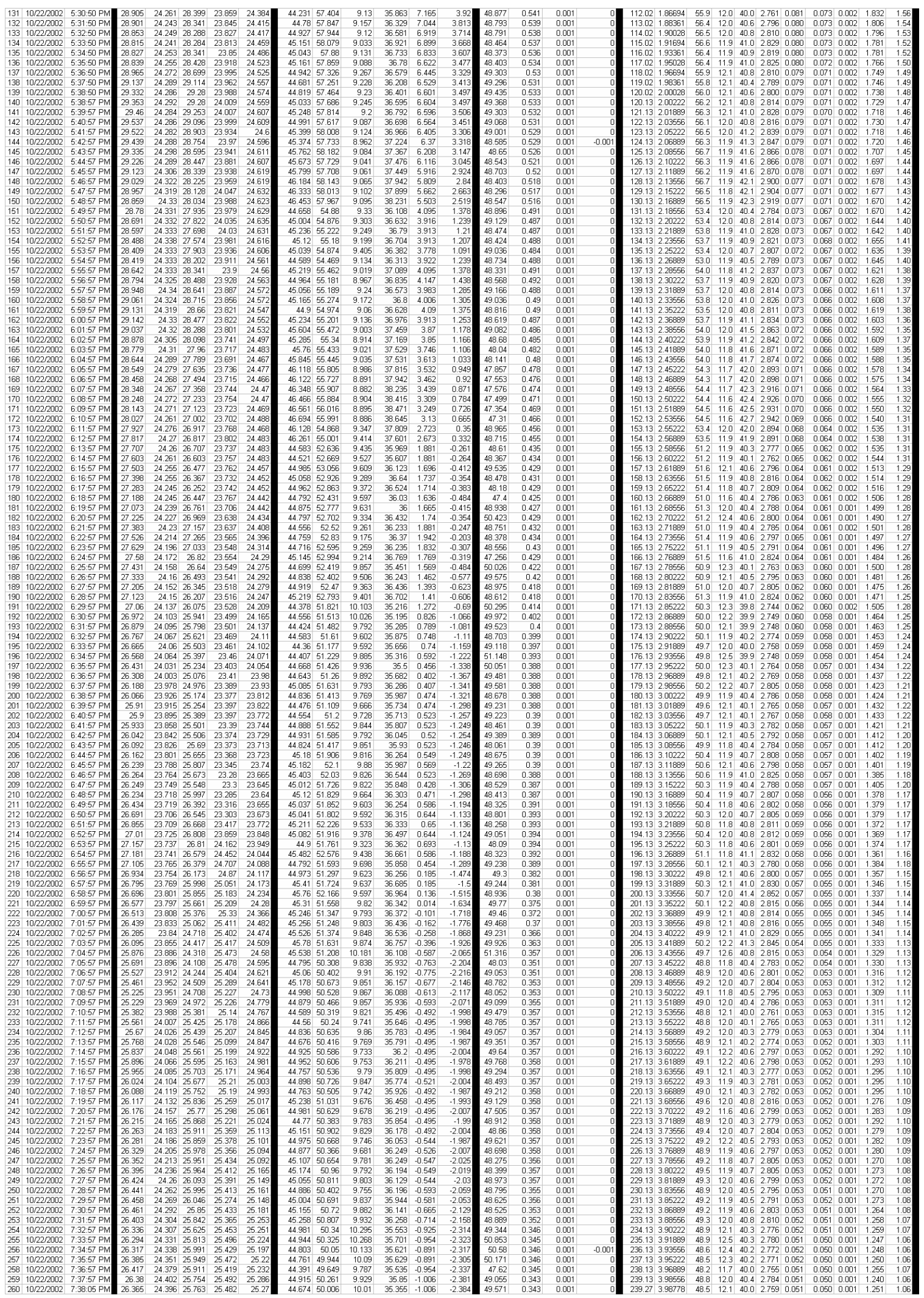

Figure D4-2: Dewatering of the AN-102R2, batch 3B, insoluble solids concentrations from 1 to 22 wt $\%$ Raw \& Calculated Data 
WSRC-TR-2003-00204, REV. 0

SRT-RPP-2003-00087, REV. 0

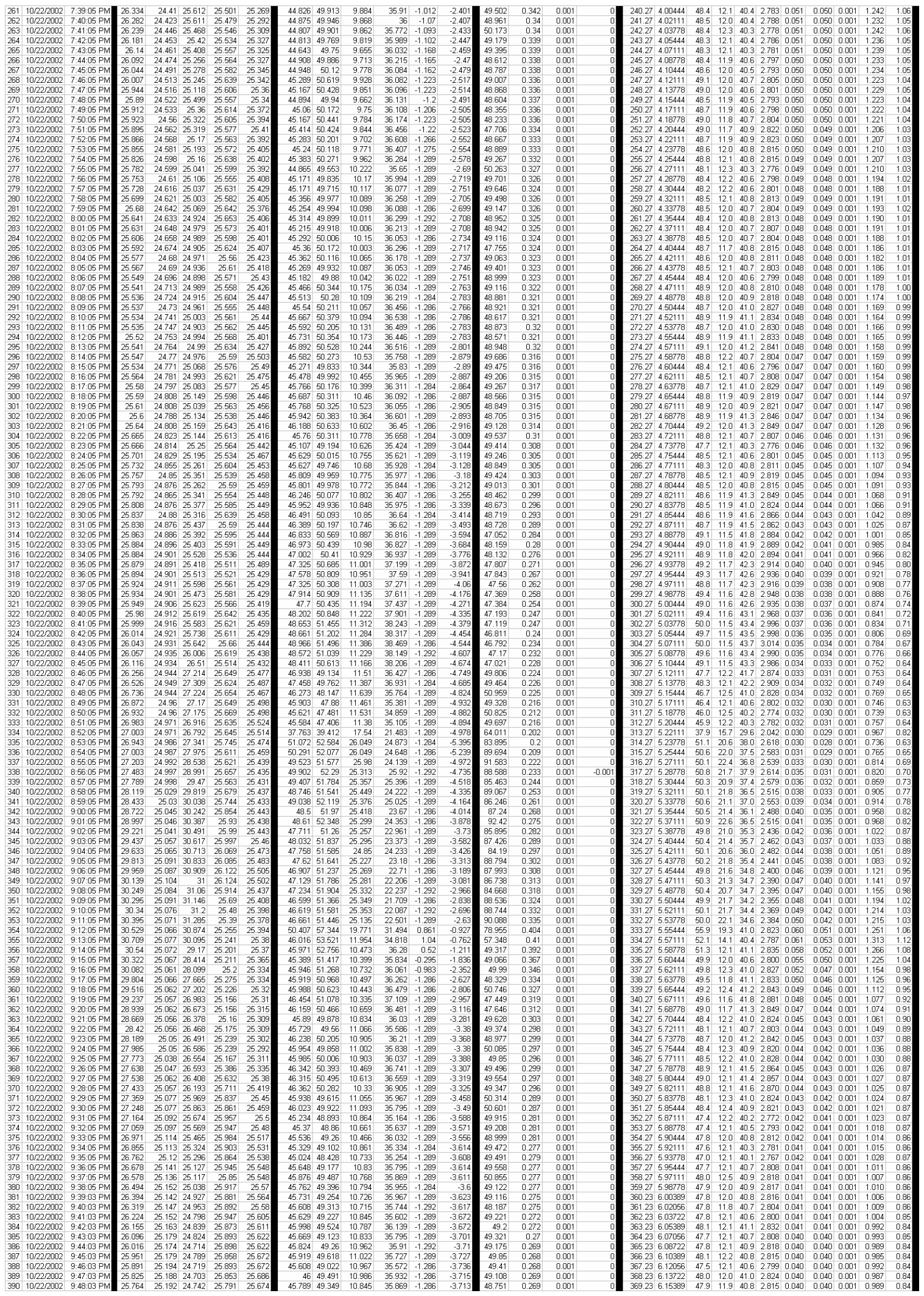

Figure D4-3: Dewatering of the AN-102R2, batch 3B, insoluble solids concentrations from 1 to 22 wt\% Raw \& Calculated Data 
WSRC-TR-2003-00204, REV. 0

SRT-RPP-2003-00087, REV. 0

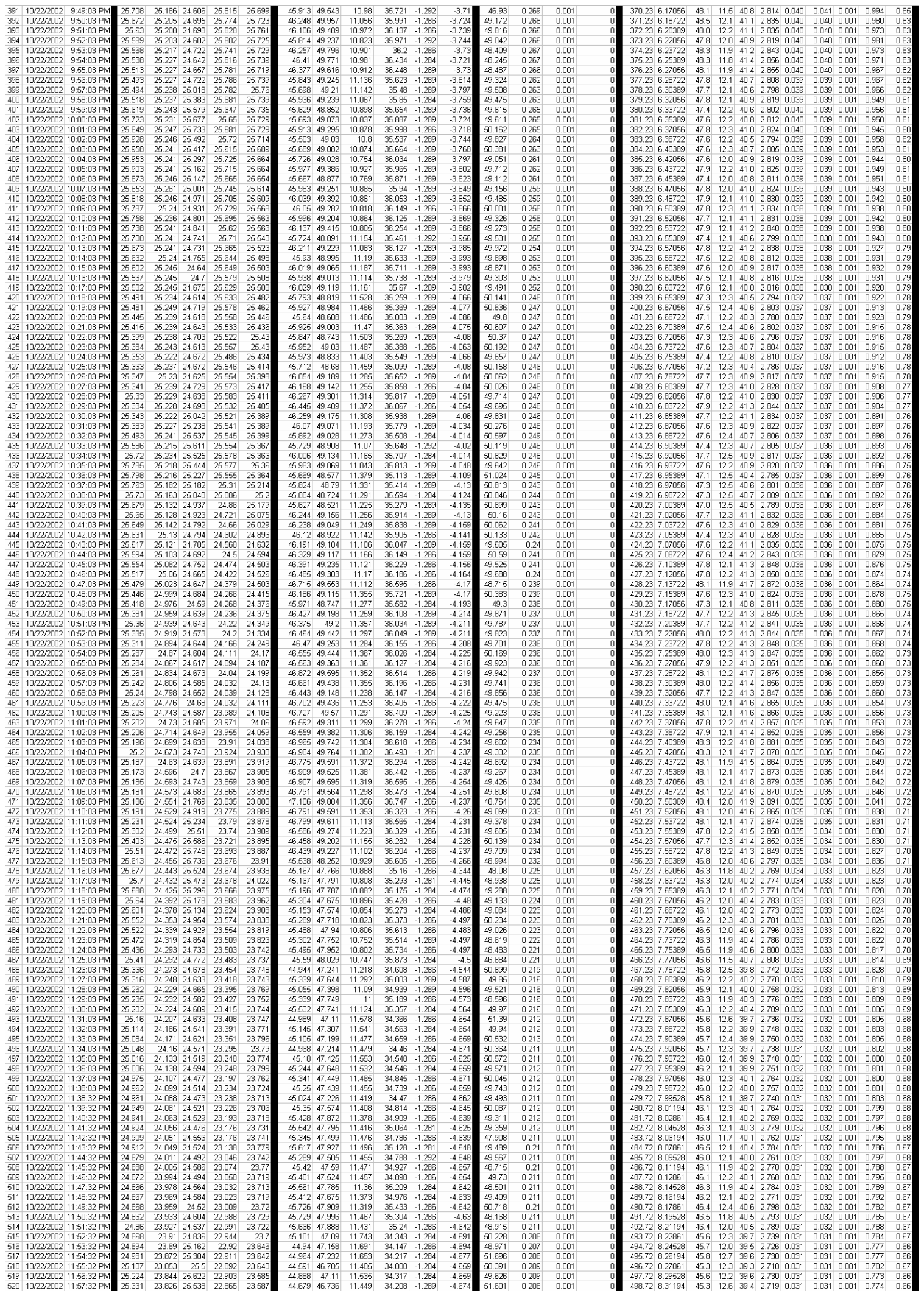

Figure D4-4: Dewatering of the AN-102R2, batch 3B, insoluble solids concentrations from 1 to 22 wt $\%$ Raw \& Calculated Data 
WSRC-TR-2003-00204, REV. 0

SRT-RPP-2003-00087, REV. 0

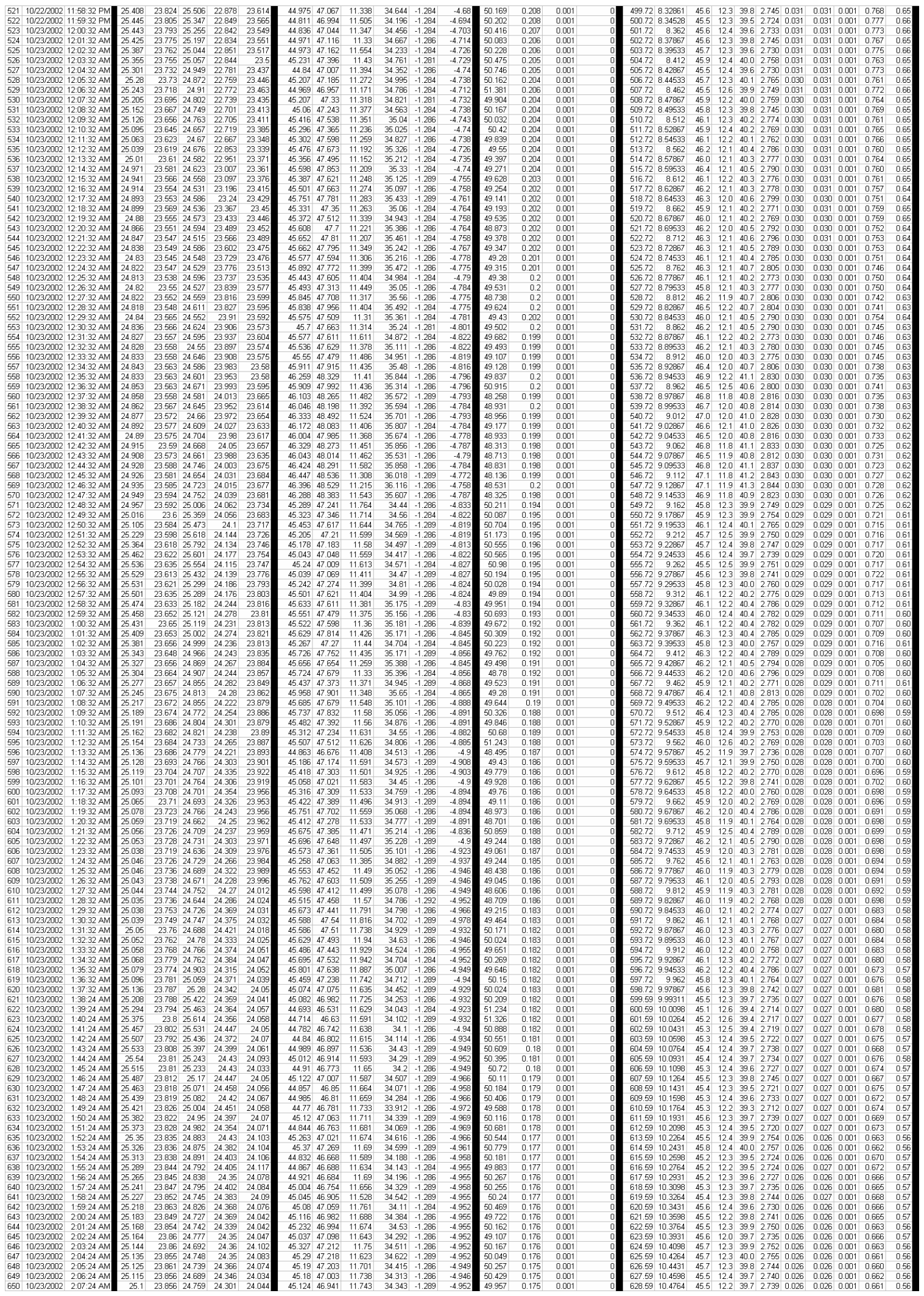

Figure D4-5: Dewatering of the AN-102R2, batch 3B, insoluble solids concentrations from 1 to 22 wt\% Raw \& Calculated Data 
WSRC-TR-2003-00204, REV. 0

SRT-RPP-2003-00087, REV. 0

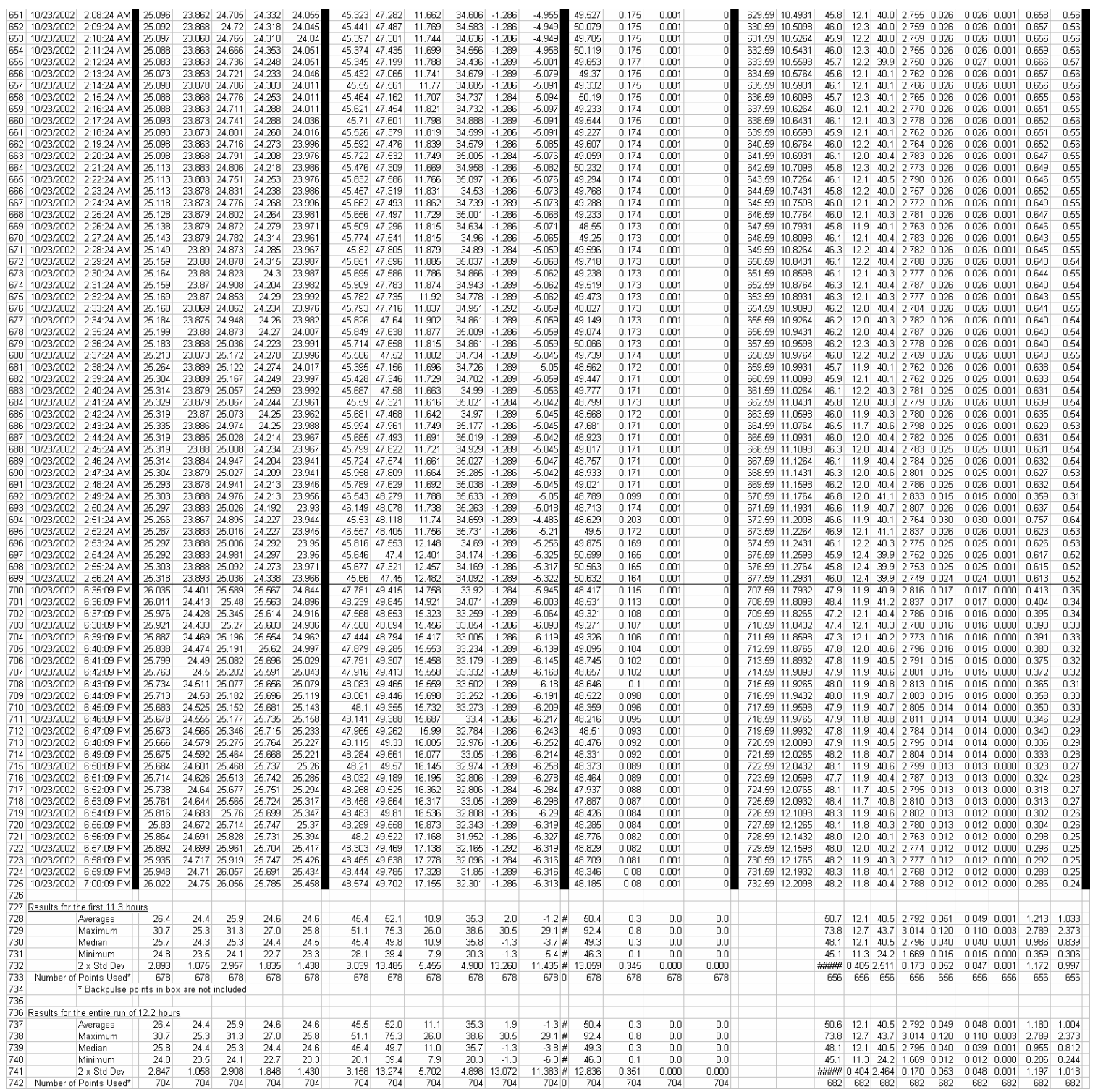

Figure D4-6: Dewatering of the AN-102R2, batch 3B, insoluble solids concentrations from 1 to 22 wt\% Raw \& Calculated Data 


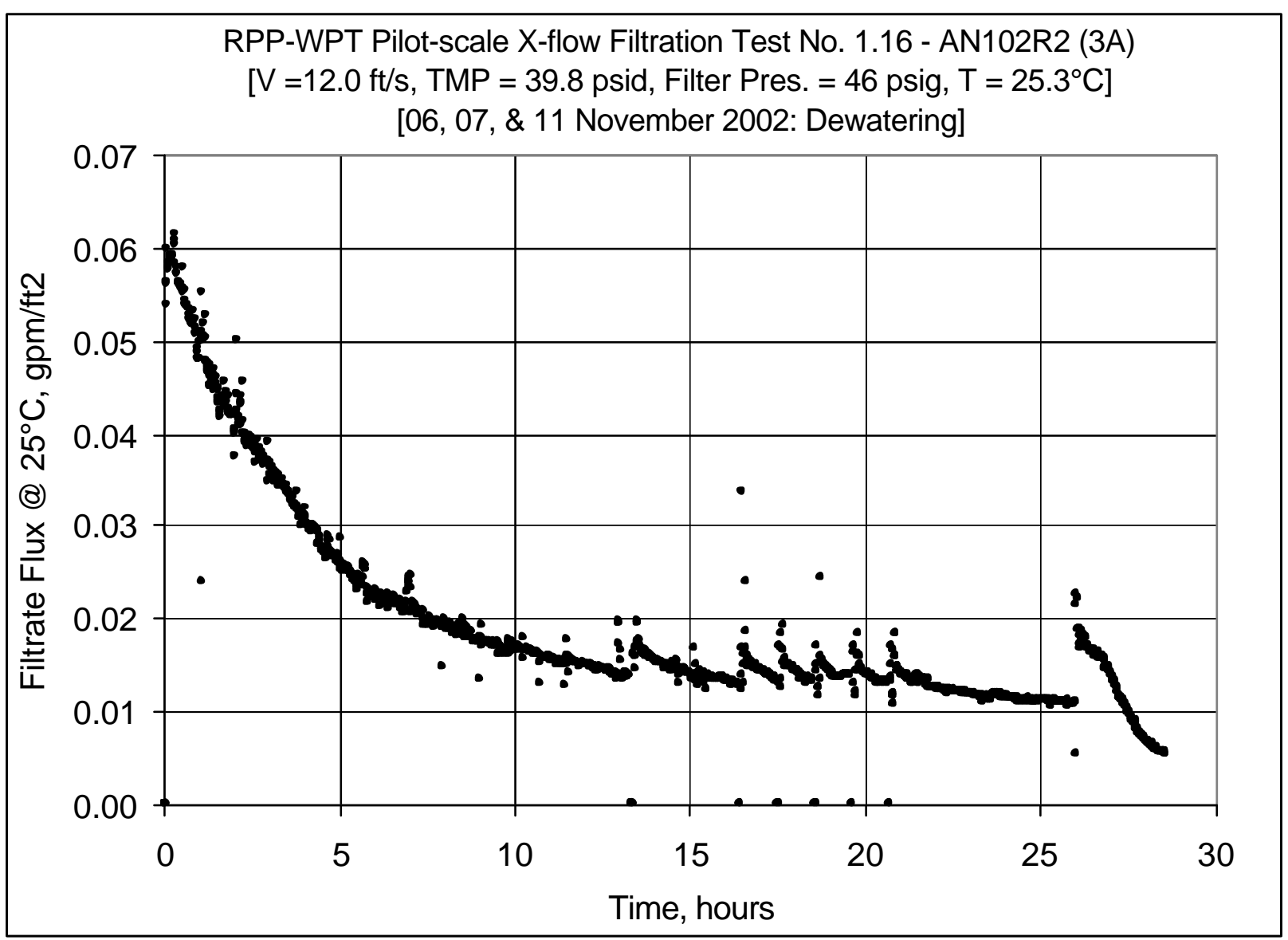

Figure D5: Dewatering of the AN-102R2, batch 3A, insoluble solids concentrations from 1.6 to $25 \mathrm{wt} \%$

(Data from 18 Files)

(1.16-3a-rpp-pxu-110602-1515, -1715, -1915, -2015, -2115, -2305)

(1.16-3a-rpp-pxu-110702-0101, -0301, -0432, -0712, -0740, -0843, -947, -1050, -1400, -1600)

(1.16-3a-rpp-pxu-111102-0855, -1055) 
WSRC-TR-2003-00204, REV. 0

SRT-RPP-2003-00087, REV. 0

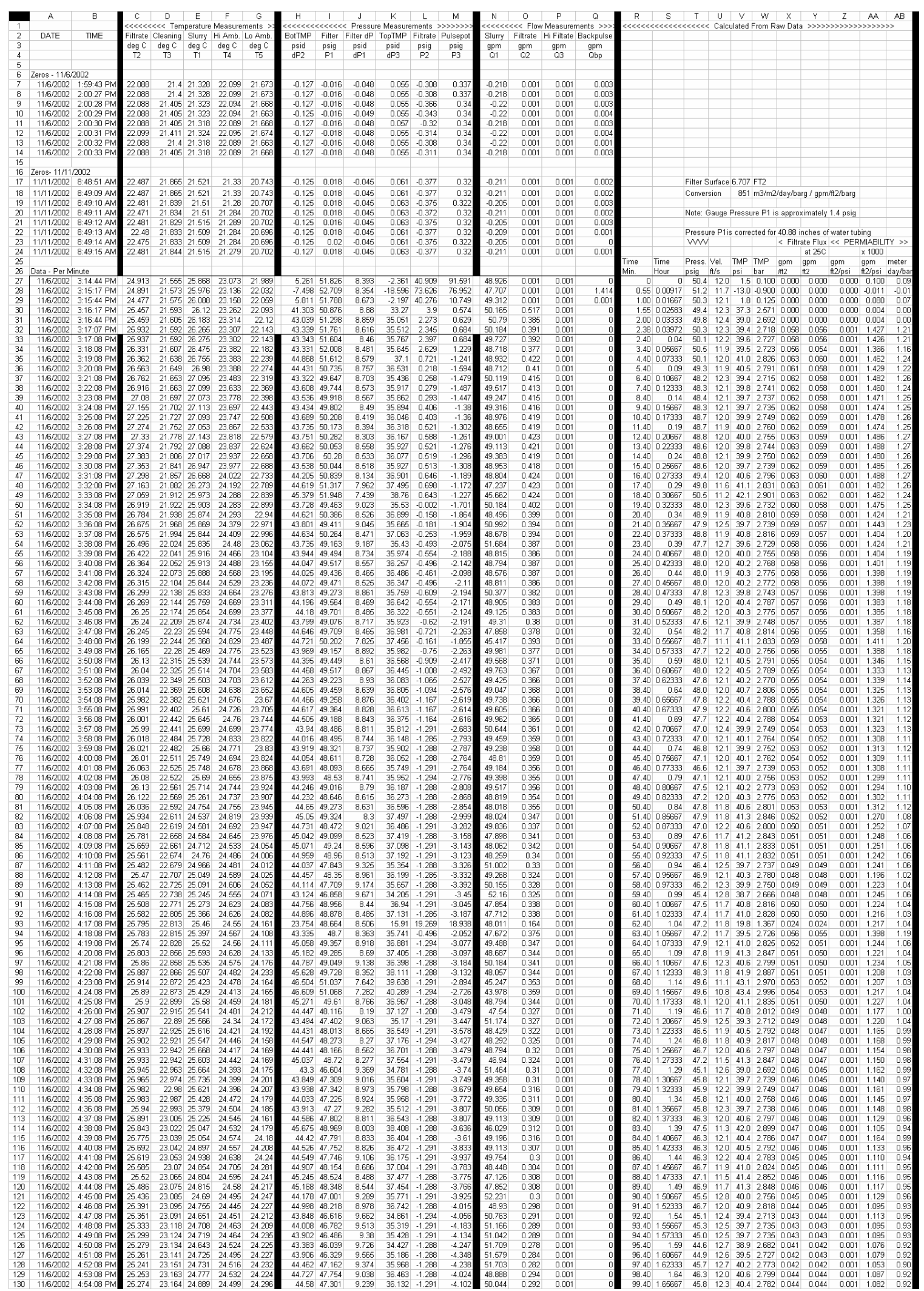

Figure D6-1: Dewatering of the AN-102R2, batch 3A, insoluble solids concentrations from 1.6 to 25 wt $\%$ Raw \& Calculated Data 
WSRC-TR-2003-00204, REV. 0 SRT-RPP-2003-00087, REV. 0

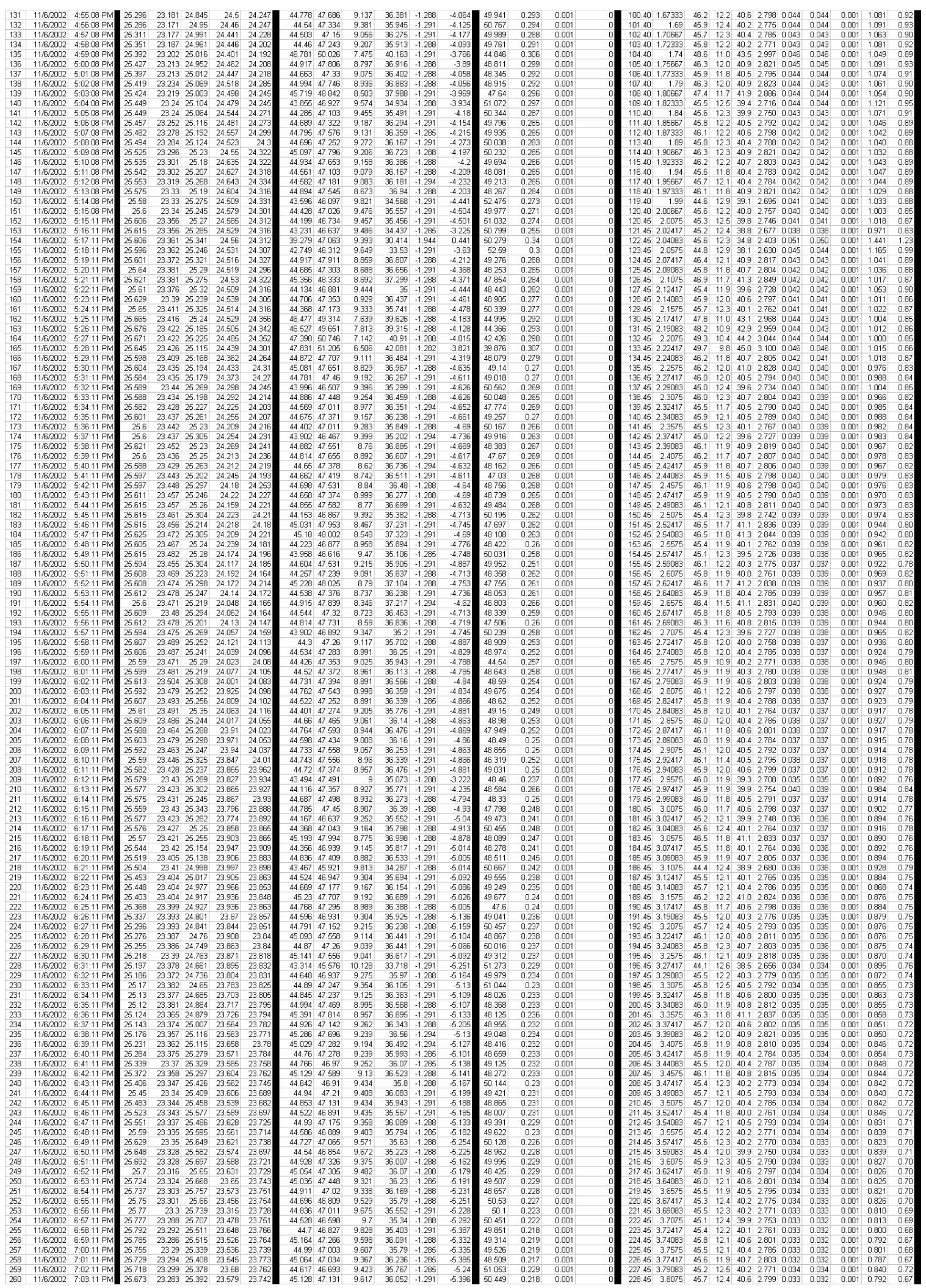

Figure D6-2: Dewatering of the AN-102R2, batch 3A, insoluble solids concentrations from 1.6 to $25 \mathrm{wt} \%$ Raw \& Calculated Data 
WSRC-TR-2003-00204, REV. 0 SRT-RPP-2003-00087, REV. 0

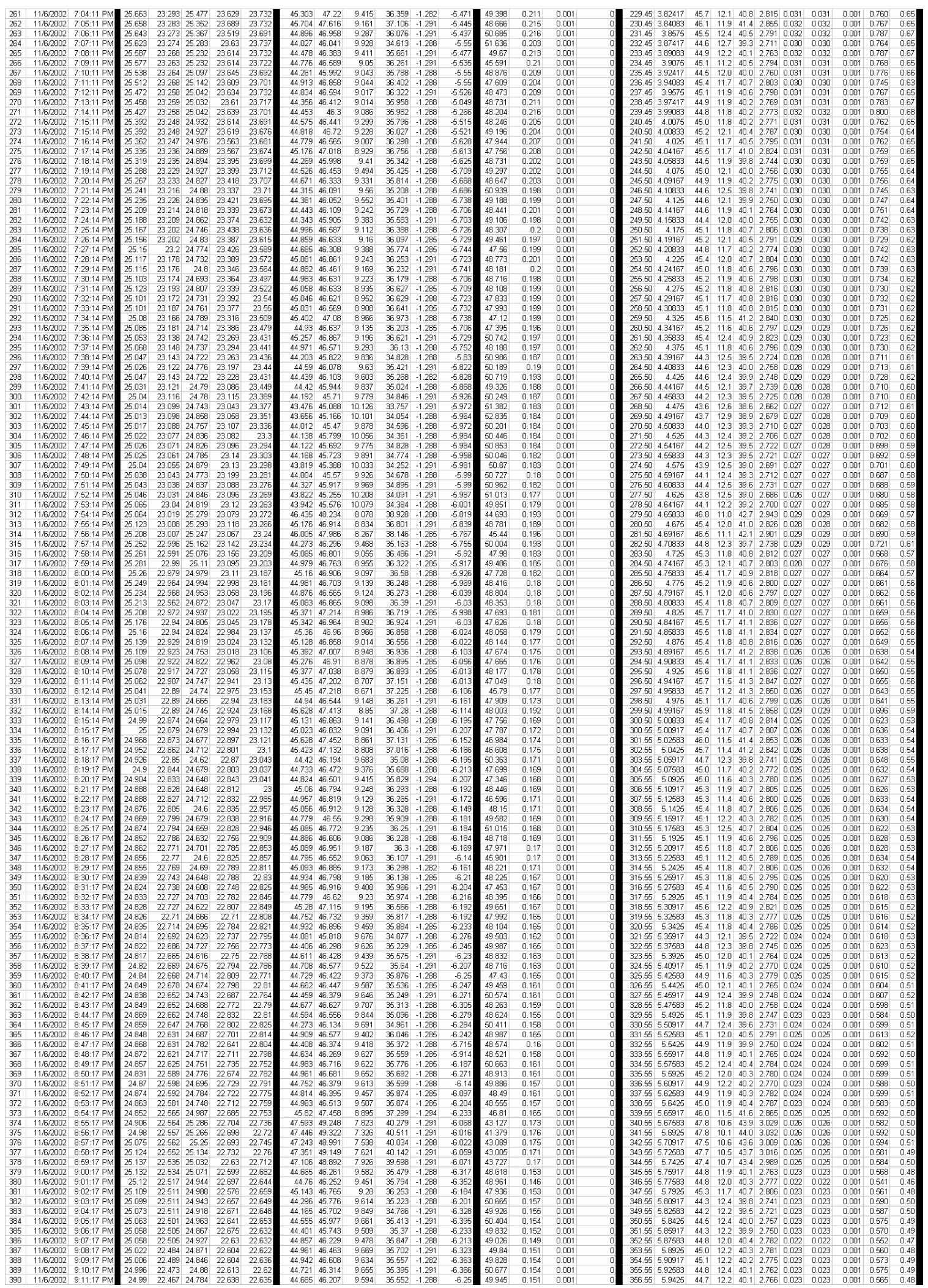

Figure D6-3: Dewatering of the AN-102R2, batch 3A, insoluble solids concentrations from 1.6 to $25 \mathrm{wt} \%$ Raw \& Calculated Data 
WSRC-TR-2003-00204, REV. 0 SRT-RPP-2003-00087, REV. 0

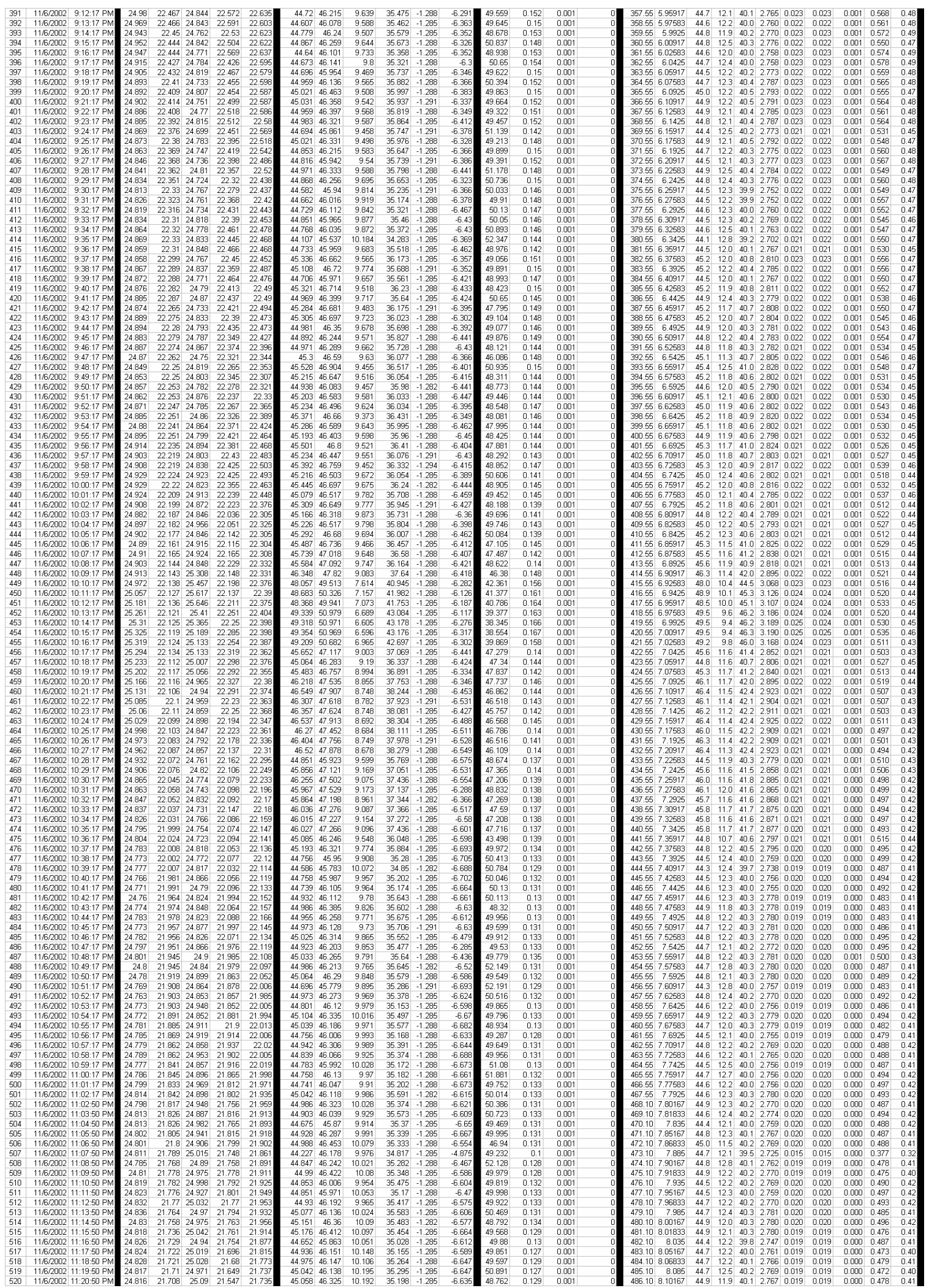

Figure D6-4: Dewatering of the AN-102R2, batch 3A, insoluble solids concentrations from 1.6 to $25 \mathrm{wt} \%$ Raw \& Calculated Data 
WSRC-TR-2003-00204, REV. 0

SRT-RPP-2003-00087, REV. 0
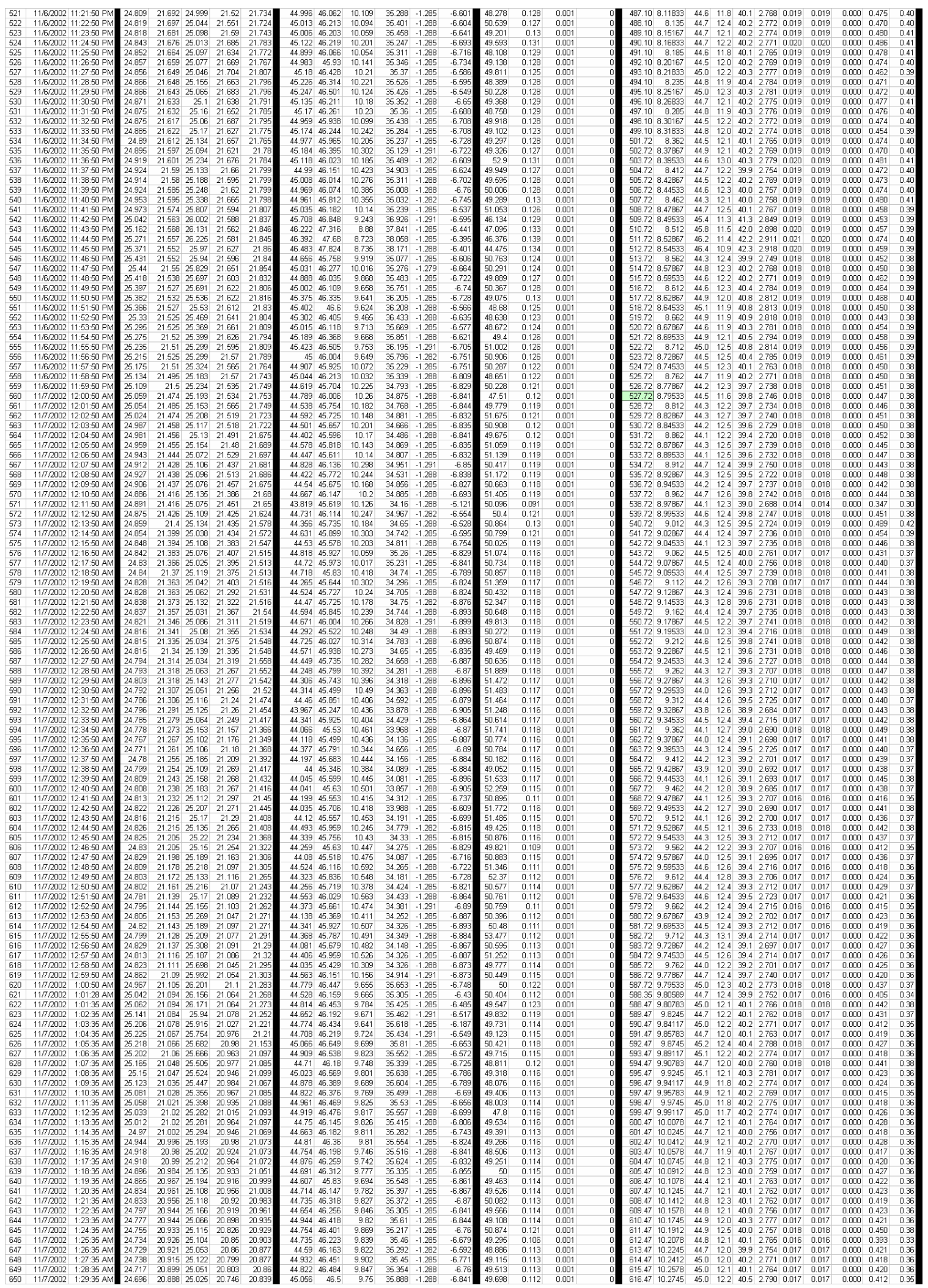

Figure D6-5: Dewatering of the AN-102R2, batch 3A, insoluble solids concentrations from 1.6 to 25 wt $\%$ Raw \& Calculated Data 
WSRC-TR-2003-00204, REV. 0 SRT-RPP-2003-00087, REV. 0

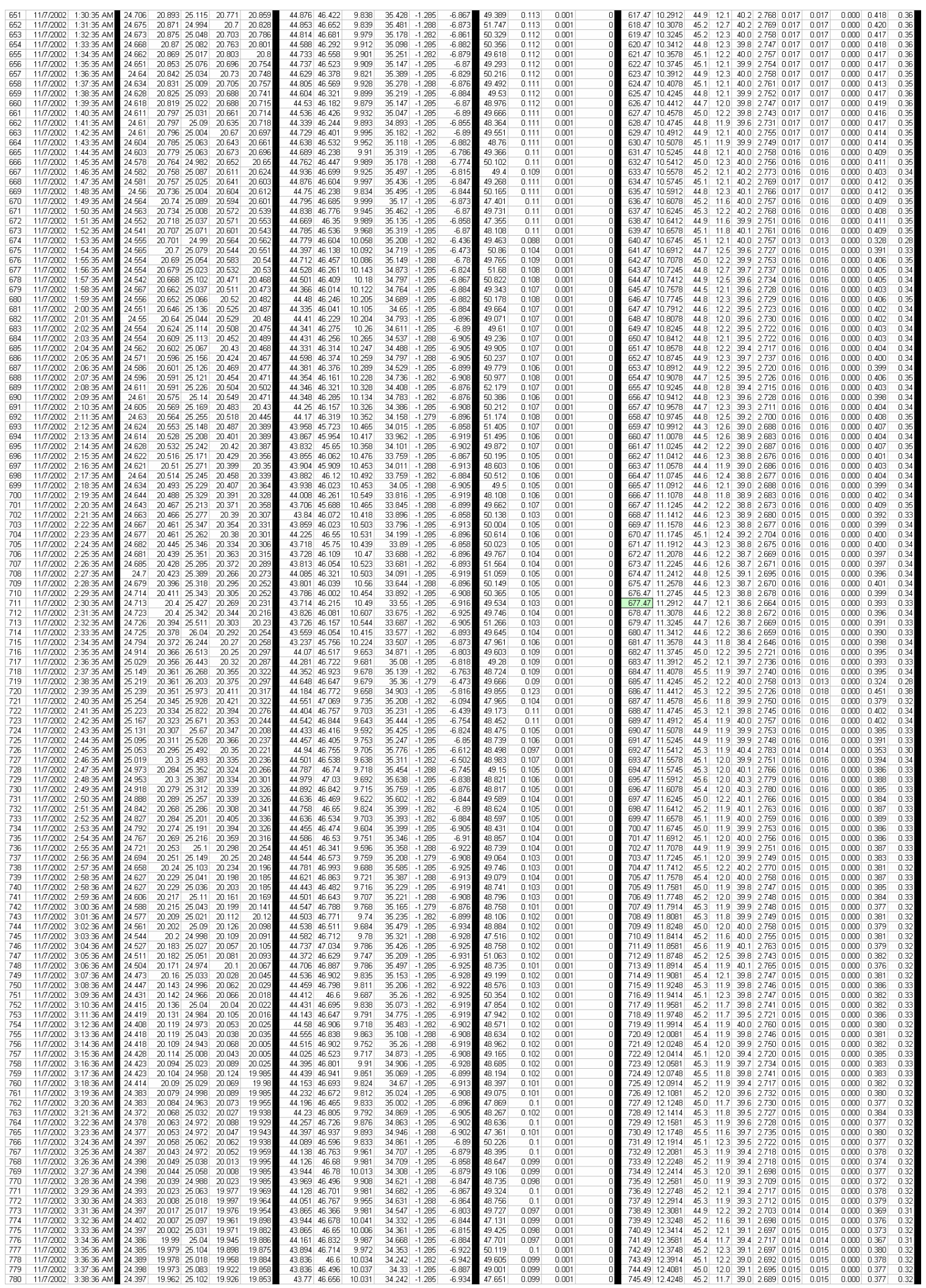

Figure D6-6: Dewatering of the AN-102R2, batch 3A, insoluble solids concentrations from 1.6 to $25 \mathrm{wt} \%$ Raw \& Calculated Data 
WSRC-TR-2003-00204, REV. 0 SRT-RPP-2003-00087, REV. 0

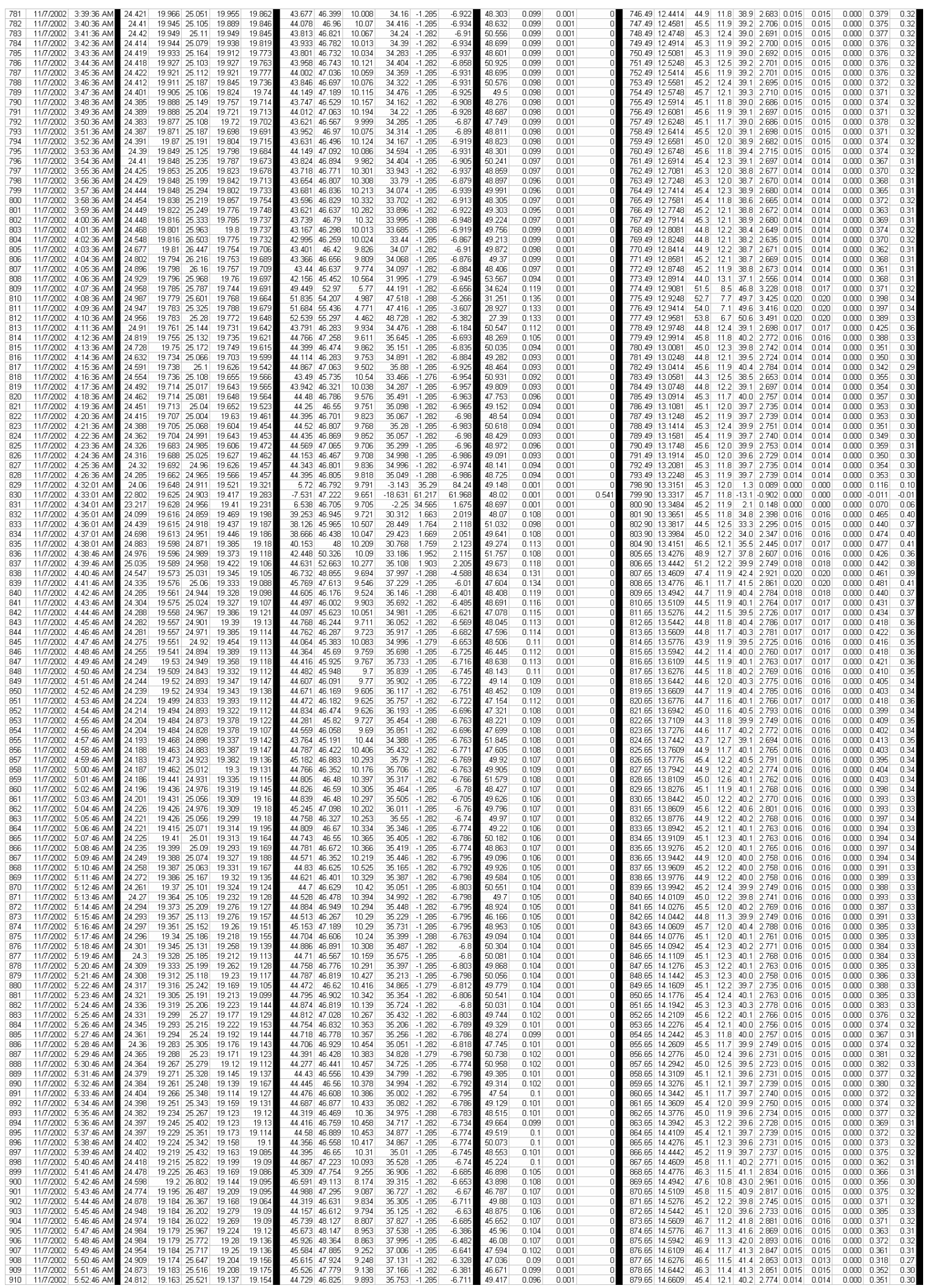

Figure D6-7: Dewatering of the AN-102R2, batch 3A, insoluble solids concentrations from 1.6 to $25 \mathrm{wt} \%$ Raw \& Calculated Data 
WSRC-TR-2003-00204, REV. 0 SRT-RPP-2003-00087, REV. 0

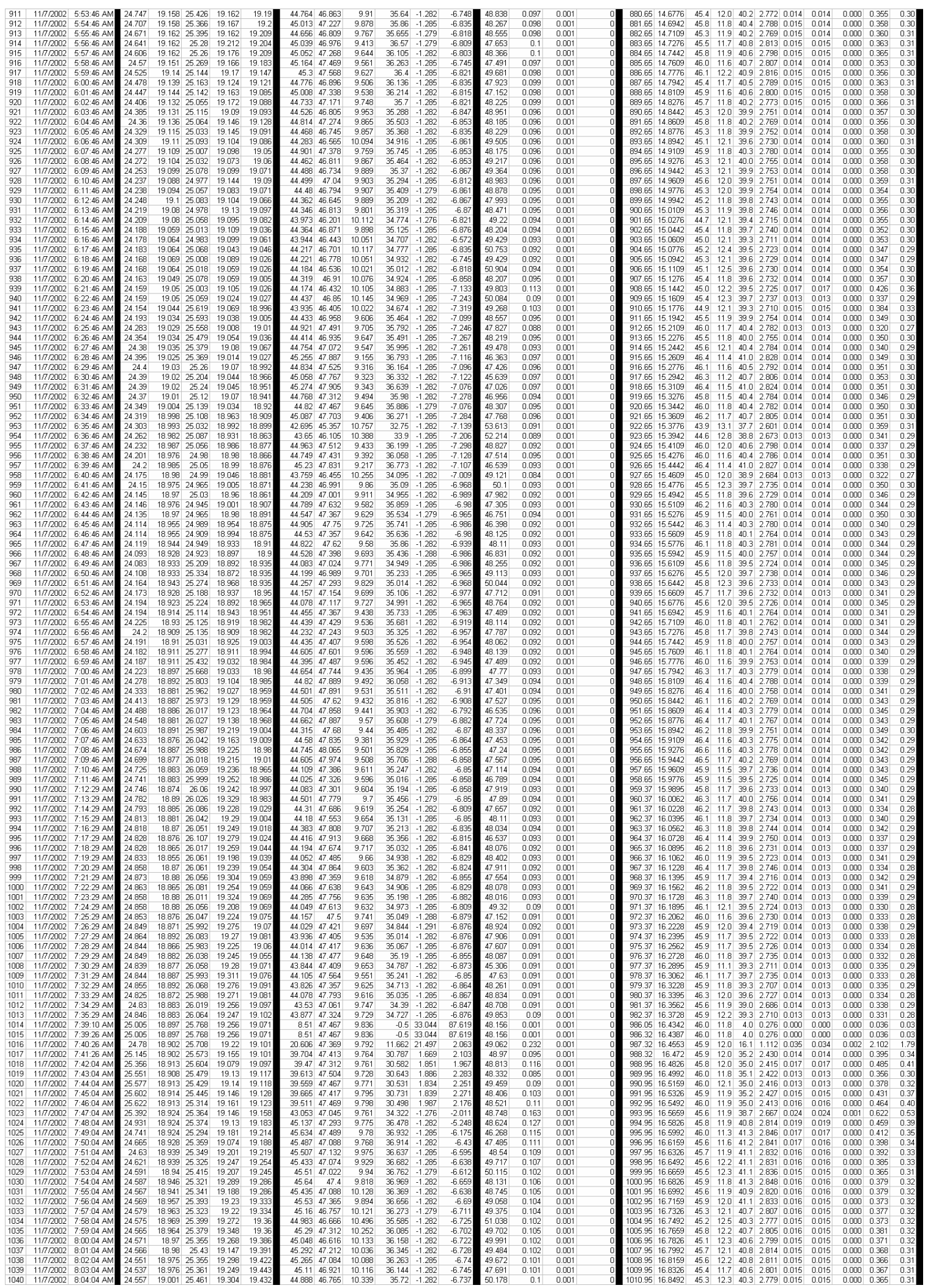

Figure D6-8: Dewatering of the AN-102R2, batch 3A, insoluble solids concentrations from 1.6 to $25 \mathrm{wt} \%$ Raw \& Calculated Data 
WSRC-TR-2003-00204, REV. 0 SRT-RPP-2003-00087, REV. 0

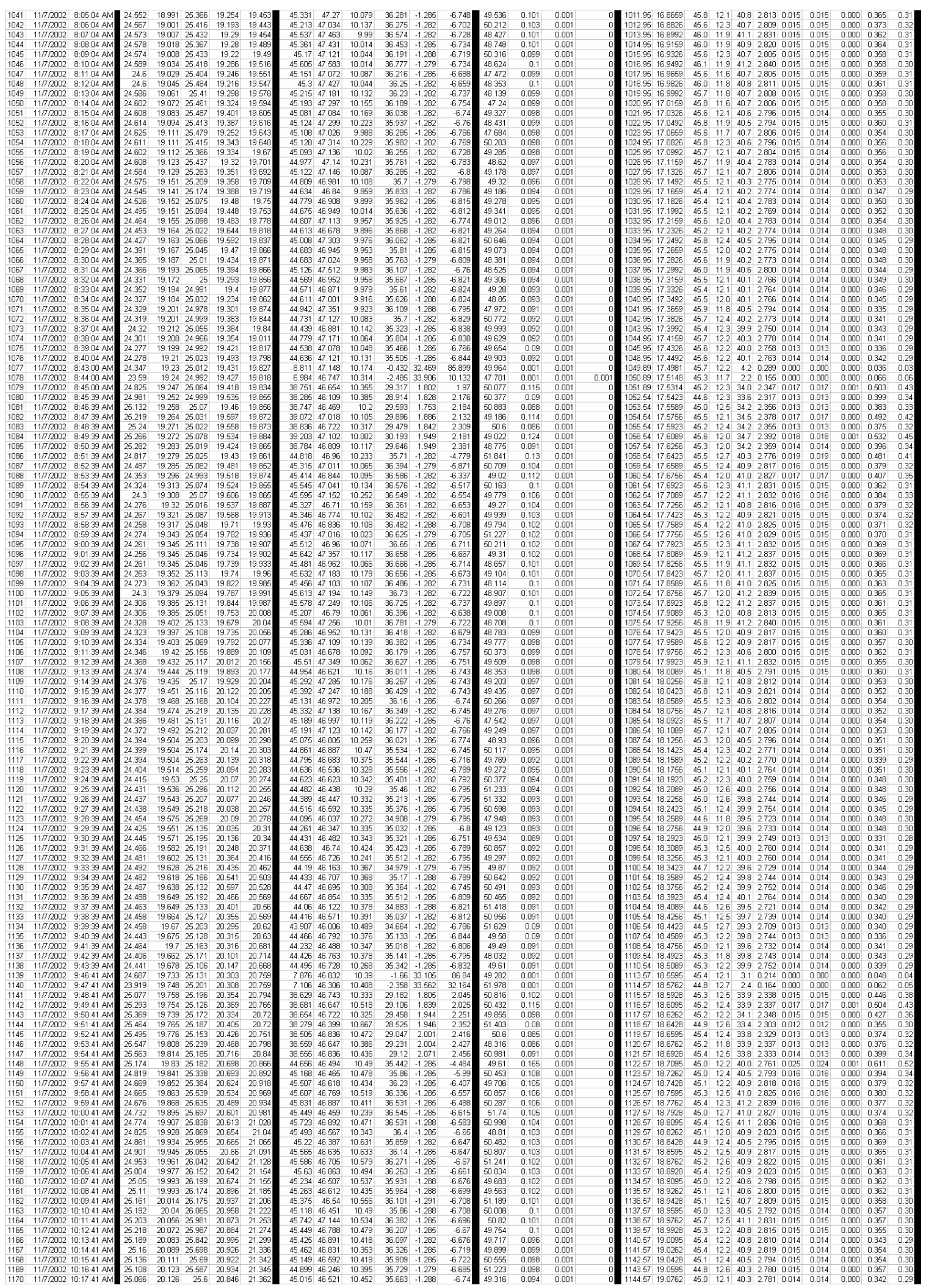

Figure D6-9: Dewatering of the AN-102R2, batch 3A, insoluble solids concentrations from 1.6 to $25 \mathrm{wt} \%$ Raw \& Calculated Data 
WSRC-TR-2003-00204, REV. 0

SRT-RPP-2003-00087, REV. 0

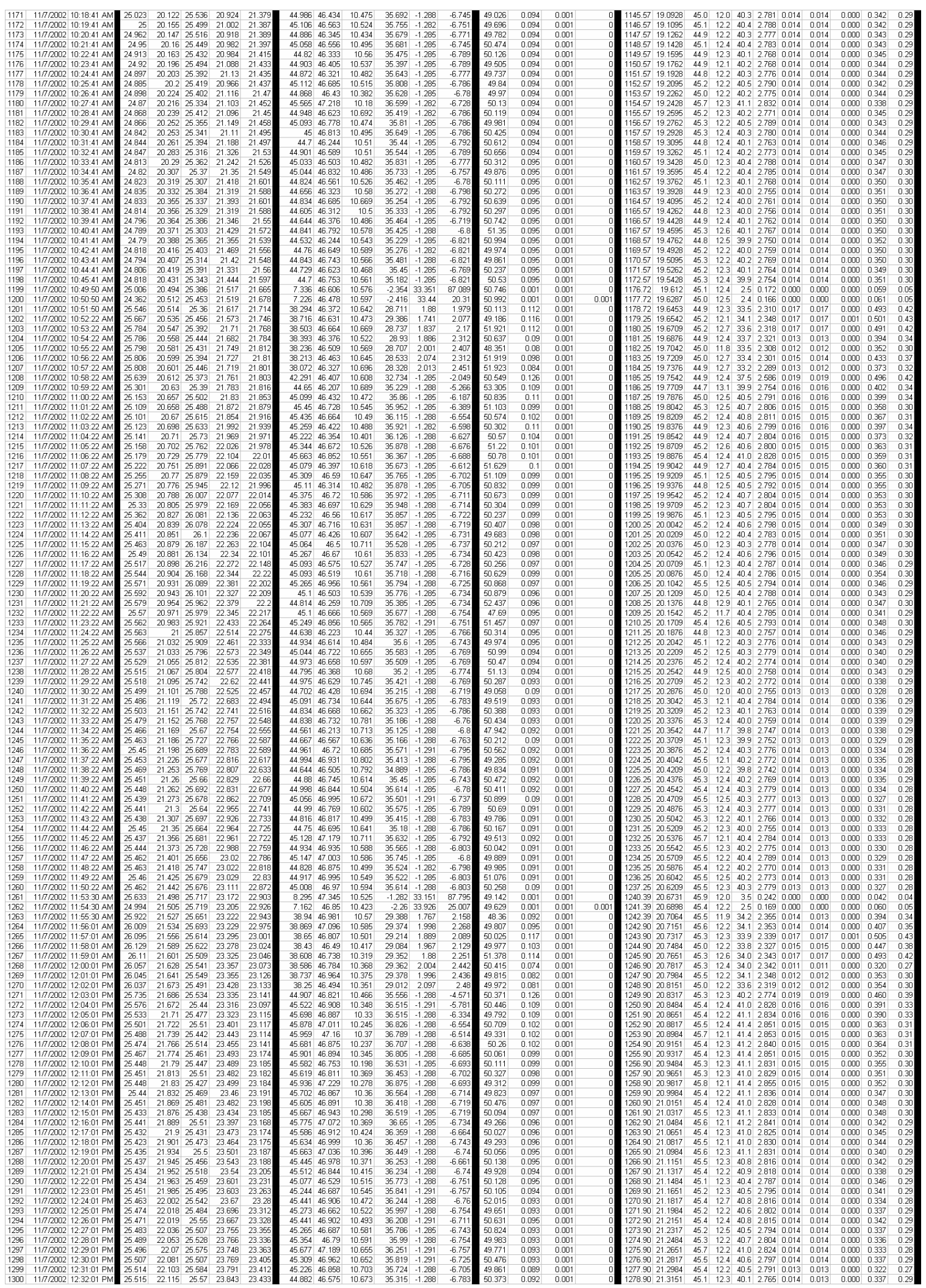

Figure D6-10: Dewatering of the AN-102R2, batch 3A, insoluble solids concentrations from 1.6 to $25 \mathrm{wt} \%$ - Raw \& Calculated Data 
WSRC-TR-2003-00204, REV. 0 SRT-RPP-2003-00087, REV. 0

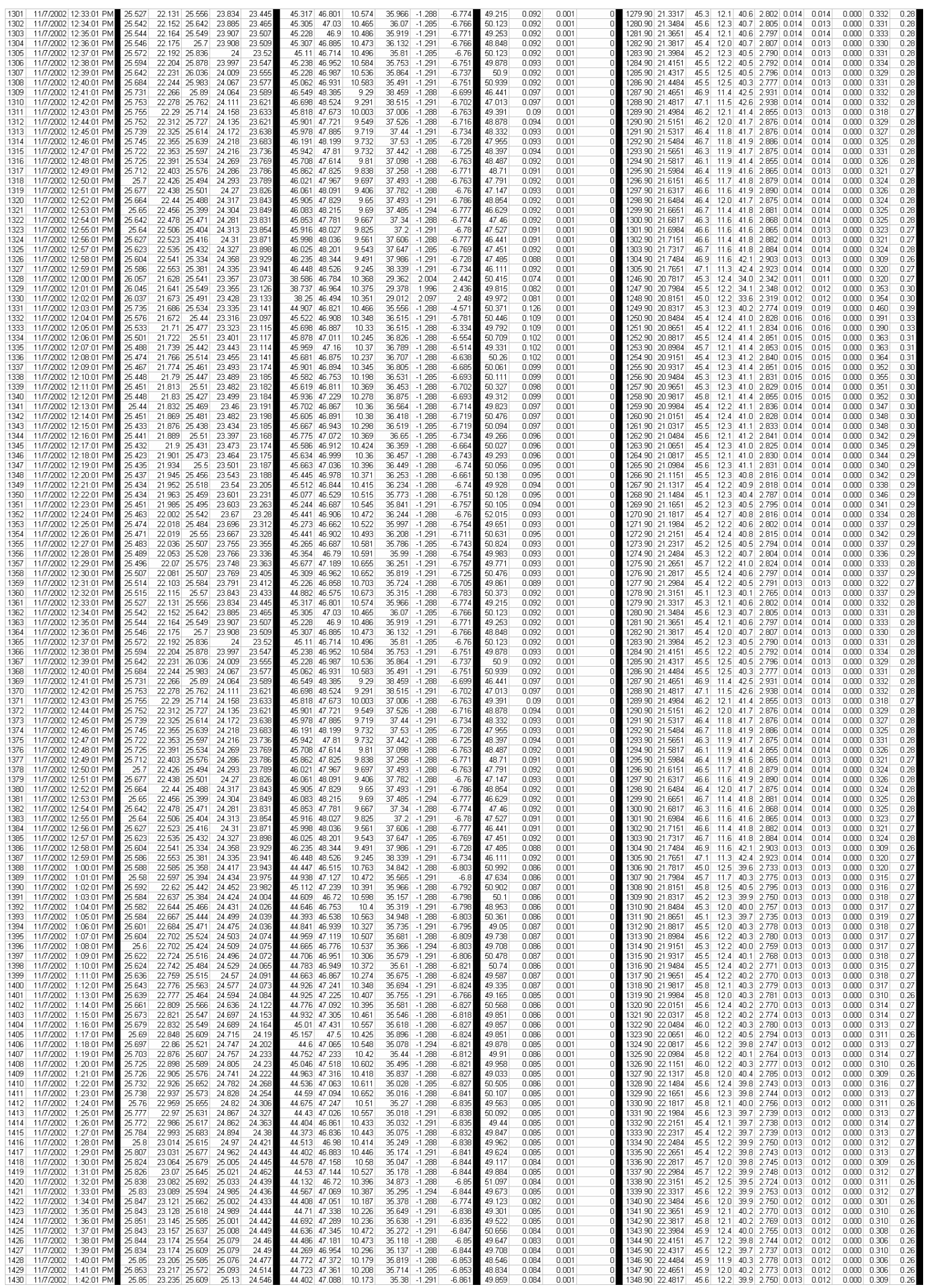

Figure D6-11: Dewatering of the AN-102R2, batch 3A, insoluble solids concentrations from 1.6 to $25 \mathrm{wt} \%$ - Raw \& Calculated Data 
WSRC-TR-2003-00204, REV. 0 SRT-RPP-2003-00087, REV. 0

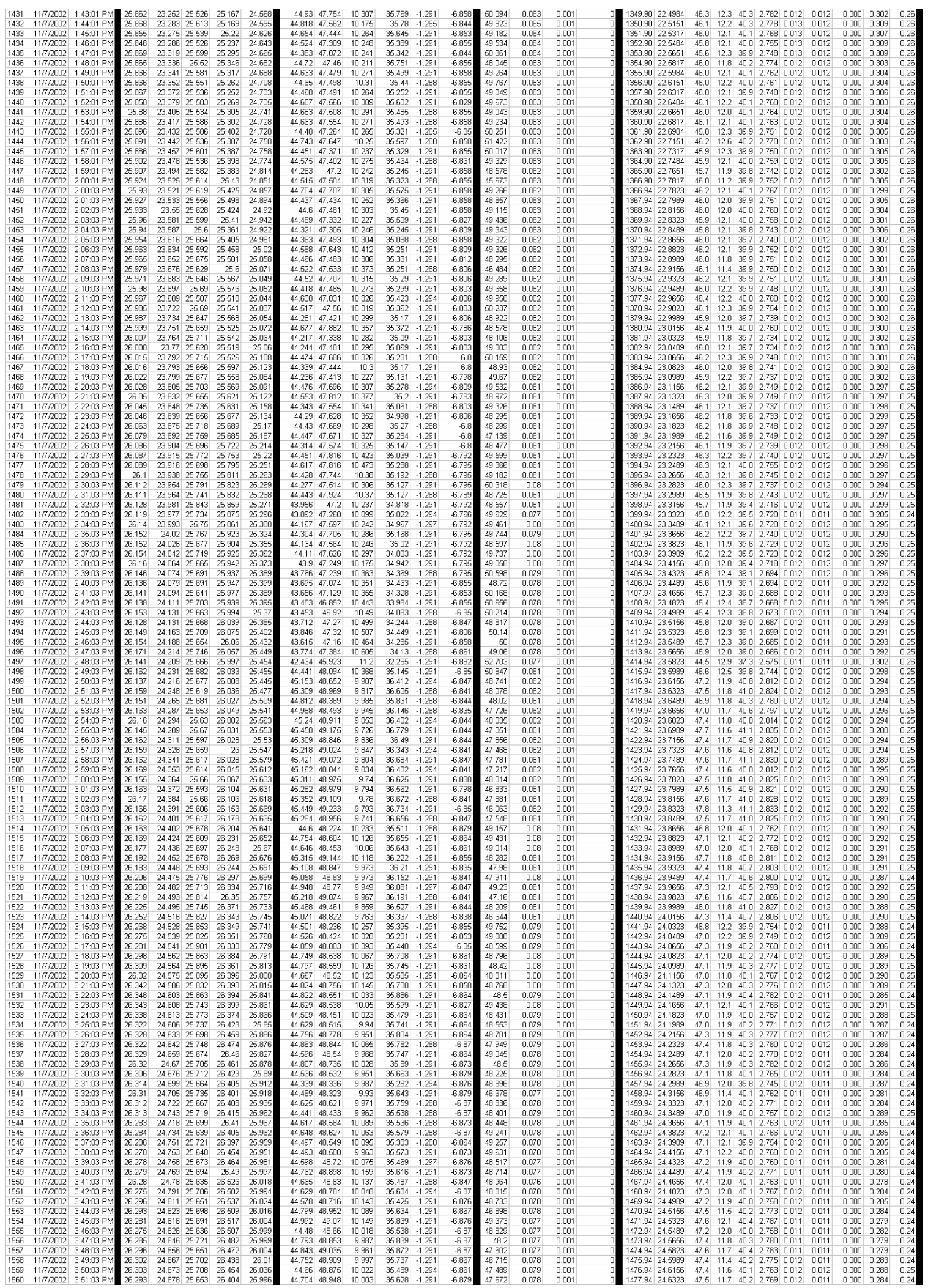

Figure D6-12: Dewatering of the AN-102R2, batch 3A, insoluble solids concentrations from 1.6 to $25 \mathrm{wt} \%$ - Raw \& Calculated Data 
WSRC-TR-2003-00204, REV. 0 SRT-RPP-2003-00087, REV. 0

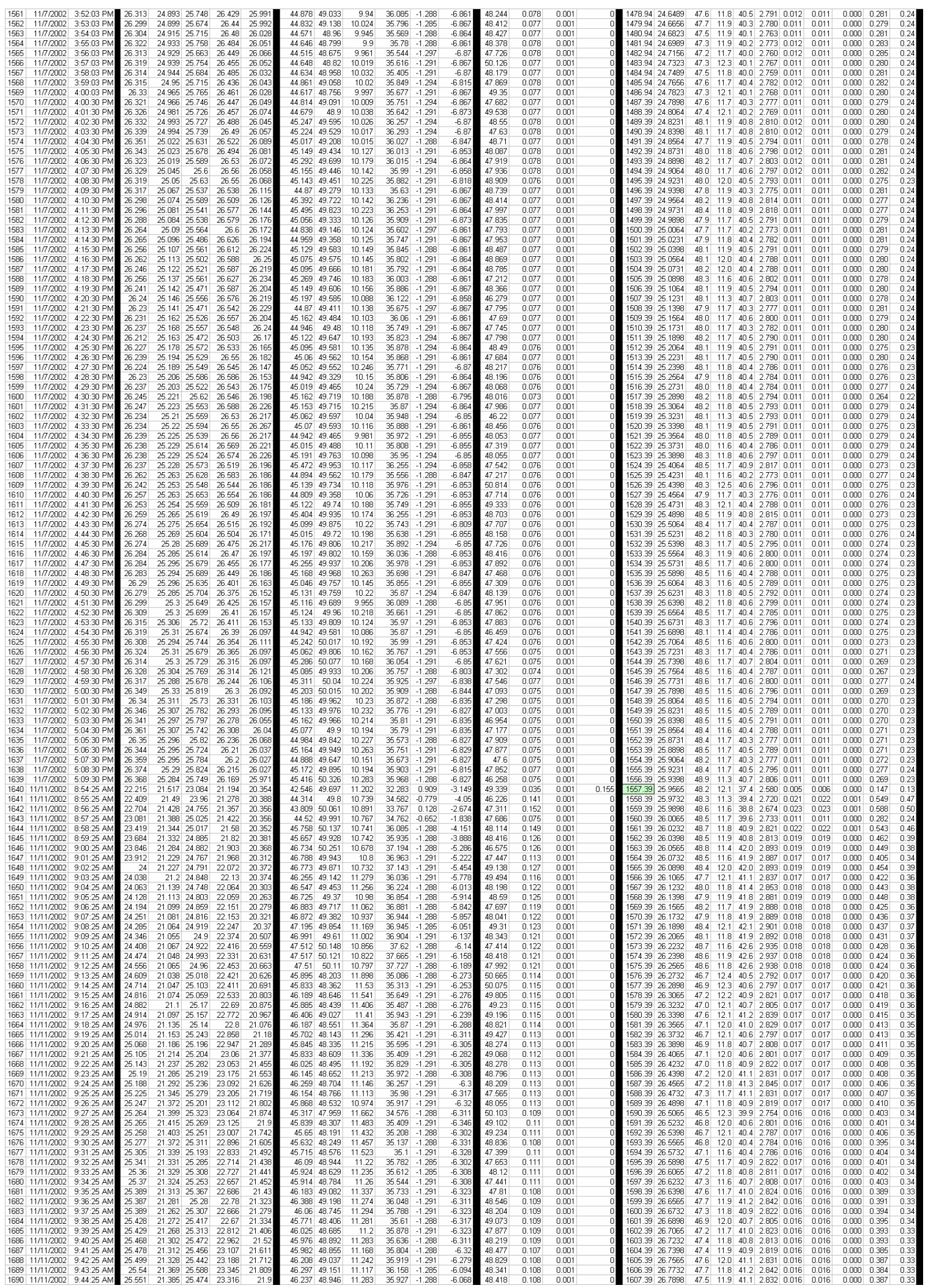

Figure D6-13: Dewatering of the AN-102R2, batch 3A, insoluble solids concentrations from 1.6 to $25 \mathrm{wt} \%$ - Raw \& Calculated Data 
WSRC-TR-2003-00204, REV. 0 SRT-RPP-2003-00087, REV. 0

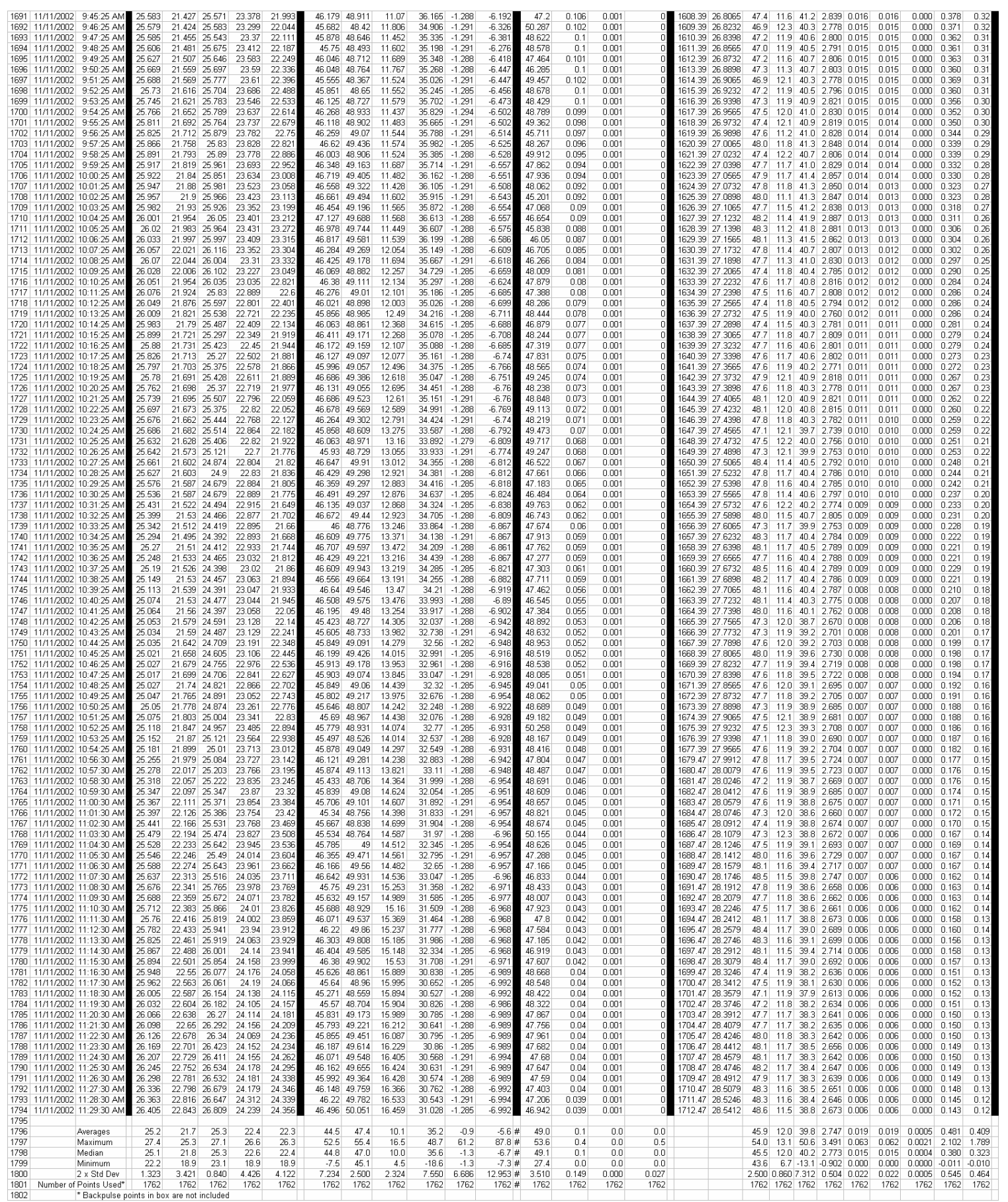

Figure D6-14: Dewatering of the AN-102R2, batch 3A, insoluble solids concentrations from 1.6 to $25 \mathrm{wt} \%$ - Raw \& Calculated Data 


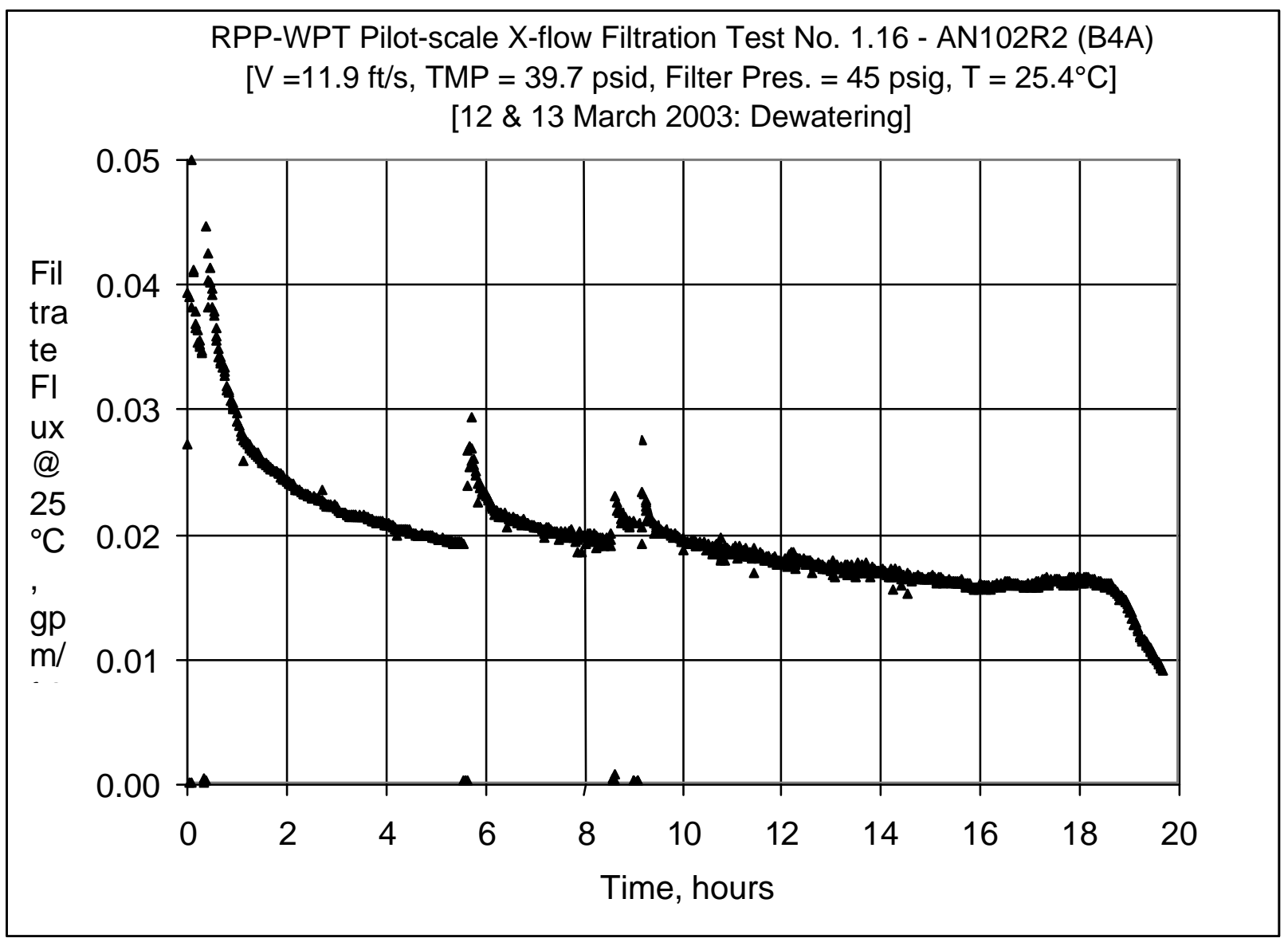

Figure D7: Dewatering of the AN-102R2, batch 4A, insoluble solids concentrations from 1.5 to $18 \mathrm{wt} \%$

(Data from 10 Files)

(1.16-4a-rpp-pxu-031203-0810, -1045, -1306, -1505, -1708, -1906, 2106, -2303)

(1.16-3a-rpp-pxu-031303-77-0101, -0301) 
WSRC-TR-2003-00204, REV. 0

SRT-RPP-2003-00087, REV. 0

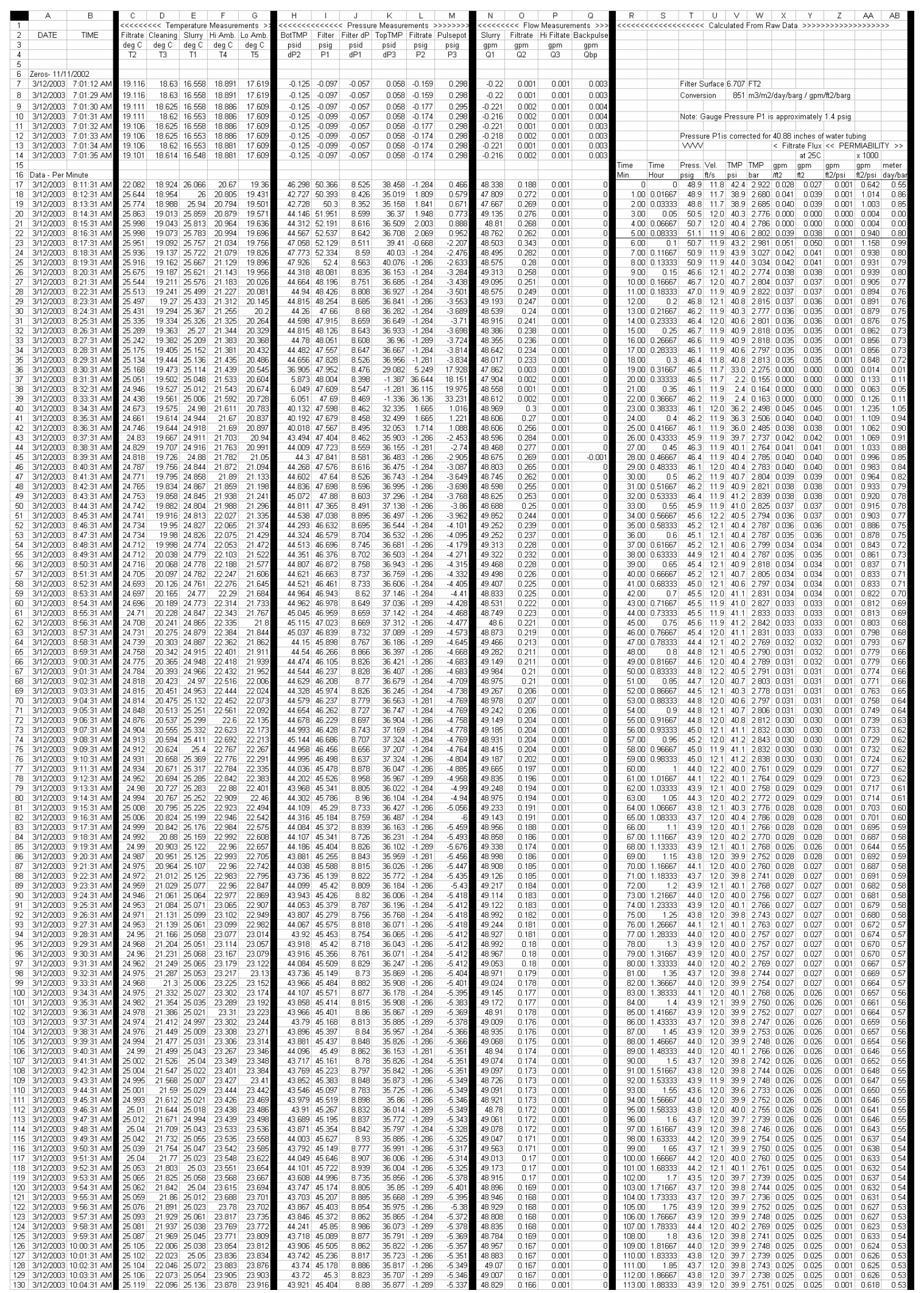

Figure D8-1: Dewatering of the AN-102R2, batch 4A, insoluble solids concentrations from 1.5 to $81 \mathrm{wt} \%$ Raw \& Calculated Data 
WSRC-TR-2003-00204, REV. 0

SRT-RPP-2003-00087, REV. 0

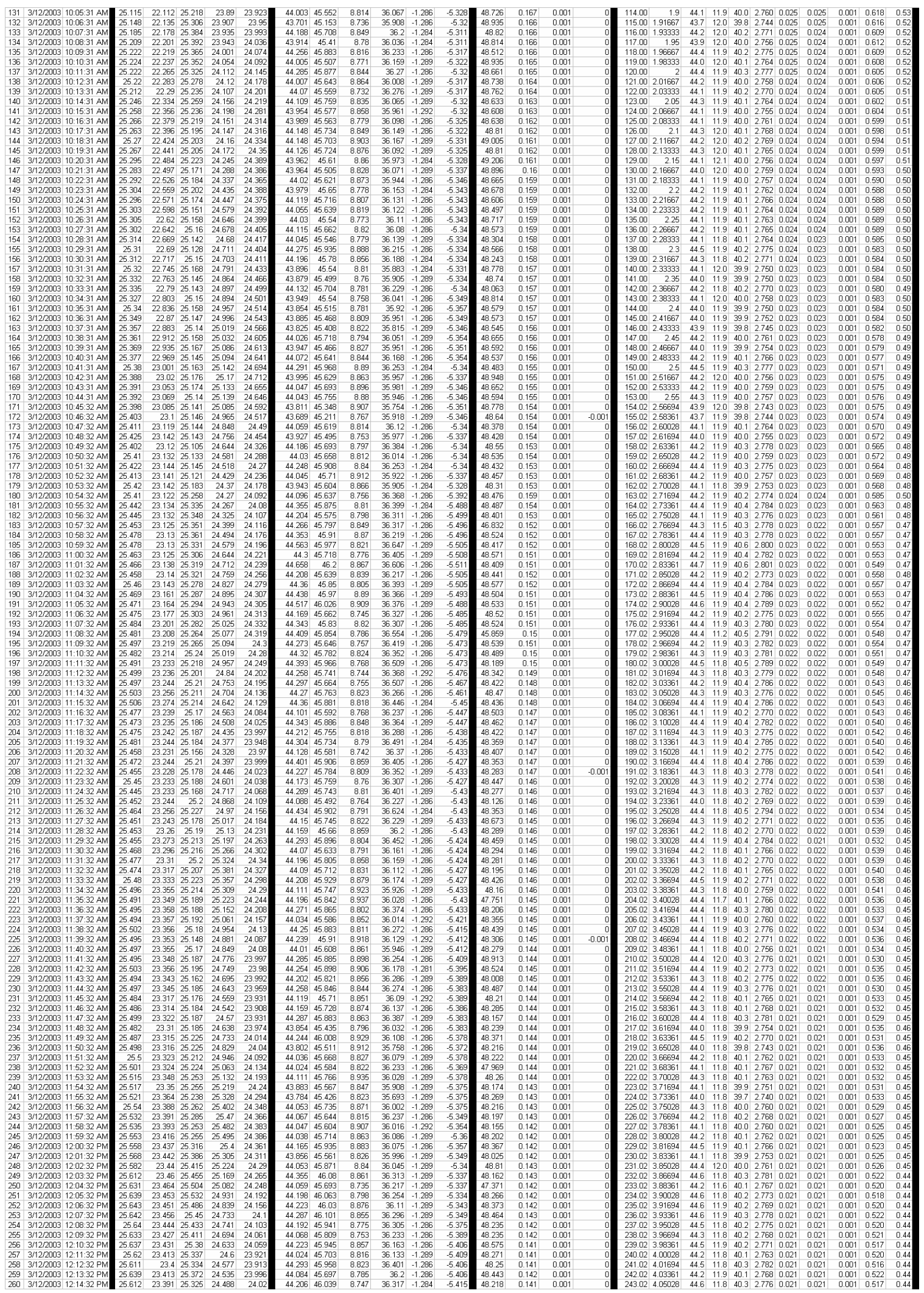

Figure D8-2: Dewatering of the AN-102R2, batch 4A, insoluble solids concentrations from 1.5 to $81 \mathrm{wt} \%$ Raw \& Calculated Data 
WSRC-TR-2003-00204, REV. 0

SRT-RPP-2003-00087, REV. 0

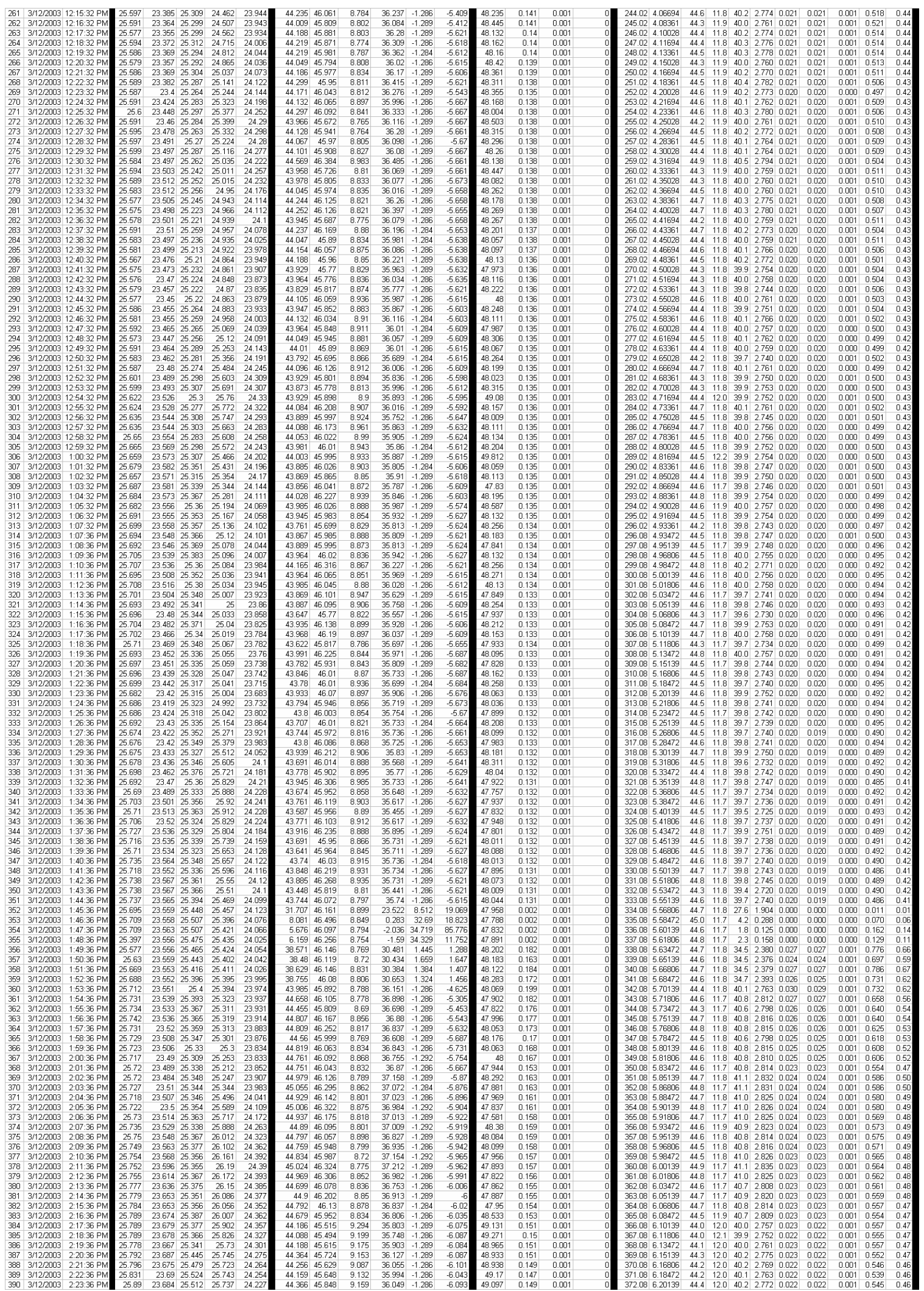

Figure D8-3: Dewatering of the AN-102R2, batch 4A, insoluble solids concentrations from 1.5 to $81 \mathrm{wt} \%$ Raw \& Calculated Data 
WSRC-TR-2003-00204, REV. 0

SRT-RPP-2003-00087, REV. 0

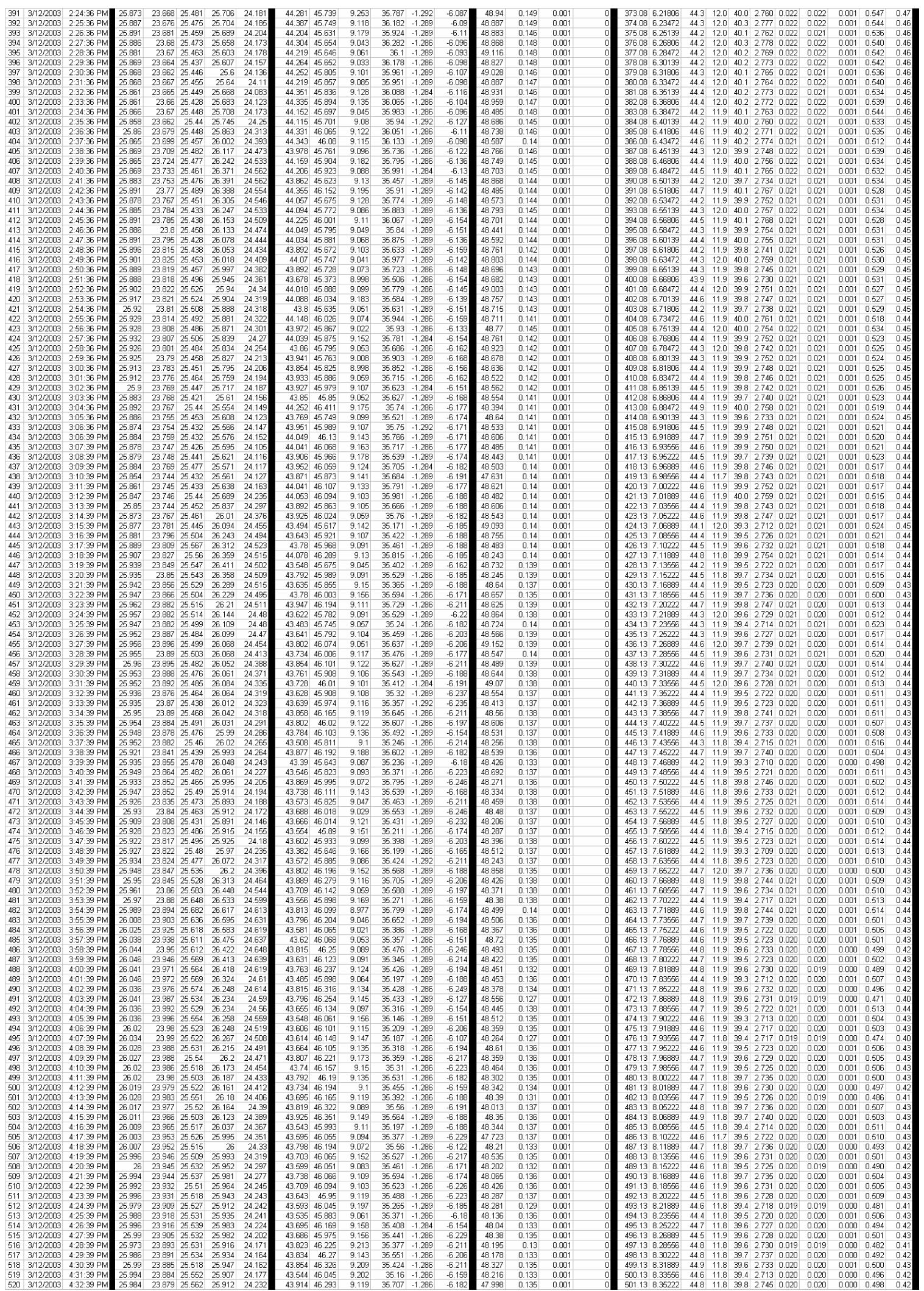

Figure D8-4: Dewatering of the AN-102R2, batch 4A, insoluble solids concentrations from 1.5 to $81 \mathrm{wt} \%$ Raw \& Calculated Data 
WSRC-TR-2003-00204, REV. 0

SRT-RPP-2003-00087, REV. 0

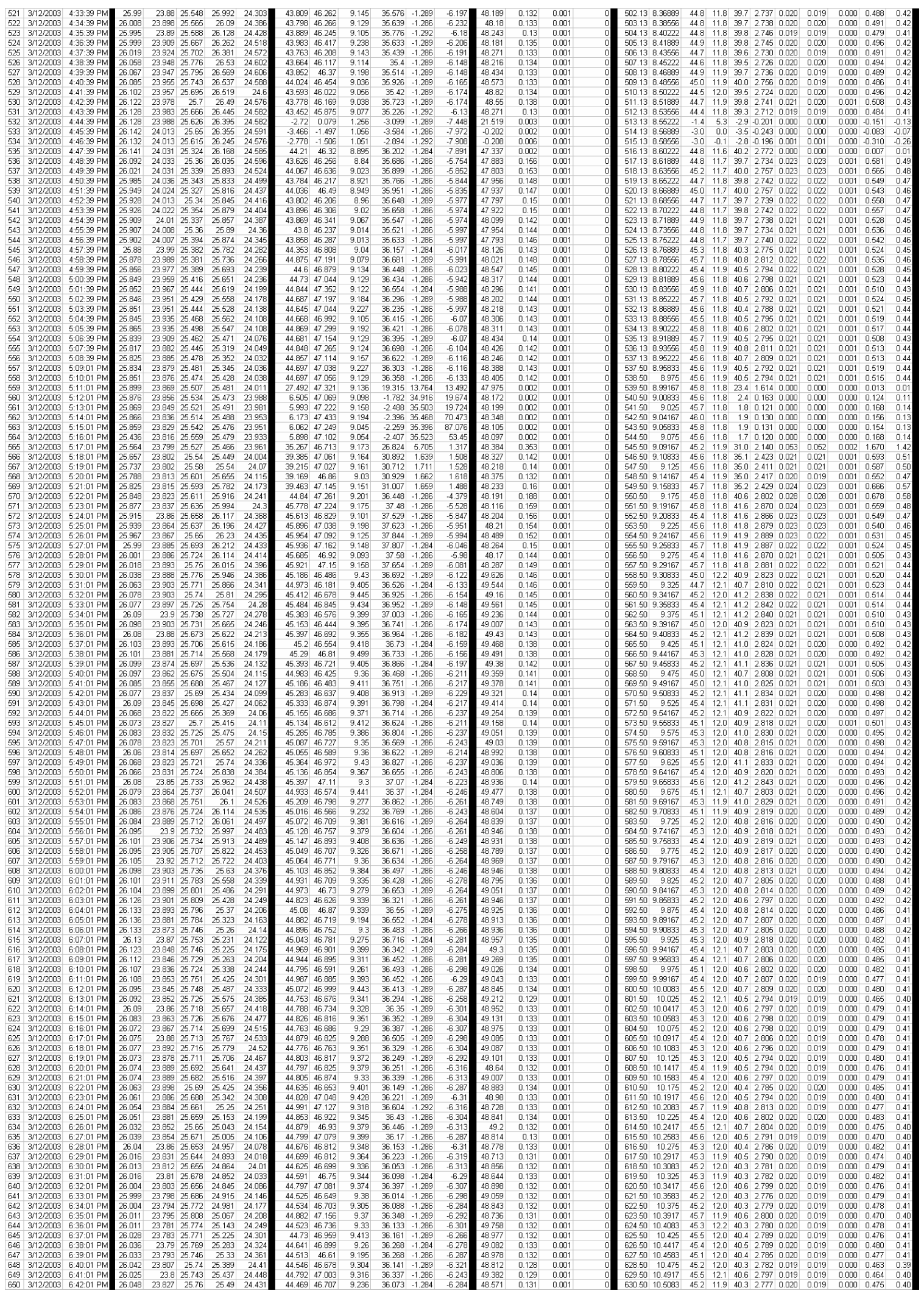

Figure D8-5: Dewatering of the AN-102R2, batch 4A, insoluble solids concentrations from 1.5 to $81 \mathrm{wt} \%$ Raw \& Calculated Data 
WSRC-TR-2003-00204, REV. 0

SRT-RPP-2003-00087, REV. 0

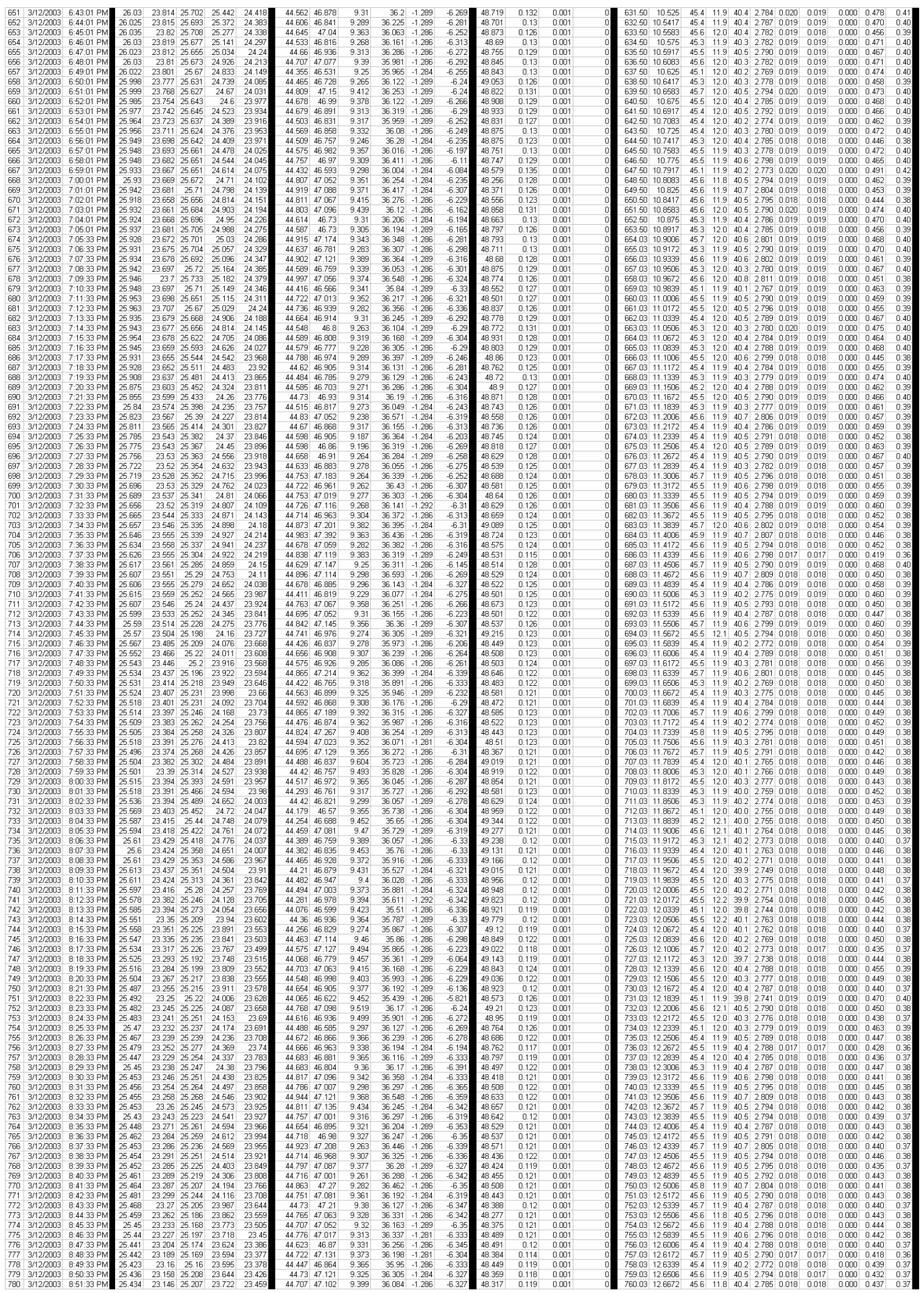

Figure D8-6: Dewatering of the AN-102R2, batch 4A, insoluble solids concentrations from 1.5 to $81 \mathrm{wt} \%$ Raw \& Calculated Data 
WSRC-TR-2003-00204, REV. 0

SRT-RPP-2003-00087, REV. 0

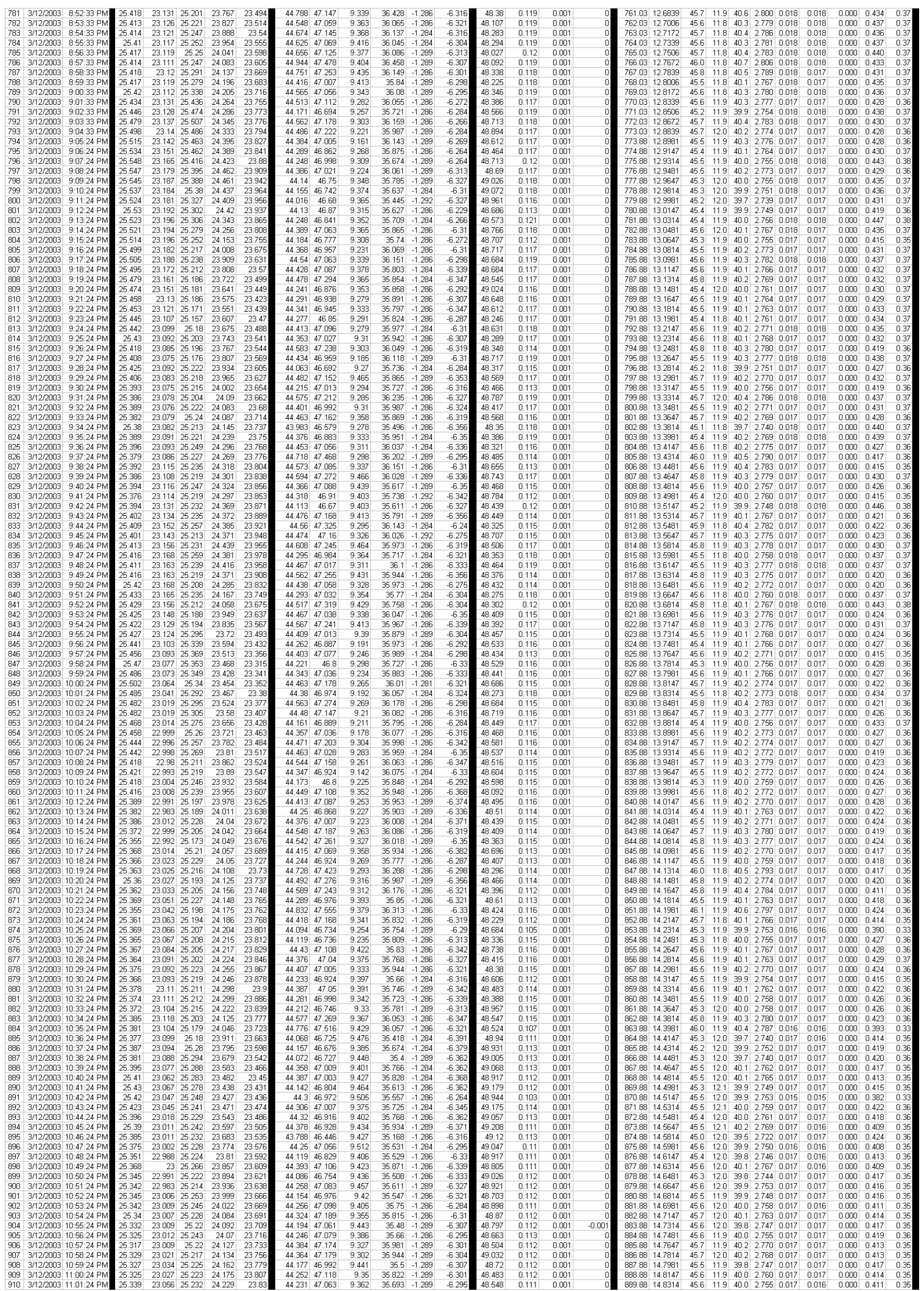

Figure D8-7: Dewatering of the AN-102R2, batch 4A, insoluble solids concentrations from 1.5 to $81 \mathrm{wt} \%$ Raw \& Calculated Data 
WSRC-TR-2003-00204, REV. 0

SRT-RPP-2003-00087, REV. 0
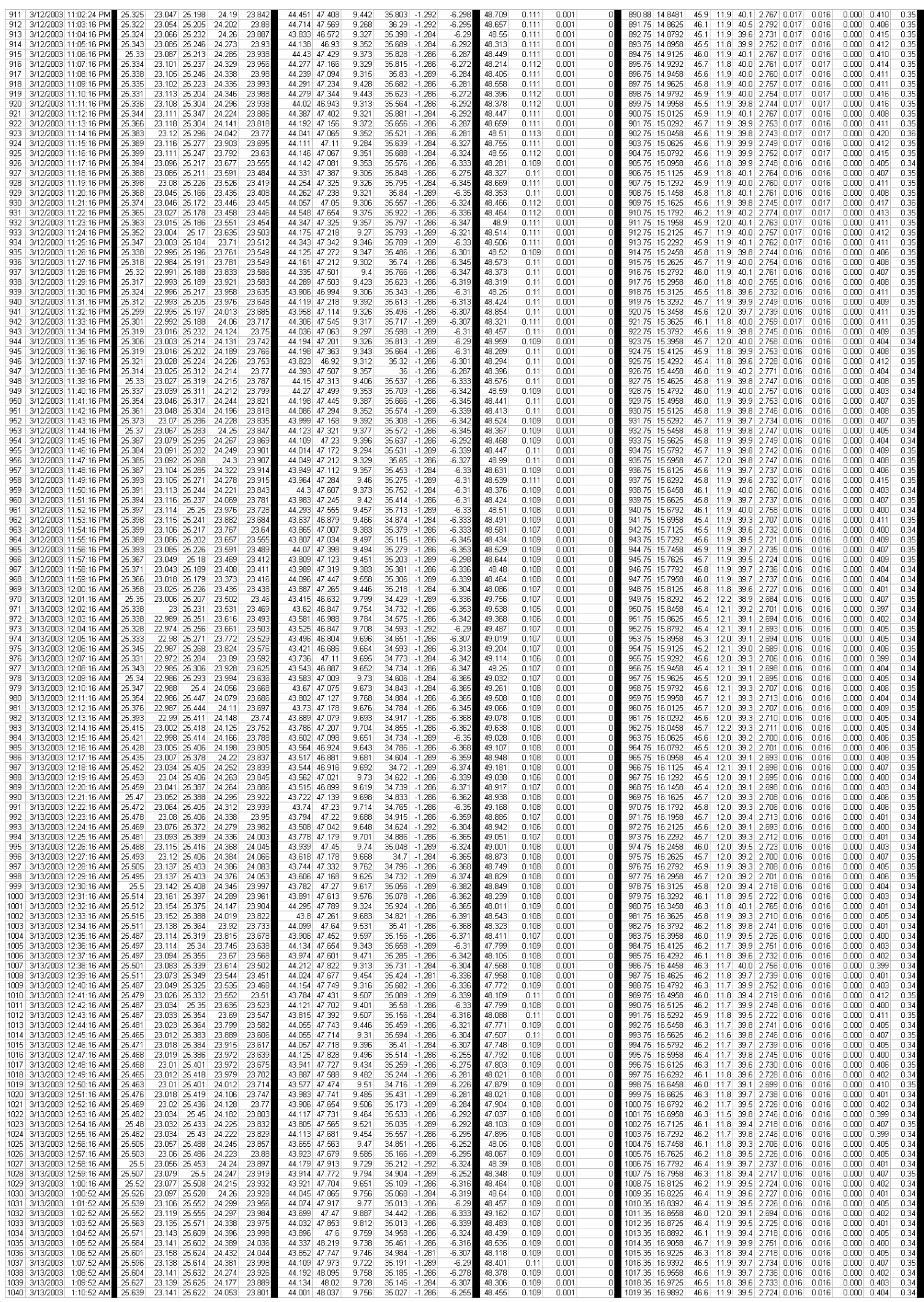

Figure D8-8: Dewatering of the AN-102R2, batch 4A, insoluble solids concentrations from 1.5 to $81 \mathrm{wt} \%$ Raw \& Calculated Data 
WSRC-TR-2003-00204, REV. 0

SRT-RPP-2003-00087, REV. 0
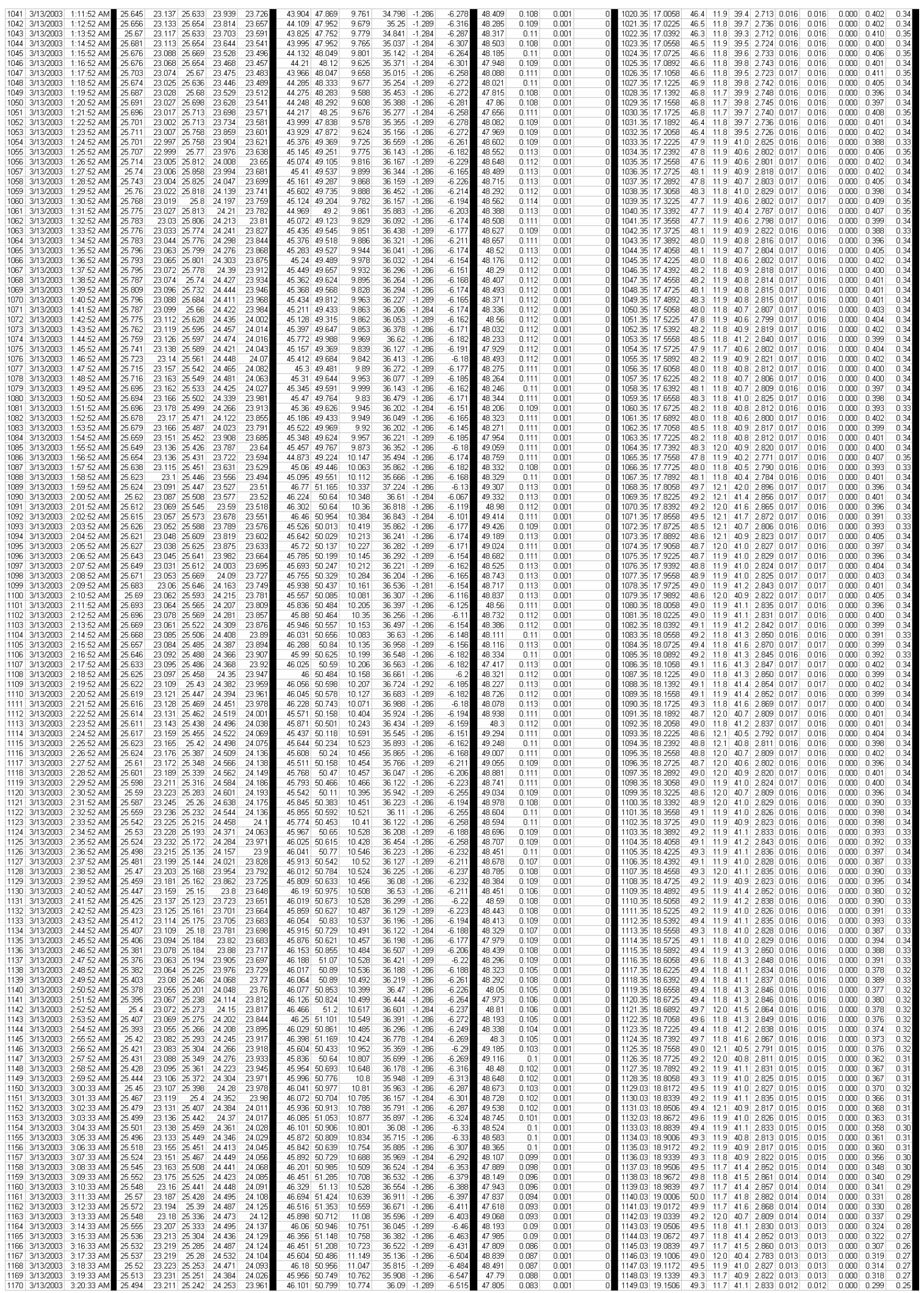

Figure D8-9: Dewatering of the AN-102R2, batch 4A, insoluble solids concentrations from 1.5 to $81 \mathrm{wt} \%$ Raw \& Calculated Data 
WSRC-TR-2003-00204, REV. 0 SRT-RPP-2003-00087, REV. 0

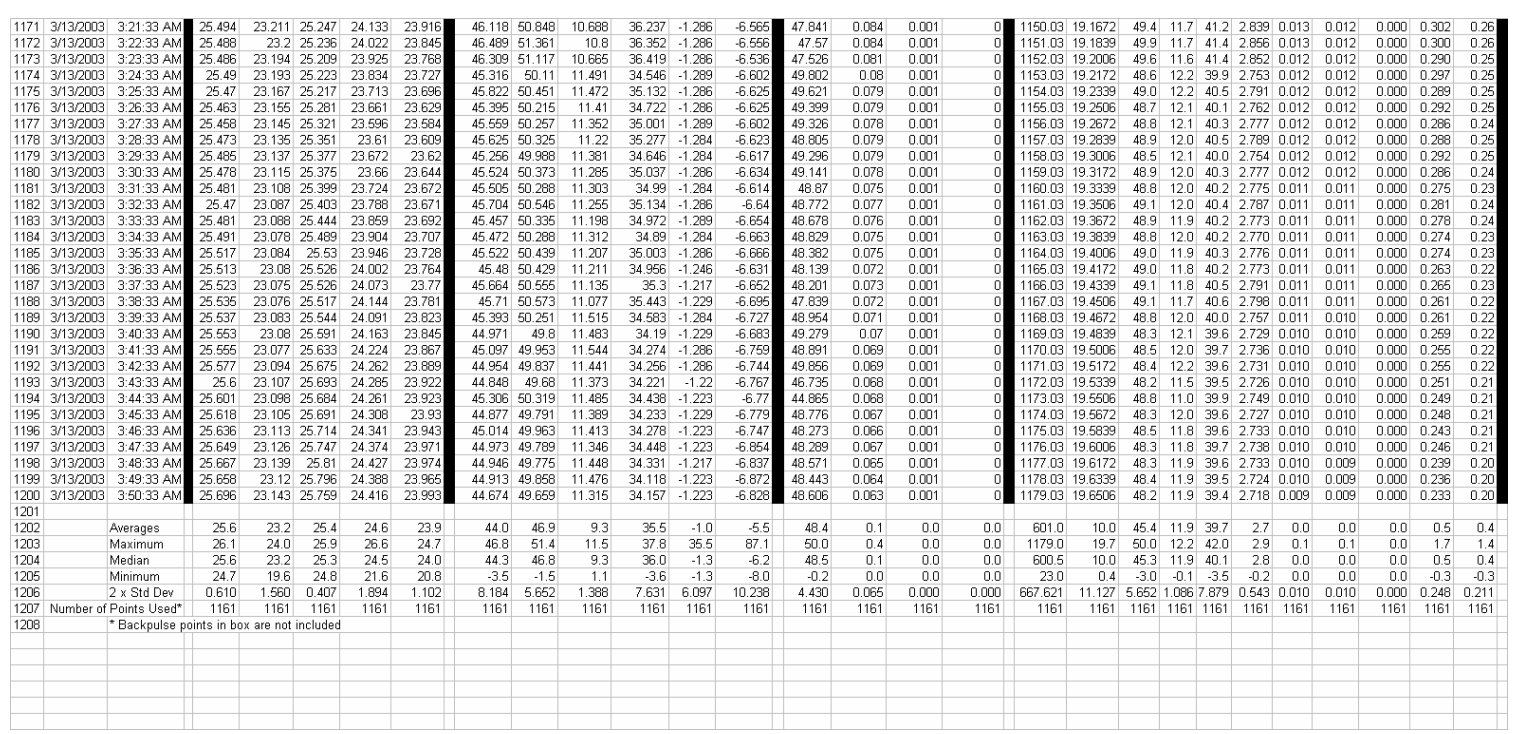

Figure D8-10: Dewatering of the AN-102R2, batch 4A, insoluble solids concentrations from 1.5 to $81 \mathrm{wt} \%$ - Raw \& Calculated Data 


\section{Appendix E: Steady-state Filtering, Test Runs 1.17 - 1.29 - AN-102R2}

After each dewatering test run (run 1.16 of Batches 3 and 4) the filter was to be operated under steady state insoluble solids concentrations. During dewatering the insoluble solids concentration of the test slurry is increased to above $20 \mathrm{wt} \%$, then while maintaining the high concentration constant 13 test runs were carried out. Those thirteen test runs were to vary the slurry axial velocity, $\mathrm{V}$, and transmembrane pressure, TMP, in order to determine the combination that would give the best filtrate flux.

Initially there were to be just two tests using an organic based slurry: Batch 3 and Batch 4 . However, problems with filtering Batch 3 led to reformulating the slurry; therefore it was followed with a Batch 3B and a Batch 3A. The first batch was named Batch 3C. Instead of having two batch it turned out to be four: Batches 3C, 3B, 3A, and 4). The Batch 3C dewatering test was not completed because of poor filterability, therefore no steady-state runs were made. The next batch, Batch 3B, went as planned, therefore all the high solids concentration steady-state runs were completed. Batch 3A did not filter as well, therefore the RPP customer did not required steady-state test, but gave permission to carry out some to quantify the filtate flux. The customer also did not require the steady-state runs for Batch 4 .

This Appendix includes the following test runs:

Steady-state test runs with simulant slurry AN-102R2 Batch 3B at insoluble solids of $22 \mathrm{wt} \%$ :

$1.17,1.18,1.19,1.20 \quad$ done on 24 October 2002

$1.21,1.22,1.23,1.24 \quad$ done on 28 October 2002

$1.25,1.26,1.27,1.28,1.29 \quad$ done on 29 October 2002

Steady-state test runs with simulant slurry AN-102R2 Batch $3 A^{*}$ at insoluble solids of 25 wt\%:

$1.17,1.20$

done on 11 November 2002

$1.23,1.25,1.28,1.29$

done on 12 November 2002

*Because the dewatering of Batch 3A did not filter as good as Batch 3B steady state runs were not going to be done for this last Batch 3 slurry, except for a few to establish filtrate fluxes. Eight runs were eventually done due to available time and slurry.

Each dewatering test in this Appendix contains a graph of the filtrate flux with time and tables of the data taken and calculated. To better understand the presented information important aspects of the graphs and tables are explained below:

Graphs - Each graph contains time averages of some of the imporant parameters taken during the run at the top of the figures, i.e. Slurry velocity in the filter tube, V; transmembrane pressure, TMP; slurry pressure at the entrance of the filter tube, Filter Pres; and the slurry temperature, T.

Tables - Each table is divided into raw data (the first 17 columns) and calculations (the last 10 columns) which are based on the raw data. The raw data section is divided into three parts: 


\title{
WSRC-TR-2003-00204, REV. 0
} SRT-RPP-2003-00087, REV. 0

\author{
Individual Entries - Raw Data \\ Temperature $\quad$ Filtrate - from the filtrate loop at the exit of the filter bundle \\ Cleaning - from the cleaning loop (this loop was not used, except for tests Clean $1 \& 2$ ) \\ Slurry - from the slurry loop in the slurry reservoir \\ Hi Amb. - outside ambient temperature at the top of the test rig \\ Lo Amb. - inside ambient temperature at the bottom of the test rig \\ Pressure BotTMP - differential pressure across the filter tube at the bottom of the vertical tube \\ Filter - gauge pressureof the slurry at the entrance of the filter tube bundle \\ Filter $\mathrm{dP}$ - differential pressure of the slurry along the height of the vertical 90-inch tube \\ TopTMP - differential pressure across the filter tube at the top the vertical 90-inch tube \\ Filtrate - gauge pressure of the filtrate at the exit of the filter tube housing \\ Pulsepot - gauge pressure of the filtrate at the bottom of the pulsepot \\ Flow Slurry - rate of slurry just before entering the seven-filter-tube bundle \\ Filtrate - rate of slurry filtrate under normal flow (max. is $1.2 \mathrm{gpm}$ ) \\ Hi Filtrate - rate of any liquid with has a high flowrate (water, acid, etc.) (max. 5 gpm) \\ Backpulse - rate of filtrate in opposite direction from normal flow, i.e., backpulse
}

Individual Entries - Calculated Data

Time - Minutes after start of test, including the backpulse (based on raw data TIME column)

Press. - Internal filter pressure of slurry at the filter bundle entrance (based on raw data Pl column and

corrected for a 40.9-inch water column that exists from the transducer to the point where P1 is measured.)

Vel. - Slurry velocity in filter tube (based on raw data Q1 column and i.d. of the tubes of 0.488 inches)

TMP - Filter transmembrane pressure (based on average of raw data columns dP2 and dP3) in psi.

TMP - Filter transmembrane pressure (based on average of raw data columns dP2 and dP3) in bar.

Filtrate Flux - Filtrate flow rate (raw data columns Q2/Q3) divided by inside filter surface area $(6.87 \mathrm{ft} 2)$

Filtrate Flux @ $25^{\circ} \mathrm{C}$ - Same as Filtrate flux, but correct for temperature with the following multiplier:

$\mathrm{T}($ corrected $)=\mathrm{T} \times \operatorname{Exp}[2500 /((1 / 273+\mathrm{T}))-(1 / 298)]$ as per Test Spec 24590-WTP-TSP-RT-01-029, Rev. 0

Permiability - Filterate flow rate per TMP (based on calculated columns Filtrate Flux @ $25^{\circ} \mathrm{C}$ and TMP)

Permiability x 1000 - Same as the above Permiability, but increase by 1000 to facilitate graphing

Permiability - Same as Permiability but in metric units, meter/day/bar

$\underline{\text { Statistics }}$

At the bottom of the last individual entry there are six rows with some data statistics to better understand the data and their fluctuations. The columns are self explained with titles of Averages, Maximum, Median, and Minimum. The next row 2 x Std Dev, is two times the population standard deviation based on the number of data points used, which is the last row. The Number of Points used generally do not included the points taken during the backpulse sequence of each test. This is explained below.

Raw Data Table Layout

The raw data is generally made up of three sections:

Zeros - These eight rows include approximately 15 to 20 seconds of data taken with the pressure transducers placed in the "zero" mode to determine if any zero drift occurred from one test to the next.

Data-Per Minute - On most of the tables the first six rows of these data are contained in a box which indicate data taken during the backpulse. This is were the filtrate flow is reversed temporarily to knock the filtrate cake of the filter surface. During these first few minutes the filtrate flow is stopped, then reversed, then stopped again, then it is finally returned to normal operation. The data during the backpulse operation were taken with a separate log, therefore those data are marked with a box to note this fact. Most or all of those data points are not counted in the statistics at the bottom of the table. The tables of some of the later tests do not show the boxed data because separate logs of data were not taken, however since a backpulse for each run still occurred only those data were not included in the statistics at the bottom of the page. 
WSRC-TR-2003-00204, REV. 0

SRT-RPP-2003-00087, REV. 0

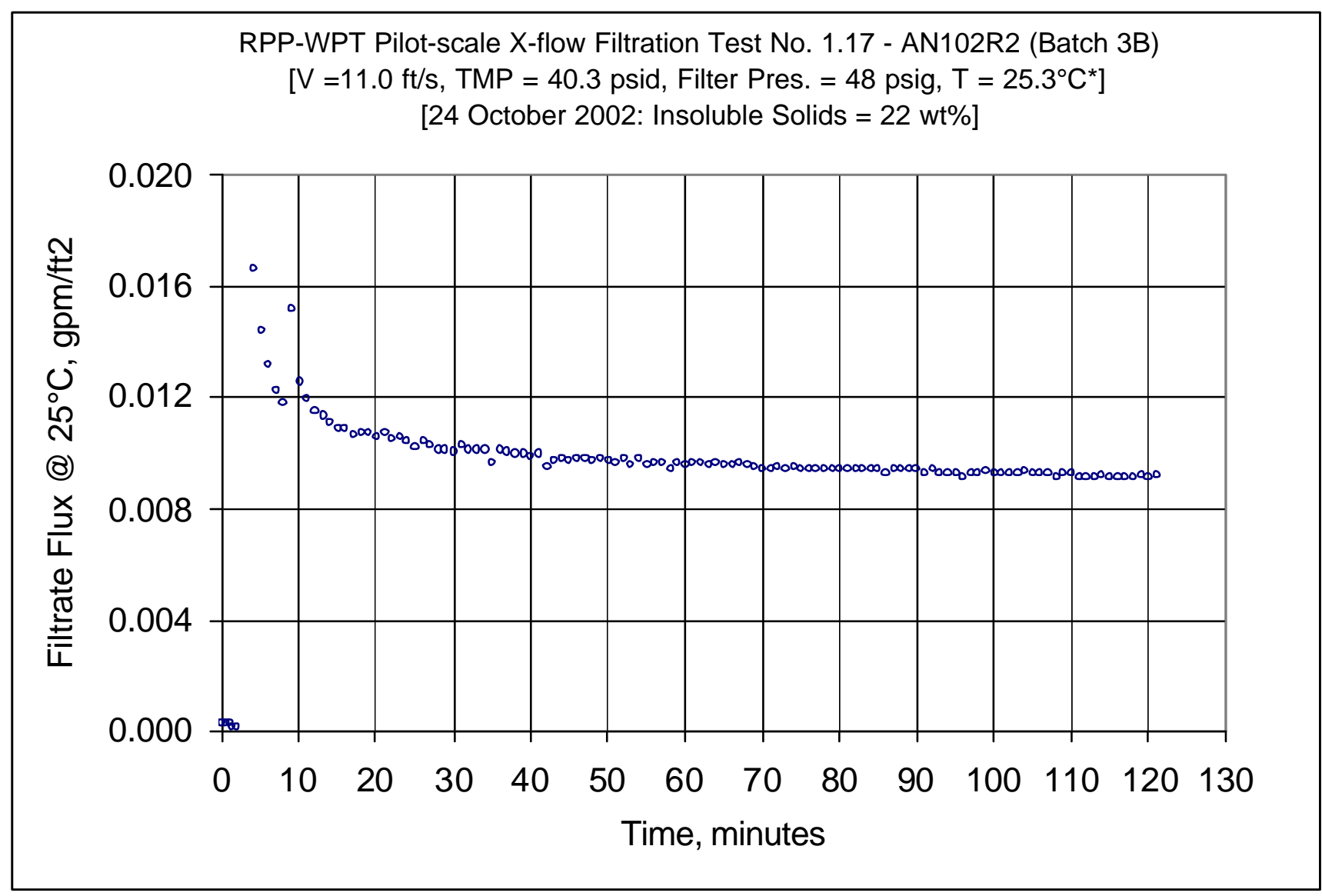

Figure E1: Steady state test run 1.17 of AN-102R2, batch 3B, insoluble solids concentration of $22 \mathrm{wt} \%$ 
WSRC-TR-2003-00204, REV. 0

SRT-RPP-2003-00087, REV. 0

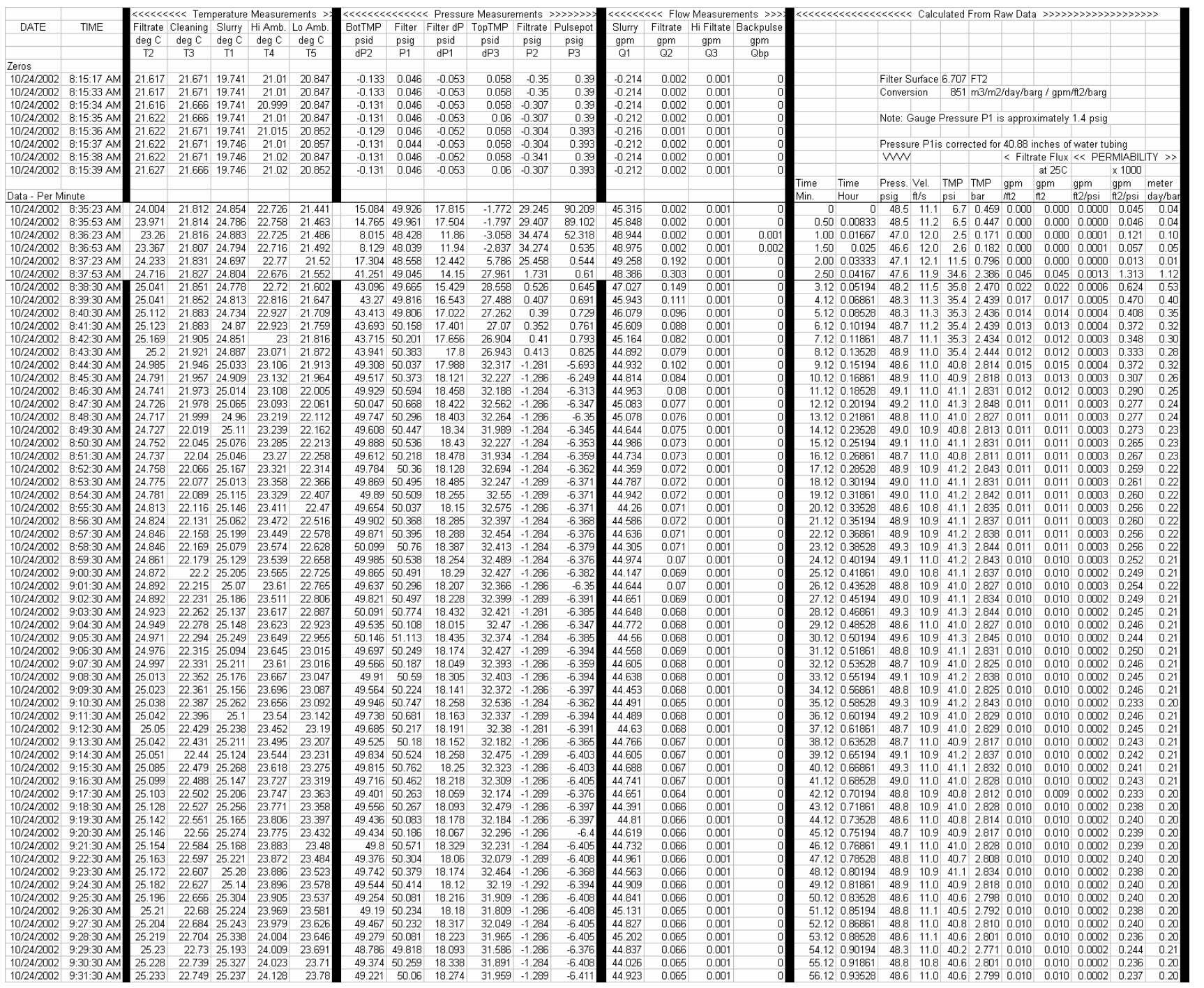

Figure E2-1: Steady state test run 1.17 of AN-102R2, batch 3B, insoluble solids concentration of 22 wt\% - Raw \& Calculated Data 
WSRC-TR-2003-00204, REV. 0

SRT-RPP-2003-00087, REV. 0

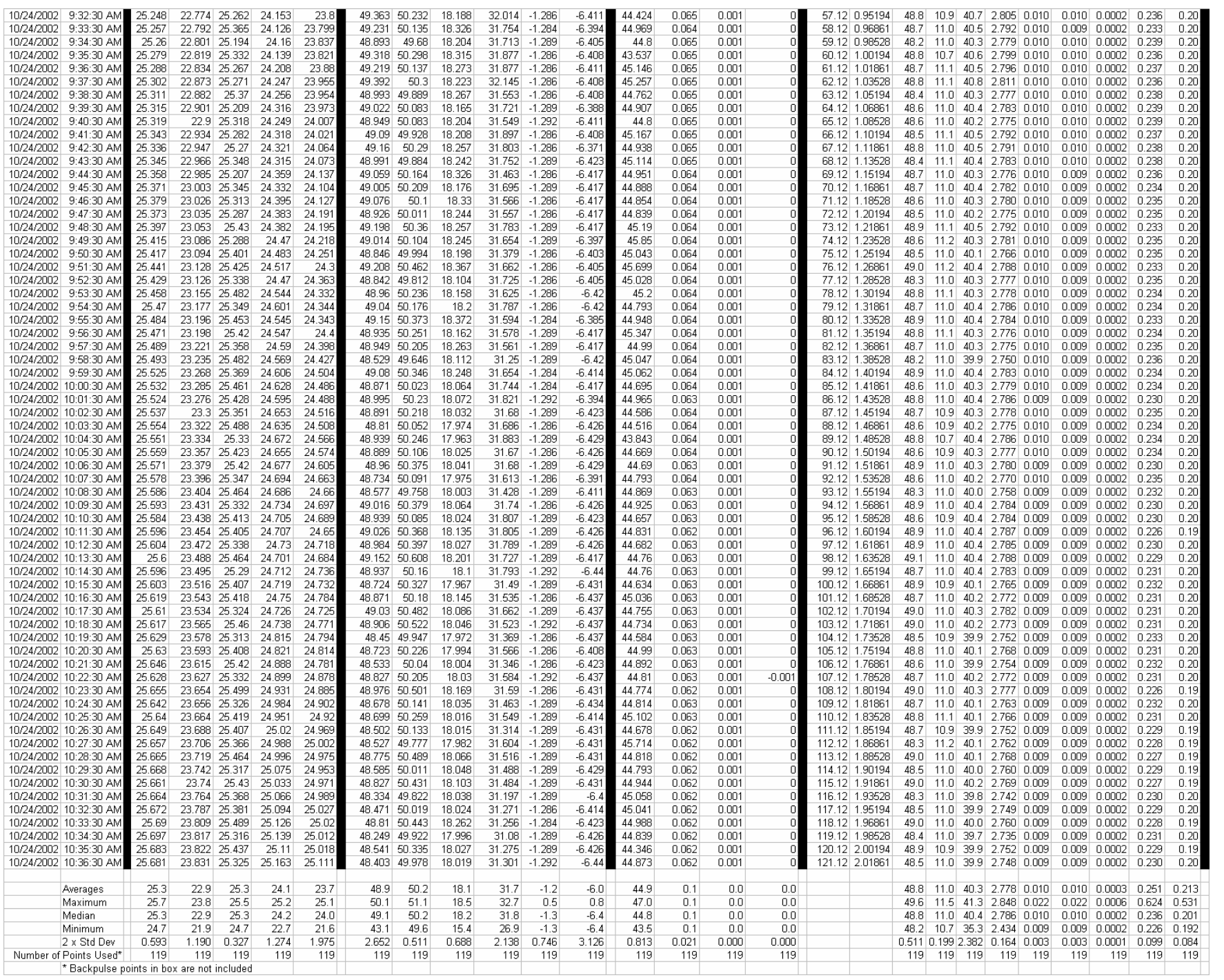

Figure E2-2: Steady state test run 1.17 of AN-102R2, batch 3B, insoluble solids concentration of 22 wt $\%$ - Raw \& Calculated Data 
WSRC-TR-2003-00204, REV. 0

SRT-RPP-2003-00087, REV. 0

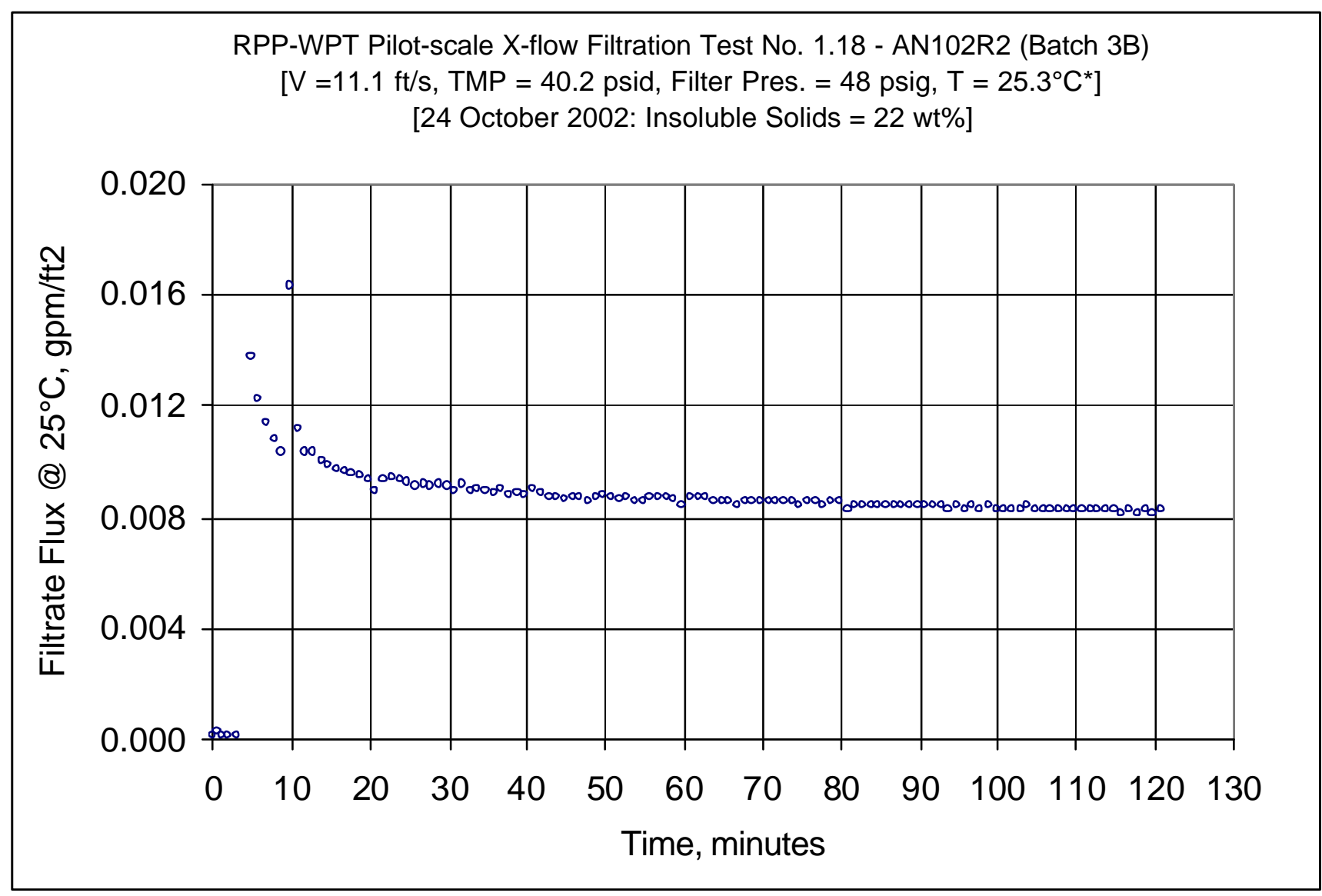

Figure E3: Steady state test run 1.18 of AN-102R2, batch 3B, insoluble solids concentration of 22 wt $\%$ 
WSRC-TR-2003-00204, REV. 0

SRT-RPP-2003-00087, REV. 0

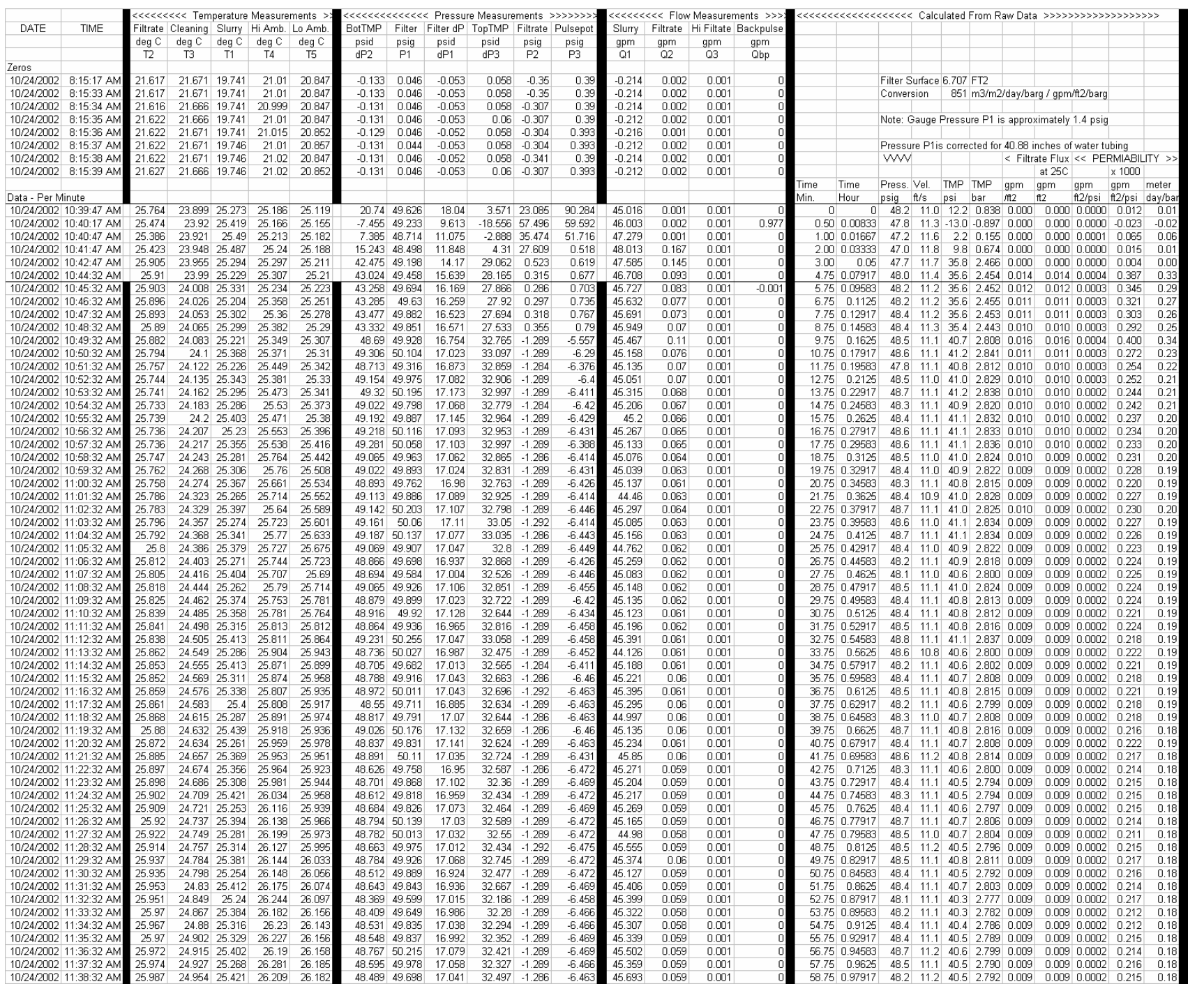

Figure E4-1: Steady state test run 1.18 of AN-102R2, batch 3B, insoluble solids concentration of 22 wt\% - Raw \& Calculated Data 
WSRC-TR-2003-00204, REV. 0

SRT-RPP-2003-00087, REV. 0

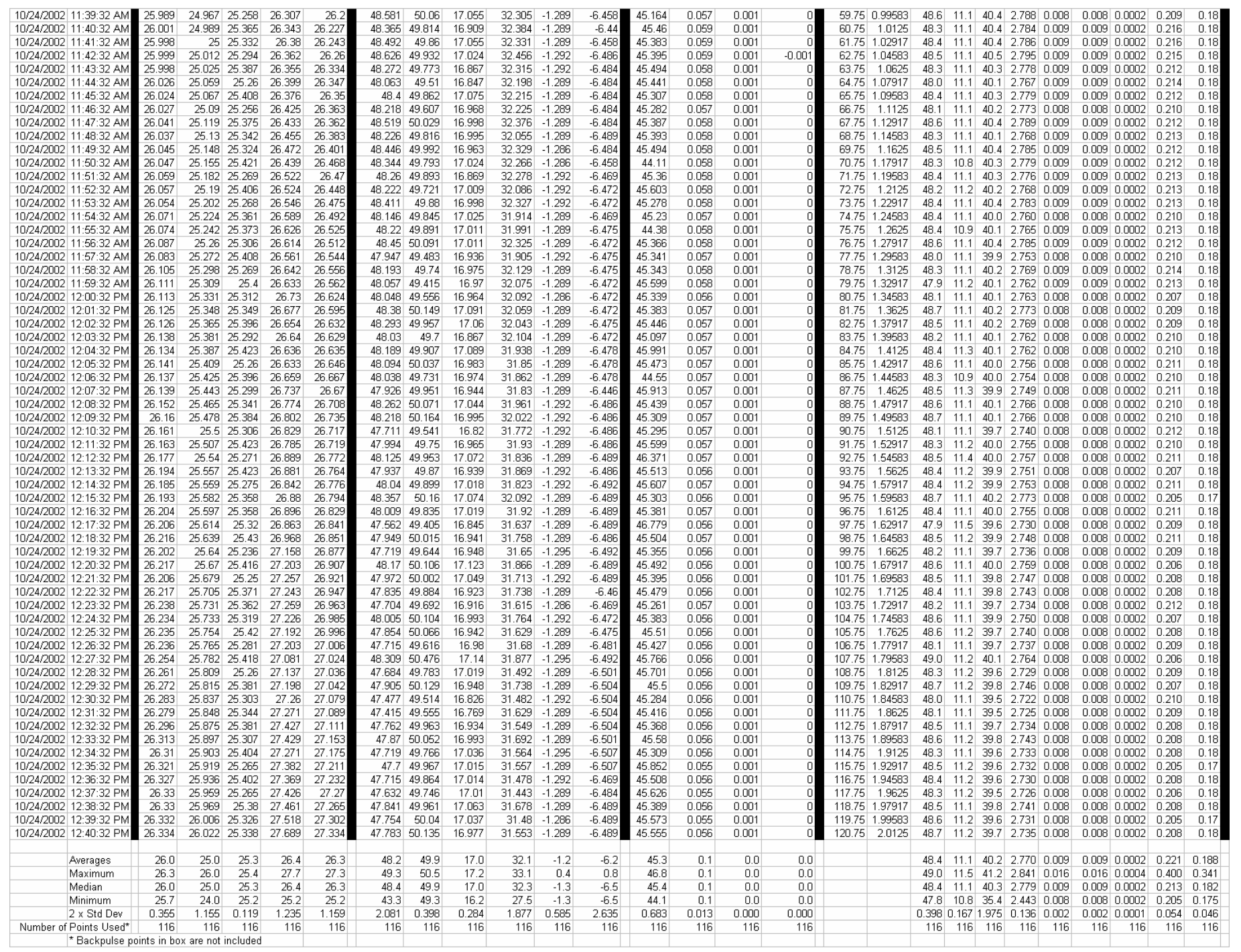

Figure E4-2: Steady state test run 1.18 of AN-102R2, batch 3B, insoluble solids concentration of $22 \mathrm{wt} \%$ - Raw \& Calculated Data 
WSRC-TR-2003-00204, REV. 0

SRT-RPP-2003-00087, REV. 0

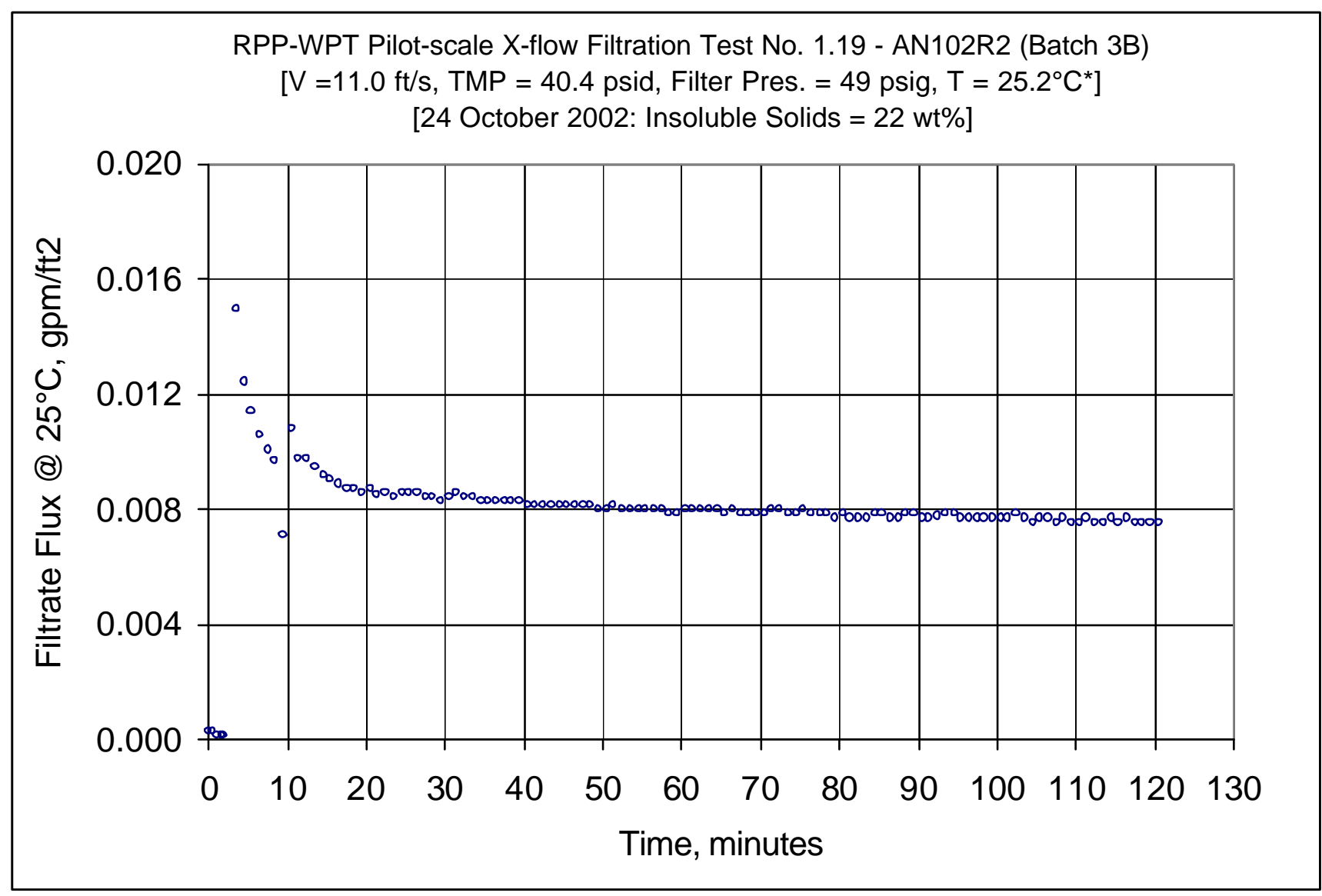

Figure E5: Steady state test run 1.19 of AN-102R2, batch 3B, insoluble solids concentration of $22 \mathrm{wt} \%$ 
WSRC-TR-2003-00204, REV. 0

SRT-RPP-2003-00087, REV. 0

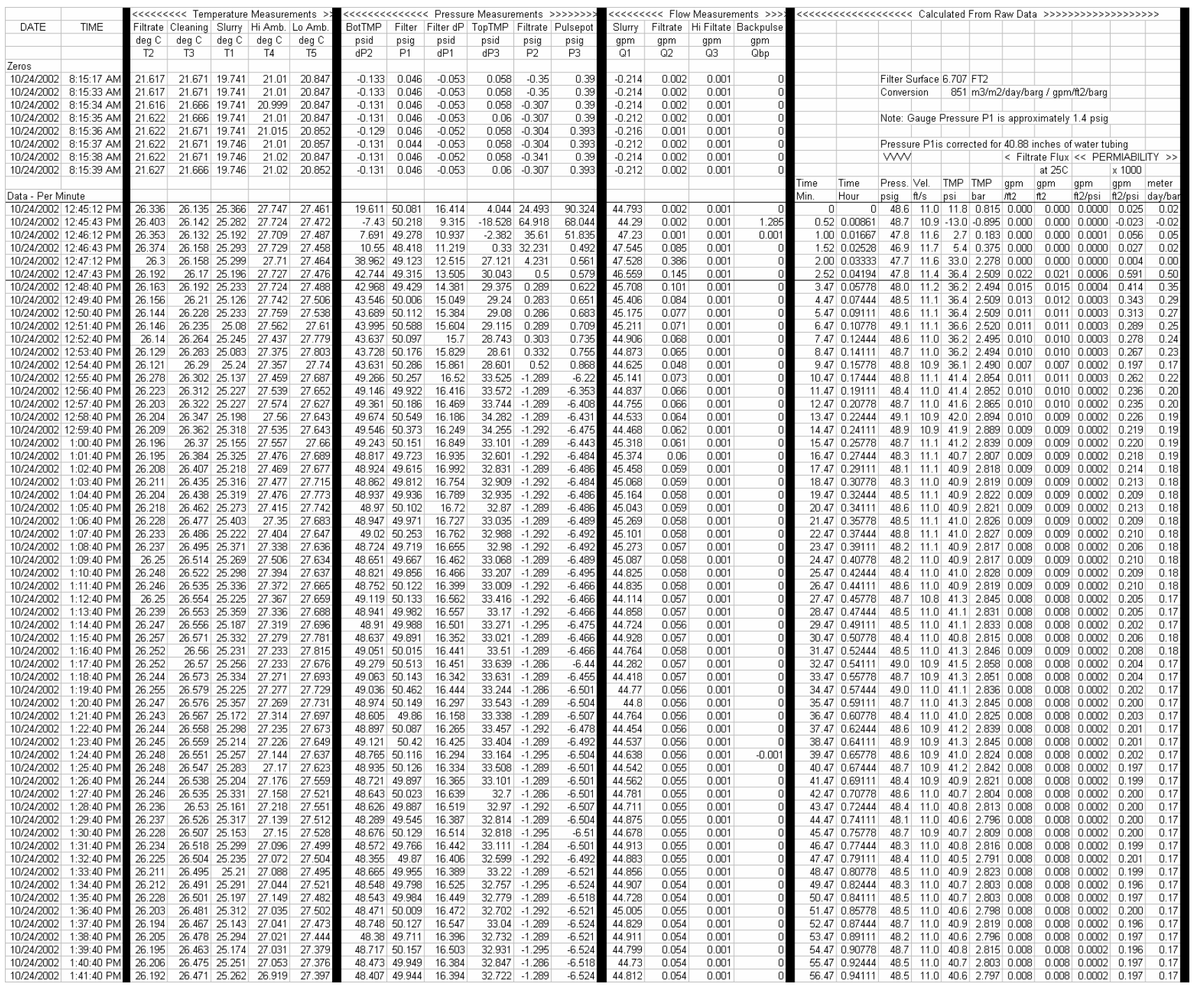

Figure E6-1: Steady state test run 1.18 of AN-102R2, batch 3B, insoluble solids concentration of 22 wt\% - Raw \& Calculated Data 
WSRC-TR-2003-00204, REV. 0

SRT-RPP-2003-00087, REV. 0

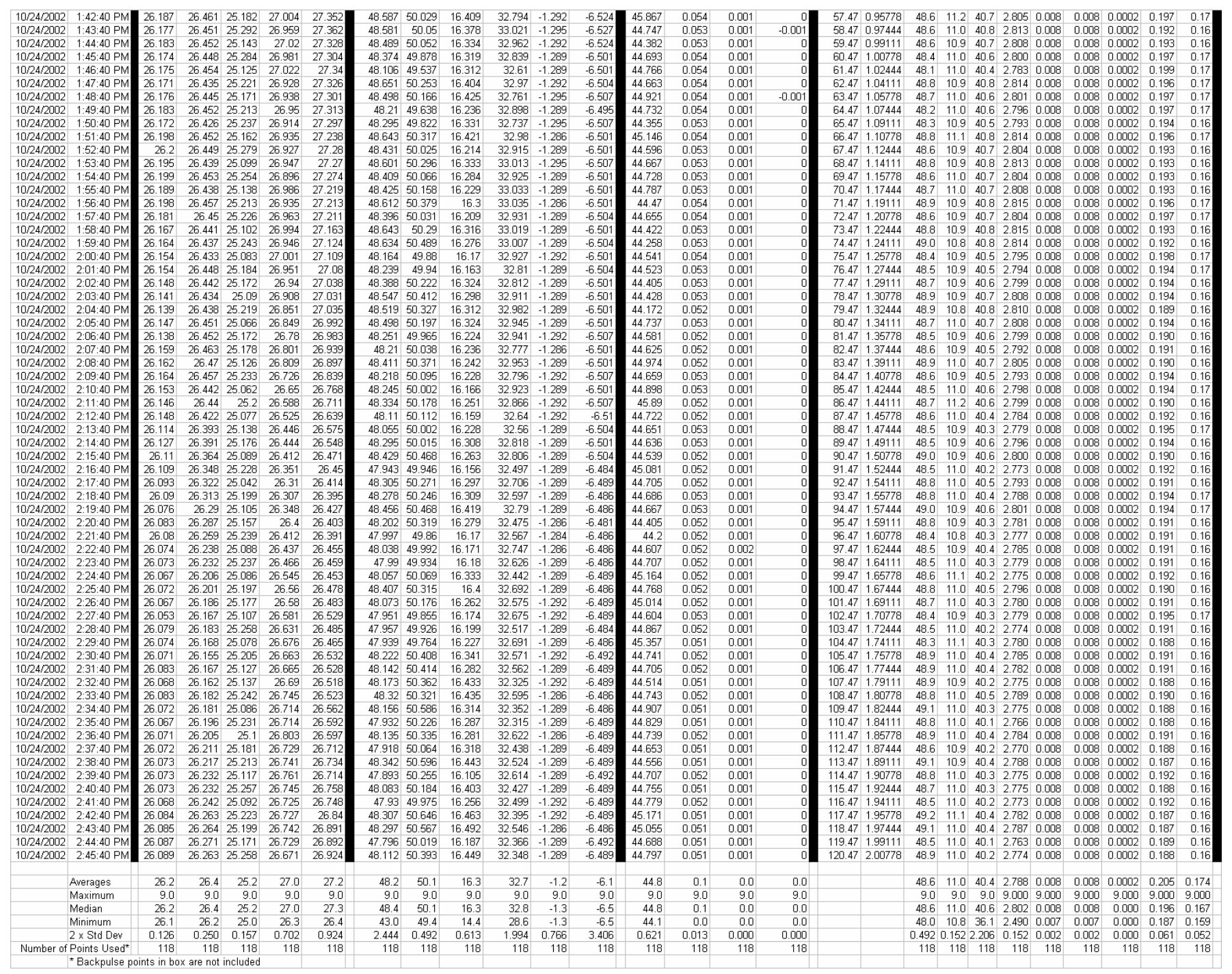

Figure E6-2: Steady state test run 1.18 of AN-102R2, batch 3B, insoluble solids concentration of 22 wt\% - Raw \& Calculated Data 
WSRC-TR-2003-00204, REV. 0

SRT-RPP-2003-00087, REV. 0

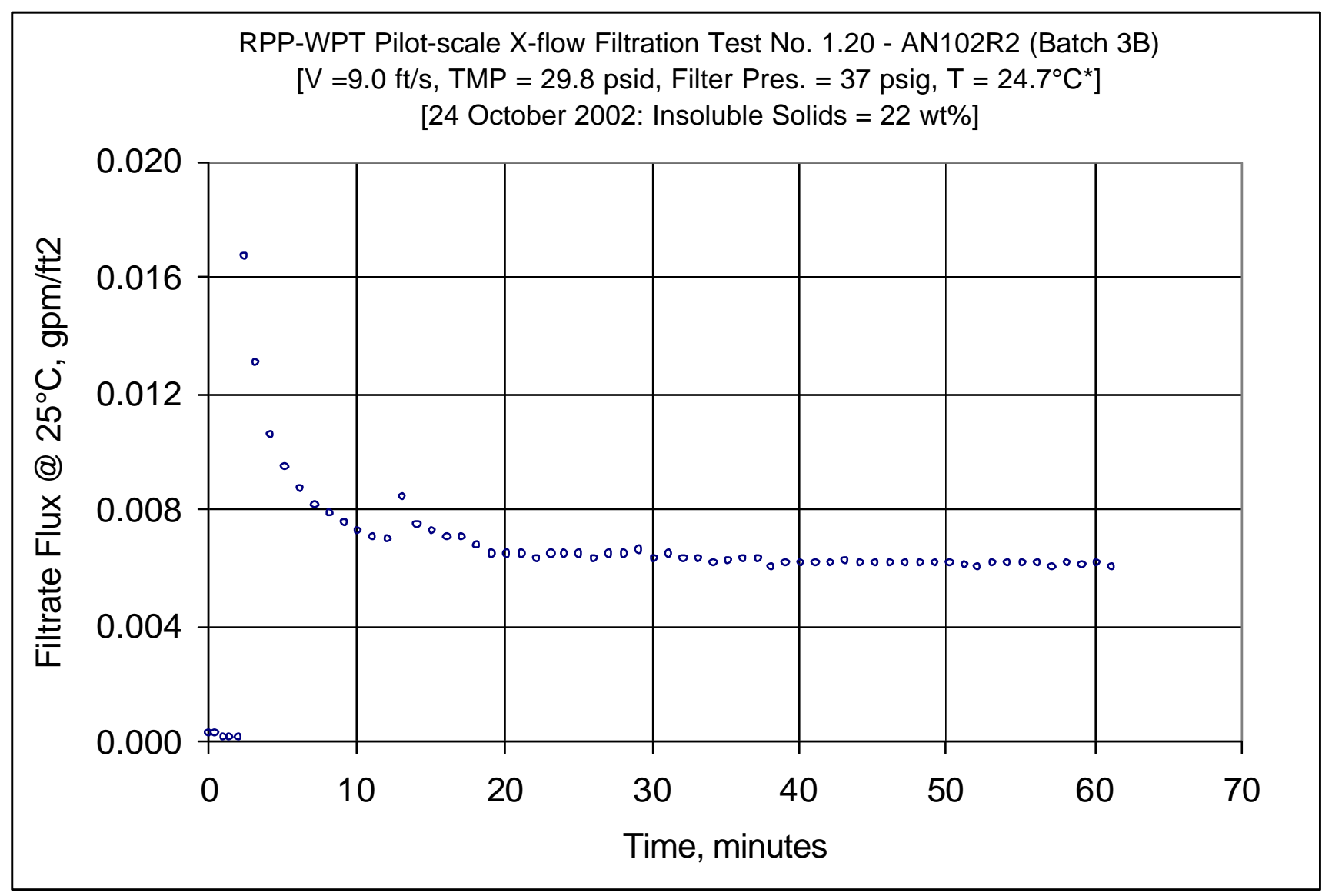

Figure E7: Steady state test run 1.20 of AN-102R2, batch 3B, insoluble solids concentration of $22 \mathrm{wt} \%$ 
WSRC-TR-2003-00204, REV. 0

SRT-RPP-2003-00087, REV. 0

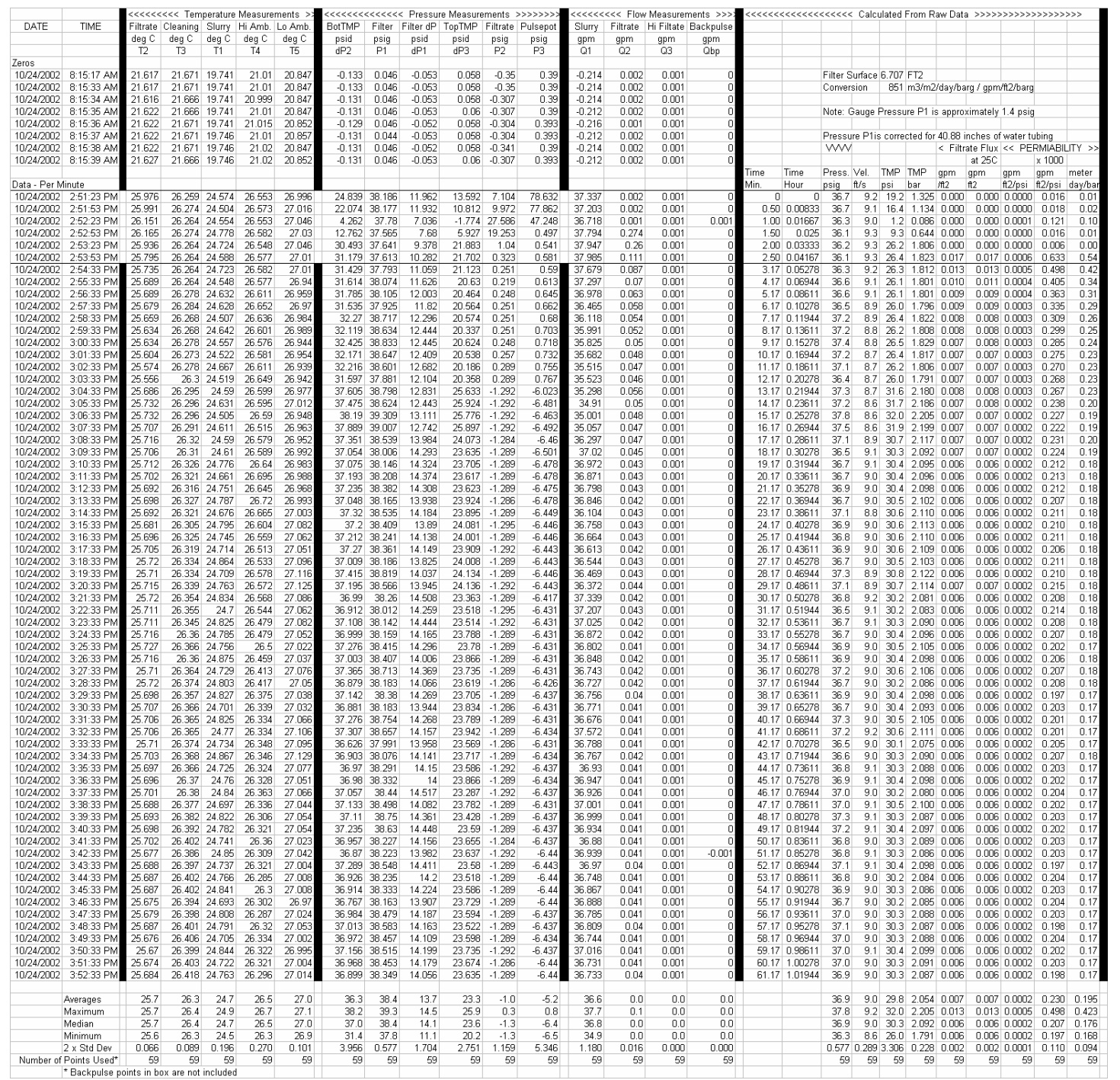

Figure E8: Steady state test run 1.20 of AN-102R2, batch 3B, insoluble solids concentration of $22 \mathrm{wt} \%$ - Raw \& Calculated Data 
WSRC-TR-2003-00204, REV. 0

SRT-RPP-2003-00087, REV. 0

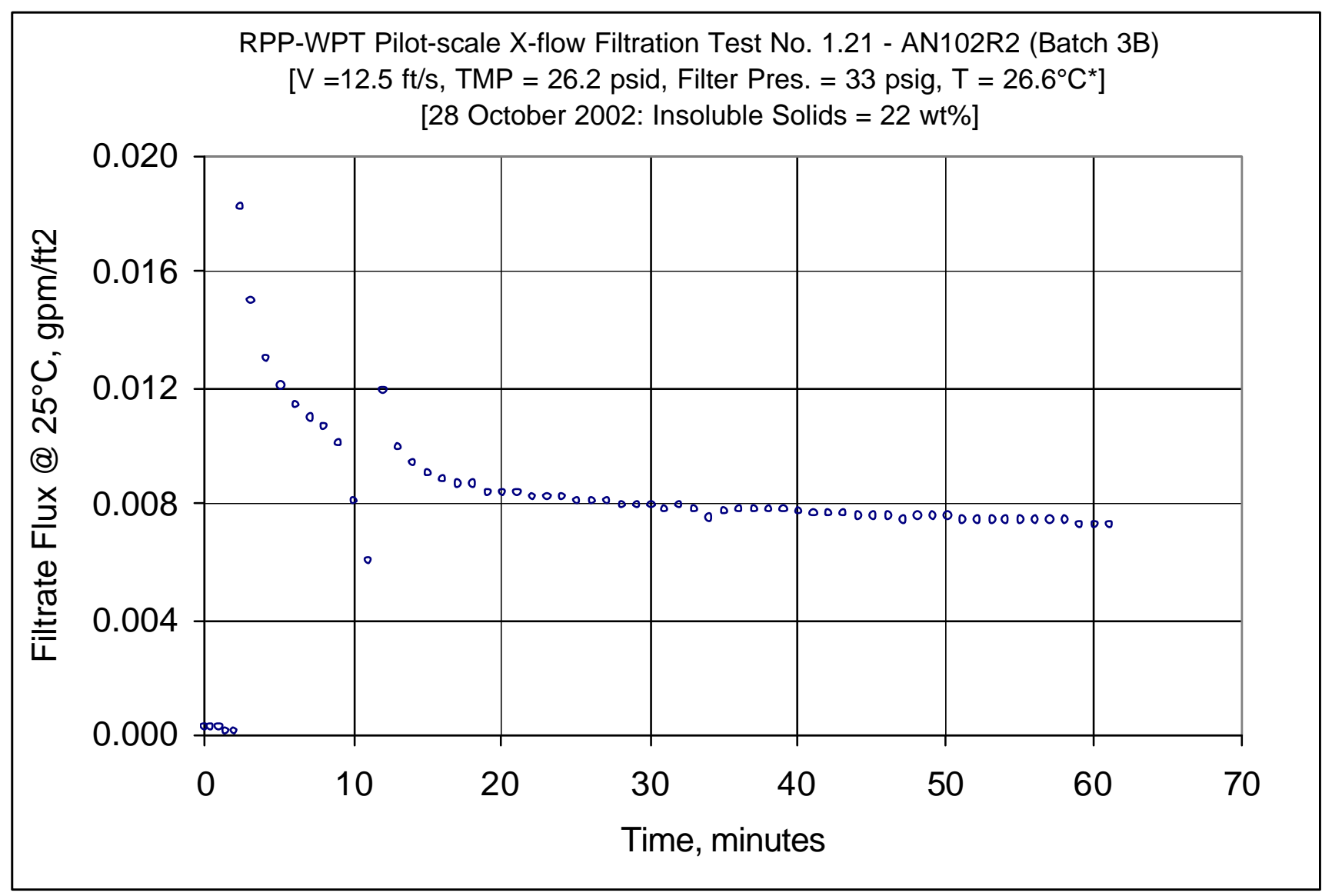

Figure E9: Steady state test run 1.21 of AN-102R2, batch 3B, insoluble solids concentration of 22 wt $\%$ 
WSRC-TR-2003-00204, REV. 0

SRT-RPP-2003-00087, REV. 0

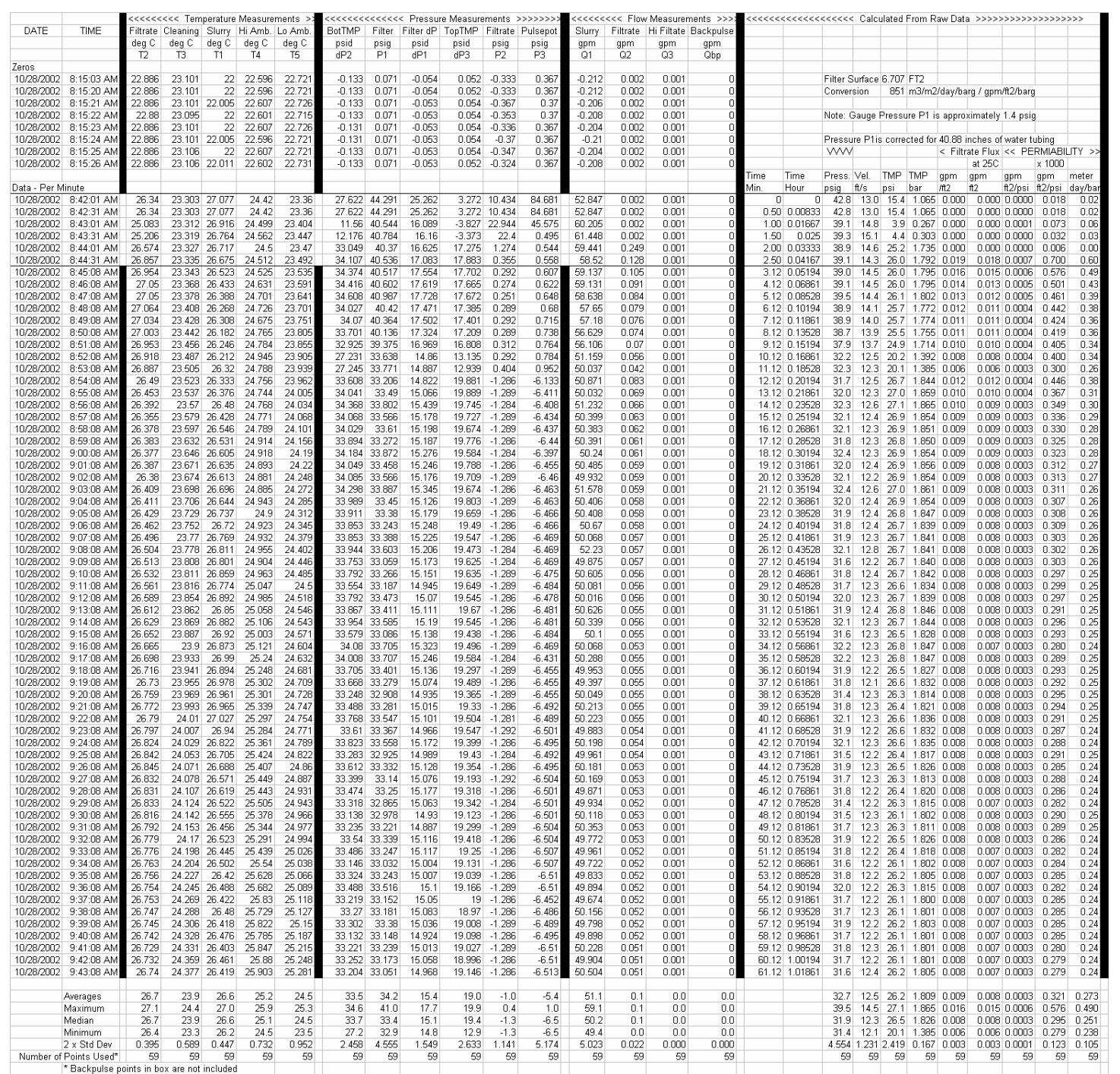

Figure E10: Steady state test run 1.21 of AN-102R2, batch 3B, insoluble solids concentration of 22 wt\% - Raw \& Calculated Data 
WSRC-TR-2003-00204, REV. 0

SRT-RPP-2003-00087, REV. 0

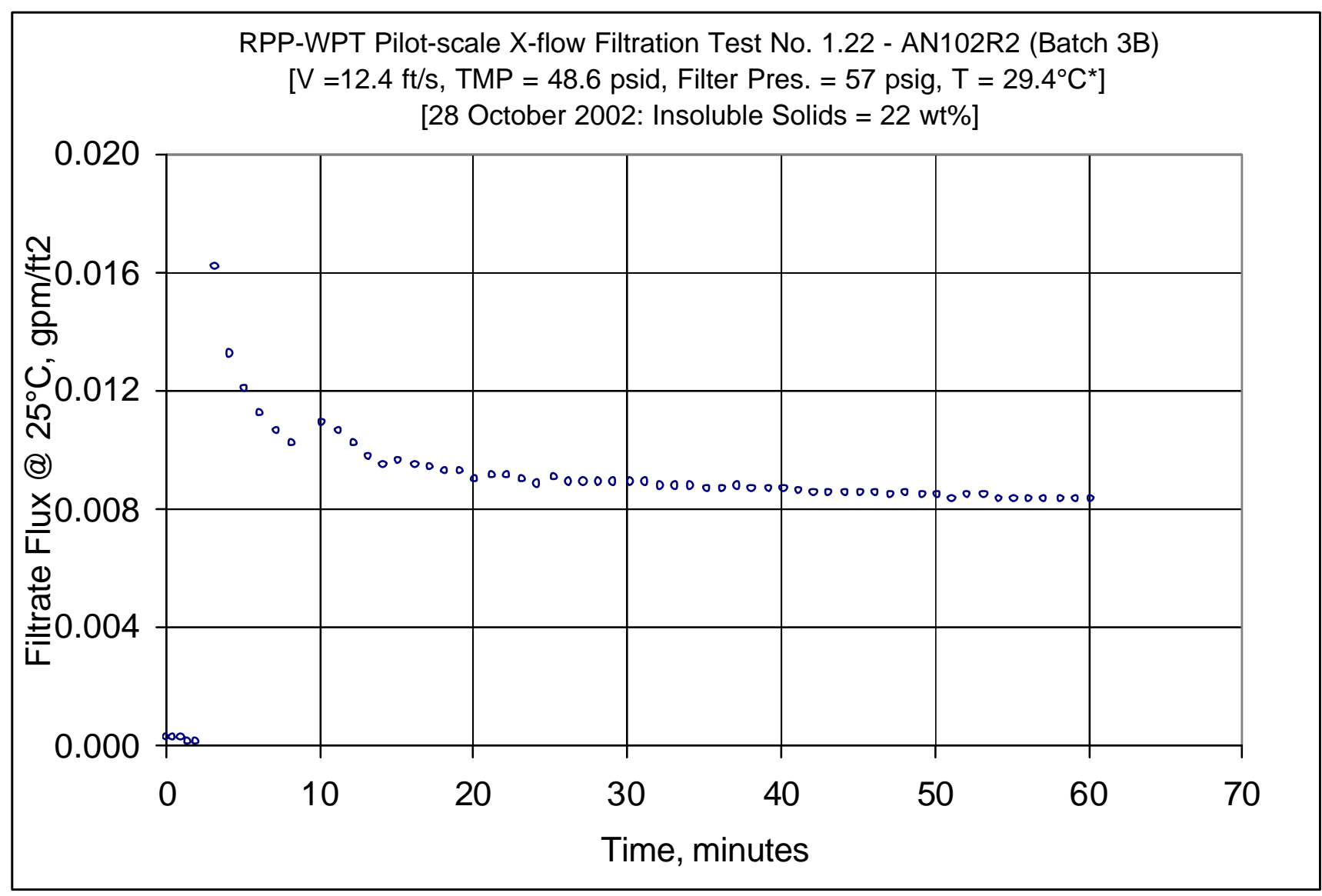

Figure E11: Steady state test run 1.22 of AN-102R2, batch 3B, insoluble solids concentration of 22 wt $\%$ 
WSRC-TR-2003-00204, REV. 0

SRT-RPP-2003-00087, REV. 0

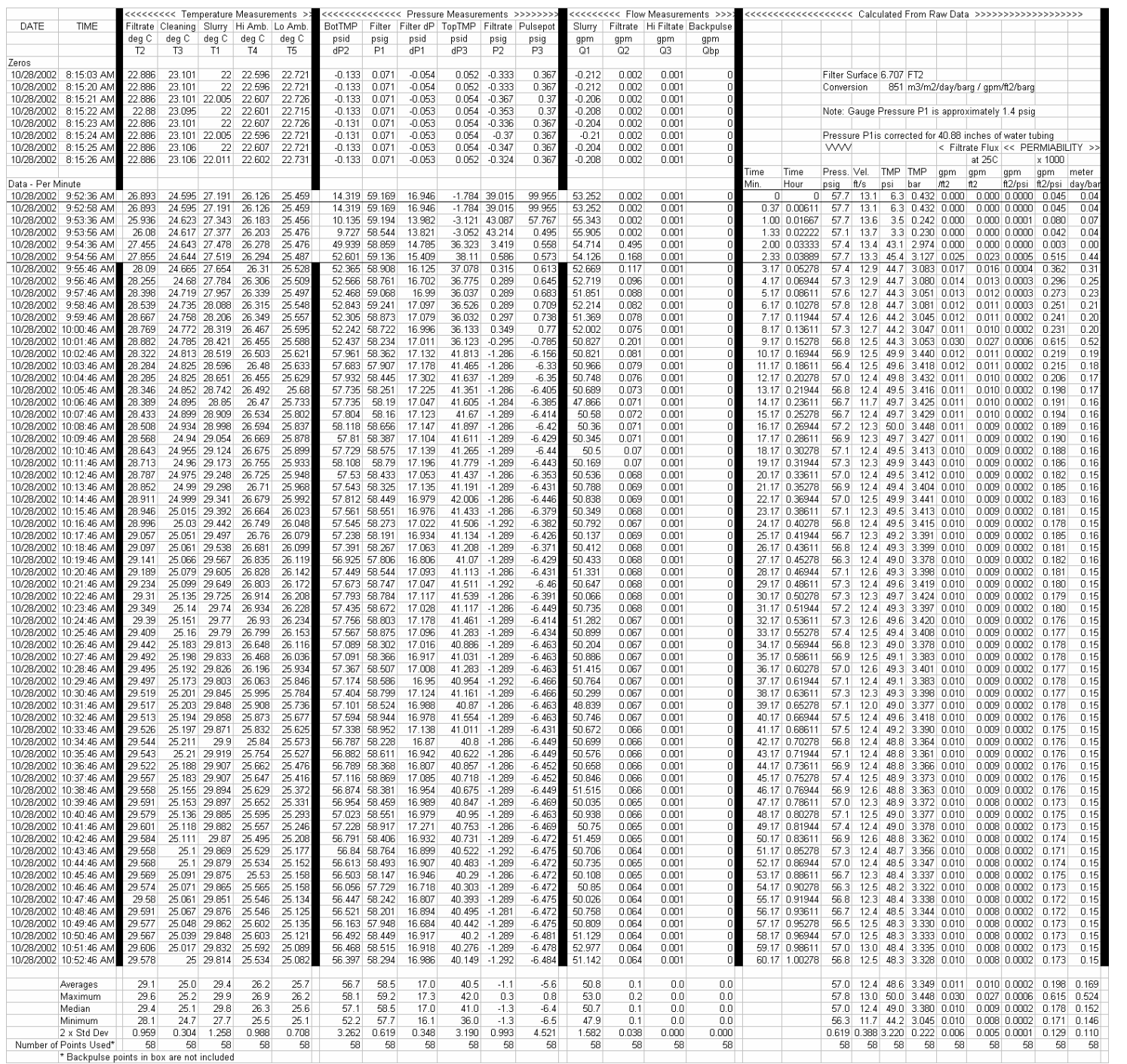

Figure E12: Steady state test run 1.22 of AN-102R2, batch 3B, insoluble solids concentration of 22 wt\% - Raw \& Calculated Data 
WSRC-TR-2003-00204, REV. 0

SRT-RPP-2003-00087, REV. 0

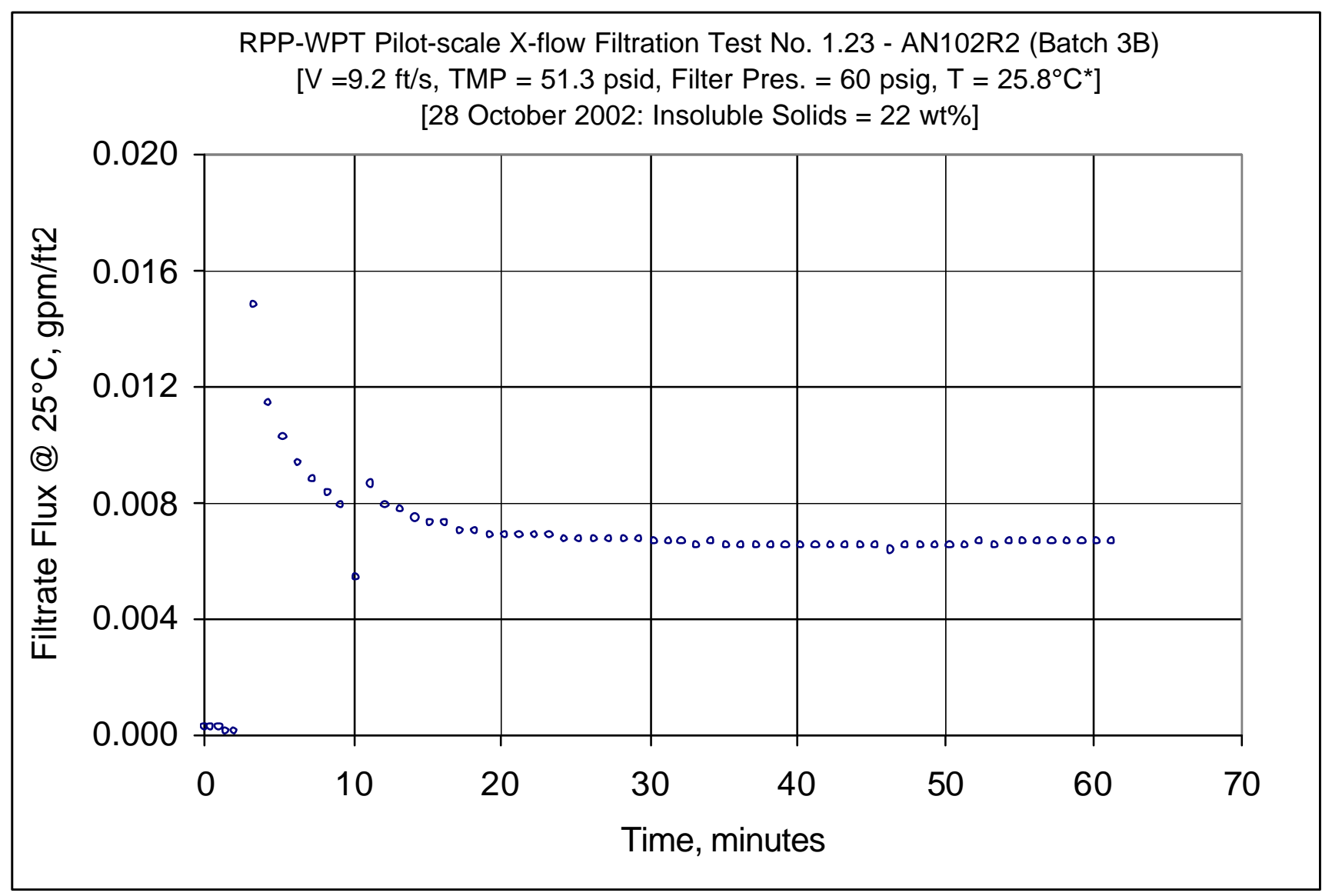

Figure E13: Steady state test run 1.23 of AN-102R2, batch 3B, insoluble solids concentration of $22 \mathrm{wt} \%$ 
WSRC-TR-2003-00204, REV. 0

SRT-RPP-2003-00087, REV. 0

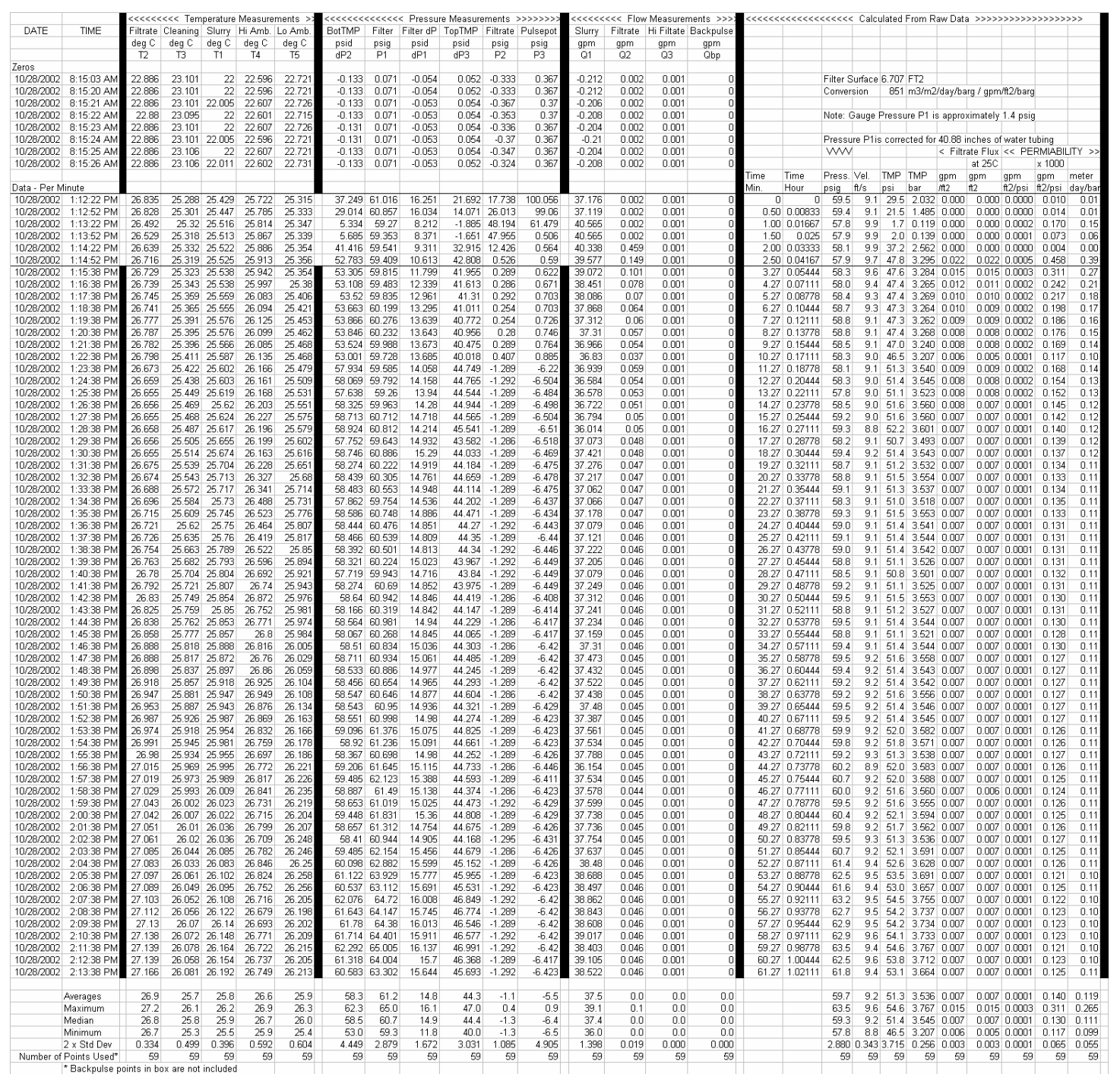

Figure E14: Steady state test run 1.23 of AN-102R2, batch 3B, insoluble solids concentration of 22 wt\% - Raw \& Calculated Data 
WSRC-TR-2003-00204, REV. 0

SRT-RPP-2003-00087, REV. 0

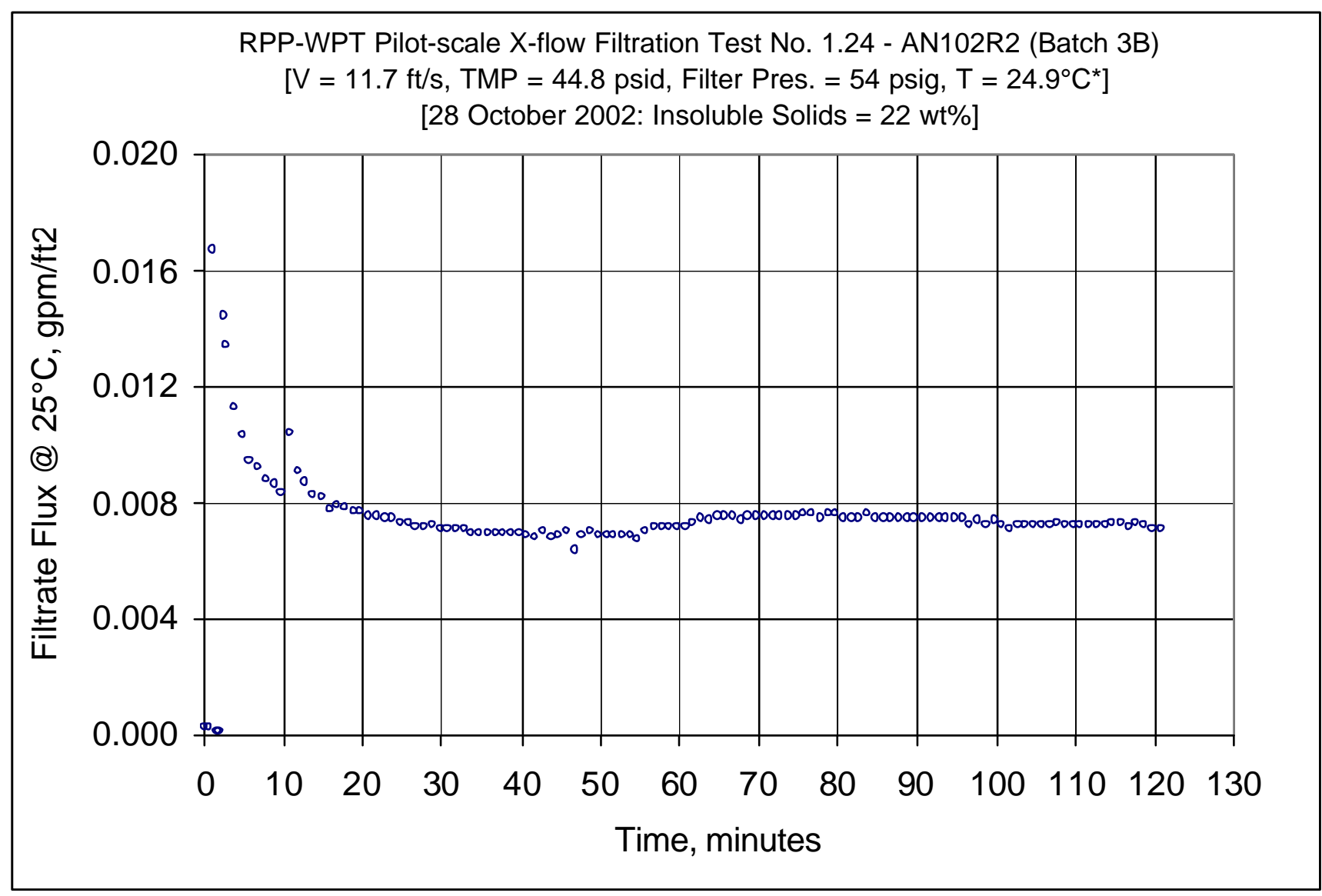

Figure E15: Steady state test run 1.24 of AN-102R2, batch 3B, insoluble solids concentration of $22 \mathrm{wt} \%$ 
WSRC-TR-2003-00204, REV. 0

SRT-RPP-2003-00087, REV. 0

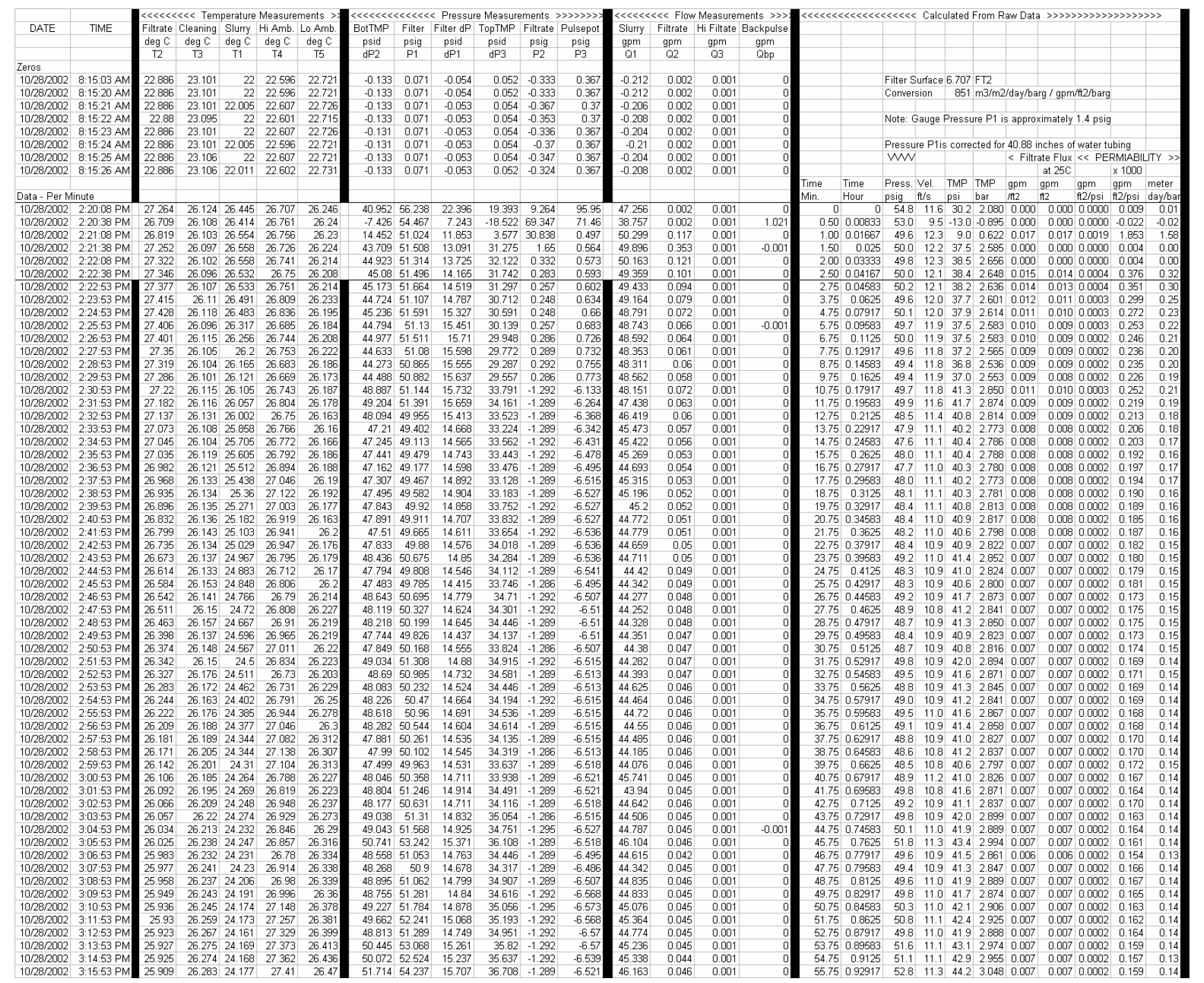

Figure E16-1: Steady state test run 1.24 of AN-102R2, batch 3B, insoluble solids concentration of 22 wt $\%$ - Raw \& Calculated Data 
WSRC-TR-2003-00204, REV. 0

SRT-RPP-2003-00087, REV. 0

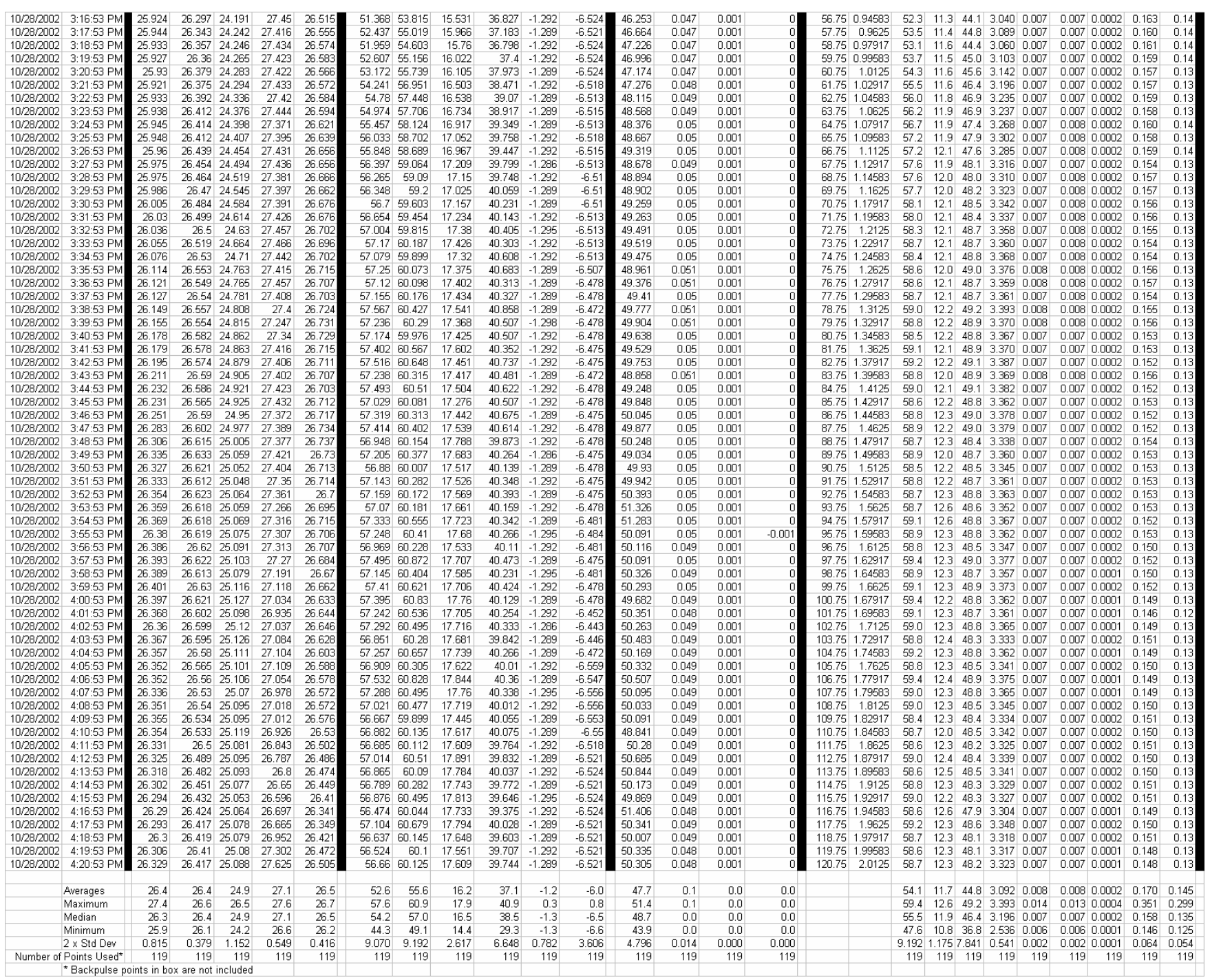

Figure E16-2: Steady state test run 1.24 of AN-102R2, batch 3B, insoluble solids concentration of 22 wt $\%$ - Raw \& Calculated Data 
WSRC-TR-2003-00204, REV. 0

SRT-RPP-2003-00087, REV. 0

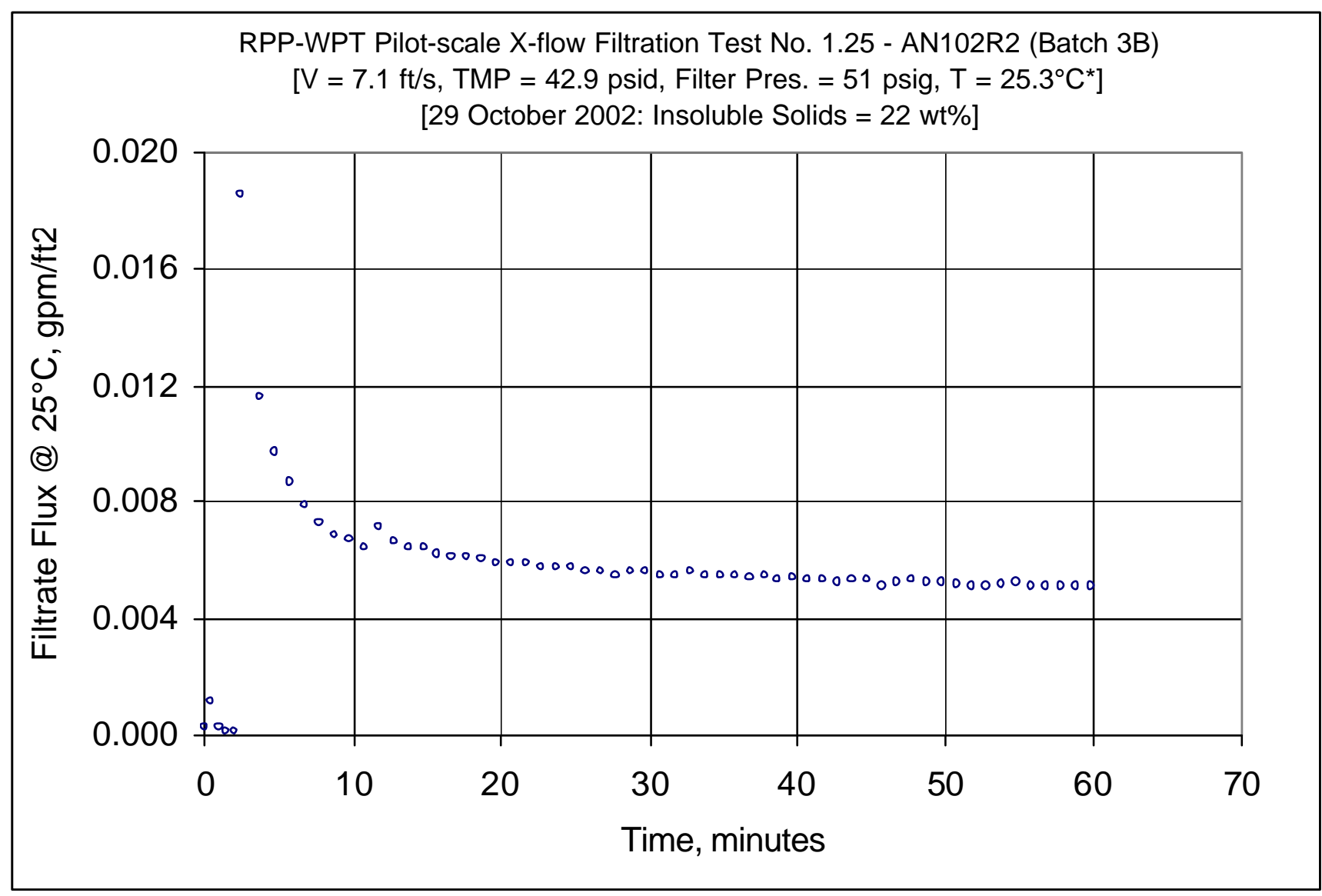

Figure E17: Steady state test run 1.25 of AN-102R2, batch 3B, insoluble solids concentration of $22 \mathrm{wt} \%$ 
WSRC-TR-2003-00204, REV. 0

SRT-RPP-2003-00087, REV. 0

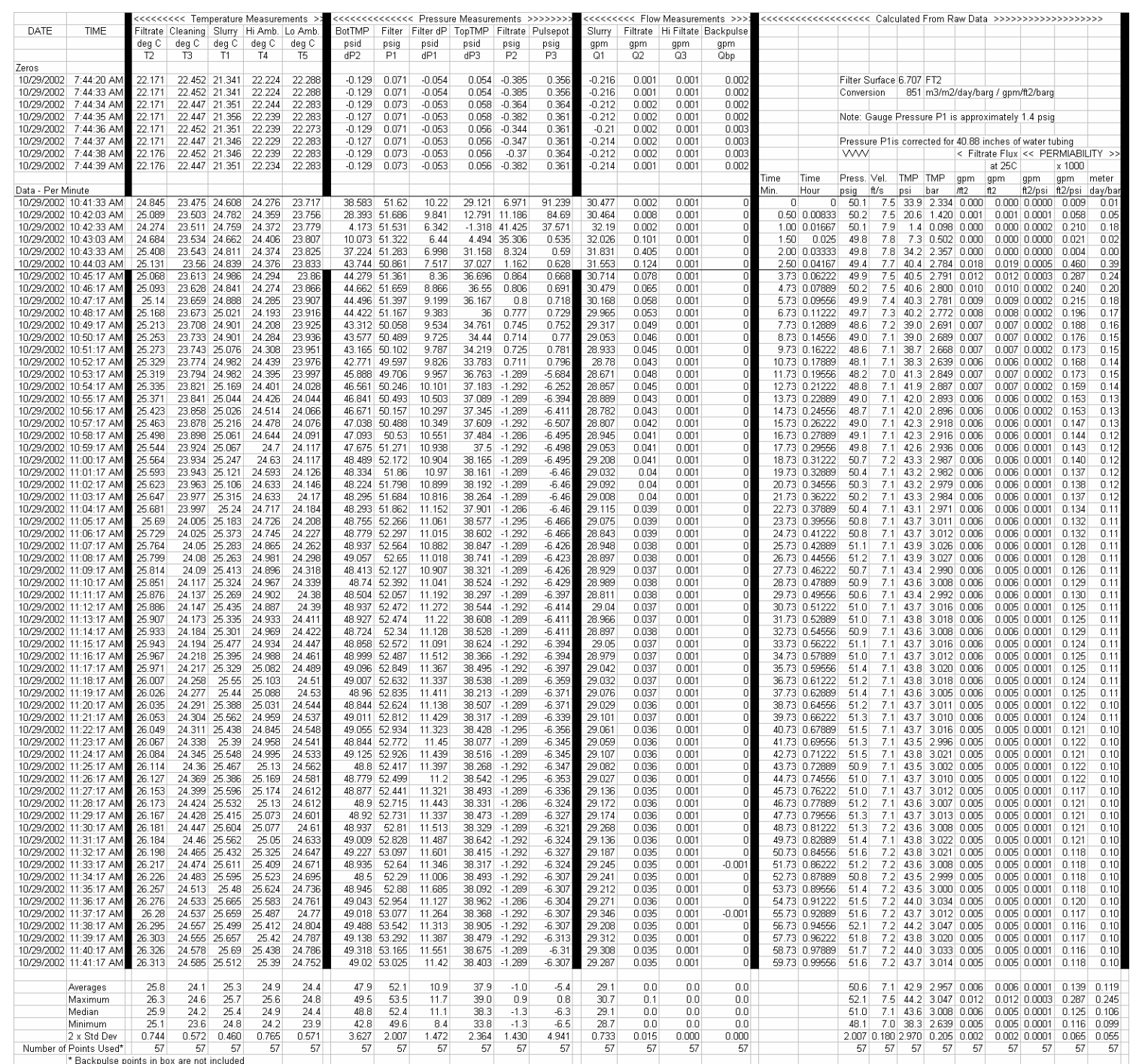

Figure E18: Steady state test run 1.25 of AN-102R2, batch 3B, insoluble solids concentration of $22 \mathrm{wt} \%$ - Raw \& Calculated Data 
WSRC-TR-2003-00204, REV. 0

SRT-RPP-2003-00087, REV. 0

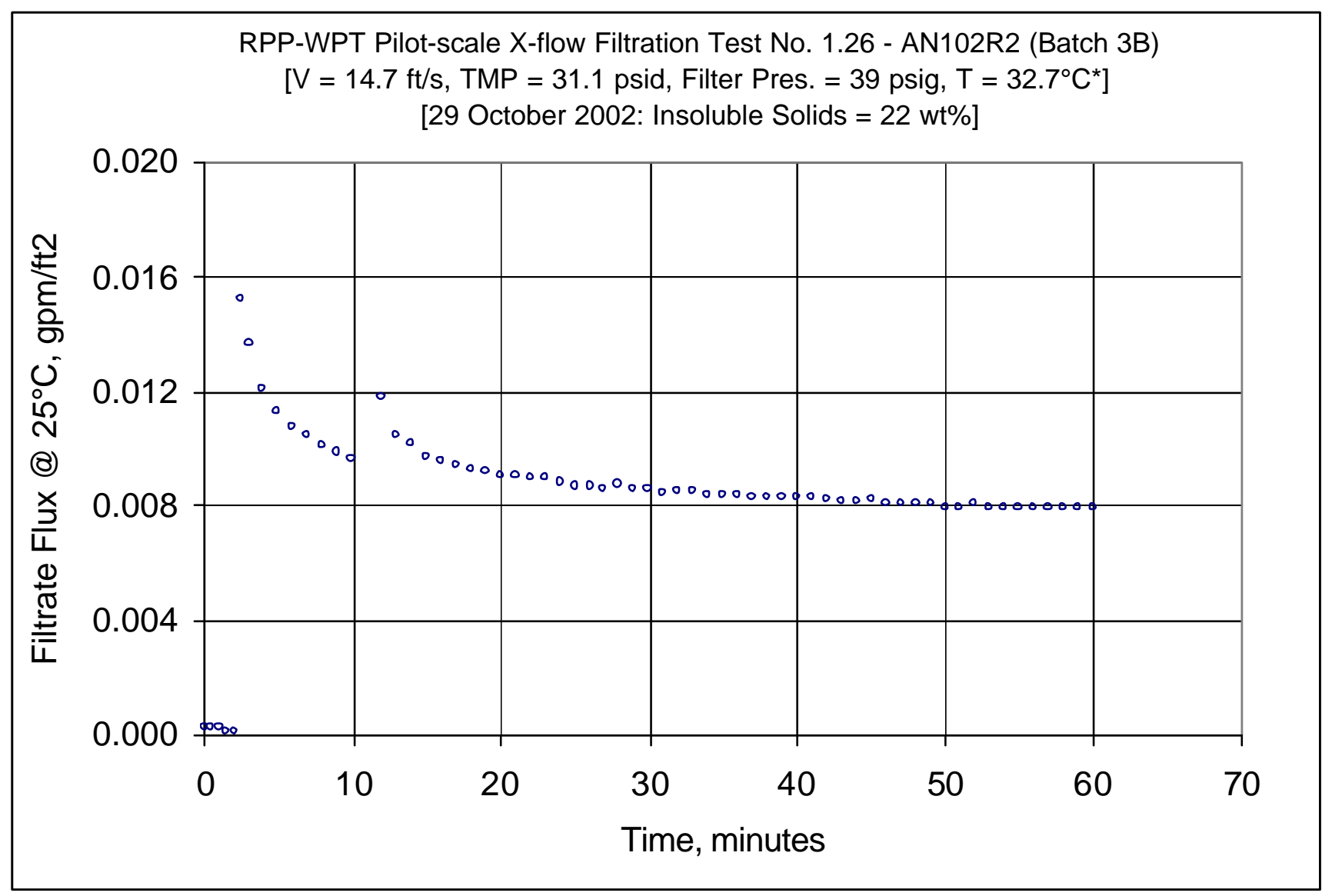

Figure E19: Steady state tes t run 1.26 of AN-102R2, batch 3B, insoluble solids concentration of $22 \mathrm{wt} \%$ 
WSRC-TR-2003-00204, REV. 0

SRT-RPP-2003-00087, REV. 0

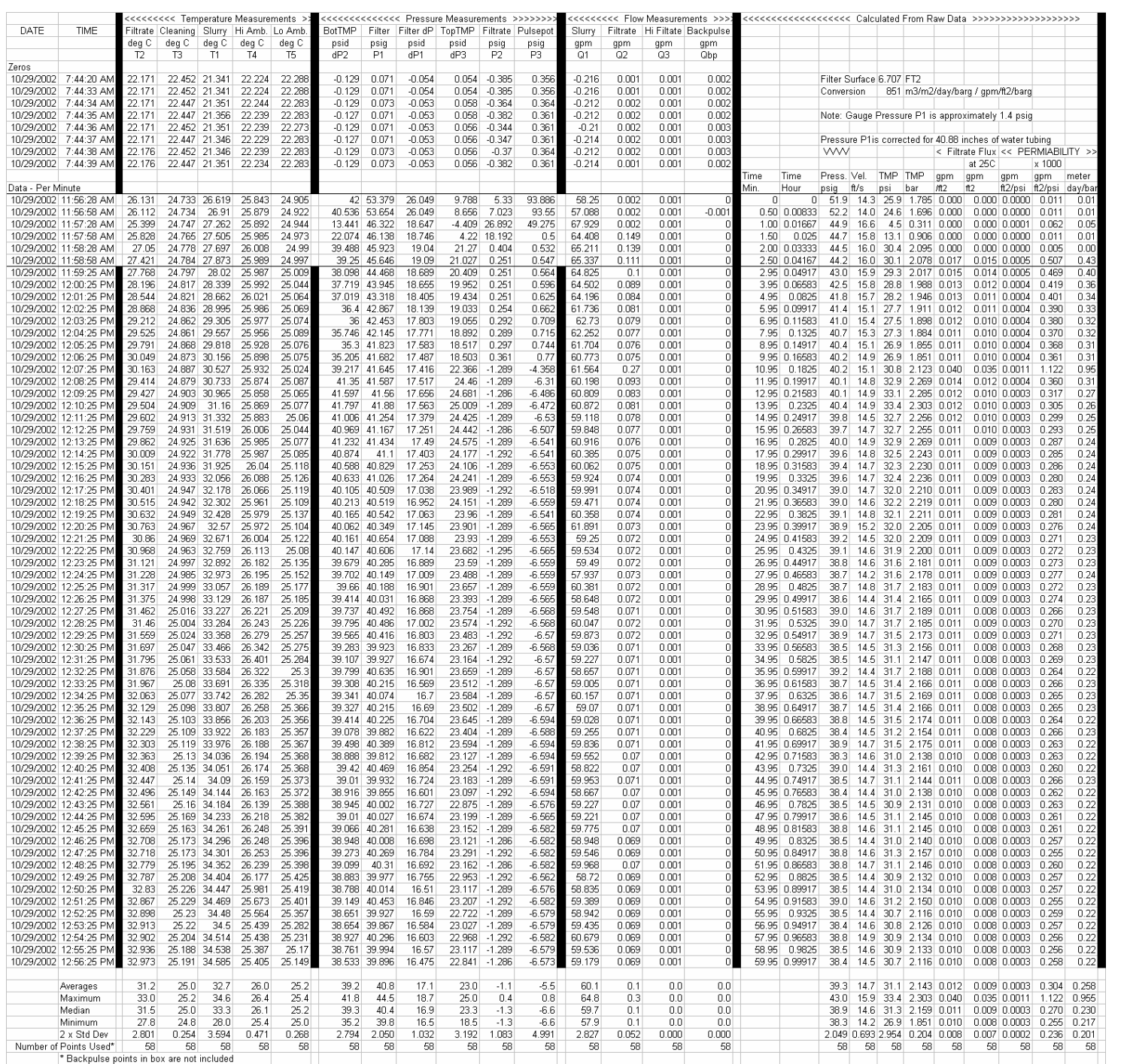

Figure E20: Steady state test run 1.26 of AN-102R2, batch 3B, insoluble solids concentration of 22 wt $\%$ - Raw \& Calculated Data 
WSRC-TR-2003-00204, REV. 0

SRT-RPP-2003-00087, REV. 0

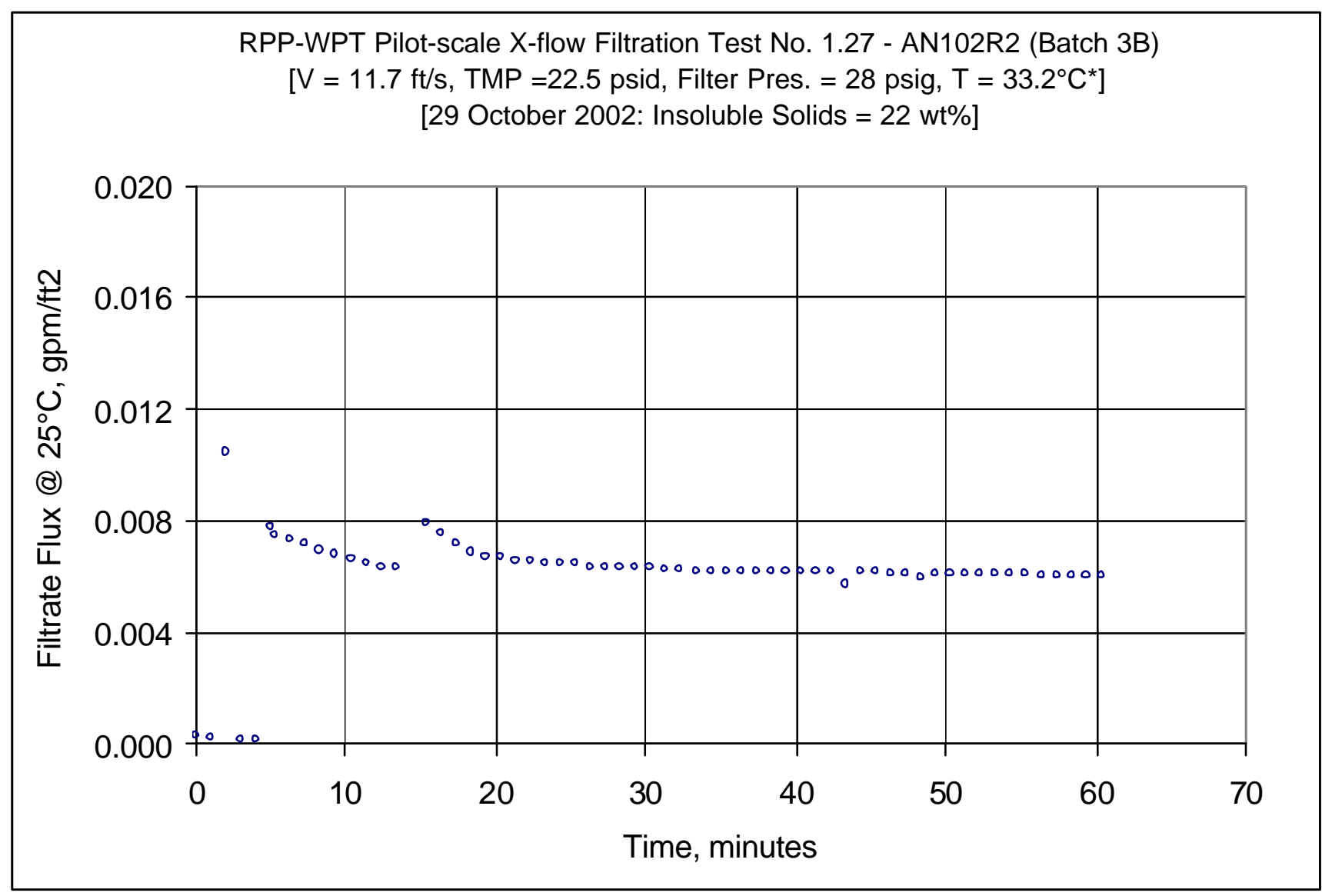

Figure E21: Steady state test run 1.27 of AN-102R2, batch 3B, insoluble solids concentration of $22 \mathrm{wt} \%$ 
WSRC-TR-2003-00204, REV. 0

SRT-RPP-2003-00087, REV. 0

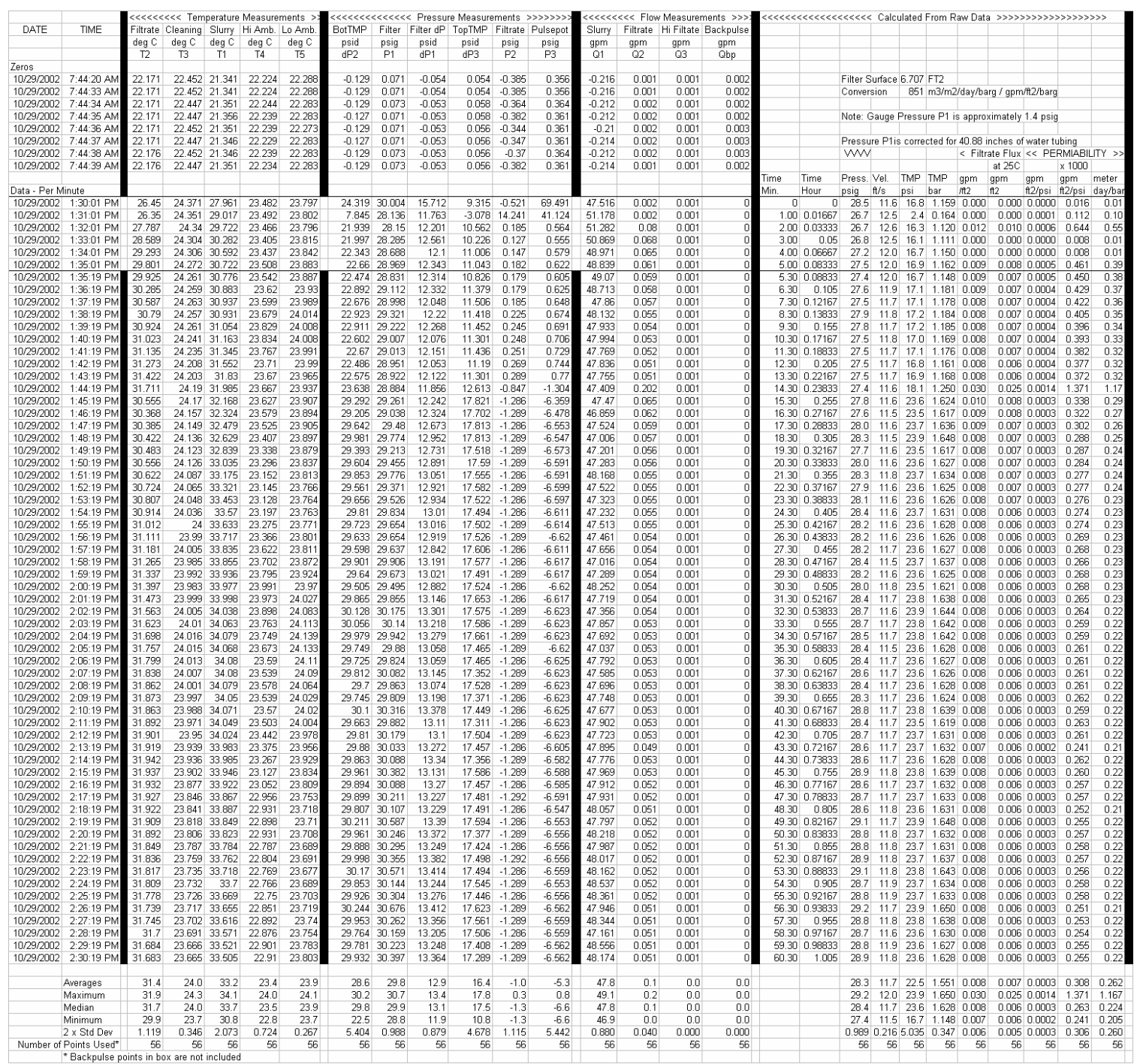

Figure E22: Steady state test run 1.27 of AN-102R2, batch 3B, insoluble solids concentration of $22 \mathrm{wt} \%$ - Raw \& Calculated Data 
WSRC-TR-2003-00204, REV. 0

SRT-RPP-2003-00087, REV. 0

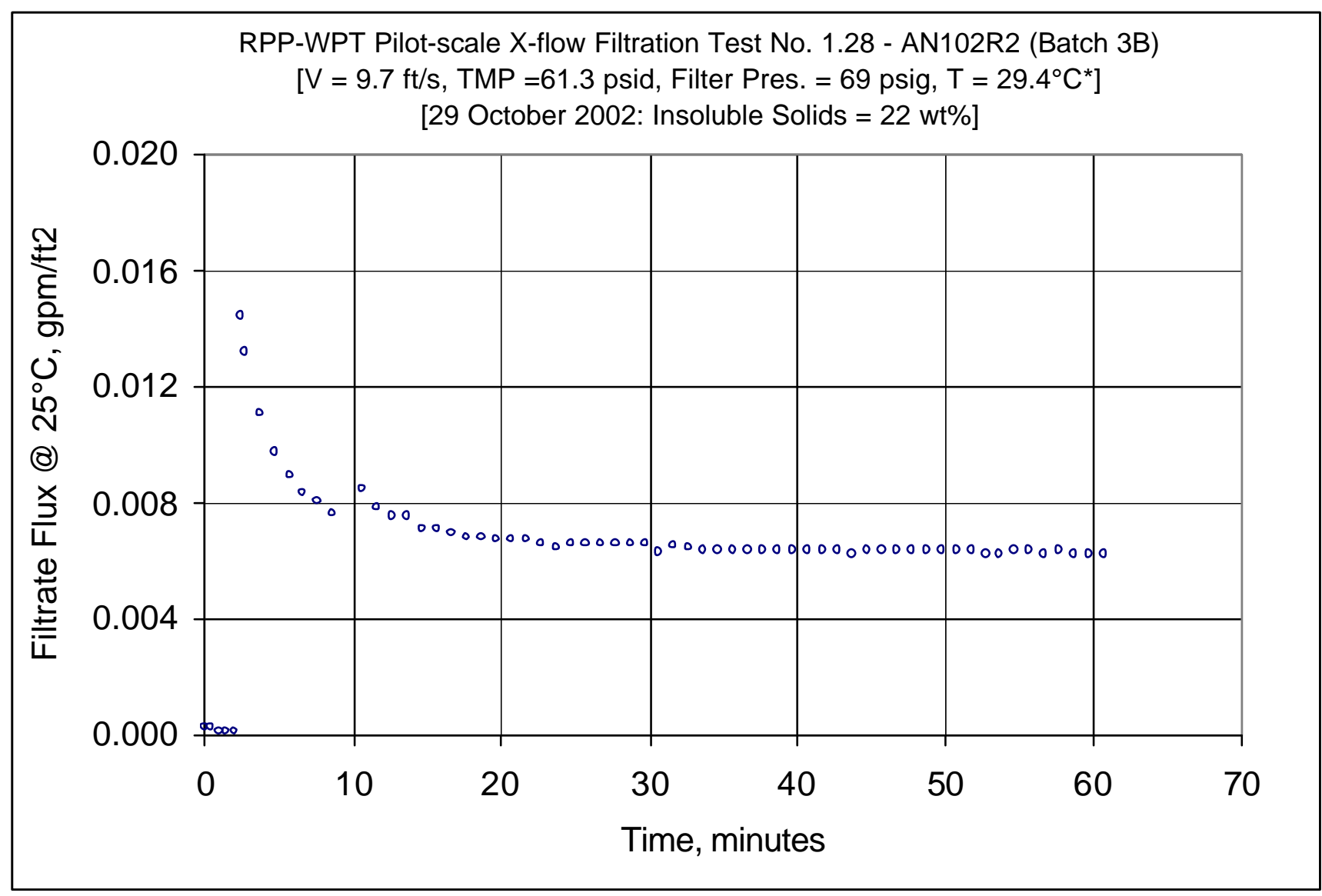

Figure E23: Steady state test run 1.28 of AN-102R2, batch 3B, in soluble solids concentration of $22 \mathrm{wt} \%$ 
WSRC-TR-2003-00204, REV. 0

SRT-RPP-2003-00087, REV. 0

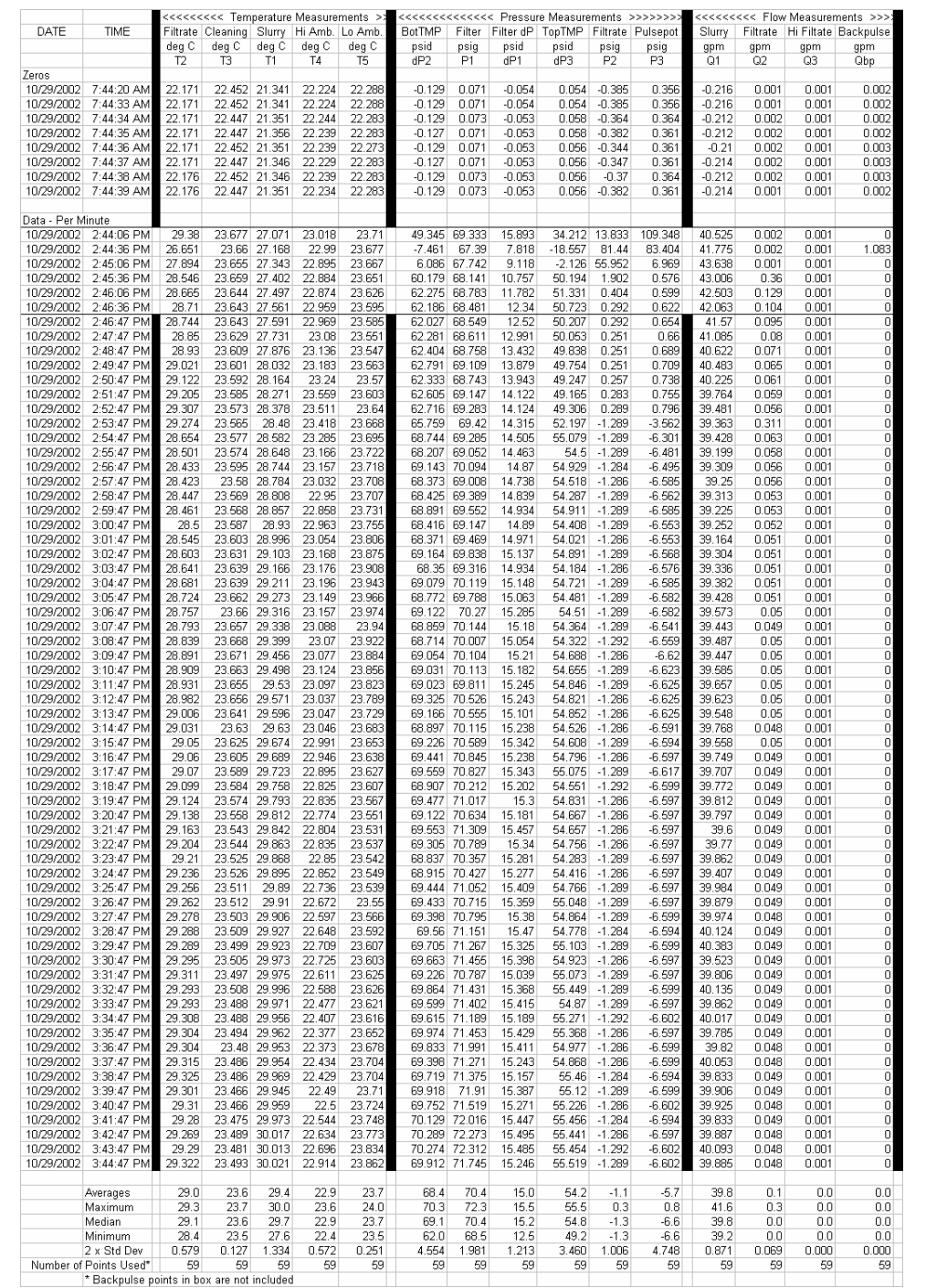

Figure E24: Steady state test run 1.28 of AN-102R2, batch 3B, insoluble solids concentration of 22 wt\% - Raw \& Calculated Data 
WSRC-TR-2003-00204, REV. 0

SRT-RPP-2003-00087, REV. 0

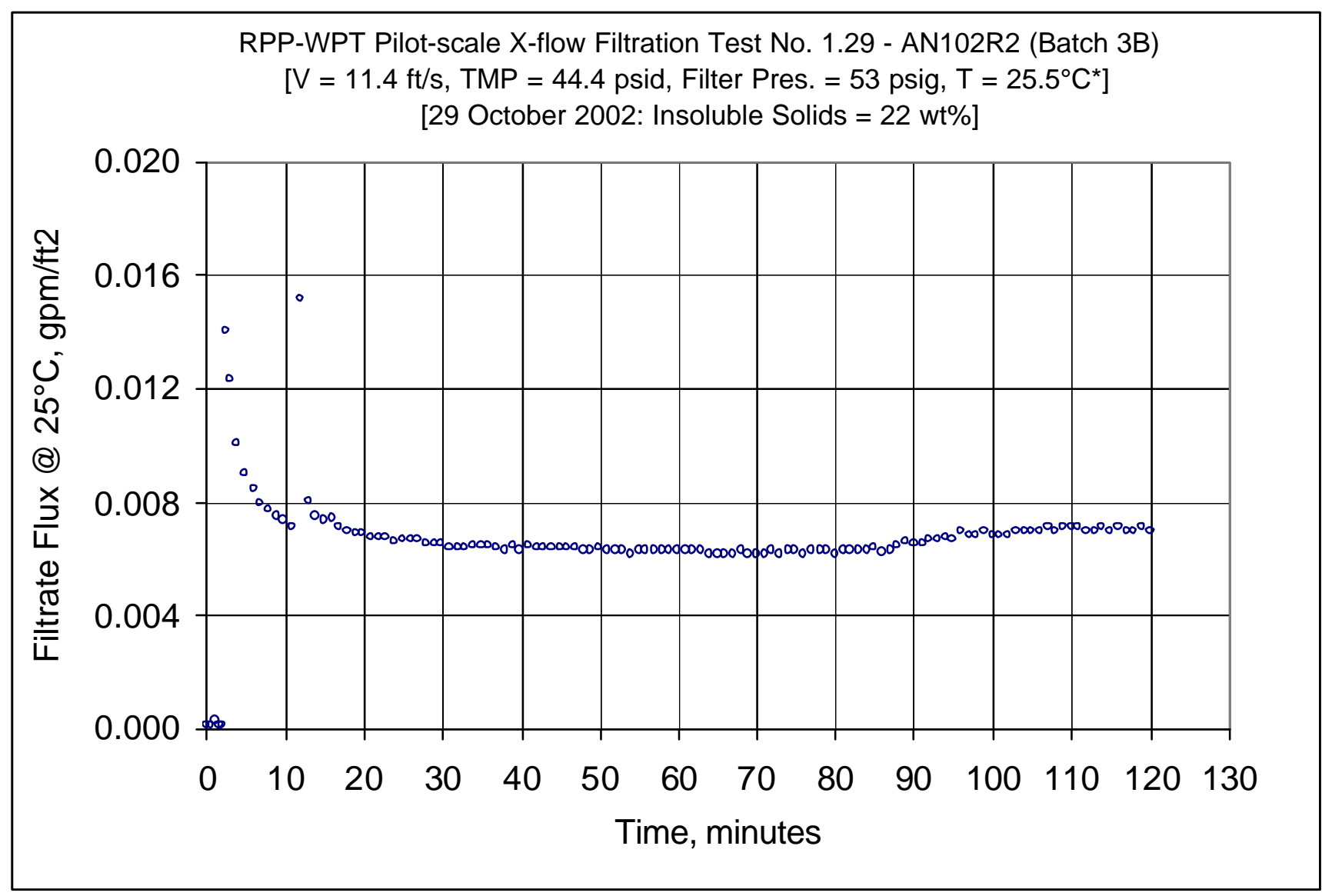

Figure E25: Steady state test run 1.29 of AN-102R2, batch 3B, insoluble solids concentration of $22 \mathrm{wt} \%$ 
WSRC-TR-2003-00204, REV. 0

SRT-RPP-2003-00087, REV. 0

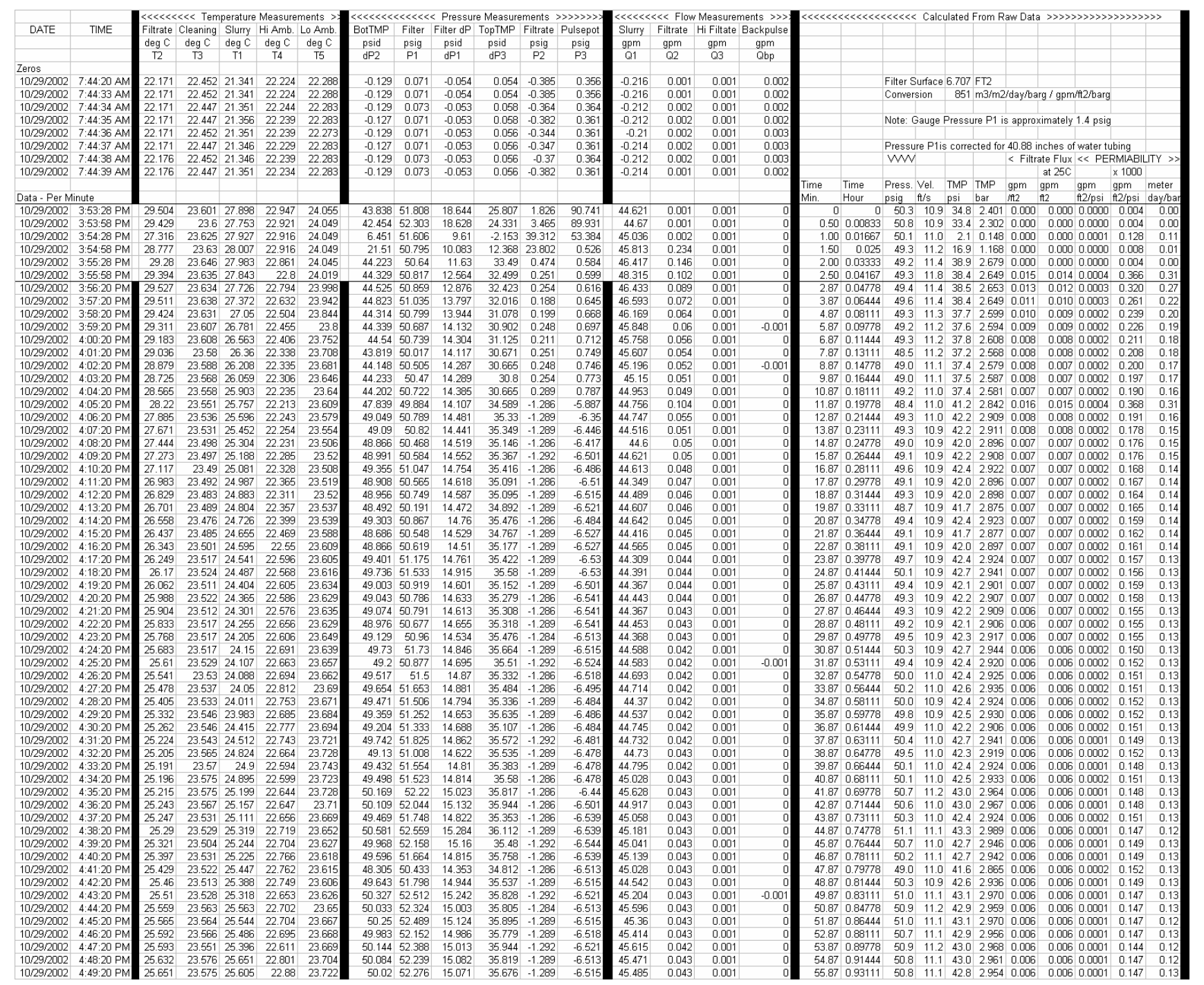

Figure E26-1: Steady state test run 1.29 of AN-102R2, batch 3B, insoluble solids concentration of 22 wt $\%$ - Raw \& Calculated Data 
WSRC-TR-2003-00204, REV. 0

SRT-RPP-2003-00087, REV. 0

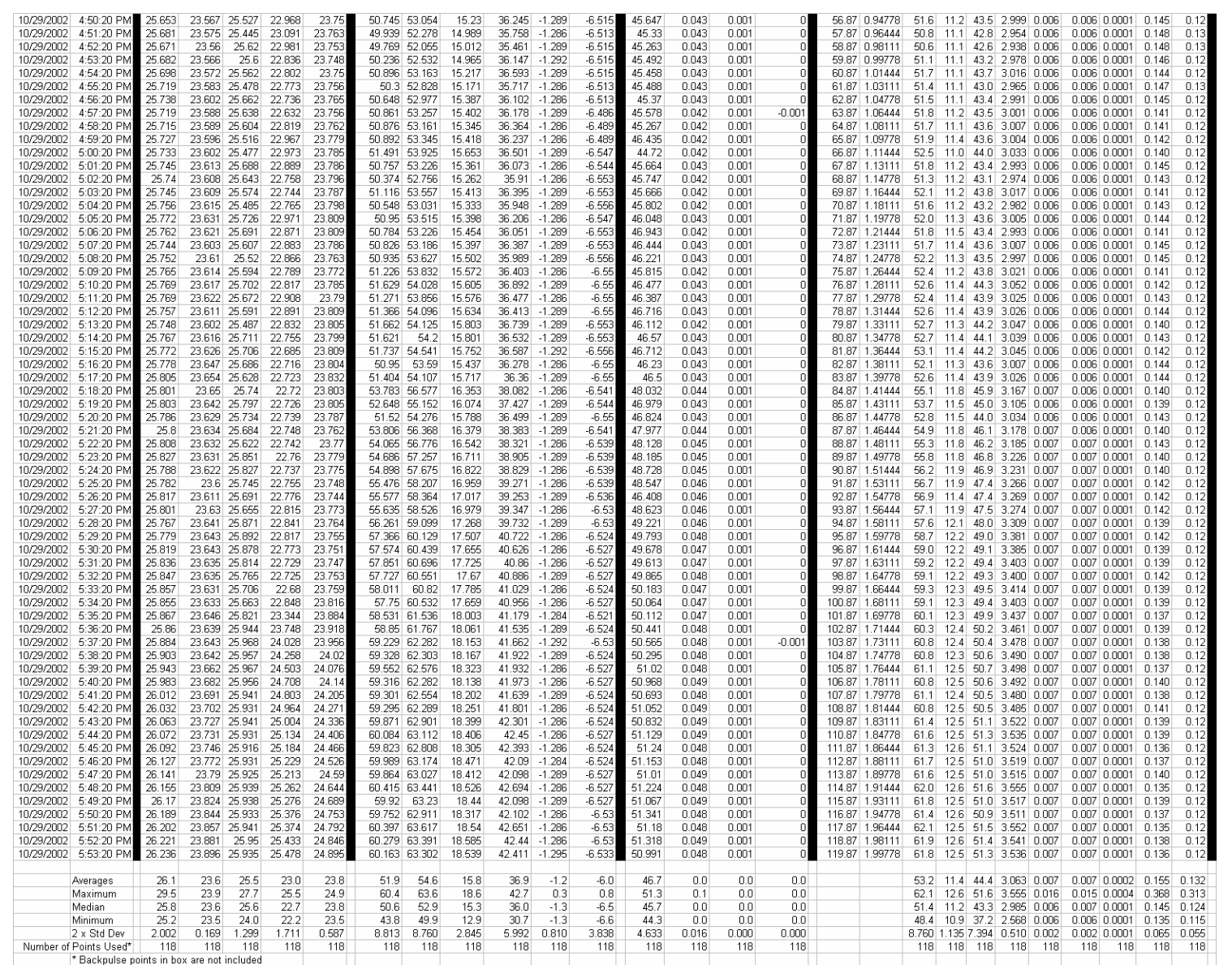

Figure E26-2: Steady state test run 1.29 of AN-102R2, batch 3B, insoluble solids concentration of 22 wt\% - Raw \& Calculated Data 
WSRC-TR-2003-00204, REV. 0

SRT-RPP-2003-00087, REV. 0

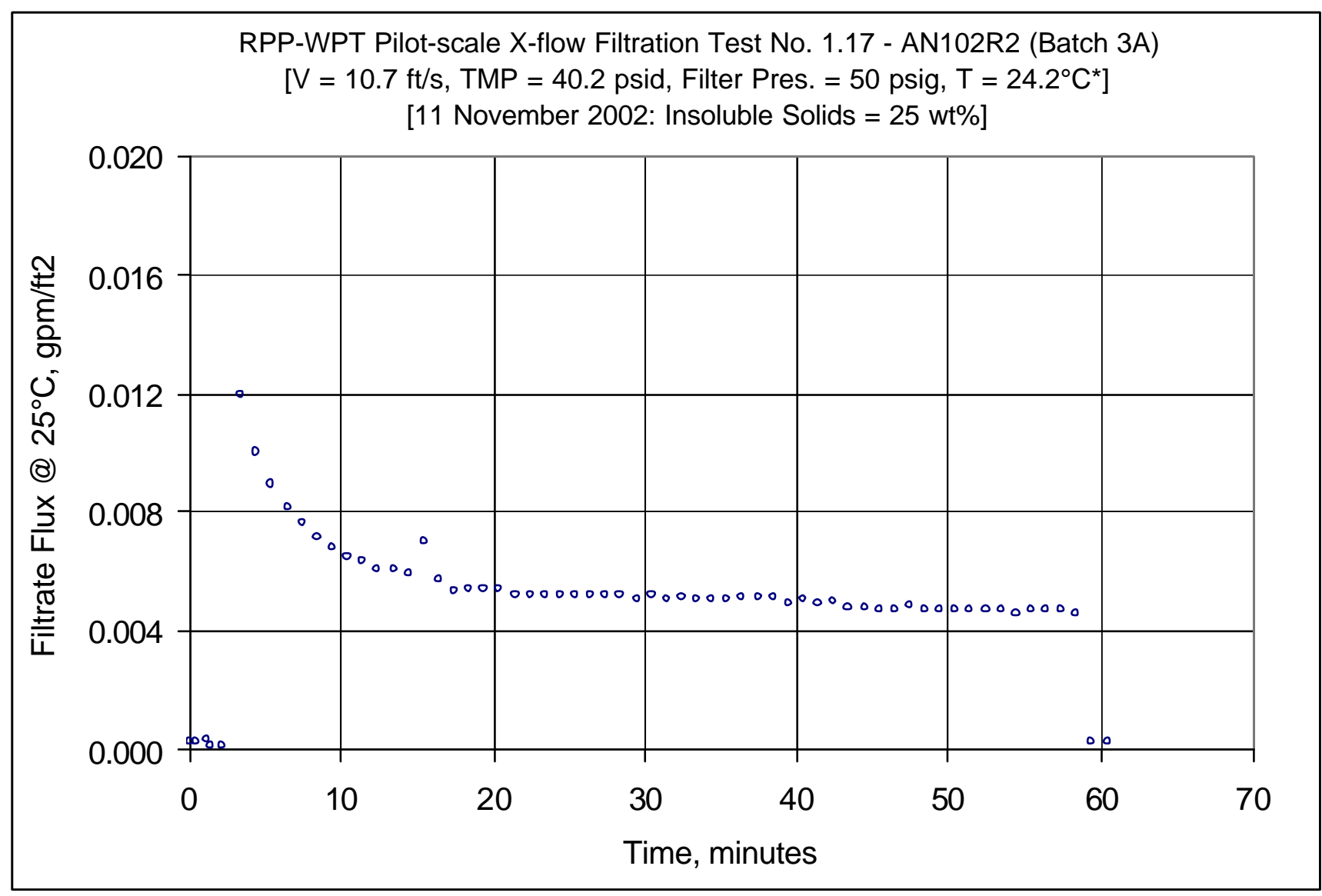

Figure E27: Steady state test run 1.17 of AN-102R2, batch 3A, insoluble solids concentration of $25 \mathrm{wt} \%$ 
WSRC-TR-2003-00204, REV. 0

SRT-RPP-2003-00087, REV. 0

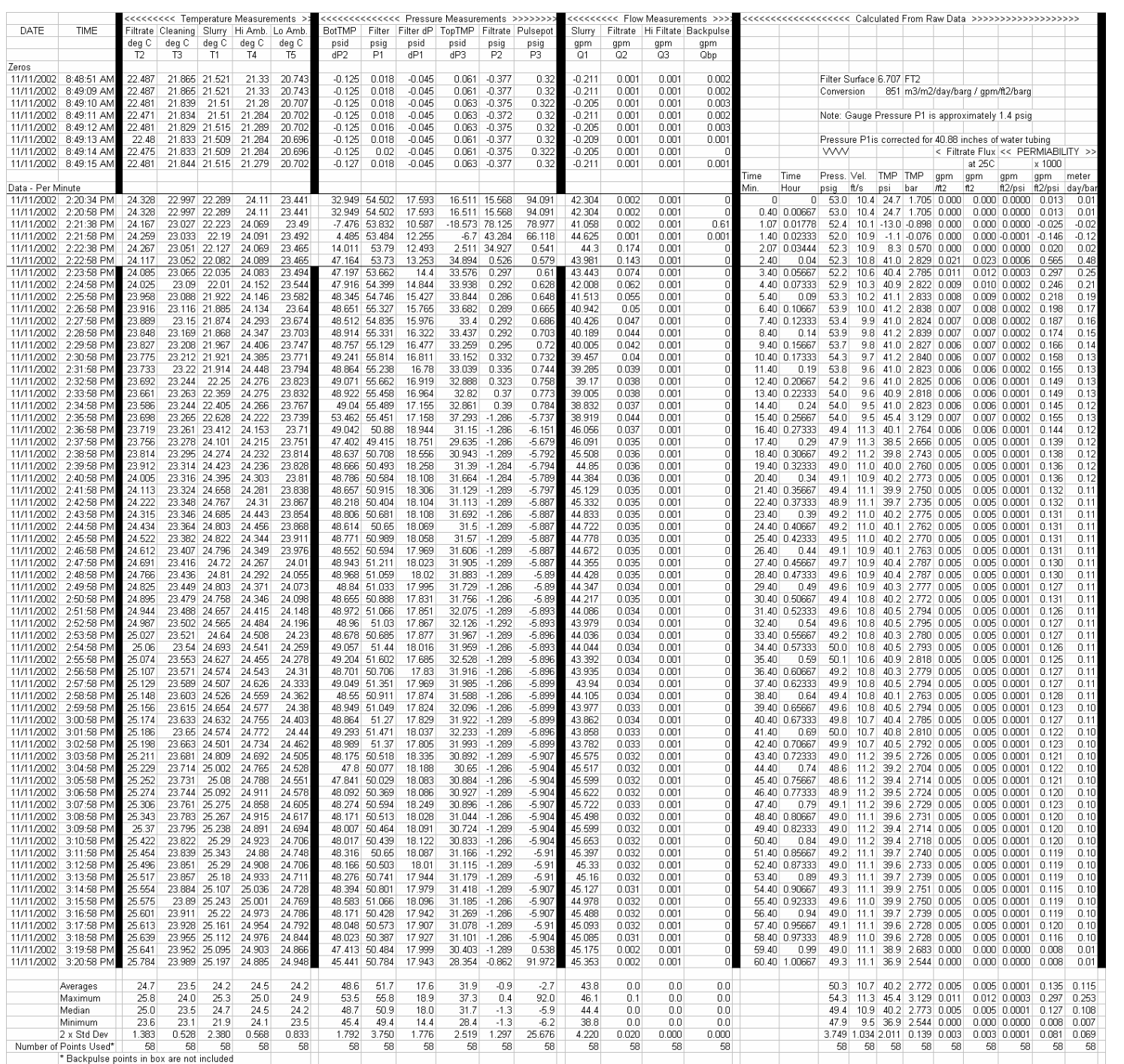

Figure E28: Steady state test run 1.17 of AN-102R2, batch 3A, insoluble solids concentration of 25 wt\% - Raw \& Calculated Data 
WSRC-TR-2003-00204, REV. 0

SRT-RPP-2003-00087, REV. 0

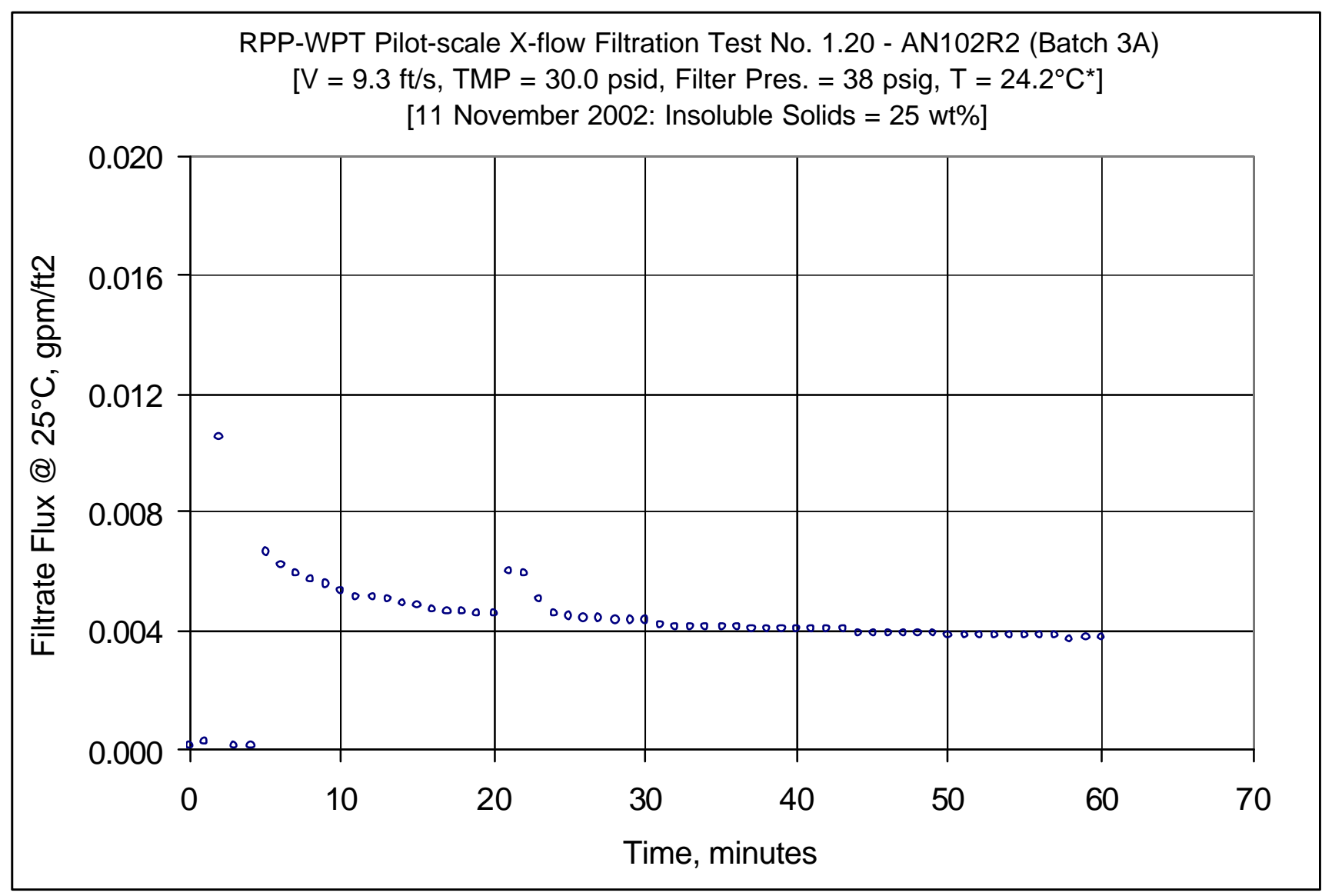

Figure E29: Steady state test run 1.20 of AN-102R2, batch 3A, insoluble solids concentration of $25 \mathrm{wt} \%$ 
WSRC-TR-2003-00204, REV. 0

SRT-RPP-2003-00087, REV. 0

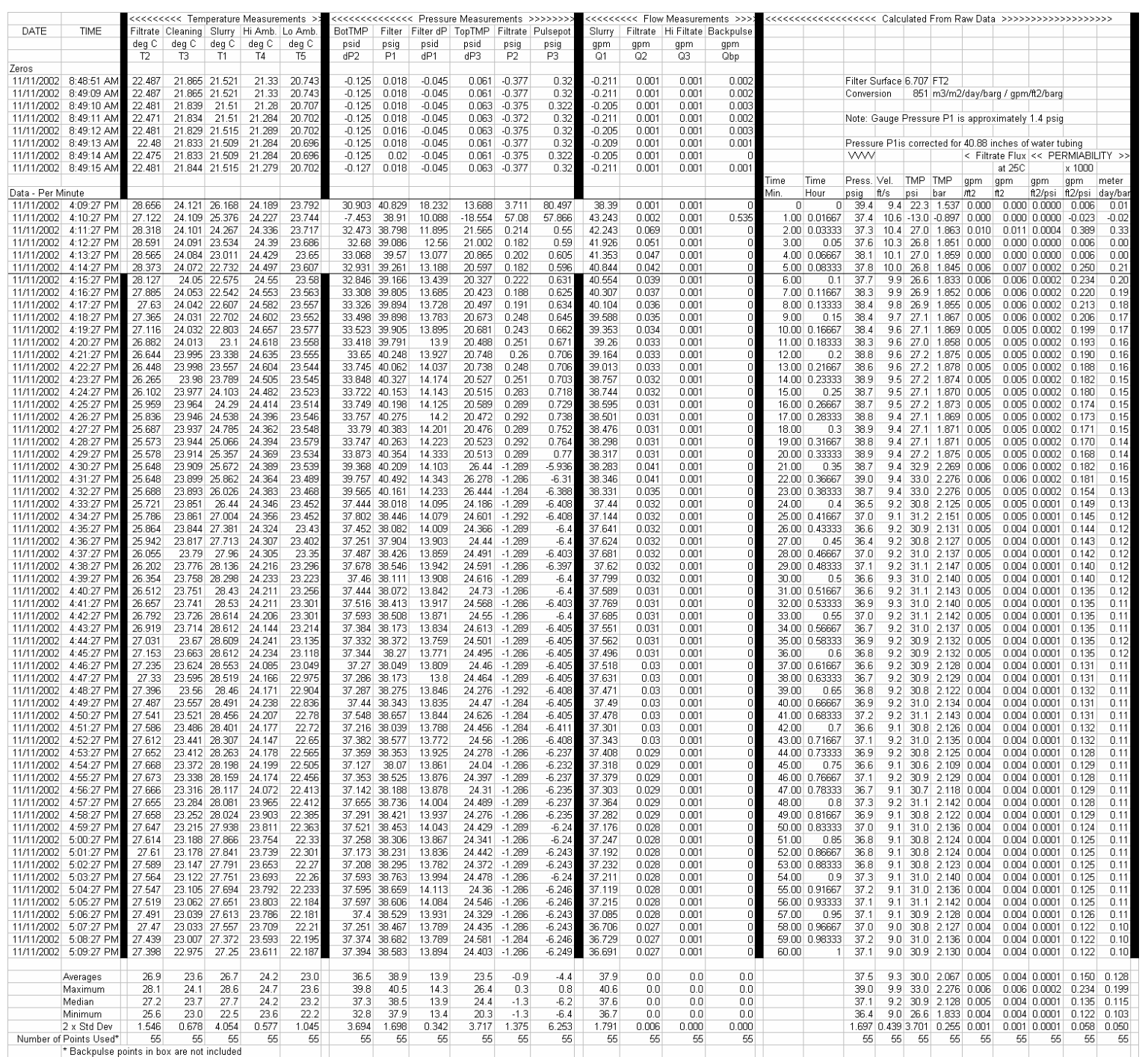

Figure E30: Steady state test run 1.20 of AN-102R2, batch 3A, insoluble solids concentration of $25 \mathrm{wt} \%$ - Raw \& Calculated Data 
WSRC-TR-2003-00204, REV. 0

SRT-RPP-2003-00087, REV. 0

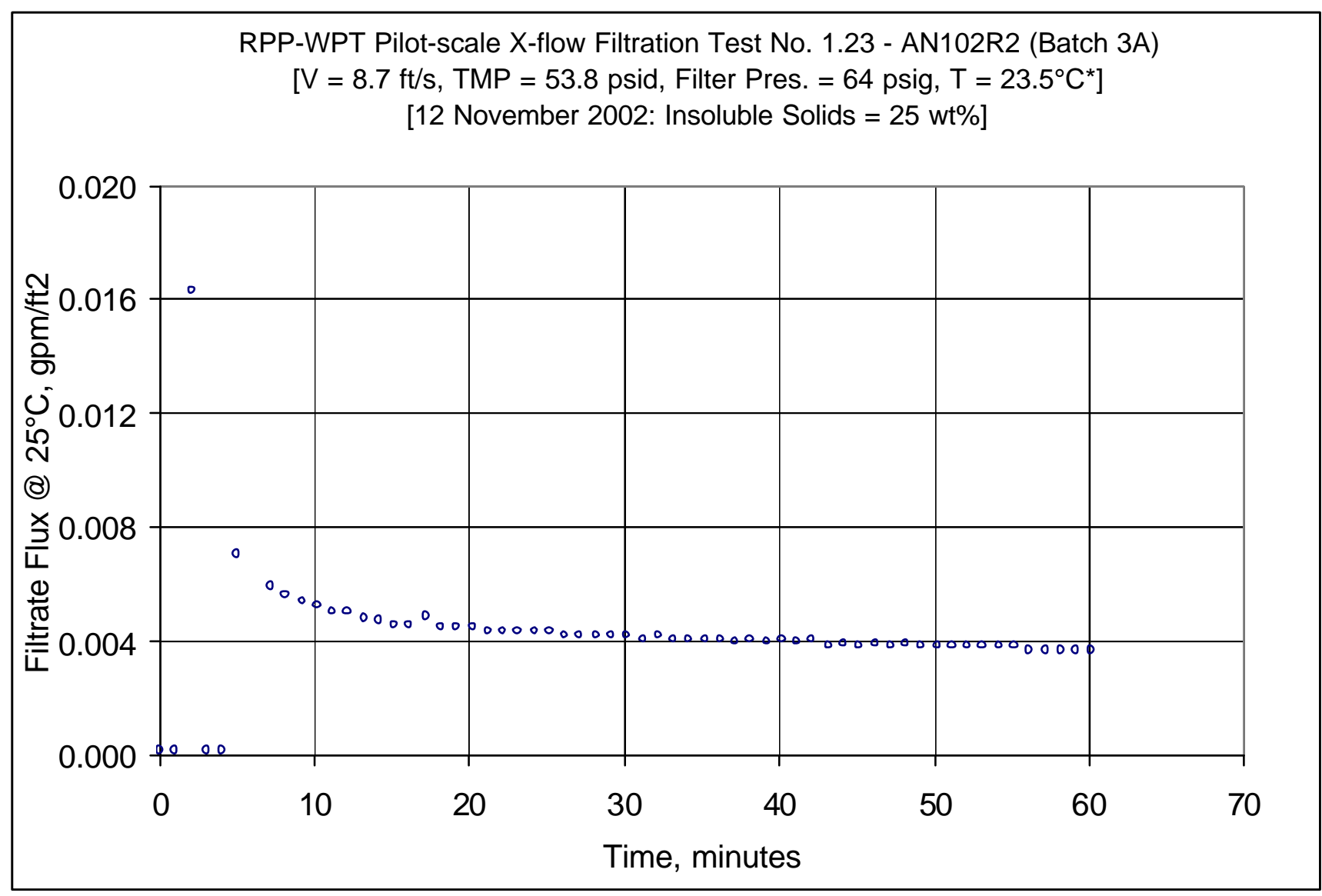

Figure E31: Steady state test run 1.23 of AN-102R2, batch 3A, insoluble solids concentration of $25 \mathrm{wt} \%$ 
WSRC-TR-2003-00204, REV. 0

SRT-RPP-2003-00087, REV. 0

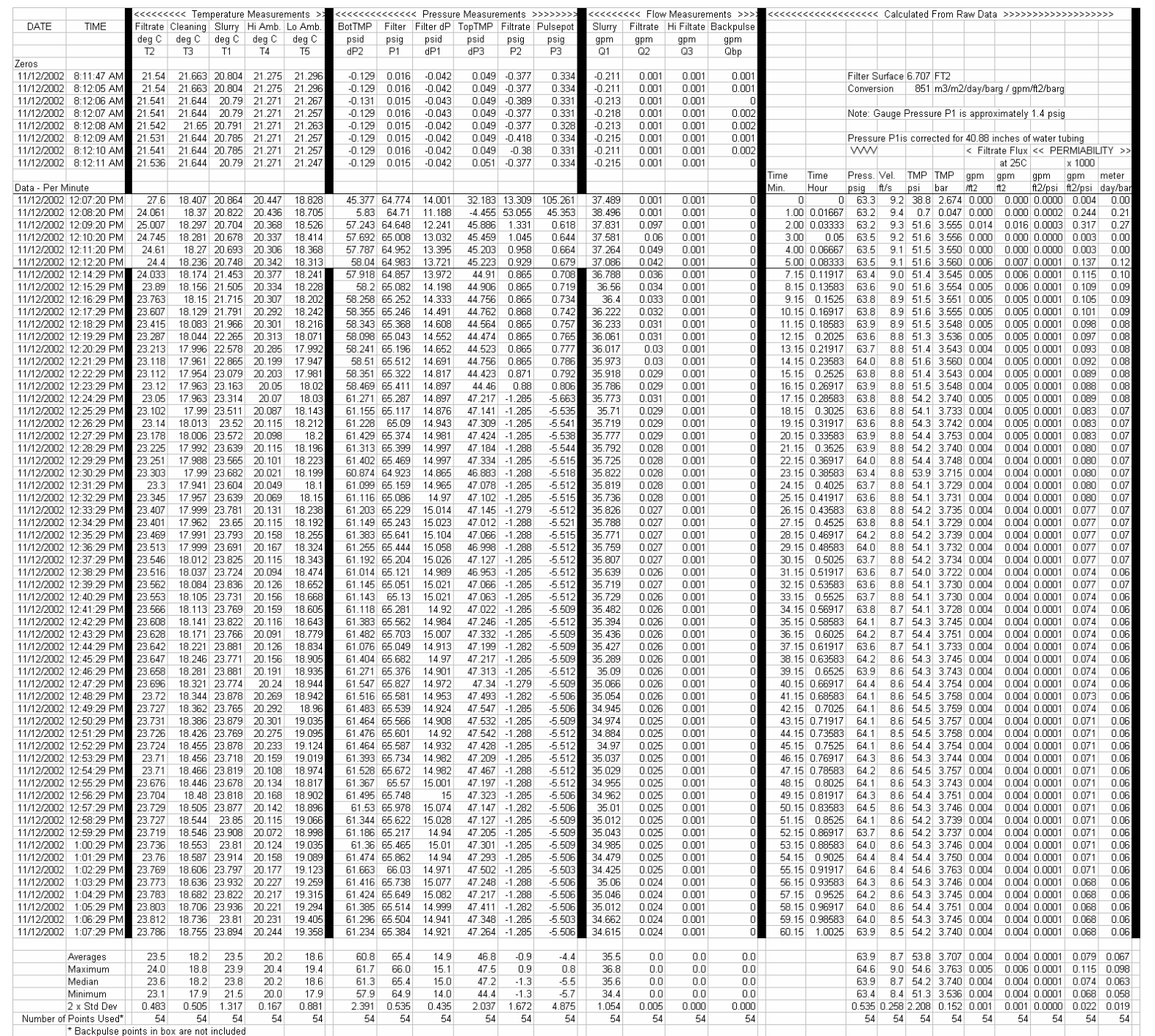

Figure E32: Steady state test run 1.23 of AN-102R2, batch 3A, insoluble solids concentration of $25 \mathrm{wt} \%$ - Raw \& Calculated Data 
WSRC-TR-2003-00204, REV. 0

SRT-RPP-2003-00087, REV. 0

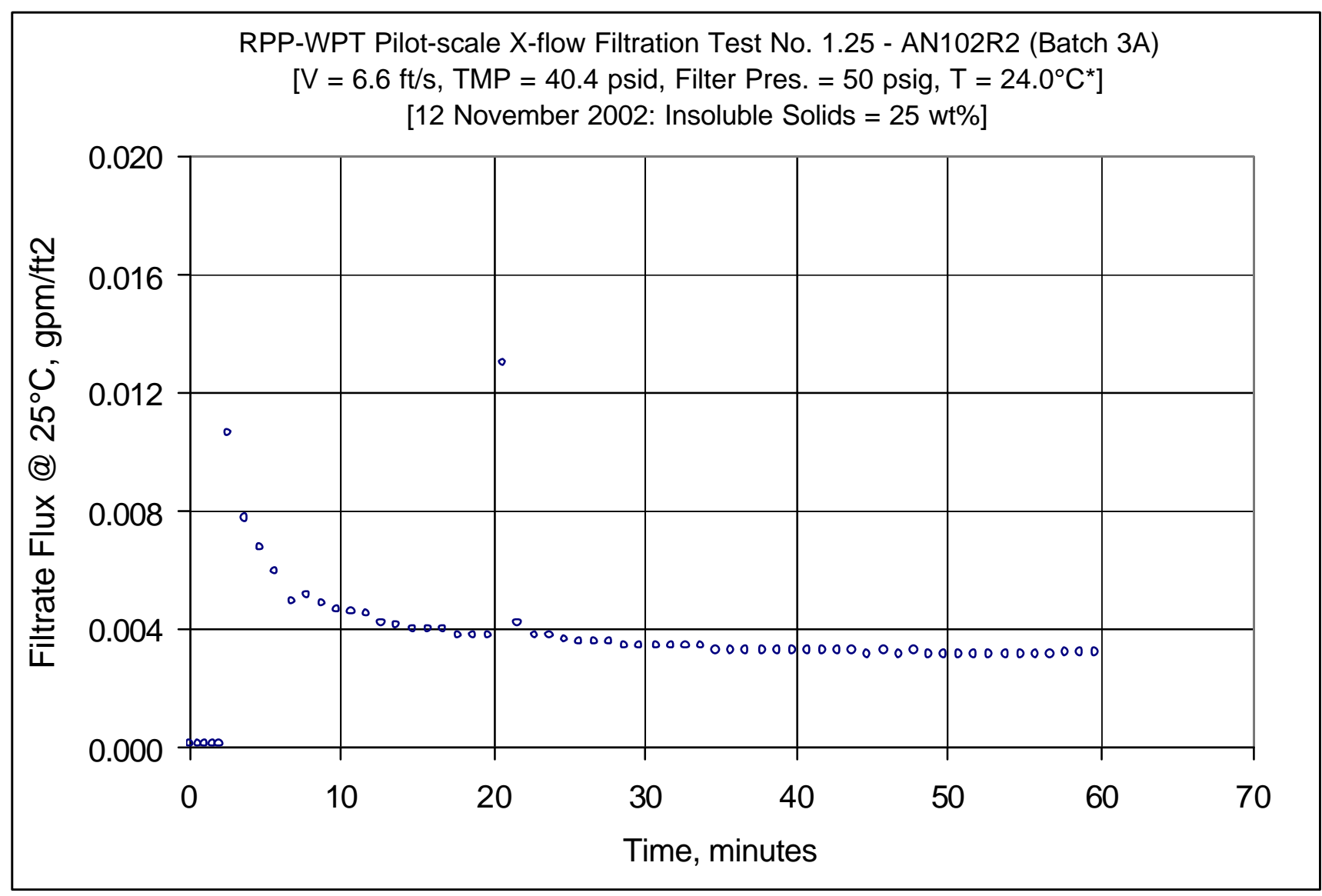

Figure E33: Steady state test run 1.25 of AN-102R2, batch 3A, insoluble solids concentration of $25 \mathrm{wt} \%$ 
WSRC-TR-2003-00204, REV. 0

SRT-RPP-2003-00087, REV. 0

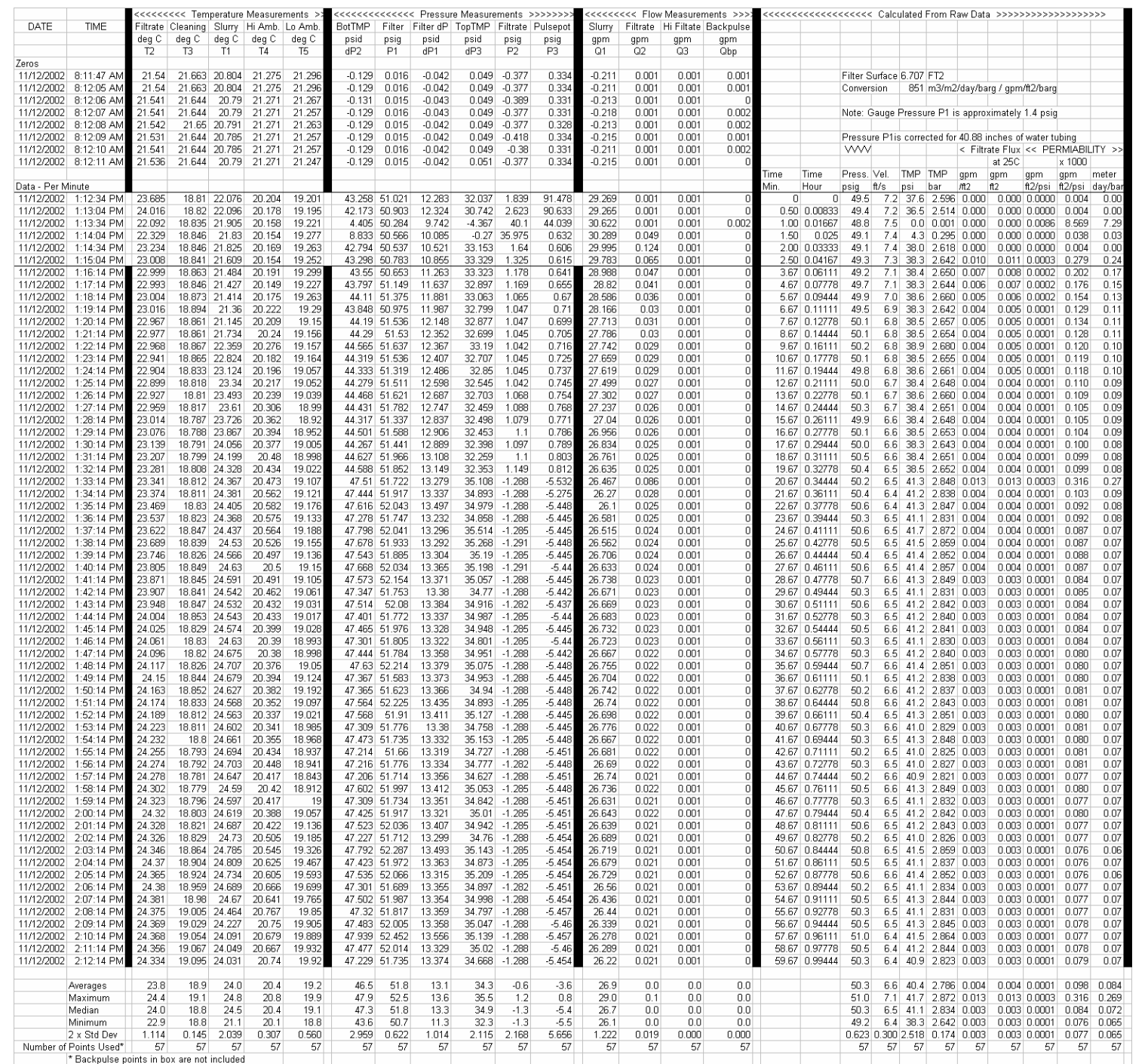

Figure E34: Steady state test run 1.25 of AN-102R2, batch 3A, insoluble solids concentration of 25 wt\% - Raw \& Calculated Data 
WSRC-TR-2003-00204, REV. 0

SRT-RPP-2003-00087, REV. 0

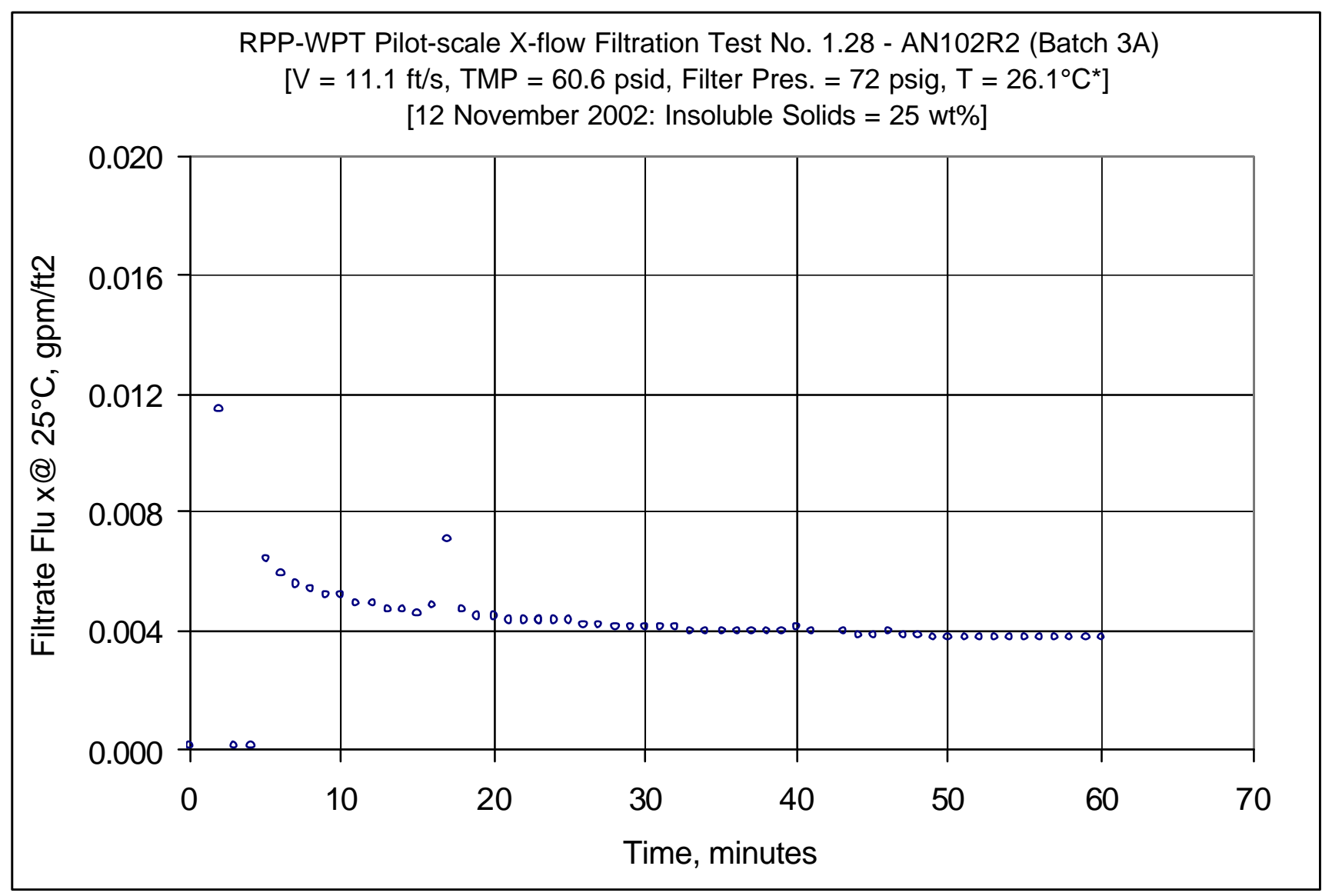

Figure E35: Steady state test run 1.28 of AN-102R2, batch 3A, insoluble solids concentration of $25 \mathrm{wt} \%$ 
WSRC-TR-2003-00204, REV. 0

SRT-RPP-2003-00087, REV. 0

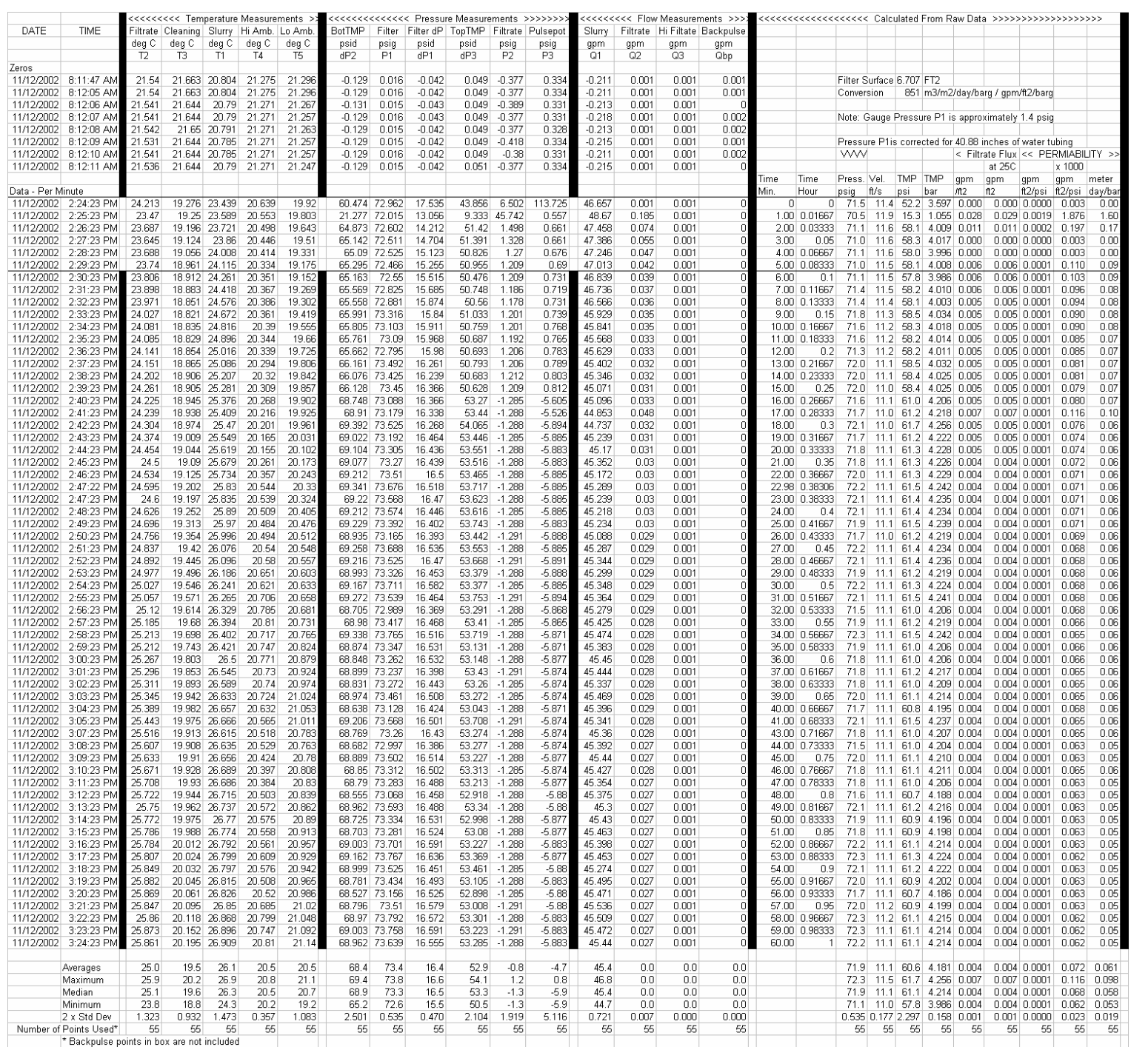

Figure E36: Steady state test run 1.28 of AN-102R2, batch 3A, insoluble solids concentration of $25 \mathrm{wt} \%$ - Raw \& Calculated Data 
WSRC-TR-2003-00204, REV. 0

SRT-RPP-2003-00087, REV. 0

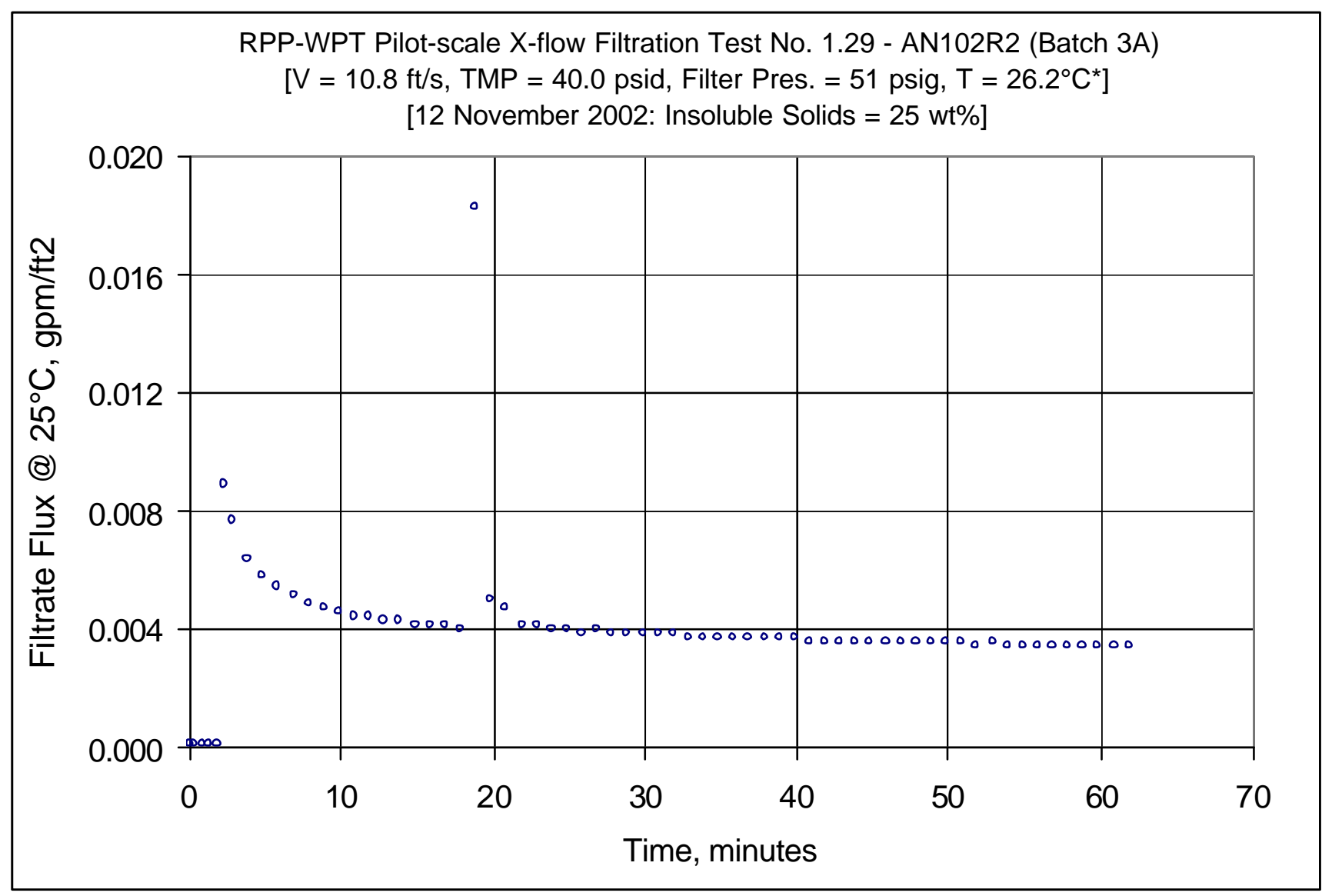

Figure E37: Steady state test run 1.29 of AN-102R2, batch 3A, insoluble solids concentration of $25 \mathrm{wt} \%$ 
WSRC-TR-2003-00204, REV. 0

SRT-RPP-2003-00087, REV. 0

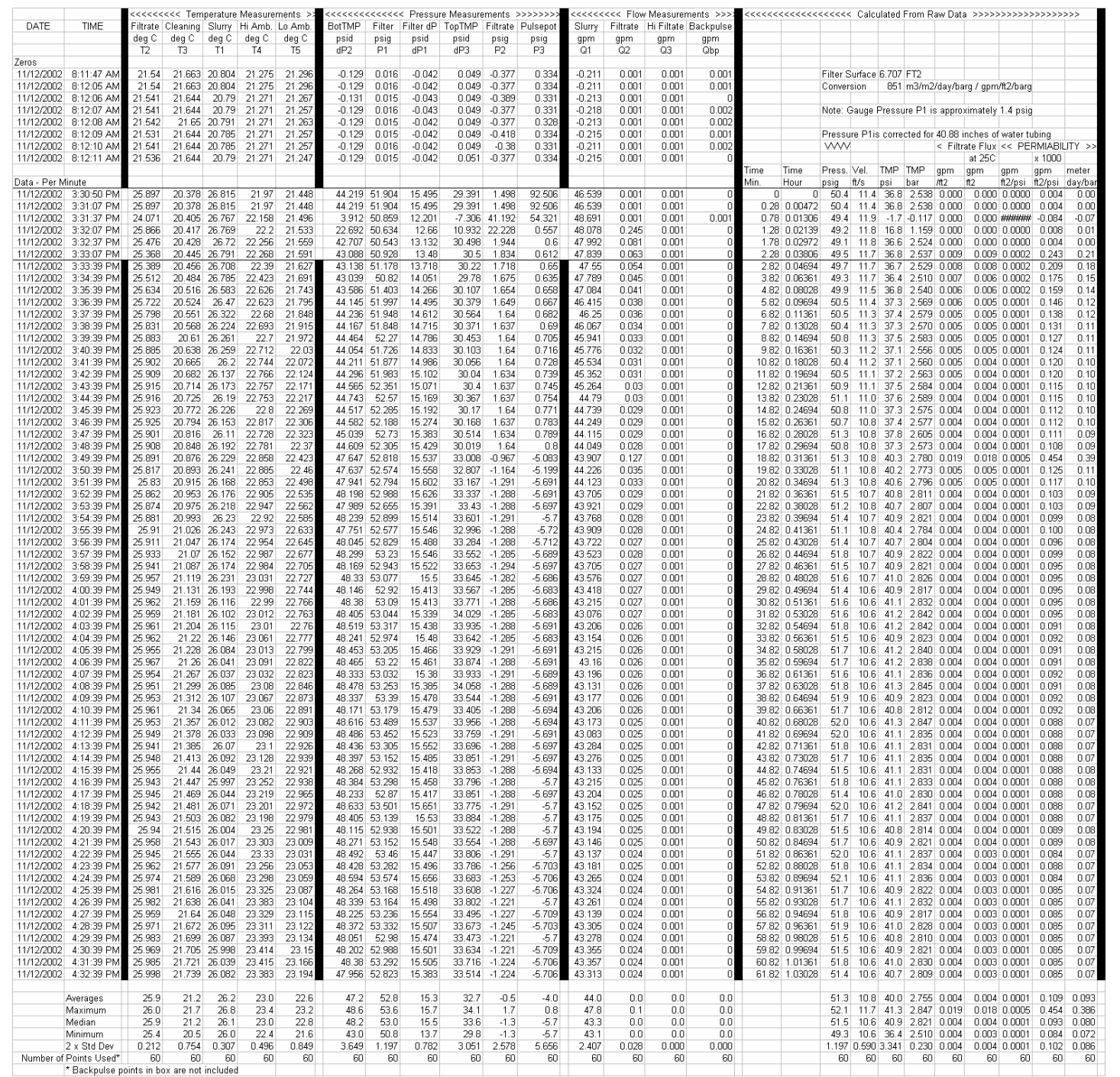

Figure E38: Steady state test run 1.29 of AN-102R2, batch 3A, insoluble solids concentration of 25 wt\% - Raw \& Calculated Data 
WSRC-TR-2003-00204, REV. 0

SRT-RPP-2003-00087, REV. 0

This page intentionally left blank

Page E46 of E46 
WSRC-TR-2003-00204, REV. 0

SRT-RPP-2003-00087, REV. 0

\section{Appendix F: Slurry Wash and Concentration of Washed Slurry, AN-102R2, Batch 3B}

While this report deals with four different batches of AN-102 simulant only after the first successful dewatering test could the steady state runs be done, and therefore, the washing test run. This occurred after the second batch, i.e., 3B. For subsequent batches most steady-state runs were not required, as well as the washing tests.

After the last steady state run with $\mathrm{AN}-102$, batch $3 \mathrm{~B}$, i.e., run 1.29 , the test rig was made ready to wash the slurry. The washing cycle followed the method to be used by WTP. That is, for the plant the concentrated slurry would have an approximately volume of 18,000 gallons and it would be washed with 21,000 gallons of inhibited water. The washing liquid would be added 1,000 gallons at a time and an equal volume of washed filtratre removed before adding the next 1,000 gallons, for a total of 21 cycles. The same was done for the pilot wash test. Of course the scale was reduced. At the end of test run 1.29 there was approximately 67 liters of concentrated slurry. As required by the test specification (Townson, 2002), the slurry insoluble solids concentration was reduced from was attained during the dewater test run, i.e., 1.16 to $20 \mathrm{wt} \%$, if $\mathbf{t}$ happened to be higher. The actual concentration was approximately $22 \mathrm{wt} \%$, therefore some of the previously removed filtate was added back to the slurry to reduce the solids concentration. The amount was $7.5 \mathrm{~kg}$, or approximately 5.8 liters. The filtrate addition reduced the solids concentration of the simulant to $18.8 \mathrm{wt} \%$. Then, to this approximate volume 72.8 liters of slurry were added 72.8 x 21/18 - 85 liters of wash water. The 85 liters were added in 4.04-liter batches while 4.04 liters of washed filtrate were removed before adding the next batch. This was done 21 times.

This appendix contain the raw data for the washing run and for test run 1.30, which concentrated the washed slurry until it could not be concentrated further.

This Appendix includes the following test runs:

Washing test run with simulant slurry AN-102R2 Batch 3B at insoluble solids of 18.8 wt\%:

Washing test run done on 31 October 2002

Concentration of washed slurry: insoluble solids of increased from $18.3 \mathrm{wt} \%$ to $25 \mathrm{wt} \%$ *:

done on 31 October 2002

Test 1.30 was stopped at $25 \mathrm{wt} \%$ because during the last 5 minutes of the test the slurry temperature began to increase very rapidly, i.e., from approximately $25^{\circ} \mathrm{C}$ to above $45^{\circ} \mathrm{C}$.

Each test in this Appendix contains a graph of the filtrate flux with time and tables of the data taken and calculated. To better understand the presented information important aspects of the graphs and tables are explained below:

Graphs - Each graph contains time averages of some of the imporant parameters taken during the run at the top of the figures, i.e. Slurry velocity in the filter tube, V; transmembrane pressure, TMP; slurry pressure at the entrance of the filter tube, Filter Pres; and the slurry temperature, T.

Tables - Each table is divided into raw data (the first 17 columns) and calculations (the last 10 columns) which are based on the raw data. The raw data section is divided into three parts: 


\title{
WSRC-TR-2003-00204, REV. 0
} SRT-RPP-2003-00087, REV. 0

\author{
Individual Entries - Raw Data \\ Temperature Filtrate - from the filtrate loop at the exit of the filter bundle \\ Cleaning - from the cleaning loop (this loop was not used, except for tests Clean $1 \& 2$ ) \\ Slurry - from the slurry loop in the slurry reservoir \\ Hi Amb. - outside ambient temperature at the top of the test rig \\ Lo Amb. - inside ambient temp erature at the bottom of the test rig \\ Pressure BotTMP - differential pressure across the filter tube at the bottom of the vertical tube \\ Filter - gauge pressureof the slurry at the entrance of the filter tube bundle \\ Filter $\mathrm{dP}$ - differential pressure of the slurry along the height of the vertical 90-inch tube \\ TopTMP - differential pressure across the filter tube at the top the vertical 90-inch tube \\ Filtrate - gauge pressure of the filtrate at the exit of the filter tube housing \\ Pulsepot - gauge pressure of the filtrate at the bottom of the pulsepot \\ Flow Slurry - rate of slurry just before entering the seven-filter-tube bundle \\ Filtrate - rate of slurry filtrate under normal flow (max. is $1.2 \mathrm{gpm}$ ) \\ Hi Filtrate - rate of any liquid with has a high flowrate (water, acid, etc.) (max. $5 \mathrm{gpm}$ ) \\ Backpulse - rate of filtrate in opposite direction from normal flow, i.e., backpulse
}

Individual Entries - Calculated Data

Time - Minutes after start of test, including the backpulse (based on raw data TIME colu mn)

Press. - Internal filter pressure of slurry at the filter bundle entrance (based on raw data P1 column and corrected for a 40.9-inch water column that exists from the transducer to the point where P1 is measured.)

Vel. - Slurry velocity in filter tube (based on raw data Q1 column and i.d. of the tubes of 0.488 inches)

TMP - Filter transmembrane pressure (based on average of raw data columns dP2 and dP3) in psi.

TMP - Filter transmembrane pressure (based on average of raw data columns dP2 and dP3) in bar.

Filtrate Flux - Filtrate flow rate (raw data columns Q2/Q3) divided by inside filter surface area $(6.87 \mathrm{ft} 2)$

Filtrate Flux @ $25^{\circ} \mathrm{C}$ - Same as Filtrate flux, but correct for temperature with the following multiplier:

$\mathrm{T}($ corrected $)=\mathrm{T} \times \operatorname{Exp}[2500 /((1 / 273+\mathrm{T}))-(1 / 298)]$ as per Test Spec 24590-WTP-TSP-RT-01-029, Rev. 0

Permiability - Filterate flow rate per TMP (based on calculated columns Filtrate Flux @ $25^{\circ} \mathrm{C}$ and TMP)

Permiability x 1000 - Same as the above Permiability, but increase by 1000 to facilitate graphing

Permiability - Same as Permiability but in metric units, meter/day/bar

$\underline{\text { Statistics }}$

At the bottom of the last individual entry there are six rows with some data statistics to better understand the data and their fluctuations. The columns are self explained with titles of Averages, Maximum, Median, and Minimum. The next row $2 \times$ Std Dev, is two times the population standard deviation based on the number of data points used, which is the last row. The Number of Points used generally do not included the points taken during the backpulse sequence of each test. This is explained below.

\section{Raw Data Table Layout}

The raw data is generally made up of three sections:

Zeros - These eight rows include approximately 15 to 20 seconds of data taken with the pressure transducers placed in the "zero" mode to determine if any zero drift occurred from one test to the next.

Data-Per Minute - On most of the tables the first six rows of these data are contained in a box which indicate data taken during the backpulse. This is were the filtrate flow is reversed temporarily to knock the filtrate cake of the filter surface. During these first few minutes the filtrate flow is stopped, then reversed, then stopped again, then it is finally returned to normal operation. The data during the backpulse operation were taken with a separate $\log$, therefore those data are marked with a box to note this fact. Most or all of those data points are not counted in the statistics at the bottom of the table. The tables of some of the later tests do not show the boxed data because separate logs of data were not taken, however since a backpulse for each run still occurred only those data were not included in the statistics at the bottom of the page. 
WSRC-TR-2003-00204, REV. 0

SRT-RPP-2003-00087, REV. 0

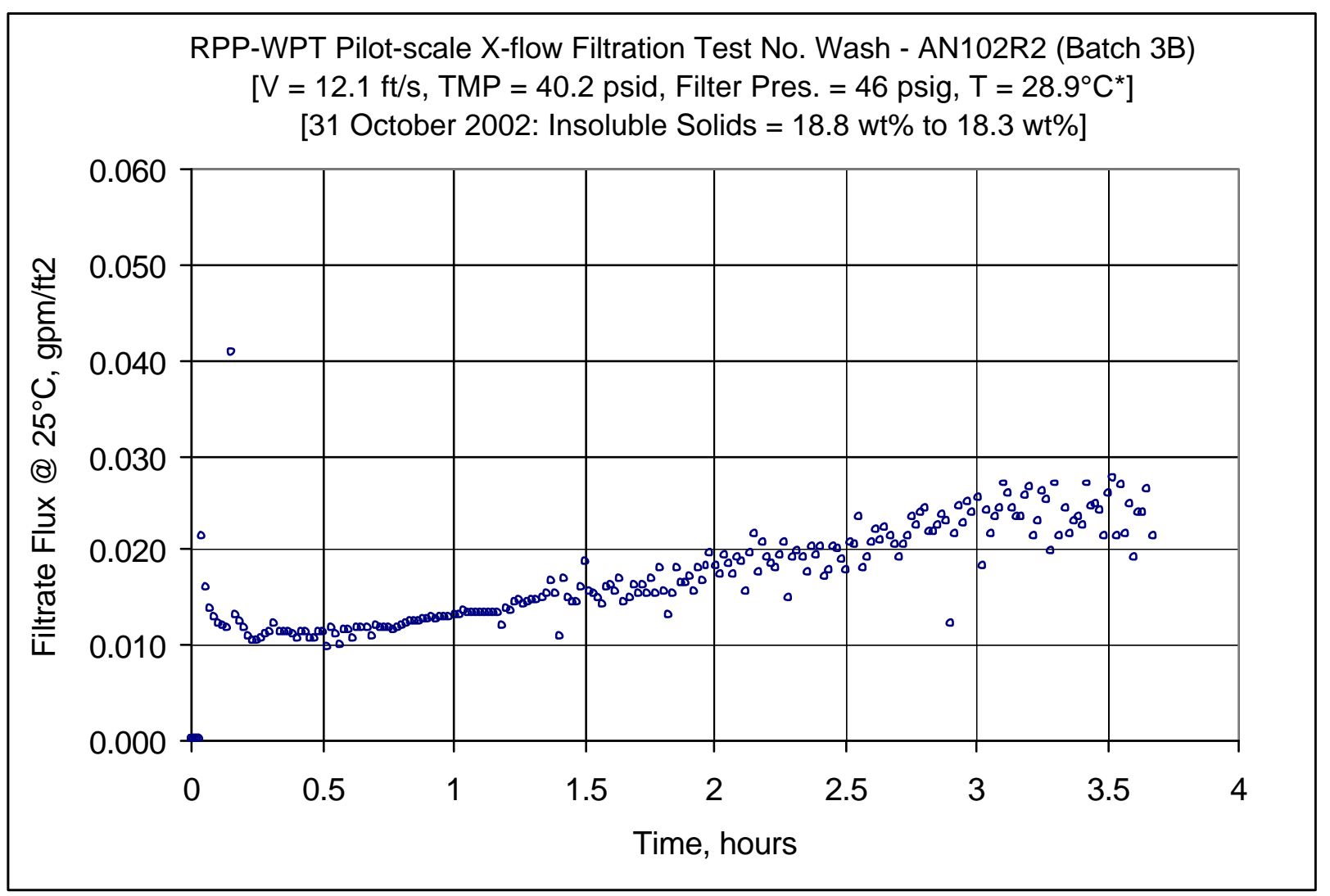

Figure F1. Complete washing cycle - 21 mini-batches of inhibited/filtered water with a

Volume $=$ Total Slurry Volume $\mathrm{x}(21 / 18) / 21$ were added and removed.

At an average filtrate flux of $0.0175 \mathrm{gpm} / \mathrm{ft} 2$ the mini-batch additions

occurred approximately, on the average, every 10 minutes 
WSRC-TR-2003-00204, REV. 0

SRT-RPP-2003-00087, REV. 0

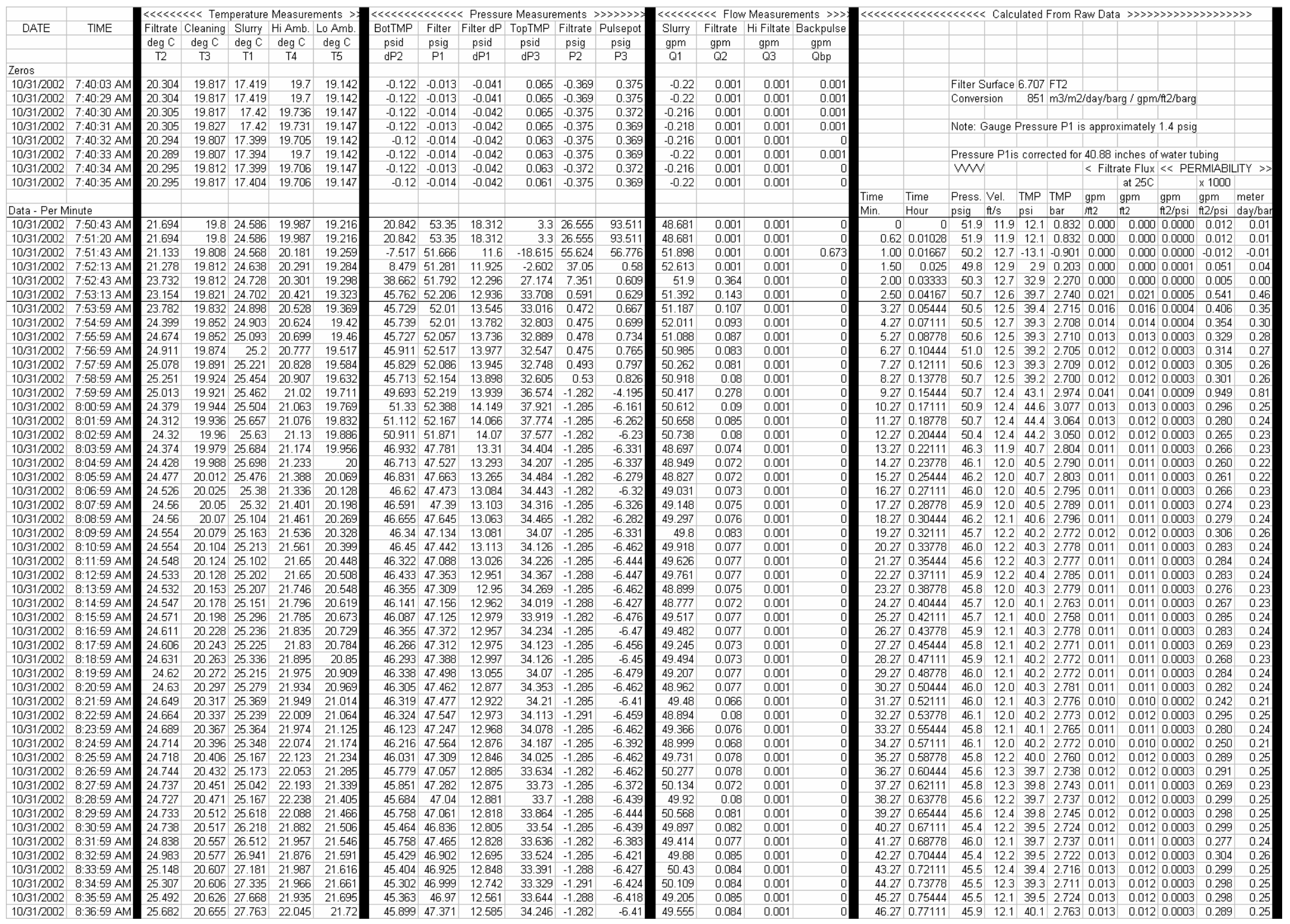

Figure F2-1: Wash run after the last steady state test run (1.29) of AN-102R2, batch 3B, insoluble solids concentration of 19 wt\% - Raw \& Calculated Data 
WSRC-TR-2003-00204, REV. 0

SRT-RPP-2003-00087, REV. 0

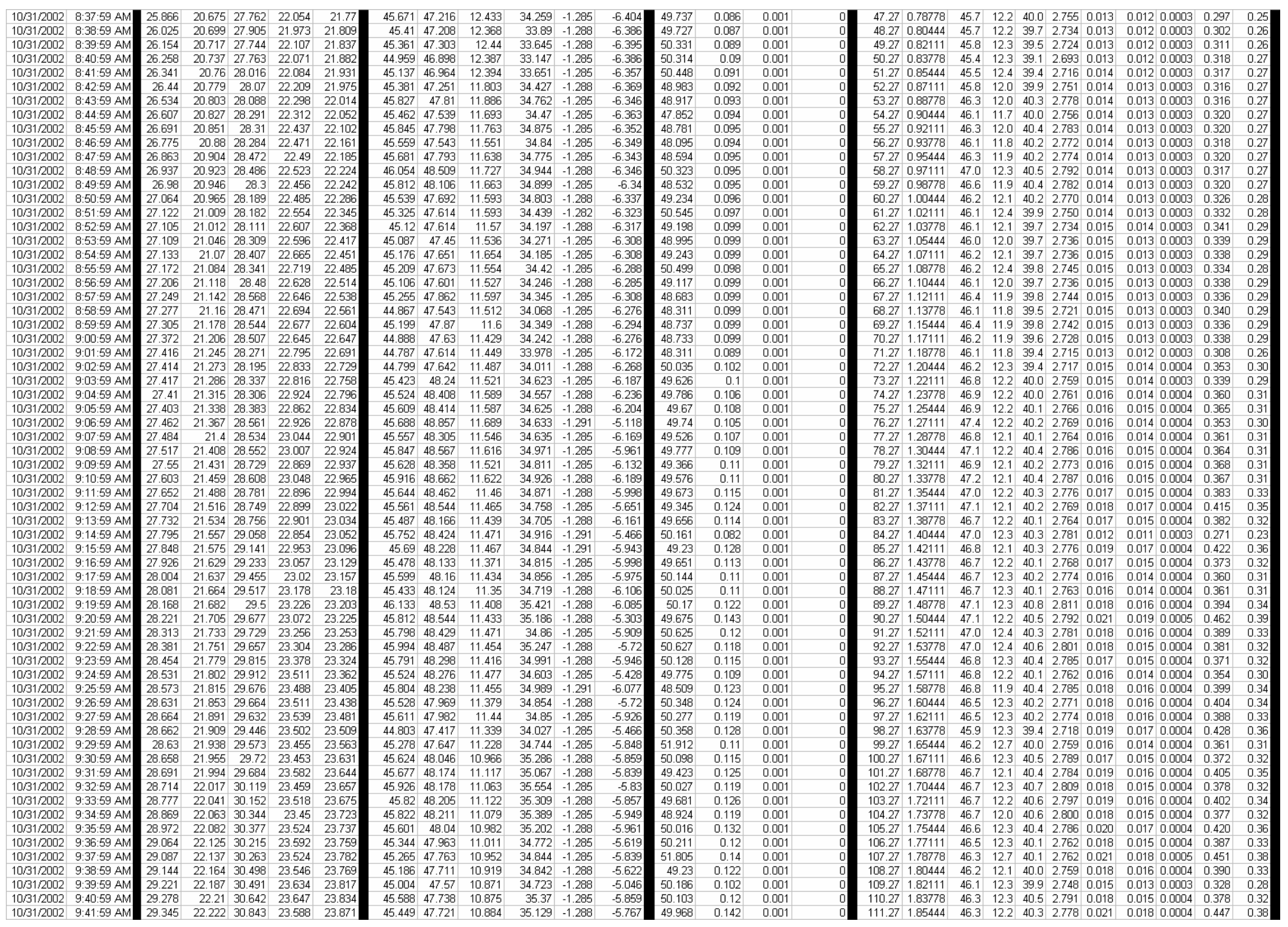

Figure F2-2: Wash run after the last steady state test run (1.29) of AN-102R2, batch 3B, insoluble solids concentration of 19 wt\% - Raw \& Calculated Data 
WSRC-TR-2003-00204, REV. 0

SRT-RPP-2003-00087, REV. 0

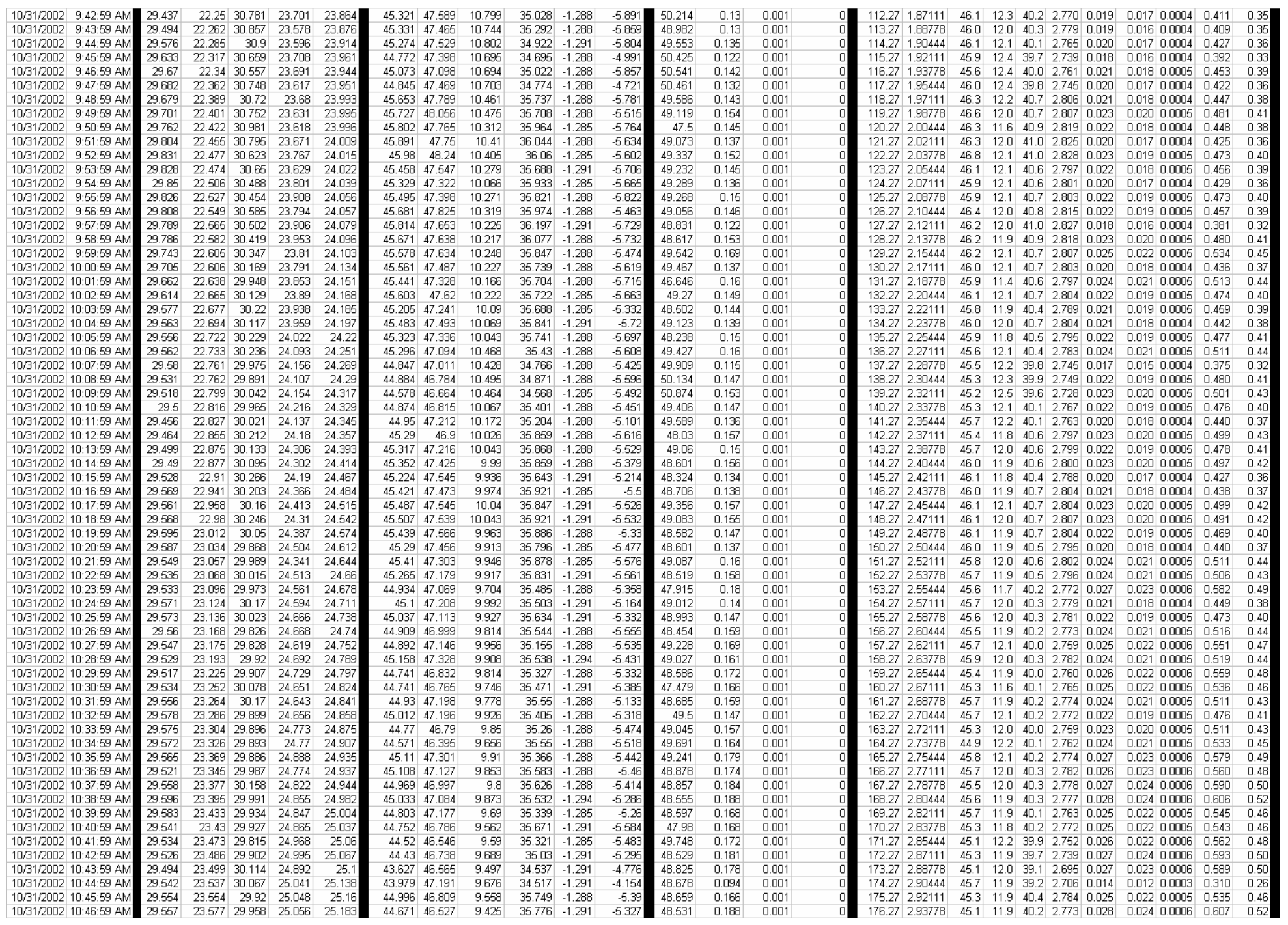

Figure F2-3: Wash run after the last steady state test run (1.29) of AN-102R2, batch 3B, insoluble solids concentration of 19 wt\% - Raw \& Calculated Data 
WSRC-TR-2003-00204, REV. 0

SRT-RPP-2003-00087, REV. 0

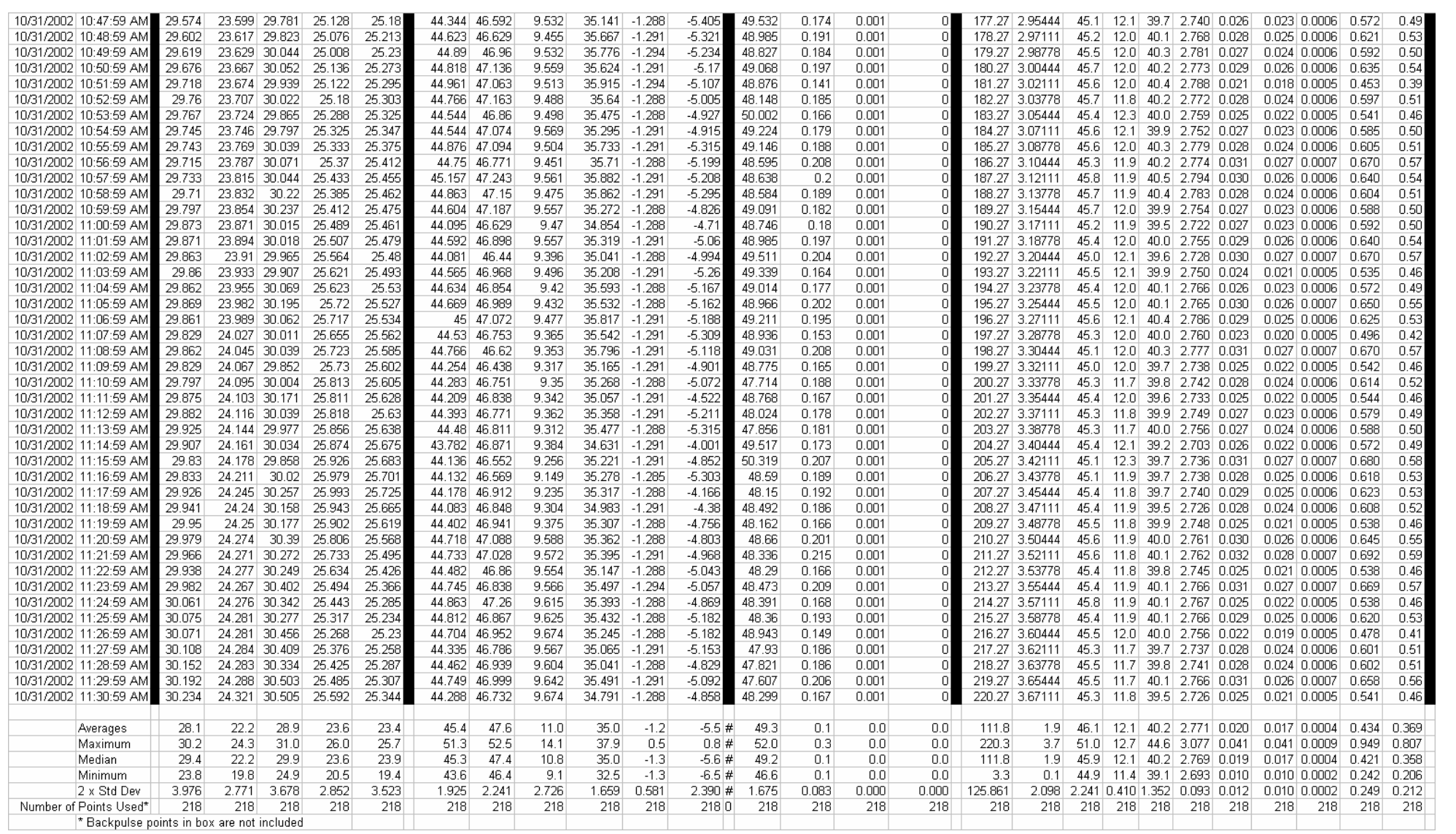

Figure F2-4: Wash run after the last steady state test run (1.29) of AN-102R2, batch 3B, insoluble solids concentration of 19 wt\% - Raw \& Calculated Data 
WSRC-TR-2003-00204, REV. 0

SRT-RPP-2003-00087, REV. 0

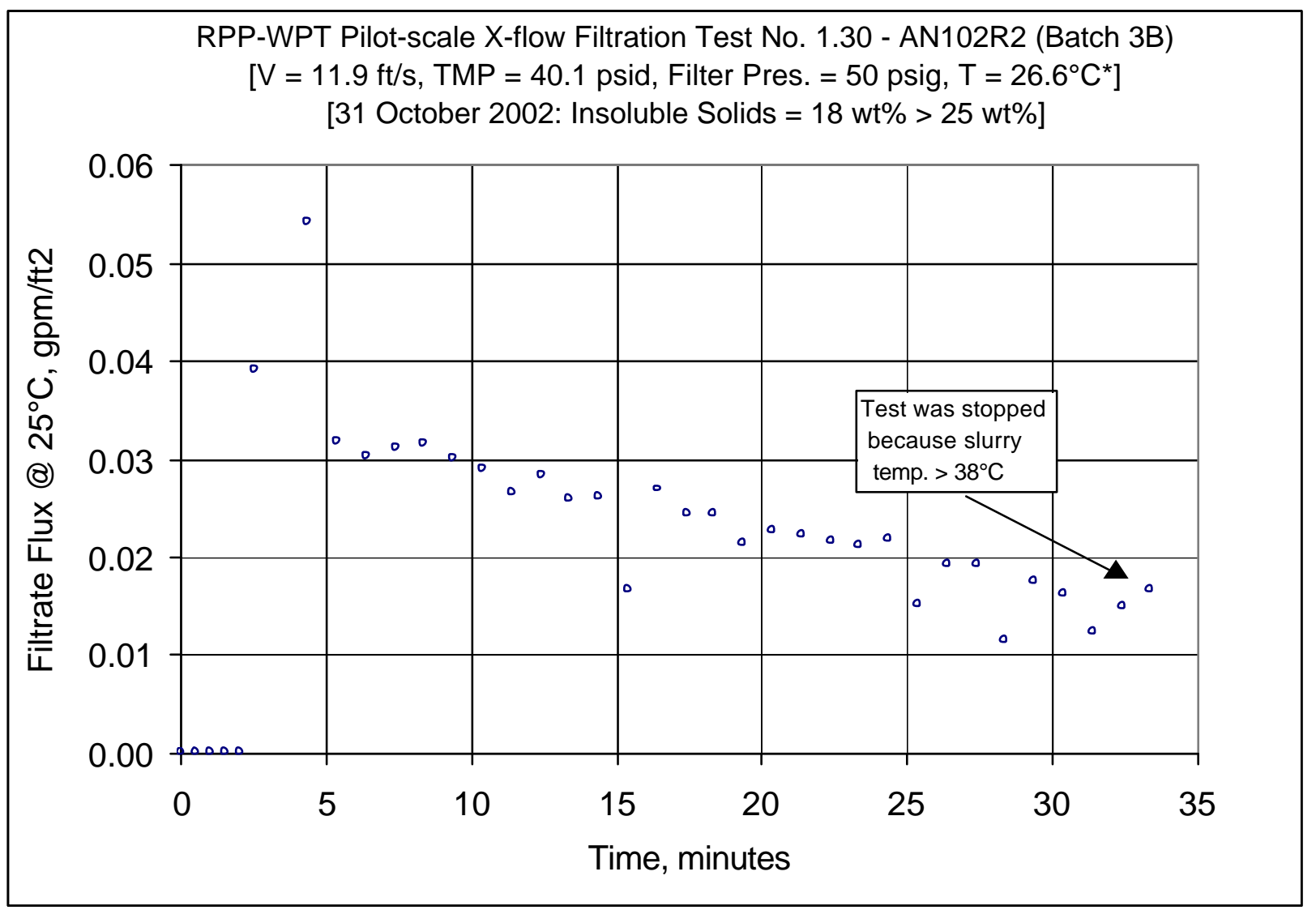

Figure F3: Concentrating washed slurry to maximum possible. It turned out that neither the slurry volume, nor a filter pluggage limited concentration. It was the slurry temperature. After the $26^{\text {th }}$ minute to slurry properties changed such that the active cooling was less and less effective. The exponential temperature increase forced termination. When the temperature passed

$38^{\circ} \mathrm{C}$, and increasing at $2.2^{\circ} \mathrm{C} / \mathrm{min}$, the test was stopped to avoid pump damage. The thermal momentum caused the temperature to reach $46^{\circ} \mathrm{C}$ before it could be controlled. Approximately 20 liters of the approximate 75 liters of washed slurry were removed. The insoluble solids concentration increased from 19 to $25 \mathrm{wt} \%$. 
WSRC-TR-2003-00204, REV. 0

SRT-RPP-2003-00087, REV. 0

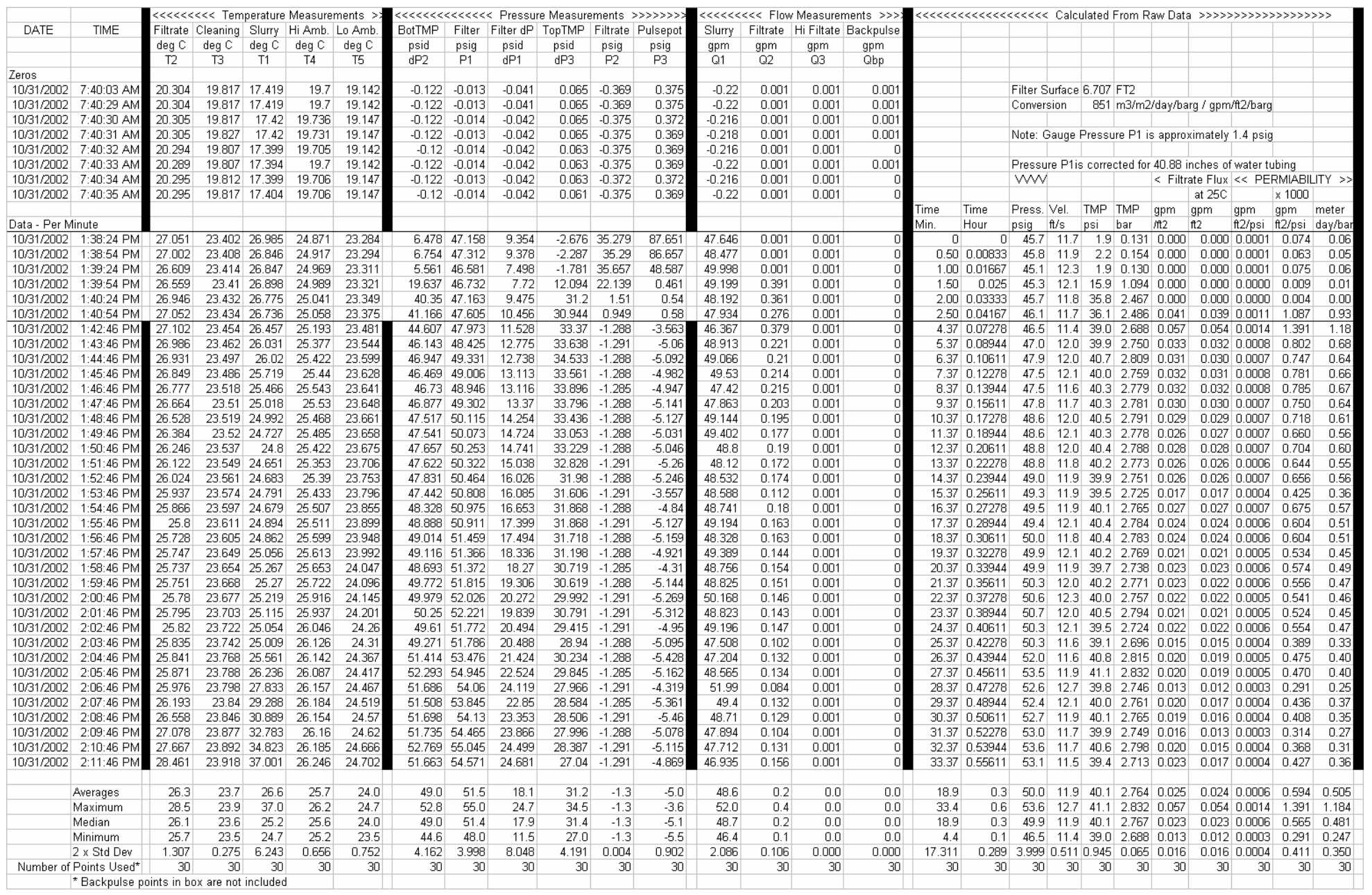

Figure F4-1: Test run no. 1.30: Concentration of washed AN-102R2, batch 3B, insoluble solids concentration of 18 wt\% 25 wt\%- Raw \& Calculated Data 
WSRC-TR-2003-00204, REV. 0

SRT-RPP-2003-00087, REV. 0

This page intentionally left blank 


\section{APPENDIX G \\ ANALYTICAL, RHEOLOGICAL, AND PARTICAL-SIZE DATA}

\section{Appendix Contents}

1. List of all samples taken during test: Table G1.

2. Notes to Table G2.

3. Table G2 contains all of the analytical data as well as some rheological and particlesize data.

4. Slurry Rheology

5. Particle-size Distribution data

Note on measurement uncertainties of the included data:

There are no measurement uncertainties listed because the measurement uncertainties for analytical data are beyond the scope and control of this task. There is reason to believe that all analytical data can be at least $15 \%$ accurate but no quantitative data are given to this effect. Density, filtrate viscosity, turbidity are the only simulant property data that were obtained at the test rig location and the uncertainty of those data can be stated as:

Density: $\pm 0.5 \%$ of reading by calibration

Viscosity: $\pm 0.34 \%$ of reading by manufacturer's statement.

Turbidity: \pm 0.04 NTU 
WSRC-TR-2003-00204, REV. 0 SRT-RPP-2003-00087, REV. 0

\begin{tabular}{|c|c|c|c|c|c|c|}
\hline Related Tes & When Samp & e was Taken & Sample & EDL & ADS or ITS & Analysis \\
\hline & Date & Time & Size $(\mathrm{mL})$ & Sample \# & Sample \# & Requested \\
\hline \multicolumn{7}{|c|}{ AN-102 Batch 3C } \\
\hline $1.16-3 \mathrm{C}$ & 1-Oct-02 & before test & 250 & rpp-pxu-filtrate-pre1.16-turbidity & none-by EDL/EDS/SRTC & turb, dens, visc \\
\hline $1.16-3 \mathrm{C}$ & 1-Oct-02 & before test & 250 & rpp-pxu-filtrate-pre1.16-archive & none & none-archive \\
\hline $1.16-3 \mathrm{C}$ & 1-Oct-02 & before test & 15 & rpp-pxu-filtrate-pre1.16-archive & none & none-archive \\
\hline $1.16-3 \mathrm{C}$ & 1-Oct-02 & before test & 15 & rpp-pxu-filtrate-pre1.16-table1 & 300188154 & ICPES, IC An, TIC/TOC \\
\hline $1.16-3 \mathrm{C}$ & 1-Oct-02 & before test & 15 & rpp-pxu-filtrate-pre1.16-solids & 300186654 & TS, dens \\
\hline $1.16-3 \mathrm{C}$ & 1-Oct-02 & before test & 250 & rpp-pxu-slurry-pre1.16-rheology & none-by PDH/ITS/SRTC & rheology \\
\hline $1.16-3 \mathrm{C}$ & 1-Oct-02 & before test & 250 & rpp-pxu-slurry-pre1.16-archive & none & none-archive \\
\hline $1.16-3 \mathrm{C}$ & 1-Oct-02 & before test & 15 & rpp-pxu-slurry-pre1.16-solids1 & 300186655 & TS, SS, dens \\
\hline $1.16-3 \mathrm{C}$ & $1-$ Oct-02 & before test & 15 & rpp-pxu-slurry-pre1.16-solids2 & 300186656 & TS, SS, dens \\
\hline $1.16-3 \mathrm{C}$ & 1-Oct-02 & before test & 15 & rpp-pxu-slurry-pre1.16-psd & 300188111 & microtrac \\
\hline $1.16-3 \mathrm{C}$ & 1-Oct-02 & before test & 15 & rpp-pxu-slurry-pre1.16-archive & none & none-archive \\
\hline $1.16-3 \mathrm{C}$ & 3-Oct-02 & test end & 15 & rpp-pxu-filtrate-mid1.16-table1 & 300188155 & ICPES, IC An, TIC/TOC, turb \\
\hline $1.16-3 \mathrm{C}$ & 3-Oct-02 & test end & 15 & rpp-pxu-filtrate-mid1.16-solids & no number, used for > & acquous for next 2 samples \\
\hline $1.16-3 \mathrm{C}$ & 3-Oct-02 & test end & 15 & rpp-pxu-slurry-mid1.16-solids1 & 300186909 & TS, SS, dens \\
\hline $1.16-3 \mathrm{C}$ & 3-Oct-02 & test end & 15 & rpp-pxu-slurry-mid1.16-solids2 & 300186910 & TS, SS, dens \\
\hline $1.16-3 \mathrm{C}$ & 3-Oct-02 & test end & 15 & rpp-pxu-slurry-mid1.16-psd & 300188112 & microtrac \\
\hline $1.16-3 \mathrm{C}$ & $3-$-Oct-02 & test end & 250 & rpp-pxu-slurry-mid1.16-archive & none & none-archive \\
\hline \multicolumn{7}{|c|}{ AN-102Batch 3B } \\
\hline $1.16-3 \mathrm{~B}$ & 22-Oct-02 & before test & 250 & Pre1.16-batch 3B - P. Townson & sent to Hanford & none \\
\hline $1.16-3 \mathrm{~B}$ & 22-Oct-02 & before test & 250 & rpp-pxu-slu-pre1.16-3b-rheology & none-by PDH/ITS/SRTC & rheology \\
\hline $1.16-3 \mathrm{~B}$ & 22-Oct-02 & before test & 250 & rpp-pxu-slu-pre1.16-3b-archive & none & none-archive \\
\hline $1.16-3 \mathrm{~B}$ & 22-Oct-02 & before test & 15 & rpp-pxu-slu-pre1.16-3b-solids1 & 300187815 & TS, SS, dens \\
\hline $1.16-3 \mathrm{~B}$ & 22-Oct-02 & before test & 15 & rpp-pxu-slu-pre1.16-3b-solids2 & 300187816 & TS, SS, dens \\
\hline $1.16-3 \mathrm{~B}$ & 22-Oct-02 & before test & 15 & rpp-pxu-slu-pre1.16-3b-psd & 300188109 & microtrac \\
\hline $1.16-3 \mathrm{~B}$ & 22-Oct-02 & before test & 15 & rpp-pxu-slu-pre1.16-3b-archive & none & none-archive \\
\hline $1.16-3 \mathrm{~B}$ & 22-Oct-02 & $1 \mathrm{hr}$ into test & 250 & rpp-pxu-filt-pre1.16-3b-turbidity & none-by EDL/EDS/SRTC & turb, dens, visc \\
\hline $1.16-3 \mathrm{~B}$ & 22-Oct-02 & $1 \mathrm{hr}$ into test & 15 & rpp-pxu-filt-pre1.16-3b-archive & none & none-archive \\
\hline $1.16-3 \mathrm{~B}$ & $22-\mathrm{Oct}-02$ & $1 \mathrm{hr}$ into test & 15 & rpp-pxu-filt-pre1.16-3b-table1 & 300188150 & ICPES, IC An, TIC/TOC \\
\hline $1.16-3 B$ & 23-Oct-02 & test end/02:56 & 250 & rpp-pxu-filt-end1.16-3b-solids & for archive & none \\
\hline $1.16-3 \mathrm{~B}$ & 23-Oct-02 & test end/02:56 & 15 & rpp-pxu-filt-end1.16-3b-solids & no number & none \\
\hline 1.16-3B & 23-Oct-02 & test end/02:56 & 15 & rpp-pxu-slu-end1.16-3b-solids1 & 300187825 & TS, SS, dens \\
\hline 1.16-3B & 23-Oct-02 & test end/02:56 & 15 & rpp-pxu-slu-end1.16-3b-solids2 & 300187826 & TS, SS, dens \\
\hline 1.16-3B & 23-Oct-02 & test end/19:00 & 250 & rpp-pxu-filt-final1.16-3b-archive & none & none-archive \\
\hline $1.16-3 \mathrm{~B}$ & 23-Oct-02 & test end/19:00 & 15 & rpp-pxu-filt-final1.16-3b-solids & no number, used for > & acquous for next solids $1 \& 2$ \\
\hline $1.16-3 \mathrm{~B}$ & 23-Oct-02 & test end/19:00 & 15 & rpp-pxu-filt-final1.16-3b-table1 & 300188152 & ICPES, IC An, TIC/TOC, turb \\
\hline $1.16-3 \mathrm{~B}$ & 23-Oct-02 & test end/19:00 & 250 & Final1.16-batch 3B - P. Townson & sent to Hanford & none \\
\hline $1.16-3 \mathrm{~B}$ & 23-Oct-02 & test end/19:00 & 250 & rpp-pxu-slu-final1.16-3b-rheology & none-by PDH/ITS/SRTC & rheology \\
\hline 1.16-3B & 23-Oct-02 & test end/19:00 & 250 & rpp-pxu-slu-final1.16-3b-archive & none & none-archive \\
\hline $1.16-3 \mathrm{~B}$ & 23-Oct-02 & test end/19:00 & 15 & rpp-pxu-slu-final1.16-3b-solids1 & 300187857 & TS, SS, dens \\
\hline $1.16-3 \mathrm{~B}$ & 23-Oct-02 & test end/19:00 & 15 & rpp-pxu-slu-final1.16-3b-solids2 & 300187858 & TS, SS, dens \\
\hline 1.16-3B & 23-Oct-02 & test end/19:00 & 15 & rpp-pxu-slu-final1.16-3b-psd & 300188110 & microtrac \\
\hline Wash-3B & 30-Oct-02 & PreWash22wt\% & 250 & rpp-pxu-filt22\%-prewash-3b-archive & none & none-archive \\
\hline Wash-3B & 30-Oct-02 & PreWash22wt\% & 250 & rpp-pxu-slu22\%-prewash-3b-rheology & none-by PDH/ITS/SRTC & rheology \\
\hline Wash-3B & 30-Oct-02 & PreWash22wt\% & 250 & rpp-pxu-slu22\%-prewash-3b-archive & none & none-archive \\
\hline Wash-3B & 30-Oct-02 & PreWash20wt\% & 250 & rpp-pxu-filt20\%-prewash-3b-archive & none & none-archive \\
\hline Wash-3B & 30-Oct-02 & PreWash20wt\% & 250 & rpp-pxu-slu20\%-prewash-3b-rheology & none-by PDH/ITS/SRTC & rheology \\
\hline Wash-3B & $30-$-Oct-02 & PreWash20wt\% & 250 & rpp-pxu-slu20\%-prewash-3b-archive & none & none-archive \\
\hline Wash-3B & 30-Oct-02 & PreWash20wt\% & 15 & rpp-pxu-filt20\%-prewash-3b-table1 & 300189078 & ICPES, IC An, TIC/TOC \\
\hline Wash-3B & $30-$ Oct-02 & PreWash20wt\% & 15 & rpp-pxu-filt20\%-prewash-3b-solids & 300189087 & TS, dens \\
\hline Wash-3B & $30-$-Oct-02 & PreWash20wt\% & 15 & rpp-pxu-slu20\%-prewash-3b-solids & 300189088 & TS, SS, dens \\
\hline Wash-3B & $30-$-Oct-02 & PreWash20wt\% & 15 & rpp-pxu-slu20\%-prewash-3b-psd & 300189271 & microtrac \\
\hline Wash-3B & $30-$-Oct-02 & Wash & 15 & rpp-pxu-filt-wash-5-3b & 300189083 & ICPES \\
\hline Wash-3B & 30-Oct-02 & Wash & 15 & rpp-pxu-filt-wash-10-3b & 300189084 & ICPES \\
\hline Wash-3B & 30-Oct-02 & Wash & 15 & rpp-pxu-filt-wash-15-3b & 300189085 & ICPES \\
\hline Wash-3B & 30-Oct-02 & PostWash & 250 & rpp-pxu-filt-postwash-3b-archive & none & none-archive \\
\hline Wash-3B & 30-Oct-02 & PostWash & 250 & rpp-pxu-slu-postwash-3b-rheology & none-by PDH/ITS/SRTC & rheology \\
\hline Wash-3B & 30-Oct-02 & PostWash & 250 & rpp-pxu-slu-postwash-3b-archive & none & none-archive \\
\hline Wash-3B & 30-Oct-02 & PostWash & 15 & rpp-pxu-filt-postwash-3b-table1 & 300189079 & ICPES, IC An, TIC/TOC \\
\hline Wash-3B & 30-Oct-02 & PostWash & 15 & rpp-pxu-filt-postwash-3b-solids & 300189089 & TS, dens \\
\hline Wash-3B & 30-Oct-02 & PostWash & 15 & rpp-pxu-slu-postwash-3b-solids & 300189090 & TS, SS, dens \\
\hline Wash-3B & 30-Oct-02 & PostWash & 15 & rpp-pxu-slu-postwash-3b-psd & 300189272 & microtrac \\
\hline $130-3 \mathrm{~B}$ & 31-Oct-02 & Test End & 250 & rpp-pxu-filt-end1.30-3b-archive & none & none-archive \\
\hline $130-3 \mathrm{~B}$ & 31-Oct-02 & Test End & 15 & rpp-pxu-filt-end1.30-3b-solids & 300189091 & TS, dens \\
\hline 130-3B & 31-Oct-02 & Test End & 15 & rpp-pxu-filt-end1.30-3b-table1 & 300189080 & TS, SS, dens \\
\hline $130-3 \mathrm{~B}$ & 31-Oct-02 & Test End & 250 & rpp-pxu-slu-end1.30-3b-rheology & none-by PDH/ITS/SRTC & rheology \\
\hline $130-3 \mathrm{~B}$ & $31-$ Oct-02 & Test End & 250 & rpp-pxu-slu-end1.30-3b-archive & none & none-archive \\
\hline $130-3 \mathrm{~B}$ & 31-Oct-02 & Test End & 15 & rpp-pxu-slu-end1.30-3b-table1 & 300189086 & ICPES, IC An, TIC/TOC \\
\hline $130-3 \mathrm{~B}$ & 31-Oct-02 & Test End & $\sim 50$ in 125 & rpp-pxu-slu-end1.30-3b-solids & 300189092 & TS, SS, dens \\
\hline
\end{tabular}

Table G1:All Samples taken during AN-102 Testing, Page 1 
WSRC-TR-2003-00204, REV. 0 SRT-RPP-2003-00087, REV. 0

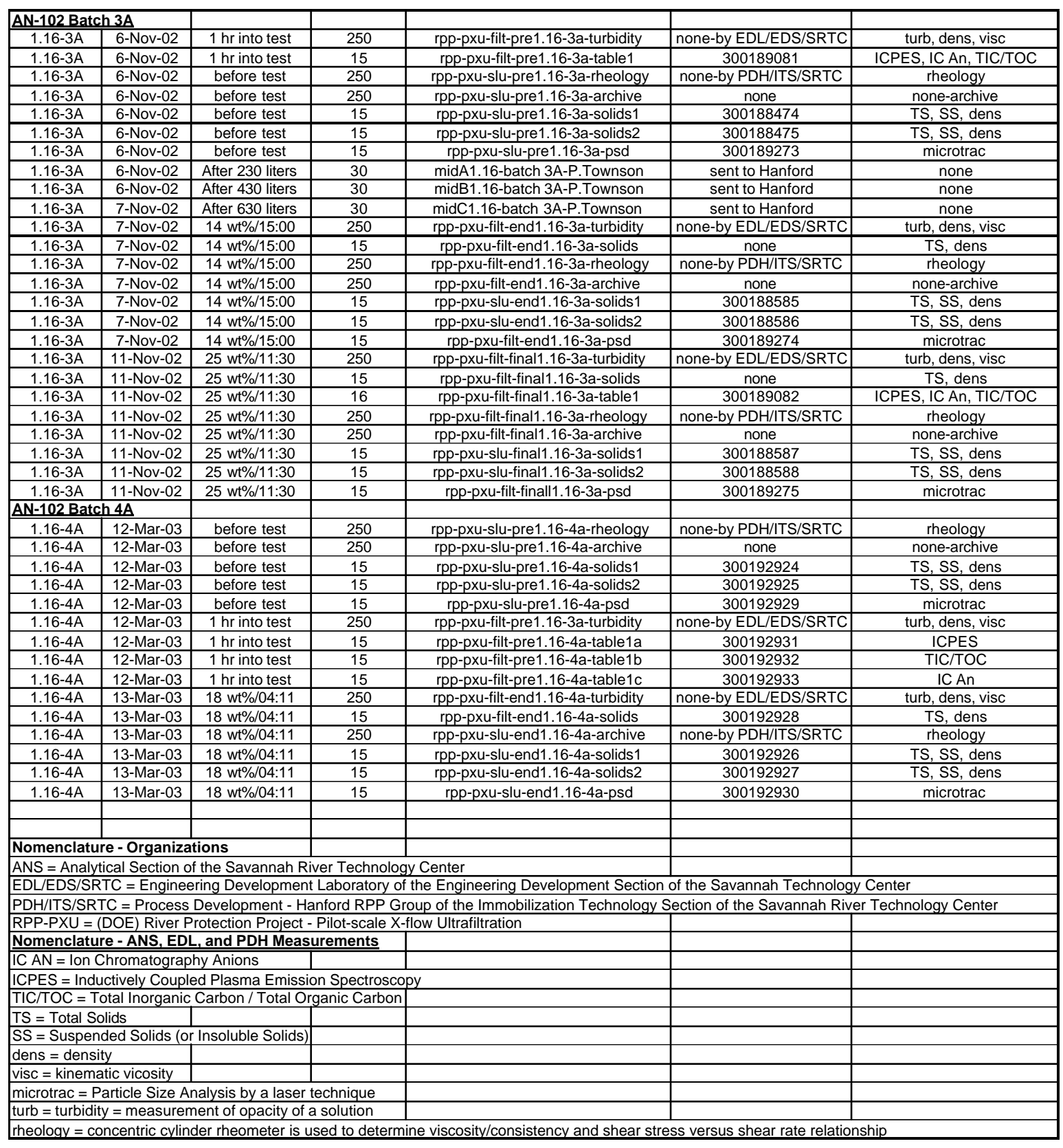

Table G1:All Samples taken during AN-102 Testing, Page 2 
2. The units listed are used for the data in each row except where otherwise noted.

3. See the sample page, which precedes this table for the nomeclature.

4. Turbidity was checked periodically: on $10 / 2 / 02$ it was $0.28 \mathrm{NTU}$, on $10 / 3 / 02$ it was 0.28 NTUon $10 / 9 / 02$ it was 1.01 NTU. This turbidity increase may be due to exposure to light because another sample was taken from a filtrate batch that was created on 10/1/02, but it was kept from light. It's turbidity was $0.16 \mathrm{NTU}$; the same as the orginal sample taken on 10/1/02.

5. Testing with batch $3 \mathrm{C}$ was stopped because of poor filterability and a dedicated slurry sample was not taken for rheological measurements as part of the filtration task. However, sample was not taken as part of the precipitation task. The results of sample RPP-WTP-PREC3C-3SL are in memo: SRTC-PDH-2002-00005 of 8-Oct-2002: 9.68cP and $9.50 \mathrm{cP}$; Yield $=1.47 \mathrm{~Pa}$ and $1.79 \mathrm{~Pa}$

6. The slurry sample was taken AFTER the filtrate sample was take. Approximately 0.75 liters of filtrate was removed from the slurry and the remaining slurry was approximately 60 liters. Therefore the solids concentration of $18.71 \mathrm{wt} \%$ of the slurry sample was slightly higher than the slurry at the end of the experiment and the actual insoluble solids contents was approximately $18.3 \mathrm{wt} \%$. 
WSRC-TR-2003-00204, REV. 0

SRT-RPP-2003-00087, REV. 0

\begin{tabular}{|c|c|c|c|c|c|c|c|c|}
\hline & $\mathrm{A}$ & $\mathrm{B}$ & $\begin{array}{c}\mathrm{C} \\
\mathrm{C}\end{array}$ & $\mathrm{D}$ & $E$ & $\mathrm{~F}$ & G & $\mathrm{H}$ \\
\hline 1 & \multicolumn{2}{|c|}{ 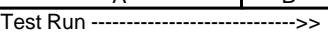 } & & $1.16-3 C$ & $1.16-3 \mathrm{C}$ & $1.16-3 \mathrm{C}$ & $1.16-3 C$ & $1.16-3 C$ \\
\hline 2 & \multirow{2}{*}{\multicolumn{2}{|c|}{ Sample Taken Date ---------->> }} & & October 1,2002 & October 1, 2002 & October 1, 2002 & October 1,2002 & October 1, 2002 \\
\hline 3 & & & & before test & before test & before test & before test & before test \\
\hline 4 & \multicolumn{2}{|c|}{$\begin{array}{l}\text { Sample Taken Tine --------->> } \\
\text { Sample ID (Prefix }=\text { rpp-pu-) ->> }\end{array}$} & & filtrate-pre1.16-turbidity & filtrate-pre1.16-table1 & filtrate-pre1.16-solids & slurry-pre1.16-rheology & slurry-pre1.16-solids1 \\
\hline 5 & \multicolumn{2}{|c|}{ ADS Sample ID -------->> } & & none & 300188154 & 300186654 & none & 300186655 \\
\hline 6 & \multicolumn{2}{|l|}{ Type Sample -- } & & filtrate & filtrate & filtrate & slurry & slurry \\
\hline 7 & \multicolumn{2}{|c|}{ Sample Size (mL) - - } & & 250 & 15 & 15 & 250 & 15 \\
\hline 8 & Item Measured & Units* & Method/Analyst Org & & & & & \\
\hline 9 & Al & $\mathrm{ug} / \mathrm{mL}$ & ICP-ES / ADS & & 7840 & & & \\
\hline 10 & & $\mathrm{ug} / \mathrm{mL}$ & ICP-ES / ADS & & 24.9 & & & \\
\hline 11 & $\mathrm{Ba}$ & $\mathrm{ug} / \mathrm{mL}$ & ICP-ES / ADS & & $<0.024$ & & & \\
\hline 12 & $\mathrm{Ca}$ & $\mathrm{ug} / \mathrm{mL}$ & ICP-ES/ADS & & 78.8 & & & \\
\hline 13 & $\mathrm{Cd}$ & $\mathrm{ug} / \mathrm{mL}$ & ICP-ES / ADS & & 35.2 & & & \\
\hline 14 & $\mathrm{Ce}$ & $\mathrm{ug} / \mathrm{mL}$ & ICP-ES / ADS & & 6.9 & & & \\
\hline 15 & $\mathrm{CI}$ & $\mathrm{ug} / \mathrm{mL}$ & IC Anions / ADS & & 3410 & & & \\
\hline 16 & Co & $\mathrm{ug} / \mathrm{mL}$ & ICP-ES / ADS & & $<1.0$ & & & \\
\hline 17 & $\mathrm{Cr}$ & $\mathrm{ug} / \mathrm{mL}$ & ICP-ES / ADS & & 146 & & & \\
\hline 18 & $\mathrm{Cu}$ & $\mathrm{ug} / \mathrm{mL}$ & ICP-ES / ADS & & $<0.25$ & & & \\
\hline 19 & $\mathrm{Fe}$ & $\mathrm{ug} / \mathrm{mL}$ & ICP-ES / ADS & & 1.5 & & & \\
\hline 20 & K & $\mathrm{ug} / \mathrm{mL}$ & ICP-ES / ADS & & 1740 & & & \\
\hline 21 & $\mathrm{La}$ & $\mathrm{ug} / \mathrm{mL}$ & ICP-ES / ADS & & 4.2 & & & \\
\hline 22 & $\mathrm{Mg}$ & $\mathrm{ug} / \mathrm{mL}$ & ICP-ES / ADS & & $<0.168$ & & & \\
\hline 23 & $\mathrm{Mn}$ & $\mathrm{ug} / \mathrm{mL}$ & ICP-ES / ADS & & 4.7 & & & \\
\hline 24 & & $\mathrm{ug} / \mathrm{mL}$ & ICP-ES / ADS & & 29.6 & & & \\
\hline 25 & $\mathrm{Na}$ & $\mathrm{ug} / \mathrm{mL}$ & ICP-ES / ADS & & 123000 & & & \\
\hline 26 & Nd & $\mathrm{ug} / \mathrm{mL}$ & ICP-ES / ADS & & 11.4 & & & \\
\hline 27 & $\mathrm{Ni}$ & $\mathrm{ug} / \mathrm{mL}$ & ICP-ES / ADS & & 240 & & & \\
\hline 28 & NO3 (Nitrate) & $\mathrm{ug} / \mathrm{mL}$ & IC Anions / ADS & & 135000 & & & \\
\hline 29 & $P$ & $\mathrm{ug} / \mathrm{mL}$ & ICP-ES / ADS & & 681 & & & \\
\hline 30 & PO4 (Phosphate) & $\mathrm{ug} / \mathrm{mL}$ & IC Anions / ADS & & 3140 & & & \\
\hline 31 & $\mathrm{~Pb}$ & $\mathrm{ug} / \mathrm{mL}$ & ICP-ES / ADS & & 20.3 & & & \\
\hline 32 & S & $\mathrm{ug} / \mathrm{mL}$ & ICP-ES / ADS & & 2840 & & & \\
\hline 33 & $\mathrm{Si}$ & $\mathrm{ug} / \mathrm{mL}$ & ICP-ES / ADS & & 21.6 & & & \\
\hline 34 & Sn & $\mathrm{ug} / \mathrm{mL}$ & ICP-ES / ADS & & 0.7 & & & \\
\hline 35 & SO4 (Sulfate) & $\mathrm{ug} / \mathrm{mL}$ & IC Anions / ADS & & 8890 & & & \\
\hline 36 & $\mathrm{Sr}$ & $\mathrm{ug} / \mathrm{mL}$ & ICP-ES / ADS & & 30.5 & & & \\
\hline 37 & $\mathrm{~W}$ & $\mathrm{ug} / \mathrm{mL}$ & ICP-ES / ADS & & 107 & & & \\
\hline 38 & $\mathrm{Zn}$ & $\mathrm{ug} / \mathrm{mL}$ & ICP-ES / ADS & & 1.2 & & & \\
\hline 39 & $\mathrm{Zr}$ & $\mathrm{ug} / \mathrm{mL}$ & ICP-ES / ADS & & 2.4 & & & \\
\hline 40 & Total Organic Carbon & $\mathrm{ug} / \mathrm{mL}$ & TIC-TOC/ADS & & 10000 & & & \\
\hline 41 & Total Inorganic Carbon & $\mathrm{ug} / \mathrm{mL}$ & TIC-TOC / ADS & & 6990 & & & \\
\hline 42 & Suspended Solids & $w t \%$ & Gravimetric / ADS & & & & & 1.00 \\
\hline 43 & \begin{tabular}{|l} 
Total Solids \\
\end{tabular} & $\mathrm{wt} \%$ & Gravimetric / ADS & & & 33.08 & & 33.70 \\
\hline 44 & \begin{tabular}{|l|} 
Particle Size by Volume \\
\end{tabular} & micron & Microtrac/ADS & & & & & \\
\hline 45 & Particle Size by Number & micron & Microtrac / ADS & & & & & \\
\hline 46 & Kin. Viscosity & $\mathrm{cSt}$ & CONE/ITS-CAPILLARY/EDL & 2.16 & & & 2.8 & \\
\hline 47 & Dyn. Visc./Consistency & $\frac{\mathrm{cP}}{\mathrm{cP}}$ & Kin. Visc. $x$ Density & 2.67 & & & 3.6 & \\
\hline 48 & Yield Stress & $\mathrm{Pa}$ & & & & & 0.0 & \\
\hline 49 & Density & $\mathrm{g} / \mathrm{mL}$ & Gravimetric / EDL & 1.238 & & & 1.279 & \\
\hline 50 & Density & $\mathrm{g} / \mathrm{mL}$ & Gravimetric / ADS & & & 1.250 & & 1.330 \\
\hline 51 & Turbidity & NTU & $/ \mathrm{EDL}$ & 0.18 (on 10/1) & & & & \\
\hline 52 & Comment - Note (1) & Note (2) & Note (3) & Note (4) & & & Note (5) & \\
\hline 53 & Note (1) - See notes on pa & ge prece & able. & & & & & \\
\hline
\end{tabular}

Table G2:AN-102 simulant analytical and other data, Page 1 
WSRC-TR-2003-00204, REV. 0

SRT-RPP-2003-00087, REV. 0

\begin{tabular}{|c|c|c|c|c|c|c|c|c|}
\hline & $\mathrm{I}$ & $\mathrm{J}$ & $\mathrm{K}$ & $\mathrm{L}$ & $\mathrm{M}$ & $\mathrm{N}$ & 0 & $P$ \\
\hline \begin{tabular}{|l|}
1 \\
\end{tabular} & Test Run --.-.-- & -.-->> & $1.16-3 \mathrm{C}$ & $1.16-3 \mathrm{C}$ & $1.16-3 \mathrm{C}$ & $1.16-3 \mathrm{C}$ & $1.16-3 \mathrm{C}$ & $1.16-3 \mathrm{C}$ \\
\hline \begin{tabular}{|l|l|}
2 \\
\end{tabular} & Sample Taken Date ----- & $\cdots>>$ & October 1,2002 & October 1,2002 & October 3,2002 & October 3,2002 & October 3,2002 & October 3,2002 \\
\hline \begin{tabular}{|l|l|}
3 \\
\end{tabular} & Sample Taken Time --.--. & -..--->> & before test & before test & test end & test end & test end & test end \\
\hline \begin{tabular}{|l|}
4 \\
\end{tabular} & Sample ID (Prefix = rpp-p & (u-) - -> & slurry-pre1.16-solids2 & slurry-pre1.16-psd & filtrate-mid1.16-table1 & \begin{tabular}{|l|} 
slurry-mid1.16-solids1 \\
\end{tabular} & slurry-mid1.16-solids2 & slurry-mid1.16-psd \\
\hline \begin{tabular}{|l|}
5 \\
\end{tabular} & ADS Sample ID & $\ldots>$ & 300186656 & 300188111 & 300188155 & 300186909 & 300186910 & 300188112 \\
\hline \begin{tabular}{|l|l|}
6 & \\
\end{tabular} & Type Sample -.--.-.-.-.-. & $-\cdots+\cdots>>>>$ & slurry & slurry & filtrate & slurry & slurry & slurry \\
\hline \begin{tabular}{|l|}
7 \\
\end{tabular} & Sample Size $(\mathrm{mL})$ - - & 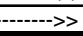 & 15 & 15 & 15 & 15 & 15 & 15 \\
\hline \begin{tabular}{|l|}
8 \\
\end{tabular} & Item Measured & Units $^{*}$ & & & & & & \\
\hline \begin{tabular}{|l|}
9 \\
\end{tabular} & $\mid \mathrm{Al}$ & $\mathrm{ug} / \mathrm{mL}$ & & & 8480 & & & \\
\hline \begin{tabular}{|l|}
10 \\
\end{tabular} & $B$ & $\mathrm{ug} / \mathrm{mL}$ & & & 27.5 & & & \\
\hline \begin{tabular}{|l|}
11 \\
\end{tabular} & $\mathrm{Ba}$ & \begin{tabular}{|l}
$\mathrm{ug} / \mathrm{mL}$ \\
\end{tabular} & & & $<0.024$ & & & \\
\hline 12 & $\mathrm{Ca}$ & $u g / m L$ & & & 102.0 & & & \\
\hline \begin{tabular}{|c|}
13 \\
\end{tabular} & $\mathrm{Cd}$ & \begin{tabular}{|l}
$\mathrm{ug} / \mathrm{mL}$ \\
\end{tabular} & & & 38.4 & & & \\
\hline \begin{tabular}{|l|}
14 \\
\end{tabular} & $\mathrm{Ce}$ & $\mathrm{ug} / \mathrm{mL}$ & & & 5.6 & & & \\
\hline \begin{tabular}{|c|}
15 \\
\end{tabular} & $\mathrm{Cl}^{-}$ & $\mathrm{ug} / \mathrm{mL}$ & & & 3610 & & & \\
\hline 16 & Co & $\mathrm{ug} / \mathrm{mL}$ & & & $<1.0$ & & & \\
\hline \begin{tabular}{|c|}
17 \\
\end{tabular} & $\mathrm{Cr}$ & $\mathrm{ug} / \mathrm{mL}$ & & & 160 & & & \\
\hline 18 & $\mathrm{Cu}$ & $\mathrm{ug} / \mathrm{mL}$ & & & $<0.25$ & & & \\
\hline \begin{tabular}{|l|}
19 \\
\end{tabular} & $\mathrm{Fe}$ & $\mathrm{ug} / \mathrm{mL}$ & & & 0.9 & & & \\
\hline \begin{tabular}{|l|}
20 \\
\end{tabular} & $K$ & $\mathrm{ug} / \mathrm{mL}$ & & & 1940 & & & \\
\hline \begin{tabular}{|l|}
21 \\
\end{tabular} & $\mathrm{La}$ & $\mathrm{ug} / \mathrm{mL}$ & & & 2.5 & & & \\
\hline 22 & $\mathrm{Mg}$ & $\mathrm{ug} / \mathrm{mL}$ & & & $<0.168$ & & & \\
\hline \begin{tabular}{|l|}
23 \\
\end{tabular} & $\mathrm{Mn}$ & $\mathrm{ug} / \mathrm{mL}$ & & & 5.4 & & & \\
\hline \begin{tabular}{|l|}
24 \\
\end{tabular} & Mo & $\mathrm{ug} / \mathrm{mL}$ & & & 31.8 & & & \\
\hline \begin{tabular}{|l|}
25 \\
25 \\
\end{tabular} & $\mathrm{Na}$ & \begin{tabular}{|l}
$\mathrm{ug} / \mathrm{mL}$ \\
\end{tabular} & & & 133000 & & & \\
\hline \begin{tabular}{|l|l|}
26 \\
\end{tabular} & Nd & \begin{tabular}{|l}
$\mathrm{ug} / \mathrm{mL}$ \\
\end{tabular} & & & 6.9 & & & \\
\hline \begin{tabular}{|l|}
27 \\
\end{tabular} & $\mathrm{Ni}$ & $\mathrm{ug} / \mathrm{mL}$ & & & 254 & & & \\
\hline \begin{tabular}{|l|}
28 \\
\end{tabular} & NO3 (Nitrate) & $\mathrm{ug} / \mathrm{mL}$ & & & 132000 & & & \\
\hline \begin{tabular}{|l|l|}
29 \\
\end{tabular} & $\mathrm{P}$ & $\mathrm{ug} / \mathrm{mL}$ & & & 669 & & & \\
\hline \begin{tabular}{|l|}
30 \\
\end{tabular} & PO4 (Phosphate) & $\mathrm{ug} / \mathrm{mL}$ & & & 3220 & & & \\
\hline \begin{tabular}{|l|}
31 \\
\end{tabular} & $\mathrm{~Pb}$ & $\mathrm{ug} / \mathrm{mL}$ & & & 35.2 & & & \\
\hline \begin{tabular}{|l|}
32 \\
\end{tabular} & s & $\mathrm{ug} / \mathrm{mL}$ & & & 3120 & & & \\
\hline 33 & Si & $\mathrm{ug} / \mathrm{mL}$ & & & 24.4 & & & \\
\hline \begin{tabular}{|l|}
34 \\
\end{tabular} & Sn & $\mathrm{ug} / \mathrm{mL}$ & & & 0.8 & & & \\
\hline \begin{tabular}{|l|l|}
35 \\
\end{tabular} & SO4 (Sulfate) & $\mathrm{ug} / \mathrm{mL}$ & & & 9380 & & & \\
\hline 36 & $\mathrm{Sr}$ & $u \mathrm{ug} / \mathrm{mL}$ & & & 10.9 & & & \\
\hline \begin{tabular}{|l|}
37 \\
\end{tabular} & w & $\mathrm{ug} / \mathrm{mL}$ & & & 120 & & & \\
\hline \begin{tabular}{|l|}
38 \\
\end{tabular} & $\mathrm{Zn}$ & $\mathrm{ug} / \mathrm{mL}$ & & & 1.4 & & & \\
\hline \begin{tabular}{|l|}
39 \\
\end{tabular} & $\mathrm{Zr}$ & $\mathrm{ug} / \mathrm{mL}$ & & & 2.4 & & & \\
\hline \begin{tabular}{|l|}
40 \\
\end{tabular} & Total Organic Carbon & $\mathrm{ug} / \mathrm{mL}$ & & & 10900 & & & \\
\hline \begin{tabular}{|l|}
41 \\
\end{tabular} & Total Inorganic Carbon & \begin{tabular}{|l}
$\mathrm{ug} / \mathrm{mL}$ \\
\end{tabular} & & & 7820 & & & \\
\hline \begin{tabular}{|l|}
42 \\
\end{tabular} & Suspended Solids & $w+\%$ & 0.68 & & & 8.51 & 8.30 & \\
\hline \begin{tabular}{|l|}
43 \\
\end{tabular} & Total Solids & $w t \%$ & 33.49 & & & 38.71 & 38.56 & \\
\hline \begin{tabular}{|l|}
44 \\
\end{tabular} & Particle Size by Volume & micron & & $2.56(32 \%), 8.59(68 \%)$ & & & & $2.47(49 \%), 6.75(51 \%)$ \\
\hline 45 & Particle Size by Number & micron & & $2.08(100 \%)$ & & & & 1.91 \\
\hline \begin{tabular}{|l|}
46 \\
\end{tabular} & Kin. Viscosity & \begin{tabular}{|c|}
$\mathrm{cSt}$ \\
\end{tabular} & & & & & & 7.5 \\
\hline \begin{tabular}{|l|}
47 \\
\end{tabular} & Dyn. Visc./Consistency & $\mathrm{cP}$ & & & & & & 9.8 \\
\hline 48 & Yield Stress & $\mathrm{Pa}$ & & & & & & 1.7 \\
\hline \begin{tabular}{|l|l|}
49 \\
\end{tabular} & Density & $\mathrm{g} / \mathrm{mL}$ & & & & & & \\
\hline \begin{tabular}{|l|}
50 \\
\end{tabular} & Density & $\mathrm{g} / \mathrm{mL}$ & 1.310 & & & 1.276 & 1.306 & \\
\hline \begin{tabular}{|l|}
51 \\
\end{tabular} & Turbidity & NTU & & & 0.14 & & & \\
\hline \begin{tabular}{|l|}
52 \\
\end{tabular} & Comment - Note (1) & Note (2) & & bimodal by volume & & & & bimodal by volume \\
\hline \begin{tabular}{|l|l|}
53 \\
\end{tabular} & Note(1)-Notes precede thi & stable & & & & & & \\
\hline
\end{tabular}

Table G2:AN-102 simulant analytical and other data, Page 2 
WSRC-TR-2003-00204, REV. 0

SRT-RPP-2003-00087, REV. 0

\begin{tabular}{|c|c|c|c|c|c|c|c|c|}
\hline & $\mathrm{R}$ & $\mathrm{s}$ & $T$ & $\mathrm{U}$ & $\mathrm{V}$ & $\mathrm{W}$ & $\bar{x}$ & $\bar{Y}$ \\
\hline 1 & \multirow{2}{*}{\multicolumn{2}{|c|}{ 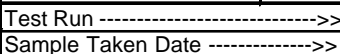 }} & $1.16-3 \mathrm{~B}$ & $1.16-3 \mathrm{~B}$ & $1.16-3 \mathrm{~B}$ & $1.16-3 \mathrm{~B}$ & $1.16-3 \mathrm{~B}$ & $1.16-3 \mathrm{~B}$ \\
\hline 2 & & & October 22, 2002 & October 22, 2002 & October 22, 2002 & October 22,2002 & October 22, 2002 & October 22,2002 \\
\hline 3 & \multicolumn{2}{|c|}{ Sample Taken Time ------------>> } & before test & before test & before test & before test & $1 \mathrm{hr}$ into test & $1 \mathrm{hr}$ into test \\
\hline 4 & \multicolumn{2}{|c|}{ Sample ID (Prefix = rpp-pxu-) ->> } & slu-pre1.16-3b-rheology & slu-pre1.16-3b-solids1 & slu-pre1.16-3b-solids2 & slu-pre1.16-3b-psd & filt-pre1.16-3b-turbidity & filt-pre1.16-3b-table1 \\
\hline 5 & \multicolumn{2}{|c|}{ 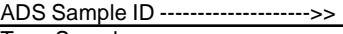 } & none & 300187815 & 300187816 & 300188109 & none & 300188150 \\
\hline 6 & & slurry & slurry & slurry & slurry & filtrate & filtrate \\
\hline 7 & & & 250 & 15 & 15 & 15 & 250 & 15 \\
\hline 8 & \multicolumn{2}{|c|}{\begin{tabular}{l|l} 
Sample Size $(\mathrm{mL})$ & $\ldots$ \\
Item Measured
\end{tabular}} & & & & & & \\
\hline 9 & \multicolumn{2}{|r|}{$\mathrm{ug} / \mathrm{mL}$} & & & & & & 7970 \\
\hline 10 & \multicolumn{2}{|r|}{$\mathrm{ug} / \mathrm{mL}$} & & & & & & 25.2 \\
\hline 11 & \multirow{2}{*}{\multicolumn{2}{|c|}{$\frac{\mathrm{ug} / \mathrm{mL}}{\mathrm{ug} / \mathrm{mL}}$}} & & & & & & $<0.024$ \\
\hline 12 & & & & & & & & 56.2 \\
\hline 13 & \multicolumn{2}{|r|}{$\mathrm{ug} / \mathrm{mL}$} & & & & & & 36.1 \\
\hline 14 & \multicolumn{2}{|r|}{$\mathrm{ug} / \mathrm{mL}$} & & & & & & 2.3 \\
\hline 15 & \multicolumn{2}{|r|}{$\mathrm{ug} / \mathrm{mL}$} & & & & & & 3510 \\
\hline 16 & \multicolumn{2}{|r|}{$\mathrm{ug} / \mathrm{mL}$} & & & & & & $<1.0$ \\
\hline 17 & \multicolumn{2}{|r|}{$\mathrm{ug} / \mathrm{mL}$} & & & & & & 134 \\
\hline 18 & \multicolumn{2}{|r|}{$\mathrm{ug} / \mathrm{mL}$} & & & & & & $<0.25$ \\
\hline 19 & \multirow{2}{*}{\multicolumn{2}{|c|}{$\frac{\mathrm{ug} / \mathrm{mL}}{\mathrm{ug} / \mathrm{mL}}$}} & & & & & & 1.1 \\
\hline 20 & & & & & & & & 1790 \\
\hline 21 & \multicolumn{2}{|r|}{$\mathrm{ug} / \mathrm{mL}$} & & & & & & $<1.4$ \\
\hline 22 & \multicolumn{2}{|r|}{$\mathrm{ug} / \mathrm{mL}$} & & & & & & $<0.168$ \\
\hline 23 & $\mathrm{Mn}$ & $\mathrm{ug} / \mathrm{mL}$ & & & & & & 8.3 \\
\hline 24 & Mo & $\mathrm{ug} / \mathrm{mL}$ & & & & & & 31.4 \\
\hline 25 & $\mathrm{Na}$ & $\mathrm{ug} / \mathrm{mL}$ & & & & & & 140000 \\
\hline 26 & $\mathrm{Nd}$ & $\mathrm{ug} / \mathrm{mL}$ & & & & & & 3.9 \\
\hline 27 & $\mathrm{Ni}$ & $\mathrm{ug} / \mathrm{mL}$ & & & & & & 229 \\
\hline 28 & NO3 (Nitrate) & $\mathrm{ug} / \mathrm{mL}$ & & & & & & 135000 \\
\hline 29 & $\mathrm{P}$ & $\mathrm{ug} / \mathrm{mL}$ & & & & & & 1130 \\
\hline 30 & PO4 (Phosphate) & $\mathrm{ug} / \mathrm{mL}$ & & & & & & 4090 \\
\hline 31 & $\mathrm{~Pb}$ & $\mathrm{ug} / \mathrm{mL}$ & & & & & & 36.4 \\
\hline 32 & 5 & $\mathrm{ug} / \mathrm{mL}$ & & & & & & 2900 \\
\hline 33 & $\mathrm{Si}$ & $\mathrm{ug} / \mathrm{mL}$ & & & & & & 23.2 \\
\hline 34 & Sn & $\mathrm{ug} / \mathrm{mL}$ & & & & & & 0.6 \\
\hline 35 & SO4 (Sulfate) & $\mathrm{ug} / \mathrm{mL}$ & & & & & & 8680 \\
\hline 36 & $\mathrm{Sr}$ & $\mathrm{ug} / \mathrm{mL}$ & & & & & & 40.6 \\
\hline 37 & $\mathrm{~W}$ & $\mathrm{ug} / \mathrm{mL}$ & & & & & & 115 \\
\hline 38 & $\mathrm{Zn}$ & $\mathrm{ug} / \mathrm{mL}$ & & & & & & 3.2 \\
\hline 39 & $\mathrm{Zr}$ & $\mathrm{ug} / \mathrm{mL}$ & & & & & & 1.0 \\
\hline 40 & Total Organic Carbon & $\mathrm{ug} / \mathrm{mL}$ & & & & & & 9620 \\
\hline 41 & Total Inorganic Carbon & $\mathrm{ug} / \mathrm{mL}$ & & & & & & 6480 \\
\hline 42 & \begin{tabular}{|l} 
Suspended Solids \\
\end{tabular} & wt\% & & 1.30 & 1.12 & & & \\
\hline 43 & Total Solids & wt $\%$ & & 32.72 & 32.71 & & & \\
\hline 44 & Particle Size by Volume & micron & & & & $2.25(52 \%), 8.55(48 \%)$ & & \\
\hline 45 & Particle Size by Number & micron & & & & 1.94 & & \\
\hline 46 & Kin. Viscosity & $\mathrm{cSt}$ & 3.1 & & & & 2.8 & \\
\hline 47 & Dyn. Visc./Consistency & $\mathrm{cP}$ & 4.0 & & & & 3.6 & \\
\hline 48 & Yield Stress & $\mathrm{Pa}$ & 0.0 & & & & & \\
\hline 49 & \begin{tabular}{|l|} 
Density \\
\end{tabular} & $\mathrm{g} / \mathrm{mL}$ & 1.285 & & & & 1.279 & \\
\hline 50 & Density & $\mathrm{g} / \mathrm{mL}$ & & 1.314 & 1.265 & & & \\
\hline 51 & Turbidity & NTU & & & & & 0.13 & \\
\hline 52 & Comment - Note (1) & Note (2) & & & & bimodal by volume & & \\
\hline 53 & Note(1)-Notes precede th & is table & & & & & & \\
\hline
\end{tabular}

Table G2:AN-102 simulant analytical and other data, Page 3 
WSRC-TR-2003-00204, REV. 0

SRT-RPP-2003-00087, REV. 0

\begin{tabular}{|c|c|c|c|c|c|c|c|c|}
\hline & $\bar{Z}$ & $\mathrm{AA}$ & $\mathrm{AB}$ & $\mathrm{AC}$ & $\mathrm{AD}$ & $\overline{\mathrm{AE}}$ & $\mathrm{AF}$ & $\overline{A G}$ \\
\hline 1 & Test Run --o- & $-\cdots-\cdots>>$ & $1.16-3 \mathrm{~B}$ & $1.16-3 \mathrm{~B}$ & $1.16-3 \mathrm{~B}$ & $1.16-3 \mathrm{~B}$ & $1.16-3 \mathrm{~B}$ & $1.16-3 \mathrm{~B}$ \\
\hline 2 & Sample Taken Date --- & $-\cdots>>$ & October 23, 2002 & October 23, 2002 & October 23, 2002 & October 23, 2002 & October 23, 2002 & October 23, 2002 \\
\hline 3 & Sample Taken Time --- & -..-->> & test end / 02:56 A.M. & test end / 02:56 A.M. & test end / 02:56 A.M. & test end / 19:00 & test end / 19:00 & test end / 19:00 \\
\hline 4 & Sample ID (Prefix $=$ rpp-p) & (-) ->> & filt-end1.16-3b-solids & slu-end1.16-3b-solids1 & slu-end1.16-3b-solids2 & filt-final1.16-3b-solids & filt-final1.16-3b-table1 & slu-final1.16-3b-rheology \\
\hline 5 & ADS Sample ID & -.--->> & none & 300187825 & 300187826 & none & 300188152 & none \\
\hline 6 & Type Sample ----------- & $-\cdots>$ & filtrate & slurry & slurry & filtrate & filtrate & slurry \\
\hline 7 & Sample Size $(\mathrm{mL})$ & $\cdots$ & 15 & 15 & 15 & 15 & 15 & 250 \\
\hline 8 & Item Measured & Units* $^{*}$ & & & & & & \\
\hline 9 & $\overline{\mathrm{Al}}$ & $\mathrm{ug} / \mathrm{mL}$ & & & & & 8150 & \\
\hline 10 & $B$ & $\mathrm{ug} / \mathrm{mL}$ & & & & & 25.6 & \\
\hline 11 & $\mathrm{Ba}$ & $\mathrm{ug} / \mathrm{mL}$ & & & & & $<0.024$ & \\
\hline 12 & $\mathrm{Ca}$ & $\mathrm{ug} / \mathrm{mL}$ & & & & & 69.3 & \\
\hline 13 & $\mathrm{Cd}$ & $\mathrm{ug} / \mathrm{mL}$ & & & & & 36.3 & \\
\hline 14 & $\mathrm{Ce}$ & $\mathrm{ug} / \mathrm{mL}$ & & & & & 1.8 & \\
\hline 15 & $\mathrm{Cl}^{-}$ & $\mathrm{ug} / \mathrm{mL}$ & & & & & 3360 & \\
\hline 16 & Co & $\mathrm{ug} / \mathrm{mL}$ & & & & & $<1.0$ & \\
\hline 17 & $\mathrm{Cr}$ & $\mathrm{ug} / \mathrm{mL}$ & & & & & 134 & \\
\hline 18 & $\mathrm{Cu}$ & $\mathrm{ug} / \mathrm{mL}$ & & & & & $<0.25$ & \\
\hline 19 & $\mathrm{Fe}$ & $\mathrm{ug} / \mathrm{mL}$ & & & & & 1.2 & \\
\hline 20 & K & $\mathrm{ug} / \mathrm{mL}$ & & & & & 1840 & \\
\hline 21 & La & $\mathrm{ug} / \mathrm{mL}$ & & & & & $<1.4$ & \\
\hline 22 & $\mathrm{Mg}$ & $\mathrm{ug} / \mathrm{mL}$ & & & & & $<0.168$ & \\
\hline 23 & $\mathrm{Mn}$ & $\mathrm{ug} / \mathrm{mL}$ & & & & & 6.2 & \\
\hline 24 & Mo & $\mathrm{ug} / \mathrm{mL}$ & & & & & 31.4 & \\
\hline 25 & $\mathrm{Na}$ & $\mathrm{ug} / \mathrm{mL}$ & & & & & 141000 & \\
\hline 26 & $\mathrm{Nd}$ & $\mathrm{ug} / \mathrm{mL}$ & & & & & 2.8 & \\
\hline 27 & $\mathrm{Ni}$ & $\mathrm{ug} / \mathrm{mL}$ & & & & & 228 & \\
\hline 28 & NO3 (Nitrate) & $\mathrm{ug} / \mathrm{mL}$ & & & & & 122000 & \\
\hline 29 & $\mathrm{P}$ & $\mathrm{ug} / \mathrm{mL}$ & & & & & 716 & \\
\hline 30 & PO4 (Phosphate) & $\mathrm{ug} / \mathrm{mL}$ & & & & & 2570 & \\
\hline 31 & $\mathrm{~Pb}$ & $\mathrm{ug} / \mathrm{mL}$ & & & & & 32.0 & \\
\hline 32 & $S$ & $\mathrm{ug} / \mathrm{mL}$ & & & & & 2910 & \\
\hline 33 & $\mathrm{Si}$ & $\mathrm{ug} / \mathrm{mL}$ & & & & & 24.0 & \\
\hline 34 & Sn & $\mathrm{ug} / \mathrm{mL}$ & & & & & 0.9 & \\
\hline 35 & SO4 (Sulfate) & $\mathrm{ug} / \mathrm{mL}$ & & & & & 7900 & \\
\hline 36 & $\mathrm{Sr}$ & $\mathrm{ug} / \mathrm{mL}$ & & & & & 35.5 & \\
\hline 37 & $\mathrm{~W}$ & $\mathrm{ug} / \mathrm{mL}$ & & & & & 112 & \\
\hline 38 & $\mathrm{Zn}$ & $\mathrm{ug} / \mathrm{mL}$ & & & & & 3.2 & \\
\hline 39 & $\mathrm{Zr}$ & $\mathrm{ug} / \mathrm{mL}$ & & & & & 1.0 & \\
\hline 40 & Total Organic Carbon & $\mathrm{ug} / \mathrm{mL}$ & & & & & 9830 & \\
\hline 41 & Total Inorganic Carbon & $\mathrm{ug} / \mathrm{mL}$ & & & & & 6470 & \\
\hline 42 & Suspended Solids & $w t \%$ & & 14.44 & 14.47 & & & \\
\hline 43 & $\begin{array}{l}\text { Total Solids } \\
\end{array}$ & $w+\%$ & & 41.83 & 41.85 & & & \\
\hline 44 & Particle Size by Volume & micron & & & & & & \\
\hline 45 & Particle Size by Number & micron & & & & & & \\
\hline 46 & Kin. Viscosity & $\mathrm{cSt}$ & & & & 2.9 & & 15 \\
\hline 47 & Dyn. Visc./Consistency & $\mathrm{CP}$ & & & & 3.7 & & 21 \\
\hline 48 & Yield Stress & $\mathrm{Pa}$ & & & & & & 8.8 \\
\hline 49 & Density & $\mathrm{g} / \mathrm{mL}$ & & & & 1.283 & & 1.423 \\
\hline 50 & Density & $\mathrm{g} / \mathrm{mL}$ & 1.277 & 1.345 & 1.404 & 1.288 & & \\
\hline 51 & Turbidity & NTU & & & & & 0.28 & \\
\hline 52 & Comment - Note (1) & Note (2) & & & & & & \\
\hline 53 & Note(1)-Notes preced thi & table $>$ > & & & & & & \\
\hline
\end{tabular}

Table G2:AN-102 simulant analytical and other data, Page 4 
WSRC-TR-2003-00204, REV. 0

SRT-RPP-2003-00087, REV. 0

\begin{tabular}{|c|c|c|c|c|c|c|c|c|}
\hline & $\overline{\mathrm{AH}}$ & $\mathrm{Al}$ & $\mathrm{AJ}$ & $\overline{A K}$ & $\overline{\mathrm{AL}}$ & $\mathrm{AM}$ & $\overline{\mathrm{AN}}$ & $\mathrm{AO}$ \\
\hline 17 & Test Run -- & & $1.16-3 \mathrm{~B}$ & $1.16-3 \mathrm{~B}$ & $1.16-3 \mathrm{~B}$ & Wash-3B & Wash-3B & Wash-3B \\
\hline 2 & \multicolumn{2}{|c|}{ Sample Taken Date --.-.-.----->> } & October 23, 2002 & October 23, 2002 & October 23, 2002 & October 30,2002 & October 30, 2002 & October 30,2002 \\
\hline 3 & \multicolumn{2}{|c|}{ Sample Taken Time --..--.--->> } & test end / 19:00 & test end / 19:00 & test end / 19:00 & prewash 22 wt $\%$ & prewash 20 wt $\%$ & prewash 20 wt $\%$ \\
\hline$\frac{4}{5}$ & \multirow{2}{*}{\multicolumn{2}{|c|}{ 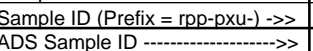 }} & slu-final1.16-3b-solids1 & slu-final1.16-3b-solids2 & slu-final1.16-3b-psd & slu22\%-prewash-3b-rheology & slu20\%-prewash-3b-rheology & filt20\%-prewash-3b-table1 \\
\hline 5 & & & 300187857 & 300187858 & 300188110 & none & none & 300189078 \\
\hline 6 & \multirow{2}{*}{\multicolumn{2}{|c|}{ Tvpe Sample }} & slurry & slurry & slurry & slurry & slurry & filtrate \\
\hline 7 & & Sample Size (mL) - & 15 & $\frac{15}{15}$ & 15 & 250 & 250 & 15 \\
\hline 8 & Item Measured & Units $^{*}$ & & & & & & \\
\hline 9 & $\mathrm{Al}$ & $\mathrm{ug} / \mathrm{mL}$ & & & & & & 7950 \\
\hline 10 & $\mathrm{~B}$ & $\mathrm{ug} / \mathrm{mL}$ & & & & & & $\mathrm{n} / \mathrm{a}$ \\
\hline 111 & $\mathrm{Ba}$ & $\mathrm{ug} / \mathrm{mL}$ & & & & & & $\mathrm{n} / \mathrm{a}$ \\
\hline 12 & $\mathrm{Ca}$ & $\mathrm{ug} / \mathrm{mL}$ & & & & & & 82.3 \\
\hline 13 & $\mathrm{Cd}$ & $\mathrm{ug} / \mathrm{mL}$ & & & & & & 38 \\
\hline 14 & $\mathrm{Ce}$ & $\mathrm{ug} / \mathrm{mL}$ & & & & & & $\mathrm{n} / \mathrm{a}$ \\
\hline 15 & $\mathrm{Cl}^{-}$ & $\mathrm{ug} / \mathrm{mL}$ & & & & & & 3260 \\
\hline 16 & Co & $\mathrm{ug} / \mathrm{mL}$ & & & & & & $\mathrm{n} / \mathrm{a}$ \\
\hline 17 & $\mathrm{Cr}$ & $\mathrm{ug} / \mathrm{mL}$ & & & & & & 84.6 \\
\hline 18 & $\mathrm{Cu}$ & $\mathrm{ug} / \mathrm{mL}$ & & & & & & 4 \\
\hline 19 & $\mathrm{Fe}$ & $\mathrm{ug} / \mathrm{mL}$ & & & & & & 1.2 \\
\hline 20 & K & $\mathrm{ug} / \mathrm{mL}$ & & & & & & 1640 \\
\hline 21 & La & $\mathrm{ug} / \mathrm{mL}$ & & & & & & $\mathrm{n} / \mathrm{a}$ \\
\hline 22 & $\mathrm{Mq}$ & $\mathrm{ug} / \mathrm{mL}$ & & & & & & n/a \\
\hline 23 & $\mathrm{Mn}$ & $u g / m L$ & & & & & & 5.5 \\
\hline 24 & Mo & $\mathrm{ug} / \mathrm{mL}$ & & & & & & $\mathrm{n} / \mathrm{a}$ \\
\hline 25 & $\mathrm{Na}$ & $\mathrm{ug} / \mathrm{mL}$ & & & & & & 146000 \\
\hline 26 & $\mathrm{Nd}$ & $\mathrm{ug} / \mathrm{mL}$ & & & & & & 2.6 \\
\hline 27 & & $\mathrm{ug} / \mathrm{mL}$ & & & & & & 222 \\
\hline 28 & NO3 (Nitrate) & $\mathrm{ug} / \mathrm{mL}$ & & & & & & 130000 \\
\hline 29 & & $\mathrm{ug} / \mathrm{mL}$ & & & & & & 590 \\
\hline 30 & PO4 (Phosphate) & $\mathrm{ug} / \mathrm{mL}$ & & & & & & 2280 \\
\hline 31 & $\mathrm{~Pb}$ & $\mathrm{ug} / \mathrm{mL}$ & & & & & & 29.1 \\
\hline 32 & & $\mathrm{ug} / \mathrm{mL}$ & & & & & & 2960 \\
\hline 33 & $\mathrm{Si}$ & $\mathrm{ug} / \mathrm{mL}$ & & & & & & $n / a$ \\
\hline 34 & Sn & $\mathrm{ug} / \mathrm{mL}$ & & & & & & $\mathrm{n} / \mathrm{a}$ \\
\hline 35 & SO4 (Sulfate) & $\mathrm{ug} / \mathrm{mL}$ & & & & & & 9030 \\
\hline 36 & Sr & $\mathrm{ug} / \mathrm{mL}$ & & & & & & 19.2 \\
\hline 37 & W & $\mathrm{ug} / \mathrm{mL}$ & & & & & & $\mathrm{n} / \mathrm{a}$ \\
\hline 38 & $\mathrm{Zn}$ & $\mathrm{ug} / \mathrm{mL}$ & & & & & & 2.9 \\
\hline 39 & $Z_{r}$ & $\mathrm{ug} / \mathrm{mL}$ & & & & & & $\mathrm{n} / \mathrm{a}$ \\
\hline 40 & Total Organic Carbon & $\mathrm{ug} / \mathrm{mL}$ & & & & & & 9380 \\
\hline 41 & Total Inorganic Carbon & $\mathrm{ug} / \mathrm{mL}$ & & & & & & 6820 \\
\hline 42 & Suspended Solids & wt\% & 21.69 & 21.65 & & & & \\
\hline 43 & Total Solids & wt\% & 47.19 & 47.17 & & & & \\
\hline 44 & Particle Size by Volume & micron & & & $0.86(87 \%), 8.22(13 \%)$ & & & \\
\hline 45 & Particle Size by Number & micron & & & 0.80 & & & \\
\hline 46 & Kin. Viscosity & $\mathrm{cSt}$ & & & & 12 & 11 & \\
\hline 47 & Dyn. Visc./Consistency & $\mathrm{cP}$ & & & & 17 & 16 & \\
\hline 48 & Yield Stress & $\mathrm{Pa}$ & & & & 12 & 7.6 & \\
\hline 49 & \begin{tabular}{|l|l} 
Density \\
\end{tabular} & $\mathrm{g} / \mathrm{mL}$ & & & & & & \\
\hline 50 & Density & $\mathrm{g} / \mathrm{mL}$ & $n / a$ & 1.430 & & & & \\
\hline 51 & Turbidity & NTU & & & & & & \\
\hline 52 & Comment - Note (1) & Note (2) & & & bimodal by volume & & & \\
\hline 53ل & Note(1)-Notes brecede th & stable & & & & & & \\
\hline
\end{tabular}

Table G2:AN-102 simulant analytical and other data, Page 5 
WSRC-TR-2003-00204, REV. 0

SRT-RPP-2003-00087, REV. 0

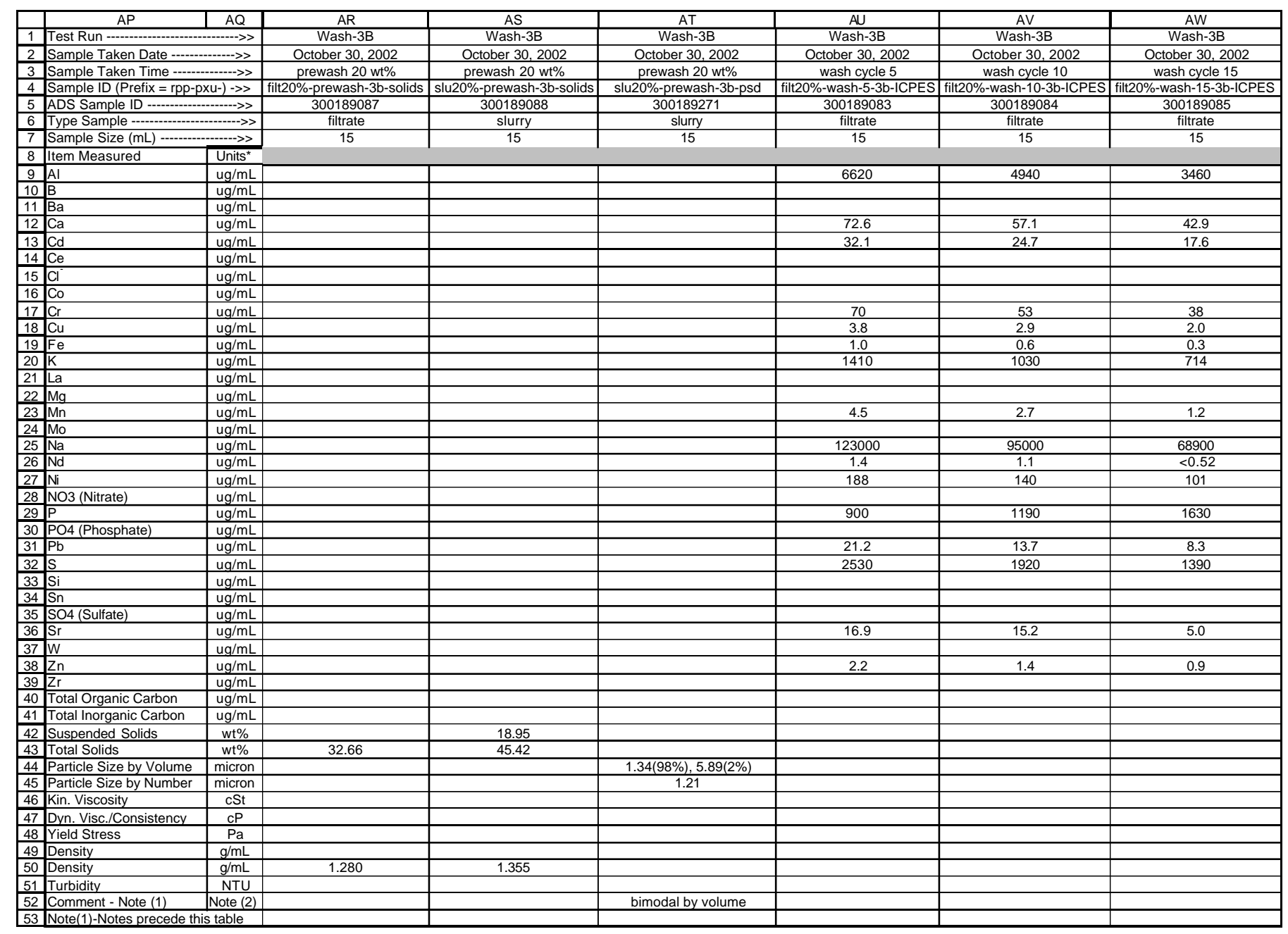

Table G2:AN-102 simulant analytical and other data, Page 6 
WSRC-TR-2003-00204, REV. 0

SRT-RPP-2003-00087, REV. 0

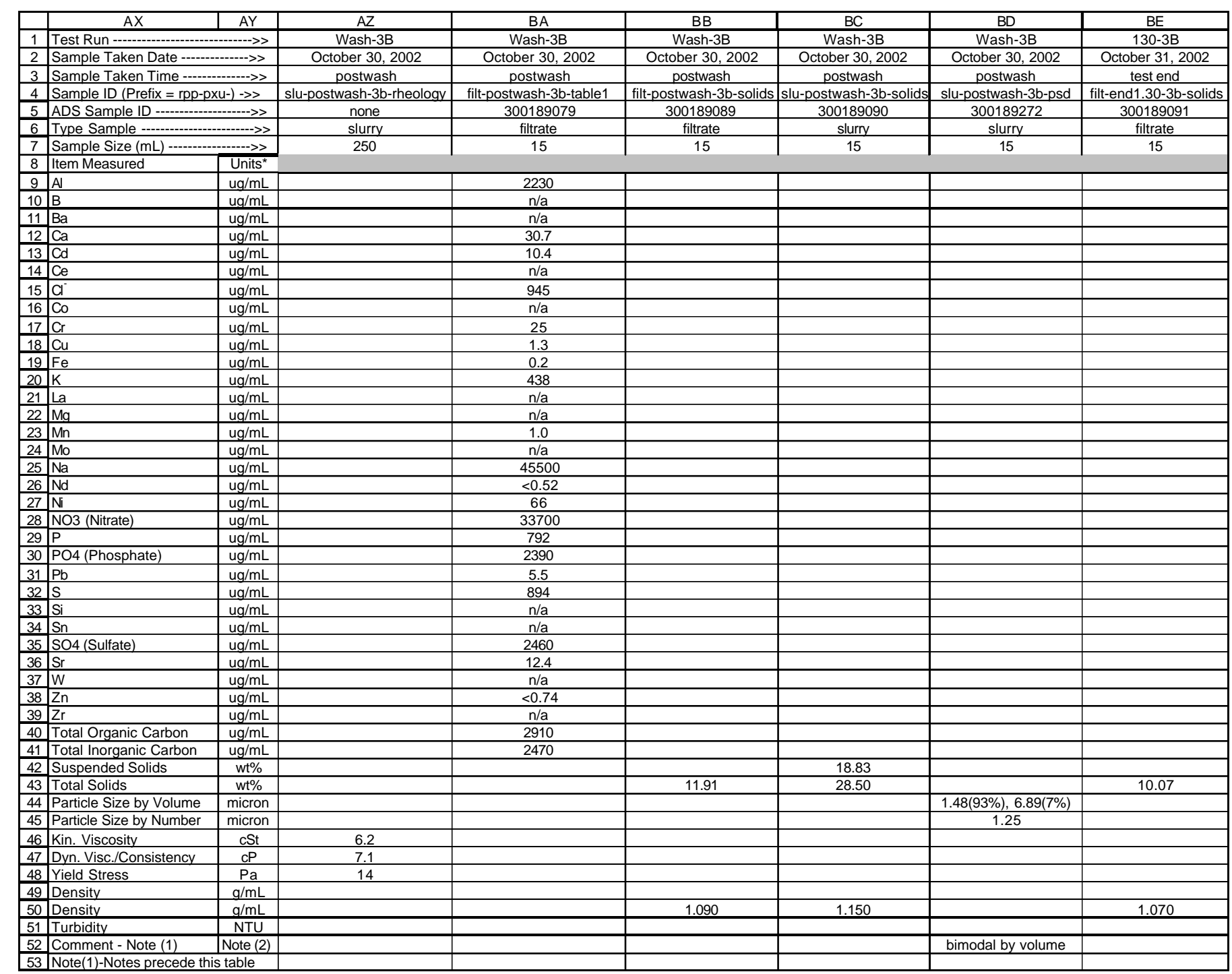

Table G2:AN-102 simulant analytical and other data, Page 7 
WSRC-TR-2003-00204, REV. 0

SRT-RPP-2003-00087, REV. 0

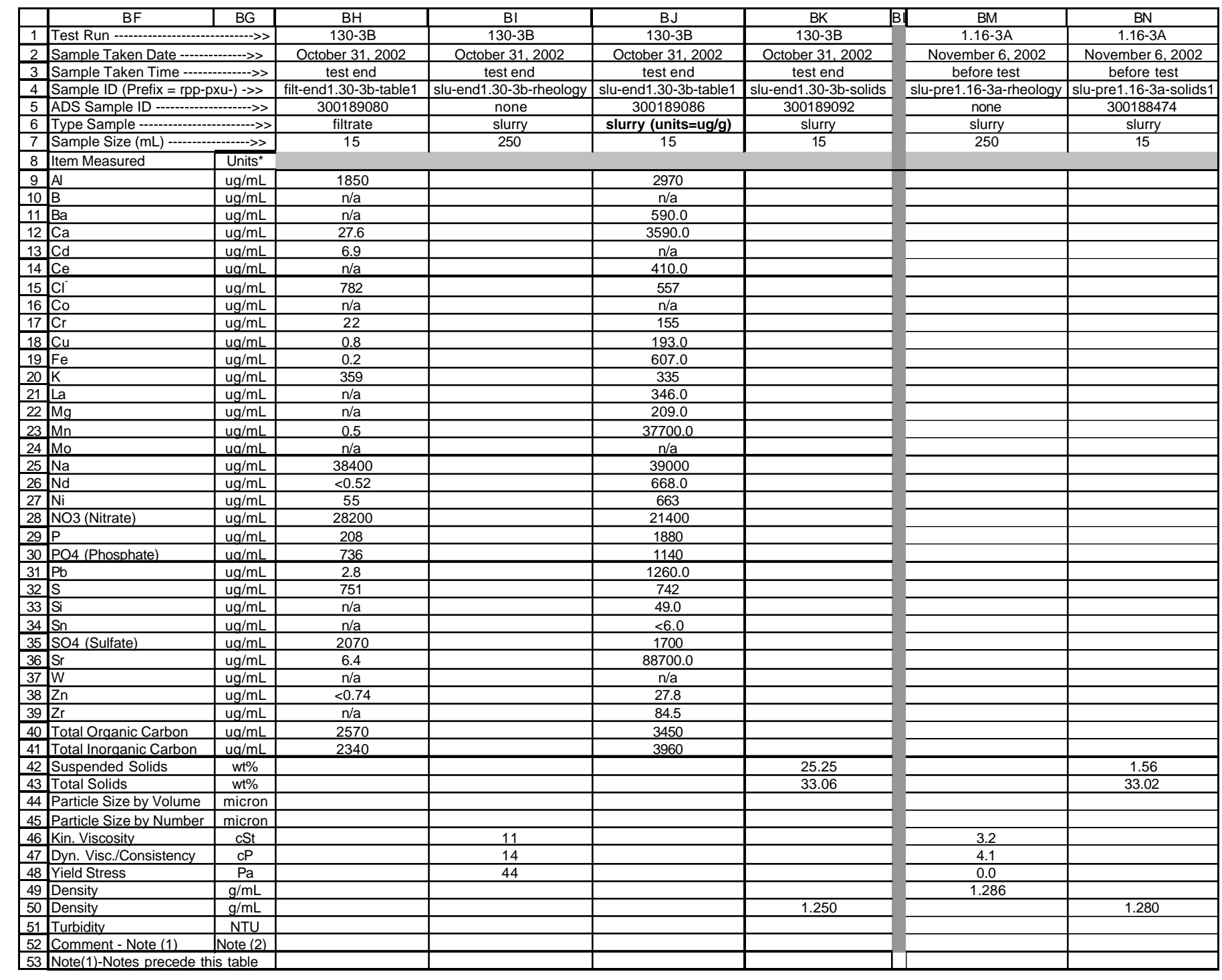

Table G2:AN-102 simulant analytical and other data, Page 8 
WSRC-TR-2003-00204, REV. 0

SRT-RPP-2003-00087, REV. 0

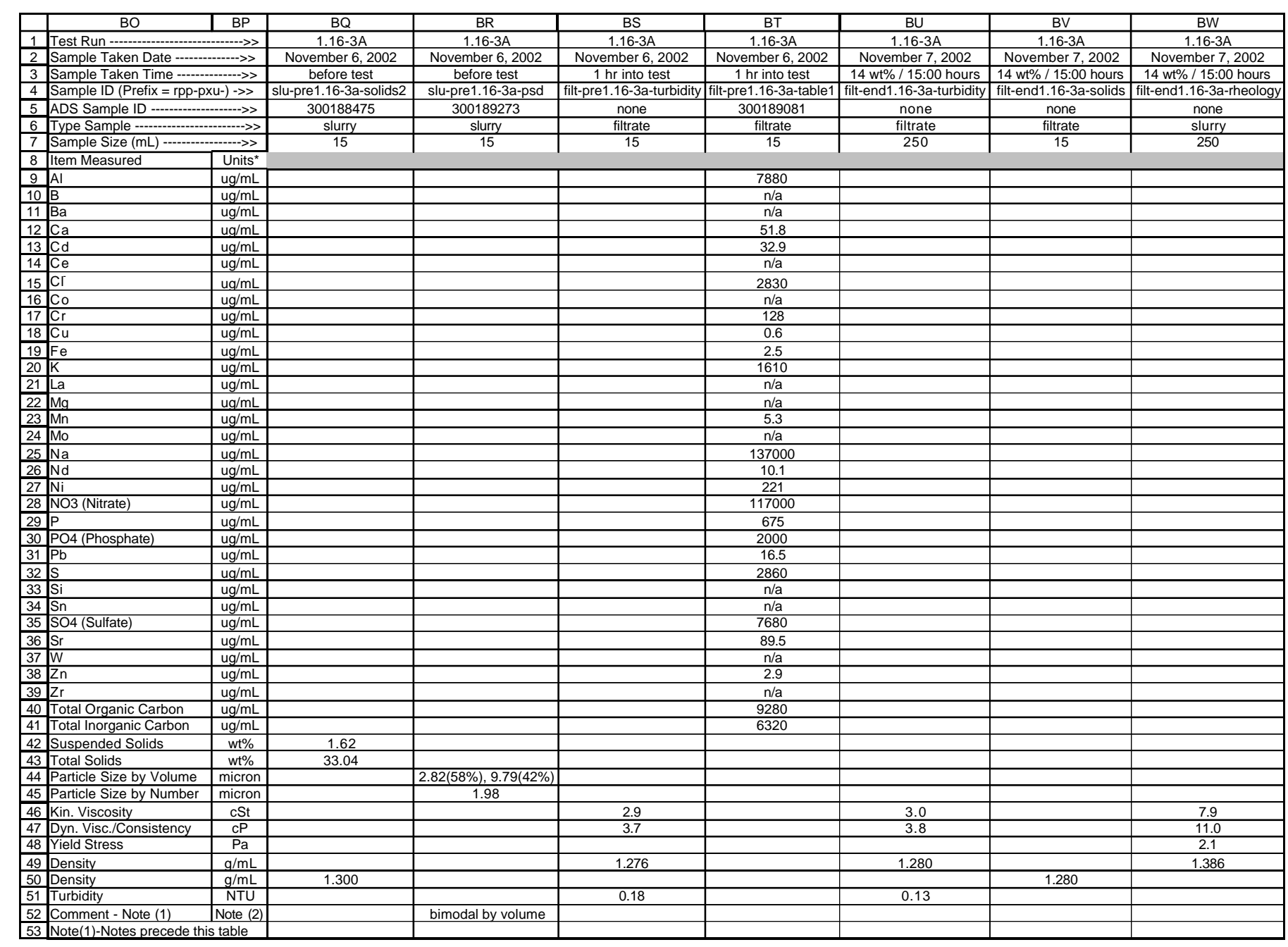

Table G2:AN-102 simulant analytical and other data, Page 9

Page G13 of G48 
WSRC-TR-2003-00204, REV. 0

SRT-RPP-2003-00087, REV. 0

\begin{tabular}{|c|c|c|c|c|c|c|c|c|}
\hline & $\mathrm{BX}$ & BY & $\mathrm{BZ}$ & $\mathrm{CA}$ & $\mathrm{CB}$ & $\mathrm{CC}$ & $\mathrm{CD}$ & $\mathrm{CE}$ \\
\hline 1 & Test Run & $-\cdots>>$ & $1.16-3 \mathrm{~A}$ & $1.16-3 \mathrm{~A}$ & $1.16-3 \mathrm{~A}$ & $1.16-3 \mathrm{~A}$ & $1.16-3 \mathrm{~A}$ & $1.16-3 \mathrm{~A}$ \\
\hline 2 & Sample Taken Date ---- & $\cdots$ & November 7, 2002 & November 7, 2002 & November 7, 2002 & November 11,2002 & November 11,2002 & November 11,2002 \\
\hline 3 & Sample Taken Time --.-- & $-\cdots+->>$ & $14 \mathrm{wt} \% / 15: 00$ hours & 14 wt $\% / 15: 00$ hours & 14 wt $\% / 15: 00$ hours & $25 \mathrm{wt} \% / 11: 30$ hours & $25 \mathrm{wt} \% / 11: 30$ hours & 25 wt $\% / 11: 30$ hours \\
\hline \begin{tabular}{l|l}
4 \\
\end{tabular} & Sample ID (Prefix = rpp-px & $u-)->>$ & slu-end1.16-3a-solids1 & slu-end1.16-3a-solids2 & slu-end1.16-3a-psd & filt-final1.16-3a-turbidity & filt-final1.16-3a-solids & slu-final1.16-3a-rheology \\
\hline 5 & ADS Sample ID -...-..-..- & $\cdots>>$ & 300188585 & 300188586 & 300189274 & none & none & none \\
\hline 6 & Type Sample ----------- & $\cdots--->>$ & slurry & slurry & slurry & filtrate & filtrate & slurry \\
\hline \begin{tabular}{l|l}
7 \\
7
\end{tabular} & Sample Size (mL) ---- & 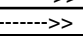 & 15 & $\frac{15}{15}$ & $\frac{15}{15}$ & 250 & 15 & 250 \\
\hline 8 & Item Measured & Units $^{*}$ & & & & & & \\
\hline 9 & $\mathrm{Al}$ & $\mathrm{ug} / \mathrm{mL}$ & & & & & & \\
\hline 10 & & $\mathrm{ug} / \mathrm{mL}$ & & & & & & \\
\hline 11 & $\mathrm{Ba}$ & $\mathrm{ug} / \mathrm{mL}$ & & & & & & \\
\hline 12 & $\mathrm{Ca}$ & $\mathrm{ug} / \mathrm{mL}$ & & & & & & \\
\hline 13 & $\mathrm{Cd}$ & $\mathrm{ug} / \mathrm{mL}$ & & & & & & \\
\hline 14 & $\mathrm{Ce}$ & $\mathrm{ug} / \mathrm{mL}$ & & & & & & \\
\hline 15 & $\mathrm{Cl}$ & $\mathrm{ug} / \mathrm{mL}$ & & & & & & \\
\hline 16 & Co & $\mathrm{ug} / \mathrm{mL}$ & & & & & & \\
\hline 17 & $\mathrm{Cr}$ & $\mathrm{ug} / \mathrm{mL}$ & & & & & & \\
\hline 18 & $\mathrm{Cu}$ & $\mathrm{ug} / \mathrm{mL}$ & & & & & & \\
\hline 19 & $\mathrm{Fe}$ & $\mathrm{ug} / \mathrm{mL}$ & & & & & & \\
\hline 20 & $\mathrm{~K}$ & $\mathrm{ug} / \mathrm{mL}$ & & & & & & \\
\hline 21 & $\mathrm{La}$ & $\mathrm{ug} / \mathrm{mL}$ & & & & & & \\
\hline 22 & $\mathrm{Mg}$ & $\mathrm{ug} / \mathrm{mL}$ & & & & & & \\
\hline 23 & $\mathrm{Mn}$ & $\mathrm{ug} / \mathrm{mL}$ & & & & & & \\
\hline 24 & Mo & $\mathrm{ug} / \mathrm{mL}$ & & & & & & \\
\hline 25 & $\mathrm{Na}$ & $\mathrm{ug} / \mathrm{mL}$ & & & & & & \\
\hline 26 & $\mathrm{Nd}$ & $\mathrm{ug} / \mathrm{mL}$ & & & & & & \\
\hline 27 & $\mathrm{Ni}$ & $\mathrm{ug} / \mathrm{mL}$ & & & & & & \\
\hline 28 & NO3 (Nitrate) & $\mathrm{ug} / \mathrm{mL}$ & & & & & & \\
\hline 29 & $\mathrm{P}$ & $\mathrm{ug} / \mathrm{mL}$ & & & & & & \\
\hline 30 & PO4 (Phosphate) & $\mathrm{ug} / \mathrm{mL}$ & & & & & & \\
\hline 31 & $\mathrm{~Pb}$ & $\mathrm{ug} / \mathrm{mL}$ & & & & & & \\
\hline 32 & 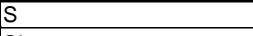 & $\mathrm{ug} / \mathrm{mL}$ & & & & & & \\
\hline 33 & $\mathrm{Si}$ & $\mathrm{ug} / \mathrm{mL}$ & & & & & & \\
\hline 34 & Sn & $\mathrm{ug} / \mathrm{mL}$ & & & & & & \\
\hline 35 & SO4 (Sulfate) & $\mathrm{ug} / \mathrm{mL}$ & & & & & & \\
\hline 36 & $\mathrm{Sr}$ & $\mathrm{ug} / \mathrm{mL}$ & & & & & & \\
\hline 37 & $\mathrm{~W}$ & $\mathrm{ug} / \mathrm{mL}$ & & & & & & \\
\hline 38 & $\mathrm{Zn}$ & $\mathrm{ug} / \mathrm{mL}$ & & & & & & \\
\hline 39 & $\mathrm{Zr}$ & $\mathrm{ug} / \mathrm{mL}$ & & & & & & \\
\hline 40 & Total Organic Carbon & $\mathrm{ug} / \mathrm{mL}$ & & & & & & \\
\hline 41 & Total Inorganic Carbon & $\mathrm{ug} / \mathrm{mL}$ & & & & & & \\
\hline 42 & Suspended Solids & wt\% & 14.14 & 13.97 & & & & \\
\hline 43 & Total Solids & wt\% & 41.67 & 41.55 & & & & \\
\hline 44 & Particle Size by Volume & micron & & & 2.79 & & & \\
\hline 45 & Particle Size by Number & micron & & & 1.79 & & & \\
\hline 46 & Kin. Viscosity & $\mathrm{cSt}$ & & & & 3.5 & & 17 \\
\hline 47 & Dyn. Visc./Consistency & $\frac{C P}{C P}$ & & & & 4.5 & & 24 \\
\hline 48 & $\begin{array}{l}\text { Yield Stress } \\
\end{array}$ & $\mathrm{Pa}$ & & & & & & 24 \\
\hline 49 & \begin{tabular}{|l} 
Density \\
\end{tabular} & $\mathrm{g} / \mathrm{mL}$ & & & & 1.283 & & 1.469 \\
\hline 50 & Density & $\mathrm{g} / \mathrm{mL}$ & 1.3 (too low?) & 1.26 (too low?) & & & 1.270 & \\
\hline 51 & Turbidity & NTU & & & & 0.23 & & \\
\hline 52 & Comment - Note (1) & Note (2) & & & & & & \\
\hline 53 & Note(1)-Notes precede thi & table & & & & & & \\
\hline
\end{tabular}

Table G2:AN-102 simulant analytical and other data, Page 10 
WSRC-TR-2003-00204, REV. 0

SRT-RPP-2003-00087, REV. 0

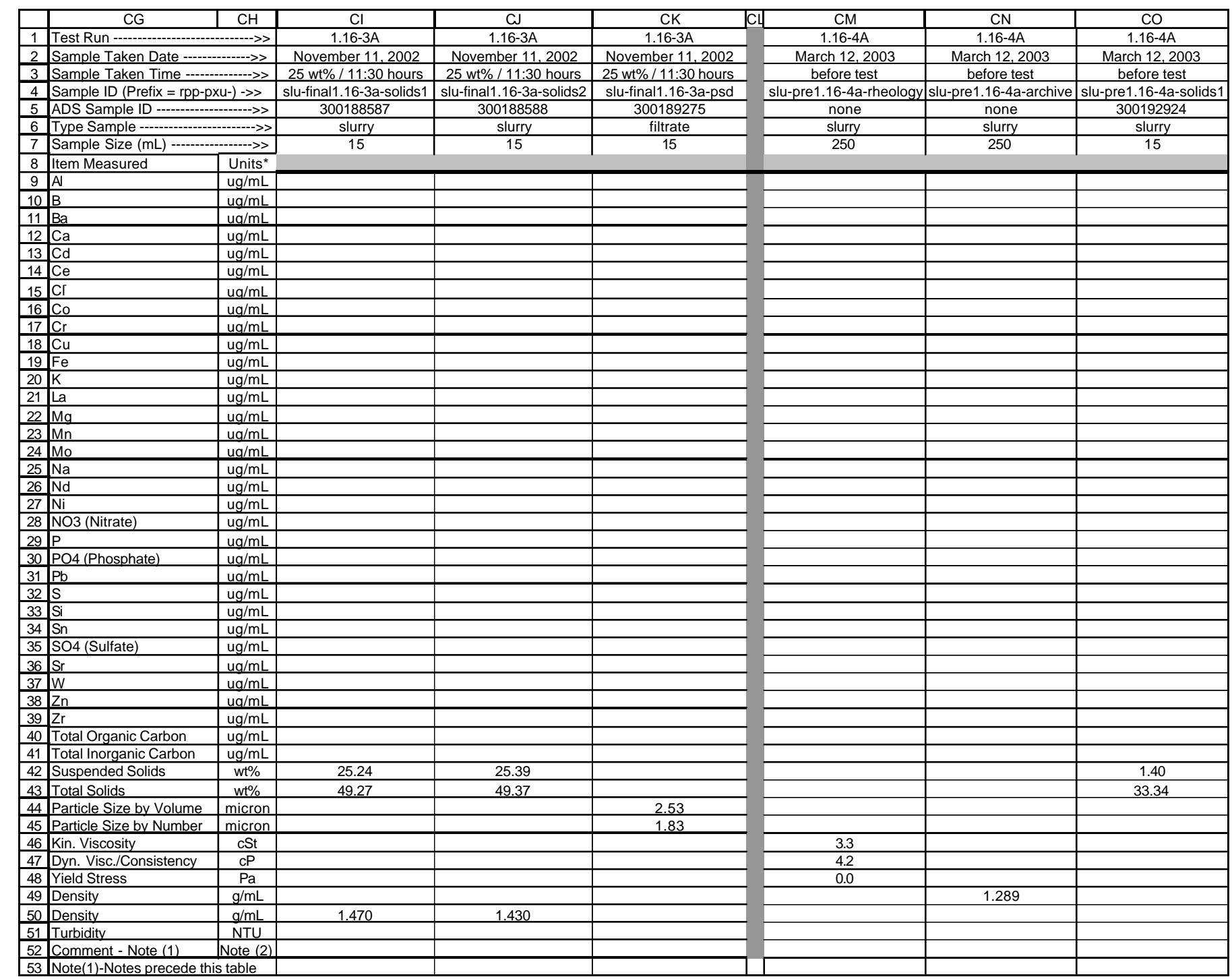

Table G2:AN-102 simulant analytical and other data, Page 11 
WSRC-TR-2003-00204, REV. 0

SRT-RPP-2003-00087, REV. 0

\begin{tabular}{|c|c|c|c|c|c|c|c|c|c|}
\hline & $\mathrm{CP}$ & $\mathrm{CQ}$ & $\mathrm{CR}$ & CS & CT & $\mathrm{CU}$ & $\mathrm{CV}$ & $\mathrm{CW}$ & $C X$ \\
\hline 11 & Test Run -- & & $1.16-4 \mathrm{~A}$ & $1.16-4 \mathrm{~A}$ & $1.16-4 \mathrm{~A}$ & $1.16-4 \mathrm{~A}$ & $1.16-4 \mathrm{~A}$ & $1.16-4 \mathrm{~A}$ & $1.16-4 \mathrm{~A}$ \\
\hline 2 & Sample Taken Date - & $\cdots$ & March 12, 2003 & March 12, 2003 & March 12, 2003 & March 12, 2003 & March 12, 2003 & March 12, 2003 & March 13, 2003 \\
\hline 3 & Sample Taken Time -....... & $\ldots \ldots$ & before test & before test & $1 \mathrm{hr}$ into test & $1 \mathrm{hr}$ into test & $1 \mathrm{hr}$ into test & $1 \mathrm{hr}$ into test & $18 \mathrm{wt} \% / 04: 11$ hours \\
\hline $4 \sqrt{3}$ & Sample ID (Prefix = rpp-p) & $(u-) \rightarrow>$ & slu-pre1.16-4a-solids2 & slu-pre1.16-4a-psd & filt-pre1.16-4a-turbidity & filt-pre1.16-4a-table1a & filt-pre1.16-4a-table1b & filt-pre1.16-4a-table1c & filt-end1.16-4a-turbidity \\
\hline 5 & ADS Sample ID & & 300192925 & 300192929 & none & 300192931 & 300192932 & 300192933 & none \\
\hline \begin{tabular}{c|c}
6 \\
7
\end{tabular} & $\begin{array}{l}\text { Type Sample -..-. } \\
\text { Sample Size (mL)--.-- }\end{array}$ & $\cdots \cdots>>$ & $\begin{array}{l}\text { slurry } \\
15\end{array}$ & $\frac{\text { slurry }}{15}$ & filtrate & filtrate & filtrate & filtrate & filtrate \\
\hline 8 & $\begin{array}{l}\text { Sample Size ( }(\text { Item Measured } \\
\end{array}$ & Units $^{*}$ & 15 & & 250 & 15 & 15 & 15 & 250 \\
\hline $9 \mathrm{~A}$ & $\mathrm{Al}$ & $\mathrm{ug} / \mathrm{mL}$ & & & & 7648 & & & \\
\hline 10$] \mathrm{E},+3$ & & $\mathrm{ug} / \mathrm{mL}$ & & & & $\mathrm{n} / \mathrm{a}$ & & & \\
\hline $11 \mathrm{E}$ & $\mathrm{Ba}$ & $\mathrm{ug} / \mathrm{mL}$ & & & & $\mathrm{n} / \mathrm{a}$ & & & \\
\hline 12( & $\mathrm{Ca}$ & $\mathrm{ug} / \mathrm{mL}$ & & & & 60.6 & & & \\
\hline $13 \bar{c}$ & $\mathrm{Cd}$ & $\mathrm{ug} / \mathrm{mL}$ & & & & $\mathrm{n} / \mathrm{a}$ & & & \\
\hline $14 \mathrm{C}$ & $\mathrm{Ce}$ & $\mathrm{ug} / \mathrm{mL}$ & & & & $n / a$ & & & \\
\hline 15 & $\mathrm{Cl}^{-}$ & $u g / m L$ & & & & & & 3280 & \\
\hline $16 \mathrm{c}$ & $\mathrm{Co}_{0}$ & $\mathrm{ug} / \mathrm{mL}$ & & & & $\mathrm{n} / \mathrm{a}$ & & & \\
\hline 17 ( & $\frac{\mathrm{Cr}}{\mathrm{Cr}}$ & $\mathrm{ug} / \mathrm{mL}$ & & & & 135 & & & \\
\hline $18 \mathrm{C}$ & $\mathrm{Cu}$ & $\mathrm{ug} / \mathrm{mL}$ & & & & 5.3 & & & \\
\hline $19 \mathrm{~F}$ & $\mathrm{Fe}$ & $\mathrm{ug} / \mathrm{mL}$ & & & & 1.8 & & & \\
\hline $20 \mathrm{k}$ & & $\mathrm{ug} / \mathrm{mL}$ & & & & 1453 & & & \\
\hline$\frac{21}{22} \frac{L}{\Lambda}$ & La & $\mathrm{ug} / \mathrm{mL}$ & & & & $\leq 1.4$ & & & \\
\hline $22 \pi$ & $\frac{\mathrm{Mg}}{\mathrm{Mn}}$ & $u g / m L$ & & 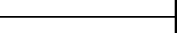 & - & $\leq 0.17$ & & & \\
\hline $23 \sqrt{1}$ & $\frac{\mathrm{Mn}}{\mathrm{Mo}}$ & $\mathrm{ug} / \mathrm{mL}$ & $\bar{C}$ & 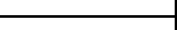 & 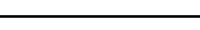 & 9.7 & & & \\
\hline $24 \sqrt{\pi}$ & $\frac{\mathrm{Mo}}{\mathrm{Na}}$ & $\mathrm{ug} / \mathrm{mL}$ & & & 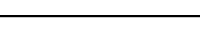 & $\frac{n / a}{12051}$ & & & \\
\hline $25 \mathrm{~N}$ & $\frac{\mathrm{Na}}{\mathrm{Nd}}$ & $\mathrm{ug} / \mathrm{mL}$ & & & & $\frac{132512}{34}$ & & & \\
\hline $26 \sqrt{\mathrm{N}} \mathrm{N}-2$ & $\frac{N d}{N d}$ & $u g / m L$ & & & & 3.4 & & & \\
\hline $27 \sqrt{1}$ & $\mathrm{Ni}$ & $\mathrm{ug} / \mathrm{mL}$ & & & & 229 & & & \\
\hline $28 \sqrt{\mathrm{N}}-2-2$ & NO3 (Nitrate) & $\mathrm{ug} / \mathrm{mL}$ & & & & & & 119000 & \\
\hline 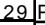 & & $\mathrm{ug} / \mathrm{mL}$ & & & & $\mathrm{n} / \mathrm{a}$ & & & \\
\hline$3 0 \longdiv { F }$ & PO4 (Phosphate) & $u g / m L$ & & & & & & 3550 & \\
\hline $31 \mathrm{~F}$ & $\mathrm{~Pb}$ & $\mathrm{ug} / \mathrm{mL}$ & & & & 66.5 & & & \\
\hline 325 & $\frac{S}{S i}$ & $\mathrm{ug} / \mathrm{mL}$ & & & & $\mathrm{n} / \mathrm{a}$ & & & \\
\hline $33 \sqrt{5}$ & $\mathrm{Si}$ & $\mathrm{ug} / \mathrm{mL}$ & & & & $\mathrm{n} / \mathrm{a}$ & & & \\
\hline $34 \sqrt{5}$ & Sn & $\mathrm{ug} / \mathrm{mL}$ & & & & $n / a$ & & & \\
\hline 35 s & SO4 (Sulfate) & $\mathrm{ug} / \mathrm{mL}$ & & & & & & 8890 & \\
\hline $36 \mathrm{~s} s, 2 \rightarrow$ & $\mathrm{sr}$ & $\mathrm{ug} / \mathrm{mL}$ & & & & 27.6 & & & \\
\hline $37 \mathrm{~V}$ & W & $\mathrm{ug} / \mathrm{mL}$ & & & & $\mathrm{n} / \mathrm{a}$ & & & \\
\hline $38 \sqrt{2}$ & $\mathrm{Zn}$ & $\mathrm{ug} / \mathrm{mL}$ & & & & 3.4 & & & \\
\hline $39 \sqrt{2}-2>$ & $\mathrm{Zr}$ & $\mathrm{ug} / \mathrm{mL}$ & & & & $\mathrm{n} / \mathrm{a}$ & & & \\
\hline 40 & Total Organic Carbon & $\mathrm{ug} / \mathrm{mL}$ & & & & & 9470 & & \\
\hline 41 & Total Inorganic Carbon & $\mathrm{ug} / \mathrm{mL}$ & & & & & 7030 & & \\
\hline 42 & Suspended Solids & wt\% & 1.61 & & & & & & \\
\hline 43 & Total Solids & $\mathrm{wt} \%$ & 33.35 & & & & & & \\
\hline 44 & Particle Size by Volume & micron & & 2.67 & & & & & \\
\hline 45 & Particle Size by Number & micron & & 1.79 & & & & & \\
\hline 46 & Kin. Viscosity & $\mathrm{cSt}$ & & & 2.9 & & & & 3.0 \\
\hline 47 & Dyn. Visc./Consistency & $d P$ & & & 3.7 & & & & 3.8 \\
\hline 48 & Yield Stress & $\mathrm{Pa}$ & & & & & & & \\
\hline 49[ & Density & $\mathrm{g} / \mathrm{mL}$ & & & 1.278 & & & & \\
\hline 50[ & Density & $\mathrm{g} / \mathrm{mL}$ & & & & 1.273 & & & \\
\hline 51 & Turbidity & NTU & & & 0.06 & & & & 0.18 \\
\hline 52 & Comment - Note (1) & Note (2) & & & & density at start & & & \\
\hline 53 & Note(1)-Notes precede thi & stable & & & & & & & \\
\hline
\end{tabular}

Table G2:AN-102 simulant analytical and other data, Page 12 
WSRC-TR-2003-00204, REV. 0

SRT-RPP-2003-00087, REV. 0

\begin{tabular}{|c|c|c|c|c|c|c|c|}
\hline & $\mathrm{CY}$ & $\mathrm{CZ}$ & $\mathrm{DA}$ & $\mathrm{DB}$ & $\mathrm{DC}$ & $\mathrm{DD}$ & $\mathrm{DE}$ \\
\hline 1 & Test Run -- & $-\cdots>>$ & $1.16-4 \mathrm{~A}$ & $1.16-4 \mathrm{~A}$ & $1.16-4 \mathrm{~A}$ & $1.16-4 \mathrm{~A}$ & $1.16-4 \mathrm{~A}$ \\
\hline 2 & Sample Taken Date -..-- & $-\cdots>$ & March 13, 2003 & March 13, 2003 & March 13, 2003 & March 13, 2003 & March 13, 2003 \\
\hline 3 & Sample Taken Time -.... & $-\cdots$ & $18 \mathrm{wt} \% / 04: 11$ hours & 18 wt\% / 04:11 hours & 18 wt $\% / 04: 11$ hours & $18 \mathrm{wt} \% / 04: 11$ hours & $18 \mathrm{wt} \% / 04: 11$ hours \\
\hline 4 & Sample ID (Prefix = rpp-p & $\langle(\mathrm{u}-)-\rangle\rangle$ & filt-end1.16-4a-solids & slu-end1.16-4a-archive & \begin{tabular}{|l|} 
slu-end1.16-4a-solids1 \\
\end{tabular} & \begin{tabular}{|l} 
slu-end1.16-4a-solids2 \\
\end{tabular} & slu-end1.16-3a-psd \\
\hline 5 & ADS Sample ID -............. & $-\cdots-\cdots>$ & 300192928 & none & 300192926 & 300192927 & 300192930 \\
\hline 6 & Type Sample -- & $-\cdots---->>$ & filtrate & filtrate & slurry & slurry & slurry \\
\hline 7 & Sample Size $(\mathrm{mL})$ & $\cdots$ & 15 & 250 & 15 & 15 & 15 \\
\hline 8 & Item Measured & \begin{tabular}{|l} 
Units $^{*}$ \\
\end{tabular} & & & & & \\
\hline 9 & $|A|$ & $\mathrm{ug} / \mathrm{mL}$ & & & & & \\
\hline 10 & $\mathrm{~B}$ & $\mathrm{ug} / \mathrm{mL}$ & & & & & \\
\hline 11 & $\mathrm{Ba}$ & $\mathrm{ug} / \mathrm{mL}$ & & & & & \\
\hline 12 & $\mathrm{Ca}$ & $\mathrm{ug} / \mathrm{mL}$ & & & & & \\
\hline 13 & $\mathrm{Cd}$ & $\mathrm{ug} / \mathrm{mL}$ & & & & & \\
\hline 14 & $\mathrm{Ce}$ & $\mathrm{ug} / \mathrm{mL}$ & & & & & \\
\hline 15 & $\mathrm{Cl}^{\top}$ & $\mathrm{ug} / \mathrm{mL}$ & & & & & \\
\hline 16 & Co & $\mathrm{ug} / \mathrm{mL}$ & & & & & \\
\hline 17 & $\mathrm{Cr}$ & $\mathrm{ug} / \mathrm{mL}$ & & & & & \\
\hline 18 & $\mathrm{Cu}$ & $\mathrm{ug} / \mathrm{mL}$ & & & & & \\
\hline 19 & $\mathrm{Fe}$ & $\mathrm{ug} / \mathrm{mL}$ & & & & & \\
\hline 20 & $K$ & $\mathrm{ug} / \mathrm{mL}$ & & & & & \\
\hline 21 & $\mathrm{La}$ & $\mathrm{ug} / \mathrm{mL}$ & & & & & \\
\hline 22 & $\mathrm{Mg}$ & $\mathrm{ug} / \mathrm{mL}$ & & & & & \\
\hline 23 & $\overline{\mathrm{Mn}}$ & $\mathrm{ug} / \mathrm{mL}$ & & & & & \\
\hline 24 & Mo & $\mathrm{ug} / \mathrm{mL}$ & & & & & \\
\hline 25 & $\mathrm{Na}$ & $\mathrm{ug} / \mathrm{mL}$ & & & & & \\
\hline 26 & $\mathrm{Nd}$ & $\mathrm{ug} / \mathrm{mL}$ & & & & & \\
\hline 27 & $\mathrm{Ni}$ & $\mathrm{ug} / \mathrm{mL}$ & & & & & \\
\hline 28 & NO3 (Nitrate) & $\mathrm{ug} / \mathrm{mL}$ & & & & & \\
\hline 29 & $\mathrm{P}$ & $\mathrm{ug} / \mathrm{mL}$ & & & & & \\
\hline 30 & PO4 (Phosphate) & $\mathrm{ug} / \mathrm{mL}$ & & & & & \\
\hline 31 & $\mathrm{~Pb}$ & $\mathrm{ug} / \mathrm{mL}$ & & & & & \\
\hline 32 & $\mathrm{~S}$ & $\mathrm{ug} / \mathrm{mL}$ & & & & & \\
\hline 33 & $\mathrm{Si}$ & $\mathrm{ug} / \mathrm{mL}$ & & & & & \\
\hline 34 & $\mathrm{sn}$ & $\mathrm{ug} / \mathrm{mL}$ & & & & & \\
\hline 35 & SO4 (Sulfate) & $\mathrm{ug} / \mathrm{mL}$ & & & & & \\
\hline 36 & $\mathrm{Sr}$ & $\mathrm{ug} / \mathrm{mL}$ & & & & & \\
\hline 37 & W & $\mathrm{ug} / \mathrm{mL}$ & & & & & \\
\hline 38 & $\mathrm{Zn}$ & $\mathrm{ug} / \mathrm{mL}$ & & & & & \\
\hline 39 & $Z r$ & $\mathrm{ug} / \mathrm{mL}$ & & & & & \\
\hline 40 & Total Organic Carbon & $\mathrm{ug} / \mathrm{mL}$ & & & & & \\
\hline 41 & Total Inorganic Carbon & $\mathrm{ug} / \mathrm{mL}$ & & & & & \\
\hline 42 & Suspended Solids & $\mathrm{wt} \%$ & & & 18.71 & 18.71 & \\
\hline 43 & Total Solids & wt $\%$ & 33.31 & & 45.79 & 45.82 & \\
\hline 44 & Particle Size by Volume & micron & & & & & 2.13 \\
\hline 45 & Particle Size by Number & micron & & & & & 1.76 \\
\hline 46 & Kin. Viscosity & $\mathrm{cSt}$ & & 11 & & & \\
\hline 47 & Dyn. Visc./Consistency & $\mathrm{cP}$ & & 15 & & & \\
\hline 48 & Yield Stress & $\mathrm{Pa}$ & & 7.6 & & & \\
\hline 49 & Density & $\mathrm{g} / \mathrm{mL}$ & & 1.315 & & & \\
\hline 50 & Density & $\mathrm{g} / \mathrm{mL}$ & 1.270 & & 1.320 & 1.330 & \\
\hline 51 & Turbidity & NTU & & & & & \\
\hline 52 & Comment - Note (1) & Note (2) & & submitted for rheologv & Note (6) & Note (6) & \\
\hline 53 & Note(1)-Notes precede thi & stable & & & & & \\
\hline
\end{tabular}

Table G2:AN-102 simulant analytical and other data, Page 13 


\section{Rheology}

At the beginning and the end (and at other points) of each pilot-scale cross-flow ultrafiltration test with AN-102 simulant slurry, samples were taken to measure some rheological properties, like shear stress versus shear rate, viscosity, or if non-Newtonian, consistency. The preceding table lists the viscosity, or consistency and yield shear stress, for each of the samples. This section shows graphical representation of the data used to determine those number.

Those simulant slurry samples at $25^{\circ} \mathrm{C}$ are: $\quad$ (Consistency, cP; Yield Stress, Pa)

Fig.G_

1. $\mathrm{AN}-102 \mathrm{R} 2$, Batch $3 \mathrm{C}$ at $0.8 \mathrm{wt} \%$ insoluble solids

2. AN-102R2, Batch $3 \mathrm{C}$ at $8.4 \mathrm{wt} \%$ insoluble solids

3. AN-102R2, Batch 3B at $1.2 \mathrm{wt} \%$ insoluble solids

4. AN-102R2, Batch 3B at $22 \mathrm{wt} \%$ insoluble solids

5. AN-102R2, Batch 3B at $19 \mathrm{wt} \%$ insoluble solids:

6. AN-102R2, Batch 3B (post-washed) at $18 \mathrm{wt} \%$ insoluble solids:

7. AN-102R2, Batch 3B (post-washed) at $25 \mathrm{wt} \%$ insoluble solids:

8. AN-102R2, Batch $3 \mathrm{~A}$ at $1.6 \mathrm{wt} \%$ insoluble solids:

9. AN-102R2, Batch $3 \mathrm{~A}$ at $14 \mathrm{wt} \%$ insoluble solids:

10. AN-102R2, Batch $3 \mathrm{~A}$ at $25 \mathrm{wt} \%$ insoluble solids:

11. AN-102R2, Batch $4 \mathrm{~A}$ at $1.5 \mathrm{wt} \%$ insoluble solids:

12. AN-102R2, Batch $4 \mathrm{~A}$ at $18 \mathrm{wt} \%$ isoluble solids:

Note on rheological character of the AN-102R2 slurry simulant

As will be seen in the flowing shear stress verus shear strain curves the simulants do not generally produce a straight line. The slurries are actually a time dependent pseudoplastics (a thixotropic,like ink and some paints). However, when the insoluble solids concentration is low (e.g., < 2 wt $\%$ ) the stress versus strain curves are approximately straight (see the next note) there is not significant shear stress at zero shear strain. That is, they behave like Newtonian fluids for which the concept viscosity is defined and are listed above when the yield stress is zero. When the concentration of the insoluble solids increases the non-Newtonian nature of the slurries becomes evident. However, for engineering purposes the slurries can be fit to a Bingham-type fluid model where over more of the strain range the stress is close to a straight line except that as the strain approaches zero there remains a finite stress, which is referred to as the Yield stress. This is the stress that must be overcome for the slurry to begin to flow; its yield point.

Note on the less viscous slurry solutions: Taylor Vorticies

The straight line for the lowest viscosity simulants is limited by the type of viscometer used. For the simulants with the lowest viscosities, i.e., less than $5 \mathrm{cP}$, the shear stress versus shear strain curves show a knee somewhere between a shear strain of 550 and $650 \mathrm{sec}^{-1}$. The data for shear strains above the knee are not used in calculating slurry viscosity because the measurement is being affected by Taylor vorticies. The type of 
viscometer used for this work utilizes concentric cylinders. The solution to be measured filled the annular gap between two cylinder; the outer cylinder is stationary while the inner one rotates. As the inner cylinder increases with speed it imparts a centrifigul force on the solution through shear and the closer the solution to the cylinder the larger the force. That is, the solution closest to the inner cyclinder feels a larger force to move away from the inner surface than the liquid further away. This secondary flow causes what is called Taylor vorticies and at some point they affect and corrupt the rheological measurement. That point is evidenced by the knee in the data as seen in Figs. G1, G3, G8, and G11. This knee is not seen for the high viscosity solutions because it would occur beyond the shear strains measure for this work. To illustrate the Taylor vorticies see Fig. G11. The shear stress caused by the Taylor vorticies has been calculated and while the stress is below the inherent shear stress of the solution itself the solution viscosity is unaffected. However, when the shear stress of the Taylor vorticies is on the same order of magnitude or large, then the rheometer measures this larger shear field, thus the knee and steeper slope of the data. If the Taylor vorticies were not present the shear stress versus shear stain slope would be unaffected.

Note on choice of data included

For each slurry the rheological measurements were done at least twice. However, only one graph for each data set is included since the extra data do not add more information. None of the repeated measurements showed significant differences from those shown in this sections. 


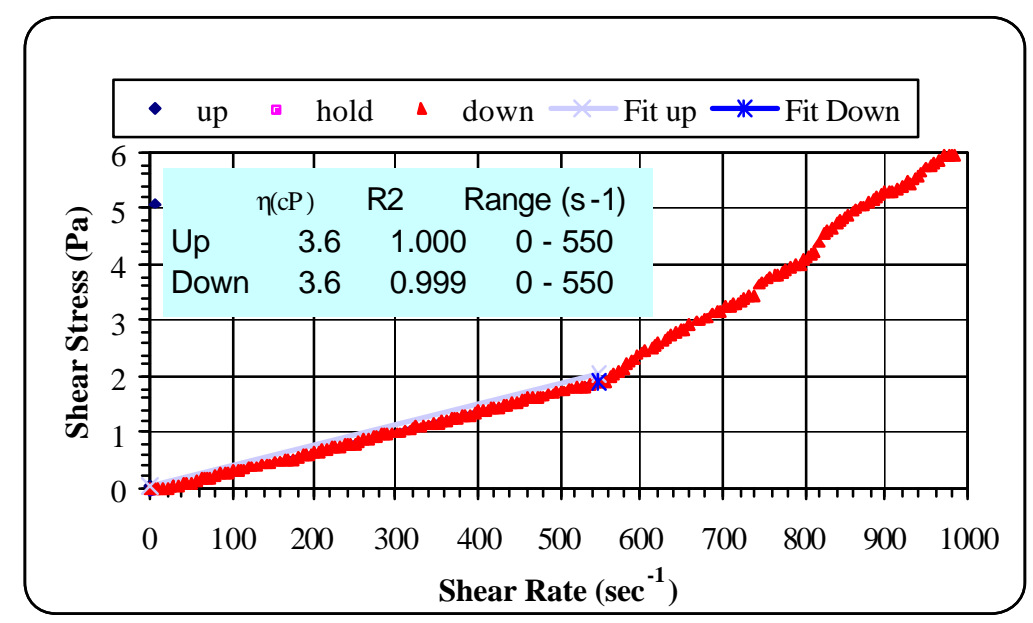

G1. AN-102R2, Batch $3 \mathrm{C}$ at $0.8 \mathrm{wt} \%$ I.S. at $25^{\circ} \mathrm{C}($ Consistency $=3.6 \mathrm{cP}$; Yield Stress $=0.0 \mathrm{~Pa})$

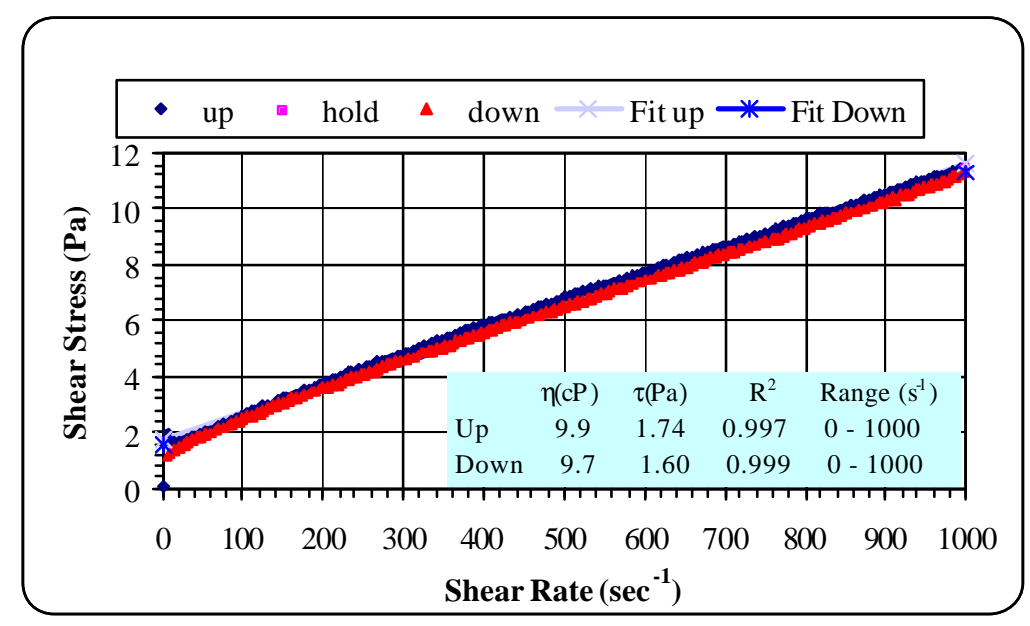

G2. AN-102R2, Batch $3 \mathrm{C}$ at $8.4 \mathrm{wt} \%$ I.S. at $25^{\circ} \mathrm{C}($ Consistency $=9.8 \mathrm{cP}$; Yield Stress $=1.7 \mathrm{~Pa})$

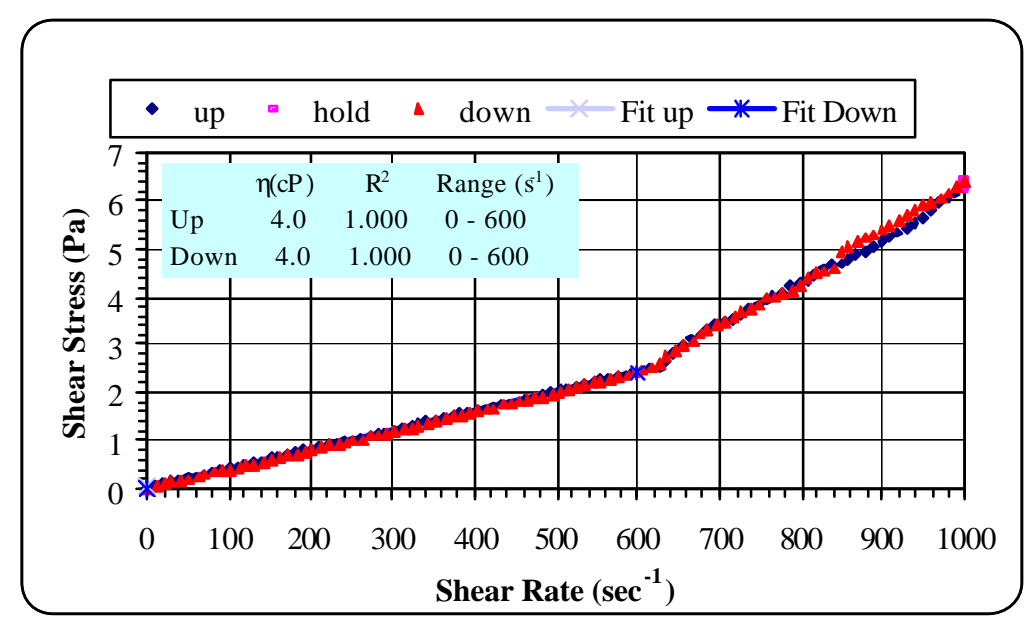

G3. AN-102R2, Batch 3B at $1.2 \mathrm{wt} \%$ I.S. at $25^{\circ} \mathrm{C}($ Consistency $=4.0 \mathrm{cP}$; Yield Stress $=0.0 \mathrm{~Pa})$ 
WSRC-TR-2003-00204, REV. 0

SRT-RPP-2003-00087, REV. 0

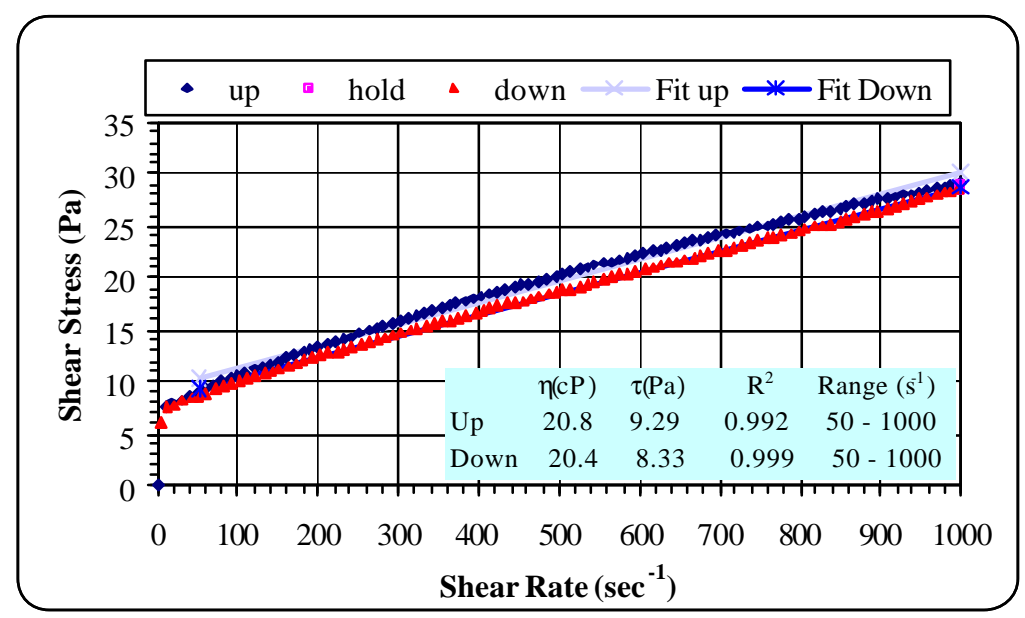

G4. AN-102R2, Batch 3B at $22 \mathrm{wt} \%$ I.S. at $25^{\circ} \mathrm{C}($ Consistency $=21 \mathrm{cP}$; Yield Stress $=8.8 \mathrm{~Pa})$

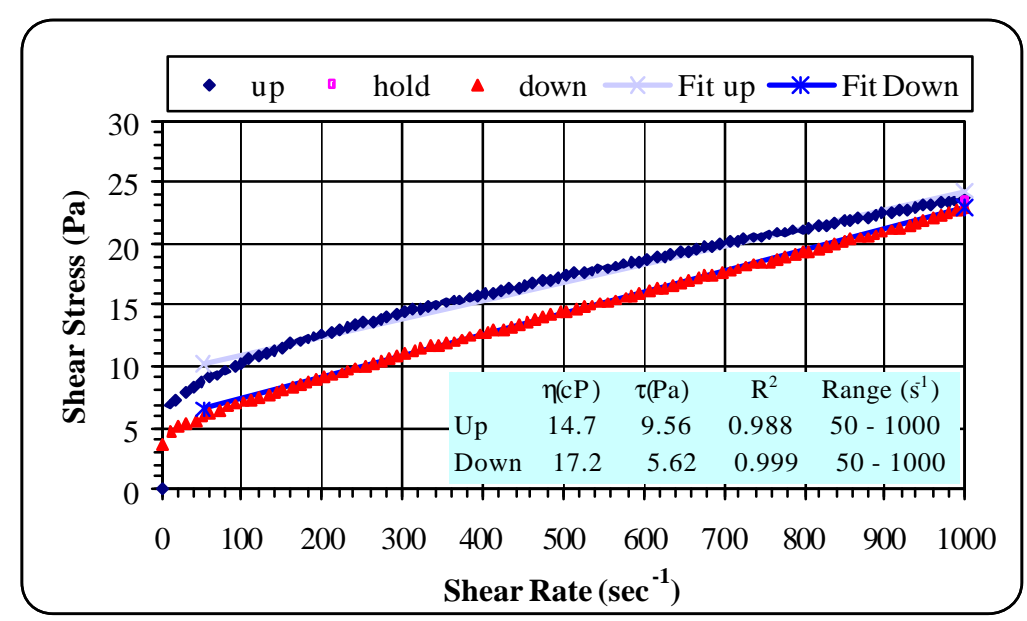

G5. AN-102R2, Batch 3B at $19 \mathrm{wt} \%$ I.S. at $25^{\circ} \mathrm{C}($ Consistency $=16 \mathrm{cP}$; Yield Stress $=7.6 \mathrm{~Pa})$

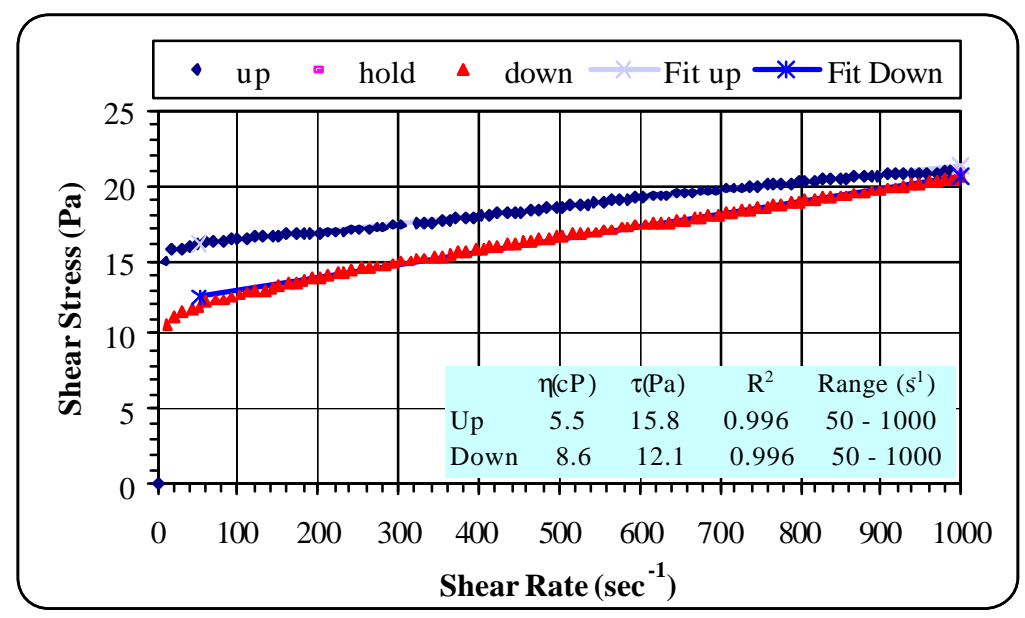

G6. AN-102R2, Batch 3B (washed) at $18 \mathrm{wt} \%$ I.S. at $25^{\circ} \mathrm{C}($ Consistency $=7.1 \mathrm{cP}$; Yield Stress $=14 \mathrm{~Pa})$ 
WSRC-TR-2003-00204, REV. 0

SRT-RPP-2003-00087, REV. 0

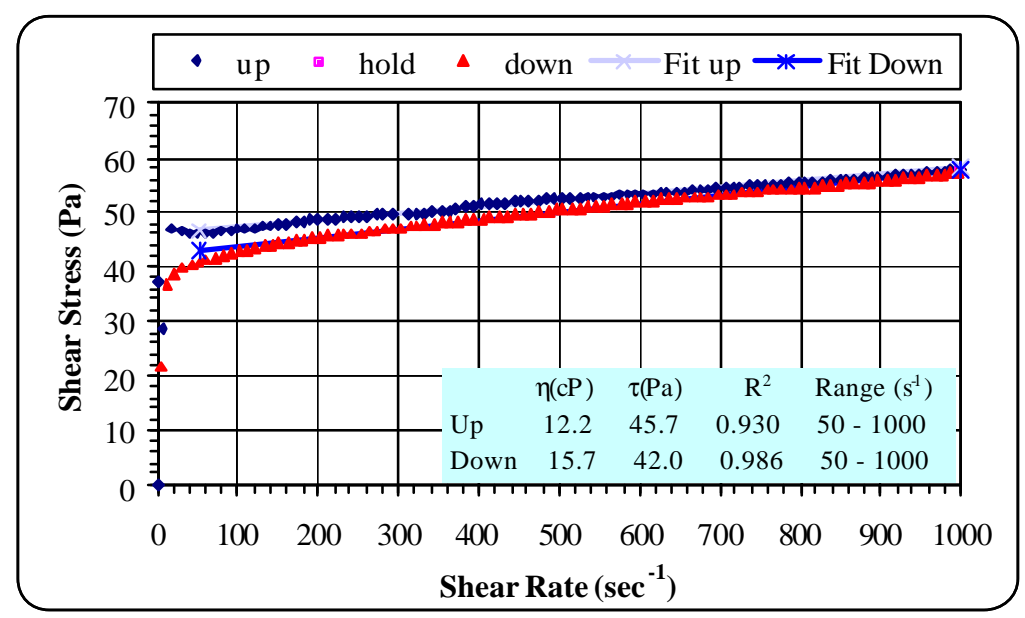

G7. AN-102R2, Batch 3B (washed) at $25 \mathrm{wt} \%$ I.S. at $25^{\circ} \mathrm{C}($ Consistency $=14 \mathrm{cP}$; Yield Stress $=44 \mathrm{~Pa})$

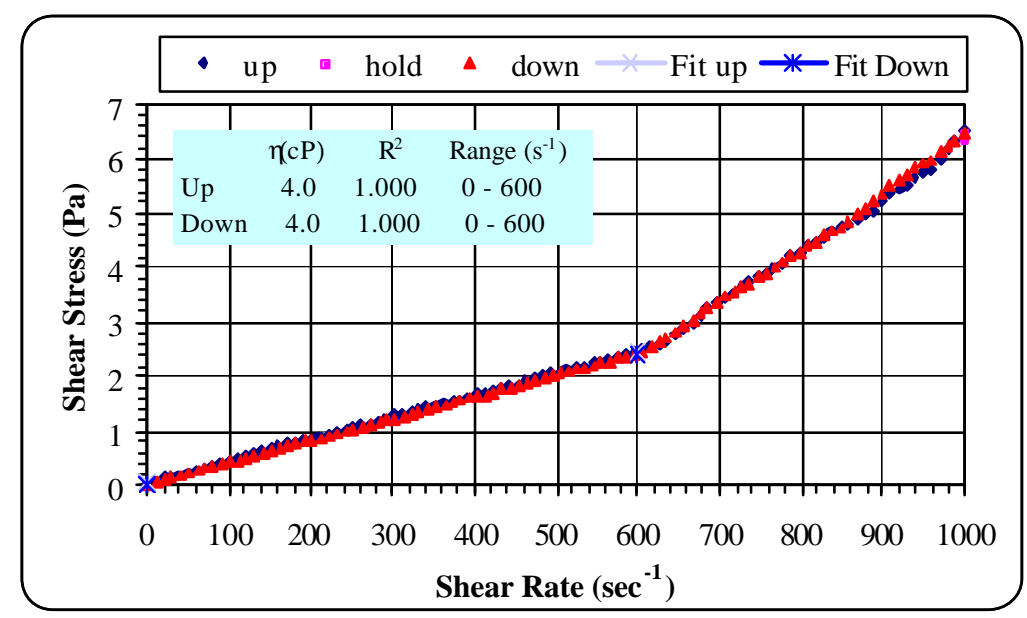

G8. AN-102R2, Batch 3A at $1.6 \mathrm{wt} \%$ I.S. at $25^{\circ} \mathrm{C}($ Consistency $=4.0 \mathrm{cP}$; Yield Stress $=0.0 \mathrm{~Pa})$

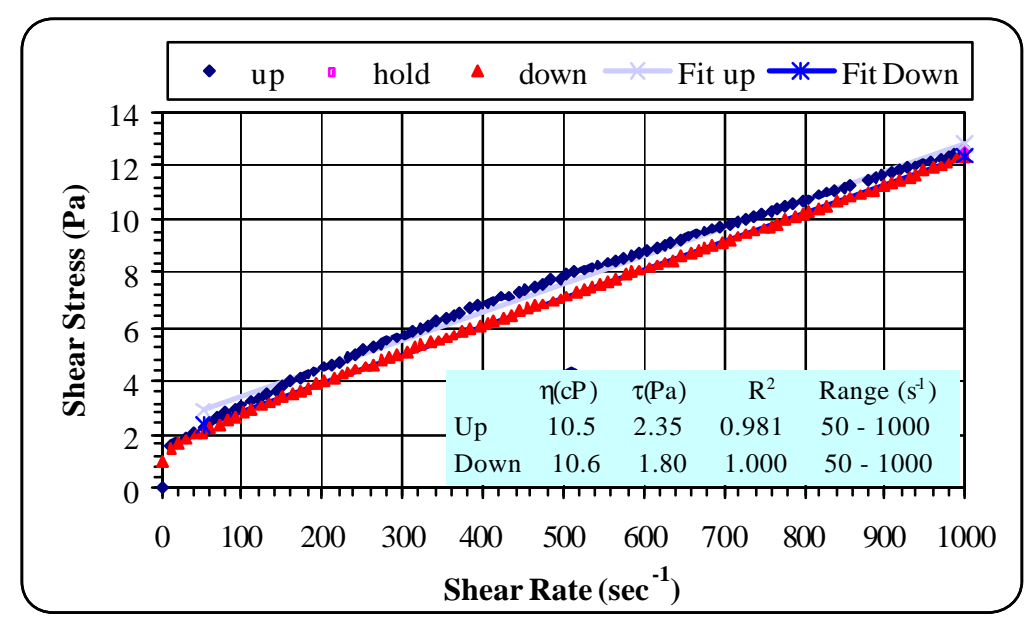

G9. AN-102R2, Batch $3 \mathrm{~A}$ at $14 \mathrm{wt} \%$ I.S. at $25^{\circ} \mathrm{C}($ Consistency $=11 \mathrm{cP}$; Yield Stress $=2.1 \mathrm{~Pa})$ 
WSRC-TR-2003-00204, REV. 0

SRT-RPP-2003-00087, REV. 0

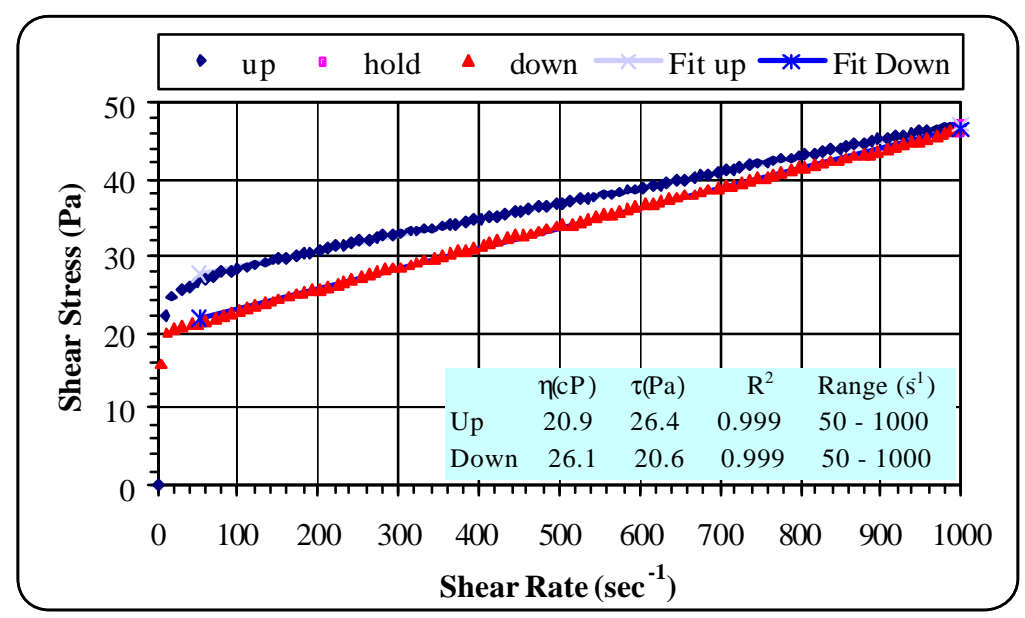

G10. AN-102R2, Batch $3 \mathrm{~A}$ at $25 \mathrm{wt} \%$ I.S. at $25^{\circ} \mathrm{C}($ Consistency $=24 \mathrm{cP}$; Yield Stress $=24 \mathrm{~Pa})$

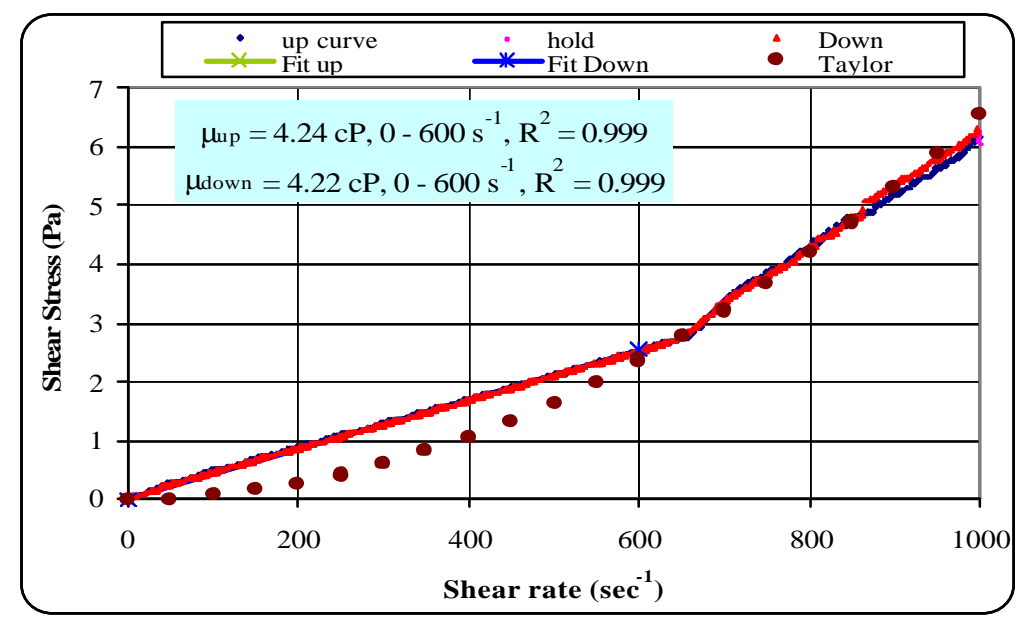

G11. AN-102R2, Batch 4A at $1.5 \mathrm{wt} \%$ I.S. at $25^{\circ} \mathrm{C}($ Consistency $=4.2 \mathrm{cP}$; Yield Stress $=0.0 \mathrm{~Pa})$

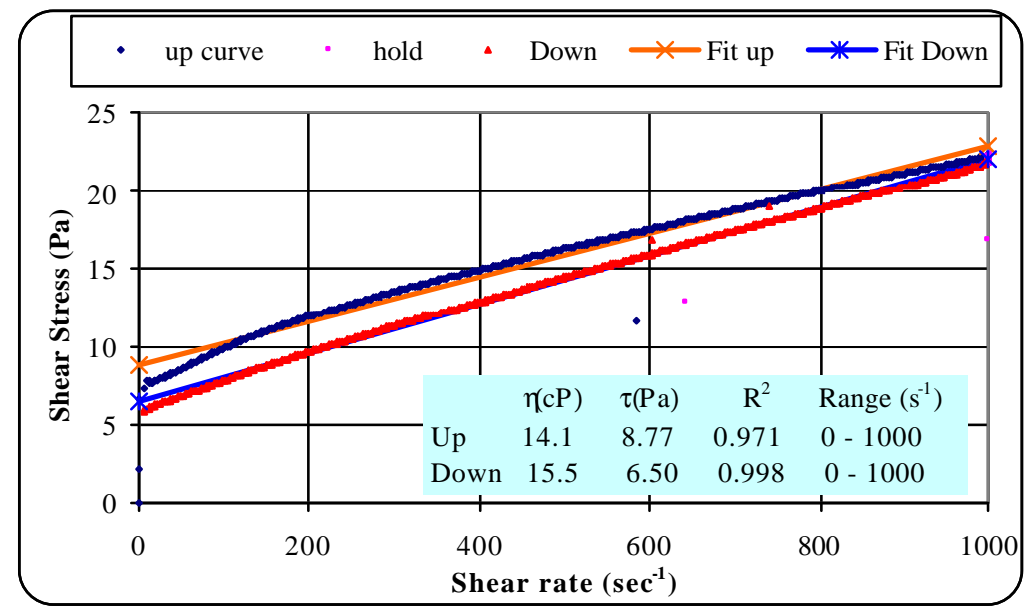

G12. AN-102R2, Batch 4A at $18 \mathrm{wt} \%$ I.S. at $25^{\circ} \mathrm{C}($ Consistency $=15 \mathrm{cP}$; Yield Stress $=7.6 \mathrm{~Pa})$ 


\section{Particle Size Distribution}

Samples were taken at the beginning and the end (and at other points) of each pilot-scale cross-flow ultrafiltration test with AN-102 simulant slurry, to measure some rheological properties, like shear stress versus shear rate, viscosity, or if non-Newtonian, consistency. The preceding table lists the viscosity, or consistency and yield shear stress, for each of the samples. This section shows graphical representation of the data used to determine those number.

Those simulant slurry samples at $25^{\circ} \mathrm{C}$ are:

Fig.G

13. AN-102R2, Batch $3 \mathrm{C}$ at $0.8 \mathrm{wt} \%$ insoluble solids: 14. AN-102R2, Batch $3 \mathrm{C}$ at $0.8 \mathrm{wt} \%$ insoluble solids: 15. AN-102R2, Batch $3 \mathrm{C}$ at $8.4 \mathrm{wt} \%$ insoluble solids: 16. AN-102R2, Batch $3 \mathrm{C}$ at $8.4 \mathrm{wt} \%$ insoluble solids: 17. AN-102R2, Batch 3B at $1.2 \mathrm{wt} \%$ insoluble solids: 18. AN-102R2, Batch 3B at $1.2 \mathrm{wt} \%$ insoluble solids: 19. AN-102R2, Batch 3B at $22 \mathrm{wt} \%$ insoluble solids: 20. AN-102R2, Batch $3 \mathrm{~B}$ at $22 \mathrm{wt} \%$ insoluble solids: 21. AN-102R2, Batch 3B at $19 \mathrm{wt} \%$ insoluble solids: 22. AN-102R2, Batch 3B at $19 \mathrm{wt} \%$ insoluble solids:

Volume Distribution Number Distribution Volume Distribution Number Distribution Volume Distribution Number Distribution Volume Distribution Number Distribution Volume Distribution Number Distribution 23. AN-102R2, Batch 3B (post-washed) at $18 \mathrm{wt} \%$ insoluble solids:Volume Distribution 24. AN-102R2, Batch 3B (post-washed) at $18 \mathrm{wt} \%$ insoluble solids:Number Distribution 25. AN-102R2, Batch $3 \mathrm{~A}$ at $1.6 \mathrm{wt} \%$ insoluble solids: 26. AN-102R2, Batch $3 \mathrm{~A}$ at $1.6 \mathrm{wt} \%$ insoluble solids: 27. AN-102R2, Batch $3 \mathrm{~A}$ at $14 \mathrm{wt} \%$ insoluble solids: 28. AN-102R2, Batch 3A at $14 \mathrm{wt} \%$ insoluble solids: 29. AN-102R2, Batch $3 \mathrm{~A}$ at $25 \mathrm{wt} \%$ insoluble solids: 30. AN-102R2, Batch 3A at $25 \mathrm{wt} \%$ insoluble solids: 31. AN-102R2, Batch $4 \mathrm{~A}$ at $1.5 \mathrm{wt} \%$ insoluble solids: 32. AN-102R2, Batch $4 \mathrm{~A}$ at $1.5 \mathrm{wt} \%$ insoluble solids: 33. AN-102R2, Batch $4 \mathrm{~A}$ at $18 \mathrm{wt} \%$ isoluble solids: 34. AN-102R2, Batch $4 \mathrm{~A}$ at $18 \mathrm{wt} \%$ isoluble solids:
Volume Distribution

Number Distribution Volume Distribution Number Distribution Volume Distribution Number Distribution Volume Distribution Number Distribution Volume Distribution Number Distribution

Note on Particle Size Distribution Method

Three of the methods available at the Savannah River Site to evaluate the particle size distribution (PSD) are performed by equipment made by Microtrac. They are:

Mono-laser diffraction analysis:

a. SRA150 standard range, 20 channels: 0.7 to 700 microns

b. SRA150 extended range, 40 channels: 0.2 to 700 microns

Tri-laser diffraction analysis

a. X100 high resolution, 40 channels: 0.04 to 700 microns

Each method has its strong and weak points. 


\section{SRA150 standard range}

Strong point: No knowledge of particle transparency is needed and gives immediate results.

Weak point: There is not enough resolution to discern between particle sizes and is on the threshold of the minimum particle size of interest.

\section{SRA150 extended range}

Strong point: It covers the range of particle size of interest

Weak point: Some knowledge of particle transparency is needed for submicron particles, however, the apparatus will make an educated guess if not known.

\section{X100 high resolution}

Strong point: Can measure to very small sizes

Weak point: The accuracy of the results is highly dependent on knowledge of particle transparency and the index of refraction of the fluid. The measurement is very sensitive and much more prone to measurement uncertainty without a detailed knowledge of the slurry's and solids'optical characteristics.

Because particle transparencies and simulants' index of refractions were not known the SRA150 extended range method was chosen. It covered the expected range of particle sizes and gave very consistent results with minimal input. On testing the first slurry, batch 3C, tests were done by choosing all three particle transparencies options, i.e., transparent, absorbtive (opaque), or reflective. For the tests with one slurry sample all three choices gave very similar results and within the significance of measurement the difference were not important. That is, a particle size averages did not vary more that $15 \%$ amongst themselves and distribution characteristics remain the same, e.g., a bimodal distribution was obtain independent of the transparency. Table G3 shows ther results of the sample, AN-102R2, Batch 3C, at $0.8 \mathrm{wt} \%$ insoluble solids. (Figs. G13 and G14 are the full Microtrac results of the absorptive PSDs.)

\begin{tabular}{|l|c|c|c|c|c|c|}
\hline Particle Transparency & Reflective & Transparent & Absorptive & Reflective & Transparent & Absorptive \\
\hline Type Distribution & Volume & Volume & Volume & Number & Number & Number \\
\hline Distribution Characteristic & Bimodal & Bimodal & Bimodal & Single & Single & Sinqle \\
\hline Large Average Particle Size & 8.5 microns & 8.6 microns & 8.6 microns & 2.3 microns & 2.4 microns & 2.1 microns \\
\hline$\%$ of Particles in Average & $69 \%$ & $71 \%$ & $68 \%$ & $100 \%$ & $100 \%$ & $100 \%$ \\
\hline Small Average Particle Size & 2.6 microns & 2.7 microns & 2.6 microns & not applicable & not applicable & not applicable \\
\hline$\%$ of Particles in Average & $31 \%$ & $29 \%$ & $32 \%$ & not applicable & not applicable & not applicable \\
\hline Particle Range (microns) & 1.6 to 74 & 1.6 to 74 & 1.0 to 74 & 1.6 to 22 & 1.6 to 22 & 1.0 to 22 \\
\hline
\end{tabular}

Table G3. Comparing particle transparency results using Microtrac SRA150 extended range with a simulant sample from AN-102R2, Batch 3C (No. 300188111)

All the following PSD analyses were done with an absorptive particle transparency because that method seemed to indicate slightly smaller particle than the other methods.

\section{Minimum size for SRA150 extended range}

The stated range for this method, as given above, is 0.2 to 700 micron. However, as seen in the following Microtrac data sheets is appears that the lower setting was set at 
0.688 micron for an unknown reason. While the smaller size was intended, a scan of the data show that for all but a few of the samples measured the PSD data tails smoothly taper off for the smallest particles, which implies that most, if not all, particle sizes were measured. However, there are a few exceptions like for the sample of Batch $3 \mathrm{~B}$ at its highest concentration of $22 \mathrm{wt} \%$, i.e., Figs 19 and 20. In most cases, the 0.688 micron cutoff was sufficient.

Note on Sample Preparation to preform a Microtrac Evaluation

Besides the methods to choose in evaluating sample it is also important to prepare the sample properly to obtain accurate results.

\section{Diluent}

The slurry sample is suspended in a large volume ( 0.3 liter $)$ of dilute. The standard diluent is distilled water but due to the neutral $\mathrm{pH}$ and the solubility of solids in water the slurry sample were suspended in it respective filtrate. Filtrate was usually optically transparent and free of solids was used.

\section{Sonication}

In the past the insoluble solids in slurry samples that sat while waiting for process tended to clump and give falsely large particle measurements. To minimize this each sample was mildly agitated with sonic waves to break up such clumps. For this task the samples were sonicated four times at 15 seconds each at a power setting of 30 watts. All samples received the same treatment. 
WSRC-TR-2003-00204, REV. 0

SRT-RPP-2003-00087, REV. 0

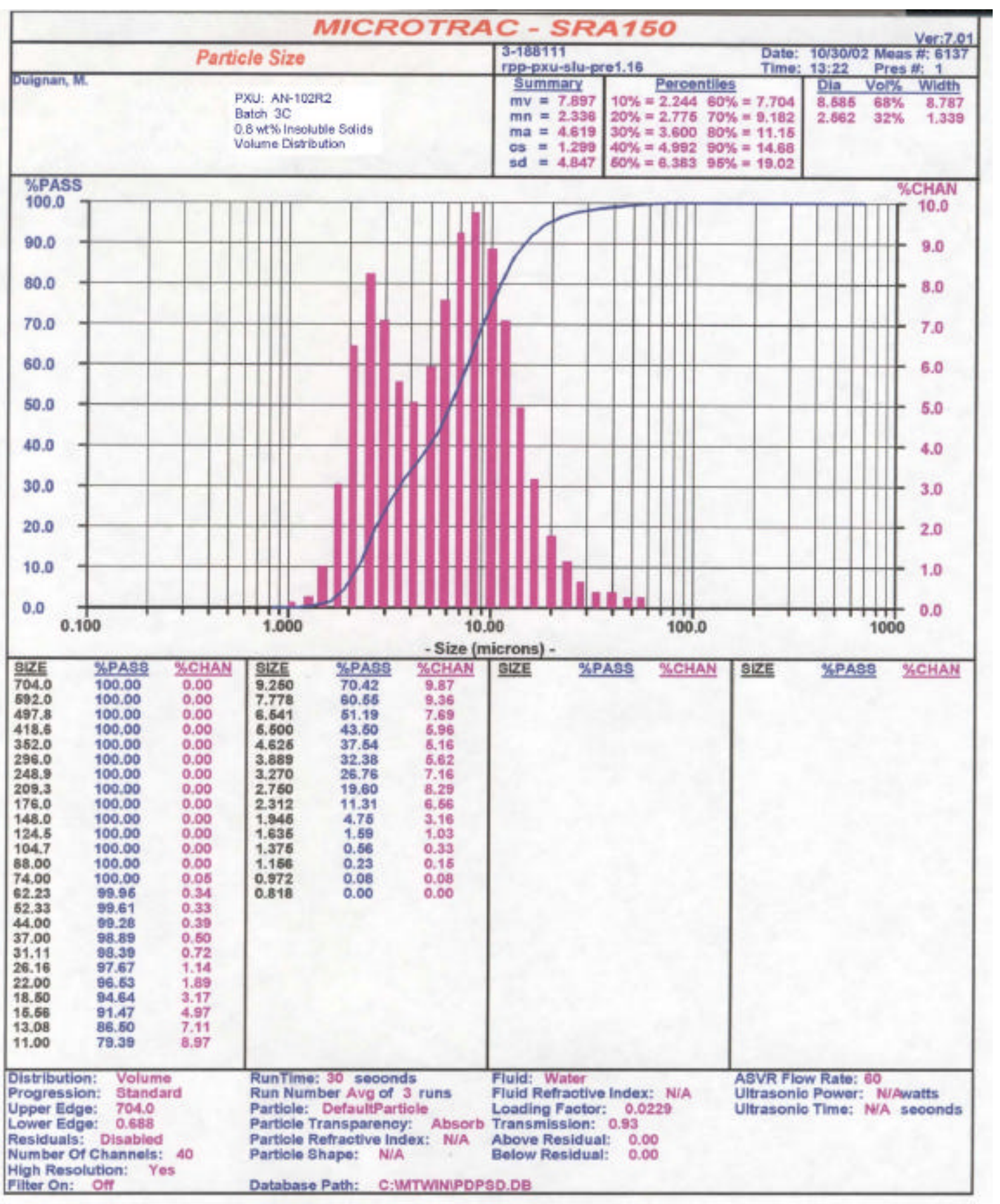

Figure G13. At start of Batch 3C dewatering: 0.8 wt\% Insoluble Solids (VOLUME Distribution) 
WSRC-TR-2003-00204, REV. 0

SRT-RPP-2003-00087, REV. 0

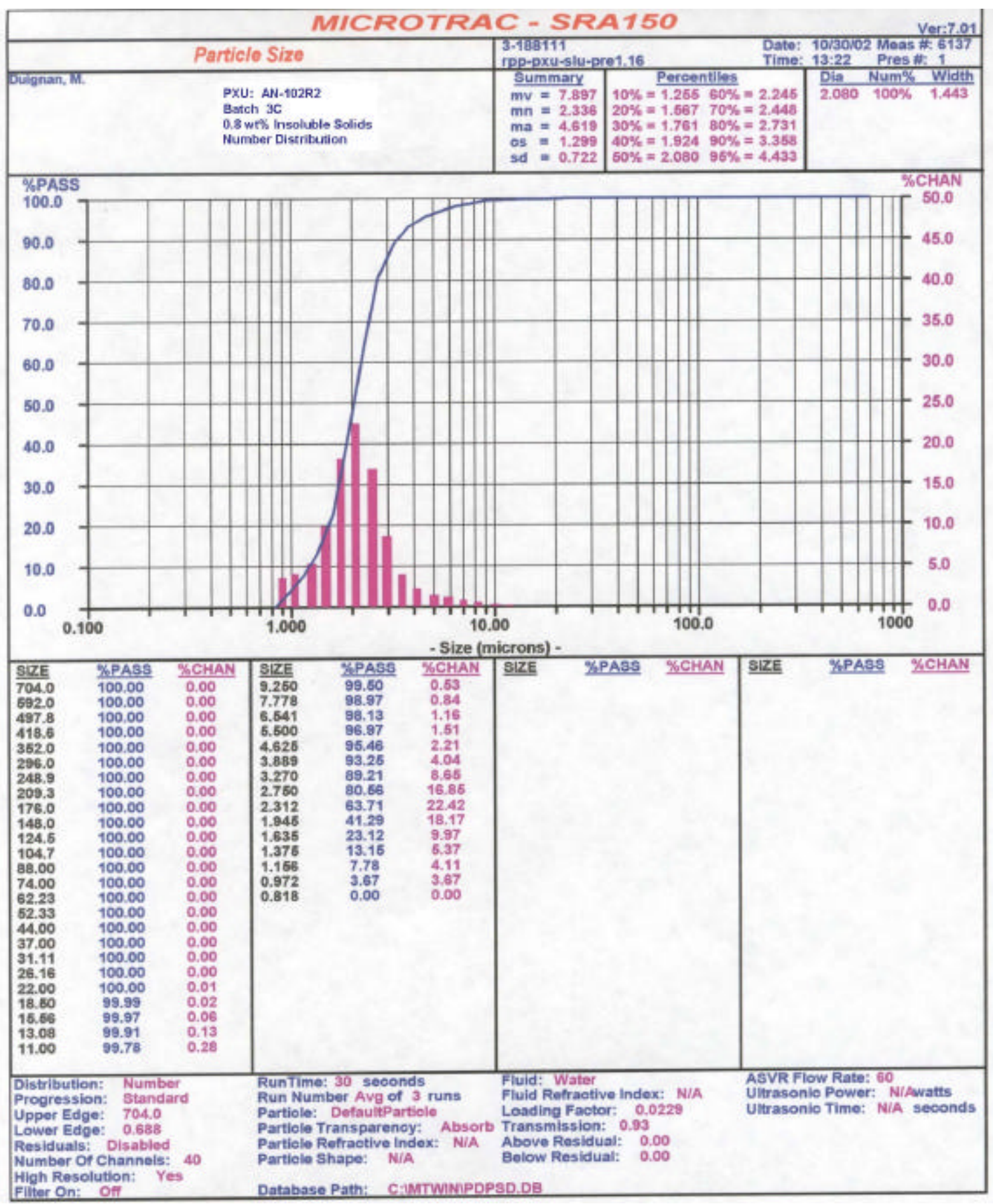

Figure G14. At start of Batch 3C dewatering: 0.8 wt\% Insoluble Solids (NUMBER Distribution) 
WSRC-TR-2003-00204, REV. 0

SRT-RPP-2003-00087, REV. 0

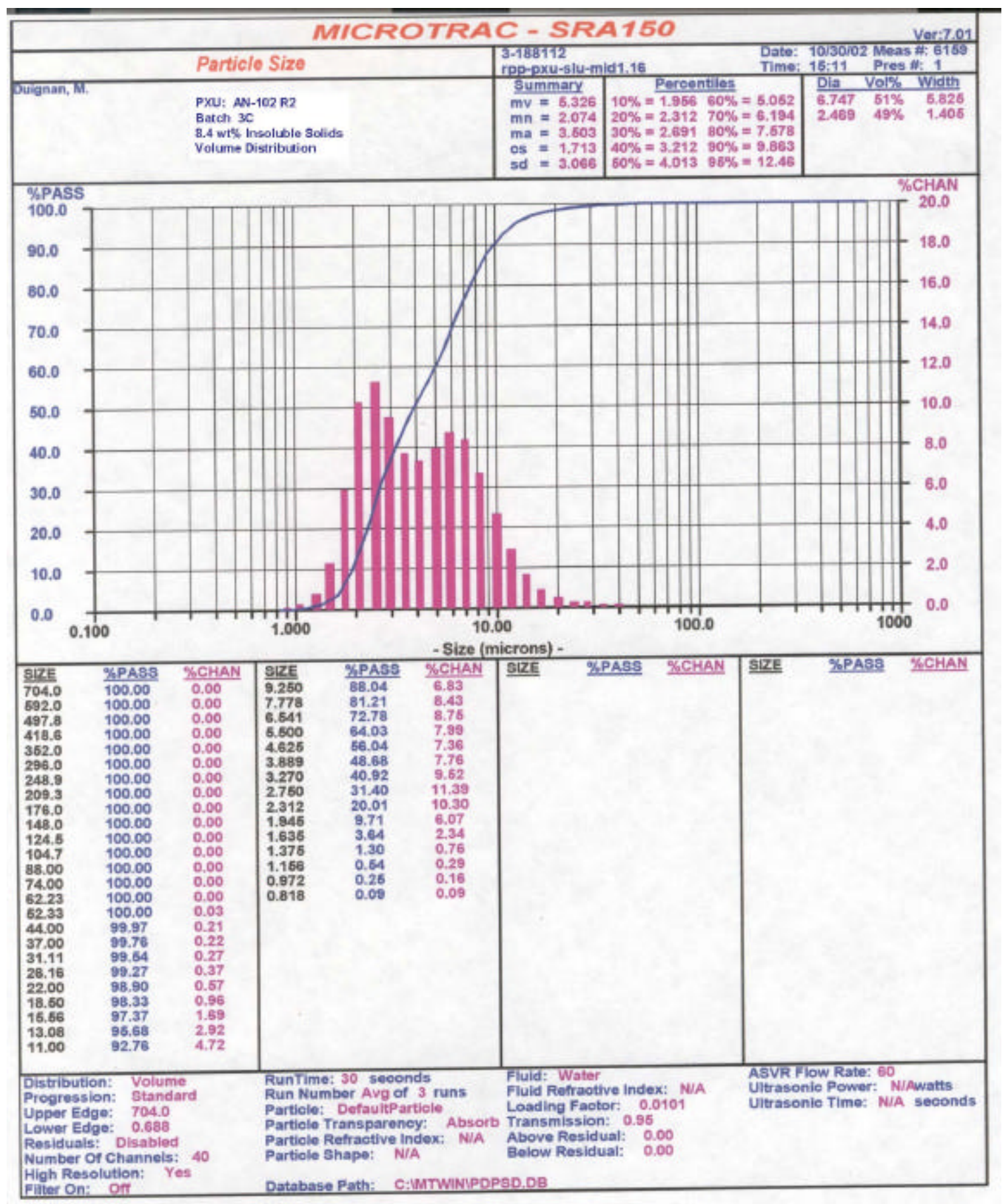

Figure G15. After 45 hours of Batch 3C dewatering: 8.4 wt\% Insoluble Solids (VOLUME Distribution) 
WSRC-TR-2003-00204, REV. 0

SRT-RPP-2003-00087, REV. 0

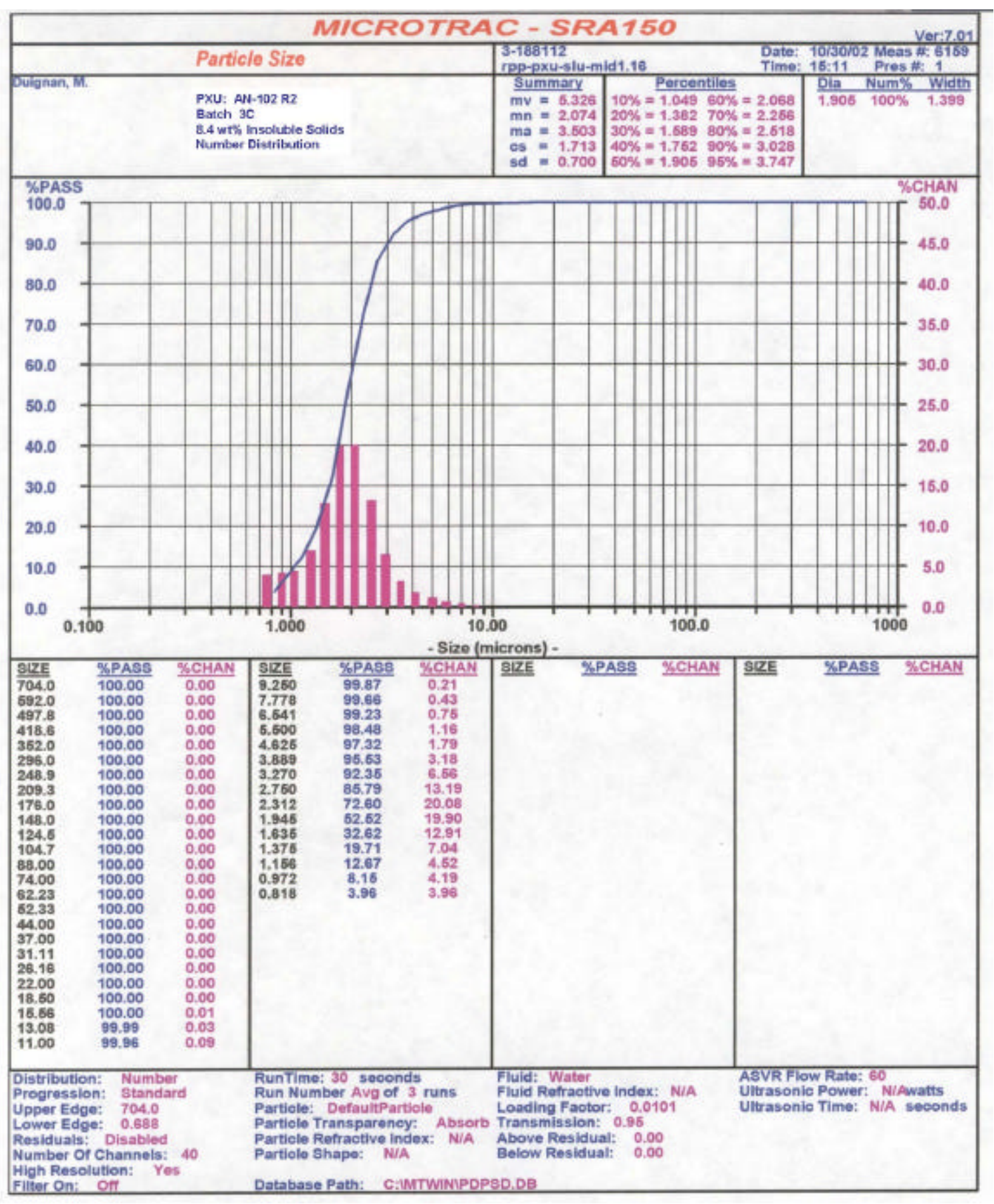

Figure G16. After 45 hours of Batch 3C dewatering: 8.4 wt\% Insoluble Solids (NUMBER Distribution) 
WSRC-TR-2003-00204, REV. 0

SRT-RPP-2003-00087, REV. 0

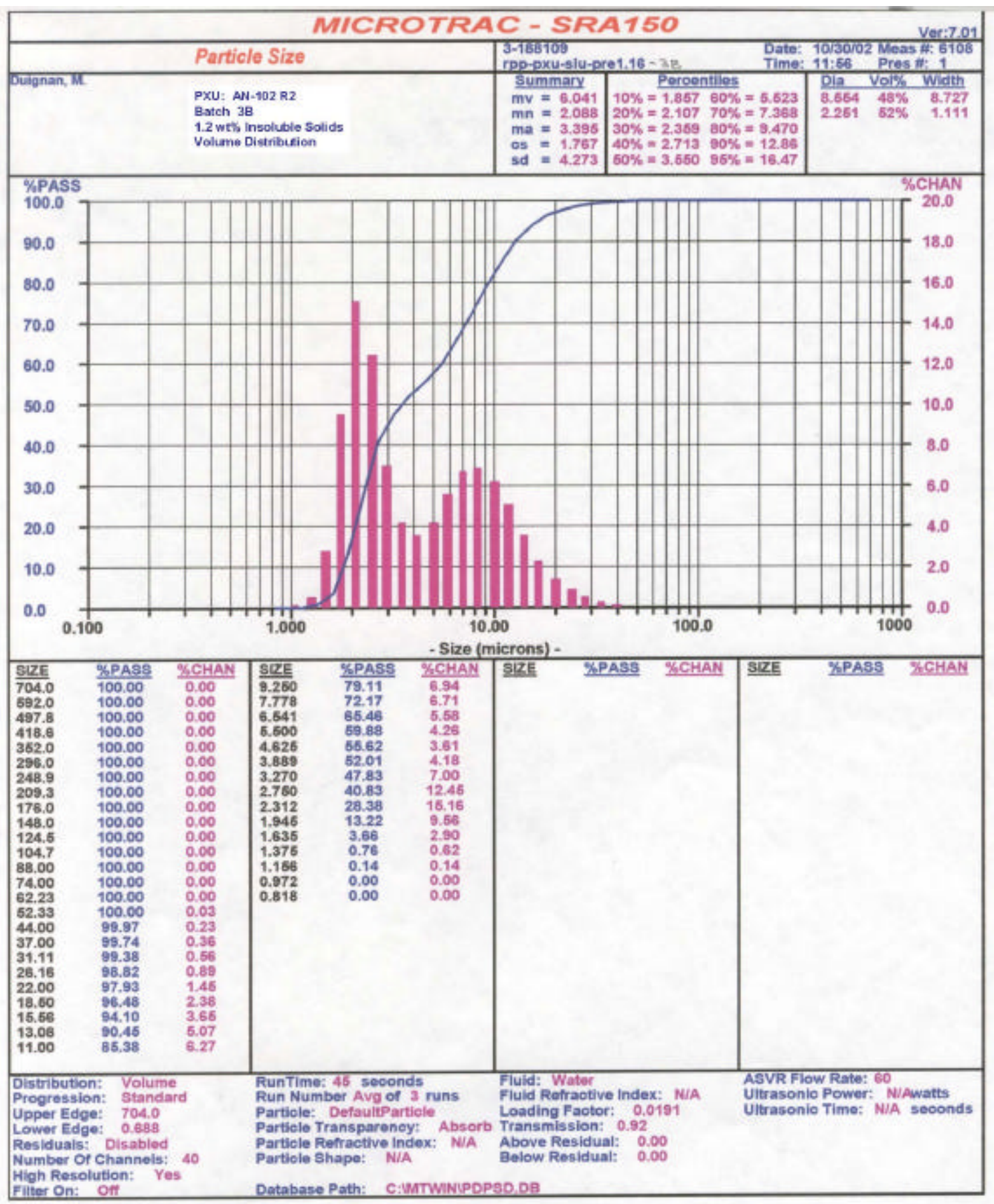

Figure G17. At start of Batch 3B dewatering: 1.2 wt\% Insoluble Solids (VOLUME Distribution) 
WSRC-TR-2003-00204, REV. 0

SRT-RPP-2003-00087, REV. 0

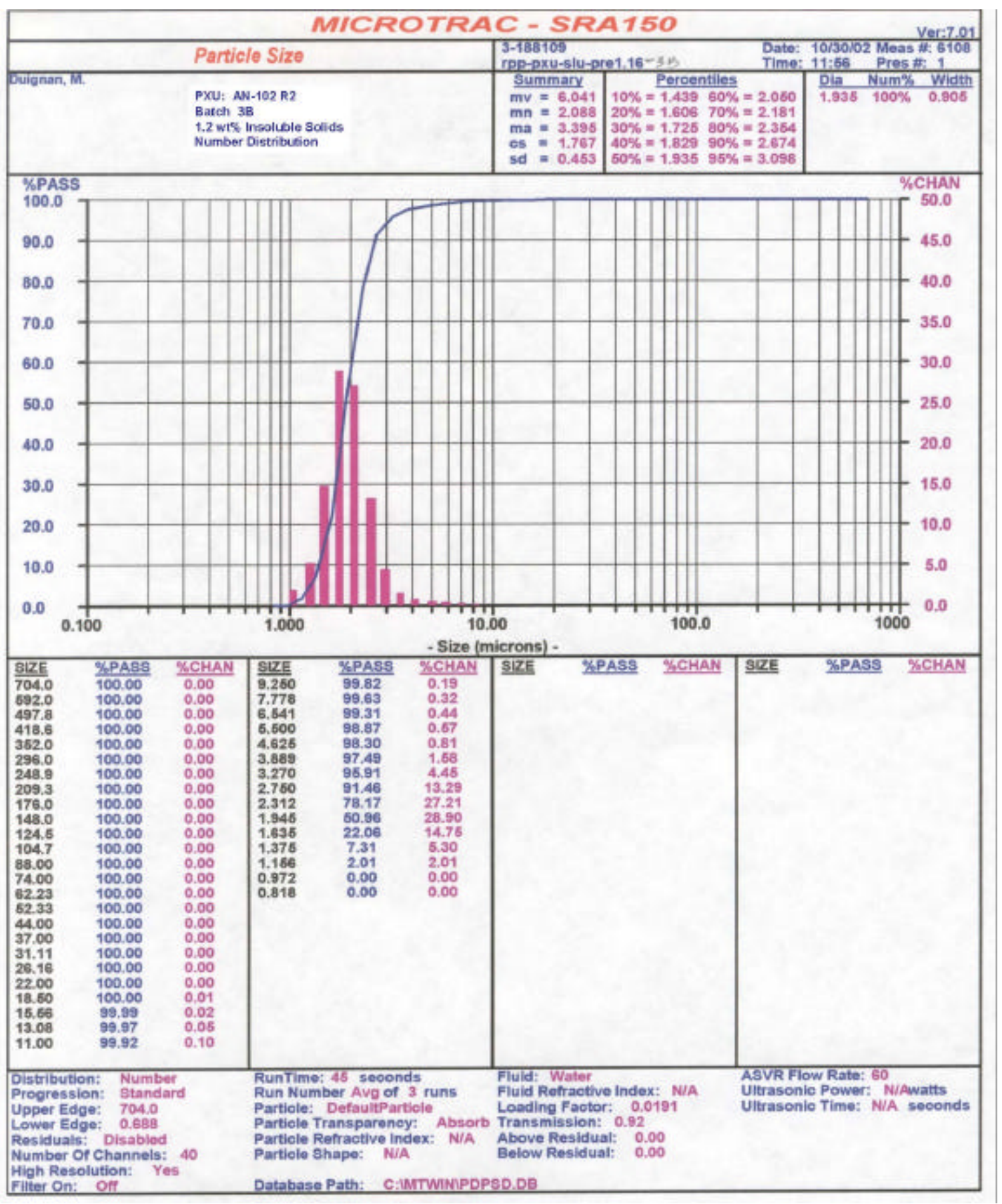

Figure G18. At start of Batch 3B dewatering: 1.2 wt\% Insoluble Solids (NUMBER Distribution) 
WSRC-TR-2003-00204, REV. 0

SRT-RPP-2003-00087, REV. 0

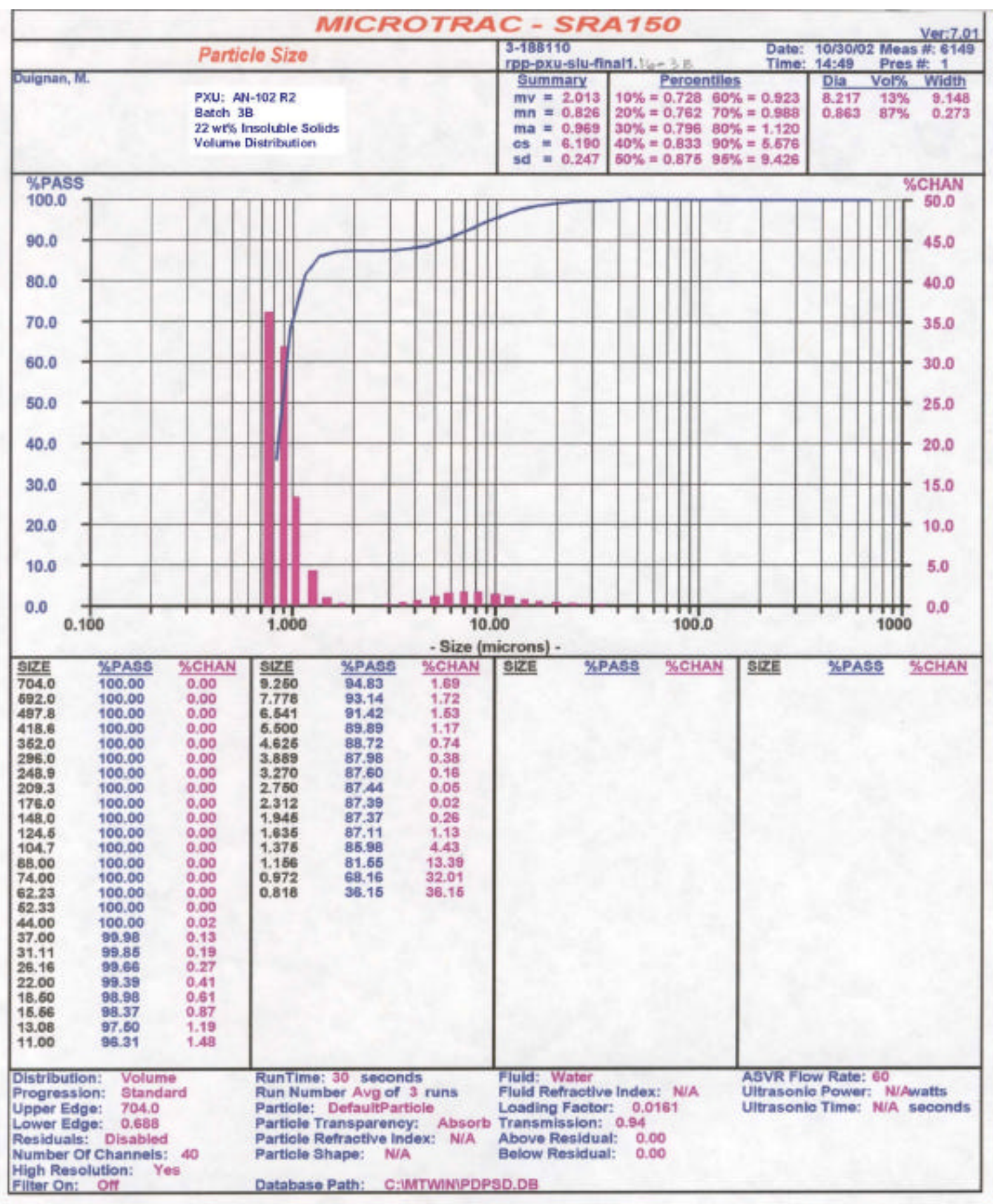

Figure G19. After 12 hours of Batch 3B dewatering: 22 wt\% Insoluble Solids (VOLUME Distribution) 
WSRC-TR-2003-00204, REV. 0

SRT-RPP-2003-00087, REV. 0

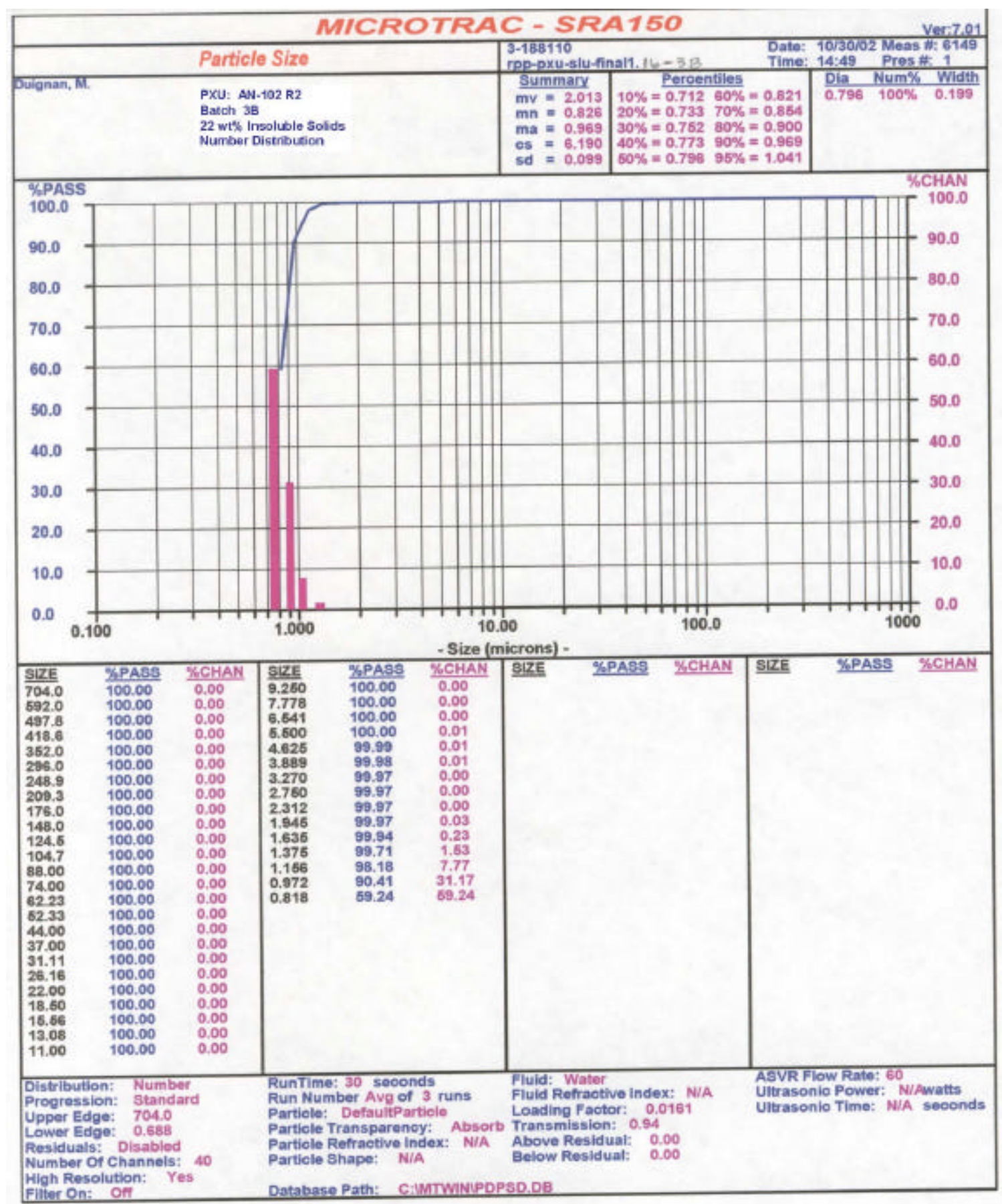

Figure G20. After 12 hours of Batch 3B dewatering: $22 \mathrm{wt} \%$ Insoluble Solids (NUMBER Distribution) 
WSRC-TR-2003-00204, REV. 0

SRT-RPP-2003-00087, REV. 0

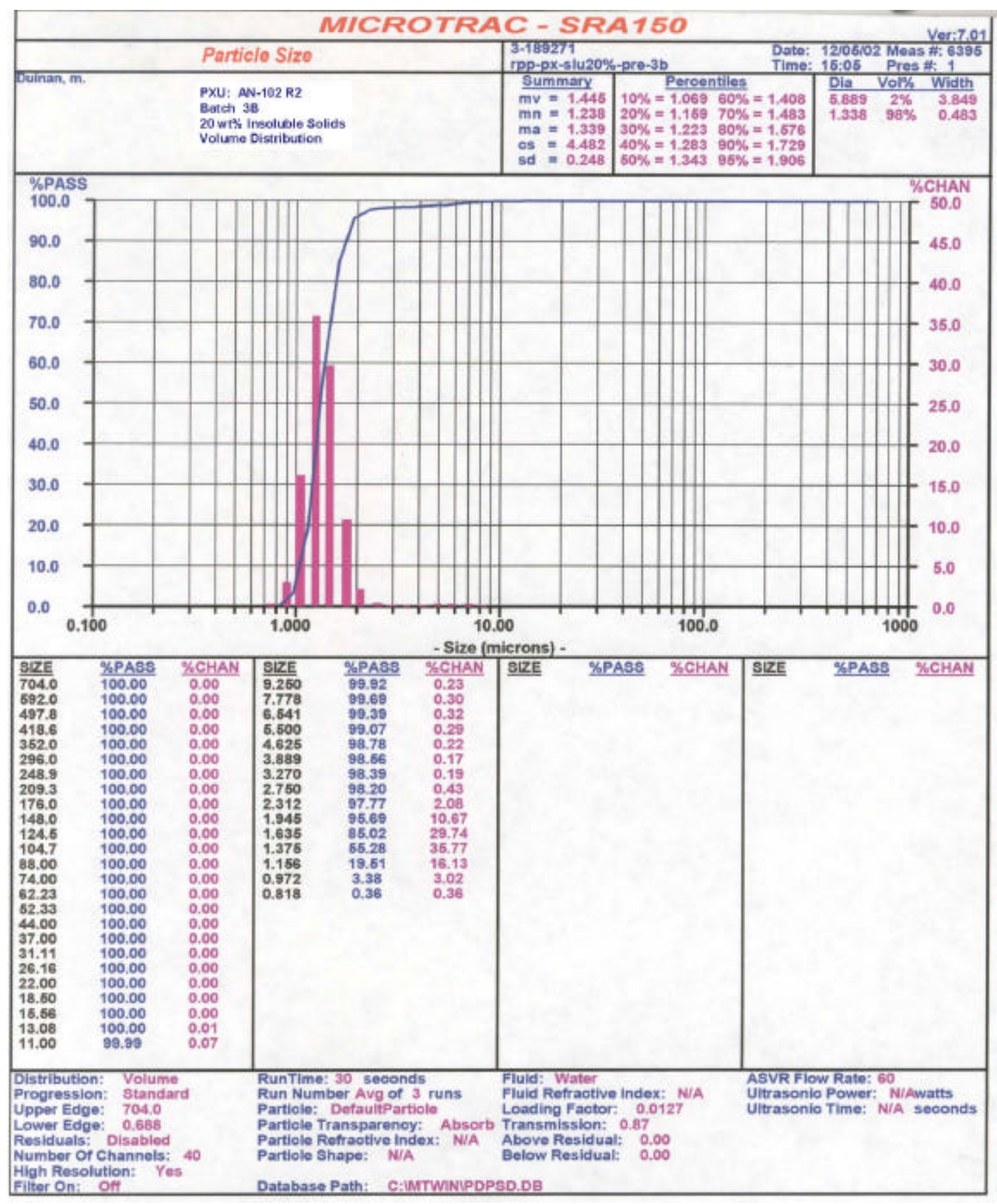

Figure G21. After 12 hours of Batch 3B dewatering: 19 wt\% Insoluble Solids (VOLUME Distribution) - Diluted with filtrate to prepare for washing 
WSRC-TR-2003-00204, REV. 0

SRT-RPP-2003-00087, REV. 0

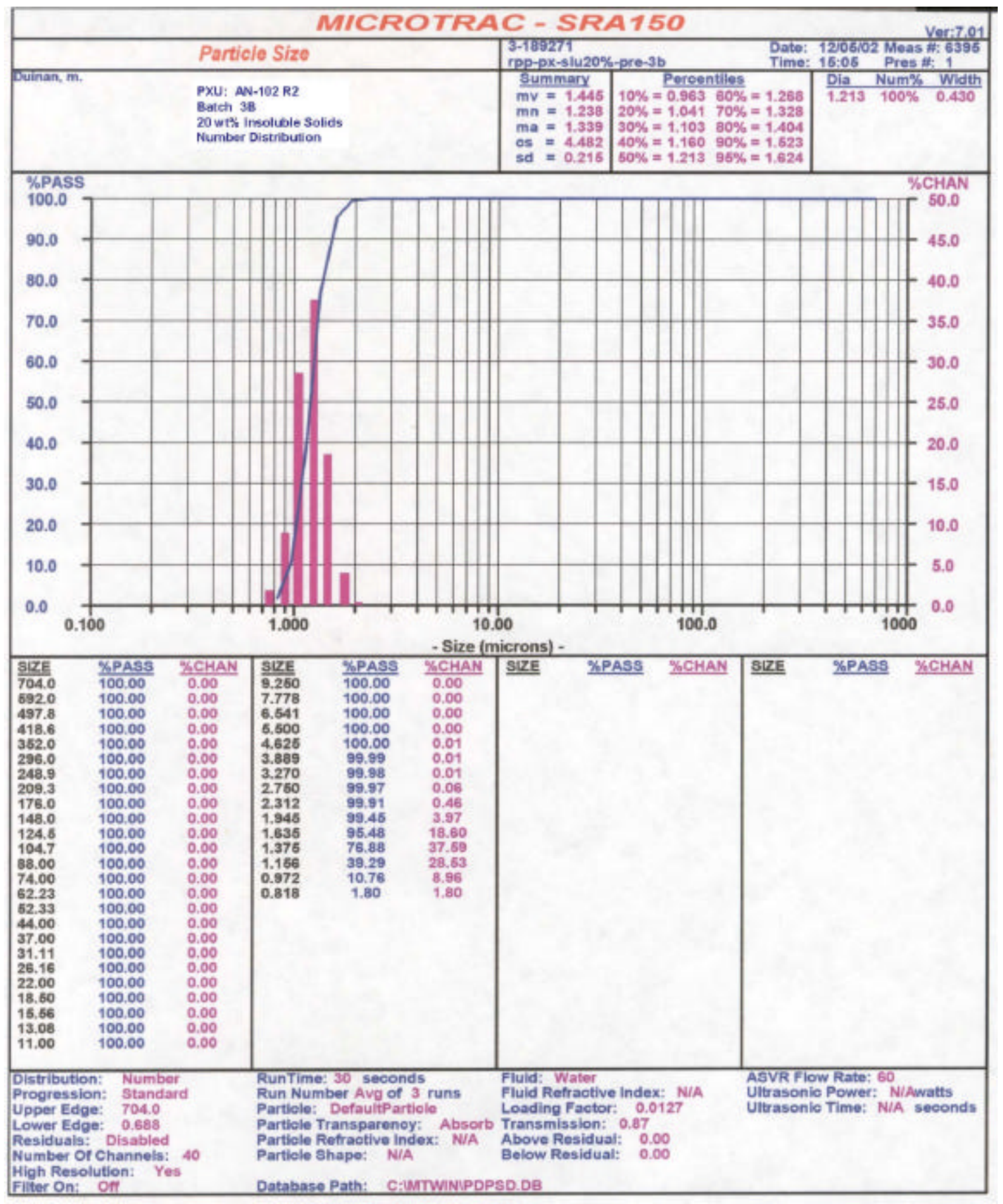

Figure G22. After 12 hours of Batch 3B dewatering: 19 wt\% Insolub le Solids (NUMBER Distribution) - Diluted with filtrate to prepare for washing 
WSRC-TR-2003-00204, REV. 0

SRT-RPP-2003-00087, REV. 0

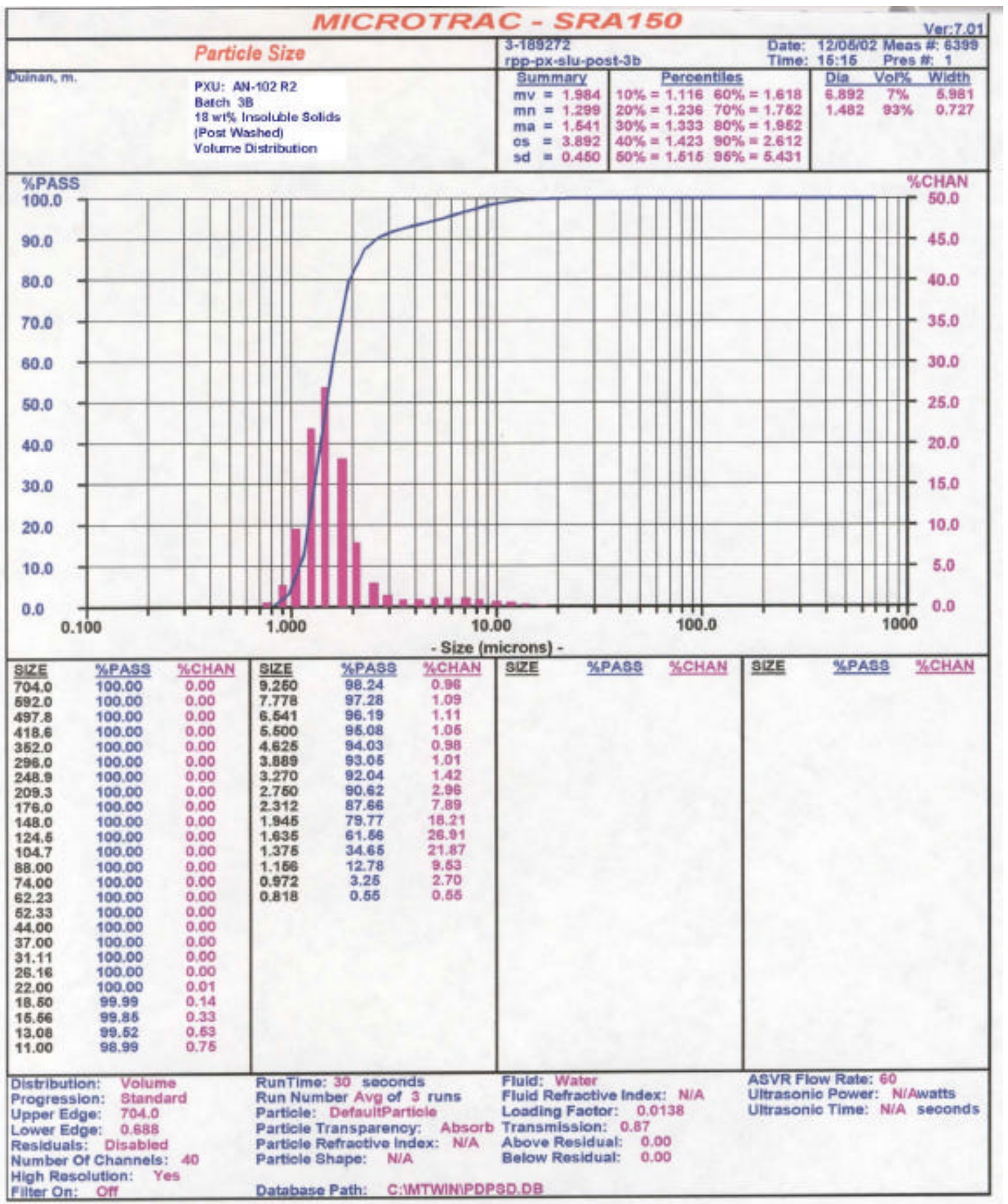

Figure G23. After washing of Batch 3B: 18 wt\% Insoluble Solids (VOLUME Distribution) 
WSRC-TR-2003-00204, REV. 0

SRT-RPP-2003-00087, REV. 0

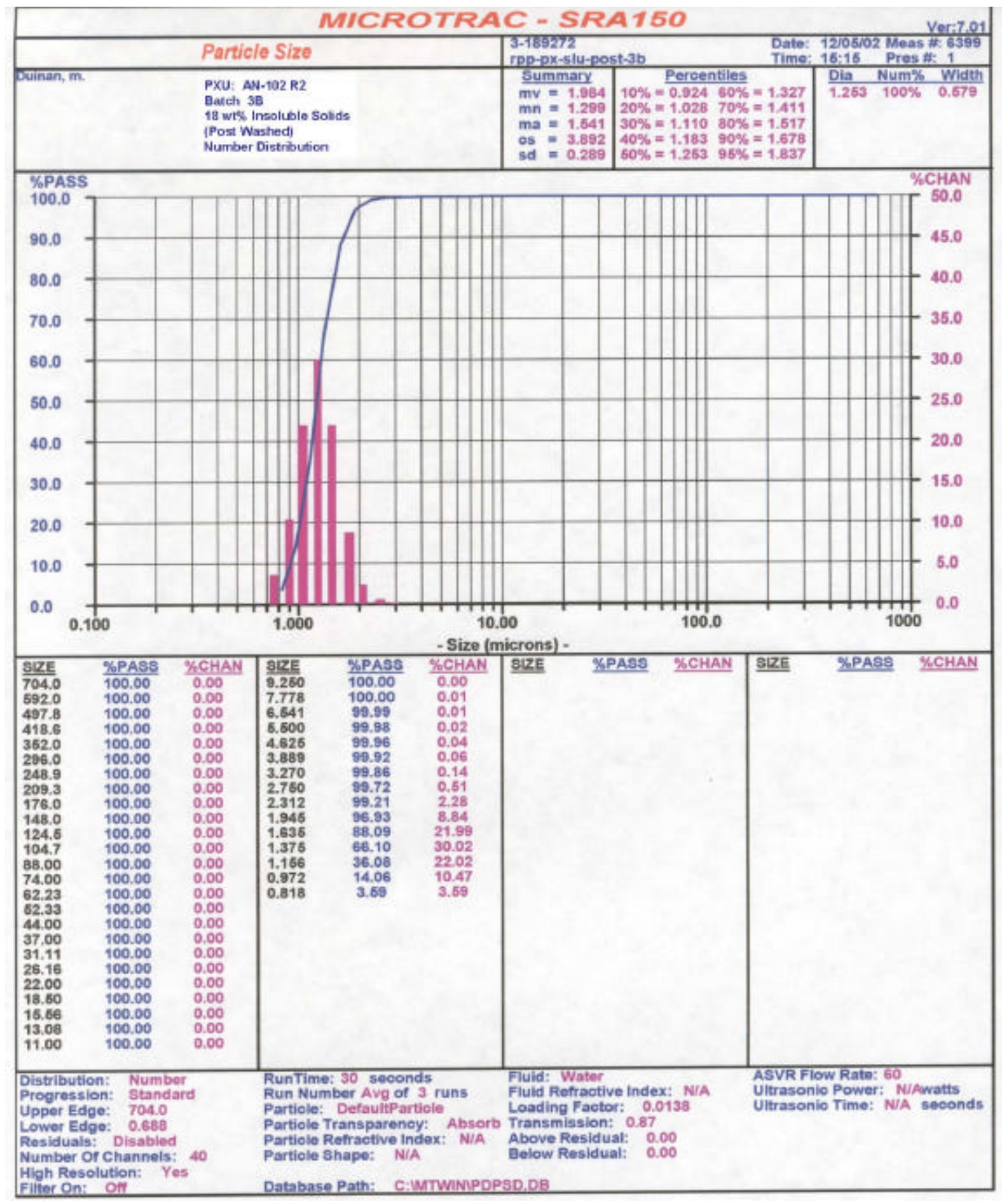

Figure G24. After washing of Batch 3B: 18 wt\% Insoluble Solids (NUMBER Distribution) 
WSRC-TR-2003-00204, REV. 0

SRT-RPP-2003-00087, REV. 0

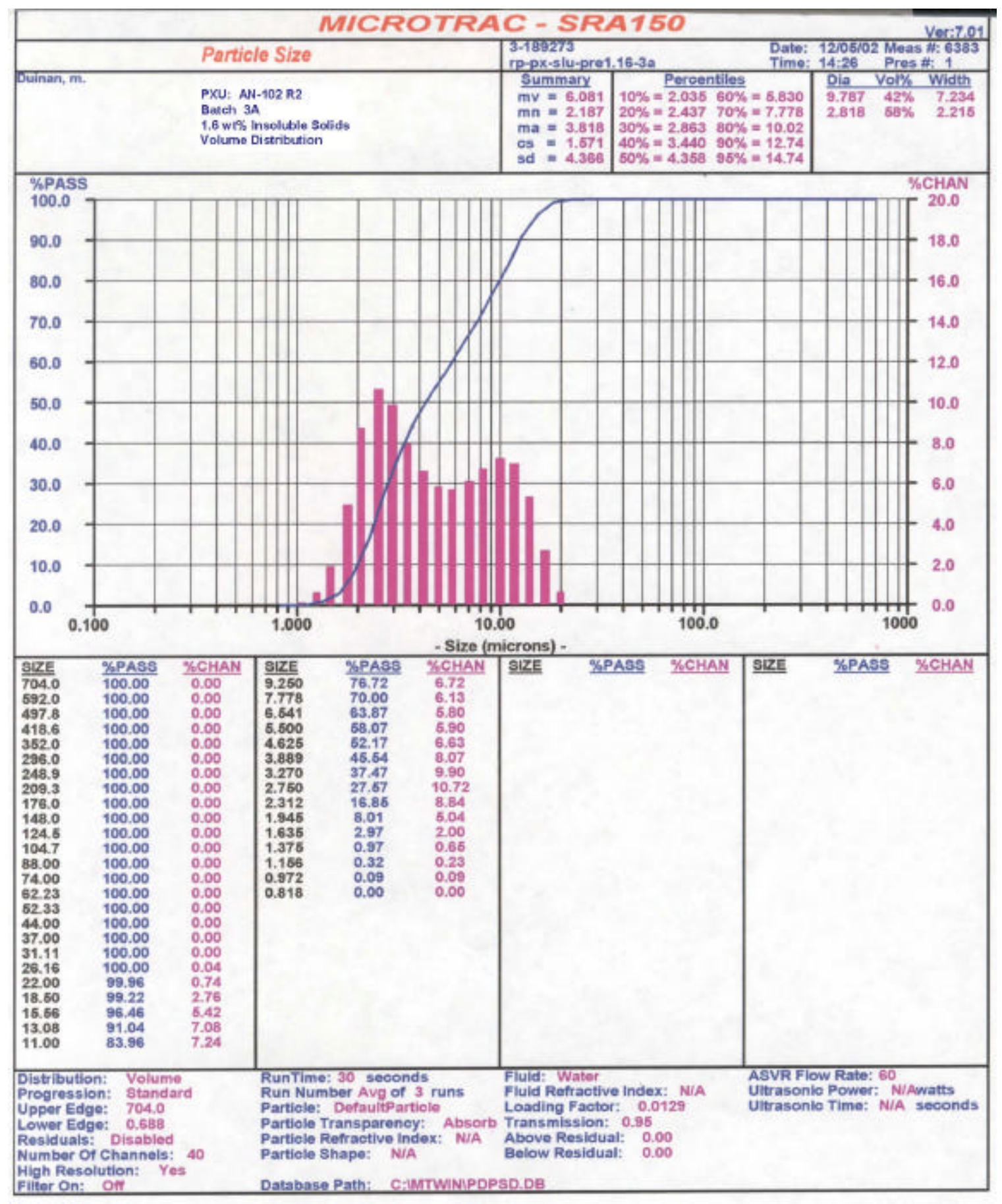

Figure G25. At start of Batch 3A dewatering: $1.6 \mathrm{wt} \%$ Insoluble Solids (VOLUME Distribution) 
WSRC-TR-2003-00204, REV. 0

SRT-RPP-2003-00087, REV. 0

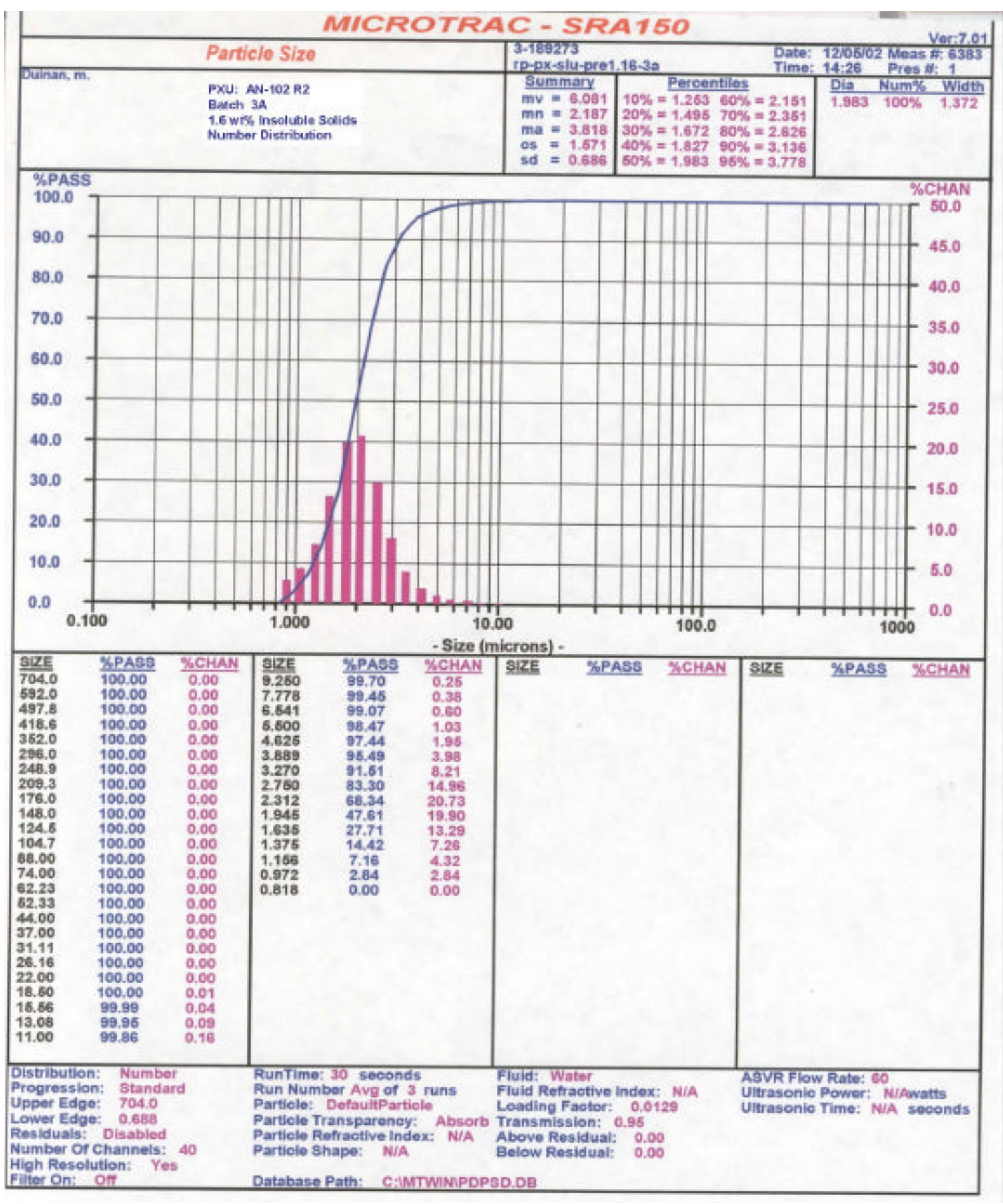

Figure G26. At start of Batch 3A: 1.6 wt\% Insoluble Solids (NUMBER Distribution) 
WSRC-TR-2003-00204, REV. 0

SRT-RPP-2003-00087, REV. 0

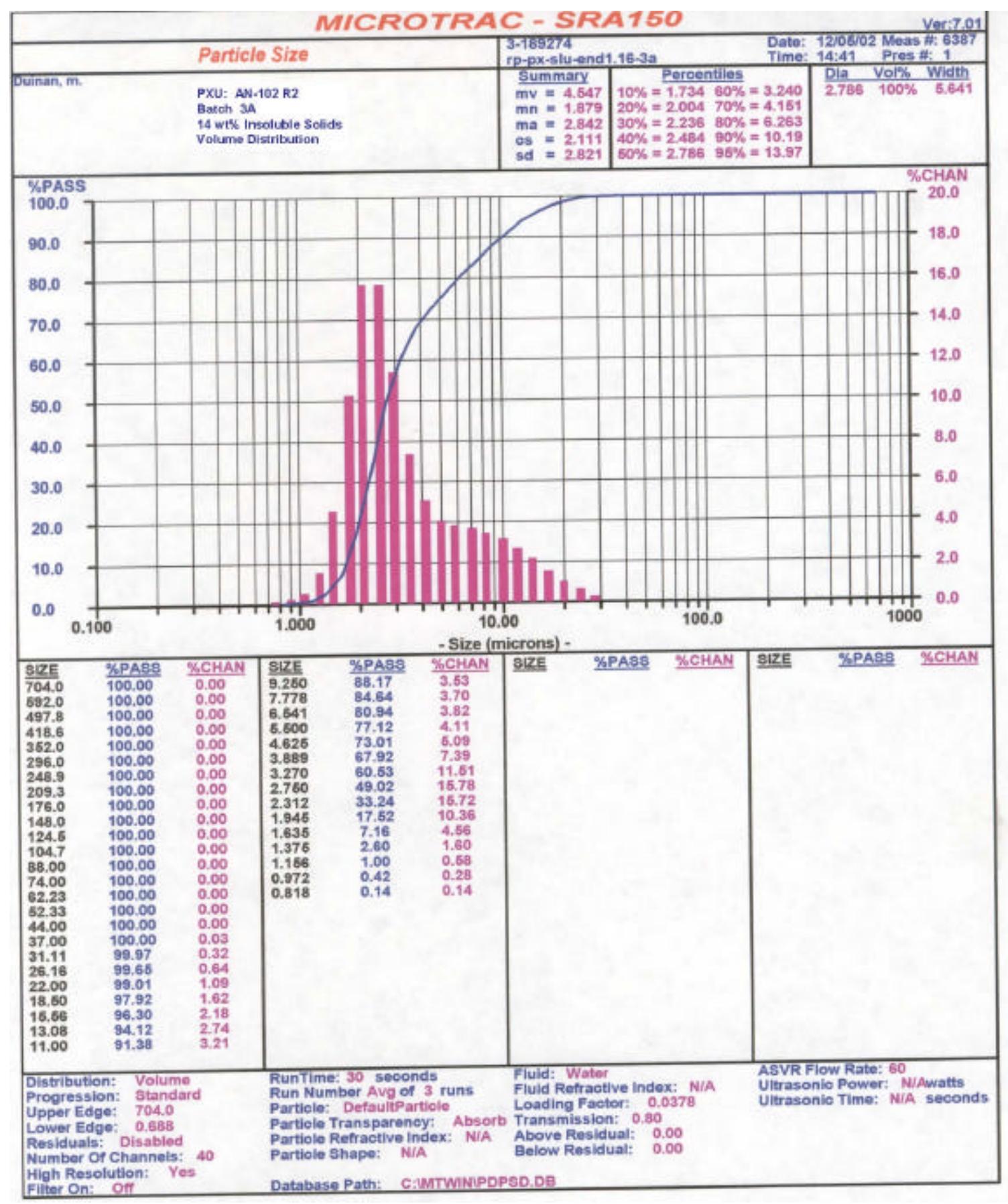

Figure G27. After 26 hours of Batch 3A dewatering: 14 wt\% Insoluble Solids (VOLUME Distribution) 
WSRC-TR-2003-00204, REV. 0

SRT-RPP-2003-00087, REV. 0

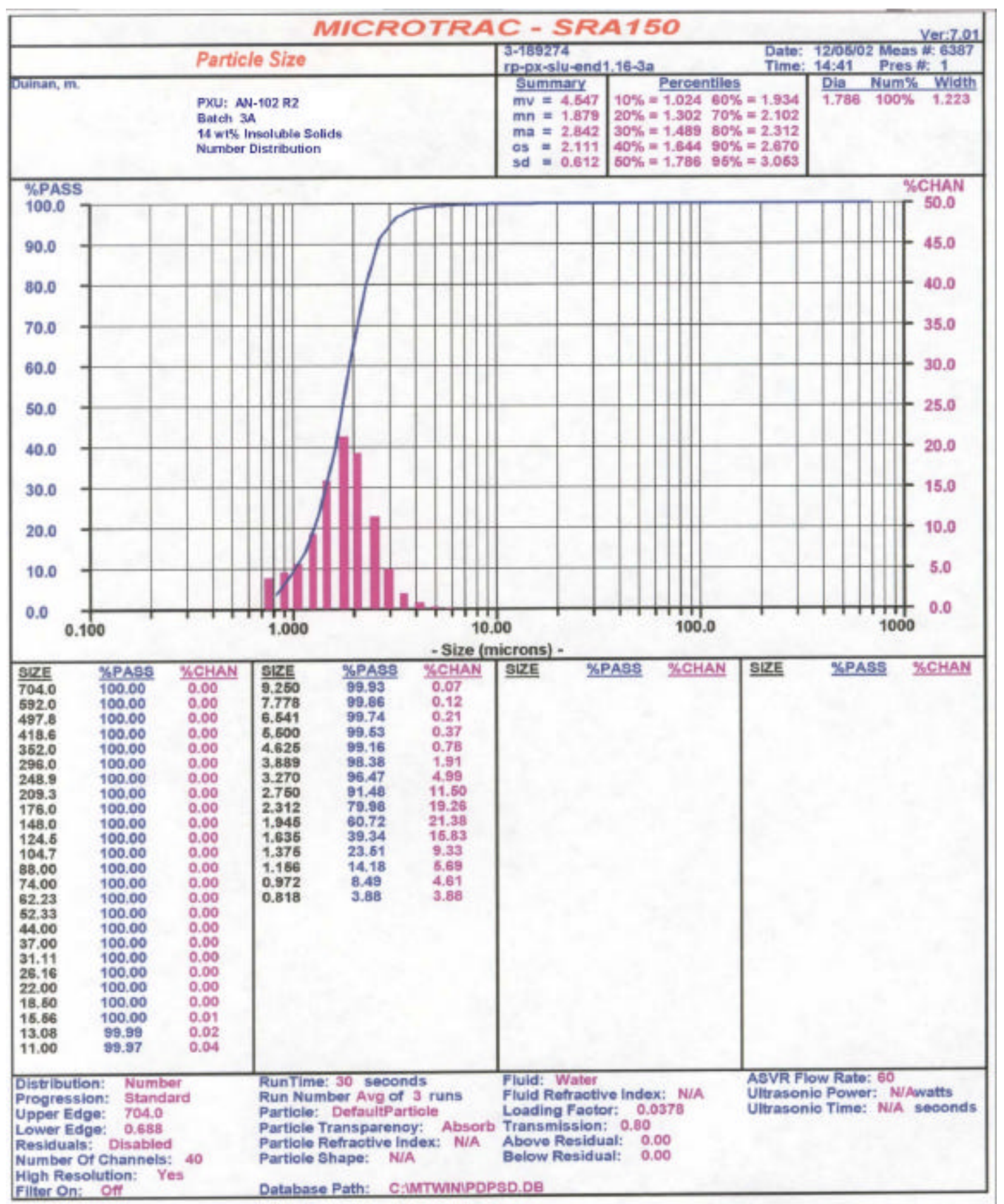

Figure G28. After 26 hours of Batch 3A: 14 wt\% Insoluble Solids (NUMBER Distribution) 
WSRC-TR-2003-00204, REV. 0

SRT-RPP-2003-00087, REV. 0

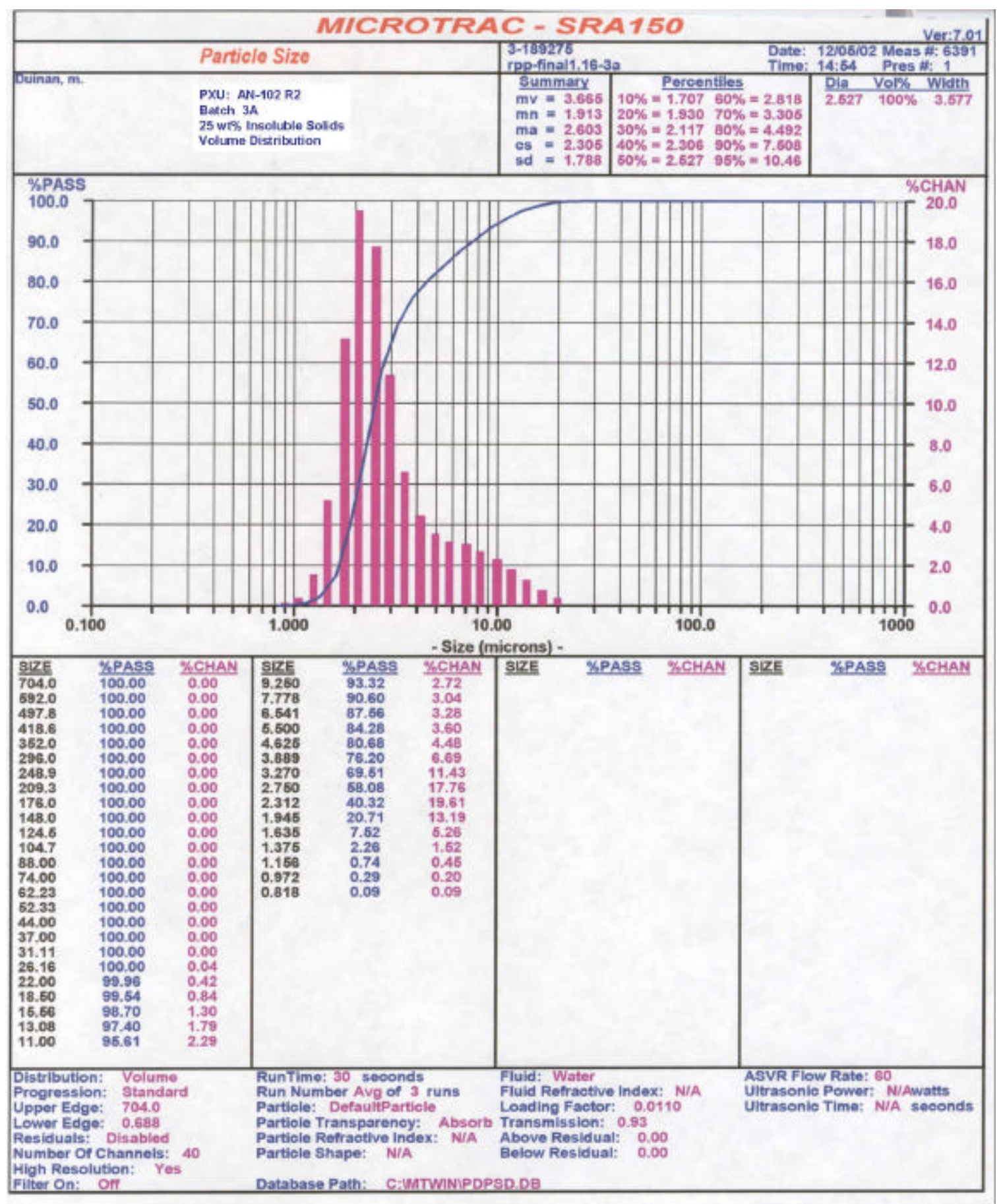

Figure G29. After 29 hours of Batch 3A dewatering: 25 wt\% Insoluble Solids (VOLUME Distribution) 
WSRC-TR-2003-00204, REV. 0

SRT-RPP-2003-00087, REV. 0

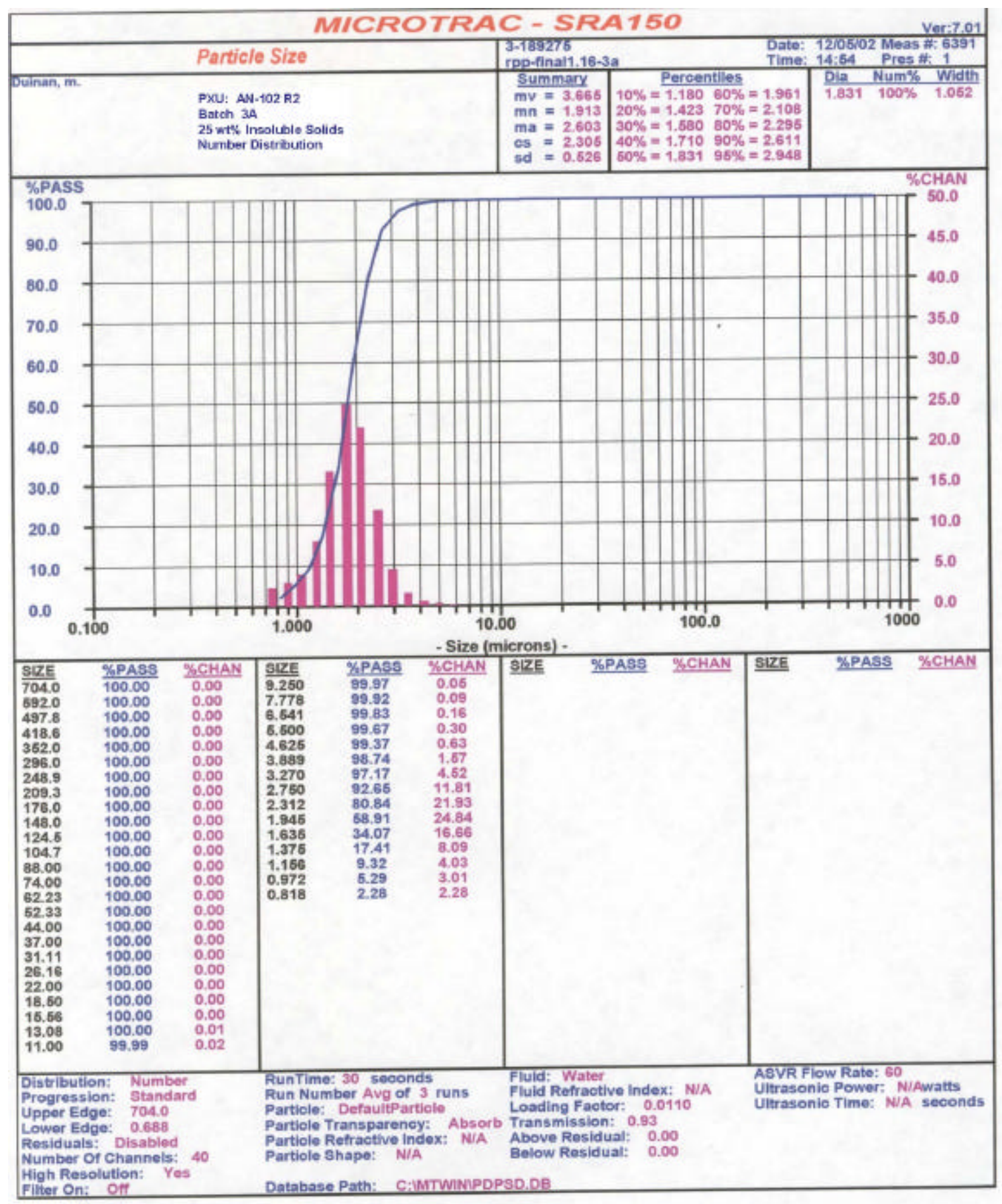

Figure G30. After 29 hours of Batch 3A: 25 wt\% Insoluble Solids (NUMBER Distribution) 
WSRC-TR-2003-00204, REV. 0

SRT-RPP-2003-00087, REV. 0

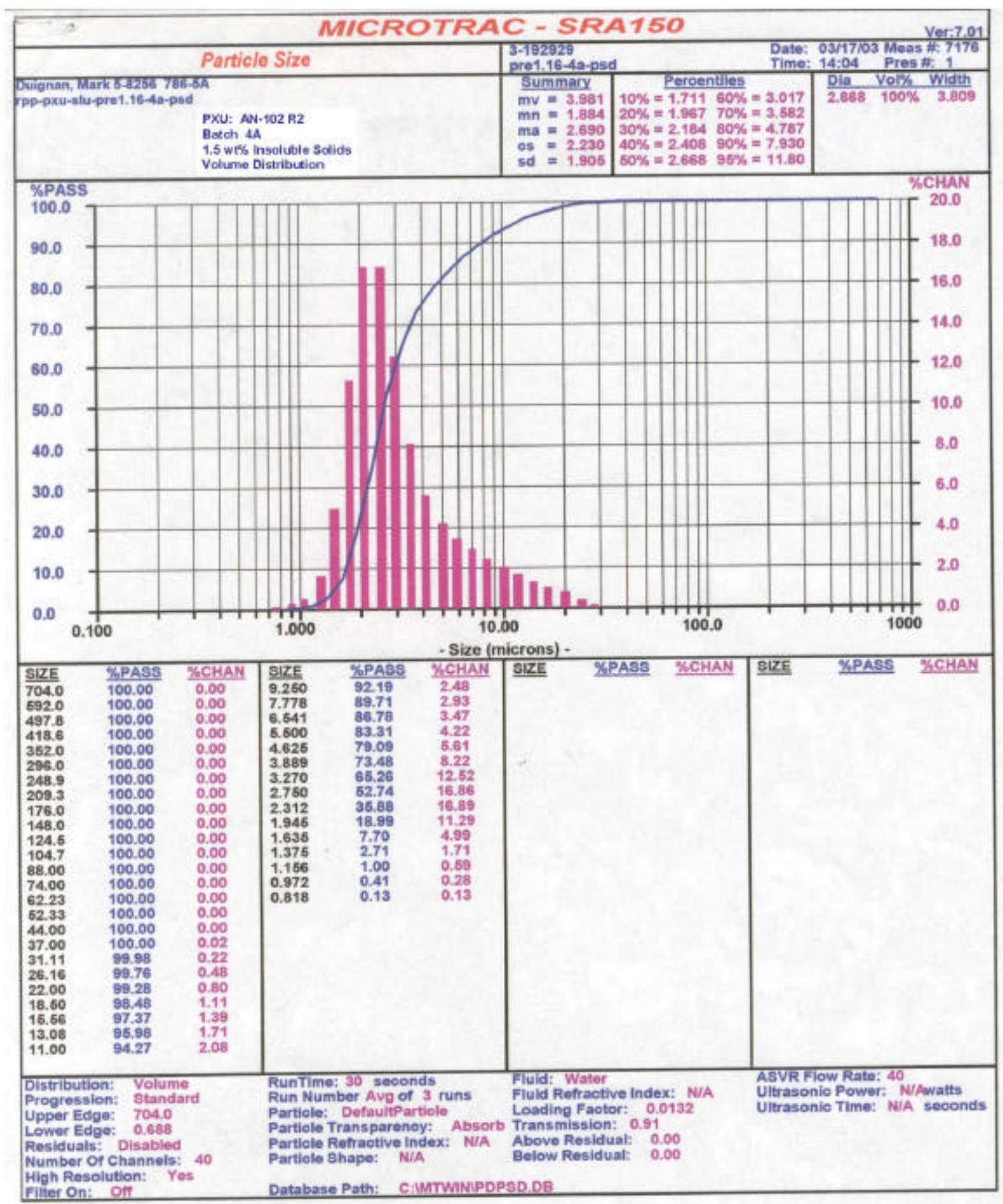

Figure G31. At start of Batch 4A dewatering: $1.5 \mathrm{wt} \%$ Insoluble Solids (VOLUME Distribution) 
WSRC-TR-2003-00204, REV. 0

SRT-RPP-2003-00087, REV. 0

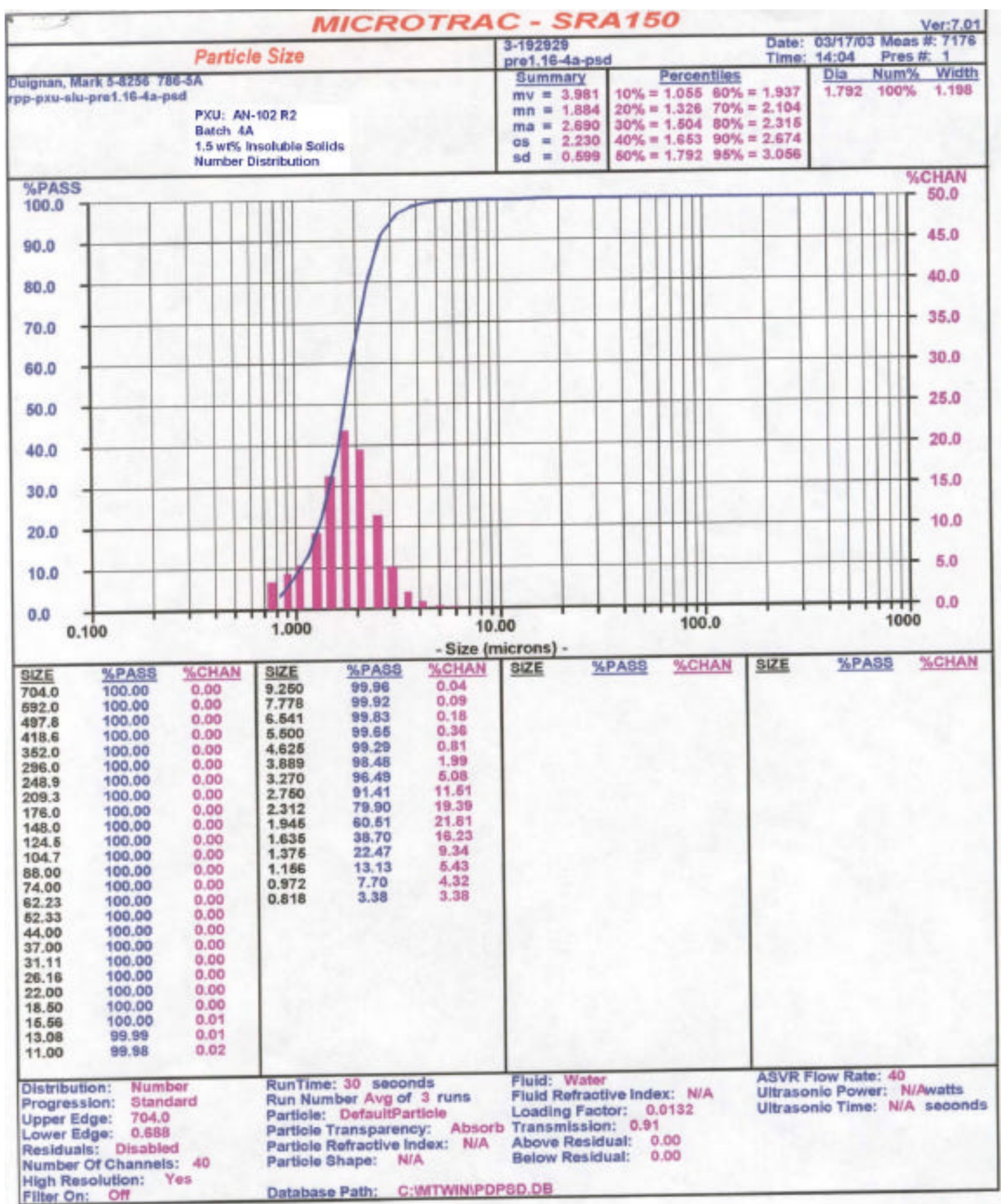

Figure G32. At start of Batch 4A: $1.5 \mathrm{wt} \%$ Insoluble Solids (NUMBER Distribution) 
WSRC-TR-2003-00204, REV. 0

SRT-RPP-2003-00087, REV. 0

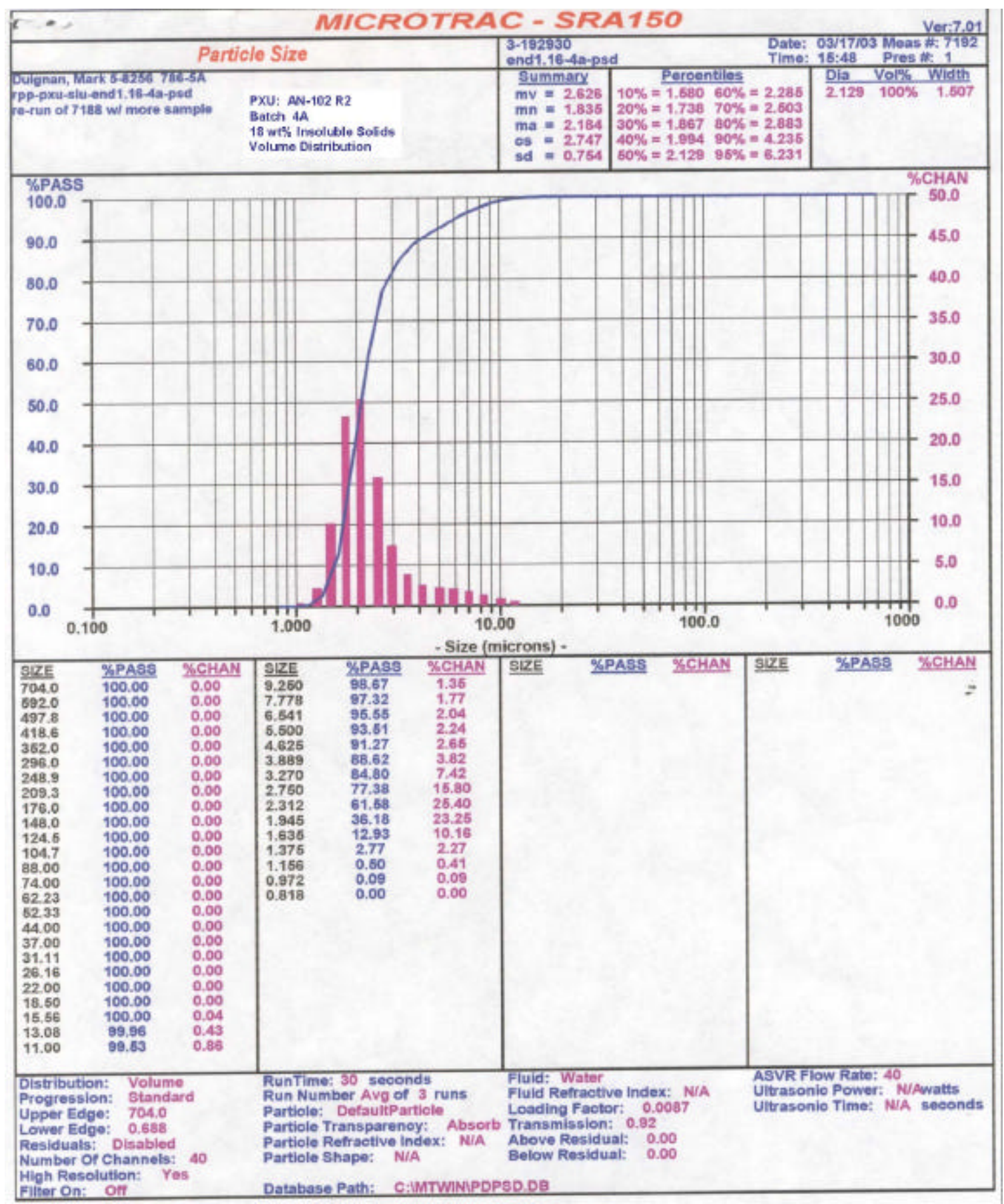

Figure G33. After 20 hours of Batch 4A dewatering: 18 wt\% Insoluble Solids (VOLUME Distribution) 
WSRC-TR-2003-00204, REV. 0

SRT-RPP-2003-00087, REV. 0

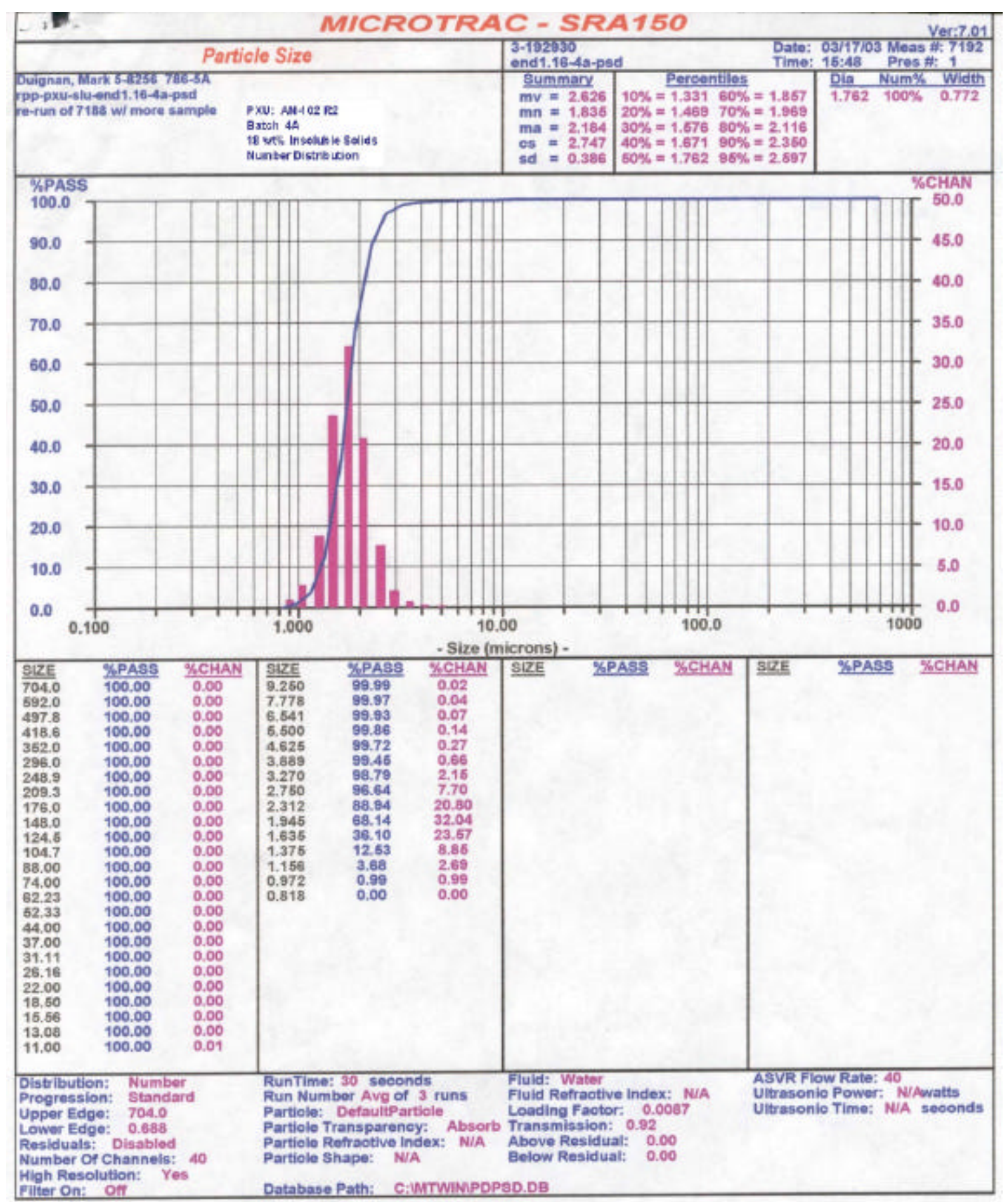

Figure G34. After 20 hours of Batch 4A: 18 wt\% Insoluble Solids (NUMBER Distribution) 


\section{APPENDIX H \\ INSTRUMENTATION AND MEASUREMENT UNCERTAINTY}

\section{Appendix Contents}

1. Experimental measurement uncertainty

2. Figure H1. Instrumentation used with their transfer functions

3. Figure H2. Pressure transducer locations

4. Figures H3 to H17. Pre-test calibration sheets ${ }^{\dagger}$ for the 15 instruments

Special Note:

As mentioned in Appendix F, there are no measurement uncertainties listed for the analytical data because those uncertainties are beyond the scope and control of this task. There is reason to believe that all analytical data can be at least $15 \%$ accurate, but no quantitative data are given to this effect.

\footnotetext{
$\dagger$ To save space the post-test calibration sheets have not been included. They show no more information than what is shown on the pre-test sheets. Moreover, the transfer functions for the pre-test calibration were used through the testing; therefore it is more appropriate to include those calibrations. However, the top table in Fig. H1 shows the measurement uncertainties for both calibrations and the resulting uncertainty used for this task.
} 


\section{EXPERIMENTAL MEASUREMENT UNCERTAINTY}

As always, any measurement made has an attributed error which must be known before a level of confidence can be attained for the results obtained. This error may come from one or all of the following: the measurement instrument, the way an instrument is set up to make a measurement in relation to the experimental phenomenon to be measured, and the person using the instrument. It is not the purpose of this section to exhaust all possible avenues of measurement uncertainty, but rather to illustrate the level of measurement uncertainty in the results presented in Appendices A, B, C, D, E. (Outside of density and capillary-viscometer measurements taken, the measurement uncertainty of the analytical data in Appendix $\mathrm{F}$ is beyond the scope of this task.) In general, the measurement uncertainties present here are for a reading or calculation at any instant. That is, the fluctuations that occur during experimentation are not addressed here. The magnitude of measurement fluctuations with time that occur during an experimental run can be seen at the bottom of each data table from the preceding appendices. Each column of data includes the average value of those data and their standard deviation.

In all the data sheets included in Appendices A, B, C, D, E there are raw data columns (for instruments, five thermocouples: T1-T5, six pressure transducers: $\mathrm{dP} 1-\mathrm{dP} 3$ and P1-P3, and four flow meters: Q1-Q3, and Qbp) and there are calculated data columns (all columns to the right Column Qbp). The uncertainty of a raw data measurement is the calibrated uncertainty of the individual instrument to a 95\% confidence level, Figs. H3 to H17.

Example to find the measurement uncertainty of a raw data point:

1. Find the data column entitled, T1, any Appendix A through E.

2. Look up Thermocouple T1 on Fig. H1 in this Appendix to see that the calibrated uncertainty is $1.2^{\circ} \mathrm{C}\left(95 \%\right.$ confidence level $\left.{ }^{*}\right)$. If a closer look on how that specific systematic error was obtained is desired, then check the appropriate calibration sheet. Thermocouple T2 is shown as Fig. H4. (The calibration sheets are in order of the fifteen instruments that are listed in Fig. H1, with the first instrument T1 shown in Fig. H3 and the last instrument Q4 (or Qbp) shown in Fig. H17.)

[*The confidence level comes from the Student's $t$ distribution function used in determining an instrument uncertainty.]

3. The magnitude of the random error can be obtained from the standard deviation shown at the bottom of each column of raw data. (The values shown are twice the magnitude of the standard deviation of the data which represents a 95\% confidence level. The standard deviation from the average value, obtained from a specific instrument for a specific test run, will be a good indication of random error for all but the filtrate flowrates, Q2, Q3, Qbp. Temperatures, pressures, and slurry flowrates were maintained constant, therefore the fluctuations around the mean should be normally distributed. The filtrate flowrates decreased with time, due to the nature of the experiment. Therefore, the random error for Q2 and Q3 should only be obtained when the filtrate flowrates reach some asymptote. (In some cases, for a thick slurry the temperature could not be held constant, but increased with time. In these cases the standard deviation will not properly represent random fluctuations. To facilitate 
the evaluation of such occurrences each column of data also has the maximum, minimum, and median values along with $2 \mathrm{x}$ the standard deviation. Note that the number of points used to determine these values is also given. Extreme points, like when backpulsing occurs were excluded.). Finally, the backpulse flowrate, Qbp, was never in steady state, as per design, therefore the standard deviation means nothing.

Measurement uncertainties for the calculated results can be obtained by the general method of the Law of Propagation of Errors (section 4.7 of Mandel, 1964). The derivation will not be given here and the following is just one example for one type of relation, albeit a common relation.

For example, a calculated entity has an uncertainty of $\delta$ a. The entity a is a function of three measured quantities: $\mathrm{b}$, $\mathrm{c}$, and $\mathrm{d}$ by the following relationship: $\mathrm{a}=\mathrm{b} \times \mathrm{c} / \mathrm{d}$ and these quantities have measurement uncertainties of $\delta b, \delta c$, and $\delta d$, respectively. The uncertainty can be shown as:

$(\delta \mathrm{a})^{2}=[(\partial \mathrm{a} / \partial \mathrm{b}) \delta \mathrm{b}]^{2}+[(\partial \mathrm{a} / \partial \mathrm{c}) \delta \mathrm{c}]^{2}+[(\partial \mathrm{a} / \partial \mathrm{d}) \delta \mathrm{d}]^{2}$,

if the error terms are independent and symmetrical. The term $\delta a$ is squared to capture both the negative and positive error terms.

Then for the relation $\mathrm{a}=\mathrm{bc} / \mathrm{d}$ the relative uncertainty can be shown to be:

$\delta \mathrm{a} / \mathrm{a}=\left[(\delta \mathrm{b} / \mathrm{b})^{2}+(\delta \mathrm{c} / \mathrm{c})^{2}+(\delta \mathrm{d} / \mathrm{d})^{2}\right]^{1 / 2}$.

Using the above the relation (1) an uncertainty for velocity, transmembrane pressure, filtrate flux, and permeability are determined. The method of determining the measurement uncertainty of any of the calculated results will the same as the following analyses. However, only one example of each calculated result is shown below. To show an example, any specific calculated quantity is sufficient. An arbitrary choice (but having a low TMP to obtain a bounding high uncertainty) of a representative group of results is: Run 1.01A at the $10.70^{\text {th }}$ minute [The data for Run 1.01A can be found in Appendix B in the Fig. B18 and the $10.70^{\text {th }}$ minute is row $23^{\text {rd }}$ row from the top or $8^{\text {th }}$ from sub-table "Data - Per Minute.]

The measurement uncertainty for the following values will be shown:

$\begin{array}{llll}\mathrm{V}, \mathrm{ft} / \mathrm{s} & \mathrm{TMP}, \mathrm{psi} & \mathrm{Fc}\left(\text { at } 25^{\circ} \mathrm{C}^{*}\right), \mathrm{gpm} / \mathrm{ft}^{2}, & \mathrm{P}, \mathrm{gpm} / \mathrm{ft}^{2} / \mathrm{psi} \\ 11.6 & 10.4 & 0.028 & 0.003\end{array}$

*actual temperature was $25.1^{\circ} \mathrm{C}$ but was adjusted to $25.0^{\circ} \mathrm{C}$ as per the customer specification, Ref. 3, therefore Fc means the corrected filtrate flux.

The results of the measurement uncertainties found below at the $95 \%$ confidence level are:
Slurry Velocity
$=\quad \mathrm{V} \quad \pm 6.3 \%$ 


$\begin{array}{llll}\text { Transmembrane Pressure } & = & \text { TMP } & \pm \mathbf{2 . 2} \% \\ \text { Temperature Corrected Filtrate Flux } & = & \text { Fc } & \mathbf{\pm 5 . 4 \%} \\ \text { Permeability } & = & \mathbf{P} & \mathbf{5 5 . 8 \%}\end{array}$

\section{H.1 Velocity [ V = Q1 / A cross-section ]}

This uncertainty is combination of the instrument, Q1, uncertainty and from the lack of knowledge of the exact inside diameters of the filter tubes. The instrument uncertainty is obtained for that instrument's calibrated uncertainty. An accurate measurement of the average inside diameter of the filtrate tubes was impossible since it may vary down the length for each filter tube and may vary from tube to tube. Even measuring the diameter at the filter tube ends is difficult because of the weldments to the tube sheets. For a Mott 1/2-inch tube the diameter was measured to be 0.488 inch and the tolerances were measured at +0.002 inch and -0.003 inch. The diameter could only be measured at the tube ends and the vendor stated tolerences for the overall tubes are +0.025 and -0.005 and the diameter of the filter tubes can presumably vary anywhere between those tolerances therefore for this task the diameter uncertainty will be taken as the average tolerance, i.e., \pm 0.015 inch.

The measurement uncertainty estimate:

Calibrated uncertainty (Figs. H1 and H14): $\delta \mathrm{Q} 1= \pm 0.5 \mathrm{gpm}$

Slurry flow rate: $\mathrm{Q} 1=47.23 \mathrm{gpm}$ [Run 1.01A, Row 23, Q1 Column: Appendix B]

Filter tube inside diameter: $\delta \mathrm{d}= \pm 0.015$ inch

Filter tube inside diameter: $\mathrm{d}=0.488$ inch

$\mathrm{V}=\mathrm{Q} / \mathrm{A}=\mathrm{Q} 1 /\left(\pi \mathrm{d}^{2} / 2\right)$

In the form of Eq. $(1): \delta \mathrm{V} / \mathrm{V}=\left[(\delta \mathrm{Q} 1 / \mathrm{Q} 1)^{2}+4(\delta \mathrm{d} / \mathrm{d})^{2}\right]^{1 / 2}$

[Note the multiplier 4. This results from the derivation $\partial \mathrm{V} / \partial \mathrm{d}$ because of the exponent]

Therefore, $\left[(0.5 / 47.23)^{2}+4(0.015 / 0.488)^{2}\right]^{1 / 2} \times 100 \%= \pm \mathbf{6 . 2 4} \%$

The uncertainty of the example $\mathbf{V}$ is: $\mathbf{1 1 . 5} \pm \mathbf{0 . 7} \mathbf{f t} / \mathbf{s}$

[since $11.5 \times 0.0624 \sim 0.72$ ]

\section{H.2 Transme mbrane Pressure $[\mathrm{TMP}=(\mathrm{dP2}+\mathrm{dP3}) / 2$ ]}

This uncertainty will come from two instruments, $\mathrm{dP} 2$ and $\mathrm{dP} 3$. Also, there is another uncertainty due to location of the pressure taps. As seen in Figs. H1 and H2, dP2 is located at the bottom to the filter housing (upstream to the filter) and $\mathrm{dP} 3$ is located at the top of the filter housing (downstream to the filter). Due to fluid being in the pressure lines (water) a liquid-filled system will give approximately* the correct pressure drops, 
however, the slurry pressures lost upstream to the filter and gained downstream of the filter are not symmetrical and therefore do not cancel out. With this said, this addition to the uncertainty is small compared to the assumption that true TMP is represented by the average of two pressures at the ends of a filter. Because the filter is oriented perpendicular to gravity and the flow causes a pressure and concentration gradient along the entire tube wall it is not clear what TMP would be representative of the entire filter unit. On the other hand, the way measurements were taken probably will be similar to the field use of this filter and therefore a good measurement for design purposes. The uncertainty is actually the uncertainty of two measurement devices, and nothing more. Finally, the Law of Propogation of Errors by Eq. (1) does not lend itself to additive contributions to uncertainties. Fortunately, the two quantities are similar in magnitude and calibrated uncertainty. Equation (1) will be used as long as it gives an uncertainty larger than the largest calibrated uncertainty for the two pressure transducers.

[*The correct pressure drop, for any $\mathrm{dP}$, is obtained when the test liquid is the same as the liquid in the pressure lines. Since the slurry was more dense than the water the readings are affected accordingly. For instance, the pressure lines to obtained dP3 are lines 3 and 4, Fig. H2, and their heights were 139.63 inches and 131.88 inches, respectively. With NO flow in a completely water-filled system, then $\mathrm{dP} 3=0$, (that is, the two water columns cancel each other) but with a different density fluid in the test rig there is a differential pressure $\mathrm{dP} 3=\mathrm{P}_{\text {line3 }}-\mathrm{P}_{\text {line } 4}=(139.63-131.88) \times($ density difference $)$. In the worst case the slurry density was approximately $1.4 \mathrm{~g} / \mathrm{cc}$, therefore the offset could be a maximum of 7.75 inches $\times 0.4=3.1$ inches $\mathrm{H}_{2} \mathrm{O}$ or 0.11 psid. However, the actual magnitude of the offest was probably smaller because the differential height, 7.8 inches, was a combination of slurry and filtrate, which had a lower density. Combining this complication of obtaining the true contribution to the overall uncertainty, along with the measurement fluctuations and other factors, then this quantity to the uncertainty was neglected.]

The measurement uncertainty estimate:

Calibrated uncertainty (Figs. $\mathrm{H} 1$ and $\mathrm{H} 12$ ): $\delta \mathrm{dP} 2= \pm 0.11 \mathrm{psi}$

Pressure drop: dP2 = 12.669 psid [Run 1.01A, Row 23, dP2 Column: Appendix B]

Calibrated uncertainty (Figs. $\mathrm{H} 1$ and $\mathrm{H} 13$ ): $\delta \mathrm{dP} 3= \pm 0.16 \mathrm{psi}$

Pressure drop: $\mathrm{dP} 3=8.23$ psid [Run 1.01A, Row 23, dP3 Column: Appendix B]

$\mathrm{TMP}=(\mathrm{dP} 2+\mathrm{dP} 3) / 2$

In the form of Eq. $(1): \delta \mathrm{TMP} / \mathrm{TMP}=\left[(\delta \mathrm{dP} 2 / \mathrm{dP} 2)^{2}+(\delta \mathrm{dP} 3 / \mathrm{dP} 3)^{2}\right]^{1 / 2}$

Therefore, $\left[(0.11 / 12.67)^{2}+(0.16 / 8.23)^{2}\right]^{1 / 2} \times 100 \%= \pm \mathbf{2 . 1 3} \%$

The uncertainty of the example TMP is: $\mathbf{1 0 . 5} \pm \mathbf{0 . 3}$ psid

[since TMP $=(12.669+8.23) / 2=10.45 \mathrm{psid}$ and $10.45 \times 0.0213 \sim 0.223$ ] 


\section{H.3 Filtrate Flux $\left[\mathrm{F}=\mathrm{Q} 2 / \mathrm{A}_{\text {inner-surface }}\right]$}

This uncertainty is combination of the instrument, Q2, uncertainty and from the lack of knowledge of the exact inside diameters and length of the filter tubes. The instrument uncertainty is obtained for that instrument's calibrated uncertainty. The uncertainty of the inside diameter of the filter tubes has already been addressed in section H.1 and it was estimated at 0.015 inch from the manufacturer's stated tolerance. The uncertainty of the length of the filter tubes was estimate from in-house measurements. The requested tube length from the manufacturer was 90 inches. Because of the center weldments (the 90-inch length was made of four 22.5-inch tube sections) the active length measured closer to $895 / 8$ inches; take the each of the three central weldments to by 1/8 inch long. Due to bowing and weldment variations an overall length uncertainty will be taken as the length of one weldment, i.e., 1/8-inch.

The measurement uncertainty estimate:

Calibrated uncertainty (Figs. H1 and H15): $\delta \mathrm{Q} 2= \pm 0.005$ gpm

Filtrate flow rate: $\mathrm{Q} 2=0.186 \mathrm{gpm}$ [Run 1.01A, Row 23, Q2 Column: Appendix B]

Tube inside diameter uncertainty: $\delta \mathrm{d}= \pm 0.015$ inch (from manufacturer)

Tube inside diameter: $\mathrm{d}=0.488$ inch

Tube length uncertainty: $\delta \mathrm{L}= \pm 0.125$ inch

Tube length: $\mathrm{L}=89.625$ inches

$\mathrm{F}=\mathrm{Q} / \mathrm{A}=\mathrm{Q} 2 / \pi \mathrm{d} \mathrm{L}$

In the form of Eq. $(1): \delta \mathrm{F} / \mathrm{F}=\left[(\delta \mathrm{Q} 2 / \mathrm{dQ} 2)^{2}+(\delta \mathrm{d} / \mathrm{d})^{2}+(\delta \mathrm{L} / \mathrm{L})^{2}\right]^{1 / 2}$

Therefore, $\left[(0.005 / 0.186)^{2}+(0.015 / 0.488)^{2}+(0.125 / 89.625)^{2}\right]^{1 / 2} \times 100 \%= \pm \mathbf{4 . 0 9} \%$

The uncertainty of the example $\mathbf{F}$ is: $\mathbf{0 . 1 9} \pm \mathbf{0 . 0 0 8} \mathbf{g p m} / \mathbf{f t}^{\mathbf{2}}$

[since $0.186 \times 0.0409 \sim 0.0076$ ]

\section{H.3.1 Effect of Temperature on the Measurement Uncertainty on $F$}

As per the customer specification the filtrate flux was to adjusted such that it would give a result at $25^{\circ} \mathrm{C}$. The equation as was stated is:

$$
\begin{aligned}
& \mathrm{F}=\mathrm{Q} 2(\text { Temperature }) / \text { Area }=\mathrm{Q} 2 \times \text { Correction Factor /Area } \\
& \mathrm{CF}=e^{[2500((1 / 273+\text { Slurry Temperature }))-(1 / 298))]}
\end{aligned}
$$

Only the correction factor's effect on measurement uncertainty is dealt with here. The equation is accepted as error free, i.e., method, constants, etc. Only the uncertainty of the temperature measurement, which leads to the correction, will be addressed. 
Generally, the adjustment to filtrate flux, $\mathbf{F}$, is small because, whenever possible, the slurry's operational temperature was maintained at $25^{\circ} \mathrm{C}$, which would result in a zero adjustment. However, the example chosen to show measurement uncertainty had a temperature difference of $0.2^{\circ} \mathrm{C}$. That is, for Run $1.01 \mathrm{~A}$ at the $20^{\text {th }}$ minute the slurry temperature was $25.076^{\circ} \mathrm{C}$. Unfortunately, the fact that a temperature correction is applied means the temperature uncertainty will effect the calculated result at any temperature. That is, even if the slurry temperature were exactly $25^{\circ} \mathrm{C}$, which would make the correction $=1.0$, the uncertainty of that temperature and thereby the correction, leads to an inherent uncertainty of value that is being corrected, i.e, F. For this task the slurry temperature was measured with thermocouple $\mathrm{T} 1$, which had a calibrated measurement uncertainty of $\pm 1.2^{\circ} \mathrm{C}$ [see Fig. H1]. If at $25^{\circ} \mathrm{C}$ the temperature has an uncertainty of $\pm 1.2^{\circ} \mathrm{C}$, then the correction can be either 0.9669 to 1.0345 , or approximately $\pm 3.38 \%$ [i.e., $((1-0.9669)+(1.0345-1)) / 2 / 1.00=0.0338]$. To show the measurement uncertainty mathematically from the correction equation is beyond the scope of this task, however it can be shown graphically. For a range of slurry temperatures from $15^{\circ} \mathrm{C}$ to $40^{\circ} \mathrm{C}$, then the correction can be shown to change from 1.34 to 0.65 , respectively. However, with a measurement uncertainty of $\pm 1.2^{\circ} \mathrm{C}$ this factor can be in error from approximately $3.62 \%$ to $3.06 \%$, respectively, see the Figure below:

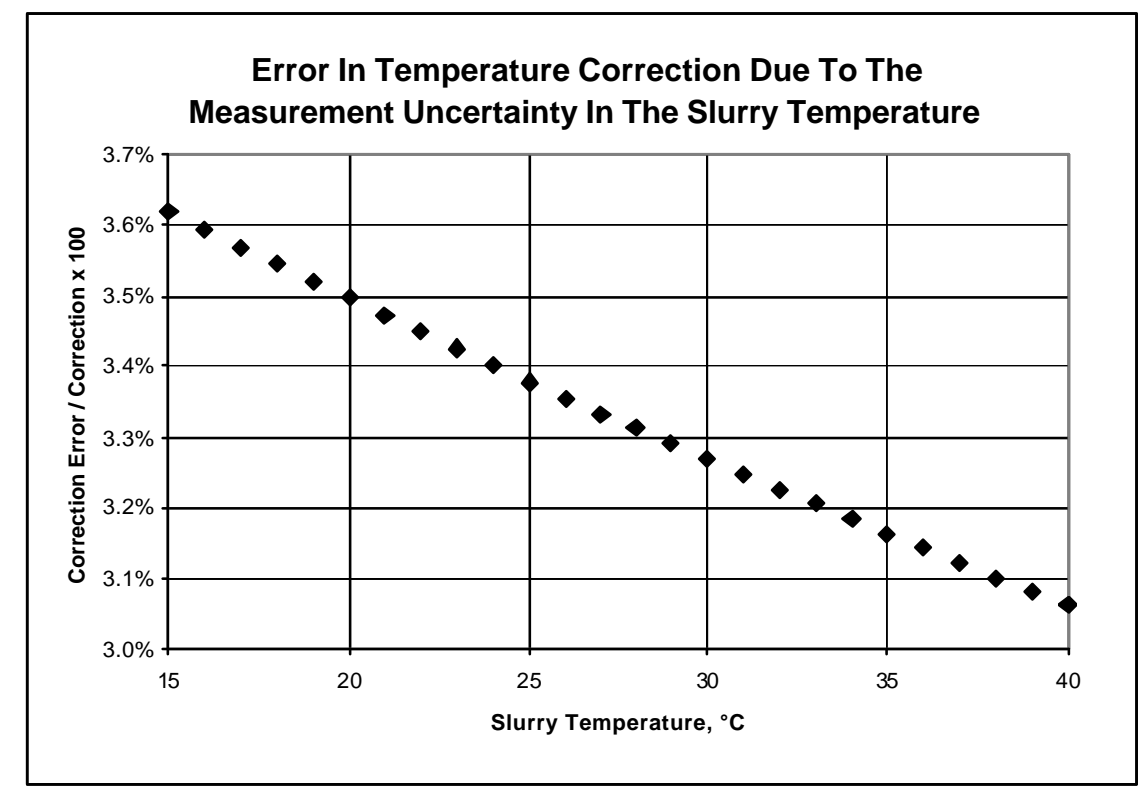

Therefore, for the sake of this example the relative uncertainty of the correction factor due to the temperature will be assumed to be $3.4 \%$, since most of the data were obtained at $25^{\circ} \mathrm{C}$. Using this constant value is not a bad assumption because between $20^{\circ} \mathrm{C}$ and $30^{\circ} \mathrm{C}$ this uncertainty only fluctuates by approximately $0.1 \%$. 
This increased uncertainty to the filtrate flux is then a combination of two uncertainties already calculated above for $\mathbf{F}$ (section H.3) and the correction factor. The analyses still follows Eq. (1) therefore:

The measurement uncertainty estimate:

Filtrate flux relative uncertainty: $\delta \mathrm{F} / \mathrm{F}= \pm 4.09 \%$ [section H.3]

Correction factor relative uncertainty: $\delta \mathrm{CF} / \mathrm{CF}= \pm 3.38 \%$ [section H.3.1]

$\mathrm{Fc}=$ Fcorrected $=\mathrm{F} \times \mathrm{CF}$

In the form of Eq. (1): $\delta \mathrm{Fc} / \mathrm{Fc}=\left[(\delta \mathrm{F} / \mathrm{F})^{2}+(\delta \mathrm{CF} / \mathrm{CF})^{2}\right]^{1 / 2}$

Therefore, $\left[(4.09 \%)^{2}+(3.38 \%)^{2}\right]^{1 / 2}= \pm \mathbf{5 . 3 1} \%$

The uncertainty of the example $\mathbf{F c}$ is: $\mathbf{0 . 1 8 6} \pm \mathbf{0 . 0 1 0} \mathbf{g p m} / \mathbf{f t}^{2}$

[since $0.186 \times 0.0531 \sim 0 . .0099$ ]

\section{H.4 Permeability [ P = Fc / TMP ]}

This uncertainty is combination of two uncertainties already calculated above, in sections H.2 and H.3. The analyses still follows Eq. (1) therefore:

The measurement uncertainty estimate:

Filtrate flux relative uncertainty: $\delta \mathrm{Fc} / \mathrm{Fc}= \pm 5.31 \%$ [section H.3.1]

TMP relative uncertainty: $\delta \mathrm{TMP} / \mathrm{TMP}= \pm 2.13 \%$ [section H.2]

$\mathrm{P}=\mathrm{Fc} / \mathrm{TMP}$

In the form of Eq. $(1): \delta \mathrm{P} / \mathrm{P}=\left[(\delta \mathrm{Fc} / \mathrm{Fc})^{2}+(\delta \mathrm{TMP} / \mathrm{TMP})^{2}\right]^{1 / 2}$

Therefore, $\left[(5.31 \%)^{2}+(2.13 \%)^{2}\right]^{1 / 2}= \pm \mathbf{5 . 7 2} \%$

The uncertainty of the example $\mathbf{P}$ is: $\mathbf{0 . 0 0 3} \pm \mathbf{0 . 0 0 0 2} \mathbf{~ g p m} / \mathbf{f t}^{2} / \mathbf{p s i}$

[since $0.003 \times 0.0572 \sim 0.00017$ ] 


\section{H.5 Second-Order Effects to Measurement Uncertainty}

There were other effects on the measurement uncertainty, which are not included because they are thought to be of second order. For example, since the test rig was very tall, approximately $30 \mathrm{feet}$, it was subjected an ambient temperature gradient. Ambient temperatures at the bottom of the rig were usually less than at the top. For the example used in this section, [Run 1.01A, Row 23, T4 and T5 Columns: Appendix B], the temperatures were $24.9^{\circ} \mathrm{C}$ and $22.8^{\circ} \mathrm{C}$, respectively. This gradient varied hourly and daily for several reasons. Most importantly, the $2.1^{\circ} \mathrm{C}$ temperature variation shown in this example is on the same order of magnitude as the calibrated uncertainties for the thermocouples. Further, the slurry in the loop generally flowed fast, so the residence time in any one section of the rig was small. For Run 1.01A the flow rate was, $\mathrm{Q} 1=47.3 \mathrm{gpm}$. The loop volume was approximately 6 gallons so a fluid particle traversed the loop every $6 / 47.3 \times 60 \sim 7.6$ seconds. [Note that the slurry flow rate for Run 1.01A was typical of most test runs.] In this example, the slurry temperature was measured to be $25.1^{\circ} \mathrm{C}$, from one thermocouple located in the suction line of the pumps. While it is certain that the ambient temperatures had an effect on the slurry temperature it was small, and at steady state the effect is incorporated in the slurry temperature. The same is true for the ambient temperature gradient but to a lesser extent and therefore not considered. 
WSRC-TR-2003-00204, REV. 0

SRT-RPP-2003-00087, REV. 0

\begin{tabular}{|c|c|c|c|c|c|c|c|c|c|}
\hline \multicolumn{10}{|c|}{ INSTRUMENTS USED ON THE RPP-WTP PILOT-SCALE CROSS-FLOW FILTRATION TEST } \\
\hline \multirow{2}{*}{\multicolumn{10}{|c|}{\begin{tabular}{|l|l|} 
(Printed: April 17, 2003) \\
\end{tabular}}} \\
\hline & & & & & & & & & \\
\hline DAS Chan. & Test Rig No. & Instrument & M\&TE \# & Make & Model/Serial & Calibrated Range & Uncertainty & Uncertainty & Uncertainty \\
\hline & & & & & & & (pre-test) & (post-test) & Average \\
\hline 0 & $\bar{T} 2$ & Thermocouple & \begin{tabular}{|c|} 
TR-02927 \\
\end{tabular} & OMEGA & TJ36-CXSS-18U-6-SB-OST-M/None & 0 to $100 \mathrm{C}$ & $1.3^{\circ} \mathrm{C}$ & $0.7^{\circ} \mathrm{C}$ & 1.0 \\
\hline 1 & T3 & Thermocouple & \begin{tabular}{|c|} 
TR-02930 \\
\end{tabular} & OMEGA & TJ36-CXSS-18U-6-SB-OST-M/None & 0 to $100 \mathrm{C}$ & $1.4^{\circ} \mathrm{C}$ & $0.6^{\circ} \mathrm{C}$ & 1.0 \\
\hline 2 & T1 & Thermocouple & TR-02929 & OMEGA & TJ36-CXSS-18U-6-SB-OST-M/None & 0 to $100 \mathrm{C}$ & $1.2^{\circ} \mathrm{C}$ & $0.6^{\circ} \mathrm{C}$ & 0.9 \\
\hline 3 & T4 & Thermocouple & \begin{tabular}{|l} 
TR-02925 \\
\end{tabular} & OMEGA & TJ36-CXSS-18U-6-SB-OST-M/None & 0 to $100 \mathrm{C}$ & $1.4^{\circ} \mathrm{C}$ & $0.6^{\circ} \mathrm{C}$ & 1.0 \\
\hline 4 & T5 & Thermocouple & \begin{tabular}{|l|} 
TR-02926 \\
\end{tabular} & OMEGA & TJ36-CXSS-18U-6-SB-OST-M/None & 0 to $100 \mathrm{C}$ & $1.5^{\circ} \mathrm{C}$ & $0.5^{\circ} \mathrm{C}$ & 1.0 \\
\hline 6 & $\mathrm{dP} 2$ & Pres. Transducer & \begin{tabular}{|l} 
TR-03553 \\
\end{tabular} & Rosemount & 1151DP6E22/1368962 & 0 to $101 \mathrm{PSID}$ & $0.11 \mathrm{PSIG}$ & $0.10 \mathrm{PSIG}$ & 0.11 \\
\hline 7 & $\mathrm{P} 1$ & \begin{tabular}{|l} 
Pres. Transducer \\
\end{tabular} & \begin{tabular}{|l|} 
TR-02917 \\
\end{tabular} & Rosemount & $1151 \mathrm{GPG} / 409543$ & 0 to 101 PSIG & $0.10 \mathrm{PSIG}$ & $0.10 \mathrm{PSIG}$ & 0.10 \\
\hline 8 & $\mathrm{dP1}$ & Pres. Transducer & \begin{tabular}{|c|} 
TR-03495 \\
\end{tabular} & Rosemount & 1151DP5E22/1368952 & 0 to 26 PSID & 0.044 PSID & 0.046 PSID & 0.045 \\
\hline 9 & $\mathrm{dP} 3$ & Pres. Transducer & \begin{tabular}{|l} 
TR-03109 \\
\end{tabular} & Rosemount & 1151DP6E22/1368963 & -11 to 91 PSID & $0.16 \mathrm{PSIG}$ & $0.12 \mathrm{PSIG}$ & 0.14 \\
\hline 10 & $\mathrm{P} 2$ & \begin{tabular}{|l} 
Pres. Transducer \\
\end{tabular} & \begin{tabular}{|l} 
TR-03115 \\
\end{tabular} & Rosemount & 1151DP7E22M3B1/402987 & 0 to 151 PSIG & 0.36 PSIG & $0.41 \mathrm{PSIG}$ & 0.39 \\
\hline 11 & P3(backpulse) & \begin{tabular}{|l} 
Pres. Transducer \\
\end{tabular} & \begin{tabular}{|l} 
TR-00532 \\
\end{tabular} & Rosemount & 1151DP7E22M3B1P1/ 1038513 & 0 to 151 PSIG & $0.15 \mathrm{PSIG}$ & $0.20 \mathrm{PSIG}$ & 0.18 \\
\hline 12 & Q2 & \begin{tabular}{|l} 
Mag. Flowmeter \\
\end{tabular} & \begin{tabular}{|l} 
TR-20353 \\
\end{tabular} & Fischer-Porter & 10D1475EN01PF29KW12CAC1/93W011793 & 0 to $1.2 \mathrm{GPM}$ & $0.005 \mathrm{GPM}$ & $0.005 \mathrm{GPM}$ & 0.005 \\
\hline 13 & Q1 & Mag. Flowmeter & \begin{tabular}{|c|} 
TR-20350 \\
\end{tabular} & Fischer-Porter & 10D1475DK11PL29KD11CAC / 90W007609 & 0 TO 100 GPM & $0.5 \mathrm{GPM}$ & 0.5 GPM & 0.5 \\
\hline 14 & Q3 & Mag. Flowmeter & \begin{tabular}{|l} 
TR-03562 \\
\end{tabular} & Fischer-Porter & 10D1475CDBC11BBL1/8710A0242A3 & 0 to $5 \mathrm{GPM}$ & $0.02 \mathrm{GPM}$ & 0.02 GPM & 0.02 \\
\hline \multirow[t]{14}{*}{15} & Q4 = Qbackpulse & Mag. Flowmeter & \begin{tabular}{|l} 
TR-03276 \\
\end{tabular} & Fischer-Porter & 10D1475EN06PL29KD11CAC1 / 92W446636 & 0 to 5 GPM & 0.02 GPM & $0.02 \mathrm{GPM}$ & 0.02 \\
\hline & & & & & & & & & \\
\hline & & & & DAS Calibration & & Transfer Function & for DAS (Con & ersion from & A to Volts) \\
\hline & DAS Channel & Instrument & & Slope $(\mathrm{V} / \mathrm{mA})$ & Intercept (V) & Output & Slope (unit/V) & & Intercept (unit) \\
\hline & 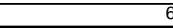 & $\mathrm{dP} 2$ & & 0.4975 & 0.0000 & Pressure $(p s i d)$ & 12.6673 & $x V+$ & -25.1790 \\
\hline & 7 & $\mathrm{P} 1$ & & 0.4975 & 0.0000 & Pressure (psig) & 12.6814 & $x V+$ & -25.1980 \\
\hline & 8 & $\mathrm{dP1}$ & & 0.4988 & 0.0030 & Pressure (psid) & 3.2562 & $x \mathrm{~V}+$ & -6.5230 \\
\hline & 9 & $\mathrm{dP3}$ & & 0.4983 & 0.0050 & Pressure $(\mathrm{psid})$ & 12.8077 & $x V+$ & -36.5748 \\
\hline & 10 & $\mathrm{P} 2$ & & 0.4983 & 0.0050 & Pressure (psig) & 18.9324 & $x V+$ & -37.9977 \\
\hline & 11 & $\mathrm{P} 3$ & & 0.4975 & 0.0000 & $\begin{array}{l}\text { Pressure (psig) } \\
\end{array}$ & 18.9688 & $x V+$ & -37.7140 \\
\hline & 12 & Q2 & & 0.4975 & 0.0100 & Flow (gpm) & 0.1487 & $x V+$ & -0.2955 \\
\hline & 13 & Q1 & & 0.4990 & 0.0020 & Flow (gpm) & 12.5210 & $x V+$ & -25.0350 \\
\hline & 14 & $\mathrm{Q} 3$ & & 0.4993 & -0.0030 & Flow (gpm) & 0.6249 & $x V+$ & -1.2381 \\
\hline & 15 & Q4 = Qbackpulse & & 0.4988 & 0.0070 & Flow $(\mathrm{gpm})$ & 0.6195 & $x V+$ & -1.2373 \\
\hline
\end{tabular}

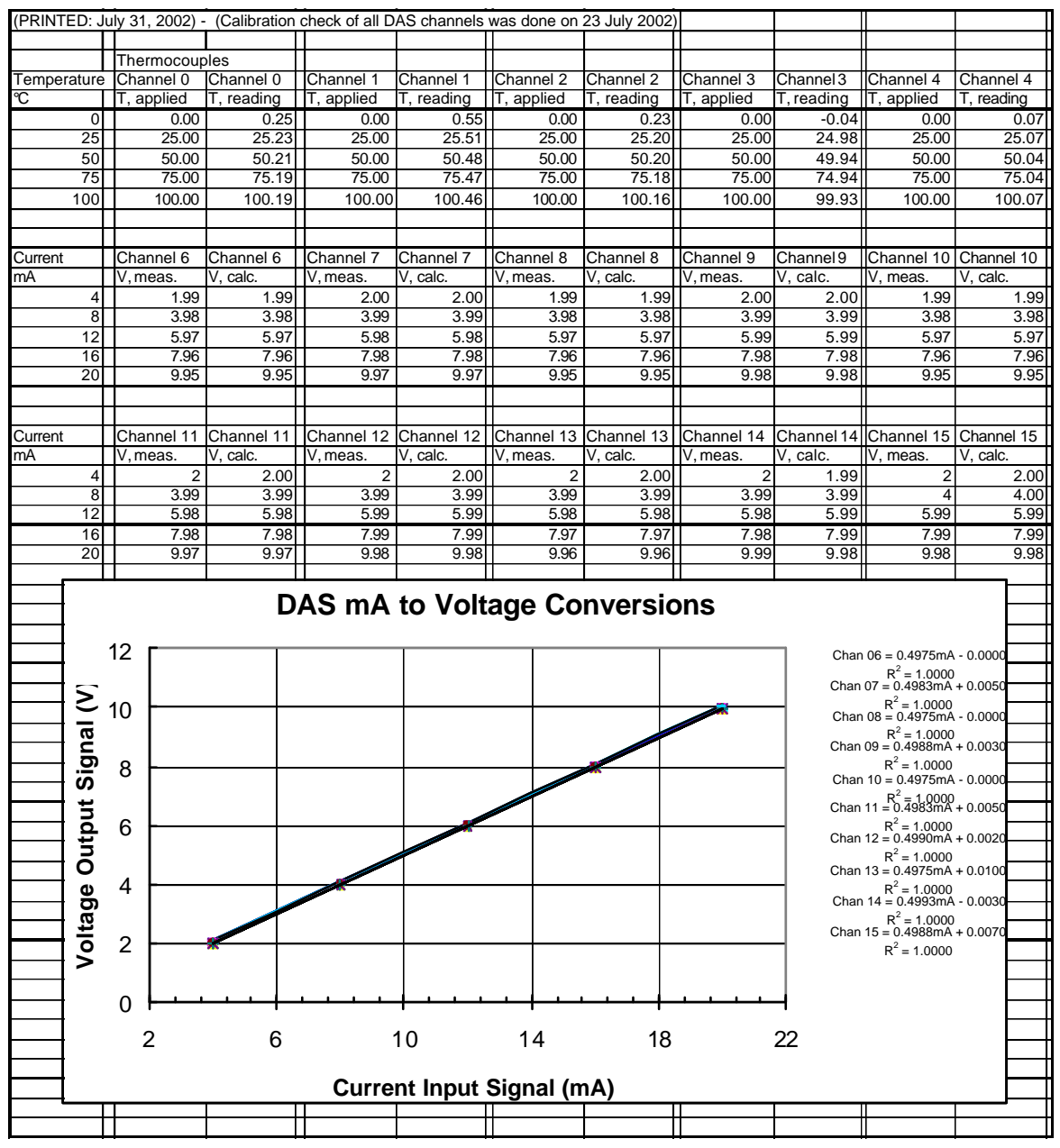

Figure H1. Instrumentation Used with their Transfer Functions 


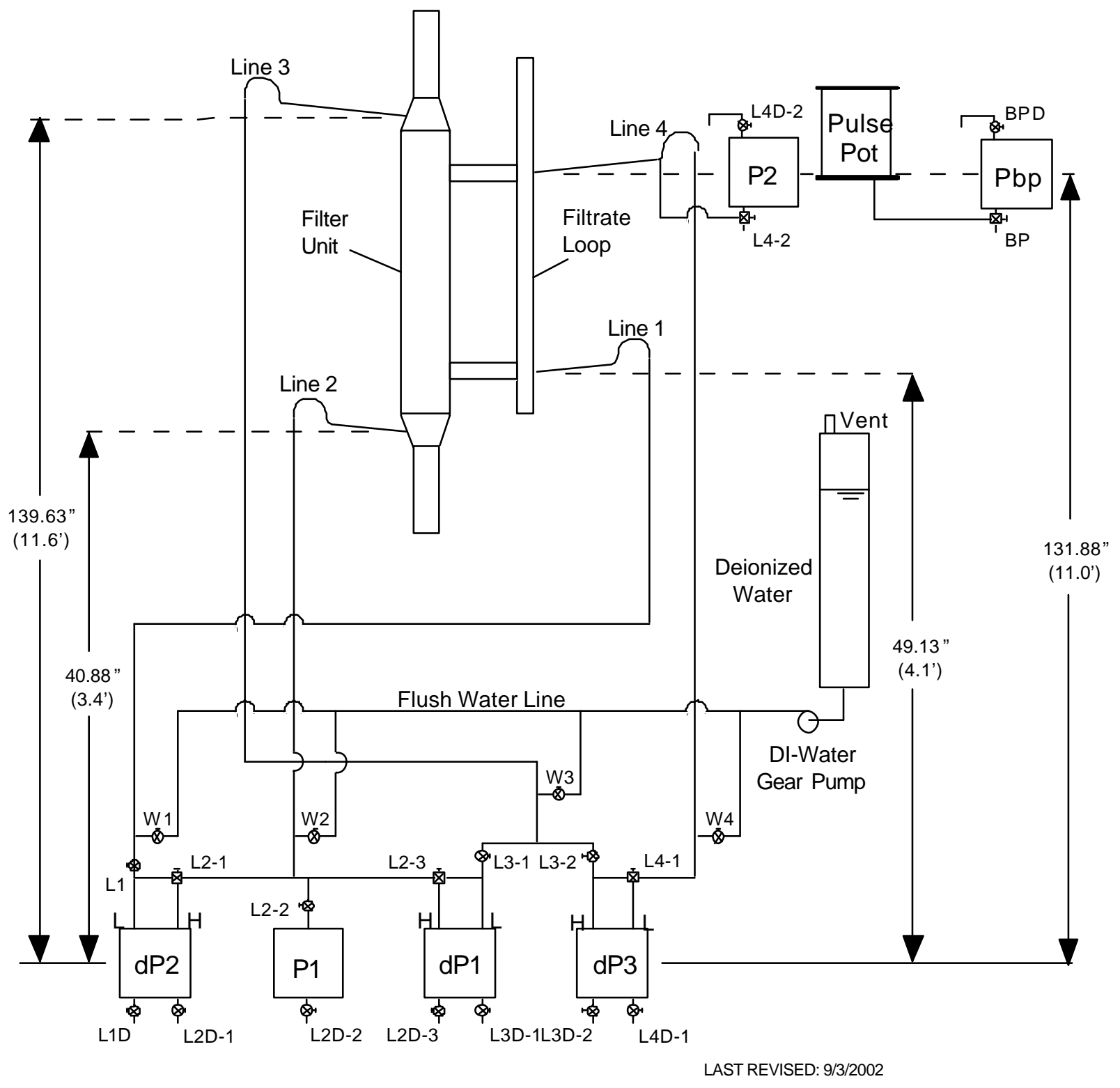

Figure H2. Pressure Transducer Locations 
WSRC-TR-2003-00204, REV. 0

SRT-RPP-2003-00087, REV. 0

UNCERTAINTY ANALYSIS REF. WSRC-TR-91-106
TR-2929

cal date: 16 April 2001

(Note: This uncertainty is the variance of the actual T from the NIST curve (Eq.1) based on the output $\mathrm{mV}$ )

\begin{tabular}{|c|c|c|c|c|}
\hline & \multicolumn{2}{|c|}{ Calibration Data } & \multicolumn{2}{|l|}{ Calculated } \\
\hline Temperature & Standard & Voltage & Temperature & \\
\hline Medium & $\begin{array}{l}\text { Temp } \\
\text { (C) }\end{array}$ & $\begin{array}{l}\text { Output } \\
\text { (mV) }\end{array}$ & $\begin{array}{l}\text { (eq. 1) } \\
\text { (C) }\end{array}$ & $\begin{array}{l}\text { Error } \\
\text { (C) }\end{array}$ \\
\hline Ice Point & 0.00 & -0.010 & -0.17 & -0.2 \\
\hline Ice Point & 0.00 & -0.025 & -0.42 & -0.4 \\
\hline Ice Point & 0.00 & -0.025 & -0.42 & -0.4 \\
\hline Ice Point & 0.00 & -0.025 & -0.42 & -0.4 \\
\hline Room Temp & 22.79 & 1.313 & 22.00 & -0.8 \\
\hline Room Temp & 23.23 & 1.439 & 24.08 & 0.8 \\
\hline Room Temp & 24.58 & 1.435 & 24.01 & -0.6 \\
\hline Room Temp & 24.07 & 1.421 & 23.78 & -0.3 \\
\hline Boiling Water & 99.53 & 6.330 & 100.16 & 0.6 \\
\hline Boiling Water & 99.53 & 6.330 & 100.16 & 0.6 \\
\hline Boiling Water & 99.44 & 6.317 & 99.97 & 0.5 \\
\hline Boiling Water & 99.45 & 6.317 & 99.97 & \\
\hline
\end{tabular}

$\mathrm{T}(\mathrm{C})=0.00483+17.040918^{*} \mathrm{mV}-0.224284^{*} \mathrm{mV}^{\wedge} 2+0.005038^{*} \mathrm{mV}^{\wedge} 3 \quad$ (eq. 1$)$

(Limited Curve Fit, 0-100 C. From N.I.S.T. Reference Tables)

Uncertainty of the Standards: Temperature Curve Fit: $+/-\quad 0.010$ C

Thermometer: $+/-\quad 0.40 \quad \mathrm{C}$

Ice Bath: +/- $0.10 \quad \mathrm{C}$

Multimeter: +/- $(0.0045 \% \mathrm{RDG}+0.0005 \mathrm{mV})$

$=+/-\quad 0.012 \quad \mathrm{C} @ \quad 6.33 \quad \mathrm{mV}$

Accepted Tolerance: +/- $\quad 1.7 \quad$ C

Statistical Info.

\begin{tabular}{|c|c|c|c|c|c|c|c|}
\hline a & $b$ & $\mathrm{n}$ & $\mathrm{T}$ & $\begin{array}{l}\text { Xbar } \\
\text { (C) }\end{array}$ & $\begin{array}{c}\text { Sxx } \\
\left(C^{\wedge} 2\right)\end{array}$ & $\begin{array}{c}\text { SEE } \\
\left(C^{\wedge} 2\right)\end{array}$ & $\begin{array}{l}\text { MSE } \\
\left(C^{\wedge} 2\right)\end{array}$ \\
\hline-0.39 & 1.01 & 12.00 & 2.228 & 41.05 & 21610.7 & 1.660 & 0.1660 \\
\hline
\end{tabular}

Calculated Uncertainties:

standard curve-fit

uncertainty uncertainty

(C) (C)

$0.41 \quad 1.00$

1.00

fixed total

uncertainty uncertainty

(C) (C)

$0.57 \quad 1.2$

PASS CALIBRATION?

YES

Figure H3. Pre-test Calibration Data of Thermocouple T1 
WSRC-TR-2003-00204, REV. 0

SRT-RPP-2003-00087, REV. 0

UNCERTAINTY ANALYSIS

REF. WSRC-TR-91-106
TR-2927

cal date: 16 April 2001

(Note: This uncertainty is the variance of the actual T from the NIST curve (Eq.1) based on the output $\mathrm{mV}$ )

\begin{tabular}{|c|c|c|c|c|}
\hline & \multicolumn{2}{|c|}{ Calibration Data } & \multicolumn{2}{|l|}{ Calculated } \\
\hline Temperature & Standard & Voltage & Temperature & \\
\hline Medium & $\begin{array}{l}\text { Temp } \\
\text { (C) }\end{array}$ & $\begin{array}{l}\text { Output } \\
\text { (mV) }\end{array}$ & $\begin{array}{l}\text { (eq. 1) } \\
\text { (C) }\end{array}$ & $\begin{array}{l}\text { Error } \\
\text { (C) }\end{array}$ \\
\hline Ice Point & 0.00 & -0.009 & -0.15 & -0.1 \\
\hline Ice Point & 0.00 & -0.014 & -0.23 & -0.2 \\
\hline Ice Point & 0.00 & -0.015 & -0.25 & -0.3 \\
\hline Ice Point & 0.00 & -0.012 & -0.20 & -0.2 \\
\hline Room Temp & 22.79 & 1.315 & 22.04 & -0.8 \\
\hline Room Temp & 23.23 & 1.438 & 24.06 & 0.8 \\
\hline Room Temp & 24.58 & 1.450 & 24.26 & -0 . \\
\hline Room Temp & 24.07 & 1.449 & 24.24 & 0.2 \\
\hline Boiling Water & 99.53 & 6.344 & 100.37 & 0.8 \\
\hline Boiling Water & 99.53 & 6.345 & 100.39 & 0.9 \\
\hline Boiling Water & 99.46 & 6.328 & 100.14 & 0.7 \\
\hline Boiling Water & 99.48 & 6.329 & 100.15 & 0.7 \\
\hline
\end{tabular}

$\mathrm{T}(\mathrm{C})=0.00483+17.040918^{\star} \mathrm{mV}-0.224284^{\star} \mathrm{mV}^{\wedge} 2+0.005038^{\star} \mathrm{mV} \wedge 3$ (eq. 1)

(Limited Curve Fit, 0-100 C. From N.I.S.T. Reference Tables)

Uncertainty of the Standards: Temperature Curve Fit: + /- $0.010 \quad$ C

Thermometer: + - $0.40 \quad \mathrm{C}$

Ice Bath: + - $0.10 \quad \mathrm{C}$

Multimeter: $+/-(0.0045 \% \mathrm{RDG}+0.0005 \mathrm{mV})$

$$
=+/-0.012 \quad \mathrm{C} @ \quad 6.35 \quad \mathrm{mV}
$$

Accepted Tolerance: + - $\quad 1.7 \quad$ C

Statistical Info.

\begin{tabular}{|c|c|c|c|c|c|c|c|}
\hline$a$ & $b$ & $\mathrm{n}$ & $\mathrm{T}$ & $\begin{array}{l}\text { Xbar } \\
(\mathrm{C})\end{array}$ & $\begin{array}{c}S x x \\
\left(C^{\wedge} 2\right)\end{array}$ & $\begin{array}{c}\text { SEE } \\
\left(C^{\wedge} 2\right)\end{array}$ & $\begin{array}{l}\text { MSE } \\
\left(C^{\wedge} 2\right)\end{array}$ \\
\hline-0.23 & 1.01 & 12.00 & 2.228 & 41.06 & 21616.6 & 1.429 & 0.1429 \\
\hline
\end{tabular}

Calculated Uncertainties:

standard curve-fit

uncertainty uncertainty

(C) $\quad(\mathrm{C})$

$0.41 \quad 0.93$

fixed total

uncertainty uncertainty

(C) (C)

$0.75 \quad 1.3$

PASS CALIBRATION?

YES

Figure H4. Pre-test Calibration Data of Thermocouple T2 
WSRC-TR-2003-00204, REV. 0

SRT-RPP-2003-00087, REV. 0

UNCERTAINTY ANALYSIS REF. WSRC-TR-91-106
TR-2930

cal date: 17 April 2001

(Note: This uncertainty is the variance of the actual T from the NIST curve (Eq.1) based on the output $\mathrm{mV}$ )

\begin{tabular}{lcccc} 
Temperature & \multicolumn{2}{c}{$\begin{array}{c}\text { Calibration Data } \\
\text { Standard } \\
\text { Voltage }\end{array}$} & $\begin{array}{c}\text { Calculated } \\
\text { Temperature }\end{array}$ \\
& $\begin{array}{c}\text { Temp } \\
\text { Medium }\end{array}$ & $\begin{array}{c}\text { Output } \\
(\mathrm{mV})\end{array}$ & $\begin{array}{c}\text { (eq. }) \\
(\mathrm{C})\end{array}$ & $\begin{array}{c}\text { Error } \\
(\mathrm{C})\end{array}$ \\
Ice Point & 0.00 & -0.016 & -0.27 & -0.3 \\
Ice Point & 0.00 & -0.015 & -0.25 & -0.3 \\
Ice Point & 0.00 & -0.015 & -0.25 & -0.3 \\
Ice Point & 0.00 & -0.015 & -0.25 & -0.3 \\
Room Temp & 21.33 & 1.222 & 20.50 & -0.8 \\
Room Temp & 20.42 & 1.210 & 20.30 & -0.1 \\
Room Temp & 21.66 & 1.268 & 21.26 & -0.4 \\
Room Temp & 21.71 & 1.367 & 22.89 & 1.2 \\
Boiling Water & 99.54 & 6.336 & 100.25 & 0.7 \\
Boiling Water & 99.51 & 6.334 & 100.22 & 0.7 \\
Boiling Water & 99.80 & 6.343 & 100.36 & 0.6 \\
Boiling Water & 99.80 & 6.343 & 100.36 & 0.6
\end{tabular}

$\mathrm{T}(\mathrm{C})=0.00483+17.040918^{*} \mathrm{mV}-0.224284^{*} \mathrm{mV}^{\wedge} 2+0.005038^{*} \mathrm{mV}^{\wedge} 3 \quad$ (eq. 1$)$ (Limited Curve Fit, 0-100 C. From N.I.S.T. Reference Tables)

Uncertainty of the Standards: Temperature Curve Fit: $+/-\quad 0.010 \quad \mathrm{C}$

Thermometer: + /- $0.40 \quad \mathrm{C}$ Ice Bath: $+/-\quad 0.10 \quad \mathrm{C}$

Multimeter: $+/-(0.0045 \% \mathrm{RDG}+0.0005 \mathrm{mV})$

$=+/-0.012 \quad \mathrm{C} @ \quad 6.34 \quad \mathrm{mV}$

Accepted Tolerance: +/- $\quad 1.7 \quad$ C

Statistical Info.

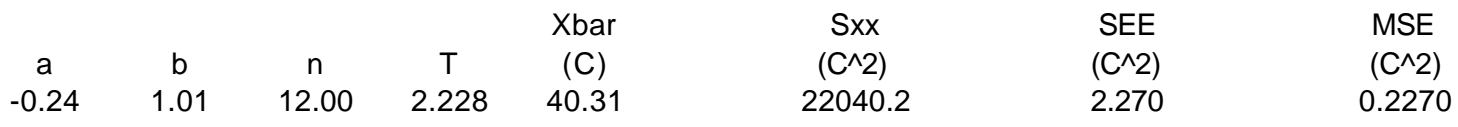

Calculated Uncertainties:

$\begin{array}{llll}\text { standard } & \text { curve-fit } & \text { fixed } & \text { total } \\ \text { uncertainty } & \text { uncertainty } & \text { uncertainty } & \text { uncertainty } \\ \text { (C) } & \text { (C) } & \text { (C) } & \text { (C) }\end{array}$

$\begin{array}{llll}0.41 & 1.17 & 0.63 & 1.4\end{array}$

PASS CALIBRATION? YES

Figure H5. Pre-test Calibration Data of Thermocouple T3 
WSRC-TR-2003-00204, REV. 0

SRT-RPP-2003-00087, REV. 0

UNCERTAINTY ANALYSIS

REF. WSRC-TR-91-106
TR-2925

cal date: 12 April 2001

(Note: This uncertainty is the variance of the actual T from the NIST curve (Eq.1) based on the output $\mathrm{mV}$ )

\begin{tabular}{|c|c|c|c|c|}
\hline & \multicolumn{2}{|c|}{ Calibration Data } & \multicolumn{2}{|l|}{ Calculated } \\
\hline Temperature & Standard & Voltage & Temperature & \\
\hline Medium & $\begin{array}{l}\text { Temp } \\
\text { (C) }\end{array}$ & $\begin{array}{l}\text { Output } \\
\text { (mV) }\end{array}$ & $\begin{array}{l}\text { (eq. 1) } \\
\text { (C) }\end{array}$ & $\begin{array}{c}\text { Error } \\
\text { (C) }\end{array}$ \\
\hline Ice Point & 0.00 & -0.013 & -0.22 & -0.2 \\
\hline Ice Point & 0.00 & -0.009 & -0.15 & -0.1 \\
\hline Ice Point & 0.00 & -0.009 & -0.15 & -0.1 \\
\hline Ice Point & 0.00 & -0.009 & -0.15 & -0.1 \\
\hline Room Temp & 23.30 & 1.384 & 23.17 & -0.1 \\
\hline Room Temp & 24.99 & 1.400 & 23.44 & -1.6 \\
\hline Room Temp & 23.48 & 1.356 & 22.71 & -0.8 \\
\hline Room Temp & 25.57 & 1.500 & 25.08 & -0.5 \\
\hline Boiling Water & 99.94 & 6.342 & 100.34 & 0.4 \\
\hline Boiling Water & 99.94 & 6.345 & 100.39 & 0.4 \\
\hline Boiling Water & 99.70 & 6.338 & 100.28 & 0.6 \\
\hline Boiling Water & 99.71 & 6.339 & 100.30 & 0.6 \\
\hline
\end{tabular}

$\mathrm{T}(\mathrm{C})=0.00483+17.040918^{\star} \mathrm{mV}-0.224284^{\star} \mathrm{mV}^{\wedge} 2+0.005038^{\star} \mathrm{mV}^{\wedge} 3$ (eq. 1) (Limited Curve Fit, 0-100 C. From N.I.S.T. Reference Tables)

Uncertainty of the Standards: Temperature Curve Fit: $+/-\quad 0.010 \quad$ C

Thermometer: + - $0.40 \quad \mathrm{C}$

Ice Bath: +/- $0.10 \quad \mathrm{C}$

Multimeter: $+/-(0.0045 \% \mathrm{RDG}+0.0005 \mathrm{mV})$

$$
=+/-0.012 \quad \mathrm{C} @ \quad 6.35 \quad \mathrm{mV}
$$

Accepted Tolerance: + - $\quad 1.7 \quad$ C

Statistical Info.

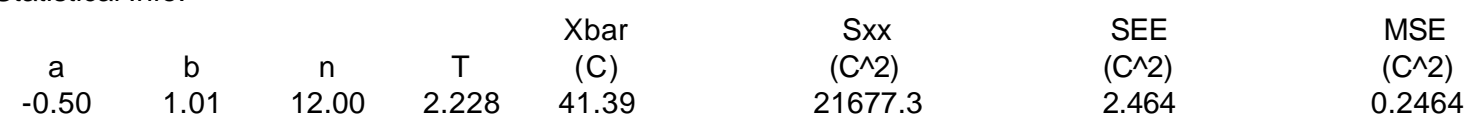

Calculated Uncertainties:

standard curve-fit

uncertainty uncertainty

(C) (C)

fixed total

$0.41 \quad 1.22$

(C) (C)

$0.50 \quad 1.4$

PASS CALIBRATION?

YES

Figure H6. Pre-test Calibration Data of Thermocouple T4 
WSRC-TR-2003-00204, REV. 0

SRT-RPP-2003-00087, REV. 0

UNCERTAINTY ANALYSIS

REF. WSRC-TR-91-106
TR-2926

cal date: 18 July 2001

(Note: This uncertainty is the variance of the actual T from the NIST curve (Eq.1) based on the output $\mathrm{mV}$ )

\begin{tabular}{|c|c|c|c|c|}
\hline & \multicolumn{2}{|c|}{ Calibration Data } & \multicolumn{2}{|l|}{ Calculated } \\
\hline Temperature & Standard & Voltage & Temperature & \\
\hline Medium & $\begin{array}{c}\text { Temp } \\
\text { (C) }\end{array}$ & $\begin{array}{l}\text { Output } \\
\text { (mV) }\end{array}$ & $\begin{array}{l}\text { (eq. 1) } \\
\text { (C) }\end{array}$ & $\begin{array}{l}\text { Error } \\
\text { (C) }\end{array}$ \\
\hline Ice Point & 0.00 & -0.017 & -0.28 & -0.3 \\
\hline Ice Point & 0.00 & -0.015 & -0.25 & -0.3 \\
\hline Ice Point & 0.00 & -0.015 & -0.25 & -0.3 \\
\hline Ice Point & 0.00 & -0.016 & -0.27 & -0.3 \\
\hline Room Temp & 24.37 & 1.402 & 23.47 & -0.9 \\
\hline Room Temp & 24.33 & 1.401 & 23.45 & -0.9 \\
\hline Room Temp & 24.43 & 1.367 & 22.89 & -1.5 \\
\hline Room Temp & 21.54 & 1.308 & 21.92 & 0.4 \\
\hline Boiling Water & 100.20 & 6.344 & 100.37 & 0.2 \\
\hline Boiling Water & 100.20 & 6.344 & 100.37 & 0.2 \\
\hline Boiling Water & 100.10 & 6.345 & 100.39 & 0.3 \\
\hline Boiling Water & 100.10 & 6.343 & 100.36 & 0.3 \\
\hline
\end{tabular}

$\mathrm{T}(\mathrm{C})=0.00483+17.040918^{\star} \mathrm{mV}-0.224284^{\star} \mathrm{mV}^{\wedge} 2+0.005038^{\star} \mathrm{mV} \wedge 3$ (eq. 1)

(Limited Curve Fit, 0-100 C. From N.I.S.T. Reference Tables)

Uncertainty of the Standards: $\quad$ Temperature Curve Fit: $+/-\quad 0.010 \quad$ C

Thermometer: + - $0.40 \quad \mathrm{C}$

Ice Bath: + - $0.10 \quad \mathrm{C}$

Multimeter: $+/-(0.0045 \% \mathrm{RDG}+0.0005 \mathrm{mV})$

$$
=+/-0.012 \quad \mathrm{C} @ \quad 6.35 \quad \mathrm{mV}
$$

Accepted Tolerance: +/- $\quad 1.7 \quad$ C

Statistical Info.

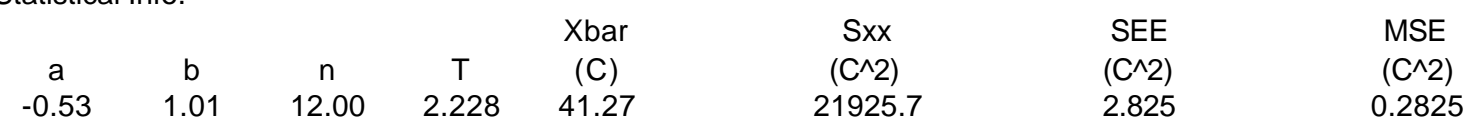

Calculated Uncertainties:

standard curve-fit

uncertainty uncertainty

(C) (C)

$0.41 \quad 1.31$

$\begin{array}{ll}\text { fixed } & \text { total } \\ \text { uncertainty } & \text { uncertainty } \\ \text { (C) } & \text { (C) } \\ 0.53 & 1.5\end{array}$

PASS CALIBRATION?

YES

Figure H7. Pre-test Calibration Data of Thermocouple T5 
WSRC-TR-2003-00204, REV. 0 SRT-RPP-2003-00087, REV. 0

UNCERTAINTY ANALYSIS

REF. WSRC-TR-91-106, REV. 0
TR-02917

Calibration Data

$\begin{array}{ccc}\begin{array}{c}\text { Nominal } \\ \text { Pressure } \\ \text { (psig) }\end{array} & \begin{array}{c}\text { Applied } \\ \text { Pressure } \\ \text { (psig) }\end{array} & \begin{array}{c}\text { Gage } \\ \text { Reading } \\ \text { (mADC) }\end{array} \\ 0.00 & 0.00 & 4.00 \\ 21.00 & 21.00 & 7.32 \\ 41.00 & 41.00 & 10.49 \\ 61.00 & 61.00 & 13.66 \\ 81.00 & 81.00 & 16.83 \\ 101.00 & 101.00 & 20.01 \\ 0.00 & 0.00 & 4.00 \\ 21.00 & 21.00 & 7.32 \\ 41.00 & 41.00 & 10.49 \\ 61.00 & 61.00 & 13.66 \\ 81.00 & 81.00 & 16.83 \\ 101.00 & 101.00 & 20.01 \\ 0.00 & 0.00 & 4.00 \\ 21.00 & 21.00 & 7.32 \\ 41.00 & 41.00 & 10.49 \\ 61.00 & 61.00 & 13.66 \\ 81.00 & 81.00 & 16.83 \\ 101.00 & 101.00 & 20.01 \\ 0.00 & 0.00 & 4.00 \\ 21.00 & 21.00 & 7.32 \\ 41.00 & 41.00 & 10.49 \\ 61.00 & 61.00 & 13.66 \\ 81.00 & 81.00 & 16.83 \\ 101.00 & 101.00 & 20.01\end{array}$

Standard Uncertainties:

Multimeter: $+/-($
Dead Weight Tester: $+/-($

0.04

Statistical Info:

\begin{tabular}{ccc}
$\mathrm{a}$ & $\mathrm{b}$ & $\mathrm{n}$ \\
3.9941 & 0.1585 & 24.00 \\
& & \\
Calculated & Uncertainties: & \\
\hline$\sigma_{\mathrm{C}}$ & $\sigma_{\mathrm{E}}$ \\
psig & psig \\
0.08 & 0.06
\end{tabular}

Accepted Tolerance: +/-
T 2.07

Xbar

psig

50.83

$\sigma_{\mathrm{F}}$

psig

0.00

psig cal. date: 22 April 2001

$\begin{array}{ccc}\begin{array}{c}\text { Error } \\ \text { (mADC) }\end{array} & \begin{array}{c}\text { Error } \\ (\mathrm{psig})\end{array} & \\ -0.006 & -0.04 & \\ 0.003 & 0.02 & \\ 0.003 & 0.02 & \\ 0.003 & 0.02 & \\ 0.003 & 0.02 & \\ -0.006 & -0.04 & \\ -0.006 & -0.04 & \\ 0.003 & 0.02 & \\ 0.003 & 0.02 & \\ 0.003 & 0.02 & \\ 0.003 & 0.02 & \\ -0.006 & -0.04 & \\ -0.006 & -0.04 & \\ 0.003 & 0.02 & \\ 0.003 & 0.02 & \\ 0.003 & 0.02 & \\ 0.003 & 0.02 & \\ -0.006 & -0.04 & \\ -0.006 & -0.04 & \\ 0.003 & 0.02 & \\ 0.003 & 0.02 & \\ 0.003 & 0.02 & \\ 0.003 & 0.02 & \\ -0.006 & -0.04 & \\ & & \\ \% \text { RDG }+ & 0.001 & \mathrm{mADC}) \\ \% \mathrm{RDG}+ & 0.03 & \mathrm{psig}) \\ & & \\ \text { Sxx } & \text { SEE } & \text { MSE } \\ \text { psig } & \mathrm{mADC} & \mathrm{mADC} \\ 28403.33 & 0.0005 & 0.0000 \\ & & \end{array}$

Total Uncertainty

$\sigma_{\mathrm{T}}$

psig

0.10

\section{PASS CALIBRATION? YES}

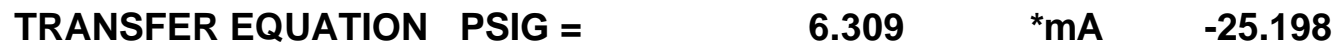

Figure H8. Pre-test Calibration Data of Gauge Pressure Transducer P1 
WSRC-TR-2003-00204, REV. 0

SRT-RPP-2003-00087, REV. 0

UNCERTAINTY ANALYSIS

REF. WSRC-TR-91-106, REV. 0

\begin{tabular}{ccc}
\multicolumn{3}{l}{ Calibration Data } \\
$\begin{array}{c}\text { Nominal } \\
\text { Pressure } \\
\text { (psig) }\end{array}$ & $\begin{array}{c}\text { Applied } \\
\text { Pressure } \\
\text { (psig) }\end{array}$ & $\begin{array}{c}\text { Gage } \\
\text { Reading } \\
\text { (mADC) }\end{array}$ \\
0 & 0.00 & 4.00 \\
31 & 31.00 & 7.30 \\
61 & 61.00 & 10.50 \\
91 & 91.00 & 13.68 \\
121 & 121.00 & 16.85 \\
151 & 151.00 & 20.00 \\
0 & 0.00 & 4.00 \\
31 & 31.00 & 7.30 \\
61 & 61.00 & 10.50 \\
91 & 91.00 & 13.68 \\
121 & 121.00 & 16.85 \\
151 & 151.00 & 20.00 \\
0 & 0.00 & 4.00 \\
31 & 31.00 & 7.30 \\
61 & 61.00 & 10.50 \\
91 & 91.00 & 13.68 \\
121 & 121.00 & 16.85 \\
151 & 151.00 & 20.00 \\
0 & 0.00 & 4.00 \\
31 & 31.00 & 7.31 \\
61 & 61.00 & 10.50 \\
91 & 91.00 & 13.68 \\
121 & 121.00 & 16.85 \\
151 & 151.00 & 20.00
\end{tabular}

Standard Uncertainties:
TR-03115

cal. date: 27 August 2002

\begin{tabular}{|c|c|c|c|}
\hline \multicolumn{4}{|l|}{ Curve } \\
\hline Fit & Error & Error & \\
\hline$(\mathrm{mADC})$ & $(\mathrm{mADC})$ & (psig) & \\
\hline 4.02 & 0.018 & 0.17 & \\
\hline 7.30 & 0.003 & 0.03 & \\
\hline 10.48 & -0.017 & -0.16 & \\
\hline 13.66 & -0.017 & -0.16 & \\
\hline 16.84 & -0.007 & -0.07 & \\
\hline 20.02 & 0.023 & 0.21 & \\
\hline 4.02 & 0.018 & 0.17 & \\
\hline 7.30 & 0.003 & 0.03 & \\
\hline 10.48 & -0.017 & -0.16 & \\
\hline 13.66 & -0.017 & -0.16 & \\
\hline 16.84 & -0.007 & -0.07 & \\
\hline 20.02 & 0.023 & 0.21 & \\
\hline 4.02 & 0.018 & 0.17 & \\
\hline 7.30 & 0.003 & 0.03 & \\
\hline 10.48 & -0.017 & -0.16 & \\
\hline 13.66 & -0.017 & -0.16 & \\
\hline 16.84 & -0.007 & -0.07 & \\
\hline 20.02 & 0.023 & 0.21 & \\
\hline 4.02 & 0.018 & 0.17 & \\
\hline 7.30 & -0.007 & -0.06 & \\
\hline 10.48 & -0.017 & -0.16 & \\
\hline 13.66 & -0.017 & -0.16 & \\
\hline 16.84 & -0.007 & -0.07 & \\
\hline 20.02 & 0.023 & 0.21 & \\
\hline 0.04 & $\%$ RDG + & 0.001 & mADC) \\
\hline 0.03 & $\%$ RDG + & 0.03 & psig) \\
\hline $\begin{array}{l}\text { Xbar } \\
\text { psig }\end{array}$ & $\begin{array}{l}\text { Sxx } \\
\text { psig }^{2}\end{array}$ & $\begin{array}{c}\text { SEE } \\
\mathrm{mADC}^{2}\end{array}$ & $\begin{array}{c}\text { MSE } \\
\text { mADC }^{2}\end{array}$ \\
\hline 75.83 & 63603.33 & 0.0059 & 0.0003 \\
\hline
\end{tabular}

Calculated Uncertainties:

$\sigma_{\mathrm{C}}$

psig

0.11
Multimeter: $+/-($
Dead Weight Tester: $+/-($

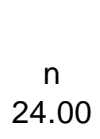

$\begin{array}{cc} & \text { Xbar } \\ \mathrm{T} & \text { psig } \\ 2.07 & 75.83\end{array}$
63603.33

Total Uncertainty

$\sigma_{\mathrm{F}}$

psig

0.00

psig

$\sigma_{\mathrm{T}}$
psig
0.36

Accepted Tolerance: +/-

1.5

psig

\section{PASS CALIBRATION? YES}

Figure H9. Pre-test Calibration Data of Gauge Pressure Transducer P2 
WSRC-TR-2003-00204, REV. 0

SRT-RPP-2003-00087, REV. 0

UNCERTAINTY ANALYSIS

REF. WSRC-TR-91-106, REV. 0

Calibration Data

$\begin{array}{ccc}\begin{array}{c}\text { Nominal } \\ \text { Pressure } \\ \text { (psig) }\end{array} & \begin{array}{c}\text { Applied } \\ \text { Pressure } \\ \text { (psig) }\end{array} & \begin{array}{c}\text { Gage } \\ \text { Reading } \\ \text { (mADC) }\end{array} \\ 0.00 & 0.00 & 4.00 \\ 31.00 & 31.00 & 7.28 \\ 61.00 & 61.00 & 10.46 \\ 91.00 & 91.00 & 13.63 \\ 121.00 & 121.00 & 16.82 \\ 151.00 & 151.00 & 20.00 \\ 0.00 & 0.00 & 4.00 \\ 31.00 & 31.00 & 7.28 \\ 61.00 & 61.00 & 10.46 \\ 91.00 & 91.00 & 13.63 \\ 121.00 & 121.00 & 16.82 \\ 151.00 & 151.00 & 20.00 \\ 0.00 & 0.00 & 4.00 \\ 31.00 & 31.00 & 7.28 \\ 61.00 & 61.00 & 10.46 \\ 91.00 & 91.00 & 13.63 \\ 121.00 & 121.00 & 16.82 \\ 151.00 & 151.00 & 20.00 \\ 0.00 & 0.00 & 4.00 \\ 31.00 & 31.00 & 7.28 \\ 61.00 & 61.00 & 10.46 \\ 91.00 & 91.00 & 13.63 \\ 121.00 & 121.00 & 16.82 \\ 151.00 & 151.00 & 20.00\end{array}$

Standard Uncertainties:

Dead Weight Tester: +/-(

Statistical Info:

$\begin{array}{ccccc}a & b & n & T & \text { psig } \\ 3.9962 & 0.1060 & 24.00 & 2.07 & 75.83\end{array}$

Calculated Uncertainties:

$\sigma_{\mathrm{C}}$

psig

0.11
$\sigma_{\mathrm{E}}$

psig

0.09
TR-00532

cal. date: 23 May 2002

$\begin{array}{ccc}\text { Fit } & \text { Error } & \text { Erro } \\ (\mathrm{mADC}) & (\mathrm{mADC}) & (\mathrm{psig})\end{array}$

$\begin{array}{lll}4.00 & -0.004 & -0.04\end{array}$

$\begin{array}{lll}7.28 & 0.001 & 0.01\end{array}$

$\begin{array}{lll}10.46 & 0.000 & 0.00\end{array}$

$\begin{array}{lll}13.64 & 0.009 & 0.08\end{array}$

$\begin{array}{lll}16.82 & -0.002 & -0.02\end{array}$

$\begin{array}{lll}20.00 & -0.004 & -0.03\end{array}$

$\begin{array}{lll}4.00 & -0.004 & -0.04\end{array}$

$\begin{array}{lll}7.28 & 0.001 & 0.01\end{array}$

$\begin{array}{lll}10.46 & 0.000 & 0.00\end{array}$

$\begin{array}{lll}13.64 & 0.009 & 0.08\end{array}$

$\begin{array}{lll}16.82 & -0.002 & -0.02\end{array}$

$20.00 \quad-0.004 \quad-0.03$

$\begin{array}{lll}4.00 & -0.004 & -0.04\end{array}$

$\begin{array}{lll}7.28 & 0.001 & 0.01\end{array}$

$\begin{array}{lll}10.46 & 0.000 & 0.00\end{array}$

$\begin{array}{lll}13.64 & 0.009 & 0.08\end{array}$

$\begin{array}{lll}16.82 & -0.002 & -0.02\end{array}$

$20.00 \quad-0.004 \quad-0.03$

$\begin{array}{lll}4.00 & -0.004 & -0.04\end{array}$

$\begin{array}{lll}7.28 & 0.001 & 0.01\end{array}$

$\begin{array}{lll}10.46 & 0.000 & 0.00\end{array}$

$\begin{array}{lll}13.64 & 0.009 & 0.08\end{array}$

$\begin{array}{lll}16.82 & -0.002 & -0.02\end{array}$

$\begin{array}{lll}20.00 & -0.004 & -0.03\end{array}$

$0.04 \% R D G+\quad 0.001 \quad m A D C)$

$0.03 \% \mathrm{RDG}+\quad 0.03 \quad$ psig)

$\begin{array}{llll}\text { Xbar } & \text { Sxx } & \text { SEE } & \text { MSE }\end{array}$

psig psig $^{2} \quad \mathrm{mADC}^{2} \quad \mathrm{mADC}^{2}$

$\begin{array}{lll}63603.33 & 0.0004 & 0.0000\end{array}$

Total Uncertainty

$\sigma_{\mathrm{F}}$

psig

0.00

$\sigma_{\mathrm{T}}$

psig

0.15

Accepted Tolerance: +/-

1.5

psig

PASS CALIBRATION? YES

TRANSFER EQUATION PSIG =

$9.437 \quad$ *mA

$-37.714$

Figure H10. Pre-test Calibration Data of Gauge Pressure Transducer P3 
WSRC-TR-2003-00204, REV. 0

SRT-RPP-2003-00087, REV. o

UNCERTAINTY ANALYSIS

REF. WSRC-TR-91-106, REV. 0

Calibration Data

$\begin{array}{cc}\begin{array}{c}\text { Nominal } \\ \text { Pressure } \\ \text { (psid) }\end{array} & \begin{array}{c}\text { Applied } \\ \text { Pressure } \\ \text { (psid) }\end{array} \\ 0.00 & 0.00 \\ 6.00 & 6.00 \\ 11.00 & 11.00 \\ 16.00 & 16.00 \\ 21.00 & 21.00 \\ 26.00 & 26.00 \\ 0.00 & 0.00 \\ 6.00 & 6.00 \\ 11.00 & 11.00 \\ 16.00 & 16.00 \\ 21.00 & 21.00 \\ 26.00 & 26.00 \\ 0.00 & 0.00 \\ 6.00 & 6.00 \\ 11.00 & 11.00 \\ 16.00 & 16.00 \\ 21.00 & 21.00 \\ 26.00 & 26.00 \\ 0.00 & 0.00 \\ 6.00 & 6.00 \\ 11.00 & 11.00 \\ 16.00 & 16.00 \\ 21.00 & 21.00 \\ 26.00 & 26.00 \\ & \end{array}$

Standard Uncertainties:

Statistical Info:

$\begin{array}{ccc}a & b & n \\ 4.0101 & 0.6157 & 24.00 \\ & & \\ & \\ \text { Calculated } & \text { Uncertainties: } & \\ \sigma_{C} & & \sigma_{E} \\ \text { psid } & & \text { psid } \\ 0.041 & & 0.017\end{array}$

TR-03495

cal. date: 22 April 2001

Gage
Reading
(mADC)
4.02
7.70
10.78
13.86
16.94
20.02
4.02
7.70
10.78
13.86
16.94
20.02
4.02
7.70
10.78
13.86
16.94
20.02
4.00
7.70
10.78
13.86
16.94
20.02

Curve

$\begin{array}{ccc}\begin{array}{c}\text { Fit } \\ (\mathrm{mADC})\end{array} & \begin{array}{c}\text { Error } \\ \text { (mADC) }\end{array} & \begin{array}{c}\text { Error } \\ \text { (psid) }\end{array} \\ 4.01 & -0.010 & -0.02 \\ 7.70 & 0.004 & 0.01\end{array}$

10.78

13.86

16.94

20.02

4.01

7.70

10.78

13.86

16.94

20.02

4.01

7.70

10.78

13.86

16.94

20.02

4.01

7.70

10.78

13.86

16.94

20.02

Multimeter: $+/-\left(\begin{array}{ll}0.04 \\ 0\end{array}\right.$ Dead Weight Tester: +/-(

0.03

Xbar

psid

13.33

2.07
$\sigma_{\mathrm{F}}$
psid
0.000

0.500 psid

$0.003 \quad 0.00$

$0.001 \quad 0.00$

$\begin{array}{ll}-0.001 & 0.00\end{array}$

$\begin{array}{ll}-0.002 & 0.00\end{array}$

$\begin{array}{ll}-0.010 & -0.02\end{array}$

$0.004 \quad 0.01$

$0.003 \quad 0.00$

$0.001 \quad 0.00$

$\begin{array}{ll}-0.001 & 0.00\end{array}$

$\begin{array}{ll}-0.002 & 0.00\end{array}$

$\begin{array}{ll}-0.010 & -0.02\end{array}$

$0.004 \quad 0.01$

$0.003 \quad 0.00$

$0.001 \quad 0.00$

$\begin{array}{ll}-0.001 & 0.00\end{array}$

$\begin{array}{ll}-0.002 & 0.00\end{array}$

$0.010 \quad 0.02$
0.004

$0.004 \quad 0.01$

$0.003 \quad 0.00$

$0.001 \quad 0.00$

$-0.001 \quad 0.00$

$\begin{array}{ll}-0.002 & 0.00\end{array}$

$\% R D G+\quad 0.001 \quad m A D C)$

$\% R D G+\quad 0.03 \quad$ psi)

$\begin{array}{ccc}\text { Sxx } & \text { SEE } & \text { MSE } \\ \text { psid } & \text { mADC }^{<} & \text {mADC }^{<} \\ 1853.33 & 0.0005 & 0.0000\end{array}$

Total Uncertainty<smiles>[87BrH]</smiles>

psid

0.044

Accepted Tolerance: +/-

PASS CALIBRATION?

YES

TRANSFER EQUATION: $\quad$ PSID $=\quad 1.6242 \quad{ }^{*} \mathrm{~mA} \quad-6.5132$

Figure H11. Pre-test Calibration Data of Differential Pressure Transducer dP1 
WSRC-TR-2003-00204, REV. 0

SRT-RPP-2003-00087, REV. 0

UNCERTAINTY ANALYSIS

REF. WSRC-TR-91-106, REV. 0

Calibration Data

$\begin{array}{ccc}\begin{array}{c}\text { Nominal } \\ \text { Pressure } \\ \text { (psid) }\end{array} & \begin{array}{c}\text { Applied } \\ \text { Pressure } \\ \text { (psid) }\end{array} & \begin{array}{c}\text { Gage } \\ \text { Reading } \\ \text { (mADC) }\end{array} \\ 0.00 & 0.00 & 4.00 \\ 21.00 & 21.00 & 7.32 \\ 41.00 & 41.00 & 10.50 \\ 61.00 & 61.00 & 13.68 \\ 81.00 & 81.00 & 16.85 \\ 101.00 & 101.00 & 20.02 \\ 0.00 & 0.00 & 4.00 \\ 21.00 & 21.00 & 7.32 \\ 41.00 & 41.00 & 10.50 \\ 61.00 & 61.00 & 13.68 \\ 81.00 & 81.00 & 16.85 \\ 101.00 & 101.00 & 20.02 \\ 0.00 & 0.00 & 4.00 \\ 21.00 & 21.00 & 7.32 \\ 41.00 & 41.00 & 10.50 \\ 61.00 & 61.00 & 13.68 \\ 81.00 & 81.00 & 16.85 \\ 101.00 & 101.00 & 20.02 \\ 0.00 & 0.00 & 4.00 \\ 21.00 & 21.00 & 7.32 \\ 41.00 & 41.00 & 10.50 \\ 61.00 & 61.00 & 13.68 \\ 81.00 & 81.00 & 16.85 \\ 101.00 & 101.00 & 20.02\end{array}$

Standard Uncertainties:
TR-03553

cal. date: 22 May 2002

$\begin{array}{ccc}\begin{array}{c}\text { Curve } \\ \text { Fit } \\ \text { (mADC) }\end{array} & \begin{array}{c}\text { Error } \\ \text { (mADC) }\end{array} & \begin{array}{c}\text { Error } \\ \text { (psid) }\end{array} \\ 4.00 & -0.005 & -0.03 \\ 7.33 & 0.008 & 0.05 \\ 10.50 & 0.001 & 0.01 \\ 13.67 & -0.005 & -0.03 \\ 16.85 & -0.001 & -0.01 \\ 20.02 & 0.002 & 0.01 \\ 4.00 & -0.005 & -0.03 \\ 7.33 & 0.008 & 0.05 \\ 10.50 & 0.001 & 0.01 \\ 13.67 & -0.005 & -0.03 \\ 16.85 & -0.001 & -0.01 \\ 20.02 & 0.002 & 0.01 \\ 4.00 & -0.005 & -0.03 \\ 7.33 & 0.008 & 0.05 \\ 10.50 & 0.001 & 0.01 \\ 13.67 & -0.005 & -0.03 \\ 16.85 & -0.001 & -0.01 \\ 20.02 & 0.002 & 0.01 \\ 4.00 & -0.005 & -0.03 \\ 7.33 & 0.008 & 0.05 \\ 10.50 & 0.001 & 0.01 \\ 13.67 & -0.005 & -0.03 \\ 16.85 & -0.001 & -0.01 \\ 20.02 & 0.002 & 0.01\end{array}$

$\begin{array}{rrrrr}\text { Multimeter: }+/-( & 0.04 & \% R D G+ & 0.001 & \text { mADC }) \\ \text { Dead Weight Tester: }+/-( & 0.03 & \% R D G+ & 0.03 & \text { psi })\end{array}$

Statistical Info:

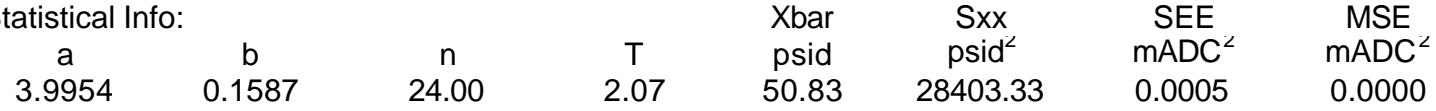

Calculated Uncertainties:

$\begin{array}{cc}\sigma_{C} & \sigma_{E} \\ \text { psid } & \text { psid } \\ 0.083 & 0.064\end{array}$

$\begin{array}{cc} & \text { Total Uncertainty } \\ \sigma_{\mathrm{F}} & \sigma_{\mathrm{T}} \\ \text { psid } & \text { psid } \\ 0.000 & 0.104\end{array}$

Accepted Tolerance: +/- $\quad 1.000$ psid

PASS CALIBRATION? YES

TRANSFER EQUATION: $\quad$ PSID $=\quad 6.3020 \quad{ }^{*} \mathrm{~mA} \quad-25.1790$

Figure H12. Pre-test Calibration Data of Differential Pressure Transducer dP2 
WSRC-TR-2003-00204, REV. 0

SRT-RPP-2003-00087, REV. 0

UNCERTAINTY ANALYSIS

REF. WSRC-TR-91-106, REV. 0
TR-03109

cal. date: 22 April 2001

Calibration Data

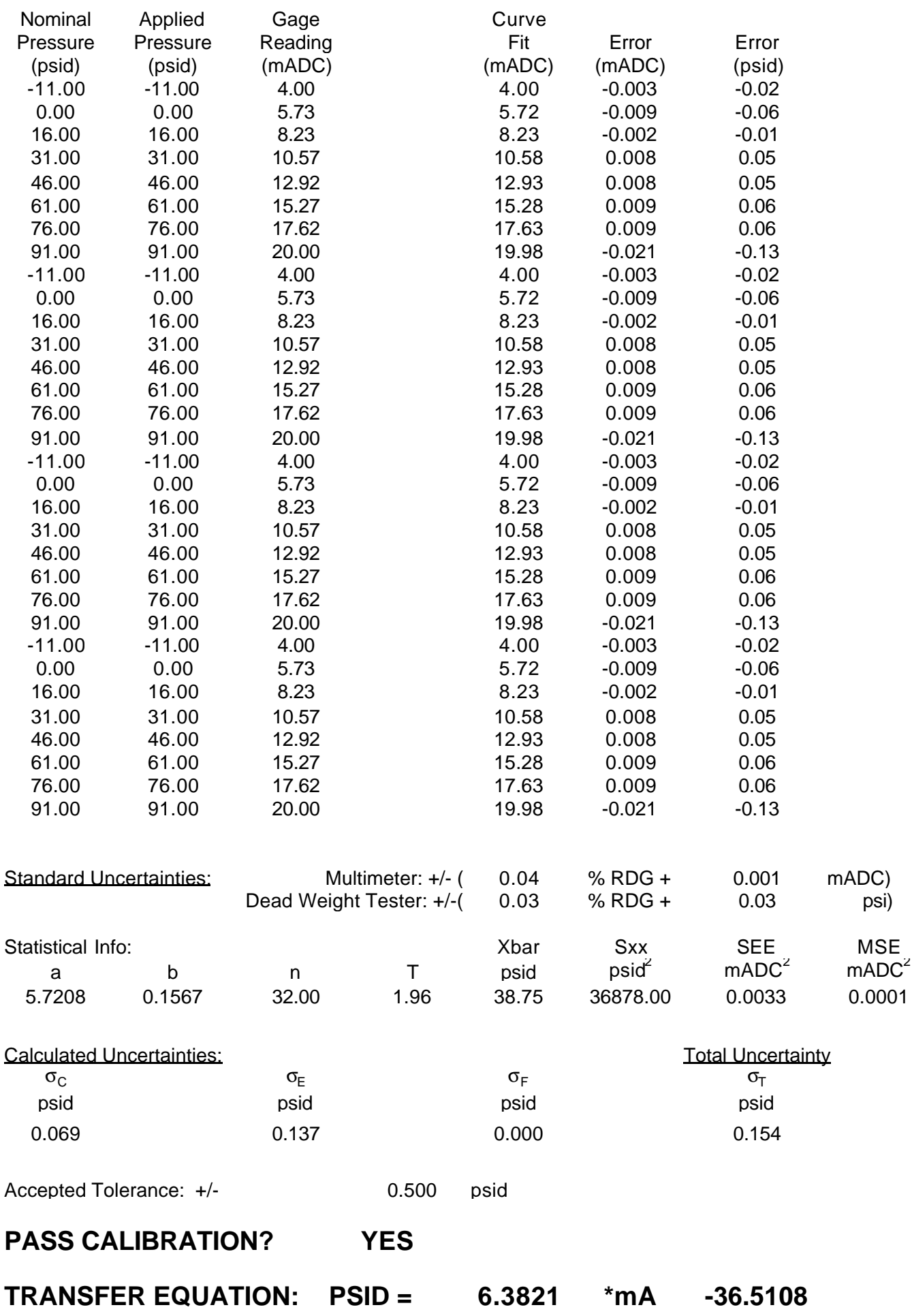

Figure H13. Pre-test Calibration Data of Differential Pressure Transducer dP3 
WSRC-TR-2003-00204, REV. 0

SRT-RPP-2003-00087, REV. 0

UNCERTAINTY ANALYSIS

REF. WSRC-TR-91-106

Calibration Data

$\begin{array}{llll}\begin{array}{l}\text { Meter } \\ \text { Output } \\ \text { (mA) }\end{array} & \begin{array}{l}\text { Water } \\ \text { Temp } \\ \text { (C) }\end{array} & \begin{array}{l}\text { Water } \\ \text { Weight } \\ \text { (lbs) }\end{array} & \begin{array}{l}\text { Time } \\ \text { Duration } \\ \text { (min) }\end{array} \\ 7.27 & 26.20 & 0.00 & 0.001 \\ 10.43 & 27.06 & 1020 & 6.01 \\ 13.63 & 27.53 & 1336 & 4.00 \\ 16.86 & 26.07 & 1501 & 3.01 \\ 19.98 & 26.50 & 2006 & 3.01 \\ 7.20 & 26.90 & 1005 & 3.01 \\ 10.49 & 26.64 & 1345 & 4.01 \\ 13.65 & 26.11 & 1505 & 3.00 \\ 16.88 & 25.90 & 2010 & 3.00 \\ 20.05 & 25.48 & 2507 & 3.00 \\ 7.23 & 24.91 & 1010 & 6.01 \\ 10.45 & 23.17 & 1340 & 4.00 \\ 13.70 & 25.21 & 1512 & 3.00 \\ 16.80 & 25.76 & 1997 & 3.00 \\ 20.06 & 28.33 & 2508 & 3.00\end{array}$

TR-20350

calibration date: 29 April 2001

Water Density: $\rho=62.441-1.374 \mathrm{E}-3^{\star} \mathrm{T}-271.818 \mathrm{E}-6^{\star} \mathrm{T}^{\wedge} 2+194.093 \mathrm{E}-9^{\star} \mathrm{T}^{\wedge} 3$

7.4805 gallons $=1 \mathrm{ft} \wedge 3$

Uncertainty of the Standards:

Weight:+/- 7.0 Temperature: $+/-\quad 0.40$

Density: $+/-\quad 0.06$

Time: + /- $(0.20$

Multimeter: $+/-(\quad 0.04$

0.5 GPM

Accepted Tolerance: +/-

Statistical Info.

$\begin{array}{ccccc}a & b & n & T & \begin{array}{c}\text { Xbar } \\ \text { (GPM) }\end{array}\end{array}$

4.003

0.160

16.00

2.145

56.48

standard

uncertainty

(GPM)

0.336

YES

PASS CALIBRATION? TRANSFER EQUATION:

\begin{tabular}{|c|c|}
\hline $\begin{array}{l}\text { Mass } \\
\text { Flow } \\
\text { (lbs/mi }\end{array}$ & $\begin{array}{l}\text { Water } \\
\text { Density } \\
\left(\mathrm{lb} / \mathrm{ft}^{\wedge} 3\right.\end{array}$ \\
\hline 0.00 & $62.2 r$ \\
\hline 169.72 & 62.2 \\
\hline 34.00 & 62.20 \\
\hline 98.67 & 62.20 \\
\hline 66.45 & 62.2 \\
\hline 28.24 & 62.2 \\
\hline 167.22 & 62.21 \\
\hline 336.25 & 62.2 \\
\hline 501.67 & 62.22 \\
\hline 670.00 & 62.23 \\
\hline 835.67 & 62.2 \\
\hline 168.05 & 62.2 \\
\hline 35.00 & 62.2 \\
\hline 4.00 & 62.2 \\
\hline & \\
\hline 6.00 & \\
\hline
\end{tabular}

Calculated Data

$\begin{array}{lllcc}\begin{array}{l}\text { Volume } \\ \text { Flow } \\ (\mathrm{ft} \wedge \text { (min) }\end{array} & \begin{array}{l}\text { Volume } \\ \text { (GPM) }\end{array} & \begin{array}{l}\text { Curve Fit } \\ \text { Ouput } \\ (\mathrm{mA})\end{array} & \begin{array}{c}\text { Error } \\ (\mathrm{mA})\end{array} & \begin{array}{c}\text { Error } \\ (\mathrm{GPM})\end{array} \\ 0.00 & 0.00 & 4.00 & 0.00 & 0.030 \\ 2.73 & 20.41 & 7.27 & 0.00 & -0.005 \\ 5.37 & 40.17 & 10.43 & 0.00 & 0.010 \\ 8.02 & 59.97 & 13.60 & -0.03 & -0.181 \\ 10.71 & 80.12 & 16.83 & -0.03 & -0.214 \\ 13.31 & 99.58 & 19.94 & -0.04 & -0.248 \\ 2.69 & 20.11 & 7.22 & 0.02 & 0.131 \\ 5.40 & 40.43 & 10.47 & -0.02 & -0.103 \\ 8.06 & 60.31 & 13.66 & 0.01 & 0.033 \\ 10.77 & 80.54 & 16.89 & 0.01 & 0.085 \\ 13.43 & 100.45 & 20.08 & 0.03 & 0.183 \\ 2.70 & 20.20 & 7.24 & 0.01 & 0.034 \\ 5.38 & 40.25 & 10.44 & -0.01 & -0.036 \\ 8.10 & 60.58 & 13.70 & 0.00 & -0.012 \\ 10.70 & 80.02 & 16.81 & 0.01 & 0.061 \\ 13.44 & 100.56 & 20.10 & 0.04 & 0.232\end{array}$

Ibs

$\mathrm{lbm} / \mathrm{ft}^{\wedge} 3$

$\mathrm{sec}+\quad 500.00 \mu \mathrm{sec} / \mathrm{sec})$

$\% R D G+0.001 \mathrm{mADC}$

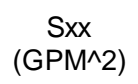

15387.47

curve-fit

uncertainty

(GPM)

0.336
GPM

$-0.005$

0.010

$-0.214$

248

$-0.103$

0.183

$-0.036$

0.061

0.232

Figure H14. Pre-test Calibration Data of Magnetic Flowmeter Q1 
WSRC-TR-2003-00204, REV. 0

SRT-RPP-2003-00087, REV. 0

UNCERTAINTY ANALYSIS

REF. WSRC-TR-91-106

\section{Calibration Data}

$\begin{array}{llll}\begin{array}{l}\text { Meter } \\ \text { Output } \\ (\mathrm{mA})\end{array} & \begin{array}{l}\text { Water } \\ \text { Temp }\end{array} & \begin{array}{l}\text { Water } \\ \text { Weight } \\ \text { (C) }\end{array} & \begin{array}{l}\text { Time } \\ \text { Duration } \\ \text { (min) }\end{array} \\ 7.00 & 21.64 & 0.00 & 0.001 \\ 10.48 & 20.92 & 10.12 & 5.00 \\ 13.65 & 21.24 & 12.09 & 3.00 \\ 16.78 & 21.38 & 17.97 & 3.00 \\ 20.05 & 21.57 & 19.76 & 2.50 \\ 7.17 & 21.39 & 19.86 & 2.00 \\ 10.43 & 21.77 & 12.11 & 3.01 \\ 13.61 & 21.42 & 17.93 & 3.01 \\ 16.79 & 21.77 & 19.83 & 2.50 \\ 20.03 & 21.93 & 19.86 & 2.00 \\ 7.17 & 21.81 & 9.96 & 5.01 \\ 10.44 & 22.01 & 12.07 & 3.01 \\ 13.65 & 22.14 & 17.98 & 3.00 \\ 16.86 & 22.02 & 19.93 & 2.50 \\ 19.96 & 21.61 & 19.77 & 2.00\end{array}$

TR-20353

page
calibration date: 29 April 2001

\section{Calculated Data}

$\begin{array}{ll}\text { Mass } & \text { Water } \\ \text { Flow } & \left.\begin{array}{c}\text { Density } \\ \text { (lbs/min }\left(\mathrm{lb} / \mathrm{ft}^{\wedge} 3\right.\end{array}\right) \\ 0.00 & 62.29 \\ 2.02 & 62.30 \\ 4.03 & 62.29 \\ 5.99 & 62.29 \\ 7.90 & 62.29 \\ 9.93 & 62.29 \\ 1.99 & 62.29 \\ 4.02 & 62.28 \\ 5.96 & 62.29 \\ 7.93 & 62.28 \\ 9.93 & 62.28 \\ 1.99 & 62.28 \\ 4.01 & 62.28 \\ 5.99 & 62.28 \\ 7.97 & 62.28 \\ 9.89 & 62.29\end{array}$

Volume Volume Curve Fit

Flow Flow Ouput Error Error

$\left(\mathrm{ft}^{\wedge} 3 / \mathrm{min}\right)(\mathrm{GPM}) \quad(\mathrm{mA}) \quad(\mathrm{mA}) \quad(\mathrm{GPM})$

$\begin{array}{lllll}0.00 & 0.00 & 3.96 & -0.04 & -0.003\end{array}$

$\begin{array}{lllll}0.03 & 0.24 & 7.23 & 0.00 & 0.000\end{array}$

$\begin{array}{lllll}0.06 & 0.48 & 10.48 & 0.00 & 0.000\end{array}$

$\begin{array}{lllll}0.10 & 0.72 & 13.66 & 0.01 & 0.000\end{array}$

$\begin{array}{lllll}0.13 & 0.95 & 16.75 & -0.03 & -0.002\end{array}$

$\begin{array}{lllll}0.16 & 1.19 & 20.03 & -0.02 & -0.001\end{array}$

$\begin{array}{lllll}0.03 & 0.24 & 7.17 & 0.00 & 0.000\end{array}$

$\begin{array}{lllll}0.06 & 0.48 & 10.47 & 0.04 & 0.003\end{array}$

$\begin{array}{lllll}0.10 & 0.72 & 13.60 & -0.01 & -0.001\end{array}$

$\begin{array}{lllll}0.13 & 0.95 & 16.80 & 0.01 & 0.001\end{array}$

$\begin{array}{lllll}0.16 & 1.19 & 20.03 & 0.00 & 0.000\end{array}$

$\begin{array}{lllll}0.03 & 0.24 & 7.18 & 0.01 & 0.001\end{array}$

$\begin{array}{lllll}0.06 & 0.48 & 10.45 & 0.01 & 0.001\end{array}$

$\begin{array}{lllll}0.10 & 0.72 & 13.66 & 0.01 & 0.001\end{array}$

$\begin{array}{lllll}0.13 & 0.96 & 16.87 & 0.01 & 0.000\end{array}$

$\begin{array}{lllll}0.16 & 1.19 & 19.96 & 0.00 & 0.000\end{array}$

Water Density: $\rho=62.441-1.374 \mathrm{E}-3^{*} \mathrm{~T}-271.818 \mathrm{E}-6^{\star} \mathrm{T}^{\wedge} 2+194.093 \mathrm{E}-9^{*} \mathrm{~T}^{\wedge} 3$

7.4805 gallons $=1 \mathrm{ft}^{\wedge} 3$

Uncertainty of the Standards:

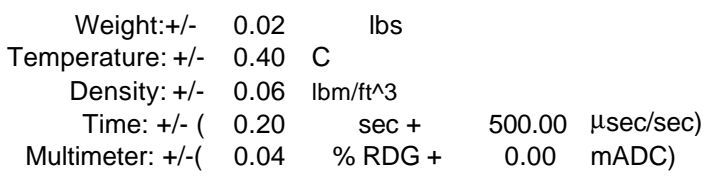

standard

uncertainty

(GPM)

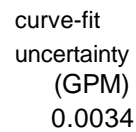

fixed

total

uncertainty

(GPM)

uncertainty

(GPM)

0.0031

0.0034

0.0000

0.0046

PASS CALIBRATION?

YES

\begin{tabular}{lllll}
\hline TRANSFER EQUATION: & GPM $=$ & 0.074 & $\mathrm{~mA}$ & -0.294 \\
\hline
\end{tabular}

Figure H15. Pre-test Calibration Data of Magnetic Flowmeter Q2 
WSRC-TR-2003-00204, REV. 0

SRT-RPP-2003-00087, REV. 0

UNCERTAINTY ANALYSIS

REF. WSRC-TR-91-106

\section{Calibration Data}

\begin{tabular}{|c|c|c|c|}
\hline Meter & Water & Water & Time \\
\hline $\begin{array}{l}\text { Output } \\
\text { (mA) }\end{array}$ & $\begin{array}{l}\text { Temp } \\
\text { (C) }\end{array}$ & $\begin{array}{l}\text { Weight } \\
\text { (lbs) }\end{array}$ & $\begin{array}{l}\text { Duration } \\
\text { (min) }\end{array}$ \\
\hline 4.00 & 21.65 & 0.00 & 0.001 \\
\hline 7.24 & 22.09 & 50.91 & 6.00 \\
\hline 10.37 & 22.13 & 66.34 & 4.00 \\
\hline 13.18 & 22.08 & 71.69 & 3.00 \\
\hline 16.77 & 22.14 & 99.58 & 3.01 \\
\hline 19.87 & 22.59 & 123.73 & 3.00 \\
\hline 7.30 & 21.19 & 51.43 & 6.00 \\
\hline 10.42 & 21.71 & 66.84 & 4.00 \\
\hline 13.75 & 21.65 & 76.15 & 3.00 \\
\hline 16.77 & 21.32 & 99.71 & 3.01 \\
\hline 19.97 & 20.89 & 124.55 & 3.00 \\
\hline 7.20 & 21.01 & 50.55 & 6.01 \\
\hline 10.40 & 20.61 & 66.80 & 4.00 \\
\hline 13.61 & 21.71 & 74.95 & 3.00 \\
\hline 16.82 & 21.03 & 100.01 & 3.00 \\
\hline 20.07 & 22.59 & 125.23 & 3.00 \\
\hline
\end{tabular}

TR-03562

page
calibration date: 07 May $\overline{2001}$

\section{Calculated Data}

\begin{tabular}{|c|c|c|c|c|c|c|}
\hline Mass & Water & Volume & Volume & Curve Fit & & \\
\hline $\begin{array}{l}\text { Flow } \\
\text { (lbs/min }\end{array}$ & $\begin{array}{r}\text { Density } \\
\left(\mathrm{lb} / \mathrm{ft}^{\wedge} 3\right)\end{array}$ & $\begin{array}{l}\text { Flow } \\
\left(\mathrm{ft}^{\wedge} 3 / \mathrm{min}\right)\end{array}$ & $\begin{array}{l}\text { Flow } \\
\text { (GPM) }\end{array}$ & $\begin{array}{l}\text { Ouput } \\
\text { (mA) }\end{array}$ & $\begin{array}{l}\text { Error } \\
(\mathrm{mA})\end{array}$ & $\begin{array}{l}\text { Error } \\
\text { (GPM) }\end{array}$ \\
\hline 0.00 & 62.29 & 0.00 & 0.00 & 3.98 & -0.02 & -0.006 \\
\hline 8.49 & 62.28 & 0.14 & 1.02 & 7.25 & 0.01 & 0.003 \\
\hline 16.59 & 62.28 & 0.27 & 1.99 & 10.37 & 0.00 & 0.001 \\
\hline 23.90 & 62.28 & 0.38 & 2.87 & 13.19 & 0.01 & 0.003 \\
\hline 33.08 & 62.28 & 0.53 & 3.97 & 16.73 & -0.04 & -0.012 \\
\hline 41.24 & 62.27 & 0.66 & 4.95 & 19.88 & 0.01 & 0.003 \\
\hline 8.57 & 62.29 & 0.14 & 1.03 & 7.28 & -0.02 & -0.005 \\
\hline 16.71 & 62.29 & 0.27 & 2.01 & 10.42 & 0.00 & 0.000 \\
\hline 25.38 & 62.29 & 0.41 & 3.05 & 13.76 & 0.01 & 0.004 \\
\hline 33.13 & 62.29 & 0.53 & 3.98 & 16.75 & -0.02 & -0.007 \\
\hline 41.52 & 62.30 & 0.67 & 4.99 & 19.98 & 0.01 & 0.003 \\
\hline 8.41 & 62.29 & 0.14 & 1.01 & 7.22 & 0.02 & 0.006 \\
\hline 16.70 & 62.30 & 0.27 & 2.01 & 10.41 & 0.01 & 0.005 \\
\hline 24.98 & 62.29 & 0.40 & 3.00 & 13.61 & 0.00 & 0.000 \\
\hline 33.34 & 62.29 & 0.54 & 4.00 & 16.83 & 0.01 & 0.002 \\
\hline 41.74 & 62.27 & 0.67 & 5.01 & 7 & 0 & 0.001 \\
\hline
\end{tabular}

Water Density: $\rho=62.441-1.374 \mathrm{E}-3^{\star} \mathrm{T}-271.818 \mathrm{E}-6^{\star} \mathrm{T}^{\wedge} 2+194.093 \mathrm{E}-9^{\star} \mathrm{T}^{\wedge} 3$

7.4805 gallons $=1 \mathrm{ft}^{\wedge} 3$

Uncertainty of the Standards:

Weight:+/- 0.2

Temperature: + /- 0.40

Density: $+/-0.06 \quad \mathrm{lbm} / \mathrm{ft}^{\wedge} 3$

Time: $+/-\left(\begin{array}{lll}0.20 & \mathrm{sec}+ & 500.00 \mu \mathrm{sec} / \mathrm{sec})\end{array}\right.$

Multimeter: $+/-\left(\begin{array}{lll}0.04 & \% \mathrm{RDG}+ & 0.001 \\ \mathrm{mADC}\end{array}\right)$

Accepted Tolerance: +/- $\quad 0.10 \quad$ GPM

Statistical Info. Xbar

$\begin{array}{ccccc}\mathrm{a} & \mathrm{b} & \mathrm{n} & \mathrm{T} & (\mathrm{GPM}) \\ 3.979 & 3.209 & 16.00 & 2.145 & 2.81\end{array}$

Sxx

(GPM^2)

37.90

SEE

$\left(\mathrm{mA}^{\wedge} 2\right)$

0.0039

MSE

$\left(\mathrm{mA}^{\wedge} 2\right)$

0.0003

Calculated Uncertainties:

standard

uncertainty

(GPM)

0.012
curve-fit
uncertainty
(GPM)
0.013

YES

PASS CALIBRATION?

TRANSFER EQUATION:

GPM =

fixed

uncertainty

(GPM)

0.000 total

uncertainty (GPM)

0.018

Figure H16. Pre-test Calibration Data of Magnetic Flowmeter Q3 
WSRC-TR-2003-00204, REV. 0

SRT-RPP-2003-00087, REV. 0

UNCERTAINTY ANALYSIS

REF. WSRC-TR-91-106

\section{Calibration Data}

\begin{tabular}{|c|c|c|c|}
\hline Meter & Water & Water & Time \\
\hline $\begin{array}{l}\text { Output } \\
\text { (mA) }\end{array}$ & $\begin{array}{l}\text { Temp } \\
\text { (C) }\end{array}$ & $\begin{array}{l}\text { Weight } \\
\text { (lbs) }\end{array}$ & $\begin{array}{l}\text { Duration } \\
\text { (min) }\end{array}$ \\
\hline 4.00 & 20.70 & 0.00 & 0.001 \\
\hline 7.27 & 18.90 & 84.35 & 10.005 \\
\hline 10.42 & 19.20 & 82.87 & 5.004 \\
\hline 13.66 & 20.60 & 99.50 & 4.003 \\
\hline 16.78 & 21.30 & 98.82 & 3.004 \\
\hline 20.04 & 21.30 & 123.96 & 3.002 \\
\hline 7.25 & 21.10 & 84.00 & 10.002 \\
\hline 10.40 & 21.10 & 82.66 & 5.001 \\
\hline 13.69 & 21.40 & 99.77 & 4.009 \\
\hline 16.80 & 21.50 & 98.99 & 3.002 \\
\hline 20.05 & 21.50 & 124.17 & 3.006 \\
\hline 7.26 & 20.80 & 84.11 & 10.001 \\
\hline 10.41 & 20.70 & 82.72 & 5.004 \\
\hline 13.65 & 19.80 & 99.49 & 4.005 \\
\hline 16.85 & 19.20 & 99.37 & 3.002 \\
\hline 20.05 & 22.10 & 124.13 & 3.004 \\
\hline
\end{tabular}

TR-03276

page
calibration date: 21
May 2002

\section{Calculated Data}

\begin{tabular}{|c|c|c|c|c|c|c|}
\hline Mass & Water & Volum & Volume & \multicolumn{3}{|l|}{ Curve Fit } \\
\hline $\begin{array}{l}\text { Flow } \\
\text { (lbs/m }\end{array}$ & $\begin{array}{l}\text { Density } \\
\left(\mathrm{lb} / \mathrm{ft}^{\wedge} 3\right)\end{array}$ & $\begin{array}{l}\text { Flow } \\
\left(f t^{\wedge} 3 / r\right.\end{array}$ & $\begin{array}{l}\text { Flow } \\
\text { (GPM) }\end{array}$ & $\begin{array}{l}\text { Ouput } \\
\text { (mA) }\end{array}$ & $\begin{array}{l}\text { Error } \\
(\mathrm{mA})\end{array}$ & $\begin{array}{l}\text { Error } \\
\text { (GPM }\end{array}$ \\
\hline 0.00 & 62.30 & 0.00 & 0.00 & 3.99 & -0.01 & -0.002 \\
\hline 8.43 & 62.32 & 0.14 & 1.01 & 7.27 & 0.00 & -0.001 \\
\hline 16.56 & 62.32 & 0.27 & 1.99 & 10.43 & 0.01 & 0.002 \\
\hline 24.86 & 62.30 & 0.40 & 2.98 & 13.65 & -0.01 & -0.003 \\
\hline 32.90 & 62.29 & 0.53 & 3.95 & 16.78 & 0.00 & 0.000 \\
\hline 41.29 & 62.29 & 0.66 & 4.96 & 20.04 & 0.00 & 0.000 \\
\hline 8.40 & 62.29 & 0.13 & 1.01 & 7.26 & 0.01 & 0.002 \\
\hline 16.53 & 62.29 & 0.27 & 1.98 & 10.42 & 0.02 & 0.005 \\
\hline 24.89 & 62.29 & 0.40 & 2.99 & 13.66 & -0.03 & -0.008 \\
\hline 32.98 & 62.29 & 0.53 & 3.96 & 16.81 & 0.01 & 0.003 \\
\hline 41.30 & 62.29 & 0.66 & 4.96 & 20.05 & 0.00 & -0.001 \\
\hline 8.41 & 62.30 & 0.13 & 1.01 & 7.26 & 0.00 & 0.000 \\
\hline 16.53 & 62.30 & 0.27 & 1.98 & 10.42 & 0.01 & 0.002 \\
\hline 24.84 & 62.31 & 0.40 & 2.98 & 13.64 & -0.01 & -0.002 \\
\hline 33.10 & 62.32 & 0.53 & 3.97 & 16.85 & 0.00 & 0.001 \\
\hline 41.33 & 62.28 & 0.66 & 4.96 & 20.06 & 0.01 & 0.002 \\
\hline
\end{tabular}

Water Density: $\rho=62.441-1.374 \mathrm{E}-3^{*} \mathrm{~T}-271.818 \mathrm{E}-6^{*} \mathrm{~T}^{\wedge} 2+194.093 \mathrm{E}-9^{*} \mathrm{~T}^{\wedge} 3$

7.4805 gallons $=1 \mathrm{ft}^{\wedge} 3$

Uncertainty of the Standards:

Weight:+/- 0.2 lbs

Temperature: $+/-\quad 0.20 \quad \mathrm{C}$

Density: $+/-0.06 \quad \mathrm{lbm} / \mathrm{ft}^{\wedge} 3$

Time: $+/-\left(\begin{array}{lll}0.20 \mathrm{sec}+ \\ \text { s }\end{array}\right.$

Multimeter: $+/-\left(\begin{array}{lll}0.04 & \% \mathrm{RDG}+ & 0.001 \mathrm{mADC}\end{array}\right.$

Accepted Tolerance: +/- $\quad 0.025$ GPM

\begin{tabular}{|c|c|c|c|c|c|c|c|}
\hline \multicolumn{4}{|c|}{ Statistical Info. } & $\begin{array}{l}\text { Xbar } \\
\text { (GPM) }\end{array}$ & $\begin{array}{c}\text { Sxx } \\
\left(\mathrm{GPM}^{\wedge} 2\right)\end{array}$ & $\begin{array}{c}\text { SEE } \\
\left(\mathrm{mA}^{\wedge} 2\right)\end{array}$ & $\begin{array}{c}\text { MSE } \\
\left(\mathrm{mA}^{\wedge} 2\right)\end{array}$ \\
\hline 3.992 & 3.236 & 15.00 & 2.160 & 2.79 & 37.60 & 0.0013 & 0.0001 \\
\hline Calculat & Uncert & ties: & & $\begin{array}{c}\text { standard } \\
\text { uncertainty } \\
\text { (GPM) } \\
0.012\end{array}$ & $\begin{array}{l}\text { curve-fit } \\
\text { uncertainty } \\
\text { (GPM) } \\
0.008\end{array}$ & $\begin{array}{l}\text { fixed } \\
\text { uncertainty } \\
\text { (GPM) } \\
0.000\end{array}$ & $\begin{array}{l}\text { total } \\
\text { uncertainty } \\
\text { (GPM) } \\
0.014\end{array}$ \\
\hline
\end{tabular}

PASS CALIBRATION?

YES

\begin{tabular}{lllll}
\hline TRANSFER EQUATION: & GPM $=$ & 0.309 & $\mathrm{~mA}$ & -1.233
\end{tabular}

Figure H17. Pre-test Calibration Data of Magnetic Flowmeter Q4 or Qbp 